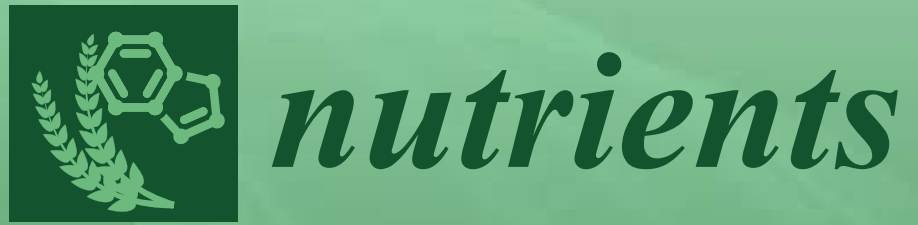

\title{
Breastfeeding and Human Lactation
}

Edited by Donna Geddes and Sharon Perrella Printed Edition of the Special Issue Published in Nutrients 


\section{Breastfeeding and Human Lactation}





\section{Breastfeeding and Human Lactation}

Special Issue Editors

Donna Geddes

Sharon Perrella 


\section{Donna Geddes}

The University of Western Australia

Australia
Sharon Perrella

The University of Western Australia

Australia

Editorial Office

MDPI

St. Alban-Anlage 66

4052 Basel, Switzerland

This is a reprint of articles from the Special Issue published online in the open access journal Nutrients (ISSN 2072-6643) from 2018 to 2019 (available at: https://www.mdpi.com/journal/nutrients / special_issues/Breastfeeding_Lactation)

For citation purposes, cite each article independently as indicated on the article page online and as indicated below:

LastName, A.A.; LastName, B.B.; LastName, C.C. Article Title. Journal Name Year, Article Number, Page Range.

ISBN 978-3-03897-930-2 (Pbk)

ISBN 978-3-03897-931-9 (PDF)

(C) 2019 by the authors. Articles in this book are Open Access and distributed under the Creative Commons Attribution (CC BY) license, which allows users to download, copy and build upon published articles, as long as the author and publisher are properly credited, which ensures maximum dissemination and a wider impact of our publications.

The book as a whole is distributed by MDPI under the terms and conditions of the Creative Commons license CC BY-NC-ND. 


\section{Contents}

About the Special Issue Editors $\ldots \ldots \ldots \ldots \ldots \ldots \ldots \ldots \ldots$

Breastfeeding and Human Lactation

Reprinted from: Nutrients 2019, 11, 802, doi:10.3390/nu11040802 . . . . . . . . . . . . . 1

Zoya Gridneva, Alethea Rea, Anna R. Hepworth, Leigh C. Ward, Ching T. Lai,

Peter E. Hartmann and Donna T. Geddes

Relationships between Breastfeeding Patterns and Maternal and Infant Body Composition over the First 12 Months of Lactation

Reprinted from: Nutrients 2018, 10, 45, doi:10.3390/nu10010045 . . . . . . . . . . . . .

Donna Geddes, Chooi Kok, Kathryn Nancarrow, Anna Hepworth and Karen Simmer

Preterm Infant Feeding: A Mechanistic Comparison between a Vacuum Triggered Novel Teat and Breastfeeding

Reprinted from: Nutrients 2018, 10,376, doi:10.3390/nu10030376 _ . . . . . . . . . . . . . 32

Alejandra M. Wiedeman, Kyly C. Whitfield, Kaitlin M. March, Nancy N. Chen, Hou Kroeun, Ly Sokhoing, Prak Sophonneary, Roger A. Dyer, Zhaoming Xu, David D. Kitts,

Timothy J. Green, Sheila M. Innis and Susan I. Barr

Concentrations of Water-Soluble Forms of Choline in Human Milk from Lactating Women in Canada and Cambodia

Reprinted from: Nutrients 2018, 10, 381, doi:10.3390/nu10030381 . . . . . . . . . . . . . 4

Elizabeth V. Asztalos

Supporting Mothers of Very Preterm Infants and Breast Milk Production: A Review of the Role of Galactogogues

Reprinted from: Nutrients 2018, 10, 600, doi:10.3390/nu10050600 _ . . . . . . . . . . . . . 57

Kirsty V. Biggs, Katherine Hurrell, Eleanor Matthews, Ekaterina Khaleva, Daniel Munblit and Robert J. Boyle

Formula Milk Supplementation on the Postnatal Ward: A Cross-Sectional Analytical Study

Reprinted from: Nutrients 2018, 10, 608, doi:10.3390/nu10050608 . . . . . . . . . . . . . . 67

Annemarie E. Bennett and John M. Kearney

Factors Associated with Maternal Wellbeing at Four Months Post-Partum in Ireland

Reprinted from: Nutrients 2018, 10, 609, doi:10.3390/nu10050609 . . . . . . . . . . . . . . 86

Veronique Demers-Mathieu, Mark A. Underwood, Robert L. Beverly, Søren D. Nielsen and David C. Dallas

Comparison of Human Milk Immunoglobulin Survival during Gastric Digestion between Preterm and Term Infants

Reprinted from: Nutrients 2018, 10, 631, doi:10.3390/nu10050631 . . . . . . . . . . . . . . 977

Carlos Zozaya, Alba García-Serrano, Javier Fontecha, Lidia Redondo-Bravo,

Victoria Sánchez-González, María Teresa Montes and Miguel Saenz de Pipaón

Fat Loss in Continuous Enteral Feeding of the Preterm Infant: How Much, What and When Is

It Lost?

Reprinted from: Nutrients 2018, 10, 809, doi:10.3390/nu10070809 _ . . . . . . . . . . . . . . 110 
Cynthia Barrera, Rodrigo Valenzuela, Rodrigo Chamorro, Karla Bascuñán, Jorge Sandoval, Natalia Sabag, Francesca Valenzuela, María-Paz Valencia, Claudia Puigrredon and Alfonso Valenzuela

The Impact of Maternal Diet during Pregnancy and Lactation on the Fatty Acid Composition of Erythrocytes and Breast Milk of Chilean Women

Reprinted from: Nutrients 2018, 10, 839, doi:10.3390/nu10070839 . . . . . . . . . . . . . 120

Camila Narváez-Caicedo, Gabriela Moreano, Bernardo A. Sandoval and Miguel Á. Jara-Palacios

Zinc Deficiency among Lactating Mothers from a Peri-Urban Community of the Ecuadorian Andean Region: An Initial Approach to the Need of Zinc Supplementation

Reprinted from: Nutrients 2018, 10, 869, doi:10.3390/nu10070869 . . . . . . . . . . . . . . 134

Pranati L. Panuganti, Stefanie N. Hinkle, Shristi Rawal, Louise G. Grunnet, Yuan Lin, Aiyi Liu, Anne C. B. Thuesen, Sylvia H. Ley, Sjurdur F. Olesen and Cuilin Zhang Lactation Duration and Long-Term Thyroid Function: A Study among Women with Gestational Diabetes

Reprinted from: Nutrients 2018, 10, 938, doi:10.3390/nu10070938 . . . . . . . . . . . . . . 143

Amal Nasser, Fadumo Omer, Fatima Al-Lenqawi, Rehab Al-awwa, Tamam Khan, Asmaa El-Heneidy, Rana kurdi and Ghadir Al-Jayyousi

Predictors of Continued Breastfeeding at One Year among Women Attending Primary

Healthcare Centers in Qatar: A Cross-Sectional Study

Reprinted from: Nutrients 2018, 10, 983, doi:10.3390/nu10080983 . . . . . . . . . . . . . . . 155

Anne-Aurelie Lopes, Valerie Champion and Delphine Mitanchez

Nutrition of Preterm Infants and Raw Breast Milk-Acquired Cytomegalovirus Infection:

French National Audit of Clinical Practices and Diagnostic Approach

Reprinted from: Nutrients 2018, 10, 1119, doi:10.3390/nu10081119 . . . . . . . . . . . . . . . 170

Zoya Gridneva, Sambavi Kugananthan, Alethea Rea, Ching Tat Lai, Leigh C. Ward, Kevin Murray, Peter E. Hartmann and Donna T. Geddes

Human Milk Adiponectin and Leptin and Infant Body Composition over the First 12 Months of Lactation

Reprinted from: Nutrients 2018, 10, 1125, doi:10.3390/nu10081125 . . . . . . . . . . . . . 183

Jacqueline C. Kent, Hazel Gardner, Ching-Tat Lai, Peter E. Hartmann, Kevin Murray, Alethea Rea and Donna T. Geddes

Hourly Breast Expression to Estimate the Rate of Synthesis of Milk and Fat

Reprinted from: Nutrients 2018, 10, 1144, doi:10.3390/nu10091144 . . . . . . . . . . . . 207

Melvin C. L. Gay, Petya T. Koleva, Carolyn M. Slupsky, Elloise du Toit, Merete Eggesbo,

Christine C. Johnson, Ganesa Wegienka, Naoki Shimojo, Dianne E. Campbell,

Susan L. Prescott, Daniel Munblit, Donna T. Geddes, Anita L. Kozyrskyj and

InVIVO LactoActive Study Investigators

Worldwide Variation in Human Milk Metabolome: Indicators of Breast Physiology and Maternal Lifestyle?

Reprinted from: Nutrients 2018, 10, 1151, doi:10.3390/nu10091151 _ . . . . . . . . . . . . . 216

Alexandra D. George, Melvin C. L. Gay, Robert D. Trengove and Donna T. Geddes

Human Milk Lipidomics: Current Techniques and Methodologies

Reprinted from: Nutrients 2018, 10, 1169, doi:10.3390/nu10091169 _ . . . . . . . . . . . . . 228 
Laura Galante, Amber M. Milan, Clare M. Reynolds, David Cameron-Smith, Mark H. Vickers and Shikha Pundir

Sex-Specific Human Milk Composition: The Role of Infant Sex in Determining Early Life Nutrition

Reprinted from: Nutrients 2018, 10, 1194, doi:10.3390/nu10091194 _ . . . . . . . . . . . . . 248

Daniel Munblit, Priya Abrol, Shreya Sheth, Li Yan Chow, Ekaterina Khaleva, Alan Asmanov, Silvana Lauriola, Ezio M. Padovani, Pasquale Comberiati, Attilio L. Boner, John O. Warner, Robert J. Boyle and Diego G. Peroni

Levels of Growth Factors and IgA in the Colostrum of Women from Burundi and Italy

Reprinted from: Nutrients 2018, 10, 1216, doi:10.3390/nu10091216 . . . . . . . . . . . . . . 259

Ching Tat Lai, Hazel Gardner and Donna Geddes

Comparison of Inductively Coupled Plasma Optical Emission Spectrometry with an Ion Selective Electrode to Determine Sodium and Potassium Levels in Human Milk

Reprinted from: Nutrients 2018, 10, 1218, doi:10.3390/nu10091218 . . . . . . . . . . . . . 272

\section{Sucheta Telang}

Lactoferrin: A Critical Player in Neonatal Host Defense

Reprinted from: Nutrients 2018, 10, 1228, doi:10.3390/nu10091228 . . . . . . . . . . . . . . 281

Anna Gustafsson, Elisabeth Granström, Christina Stecksén-Blicks, Christina E. West and Sven-Arne Silfverdal

The Antisecretory Factor in Plasma and Breast Milk in Breastfeeding Mothers-A Prospective Cohort Study in Sweden

Reprinted from: Nutrients 2018, 10, 1227, doi:10.3390/nu10091227 . . . . . . . . . . . . . 297

Alecia-Jane Twigger, Gwendoline K. Küffer, Donna T. Geddes and Luis Filgueria

Expression of Granulisyn, Perforin and Granzymes in Human Milk over Lactation and in the Case of Maternal Infection

Reprinted from: Nutrients 2018, 10, 1230, doi:10.3390/nu10091230 . . . . . . . . . . . . . 308

Christine A. Butts, Duncan I. Hedderley, Thanuja D. Herath, Gunaranjan Paturi, Sarah Glyn-Jones, Frank Wiens, Bernd Stahl and Pramod Gopal

Human Milk Composition and Dietary Intakes of Breastfeeding Women of Different Ethnicity from the Manawatu-Wanganui Region of New Zealand

Reprinted from: Nutrients 2018, 10, 1231, doi:10.3390/nu10091231 . . . . . . . . . . . . . 323

Emily E. Little, Cristine H. Legare and Leslie J. Carver

Mother-Infant Physical Contact Predicts Responsive Feeding among U.S.

Breastfeeding Mothers

Reprinted from: Nutrients 2018, 10, 1251, doi:10.3390/nu10091251 . . . . . . . . . . . . . 339

Adriana V. Gaitán, JodiAnne T. Wood, Fan Zhang, Alexandros Makriyannis and Carol J. Lammi-Keefe

Endocannabinoid Metabolome Characterization of Transitional and Mature Human Milk

Reprinted from: Nutrients 2018, 10, 1294, doi:10.3390/nu10091294 . . . . . . . . . . . . . 355

Zoya Gridneva, Wan J. Tie, Alethea Rea, Ching Tat Lai, Leigh C. Ward, Kevin Murray,

Peter E. Hartmann and Donna T. Geddes

Human Milk Casein and Whey Protein and Infant Body Composition over the First 12 Months

of Lactation

Reprinted from: Nutrients 2018, 10, 1332, doi:10.3390/nu10091332 . . . . . . . . . . . . . 363 
Carlos Gómez-Gallego, Jose Manuel Morales, Daniel Monleón, Elloise du Toit, Himanshu Kumar, Kaisa M. Linderborg, Yumei Zhang, Baoru Yang, Erika Isolauri, Seppo Salminen and Maria Carmen Collado

Human Breast Milk NMR Metabolomic Profile across Specific Geographical Locations and Its Association with the Milk Microbiota

Reprinted from: Nutrients 2018, 10, 1355, doi:10.3390/nu10101355 . . . . . . . . . . . . . . 389

Agnieszka Bzikowska-Jura, Aneta Czerwonogrodzka-Senczyna, Gabriela Olędzka,

Dorota Szostak-Węgierek, Halina Weker and Aleksandra Wesołowska

Maternal Nutrition and Body Composition During Breastfeeding: Association with Human Milk Composition

Reprinted from: Nutrients 2018, 10, 1379, doi:10.3390/nu10101379 . . . . . . . . . . . . . . . . 409

Signe Bruun, Sandra Gouveia-Figueira, Magnus Domellöf, Steffen Husby,

Lotte Neergaard Jacobsen, Kim F. Michaelsen, Christopher J. Fowler and Gitte Zachariassen

Satiety Factors Oleoylethanolamide, Stearoylethanolamide, and Palmitoylethanolamide in Mother's Milk Are Strongly Associated with Infant Weight at Four Months of Age—Data from the Odense Child Cohort

Reprinted from: Nutrients 2018, 10, 1747, doi:10.3390/nu10111747 . . . . . . . . . . . . . . 424 


\section{About the Special Issue Editors}

Donna Geddes is currently working at the University of Western Australia. Dr Geddes's research focuses on milk synthesis, milk removal and milk composition in breastfeeding women. She is also interested in the maternal influence on milk composition and subsequent impact on the growth and development of the breastfed infant. Her research group has shown the dose of a component in human milk, rather than the concentration of a component measured in one sample, is related to the differential development of infant body composition in the first year of life. In addition, her research encompasses the effect of pregnancy complications on both milk production and milk composition with an emphasis on infant outcomes.

Sharon Perrella is currently working at the University of Western Australia. Dr Perrella's research focuses on the effect of differing human milk composition on gastric emptying in preterm infants. Despite differences in milk macronutrient composition between mothers, there is no evidence of a clinically significant impact on gastric emptying. However, some bovine-based human milk fortifiers may slow gastric emptying. Sharon is also interested in maternal factors that impact milk production. A higher incidence of low milk production is reported in women after preterm birth, although measured milk productions range from low to well above the reference range. Similarly, low milk production appears to be more common in women with metabolic dysfunction, with some evidence of an effect on milk composition. 



\title{
Editorial
}

\section{Breastfeeding and Human Lactation}

\author{
Donna Geddes * and Sharon Perrella \\ School of Molecular Sciences, M310, The University of Western Australia, Crawley, Perth, WA 6009, Australia; \\ sharon.perrella@uwa.edu.au \\ * Correspondence: donna.geddes@uwa.edu.au; Tel.: +61-8-6488-7006
}

Received: 27 March 2019; Accepted: 2 April 2019; Published: 9 April 2019

check for updates

Keywords: Breastfeeding; human lactation; lactation; human milk; breast milk; milk composition

\section{Introduction}

Breastfeeding is the very means by which humans have thrived and developed as a species. Indeed, the Developmental Origins of Health and Disease Hypothesis recognises that the breastfeeding phase, which can continue to 2 years and beyond, plays a major role in the continuum of optimal programming of the lifelong health and development of the infant. Early life nutrition therefore presents a window of opportunity where the infant's short and long-term health can potentially be improved in the face of escalating rates of chronic disease that have reached epidemic proportions.

This special issue "Breastfeeding and Human Lactation" is thus timely, in an era of resurgence of lactation research, and is comprised of 30 manuscripts that cover a wide range of areas. This research will contribute to a growing scientific knowledge base that is critical to improving breastfeeding rates and the delivery of human milk (HM) to all infants, including those that cannot breastfeed, such as the vulnerable preterm infant. The majority of the papers in this issue address one of two broad themes; factors influencing milk composition, or relationships between milk composition and infant development. Findings from these research papers further elucidate the variability of milk composition and its impact on infant health.

\section{Factors Influencing Milk Composition}

It is evident that mammalian milk evolved as a protective fluid harbouring antimicrobial proteins predominantly for the protection of the offspring, with nutrition developing later. As such, many components of milk have dual roles, working synergistically to protect and nourish the infant. Indeed, the footprints of evolution are apparent in the presence of immune cells in HM that increase significantly in response to both maternal and infant infections. Twigger et al. [1] have identified antimicrobial proteins, granulysin and perforin along with other granzymes released by leukocytes in $\mathrm{HM}$, that are elevated in maternal breast infection. Milk immune cells may therefore be beneficial for protection of both the infant and the breast.

Anti-secretory factor (AF) is involved in the regulation of secretory processes and inflammation and is expressed in immune cells: B-cells, macrophages and dendritic cells. AF concentrations in HM are lower than that of maternal plasma, with a positive relationship between milk AF concentration and maternal body mass index (BMI), which might be due to a greater level of maternal inflammation associated with obesity [2].

It is increasingly apparent that maternal factors such as body composition, diet, ethnicity, geography, genetics and lifestyle all contribute to the unique milk signature of each woman. In this issue, a number of papers have shown differences in milk composition with respect to geographical location. In particular, concentrations of the immune active molecules transforming growth factor- $\beta 2$ (TGF- $\beta 2)$, immunoglobulin A (IgA), and hepatocyte growth factor (HGF) were higher in African 
women than in Italian women, suggesting a stronger response to the environment and thus greater infant protection against infection [3].

With cutting-edge technologies, it is possible to study metabolites in all bodily fluids. Variability of the metabolite profile of HM has not been comprehensively explored, however three papers have attempted this ambitious task. It was shown in two studies that the milk metabolome differs according to country [4,5] and mode of birth. Further interactions between the milk metabolites and microbes in the milk were also discovered, indicating the importance of the milk microbiome [5]. The third paper studied the endocannabinoid metabolome, for which there are receptors in the infant brain with evidence of a role in appetite and food intake. The study aimed to determine differences in endocannabinoids between transitional and mature milk, of which only one was significant [6]. The impact of these components on infant growth and development is yet to be studied.

Variability in HM composition would logically depend on maternal diet to some extent, although few studies have been carried out in this area. Studies that attempted this difficult task have provided conflicting results, largely due to the observational nature of the research. Two papers in this issue demonstrated an absence of relationship between diet and macronutrients [7,8]. Similarly, no relationships were observed between maternal dietary intakes of the micronutrients choline and zinc and their respective HM concentrations [9]. For breastfeeding women in a population with a high prevalence of zinc deficiency, zinc supplementation during pregnancy did not impact postnatal maternal serum zinc levels, which likely reflect HM concentrations [10]. Relationships were noted between diet and HM fatty acid profiles, as previously documented [8]. Interestingly, Bzikowska-Jura et al made the observation that maternal adiposity was related to HM protein and energy content at 3 months lactation, irrespective of diet [11]. Appropriate HM sampling methods are imperative when examining variability of milk components. In this context, Bzikowska-Jura et al found a weak relationship between HM fat content and maternal BMI using an intense sampling regime to account for changes in fat over the course of $24 \mathrm{~h}$. Kent et al trialed hourly expression of breast milk over $3 \mathrm{~h}$ ( 4 expressions) in an effort to estimate rates of milk fat synthesis. Unfortunately, this was not a reliable measure when compared to $24 \mathrm{~h}$ milk sampling [12]. George et al has highlighted sampling as one of the major challenges when examining milk lipids [13].

The idea of maternal-infant signaling via milk is attractive to explain both milk composition and infant outcome variability. Maternal adiposity is related to lower lean infant mass across 12 months of lactation [14], and while a review in the issue suggests that milk is tailored according to sex of the infant, there is yet to be strong evidence of this in humans [15].

\section{Relationships of Milk Composition with Infant Protection, Growth and Development}

Historically very few milk components have been associated with infant outcomes. Two papers in this issue highlight that the dose, rather than concentrations, of milk components are associated with infant body composition development over the first 12 months of life. Specifically, Gridneva and colleagues showed that the $24 \mathrm{~h}$ dose of appetite hormones adiponectin and whole milk leptin [16], along with casein [17], are differentially related to the development of infant body composition. The mechanisms by which the components exert their effects are still not clear.

Interestingly, endogenous satiety factors produced in the small intestine have been detected in $\mathrm{HM}$ and have been related to infant weight gain and weight for age z scores [18]. Whilst more work has to be done to verify the results, it is becoming increasingly clear that both the composition and volume of milk consumed by the infant modulates growth and development.

Growth of the preterm infant is critical, as these vulnerable infants are at high risk for morbidities both early and later in life. Whilst HM is recommended as the optimal nutrition for preterm infants, fortification is almost universal to ensure adequate growth of those born $<33$ weeks gestation. The delivery of human milk during continuous enteral feeding therefore is an area where enhancement may be needed to avoid the potential loss of nutrients to the infant. Zozaya et al. [19] found a reduction in the total fat delivered to the preterm infant via continuous enteral feeding, with long chain fatty 
acids more likely to be adsorbed to the feeding tube. These losses, while statistically significant, were considered clinically small. Once preterm infants are able to feed orally a dilemma exists about how to feed the infant in the absence of the breastfeeding mother. Geddes et al compared breastfeeding with use of a novel teat that required the infant to apply a vacuum and use a tongue movement mimicking that of breastfeeding to remove milk. They observed that although the infants' intra-oral vacuums were lower with the teat than at the breast, more milk was transferred [20]. This finding is indicative of the immaturity of the preterm infant's oral motor systems and should be taken into account when transitioning to full breastfeeding.

Many of the preterm infant's systems are immature, in particular the gastrointestinal system. This increases the preterm infant's susceptibility to infection and may impact the digestion of milk and subsequent absorption of nutrients and immune components. Indeed, Demers-Mathiew et al. [21] have described differences in the digestion of HM immunoglobulins between the preterm and term infant. The impacts of these findings are yet to be determined.

One of the major reasons HM feeding is recommended for preterm infants is that it markedly decreases the risk for necrotizing enterocolitis. However, controversy exists over whether raw or pasteurized HM should be fed to infants less than 32 weeks corrected age or less than 1500g in weight due to the high prevalence of cytomegalovirus in the milk. Lopes et al. [22] describe the heterogeneity in feeding practices between French neonatal units highlighting lack of consensus within the medical field. Pasteurization of HM is of concern because it reduces the impact of several immune factors in milk, including lactoferrin, which plays a significant role in antimicrobial and immunomodulatory functions. Telang provided a comprehensive review of the structure and functions of lactoferrin, and discussed the importance of continued clinical trials in determining the role of lactoferrin in prevention of neonatal sepsis [23].

An in-depth understanding of both the complex processes that impact HM and the impact of $\mathrm{HM}$ on the infant is critical to understanding lactation dysfunction, and may inform the identification of windows of potential intervention. An understanding of physiological and clinical dilemmas in lactation is also important.

In this context, insufficient milk supply is the most common reason for early weaning. Currently evidence-based treatments are limited for women with low milk supply. While galactogogues are often prescribed, the effect is modest for pharmacologic galactogogues as reviewed by Asztalos [24]. In light of this review, much more research is required to understand the causes of low milk supply along with more controlled studies of the efficacy of galactogogues.

Low milk supply may follow delayed secretory activation, or may be associated with breast inflammation. While both conditions are characterised by an elevated HM sodium concentration and sodium:potassium ratio, to date there are no clinical tools available to track these complications of lactation. Lai et al validation of handheld devices for determining sodium and potassium levels in HM indicates these may offer a promising point of care tool for monitoring secretory activation, the onset of mastitis and evaluation of treatment [25].

Mothers face many other barriers to successful breastfeeding, including their perceptions and own wellbeing [26,27]. Early hospital practices can also impact lactation, including early introduction of formula in the hospital, which was estimated at $28 \%$ in the UK [28]. The authors found many of the factors implicated in early supplementation to be modifiable. Further early recognition of infant feeding cues and responsive feeding is facilitated by increased mother-infant contact [29].

Finally, one must not discount the health benefits reaped by the lactating mother. The incidence of gestational diabetes mellitus is increasing and is associated with greater maternal risk for type 2 diabetes. However, breastfeeding is associated with lower risk of maternal type 2 diabetes, and in a new analysis maternal thyroid function also appears to be positively affected out to 6-16 years post-partum [30]. 


\section{Conclusions}

While HM is traditionally thought of primarily as a source of infant nutrition, evidence from lactation research shows a diverse range of functions, including protection from infection and disease, and programming of future health and development of both mother and infant through microbial and hormonal signaling. Interactions between maternal endocrine and mammary function, as well as diet, also impact milk composition and production. New evidence presented in this special edition of Nutrients contributes to the growing body of lactation and breastfeeding research, and informs our understanding of the complex composition of HM and its impact on infant health.

Conflicts of Interest: The authors declare no conflict of interest. Both authors receive a salary from an unrestricted research grant from Medela AG. The funders had no influence or input into this editorial.

\section{References}

1. Twigger, A.J.; Kuffer, G.K.; Geddes, D.T.; Filgueria, L. Expression of Granulisyn, Perforin and Granzymes in Human Milk over Lactation and in the Case of Maternal Infection. Nutrients 2018, 10, 1230. [CrossRef] [PubMed]

2. Gustafsson, A.; Granstrom, E.; Stecksen-Blicks, C.; West, C.E.; Silfverdal, S.A. The Antisecretory Factor in Plasma and Breast Milk in Breastfeeding Mothers-A Prospective Cohort Study in Sweden. Nutrients 2018, 10, 1227. [CrossRef]

3. Munblit, D.; Abrol, P.; Sheth, S.; Chow, L.; Khaleva, E.; Asmanov, A.; Lauriola, S.; Padovani, E.; Comberiati, P.; Boner, A.; et al. Levels of Growth Factors and IgA in the Colostrum of Women from Burundi and Italy. Nutrients 2018, 10, 1216. [CrossRef]

4. Gay, M.; Koleva, P.; Slupsky, C.; Toit, E.; Eggesbo, M.; Johnson, C.; Wegienka, G.; Shimojo, N.; Campbell, D.; Prescott, S.; et al. Worldwide Variation in Human Milk Metabolome: Indicators of Breast Physiology and Maternal Lifestyle? Nutrients 2018, 10, 1151. [CrossRef] [PubMed]

5. Gómez-Gallego, C.; Morales, J.; Monleón, D.; Du Toit, E.; Kumar, H.; Linderborg, K.; Zhang, Y.; Yang, B.; Isolauri, E.; Salminen, S.; et al. Human Breast Milk NMR Metabolomic Profile across Specific Geographical Locations and Its Association with the Milk Microbiota. Nutrients 2018, 10, 1355. [CrossRef]

6. Gaitan, A.V.; Wood, J.T.; Zhang, F.; Makriyannis, A.; Lammi-Keefe, C.J. Endocannabinoid Metabolome Characterization of Transitional and Mature Human Milk. Nutrients 2018, 10, 1294. [CrossRef] [PubMed]

7. Butts, C.A.; Hedderley, D.I.; Herath, T.D.; Paturi, G.; Glyn-Jones, S.; Wiens, F.; Stahl, B.; Gopal, P. Human Milk Composition and Dietary Intakes of Breastfeeding Women of Different Ethnicity from the Manawatu-Wanganui Region of New Zealand. Nutrients 2018, 10, 1231. [CrossRef]

8. Barrera, C.; Valenzuela, R.; Chamorro, R.; Bascunan, K.; Sandoval, J.; Sabag, N.; Valenzuela, F.; Valencia, M.P.; Puigrredon, C.; Valenzuela, A. The Impact of Maternal Diet during Pregnancy and Lactation on the Fatty Acid Composition of Erythrocytes and Breast Milk of Chilean Women. Nutrients 2018, 10, 839. [CrossRef]

9. Wiedeman, A.M.; Whitfield, K.C.; March, K.M.; Chen, N.N.; Kroeun, H.; Sokhoing, L.; Sophonneary, P.; Dyer, R.A.; Xu, Z.; Kitts, D.D.; et al. Concentrations of Water-Soluble Forms of Choline in Human Milk from Lactating Women in Canada and Cambodia. Nutrients 2018, 10, 381. [CrossRef]

10. Narvaez-Caicedo, C.; Moreano, G.; Sandoval, B.A.; Jara-Palacios, M.A. Zinc Deficiency among Lactating Mothers from a Peri-Urban Community of the Ecuadorian Andean Region: An Initial Approach to the Need of Zinc Supplementation. Nutrients 2018, 10, 869. [CrossRef]

11. Bzikowska-Jura, A.; Czerwonogrodzka-Senczyna, A.; Oledzka, G.; Szostak-Wegierek, D.; Weker, H.; Wesolowska, A. Maternal Nutrition and Body Composition During Breastfeeding: Association with Human Milk Composition. Nutrients 2018, 10, 1379. [CrossRef]

12. Kent, J.C.; Gardner, H.; Lai, C.T.; Hartmann, P.E.; Murray, K.; Rea, A.; Geddes, D.T. Hourly Breast Expression to Estimate the Rate of Synthesis of Milk and Fat. Nutrients 2018, 10, 1144. [CrossRef]

13. George, A.D.; Gay, M.C.L.; Trengove, R.D.; Geddes, D.T. Human Milk Lipidomics: Current Techniques and Methodologies. Nutrients 2018, 10, 1169. [CrossRef]

14. Gridneva, Z.; Rea, A.; Hepworth, A.R.; Ward, L.C.; Lai, C.T.; Hartmann, P.E.; Geddes, D.T. Relationships between Breastfeeding Patterns and Maternal and Infant Body Composition over the First 12 Months of Lactation. Nutrients 2018, 10, 45. [CrossRef] 
15. Galante, L.; Milan, A.M.; Reynolds, C.M.; Cameron-Smith, D.; Vickers, M.H.; Pundir, S. Sex-Specific Human Milk Composition: The Role of Infant Sex in Determining Early Life Nutrition. Nutrients 2018, 10, 1194. [CrossRef]

16. Gridneva, Z.; Kugananthan, S.; Rea, A.; Lai, C.T.; Ward, L.C.; Murray, K.; Hartmann, P.E.; Geddes, D.T. Human Milk Adiponectin and Leptin and Infant Body Composition over the First 12 Months of Lactation. Nutrients 2018, 10, 1125. [CrossRef]

17. Gridneva, Z.; Tie, W.J.; Rea, A.; Lai, C.T.; Ward, L.C.; Murray, K.; Hartmann, P.E.; Geddes, D.T. Human Milk Casein and Whey Protein and Infant Body Composition over the First 12 Months of Lactation. Nutrients 2018, 10, 1332. [CrossRef]

18. Bruun, S.; Gouveia-Figueira, S.; Domellof, M.; Husby, S.; Neergaard Jacobsen, L.; Michaelsen, K.F.; Fowler, C.J.; Zachariassen, G. Satiety Factors Oleoylethanolamide, Stearoylethanolamide, and Palmitoylethanolamide in Mother's Milk Are Strongly Associated with Infant Weight at Four Months of Age-Data from the Odense Child Cohort. Nutrients 2018, 10, 1747. [CrossRef]

19. Zozaya, C.; Garcia-Serrano, A.; Fontecha, J.; Redondo-Bravo, L.; Sanchez-Gonzalez, V.; Montes, M.T.; Saenz de Pipaón, M. Fat Loss in Continuous Enteral Feeding of the Preterm Infant: How Much, What and When Is It Lost? Nutrients 2018, 10, 809. [CrossRef]

20. Geddes, D.; Kok, C.; Nancarrow, K.; Hepworth, A.; Simmer, K. Preterm Infant Feeding: A Mechanistic Comparison between a Vacuum Triggered Novel Teat and Breastfeeding. Nutrients 2018, 10, 376. [CrossRef]

21. Demers-Mathieu, V.; Underwood, M.A.; Beverly, R.L.; Nielsen, S.D.; Dallas, D.C. Comparison of Human Milk Immunoglobulin Survival during Gastric Digestion between Preterm and Term Infants. Nutrients 2018, 10, 631. [CrossRef]

22. Lopes, A.A.; Champion, V.; Mitanchez, D. Nutrition of Preterm Infants and Raw Breast Milk-Acquired Cytomegalovirus Infection: French National Audit of Clinical Practices and Diagnostic Approach. Nutrients 2018, 10, 1119. [CrossRef]

23. Telang, S. Lactoferrin: A Critical Player in Neonatal Host Defense. Nutrients 2018, 10, 1228. [CrossRef]

24. Asztalos, E.V. Supporting Mothers of Very Preterm Infants and Breast Milk Production: A Review of the Role of Galactogogues. Nutrients 2018, 10, 600. [CrossRef]

25. Lai, C.T.; Gardner, H.; Geddes, D. Comparison of Inductively Coupled Plasma Optical Emission Spectrometry with an Ion Selective Electrode to Determine Sodium and Potassium Levels in Human Milk. Nutrients 2018, 10, 1218. [CrossRef]

26. Nasser, A.; Omer, F.; Al-Lenqawi, F.; Al-Awwa, R.; Khan, T.; El-Heneidy, A.; Kurdi, R.; Al-Jayyousi, G. Predictors of Continued Breastfeeding at One Year among Women Attending Primary Healthcare Centers in Qatar: A Cross-Sectional Study. Nutrients 2018, 10, 983. [CrossRef]

27. Bennett, A.E.; Kearney, J.M. Factors Associated with Maternal Wellbeing at Four Months Post-Partum in Ireland. Nutrients 2018, 10, 609. [CrossRef]

28. Biggs, K.V.; Hurrell, K.; Matthews, E.; Khaleva, E.; Munblit, D.; Boyle, R.J. Formula Milk Supplementation on the Postnatal Ward: A Cross-Sectional Analytical Study. Nutrients 2018, 10, 608. [CrossRef]

29. Little, E.E.; Legare, C.H.; Carver, L.J. Mother(-)Infant Physical Contact Predicts Responsive Feeding among U.S. Breastfeeding Mothers. Nutrients 2018, 10, 1251. [CrossRef]

30. Panuganti, P.L.; Hinkle, S.N.; Rawal, S.; Grunnet, L.G.; Lin, Y.; Liu, A.; Thuesen, A.C.B.; Ley, S.H.; Olesen, S.F.; Zhang, C. Lactation Duration and Long-Term Thyroid Function: A Study among Women with Gestational Diabetes. Nutrients 2018, 10, 938. [CrossRef] 


\title{
Relationships between Breastfeeding Patterns and Maternal and Infant Body Composition over the First 12 Months of Lactation
}

\author{
Zoya Gridneva $^{1, *}$, Alethea Rea ${ }^{2}$, Anna R. Hepworth ${ }^{1}$, Leigh C. Ward ${ }^{3}$, Ching T. Lai ${ }^{1}$, \\ Peter E. Hartmann ${ }^{1}$ and Donna T. Geddes ${ }^{1}$ \\ 1 School of Molecular Sciences, M310, The University of Western Australia, Crawley, \\ Perth, WA 6009, Australia; arhepworth@westnet.com.au (A.R.H.); ching-tat.lai@uwa.edu.au (C.T.L.); \\ peter.hartmann@uwa.edu.au (P.E.H.); donna.geddes@uwa.edu.au (D.T.G.) \\ 2 Centre for Applied Statistics, The University of Western Australia, Crawley, Perth, WA 6009, Australia; \\ alethea.rea@uwa.edu.au \\ 3 School of Chemistry and Molecular Biosciences, The University of Queensland, St. Lucia, \\ Brisbane, QLD 4072, Australia; l.ward@uq.edu.au \\ * Correspondence: zoya.gridneva@uwa.edu.au; Tel.: +61-8-6488-4467
}

Received: 30 November 2017; Accepted: 2 January 2018; Published: 5 January 2018

\begin{abstract}
Breastfeeding has been implicated in the establishment of infant appetite regulation, feeding patterns and body composition (BC). A holistic approach is required to elucidate relationships between infant and maternal $\mathrm{BC}$ and contributing factors, such as breastfeeding parameters. Associations between maternal and breastfed term infant BC $(n=20)$ and feeding parameters during first 12 months of lactation were investigated. BC was measured at 2, 5, 9 and/or 12 months postpartum with ultrasound skinfolds (US; infants only) and bioimpedance spectroscopy (infants and mothers). 24-h milk intake (MI) and feeding frequency (FFQ) were measured. Higher FFQ was associated with larger 24-h MI ( $p \leq 0.003)$. Higher 24-h MI was associated with larger infant fat mass (FM) (US: $p \leq 0.002$ ), greater percentage FM (US: $p \leq 0.008$ ), greater FM index (FMI) (US: $p \leq 0.001$ ) and lower fat-free mass index (FFMI) (US: $p=0.015$ ). Lower FFQ was associated with both larger FFM (US: $p \leq 0.001$ ) and FFMI (US: $p<0.001$ ). Greater maternal adiposity was associated with smaller infant FFM measured with US (BMI: $p<0.010$; $\%$ FM: $p=0.004$; FMI: $p<0.011$ ). Maternal BC was not associated with FFQ or 24-h MI. These results reinforce that early life is a critical window for infant programming and that breastfeeding may influence risk of later disease via modulation of BC.
\end{abstract}

Keywords: human milk; breastfed infants; body composition; anthropometrics; milk intake; bioelectrical impedance spectroscopy; ultrasound skinfolds; maternal factors

\section{Introduction}

The importance of lactocrine programming has been highlighted recently, with breastfeeding identified as one of the most economical preventative measures for non-communicable diseases (NCD) including obesity later in life [1-5]. The development of body composition (BC) in early life is known to play an important role in the programming of these health outcomes [6]. This reduction in risk may be a result of multiple mechanisms associated not only with composition of human milk (HM) but also with infant breastfeeding patterns and behavior [7-9], all of which may influence the growth and development of breastfed infants. Differences in the weight and BC between breastfed and formula-fed infants have been attributed to the stark compositional differences of HM and formula [10,11]. Despite the evidence that volume of HM is a main driver of growth [11-13], a major focus of at present limited research on infant growth and $\mathrm{BC}$ development has been on the composition of HM and maternal 
pre-pregnancy body mass index (BMI), and to a lesser extent on the effect of the volume of HM and maternal adiposity. Although these findings suggest a dose-dependent effect of breastfeeding on development of infant $\mathrm{BC}$, the pathways of this effect are not fully understood.

Indeed, in utero maternal influences are apparent in obese women who generally deliver heavier infants with greater adiposity [14] thus maternal weight is a major predictor of infant birth weight (BW) $[15,16]$. However, recent studies have shown the infant BW is not associated with increased maternal BMI in women with BMI above $24 \mathrm{~kg} / \mathrm{m}^{2}$ [17]. To further complicate our understanding, overweight women deliver infants with higher adiposity [18] but not fat-free mass (FFM) [19,20]. Unfortunately, the majority of the studies have analyzed maternal pre-pregnancy BMI or gestational weight gain (parameters that are often self-reported and potentially misleading) as measures of adiposity. Considering that HM composition is influenced by the current maternal BC [21] rather than pre-pregnancy BMI, longitudinal studies with multiple measures of both maternal and breastfed infant $\mathrm{BC}$ are necessary [22] to elucidate the positive mechanistic effects of breastfeeding.

In a few recent studies, maternal BC was measured during pregnancy and a positive association between maternal BC and infant BW was found [23-26], showing maternal FFM or total body water, but not fat mass (FM) are the strongest predictors. Interestingly, longer duration of breastfeeding is shown to attenuate the adverse effects of BW and early weight gain on infant FM gain [27]. Further, the majority of participants are either newborns or children between 2 and 11 years of age, and infant adiposity (fat mass (FM) and percentage FM (\%FM)) has been measured rather than FFM, yet the metabolic rate is largely determined by the FFM [28,29]. Furthermore, despite 24-h milk intake (MI) having a strong positive relationship with infant weight gain $[12,13,30]$, there has been no investigation of the effect of either 24-h MI or feeding frequency (FFQ) on infant BC, yet these factors are highly variable between infants [31]. Our recent research of gastric emptying in term breastfed infants indicated that shorter, smaller and leaner infants fed more frequently (maternal self-report) [32], highlighting the need to connect not only maternal and infant $\mathrm{BC}$, but also contributing factors, such as milk production and composition, infant $\mathrm{MI}$ and FFQ and, in turn, the development of the breastfed infant BC (Figure 1).

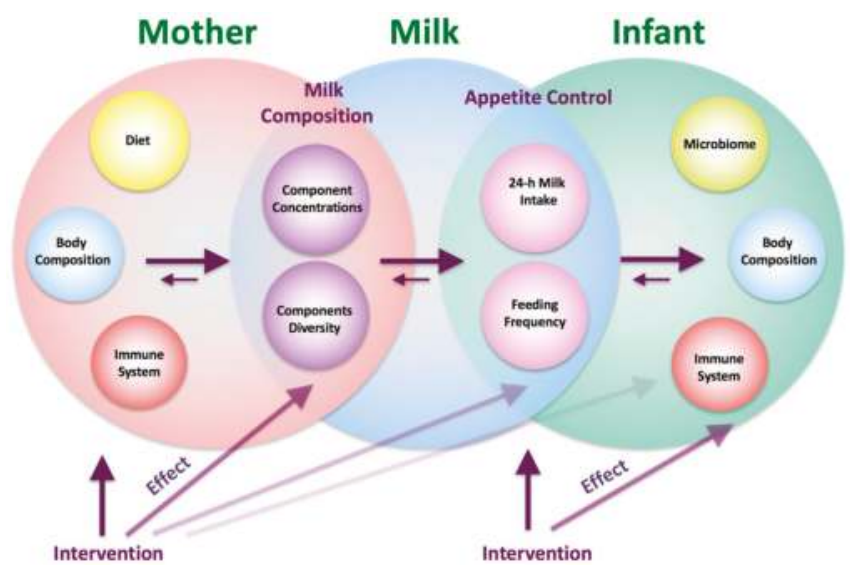

Figure 1. Framework for possible interconnecting pathways of lactocrine programming of the infant, and points of intervention for potential improvement of infant growth, development and health, based on available research.

It is important to understand the mechanisms by which maternal $\mathrm{BC}$, breastfeeding and $\mathrm{HM}$ components may influence infant $\mathrm{BC}$, as this will allow for more targeted interventions that may potentially reduce both infant and adult overweight and obesity. Therefore, the aim of this longitudinal study was to investigate relationships between maternal and infant $B C$ during the first 12 months of 
lactation. Further, exploration of relationships of infant 24-h MI and FFQ with maternal and infant BC was carried out.

\section{Materials and Methods}

\subsection{Study Participants}

Breastfed infants ( $n=20 ; 10$ males, 10 females) of English-speaking, predominantly Caucasian, mothers of higher social-economic status from a developed country were recruited from the community, primarily from the West Australian branch of the Australian Breastfeeding Association. Inclusion criteria were: healthy singletons, gestational age $\geq 37$ weeks, exclusively breastfed [33] at 2 and 5 months, and maternal intention to breastfeed until 12 months. Exclusion criteria were: infant factors that could potentially influence growth and development of BC, maternal smoking, and low milk supply. All mothers provided written informed consent to participate in the study, which was approved by The University of Western Australia Human Research Ethics Committee (RA/1/4253, RA/4/1/2639) and registered with the Australian New Zealand Clinical Trials Registry (ACTRN12616000368437).

\subsection{Study Session}

Measurements were made when the infants were 2 and/or 5, 9 and 12 months of age. Participants visited our laboratory at King Edward Memorial Hospital for Women (Subiaco, Perth, WA, Australia) for up to four monitored breastfeeding sessions between March 2013 and September 2015. At each study session, the infant was weighed pre-feed, and then the mother breastfed her infant. Infant bioelectrical impedance spectroscopy (BIS) measurements were made pre-feed, unless impractical, then they were made post-feed [34]. Ultrasound skinfold (US) and anthropometric measurements were made post-feed. This combination of methods for measuring infant BC was used to ensure safe, non-invasive and accurate assessment, and to avoid the inherent limitations of a singular technique [35]. Clothing was removed for the measurements except for a dry diaper and a singlet.

Maternal weight, height and BIS measurements were recorded. Current FFQ of the infants was self-reported by mothers.

\subsection{Anthropometric Measurements}

Infants weight was determined before breastfeeding using Medela Electronic Baby Weigh Scales ( \pm 2.0 g; Medela Inc., McHenry, IL, USA). Infant crown-heel length was measured once to the nearest $0.1 \mathrm{~cm}$ using non-stretch tape and a headpiece and a footpiece, both applied perpendicularly to a hard surface. Infant head circumference was measured with a non-stretch tape to the nearest $0.1 \mathrm{~cm}$.

Maternal weight was measured using an electronic scale ( $\pm 0.1 \mathrm{~kg}$; Seca, Chino, CA, USA). Height was self-reported by participants or measured against a calibrated marked wall (accuracy $\pm 0.1 \mathrm{~cm}$ ).

Infant and maternal BMI were calculated as $\mathrm{kg} / \mathrm{m}^{2}$.

\subsection{Body Composition with Bioelectrical Impedance Spectroscopy}

Whole body bioimpedance (wrist to ankle) of infants and mothers was measured using the Impedimed SFB7 bioelectrical impedance analyzer (ImpediMed, Brisbane, QLD, Australia) according to the manufacturer's instructions.

Mothers were measured in supine position on a non-conductive surface. A series of ten consecutive measurements (fat mass (FM), percentage fat mass (\%FM) and fat-free mass (FFM)) were taken within 1-2 min and averaged for data analysis. The within participant coefficient of variation (CV) for maternal \%FM was $0.21 \%$ [21].

Infants were measured by applying an adult protocol as used previously in infants but with data analyzed using settings customized for infants [35,36]. Resistance (ohm) at $50 \mathrm{kHz}\left(\mathrm{R}_{50}\right)$ was determined from the curve of best fit, averaged for analysis purposes and used in the Lingwood et al. [36] 
age-matched (3 and 4.5 month-old infants) BIS equations for FFM of 2 and 5 month-old infants respectively, and Bocage [37] total body water (TBW) equations for 9 and 12 month-old infants:

$$
\text { TBW }=\left(0.418 \times \text { Weight }(\mathrm{kg})+1936 / \mathrm{R}_{50}+0.8649\right) \times \text { Length }(\mathrm{cm}) / 100
$$

FFM was further determined using sex and age-appropriate hydration factors (HF) calculated from Butte et al. [38]:

$$
\mathrm{FFM}=\mathrm{TBW} / \mathrm{HF} \text {. }
$$

\%FM was calculated as follows:

$$
\% \mathrm{FM}=100 \times(\text { Weight }(\mathrm{kg})-\mathrm{FFM}(\mathrm{kg})) / \text { Weight }(\mathrm{kg}) .
$$

Within participant $\mathrm{CV}$ for infant $\mathrm{R}_{50}$ was $1.5 \%$ [34].

\subsection{Ultrasound Skinfold Measurements}

Infant skinfolds were measured using the Aplio XG (Toshiba, Tokyo, Japan) US machine with a 14-8 MHz transducer (PLT-1204BX) and sterile water-based ultrasonic gel (Parker Laboratories Inc., Fairfield, NJ, USA) as described previously [35]. Single US scans of four anatomical sites (biceps, subscapular, suprailiac and triceps) were performed on the left side of the body with minimal compression. Subcutaneous tissue thickness (skin thickness and the skin-fat interface to fat-muscle interface distance) was measured directly from images on the screen using electronic calipers. One experienced sonographer with good interrater reliability [39] performed all of the measurements. US measurements were doubled [40] for use in skinfold equations developed for subcutaneous tissue thickness measurement with skinfold calipers. At all time points, infant \%FM with 2-skinfolds (US 2SF: triceps, subscapular; Slaughter et al. [41]) and density $(d ; \mathrm{kg} / \mathrm{L})$ with 4-skinfolds (US 4SF: biceps, subscapular, suprailiac and triceps; Brook [42]) were calculated with \%FM further determined using Lohman equation [43]:

$$
\% \mathrm{FM}=100 \times(5.28 / d-4.89) .
$$

\subsection{Body Composition Indices}

The indices of height-normalized BC were calculated for mothers and infants: fat mass index (FMI) was calculated as FM/length ${ }^{2}$, and fat-free mass index (FFMI) was calculated as FFM/length² ; both expressed as $\mathrm{kg} / \mathrm{m}^{2}$ [44].

\subsection{4-H Milk Intake and Feeding Frequency}

Infant MI was measured by mothers using the 24-h milk production (MP) protocol, weighing infants at home with the Medela Electronic Baby Weigh Scales pre- and post each breastfeed during a 24-h period plus one breastfeeding, and recording amounts of HM (g) consumed by the infant (including expressed HM if any) [45]. 24-h MI was determined as previously described with potential underestimation of 3-10\% [45] and FFQ (meals per day) was recorded [31]. 24-h MI was measured at three time points: between 2 and $5(4.0 \pm 1.3)$ months, when MI is shown to be stable [31], and within two weeks of 9 (9.4 \pm 0.3$)$ and $12(12.2 \pm 0.4)$ months. Given that measuring 24-h MI is not always practical, particularly at the later stages of lactation, mothers were also asked how frequently the infant fed, and self-reported the typical time between the feeds (e.g., each $2 \mathrm{~h}$ ) during the week prior to the study session as a proxy measure of FFQ.

\subsection{Statistical Analyses}

Statistical analysis was performed in R 3.1.2 for Mac OSX [46]. Additional packages were used for linear mixed effects models (nlme, lme4 and car) [47-49], intra-class correlations (irr) [50], Tukey's all 
pair comparisons (multcomp) [51] and graphics (ggplot2) [52]. Descriptive statistics are reported as mean \pm standard deviation (SD) (range); model parameters as estimate $\pm \mathrm{SE}$ (standard error).

During this longitudinal study infants were measured at four time points ( 2 and/or 5, 9 and 12 months). An approximate sample size was calculated using the 'F tests-Linear multiple regression: Fixed model: $R^{2}$ increase' option in $G^{*}$ Power [53] as if this was a cross-sectional study with equal numbers at each time. Allowing four predictors ( 3 for age comparisons), $\alpha=0.05$ and 14 participants (56 sample points $=14$ participants $\times 4$ time points) gave the study power of 0.80 to detect an effect size of 0.15 . This approach was selected, as there is no closed form expression suitable for the calculation of sample sizes for this research design [54], with the consideration that longitudinal study design is more powerful. Recruitment of participants at the 5 months point was introduced, as many mothers would not commit to a study that required breastfeeding to 12 months, when approached at 2 months $(n=8)$. As a result, required number of participants was increased to 20 in order to maintain predicted power; this also addressed issues relating to missed visits. Missing data was dealt with using available case analysis.

Maternal BC was analysed using an intercept only linear mixed effects model for the calculation of CV for maternal \%FM measurements ( $n=10,10$ measurements each).

Infant BC was analysed using linear mixed effects models with random intercept per participant to determine whether BC measurements (\%FM, FM, FMI, FFM and FFMI) differed systematically by age, measurement method (US 2SF, US 4SF and BIS) and infant sex. As interactions between sex and methods were non-significant $(p>0.52)$, reported associations are for pooled data. Months after birth were accounted for in all models; results reported account for this, regardless of significance.

Survey responses relating to FFQ were analysed using a one-way intra-class correlation for agreement of single measures.

The analyses for systematic differences in all measured parameters (maternal characteristics, infant characteristics and breastfeeding characteristics) at different months after birth and between different measurement methods used general linear hypothesis tests (Tukey's all pair comparisons).

Relationships between infant BW and maternal and infant BC at four time points after birth were analysed using linear regression models accounting for gestational age and sex, which were identified as significant covariates using a stepwise regression analysis. Since major postpartum weight/adiposity loss happens during the first 4 to 6 months in women of high social-economic status [55,56], maternal BC at 5, 9 and 12 months was considered instead of unavailable pre-pregnancy BMI.

Relationships between: (a) infant BC and breastfeeding characteristics; (b) breastfeeding characteristics and maternal BC; and (c) infant and maternal BC were analysed using linear mixed effects models. Each breastfeeding characteristic or infant BC measure/index was considered separately as the response variable, and each model contained fixed effects of infant age (months), a predictor (breastfeeding measure or maternal BC measure/index) and an interaction between infant age and predictor, as well as a random intercept per participant.

Owing to the large number of comparisons, a false discovery rate adjustment [57] was performed on associated subgroupings of results. $p$-values were considered to be significant below 0.018 for associations between infant BW and maternal BC; below 0.047 for associations between infant BW and infant BC; below 0.018 for associations between infant FFM and maternal BC; below 0.038 for associations between infant FFMI and maternal BC; below 0.029 for associations between infant BC and MI; below 0.040 for associations between infant BC and self-reported FFQ; below 0.05 for associations between infant BC and 24-h MP FFQ; below 0.0004 for associations between infant BC changes between the time points and 24-h MI; below 0.001 for associations between infant BC changes between the time points and self-reported FFQ; below 0.004 for associations between infant BC changes between the time points and 24-h MP FFQ; below 0.009 for associations between maternal BC changes between time points and 24-h MI; below 0.014 for associations between maternal BC changes between time points and self-reported FFQ; and below 0.05 for associations between maternal BC changes between time points and 24-h MP FFQ. The significance was set at the 5\% level otherwise. 


\section{Results}

\subsection{Subjects}

Twenty-two infants were recruited; 2 infants ( 1 male, 1 female) were excluded from the study after the 2-month visit (starting supplementation with formula; personal circumstances). One female infant started supplementation with formula/weaning at 6 months and was excluded from further analysis. Nineteen remaining infants were breastfed at 2, 5 and 9 months. Seventeen infants (94\%) continued to breastfeed at 12 months, but one male was too sick to attend the last session. Out of 18 infants measured at 12 months 16 infants (89\%) still continued to breastfeed. One male infant ceased breastfeeding 2 weeks before the 12-month appointment and one female infant stopped at 10 months after birth. Both infants and their mothers were measured at 12 months.

Some sessions were not attended by some participants leading to incomplete data. Five infants did not start at 2 months, two did not attend at 9 months and two at 12 months. Overall 80 measures were expected, however some were missing, specifically: infant weight $(n=9)$; infant \%FM, FM, FMI, FFM and FFMI measured with US 2SF, and maternal age, weight, height, BMI, \%FM, FM, FMI, FFM and FFMI $(n=10)$; infant head circumference $(n=11)$; infant length, BMI and \%FM, FM, FMI, FFM and FFMI measured with US 4SF ( $n=12$ ); infant \%FM, FM, FMI, FFM and FFMI measured with BIS $(n=13)$; self-reported FFQ $(n=20)$. Missing data also occurred due to difficulties with conducting 24-h MI measurements at later stages of lactation. The following measurements from the 60 expected were missing: FFQ from 24-h MP $(n=26)$ and 24-h MI $(n=27)$. Missing data were spread across the time points (Table 1).

Breastfeeding characteristics, infant and maternal demographics and anthropometrics as well as maternal BC measures at the four study sessions are presented in Table 1. Mean maternal age at the start of the study was $33.3 \pm 4.7(24-44)$ years, mean height was $167.4 \pm 7.4(150-181) \mathrm{cm}$ and mean parity was $2.3 \pm 0.9$ (1-4). Infant male/female ratio was 10/10, mean BW was $3.486 \pm 0.498$ (2.660-4.455) kg and mean gestational age was 39.4 (37.6-43) weeks. After accounting for infant age males were heavier $(0.85[0.12,1.57], p=0.025)$ and had larger head circumferences than females $(1.89[0.81,2.96], p=0.002)$, while no significant difference between sexes was seen for either length $(1.68[-0.24,3.59], p=0.083)$ or BMI $(1.09[-0.15,2.32], p=0.081)$.

Table 1. Participant anthropometric and breastfeeding characteristics.

\begin{tabular}{|c|c|c|c|c|}
\hline \multirow{3}{*}{ Characteristic } & 2 Months ${ }^{a}$ & 5 Months ${ }^{b}$ & 9 Months $^{c}$ & 12 Months ${ }^{d}$ \\
\hline & Mean \pm SD & Mean \pm SD & Mean \pm SD & Mean \pm SD \\
\hline & (Range) & (Range) & (Range) & (Range) \\
\hline \multicolumn{5}{|c|}{ Mothers } \\
\hline \multirow{2}{*}{ Weight $(\mathrm{kg})$} & $78.8 \pm 19.3$ & $70.1 \pm 17.8$ & $63.0 \pm 10.0$ & $64.2 \pm 17.3$ \\
\hline & $(57.5-116.2)$ & $(53.7-115.3)$ & $(50.4-121.9)$ & (51.4-121.9) \\
\hline \multirow{2}{*}{$\operatorname{BMI}\left(\mathrm{kg} / \mathrm{m}^{2}\right)$} & $27.2 \pm 5.5$ & $24.8 \pm 5.0$ & $22.7 \pm 3.9$ & $23.9 \pm 5.9$ \\
\hline & $(20.4-35.5)$ & $(19.0-35.2)$ & $(17.9-37.2)$ & $(18.2-37.2)$ \\
\hline \multirow{2}{*}{ Fat-free Mass ${ }^{e}(\mathrm{~kg})$} & $49.5 \pm 8.2$ & $45.4 \pm 6.6$ & $44.1 \pm 4.1$ & $45.4 \pm 6.7$ \\
\hline & $(38.2-66.2)$ & $(37.4-60.9)$ & $(35.1-68.5)$ & $(35.9-67.7)$ \\
\hline \multirow{2}{*}{ Fat Mass e $(\mathrm{kg})$} & $29.3 \pm 11.8$ & $24.6 \pm 12.0$ & $18.9 \pm 7.4$ & $18.8 \pm 11.0$ \\
\hline & $(15.2-50.0)$ & $(13.9-54.4)$ & $(11.4-53.4)$ & $(10.0-54.3)$ \\
\hline \multirow{2}{*}{ Fat Mass ${ }^{\mathrm{e}}(\%)$} & $36.0 \pm 6.4$ & $33.8 \pm 7.0$ & $29.2 \pm 6.7$ & $27.7 \pm 7.9$ \\
\hline & $(25.7-44.7)$ & $(23.2-47.2)$ & $(20.0-44.3)$ & $(19.4-44.5)$ \\
\hline \multirow{2}{*}{ FFMI $^{\mathrm{e}}\left(\mathrm{kg} / \mathrm{m}^{2}\right)$} & $16.8 \pm 2.1$ & $16.2 \pm 1.9$ & $16.1 \pm 2.3$ & $16.4 \pm 2.4$ \\
\hline & $(13.7-20.2)$ & $(13.2-20.0)$ & $(12.4-20.9)$ & $(12.7-20.7)$ \\
\hline
\end{tabular}


Table 1. Cont.

\begin{tabular}{|c|c|c|c|c|}
\hline \multirow{3}{*}{ Characteristic } & 2 Months $^{a}$ & 5 Months ${ }^{b}$ & 9 Months ${ }^{c}$ & 12 Months ${ }^{d}$ \\
\hline & Mean \pm SD & Mean \pm SD & Mean \pm SD & Mean \pm SD \\
\hline & (Range) & (Range) & (Range) & (Range) \\
\hline \multicolumn{5}{|c|}{ Mothers } \\
\hline \multirow{2}{*}{$\mathrm{FMI}^{\mathrm{e}}\left(\mathrm{kg} / \mathrm{m}^{2}\right)$} & $9.5 \pm 3.3$ & $8.4 \pm 3.3$ & $7.9 \pm 3.5$ & $7.5 \pm 3.5$ \\
\hline & $(6.2-15.3)$ & $(4.8-16.6)$ & $(3.9-16.3)$ & $(3.6-16.6)$ \\
\hline \multicolumn{5}{|c|}{ Infants } \\
\hline $\operatorname{Sex}(M / F)$ & $9 \mathrm{M} / 6 \mathrm{~F}$ & $10 \mathrm{M} / 10 \mathrm{~F}$ & $10 \mathrm{M} / 9 \mathrm{~F}$ & $9 \mathrm{M} / 9 \mathrm{~F}$ \\
\hline \multirow{2}{*}{ Age (months) } & $2.04 \pm 0.14$ & $5.16 \pm 0.22$ & $9.22 \pm 0.27$ & $12.26 \pm 0.28$ \\
\hline & $(1.87-2.33)$ & $(4.77-5.47)$ & $(8.83-9.77)$ & $(11.63-12.67)$ \\
\hline \multirow{2}{*}{ Length $(\mathrm{cm})$} & $58.1 \pm 1.9$ & $64.8 \pm 2.3$ & $71.7 \pm 1.9$ & $73.6 \pm 3.2$ \\
\hline & $(54.2-60.0)$ & $(60.5-69.5)$ & $(66.0-74.0)$ & $(69.0-78.5)$ \\
\hline \multirow{2}{*}{ Weight $(\mathrm{kg})$} & $5.630 \pm 0.660$ & $7.431 \pm 1.134$ & $8.836 \pm 0.975$ & $9.650 \pm 0.618$ \\
\hline & $(4.420-7.400)$ & $(5.808-9.510)$ & $(6.675-10.095)$ & $(7.165-11.085)$ \\
\hline \multirow{2}{*}{ BMI $\left(\mathrm{kg} / \mathrm{m}^{2}\right)$} & $16.6 \pm 1.2$ & $17.6 \pm 1.9$ & $17.7 \pm 1.7$ & $17.8 \pm 0.9$ \\
\hline & $(14.5-18.1)$ & $(14.9-20.4)$ & $(14.2-20.2)$ & $(13.7-19.2)$ \\
\hline \multirow{2}{*}{ Head circumference (cm) } & $39.7 \pm 1.6$ & $42.1 \pm 1.5$ & $45.6 \pm 1.7$ & $46.6 \pm 1.7$ \\
\hline & $(37.0-42.0)$ & $(40.0-45.9)$ & $(43.0-48.5)$ & $(44.2-49.5)$ \\
\hline \multicolumn{5}{|c|}{ Breastfeeding characteristics } \\
\hline \multirow{2}{*}{ 24-h milk intake (g) } & \multirow{2}{*}{$n / a^{f}$} & $818.8 \pm 204.9$ & $502.3 \pm 157.8$ & $445.5 \pm 200.4$ \\
\hline & & $(498-1185)$ & $(300-775)$ & $(255-795)$ \\
\hline \multirow{2}{*}{ 24-h feeding frequency (MP) } & \multirow{2}{*}{$n / a^{f}$} & $8.1 \pm 1.4$ & $5.4 \pm 1.2$ & $4.4 \pm 1.9$ \\
\hline & & $(6-11)$ & $(4-7)$ & $(2-8)$ \\
\hline \multirow{2}{*}{ Feeding frequency (SR) } & $2.3 \pm 0.4^{g}$ & $2.8 \pm 0.8$ & $3.7 \pm 1.2$ & $5.4 \pm 2.9$ \\
\hline & $(1.5-3.0)$ & $(1.5-4.0)$ & $(2.0-6.0)$ & $(2.2-12.0)$ \\
\hline
\end{tabular}

Data are mean \pm SD and ranges. ${ }^{\mathrm{a}} n=15 ;{ }^{\mathrm{b}} n=20 ;{ }^{\mathrm{c}} n=19 ;{ }^{\mathrm{d}} n=18 .{ }^{\mathrm{e}}$ Maternal body composition as measured with bioelectrical impedance spectroscopy. ${ }^{\mathrm{f}}$ Milk intake and feeding frequency as meals per 24-h was determined from 24-h milk production (MP) measured between 2 and 5 months (presented at 5 months here, $n=17$ ) and within 2 weeks of $9(n=8)$ and 12 months ( $n=9$ for feeding frequency, $n=8$ for milk intake). ${ }^{8}$ Maternal self-report (SR) of feeding frequency at the time of the visit as a typical time between feeds (e.g., each $2 \mathrm{~h})(n=11, n=19, n=17, n=13$ at 2, 5, 9 and 12 months respectively). BMI—body mass index; FFMI—fat-free mass index; FMI—fat mass index, $\mathrm{n} / \mathrm{a}-$ not applicable.

\subsection{Maternal Body Composition}

Maternal $\mathrm{BC}$ is presented in Table 1. At the session attended at 5 months postpartum none of the participants were classified as being underweight $(\mathrm{BMI}<18.5 ; \% \mathrm{FM}<21)$. They were classified as: normal weight (BMI 18.5-24.9, 65\%, $n=13$; \%FM 21-32.9, 55\%, $n=11$ ), overweight (BMI 25-29.9, 20\%, $n=4 ; \% \mathrm{FM} 33-38.9,30 \%, n=6)$ or obese (BMI $>30,15 \%, n=3 ; \% \mathrm{FM}>39,15 \%, n=3$ ) [58].

\subsection{Infant Body Composition}

Infant BC measured with three measurement techniques (BIS, US 2SF and US 4SF) is presented in Table S1.

Male infants were compared to female infants using all three measurement techniques. FFM was significantly greater in males overall $(0.66[0.19,1.14] \mathrm{kg}, p=0.009)$ and when the methods were considered separately (US 2SF: $0.55[0.07,1.03] \mathrm{kg}, p=0.027$; US 4SF: $0.70[0.20,1.20] \mathrm{kg}, p=0.009$; BIS: $0.74[0.25,1.22] \mathrm{kg}, p=0.005)$. FFMI was significantly higher in males overall $(0.95[0.21,1.69] \mathrm{kg}$, 
$p=0.015$ ) and when determined with US 4SF and BIS (US 4SF: $1.01[0.29,1.73] \mathrm{kg}, p=0.009$; BIS: $1.09[0.37,1.81] \mathrm{kg}, p=0.005)$ but not with US 2SF $(0.70[-0.12,1.52] \mathrm{kg}, p=0.089)$.

Differences were not seen for \%FM, FM and FMI overall (males \%FM: $-0.38[-3.02,2.26] \%$, $p=0.77$; FM: $0.19[-0.17,0.55] \mathrm{kg}, p=0.27$; FMI: $\left.0.16[-0.52,0.85] \mathrm{kg} / \mathrm{m}^{2}, p=0.62\right)$ or when the methods were considered separately (\%FM: $p \geq 0.30$; FM: $p \geq 0.095$; FMI: $p \geq 0.25$ ).

A comparison of measurement methods showed no difference for $\% \mathrm{FM}(p \geq 0.074), \operatorname{FM}(p \geq 0.11)$, FMI $(p \geq 0.077)$ and FFM $(p \geq 0.15)$. Overall FFMI determined with BIS was significantly higher compared with US 2SF $(0.24 \pm 0.10, p>0.039)$ with no further differences between the methods $(p \geq 0.24)$.

\subsection{Infant Birth Weight and Maternal and Infant Body Composition}

After accounting for infant sex and gestational age no significant associations between $\mathrm{BW}$ and any maternal $\mathrm{BC}$ parameter were seen at any time point after birth after adjusting for the false discovery rate $(p \geq 0.018)$ (the raw $p$-values for negative associations between maternal adiposity / BC indices at 5 , 9 and 12 months postpartum and infant BW before the adjustment were: \%FM ( 5 months: $-0.03 \pm 0.01$, $p=0.026$; 9 months: $-0.03 \pm 0.01, p=0.021 ; 12$ months: $-0.03 \pm 0.01, p=0.018)$, BMI (5 months: $-0.04 \pm 0.02, p=0.024 ; 9$ months: $-0.03 \pm 0.02, p=0.046 ; 12$ months: $-0.04 \pm 0.02, p=0.019$ ), FMI (5 months: $-0.06 \pm 0.02, p=0.032$; 9 months: $-0.05 \pm 0.02, p=0.038 ; 12$ months: $-0.06 \pm 0.02$, $p=0.023)$, and FFMI (5 months: $-0.10 \pm 0.04, p=0.042 ; 12$ months: $-0.09 \pm 0.04, p=0.032)$ ).

After accounting for infant sex and gestational age and adjusting for the false discovery rate $(p \geq 0.047)$ larger BW was associated with larger infant FFM measured at all-time points and with all three methods ( 2 months: US 2SF, $0.81 \pm 0.17, p=0.001$; US 4SF, $0.95 \pm 0.23, p=0.004$; BIS, $0.75 \pm 0.22$, $p=0.010$; 5 months: US 2SF, $1.03 \pm 0.30, p=0.004$; US 4SF, $1.24 \pm 0.29, p<0.001$; BIS, $0.87 \pm 0.17$, $p<0.001$; 9 months: US 2SF, $1.20 \pm 0.32, p=0.002$; US 4SF, $1.17 \pm 0.33, p=0.004$; BIS, $1.37 \pm 0.39$, $p=0.004$; 12 months: US 2SF, $1.46 \pm 0.32, p<0.001$; US 4SF, $1.55 \pm 0.32, p<0.001$; BIS, $1.42 \pm 0.42$, $p=0.006$ ). Also, larger BW was associated with larger FM only at 5 months and only when measured with BIS $(0.70 \pm 0.25, p=0.014)$.

\subsection{4-H Milk Intake and Feeding Frequency}

A moderate level of agreement (ICC $=0.602[0.339,0.779], p<0.001$ ) was seen between FFQ measured with 24-h MP as meals per 24-h and FFQ self-reported by mothers as hours between meals. Short intervals between feeds were associated with higher self-reported values than 24-h MP values; this effect was not seen with longer intervals between feeds.

FFQ and 24-h MI did not differ by infant sex ( $p \geq 0.54)$. Greater FFQ was associated with larger 24-h MI (24-h MP FFQ: $81.1 \pm 18.5, p<0.001$; self-reported FFQ: $-50.6 \pm 13.3, p=0.003$ ).

\subsection{Longitudinal Changes in Maternal, Breastfeeding and Infant Characteristics}

Maternal weight, BMI, \%FM, FM and FMI decreased significantly between 2 and 12 months (Table S2), while FFM and FFMI did not differ (FFM: $p=0.10$; FFMI: $p=0.076$ ). Over the first year of lactation, maternal adiposity decreased (Figure 2 ) (\%FM: $-2.03 \% \pm 0.59, p=0.001$, month of lactation: $p<0.001$; BMI: $-0.78 \pm 0.24, p=0.002$, month of lactation: $p<0.001$; FMI: $-0.64 \pm 0.18, p<0.001$, month of lactation: $p=0.001$ ), after accounting for the month of lactation as a factor.

FFQ and 24-h MI decreased significantly across the lactation (Table S2).

Infant anthropometrics and both FM and FFM measured with all methods increased significantly as age increased (Table S3). BMI, FFMI determined with US 4SF, FMI determined with US 2SF and FM determined with BIS initially increased and then plateaued, while \%FM and FMI measured with BIS initially increased and then decreased (Figure 3). \%FM measured with US skinfolds and FFMI determined with US 2 SF did not differ significantly (\%FM US 2SF: $p=0.56$; \%FM US 4 SF: $p=0.11$; FFMI US 2SF: $p=0.13$ ). 

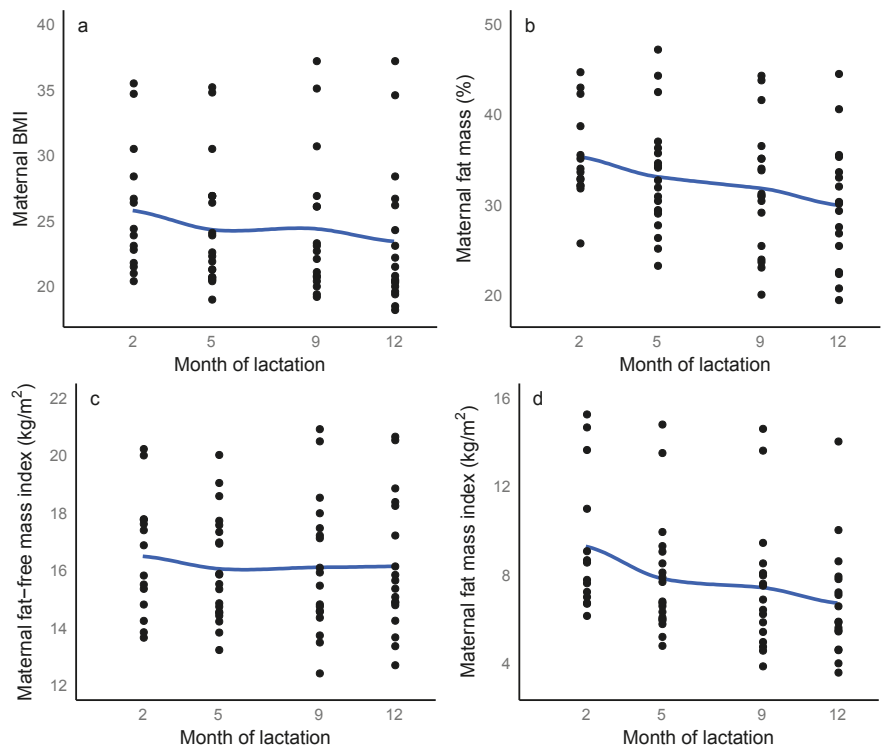

Figure 2. Longitudinal changes in: (a) maternal body mass index (BMI); (b) maternal percentage fat mass, (c) fat-free mass index and (d) fat mass index measured with bioelectrical impedance spectroscopy from 2 to 12 months of lactation. Blue line represents local regression smoother (LOESS), grey areas represent \pm confidence interval, ( $n=14, n=20, n=18, n=18$ at 2, 5, 9 and 12 months respectively).
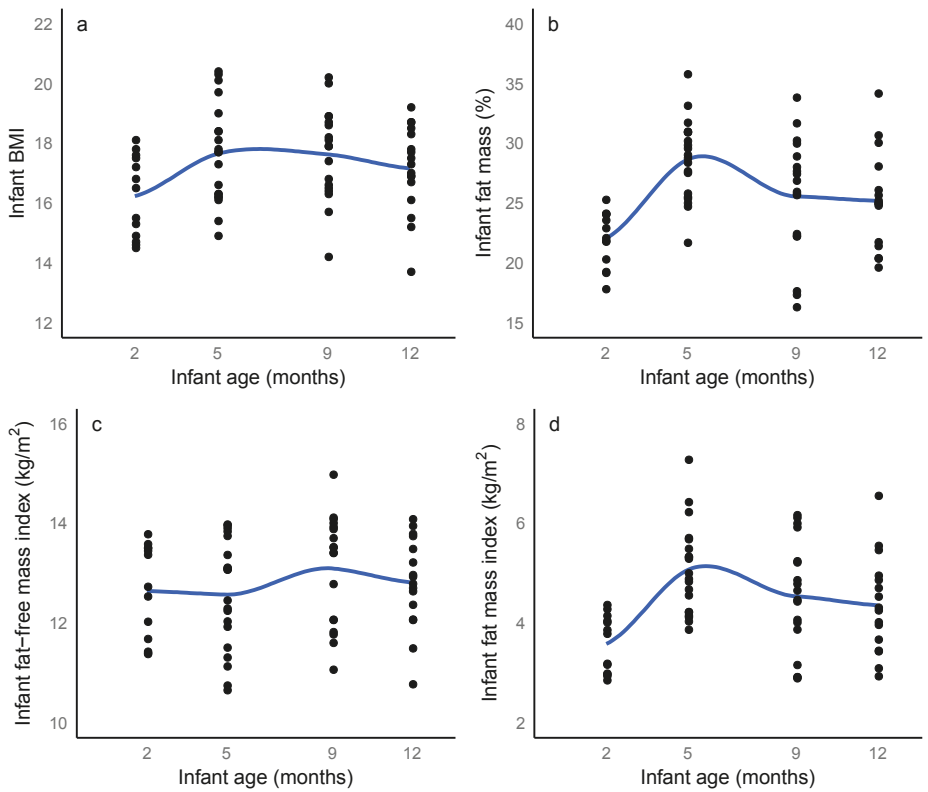

Figure 3. Longitudinal changes in: (a) infant body mass index (BMI); (b) percentage fat mass, (c) infant fat-free mass index and (d) infant fat mass index from 2 to 12 months after birth measured with bioelectrical impedance spectroscopy. Blue line represents local regression smoother (LOESS), grey areas represent \pm confidence interval, ( $n=14, n=20, n=18, n=18$ at 2, 5, 9 and 12 months respectively). 


\subsection{Relationships between Infant and Maternal Body Composition}

Significant negative associations between infant FFM and maternal adiposity were seen after accounting for month after birth and interaction between month after birth and maternal characteristic (Table A1). After adjusting for the false discovery rate, higher maternal BMI was associated with smaller infant FFM measured with both US 2SF ( $p=0.007)$ and US 4SF ( $p=0.010)$ (Figure 4a); greater maternal FM was associated with smaller infant FFM measured with US 2SF ( $p=0.004$ ) (Figure 4b); and greater maternal FMI was associated with smaller infant FFM measured with both US 2SF $(p=0.005)$ and US 4SF ( $p=0.011$ ). There were no other significant associations between the measured maternal and infant BC parameters. No significant interactions between month after birth and maternal predictors were seen.
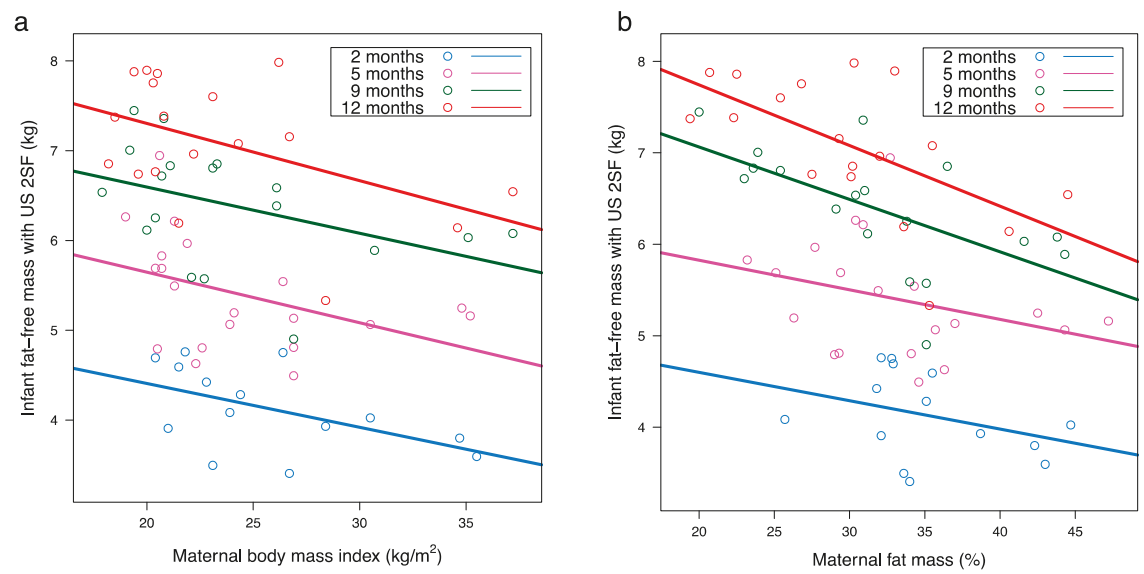

Figure 4. Significant negative associations between: (a) maternal body mass index and infant fat-free mass measured with ultrasound (2 skinfolds; US 2SF); (b) maternal percentage fat mass (\%FM) and infant fat-free mass measured with US 2SF. Lines represent linear regression, one line for each time point ( $n=14, n=20, n=18, n=18$ at 2, 5, 9 and 12 months respectively).

\subsection{Infant Body Composition and Breastfeeding Parameters}

Significant associations between infant BC and feeding parameters (FFQ, 24-h MI) were seen after accounting for the month after birth and interaction between month after birth and feeding parameters (Table A2).

After adjusting for false discovery rate, higher 24-h MI was associated with greater infant FM measured with both US 2SF ( $p=0.004)$ and US 4SF $(p=0.002)$, greater \%FM measured with both US 2SF ( $p=0.008)$ and US 4SF $(p<0.001)$, greater FMI measured with US 2SF $(p=0.001)$ and US 4SF $(p<0.001)$ and lower FFMI measured with US 4SF ( $p=0.015)$ (Table A2, Figure 5).

After adjusting for false discovery rate, longer intervals between feeds (self-reported FFQ) were associated with larger infant FFM (US 2SF: $p=0.001$; US 4SF: $p<0.001$; BIS: $p=0.019$ ) and FFMI (US 2SF: $p=0.013$; US 4SF: $p<0.001$; BIS: $p=0.017$ ) (Table A2, Figure 6). No significant associations were seen for 24-h MP FFQ (meals per $24 \mathrm{~h}$ ).

Significant interactions between breastfeeding parameters and the month after birth were seen for infant BC characteristics (Table A2). 24-h MI and the month after birth: the slope for infant BMI changes from positive ( 5 months) to flat ( 9 months) and then negative (12 months) ( $p=0.018)$ indicating that associations between 24-h MI and infant BMI weakens over the first 12 months of lactation; the slope for infant FFMI measured with US 4SF changes from flat (5 and 9 months) to negative (12 months) ( $p=0.024)$ indicating that associations between 24-h MI and infant FFMI strengthens over the first 12 months of lactation. 24-h MP FFQ and the month after birth: the slope for infant FM measured with 
US 2 SF changes from negative ( 5 months) to positive ( 9 and 12 months) $(p=0.014)$ indicating that associations between FFQ and infant FM strengthens over the first 12 months of lactation.
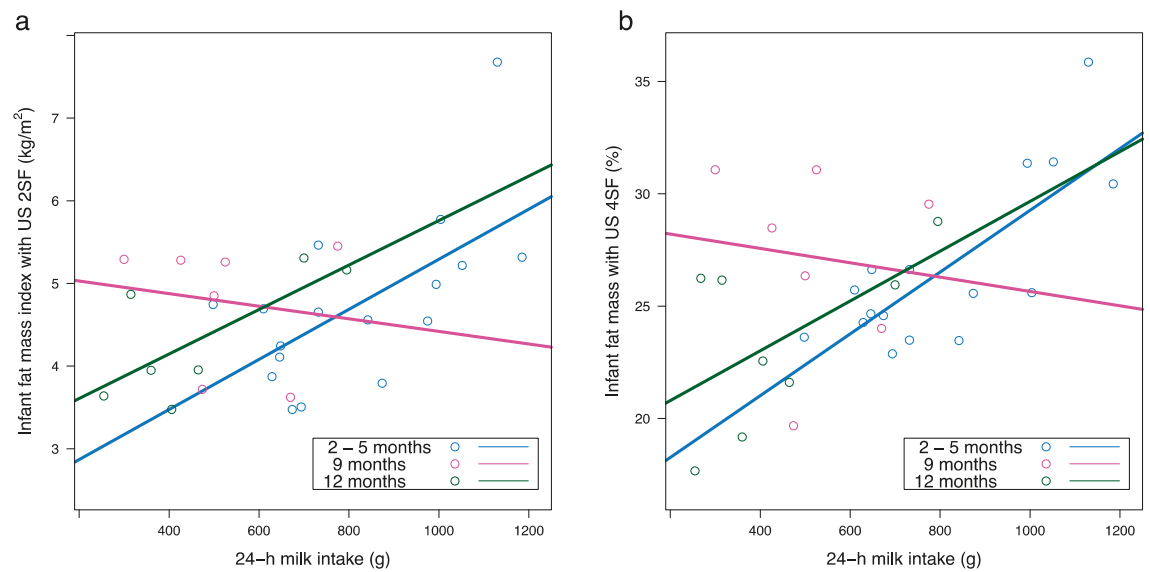

Figure 5. Significant positive associations between: (a) 24-h milk intake and infant fat mass index measured with ultrasound ( 2 skinfolds; US 2SF) $(n=17, n=7, n=7$ between 2 and 5 , and at 9 and 12 months respectively); (b) 24-h milk intake and infant percentage fat mass (\%FM) measured with ultrasound ( 4 skinfolds; US 4SF) ( $n=16, n=7, n=7$ between 2 and 5, and at 9 and 12 months respectively). Lines represent linear regression, one line for each time point.
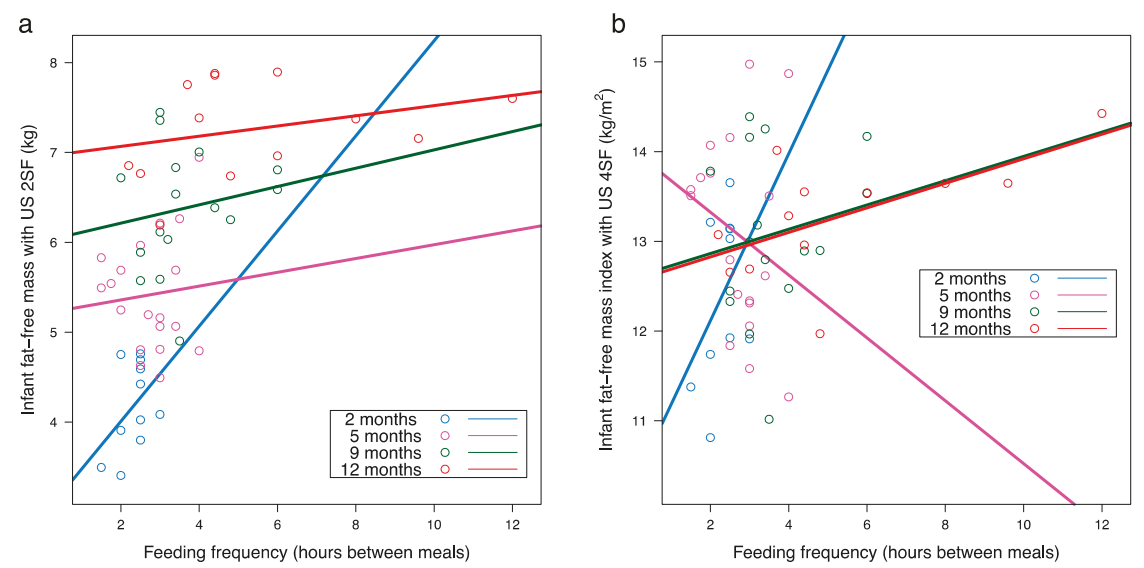

Figure 6. Significant positive associations between: (a) self-reported feeding frequency (hours between feeds) and infant fat-free mass measured with ultrasound (2 skinfolds; US 2SF) ( $n=11, n=19, n=16$, $n=13$ at 2, 5, 9 and 12 months respectively); (b) self-reported feeding frequency and infant fat-free mass index measured with ultrasound (4 skinfolds; US 4SF) ( $n=10, n=18, n=16, n=12$ at 2, 5, 9 and 12 months respectively). Lines represent linear regression, one line for each time point.

After adjusting for false discovery rate, no associations were seen between changes in infant BC and either 24-h MI ( $p \geq 0.0004$ ) or FFQ ( $p \geq 0.001$ for self-reported FFQ, and $p \geq 0.004$ for 24-h MP FFQ) at any practical time points (Tables $\mathrm{A} 3$ and $\mathrm{A} 4$ ). 


\subsection{Maternal Body Composition and Breastfeeding Parameters}

No associations were seen between maternal BC and 24-h MI and both FFQ (24-h MP and self-reported) (BMI: $p \geq 0.45$; FFM: $p \geq 0.51$; FFMI: $p \geq 0.13$; FM: $p \geq 0.82$; FMI: $p \geq 0.69$; \%FM: $p \geq 0.67)$ after accounting for the month after birth $(p<0.001$ for all $)$ and interaction between month after birth and maternal characteristics.

Significant interaction was seen between maternal BMI and the month after birth ( 2 months: reference; 5 months: $-0.03 \pm 0.07 ; 9$ months: $0.01 \pm 0.07 ; 12$ months: $0.76 \pm 0.17, p<0.001$; month after birth: $p<0.001)$ and maternal FFMI and the month after birth ( 2 months: reference; 5 months: $-0.13 \pm 0.16$; 9 months: $0.11 \pm 0.16 ; 12$ months: $1.18 \pm 0.22, p<0.001$; month after birth: $p<0.001)$ for self-reported FFQ indicating that the association between both, BMI and FFMI, and self-reported FFQ (hours between feeds) strengthens over the first 12 months of lactation. No significant interaction with month after birth was seen for other maternal characteristics.

After adjusting for the false discovery rate, no associations were seen between decrease in maternal BMI, FFM, FFMI, FM, \%FM and FMI and either 24-h MI ( $p \geq 0.009$ ) or FFQ (self-reported FFQ: $p \geq 0.014 ; 24$-h MP FFQ: $p \geq 0.068$ ) at any practical time points (Table A5).

\section{Discussion}

The life period spanning from pre-conception to early life is a critical period when appetite control and $\mathrm{BC}$ are programmed and is the greatest window of opportunity for intervention to significantly improve infant outcome. This period is influenced by the maternal factors and early nutrition [5] and breastfeeding can have long-term beneficial health effects at both the individual and population levels [4]. Furthermore, longer duration of breastfeeding is shown to reduce risk for rapid growth patterns in early childhood [59] and attenuate the adverse effects of BW and early weight gain on infant FM gain [27], suggesting dose-dependent effect of breastfeeding on development of infant BC, but the mechanisms of this effect are not fully understood. Our study expands previous research, identifies specific risk factors and critical periods and sheds new light on the mechanisms by which breastfeeding influences infant BC. FFQ and 24-h MI are implicated in development of infant FM while maternal BC is associated with infant FFM, all of these emphasizing the critical role of breastfeeding in programming growth in the first 12 months of life (Figure 7).

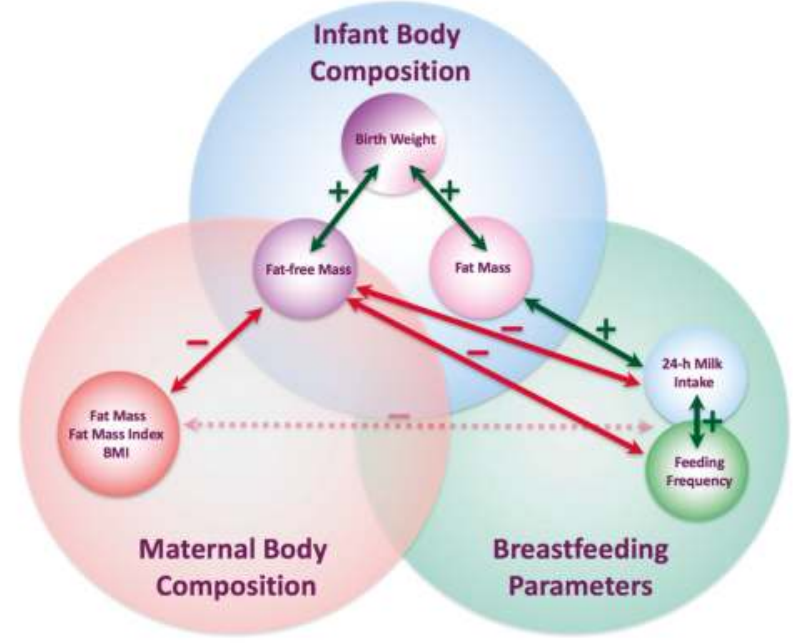

Figure 7. Interconnecting pathways of lactocrine programming of the infant as researched. Green arrows indicate positive associations between measured parameters; red arrows indicate negative associations; red dotted arrow indicates significant interaction terms (negative). BMI—body mass index. 
Milk intake is a major driver of infant growth and here we show a link between infant breastfeeding behavior, as in FFQ, where more frequent feeders consumed more milk and subsequently had greater adiposity (FM, \%FM, FMI) and less lean mass (FFM, FFMI) over the first 12 months of life. This supports a study, in which 24-h MI had no associations with infant weight, but was positively associated with both weight-for-length and weight-for-age [60], suggesting infant BC rather than weight drives this relationship [61]. The lack of association between FFQ and infant 24h-MI in previous studies of the exclusive breastfeeding period is likely due to no consensus on definitions of a breastfeed or meal and non-compliance to demand feeding [31,62]. One cross-sectional study has reported a positive association between FFQ and 24-h MI, however, not all mothers exclusively breastfed for 6 months and study did not account for FFQ in early life [63], which is known to reduce in established lactation [64]. In our study, the association between FFQ and infant adiposity strengthened with duration of lactation, similar to the study, which found that the later breastfeeding was discontinued the more infant \%FM was observed at 6 months [65], further highlighting the importance of breastfeeding in the weaning period during the gradual introduction of food.

It is not fully understood what influences infant FFQ, which generally declines with the duration of lactation [62,64]. We have previously reported that smaller, shorter and leaner (less \%FM), but not younger infants fed more frequently in a cohort of 2 and 5 month-old fully breastfed infants [32] and we have now extended this relationship to FFM, although it is not clear which comes first: are smaller infants in greater demand for nutrients, or is higher FFQ/MI provide more HM components that may regulate/slow down the growth? The results of this study were not uniform between self-reported FFQ and 24-h MP FFQ. This could be explained by the fact that both methods of measuring FFQ have some limitations: self-reported FFQ was shown to be biased towards reporting higher numbers of feeds in frequent feeders compared with 24-h MP FFQ, which itself is limited to one measure at the time point of data collection. Nevertheless, the associations between infant BC and both FFQ and 24-h $\mathrm{MI}$ indicate that, with more comprehensive investigation, these provide a window for adjustment of infant growth rate, during both, exclusive breastfeeding period and specifically from 9 to 12 months, where HM could potentially make a greater contribution to optimal infant growth rate and adiposity (Figure 1).

Previous studies based on maternal BMI $[14,15]$ or BC measured during pregnancy $[23-26,66]$ reported a positive relationship with infant BW. Our study showed no relationship of BW with either maternal BMI or BC, although none of our mothers were categorised as underweight and only $15 \%$ were obese, and correction for multiple comparisons eliminated statistical significance. However, we did find that increased maternal adiposity was related to lower infant FFM over the course of the first year of life whereas only one previous cross-sectional study has found this at 5 to 35 days of life [67]. Therefore, this sustained relationship suggests maintenance of healthy maternal pre-pregnancy and possibly lactational adiposity is beneficial for the development of infant lean body mass. Optimal lean body mass is desirable, since development of obesity is associated with increased FFM [68] and compositional changes of the lean tissue [69]. Further, we show in breastfed infants increased BW is associated with increased FFM throughout the first year of life, which further emphasizes the necessity for maternal BC to remain within the normal range. Interestingly, FFM at birth has also been shown to explain most of the variability in BW [70-72], although this finding is not consistent $[19,73]$.

Previous studies also have linked maternal pre-pregnancy BMI or gestational weight gain to increased infant FM or \%FM either very early in life (birth to 4 months) or later in childhood (2-11 years) $[19,20,67,74,75]$. Mechanistic studies have not been attempted to understand how breastfeeding in early life is related to development of infant adiposity. Similar to one recent longitudinal study that showed no relationship between pre-pregnancy BMI and infant postpartum $\%$ FM during first 6 months [76], we found no relationship of estimated pre-pregnancy/current maternal BC and infant FM, leading us to speculate that breastfeeding may modulate infant FM development, contributing in part to protection against obesity. Further, other studies have not measured breastfeeding parameters and have included formula-fed infants, this combined with the 
historic cohorts including mothers with BMI significantly lower than current cohorts [77] may have influenced their findings.

BW is not a reliable predictor of newborn adiposity [78]. We found no association between BW and infant FM during the first 12 months of life, with the exception of a positive association at 5 months, when measured with BIS only. Similarly, Chomtho et al. have reported positive association between BW and infant FM measured with stable isotope at 3 months [73]. The emergence of positive associations of BW with infant FM only at 3 months [73] and 5 months (our study) in the first year of life might be explained by relative proximity of the measures to the peaking of infant adiposity at around 6-7 months [38,79] and a reduction in the adverse effect of BW and early infant weight gain on FM later in infancy with increased duration of breastfeeding $[27,80]$. Our findings therefore point to maternal $\mathrm{BC}$ being implicated in lean tissue development rather than fat accrual in the infant post birth, while breastfeeding parameters appear to be involved in development of adipose tissue.

Higher FM in infants is usually considered as a negative result [81], however some studies observed \%FM to be consistently higher due to lower FFM in breastfed infants compared to formula-fed [82] which may be related to the neurodevelopmental and cognitive differences between these two groups [83]. Curiously, duration of breastfeeding has been found to associate positively with infant subcutaneous but not visceral fat [76], indicating that breastfeeding may ensure a beneficial adipose phenotype, associated with a reduced risk of NCD and obesity $[76,84]$. It will be necessary to study the development of visceral fat in breastfed infants over time, since it cannot be extrapolated to $\%$ FM [76].

Infant $\mathrm{BC}$ also influenced by the infant sex. In our recent cross-sectional study of 2, 5, 9 and 12 months old infants we showed that \%FM was lower in males than in females [35]. Similar to some longitudinal studies $[85,86]$ and contrary to others $[38,87,88]$, we have seen no difference in adiposity between sexes in this study, although, as expected, we observed that lean mass in males was greater (FFM and FFMI). Larger sample sizes will allow for more robust findings with respect to sex and BC of breastfeeding infants.

Given the evidence confirming that obese mothers experience greater physical difficulties in breastfeeding as well as being at higher risk of not producing adequate volumes of milk [60,61,89-91], one might expect maternal BC to influence breastfeeding parameters. Our study, however, did not find any associations between maternal BC or indicators of milk production (24-h MI) and breastfeeding behaviour (FFQ). This may be because all of our mothers produced enough milk for their infants [31] and that only $15 \%$ of our mothers were obese. Furthermore, infants in our study displayed appropriate patterns of growth according to WHO growth standards [92], with 18 out of 20 infants residing between 15th and 97th weight-for-age centiles over the first year of life, and only three infants crossing two major centiles in downward and one in upward trend from birth to 12 months. Before correction for multiple comparisons however, we found that the reduction in maternal adiposity was associated with higher 24-h MI in the later stages of lactation, these findings are further supported by significant interaction between maternal BMI and the month after birth, which indicated the strengthening of the association between BMI and FFQ at the later stages of lactation. It is biologically plausible that higher FFQ and MI at the later stages of lactation/during weaning may contribute to greater reduction in maternal adiposity due to the energetic demand of lactation. Indeed, exclusive breastfeeding promotes greater maternal \%FM loss than mixed feeding during the early postpartum period [93], and at the later stages of lactation $[56,94]$, with more frequent feeding associating with greater fat reduction at 6 months postpartum [94]. One must be cautious, as FFQ is also related to 24-h MI. These findings support the limited data that suggests that duration of lactation is associated with protection against incidence of obesity, CVD, type 2 diabetes and prevalence of the metabolic syndrome [95], enabled by the mobilization of the stored fat and persisting beyond weaning.

The strength of this proof-of-concept study is the wide variation of adiposity levels among the mothers and that measurements were performed on breastfeeding dyads feeding on demand over a wide period of lactation, from 2 to 12 months. The limitations are the small number of $24-\mathrm{h} \mathrm{MP}$ at the 
later stages of lactation and the modest number of participants as a result of time constraints associated with multiple measurement time points. Our population was predominantly Caucasian term healthy fully breastfed singletons from mothers of higher social-economic status therefore, the results may not be applicable to dyads from other backgrounds. Further analysis including a holistic approach is required to understand multiple levels of breastfeeding programing and regulatory effects. Elucidation of the effect of maternal BC on infant BC via both composition and the quantity of HM will help to understand the intergenerational nature of obesity allowing for the possibility of interventions. These interventions would include maintaining/achieving healthy maternal BC pre-conception and during pregnancy and lactation, as well as supporting breastfeeding from 9 to 12 months and beyond, as outlined in the NHMRC [96] and WHO infant feeding guidelines [97], to improve the outcomes for breastfeeding dyads. An example of an 'intervention' is Norway's promotion of breastfeeding (and extended breastfeeding), which is deployed by overweight/obese mothers as a weight loss strategy, since Norwegian women with greater pre-pregnancy weight concerns are more likely to initiate breastfeeding and breastfeed for longer [98].

\section{Conclusions}

This study found that infant BC was associated with both maternal adiposity and breastfeeding patterns over the first 12 months of lactation. These results confirm that the first year of life is a critical window of infant developmental programming that has the potential for intervention to improve outcomes for the infant, and emphasise the importance of including quantitative measures in order to elucidate the mechanisms by which breastfeeding affects infant BC (Figure 1).

Supplementary Materials: The following are available online at www.mdpi.com/2072-6643/10/1/45/s1, Table S1: Body composition of breastfed infants aged 2 to 12 months calculated with ultrasound skinfolds and bioelectrical impedance equations, Table S2: Significant differences by lactation duration within measured maternal and breastfeeding characteristics, Table S3: Significant differences by age within measured infant characteristics.

Acknowledgments: All authors critically reviewed and approved the final manuscript. This study was supported by an unrestricted research grant from Medela AG (Switzerland). Zoya Gridneva was supported by an Australian Postgraduate Award from The University of Western Australia (Australia). Many thanks are extended to all mothers who participated in this study.

Author Contributions: Zoya Gridneva designed the study, collected the data, conducted data analysis and interpreted results, and wrote the manuscript; Alethea Rea conducted data analysis and interpreted results; Anna R. Hepworth provided consultation for research design and conducted data analysis and interpretation; Ching T. Lai provided technical and analytical support; Leigh C. Ward contributed to the design of the study and provided technical and educational support; Peter E. Hartmann substantially contributed to the conception and design of the study and contributed reagents/materials/analysis tools for research; Donna T. Geddes designed the study, collected the data and interpreted results.

Conflicts of Interest: The authors declare that Medela AG provided an unrestricted research grant to Donna T. Geddes, from which salaries to Donna T. Geddes, Anna R. Hepworth, Ching T. Lai, and Peter E. Hartmann were paid. Medela AG provided a Top-up Scholarship for Zoya Gridneva, and has provided speaker's fees to Donna T. Geddes for educational lectures. The funding sponsors had no role in the design of the study; in the collection, analyses, or interpretation of data; in the writing of the manuscript, or in the decision to publish the results. Author Leigh C. Ward provides consultancy services to ImpediMed Ltd. ImpediMed Ltd. had no involvement in the inception and conducting of this research or in the writing of the manuscript. 


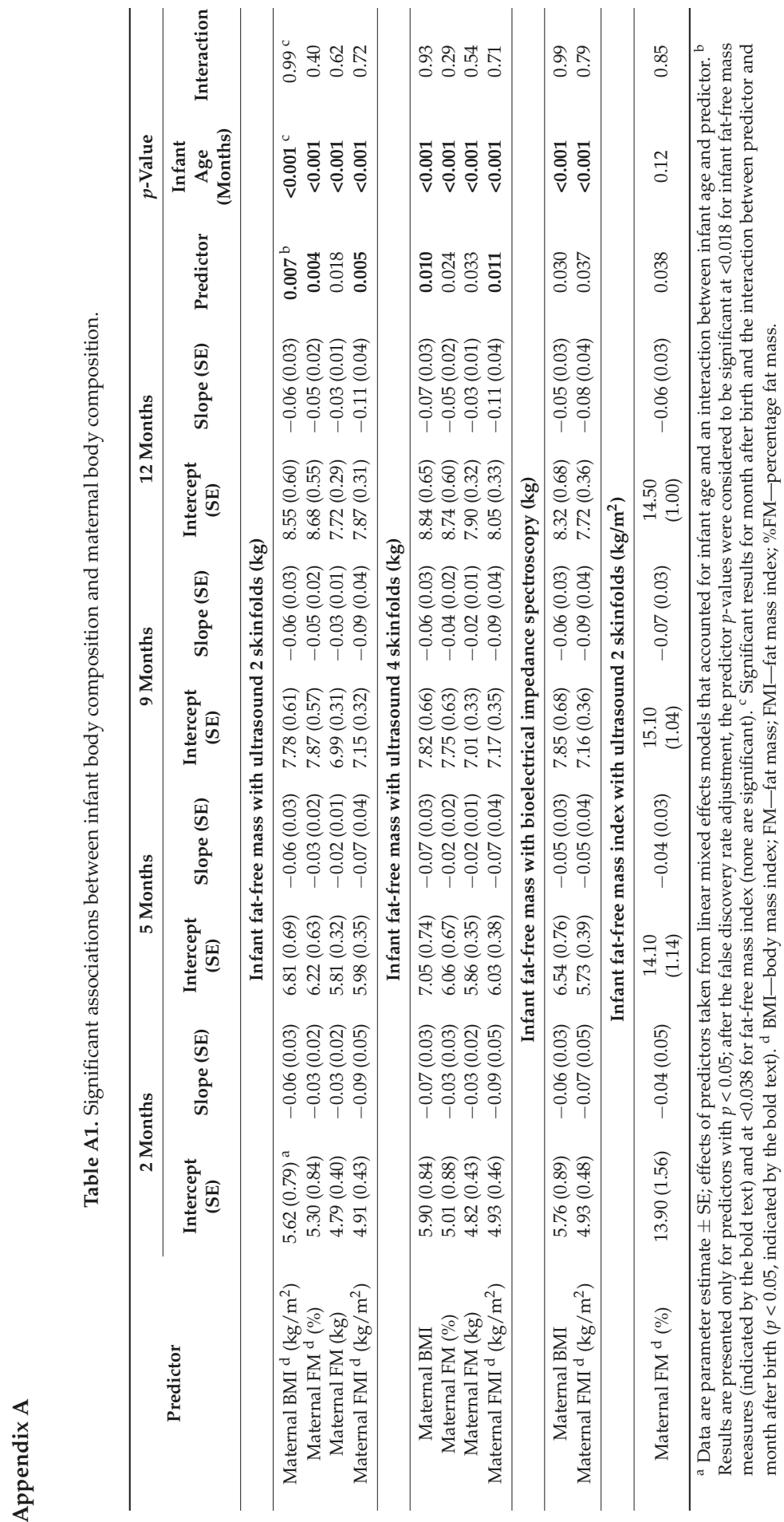




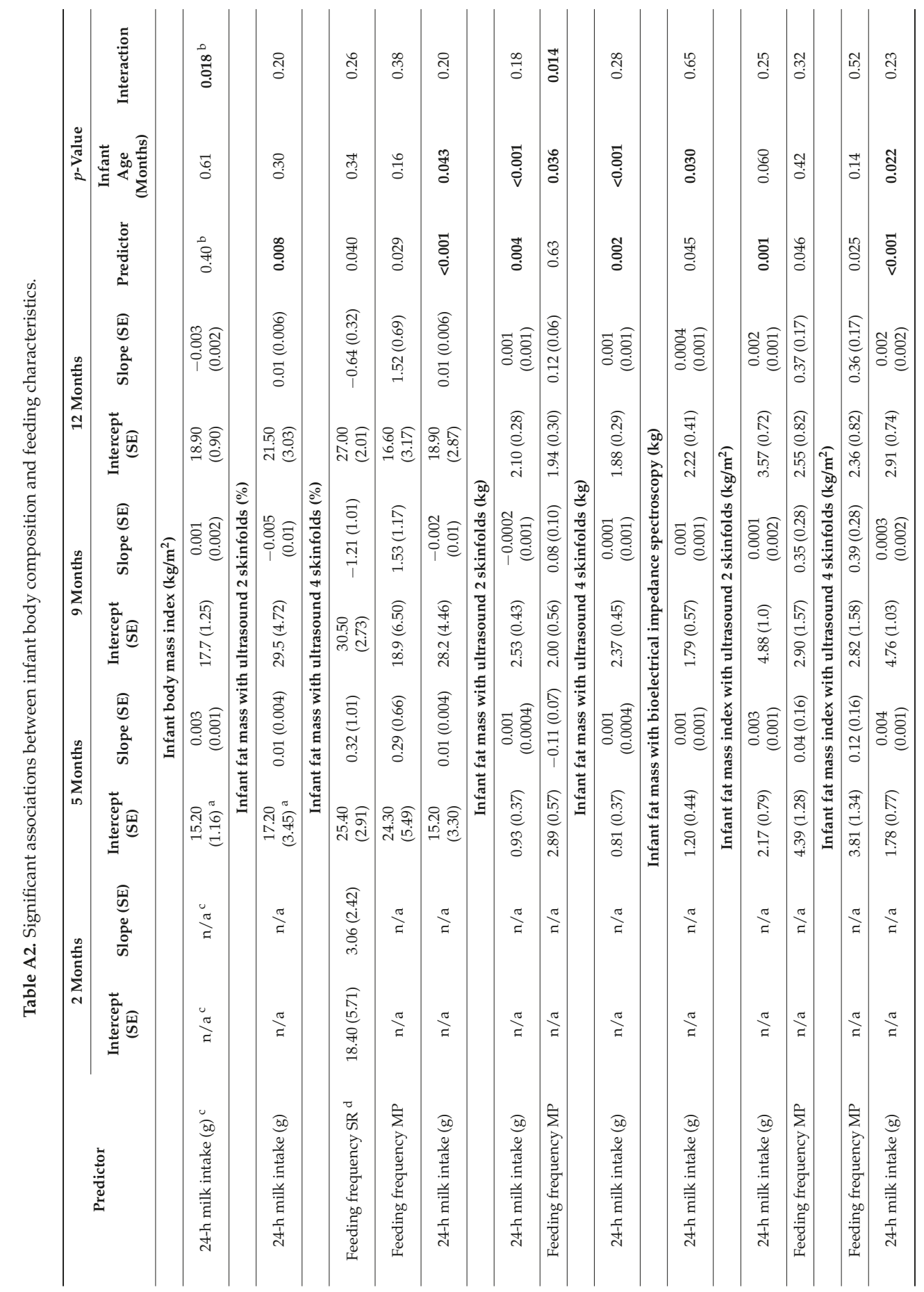




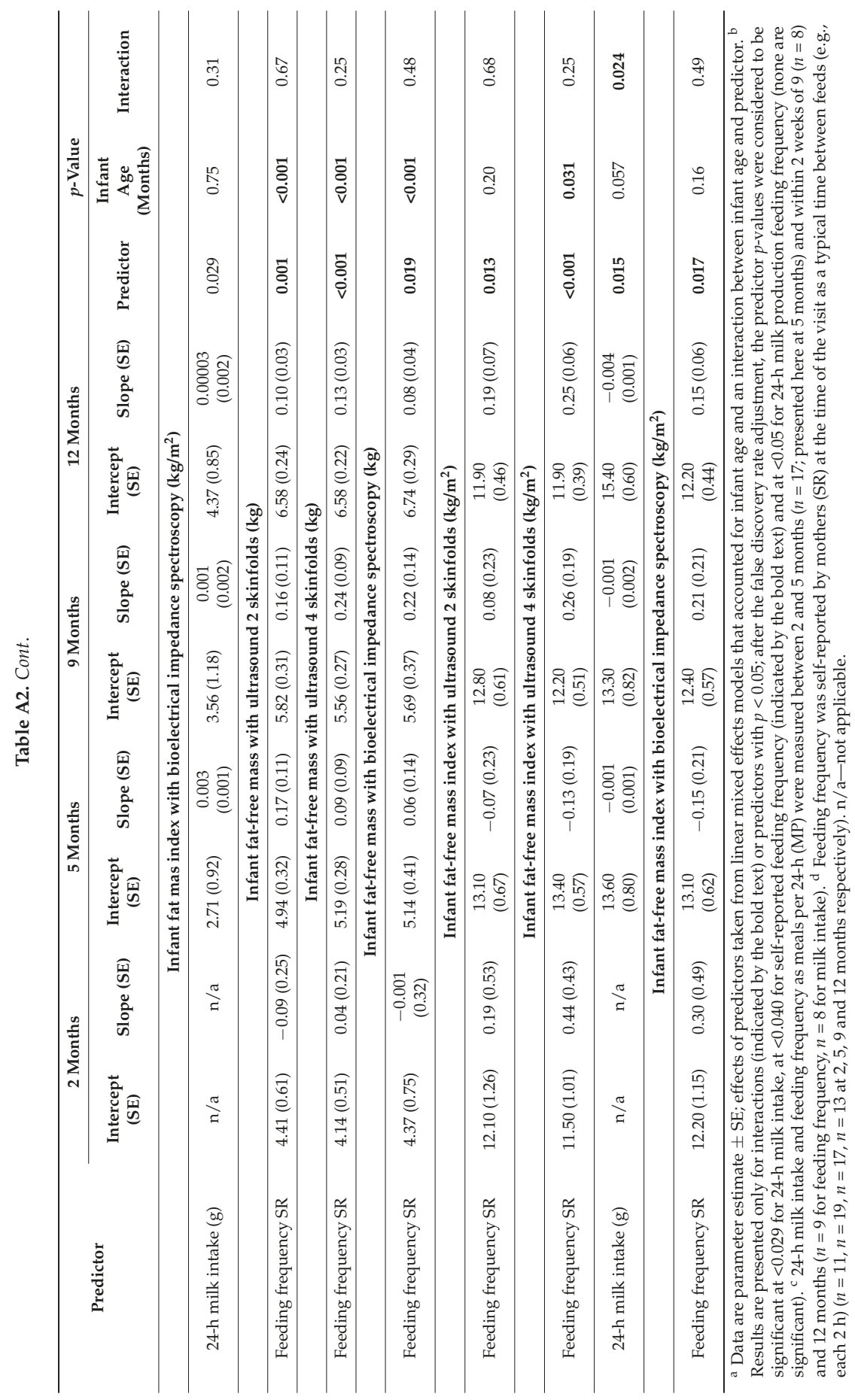


Table A3. Associations between 24-h milk intake at given time points and infant body composition changes between time points.

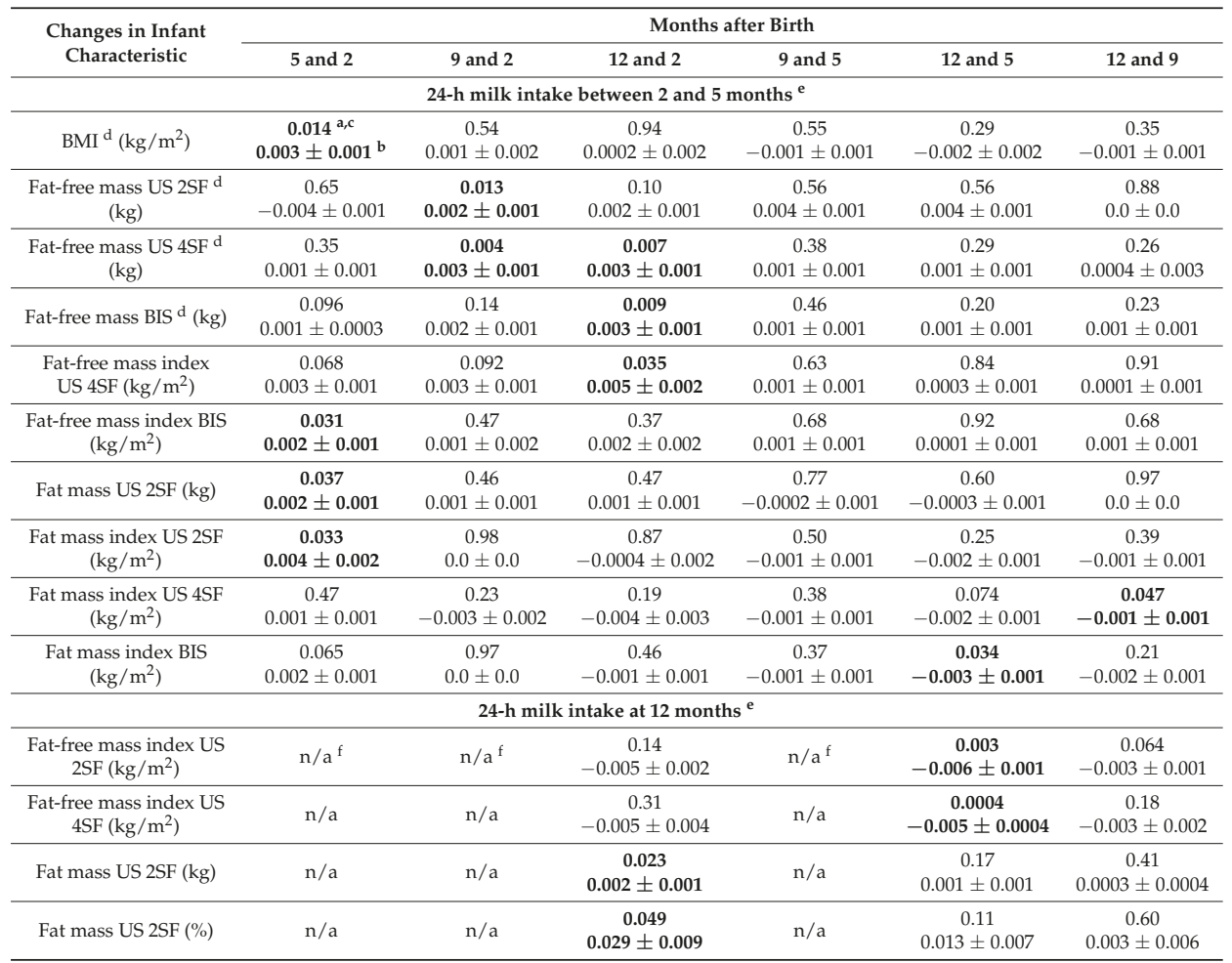

${ }^{\mathrm{a}} p$-values, ${ }^{\mathrm{b}}$ parameter estimates and standard errors of estimate for significant associations between feeding frequency at given time points and the changes in measured variables between different months after birth. ${ }^{c}$ Results are presented only for variables with at least one significant raw $p$-value $(p<0.05$, indicated by the bold text); after the false discovery rate adjustment, the predictor $p$-values were considered to be significant at $<0.0004$ for $24-\mathrm{h}$ milk intake (none are significant). ${ }^{\mathrm{d}}$ BIS —-bioimpedance spectroscopy; BMI—-body mass index; US 2SF-ultrasound 2-skinfolds; US 4SF-ultrasound 4-skinfolds. ${ }^{\text {e }}$ 24-h milk intake was measured at 24-h milk production between 2 and 5 months $(n=17)$ and within 2 weeks of $9(n=8)$ and 12 months $(n=8)$. ${ }^{\mathrm{f}}$ Results are not presented for impractical combinations, $\mathrm{n} / \mathrm{a}-$ not applicable.

Table A4. Associations between feeding frequency at given time points and infant body composition changes between time points.

\begin{tabular}{|c|c|c|c|c|c|c|}
\hline \multirow{2}{*}{$\begin{array}{l}\text { Changes in Infant } \\
\text { Characteristic }\end{array}$} & \multicolumn{6}{|c|}{ Months after Birth } \\
\hline & 5 and 2 & 9 and 2 & 12 and 2 & 9 and 5 & 12 and 5 & 12 and 9 \\
\hline \multicolumn{7}{|c|}{ Self-reported feeding frequency at 2 months ${ }^{e}$} \\
\hline $\mathrm{BMI}^{\mathrm{d}}\left(\mathrm{kg} / \mathrm{m}^{2}\right)$ & $\begin{array}{c}0.83^{\mathrm{a}} \\
0.14 \pm 064^{\mathrm{b}}\end{array}$ & $\begin{array}{c}0.067 \\
-1.95 \pm 0.92\end{array}$ & $\begin{array}{c}0.42 \\
-0.99 \pm 1.15\end{array}$ & $\begin{array}{c}0.013^{c} \\
-2.09 \pm 0.66\end{array}$ & $\begin{array}{c}0.25 \\
-1.13 \pm 0.87\end{array}$ & $\begin{array}{c}0.027 \\
1.03 \pm 0.37\end{array}$ \\
\hline $\begin{array}{c}\text { Fat mass US } 4 S F^{d} \\
(\%)\end{array}$ & $\begin{array}{c}0.55 \\
1.34 \pm 2.14\end{array}$ & $\begin{array}{c}0.058 \\
-7.19 \pm 3.25\end{array}$ & $\begin{array}{c}0.25 \\
-4.67 \pm 3.79\end{array}$ & $\begin{array}{c}0.027 \\
-9.11 \pm 3.37\end{array}$ & $\begin{array}{c}0.13 \\
-5.80 \pm 3.40\end{array}$ & $\begin{array}{c}0.28 \\
3.20 \pm 2.74\end{array}$ \\
\hline $\begin{array}{l}\text { Fat mass US 4SF } \\
(\mathrm{kg})\end{array}$ & $\begin{array}{c}0.37 \\
0.14 \pm 0.15\end{array}$ & $\begin{array}{c}0.12 \\
-0.59 \pm 0.34\end{array}$ & $\begin{array}{c}0.23 \\
-0.44 \pm 0.33\end{array}$ & $\begin{array}{c}0.028 \\
-0.76 \pm 0.28\end{array}$ & $\begin{array}{c}0.076 \\
-0.56 \pm 0.28\end{array}$ & $\begin{array}{c}0.42 \\
0.17 \pm 0.20\end{array}$ \\
\hline $\begin{array}{l}\text { Fat mass index } \\
\text { US } 4 \mathrm{SF}\left(\mathrm{kg} / \mathrm{m}^{2}\right)\end{array}$ & $\begin{array}{c}0.28 \\
0.40 \pm 0.35\end{array}$ & $\begin{array}{c}0.039 \\
-1.73 \pm 0.70\end{array}$ & $\begin{array}{c}0.27 \\
-0.97 \pm 0.81\end{array}$ & $\begin{array}{c}0.009 \\
-2.13 \pm 0.62\end{array}$ & $\begin{array}{c}0.094 \\
-1.35 \pm 0.70\end{array}$ & $\begin{array}{c}0.14 \\
0.83 \pm 0.49\end{array}$ \\
\hline
\end{tabular}


Table A4. Cont.

\begin{tabular}{|c|c|c|c|c|c|c|}
\hline \multirow{2}{*}{$\begin{array}{l}\text { Changes in Infant } \\
\text { Characteristic }\end{array}$} & \multicolumn{6}{|c|}{ Months after Birth } \\
\hline & 5 and 2 & 9 and 2 & 12 and 2 & 9 and 5 & 12 and 5 & 12 and 9 \\
\hline \multicolumn{7}{|c|}{ Self-reported feeding frequency at 9 months ${ }^{e}$} \\
\hline $\begin{array}{c}\text { Fat-free mass } \\
\text { US 4SF (kg) }\end{array}$ & $\mathrm{n} / \mathrm{a}^{\mathrm{g}}$ & $\begin{array}{c}0.055 \\
0.21 \pm 0.10\end{array}$ & $\begin{array}{c}0.25 \\
0.21 \pm 0.17\end{array}$ & $\begin{array}{c}0.003 \\
0.25 \pm 0.07\end{array}$ & $\begin{array}{c}0.13 \\
0.18 \pm 0.11 \\
\end{array}$ & $\begin{array}{c}0.93 \\
-0.01 \pm 0.09\end{array}$ \\
\hline $\begin{array}{l}\text { Fat-free mass index } \\
\text { US } 4 S F\left(\mathrm{~kg} / \mathrm{m}^{2}\right)\end{array}$ & $\mathrm{n} / \mathrm{a}$ & $\begin{array}{c}0.10 \\
0.29 \pm 0.16\end{array}$ & $\begin{array}{c}0.37 \\
0.29 \pm 0.31\end{array}$ & $\begin{array}{c}0.014 \\
0.36 \pm 0.13\end{array}$ & $\begin{array}{c}0.025 \\
0.48 \pm 0.19\end{array}$ & $\begin{array}{c}0.49 \\
0.13 \pm 0.18\end{array}$ \\
\hline \multicolumn{7}{|c|}{ Self-reported feeding frequency at 12 months ${ }^{e}$} \\
\hline $\operatorname{BMI}\left(\mathrm{kg} / \mathrm{m}^{2}\right)$ & $\mathrm{n} / \mathrm{a}^{\mathrm{g}}$ & $\mathrm{n} / \mathrm{a}^{\mathrm{g}}$ & $\begin{array}{c}0.45 \\
0.15 \pm 0.18\end{array}$ & $\mathrm{n} / \mathrm{a}^{\mathrm{g}}$ & $\begin{array}{c}0.12 \\
0.21 \pm 0.12\end{array}$ & $\begin{array}{c}0.044 \\
0.14 \pm 0.06\end{array}$ \\
\hline 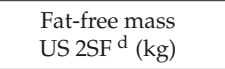 & $\mathrm{n} / \mathrm{a}$ & $\mathrm{n} / \mathrm{a}$ & $\begin{array}{c}0.031 \\
0.13 \pm 0.04\end{array}$ & $\mathrm{n} / \mathrm{a}$ & $\begin{array}{c}0.022 \\
0.12 \pm 0.05\end{array}$ & $\begin{array}{c}0.43 \\
0.03 \pm 0.03\end{array}$ \\
\hline $\begin{array}{l}\text { Fat-free mass } \\
\text { US 4SF (kg) }\end{array}$ & $\mathrm{n} / \mathrm{a}$ & $\mathrm{n} / \mathrm{a}$ & $\begin{array}{c}0.047 \\
0.15 \pm 0.06\end{array}$ & $\mathrm{n} / \mathrm{a}$ & $\begin{array}{c}0.001 \\
0.15 \pm 0.03\end{array}$ & $\begin{array}{c}0.21 \\
0.04 \pm 0.03\end{array}$ \\
\hline $\begin{array}{l}\text { Fat-free mass index } \\
\text { US } 2 S F\left(\mathrm{~kg} / \mathrm{m}^{2}\right)\end{array}$ & $\mathrm{n} / \mathrm{a}$ & $\mathrm{n} / \mathrm{a}$ & $\begin{array}{c}0.024 \\
0.24 \pm 0.07\end{array}$ & $\mathrm{n} / \mathrm{a}$ & $\begin{array}{c}0.020 \\
0.24 \pm 0.09\end{array}$ & $\begin{array}{c}0.052 \\
0.12 \pm 0.06\end{array}$ \\
\hline $\begin{array}{l}\text { Fat-free mass index } \\
\text { US } 4 \mathrm{SF}\left(\mathrm{kg} / \mathrm{m}^{2}\right)\end{array}$ & $\mathrm{n} / \mathrm{a}$ & $\mathrm{n} / \mathrm{a}$ & $\begin{array}{c}0.043 \\
0.31 \pm 0.10 \\
\end{array}$ & $\mathrm{n} / \mathrm{a}$ & $\begin{array}{c}0.003 \\
0.27 \pm 0.07 \\
\end{array}$ & $\begin{array}{c}0.073 \\
0.15 \pm 0.08 \\
\end{array}$ \\
\hline $\begin{array}{l}\text { Fat-free mass index } \\
\text { BIS }^{\mathrm{d}}\left(\mathrm{kg} / \mathrm{m}^{2}\right)\end{array}$ & $\mathrm{n} / \mathrm{a}$ & $\mathrm{n} / \mathrm{a}$ & $\begin{array}{c}0.051 \\
0.25 \pm 0.09\end{array}$ & $\mathrm{n} / \mathrm{a}$ & $\begin{array}{c}0.030 \\
0.16 \pm 0.06 \\
\end{array}$ & $\begin{array}{c}0.37 \\
0.07 \pm 0.07\end{array}$ \\
\hline Fat mass US 4SF (\%) & $\mathrm{n} / \mathrm{a}$ & $\mathrm{n} / \mathrm{a}$ & $\begin{array}{c}0.043 \\
-1.05 \pm 0.39 \\
\end{array}$ & $\mathrm{n} / \mathrm{a}$ & $\begin{array}{c}0.11 \\
-0.67 \pm 0.38 \\
\end{array}$ & $\begin{array}{c}0.44 \\
-0.26 \pm 0.32 \\
\end{array}$ \\
\hline \multicolumn{7}{|c|}{ 24-h MP feeding frequency between 2 and 5 months ${ }^{f}$} \\
\hline $\begin{array}{l}\text { Fat-free mass index } \\
\text { US } 2 \mathrm{SF}\left(\mathrm{kg} / \mathrm{m}^{2}\right)\end{array}$ & $\begin{array}{c}0.028 \\
0.45 \pm 0.17\end{array}$ & $\begin{array}{c}0.16 \\
-0.27 \pm 0.17\end{array}$ & $\begin{array}{c}0.52 \\
-0.17 \pm 0.25\end{array}$ & $\begin{array}{c}0.13 \\
-0.28 \pm 0.17\end{array}$ & $\begin{array}{c}0.29 \\
-0.21 \pm 0.19\end{array}$ & $\begin{array}{c}0.64 \\
0.06 \pm 0.12\end{array}$ \\
\hline $\begin{array}{l}\text { Fat-free mass index } \\
\text { US } 4 S F\left(\mathrm{~kg} / \mathrm{m}^{2}\right)\end{array}$ & $\begin{array}{c}0.012 \\
0.45 \pm 0.13\end{array}$ & $\begin{array}{c}0.51 \\
-0.16 \pm 0.23\end{array}$ & $\begin{array}{c}0.80 \\
-0.09 \pm 0.36\end{array}$ & $\begin{array}{c}0.065 \\
-0.25 \pm 0.12\end{array}$ & $\begin{array}{c}0.63 \\
-0.09 \pm 0.18\end{array}$ & $\begin{array}{c}0.46 \\
0.11 \pm 0.15\end{array}$ \\
\hline Fat mass US 2SF (\%) & $\begin{array}{c}0.39 \\
-1.60 \pm 1.75\end{array}$ & $\begin{array}{c}0.24 \\
1.20 \pm 0.94 \\
\end{array}$ & $\begin{array}{c}0.48 \\
1.34 \pm 1.81 \\
\end{array}$ & $\begin{array}{c}0.012 \\
2.23 \pm 0.78\end{array}$ & $\begin{array}{c}0.068 \\
1.70 \pm 0.86\end{array}$ & $\begin{array}{c}0.21 \\
-0.51 \pm 0.38\end{array}$ \\
\hline Fat mass US 4SF (\%) & $\begin{array}{c}0.23 \\
-0.96 \pm 0.73 \\
\end{array}$ & $\begin{array}{c}0.53 \\
0.93 \pm 1.38 \\
\end{array}$ & $\begin{array}{c}0.62 \\
0.90 \pm 1.73\end{array}$ & $\begin{array}{c}0.010 \\
2.09 \pm 0.69\end{array}$ & $\begin{array}{c}0.12 \\
1.26 \pm 0.77 \\
\end{array}$ & $\begin{array}{c}0.086 \\
-0.76 \pm 0.41\end{array}$ \\
\hline $\begin{array}{l}\text { Fat mass US 2SF } \\
(\mathrm{kg})\end{array}$ & $\begin{array}{c}0.62 \\
-0.08 \pm 0.15\end{array}$ & $\begin{array}{c}0.23 \\
0.14 \pm 0.10\end{array}$ & $\begin{array}{c}0.48 \\
0.13 \pm 0.17\end{array}$ & $\begin{array}{c}0.007 \\
0.21 \pm 0.07 \\
\end{array}$ & $\begin{array}{c}0.033 \\
0.17 \pm 0.07 \\
\end{array}$ & $\begin{array}{c}0.34 \\
-0.04 \pm 0.04\end{array}$ \\
\hline $\begin{array}{l}\text { Fat mass US 4SF } \\
(\mathrm{kg})\end{array}$ & $\begin{array}{c}0.81 \\
-0.01 \pm 0.05 \\
\end{array}$ & $\begin{array}{c}0.24 \\
0.14 \pm 0.11 \\
\end{array}$ & $\begin{array}{c}0.38 \\
0.12 \pm 0.13\end{array}$ & $\begin{array}{c}0.006 \\
0.21 \pm 0.06 \\
\end{array}$ & $\begin{array}{c}0.054 \\
0.14 \pm 0.07\end{array}$ & $\begin{array}{c}0.17 \\
-0.05 \pm 0.04 \\
\end{array}$ \\
\hline $\begin{array}{c}\text { Fat mass index US } \\
2 \mathrm{SF}\left(\mathrm{kg} / \mathrm{m}^{2}\right)\end{array}$ & $\begin{array}{c}0.64 \\
-0.16 \pm 0.32 \\
\end{array}$ & $\begin{array}{c}0.40 \\
0.20 \pm 0.23\end{array}$ & $\begin{array}{c}0.40 \\
0.30 \pm 0.34 \\
\end{array}$ & $\begin{array}{c}0.016 \\
0.44 \pm 0.16\end{array}$ & $\begin{array}{c}0.11 \\
0.33 \pm 0.19\end{array}$ & $\begin{array}{c}0.22 \\
-0.11 \pm 0.08\end{array}$ \\
\hline $\begin{array}{c}\text { Fat mass index US } \\
4 \mathrm{SF}\left(\mathrm{kg} / \mathrm{m}^{2}\right)\end{array}$ & $\begin{array}{c}0.81 \\
-0.03 \pm 0.13\end{array}$ & $\begin{array}{c}0.53 \\
0.19 \pm 0.28\end{array}$ & $\begin{array}{c}0.60 \\
0.20 \pm 0.36\end{array}$ & $\begin{array}{c}0.023 \\
0.42 \pm 0.16\end{array}$ & $\begin{array}{c}0.17 \\
0.26 \pm 0.18\end{array}$ & $\begin{array}{c}0.053 \\
-0.16 \pm 0.08\end{array}$ \\
\hline
\end{tabular}


Table A4. Cont.

\begin{tabular}{|c|c|c|c|c|c|c|}
\hline \multirow{2}{*}{$\begin{array}{c}\text { Changes in Infant } \\
\text { Characteristic }\end{array}$} & \multicolumn{6}{|c|}{ Months after Birth } \\
\hline & 5 and 2 & 9 and 2 & 12 and 2 & 9 and 5 & 12 and 5 & 12 and 9 \\
\hline \multicolumn{7}{|c|}{ 24-h MP feeding frequency at 9 months $f$} \\
\hline $\begin{array}{l}\text { Fat-free mass US } \\
2 \mathrm{SF}(\mathrm{kg})\end{array}$ & $\mathrm{n} / \mathrm{a}^{\mathrm{g}}$ & $\begin{array}{c}0.030 \\
-0.28 \pm 0.05 \\
\end{array}$ & $\begin{array}{c}0.33 \\
-0.32 \pm 0.28 \\
\end{array}$ & $\begin{array}{c}0.013 \\
-0.33 \pm 0.09 \\
\end{array}$ & $\begin{array}{c}0.16 \\
-0.33 \pm 0.20\end{array}$ & $\begin{array}{c}0.85 \\
-0.03 \pm 0.15 \\
\end{array}$ \\
\hline $\begin{array}{l}\text { Fat-free mass index } \\
\text { US } 4 \mathrm{SF}\left(\mathrm{kg} / \mathrm{m}^{2}\right)\end{array}$ & $\mathrm{n} / \mathrm{a}$ & $\begin{array}{c}0.33 \\
-0.45 \pm 0.35 \\
\end{array}$ & $\begin{array}{c}0.43 \\
-0.55 \pm 0.57 \\
\end{array}$ & $\begin{array}{c}0.14 \\
-0.35 \pm 0.19 \\
\end{array}$ & $\begin{array}{c}0.044 \\
-0.68 \pm 0.23 \\
\end{array}$ & $\begin{array}{c}0.15 \\
-0.35 \pm 0.20 \\
\end{array}$ \\
\hline \multicolumn{7}{|c|}{ 24-h MP feeding frequency at 12 months ${ }^{f}$} \\
\hline $\begin{array}{l}\text { Fat-free mass US } \\
2 \mathrm{SF}(\mathrm{kg})\end{array}$ & $\mathrm{n} / \mathrm{a}^{\mathrm{g}}$ & $\mathrm{n} / \mathrm{a}^{\mathrm{g}}$ & $\begin{array}{c}0.029 \\
-0.23 \pm 0.06 \\
\end{array}$ & $\mathrm{n} / \mathrm{a}^{\mathrm{g}}$ & $\begin{array}{c}0.097 \\
-0.20 \pm 0.11 \\
\end{array}$ & $\begin{array}{c}0.55 \\
-0.05 \pm 0.07 \\
\end{array}$ \\
\hline $\begin{array}{l}\text { Fat-free mass index } \\
\text { US } 2 \mathrm{SF}\left(\mathrm{kg} / \mathrm{m}^{2}\right)\end{array}$ & $\mathrm{n} / \mathrm{a}$ & $\mathrm{n} / \mathrm{a}$ & $\begin{array}{c}0.070 \\
-0.41 \pm 0.12 \\
\end{array}$ & $\mathrm{n} / \mathrm{a}$ & $\begin{array}{c}0.037 \\
-0.48 \pm 0.18 \\
\end{array}$ & $\begin{array}{c}0.45 \\
-0.13 \pm 0.15 \\
\end{array}$ \\
\hline Fat mass US 2SF (\%) & $\mathrm{n} / \mathrm{a}$ & $\mathrm{n} / \mathrm{a}$ & $\begin{array}{c}0.13 \\
2.00 \pm 0.97\end{array}$ & $\mathrm{n} / \mathrm{a}$ & $\begin{array}{c}0.004 \\
2.12 \pm 0.52 \\
\end{array}$ & $\begin{array}{c}0.96 \\
0.03 \pm 0.61\end{array}$ \\
\hline $\begin{array}{l}\text { Fat mass US 2SF } \\
(\mathrm{kg})\end{array}$ & $\mathrm{n} / \mathrm{a}$ & $\mathrm{n} / \mathrm{a}$ & $\begin{array}{c}0.090 \\
0.15 \pm 0.06\end{array}$ & $\mathrm{n} / \mathrm{a}$ & $\begin{array}{c}0.009 \\
0.19 \pm 0.05\end{array}$ & $\begin{array}{c}0.90 \\
0.01 \pm 0.04\end{array}$ \\
\hline $\begin{array}{c}\text { Fat mass index US } \\
2 \mathrm{SF}\left(\mathrm{kg} / \mathrm{m}^{2}\right)\end{array}$ & $\mathrm{n} / \mathrm{a}$ & $\mathrm{n} / \mathrm{a}$ & $\begin{array}{c}0.30 \\
0.24 \pm 0.17\end{array}$ & $\mathrm{n} / \mathrm{a}$ & $\begin{array}{c}0.015 \\
0.41 \pm 0.12\end{array}$ & $\begin{array}{c}0.75 \\
-0.04 \pm 0.11\end{array}$ \\
\hline
\end{tabular}

${ }^{\mathrm{a}} p$-values, ${ }^{\mathrm{b}}$ parameter estimates and standard errors of estimate for significant associations between feeding frequency at given time points and the changes in measured variables between different months after birth. ${ }^{c}$ Results are presented only for variables with at least one significant raw $p$-value $(p<0.05$, indicated by the bold text); after the false discovery rate adjustment, the predictor $p$-values were considered to be significant at $<0.001$ for self-reported feeding frequency, and $<0.004$ for $24-\mathrm{h}$ milk production feeding frequency (none are significant). d BIS-bioimpedance spectroscopy; BMI-body mass index; MP-milk production; US 2SF-ultrasound 2-skinfolds; US 4SF-ultrasound 4-skinfolds. ${ }^{e}$ Feeding frequency was self-reported by mothers at the time of the visit as a typical time between feeds (e.g., each $2 \mathrm{~h}$ ) ( $n=11, n=19, n=17, n=13$ at 2, 5, 9 and 12 months respectively). ${ }^{\mathrm{f}}$ Feeding frequency as meals per 24-h was measured at 24-h milk production between 2 and 5 months (presented at 5 months here, $n=17)$ and within 2 weeks of $9(n=8)$ and 12 months $(n=9)$. ${ }^{8}$ Results are not presented for impractical combinations, $\mathrm{n} / \mathrm{a}$ - not applicable.

Table A5. Associations between breastfeeding parameters at given time points and maternal body composition changes between time points.

\begin{tabular}{|c|c|c|c|c|c|c|}
\hline \multirow{2}{*}{$\begin{array}{c}\text { Changes in Maternal } \\
\text { Characteristic }\end{array}$} & \multicolumn{6}{|c|}{ Months after Birth } \\
\hline & 5 and 2 & 9 and 2 & 12 and 2 & 9 and 5 & 12 and 5 & 12 and 9 \\
\hline \multicolumn{7}{|c|}{ 24-h milk intake at 9 months ${ }^{e}$} \\
\hline Fat mass BIS ${ }^{d}(\%)$ & $\mathrm{n} / \mathrm{a}^{\mathrm{g}}$ & $\begin{array}{c}0.075^{\mathrm{a}} \\
0.04 \pm 0.01^{\mathrm{b}}\end{array}$ & $\begin{array}{c}0.33 \\
0.01 \pm 0.01\end{array}$ & $\begin{array}{c}0.042^{\mathrm{c}} \\
-0.01 \pm 0.004\end{array}$ & $\begin{array}{c}0.013 \\
-0.02 \pm 0.005\end{array}$ & $\begin{array}{c}0.16 \\
-0.007 \pm 0.004 \\
\end{array}$ \\
\hline Fat mass BIS $(\mathrm{kg})$ & $\mathrm{n} / \mathrm{a}$ & $\begin{array}{c}0.026 \\
0.04 \pm 0.01\end{array}$ & $\begin{array}{c}0.28 \\
0.01 \pm 0.01\end{array}$ & $\begin{array}{c}0.10 \\
-0.006 \pm 0.003\end{array}$ & $\begin{array}{c}0.011 \\
-0.01 \pm 0.003\end{array}$ & $\begin{array}{c}0.16 \\
-0.005 \pm 0.003\end{array}$ \\
\hline $\begin{array}{l}\text { Fat mass index BIS } \\
\left(\mathrm{kg} / \mathrm{m}^{2}\right)\end{array}$ & $\mathrm{n} / \mathrm{a}$ & $\begin{array}{c}0.020 \\
0.02 \pm 0.002\end{array}$ & $\begin{array}{c}0.26 \\
0.003 \pm 0.002\end{array}$ & $\begin{array}{c}0.11 \\
-0.002 \pm 0.001\end{array}$ & $\begin{array}{c}0.009 \\
-0.004 \pm 0.001\end{array}$ & $\begin{array}{c}0.15 \\
-0.002 \pm 0.001\end{array}$ \\
\hline
\end{tabular}


Table A5. Cont.

\begin{tabular}{|c|c|c|c|c|c|c|}
\hline \multirow{2}{*}{$\begin{array}{c}\text { Changes in Maternal } \\
\text { Characteristic }\end{array}$} & \multicolumn{6}{|c|}{ Months after Birth } \\
\hline & 5 and 2 & 9 and 2 & 12 and 2 & 9 and 5 & 12 and 5 & 12 and 9 \\
\hline \multicolumn{7}{|c|}{ Self-reported feeding frequency at 12 months ${ }^{f}$} \\
\hline $\mathrm{BMI}{ }^{\mathrm{d}}\left(\mathrm{kg} / \mathrm{m}^{2}\right)$ & $\mathrm{n} / \mathrm{a}^{\mathrm{g}}$ & $\mathrm{n} / \mathrm{a}^{\mathrm{g}}$ & $\begin{array}{c}0.025 \\
0.15 \pm 0.05\end{array}$ & $\mathrm{n} / \mathrm{a} \mathrm{g}$ & $\begin{array}{c}0.96 \\
-0.003 \pm 0.05\end{array}$ & $\begin{array}{c}0.74 \\
0.02 \pm 0.05 \\
\end{array}$ \\
\hline Fat mass BIS (\%) & $\mathrm{n} / \mathrm{a}$ & $\mathrm{n} / \mathrm{a}$ & $\begin{array}{c}0.048 \\
0.38 \pm 0.15\end{array}$ & $\mathrm{n} / \mathrm{a}$ & $\begin{array}{c}0.83 \\
0.07 \pm 0.32\end{array}$ & $\begin{array}{c}0.63 \\
0.10 \pm 0.20\end{array}$ \\
\hline Fat mass BIS $(\mathrm{kg})$ & $\mathrm{n} / \mathrm{a}$ & $\mathrm{n} / \mathrm{a}$ & $\begin{array}{c}0.020 \\
0.38 \pm 0.11\end{array}$ & $\mathrm{n} / \mathrm{a}$ & $\begin{array}{c}0.71 \\
0.08 \pm 0.20\end{array}$ & $\begin{array}{c}0.59 \\
0.08 \pm 0.15\end{array}$ \\
\hline $\begin{array}{l}\text { Fat mass index BIS } \\
\qquad\left(\mathrm{kg} / \mathrm{m}^{2}\right)\end{array}$ & $\mathrm{n} / \mathrm{a}$ & $\mathrm{n} / \mathrm{a}$ & $\begin{array}{c}0.014 \\
0.12 \pm 0.03\end{array}$ & $\mathrm{n} / \mathrm{a}$ & $\begin{array}{c}0.78 \\
0.02 \pm 0.07\end{array}$ & $\begin{array}{c}0.58 \\
0.03 \pm 0.05\end{array}$ \\
\hline
\end{tabular}

${ }^{\mathrm{a}} p$-values, ${ }^{\mathrm{b}}$ parameter estimates and standard errors of estimate for significant associations between breastfeeding parameters at given time points and the changes in measured variables between different months after birth. ${ }^{c}$ Results are presented only for variables with at least one significant raw $p$-value $(p<0.05$; indicated by the bold text; none were significant for 24-h milk production feeding frequency); after the false discovery rate adjustment, the predictor $p$-values were considered to be significant at $<0.009$ for 24 -h milk intake and $<0.014$ for self-reported feeding frequency (none are significant). ${ }^{\mathrm{d}}$ BIS—bioimpedance spectroscopy; BMI—body mass index. ${ }^{\mathrm{e}}$ 24-h milk intake was measured at 24-h milk production between 2 and 5 months $(n=17)$ and within 2 weeks of $9(n=8)$ and 12 months $(n=8) .{ }^{\mathrm{f}}$ Feeding frequency was self-reported by mothers at the time of the visit as a typical time between feeds (e.g., each $2 \mathrm{~h}$ ) $\left(n=11, n=19, n=17, n=13\right.$ at 2, 5, 9 and 12 months respectively). ${ }^{g}$ Results are not presented for impractical combinations, $\mathrm{n} / \mathrm{a}-$ not applicable.

\section{References}

1. Geddes, D.; Prescott, S. Developmental origins of health and disease: The role of human milk in preventing disease in the 21(st) century. J. Hum. Lact. 2013, 29, 123-127. [CrossRef] [PubMed]

2. Prescott, S.L. Early nutrition as a major determinant of 'immune health': Implications for allergy, obesity and other noncommunicable diseases. In Preventive Aspects of Early Nutrition; Fewtrell, M.S., Haschke, F., Prescott, S.L., Eds.; Vevey/S. Kager AG: Basel, Switzerland, 2016; Volume 85, pp. 1-17.

3. Socha, P.; Hellmuth, C.; Gruszfeld, D.; Demmelmair, H.; Rzehak, P.; Grote, V.; Weber, M.; Escribano, J.; Closa-Monasterolo, R.; Dain, E.; et al. Endocrine and metabolic biomarkers predicting early childhood obesity risk. In Preventive Aspects of Early Nutrition; Fewtrell, M.S., Haschke, F., Prescott, S.L., Eds.; Vevey/S. Kager AG: Basel, Switzerland, 2016; Volume 85, pp. 81-88.

4. Kelishadi, R.; Farajian, S. The protective effects of breastfeeding on chronic non-communicable diseases in adulthood: A review of evidence. Adv. Biomed. Res. 2014, 3, 3:1-3:5. [CrossRef] [PubMed]

5. Godfrey, K.M.; Costello, P.M.; Lillycrop, K.A. Development, epigenetics and metabolic programming. In Preventive Aspects of Early Nutrition; Fewtrell, M.S., Haschke, F., Prescott, S.L., Eds.; Vevey/S. Kager AG: Basel, Switzerland, 2016; Volume 85, pp. 71-80.

6. Wells, J.C.; Chomoto, S.; Fewtrell, M.S. Programming of body composition by early growth and nutrition. Proc. Nutr. Soc. 2007, 66, 423-434. [CrossRef] [PubMed]

7. Sievers, E.; Oldigs, H.D.; Santer, R.; Schaub, J. Feeding patterns in breast-fed and formula-fed infants. Ann. Nutr. Metab. 2002, 46, 243-248. [CrossRef] [PubMed]

8. Savino, F.; Liguori, S.A.; Fissore, M.F.; Oggero, R. Breast milk hormones and their protective effect on obesity. Int. J. Pediatr. Endocrinol. 2009, 2009, 327505:1-327505:8. [CrossRef]

9. Bartok, C. Babies fed breastmilk by breast versus by bottle: A pilot study evaluating early growth patterns. Breastfeed. Med. 2011, 6, 117-124. [CrossRef] [PubMed]

10. Luque, V.; Closa-Monasterolo, R.; Escribano, J.; Ferre, N. Early programming by protein intake: The effect of protein on adiposity development and the growth and functionality of vital organs. Nutr. Metab. Insights 2015, 8, 49-56. [CrossRef] [PubMed]

11. Butte, N.; Wong, W.; Hopkinson, J.; Smith, E.; Ellis, K. Infant feeding mode affects early growth and body composition. Pediatrics 2000, 16, 1355-1366. [CrossRef]

12. Kent, J.; Mitoulas, L.; Cox, D.B.; Owens, R.; Hartmann, P. Breast volume and milk production during extended lactation in women. Exp. Physiol. 1999, 84, 435-447. [CrossRef] [PubMed] 
13. Mitoulas, L.R.; Kent, J.C.; Cox, D.B.; Owens, R.A.; Sherriff, J.L.; Hartmann, P.E. Variation in fat, lactose and protein in human milk over $24 \mathrm{~h}$ and throughout the first year of lactation. Br. J. Nutr. 2002, 88, $29-37$. [CrossRef] [PubMed]

14. Leddy, M.A.; Power, M.L.; Schulkin, J. The impact of maternal obesity on maternal and fetal health. Rev. Obstet. Gynecol. 2008, 1, 170-178. [PubMed]

15. Nahar, S.; Mascie-Taylor, C.G.; Begum, H.A. Maternal anthropometry as a predictor of birth weight. Public Health Nutr. 2007, 10, 965-970. [CrossRef] [PubMed]

16. Yu, Z.; Han, S.; Zhu, J.; Sun, X.; Ji, C.; Guo, X. Pre-pregnancy body mass index in relation to infant birth weight and offspring overweight/obesity: A systematic review and meta-analysis. PLoS ONE 2013, 8, e61627. [CrossRef] [PubMed]

17. Rossner, S.; Ohlin, A. Maternal body weight and relation to birth weight. Acta Obstet. Gynaecol Scand. 1990, 69, 475-478. [CrossRef]

18. Moussa, H.N.; Alrais, M.A.; Leon, M.G.; Abbas, E.L.; Sibai, B.M. Obesity epidemic: Impact from preconception to postpartum. Future Sci. OA 2016, 2, FSO137:1-FSO137:12. [CrossRef] [PubMed]

19. Sewell, M.F.; Huston-Presley, L.; Super, D.M.; Catalano, P. Increased neonatal fat mass, not lean body mass, is associated with maternal obesity. Am. J. Obstet. Gynecol. 2006, 195, 1100-1103. [CrossRef] [PubMed]

20. Castillo-Laura, H.; Santos, I.S.; Quadros, L.C.M.; Matijasevich, A. Maternal obesity and offspring body composition by indirect methods: A systematic review and meta-analysis. Cad. Saude Publica 2015, 31, 2073-2092. [CrossRef] [PubMed]

21. Kugananthan, S.; Gridneva, Z.; Lai, C.T.; Hepworth, A.R.; Mark, P.J.; Kakulas, F.; Geddes, D.T. Associations between maternal body composition and appetite hormones and macronutrients in human milk. Nutrients 2017, 9, 252. [CrossRef] [PubMed]

22. Fields, D.A.; Schneider, C.R.; Pavela, G. A narrative review of the associations between six bioactive components in breast milk and infant adiposity. Obesity 2016, 24, 1213-1221. [CrossRef] [PubMed]

23. Kent, E.; O’Dwyer, V.; Fattah, C.; Farah, N.; O'Connor, C.; Turner, M.J. Correlation between birth weight and maternal body composition. Obstet. Gynecol. 2013, 121, 46-50. [CrossRef] [PubMed]

24. O'Connor, C.; O'Higgins, A.; Segurado, R.; Turner, M.J.; Stuart, B.; Kennelly, M.M. Maternal body composition and birth weight. Prenat. Diagn. 2014, 34, 605-607. [PubMed]

25. Larciprete, G.; Valensise, H.; Vasapollo, B.; Di Pierro, G.; Menghini, S.; Magnani, F.; De Lorenzo, A.; Arduini, D. Maternal body composition at term gestation and birth weight: Is there a link? Acta Diabetol. 2003, 40, 222-224. [CrossRef] [PubMed]

26. Sanin Aguirre, L.H.; Reza-Lopez, S.; Levario-Carrillo, M. Relation between maternal body composition and birth weight. Biol. Neonate 2004, 86, 55-62. [CrossRef] [PubMed]

27. Ejlerskov, K.T.; Christensen, L.B.; Ritz, C.; Jensen, S.M.; Molgaard, C.; Michaelsen, K.F. The impact of early growth patterns and infant feeding on body composition at 3 years of age. Br. J. Nutr. 2015, 114, 316-327. [CrossRef] [PubMed]

28. Holliday, M.A. Metabolic rate and organ size during growth from infancy to maturity and during late gestation and early infancy. Pediatrics 1971, 47, 169-179.

29. Elia, M. Organ and tissue contribution to metabolic rate. In Energy Metabolism. Tissue Determinants and Cellular Corollaries; Kinney, J.M., Tucker, H.N., Eds.; Raven: New York, NY, USA, 1992; pp. 61-79.

30. Dewey, K.; Heinig, M.; Nommsen, L.; Lonnerdal, B. Maternal versus infant factors related to breast milk intake and residual milk volume: The DARLING study. Pediatrics 1991, 87, 829-837. [PubMed]

31. Kent, J.C.; Mitoulas, L.R.; Cregan, M.D.; Ramsay, D.T.; Doherty, D.A.; Hartmann, P.E. Volume and frequency of breastfeedings and fat content of breast milk throughout the day. Pediatrics 2006, 117, e387-e395. [CrossRef] [PubMed]

32. Gridneva, Z.; Kugananthan, S.; Hepworth, A.R.; Tie, W.J.; Lai, C.T.; Ward, L.C.; Hartmann, P.E.; Geddes, D.T. Effect of human milk appetite hormones, macronutrients, and infant characteristics on gastric emptying and breastfeeding patterns of term fully breastfed infants. Nutrients 2017, 9, 15. [CrossRef] [PubMed]

33. Binns, C.W.; Fraser, M.L.; Lee, A.H.; Scott, J. Defining exclusive breastfeeding in Australia. J. Paediatr. Child Health 2009, 45, 174-180. [CrossRef] [PubMed]

34. Gridneva, Z.; Hepworth, A.; Ward, L.; Lai, C.T.; Hartmann, P.; Geddes, D.T. Bioimpedance spectroscopy in the infant: Effect of milk intake and extracellular fluid reservoirs on resistance measurements in term breastfed infants. Eur. J. Clin. Nutr. 2016, 70, 843-851. [CrossRef] [PubMed] 
35. Gridneva, Z.; Hepworth, A.R.; Ward, L.C.; Lai, C.T.; Hartmann, P.E.; Geddes, D.T. Determinants of body composition in breastfed infants using bioimpedance spectroscopy and ultrasound skinfolds-Methods comparison. Pediatr. Res. 2016, 81, 423-433. [CrossRef] [PubMed]

36. Lingwood, B.E.; van Leeuwen, A.M.S.; Carberry, A.E.; Fitzgerald, E.C.; Callaway, L.K.; Colditz, P.B.; Ward, L.C. Prediction of fat-free mass and percentage of body fat in neonates using biolelectrical impedance analysis and anthropometric measures: Validation against Pea Pod. Br. J. Nutr. 2012, 107, 1545-1552. [CrossRef] [PubMed]

37. Bocage, C. Impedance Measurements of Body Composition in Children. Master's Thesis, University of West Indies, Kingston, Jamaica, 1988.

38. Butte, N.; Hopkinson, J.; Wong, W.; Smith, E.; Ellis, K. Body composition during the first 2 years of life: An updated reference. Pediatr. Res. 2000, 47, 578-585. [CrossRef] [PubMed]

39. McLeod, G.; Geddes, D.; Nathan, E.; Sherriff, J.; Simmer, K.; Hartmann, P. Feasibility of using ultrasound to measure preterm body composition and to assess macronutrient influences on tissue accretion rates. Early Hum. Dev. 2013, 89, 577-582. [CrossRef] [PubMed]

40. Ulbricht, L.; Neves, E.; Ripka, W.; Romaneli, E. Comparison between Body Fat Measurements Obtained by Portable Ultrasound and Caliper in Young Adults. In Proceedings of the 2012 Annual International Conference of the IEEE Engineering in Medicine and Biology Society (EMBS), San Diego, CA, USA, 28 August-1 September 2012; pp. 1952-1955.

41. Slaughter, M.; Lohman, T.; Boileau, R.; Horswill, C.; Stillman, R.; Van Loan, M.; Bemben, D. Skinfold equations for estimation of body fatness in children and youth. Hum. Biol. 1988, 60, 709-723. [PubMed]

42. Brook, C. Determination of body composition of children from skinfold measurements. Arch. Dis. Child. 1971, 46, 182-184. [CrossRef] [PubMed]

43. Lohman, T. Body composition in children and youth. In Advances in Pediatric Sport Sciences; Boileau, R.A., Ed.; Human Kinetics Publishers: Champaign, IL, USA, 1984; pp. $29-57$.

44. Van Itallie, T.B.; Yang, M.U.; Heymsfield, S.B.; Funk, R.C.; Boileau, R.A. Height-normalized indices of the body's fat-free mass and fat mass: Potentially useful indicators of nutritional status. Am. J. Clin. Nutr. 1990, 52, 953-959.

45. Arthur, P.; Hartmann, P.; Smith, M. Measurement of the milk intake of breast-fed infants. J. Pediatr. Gastroenterol. Nutr. 1987, 6, 758-763. [CrossRef] [PubMed]

46. R Core Team. R: A Language and Environment for Statistical Computing; R Foundation for Statistical Computing: Vienna, Austria, 2017.

47. Pinheiro, J.B.; Bates, D.; DebRoy, S.; Sarkar, D.; R Development Core Team. nlme: Linear and Nonlinear Mixed Effects Models; R Package Version 3.1-131; R Foundation for Statistical Computing: Vienna, Austria, 2017.

48. Bates, D.; Maechler, M.; Bolker, B.; Walker, S. Lme4: Linear Mixed-Efects Models Using Eigen and S4; R Package Version 1.1-7; R Foundation for Statistical Computing: Vienna, Austria, 2014.

49. Fox, J.; Weisberg, S. An R Companion to Applied Regression; Sage: Thousand Oaks, CA, USA, 2011.

50. Gamer, M.; Lemon, J.; Fellows, I.; Singh, P. Various Coefficients of Interrater Reliability and Agreement; R Package Version 0.84; R Foundation for Statistical Computing: Vienna, Austria, 2012.

51. Hothorn, T.; Bretz, F.; Westfall, P. Simultaneous inference in general parametric models. Biom. J. 2008, 50, 346-363. [CrossRef] [PubMed]

52. Wickham, H. ggplot2: Elegant Graphics for Data Analysis; Springer: New York, NY, USA, 2009.

53. Faul, F.; Erdfelder, E.; Buchner, A.; Lang, A.-G. Statistical power analyses using G*Power 3.1: Tests for correlation and regression analyses. Behav. Res. Methods 2009, 41, 1149-1160. [CrossRef] [PubMed]

54. Diggle, P.J.; Heagerty, P.J.; Liang, K.-Y.; Zeger, S.L. Analysis of Longitudinal Data; Oxford University Press Inc.: New York, NY, USA, 2002.

55. Institute of Medicine; Subcommittee on Nutrition During Lactation. Nutrition During Lactation; National Academy Press: Washington, DC, USA, 1991.

56. Nommsen, L.A.; Lovelady, C.A.; Heinig, M.; Lonnerdal, B.; Dewey, K.G. Determinants of energy, protein, lipid, and lactose concentrations in human milk during the first 12 mo of lactation: The DARLING study. Am. J. Clin. Nutr. 1991, 53, 457-465. [PubMed]

57. Curran-Everett, D. Multiple comparisons: Philosophies and illustrations. Am. J. Physiol. Regul. Integr. Comp. Physiol. 2000, 279, R1-R8. [CrossRef] [PubMed] 
58. Gallagher, D.; Heymsfield, S.B.; Heo, M.; Jebb, S.A.; Murgatroyd, P.R.; Sakamoto, Y. Healthy percentage body fat ranges: An approach for developing guidelines based on body mass index. Am. J. Clin. Nutr. 2000, 72, 694-701. [PubMed]

59. Rzehak, P.; Oddy, W.H.; Mearin, M.L.; Grote, V.; Mori, T.A.; Szajewska, H.; Shamir, R.; Koletzko, S.; Weber, M.; Beilin, L.J.; et al. Infant feeding and growth trajectory patterns in childhood and body composition in young adulthood. Am. J. Clin. Nutr. 2017, 106, 568-580. [CrossRef] [PubMed]

60. Nazlee, N.; Bilal, R.; Latif, Z.; Bluck, L. Maternal body composition and its relationship to infant breast milk intake in rural Pakistan. Food Nutr. Sci. 2011, 2, 932-937. [CrossRef] [PubMed]

61. Galpin, L.; Thakwalakwa, C.; Phuka, J.; Ashorn, P.; Maleta, K.; Wong, W.W.; Manary, M.J. Breast milk intake is not reduced more by the introduction of energy dense complementary food than by typical infant porridge. J. Nutr. 2007, 137, 1828-1833. [PubMed]

62. Butte, N.F.; Wills, C.; Jean, C.A.; Smith, E.O.; Garza, C. Feeding patterns of exclusively breast-fed infants during the first four months of life. Early Hum. Dev. 1985, 12, 291-300. [CrossRef]

63. Rattigan, S.; Ghisalberti, A.V.; Hartmann, P.E. Breast-milk production in Australian women. Br. J. Nutr. 1981, 45, 243-249. [CrossRef] [PubMed]

64. Kent, J.; Hepworth, A.; Sherriff, J.; Cox, D.; Mitoulas, L.; Hartmann, P. Longitudinal changes in breastfeeding patterns from 1 to 6 months of lactation. Breastfeed. Med. 2013, 8, 401-407. [CrossRef] [PubMed]

65. Ay, L.; Van Houten, V.A.; Steegers, E.A.; Hofman, A.; Witteman, J.C.; Jaddoe, V.W.; Hokken-Koelega, A.C. Fetal and postnatal growth and body composition at 6 months of age. J. Clin. Endocrinol. Metab. 2009, 94, 2023-2030. [CrossRef] [PubMed]

66. Forsum, E.; Lof, M.; Olausson, H.; Olhager, E. Maternal body composition in relation to infant birth weight and subcutaneous adipose tissue. Br. J. Nutr. 2006, 96, 408-414. [CrossRef] [PubMed]

67. Hull, H.R.; Dinger, M.K.; Knehans, A.W.; Thompson, D.M.; Fields, D.A. Impact of maternal body mass index on neonate birthweight and body composition. Am. J. Obstet. Gynecol. 2008, 198, 416.e411-416.e416. [CrossRef] [PubMed]

68. Dulloo, A.G.; Jacquet, J.; Miles-Chan, J.L.; Schutz, Y. Passive and active roles of fat-free mass in the control of energy intake and body composition regulation. Eur. J. Clin. Nutr. 2017, 71, 353-357. [CrossRef] [PubMed]

69. Haroun, D.; Wells, J.C.K.; Williams, J.E.; Fuller, N.J.; Fewtrell, M.S.; Lawson, M.S. Composition of the fat-free mass in obese and nonobese children: Matched case-control analyses. Int. J. Obes. 2005, 29, 29-36. [CrossRef] [PubMed]

70. Catalano, P.M.; Tyzbir, E.D.; Allen, S.R.; McBean, J.H.; McAuliffe, T.L. Evaluation of fetal growth by estimation of neonatal body composition. Obstet. Gynecol. 1992, 79, 46-50. [PubMed]

71. Singh, K.A.; Huston-Presley, L.P.; Mencin, P.; Thomas, A.; Amini, S.B.; Catalano, P.M. Birth weight and body composition of neonates born to Caucasian compared with African-American mothers. Obstet. Gynecol. 2010, 115, 998-1002. [CrossRef] [PubMed]

72. Andersen, G.S.; Girma, T.; Wells, J.C.K.; Kastel, P.; Michaelsen, K.F.; Friis, H. Fat and fat-free mass at birth: Air displacement plethysmography measurements on 350 Ethiopian newborns. Pediatr. Res. 2011, 70, 501-506. [CrossRef] [PubMed]

73. Chomtho, S.; Wells, J.C.K.; Davies, P.S.; Lucas, A.; Fewtrell, M.S. Early growth and body composition in infancy. In Early Nutrition Programming and Health Outcomes in Later Life. Advances in Experimental Medicine and Biology; Koletzko, B., Decsi, T., Molnar, D., de la Hunty, A., Eds.; Springer: Dordrecht, The Netherlands, 2009; Volume 646, pp. 165-168.

74. Andres, A.; Hull, H.R.; Shankar, K.; Casey, P.H.; Cleves, M.A.; Badger, T.M. Longitudinal body composition of children born to mothers with normal weight, overweight, and obesity. Obesity 2015, 23, 1252-1258. [CrossRef] [PubMed]

75. Silliman, K.; Kretchmer, N. Maternal obesity and body composition of the neonate. Biol. Neonate 1995, 68, 384-393. [CrossRef] [PubMed]

76. Breij, L.M.; Abrahamse-Berkeveld, M.; Acton, D.; De Lucia Rolfe, E.; Ong, K.K.; Hokken-Koelega, A.C.S. Impact of early infant growth, duration of breastfeeding and maternal factors on total body fat mass and visceral fat at 3 and 6 months of age. Ann. Nutr. Metab. 2017, 71, 203-210. [CrossRef] [PubMed]

77. Badon, S.E.; Dyer, A.R.; Josefson, J.L.; HAPO Study Coopwrative Research Group. Gestational weight gain and neonatal adiposity in the hyperglycemia and adverse pregnancy outcome study-North American region. Obesity 2014, 22, 1731-1738. [CrossRef] [PubMed] 
78. Anne, D.; O’Higgins, A.; O'Connor, C.; Mullaney, L.; Bennet, A.M.; Sheridan-Pereira, M.; Turner, M.J. PS-275 The relationship between infant birth weight and neonatal body composition. Arch. Dis. Child. 2014, 99, A211. [CrossRef]

79. Fomon, S.; Nelson, S. Body composition of the male and female reference infants. Ann. Rev. Nutr. 2002, 22, 1-17. [CrossRef] [PubMed]

80. Jensen, S.M.; Ritz, C.; Ejlerskov, K.; Molgaard, C.; Michaelsen, K.F. Infant BMI peak, breastfeeding, and body composition at age 3 years. Am. J. Clin. Nutr. 2015, 101, 319-325. [CrossRef] [PubMed]

81. Roy, S.M.; Spivack, J.G.; Faith, M.S.; Chesi, A.; Mitchell, J.A.; Kelly, A.; Grant, S.F.; McCormack, S.E.; Zemel, B.S. Infant BMI or weight-for-length and obesity risk in early childhood. Pediatrics 2016, 137, e20153492:1-e20153492:9. [CrossRef] [PubMed]

82. Gale, C.; Logan, K.; Santhakumaran, S.; Parkinson, J.; Hyde, M.; Modi, N. Effect of breastfeeding compared with formula feeding on infant body composition: A systematic review and meta-analysis. Am. J. Clin. Nutr. 2012, 95, 656-669. [CrossRef] [PubMed]

83. Lee, H.; Park, H.; Ha, E.; Hong, Y.-C.; Ha, M.; Park, H.; Kim, B.-N.; Lee, B.; Lee, S.-J.; Lee, K.Y.; et al. Effect of breastfeeding duration on cognitive development in infants: 3-year follow-up study. J. Korean Med. Sci. 2016, 31, 579-584. [CrossRef] [PubMed]

84. Booth, A.; Magnuson, A.; Foster, M. Detrimental and protective fat: Body fat distribution and its relation to metabolic disease. Horm. Mol. Biol. Clin. Investig. 2014, 17, 13-27. [CrossRef] [PubMed]

85. Roggero, P.; Gianni, M.; Orsi, A.; Piemontese, P.; Amato, O.; Liotto, N.; Morlacchi, L.; Taroni, F.; Fields, D.; Catalano, P.; et al. Quality of growth in exclusively breast-fed infants in the first six months of life: An Italian study. Pediatr. Res. 2010, 68, 542-544. [CrossRef] [PubMed]

86. Gilchrist, J. Body Composition Reference Data for Exclusively Breast-Fed Infants. In Proceedings of the Pediatric Academic Society Annual Meeting, Toronto, ON, Canada, 5-7 May 2007.

87. Carberry, A.; Golditz, P.; Lingwood, B. Body composition from birth to 4.5 months in infants born to non-obese women. Pediatr. Res. 2010, 68, 84-88. [CrossRef] [PubMed]

88. Gilchrist, J.; Andres, A.; Badger, T. Body composition of infants fed breast-milk, milk-based formula or soy-based formula during the first 6 months of life. Obesity 2008, 16, 434.

89. Twigger, A.J.; Hepworth, A.R.; Lai, C.T.; Chetwynd, E.; Stuebe, A.M.; Blancafort, P.; Hartmann, P.E.; Geddes, D.T.; Kakulas, F. Gene expression in breastmilk cells is associated with maternal and infant characteristics. Sci. Rep. 2015, 5, 12933:1-12933:14. [CrossRef] [PubMed]

90. Lepe, M.; Bacardi Gascon, M.; Castaneda-Gonzalez, L.M.; Perez Morales, M.E.; Jimenez Cruz, A. Effect of maternal obesity on lactation: Systematic review. Nutr. Hosp. 2011, 26, 1266-1269. [PubMed]

91. Villalpando, S.F.; Butte, N.F.; Wong, W.W.; Flores-Huerta, S.; Hernandez-Beltran, M.J.; Smith, E.O.; Garza, C. Lactation performance of rural Mesoamerindians. Eur. J. Clin. Nutr. 1992, 46, 337-348. [PubMed]

92. World Health Organization. Child Growth Standards: Weight-for-Age Pecentiles. Available online: http: //www.who.int/childgrowth/standards/weight_for_age/en/ (accessed on 21 December 2017).

93. Hatsu, I.E.; McDougald, D.M.; Anderson, A.K. Effect of infant feeding on maternal body composition. Int. Breastfeed. J. 2008, 3, 18:1-18:8. [CrossRef] [PubMed]

94. Quandt, S.A. Changes in maternal postpartum adiposity and infant feeding patterns. Am. J. Phys. Anthropol. 1983, 60, 455-461. [CrossRef] [PubMed]

95. Stuebe, A.M.; Rich-Edwards, J.W. The reset hypothesis: Lactation and maternal metabolism. Am. J. Perinatol. 2009, 26, 81-88. [CrossRef] [PubMed]

96. National Health and Medical Research Council. Infant Feeding Guidelines Information for Health Workers; National Health and Medical Research Council: Canberra, Australia, 2012.

97. World Health Organization. Planning Guide for National Implementation of the Global Strategy for Infant and Young Child Feeding; World Health Organization: Geneva, Switzerland, 2007.

98. Han, S.-Y.; Brewis, A.A. Influence of weight concerns on breastfeeding: Evidence from the Norwegian mother and child cohort study. Am. J. Hum. Biol. 2017, e23086:1-e23086:4. [CrossRef] [PubMed]

(C) 2018 by the authors. Licensee MDPI, Basel, Switzerland. This article is an open access article distributed under the terms and conditions of the Creative Commons Attribution (CC BY) license (http:/ / creativecommons.org/licenses/by/4.0/). 
Article

\title{
Preterm Infant Feeding: A Mechanistic Comparison between a Vacuum Triggered Novel Teat and Breastfeeding
}

\author{
Donna Geddes ${ }^{1, *}$, Chooi Kok ${ }^{2,3}$, Kathryn Nancarrow ${ }^{2,3}$, Anna Hepworth ${ }^{1}$ and \\ Karen Simmer ${ }^{2,3}$ \\ 1 School of Molecular Sciences, Faculty of Science, The University of Western Australia, Perth, WA 6009, \\ Australia; arhepworth@westnet.com.au \\ 2 Centre for Neonatal Research and Education, School of Medicine, Faculty of Health and Medical Sciences, \\ The University of Western Australia, Perth, WA 6009, Australia; Chooi.Kok@health.wa.gov.au (C.K.); \\ Nancarrow.Kathryn@health.wa.gov.au (K.N.); Karen.Simmer@health.wa.gov.au (K.S.) \\ 3 The Neonatal Directorate, King Edward Memorial Hospital, Perth, WA 6008, Australia \\ * Correspondence: donna.geddes@uwa.edu.au; Tel.: +61-8-64887006
}

Received: 12 February 2018; Accepted: 15 March 2018; Published: 19 March 2018

\begin{abstract}
The goal for preterm infants is to achieve full oral feeds quickly and ultimately progress to full breastfeeding. Supplementary oral feeds are often given when the mother is not available to breastfeed. Bottles typically deliver milk in a different fashion compared to breastfeeding, which is thought to hamper transition to full breastfeeding. The aim of this study was to compare the sucking dynamics of preterm infants fed at the breast to feeding with an experimental novel teat (NT) designed to release milk only upon the application of vacuum. Simultaneous ultrasound imaging of the infant oral cavity and measurement of intra-oral vacuum was performed during a breastfeed and a feed with the NT. Test weighs were used to measure milk intake. Linear mixed effects models were performed to investigate differences by feed type, and simultaneous linear regression was performed to investigate individual patterns. Tongue movement was not different between breastfeeding and the NT. Intra-oral vacuums (median (interquartile range: IQR)) were significantly lower with the NT (Baseline vacuum: $-5.8 \mathrm{mmHg}(-11.0,0.1)$; Peak: $40.0 \mathrm{mmHg}(-54.6,-27.1)$ ) compared to breastfeeding (Baseline: $-31.1 \mathrm{mmHg}(-60.0,-12.7)$; Peak: $-106.2 \mathrm{mmHg}(-153.0,-65.5))$. Milk intake was significantly higher with the NT $(33 \mathrm{~mL}(22.5,42.5))$ compared to the breastfeed $(12 \mathrm{~mL}$ $(3,15.5))$. The novel teat encouraged a similar tongue action to breastfeeding, and infants transferred a greater volume of milk with the novel teat. Intra-oral vacuums were lower in strength with the novel teat compared to the breast. Use of the novel teat for the training of sucking dynamics in preterm infants has the potential to improve breastfeeding success and requires further investigation.
\end{abstract}

Keywords: infant; feeding; preterm; premature; bottle; human milk; breastfeeding; nipple shield; infant feeding

\section{Introduction}

The importance of human milk (HM) for the preterm infant cannot be understated. In addition to protecting the infant from infection, which can often be life threatening for these infants, and providing nutrients for growth, several studies have linked HM with improved long-term health outcomes. Most recently, donor HM-fed preterm infants have been shown to have better cardiac morphology in adulthood compared to those fed high protein formulas, and these differences potentially increase the likelihood of better cardiopulmonary outcomes later in life [1]. Further, improved neurodevelopment [2,3] later in life has been documented in this population despite predominantly HM-fed preterm infants having 
slower growth rates than those that are formula-fed [4]. Predominant HM feeds in the first 28 days of life has been linked to a greater volume of deep gray matter volume at term along with higher IQ, working memory, academic achievement, and motor function at seven years of age in infants born less than 30 weeks gestation [5].

These important benefits of HM often overshadow the development of the oro-facial structures due to the necessity for provision of adequate infant nutrition. Preterm infant nutrition is a tenuous balance of type of nutrition, fortification, mode of delivery, and volume of feeds the infant can tolerate. As such, the mechanics of feeding or the delivery of milk are unfortunately largely underemphasized. The bones of the skull and face are malleable in early life, and stresses or lack of stress impacts bone growth [6]. Indeed, there are suggestions that extrinsic factors such as changes in head shape due to infant position and orotracheal intubation $[7,8]$ can alter both palate size and shape, with the potential to affect the entire nasomaxillary complex, particularly in the preterm infant. Further, intrinsic factors such as under nutrition, endocrine anomalies, central nervous system impairments, metabolic dysfunction, and particular medications may impact development of the nasomaxillary complex [9].

Thus, the positive associations of HM and breastfeeding with both short- and long-term health outcomes as well as better development of infant structure and form [10] underpins the goal of the Neonatal Intensive Care Unit (NICU) to facilitate full breastfeeding. Unfortunately, achievement of this goal is hampered by the immaturity of the infant as well as infant co-morbidities such as bronchopulmonary dysplasia, gastrointestinal surgeries, sepsis, neurological impairment, and growth [11], which is further compounded by a range of feed competence between infants [12]. Since discharge of the infant home is also a major goal of the NICU due to evidence suggesting preterm infant health is improved [13], achievement of full oral feeds that are both safe and efficient is imperative. Independent oral feeding is a requirement for discharge home, and often, this may be the last milestone the infant met prior to discharge [14]. More rapid discharge can be achieved by supplementing infants with expressed HM milk via a bottle whilst breastfeeding is being established [12], and this strategy is commonly employed in many units due to infant health, availability of the mother to breastfeed, and economic constraints.

In the pursuit of full breastfeeding in the preterm infant, one must recognise that marked differences in the delivery of milk exist between breastfeeding and bottle-feeding. During bottle-feeding, milk is constantly available to the infant. However, during breastfeeding, milk is only available at milk ejection, and during this transient period, milk flow rates vary markedly. Milk flow rates are important as preterm infants may not be mature enough to regulate milk flow and co-ordinate sucking, swallowing, and breathing at the same time [15]. High flow rates require the infant to swallow frequently to clear the milk from the oral cavity and increase the risk of aspiration [16]. A bottle designed for term infants to release milk only when the infant sucks (applies vacuum; Calma, Medela AG, Baar, Switzerland) has been shown to have a similar tongue action, suck-swallow-breathe patterns, and equivalent heart rate and oxygen saturation to breastfeeding [17]. It was speculated that a similar bottle design for preterm infants would encourage a similar tongue action to breastfeeding when intra-oral vacuum was applied.

The aim of this study was to compare the sucking dynamics of preterm infants fed at the breast and with an experimental novel teat (NT) designed to release milk only upon the application of vacuum and stimulate a tongue movement similar to that of breast feeding.

\section{Materials and Methods}

\subsection{Participants}

A convenience sample of 17 mothers and infants (birth gestation age: 23.6-33.3 weeks; post menstrual age (PMA) 32.7-39.9 weeks) whose mothers intended to breastfeed were examined in the special care nurseries of King Edward Memorial Hospital for Women (KEMH), Perth between 1 August 2011 and 30 June 2012. These participants were part of a randomized controlled trial [18] to assess the 
efficacy of a novel teat designed compared to a conventional teat to meet the needs of the developing preterm infant (Australian New Zealand Clinical Trials Registry, ACTRN12614000875606 [19]).

Inclusion criteria were infants of gestational age (GA) 25 to 34 weeks whose mothers intended to breastfeed and who required $75 \%$ enteral feeds by intragastric tube with the remainder provided by intravenous fluids. Exclusion criteria were congenital anomalies, grade 4 intra-cerebral hemorrhage, and periventricular leukomalacia and oral anomalies (for example, ankyloglossia, cleft palate) [18].

The study period was from 1 August 2011 to 30 June 2012. Breastfeeds were timed when the mother was available to feed and the infant could attach to the breast. 15/17 infants therefore were monitored during a breastfeed prior to the NT as it is policy not to introduce bottle feeds until after an infant has demonstrated the ability to attach and suckle at the breast. Monitored sessions were one day apart for eight infants and the same day for two infants, with the remainder ranging between two and seven days. Infant sucking dynamics has previously been shown to be similar to feeding without a nipple shield in a cross-sectional study [20].

For this sub analysis, due to limited resources, only the NT was assessed to determine if the incorporated features encouraged a suck mechanism was similar to breastfeeding. As per standard clinical practice of the study hospital, a nipple shield was used for all breastfeeds where the infant was unable to sustain attachment at the breast. Typically, mothers regularly attempted attachment without a nipple shield and proceeded with a nipple shield if the infant was unable to achieve and/or sustain attachment.

Exclusion criteria included oro-facial anomalies with the potential to affect feeding, grade IV intra-cranial haemorrhage, and other congenital anomalies. All infants were latching and sucking at the breast before participating.

Mothers supplied written, informed consent to participate in the study, which was approved by the Scientific Research Ethics Committee of King Edward Memorial Hospital.

\subsection{Experimental Novel Teat}

The infants were fed with a novel teat (Medela AG, Baar, Switzerland) that was designed to integrate oral feeding skills known to improve outcomes: development of vacuum [21] and self-paced feeding [22]. A shut-off valve ensured milk flowed only when the infant created a vacuum, and venting ensured the NT did not collapse. All infants fed with the valve threshold level of $-10 \pm 5 \mathrm{mmHg}$ [18].

\subsection{Ultrasound Imaging and Measurement of Intra-Oral Pressure}

Submental ultrasound scans of the midline of the infant's oral cavity as described previously [23-25] were acquired with a Sonsite TITAN system and an endocavity convex transducer ICT 8-5 MHz (SonoSite Inc., Bothell, WA, USA). Infant intra-oral pressures were measured via a small Silastic tube filled with sterile water and taped alongside the nipple and attached to a disposable pressure transducer (Cobe Laboratories, Frenchs Forest, NSW, Australia). The transducer was connected to the amp bridge (ADInstruments, Castle Hill, NSW, Australia), and the output was recorded using MacLab (ADInstruments) and software package Chart v5.0.2 (ADInstruments) on a laptop computer (Mac OS X v10.3.8, Apple Inc., Kupatino, CA, USA). Signals from the ultrasound machine and pressure transducer were recorded simultaneously with a Video Capture Module (ADInstruments) for the entire feed.

\subsection{Ultrasound and Intra-Oral Vacuum Measurement}

The first three well-visualized nutritive suck cycles were selected from each feed. Tongue and nipple movement were measured from two images of each suck cycle when the mid tongue was at its highest (TU) and lowest point (TD) [26] using Screen Calipers v 3.2 (Iconico Inc., New York, NY, USA). Measurements made included nipple to hard-soft palate junction (N-HSPJ), intra-oral depth (IOD; vertical measurement of the mid tongue lowering creating the space accommodating the milk bolus), and nipple diameter at 2, 5, 10, 15, and $20 \mathrm{~mm}$ from the tip of the nipple. 
Measures of suck bursts included mean minimum pressure (peak vacuum) and mean maximum pressure (baseline vacuum), mean pressure, maximum and minimum pressure, suck rate, and duration of the burst. Mean pressure and pause duration were also measured.

\subsection{Infant Milk Intake}

Milk intake was measured by test weights made before and after the breastfeed [27] (Baby Weigh Scale, Medela AG, Baar, Switzerland). Milk transfer $(\mathrm{mL} / \mathrm{min}$ ) was calculated by dividing the volume of milk consumed by the duration of the feed (minutes).

\subsection{Bradycardia and Desaturation Events}

Bradycardia was defined as $<90 \mathrm{bpm}$ and desaturation episodes defined as $<91 \%$ were recorded during each of the monitored feeds.

\subsection{Statistical Analysis}

Data analysis was run in R v3.0.3 for Mac OS X (Apple Inc., Cupertino, CA, USA), with additional packages nlme and lattice. Unless otherwise specified, summary statistics are presented as mean \pm SD (range), while model parameters are presented as estimate (95\% confidence interval (95\% CI)). Model parameters are not presented for transformed data.

Summary variables presented in Table 1 were compared using either paired $t$-tests or paired Wilcoxon signed rank tests. Comparison of infants included in this study with those omitted used either independent samples $t$-tests or Fisher's Exact test. All other analyses used linear mixed effects models to investigate differences by feed type, and simultaneous linear regression to investigate individual patterns, using the same fixed effects as the final mixed effects model. Model fit was assessed with residual plots. Where more than one random effects grouping was considered, models were compared using likelihood ratio tests. Multiple comparisons used Tukey's all pair comparisons.

Table 1. Infant and monitored feed characteristics for breastfeeding and the novel teat.

\begin{tabular}{ccccc}
\hline Infant and Feed Characteristics & Breast & Novel Teat & Difference & $p$-Value \\
\hline Feed duration (min) & $10.6(9.0,20.3)$ & $12.3(9.1,16.6)$ & $1.9(-4.0,9.4)$ & 0.28 \\
Time sucking (min) & $3.4(2.4,7.8)$ & $2.7(1.8,3.7)$ & $1.4(0.0,4.6)$ & 0.027 \\
Time Pausing (min) & $7.9(5.2,11.7)$ & $9.5(5.8,11.4)$ & $0.3(-3.6,2.4)$ & 0.078 \\
Mean vacuum (mmHg) & $-30.7(-50.0,-21.2)$ & $-6.0(-14.0,-3.4)$ & $-24.6(-46.4,-9.2)$ & $<0.001$ \\
Proportion of feed spent sucking & $0.36 \pm 0.16$ & $0.27 \pm 0.15$ & $0.14(-0.07,0.09)$ & 0.072 \\
Number of suck bursts & $35(29,66)$ & $34(25,51)$ & $6(-5,29)$ & 0.28 \\
Prescribed volume (mL) & $46.1 \pm 8.2$ & $45.7 \pm 7.1$ & $0(0,0)$ & 0.36 \\
Milk intake (mL) & $12(3,15.5)$ & $33(22.5,42.5)$ & $-19(-35,-4)$ & 0.007 \\
Milk transfer (mL/min) & $2.2(1.2,3.2)$ & $9.2(7.0,13.2)$ & $-7.9(-12.6,-3.4)$ & $<0.001$ \\
Age at monitored feed PMA (weeks) & $36.3 \pm 1.8$ & $36.4 \pm 1.5$ & $-0.14(-0.3,0)$ & 0.74 \\
Post-natal (weeks) & $7.1 \pm 4.2$ & $7.2 \pm 3.9$ & & \\
Weight at monitored feed (g) & $2120 \pm 421.7$ & $2146 \pm 294.8$ & $-65(-129,0)$ & 0.50 \\
\hline
\end{tabular}

Intra-oral vacuum data consisted of 1641 suck burst and 1607 pause records. Single sucks (102 breast, 193 NT) were omitted from vacuum analyses (other than mean pressure) as these measures cannot be made on single sucks. Five records (three breast, two NT) with a sucking rate $\geq 200$ sucks/min were omitted as this indicates measurement artifacts. Missing data included tongue movement for two NT feeds; nipple diameters at $15 \mathrm{~mm}$ in 37 records, as insufficient nipple was drawn into the mouth to make the measurement and milk intakes for four feeds in three infants.

\subsection{Tongue Movement}

Linear mixed effects models were used to investigate relationships between feed type and N-HSPJ, IOD, and nipple diameter after accounting for tongue position and measurement location. Main effect, two-way interaction involving feed, and three way interaction models investigated whether there was 
differing movement patterns between feed types. For each fixed effects model, four random intercept groupings were compared, allowing for differences between infants, amount of tongue movement between infants, and between feed types between infants, in the amount of tongue movement by feed type within the infant.

\subsection{Intra-Oral Vacuum}

All vacuum and sucking measures were analysed with linear mixed effects models. Minimal random effects considered were random intercepts by feed type within infant. Random slopes by time (linear or second order polynomial), burst type, and suck burst vacuum were also considered as random effects when included in the fixed effects; groupings were by infant or by feed within infant. Fixed effects are detailed for each analysis. Models, which did not converge, were considered to be misspecified, and alternate fixed and/or random effects were considered. Suck burst/pause duration and sucking events data were log transformed before analysis. Measurements of vacuum strength were square root transformed.

Effect of feed type on suck burst/pause durations considered both main effects of and interactions between feed type and burst type. Effect of feed type on changes over the course of the feed for suck bursts, for duration, sucking rate, and number of sucking events per burst, were analysed with either main effects or interaction models, with feed type and time since the beginning of the feed (linear or second order polynomial) as the fixed effects of interest. Effect of baseline and peak suck burst vacuum on overall sucking rate was analysed with either main effects or interaction models for feed type and vacuum. Number of sucks per burst was grouped as 'single' (1 suck), 'immature' (2-9 sucks), and 'mature' (10+ sucks). Number and proportion of suck bursts in the 'single' and 'mature' categories were compared using paired Wilcoxon signed rank tests.

Variability of vacuum within a feed was characterised by calculating the width of the inter-quartile range across all suck bursts for each of the pressures and compared with fixed effects of measure type and feed type, and random intercepts for feed type within infant.

\subsection{Milk Intake}

The association between milk intake and feed type was tested using a linear mixed effects model with random intercepts by infant and a fixed effect of feed type. Covariates are listed in Table 1 , and vacuum variability measures; main effect and interactions with feed type were considered separately for each covariate. Milk intake data was square root transformed prior to analysis.

\section{Results}

\subsection{Participant Characteristics}

Seventeen mothers and infants participated in this study. These infants were more likely than the others in the NT group of the larger study to be discharged home rather than back transferred $(p=0.002)$ and had spent longer on continuous positive airway pressure (CPAP) $(p=0.004)$.

Infant (11 female, 6 male; 8 singletons, 9 twins from 5 twin pairs) characteristics were birth gestation $29.2 \pm 3.0$ weeks (23.6-33.1 weeks), birth weight $1218 \pm 43 \mathrm{~g}$ (540-1940 g), post-menstrual age (PMA) $33.6 \pm 0.8$ weeks (31.9-35.3 weeks)/post-natal age $4.4 \pm 2.8$ weeks (1.0-10.6 weeks) at introduction of full oral feeds, post-menstrual age $37.1 \pm 1.2$ weeks (35.9-40.1 weeks)/post-natal age $7.9 \pm 3.6$ weeks (2.7-16.6 weeks) at achievement of full oral feeds. Fifteen infants had been on respiratory support (CPAP: 3 infants for $<1$ week, 12 for longer; ventilation: 7 for $<48$ h, 4 for longer; oxygen: 13 for $1 \mathrm{~h}$ or longer). 


\subsection{Characteristics of Monitored Feeds}

Characteristics of the monitored feeds are presented in Table 1. Monitored feeds occurred $2.7 \pm 1.7(0,5.9)$ weeks after introduction of suck feeds and $-0.8 \pm 1.0(-2.7,1.1)$ weeks after achievement of full suck feeds. Nipple shields were used during 15/17 breastfeeds.

\subsection{Tongue Movement}

Nipple diameters for the breastfeed and the NT are documented in Table 2.

Table 2. Measures of ultrasound images during breastfeeding and feeding with the novel teat. N-HSPJ: nipple-hard soft palate junction.

\begin{tabular}{ccccc}
\hline \multirow{2}{*}{$\begin{array}{c}\text { Infant Intra-Oral and } \\
\text { Nipple Diameter Measures }\end{array}$} & \multicolumn{2}{c}{ Breast } & \multicolumn{2}{c}{ Novel Teat } \\
\cline { 2 - 5 } & Tongue Up & Tongue Down & Tongue Up & Tongue Down \\
\hline N-HSPJ distance (mm) & $7.1 \pm 2.9$ & $5.2 \pm 2.6$ & $5.6 \pm 1.4$ & $4.7 \pm 1.4$ \\
Intra-oral depth (mm) & $0.3 \pm 0.5$ & $4.2 \pm 2.0$ & $0.1 \pm 0.2$ & $4.1 \pm 1.3$ \\
Nipple diameters (mm) & & & & \\
$2 \mathrm{~mm}$ & $10.0 \pm 3.0$ & $11.3 \pm 2.3$ & $8.0 \pm 1.1$ & $8.8 \pm 1.1$ \\
$5 \mathrm{~mm}$ & $11.4 \pm 2.6$ & $12.8 \pm 2.5$ & $9.2 \pm 0.9$ & $9.6 \pm 1.3$ \\
$10 \mathrm{~mm}$ & $12.2 \pm 2.5$ & $13.2 \pm 2.4$ & $9.8 \pm 1.0$ & $10.3 \pm 1.3$ \\
$15 \mathrm{~mm}$ & $12.5 \pm 2.7$ & $13.5 \pm 2.6$ & $10.0 \pm 1.0$ & $10.2 \pm 1.5$ \\
\hline
\end{tabular}

Effect sizes (95\% CI, associated $p$-value) for the main effects models and models with interactions involving feed type are presented in Table 3. No associations were seen between feed type and either N-HSPJ distance or intra-oral depth $(p>0.21)$. The amount of inferior movement (IOD) was between $-2.4 \pm 0.8$ and $3.4 \pm 0.8 \mathrm{~mm}$ greater during NT feeds.

Nipple diameters were on average $2.3 \mathrm{~mm}(1.0,3.6)$ smaller during NT feeds (Table $3 ; p=0.002$ ). The amount of change between tongue up and tongue down was on average $0.6(-0.1,1.3) \mathrm{mm}$ greater during NT feeds. Overall, the pattern of nipple diameter measurements did not differ by feed, except at $2 \mathrm{~mm}$ from the tip of the nipple, where larger values were seen during NT feeds (Figure 1). The three-way interaction between measurement location, tongue position, and feed type was not significant $(p=0.80)$. Individual differences were seen in the relative patterns between NT and breastfeeds.

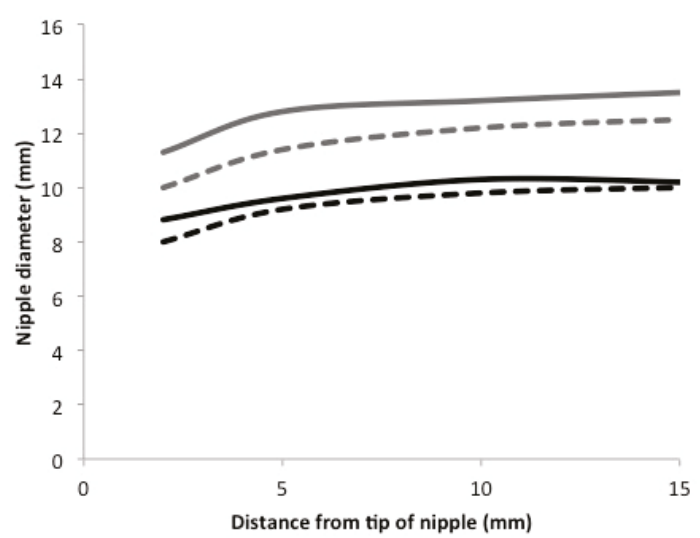

Figure 1. The movement of the infant tongue from its uppermost point to its lowest point for a breastfeed and feed with the novel teat. Solid grey line: breastfeed tongue down; dashed grey line: breastfeed tongue up; solid black line: novel teat tongue down; dashed black line: novel teat tongue up. 
Table 3. Effect (parameter \pm SE) of tongue position (tongue down) and feed type (NT) on N-HSPJ distance, and intra oral depth; and tongue position, feed type, and measurement location (location) on nipple diameters. Reference levels are tongue position is up, feed type is breastfeed, and location is $5 \mathrm{~mm}$ from the nipple tip. Random effects indicates the sub-groups of the data for which random intercepts were fitted, allowing for individual (infant) differences between measurements. The main effects models include all considered terms; interaction models consider only interactions, which include feed type.

\begin{tabular}{|c|c|c|c|c|c|c|}
\hline \multirow{3}{*}{ Random Effects } & \multicolumn{2}{|l|}{ N-HSPJ } & \multicolumn{2}{|l|}{ IOD } & \multicolumn{2}{|c|}{ Nipple Diameters } \\
\hline & Coeff $(95 \%$ CI $)$ & $p$-Value & Coeff $(95 \%$ CI) & $p$-Value & Coeff $(95 \%$ CI) & $p$-Value \\
\hline & \multicolumn{2}{|c|}{ Feed within Infant } & \multicolumn{2}{|c|}{$\begin{array}{l}\text { Tongue Position within } \\
\text { Feed within Infant }\end{array}$} & \multicolumn{2}{|c|}{$\begin{array}{c}\text { Tongue Position within Feed } \\
\text { within Infant }\end{array}$} \\
\hline \multicolumn{7}{|c|}{ Main effects models } \\
\hline Reference $^{a}$ & $5.2(4.1,6.2)$ & - & $0.3(-0.2,0.7)$ & 0.30 & $11.2(10.3,12.2)$ & - \\
\hline Tongue down & $1.6(1.3,1.8)$ & $<0.001$ & $3.9(3.4,4.5)$ & $<0.001$ & $1.0(0.7,1.4)$ & $<0.001$ \\
\hline Novel Teat & $-0.5(-1.9,0.8)$ & 0.42 & $-0.1(-0.7,0.5)$ & 0.72 & $-2.3(-3.6,-1.0)$ & 0.002 \\
\hline Location & & & & & & $<0.001^{b}$ \\
\hline $2 \mathrm{~mm}$ & - & - & - & - & $-1.2(-1.4,-1.0)$ & $<0.001$ \\
\hline $10 \mathrm{~mm}$ & - & - & - & - & $0.6(0.4,0.8)$ & $<0.001$ \\
\hline $15 \mathrm{~mm}$ & - & - & - & - & $0.8(0.6,1.0)$ & $<0.001$ \\
\hline \multicolumn{7}{|c|}{ Interaction models } \\
\hline Reference $^{a}$ & $5.1(4.0,6.1)$ & - & $0.3(-0.5,0.8)$ & 0.28 & $11.1(10.1,12.1)$ & \\
\hline Tongue down & $1.7(1.3,2.1)$ & $<0.001$ & $3.8(3.1,4.6)$ & $<0.001$ & $1.3(0.9,1.8)$ & $<0.0001$ \\
\hline $\begin{array}{l}\text { Novel Teat feed } \\
\text { Location }\end{array}$ & $-0.4(-1.8,1.1)$ & 0.60 & $-0.2(-1.0,0.6)$ & 0.61 & $-2.0(-3.4,-0.6)$ & $\begin{array}{c}0.007 \\
<0.0001 \mathrm{~b}\end{array}$ \\
\hline 2 & - & - & - & - & $-1.4(-1.7,-1.1)$ & $<0.0001$ \\
\hline 10 & - & - & - & - & $0.6(0.3,0.9)$ & $<0.0001$ \\
\hline 15 & - & - & - & - & $0.9(0.6,1.2)$ & $<0.0001$ \\
\hline Tongue down Teat & $-0.4(-0.9,0.2)$ & 0.21 & $0.2(-0.9,1.3)$ & 0.72 & $-0.6(-1.3,0.1)$ & 0.069 \\
\hline Location Teat & & & & & & $0.010^{\mathrm{b}}$ \\
\hline $2 \mathrm{~mm}$ & - & - & - & - & $0.4(0.002,0.8)$ & 0.039 \\
\hline $10 \mathrm{~mm}$ & - & - & - & - & $-0.1(-0.5,0.3)$ & 0.56 \\
\hline $15 \mathrm{~mm}$ & - & - & - & - & $0.3(-0.7,0.2)$ & 0.26 \\
\hline
\end{tabular}

a $p$-Values not included for reference levels except models for IOD, as the question 'is this significantly different from zero' is not meaningful for other analyses. ${ }^{b}$ Omnibus $p$-values, indicating the overall chance that there is at least measurement points with significantly different measurements. $p$-Values for each of the measurement locations show significance of difference with respect to measurement made at $5 \mathrm{~mm}$ from nipple tip.

\subsection{Intra-Oral Vacuum}

Summary statistics for intra-oral vacuum are presented in Table 4.

Changes in vacuum and sucking measures over the feed differed between infants $(p<0.001$, all), with effects of feed on the pattern of change differing between infants $(p<0.001)$. Effect of feed type on burst (suck vs. pause) duration differed by infant $(p<0.001)$. Effect of suck burst vacuums on sucking rate differed by infant $(p \leq 0.001)$.

Mean suck burst vacuums, mean pause vacuums, baseline suck burst vacuums, and peak suck burst vacuums were all stronger during breastfeeding $(p<0.001, p=0.003, \mathrm{p}<0.001, \mathrm{p}<0.001$, respectively). Mean suck burst vacuums varied over the course of breast $(p=0.011)$ but not NT $(p=0.68)$ feeds. Mean pause vacuums and peak suck burst vacuums did not display consistent patterns of change over the feed ( $p=0.12, p=0.22$, respectively). Baseline suck burst vacuums showed consistent patterns over the feed, which differed by feed type ( $p=0.043$; Figure 2$)$. Variability of all three measures was greater during breastfeeds $(p<0.008)$. Peak suck burst vacuums are more variable than baseline or mean suck burst vacuums $(p<0.001)$. The difference was smaller in NT feeds than breastfeeds but only significant for the comparison between peak and baseline suck burst vacuums $(p=0.021)$. 
Table 4. Summary statistics for infant intra-oral vacuum measures, separated by feed and burst type. Data is presented as median (IQR) (range).

\begin{tabular}{|c|c|c|c|c|}
\hline & \multicolumn{2}{|c|}{ Breast } & \multicolumn{2}{|c|}{ Novel Teat } \\
\hline & Sucks $(n=912)$ & Pauses $(n=895)$ & Sucks $(n=729)$ & Pauses $(n=712)$ \\
\hline Duration * (s) & $\begin{array}{c}4.5(2.7,7.4) \\
(0.5,52.4)\end{array}$ & $\begin{array}{l}5.4(2.7,9.0) \\
(0.5,712.6)\end{array}$ & $\begin{array}{c}2.4(1.3,4.6) \\
(0.4,132.8)\end{array}$ & $\begin{array}{c}5.2(2.4,11.6) \\
(0.6,379.7)\end{array}$ \\
\hline Sucking Events * $(n)$ & $\begin{array}{c}6(3.8,9) \\
(1,54)\end{array}$ & - & $\begin{array}{l}3(1,5) \\
(1,147)\end{array}$ & - \\
\hline Sucking Rate $(n / \mathrm{min})$ & $\begin{array}{c}88.0(73.7,102.6) \\
(19.0,194.4)\end{array}$ & - & $\begin{array}{c}77.6(62.4,96.6) \\
(14.2,191.2)\end{array}$ & - \\
\hline \multicolumn{5}{|c|}{ Vacuum (mmHg) } \\
\hline Baseline & $\begin{array}{c}-31.1(-60.0,-12.7) \\
(-181.0,12.4)\end{array}$ & - & $\begin{array}{c}-5.8(-11.0,0.1) \\
(-53.3,14.3)\end{array}$ & - \\
\hline Mean * & $\begin{array}{c}-53.6(-89.3,-31.5) \\
(-199.3,0.4)\end{array}$ & $\begin{array}{c}-23.9(-48.8,-11.7) \\
(-185.1,1.9)\end{array}$ & $\begin{array}{c}-15.5(-21.8,-8.9) \\
(-77.6,2.2)\end{array}$ & $\begin{array}{c}-5.1(-11.0,-0.6) \\
(-79.6,5.3)\end{array}$ \\
\hline Peak & $\begin{array}{c}-106.2(-153.0,-65.5) \\
(-325.7,-9.6)\end{array}$ & - & $\begin{array}{c}-40.0(-54.6,-27.1) \\
(-116.7,-3.1)\end{array}$ & - \\
\hline \multicolumn{5}{|c|}{ Vacuum variability } \\
\hline Baseline & $\begin{array}{l}23.4(17.3,37.0) \\
\quad(5.3,47.3)\end{array}$ & - & $\begin{array}{c}5.5(4.4,6.1) \\
(1.4,12.0)\end{array}$ & - \\
\hline Mean * & $\begin{array}{l}26.2(25.0,41.6) \\
\quad(11.2,73.9)\end{array}$ & - & $\begin{array}{c}9.3(7.8,10.5) \\
(3.6,18.9)\end{array}$ & - \\
\hline Peak & $\begin{array}{c}50.0(33.0,60.7) \\
(18.9,154.3)\end{array}$ & - & $\begin{array}{c}25.4(20.6,29.3) \\
\quad(3.9,39.4)\end{array}$ & - \\
\hline
\end{tabular}

* includes single sucks.

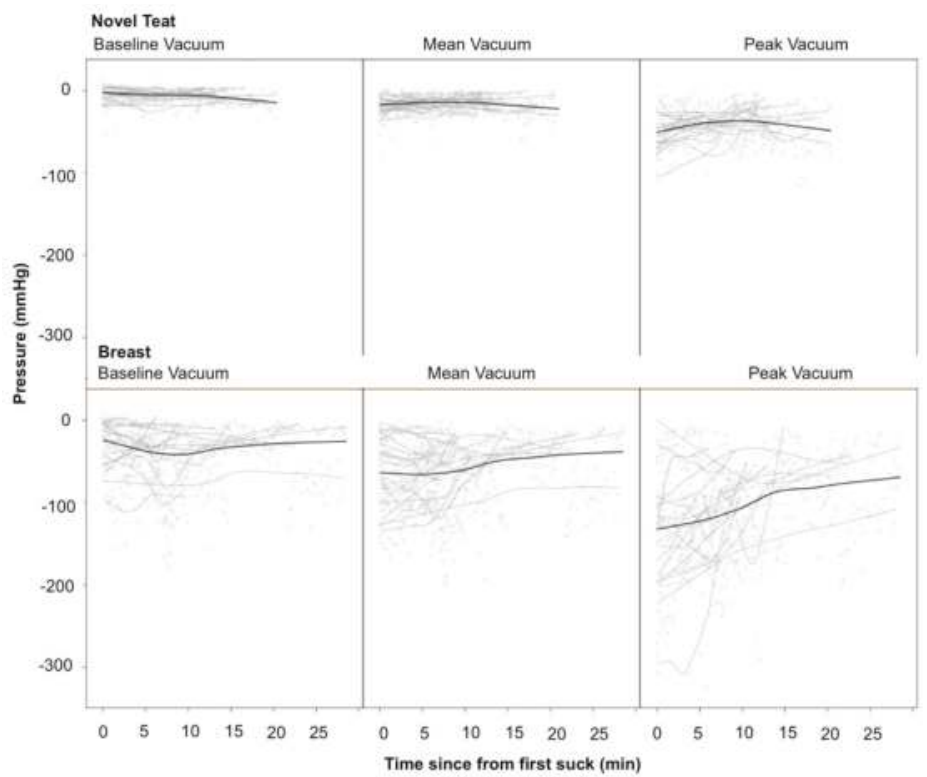

Figure 2. Sucking vacuums over the course of both the breastfeed and novel teat feed. Black lines are local regression smoothers for the full data set; grey lines are local regression smoothers indicating individual infant patterns. 
Suck bursts were shorter than pauses $(p<0.001)$, and no overall association with feed type was seen $(p>0.13)$. Suck burst durations did not change over the course of the feed $(p=0.29)$. At the beginning of the feeds, pause durations did not differ significantly by feed type $(p=0.063)$. Pause durations decreased over the course of NT feeds $(p=0.017)$, but no change was seen during breastfeeds $(p=0.37)$. Breastfeeds had more sucks per burst $(p=0.001)$ and higher sucking rates $(10.4(2.1,18.6)$ sucks $/$ minute, $p=0.001$ ) than NT feeds; neither showed a consistent pattern of change over the feed $(p=0.32, p=0.93$, respectively). Higher sucking rates were associated with few sucks per burst $(p=0.004)$, but this association did not differ by feed type $(p=0.88)$. No association was seen between suck burst vacuums and sucking rate (peak, $p>0.54$; baseline, $p>0.082$ ).

Suck bursts of $\geq 10$ sucks occurred during all breast and 15 of the 17 NT feeds; single sucks occurred during $15 / 17$ of the breast and NT feeds. The number $(p=0.002)$ and proportion $(p=0.017)$ of suck bursts with $\geq 10$ sucks were higher in breastfeeds $9(6,12)(22.2 \%(15.8,31.0))$ than NT feeds $3(1,7)$ $(8.8 \%(3.3,12.5))$. The number $(p=0.031)$ and proportion $(p=0.008)$ of single sucks were higher during NT feeds: $6(3,8)(10.0 \%(6.7,15.2))$ than during breastfeeds; $10(4,14)(24.1 \%(15.8,32.9))$. Proportion of bursts with $2-9$ sucks did not differ $(p=0.78)$, being $64.0 \%(58.8,69.5)$ and $63.3 \%(56.2,68.2)$ of NT and breastfeed suck bursts respectively.

\subsection{Bradycardia and Desaturation Events}

Desaturation events were reported for 4 infants for 6 of the 32 feeds. The breast:NT incidence for the four infants with desaturations was 3:3, 1:3, 0:1, and 1:0. Bradycardia events were recorded for three infants only during breastfeeding; one infant had two episodes, and two infants had one episode. Data was not recorded for two feeds.

\subsection{Milk Intake}

No measureable milk intake occurred for one breastfeed; three breastfeeds had milk intakes of $4 \mathrm{~mL}$ or less, whereas milk intake from the NT feeds was never this low. Milk intakes were larger during NT feeds $(p=0.003)$ and for longer feeds $(p=0.011)$, when a greater proportion of the feed was spent sucking $(p=0.023)$ and / or had a greater number of suck bursts $(p=0.007)$. The association between milk intake and feed type remained significant after accounting for each of the covariates $(p<0.004)$; no significant interactions were seen $(p>0.10)$. No association was seen between milk intake and any of the vacuum variability measures $(p>0.32)$.

\section{Discussion}

This study has found that movement of the tongue to create a vacuum with the NT was similar to that of breastfeeding. Whilst infants were required to exert a vacuum to remove milk from the NT, the intra-oral vacuums were weaker compared to the breast. Infants, however, consistently removed more milk from the NT than the breast.

\subsection{Tongue Movement}

The introduction of bottles during the establishment of breastfeeding in the term infant is considered detrimental due to the potential of nipple confusion, where the infant refuses the breast $[28,29]$. Whilst there is little evidence of nipple confusion, the infant has been shown to be highly adaptable. Infants will elect to use compression of the tongue to remove milk from a teat if it is easier than using vacuum. However, if the teat is designed to minimise or exclude the compression effect [30], the infant will employ vacuum to remove milk. Healthy preterm infants fed with an NT that only released milk with the application of vacuum moved their tongue in a similar fashion to breastfeeding (Figure 1). During a breast or NT feed, the infant drew their tongue downward, the nipple/NT expanded evenly, and intra-oral vacuum strength increased to peak vacuum (minimum pressure). Milk flowed into the cavity bounded by the hard and soft palate, the nipple, and the tongue surface. As the tongue was raised, intra-oral vacuum strength reduced to baseline vacuum (maximum 
pressure), milk was cleared under the soft palate to the phalangeal area and the nipple/NT diameter decreased (Figure 1). The only difference was that the tip of the NT was larger than the nipple (Figure 1) due to its shape and being less compressible than the human nipple. The absence of peristaltic tongue action with the NT compared to other teats [31,32] can be attributed greater thickness of silicone at the base of the NT, which minimised infant tongue compression. While the pattern of tongue movement was similar to breastfeeding, the degree of movement was greater during the breastfeed (Figure 1), which was likely due to the difference in elasticity between the nipple and the NT. Further, whilst most of the infants in this study used a nipple shield to feed, we have shown previously that tongue movement with a nipple shield is not different to tongue movement without [20].

Nipple shields are often used in our NICU to facilitate breastfeeding in preterm infants, enabling them to remain attached to the breast. Evidence shows that for the hospitalised preterm infant, nipple shield (NS) use is associated with improved milk removal [33]. There is limited evidence regarding the impact of NS on breastfeeding exclusivity and duration beyond discharge from hospital. The absence of a relationship between nipple shield use and age at achievement of exclusive breastfeeding [34] has been reported, while lower rates of exclusive breastfeeding have been reported in infants that have used a shield ( $49 \%$ with nipple shield use and $66 \%$ without) [35]. Indeed, several factors impact the achievement of exclusive breastfeeding in preterm infants [35]. Complicating factors such as low maternal milk production and/or low infant intraoral vacuum with subsequent insufficient milk transfer are rarely measured. Thus there is a critical need to understand the mechanisms by which nipple shields function to enhance transfer of milk from the breast in the context complicating maternal and infant factors.

The tongue movements of the preterm infants studied are also comparable to that documented in term breastfeeding infants [26,36-38] as well as a larger cohort of breastfeeding preterm infants also recruited from this study [20]. The absence of any marked peristaltic tongue action during breastfeeding or feeding with the NT is in contrast to other studies of bottle-feeding, implying the design of the teat influences tongue movement [31,39]. The unique stresses of tongue movement during feeding likely impact the development of the form and structure of facial structures, particularly the palate [40,41], evidenced by a $68 \%$ reduction of malocclusion if infants are breastfed [42]. Further, anthropological observations of prehistoric skulls show broader flatter palates suggesting the forces exerted during breastfeeding influences developing structures [43]. Given the malleability of preterm infants' facial structure and immaturity of muscular control and strength, it could be potentially beneficial to emulate the tongue motion of breastfeeding in the event of the unavailability of the mother to breastfeed.

Positioning of the teat/nipple in the oral cavity is important in order to avoid gagging, which may progress to oral aversion and also to ensure optimal placement of the milk bolus so that it can be easy cleared in co-ordination with breathing and swallowing [44]. We found no difference in the positioning of the nipple and NT in relation to the N-HSPJ (Table 2), indicating good positioning [45]. The infants, however, drew their tongue down more (IOD) during the NT feed, which may be due to not having to apply as much vacuum to obtain a bolus. Thus, greater volumes of milk were transferred during the NT feeds.

\subsection{Intra-Oral Vacuum}

Preterm infants are recognised to have individual trajectories to attainment of feeding milestones that are complicated by immaturity and associated co-morbidities $[28,46]$. Commensurate with this notion, measures of preterm intra-oral vacuum were variable across the course of a feed, and patterns were different between individual infants in this study.

One of the factors implicated in more effective and efficient bottle-feeding is increased strength of vacuum [47]. The breastfeeding preterm infants in this study displayed vacuums approximately a half to two-thirds that applied by term infants [36,48] (Table 4). This is consistent with our larger descriptive study of preterm breastfeed infants [20] and also the low milk transfer from the breast 
(Table 1). Peak vacuums were also weaker when infants fed at the NT likely due the threshold set for milk flow from the NT while peak vacuums at the breast are due to the greater flexibility of the human nipple. With the NT the infant may also adjust vacuums applied to modulate milk flow rate so that suck-swallow-breathe co-ordination is not compromised.

To suck effectively, infants latch to the breast or the teat, and this is measured as the baseline vacuum. It is important to form a seal to the breast/teat to stretch and position the nipple/teat optimally in the oral cavity in relation to the N-HSPJ to remove milk [49]. If this is not possible, the infant may compensate by allowing milk to spill out of the mouth [50]. The baseline seal to the NT increased in strength over the feed and weakened during the breastfeed (Figure 2), perhaps reflecting fatigue due to the stronger applied vacuums. In contrast, term breastfed infants increase their baseline vacuums over a feed [49]. These changes might be due to changes in breast elasticity due to the drainage of milk, whereas preterm infants remove small volumes from the breast (Table 1).

Mature sucking patterns are frequently described as the ability to extend suck bursts and reduce the lengths of pauses [21]. In contrast, our preterm infants exhibited suck bursts that were shorter than the pauses for both the breast and NT feeds. This apparent reversal suggests a lack of feeding maturity and is further reflected by low milk transfer along with the low proportion of suck bursts comprised of $>10$ sucks, which is also considered a marker of feed progression. Single sucks were also frequently identified in nearly all breast and NT feeds which is rarely seen during a term breastfeed and itself perhaps a gauge of immaturity of oral feeding. Interestingly, the frequency and proportion of 'mature' suck bursts was higher in breastfeeds (nine per feed; $22.2 \%$ ) than NT feeds (three per feed; $8.8 \%$ ), yet milk transfer was more effective in the NT feeds.

Often, clinical observations of slowing of sucking rate are interpreted as a change from non-nutritive to nutritive sucking [17]. Infants in this study did not change their suck frequency across a feed but sucked more rapidly during breastfeeding. Conversely, sucking rates increase across a breastfeed in term infants [49]. This absence of change across a feed makes sucking rate an unreliable indicator of milk intake particularly during breastfeeding. Further, suck rate is not indicative of vacuum strength, with no relationships demonstrated with either peak or baseline vacuum.

Little attention is paid to the length of time a preterm infant pauses at the breast/teat, and long pauses are considered to be due to fatigue or state. We found that pause durations decreased over the course of NT feeds whilst remaining constant during breastfeeds. This may be a congruent with better suck-swallow-breathe co-ordination as the NT feed progressed. Further studies identifying the nutritive and non-nutritive portions of the feed as well as measurement of swallowing and breathing would serve to clarify these differences.

Cardiorespiratory stability is a major concern during oral feeding of the preterm infant. It is well documented that bottle-feeding is associated with more episodes of desaturation and bradycardias compared to breastfeeding [51]. Due to the design of the NT, we expected that the cardiorespiratory stability of the infant fed with the NT would be similar to breastfeeding due to the infant having the opportunity to self regulate their intake from the NT as they would from the breast. However, desaturation occurred overall in only six of 32 feeds $(20 \%)$, and these were in only four infants. Similarly, bradycardia events were rare and occurred in three infants where one had two episodes and two had one episode during breastfeeding only. Although the numbers are too small to conduct meaningful statistical analysis, there did not appear to be more desaturations during NT feeds.

Good growth is critical for the preterm infant as under nutrition is associated deficits in neurodevelopment and increased risk of sepsis and necrotizing enterocolitis [52]. Thus, the ingestion of adequate volumes of milk from every oral feed is imperative. We have demonstrated in a previous study that intakes range from 0 to $40 \mathrm{~mL}$ in preterm breastfed infants [18], which is consistent with Meier et al. [33], who showed intakes from 2-62 mL, and this study. Typically, infants are supplemented to achieve the prescribed volume but without objective measures of the volume of milk ingested at a breastfeed. Overall, infants received significantly greater volumes from the NT that were much closer to the prescribed volume by the neonatologist. As aforementioned, the small volumes of milk 
removed from the breast is likely due to the low strength of vacuum generated, as [36,49] (Figure 2) this has been associated positively associated with milk intake in term breastfed infants [53]. Longer breastfeeds and a greater proportion of the breastfeed spent sucking usually resulted in increased milk intake, which is in contrast to the NT, where any suck resulted in milk transfer. Clearly, more research is required to identify factors that would improve milk intakes from the breast.

Clinically encouragement of a sucking action similar to breastfeeding during bottle-feeding is likely to confer immediate advantages such as increased infant feed regulation without reduction in feed volume and potentially less nipple 'confusion'. In the longer term, it may also impact development of the oro-facial development by reducing the incidence of conditions such as malocclusion, which is higher in preterm infants [54,55], and sleep disordered breathing where factors such as palate shape, hypotonia, and bottle-feeding appear to contribute [56].

The limitations of the study include the small numbers of participants that were not using a nipple shield to feed; thus, we were not able to directly compare breastfeeding without a shield and with the NT. A more extensive study is required to assess the sucking dynamics with and without a nipple shield. Further, these infants represent a relatively 'healthy' population that began oral feeding at approximately 34 to 35 weeks post menstrual age therefore the results are not applicable to younger infants or those with co-morbidities that are known to adversely affect feeding trajectories. It is anticipated that future studies will investigate suck, swallow, breathe co-ordination, and sucking during breastfeeding and feeding with the NT and conventional teat.

\section{Conclusions}

The novel teat, which only released milk when the preterm infant applied vacuum, encouraged a similar tongue action to breastfeeding and infants transferred a greater volume of milk compared to breastfeeding. Intra-oral vacuums were lower in strength with the novel teat compared to the breast. Further research is required to compare suck-swallow-breathe patterns with the novel teat and breastfeeding and improve transfer volumes at the breast.

Acknowledgments: This study was supported by an unrestricted research grant from Medela AG (Switzerland). Zoya Gridneva was supported by an Australian Postgraduate Award from the University of Western Australia (Australia). Many thanks are extended to all mothers who participated in this study.

Author Contributions: D.G. designed the study, conducted experiments and data analysis and interpretation, and wrote the manuscript; C.K. designed the study, collected the data and provided input to the manuscript; K.N. designed the study, collected the data and provided input to the manuscript; A.H. provided consultation for research design, conducted data analysis and interpretation, and critically reviewed the manuscript, K.S. designed the study, interpreted results, and critically reviewed the manuscript. All authors read and approved the final manuscript.

Conflicts of Interest: The authors declare no conflict of interest and that Medela AG (Switzerland) provides an unrestricted research grant to Donna Geddes from which salaries to Donna Geddes and Anna Hepworth are paid. Medela AG provided a Top-up Scholarship for Zoya Gridneva and has provided speaker's fees to Donna Geddes for educational lectures. The funding sponsors had no role in the design of the study; in the collection, analyses, or interpretation of data; in the writing of the manuscript, or in the decision to publish the results.

\section{References}

1. Lewandowski, A.J.; Lamata, P.; Francis, J.M.; Piechnik, S.K.; Ferreira, V.M.; Boardman, H.; Neubauer, S.; Singhal, A.; Leeson, P.; Lucas, A. Breast milk consumption in preterm neonates and cardiac shape in adulthood. Pediatrics 2016, 138, e20160050. [CrossRef] [PubMed]

2. Isaacs, E.B.; Gadian, D.G.; Sabatini, S.; Chong, W.K.; Quinn, B.T.; Fischl, B.R.; Lucas, A. The effect of early human diet on caudate volumes and IQ. Pediatr. Res. 2008, 63, 308-314. [CrossRef] [PubMed]

3. Isaacs, E.B.; Fischl, B.R.; Quinn, B.T.; Chong, W.K.; Gadian, D.G.; Lucas, A. Impact of breast milk on intelligence quotient, brain size, and white matter development. Pediatr. Res. 2010, 67, 357-362. [CrossRef] [PubMed] 
4. O'Connor, D.L.; Jacobs, J.; Hall, R.; Adamkin, D.; Auestad, N.; Castillo, M.; Connor, W.E.; Connor, S.L.; Fitzgerald, K. Growth and development of premature infants fed predominantly human milk, predominantly premature infant formula, or a combination of human milk and premature formula. J. Pediatr. Gastroenterol. Nutr. 2003, 37, 437-446. [CrossRef] [PubMed]

5. Belfort, M.B.; Anderson, P.J.; Nowak, V.A.; Lee, K.J.; Molesworth, C.; Thompson, D.K.; Doyle, L.W.; Inder, T.E. Breast milk feeding, brain development, and neurocognitive outcomes: A 7-year longitudinal study in infants born at less than 30 weeks' gestation. J. Pediatr. 2016, 177, 133-139. [CrossRef] [PubMed]

6. Bronfin, D.R. Misshapen heads in babies: Position or pathology? Ochsner J. 2001, 3, 191-199. [PubMed]

7. Morris, K.M.; Burns, Y.R. Reduction of craniofacial and palatal narrowing in very low birthweight infants. J. Paediatr. Child Health 1994, 30, 518-522. [CrossRef] [PubMed]

8. Ash, S.P.; Moss, J.P. An investigation of the features of the preterm infant palate and the effect of prolonged orotracheal intubation with and without protective appliances. Br. J. Orthod. 1987, 14, 253-261. [CrossRef] [PubMed]

9. De Curtis, M.; Rigo, J. Extrauterine growth restriction in very low-birthweight infants. Acta Paediatr. 2004, 93, 1563-1568. [CrossRef] [PubMed]

10. Geddes, D.T.; Prescott, S.L. Developmental origins of health and disease: The role of human milk in preventing disease in the 21st century. J. Hum. Lact. 2013, 29, 123-127. [CrossRef] [PubMed]

11. Callen, J.; Pinelli, J. A review of the literature examining the benefits and challenges, incidence and duration, and barriers to breastfeeding in preterm infants. Adv. Neonatal Care 2005, 5, 72-88. [CrossRef] [PubMed]

12. Garber, J. Oral-motor function and feeding intervention. Phys. Occup. Ther. Pediatr. 2013, 33, 111-138. [CrossRef] [PubMed]

13. Casiro, O.G.; McKenzie, M.E.; McFadyen, L.; Shapiro, C.; Seshia, M.M.; MacDonald, N.; Moffatt, M.; Cheang, M.S. Earlier discharge with community-based intervention for low birth weight infants: A randomized trial. Pediatrics 1993, 92, 128-134. [PubMed]

14. Bertoncelli, N.; Cuomo, G.; Cattani, S.; Mazzi, C.; Pugliese, M.; Coccolini, E.; Zagni, P.; Mordini, B.; Ferrari, F. Oral feeding competences of healthy preterm infants: A review. Int. J. Pediatr. 2012, 2012, 896257. [CrossRef] [PubMed]

15. Mathew, O.P. Breathing patterns of preterm infants during bottle feeding: Role of milk flow. J. Pediatr. 1991, 119, 960-965. [CrossRef]

16. Al-Sayed, L.E.; Schrank, W.I.; Thach, B.T. Ventilatory sparing strategies and swallowing pattern during bottle feeding in human infants. J. Appl. Physiol. 1994, 77, 78-83. [CrossRef] [PubMed]

17. Sakalidis, V.S.; McClellan, H.L.; Hepworth, A.R.; Kent, J.C.; Lai, C.T.; Hartmann, P.E.; Geddes, D.T. Oxygen saturation and suck-swallow-breathe coordination of term infants during breastfeeding and feeding from a teat releasing milk only with vacuum. Int. J. Pediatr. 2012. [CrossRef] [PubMed]

18. Simmer, K.; Kok, C.; Nancarrow, K.; Hepworth, A.R.; Geddes, D.T. Novel feeding system to promote establishment of breastfeeds after preterm birth: A randomized controlled trial. J. Perinatol. 2016, 36, 210-215. [CrossRef] [PubMed]

19. Trial Registered on ANZCTR. Available online: http://www.ANZCTR.org.au/ACTRN12614000875606.aspx (accessed on 19 March 2018).

20. Geddes, D.T.; Chooi, K.; Nancarrow, K.; Hepworth, A.R.; Gardner, H.; Simmer, K. Characterisation of sucking dynamics of breastfeeding preterm infants: A cross sectional study. BMC 2017, 17, 386. [CrossRef] [PubMed]

21. Lau, C.; Alagugurusamy, R.; Schanler, R.J.; Smith, E.O.; Shulman, R.J. Characterization of the developmental stages of sucking in preterm infants during bottle feeding. Acta Paediatr. 2000, 89, 846-852. [CrossRef] [PubMed]

22. Lau, C.; Schanler, R.J. Oral feeding in premature infants: Advantage of a self-paced milk flow. Acta Paediatr. 2000, 89, 453-459. [CrossRef] [PubMed]

23. Weber, F.; Woolridge, M.; Baum, J. An ultrasonographic study of the organisation of sucking and swallowing by newborn infants. Dev. Med. Child Neurol. 1986, 28, 19-24. [CrossRef] [PubMed]

24. Ardran, G.; Kemp, F. A correlation between suckling pressures and the movements of the tongue. Acta Paediatr. 1959, 48, 261-272. [PubMed]

25. Prieto, C.; Cárdenas, H.; Salvatierra, A.; Boza, C.; Montes, C.; Croxatto, H. Sucking pressure and its relationship to milk transfer during breastfeeding in humans. J. Reprod. Fertil. 1996, 108, 69-74. [CrossRef] [PubMed] 
26. McClellan, H.L.; Sakalidis, V.S.; Hepworth, A.R.; Hartmann, P.E.; Geddes, D.T. Validation of nipple diameter and tongue movement measurements with B-mode ultrasound during breastfeeding. Ultrasound Med. Biol. 2010, 36, 1797-1807. [CrossRef] [PubMed]

27. Kent, J.C.; Mitoulas, L.R.; Cregan, M.D.; Ramsay, D.T.; Doherty, D.A.; Hartmann, P.E. Volume and frequency of breastfeedings and fat content of breast milk throughout the day. Pediatrics 2006, 117, e387-e395. [CrossRef] [PubMed]

28. Bagnall, A. Feeding problems. In Feeding and Nutrition in the Preterm Infant; Jones, E., King, C., Eds.; Elsevier Churchill Livingstone: Edinburgh, UK, 2005; pp. 165-183.

29. Zimmerman, E.; Thompson, K. Clarifying nipple confusion. J. Perinatol. 2015, 35, 895-899. [CrossRef] [PubMed]

30. Geddes, D.T.; Sakalidis, V.S.; Hepworth, A.R.; McClellan, H.L.; Kent, J.C.; Lai, C.T.; Hartmann, P.E. Tongue movement and intra-oral vacuum of term infants during breastfeeding and feeding from an experimental teat that released milk under vacuum only. Early Hum. Dev. 2012, 88, 443-449. [CrossRef] [PubMed]

31. Miller, J.L.; Kang, S.M. Preliminary ultrasound observation of lingual movement patterns during nutritive versus non-nutritive sucking in a premature infant. Dysphagia 2007, 22, 150-160. [CrossRef] [PubMed]

32. Eishima, K. The analysis of sucking behaviour in newborn infants. Early Hum. Dev. 1991, 27, $163-173$. [CrossRef]

33. Meier, P.P.; Brown, L.P.; Hurst, N.M.; Spatz, D.L.; Engstrom, J.L.; Borucki, L.C.; Krouse, A.M. Nipple shields for preterm infants: Effect on milk transfer and duration of breastfeeding. J. Hum. Lact. 2000, 16, 106-114. [CrossRef] [PubMed]

34. Maastrup, R.; Hansen, B.M.; Kronborg, H.; Bojesen, S.N.; Hallum, K.; Frandsen, A.; Kyhnaeb, A.; Svarer, I.; Hallström, I. Breastfeeding progression in preterm infants is influenced by factors in infants, mothers and clinical practice: The results of a national cohort study with high breastfeeding initiation rates. PLoS ONE 2014, 9, e108208. [CrossRef] [PubMed]

35. Maastrup, R.; Hansen, B.M.; Kronborg, H.; Bojesen, S.N.; Hallum, K.; Frandsen, A.; Kyhnaeb, A.; Svarer, I.; Hallström, I. Factors associated with exclusive breastfeeding of preterm infants. Results from a prospective national cohort study. PLoS ONE 2014, 9, e89077. [CrossRef] [PubMed]

36. Geddes, D.T.; Kent, J.C.; Mitoulas, L.R.; Hartmann, P.E. Tongue movement and intra-oral vacuum in breastfeeding infants. Early Hum. Dev. 2008, 84, 471-477. [CrossRef] [PubMed]

37. Sakalidis, V.S.; Williams, T.M.; Garbin, C.P.; Hepworth, A.R.; Hartmann, P.E.; Paech, M.J.; Geddes, D.T. Ultrasound imaging of infant sucking dynamics during the establishment of lactation. J. Hum. Lact. 2013, 29, 205-213. [CrossRef] [PubMed]

38. Elad, D.; Kozlovsky, P.; Blum, O.; Laine, A.F.; Po, M.J.; Botzer, E.; Dollberg, S.; Zelicovich, M.; Ben Sira, L. Biomechanics of milk extraction during breast-feeding. Proc. Natl. Acad. Sci. USA 2014, 111, 5230-5235. [CrossRef] [PubMed]

39. Nowak, A.J.; Smith, W.L.; Erenberg, A. Imaging evaluation of breast-feeding and bottle-feeding systems. J. Pediatr. 1995, 126, S130-S134. [CrossRef]

40. Hohoff, A.; Rabe, H.; Ehmer, U.; Harms, E. Palatal development of preterm and low birthweight infants compared to term infants-What do we know? Part 1: The palate of the term newborn. Head Face Med. 2005, 1, 8. [CrossRef] [PubMed]

41. Hohoff, A.; Rabe, H.; Ehmer, U.; Harms, E. Palatal development of preterm and low birthweight infants compared to term infants-What do we know? Part 2: The palate of the preterm/low birthweight infant. Head Face Med. 2005, 1, 9. [CrossRef] [PubMed]

42. Victora, C.G.; Bahl, R.; Barros, A.J.; França, G.V.; Horton, S.; Krasevec, J.; Murch, S.; Sankar, M.J.; Walker, N.; Rollins, N.C.; et al. Breastfeeding in the 21st century: Epidemiology, mechanisms, and lifelong effect. Lancet 2016, 387, 475-490. [CrossRef]

43. Palmer, B. The influence of breastfeeding on the development of the oral cavity: A commentary. J. Hum. Lact. 1998, 14, 93-98. [CrossRef] [PubMed]

44. Arvedson, J.C. Assessment of pediatric dysphagia and feeding disorders: Clinical and instrumental approaches. Dev. Disabil. Res. Rev. 2008, 14, 118-127. [CrossRef] [PubMed]

45. Delaney, A.; Arvedson, J.C. Development of swallowing and feeding: Prenatal through first year of life. Dev. Disabil. Res. Rev. 2008, 14, 105-117. [CrossRef] [PubMed] 
46. Barlow, S.M. Oral and respiratory control for preterm feeding. Curr. Opin. Otolaryngol. Head Neck Surg. 2009, 17, 179-186. [CrossRef] [PubMed]

47. Lau, C. Development of suck and swallow mechanisms in infants. Ann. Nutr. Metab. 2015, 66, 7-14. [CrossRef] [PubMed]

48. McClellan, H.; Geddes, D.; Kent, J.; Garbin, C.; Mitoulas, L.; Hartmann, P. Infants of mothers with persistent nipple pain exert strong sucking vacuums. Acta Paediatr. 2008, 97, 1205-1209. [CrossRef] [PubMed]

49. Sakalidis, V.S.; Kent, J.C.; Garbin, C.P.; Hepworth, A.R.; Hartmann, P.E.; Geddes, D.T. Longitudinal changes in suck-swallow-breathe, oxygen saturation, and heart rate patterns in term breastfeeding infants. J. Hum. Lact. 2013, 29, 236-245. [CrossRef] [PubMed]

50. Schrank, W.; Al-Sayed, L.E.; Beahm, P.H.; Thach, B.T. Feeding responses to free-flow formula in term and preterm infants. J. Pediatr. 1998, 132, 426-430. [CrossRef]

51. Chen, C.H.; Wang, T.M.; Chang, H.M.; Chi, C.S. The effect of breast- and bottle-feeding on oxygen saturation and body temperature in preterm infants. J. Hum. Lact. 2000, 16, 21-27. [CrossRef] [PubMed]

52. Hsiao, C.C.; Tsai, M.L.; Chen, C.C.; Lin, H.C. Early optimal nutrition improves neurodevelopmental outcomes for very preterm infants. Nutr. Rev. 2014, 72, 532-540. [CrossRef] [PubMed]

53. Cannon, A.M.; Sakalidis, V.S.; Lai, C.T.; Perrella, S.L.; Geddes, D.T. Vacuum characteristics of the sucking cycle and relationships with milk removal from the breast in term infants. Early Hum. Dev. 2016, 96, 1-6. [CrossRef] [PubMed]

54. Paulsson, L.; Soderfeldt, B.; Bondemark, L. Malocclusion traits and orthodontic treatment needs in prematurely born children. Angle Orthod. 2008, 78, 786-792. [CrossRef] [PubMed]

55. Zaidi, I.; Thayath, M.N.; Singh, S.; Sinha, A. Preterm birth: A primary etiological factor for delayed oral growth and development. Int. J. Clin. Pediatr. Dent. 2015, 8, 215-219. [CrossRef] [PubMed]

56. Huang, Y.S.; Guilleminault, C. Pediatric obstructive sleep apnea and the critical role of oral-facial growth: Evidences. Front. Neurol. 2012, 3, 184. [CrossRef] [PubMed]

(C) 2018 by the authors. Licensee MDPI, Basel, Switzerland. This article is an open access article distributed under the terms and conditions of the Creative Commons Attribution (CC BY) license (http:/ / creativecommons.org/licenses/by/4.0/). 
Article

\title{
Concentrations of Water-Soluble Forms of Choline in Human Milk from Lactating Women in Canada and Cambodia
}

\author{
Alejandra M. Wiedeman ${ }^{1,2}$, Kyly C. Whitfield ${ }^{1,2,3}$, Kaitlin M. March ${ }^{1,2}$, Nancy N. Chen ${ }^{1,2}$, \\ Hou Kroeun ${ }^{4}$, Ly Sokhoing ${ }^{4}$, Prak Sophonneary ${ }^{5}$, Roger A. Dyer ${ }^{2}$, Zhaoming Xu ${ }^{1}$, \\ David D. Kitts ${ }^{1}$, Timothy J. Green ${ }^{1,2,6}$, Sheila M. Innis ${ }^{2,+}$ and Susan I. Barr ${ }^{1, *}$ \\ 1 Food, Nutrition, and Health Program, The University of British Columbia, Vancouver, BC V6T 1Z4, Canada; \\ awiedeman@bcchr.ca (A.M.W.); kyly.whitfield@msvu.ca (K.C.W.); kaitlin.march@cw.bc.ca (K.M.M.); \\ nnchen30@gmail.com (N.N.C.); zhaoming.xu@ubc.ca (Z.X.); david.kitts@ubc.ca (D.D.K.); \\ tim.green@sahmri.com (T.J.G.) \\ 2 BC Children's Hospital Research Institute, Vancouver, BC V5Z 4H4, Canada; radyer@mail.ubc.ca (R.A.D.); \\ sinnis@mail.ubc.ca (S.M.I.) \\ 3 Department of Applied Human Nutrition, Mount Saint Vincent University, Halifax, NS B3M 2J6, Canada \\ 4 Helen Keller International—Cambodia Country Office, Phnom Penh 12301, Cambodia; \\ hkroeun@hki.org (H.K.); lsokhoing@hki.org (L.S.) \\ 5 National Nutrition Programme, Maternal and Child Health Centre, Ministry of Health, \\ Phnom Penh 12202, Cambodia; sophonprak@gmail.com \\ 6 South Australia Health and Medical Research Institute, Adelaide, SA 5000, Australia \\ * Correspondence: susan.barr@ubc.ca; Tel.: +1-604-822-6766 \\ + Deceased.
}

Received: 15 February 2018; Accepted: 16 March 2018; Published: 20 March 2018

\begin{abstract}
Choline has critical roles during periods of rapid growth and development, such as infancy. In human milk, choline is mostly present in water-soluble forms (free choline, phosphocholine, and glycerophosphocholine). It is thought that milk choline concentration is influenced by maternal choline intake, and the richest food sources for choline are of animal origin. Scarce information exists on milk choline from countries differing in animal-source food availability. In this secondary analysis of samples from previous trials, the concentrations of the water-soluble forms of choline were quantified by liquid chromatography-tandem mass spectrometry in mature milk samples collected from lactating women in Canada $(n=301)$ and in Cambodia $(n=67)$. None of the water-soluble forms of choline concentrations in milk differed between Canada and Cambodia. For all milk samples $(n=368)$, free choline, phosphocholine, glycerophosphocholine, and the sum of water-soluble forms of choline concentrations in milk were (mean $(95 \% \mathrm{CI})) 151(141,160,540(519,562), 411(396,427)$, and $1102(1072,1133) \mu \mathrm{mol} / \mathrm{L}$, respectively. Theoretically, only $19 \%$ of infants would meet the current Adequate Intake (AI) for choline. Our findings suggest that the concentrations in milk of water-soluble forms of choline are similar in Canada and Cambodia, and that the concentration used to set the infant AI might be inaccurate.
\end{abstract}

Keywords: choline; phosphocholine; glycerophosphocholine; lactation; human milk; infants; adequate intake; dietary recommendations; Canada; Cambodia

\section{Introduction}

Choline is an essential nutrient with important roles during periods of rapid growth and development, such as early infancy [1-3]. Choline has a wide range of functions, including serving as a precursor for the synthesis of acetylcholine, phospholipids critical for cell membranes, surfactants important in 
lung maturity, bile formation, and betaine, which is an osmolyte and a methyl group donor [4,5]. In humans, large amounts of choline are present in milk, mainly as the water-soluble forms free choline, phosphocholine, and glycerophosphocholine, contributing an average of $84 \%$ of the total choline, whereas the lipid-soluble (phosphatidylcholine and sphingomyelin) forms account for the remaining $16 \%$ [6-10]. It has been reported that milk choline concentrations are positively associated with circulating choline concentrations in infants [7], and elevated circulating choline, as free choline, might enhance free choline uptake across the blood-brain barrier [11,12]. The authors of a recent observational study suggest that higher milk free choline concentration (mean $158 \mu \mathrm{mol} / \mathrm{L}$ ) is associated with better infant recognition memory (latency at central leads, $\beta=1.90, R^{2}=0.30, p<0.01$ ) adjusted by other milk nutrients at an age of six months [13].

The World Health Organization recommends exclusive breastfeeding for the first six months to promote optimal growth of the infant [14]. Generally, the average concentration of nutrients in mature milk from healthy, well-nourished mothers, in conjunction with the average volume consumed by fully breast-fed infants, is used to set dietary recommendations for infancy and to determine the additional amount of the nutrient over the recommendation for non-lactating women for lactation [1]. For early infancy ( $0-6$ months), the Adequate Intake (AI) for choline was set at $125 \mathrm{mg} /$ day (equivalent to $1500 \mu \mathrm{mol} / \mathrm{L}$ and an assumed volume of $780 \mathrm{~mL}$ ) [1]. Milk choline concentrations are thought to reflect habitual maternal dietary choline intake $[15,16]$, with foods of animal origin being the richest source of choline [17].

Limited data are available on choline concentration in mature human milk, including reports from the United States [6,8,10,18], Turkey [7,9], Canada [19], Japan [20], Korea [21], Sweden [22], and Ecuador [15]. Nevertheless, sample sizes in these studies are small $(n=1-75)$, and include milk samples collected after a full-term, pre-term, or mixed birth term pregnancy. Moreover, the available data on milk choline are generally from high-income countries where maternal animal-source food availability [23] and consumption is greater than in middle- and low-income countries [24,25].

In this study, we used secondary data from recent randomized controlled trials (none of which provided choline), in which mature milk samples were collected from lactating women in Canada and Cambodia, two countries differing in dietary food source availability [23]. Our primary aim was to assess the concentration of the water-soluble forms of choline. In addition, we also explored the associations between maternal dietary choline intake and milk concentrations of water-soluble forms of choline in a subset of the Canadian participants. We found that the concentrations of milk water-soluble forms of choline did not significantly differ between Canada and Cambodia, despite lower likely choline intakes in Cambodia, and that maternal total dietary choline intake was not significantly associated with the concentration of water-soluble forms of choline in milk in a subset of the Canadian participants.

\section{Materials and Methods}

\subsection{Study Participants and Sample Collection}

The present study was a secondary cross-sectional analysis of previous randomized controlled trials conducted in Canada and Cambodia [26-28]. In all the trials, convenience samples of mature milk were collected from apparently healthy lactating women (18-45 year) who had low-risk pregnancies, uncomplicated deliveries, and gave birth to healthy, full-term infants. All subjects gave their informed consent for inclusion before they participated in the studies. The studies were conducted in accordance with the Declaration of Helsinki. Demographic characteristics including age, ethnicity, education, and household income were collected from the participants.

\subsubsection{Canadian Samples}

In Canada, participants were enrolled in two supplementation trials (neither of which contained choline) and have been previously described [26,27]. In the first trial, women had consumed a daily 
supplement containing docosahexaenoic acid or placebo from 16 weeks gestation through to the end of pregnancy [26]. In the second trial, women had consumed a daily prenatal multivitamin and mineral supplement and were randomly assigned to one of three vitamin D supplement groups between 13-24 weeks gestation through to eight weeks postpartum [27]. The University of British Columbia Children's and Women's Research Ethics Board approved these trials (H08-70242 and H09-01261).

Canadian participants provided a single mature milk sample at eight weeks postpartum. In the first trial, hindmilk samples were collected at the participant's house into pre-labeled tubes and stored in a home freezer for up to three days before being stored at $-80^{\circ} \mathrm{C}$ [26]. For the present study, a total of 147 milk samples from the first trial were available for analysis. In the second Canadian trial, a full breast expression from the breast that had not been most recently emptied was collected during a clinical visit and stored at $-80^{\circ} \mathrm{C}$ [27]. For the present study, a total of 154 milk samples from the second trial were available for analysis.

\subsubsection{Cambodian Samples}

In Cambodia, women had been consuming either a thiamine-fortified or placebo fish sauce, containing insignificant choline content [17], ad libitum for six months before milk collection [28]. The Cambodian National Ethics Committee for Health Research (245 NECHR) approved this trial.

Participants provided a single mature milk sample between three to 28 weeks postpartum as a full breast expression from the breast that had not been most recently emptied in their villages in Prey Veng. The milk samples were transported on ice to the National Institute of Public Health in Phnom Penh $(<5 \mathrm{~h})$ for storage at $-80^{\circ} \mathrm{C}$ until being shipped on dry ice to the University of British Columbia for analysis. For the present study, a total of 67 milk samples from Cambodia were available for analysis.

\subsection{Biochemical Analysis}

For all milk samples $(n=368)$, the concentrations of the water-soluble forms of choline (free choline, phosphocholine, and glycerophosphocholine) were quantified in $20 \mu \mathrm{L}$ aliquots by stable isotope dilution liquid chromatography-tandem mass spectrometry (LC-MS/MS), according to the method described in detail elsewhere [19]. The inter-assay and intra-assay coefficients of variation were: $4.8 \%$ and $1.4 \%$ for free choline, $5.4 \%$ and $0.2 \%$ for phosphocholine, and $3.6 \%$ and $2.1 \%$ for glycerophosphocholine, respectively.

\subsection{Dietary Choline Intake Assessment}

For all infants $(n=368)$, dietary choline intake was estimated using milk total choline concentration and daily reference intake volume, based on the methods used to set the AI for early infancy [1]. First, total choline in milk was calculated by multiplying the concentration of the sum of the water-soluble forms of choline of each participant by a conversion factor (1.19), based on the assumption that on average the water-soluble forms of choline make up $84 \%$ of total choline in human milk $[6-9,13]$. Then, dietary choline intake from milk was estimated using $780 \mathrm{~mL} /$ day as the reference volume of milk consumption of breastfed infants during their first six months [1].

In Canada, dietary intake during lactation was available for 143 of the participants enrolled in the first trial. Intake was estimated using a semi-quantitative food frequency questionnaire (FFQ), covering the intake of the previous month, administered at 16 and 36 weeks of gestation. Dietary intakes of choline were estimated using a nutrient analysis software (ESHA Food Processor SQL, version 10.14.41; Salem, OR) and the United States Department of Agriculture (USDA) database on the Choline Content of Common Foods (Version 2) [17]. A validation analysis indicated that compared to the mean of three $24 \mathrm{~h}$ recalls, the FFQ was a valid instrument to assess dietary choline intake: the energy-adjusted de-attenuated correlation coefficient was 0.70 , only $7.5 \%$ were grossly misclassified (e.g., opposite tertiles), and weighted Cohen's kappa was 0.32 [29]. 


\subsection{Statistical Analysis}

All statistical analyses were performed using SPSS 22.0 (SPSS Inc., Chicago, IL, USA) and statistical significance was set at $p<0.05$ for two-sided testing. Data normality was determined using the Kolmogorov-Smirnov test, and skewed distributed data was log-transformed before further analyses. The concentrations of water-soluble forms of choline in milk were compared within each trial, by randomization groups, using independent samples one-way analysis of variance (ANOVA) or Student's t-test, as appropriate. In Canada, the concentrations of water-soluble forms of choline in milk were compared between the two trials, by milk fraction collected (i.e., hindmilk versus a full breast expression), using independent samples Student's $t$-test. In Cambodia, the relationship between the concentration of water-soluble forms of choline in milk and weeks postpartum was assessed by Pearson's correlation coefficient.

Demographic characteristics including age, ethnicity, education, and household income were compared between the two groups, Canada and Cambodia, using independent samples Student's $t$-test for continuous variable and Fisher's exact test for categorical variables. Water-soluble forms of choline concentrations in milk were compared between Canadian and Cambodian participants using independent samples Student's $t$-test. The estimated total choline intake was compared with the corresponding AI by one sample Student's $t$-test. The relationships between maternal dietary choline intake during pregnancy and the concentrations of water-soluble forms of choline in milk samples at eight weeks postpartum were explored using Pearson's correlation coefficient, and Bonferroni's correction was used to adjust for multiple comparisons.

\section{Results}

A total of 301 milk samples from Canadian and 67 milk samples from Cambodian lactating women were included in this analysis. In Canada, the concentrations of individual and total water-soluble forms of choline in milk compared within each trial (randomization groups) and between the two trials (milk fraction collected) did not differ ( $p>0.05$ for all, Table S1), and therefore the participants from both Canadian trials are presented as one group. Among the Cambodian sample, the concentrations of individual and total water-soluble forms of choline in milk compared by randomization groups did not differ, and there was no need to control for weeks postpartum, as no statistically significant correlations were found with the concentrations of individual and total water-soluble forms of choline in milk ( $p>0.05$ for all, Table S2). Participant characteristics are shown in Table 1. Compared to Cambodian women, Canadian women differed in age, education, ethnicity, and household income $(p<0.001)$.

The concentrations of the individual and the sum of the water-soluble forms of choline in milk are presented in Table 2, and did not differ significantly when comparing milk samples between Canada and Cambodia ( $p>0.05$, for all). Although Canadian women were older than Cambodian women, maternal age was not correlated with milk choline concentrations ( $r=0.014$ to $0.065, p>0.05$, for all). Among all samples $(n=368)$, phosphocholine and glycerophosphocholine were the predominant compounds, contributing $49 \%$ and $37 \%$ to the sum of the water-soluble forms of choline in milk, respectively.

For infants, the estimated total choline intakes in Canada, Cambodia, and in both countries combined are presented in Table 3, and were significantly below the AI for choline for early infancy ( $125 \mathrm{mg} /$ day; $p<0.001$, for all). Based on these data, only $19 \%$ of infants in Canada and Cambodia would have met the current choline AI recommendation, compared to an expected value of $\sim 50 \%$ [1]. For maternal intake, since the estimated choline intakes between gestational time points (16 and 36 weeks of gestation) did not differ ( $p=0.927$, Table S3) and were significantly correlated $(r=0.590, p<0.001)$, only the second time point was used, as it was the closest to the milk collection. The estimated maternal total choline intake was significantly below the AI for choline for both pregnancy ( $450 \mathrm{mg} /$ day) and lactation $(550 \mathrm{mg} /$ day $)$ $(p<0.001$ for both).

The correlation coefficients between the estimated choline intake and the concentrations of the water-soluble forms of choline in milk are presented in Table 4 . The sum of the water-soluble forms of choline in milk was positively, but weakly, correlated with dietary total choline intake $(r=0.166$, 
$p=0.048)$, dietary free choline intakes $(r=0.223, p=0.007)$, and dietary water-soluble forms of choline intake $(r=0.182, p=0.029)$. After adjusting for multiple comparisons, the correlations were no longer significant (Bonferroni's correction $p>0.006$, for all).

Table 1. Demographic characteristics of lactating women in Canada and Cambodia.

\begin{tabular}{|c|c|c|c|}
\hline Demographic Characteristics & Canada $(n=301)$ & Cambodia $(n=67)$ & $p$ Value $^{2}$ \\
\hline Age (year) ${ }^{1}$ & $33.3 \pm 4.1$ & $26.1 \pm 4.7$ & $<0.001$ \\
\hline \multicolumn{4}{|l|}{ Parity $(n ; \%)$} \\
\hline 1 & $161 ; 53 \%$ & $34 ; 51 \%$ & \multirow{3}{*}{0.720} \\
\hline 2 & $112 ; 37 \%$ & $24 ; 36 \%$ & \\
\hline 3 or more & $28 ; 9 \%$ & $9 ; 12 \%$ & \\
\hline \multicolumn{4}{|l|}{ Education $(n ; \%)$} \\
\hline None & $0 ; 0 \%$ & $6 ; 9 \%$ & \multirow{4}{*}{$<0.001$} \\
\hline Some primary & $0 ; 0 \%$ & $31 ; 46 \%$ & \\
\hline Some secondary & $11 ; 4 \%$ & $30 ; 44 \%$ & \\
\hline Some postsecondary & $290 ; 96 \%$ & $0 ; 0 \%$ & \\
\hline \multicolumn{4}{|l|}{ Ethnicity $(n ; \%)$} \\
\hline European & $234 ; 78 \%$ & - & \multirow{4}{*}{$<0.001$} \\
\hline Asian & $40 ; 13 \%$ & - & \\
\hline Khmer & - & $67 ; 100 \%$ & \\
\hline Other & $27 ; 9 \%$ & - & \\
\hline Household income $(n ; \%)$ & & & $<0.001$ \\
\hline$<\$ 20000 \mathrm{CAD}$ & $10 ; 3 \%$ & $67 ; 100 \%$ & \\
\hline$\$ 20000-50000$ CAD & $47 ; 16 \%$ & - & \\
\hline$>\$ 50000 \mathrm{CAD}$ & $244 ; 81 \%$ & - & \\
\hline
\end{tabular}

${ }^{1}$ Data are presented as mean $\pm \mathrm{SD} ;{ }^{2}$ Continuous data were analyzed by independent samples Student's $t$-test, and categorical data analyzed by Fisher's exact test.

Table 2. Concentration of water-soluble forms of choline in milk samples of lactating women in Canada and Cambodia ${ }^{1}$.

\begin{tabular}{|c|c|c|c|c|}
\hline Forms of Choline Concentration $(\mu \mathrm{mol} / \mathrm{L})$ & $\begin{array}{l}\text { All Women } \\
\quad(n=368)\end{array}$ & $\begin{array}{c}\text { Canada } \\
(n=301)\end{array}$ & $\begin{array}{l}\text { Cambodia } \\
\quad(n=67)\end{array}$ & $p$ Value $^{2}$ \\
\hline Free choline & $151(141,160)$ & $155(144,165)$ & $143(132,154)$ & 0.071 \\
\hline Phosphocholine & $540(519,562)$ & $535(512,559)$ & $562(513,612)$ & 0.336 \\
\hline Glycerophosphocholine & $411(396,427)$ & $416(399,434)$ & $390(356,423)$ & 0.178 \\
\hline Water-soluble choline ${ }^{3}$ & $1102(1072,1133)$ & $1106(1071,1140)$ & $1095(1018,1150)$ & 0.686 \\
\hline
\end{tabular}

${ }^{1}$ Data are presented as mean (95\% CI), and concentrations were quantified using liquid chromatography-tandem mass spectrometry; ${ }^{2}$ Group differences were analyzed by independent samples Student $t$-test after log-transformation;

${ }^{3}$ Water-soluble choline corresponds to the sum of free choline, phosphocholine, and glycerophosphocholine.

Table 3. Estimated dietary total choline intake in Canada and Cambodia ${ }^{1}$.

\begin{tabular}{|c|c|c|c|c|}
\hline \multirow[b]{2}{*}{ Dietary Intake } & \multicolumn{3}{|c|}{ Infants $^{2}$} & \multirow[b]{2}{*}{$\begin{array}{l}\text { Maternal Canada } \\
\qquad(n=143)^{3}\end{array}$} \\
\hline & $\begin{array}{c}\text { All } \\
(n=368)\end{array}$ & $\begin{array}{c}\text { Canada } \\
(n=301)\end{array}$ & $\begin{array}{l}\text { Cambodia } \\
(n=67)\end{array}$ & \\
\hline Total choline (mg/day) & $106(103,109)$ & $107(103,110)$ & $105(98,111)$ & $408(390,427)$ \\
\hline AI (mg/day) & 125 & 125 & 125 & 450 \\
\hline Above $\mathrm{AI}^{4}(n ; \%)$ & $71 ; 19$ & $58 ; 19$ & $13 ; 19$ & $46 ; 32$ \\
\hline
\end{tabular}

${ }^{1}$ Dietary intake data are presented as mean $(95 \% \mathrm{CI}) ;{ }^{2}$ Total choline intakes were estimated assuming that water-soluble forms of choline contribute $84 \%$ to total choline and a reference milk intake of $780 \mathrm{~mL} / \mathrm{day} ;{ }^{3}$ Total choline intakes were estimated at 36 weeks of gestation using a food frequency questionnaire and the USDA database on choline content in common foods (version 2); ${ }^{4}$ Since the AI reflects the average choline concentration of breastmilk from healthy lactating women, it is expected that $\sim 50 \%$ of infants would have intakes above the AI. When mean intake equals the AI, it is assumed that the prevalence of inadequacy in the group is low; when mean intake is below the $\mathrm{AI}$, no conclusions regarding adequacy can be drawn. 
Table 4. Correlation between dietary choline intake during pregnancy and water-soluble forms of choline in milk from a subset of Canadian participants ${ }^{1}$.

\begin{tabular}{|c|c|c|c|c|}
\hline \multirow{2}{*}{$\begin{array}{l}\text { Dietary Intakes } \\
\quad(n=143)\end{array}$} & \multicolumn{4}{|c|}{ Milk Choline Metabolites } \\
\hline & Free Choline & Phospho-Choline & Glycerophospho-Choline & Water-Soluble Choline \\
\hline Free choline & 0.054 & 0.125 & 0.129 & $0.223 * *$ \\
\hline Phosphocholine & 0.134 & 0.063 & -0.007 & 0.128 \\
\hline Glycerophosphocholine & 0.105 & 0.034 & 0.101 & 0.130 \\
\hline Water-soluble choline ${ }^{2}$ & 0.102 & 0.094 & 0.093 & $0.182 *$ \\
\hline Phosphatidylcholine & -0.016 & 0.145 & -0.056 & 0.091 \\
\hline Sphingomyelin & -0.031 & 0.147 & -0.109 & 0.036 \\
\hline Lipid-soluble choline ${ }^{3}$ & -0.016 & 0.150 & -0.058 & 0.093 \\
\hline Total choline ${ }^{4}$ & 0.043 & 0.150 & 0.017 & $0.166^{*}$ \\
\hline
\end{tabular}

${ }^{1}$ Data are presented as Pearson's correlation coefficients after log-transformation, none of the observed associations were significant after Bonferroni's correction $\left(0.05 / 8=0.006 ; p>0.006\right.$, for all comparisons); ${ }^{2}$ Water-soluble choline corresponds to the sum of free choline, phosphocholine, and glycerophosphocholine; ${ }^{3}$ Lipid-soluble choline corresponds to the sum of phosphatidylcholine and sphingomyelin; ${ }^{4}$ Total choline corresponds to the sum of all individual forms of choline; ${ }^{*} p<0.05,{ }^{* *} p<0.01$.

\section{Discussion}

An interesting finding from our study was that concentrations of the water-soluble forms of choline in mature milk did not differ between lactating women in Canada and Cambodia (1106 and $1095 \mu \mathrm{mol} / \mathrm{L}$, respectively), regardless of differences in animal food availability [23] and different likely dietary choline intakes, as described for other low- and middle-income countries [24,25]. An earlier study, in 1982, reported that free choline concentration in milk was lower in lactating women in Ecuador $(40 \%<100 \mu \mathrm{mol} / \mathrm{L})$ compared to the United States $(55 \%>300 \mu \mathrm{mol} / \mathrm{L})$ [15]. However, free choline contributes an average of only $10 \%$ of the total choline in human milk [6-10], and it is unknown whether this difference would be physiologically relevant. Although dietary information was not collected, the authors hypothesized that the difference was a result of the low dietary choline intake in Ecuador [15].

Two recent choline supplementation studies reported that lactating women consuming choline supplemented diets (means of 930 or $1088 \mathrm{mg} /$ day) with close to two-times the corresponding AI (550 mg/day) had significantly higher milk total choline concentrations (20\%) compared to control groups (mean intakes of 364 or $480 \mathrm{mg} /$ day) [8,10]. In our study, the mean dietary choline intake in Canada (408 mg/day) was closer to the amount of choline consumed in the control than the supplemented groups of previous studies $[8,10]$. Thus, the difference in the dietary choline intake between Canada and Cambodia may not have reached the range in which a difference in milk choline concentration would be observed. An alternative explanation for similar milk choline concentrations between Canadian and Cambodian women in our study may be that the mammary gland can obtain choline from both maternal circulation [30] and the endogenous synthesis of phosphatidylcholine through the phosphatidylethanolamine $N$-methyl transferase (PEMT) pathway [31]. Although it has been described that PEMT is mostly active in liver and kidney [32,33], its activity has also been identified in the epithelial cells of the mammary gland [34]. Consequently, this may contribute to ensuring that sufficient choline is excreted in milk to meet the choline demand for the rapidly growing and developing infant.

The estimated mean concentration of water-soluble forms of choline reported in our study was $1102 \mu \mathrm{mol} / \mathrm{L}(n=368)$, which is in agreement with previously reported values in mature milk samples. In 1996, the first study including all individual forms of choline in mature milk from the United States reported a concentration of water-soluble forms of choline of $1048 \mu \mathrm{mol} / \mathrm{L}(n=16)$ [6]. Two later studies from the same country have reported mean concentrations of water-soluble forms of choline of 987 and $1024 \mu \mathrm{mol} / \mathrm{L}(n=28$ and $n=48)$ [8,10], where choline quantification was conducted by LC-MS/MS as in our analysis. In Canada, our group recently reported on the concentration of water-soluble forms of choline in donor's milk with a slightly higher mean concentration of water-soluble forms of choline of $1275 \mu \mathrm{mol} / \mathrm{L}(n=30)$ [19]. However, it is unknown whether the pasteurization process, which is routinely used in milk banks, has an impact on the total or individual forms of choline concentration in milk. In Turkey, slightly higher concentrations of water-soluble forms of choline in mature milk 
(1189 and $1402 \mu \mathrm{mol} / \mathrm{L})$ were reported in two studies $(n=12, n=54)$, where choline was analyzed by less sensitive enzymatic methods [7,9].

Based on our results, the estimated total choline intake of the infants was $106 \mathrm{mg} /$ day $(n=368)$, which is significantly below the corresponding AI for choline (125 mg/day) [1]. However, it is important to note that the AI for choline for infants of 0-6 months reflected a milk total choline concentration of $1500 \mu \mathrm{mol} / \mathrm{L}$, which in fact was $20 \%$ higher than the mean concentration of total choline in human milk published at that time $(1254 \mu \mathrm{mol} / \mathrm{L})[6]$, and no rationale was provided as to why a higher concentration was used to set the AI during infancy instead of the observed average as per definition [35]. Accordingly, results categorized using the current AI for choline for infants of 0-6 months as a cut-off must be interpreted with caution, as the current recommendation may not accurately reflect the mean total choline concentration in human milk.

The milk choline concentrations reported in the present study are also relevant from a clinical perspective, as the nutrient content in human milk may be used as a guideline to develop human milk substitutes and enteral formulas for infants. Current guidelines for choline content in infant formula are 7-50 mg/100 kcal [36,37], which is equivalent to a wide range of choline intake ranging between 37-265 mg/day, based on milk consumption of $780 \mathrm{~mL} /$ day and energy content of $68 \mathrm{kcal} / 100 \mathrm{~mL}$ [36]. Most of the commercially available infant formulas add choline as free choline, which contributes $57 \%$ of total choline [17], compared to only $10 \%$ in human milk [6-10]. Accordingly, current infant formula composition does not mimic the profile of choline forms present in human milk; whether this is physiologically relevant is not known.

In this study, we found that the concentration of water-soluble forms of choline in milk was not significantly associated with maternal choline intake when estimated during pregnancy from a subset of the Canadian participants. In line with this finding, another study reported that choline intake was not associated with choline concentration in milk, except for a weak association with milk phosphatidylcholine $\left(\mathrm{R}^{2}=0.11, p=0.007\right)$ [8]. It also could be possible that maternal intake during pregnancy was not the same as when the milk samples were collected at eight weeks postpartum. However, previous studies have observed a minor and non-significant variation in the maternal diet when comparing intakes during pregnancy and lactation periods ( $2-4 \%$ change) $[38,39]$. This is in agreement with the finding that total choline intakes at 16 and 36 weeks of gestation did not differ $(p=0.927)$ and were positively associated $(\mathrm{r}=0.590, p<0.001)$ in our study.

A potential limitation of the present study was that milk samples were collected at different time points during lactation among the Cambodian participants (3-28 weeks postpartum). However, it has been described that total choline concentration in milk increases from colostrum at birth to two weeks postpartum, and then stays stable beyond six months $[7,20,21,40]$, and we found no association between total water-soluble choline concentration and week postpartum of the milk collection $(p=0.915)$. Additionally, we compared different milk fractions, hindmilk versus a full breast expression, which, at least for the water-soluble forms of choline, did not differ in the Canadian trials (Table S1, $p>0.05$ ) and is in agreement with an earlier study that compared different milk fractions [18]. Another limitation was that only the water-soluble forms of choline were quantified, without including the lipid-soluble forms. However, the water-soluble forms of choline contribute an average of $84 \%$ of the total choline concentration in human milk, which is relatively stable based on the published data even among the supplemented groups [6-10]. In addition, the actual infant daily volume intake of human milk is unknown, and the reference intake volume $(780 \mathrm{~mL} /$ day) used to set the $\mathrm{AI}$ for different nutrients, including choline, for early infancy was selected [1], which was obtained from earlier studies assessing infant's body weight before and after breastfeeding during the first six months of life [41-44]. Recently, a comparable value of the daily volume of human milk intake between two and five months has been published [45]. Moreover, no dietary information was collected in Cambodia and further studies need to be conducted to confirm the differences assumed in total choline intake. 


\section{Conclusions}

The present study is the first to report water-soluble forms of choline concentrations in mature milk samples collected from lactating women in Cambodia and could be considered the largest study to date for choline assessment in human milk. In summary, we found that the concentrations of the water-soluble forms of choline in mature human milk samples did not differ between Canadian and Cambodian lactating women, despite lower likely choline intakes in Cambodia. The finding that the estimated dietary choline intake during early infancy was below the corresponding AI suggest that the current AI may not reflect the average choline concentration in human milk of healthy lactating women. Among a subset of the Canadian participants, maternal dietary choline was not significantly correlated to the milk water-soluble forms of choline.

Supplementary Materials: The following are available online at http://www.mdpi.com/2072-6643/10/3/381/s1, Table S1: Comparison of the concentrations of water-soluble forms of choline in milk samples in the Canadian trials, Table S2: Comparison of the concentrations of water-soluble forms of choline in milk samples in the Cambodian trial, and Table S3: Estimated dietary choline intake during pregnancy from a subset of Canadian participants.

Acknowledgments: Funding: Supported by the Canadian Institutes of Health Research and Grand Challenges Canada Stars in Global Health Round VI Phase I grant. A.M.W. received funding support through Doctoral Scholarship to study abroad, the Government of Chile, provided through Becas-Chile, and the Nutritional Research Fellowship, University of British Columbia. K.C.W. gratefully acknowledges funding support through the International Development Research Centre Doctoral Research Award, and K.C.W. and K.M.M. were supported by Canadian Institutes of Health Research Frederick Banting and Charles Best Canada Graduate Scholarships.

Author Contributions: The authors' contributions were as follows: S.M.I. and T.J.G. designed the research; K.C.W., K.M.M., N.N.C., H.K, L.S, and P.S. coordinated and conducted the studies from which the milk samples were obtained; R.A.D. contributed to laboratory analysis; and A.M.W. conducted laboratory and dietary analyses, analyzed data, and prepared the initial draft of the manuscript; Z.X., D.D.K., T.J.G., and S.I.B. provided critical input into the manuscript. All authors have read and approved the final version of the manuscript.

Conflicts of Interest: The authors declare no conflict of interest.

\section{References}

1. Institute of Medicine. Dietary Reference Intakes for Thiamin, Riboflavin, Niacin, Vitamin B6, Folate, Vitamin B12, Pantothenic Acid, Biotin, and Choline; The National Academy Press: Washington, DC, USA, 1998.

2. Zeisel, S.H.; Niculescu, M.D. Perinatal choline influences brain structure and function. Nutr. Rev. 2006, 64, 197-203. [CrossRef] [PubMed]

3. Zeisel, S.H.; da Costa, K.A. Choline: An essential nutrient for public health. Nutr. Rev. 2009, 67, 615-623. [CrossRef] [PubMed]

4. Zeisel, S.H.; Blusztajn, J.K. Choline and human nutrition. Annu. Rev. Nutr. 1994, 14, 269-296. [CrossRef] [PubMed]

5. Ueland, P.M. Choline and betaine in health and disease. J. Inherit. Metab. Dis. 2011, 34, 3-15. [CrossRef] [PubMed]

6. Holmes-McNary, M.Q.; Cheng, W.L.; Mar, M.H.; Fussell, S.; Zeisel, S.H. Choline and choline esters in human and rat milk and in infant formulas. Am. J. Clin. Nutr. 1996, 64, 572-576. [CrossRef] [PubMed]

7. Ilcol, Y.O.; Ozbek, R.; Hamurtekin, E.; Ulus, I.H. Choline status in newborns, infants, children, breast-feeding women, breast-fed infants and human breast milk. J. Nutr. Biochem. 2005, 16, 489-499. [CrossRef] [PubMed]

8. Fischer, L.M.; da Costa, K.A.; Galanko, J.; Sha, W.; Stephenson, B.; Vick, J.; Zeisel, S.H. Choline intake and genetic polymorphisms influence choline metabolite concentrations in human breast milk and plasma. Am. J. Clin. Nutr. 2010, 92, 336-346. [CrossRef] [PubMed]

9. Ozarda, Y.; Cansev, M.; Ulus, I.H. Breast milk choline contents are associated with inflammatory status of breastfeeding women. J. Hum. Lact. 2014, 30, 161-166. [CrossRef] [PubMed]

10. Davenport, C.; Yan, J.; Taesuwan, S.; Shields, K.; West, A.A.; Jiang, X.; Perry, C.A.; Malysheva, O.V.; Stabler, S.P.; Allen, R.H.; et al. Choline intakes exceeding recommendations during human lactation improve breast milk choline content by increasing PEMT pathway metabolites. J. Nutr. Biochem. 2015, 26, 903-911. [CrossRef] [PubMed] 
11. Klein, J.; Koppen, A.; Loffelholz, K. Regulation of free choline in rat brain: Dietary and pharmacological manipulations. Neurochem. Int. 1998, 32, 479-485. [CrossRef]

12. Babb, S.M.; Ke, Y.; Lange, N.; Kaufman, M.J.; Renshaw, P.F.; Cohen, B.M. Oral choline increases choline metabolites in human brain. Psychiatry Res. 2004, 130, 1-9. [CrossRef]

13. Cheatham, C.L.; Sheppard, K.W. Synergistic effects of human milk nutrients in the support of infant recognition memory: An observational study. Nutrients 2015, 7, 9079-9095. [CrossRef] [PubMed]

14. World Health Organization. The World Health Organization's Infant Feeding Recommendation; WHO: Geneva, Switzerland, 2001.

15. Zeisel, S.H.; Stanbury, J.B.; Wurtman, R.J.; Brigida, M.; Fierro-Benitez, R. Choline content of mothers' milk in Ecuador and Boston. N. Engl. J. Med. 1982, 306, 175-176. [PubMed]

16. Allen, L.H. B vitamins in breast milk: Relative importance of maternal status and intake, and effects on infant status and function. Adv. Nutr. 2012, 3, 362-369. [CrossRef] [PubMed]

17. United States Department of Agriculture. Database for the Choline Content of Common Foods, Release 2; Agricultural Research Service: Washington, DC, USA, 2008.

18. Zeisel, S.H.; Char, D.; Sheard, N.F. Choline, phosphatidylcholine and sphingomyelin in human and bovine milk and infant formulas. J. Nutr. 1986, 116, 50-58. [CrossRef] [PubMed]

19. Moukarzel, S.; Soberanes, L.; Dyer, R.A.; Albersheim, S.; Elango, R.; Innis, S.M. Relationships among different water-soluble choline compounds differ between human preterm and donor milk. Nutrients 2017, 9, 696. [CrossRef] [PubMed]

20. Sakurai, T.; Furukawa, M.; Asoh, M.; Kanno, T.; Kojima, T.; Yonekubo, A. Fat-soluble and water-soluble vitamin contents of breast milk from Japanese women. J. Nutr. Sci. Vitaminol. 2005, 51, 239-247. [CrossRef] [PubMed]

21. Hanok Jeong, Y.S.; Chung, Y.-J. Choline and betaine concentrations in breast milk of Korean lactating women and the choline and betaine intakes of their infants. Korean J. Nutr. 2010, 43, 588-596. [CrossRef]

22. Wu, J.; Domellof, M.; Zivkovic, A.M.; Larsson, G.; Ohman, A.; Nording, M.L. NMR-based metabolite profiling of human milk: A pilot study of methods for investigating compositional changes during lactation. Biochem. Biophys. Res. Commun. 2016, 469, 626-632. [CrossRef] [PubMed]

23. Food and Agriculture Organization of the United Nations, Statistical Division. FAOSTAT Statistics Database. 2013. Available online: www.fao.org/faostat/en/\#compare (accessed on 20 August 2017).

24. Gossell-Williams, M.; Fletcher, H.; McFarlane-Anderson, N.; Jacob, A.; Patel, J.; Zeisel, S. Dietary intake of choline and plasma choline concentrations in pregnant women in Jamaica. West Indian Med. J. 2005, 54, 355-359. [CrossRef] [PubMed]

25. Goon, S.; Dey, S.R. A 24-hour dietary recall for assessing the intake pattern of choline among Bangladeshi pregnant women at their third trimester of pregnancy. Cent. Asian J. Glob. Health 2014, 3. [CrossRef]

26. Mulder, K.A.; King, D.J.; Innis, S.M. Omega-3 fatty acid deficiency in infants before birth identified using a randomized trial of maternal dha supplementation in pregnancy. PLoS ONE 2014, 9, e83764. [CrossRef] [PubMed]

27. March, K.M.; Chen, N.N.; Karakochuk, C.D.; Shand, A.W.; Innis, S.M.; von Dadelszen, P.; Barr, S.I.; Lyon, M.R.; Whiting, S.J.; Weiler, H.A.; et al. Maternal vitamin D(3) supplementation at $50 \mu \mathrm{g} / \mathrm{d}$ protects against low serum 25-hydroxyvitamin D in infants at $8 \mathrm{wk}$ of age: A randomized controlled trial of 3 doses of vitamin D beginning in gestation and continued in lactation. Am. J. Clin. Nutr. 2015, 102, 402-410. [CrossRef] [PubMed]

28. Whitfield, K.C.; Karakochuk, C.D.; Kroeun, H.; Hampel, D.; Sokhoing, L.; Chan, B.B.; Borath, M.; Sophonneary, P.; McLean, J.; Talukder, A.; et al. Perinatal consumption of thiamine-fortified fish sauce in rural Cambodia: A randomized clinical trial. JAMA Pediatr. 2016, 170, e162065. [CrossRef] [PubMed]

29. Wiedeman Manriquez, A.M. Dietary Choline Intake and Biomarkers of Choline Status across the Life Cycle. Ph.D. Thesis, University of British Columbia, Vancouver, BC, Canada, 2017.

30. Chao, C.K.; Pomfret, E.A.; Zeisel, S.H. Uptake of choline by rat mammary-gland epithelial cells. Biochem. J. 1988, 254, 33-38. [CrossRef] [PubMed]

31. Vance, J.E.; Vance, D.E. Phospholipid biosynthesis in mammalian cells. Biochem. Cell Biol. 2004, 82, 113-128. [CrossRef] [PubMed]

32. Bremer, J.; Greenberg, D.M. Biosynthesis of choline in vitro. Biochim. Biophys. Acta 1960, 37, 173-175. [CrossRef]

33. Vance, D.E.; Walkey, C.J.; Cui, Z. Phosphatidylethanolamine N-methyltransferase from liver. Biochim. Biophys. Acta 1997, 1348, 142-150. [CrossRef] 
34. Yang, E.K.; Blusztajn, J.K.; Pomfret, E.A.; Zeisel, S.H. Rat and human mammary tissue can synthesize choline moiety via the methylation of phosphatidylethanolamine. Biochem. J. 1988, 256, 821-828. [CrossRef] [PubMed]

35. Institute of Medicine. Dietary Reference Intakes: Applications in Dietary Assessment; The National Academies Press: Washington, DC, USA, 2000.

36. Koletzko, B.; Baker, S.; Cleghorn, G.; Neto, U.F.; Gopalan, S.; Hernell, O.; Hock, Q.S.; Jirapinyo, P.; Lonnerdal, B.; Pencharz, P.; et al. Global standard for the composition of infant formula: Recommendations of an ESPGHAN coordinated international expert group. J. Pediatr. Gastroenterol. Nutr. 2005, 41, 584-599. [CrossRef] [PubMed]

37. Codex Alimentarious Commision. CODEX STAN 72-1981 for Infant Formula and Formulas for Special Medical Purposes Intended for Infants; Codex Alimentarius: Geneva, Switzerland, 2007.

38. Lewis, E.D.; Subhan, F.B.; Bell, R.C.; McCargar, L.J.; Curtis, J.M.; Jacobs, R.L.; Field, C.J.; APoON Team. Estimation of choline intake from $24 \mathrm{~h}$ dietary intake recalls and contribution of egg and milk consumption to intake among pregnant and lactating women in Alberta. Br. J. Nutr. 2014, 112, 112-121. [CrossRef] [PubMed]

39. Pauwels, S.; Ghosh, M.; Duca, R.C.; Bekaert, B.; Freson, K.; Huybrechts, I.; Langie, S.A.S.; Koppen, G.; Devlieger, R.; Godderis, L. Maternal intake of methyl-group donors affects DNA methylation of metabolic genes in infants. Clin. Epigenetics 2017, 9, 16. [CrossRef] [PubMed]

40. Holmes, H.C.; Snodgrass, G.J.; Iles, R.A. Changes in the choline content of human breast milk in the first 3 weeks after birth. Eur. J. Pediatr. 2000, 159, 198-204. [CrossRef] [PubMed]

41. Hofvander, Y.; Hagman, U.; Hillervik, C.; Sjolin, S. The amount of milk consumed by 1-3 months old breastor bottle-fed infants. Acta Paediatr. Scand. 1982, 71, 953-958. [CrossRef] [PubMed]

42. Butte, N.F.; Garza, C.; Smith, E.O.; Nichols, B.L. Human milk intake and growth in exclusively breast-fed infants. J. Pediatr. 1984, 104, 187-195. [CrossRef]

43. Neville, M.C.; Keller, R.; Seacat, J.; Lutes, V.; Neifert, M.; Casey, C.; Allen, J.; Archer, P. Studies in human lactation: Milk volumes in lactating women during the onset of lactation and full lactation. Am. J. Clin. Nutr. 1988, 48, 1375-1386. [CrossRef] [PubMed]

44. Allen, J.C.; Keller, R.P.; Archer, P.; Neville, M.C. Studies in human lactation: Milk composition and daily secretion rates of macronutrients in the first year of lactation. Am. J. Clin. Nutr. 1991, 54, 69-80. [CrossRef] [PubMed]

45. Gridneva, Z.; Rea, A.; Hepworth, A.R.; Ward, L.C.; Lai, C.T.; Hartmann, P.E.; Geddes, D.T. Relationships between breastfeeding patterns and maternal and infant body composition over the first 12 months of lactation. Nutrients 2018, 10, 45. [CrossRef] [PubMed]

(C) 2018 by the authors. Licensee MDPI, Basel, Switzerland. This article is an open access article distributed under the terms and conditions of the Creative Commons Attribution (CC BY) license (http:/ / creativecommons.org/licenses/by/4.0/). 


\title{
Review \\ Supporting Mothers of Very Preterm Infants and Breast Milk Production: A Review of the Role of Galactogogues
}

\author{
Elizabeth V. Asztalos \\ Department of Newborn and Developmental Paediatrics, Sunnybrook Health Sciences Centre, \\ University of Toronto, M4N 3M5 Toronto, ON, Canada; elizabeth.asztalos@sunnybrook.ca; \\ Tel.: +1-416-480-6100 (ext. 87791); Fax: +1-416-480-5612
}

Received: 8 April 2018; Accepted: 10 May 2018; Published: 12 May 2018

\begin{abstract}
Human milk, either mother's own milk or donor human milk, is recommended as the primary source of nutrition for very preterm infants. Initiatives should be in place in neonatal units to provide support to the mother as she strives to initiate and maintain a supply of breast milk for her infant. The use of galactogogues are considered when these initiatives alone may not be successful in supporting mothers in this endeavor. Although there are non-pharmacologic compounds, this review will focus on the pharmacologic galactogogues currently available and the literature related to their use in mothers of very preterm infants.
\end{abstract}

Keywords: breast milk; galactogogues; mothers of preterm infants

\section{Introduction}

The very preterm infant ( $<30$ weeks gestation) is faced with an array of serious morbidities, which can include sepsis (late-onset), necrotizing enterocolitis (NEC), retinopathy of prematurity, bronchopulmonary dysplasia (BPD), and intracranial white matter injury [1-6]. Human milk is the recommended nutritional support for the very preterm infant as it aids in reducing these morbidities and improves the neurodevelopmental outcomes for these infants [7-10]. The bioactive components found in breast milk are thought to promote gastrointestinal development, provide substrate for brain development and reduce the incidence of sepsis and necrotizing enterocolitis, both of which are linked in part to a negative impact on neurodevelopment [11-14]. Based on these clinical information, it is recommended that very preterm infants receive breast milk, preferably mother's own milk, as the primary source of nutrition rather than rely on preterm formula [15]. Consequently, mothers are encouraged to initiate hand expression and pumping within hours of giving birth to provide breast milk for their infants. With very preterm infants requiring hospitalization for anywhere from $10-16$ weeks, continued and sustained breast milk volumes can prove to be a challenge to even the most dedicated of mothers. Many mothers of very preterm infants, for a variety of reasons such as illness, stress and other factors related to preterm birth, are unable to exclusively feed their children [16-21].

\section{Breast Milk Production in Mothers of Preterm Infants}

Lactogenesis (milk synthesis) is noted to start around mid-pregnancy and has been referred to as having 2 stages (lactogenesis I and II) which are under the influence of hormones, namely estrogen, insulin, cortisol, progesterone, prolactin, and human placental lactogen [22-25]. Lactogenesis I represents the secretory differentiation phase where the mammary epithelial cells differentiate into secretory mammary epithelial cells with the capacity to synthesize milk constituents such as lactose, total proteins and immunoglobulins. After parturition, the secretory phase of lactogenesis 
or lactogenesis II is triggered by the rapid decline of serum maternal progesterone that occurs with the expulsion of the placenta; in addition, this leads to a drop in estrogen levels while prolactin levels remain high along with insulin and cortisol $[24,25]$. Colostrum is produced during the first 4 days postpartum, followed by transitional milk secretion for the next 10 days followed by mature milk production [26]. Milk volume rapidly increases after the first $24 \mathrm{~h}$ postpartum and stabilizes after 1 month postpartum to an average volume of $750-800 \mathrm{~mL} / 24 \mathrm{~h}$ for the term infant $[27,28]$. Milk production is increased by efficient and timely removal of milk, with adequate milk removal by day 3 postpartum being critical to the establishment of ongoing successful lactation [29]. Milk production is regulated by endocrine hormones (prolactin and oxytocin) as well as adequate and regular milk removal. Prolactin is required to maintain milk yield while oxytocin is released in response to suckling and induces the contraction of myoepithelial cells surrounding mammary alveoli triggering milk ejection, "milk let-down" [25]. Once milk secretion is established, hormone levels are maintained at low levels and ongoing production is regulated by consistent and regular milk removal (autocrine control); in the term infant, the volume of milk produced is determined by how the breast is emptied at feedings which, in turn, is determined by the infant's appetite [29-32].

Preterm birth may alter the normal sequence of lactogenesis. A delay in secretory activation can be associated with a negative impact on successful lactation [33,34]. Mothers of preterm infants can have problems at this stage as a result of their preterm delivery, antenatal corticosteroids, stress, maternal illness and operative delivery [19-21]. Mothers of very preterm infants must establish their milk supply through mechanical expression as the normal mechanism of infant suckling is limited in the very preterm infant $[29,35]$.

Studies have emphasized the importance of establishing an adequate milk production in the early postpartum period for mothers of preterm infants. In a study involving 95 mothers from four tertiary care centers in the Midwest United States, the milk volume expressed on day 4 postpartum was found to be predictive of an inadequate milk supply at 6 weeks postpartum. Mothers producing less than $140 \mathrm{~mL} /$ day on day 4 were found to be 9.5 times more at risk of low or inadequate milk production by 6 weeks postpartum [36].

Maintaining a milk volume in amounts sufficient to meet the nutritional needs of their very preterm infants can be challenging for many mothers [17,18,37,38]. A volume of $500 \mathrm{~mL} /$ day or $3500 \mathrm{~mL}$ /week (equivalent to a mother pumping $80-100 \mathrm{~mL} /$ pumping, six times a day) has been identified as the minimum milk volume a mother of a preterm infant should pump in order to meet the needs of her infant at discharge [39]. If a mother is producing $>3500 \mathrm{~mL}$ by week 2 , it can be expected that she will produce this ongoing adequate amount in weeks 4 and 5 . If a mother is producing $\geq 1700 \mathrm{~mL} /$ week but $<3500 \mathrm{~mL}$ by week 2 , she has approximately a $50 \%$ likelihood of reaching the minimum of $3500 \mathrm{~mL} /$ week by week 5 postpartum. For a mother who is producing $<1700 \mathrm{~mL} /$ week ( $<40 \mathrm{~mL} /$ pumping), the outlook is grim with $100 \%$ not achieving the goal of $500 \mathrm{~mL} /$ day by weeks $4-5$ postpartum.

The inadequate milk volume and declining production over the subsequent weeks pose challenges for the mother eager to provide milk for her infant Additional approaches may need to be explored for those mothers who show a decline in production and will likely stop expression of breast milk for their infant.

\section{Use of Galactogogues for Breast Milk Production-A Review of the Literature}

Many non-pharmacological measures have been found to contribute to variable levels of success in augmenting the breast milk production in mothers of preterm infants [38]. While these approaches may be helpful, it is critical to emphasize that the primary effective strategy for optimizing breast milk volume is frequent and effective breast emptying [29]. In the setting of reduced breast milk volume, galactogogues can be added to an increased pumping regime to augment breast milk volume.

Medications which have galactogogue capabilities generally augment lactation by exerting its effects through either oxytocin or prolactin $[40,41]$. Oxytocin nasal spray has been evaluated in 
3 clinical trials, but negative clinical experience and low use led to the spray being discontinued in many countries thereby limiting its use on a widespread nature [41]. Sulpiride is a substituted benzamide antipsychotic medication. It is an antagonist of dopamine that increases serum prolactin levels similar to other galactogogues. It has poor bioavailability (35\%) and has many of the same side effects and complications as other antipsychotics including sedation, extrapyramidal effects, tardive dyskinesia, and neuroleptic malignant syndrome making its use less appealing [41].

The primary medications used today for prolactin production are, like sulpiride, dopamine antagonists. They increase serum prolactin by counteracting the inhibitory influence of dopamine on prolactin secretion. The medications studied most widely for their galactogogue capabilities have been metoclopramide and domperidone. Both medications are used in an "off-label" capacity, i.e., they have not been authorized for use in lactation support. In addition, availability of these medications vary; domperidone, in particular, is available in most countries but not in the United States. A search in the common literature databases (Medline, CINAHL, EMBASE, OVID, Cochrane Library) was done to identify studies or trials evaluating these two pharmacologic galactogogues in mothers of preterm infants.

Metoclopromide augments lactation by antagonizing the release of dopamine in the central nervous system. Because the medication exerts its effects centrally, it can cause extrapyramidal side effects which may include tremor, bradykinesia and other dystonic reactions [40,41].

Seventeen studies were identified evaluating metoclopromide to improve breast milk production (Table 1).

Table 1. Studies evaluating metoclopromide and breast milk production.

\begin{tabular}{|c|c|c|c|c|c|c|}
\hline Study & Year & $\mathbf{N}$ & Placebo & Randomization & Intervention & Findings \\
\hline Guzmán [42] & 1979 & 21 & $\mathrm{Y}$ & $\mathrm{Y}$ & 20 mg TID 4 weeks & $\uparrow \mathrm{BM}, \mathrm{PRL}$ \\
\hline Lewis [43] & 1980 & 20 & Y & $\mathrm{Y}$ & $10 \mathrm{mg}$ TID 4 days & $\uparrow \mathrm{BM}$ \\
\hline Tolino [44] & 1981 & 10 & $\mathrm{~N}$ & $\mathrm{~N}$ & $10 \mathrm{mg}$ TID 7 days & $\uparrow \mathrm{BM}, \mathrm{PRL}$ \\
\hline Kauppila [45] & 1981 & 37 & Y & Y & 5-15 mg TID 2 weeks & $\uparrow \mathrm{BM}, \mathrm{PRL}$ \\
\hline Kauppila [46] & 1981 & 17 & $\mathrm{~N}$ & $\mathrm{~N}$ & $10 \mathrm{mg}$ TID 5 weeks & $\uparrow \mathrm{BM}, \mathrm{PRL}$ \\
\hline Kauppila [47] & 1983 & 5 & $\mathrm{~N}$ & $\mathrm{~N}$ & $10 \mathrm{mg}$ TID 5 days & $\uparrow$ plasma levels in infant \\
\hline de Gezelle [48] & 1983 & 13 & Y & Y & $10 \mathrm{mg}$ TID 8 days & $\uparrow \mathrm{BM}$ \\
\hline Kauppila [49] & 1985 & 24 & Y & $\mathrm{Y}$ & $10 \mathrm{mg}$ TID 3 weeks & $\uparrow \mathrm{BM}$ \\
\hline Gupta [50] & 1985 & 32 & $\mathrm{~N}$ & $\mathrm{~N}$ & $10 \mathrm{mg}$ TID & $\uparrow$ lactation \\
\hline Ehrenkranz [51] & 1986 & 23 & $\mathrm{~N}$ & $\mathrm{~N}$ & $10 \mathrm{mg}$ TID 7 days & $\uparrow \mathrm{BM}$, basal PRL \\
\hline Ertl [52] & 1991 & 22 & $\mathrm{~N}$ & $\mathrm{~N}$ & $10 \mathrm{mg}$ TID 5 days & $\uparrow \mathrm{BM}$ \\
\hline Nemba [53] & 1994 & 37 & $\mathrm{~N}$ & $\mathrm{~N}$ & $10 \mathrm{mg}$ QID 5-11 days & $\uparrow$ lactation \\
\hline Toppare [54] & 1994 & 60 & $\mathrm{~N}$ & $\mathrm{~N}$ & $10 \mathrm{mg}$ TID & $\uparrow$ lactation \\
\hline Seema [55] & 1997 & 50 & $\mathrm{~N}$ & $\mathrm{~N}$ & $10 \mathrm{mg}$ TID 10 days & $\uparrow$ lactation \\
\hline Hansen [56] & 2005 & 57 & $\mathrm{Y}$ & $\mathrm{Y}$ & $10 \mathrm{mg}$ TID 10 days & No difference \\
\hline Sakha [57] & 2008 & 20 & Y & Y & $10 \mathrm{mg}$ TID 8 days & No difference \\
\hline Fife [58] & 2011 & 19 & Y & Y & $10 \mathrm{mg}$ TID 8 days & No difference \\
\hline
\end{tabular}

$\mathrm{BM}=$ breast milk; $\mathrm{PRL}=$ prolactin; $\mathrm{TID}=$ three times daily; $\mathrm{Y}=$ yes; $\mathrm{N}=$ no.

Many studies were done well before 2000 and mostly in mothers with term infants. However, three were conducted with mothers of preterm infants, Ehrenkranz et al. [51], Hansen et al. [56], and Fife et al. [58]. Although not a randomized clinical trial (RCT), Ehrenkranz demonstrated an increase in daily breast milk production with metoclopramide from $93.3 \pm 18.0 \mathrm{~mL} /$ day to $197.4 \pm 32.3 \mathrm{~mL} /$ day between the first and seventh day of therapy [51]. The other two, Hansen et al. [56] and Fife [58], found no difference in breast milk volume. These two studies had methodological concerns in that all mothers were enrolled without any evaluation of their ability to produce milk. The inclusion of mothers who would not have had any difficulty in breast milk production may have minimized differences between the groups.

Domperidone is a potent dopamine $\mathrm{D}_{2}$ receptor antagonist and was developed and marketed as a prokinetic and antiemetic agent. By blocking dopamine $\mathrm{D}_{2}$ receptors in the anterior pituitary, domperidone stimulates the release of prolactin. Domperidone is less lipid soluble, has a higher molecular weight and has lower protein binding $(>90 \%)$ than metoclopromide $(40 \%)$. These characteristics appear to prevent domperidone from crossing the blood brain barrier and therefore less likely to cause the extra 
pyramidal effects often seen with metoclopromide $[59,60]$. This characteristic made domperidone more appealing in use compared to metoclopramide. In addition, early studies in the 1980's evaluating its efficacy in augmenting breast milk production $[50,51]$ made this medication more enticing to consider, particularly in mothers of preterm infants.

Nine studies involving domperidone are outlined in Table 2. Seven of these studies were conducted in mothers of preterm infants. All of the studies were small in terms of number of mothers enrolled.

Table 2. Studies evaluating domperidone and breast milk production.

\begin{tabular}{|c|c|c|c|c|c|c|}
\hline Study & Year & $\mathbf{N}$ & Placebo & Randomization & Intervention & Findings \\
\hline De Leo [61] & 1986 & 15 & Y & $\mathrm{N}$ & $10 \mathrm{mg}$ TID 4 days & $\uparrow$ lactation \\
\hline Petraglia [62] & 1985 & 17 & Y & $\mathrm{N}$ & $10 \mathrm{mg}$ TID 10 days & $\uparrow \mathrm{PRL}, \mathrm{BM}$ \\
\hline da Silva [63] & 2001 & 20 & $\mathrm{Y}$ & $\mathrm{Y}$ & $10 \mathrm{mg}$ TID 7 days & $\uparrow \mathrm{PRL}, \mathrm{BM}$ \\
\hline Wan [64] & 2008 & 6 & $\mathrm{~N}$ & Y & $10 \mathrm{mg}$ vs. $20 \mathrm{mg}$ TID $1-2$ weeks & $\uparrow \mathrm{PRL}, \mathrm{BM}$ \\
\hline Campbell-Yeo [65] & 2010 & 46 & Y & Y & $10 \mathrm{mg}$ TID 14 days & $\uparrow \uparrow P R L, B M$ \\
\hline Ingram [66] & 2012 & 80 & $\mathrm{~N}$ & $\mathrm{Y}$ & $\begin{array}{l}10 \mathrm{mg} \text { TID } 10 \mathrm{~d} \text { or Metoclopramide } \\
10 \mathrm{mg} \text { TID } 10 \text { days }\end{array}$ & $\uparrow \mathrm{BM}$ \\
\hline Knoppert [67] & 2013 & 15 & $\mathrm{~N}$ & Y & $10 \mathrm{mg}$ vs. $20 \mathrm{mg}$ TID 4 weeks & $\uparrow \mathrm{BM}$ \\
\hline Rai [68] & 2016 & 32 & Y & $\mathrm{N}$ & Unknown dose for 8 days & $\uparrow \mathrm{BM}$ \\
\hline Asztalos [69] & 2017 & 90 & $\mathrm{Y}$ & Y & $10 \mathrm{mg}$ TID 14 days & $\uparrow \mathrm{BM}$ \\
\hline
\end{tabular}

$\mathrm{BM}=$ breast milk; $\mathrm{PRL}=$ prolactin; $\mathrm{TID}=$ three times daily; $\mathrm{Y}=$ yes; $\mathrm{N}=$ no.

da Silva et al. was the first RCT to evaluate the efficacy of domperidone in mothers of preterm infants [63]. In this study, there was a mean increase in breast milk yield from days 2 to 7 in the domperidone group $(49.5 \mathrm{~mL}$, standard deviation $29.4 \mathrm{~mL})$ compared to the placebo group $(8.0 \mathrm{~mL}$, standard deviation $39.5 \mathrm{~mL})(p<0.05)$ as well as an increase in serum prolactin $(p=0.008)$. Wan et al. evaluated a dose-response relationship between 30 and $60 \mathrm{mg}$ daily [64]. Serum prolactin increased for both doses but was not dose-dependent. In addition, only two-thirds of the mothers (4 out of 6) were identified as "responders" and showed a significant increase in milk production which was also dose-dependent. Campbell-Yeo et al. randomized 46 mothers to either domperidone $10 \mathrm{mg}$ three times daily or placebo equivalent for 14 days [65]. Although the study's primary goal was to evaluate the effect of domperidone on the nutrient composition of preterm human milk compared to those mothers having received a placebo, there was a significant increase in serum prolactin $(p=0.07)$ and breast milk volumes $(p=0.005)$ in the domperidone group. The mean within-subject increase by day 14 was $267 \%$ in the domperidone group (184 to $380 \mathrm{~mL}$ ) compared to $19 \%$ in placebo group (218 to $250 \mathrm{~mL}$ ). This trial did suggest that a larger yield in breast milk production could be achieved with the additional week as compared to the earlier trial.

Ingram et al. compared the effects of domperidone and metoclopramide on breast milk output in mothers of preterm infants and found no significant differences between the two galactogogues [66]. Both groups showed an increase in breast milk volume. Mothers in the domperidone group achieved a mean of $96.3 \%$ in milk volume compared to $93.7 \%$ increase for metoclopramide.

Knoppert et al. enrolled 12 mothers between 14-21 days post-delivery to evaluate the effectiveness of two dosing strategies, $10 \mathrm{mg}$ compared to $20 \mathrm{mg}$ three times daily for 28 days, on milk production in mothers of preterm infants [67]. Both dosing strategies showed breast milk volumes increasing with a clinically higher amount in the higher dosing approach, but the actual volumes were not given.

More recently, the EMPOWER trial by Asztalos et al. enrolled 90 mothers, who gave birth to preterm infants $<30$ weeks gestation, to receive domperidone $30 \mathrm{mg}$ daily compared to a placebo for 14 days followed by all mothers receiving domperidone $30 \mathrm{mg}$ daily for another 14 days [69]. More mothers achieved a 50\% increase in milk volume after 14 days in the treated group $(77.8 \%)$ compared to placebo (57.8\%) (odds ratios 2.56; 95\% confidence interval 1.02, 6.25; $p=0.04$ ) ; however, the gain in actual volume was modest and not significantly different.

Each of the described studies evaluating domperidone as a means to augment breast milk production were significantly different in design and did not allow a more direct comparison. The studies were different in dosing approaches, timing and duration of treatment and the use or 
non-use of a placebo as well as outcome measures. The response to the interventions in the individual studies were different. Most, but not all, provided 24-h volumes as a measure for determining a response to domperidone. Two studies did not give actual values [64,67].

\section{Clinical Efficacy}

Overall, study findings indicate that metoclopramide is less efficacious than domperidone in augmenting breast milk production in mothers of preterm infants. Domperidone studies showed a modest increase in breast milk production but the approaches in dosing, timing and duration of treatment varied considerably in each trial. The cumulative dose in the trial by da Silva varied greatly compared to the trial by Knoppert $[63,67]$. In addition, even within a trial, mothers varied with respect to the cumulative dose [69]. Because the objectives of the individual studies varied, how breast milk volume was measured varied as well: 24 -h volumes vs. percentages vs. volume per pump session.

Despite the varied approaches in outcome measures, the studies all demonstrated an increase in breast milk volume. However, it is important to note that 24 -h volumes on average still remained below the target of $500 \mathrm{~mL} /$ day $[63,65,66,68,69]$. Recently, Grzeskowiak et al. conducted a meta-analysis which pooled five trials $[63,65,68-70]$ which showed that short-term use of domperidone resulted in a modest $86 \mathrm{~mL} /$ day increase in expressed breast milk [71]. For a mother of a preterm infant weighing $1000 \mathrm{~g}$ and receiving enteral feeds at $160 \mathrm{~mL} / \mathrm{kg} /$ day, this represents an opportunity to meet half of her infant's feeds with her own breast milk, if not more, depending what her starting baseline volume had been. However, this modest volume increase may still fall short of the volume that an infant will need by term corrected age.

Whether there is a sustained effect on volume maintenance with galactogogues, and, in particular domperidone, is not clear. The EMPOWER study did follow the mothers to 6 weeks post term gestation. However, regardless of the assigned grouping, almost $60 \%$ of the study participants attempted to continue to provide breast milk and continued with some form of lactation inducing compounds at term gestation with the numbers dropping to just over $40 \%$ for the combined groups at 6 weeks post term gestation suggesting there was no sustained effect on breast milk production for the mothers in the trial [69]. At present, there are no studies that have looked at long-term use of domperidone beyond two or four weeks and whether it has an effect on sustained breast milk provision post initial hospital discharge.

\section{Safety Issues}

As noted earlier, metoclopromide exerts its effects centrally and can cause extrapyramidal side effects which may include tremor, bradykinesia and other dystonic reactions which are both dose and duration related $[40,41]$. These centrally-based side effects have prompted many clinicians to use domperidone rather than metoclopramide as their primary galactogogue. Domperidone's use has grown exponentially for supporting mothers in breast milk production [72,73]. However, over the past decade, concerns have risen regarding the increased risk of prolongation of the Q-Tc interval, the risk of cardiac arrhythmias, and sudden cardiac death in the general adult population [74-77]. The relevance of these findings to women who are receiving this medication for lactation support is not clear and has been questioned [75]. However, given the wide use of domperidone to augment breast milk volumes, these concerns have led regulatory agencies, in particular the European Medicines Agency and Health Canada, to recommend caution in the use of domperidone and have provided dosing recommendations $[78,79]$. The most recent study to demonstrate these concerns, Smolina et al. identified 45,518 women from a provincial database who were dispensed domperidone in the first 6 months of their postpartum period [80]. Of these women, there were 21 women hospitalized for ventricular arrhythmia. The authors concluded that that there was a possible association between exposure to domperidone and hospitalization for ventricular arrhythmia (adjusted $H R=2.25$, $95 \%$ CI 0.84-6.01), but that further research was needed to confirm this association. More recent studies have attempted to demonstrate an element of reassurance. In the EMPOWER trial, all of the 90 women 
enrolled had an ECG at study entry and at the end of the 4-week study period. Although not powered to detect a significant increase in cardiac arrhythmias, no women demonstrated any evidence of a QTC prolongation [69]. In addition, a recent review assessing QTc prolongation concluded that domperidone was not associated with QTc prolongation in healthy female volunteers [81]. A second major concern for safety related to domperidone is that of sudden cardiac death. Domperidone has been shown to have a 2.8-fold increased risk for sudden cardiac death in the general population [82,83]. However, no data to date demonstrates the risk specific to postpartum women. At present, recommendations from regulatory agencies suggest that a very small risk of cardiac arrhythmias and sudden cardiac death is associated with domperidone and clinician. If clinicians do prescribe domperidone for a non-authorized use such as lactation support, they should only use the established dosing guidelines of $30 \mathrm{mg}$ daily $[78,79]$.

As with any medication, there is always concern of transfer into breast milk. Lewis et al. evaluated the extent to which metoclopramide passed into breast milk in 10 mothers with full term infants. Maternal blood and milk samples collected $2 \mathrm{~h}$ after a single oral dose of $10 \mathrm{mg}$ had plasma concentration of $69 \pm 30 \mathrm{ng} / \mathrm{mL}$ and milk concentration $126 \pm 42 \mathrm{ng} / \mathrm{mL}$. The authors calculated that the average intake of metoclopramide by an infant would be less than $0.045 \mathrm{mg} / \mathrm{kg} /$ day which is well below the therapeutic doses used in preterm and term newborn infants [43]. Similarly, when evaluating 30 and $60 \mathrm{mg}$ total daily doses of domperidone, the amounts transferred to breast milk were extremely low with median infant dose via milk being 0.04 and $0.07 \mu \mathrm{g} / \mathrm{kg} /$ day, respectively; this is far below the dose of 100-300 $\mu \mathrm{g}$ 3-4 times-a-day infants receive for gastrointestinal stasis [64]. With oral bioavailability at $15 \%$, it is unlikely that pharmacologically meaningful amounts of domperidone reach the infant through the breast milk. No measures of infant serum concentrations of domperidone have been reported.

\section{Summary}

Following preterm delivery of an infant that is unable to breastfeed, measures should be in place to facilitate breast milk expression within one to six hours of birth as well as maintaining milk production $[84,85]$. The use of a galactogogue can be considered if additional support in breast milk production is needed especially in the presence of optimized pumping strategies. Although the trials demonstrate a modest efficacy in augmenting breast milk production at any point during the first 5 weeks of the postpartum period, earlier initiation by the end of the first week postpartum can be considered in order to optimize support for the mother. Based on the current literature and recommendations, domperidone, where available, should be the galactogogue of choice, with the dose of $10 \mathrm{mg}$ three time daily for 14 days. There is inadequate evidence to guide treatment beyond 14 days. Careful history-taking and assessment are required to ensure domperidone is not administered to mothers at risk of cardiac arrhythmia. Mothers need to maintain pumping to facilitate the autocrine regulatory mechanism. Mothers should be assessed after $48-72 \mathrm{~h}$ of initiating domperidone to determine a response as evidence by an increase in breast milk volume.

Author Contributions: The author was responsible for the conception and design of this review.

Acknowledgments: The author received funding from the Canadian Institutes of Health Research (CIHR) grant MOP\#114980 for the conduct of the EMPOWER trial and costs to publish in open access.

Conflicts of Interest: The author is the lead investigator for one of the trials presented in the review but otherwise declares no other conflict of interest.

\section{References}

1. Maffei, D.; Schanler, R.J. Human milk is the feeding strategy to prevent necrotizing enterocolitis! Semin. Perinatol. 2017, 41, 36-40. [CrossRef] [PubMed] 
2. Abrams, S.A.; Schanler, R.J.; Lee, M.L.; Rechtman, D.J. Greater mortality and morbidity in extremely preterm infants fed a diet containing cow milk protein products. Breastfeed. Med. 2014, 9, 281-285. [CrossRef] [PubMed]

3. Schanler, R.J. Mother's own milk, donor human milk, and preterm formulas in the feeding of extremely premature infants. J. Pediatr. Gastroenterol. Nutr. 2007, 45, 175S-177S. [CrossRef] [PubMed]

4. Morales, Y.; Schanler, R.J. Human milk and clinical outcomes in VLBW infants: How compelling is the evidence of benefit? Semin. Perinatol. 2007, 31, 83-88. [CrossRef] [PubMed]

5. Porcelli, P.J.; Weaver, R.G., Jr. The influence of early postnatal nutrition on retinopathy of prematurity in extremely low birth weight infants. Early Hum. Dev. 2010, 86, 391-396. [CrossRef] [PubMed]

6. Furman, L.; Taylor, G.; Minich, N.; Hack, M. The effect of maternal milk on neonatal morbidity of very low-birth-weight infants. Arch. Pediatr. Adolesc. Med. 2003, 157, 66-71. [CrossRef] [PubMed]

7. Lucas, A.; Morley, R.; Cole, T.J. Randomised trial of early diet in preterm babies and later intelligence quotients. BMJ 1996, 317, 1481-1487. [CrossRef]

8. Horwood, L.J.; Darlow, B.A.; Mogridge, N. Breast milk feeding and cognitive ability at 7-8 years. Arch. Dis. Child. Fetal Neonatal Ed. 2001, 84, 23F-27F. [CrossRef]

9. Vohr, B.R.; Poindexter, B.B.; Dusick, A.M.; McKinley, L.T.; Wright, L.L.; Langer, J.C.; Poole, W.K. NICHD Neonatal Research Network. Beneficial effects of breast milk in the neonatal intensive care unit on the developmental outcome of extremely low birth weight infants at 18 months of age. Pediatrics 2006, 118, e115-e123. [CrossRef] [PubMed]

10. Vohr, B.R.; Poindexter, B.B.; Dusick, A.M.; McKinley, L.T.; Higgins, R.D.; Langer, J.C.; Poole, W.K. NICHD Neonatal Research Network. Persistent beneficial effects of breast milk ingested in the neonatal intensive care unit on outcomes of extremely low birth weight infants at 30 months of age. Pediatrics 2007, 12, e953-e959. [CrossRef] [PubMed]

11. O'Connor, D.L.; Jacobs, J.; Hall, R.; Adamkin, D.; Auestad, N.; Castillo, M.; Connor, W.E.; Connor, S.L.; Fitzgerald, K.; Groh-Wargo, S.; et al. Growth and development of premature infants fed predominantly human milk, predominantly premature infant formula, or a combination of human milk and premature formula. J. Pediatr. Gastroenterol. Nutr. 2003, 37, 437-446. [CrossRef] [PubMed]

12. Ehrenkranz, R.A.; Dusick, A.M.; Vohr, B.R.; Wright, L.L.; Wrage, L.A.; Poole, W.K. Growth in the neonatal intensive care unit influences neurodevelopmental and growth outcomes of extremely low birth weight infants. Pediatrics 2006, 117, 1253-1261. [CrossRef] [PubMed]

13. Chirico, G.; Marzollo, R.; Cortinovis, S.; Fonte, C.; Gasparoni, A. Antiinfective properties of human milk. J. Nutr. 2008, 138, 1801S-1806S. [CrossRef] [PubMed]

14. Victora, C.G.; Bahl, R.; Barros, A.J.; França, G.V.; Horton, S.; Krasevec, J.; Murch, S.; Sankar, M.J.; Walker, N.; Rollins, N.C. Lancet Breastfeeding Series Group. Breastfeeding in the 21st century: Epidemiology, mechanisms, and lifelong effect. Lancet 2016, 387, 475-490. [CrossRef]

15. Moro, G.E.; Arslanoglu, S.; Bertino, E.; Corvaglia, L.; Montirosso, R.; Picaud, J.C.; Polberger, S.; Schanler, R.J.; Steel, C.; van Goudoever, J.; et al. American Academy of Pediatrics; European Society for Pediatric Gastroenterology, Hepatology, and Nutrition. Human milk in feeding premature infants: Consensus statement. J. Pediatr. Gastroenterol. Nutr. 2015, 61, S16-S19. [CrossRef] [PubMed]

16. Callen, J.; Pinelli, J. A review of the literature examining the benefits and challenges, incidence and duration, and barriers to breastfeeding in preterm infants. Adv. Neonatal Care 2005, 5, 72-88. [CrossRef] [PubMed]

17. Hill, P.D.; Ledbetter, R.J.; Kavanaugh, K.L. Breastfeeding patterns of low-birth-weight infants after hospital discharge. J. Obstet. Gynecol. Neonatal Nurs. 1997, 26, 189-197. [CrossRef] [PubMed]

18. Hill, P.D.; Aldag, J.C.; Chatterton, R.T.; Zinaman, M.J. Comparison of milk production between mothers of preterm and term mothers: The first six weeks after birth. J. Hum. Lact. 2005, 21, 22-30. [CrossRef] [PubMed]

19. Sievers, E.; Haase, S.; Oldigs, H.D.; Schaub, J. The impact of peripartum factors on the onset and duration of lactation. Biol. Neonatol. 2003, 83, 246-252. [CrossRef] [PubMed]

20. Houston, M.J.; Howie, P.W.; McNeilly, A.S. Factors affecting the duration of breast feeding: 1. Measurement of breast milk intake in the first week of life. Early Hum. Dev. 1983, 8, 49-54. [CrossRef]

21. Henderson, J.J.; Hartmann, P.E.; Newnham, J.P.; Simmer, K. Effects of preterm birth and antenatal corticosteroid treatment on lactogenesis II in women. Pediatrics 2008, 121, e92-e100. [CrossRef] [PubMed]

22. Neville, M.C.; McFadden, T.B.; Forsyth, I. Hormonal regulation of mammary differentiation and milk secretion. J. Mammary Gland Biol. Neoplasia 2002, 7, 49-66. [CrossRef] [PubMed] 
23. Neville, M.C.; Morton, J. Physiology and endocrine changes underlying human lactogenesis II. J. Nutr. 2001, 131, 3005S-3008S. [CrossRef] [PubMed]

24. Pang, W.W.; Hartman, P.E. Initiation of human lactation: Secretory differentiation and secretory activation. J. Mammary Gland Biol. Neoplasia 2007, 12, 211-231. [CrossRef] [PubMed]

25. Truchet, S.; Honvo-Houéto, E. Physiology of milk secretion. Best Pract. Res. Clin. Endocrinol. Metab. 2017, 31, 367-384. [CrossRef] [PubMed]

26. Kulski, J.K.; Hartmann, P.E. Changes in human milk composition during the initiation of lactation. Aust. J. Exp. Biol. Med. Sci. 1981, 59, 101-114. [CrossRef] [PubMed]

27. Neville, M.C.; Allen, J.C.; Archer, P.C.; Casey, C.E.; Seacat, J.; Keller, R.P.; Lutes, V.; Rasbach, J.; Neifert, M. Studies in human lactation: Milk volume and nutrient composition during weaning and lactogenesis. Am. J. Clin. Nutr. 1991, 54, 81-92. [CrossRef] [PubMed]

28. Kent, J.C. How breastfeeding works. J. Midwifery Womens Health 2007, 52, 564-570. [CrossRef] [PubMed]

29. Kent, J.C.; Prime, D.K.; Garbin, C.P. Principles for maintaining or increasing breast milk production. J. Obstet. Gynecol. Neonatal Nurs. 2012, 41, 114-121. [CrossRef] [PubMed]

30. Daly, S.E.; Owens, R.A.; Hartmann, P.E. The short-term synthesis and infant-regulated removal of milk in lactating women. Exp. Physiol. 1993, 78, 209-220. [CrossRef] [PubMed]

31. Peaker, M.; Wilde, C.J. Feedback control of milk secretion from milk. J. Mammary Gland Biol Neoplasia 1996, 1, 307-315. [CrossRef] [PubMed]

32. DeCoopman, J. Breastfeeding after pituitary resection: Support for a theory of autocrine control of milk supply? J. Hum. Lact. 1993, 9, 35-40. [CrossRef] [PubMed]

33. Whichelow, M.J. Factors associated with the duration of breast feeding in a privileged society. Early Hum. Dev. 1982, 7, 273-280. [CrossRef]

34. Chapman, D.J.; Pérez-Escamilla, R. Identification of risk factors for delayed onset of lactation. J. Am. Diet. Assoc. 1999, 99, 450-454. [CrossRef]

35. Case-Smith, J.; Cooper, P.; Scala, V. Feeding efficiency of premature neonates. Am. J. Occup. Ther. 1989, 43, 245-250. [CrossRef] [PubMed]

36. Hill, P.D.; Aldag, J.C. Milk volume on day 4 and income predictive of lactation adequacy at 6 weeks of mothers of non-nursing preterm infants. J. Perinat. Neonatal Nurs. 2005, 19, 273-282. [CrossRef] [PubMed]

37. Ehrenkranz, R.A.; Ackerman, B.A.; Mesger, J.; Bracken, M.B. Breast-feeding and premature infants: Incidence and success. Pediatr. Res. 1985, 19, 199A. [CrossRef]

38. Meier, P.P.; Johnson, T.J.; Patel, A.L.; Rossman, B. Evidence-Based Methods That Promote Human Milk Feeding of Preterm Infants: An Expert Review. Clin. Perinatol. 2017, 44, 1-22. [CrossRef] [PubMed]

39. Hill, P.D.; Aldag, J.C.; Chattertoon, R.T. Effects of pumping style on milk production in mothers of non-nursing preterm infants. J. Hum. Lact. 1993, 15, 209-216. [CrossRef] [PubMed]

40. Gabay, P.G. Galactogogues: Medications that induce lactation. J. Hum. Lact. 2002, 18, 274-279. [CrossRef] [PubMed]

41. Forinash, A.B.; Yancey, A.M.; Barnes, K.N.; Myles, T.D. The use of galactogogues in the breastfeeding mother. Ann. Pharmacother. 2012, 46, 1392-1404. [CrossRef] [PubMed]

42. Guzmán, V.; Toscano, G.; Canales, E.S.; Zárate, A. Improvement of defective lactation by using oral metoclopramide. Acta Obstet. Gynecol. Scand. 1979, 58, 53-55. [CrossRef] [PubMed]

43. Lewis, P.J.; Devenish, C.; Kahn, C. Controlled trial of metoclopromide in the initiation of breastfeeding. Br. J. Clin. Pharmacol. 1980, 9, 217-219. [CrossRef] [PubMed]

44. Tolino, A.; Tedeschi, A.; Farace, R.; Granata, P. The relationship between metoclopramide and milk secretion in puerperium. Clin. Exp. Obstet. Gynecol. 1981, 8, 93-95. [PubMed]

45. Kauppila, A.; Kivinen, S.; Ylikorkala, O. A dose response relation between improved lactation and metoclopromide. Lancet 1981, 1, 1175-1177. [CrossRef]

46. Kauppila, A.; Kivinen, S.; Ylikorkala, O. Metoclopromide increases prolactin release and ilk secretion in puerperium without stimulating the secretion of thyrotropin and thyroid hormones. J. Clin. Endocrinol. Metab. 1981, 52, 436-439. [CrossRef] [PubMed]

47. Kauppila, A.; Arvela, P.; Koivisto, M.; Kivinen, S.; Ylikorkala, O.; Pelkonen, O. Metoclopramide and breast feeding: Transfer into milk and the newborn. Eur. J. Clin. Pharmacol. 1983, 25, 819-823. [CrossRef] [PubMed]

48. de Gezelle, H.; Ooghe, W.; Thierry, M.; Dhont, M. Metoclopramide and breast milk. Eur. J. Obstet. Gynecol. Reprod. Biol. 1983, 15, 31-36. [CrossRef] 
49. Kauppila, A.; Anunti, P.; Kivinen, S.; Koivisto, M.; Ruokonen, A. Metoclopramide and breast feeding: Efficacy and anterior pituitary responses of the mother and the child. Eur. J. Obstet. Gynecol. Reprod. Biol. 1985, 19, 19-22. [CrossRef]

50. Gupta, A.P.; Gupta, P.K. Metoclopromide as a galactogogue. Clin. Pediatr. 1985, 24, 269-272. [CrossRef] [PubMed]

51. Ehrenkranz, R.A.; Ackerman, B.A. Metoclopromide effect of faltering milk production by mothers of premature infants. Pediatrics 1986, 78, 614-620. [PubMed]

52. Ertl, T.; Sulyok, E.; Ezer, E.; Sárkány, I.; Thurzó, V.; Csaba, I.F. The influence of metoclopramide on the composition of human breast milk. Acta Paediatr. Hung. 1991, 31, 415-422. [PubMed]

53. Nemba, K. Induced lactation: A study of 37 non-puerperal mothers. J. Trop. Pediatr. 1994, 40, $240-242$. [CrossRef] [PubMed]

54. Toppare, M.F.; Laleli, Y.; Senses, D.A.; Kitapci, F.; Kaya, I.S.; Dilmen, U. Metoclopromide for breast milk production. Nutr. Res. 1994, 14, 1019-1029. [CrossRef]

55. Seema; Patwari, A.K.; Satyanarayana, I. Relactation: An effective intervention to promote exclusive breastfeeding. J. Trop. Pediatr. 1997, 43, 213-216. [CrossRef] [PubMed]

56. Hansen, W.F.; McAndrew, S.; Harris, K.; Zimmerman, M.B. Metoclopromide effect on breastfeeding the preterm infants: A randomized trial. Obstet. Gynecol. 2005, 105, 383-389. [CrossRef] [PubMed]

57. Sakha, K.; Behbahan, A.G. Training for perfect breastfeeding or metoclopramide: Which one can promote lactation in nursing mothers? Breastfeed. Med. 2008, 3, 120-123. [CrossRef] [PubMed]

58. Fife, S.; Gill, P.; Hopkins, M.; Angello, C.; Boswell, S.; Nelson, K.M. Metoclopramide to augment lactation, does it work? A randomized trial. J. Matern. Fetal Neonatal Med. 2011, 24, 1317-1320. [CrossRef] [PubMed]

59. Brogden, R.N.; Carmine, A.A.; Heel, R.C.; Speight, T.M.; Avery, G.S. Domperidone: A review of it pharmacological activity, pharmacokinetics and therapeutic efficacy in the symptomatic treatment of chronic dyspepsia and as an antiemetic. Drugs 1982, 24, 360-400. [CrossRef] [PubMed]

60. Barone, J. Domperidone: A peripherally acting dopamine2-receptor antagonist. Ann. Pharmacother. 1999, 33, 429-440. [CrossRef] [PubMed]

61. De Leo, V.; Petraglia, F.; Sardelli, S.; Pieroni, M.L.; Bruno, G.; Gioffrè, W.R.; Genazzani, A.R.; D’Antona, N. Use of domperidone in the induction and maintenance of maternal breast feeding. Minerva Ginecol. 1986, 38, 311-315. [PubMed]

62. Petraglia, F.; De Leo, V.; Sardelli, S.; Pieroni, M.L.; D'Antona, N.; Genazzani, A.R. Domperidone in defective and insufficient lactation. Eur. J. Obstet. Gynecol. Reprod. Biol. 1985, 19, 281-287. [CrossRef]

63. da Silva, O.P.; Knoppert, D.C.; Angelini, M.M.; Forret, P.A. Effect of domperidone on milk production in mothers of premature newborn: A randomized, double-blind, placebo-controlled trial. Can. Med. Assoc. J. 2001, 164, 17-21.

64. Wan, E.W.; Davey, K.; Page-Sharp, M.; Hartmann, P.E.; Simmer, K.; Ilett, K.F. Dose-effect study of domperidone as a galactagogue in preterm mothers with insufficient milk supply, and its transfer into milk. Br. J. Clin. Pharmacol. 2008, 66, 283-289. [CrossRef] [PubMed]

65. Campbell-Yeo, M.; Allen, A.C.; Joseph, K.S.; Ledwidge, J.; Allen, V.; Dooley, K. Effect of Domperidone on the Composition of Preterm Human Breast Milk: Randomized, Double Blind, Placebo-Controlled Trial. Pediatrics 2010, 125, e107-e114. [CrossRef] [PubMed]

66. Ingram, J.; Taylor, H.; Churchill, C.; Pike, A.; Greenwood, R. Metoclopramide or domperidone for increasing maternal breast milk output: A randomized controlled trial. Arch. Dis. Child. Fetal Neonatal Ed. 2012, 97, F241-F245. [CrossRef] [PubMed]

67. Knoppert, D.C.; Page, A.; Warren, J.; Seabrook, J.A.; Carr, M.; Angelini, M.; Killick, D.; DaSilva, O.P. The effect of two different doses on maternal milk production. J. Hum. Lact. 2013, 29, 38-44. [CrossRef] [PubMed]

68. Rai, R.; Mishra, N.; Singh, D. Effect of domperidone in 2nd week postpartum on milk output in mothers of preterm infants. Indian J. Pediatr. 2016, 83, 894-895. [CrossRef] [PubMed]

69. Asztalos, E.V.; Campbell-Yeo, M.; da Silva, O.P.; Ito, S.; Kiss, A.; Knoppert, D. EMPOWER Study Collaborative Group. Enhancing Human Milk Production with Domperidone in Mothers of Preterm Infants. J. Hum. Lact. 2017, 33, 181-187. [CrossRef] [PubMed]

70. Blank, C.; Eaton, V.; Bennett, J.; James, S.L. A double blind RCT of domperidone and metoclopramide as pro-lactational agents in mothers of preterm infants. In Proceedings of the Perinatal Society of Australia and New Zealand 5th Annual Conference, Canberra, Australia, 13-16 March 2001; p. 73. 
71. Grzeskowiak, L.E.; Smithers, L.G.; Amir, L.H.; Grivell, R.M. Domperidone for increasing breast milk volume in mothers expressing breast milk for their preterm infants: A systematic review and meta-analysis. BJOG 2018. [CrossRef] [PubMed]

72. Smolina, K.; Morgan, S.G.; Hanley, G.E.; Oberlander, T.F.; Mintzes, B. Postpartum domperidone use in British Columbia: A retrospective cohort study. CMAJ Open 2016, 4, E13-E19. [CrossRef] [PubMed]

73. Grzeskowiak, L.E.; Lim, S.W.; Thomas, A.E.; Ritchie, U.; Gordon, A.L. Audit of domperidone use as a galactogogue at an Australian tertiary teaching hospital. J. Hum. Lact. 2013, 29, 32-37. [CrossRef] [PubMed]

74. Grzeskowiak, L. Use of domperidone to increase breast milk supply: Are women really dying to breastfeed? J. Hum. Lact. 2014, 30, 498-499. [CrossRef] [PubMed]

75. Paul, C.; Zénut, M.; Dorut, A.; Coudoré, M.A.; Vein, J.; Cardot, J.M.; Balayssac, D. Use of domperidone as a galactagogue drug: A systematic review of the benefit-risk ratio. J. Hum. Lact. 2015, 31, 57-63. [CrossRef] [PubMed]

76. Sewell, C.A.; Chang, C.Y.; Chehab, M.M.; Nguyen, C.P. Domperidone for Lactation: What Health Care Providers Need to Know. Obstet. Gynecol. 2017, 129, 1054-1058. [CrossRef] [PubMed]

77. Hondeghem, L.M.; Logghe, N.H. Should Domperidone be Used as a Galactagogue? Possible Safety Implications for Mother and Child. Drug Saf. 2017, 40, 109-113. [CrossRef] [PubMed]

78. European Medicines Agency. Domperidone-Containing Medicines; European Medicines Agency: London, UK, 2014. Available online: http://www.ema.europa.eu/ema/index.jsp?curl=pages/medicines/human/ referrals/Domperidone-containing_medicines/human_referral_prac_000021.jsp\&mid=WC0b01ac05805c516f (accessed on 31 March 2018).

79. Health Canada. Domperidone Maleate-Association with Serious Abnormal Heart Rhythms and Sudden Death (Cardiac Arrest)_For Health Care Professionals; Health Canada: Ottawa, ON, Canada, 2015. Available online: http://hc-sc.gc.ca/dhp-mps/medeff/advisories-avis/prof/_2015/domperidone_hpccps-eng.php (accessed on 31 March 2018).

80. Smolina, K.; Mintzes, B.; Hanley, G.E.; Oberlander, T.F.; Morgan, S.G. The association between domperidone and ventricular arrhythmia in the postpartum period. Pharmacoepidemiol. Drug. Saf. 2016, 25, 1210-1214. [CrossRef] [PubMed]

81. Buffery, P.J.; Strother, R.M. Domperidone safety: A mini-review of the science of QT prolongation and clinical implications of recent global regulatory recommendations. N. Z. Med. J. 2015, 128, 66-74. [PubMed]

82. Hondeghem, L.M. Domperidone: Limited benefits with significant risk for cardiac death. J. Cardiovasc. Pharmacol. 2013, 61, 218-225. [CrossRef] [PubMed]

83. van Noord, C.; Dielman, J.P.; van Herpen, G.; Verhamme, K.; Sturkenboom, M.C. Domperidome and ventricular arrhythmia on sudden cardiac death: A population-based case-control study in the Netherlands. Drug Saf. 2010, 17, 1131-1136.

84. Parker, L.A.; Sullivan, S.; Krueger, C.; Kelechi, T.; Mueller, M. Effect of early breast milk expression on milk volume and timing of lactogenesis stage II among mothers of very low birth weight infants: A pilot study. J. Perinatol. 2012, 32, 205-209. [CrossRef] [PubMed]

85. Parker, L.A.; Sullivan, S.; Krueger, C.; Kelechi, T.; Mueller, M. Strategies to increase milk volume in mothers of VLBW infants. MCN Am. J. Matern. Child. Nurs. 2013, 38, 385-390. [CrossRef] [PubMed]

(C) 2018 by the author. Licensee MDPI, Basel, Switzerland. This article is an open access article distributed under the terms and conditions of the Creative Commons Attribution (CC BY) license (http:/ / creativecommons.org/licenses/by/4.0/). 
Article

\title{
Formula Milk Supplementation on the Postnatal Ward: A Cross-Sectional Analytical Study
}

\author{
Kirsty V. Biggs ${ }^{1}$, Katherine Hurrell ${ }^{2}$, Eleanor Matthews ${ }^{3}$, Ekaterina Khaleva ${ }^{4,5}$, \\ Daniel Munblit ${ }^{3,5,6, \dagger}$ and Robert J. Boyle ${ }^{3,5, *, \dagger}$ \\ 1 Brighton and Sussex Medical School, Brighton BN2 5BE, UK; K.Biggs1@uni.bsms.ac.uk \\ 2 Royal United Hospital, Combe Park, Bath BA1 3NG, UK; Katherine.hurrell@nhs.net \\ 3 Department of Paediatrics, Imperial College Healthcare NHS Trust, London W2 1NY, UK; \\ ellie.matthews@imperial.nhs.uk (E.M.); daniel.munblit08@imperial.ac.uk (D.M.) \\ 4 Department of Paediatrics, Saint-Petersburg State Paediatric Medical University, 194353 Saint-Petersburg, \\ Russia; doctor.khaleva@gmail.com \\ 5 inVIVO Planetary Health, Group of the Worldwide Universities Network (WUN), 6010 Park Ave, \\ West New York, NJ 07093, USA \\ 6 Faculty of Pediatrics, I. M. Sechenov First Moscow State Medical University, 119991 Moscow, Russia \\ * Correspondence: r.boyle@nhs.net; Tel.: +44-207-594-3990 \\ + Authors contributed equally to this work.
}

Received: 6 April 2018; Accepted: 9 May 2018; Published: 14 May 2018

\begin{abstract}
Breastfeeding rates are low in the UK, where approximately one quarter of infants receive a breastmilk substitute (BMS) in the first week of life. We investigated the reasons for early BMS use in two large maternity units in the UK, in order to understand the reasons for the high rate of early BMS use in this setting. Data were collected through infant feeding records, as well as maternal and midwife surveys in 2016. During 2016, 28\% of infants received a BMS supplement prior to discharge from the hospital maternity units with only $10 \%$ supplementation being clinically indicated. There was wide variation in BMS initiation rates between different midwives, which was associated with ward environment and midwife educational level. Specific management factors associated with non-clinically indicated initiation of BMS were the absence of skin-to-skin contact within an hour of delivery $(p=0.01)$, and no attendance at an antenatal breastfeeding discussion $(p=0.01)$. These findings suggest that risk of initiating a BMS during postnatal hospital stay is largely modifiable. Concordance with UNICEF Baby Friendly 10 steps, attention to specific features of the postnatal ward working environment, and the targeting of midwives and mothers with poor educational status may all lead to improved exclusive breastfeeding rates at hospital discharge.
\end{abstract}

Keywords: breastfeeding; attitudes; knowledge; midwifery; formula supplementation; justification of supplementation

\section{Introduction}

Breastfeeding has long been regarded as the preferred infant feeding choice with established long- and short-term benefits for mothers, infants, and society [1,2]. Many high-quality studies from developed countries similar and applicable to the United Kingdom (UK) show that breastfed babies are less likely to develop infections than babies fed with breastmilk substitutes (BMS), specifically those of the lower respiratory tract, gastrointestinal system, and otitis media [3-9]. Indeed, a meta-analysis of 33 studies in developed counties concluded formula-fed infants had three times more severe infections compared to infants exclusively breastfed to four months [10]. 
Studies have found other beneficial health associations of breastfed infants compared to infants fed with BMS, including lower rates of Sudden Infant Death Syndrome, necrotizing enterocolitis, atopic diseases, childhood obesity, and enhanced neurocognitive function [11-20].

Breastfeeding not only confers health benefits to the infant, but also to the mother, for example by incurring faster weight loss and as a natural contraceptive [21,22]. The longer the duration of breastfeeding, the increased protection against breast and ovarian cancer, and reduced post-menopausal hip fractures [23-27]. Emerging evidence suggests that breastfeeding positively impacts mother-baby relationships, bonding, and post-natal depression [28-30]. From an economic perspective, the improvements in child and maternal morbidity and mortality, plus reduced workforce productivity loss are estimated to be substantial [1].

Exclusive breastfeeding (EBF) is defined as an infant who receives only breastmilk, or expressed breastmilk, but no other solids or liquids except prescribed medicines, vitamins, or mineral supplements [31].

The World Health Organisation (WHO) recommends all infants to be breastfed exclusively for the first six months of life, and for breastfeeding to continue until age 2 years or beyond [31]. Despite this, data from the National Infant Feeding survey 2010 demonstrates $<1 \%$ of mothers in the UK reach this target. The greatest decline in exclusive breastfeeding $(23 \%)$ occurred within the infant's first week of life, with infants who exclusively breastfed for the first two days having a greater chance of achieving the six-month target, with greater self-efficacy [32-34]. This demonstrates the importance of providing adequate in-hospital support.

The literature has highlighted associations between the use of BMS and negative impacts on breastfeeding. In a prospective cohort study of mothers with the intention to exclusively breastfeed, the introduction of BMS on post-natal wards, alongside intrapartum opiate-use, were the only modifiable predictors of non-exclusivity at three months [35]. Chantry et al. found a three-fold increase in the risk of breastfeeding cessation by day 60 for those receiving in-hospital BMS supplementation, regardless of the mother's intention [36]. There are also concerns about BMS use and its impact on the neonatal gut microbiota, with long-term health implications [37]. Current evidence has demonstrated that early in-hospital supplementation is commonplace in developed countries [34].

The use of BMS supplementation within the early hospital environment is a multifactorial practice. The most commonly cited reason for BMS supplementation was due to breastfeeding difficulties, demonstrating that a high proportion of supplementation occurs without medical explanation [38]. Significant variation in BMS supplementation of breastfed infants was also reported in concordance with maternal education and ethnicity [39]. Within the early postnatal environment, midwives are responsible for the initial administration of supplementary BMS. Previous work has identified a lack of time, poor staffing levels, and a resistance to change as key contributing factors to non-compliance of a breastfeeding support intervention on UK postnatal wards [40].

The training a midwife receives has also been suggested to be clinically relevant to infant feeding practices in the hospital, with those demonstrating higher breastfeeding knowledge reporting best clinical practice [41]. A UK comparative study identified negative staff attitudes as a contributing factor to the early cessation of breastfeeding [42]. However, qualitative research suggests that midwives underestimate the influence they have on maternal feeding choices $[43,44]$. Overall, these studies highlight the need to investigate the influence of maternal and midwife factors on rates of neonatal infant-formula supplementation in the absence of medical indication (NIFS-AMI).

\section{Materials and Methods}

\subsection{Study Design}

A cross-sectional analytic study evaluated features of mothers, midwives, and postnatal care which are associated with NIFS-AMI on the postnatal ward (Figure 1). Data were routinely collected over one year about feeding method at discharge at Queen Charlotte's and Chelsea Hospital (QCCH) and St Mary's Hospital (SMH), UK to determine the recorded supplementation rate within the 
Trust. A detailed analysis of one month's data from the hospital's patient records determined infant feeding method and further categorisation of supplementation into either medical or non-medical reasons (NIFS-AMI). Bedside interviews of mothers were conducted to determine maternally reported supplementation rate and explore the relationship between features of birth, mother, and family and risk of NIFS-AMI. Interviews of midwives were used to determine the range and values of midwife NIFS-AMI rate and explore midwife characteristics associated with this rate.

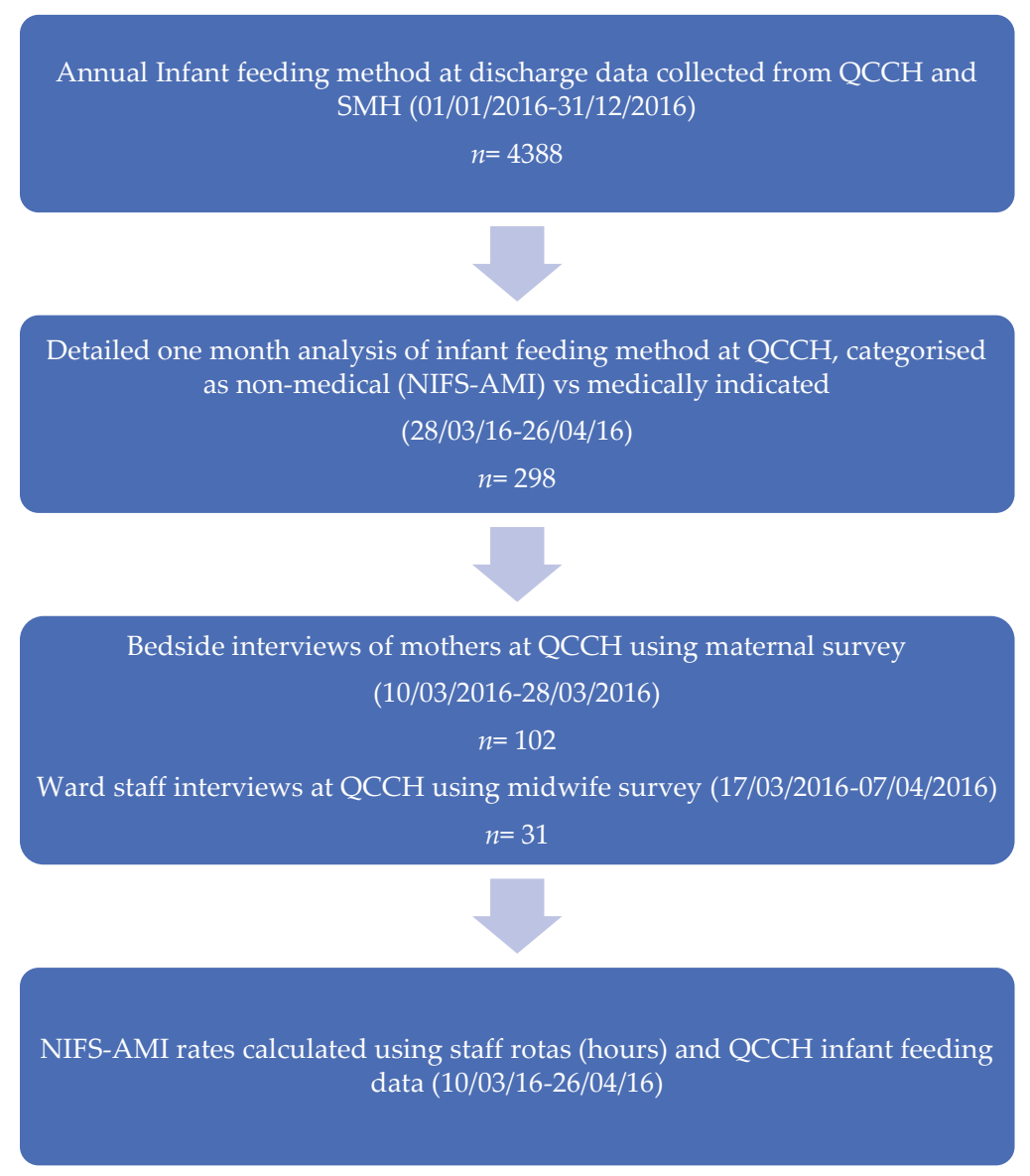

Figure 1. Study design. QCCH: Queen Charlotte's and Chelsea Hospital; SMH: Saint Mary's Hospital; NIFS-AMI: neonatal infant-formula supplementation in the absence of medical indication.

\subsection{Study Participants}

We undertook two separate cross-sectional surveys. In the maternal survey, mothers of newborn infants were surveyed at the bedside between 10 March 2016 and 28 March 2016. Women with conditions or medications that prevented breastfeeding, who had multiple pregnancies, or whose infants were inpatients on the neonatal intensive care unit were excluded. All participating mothers gave informed consent.

In the midwife survey, midwives working on the postnatal wards between 17 March 2016 and 7 April 2016 were assessed. Royal College of Midwifery registered midwives working at least one 
shift during this period, either as agency, temporary, or permanent staff members, were included. The postnatal department is divided into three distinct wards; the public National Health Service (NHS) facility (43 beds), the self-pay (private) ward (eight private rooms), and the NHS birth centre (eight birthing rooms). Care is primarily delivered by midwives and maternal support workers. At the study site, BMS cannot be initially administered without the involvement and approval of a registered midwife. An obstetrician may be present on the private and NHS postnatal wards; however, the birth centre is an entirely midwife-led provision for labour and post-natal care. As part of the NHS screening programme, an in-hospital newborn and infant physical examination (NIPE) is conducted on all neonates by a paediatrician prior to discharge. Typically in the UK, mother-infant dyads are discharged to the community midwifery team once assessed as healthy, having established feeding and having received appropriate education on caring for their infant. Home visits are provided by community midwives for the first 10 days until care transitions to the responsibility of the health visitor. A general practitioner will provide a six-week postnatal check.

All participating midwives provided verbal consent and were assured that their responses would remain anonymous. They were informed of the purpose of the study-to examine behaviours associated with formula supplementation. The project was conducted as a clinical audit, as part of the requirements of the Baby Friendly Initiative. Formal review by an ethics committee was therefore not required, but the audit was approved by the clinical department and was approved by the Queen Charlotte's and Chelsea Hospital, London, UK audit office (March 2016).

\subsection{Data Collection}

Data were collected through infant feeding records, as well as maternal and midwife surveys. Annual infant feeding data were retrieved from CERNER electronic patient records, demonstrating the feeding method at discharge for 4388 mother-infant dyads at QCCH and SMH during the study period (1 January 2016 to 31 December 2016). Data from SMH were included to better assess the generalisability of the participants recruited from $\mathrm{QCCH}$. Length of admission, ethnicity, delivery methods, parity, birth weight, gestation, and ward type were compared between the two hospital sites. To determine infant feeding method at inpatient discharge, data from ward records on the postnatal unit were collected from 298 patients between 28 March 2016 and 26 April 2016. CERNER medical and nursing notes for both mother and infant were accessed to assess any recorded medical reasons for supplementation. Reasons for supplementation were categorised as either medical or non-medical (NIFS-AMI), according to WHO criteria. Accepted clinical reasons for using BMS included the following infant conditions: classic galactosemia, maple syrup urine disease, and phenylkenonuria. Infants who may require BMS in addition to breastmilk for a limited period include very low birth weight $(<1500 \mathrm{~g})$, very pre-term ( $<32$ weeks gestation), and newborn infants who are at risk of hypoglycaemia if their blood glucose fails to respond to optimal breastmilk feeding. Maternal conditions that justify the temporary avoidance of breastfeeding included severe illness that prevents a mother from caring for her infant (e.g., sepsis), herpes simplex virus type 1 lesions on the mother's breasts, and certain maternal medications. HIV infection was the only named justification for permanent avoidance of breastfeeding [45].

\subsubsection{Maternal Survey}

All participating women were interviewed with the UNICEF Baby Friendly Hospital Initiative (BFHI) accredited audit tool [46]. Data were collected with regard to the education received on breastfeeding and relationship building from a healthcare professional, their birth experience, and the antenatal and postnatal support received. Original additional questions were included regarding maternal demographic data, infant feeding methods, durations of breastfeeding both as a mother and as an infant, and general attitudes of their close friends and relatives. Maternal levels of breastfeeding (BF) confidence were assessed using a Likert scale (1-10) for both before and after the birth of their infant. Maternal demographic data, gestation at delivery, and infant birth weight were obtained 
from patient electronic records. Ethnicity was categorised according to Office of National Statistics England [47].

\subsubsection{Midwife Survey}

Quantitative and qualitative data were obtained through staff questionnaires to assess attitudes as well as personal and professional experiences of infant feeding. The survey instrument was developed based on expert opinion and previously published literature [40-44]. Within the questionnaire, staff were asked to describe three scenarios in which they had supplemented a breastfed infant and to discuss the barriers they encountered with regard to supporting breastfeeding.

We also obtained data on the frequency of initiated NIFS-AMI across the three wards and for each midwife. Infants supplemented for medical reasons were excluded from analysis. To control for the inevitable influence of working hours, a supplementation rate was calculated. Staff rotas from the corresponding seven-week period were reviewed to total the number of hours each midwife worked, which was divided by the supplementation frequency to produce an hourly percentage rate. The hourly rate was used to estimate a monthly rate; the hourly rate was multiplied by 37.5 and divided by 7 before multiplying the value by 30 .

\subsection{Data Analysis}

Data were tested for normality to determine the choice of statistical methods using a visual inspection of histograms and the Shapiro-Wilk test. For univariate analysis, the Mann-Whitney U test for non-parametric data was employed. For categorical data, Pearson's Chi-squared test was used to determine statistically significant differences between groups. Fisher's Exact Test was used if cells had an expected count of less than 5. A $p$-value of $<0.05$ was considered significant. The Spearman Correlation coefficient was used to test associations between variables with a non-parametric distribution.

Due to the relatively small sample size, the private ward and birth centre were grouped due to similarities in size, facilities, staff to patient ratios, and working environment when using 'postnatal ward type' as a variable in analyses.

Analysis of covariance (ANCOVA) was used for multivariate analysis, considering cofounding variables that had been identified through correlation coefficients and a review of the literature, to produce the final model.

IBM SPSS 23 statistical software (IBM, Armonk, NC, USA) was used for all statistical analysis.

\section{Results}

\subsection{Maternal Descriptive Characteristics}

Table 1 presents the clinical and demographic characteristics of mothers and infants surveyed, in comparison to QCCH and SMH electronic records during April 2016. Of the 102 women included in the study, 55 were exclusively breastfeeding (EBF) their baby (54\%), 41 were supplementing with BMS (40\%), and six were exclusively receiving BMS (6\%). The monthly rates of exclusive breastfeeding at discharge for 2016 are displayed in Figure S1. The median maternal age was 32 years $(28,36$ IQR) and infant age was 1 day $(1,2)$. Primiparous women were overrepresented in our study sample $(58 \%)$; however, other characteristics were illustrative of the wider cohort.

Table 1. Clinical and demographic characteristics of mothers and infants.

\begin{tabular}{lcccc}
\hline & Survey & QCCH & SMH & Both Sites \\
\hline & $n=102$ & $n=486$ & $n=329$ & $n=815$ \\
Maternal age (years) & $32(28,36)$ & $32(29,36)$ & $33(28,36)$ & $33(29,36)$ \\
Feeding status & & $n=220^{*}$ & $n=117^{*}$ & $n=337^{*}$ \\
$\quad$ Exclusively BF & $55(54)$ & $138(63)$ & $75(64)$ & $213(63)$ \\
Supplemented with medical justification & $3(3)$ & $67(30)$ & $28(24)$ & $95(28)$ \\
\hline
\end{tabular}


Table 1. Cont.

\begin{tabular}{|c|c|c|c|c|}
\hline & Survey & QCCH & SMH & Both Sites \\
\hline \multicolumn{5}{|l|}{ Supplemented without medical justification } \\
\hline NIFS-AMI & $38(37)$ & & & \\
\hline Exclusive BMS & $6(6)$ & $15(7)$ & $14(12)$ & $29(9)$ \\
\hline Baby age (days) & $1(1,2)$ & & & \\
\hline Length of admission (days) & & $2(1,3)$ & $2(1,4)$ & $2(1,3)$ \\
\hline Age left education (years) & $23(21,24)$ & & & \\
\hline Highest level of education: University degree & $66(65)$ & & & \\
\hline Ethnicity: White Caucasian & $53 / 88(60)$ & $149 / 295(51)$ & $106 / 182(58)$ & $255 / 477(53)$ \\
\hline Vaginal delivery & $64(63)$ & $357(73)$ & $201(61)$ & $558(68)$ \\
\hline Laboured $<2 \mathrm{~h}$ & $82(80)$ & $410(84)$ & $280(85)$ & $690(85)$ \\
\hline Primiparous & $59(58)$ & $83(17)$ & $42(13)$ & $125(15)$ \\
\hline Weight of infant & $3.2(2.9,3.6)$ & $3.2(2.9,3.5)$ & $3.3(3.0,3.6)$ & $3.3(2.9,3.6)$ \\
\hline $\operatorname{Low}(<2.5 \mathrm{~kg})$ & $8(8)$ & $41(8)$ & $20(7)$ & $61(8)$ \\
\hline Gestational age (weeks) & $39(38,40)$ & $39(38,40)$ & $39(39,40)$ & $39(38,40)$ \\
\hline If multiparous, EBF previous infants & $26 / 43(61)$ & & & \\
\hline \multicolumn{5}{|l|}{ Ward type } \\
\hline Postnatal & $82(80)$ & $406(84)$ & $216(66)$ & $622(76)$ \\
\hline Private Ward & $9(9)$ & $0(0)$ & $70(21)$ & $70(9)$ \\
\hline Birth Centre & $11(11)$ & $80(16)$ & $43(13)$ & $123(15)$ \\
\hline Private room & $25(25)$ & & & \\
\hline Infant remained with mother in hospital & $92(90)$ & & & \\
\hline Partner present at night & $42(41)$ & & & \\
\hline Any skin-to-skin after birth & $84(82)$ & & & \\
\hline Skin-to-skin for $1 \mathrm{~h}$ after birth & $50(49)$ & & & \\
\hline Supported to BF after birth & $68(67)$ & & & \\
\hline Assisted with positioning and attachment & $82(80)$ & & & \\
\hline $\begin{array}{l}\text { Reports having received education about signs that } \\
\text { infant is receiving enough milk }\end{array}$ & $50(49)$ & & & \\
\hline $\begin{array}{l}\text { Reports having received education about hand } \\
\text { expression }\end{array}$ & $39(38)$ & & & \\
\hline Reports having antenatal $\mathrm{BF}$ discussion with $\mathrm{HCP}$ & $39(38)$ & & & \\
\hline
\end{tabular}

All data are displayed as number (\% or IQR) as shown. Single numerical values $(n)$ in brackets indicate \%, double numerical values $(n, n)$ indicate IQR. Ethnic groups represented in the non-Caucasian sample were categorised according to National Statistics recommended criteria and were represented as follows; surveyed mothers: Black 13 (15), Asian 22 (25), Mixed 0 (0); QCCH: Black 50 (17), Asian 80 (27), Mixed 16 (5); SMH: Black 25 (14), Asian 37 (20), Mixed 14 (8). BF-breastfeeding; BMS—breastmilk substitute; EBF-exclusive breastfeeding; HCP — healthcare professional; NIFS-AMI - neonatal infant-formula supplementation in the absence of medical indication; QCCH-Queen Charlotte's and Chelsea Hospital; SMH—Saint Mary's Hospital. * percentages in the corresponding columns were calculated on the available data for statistical analysis.

\subsection{Maternal Characteristics and NIFS-AMI}

Univariate analysis was conducted to determine which variables of maternal characteristics were associated with NIFS-AMI (Table 2). Skin-to-skin contact for at least one hour $(p=0.01)$, higher education $(p<0.01)$, and antenatal discussions regarding breastfeeding $(p=0.01)$ were all inversely associated with non-clinical supplementation behaviour on the postnatal ward.

Table 2. Association between maternal characteristics and NIFS-AMI: univariate analysis $(n=96)$.

\begin{tabular}{|c|c|c|c|}
\hline & $\begin{array}{l}\text { Number of Infants } \\
\text { Receiving NIFS-AMI }\end{array}$ & $\begin{array}{c}\text { Number of Infants EBF } \\
\text { + Medically Justified } \\
\text { Supplementation }\end{array}$ & $p$ Value \\
\hline & $n=38$ & $n=55+3$ & \\
\hline Maternal age (years) & $29(25,37)$ & $34(29,36)$ & 0.13 \\
\hline Baby age (days) & $2(1,3)$ & $1(1,1)$ & 0.01 \\
\hline Age left education (years) & $22(19,24)$ & $23(21,24)$ & 0.19 \\
\hline Highest level of education: University degree & $18(47)$ & $44(76)$ & $<0.01$ \\
\hline Employed prior to pregnancy & $21(55)$ & $43(74)$ & 0.08 \\
\hline Ethnicity: White Caucasian & $23(61)$ & $33(57)$ & 0.29 \\
\hline Vaginal delivery & $23(61)$ & $38(66)$ & 0.62 \\
\hline Laboured $>2 \mathrm{~h}$ & $31(82)$ & $45(78)$ & 0.64 \\
\hline Primiparous & $24(63)$ & $32(55)$ & 0.44 \\
\hline
\end{tabular}


Table 2. Cont.

\begin{tabular}{|c|c|c|c|}
\hline & $\begin{array}{l}\text { Number of Infants } \\
\text { Receiving NIFS-AMI }\end{array}$ & $\begin{array}{l}\text { Number of Infants EBF } \\
+ \text { Medically Justified } \\
\text { Supplementation }\end{array}$ & $p$ Value \\
\hline Weight of infant & $3.3(2.8,3.6)$ & $3.3(3.0,3.5)$ & \\
\hline Gestational age (weeks) & $39(38,40)$ & $40(39,41)$ & 0.07 \\
\hline If multiparous, EBF previous infants & $6 / 14(43)$ & $19 / 26(73)$ & 0.06 \\
\hline $\mathrm{BF}$ duration with previous infants & $12(5,24)$ & $7(6,9)$ & 0.39 \\
\hline Ward type & & & 0.04 \\
\hline Postnatal & $34(45)$ & $42(55)$ & \\
\hline Birth Centre/Private Ward & $4(25)$ & $16(75)$ & \\
\hline Private room & $7(18)$ & $18(31)$ & 0.17 \\
\hline Infant remained with mother in hospital & $36(95)$ & $52(90)$ & 0.47 \\
\hline Partner present at night & $18(47)$ & $20(35)$ & 0.13 \\
\hline Any skin-to-skin after birth & $26(68)$ & $54(93)$ & $<0.01$ \\
\hline Skin-to-skin for $1 \mathrm{~h}$ after birth & $13(34)$ & $36(62)$ & 0.01 \\
\hline Supported to BF after birth & $27(71)$ & $41(71)$ & 0.97 \\
\hline Assisted with positioning and attachment & $33(87)$ & $47(81)$ & 0.46 \\
\hline $\begin{array}{l}\text { Mother reported antenatal education about signs that infant } \\
\text { is receiving enough milk }\end{array}$ & $22(58)$ & $25(43)$ & 0.16 \\
\hline Mother reported antenatal education on responsive feeding & $10(26)$ & $33(57)$ & 0.10 \\
\hline Mother reported antenatal education about hand expression & $23(61)$ & $15(26)$ & $<0.01$ \\
\hline $\begin{array}{l}\text { Mother reported antenatal education on benefits of } \\
\text { developing relationship with infant }\end{array}$ & $14(37)$ & $32(55)$ & 0.01 \\
\hline Informed of support available within the community & $17(45)$ & $24(41)$ & 0.75 \\
\hline Attended $\mathrm{BF}$ antenatal class at $\mathrm{QCCH}$ & $3(8)$ & $7(12)$ & 0.74 \\
\hline Attended BF antenatal class at another site & $7(18)$ & $16(28)$ & 0.30 \\
\hline Antenatal BF discussion & $9(24)$ & $29(50)$ & 0.01 \\
\hline Mother reports having been EBF as infant & $16(43)$ & $23(40)$ & 0.99 \\
\hline Duration EBF as infant & $12(12,24)$ & $7(6,12)$ & 0.05 \\
\hline BF confidence on ward (1-10) & $7(6,8)$ & $7(6,9)$ & 0.28 \\
\hline BF confidence before birth (1-10) & $8(6,10)$ & $6(5,9)$ & 0.09 \\
\hline BMS first introduced (hours) & $6(4,24)$ & $5(3,39)$ & 0.94 \\
\hline Positive attitude to $\mathrm{BF}$ in social/cultural background & $29(76)$ & $46(79)$ & 0.73 \\
\hline
\end{tabular}

All data are displayed as number (\% or IQR) as shown. Single numerical values $(n)$ in brackets indicate $\%$, double numerical values $(n, n)$ indicate IQR. Mothers exclusively feeding their infants with BMS have been excluded from the multivariate analysis $(n=6)$. Significant results are indicated by the bold text. BF-breastfeeding; BMS — breastmilk substitute; EBF- exclusively breastfed; NIFS-AMI—neonatal infant-formula supplementation in the absence of medical indication; QCCH—Queen Charlotte's and Chelsea Hospital.

\subsection{Midwife Descriptive Characteristics}

The demographics of the participating midwives are detailed in Table S1. The median age was 38 years (28-50 IQR) with the majority having studied midwifery at the university level $(87 \%)$. The median years' experience as a midwife was 7 years (4-18). All the participating staff members had received mandatory breastfeeding training provided by Imperial College London NHS Hospital Trust (100\%). Of the 31 midwives available for interview, 20 had initiated NIFS-AMI within the seven-week study period $(65 \%)$, with a median hourly rate of $0.62 \%(0-2.8)$.

\subsection{Midwife Attitudes and Recalled Infant Feeding Behaviour}

Twenty-four midwives reported their cultural and social backgrounds to have a positive attitude towards breastfeeding (77\%) (Table 3). The mean perceived impact of BMS supplementation was $8 / 10$ (6-10), with 1 being lowest impact and 10 greatest. Of all the supplementation scenarios described by staff, $62 \%(n=111)$ violated WHO criteria, with only a third able to describe three scenarios appropriately and $13 \%$ unable to provide any acceptable answers. Inappropriate reasons cited for BMS supplementation are shown as number (\%); hungry infant 15 (21.7); medical recommendation 13 (18.8); jaundice 12 (17.4); latching problems 10 (14.5); low birth weight or weight loss 8 (11.6); prematurity 7 (10.1); reluctant feeders 1 (1.5); nasogastric tube-use 1 (1.5); transitional care 1 (1.5); $12 \mathrm{~h}$ without feeding 1 (1.5). 
Table 3. Midwife attitude and knowledge of breastfeeding.

\begin{tabular}{lc}
\hline Midwife Attitude and Knowledge of Breastfeeding & $n$ (IQR) \\
\hline Perceived importance of role in BF (1-10)* & $10(10,10)$ \\
Perceived impact of BMS supplements $(1-10) *$ & $8(6,10)$ \\
Perceived time satisfaction (1-10) * & $5(1,8)$ \\
Confidence in FF (1-10) * & $8(4,10)$ \\
& $n(\%)$ \\
Positive attitudes to BF in social/cultural background & $24(77)$ \\
Staff satisfied with time for FF & $10(32)$ \\
Received training on FF & $19(61)$ \\
Supplementation practice violates WHO criteria & $20(65)$ \\
One of three examples violated criteria & $6(19)$ \\
Two of three examples violated criteria & $10(32)$ \\
All examples described violated criteria & $4(13)$ \\
Percentage of time allocation to feeding support & $\%(I Q R)$ \\
versus other tasks during a typical day & $12(10,20)$ \\
Specific feeding support & $25(20,40)$ \\
General patient care & $50(30,60)$ \\
Computer/paperwork & $10(10,15)$ \\
Taking bedside observations & \\
\hline
\end{tabular}

BF-breastfeeding; BMS—breastmilk substitute; FF—finger feeding; WHO_World Health Organisation. * — Likert scale was used to assess these items.

\subsection{Assessment of Real-Life BMS Prescription}

Figure 2 illustrates the frequency of supplementation behaviour of midwives on the postnatal unit at QCCH. The vast majority of supplements administered during the study period were for non-medically indicated reasons (90\%), which was consistent with current literature [48]. Fifty-nine percent of NIFS-AMI instances occurred during between the hours of 22.00-06.00. Medical reasons to supplement included infant hypoglycaemia and very low birth weight $(<1500 \mathrm{~g})$. The most commonly recorded reason for NIFS-AMI was maternal request.

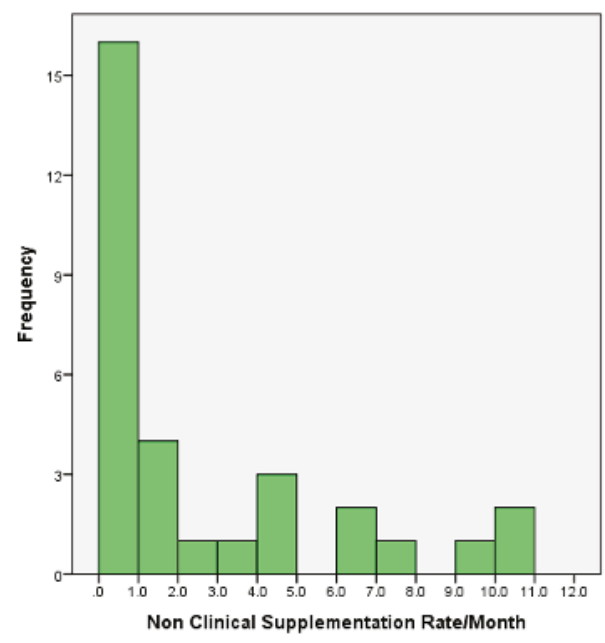

Figure 2. Midwives' estimated monthly rates of neonatal infant-formula supplementation in the absence of medical indication (NIFS-AMI) as defined by WHO $(n=31)$. Monthly rates were extrapolated from hourly rates. Midwives worked for a median of $212.5(161.3,248)$ hours during the seven-week study period (9 March 2016-26 April 2016). 


\subsection{What Influences NIFS-AMI?}

Table 4 illustrates the univariate analysis of midwife variables in relation to rates of NIFS-AMI. Statistical differences in hourly rates were observed according to ward types $(p<0.01)$, time satisfaction with breastfeeding $(p=0.01)$, and finger feeding support $(p=0.02)$, alongside finger feeding confidence $(p=0.03)$. Borderline significant values were found regarding level of education, perceived impact of BMS supplementation $(p=0.07)$, and the ability to correctly describe three appropriate scenarios $(p=0.07)$.

Table 4. Hourly rates of NIFS-AMI (\%) according to midwife variables $(n=31)$.

\begin{tabular}{|c|c|c|c|}
\hline Descriptive Characteristics & Median/IQR & $\mathbf{r}$ & $p$ Value \\
\hline Staff age & & 0.2 & 0.28 \\
\hline Years' experience as a midwife & & 0.03 & 0.89 \\
\hline \multicolumn{4}{|l|}{ Ward Type } \\
\hline Postnatal & $1.9(0.7,4.2)$ & & $<0.01$ \\
\hline Birth Centre/Private Ward & $0.0(0.0,0.4)$ & & \\
\hline \multicolumn{4}{|l|}{ Ethnic Background } \\
\hline White Caucasian & $0.4(0.0,0.7)$ & & 0.26 \\
\hline Non-Caucasian & $1.9(0.6,3.0)$ & & \\
\hline \multicolumn{4}{|l|}{ Highest level of education } \\
\hline Degree & $0.4(0.0,1.9)$ & & 0.07 \\
\hline Non-degree & $3.4(2.2,4.7)$ & & \\
\hline \multicolumn{4}{|l|}{ Personal experiences } \\
\hline \multicolumn{4}{|l|}{ Attitudes to $\mathrm{BF}$ in social/cultural background } \\
\hline Positive & $0.7(0.0,2.7)$ & & 0.84 \\
\hline Negative or mixed & $0.4(0.0,2.7)$ & & \\
\hline \multicolumn{4}{|l|}{ Parity } \\
\hline Primiparous/multiparous & $0.7(0.4,4.2)$ & & 0.22 \\
\hline Nulliparous & $0.4(0.0,1.8)$ & & \\
\hline \multicolumn{4}{|l|}{ Infant feeding method } \\
\hline EBF & $0.6(0.0,1.9)$ & & 0.57 \\
\hline Non-EBF & $2.5(0.5,4.2)$ & & \\
\hline Longest $\mathrm{BF}$ duration (months) & & -0.29 & 0.32 \\
\hline Earliest age child received non-breastmilk (months) & & -0.79 & 0.58 \\
\hline Midwife's reported feeding method as infant & & & 0.62 \\
\hline$E B F$ & $0.6(0.0,1.8)$ & & \\
\hline Mon-EBF & $0.8(0.0,2.7)$ & & \\
\hline Duration of BF as infant (months) & & 0.43 & 0.11 \\
\hline \multicolumn{4}{|l|}{ Staff perceptions and professional experiences } \\
\hline Perceived impact of BMS supplementation (1-10) & & -0.33 & 0.07 \\
\hline Happiness with time for infant feeding support (1-10) & & -0.47 & 0.01 \\
\hline \multicolumn{4}{|l|}{ Attended training sponsored by a formula company } \\
\hline Yes & $0.8(0.3,2.7)$ & & 0.62 \\
\hline No & $0.5(0.0,2.6)$ & & \\
\hline \multicolumn{4}{|l|}{ Received training on finger feeding } \\
\hline Yes & $0.4(0.0,3.0)$ & & 0.86 \\
\hline No & $0.9(0.0,2.7)$ & & \\
\hline \multicolumn{4}{|l|}{ Satisfied with time available for finger feeding } \\
\hline Yes & $0.0(0.0,0.6)$ & 0.43 & 0.02 \\
\hline No & $1.2(0.3,4.2)$ & & \\
\hline Confidence in finger feeding (1-10) & & -0.40 & 0.03 \\
\hline \multicolumn{4}{|l|}{ Correctly identify three correct reasons for supplementing } \\
\hline Yes & $0.6(0.0,4.6)$ & & 0.07 \\
\hline No & $0.6(0.0,2.1)$ & & \\
\hline
\end{tabular}


Table 4. Cont.

\begin{tabular}{|c|c|c|c|}
\hline Descriptive Characteristics & Median/IQR & $\mathbf{r}$ & $p$ Value \\
\hline \multicolumn{4}{|c|}{$\begin{array}{l}\text { Percentage of time allocation to feeding support versus } \\
\text { other tasks during a typical day (\%) }\end{array}$} \\
\hline Infant feeding support & & 0.56 & 0.77 \\
\hline General patient care & & -0.01 & 0.96 \\
\hline Computer/paperwork & & -0.01 & 0.95 \\
\hline Taking bedside observations & & 0.14 & 0.47 \\
\hline
\end{tabular}

Univariate analysis according to supplementation categorisation is displayed in Table S2. As a recurring theme, time satisfaction and ward type were significantly associated.

To assess the association between the midwives' rate of NIFS-AMI and most important exposures, a bootstrapping regression model was used in the multivariate analysis (Table 5). Ward type $(p=0.01)$ and midwife education ( $p=0.01)$ were statistically significant.

Table 5. Multivariate analysis of factors influencing midwives' decision for NIFS-AMI.

\begin{tabular}{clccc}
\hline Parameter & \multirow{2}{*}{ B } & \multirow{2}{*}{ V Value } & \multicolumn{2}{c}{ 95\% Confidence Interval } \\
\cline { 5 - 5 } & & & Lower Bound & Upper Bound \\
\hline Education & 2.53 & $\mathbf{0 . 0 1}$ & 0.95 & 4.12 \\
Breastmilk substitute impact (1-10) & -0.19 & 0.22 & -0.48 & 0.07 \\
Ability to provide three correct scenarios (100\%) & 1.01 & 0.18 & -2.47 & 0.23 \\
Ward & -1.63 & $\mathbf{0 . 0 1}$ & -2.50 & -0.66 \\
\hline
\end{tabular}

Dependent variable; rate of NIFS-AMI (\%), a; this parameter is set to zero because it is redundant. Significant results are indicated by the bold text.

\subsection{Perceived Barriers to Supporting Breastfeeding}

When asked about barriers encountered on the wards, $78 \%$ of the 31 staff members reported time constraints as a key factor affecting breastfeeding support (Figure S2). According to self-reported time allocation, midwives allocated a median of only $12 \%$ of their shift to supporting infant feeding, with the largest proportion of their time allocated to computer and paperwork (50\%). This was consistent across wards and NIFS-AMI categories. of time:

As a key theme, many midwives expressed emotive responses relating to their perceived lack

"Sometimes I get depressed, it is a terrible thing not being able to help". Midwife 4

"The training courses are pointless if you don't have the time to use it". Midwife 12

"It's never them, the mothers want the support; it's the time". Midwife 30

"It has repercussions on our own health, not being able to help, as well as of course the baby and the mothers. The mothers get so depressed". Midwife 23

There were also several members of staff expressing the mothers' poor antenatal preparation as an interfering factor (27.5\%). This was expressed in relation to a lack of knowledge and exposure to breastfeeding videos:

"They [the mothers] genuinely don't know how good it is! They underestimate the difference between breast and formula, it is such a shame". Midwife 24 
"The videos they show in antenatal classes give women unrealistic expectations of breastfeeding, it is harder than they make it look". Midwife 7

Additionally, workload and staff shortage related issues were common complaints, as reported by $55 \%$ of staff. Alongside this, the requirement for more specific staff with dedicated roles to infant feeding and one-to-one opportunities was expressed by $38.5 \%$ :

"You cave in sometimes due to the workload, giving the bottle is so easy and that is why it is so sad". Midwife 23

"I tell the women breastfeeding is hard and needs perseverance. One-to-one support is so important and 30 min isn't enough". Midwife 31

"It is exhausting helping women one-to-one to breastfeed all the time, especially when the ward is busy, it takes a lot of effort". Midwife 18

Although finger feeding was included in the compulsory training day, $38.7 \%$ of midwives regarded themselves to have not received training, which was relatively constant across the groups. Time satisfaction and confidence for this technique varied significantly according to ward type. In addition, six staff members (15\%) expressed negative attitudes towards finger feeding, describing the practice as "unhygienic", "dangerous", and "a waste of time".

\section{Discussion}

With significant evidence that NIFS-AMI has detrimental consequences on breastfeeding, the factors influencing early BMS use by staff in the post-natal environment is highly relevant. In this study, maternal and midwife factors were sought to determine possible impacts upon NIFS-AMI activity on the postnatal ward.

\subsection{The Use of BMS Supplementation on the Postnatal Ward}

Our data demonstrates that a significant proportion of BMS supplementation occurs for no defined medical reason. In nine out of 10 cases of BMS supplementation, breastmilk substitute was provided by the staff without appropriate medical indication. Local statistics from 2015 Good Food for London Report show a good level of community breastfeeding support, achieving full BFHI accreditation, which further highlights the need to change the current infant feeding practice in the postnatal unit [49]. This recommendation was supported further in the report from the National Maternity and Perinatal Audit 2017, which advised that "Commissioners, together with clinicians, services, and policymakers should strongly prioritise the provision of resources to support breastfeeding, both in maternity units and in the community, to reduce the variation in the proportion of babies receiving breastmilk at their first feed and at discharge from the maternity unit" [50].

\subsection{Maternal Factors}

Maternal characteristics protective against NIFS-AMI were associated with higher educational status, one hour of uninterrupted skin-to-skin after delivery, and breastfeeding discussions during pregnancy with a healthcare professional. These findings are reflective of previous work [51]. The evidence for skin-to-skin as an intervention to improve breastfeeding outcomes is particularly well studied. A 2016 Cochrane review concluded with good evidence that skin-to-skin was beneficial to healthy infants [52]. Our results are supportive of the UNICEF recommendations, which is in agreement with the BFHI 10 steps to successful breastfeeding.

\subsection{Midwife Factors}

Our findings suggest that the staff at QCCH experience many barriers to supporting breastfeeding. One key theme arising from this exploration regards the lack of suitable antenatal preparation. Previous 
research shows that under-preparation and the availability of BMS are key reasons why mothers requested artificial supplements [53]. In addition, prior expectations, formed from healthcare staff and the media, influence maternal breastfeeding confidence to a major extent [54].

As described by the midwives at QCCH, antenatal classes have been named as partly responsible for these unrealistic expectations $[55,56]$. This is consistent with the available evidence worldwide, demonstrating antenatal education to have negative or inconclusive impact on breastfeeding rates [57-59]. Consequently, a lack of concordance between a mother's expectations and the reality of breastfeeding can lead to feelings of guilt, failure, and a lack of confidence in breastfeeding and motherhood [60,61].

There have been several studies investigating the factors likely to affect midwives' behaviour in relation to infant feeding. Lack of time, poor staffing levels, and a resistance to change were all identified as contributing factors to non-compliance of a breastfeeding support intervention on UK postnatal wards [40]. Our data support previous findings in the UK, as the barriers most commonly reported by QCCH maternity staff were a lack of time alongside workload issues and the need for more specific feeding support with one-to-one care. The number of hospitals applying the BFHI policy is on the rise, but the real-life effectiveness of this strategy is somehow limited due to the increasing issue of staff shortages [62,63].

The views of inadequate availability of staff support appear to be consistent across cultural and ethnic backgrounds, with evidence that one-to-one feeding support is effective at significantly reducing supplementation rates [64-66]. The perception of active support from midwives postnatally in England was predictive of lower odds of breastfeeding cessation as assessed by maternal questionnaires at day 10 [67]. In this study, many midwives reported computer work, particularly discharges, to take up a considerable percentage of their day. Bowers et al. revealed that very little staff time is spent educating and supporting mothers with infant feeding; this was concordant with the midwives' time allocation at QCCH. Recent literature has demonstrated a reduction in the duration of postnatal admissions with proportionate reductions in staffing, resulting in an increased workload which negatively impacted quality of care and staff morale [68].

In our study, the most-cited reason for NIFS-AMI was maternal request for BMS. In the literature, reasons for parents requesting BMS supplementation included a lack of preparation for breastfeeding and viewing infant-formula as the answer to breastfeeding problems [24,53].

The UNICEF Maternity Standards document states that clear documentation of the mother's reason, alternative options, and information provided by midwife must be recorded when BMS supplementation is initiated for maternal requests. The recommended care includes hand expression education, support with positioning, and plans made for future feeding to maximise breastfeeding (or the use of breastmilk) [69]. In reality, this was rarely the case at $\mathrm{QCCH}$; documented notes provided very little details around the initiation of NIFS-AMI. Of the 102 mothers surveyed, only $38 \%$ could recall a discussion on hand expressing. The reported midwife dissatisfaction in the workplace appears to be a significant driver in the promotion of breastfeeding and time allocation for appropriate counselling.

As time and workload appear to have significant impacts on patients' postnatal support, the different working environments may explain the differences reported across ward types [70]. With a lower staff/patient ratio on the NHS postnatal ward, the perceived time satisfaction unsurprisingly differs to those working on private wards or at birth centres. Our study reveals that finger feeding confidence and time satisfaction were negatively associated with higher supplementation rates, which staff attributed to their lack of time. Although time availability appears to play a role in the interference of infant feeding support, a multivariate analysis identified staff perceptions of BMS to be the most influential factor upon rates of NIFS-AMI. This is supported by the inconsistencies identified across shift type and BMS use. For example, many staff reported having more time to support mothers during the night shift; however, a higher frequency of NIFS-AMI instances were reported, suggesting the current practice to be a complex, multifactorial issue. 
The next section of the survey was concerned with midwives' perceptions in relation to supplementation behaviour. Existing evidence suggests that midwives' behaviour is influential upon a mother's feeding decisions, with regard to their perceived attitudes and the support offered [71-73]. However, the current literature into midwives' supplementation perceptions and behaviour has not been thoroughly explored. An association between support offered by staff and their attitudes has been shown, with evidence of midwives with poor breastfeeding attitudes failing to support mothers [74]. Mothers usually perceiving maternity staff to have 'no preference' with regard to breastfeeding were less likely to breastfeed beyond six weeks [75]. Midwife characteristics associated with greater breastfeeding knowledge in other studies included midwives over 30 years old, with higher qualifications, as well as more clinical and personal experience of breastfeeding for over three months $[76,77]$. The training a midwife receives has also been suggested to be clinically relevant to breastfeeding practices in the hospital, with those demonstrating higher breastfeeding knowledge reporting best clinical practice [41].

Despite the fact that all maternity staff at QCCH had attended the Trust's breastfeeding training, only a third of them were able to successfully describe three valid scenarios in which they had supplemented a breastfed infant. This data is in agreement with the outcomes of an American study, reporting high rates of non-medically indicated supplementation, with staff often providing inaccurate information and failing to correct mothers who possessed incorrect information [48]. Although this may indicate that staff lack the knowledge to best advise mothers, there is evidence of non-adherence to the BFHI standards with deviant behaviour described such as undercover supplementation and concealing their actions by recording the supplementation as a 'maternal choice' rather than a 'midwife suggestion' [78]. This would suggest that midwives may be aware that their behaviour is not the best clinical practice.

\subsection{Application of BFHI Policy into Clinical Practice}

In order to explain the gap between evidence-based knowledge and clinical practice, the knowledge translation theory can be applied to the BFHI policy implementation using five stages from acceptance to adherence $[79,80]$. Our data demonstrate lack of knowledge as a crucial factor in real-life decision-making on whether to supplement an infant with BMS. In addition, emotional responses expressed by several staff members reflecting personal beliefs on breastfeeding support are consistent with previous qualitative studies in the UK and is thought to have a negative impact on clinical reasoning and adherence to BFHI criteria [81]. It has therefore been suggested that for midwives to develop new behaviour, their attitudes and belief systems need to be reframed [82,83]. This can be particularly difficult to achieve for those who have had positive experiences of supplementing, as beliefs associated with personal involvement most strongly predict forthcoming behaviour [84].

It is evident that for hospital practice modification, active participation and planning for change, alongside establishing targets, are necessary [85]. The midwife-identified barriers, such as those relating to the workplace, require consideration from the establishment to address issues surrounding infant feeding. Making BFHI an organisational priority, reducing the accessibility of BMS, and establishing financial support dedicated to the initiative are all suggestions to overcome barriers from an integrative review [86]. Enforcing the requirement for all parents to read and complete paperwork before BMS is permitted may also be successful at reducing both staff suggested and maternally requested supplementation with greater knowledge communication to ensure that parents give informed consent [87]. This also agrees with earlier results, which shows that support and encouragement from other midwives and managerial staff can promote adherence to BFHI practices, and indicates that involving staff in processes such as mentoring schemes can help promote positive attitudes towards the BFHI [79,87]. Conversely, midwives reverting back to outdated protocols have been demonstrated to influence the behaviour of newly qualified midwives and mothers, highlighting the potential for a cascade of incorrect conduct and the vital requirement for a committed workforce $[83,88]$. 


\subsection{Study Limitations}

The main limitations of this study surround the cross-sectional design, with data collection only occurring within a defined point in time. It is therefore impossible to infer causality between outcomes and exposures [89]. A prospective study assessing the attitudes of newly qualified midwives before starting work may therefore provide better insight into the temporal relationship. With limited time available for the interview period, it was not possible to survey all postnatal staff at the QCCH, which could therefore increase the risk of a non-representative sample. Additionally, due to the sample size, only a limited number of confounders could be accounted for in the multivariate analysis. However, the statistically significant correlation between midwives' education and work placement and the use of NIFS-AMI is worth further investigation. As the survey was presented in English and staff in a single hospital were approached, results cannot be generalised to all medical professionals across the globe. However, we believe that our results are closer to a best-case interpretation and therefore are even more concerning.

\section{Conclusions}

Known modifiable maternal factors confirmed in this study included the protective value of skin-to-skin and antenatal discussions against unnecessary BMS supplementation. A striking number of midwives failing to name medically appropriate scenarios for BMS introduction, which highlights failure in the education system, even when provided in accordance with the latest BFHI guidelines. Available evidence on BFHI effectiveness is somehow conflicting and cannot fully support existing training in its present form. The main outcome of BFHI efficiency is normally limited to breastfeeding initiation rates, which has been continuously critisised as an inappropriate measure to measure the success of the BFHI $[90,91]$.

With an association between the lack of knowledge and greater rates of NIFS-AMI, this study confirms the necessary requirement to reduce in-hospital supplementary feeds, through the development of a better model of breastfeeding support, which may include an extensive use of cell phone-based applications, educational programme development, and postnatal staff training improvement. Midwife interviews in this study suggested a need for more one-to-one support of women in the immediate postnatal period. Maternal request for BMS is a major factor influencing non-clinical supplementation and this should be tackled by developing strategies to train staff to respond constructively to such requests as well as by a more effective education of pregnant women, using online resources and distance learning tools. If changes could be successfully implemented, a reduction in non-clinical supplementation, with regard to both staff suggestion and maternal requests, could be achieved with predicted benefits in breastfeeding outcomes.

Key Messages:

- The use of in-hospital breastmilk substitute supplementation for breastfed infants is commonplace in the study hospital, as 95 (28\%) of infants received a BMS supplement prior to discharge from maternity unit and $90 \%$ of BMS supplementation was given without medical indication.

- Maternal factors protective of neonatal infant-formula supplementation in the absence of medical indication included skin-to-skin and antenatal breastfeeding discussions.

- Although midwives were appropriately trained in accordance with BFHI protocols, they identified a number of workplace barriers in breastfeeding support, with lack of time and heavy workload being the major contributors.

- We found significant associations between the midwives' decisions on non-medical infant-formula supplementation and educational status, which suggests a need to focus the existing BFHI training programme.

- In order to reduce unnecessary supplementation as well as address attitudes and perceived barriers, additional educational programmes should be developed, with a focus on one-to-one support for establishing lactation in the postnatal environment. 
Supplementary Materials: The following are available online at http:/ /www.mdpi.com/2072-6643/10/5/608/s1, Figure S1: The monthly rates of exclusive breastfeeding at discharge for 2016, Figure S2: Staff's perceived barriers to supporting breastfeeding on the wards, Table S1: Midwife characteristics, Table S2: Univariate analysis according to supplementation categorisation.

Author Contributions: K.B., K.H., E.M., R.J.B., and D.M. conceived and designed the study; K.B. designed the survey tool; K.B. and K.H. collected the data, K.B. and D.M. analysed the data and interpreted results; K.B. wrote the paper; E.K., D.M., and R.J.B. formatted and edited the manuscript.

Acknowledgments: All authors critically reviewed and approved the final manuscript. Many thanks are extended to all of the mothers and midwives who participated in this study.

Conflicts of Interest: K.B., K.H., E.M., and E.K. declare no conflict of interest. R.J.B. is supported by the National Institute for Health Research Biomedical Research Centre (BRC), having received research grant income from Danone in relation to studies of the value of prebiotics in allergy prevention, and Airsonette to evaluate temperature-controlled laminar airflow for asthma. He has also given paid lectures for the companies. D.M. has received consultancy payment from Dairy Goat Co-Operative (NZ) Ltd. and has given paid lectures for the Merck Sharp \& Dohme (MSD). The founding sponsors/pharmaceutical companies had no role in the design of the study; in the collection, analyses, or interpretation of data; in the writing of the manuscript, and in the decision to publish the results.

\section{References}

1. Rollins, N.C.; Bhandari, N.; Hajeebhoy, N.; Horton, S.; Lutter, C.K.; Martines, J.C.; Piwoz, E.G.; Richter, L.M.; Victora, C.G. Why invest, and what it will take to improve breastfeeding practices? Lancet 2016, 387, 491-504. [CrossRef]

2. Victora, C.G.; Bahl, R.; Barros, A.J.; França, G.V.; Horton, S.; Krasevec, J.; Murch, S.; Sankar, M.J.; Walker, N.; Rollins, N.C. Breastfeeding in the 21st century: Epidemiology, mechanisms, and lifelong effect. Lancet 2016, 387, 475-490. [CrossRef]

3. Kramer, M.S.; Kakuma, R. Optimal duration of exclusive breastfeeding. Cochrane Database Syst. Rev. 2012, 15, CD003517. [CrossRef] [PubMed]

4. Duijts, L.; Jaddoe, V.W.; Hofman, A.; Moll, H.A. Prolonged and exclusive breastfeeding reduces the risk of infectious diseases in infancy. Pediatrics 2010, 126, e18-e25. [CrossRef] [PubMed]

5. Quigley, M.A.; Kelly, Y.J.; Sacker, A. Breastfeeding and hospitalization for diarrheal and respiratoryinfection in the United Kingdom Millennium Cohort Study. Pediatrics 2007, 119, e837-e842. [CrossRef] [PubMed]

6. Klement, E.; Cohen, R.V.; Boxman, J.; Joseph, A.; Reif, S. Breastfeeding and risk of inflammatory bowel disease: A systematic review with meta-analysis. Am. J. Clin. Nutr. 2004, 80, 1342-1352. [CrossRef] [PubMed]

7. Akobeng, A.K.; Ramanan, A.V.; Buchan, I.; Heller, R.F. Effect of breast-feeding on risk of coeliac disease: A systematic review and meta-analysis of observational studies. Arch. Dis. Child. 2006, 91, 39-43. [CrossRef] [PubMed]

8. World Health Organisation. Evidence on the Long-Term Effects of Breastfeeding, Systematic Reviews and Meta-Analysis, Department of Child and Adolescent Health and Development (CAH). 2007. Available online: http:/ /apps.who.int/iris/bitstream/10665/43623/1/9789241595230_eng.pdf (accessed on 20 April 2018).

9. WHO Collaborative Study Team on the Role of Breastfeeding on the Prevention of Infant Mortality. Effect of breastfeeding on infant and child mortality due to infectious diseases in less developed countries: A pooled analysis. Lancet 2000, 355, 451-455.

10. Bachrach, V.R.; Schwarz, E.; Bachrach, L.R. Breastfeeding and the risk of hospitalization for respiratory disease in infancy: A meta-analysis. Arch. Pediatr. Adolesc. Med. 2003, 157, 237-243. [CrossRef] [PubMed]

11. Hauck, F.R.; Thompson, J.M.; Tanabe, K.O.; Moon, R.Y.; Vennemann, M.M. Breastfeeding and reduced risk of sudden infant death syndrome: A meta-analysis. Pediatrics 2011, 128, 103-110. [CrossRef] [PubMed]

12. Rothenbacher, D.; Weyermann, M.; Beermann, C.; Brenner, H. Breastfeeding, soluble CD14 concentration in breast milk and risk of atopic dermatitis and asthma in early childhood: Birth cohort study. Clin. Exp. Allergy 2005, 35, 1014-1021. [CrossRef] [PubMed]

13. Greer, F.R.; Sicherer, S.H.; Burks, A.W.; American Academy of Pediatrics Committee on Nutrition; American Academy of Pediatrics Section on Allergy and Immunology. Effects of early nutritional interventions on the development of atopic disease in infants and children: The role of maternal dietary restriction, breastfeeding, timing of introduction of complementary foods, and hydrolyzed formulas. Pediatrics 2008, 121, 183-191. [CrossRef] [PubMed] 
14. Horta, B.L.; Bahl, R.; Martines, J.C.; Victora, C.G. Evidence on the Long-Term Effects of Breastfeeding. Geneva: World Health Organization. 2007. Available online: http:/ /apps.who.int/iris/bitstream/handle/10665/ 43623/9789241595230_eng.pdf;jsessionid=96C96746137B09E506E1051FAAA5CDDC? sequence=1 (accessed on 23 April 2018).

15. Harder, T.; Bergmann, R.; Kallischnigg, G.; Plagemann, A. Duration of breastfeeding and risk of overweight: A meta-analysis. Am. J. Epidemiol. 2005, 162, 397-403. [CrossRef] [PubMed]

16. Arenz, S.; Ruckeri, R.; Koletzko, B.; von Kries, R. Breastfeeding and Childhood obesity: A systematic review. Int. J. Obes. Relat. Metab. Disord. 2004, 28, 1247-1256. [CrossRef] [PubMed]

17. Kramer, M.S.; Aboud, F.; Mironova, E.; Vanilovich, I.; Platt, R.W.; Matush, L.; Igumnov, S.; Fombonne, E.; Bogdanovich, N.; Ducruet, T.; et al. Breastfeeding and child cognitive development: New evidence from a large randomized trial. Arch. Gen. Psychiatry 2008, 65, 578-584. [CrossRef] [PubMed]

18. Heikkilä, K.; Sacker, A.; Kelly, Y.; Renfrew, M.J.; Quigley, M.A. Breastfeeding and child behaviour in the Millennium Cohort Study. Arch. Dis. Child. 2011, 96, 635-642. [CrossRef] [PubMed]

19. Quigley, M.A.; Hockley, C.; Carson, C.; Kelly, Y.; Renfrew, M.J.; Sacker, A. Breastfeeding is Associated with Improved Child Cognitive Development: A Population-Based Cohort Study. J. Pediatr. 2012, 160, $25-32$. [CrossRef] [PubMed]

20. Kramer, M.S.; Chalmers, B.; Hodnett, E.D.; Sevkovskaya, Z.; Dzikovich, I.; Shapiro, S.; Collet, J.P.; Vanilovich, I.; Mezen, I.; Ducruet, T.; et al. Promotion of Breastfeeding Intervention Trial (PROBIT). A randomized trial in the Republic of Belarus. JAMA 2001, 285, 413-420. [CrossRef] [PubMed]

21. Dewey, K.G.; Heinig, M.J.; Nommsen, L.A. Maternal weight-loss patterns during prolonged lactation. Am. J. Clin. Nutr. 1993, 58, 162-166. [CrossRef] [PubMed]

22. Short, R.V. Lactational infertility in family planning. Ann. Med. 1993, 25, 175-180. [CrossRef] [PubMed]

23. Collaborative Group on Hormonal Factors in Breast Cancer. Breast cancer and breastfeeding: Collaborative reanalysis of individual data from 47 epidemiological studies in 30 countries, including 50302 women with breast cancer and 96973 women without the disease. Lancet 2002, 360, 187-195.

24. Islami, F.; Liu, Y.; Jemal, A.; Zhou, J.; Weiderpass, E.; Colditz, G.; Boffetta, P.; Weiss, M. Breastfeeding and breast cancer risk by receptor status-A systematic review and meta-analysis. Ann. Oncol. 2015, 26, 2398-2407. [CrossRef] [PubMed]

25. Tung, K.H.; Goodman, M.T.; Wu, A.H.; McDuffie, K.; Wilkens, L.R.; Kolonel, L.N.; Nomura, A.M.; Terada, K.Y.; Carney, M.E.; Sobin, L.H. Reproductive factors and epithelial ovarian cancer risk by histologic type:a multiethnic case-control study. Am. J. Epidemiol. 2003, 158, 629-638. [CrossRef] [PubMed]

26. Rosenblatt, K.A.; Thomas, D.B. Lactation and the risk of epithelial ovarian cancer-The WHO Collaborative Study of Neoplasia and Steroid Contraceptives. Int. J. Epidemiol. 1993, 22, 192-197. [CrossRef] [PubMed]

27. Bjornerem, A.; Ahmed, L.A.; Jorgensen, L.; Stormer, J.; Joakimsen, R.M. Breastfeeding protects against hip fracture in postmenopausal women: The Tromso study. J. Bone Miner. Res. 2011, 26, 2843-2850. [CrossRef] [PubMed]

28. Stafford, M.; Kuh, D.L.; Gale, C.R.; Mishra, G.; Richards, M. Parent-child relationships and offspring's positive mental wellbeing from adolescence to early older age. J. Posit. Psychol. 2016, 11, 326-337. [CrossRef] [PubMed]

29. Puckering, C.; Allely, C.S.; Doolin, O.; Purves, D.; McConnachie, A.; Johnson, P.C.; Marwick, H.; Heron, J.; Golding, J.; Gillberg, C.; et al. Association between parent-infant interactions in infancy and disruptive behaviour disorders at age seven: A nested, case-control ALSPAC study. BMC Pediatr. 2014, 14, 223. [CrossRef] [PubMed]

30. Brown, A.; Rance, J.; Bennett, P. Understanding the relationship between breastfeeding and postnatal depression: The role of pain and physical difficulties. J. Adv. Nurs. 2016, 72, 273-282. [CrossRef] [PubMed]

31. World Health Organisation. Up to What Age Can a Baby Stay Well Nourished by Just Being Breastfed? 2015. Available online: http://www.who.int/features/qa/21/en/ (accessed on 24 April 2018).

32. K1lc1, H.; Çoban, A. The correlation between breastfeeding success in the early postpartum period and the perception of self-efficacy in breastfeeding and breast problems in the late postpartum. Breastfeed. Med. 2016, 11, 188-195. [CrossRef] [PubMed]

33. Forster, D.A.; Johns, H.M.; McLachlan, H.L.; Moorhead, A.M.; McEgan, K.M.; Amir, L.H. Feeding infants directly at the breast during the postpartum hospital stay is associated with increased breastfeeding at 6 months postpartum: A prospective cohort study. BMJ Open 2015, 5, e007512. [CrossRef] [PubMed] 
34. National Infant Feeding Survey 2010. Chapter 2: Incidence Prevalence and Duration of Breastfeeding. 2012. Available online: http:/ / www.hscic.gov.uk/catalogue/PUB08694/ifs-uk-2010-chap2-inc-prev-dur. pdf (accessed on 6 May 2016).

35. O'Connor, M.; Allen, J.; Kelly, J.; Gao, Y.; Kildea, S. Predictors of breastfeeding exclusivity and duration in a hospital without Baby Friendly Hospital Initiative accreditation: A prospective cohort study. Women Birth 2017. [CrossRef] [PubMed]

36. Chantry, C.J.; Dewey, K.G.; Peerson, J.M.; Wagner, E.A.; Nommsen-Rivers, L.A. In-hospital formula use increases early breastfeeding cessation among first-time mothers intending to exclusively breastfeed. J. Pediatr. 2014, 164, 1339-1345. [CrossRef] [PubMed]

37. Gaufin, T.; Tobin, N.H.; Aldrovandi, G.M. The importance of the microbiome in pediatrics and pediatric infectious diseases. Curr. Opin. Pediatr. 2018, 30, 117-124. [CrossRef] [PubMed]

38. Bentley, J.P.; Nassar, N.; Porter, M.; de Vroome, M.; Yip, E.; Ampt, A.J. Formula supplementation in hospital and subsequent feeding at discharge among women who intended to exclusively breastfeed: An administrative data retrospective cohort study. Birth 2017, 44, 352-362. [CrossRef] [PubMed]

39. Nguyen, T.; Dennison, B.A.; Fan, W.; Xu, C.; Birkhead, G.S. Variation in Formula Supplementation of Breastfed Newborn Infants in New York Hospitals. Pediatrics 2017, 140, E20170142. [CrossRef] [PubMed]

40. Hunter, L.; Magill-Cuerden, J.; McCourt, C. 'Oh no, no, no, we haven't got time to be doing that': Challenges encountered introducing a breast-feeding support intervention on a postnatal ward. Midwifery 2015, 31, 798-804. [CrossRef] [PubMed]

41. Cantrill, R.; Creedy, D.; Cooke, M. Midwives' knowledge of newborn feeding ability and reported practice managing the first breastfeed. Breastfeed. Rev. 2004, 12, 25-33. [PubMed]

42. Darwent, K.L.; Kempenaar, L.E. A comparison of breastfeeding women's, peer supporters' and student midwives' breastfeeding knowledge and attitudes. Nurse Educ. Pract. 2014, 14, 319-325. [CrossRef] [PubMed]

43. Marks, D.; O'Connor, R. Breastfeeding support and promotion: The health professional's perspective. J. Health Visit. 2015, 3, 38-46. [CrossRef]

44. Marks, D.; O'Connor, R. Health professionals' attitudes towards the promotion of breastfeeding. Br. J. Midwifery 2015, 23, 50-58. [CrossRef]

45. World Health Organization. Acceptable Medical Reasons for Use of Breast Milk Substitutes; WHO: Geneva, Switzerland, 2009. Available online: http://apps.who.int/iris/bitstream/10665/69938/1/WHO_FCH_ CAH_09.01_eng.pdf (accessed on 4 May 2016).

46. UNICEF. Tools and Forms to Help Support Baby Friendly. Available online: https://www.unicef.org.uk/ babyfriendly / baby-friendly-resources / guidance-for-health-professionals / tools-and-forms-for-healthprofessionals/ (accessed on 4 May 2016).

47. Office of National Statistics-Ethnicity Groups. Available online: https://www.

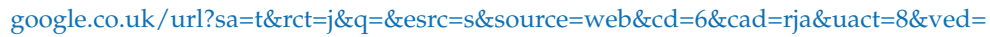
0ahUKEwjxqNqfxfzWAhXBPBoKHXa3AVQQFgg0MAU\&url=https\%3A\%2F\%2Fwww.ons.gov.uk\% 2Fons $\% 2$ Fguide-method $\% 2$ Fharmonisation $\% 2$ Fprimary-set-of-harmonised-concepts-and-questions $\%$ 2Fethnic-group.pdf\&usg=AOvVaw3O-S-j3HqaDLWP0_wT-jhs (accessed on 4 May 2016).

48. Tender, J.A.; Janakiram, J.; Arce, E.; Mason, R.; Jordan, T.; Marsh, J.; Kin, S.; He, J.; Moon, R.Y. Reasons for in-hospital formula supplementation of breastfed infants from low-income families. J. Hum. Lact. 2009, 25, 11-17. [CrossRef] [PubMed]

49. Good Food for London. 2015. Available online: http://www.sustainweb.org/londonfoodlink/ goodfoodforlondon2015/ (accessed on 6 May 2016).

50. National Maternity and Perinatal Audit 2017. Available online: http://www.maternityaudit.org.uk/ downloads/RCOG\%20NMPA\%20Clinical\%20Report(web).pdf (accessed on 13 February 2018).

51. Flacking, R.; Nyqvist, K.H.; Ewald, U. Effects of socioeconomic status on breastfeeding duration in mothers of preterm and term infants. Eur. J. Public Health 2007, 17, 579-584. [CrossRef] [PubMed]

52. Moore, E.R.; Bergman, N.; Anderson, G.C.; Medley, N. Early skin-to-skin contact for mothers and their healthy newborn infants. Cochrane Database Syst. Rev. 2016, 11, CD003519. [CrossRef] [PubMed]

53. DaMota, K.; Bañuelos, J.; Goldbronn, J.; Vera-Beccera, L.E.; Heinig, M.J. Maternal request for in-hospital supplementation of healthy breastfed infants among low-income women. J. Hum. Lact. 2012, 28, 476-482. [CrossRef] [PubMed] 
54. Larsen, J.S.; Hall, E.O.; Aagaard, H. Shattered expectations: When mothers' confidence in breastfeeding is undermined-a metasynthesis. Scand. J. Caring Sci. 2008, 22, 653-661. [CrossRef] [PubMed]

55. Bailey, C.; Pain, R.H.; Aarvold, J.E. A 'give it a go' breast-feeding culture and early cessation among low-income mothers. Midwifery 2004, 20, 240-250. [CrossRef] [PubMed]

56. Trickey, H.; Newburn, M. Goals, dilemmas and assumptions in infant feeding education and support. Applying theory of constraints thinking tools to develop new priorities for action. Matern. Child Nutr. 2014, 10, 72-91. [CrossRef] [PubMed]

57. Lumbiganon, P.; Martis, R.; Laopaiboon, M.; Festin, M.R.; Ho, J.J.; Hakimi, M. Antenatal breastfeeding education for increasing breastfeeding duration. Cochrane Database Syst. Rev. 2012, 9, CD006425. [CrossRef]

58. Wong, K.L.; Fong, D.Y.; Lee, I.L.; Chu, S.; Tarrant, M. Antenatal education to increase exclusive breastfeeding: A randomized controlled trial. Obstet. Gynecol. 2014, 124, 961-968. [CrossRef] [PubMed]

59. Lavender, T.; Baker, L.; Smyth, R.; Collins, S.; Spofforth, A.; Dey, P. Breastfeeding expectations versus reality: A cluster randomised controlled trial. BJOG 2005, 112, 1047-1053. [CrossRef] [PubMed]

60. Hauck, Y.L.; Irurita, V.F. Incompatible expectations: The dilemma of breastfeeding mothers. Health Care Women Int. 2003, 24, 62-78. [CrossRef] [PubMed]

61. Fox, R.; McMullen, S.; Newburn, M. UK women's experiences of breastfeeding and additional breastfeeding support: A qualitative study of Baby Café services. BMC Pregnancy Childbirth 2015, 15, 147. [CrossRef] [PubMed]

62. Kmietowicz, Z. Staff shortages and lack of data continue to blight England's NHS and care regulator. BMJ 2015, 351, h4000. [CrossRef] [PubMed]

63. Triggle, N. Staff shortage puts neonatal services under strain. Nurs. Child. Young People 2015, 27, 7. [CrossRef] [PubMed]

64. Furber, C.M.; Thomson, A.M. Midwives in the UK: An exploratory study of providing newborn feeding support for postpartum mothers in the hospital. J. Midwifery Womens Health 2007, 52, 142-147. [CrossRef] [PubMed]

65. Ockleford, E.M.; Berryman, J.C.; Hsu, R. Postnatal care: What new mothers say. Br. J. Midwifery 2004, 12, 166-170. [CrossRef]

66. Sharp, D.; Entwistle, F. Why women stop breastfeeding in the early days. Pract. Midwife 2015, 18, 30-33. [PubMed]

67. Oakley, L.L.; Henderson, J.; Redshaw, M.; Quigley, M.A. The role of support and other factors in early breastfeeding cessation: An analysis of data from a maternity survey in England. BMC Pregnancy Childbirth 2014, 14, 88. [CrossRef] [PubMed]

68. Bowers, J.; Cheyne, H. Reducing the length of postnatal hospital stay: Implications for cost and quality of care. BMC Health Serv. Res. 2016, 16, 16. [CrossRef] [PubMed]

69. UNICEF/BFI Supplementation Guidance: Maternity Standard 4. March 2016. Available online: https://www.unicef.org.uk/babyfriendly/wp-content/uploads/sites/2/2016/10/Supplementationguidance.pdf (accessed on 17 April 2018).

70. Forster, D.A.; McLachlan, H.L.; Yelland, J.; Rayner, J.; Lumley, J.; Davey, M.A. Staffing in postnatal units: Is it adequate for the provision of quality care? Staff perspectives from a state-widereview of postnatal care in Victoria, Australia. BMC Health Serv. Res. 2006, 6, 83. [CrossRef] [PubMed]

71. Odom, E.C.; Li, R.; Scanlon, K.S.; Perrine, C.G.; Grummer-Strawn, L. Association of family and health care provider opinion on infant feeding with mother's breastfeeding decision. J. Acad. Nutr. Diet. 2014, 114, 1203-1207. [CrossRef] [PubMed]

72. Swanson, V.; Power, K.G. Initiation and continuation of breastfeeding: Theory of planned behaviour. J. Adv. Nurs. 2005, 50, 272-282. [CrossRef] [PubMed]

73. Radzyminski, S.; Callister, L.C. Mother's beliefs, attitudes, and decision making related to infant feeding choices. J. Perinat. Educ. 2016, 25, 18-28. [CrossRef] [PubMed]

74. Taylor, A.M.; Hutchings, M. Using video narratives of women's lived experience of breastfeeding in midwifery education: Exploring its impact on midwives' attitudes to breastfeeding. Matern. Child Nutr. 2012, 8, 88-102. [CrossRef] [PubMed]

75. DiGirolamo, A.M.; Grummer-Strawn, L.M.; Fein, S.B. Do perceived attitudes of physicians and hospital staff affect breastfeeding decisions? Birth 2003, 30, 94-100. [CrossRef] [PubMed] 
76. Creedy, D.K.; Cantrill, R.M.; Cooke, M. Assessing midwives' breastfeeding knowledge: Properties of the Newborn Feeding Ability questionnaire and Breastfeeding Initiation Practices scale. Int. Breastfeed. J. 2008, 3, 7. [CrossRef] [PubMed]

77. Cantrill, R.M.; Creedy, D.K.; Cooke, M. An Australian study of midwives' breast-feeding knowledge. Midwifery 2003, 19, 310-317. [CrossRef]

78. Pierro, J.; Abulaimoun, B.; Roth, P.; Blau, J. Factors associated with supplemental formula feeding of breastfeeding infants during postpartum hospital stay. Breastfeed. Med. 2016, 11, 196-202. [CrossRef] [PubMed]

79. Atchan, M.; Davis, D.; Foureur, M. Applying a knowledge translation model to the uptake of the Baby Friendly Health Initiative in the Australian health care system. Women Birth. 2014, 27, 79-85. [CrossRef] [PubMed]

80. Glasziou, P.; Haynes, B. The paths from research to improved health outcomes. Evid. Based Nurs. 2005, 8, 36-38. [CrossRef] [PubMed]

81. Furber, C.M.; Thomson, A.M. The emotions of integrating breastfeeding knowledge into practice for English midwives: A qualitative study. Int. J. Nurs. Stud. 2008, 45, 286-297. [CrossRef] [PubMed]

82. Crano, W.D.; Prislin, R. (Eds.) Attitudes and Attitude Change; Psychology Press, Taylor and Francis Group: New York, NY, USA, 2008; p. 11. Available online: https://books.google.co.uk/books?hl= en\&lr=\&id=HpF5AgAAQBAJ\&oi=fnd\&pg=PP1\&dq=change+attitudes+\&ots=dyeEeCvkgp\&sig=6rxaq rrILyrOBbwYRkpRw4izOs\#v=onepage\&q=behavior\&f=false (accessed on 14 May 2016).

83. Schwarz, J.; Gaito, P.; Lennick, D. That's the way we (used to) do things around here. Strateg. Bus. 2011, 62, 45-53.

84. Fazio, R.H.; Zanna, M.P.; Cooper, J. Direct experience and attitude-behavior consistency: An information processing analysis. Pers. Soc. Psychol. Bull. 1978, 4, 48-51. [CrossRef]

85. Russell, K.; Walsh, D. Can the use of behavioural intervention studies support change in professional practice behaviours? Evid. Based Midwifery 2009, 7, 54-59.

86. Salvador, A.; Dumas, L.; Davies, B.; Emard, M.J.; Lortie, K. A dozen strategies along the ten steps Baby-Friendly Initiative journey. Healthc. Q. 2016, 18, 80-86. [CrossRef] [PubMed]

87. Rogers, M. Baby Friendly: A way to accreditation. Part 2. Br. J. Midwifery 2003, 11, 657-662. [CrossRef]

88. Reddin, E.; Pincombe, J.; Darbyshire, P. Passive resistance: Early experiences of midwifery students/graduates and the Baby Friendly Health Initiative 10 steps to successful breastfeeding. Women Birth 2007, 20, 71-76. [CrossRef] [PubMed]

89. Levin, K.A. Study design III: Cross-sectional studies. Evid. Based Dent. 2006, 7, 24-25. [CrossRef] [PubMed]

90. Gomez-Pomar, E.; Blubaugh, R. The Baby Friendly Hospital Initiative and the ten steps for successful breastfeeding. A critical review of the literature. J. Perinatol. 2018, 7. [CrossRef] [PubMed]

91. Howe-Heyman, A.; Lutenbacher, M. The Baby-Friendly Hospital Initiative as an Intervention to Improve Breastfeeding Rates: A Review of the Literature. J. Midwifery Womens Health 2016, 61, 77-102. [CrossRef] [PubMed]

(C) 2018 by the authors. Licensee MDPI, Basel, Switzerland. This article is an open access article distributed under the terms and conditions of the Creative Commons Attribution (CC BY) license (http://creativecommons.org/licenses/by/4.0/). 
Article

\title{
Factors Associated with Maternal Wellbeing at Four Months Post-Partum in Ireland
}

\author{
Annemarie E. Bennett ${ }^{1, *}$ and John M. Kearney ${ }^{2}$ \\ 1 Department of Clinical Medicine, Trinity Centre for Health Sciences, St. James' Hospital Campus, \\ Dublin 8, Ireland \\ 2 School of Biological Sciences, Dublin Institute of Technology, Kevin Street, Dublin 8, Ireland; \\ john.kearney@dit.ie \\ * Correspondence: abennet@tcd.ie; Tel.: +353-1-896-2100
}

Received: 4 March 2018; Accepted: 10 May 2018; Published: 14 May 2018

check for updates

\begin{abstract}
This study aimed to examine factors associated with maternal wellbeing at four months post-partum in the Irish context. Socio-demographic, health behaviour and infant feeding data were collected in pregnancy, at birth and at 17 weeks post-partum. Maternal distress, body image and resilience were measured at 17 weeks post-partum. Binary logistic regression predicted maternal distress and statistical significance was taken at $p<0.05$. One hundred and seventy-two women were followed-up in pregnancy, at birth and at 17 weeks post-partum. Three in five $(61.6 \%, n 106)$ initiated breastfeeding. At 17 weeks post-partum, $23.8 \%(n 41)$ were exclusively or partially breastfeeding and over a third $(36.0 \%, n 62)$ of all mothers were at risk of distress. In multivariate analyses, independent predictors of distress included: low maternal resilience ( $p<0.01$, odds ratio (OR): 7.22 (95\% confidence interval [CI]: 2.49-20.95)); unsatisfactory partner support ( $p=0.02$, OR: 3.89 (95\% CI: $1.20-12.65)$ ); older age ( $p=0.02$, OR: 1.11 (95\% CI: 1.02-1.21)); and breastfeeding ( $p=0.01$, OR: 2.89 (95\% CI: 1.29-6.47)). Routine assessment of emotional wellbeing and targeted interventions are needed to promote a more healthful transition to motherhood among women in Ireland.
\end{abstract}

Keywords: maternal wellbeing; maternal distress; post-partum distress; breastfeeding support; paternal role; partner support; infant; Ireland

\section{Introduction}

The transition to motherhood is associated with, and most often accompanied by, feelings of maternal joy. However, it is also a time of increased vulnerability and possible distress for women [1], as they process intense and potentially rapid shifts in their emotional state in the period shortly after giving birth [2-4]. The postpartum period presents many physiological and psychological challenges to women, such as interrupted sleep, readjustment within the parental relationship, and the need to quickly adapt to new routines and acquire new skills [1]. Challenges such as these can precipitate or amplify maternal distress, particularly if they are accompanied by a lack of social support, low self-esteem or dissatisfaction with the parenting relationship [5].

Maternal distress impairs daily functioning $[1,6]$ and increases the risk of post-partum depression and suboptimal behavioural and emotional development in an infant [6]. Given these possible consequences, it is critical to identify factors associated with maternal distress, in order to understand how it may be attenuated and how the healthy functioning of a mother and her infant may be safeguarded.

Compared to other European countries, Irish women are a singular population when breastfeeding practices are examined [7]. Irish women have the lowest breastfeeding initiation rate in Europe [8,9], rapid declines in rates of exclusive breastfeeding [10], and poor rates of breastfeeding duration beyond the first few weeks post-partum [9]. So extensive and resilient is our formula feeding culture [11], 
that government investment and policies over the past decade have proved ineffective in increasing breastfeeding rates amongst Irish mothers [7]. As such, it is clear that there are fundamental elements of the breastfeeding environment in Ireland which remain to be understood.

Breastfeeding is a unique component of the post-partum period, where its presence and absence both carry physical, psychological and socio-cultural implications for mothers [12,13]. Given the lack of research in the Irish context, this study aimed to obtain measures of maternal emotional wellbeing and to examine the associations between these measures, if any, with infant feeding outcomes.

\section{Materials and Methods}

Ethical approval for this prospective observational study was obtained from the Coombe Women and Infant's University Hospital and Dublin Institute of Technology.

Women were recruited by the lead author whilst waiting in community antenatal clinics in the Coombe Women and Infants University Hospital. Eligible women were those who: had a healthy singleton pregnancy; were at least 24 weeks pregnant; were available in hospital for follow-up after labour; and were willing to be contacted in the post-partum period for follow-up via a home visit. Written informed consent was obtained from all participants for all elements of data collection.

A questionnaire was administered in the clinic for pregnant participants to self-complete. This questionnaire collected socio-demographic and health behaviour data, to include: age; nationality; parity; education level; marital status; folic acid supplementation practices; and smoking and alcohol status. The questionnaire also included the validated Tilburg Pregnancy Distress Scale (TPDS) [14].

The TPDS [14] is a 16-item pregnancy-specific scale which measures maternal distress over the 7 days prior to its completion. The overall scale is comprised of two subscales. The negative affect subscale measures distress with respect to confinement, the post-partum period and general health. The partner involvement subscale measures distress with respect to perceived partner involvement during pregnancy. Participants rated on a four-point scale how often they felt as described by each item of the TPDS (i.e., very often; fairly often; now and then; or, rarely or never). Cut-off scores for distress were $>17$ for the overall scale, $>12$ for the negative affect subscale and $>7$ for the partner involvement subscale.

To reduce withdrawal rates and record infant feeding practices, participants were followed-up in hospital after giving birth. The lead author checked hospital records daily to identify those women who had consented to participate in the study and had given birth to a healthy term infant in the previous $24 \mathrm{~h}$. The lead author visited these women on the ward, re-introduced herself and asked them if they were still happy to be contacted at 17 weeks post-partum. The method of infant feeding initiated and the method of infant feeding on discharge from hospital were documented from each mother's medical record. Standard definitions were used to categorise infant feeding methods [15].

A home visit was arranged during the week in which infants turned 17 weeks of age. During the visit, mothers completed quantitative questions on infant feeding practices and maternal health behaviours. Maternal wellbeing was also assessed using the Mother and Baby Interaction Scale (MABISC) [16], an eight-item Body Shape Questionnaire (BSQ) [17] and the 14-item Resilience Scale [18].

The ten-item MABISC assesses maternal distress and potentially suboptimal mother-infant bonding over the month prior to its completion [16]. Scale items assess worry over bonding, infant caretaking and routine, and separation from the infant. Participants used a five-point scale to rate how often they felt as described by each item (i.e., always; most of the time; occasionally; not often; never). Cut-off scores were $\leq 7$ for no distress, $8-11$ for at risk of distress and $\geq 12$ for a high probability of distress. Given the sample size available for multivariate analysis, participants were assigned to the category of no distress $(\leq 7)$ or the aggregated category of at risk of, or high probability of, distress $(\geq 8)$.

The eight-item BSQ (version $8 \mathrm{~b}$ ) measures body shape concern over the 7 days prior to its completion [17]. Participants rated on a six-point scale how often they felt as described by each item of the scale (i.e., always; very often; often; sometimes; rarely; never). Cut-off scores for body shape concern were $<19$ for no concern, 19-25 for mild concern, 26-33 for moderate concern and $>33$ for 
marked concern. Given the sample size for analysis, participants were assigned to the category of no concern $(<19)$ or the aggregated category of mild, moderate or marked concern $(\geq 19)$.

The 14-item Resilience Scale measures resilience, confidence and ability to persevere [18]. Resilience refers to an individual's 'emotional stamina' and their ability to adapt in the face of challenges. Participants rated their agreement with each item on a seven-point scale, where $1=$ strongly disagree and $7=$ strongly agree. The cut-off scores used in this study were $\leq 73$ for low resilience and $\geq 74$ for moderate to high resilience.

IBM SPSS for Windows, version 22 (IBM, New York, NY, USA) was used for analysis. Normally distributed data were summarised numerically using the mean and standard deviation (SD). Non-normally distributed data were summarised numerically using the median and interquartile range (IQR). To identify factors associated with maternal distress at 17 weeks post-partum, univariate and multivariate analyses were conducted. Associations with continuous variables were determined using Independent Samples $t$-tests, and associations with categorical variables were assessed using $2 \times 2$ cross-tabulations, where the Chi-squared statistics test assessed statistical significance. Variables which were significantly associated with maternal distress in these univariate analyses were included in multivariate analyses. Binary logistic regression was used to predict maternal distress. The Forced Entry Method was used, whereby all predictor variables were tested in one block to assess their predictive ability whilst controlling for the effects of other predictors in the model. Statistical significance was taken at $p<0.05$.

\section{Results}

Of the 270 women recruited in pregnancy, 233 were eligible for follow-up in hospital after giving birth ( $n 8$ infants admitted to intensive care and $n 29$ mothers availed of the early discharge service). Of the 233 eligible mothers, 172 (73.8\% follow-up rate) consented to follow-up at 17 weeks post-partum (Table 1). Aside from the gestational age of the infant, there were no significant differences between mothers who did and did not consent to follow-up at 17 weeks post-partum.

Table 1. Socio-demographic and health behaviour characteristics of 172 participating women.

\begin{tabular}{|c|c|c|}
\hline & \multicolumn{2}{|c|}{ Mean \pm SD } \\
\hline \multirow[t]{2}{*}{ Age on Delivery (years) } & \multicolumn{2}{|c|}{$32.0 \pm 4.8$} \\
\hline & $n$ & $\%$ \\
\hline \multicolumn{3}{|l|}{ Nationality } \\
\hline Irish & 169 & 98.3 \\
\hline British & 3 & 1.7 \\
\hline \multicolumn{3}{|l|}{ Highest education level } \\
\hline Third level (university) & 111 & 64.6 \\
\hline Vocational qualification & 19 & 11 \\
\hline Second level (secondary school) & 42 & 24.4 \\
\hline \multicolumn{3}{|l|}{ Marital status } \\
\hline Married or cohabiting & 151 & 87.8 \\
\hline Single & 21 & 12.2 \\
\hline \multicolumn{3}{|l|}{ Health insurance } \\
\hline Semi-private & 57 & 33.1 \\
\hline Public & 115 & 66.9 \\
\hline \multicolumn{3}{|l|}{ Planned pregnancy } \\
\hline Yes, planned & 127 & 73.8 \\
\hline No, unplanned & 45 & 26.2 \\
\hline \multicolumn{3}{|l|}{ Body mass index } \\
\hline Healthy & 83 & 48.3 \\
\hline Overweight & 68 & 39.5 \\
\hline Obese & 21 & 12.2 \\
\hline
\end{tabular}

SD: Standard deviation. 
Over half $(56.4 \%, n 97)$ of this sample of 172 women were multiparous. A quarter $(25.0 \%, n 43)$ smoked until their pregnancy was confirmed, $7.6 \%(n 13)$ smoked all throughout pregnancy, and $22.1 \%$ (n38) consumed alcohol during their pregnancy. Only a third $(33.1 \%, n 57)$ supplemented with folic acid in line with recommendations [19].

\subsection{Milk-Feeding Practices}

Three in five $(61.6 \%, n 106)$ mothers initiated breastfeeding upon the birth of their infant. The median length of stay in hospital was $48.2(37.8) \mathrm{h}$, and by discharge, the proportion of women exclusively breastfeeding had decreased to $37.2 \%(n 64)$. A further $15.7 \%(n 27)$ were combination feeding, and almost half $(47.1 \%, n 81)$ were solely formula feeding upon discharge.

Of those who initiated breastfeeding $(n 106)$, the majority $(86.8 \%, n 92)$ reported that their partner was completely supportive of their decision to breastfeed. One in eight $(12.3 \%, n 13)$ reported that their partner was mostly supportive and one mother $(0.9 \%)$ reported that her partner was not supportive of her decision to breastfeed. Less than half $(44.3 \%, n 47)$ reported putting specific breastfeeding supports in place prior to the birth of their infant (e.g., making contact with local breastfeeding support groups).

By 17 weeks post-partum, the proportion of mothers exclusively breastfeeding had decreased to $15.1 \%(n 26)$, with a further $8.7 \%(n 15)$ combination feeding their infant. Over three-quarters $(76.2 \%$, $n 131$ ) were solely formula-feeding their infant at this time.

\subsection{Maternal Wellbeing in Pregnancy and at 17 Weeks Post-Partum}

A quarter $(25.0 \%, n 43)$ of the 172 women followed-up in the post-partum period had met or exceeded the cut-off [14] for distress in pregnancy (Table 2). Over a quarter $(27.9 \%, n 48)$ were distressed according to the negative affect subscale of the TPDS, and one in ten $(9.9 \%, n 17)$ were distressed on the partner involvement subscale.

Table 2. Distress in pregnancy and at 17 weeks post-partum in a sample of 172 women giving birth in Ireland.

\begin{tabular}{lcc}
\hline & $n$ & $\%$ \\
\hline Distress in pregnancy & & \\
\hline Tilburg Pregnancy Distress Scale (TPDS) & & \\
$\quad$ Significant distress & 43 & 25 \\
$\quad$ No significant distress & 129 & 75 \\
\hline Negative affect subscale of TPDS & & \\
$\quad$ Significant distress & 48 & 27.9 \\
$\quad$ No significant distress & 124 & 72.1 \\
\hline Partner involvement subscale of TPDS & & \\
$\quad$ Significant distress & 17 & 9.9 \\
$\quad$ No significant distress & 155 & 90.1 \\
\hline Distress at 17 weeks post-partum & & \\
\hline Mother and Baby Interaction scale & & \\
$\quad$ High probability of distress & 14 & 8.1 \\
$\quad$ At risk of distress & 48 & 27.9 \\
$\quad$ No distress & 110 & 64 \\
\hline
\end{tabular}

At 17 weeks post-partum, almost two-thirds $(64.0 \%, n 110)$ were coping well and not distressed (Table 2) according to the scoring of the MABISC [16]. Over a quarter $(27.9 \%, n 48)$ were at risk of distress and one in twelve $(8.1 \%, n 14)$ met cut-offs for a high probability of distress. Of note, different scales were used to determine the prevalence of distress at each time point.

Over half $(52.9 \%, n 91)$ of mothers had no body shape concern at 17 weeks post-partum. A quarter $(26.2 \%, n 45)$ had mild body shape concern and $14.0 \%(n 24)$ and $7.0 \%(n 12)$ had moderate and marked 
body shape concern, respectively. At 17 weeks post-partum, 13.3\% (n23) of mothers had low levels of resilience and $86.6 \%(n 149)$ had high levels of resilience.

\subsection{Factors Associated with Maternal Wellbeing at 17 Weeks Post-Partum}

The characteristics of women who experienced no distress at 17 weeks post-partum $(64.0 \%, n 110)$ were compared with those of mothers experiencing some degree (high probability/at risk) of distress at this time $(36.0 \%, n 62)$ (Table 3$)$. From these univariate analyses, distress at 17 weeks post-partum was more likely if a mother was: older $(p<0.01)$; multiparous $(p=0.02)$; breastfeeding $(p<0.01)$; or distressed by her partner's involvement (or lack thereof) in pregnancy $(p=0.02)$. Distress was also more likely if a mother had low resilience $(p<0.01)$.

Table 3. Comparison of maternal characteristics associated with distress at 4 months post-partum.

\begin{tabular}{|c|c|c|c|c|c|}
\hline \multirow[b]{2}{*}{ Maternal age at delivery (years) } & \multicolumn{2}{|c|}{$\begin{array}{l}\text { Significant Distress at } 4 \\
\text { Months Post-Partum (n62) }\end{array}$} & \multicolumn{2}{|c|}{$\begin{array}{l}\text { No Significant Distress at } 4 \\
\text { Months Post-Partum }(n 110)\end{array}$} & \multirow{2}{*}{$\begin{array}{c}p \text {-Value * } \\
<0.01 \ddagger\end{array}$} \\
\hline & $\begin{array}{c}n \\
62\end{array}$ & $\begin{array}{c}\text { Mean } \pm \mathrm{SD} \\
33.8 \pm 4.2\end{array}$ & $\begin{array}{c}n \\
110\end{array}$ & $\begin{array}{c}\text { Mean } \pm \mathrm{SD} \\
31.0 \pm 4.9\end{array}$ & \\
\hline & $n$ & $\%$ & $n$ & $\%$ & \\
\hline \multicolumn{6}{|l|}{ Maternal education } \\
\hline Third level education & 45 & 72.6 & 66 & 60 & $0.14+$ \\
\hline No third level education & 17 & 27.4 & 44 & 40 & \\
\hline Unplanned pregnancy & 18 & 29 & 27 & 24.5 & $0.64+$ \\
\hline No partner involved in pregnancy & 3 & 4.8 & 2 & 1.8 & $0.26+$ \\
\hline \multicolumn{6}{|l|}{ Parity } \\
\hline Nulliparous & 20 & 32.3 & 55 & 50 & $0.02+$ \\
\hline Multiparous & 42 & 67.7 & 55 & 50 & \\
\hline Smoked in all three trimesters & 4 & 6.5 & 9 & 8.2 & $0.91+$ \\
\hline Consumed alcohol in pregnancy & 15 & 24.2 & 23 & 20.9 & $0.76+$ \\
\hline \multicolumn{6}{|l|}{ Maternal body mass index } \\
\hline$\leq 24.9 \mathrm{~kg} / \mathrm{m}^{2}$ & 32 & 51.6 & 51 & 46.4 & $0.62+$ \\
\hline$\geq 25.0 \mathrm{~kg} / \mathrm{m}^{2}$ & 30 & 48.4 & 59 & 53.6 & \\
\hline \multicolumn{6}{|l|}{ Significantly distressed on TPDS } \\
\hline Overall scale & 20 & 37.7 & 23 & 41.8 & $0.14+$ \\
\hline Negative affect subscale & 22 & 41.5 & 26 & 47.3 & $0.13+$ \\
\hline Partner involvement subscale & 11 & 20.7 & 6 & 10.9 & $0.02+$ \\
\hline \multicolumn{6}{|l|}{ First milk } \\
\hline Breast milk & 43 & 69.4 & 69 & 62.7 & $0.48+$ \\
\hline Formula milk & 19 & 30.6 & 41 & 37.3 & \\
\hline Breastfeeding at 4 months post-partum & 24 & 38.7 & 17 & 15.5 & $<0.01+$ \\
\hline Has supports in place to breastfeed & 16 & 41 & 31 & 46.3 & $0.75+$ \\
\hline \multicolumn{6}{|l|}{ Post-partum body shape concern } \\
\hline No concern & 32 & 51.6 & 49 & 44.5 & $0.46+$ \\
\hline Mild, moderate or marked concern & 30 & 48.4 & 61 & 55.5 & \\
\hline \multicolumn{6}{|l|}{ Post-partum resilience category } \\
\hline High resilience & 46 & 74.2 & 103 & 93.6 & $<0.01+$ \\
\hline Low resilience & 16 & 25.8 & 7 & 6.4 & \\
\hline Not weaned before 17 weeks of age & 52 & 89.7 & 87 & 83.7 & $0.42+$ \\
\hline
\end{tabular}

Multivariate analyses were conducted to examine the characteristics independently associated with distress at 17 weeks post-partum (Table 4). All variables entered into the model were categorical, except for age, which was a continuous variable. As shown in the statistically significant adjusted model $\left(\chi^{2}(6, n 172)=43.15, p<0.01\right)$, four of the six independent variables included made a statistically significant contribution to the model. The strongest predictor of distress at 17 weeks post-partum was low resilience. Mothers with low resilience scores were over seven times more likely (Table 4) 
to be at risk of distress when compared with mothers with high resilience scores. Mothers were also significantly more likely to feel distressed at 17 weeks post-partum if they were older, breastfeeding at this time or had been distressed by partner involvement (or lack thereof) in pregnancy (Table 4).

Table 4. Binary logistic regression model examining factors associated with distress at 17 weeks post-partum in a sample of 172 mothers who gave birth in Ireland.

\begin{tabular}{|c|c|c|c|c|}
\hline & $n$ & OR & $95 \% \mathrm{CI}$ & $p$-Value * \\
\hline \multicolumn{5}{|c|}{ Third level education } \\
\hline Yes & 111 & 1.4 & $0.63-3.12$ & 0.41 \\
\hline No & 61 & 1 & Ref. & \\
\hline \multicolumn{5}{|l|}{ Parity } \\
\hline Primiparous & 75 & 1 & Ref. & 0.24 \\
\hline Multiparous & 97 & 1.6 & $0.74-3.47$ & \\
\hline \multicolumn{5}{|c|}{ Distressed by partner involvement in pregnancy $\dagger$} \\
\hline Yes & 17 & 3.89 & $1.20-12.65$ & 0.02 \\
\hline No & 155 & 1 & Ref. & \\
\hline \multicolumn{5}{|c|}{ Resilience level at 17 weeks post-partum } \\
\hline High & 149 & 1 & Ref. & $<0.01$ \\
\hline Low & 23 & 7.22 & $2.49-20.95$ & \\
\hline \multicolumn{5}{|c|}{ Breastfeeding at 17 weeks post-partum } \\
\hline Yes & 41 & 2.89 & $1.29-6.47$ & 0.01 \\
\hline No & 131 & 1 & Ref. & \\
\hline Maternal age & 172 & 1.11 & $1.02-1.21$ & 0.02 \\
\hline
\end{tabular}

Model summary
$\mathrm{R}^{2}=0.16$, Cox \& Snell $\mathrm{R}$ Square $=22.2$, Nagelkerke $\mathrm{R}$ Square $=30.4,72.7 \%$ predictive of variance

${ }^{*} p$-value significant at $<0.05$; OR: odds ratio; CI: confidence interval; + Distress measured by the Tilburg Pregnancy Distress Scale [14]

\section{Discussion}

Giving birth and caring for a new infant marks an important transition from one stage of a woman's life to another. Although this transition is often joyous, it is also a particularly challenging chapter of parenting which involves readjustment of roles and rapid acquisition of new skills. The unpredictability and upheaval associated with this time can, in turn, increase the risk of emotional distress amongst women. In this study, several factors were independently associated with post-partum distress (as measured by the MABISC), to include: low maternal resilience; suboptimal partner support; older age; and, breastfeeding. In particular, the association between breastfeeding and distress was somewhat unexpected, where breastfeeding women were almost 3 times more likely to experience distress at 17 weeks post-partum when compared with non-breastfeeding women.

Breastfeeding is often regarded as a challenging demand of motherhood [4], particularly in Ireland, which has a long-reigning formula feeding culture $[7,11,20]$ and widely reported inadequate breastfeeding support [21-23]. Women who breastfeed at 17 weeks post-partum are in the minority, with only one in five Irish women breastfeeding, exclusively or otherwise, at this time. If suboptimal breastfeeding rates [8] are to be subverted, a more comprehensive understanding of the feeding experiences of women who persevere with breastfeeding beyond the first few weeks post-partum must be obtained.

Meedya and colleagues [24] identified three factors which lend themselves to a breastfeeding experience in which maternal distress is less likely to occur, namely: a positive antenatal intention to breastfeed; strong social support; and high maternal resilience.

A positive antenatal intention to breastfeed has been strongly and consistently associated with breastfeeding initiation and duration $[9,24]$. Pregnancy is an important opportunity to prepare for breastfeeding, but less than half of the women who intended to breastfeed in this study put supports in place to increase the likelihood of a positive breastfeeding experience. Given that breastfeeding 
impacts on so many aspects of a woman's day-to-day life (i.e., her physical health, mental health, and social and cultural activities), women must be as prepared as possible for the challenges that breastfeeding may pose to these aspects of life after pregnancy $[12,13]$. Such preparation is needed to minimise any discrepancies between a woman's expectations of breastfeeding and the reality of breastfeeding [25].

When significant discrepancies between expectations and reality arise, disillusionment with breastfeeding can manifest, increasing the likelihood of maternal distress [26]. However, pregnancy presents an ample opportunity for health services to capitalise upon positive intentions to breastfeed. During their frequent points of contact with pregnant women, health professionals should help women to develop not only a positive intention to breastfeed-but an informed positive intention to breastfeed. Educating pregnant women on breastfeeding can empower them to identify suitable breastfeeding supports that they can independently access in a timely manner to help them to acquire the skill of breastfeeding upon their infant's arrival [12,27,28].

Supporting women to support themselves is especially important in light of the recent decision to cease all funding for, and activities related to, the Baby Friendly Health Initiative (BFHI) in Ireland [29]. The BFHI aims to introduce evidence-based and sustainable practices which promote and protect breastfeeding in maternity hospitals [30]. However, despite being in place for almost two decades in Ireland, the progress made by the National Committee of the BFHI was deemed insufficient by the relevant government agency in 2017, resulting in the controversial withdrawal of all funding for BFHI activities [29]. At its best, approximately one in three infants in Ireland were born in BFHI hospitals [30], so a deficit in breastfeeding supports in the immediate aftermath of birth has long existed here, and unfortunately has now only deepened with the absence of any formal implementation of an evidence-based breastfeeding support system.

Given the widespread lack of standardised support at hospital-level [29], social support is a particularly important element of a positive breastfeeding experience $[9,21,24,31]$. Specifically, the support from a woman's partner impacts on her feeding experience [24], and it is positive that in this study, most partners supported the decision to breastfeed. However, whilst partners may support this decision based on a superficial understanding of the immense value of breastfeeding, they often do not have an informed understanding of the process of breastfeeding and of the unique support it requires [21]. Several studies have reported that educating fathers on the fundamentals of breastfeeding results in improvements in rates of breastfeeding initiation and duration, and fewer technical difficulties with feeding [24,32-35]. Breastfeeding women have also reported that teaching fathers to provide specific types of practical (such as help with cooking, housework and caring for other children) and emotional support (such as praising their breastfeeding efforts and defending them from suggestions to formula-feed) enhances their breastfeeding experience and their ability to cope with breastfeeding challenges, should they arise [1]. Fathers can provide a continuity of care to a mother which no health professional can offer, and as such, educating fathers on how to best participate in the breastfeeding process can serve as a valuable means of promoting a more supportive and less isolating breastfeeding experience for a woman.

In addition to external support from health professionals and partners, it is also important that women view themselves as a source of support, in that they possess the resilience and equanimity to manage the responsibility of being the sole food provider for an infant [13]. Low maternal resilience was a separate independent predictor of maternal distress in this study, but it is also a factor which can impact on the breastfeeding experience.

Research has demonstrated the effectiveness of using mindfulness and cognitive behavioural therapy techniques to enhance maternal resilience and elicit clinically significant reductions in distress, depression and anxiety [6,36-38]. For example, mindfulness-based cognitive behavioural therapy has been shown to help pregnant women to foster acceptance, manage negative thoughts and deal with obstacles [38]. Helping pregnant women to strengthen their mental capacity to cope with the 
many elements that comprise the transition to motherhood could be a valuable means of preventing or attenuating distress which may arise during this time.

Perseverance with breastfeeding, despite its associated physical and emotional challenges, has been well-documented $[4,12,26]$. Although it may seem counterintuitive for a woman to persevere with an activity which may be a source of distress, it is likely that the commitment to, and value placed upon, breastfeeding by some women is so great that distress related to an aspect or aspects of breastfeeding is made tolerable or at least reduced. This commitment to breastfeeding may enable some women to continue to breastfeed despite any shortcomings in their support network or resilience [3,39]. However, while it is important to protect breastfeeding, equal emphasis should be placed on protecting the emotional wellbeing of women as they meet the demands of breastfeeding.

Finally, regardless of the infant feeding decision made, it is important that all women are appropriately supported throughout their transition to motherhood. Women with low resilience levels, suboptimal partner support, or who were older, were also more likely to feel distressed in this study. Many of the factors discussed here, support breastfeeding secondary to supporting a woman's overall emotional wellbeing [36,37,40]. A healthy emotional state underpins a woman's ability to carry out any element of her mothering role, breastfeeding or otherwise; breastfeeding is one element of motherhood, and just as it requires empowerment, education, resilience and support, so too do many other parts of this role. Routine assessment of emotional wellbeing and targeted interventions are needed to promote a more healthful transition to motherhood among all women who choose to have children.

Before drawing conclusions, the study strengths and limitations must be considered. The data presented were collected as part of a longitudinal observational study conducted in County Dublin and its surrounding counties. Strengths of the study include the lack of inter-observer variation and the use of validated instruments to measure different aspects of maternal wellbeing. The MABISC has been shown to have satisfactory internal consistency and convergent validity with the more widely used Edinburgh Post-partum Depression Scale (EPDS) and Post-partum Bonding Questionnaire (PBQ) [16]. Although the EPDS and PBQ are more helpful for the detection of the most serious maternal distress and rejection problems, the MABISC was suitable for use in this study due to its brevity and the inoffensive phrasing of items. However, when interpreting the findings, it is important to note that the observational study design precludes causal inferences, involves only women of Irish or British nationality, and is not nationally representative.

The relationship between breastfeeding and many sociodemographic and health behaviour characteristics has been examined in the Irish context $[7,8,11,23,41]$. Bearing the study limitations in mind, this is one of the first studies to provide insights into the relationship between emotional wellbeing and infant feeding practices amongst a cohort of mothers in Ireland.

\section{Conclusions}

Adequate physical, emotional and practical preparation for breastfeeding is paramount to its successful initiation and maintenance. Pregnant women planning to breastfeed should be empowered with practical and evidence-based information on feeding techniques and positioning, in addition to information on troubleshooting common breastfeeding challenges. All women should also be supported to attain and maintain a healthy body and frame of mind prior to giving birth. If this preparation among women is further bolstered by a well-prepared maternal support network, women can approach motherhood, whether it is for the first or subsequent time, in an informed manner which safeguards their wellbeing and that of their infant.

Author Contributions: A.E.B. was responsible for the study design, data collection, data analysis, drafting the manuscript and making amendments to the manuscript. J.M.K. was the study supervisor and was responsible for making amendments to the manuscript.

Acknowledgments: The authors wish to acknowledge the mothers who participated in this study; their time was greatly appreciated. The lead author was funded by a Dublin Institute of Technology Fiosraigh Scholarship. 
This scholarship was 50\% funded by Dublin Institute of Technology and 50\% funded by Danone Nutricia Early Life Nutrition. No funds were received to cover the costs of publishing in an open access journal.

Conflicts of Interest: The authors declare no conflict of interest. The commercial funder had no role in the design of the study; in the collection, analyses, and interpretation of data; in the writing of the manuscript, or in the decision to publish the results.

\section{References}

1. Coates, R.; Ayers, S.; de Visser, R. Women's experiences of postnatal distress: A qualitative study. BMC Pregnancy Childbirth 2014, 14, 359. [CrossRef] [PubMed]

2. Coates, R.; de Visser, R.; Ayers, S. Not identifying with postnatal depression: A qualitative study of women's postnatal symptoms of distress and need for support. J. Psychosom. Obstet. Gynaecol. 2015, 36, 114-121. [CrossRef] [PubMed]

3. Cooke, M.; Schmied, V.; Sheehan, A. An exploration of the relationship between postnatal distress and maternal role attainment, breast feeding problems and breast feeding cessation in Australia. Midwifery 2007, 23, 66-76. [CrossRef] [PubMed]

4. Thomson, G.; Ebisch-Burton, K.; Flacking, R. Shame if you do-Shame if you don't: Women's experiences of infant feeding. Matern. Child Nutr. 2015, 11, 33-46. [CrossRef] [PubMed]

5. Goyal, D.; Gay, C.; Lee, K. Fragmented maternal sleep is more strongly correlated with depressive symptoms than infant temperament at three months postpartum. Arch. Womens Ment. Health 2009, 12, 229-237. [CrossRef] [PubMed]

6. Goodman, J.H.; Guarino, A.; Chenausky, K.; Klein, L.; Prager, J.; Peterson, R.; Forget, A.; Freeman, M. CALM Pregnancy: Results of a pilot study of mindfulness-based cognitive therapy for perinatal anxiety. Arch. Womens Ment. Health 2014, 17, 373-387. [CrossRef] [PubMed]

7. Brick, A.; Nolan, A. Explaining the increase in breastfeeding at hospital discharge in Ireland, 2004-2010. Irish J. Med. Sci. 2014, 183, 333-339. [CrossRef] [PubMed]

8. Gallagher, L.; Begley, C.; Clarke, M. Determinants of breastfeeding initiation in Ireland. Irish J. Med. Sci. 2016, 185, 663-668. [CrossRef] [PubMed]

9. Tarrant, R.C.; Younger, K.M.; Sheridan-Pereira, M.; White, M.J.; Kearney, J.M. The prevalence and determinants of breast-feeding initiation and duration in a sample of women in Ireland. Public Health Nutr. 2010, 13, 760-770. [CrossRef] [PubMed]

10. Nolan, A.; Layte, R. The 'healthy immigrant effect': Breastfeeding behaviour in Ireland. Eur. J. Public Health 2015, 25, 626-631. [CrossRef] [PubMed]

11. Leahy-Warren, P.; Creedon, M.; O’Mahony, A.; Mulcahy, H. Normalising breastfeeding within a formula feeding culture: An Irish qualitative study. Women Birth 2017, 30, e103-e110. [CrossRef] [PubMed]

12. McInnes, R.J.; Chambers, J.A. Supporting breastfeeding mothers: Qualitative synthesis. J. Adv. Nurs. 2008, 62, 407-427. [CrossRef] [PubMed]

13. Perez-Blasco, J.; Viguer, P.; Rodrigo, M.F. Effects of a mindfulness-based intervention on psychological distress, well-being, and maternal self-efficacy in breast-feeding mothers: Results of a pilot study. Arch. Womens Ment. Health 2013, 16, 227-236. [CrossRef] [PubMed]

14. Pop, V.J.; Pommer, A.M.; Pop-Purceleanu, M.; Wijnen, H.A.; Bergink, V.; Pouwer, F. Development of the Tilburg Pregnancy Distress Scale: The TPDS. BMC Pregnancy Childbirth 2011, 11, 80. [CrossRef] [PubMed]

15. Kramer, M.S.; Kakuma, R. Optimal duration of exclusive breastfeeding. Cochrane Database Syst. Rev. 2012, 8, Cd003517. [CrossRef] [PubMed]

16. Hoivik, M.S.; Burkeland, N.A.; Linaker, O.M.; Berg-Nielsen, T.S. The Mother and Baby Interaction Scale: A valid broadband instrument for efficient screening of postpartum interaction? A preliminary validation in a Norwegian community sample. Scand. J. Caring Sci. 2013, 27, 733-739. [CrossRef] [PubMed]

17. Evans, C.; Dolan, B. Body Shape Questionnaire: Derivation of shortened "alternate forms". Int. J. Eat. Disord. 1993, 13, 315-321. [CrossRef]

18. Wagnild, G.M.; Young, H.M. Development and psychometric evaluation of the Resilience Scale. J. Nurs. Meas. 1993, 1, 165-178. [PubMed]

19. McKeating, A.; Farren, M.; Cawley, S.; Daly, N.; McCartney, D.; Turner, M.J. Maternal folic acid supplementation trends 2009-2013. Acta Obstet. Gynecol. Scand. 2015, 94, 727-733. [CrossRef] [PubMed] 
20. Carroll, M.; Gallagher, L.; Clarke, M.; Millar, S.; Begley, C. Artificial milk-feeding women's views of their feeding choice in Ireland. Midwifery 2015, 31, 640-646. [CrossRef] [PubMed]

21. Bennett, A.E.; McCartney, D.; Kearney, J.M. Views of fathers in Ireland on the experience and challenges of having a breast-feeding partner. Midwifery 2016, 40, 169-176. [CrossRef] [PubMed]

22. Shortt, E.; McGorrian, C.; Kelleher, C. A qualitative study of infant feeding decisions among low-income women in the Republic of Ireland. Midwifery 2013, 29, 453-460. [CrossRef] [PubMed]

23. Whelan, B.; Kearney, J.M. Breast-feeding support in Ireland: A qualitative study of health-care professionals' and women's views. Public Health Nutr. 2015, 18, 2274-2282. [CrossRef] [PubMed]

24. Meedya, S.; Fahy, K.; Kable, A. Factors that positively influence breastfeeding duration to 6 months: A literature review. Women Birth 2010, 23, 135-145. [CrossRef] [PubMed]

25. Salonen, A.H.; Kaunonen, M.; Astedt-Kurki, P.; Järvenpää, A.L.; Isoaho, H.; Tarkka, M.T. Parenting self-efficacy after childbirth. J. Adv. Nurs. 2009, 65, 2324-2336. [CrossRef] [PubMed]

26. O'Brien, M.; Buikstra, E.; Hegney, D. The influence of psychological factors on breastfeeding duration. J. Adv. Nurs. 2008, 63, 397-408. [CrossRef] [PubMed]

27. Fox, R.; McMullen, S.; Newburn, M. UK women's experiences of breastfeeding and additional breastfeeding support: A qualitative study of Baby Café services. BMC Pregnancy Childbirth 2015, 15, 147. [CrossRef] [PubMed]

28. Ingram, J.; Johnson, D.; Greenwood, R. Breastfeeding in Bristol: Teaching good positioning, and support from fathers and families. Midwifery 2002, 18, 87-101. [CrossRef] [PubMed]

29. National Committee of the Baby Friendly Health Initiative. The National Committee of the Baby Friendly Health Initiative in Ireland Announce It Is Ceasing Its Activities. Media Report. 2017. Available online: www.babyfriendly.ie/images/BFHI\%20cessation\%20statement $\% 20$ Sept $\% 2026 \% 202017$.pdf (accessed on 21 November 2017).

30. Institute of Public Health in Ireland. Review and Evaluation of "Breastfeeding in Ireland-A 5-Year Strategic Action Plan 2005-2010"; Institute of Public Health in Ireland: Dublin, Ireland, 2014; Available online: www.breastfeeding.ie/Uploads / files/Breastfeeding-in-Ireland-Review-and-Evaluation.pdf (accessed on 23 January 2018).

31. Thomson, G.; Balaam, M.C.; Hymers, K. Building social capital through breastfeeding peer support: Insights from an evaluation of a voluntary breastfeeding peer support service in North-West England. Int. Breastfeed. J. 2015, 10, 15. [CrossRef] [PubMed]

32. Lovera, D.; Sanderson, M.; Bogle, M.L.; Vela Acosta, M.S. Evaluation of a breastfeeding peer support program for fathers of Hispanic participants in a Texas special supplemental nutrition program for women, infants, and children. J. Am. Diet. Assoc. 2010, 110, 1696-1702. [CrossRef] [PubMed]

33. Pisacane, A.; Continisio, G.I.; Aldinucci, M.; D'Amora, S.; Continisio, P. A controlled trial of the father's role in breastfeeding promotion. Pediatrics 2005, 116, e494-e498. [CrossRef] [PubMed]

34. Tohotoa, J.; Maycock, B.; Hauck, Y.; Howat, P.; Burns, S.; Binns, C. Supporting mothers to breastfeed: The development and process evaluation of a father inclusive perinatal education support program in Perth, Western Australia. Health Promot. Int. 2011, 26, 351-361. [CrossRef] [PubMed]

35. Tohotoa, J.; Maycock, B.; Hauck, Y.L.; Howat, P.; Burns, S.; Binns, C.W. Dads make a difference: An exploratory study of paternal support for breastfeeding in Perth, Western Australia. Int. Breastfeed. J. 2009, 4, 15. [CrossRef] [PubMed]

36. Dimidjian, S.; Goodman, S.H.; Felder, J.N.; Gallop, R.; Brown, A.P.; Beck, A. An open trial of mindfulness-based cognitive therapy for the prevention of perinatal depressive relapse/recurrence. Arch. Womens Ment. Health 2015, 18, 85-94. [CrossRef] [PubMed]

37. Dimidjian, S.; Goodman, S.H.; Felder, J.N.; Gallop, R.; Brown, A.P.; Beck, A. Staying well during pregnancy and the postpartum: A pilot randomized trial of mindfulness-based cognitive therapy for the prevention of depressive relapse/recurrence. J. Consult. Clin. Psychol. 2016, 84, 134-145. [CrossRef] [PubMed]

38. Dunn, C.; Hanieh, E.; Roberts, R.; Powrie, R. Mindful pregnancy and childbirth: Effects of a mindfulness-based intervention on women's psychological distress and well-being in the perinatal period. Arch. Womens Ment. Health 2012, 15, 139-143. [CrossRef] [PubMed]

39. Schmied, V.; Sheehan, A.; Barclay, L. Contemporary breast-feeding policy and practice: Implications for midwives. Midwifery 2001, 17, 44-54. [CrossRef] [PubMed] 
40. Cruise, S.M.; Layte, R.; Stevenson, M.; O'Reilly, D. Prevalence and factors associated with depression and depression-related healthcare access in mothers of 9-month-old infants in the Republic of Ireland. Epidemiol. Psychiatr. Sci. 2017. [CrossRef] [PubMed]

41. Bennett, A.E.; Kearney, J.M. Predictors of vitamin D supplementation amongst infants in Ireland throughout the first year of life. J. Public Health 2018. [CrossRef]

(C) 2018 by the authors. Licensee MDPI, Basel, Switzerland. This article is an open access article distributed under the terms and conditions of the Creative Commons Attribution (CC BY) license (http:/ / creativecommons.org/licenses/by/4.0/). 
Article

\title{
Comparison of Human Milk Immunoglobulin Survival during Gastric Digestion between Preterm and Term Infants
}

\author{
Veronique Demers-Mathieu ${ }^{1}$, Mark A. Underwood ${ }^{2}$, Robert L. Beverly ${ }^{1}$, Søren D. Nielsen ${ }^{1}$ and \\ David C. Dallas ${ }^{1, *}$ \\ 1 Nutrition Program, School of Biological and Population Health Sciences, College of Public Health and \\ Human Sciences, Oregon State University, Corvallis, OR 97331, USA; \\ Veronique.Demers-Mathieu@oregonstate.edu (V.D.-M.); \\ beverlyr@oregonstate.edu (R.L.B.); sodn@food.au.dk (S.D.N.) \\ 2 Department of Pediatrics, University of California, Davis, Sacramento, CA 95817, USA; \\ munderwood@ucdavis.edu \\ * Correspondence: Dave.Dallas@oregonstate.edu; Tel.: +1-541-737-1751
}

Received: 6 April 2018; Accepted: 14 May 2018; Published: 17 May 2018

check for

\begin{abstract}
Human milk provides immunoglobulins (Igs) that supplement the passive immune system of neonates; however, the extent of survival of these Igs during gastric digestion and whether this differs between preterm and term infants remains unknown. Human milk, and infant gastric samples at $2 \mathrm{~h}$ post-ingestion were collected from 15 preterm (23-32 week gestational age (GA)) mother-infant pairs and from 8 term (38-40 week of GA) mother-infant pairs within 7-98 days postnatal age. Samples were analyzed via ELISA for concentration of total IgA (secretory $\operatorname{IgA}(\mathrm{SIgA}) / \operatorname{IgA})$, total secretory component (SC/SIgA/SIgM), total $\operatorname{IgM}(\mathrm{SIgM} / \operatorname{IgM})$, and $\operatorname{IgG}$ as well as peptidomics. Total IgA concentration decreased by $60 \%$ from human milk to the preterm infant stomach and decreased by $48 \%$ in the term infant stomach. Total IgM and IgG concentrations decreased by 33\% and $77 \%$, respectively, from human milk to the term infant stomach but were stable in the preterm infant stomach. Release of peptides from all Ig isotypes in the term infant stomach was higher than in the preterm stomach. Overall, the stability of human milk Igs during gastric digestion is higher in preterm infant than in term infants, which could be beneficial for assisting the preterm infants' immature immune system.
\end{abstract}

Keywords: passive immunity; antibodies; lactation; peptidomics; prematurity; proteolysis; breast milk

\section{Introduction}

Immunoglobulins (Igs) are important effectors of the adaptive immune system [1]. During the third trimester, the mother's placenta transports IgG to the fetus via a neonatal Fc receptor. These maternal IgG antibodies protect the infant during the first 6 months of postnatal age while the infant's own immune system is developing [2]. After birth, human milk provides another form of protection against pathogens for infants, as it contains an array of Igs, including IgA, secretory IgA (SIgA), IgM, secretory $\operatorname{IgM}(\mathrm{SIgM})$, and IgG [3,4]. Indeed, feeding mother's milk reduces risks of infectious diseases in the respiratory and gastrointestinal tract in infancy [5]. Though human milk provides different Ig isotypes, milk SIgA is thought to be the most important in the infant gut as it neutralizes bacterial and viral pathogens by binding to them, thus reducing their ability to interact with epithelial cells and infect [6,7]. The presence of SIgA in human milk temporarily replaces the normal intestinal SIgA secretion that is lacking in the infant until 4 weeks of postnatal age [8]. 
SIgA is the first line of defense in protecting the intestinal epithelium from pathogens by immune exclusion [9]. Though SIgA is the most well-known and abundant Ig of intestinal secretions [10], IgG and IgM are also secreted by plasma cells in neonatal intestinal mucosa [11,12]. IgG may have function in the gut: passive administration of virus-neutralizing $\operatorname{IgG}$ prevented mucosal immunodeficiency virus transmission from mother to infant in macaques [13], suggesting potential transmission of these IgG in the gut. However, the ability of IgG to bind to viruses to prevent attachment to the mucosal surface or trap pathogens in mucus appears to be inefficient compared with SIgA [13]. Human neonatal Fc receptor ( $\mathrm{FCRn}$ ) may be able to transport IgG (alone or bound to an antigen) across the intestinal epithelial barrier into the lamina propria [11]. Though IgM-secreting cells have been identified in the infant gut [12] and IgM was shown to be synthesized in amounts similar to IgA in pig small intestinal mucosal explants $[14,15]$, no study has determined the extent to which IgM plays a role in the adult or infant intestinal mucosal immune defense.

In order to function in immunoprotection in the gut, $\operatorname{IgA}$ and potentially the other Ig must resist proteolytic degradation and remain intact and able to bind to pathogens through the digestive system. Though the provision of milk Igs to the infant is known to reduce infection risk, the degree to which Igs survive in the gastrointestinal tract remains unknown. Few studies have investigated the stability of Igs in digestion. Two oral supplementation studies (in adults fed bovine colostrum SIgA/IgA, IgM and IgG [16] and in preterm infants fed serum IgA and IgG [17]) demonstrated that IgG and IgM survive intact to the stool, whereas SIgA/IgA does not. However, some studies have demonstrated that human milk-derived SIgA survived intact to the infant stool and urine $[8,18,19]$.

Our previous study demonstrated that preterm infants had lower gastric digestion capacity for human milk proteins than term infants [20]. This difference in digestion capacity could affect the survival of immunoglobulins. Our recent studies demonstrated that preterm infants partially degrade IgA but not IgG and IgM in the stomach [21,22]. Whether differences in preterm and term infant gastric digestion result in differences in Ig survival remain unknown. The aim of the present study was to determine whether preterm infant Ig survival in the stomach is higher than in term infants.

As preterm infants are born early and miss some of the placenta-fetal IgG transfer [23], produce less diverse of antibodies in their gut in comparison with term infants [24], and are at higher risk for bacterial [25] and viral infections [26] than term infants, the presence of Ig in human milk may be even more critically important to their health outcomes than for term infants. Therefore, milk-provided Ig may be even more critically important than for term infants. Increased survival of milk Igs in the preterm infant stomach due to lower protein digestion capacity could provide increased direct support of their naïve immune systems through immune exclusion.

\section{Materials and Methods}

\subsection{Participants and Sample Collection}

This study was approved by the Institutional Review Board of the University of California, Davis (UC Davis) and Oregon State University (OSU). Inclusion criteria included inpatient admission to the neonatal intensive care unit (NICU), an indwelling nasogastric or orogastric feeding tube and tolerance of full enteral feeding. Most of the enrolled infants required a feeding tube because of uncoordinated or immature capacity to suck and swallow. Exclusion criteria were anatomic or functional gastrointestinal disorders. Enrolled infants had a variety of medical conditions, including respiratory distress syndrome, chronic lung disease, apnea of prematurity, and Dandy-Walker malformation in the premature infants and cleft palate, respiratory distress syndrome, hypoxic-ischemic encephalopathy, and congenital diaphragmatic hernia in the term infants, but no overt gastrointestinal tract issues. Respiratory distress syndrome and congenital diaphragmatic hernia can delay gastric emptying in preterm infants $[27,28]$; however, the other medical conditions in this cohort have not been associated with an effect on gastric emptying or digestion capacity. Hypoxic-ischemic encephalopathy may result in gastrointestinal tissue damage [29]; however, the effects on gastric emptying and protein digestion 
are unknown. The infants with hypoxic ischemic encephalopathy were fasted and underwent whole body cooling for $72 \mathrm{~h}$ starting shortly after birth, however this treatment was completed and the infants advanced to full enteral feeding prior to enrollment. The infant with congenital diaphragmatic hernia had the defect repaired and was advanced to full enteral feeding prior to enrollment. Gastroesophageal reflux is almost universal in this population; however, none of the infants sampled received medications known to affect gastric $\mathrm{pH}$ or gastric digestion capacity, including prokinetics, $\mathrm{H} 2$ blockers/antagonists or proton-pump inhibitors. The enrolled infants were clinically stable at the time of sample collection (not on mechanical ventilation, stable vital signs). Samples were collected from 15 premature-delivering mother-infant pairs ranging in gestational age (GA) at birth from 23 to 32 weeks and 8 term-delivering mother-infant pairs ranging in GA at birth from 38 to 40 weeks (Table 1) over 7-98 days of postnatal age at the UC Davis Children's Hospital NICU in Sacramento, California. Enrolled infants had a variety of medical conditions, but no overt gastrointestinal tract issues or other medical conditions that have been associated with an effect on gastric emptying or digestion capacity. The enrolled infants were clinically stable at the time of sample collection (not on mechanical ventilation, stable vital signs). Human milk samples were collected as described in our previous study [30]. The preterm infants were fed their mother's milk (raw, not pasteurized) with fortifier (Similac Human Milk Fortifier Powder, Abbott Park, IL). The powdered fortifier contained intact bovine milk proteins and its protein composition was designed to match the whey:casein of human milk (60:40) using non-fat milk and whey protein concentrate. Each $25 \mathrm{~mL}$ of human milk was fortified with $0.25 \mathrm{~g}$ of bovine milk proteins (adding $10 \mathrm{mg}$ protein $/ \mathrm{mL}$ ). Term infants were fed their mother's milk (raw, not pasteurized) without fortification. The human milk feedings were delivered via the nasogastric tubes over $30 \mathrm{~min}$. Two hours after the initiation of feeding, $2 \mathrm{~mL}$ of each preterm and term infant's gastric contents were collected in a syringe back through the feeding tube via suction as previously described in our studies [21]. Gastric samples were aspirated at $2 \mathrm{~h}$ postprandial to obtain samples that represented a compromise between when adequate sample remained recoverable from the stomach and a maximum feasible length of gastric digestion time had passed based on gastric emptying times [31]. Human milk and gastric samples were placed into sterile vials and stored at $-20{ }^{\circ} \mathrm{C}$ and were transported to OSU on dry ice and stored at $-80{ }^{\circ} \mathrm{C}$.

Table 1. Demographics of preterm- and term-delivering mother-infant pairs sampled for human milk and gastric contents at $2 \mathrm{~h}$ postprandial.

\begin{tabular}{ccc}
\hline Demographics & Preterm-Delivering Mother Infant Pairs ${ }^{1-2}$ & Term-Delivering Mother-Infant Pairs $^{\text {1-2 }}$ \\
\hline GA, weeks & $27 \pm 3(23-32)$ & $38.9 \pm 0.5(38-40)$ \\
Postnatal age, day & $39 \pm 28(7-98)$ & $23 \pm 11(16-42)$ \\
Postmenstrual age, day & $32 \pm 2(30-37)$ & $42 \pm 2(41-45)$ \\
Birth weight, kg & $1.0 \pm 0.4(0.5-1.6)$ & $3.5 \pm 0.3(3.0-3.8)$ \\
Infant sex & 13 females; 2 males & $24 \pm 10(17-42)$ \\
Mother's age, year & $35 \pm 3(32-39)$ & 24 males \\
\hline
\end{tabular}

${ }^{1}$ Values are mean $\pm \mathrm{SD}$ (range); ${ }^{2}$ Number of paired milk and gastric samples from preterm and term infants is $n=15$ and $n=8$, respectively.

\subsection{Sample Reparation and ELISAs}

Samples were thawed at $4{ }^{\circ} \mathrm{C}$, $\mathrm{pH}$ was determined, and samples were centrifuged at $4226 \times g$ for $10 \mathrm{~min}$ at $4{ }^{\circ} \mathrm{C}$. The infranate was collected, separated into aliquots and stored at $-80^{\circ} \mathrm{C}$. The $\mathrm{pH}$ of the samples was measured with an S220 SevenCompact $\mathrm{pH} /$ Ion meter (Mettler-Toledo, Columbus, $\mathrm{OH}, \mathrm{USA}$ ) equipped with a combined sealed glass electrode.

The spectrophotometric ELISAs were recorded with a microplate reader (Spectramax M2, Molecular Devices, Sunnyvale, CA, USA) with two replicates of blanks, standards, and samples. SoftMax Pro 7.0 Microplate Data Analysis Software (Molecular Devices) was used to create a standard curve with a Four Parameters Logistic curve fit. ELISAs were performed according to the methods described by the manufacturers with some modifications as described (Table S1). The specific 
Ig concentrations in the samples were determined with antibody specificities as follows: human anti-alpha-chain antibody for total IgA (SIgA/IgA), anti-SC antibody for total SC (SC/SIgA/SIgM), gamma-chain antibody for IgG and mu-chain antibody for total IgM (SIgM/IgM). Concentrations of total IgA, total SC, total IgM and IgG were determined in human milk and gastric samples as well as in the fortifier alone.

For a separate test of the effect of the gastric $\mathrm{pH}$ change on Igs, ELISA were performed on standard Igs (total IgA, total SC, total IgM and IgG) in milk before and after incubation at $\mathrm{pH} 4.5$ for $1 \mathrm{~h}$ at $37^{\circ} \mathrm{C}$. $\mathrm{HCl}(10 \mathrm{mM})$ was used to adjust the $\mathrm{pH}$ to 4.5 .

\subsection{Peptidomic Analysis}

Peptide extraction from human milk and gastric samples was performed as described previously [32]. Mass spectrometric parameters were as described previously [33]. Spectra were analyzed by database searching in Thermo Proteome Discoverer (v2.1.0.81) using an in-house human milk protein sequence database. The tandem spectra were used to determine the counts and abundance of Ig alpha-chain (from IgA or SIgA), Ig gamma-chain (from IgG), Ig mu-chain (from IgM or SIgM), Ig J-chain (from IgA, SIgA, IgM or SIgM), Ig kappa-chain and Ig lambda-chain (from IgA, SIgA, IgM, SIgM or IgG), SC (f19-603 of total polymeric immunoglobulin receptor (PIgR)), and neonatal FC receptors $(\mathrm{FcRn})$. Only peptides identified with high confidence $(p<0.01)$ were included, and peptide sequences with multiple modifications were grouped into a single peptide for counts. Peptide counts measured the number of unique peptides identified in a sample whereas peptide abundance measured the ion intensity of the peak in a sample.

\subsection{Statistical Analyses}

Wilcoxon matched-pairs signed-rank test for paired sample comparisons (across milk and gastric samples within the same infant) and Mann-Whitney tests for unpaired sample comparisons (preterm versus term infants) were applied using GraphPad Prism software (version 7.03). All tests were nonparametric as some of the values did not pass the D 'Agostino \& Pearson normality test. Linear regression models were applied to determine if the concentrations of total IgA, total SC, total IgM and IgG in human milk and in gastric samples in both infant groups changed across postnatal age, GA, postmenstrual age (PMA), body weight at birth $\left(\mathrm{BW}_{\mathrm{b}}\right)$, body weight at sampling $\left(\mathrm{BW}_{\mathrm{s}}\right)$, and feed volume. Differences were designated significant at $p<0.05$. Pearson correlation coefficients $(\mathrm{r})$ were determined when $p<0.1$. The sample size of preterm $(n=15)$ and term $(n=8)$ paired milk and gastric samples was selected based on our previous study [20] and proved to be adequately powered to detect differences based on the results.

\section{Results}

\subsection{Infant Demographics}

Demographic details for the preterm- and term-delivering mother-infant pairs are presented in Table 1.

\subsection{Ig Concentrations}

\subsubsection{Total IgA Concentration}

Total $\operatorname{Ig} \mathrm{A}(\mathrm{S} \operatorname{Ig} \mathrm{A} / \operatorname{Ig} \mathrm{A})$ concentration decreased $60 \%(p=0.001$, Figure $1 \mathrm{~A})$ from human milk to the preterm infant stomach, and $47.8 \%$ from human milk to the term infant stomach $(p=0.016$, Figure 1B). Total IgA concentration was similar between preterm and term milks and between preterm and term gastric contents $(p>0.05$, Table S2). Total IgA concentration in preterm milk and gastric samples decreased with increased postnatal age, PMA, and $\mathrm{BW}_{\mathrm{s}}(p<0.05)$ but did not change in term samples $(p>0.05)$ (Table S3). Total IgA concentration in human milk or gastric contents did not change 
across GA and feed volume within preterm and term infants ( $p>0.05$, Table S3). When human milk was incubated under in vitro acidic conditions ( $\mathrm{pH} 4.5)$ to match stomach conditions but without the proteases, there was no decrease in IgA concentration. No IgA was detected in the fortifier.

\subsubsection{Total SC Concentration}

Human milk total SC (SC/SIgA/SIgM) concentration significantly decreased $62 \%(p=0.031$, Figure 1D) from human milk to the term stomach but did not change in the preterm infant stomach $(p=0.33$, Figure 1C). Total SC concentration did not differ between human milk from preterm- and term-delivering mothers $(p=0.22)$, nor between preterm and term gastric contents $(p=0.11)$. Total SC concentration in milk and gastric samples in term and preterm infants did not change across GA, postnatal age, or PMA ( $p>0.05$, Table S3). No total SC was detected in the fortifier.
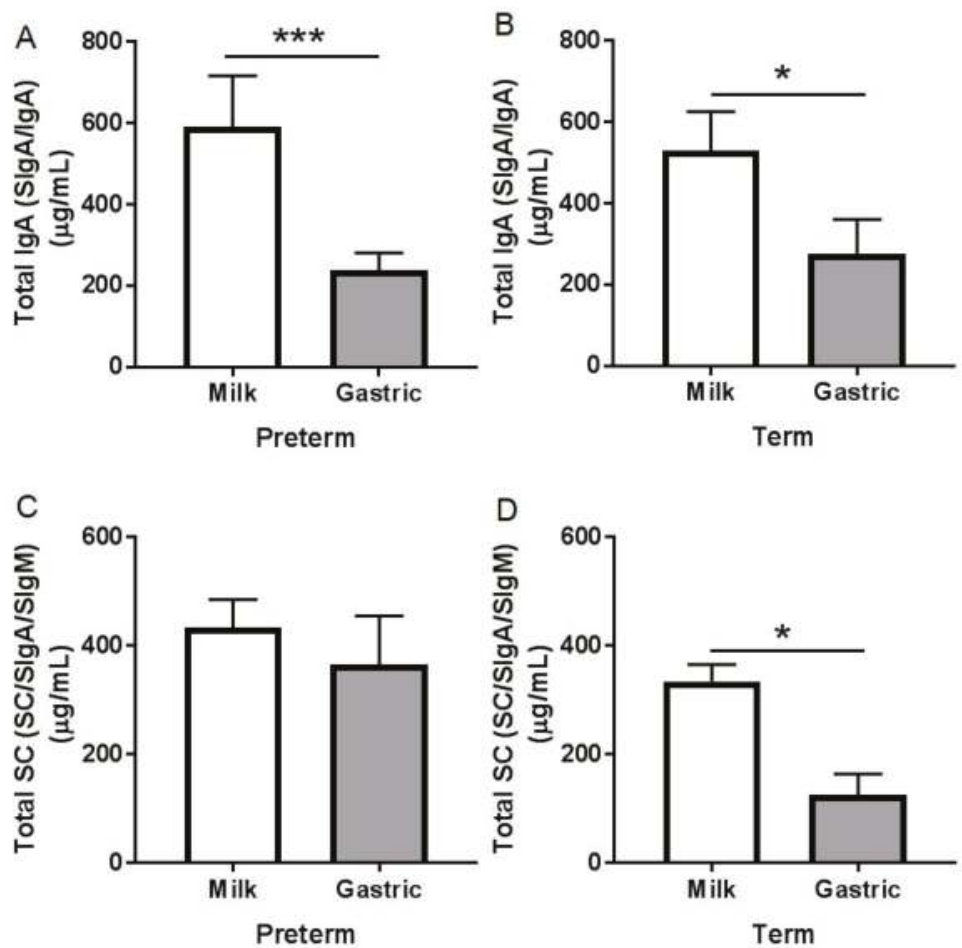

Figure 1. Immunoglobulin concentrations in human milk and gastric samples at $2 \mathrm{~h}$ postprandial time from paired mother-infant delivered prematurely (23-32 week of gestational age (GA), 7-98 days of postnatal age) and at term (38-40 week of GA, 16-42 days of postnatal age). Concentration of total $\operatorname{IgA}(\mathrm{SIgA} / \operatorname{IgA})(\mathbf{A})$ in preterm infant samples and (B) in term infant samples; Concentration of total secretory component (SC/SIgA/SIgM) (C) in preterm infant samples and (D) in term infant samples. Values are mean $\pm \mathrm{SEM}, n=15$ for preterm infants and $n=8$ for term infants. Asterisks show statistical significant differences between variables $\left({ }^{* * *} p<0.001 ; * p 0.05\right)$ using the Wilcoxon matched-pairs signed-rank test.

\subsubsection{Total IgM Concentration}

Total IgM (SIgM/IgM) concentration decreased significantly ( $p=0.016$, Figure 2B) 33\% from human milk to the term infant stomach but did not change in the preterm infant stomach $(p=0.54$, Figure 2A). Total IgM concentration in human milk from preterm-delivering mothers was $77 \%$ lower 
than in milks from term-delivering mothers $(p<0.001$, Figure S1A) but did not differ in the gastric contents between preterm and term infants $(p=0.56)$. Total IgM concentration in either milk or gastric samples did not change across postnatal age, GA, PMA, or BW $\mathrm{b}_{\mathrm{b}}$ for term or preterm infants $(p>0.05$, Table S3). Total IgM concentration decreased in preterm milk with increasing postnatal age or $\mathrm{BW}_{\mathrm{S}}$ $(p<0.05)$ but did not change in term milk or in either preterm or term stomach samples $(p>0.05$, Table S3). Total IgM concentration increased with increasing feed volume in preterm milk and gastric samples but did not change in term samples. No total IgM was detected in the fortifier.
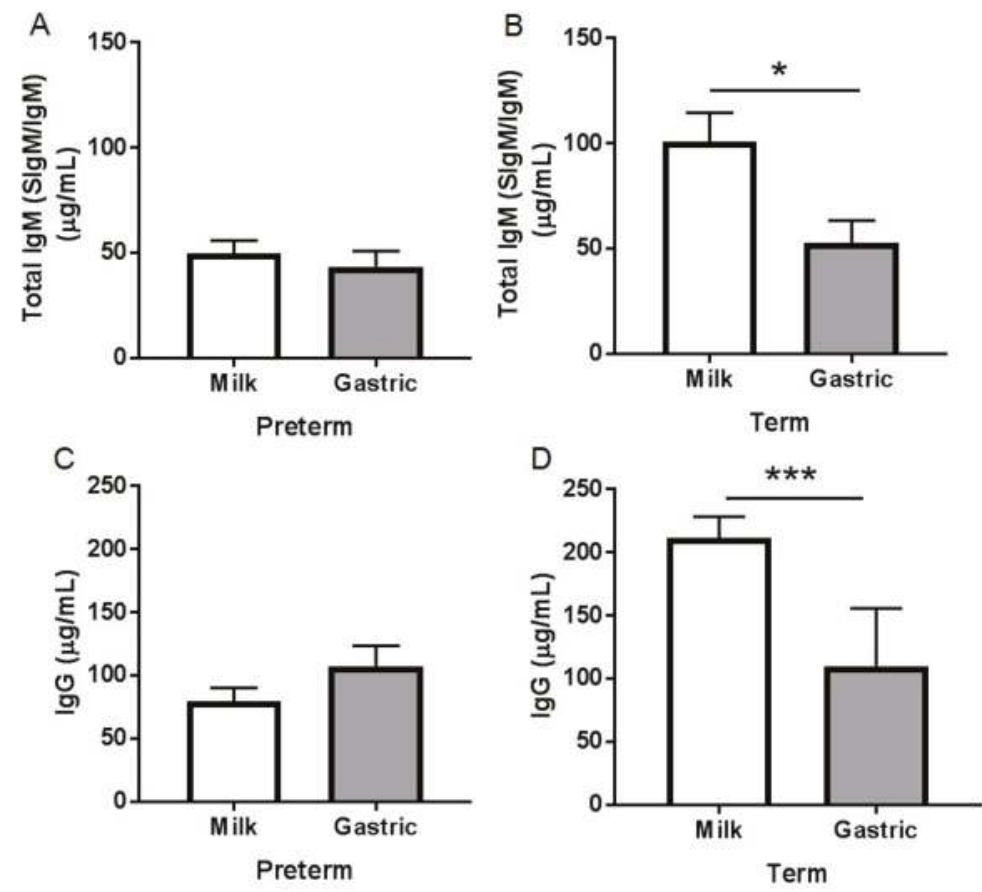

Figure 2. Immunoglobulin concentrations in human milk and gastric samples at $2 \mathrm{~h}$ postprandial time from paired mother-infant delivered prematurely (23-32 week of gestational age (GA), 7-98 days of postnatal age) and at term (38-40 week of GA, 16-42 days of postnatal age). Concentration of total IgM $(\mathrm{SIgM} / \operatorname{IgM})(\mathbf{A})$ in preterm infant samples and (B) in term infant samples; Concentration of $\operatorname{IgG}(\mathbf{C})$ in preterm infant samples and (D) in term infant samples. Values are mean $\pm \mathrm{SEM}, n=15$ for preterm infants and $n=8$ for term infants. Asterisks show statistical significant differences between variables $\left({ }^{* * *} p<0.001 ;{ }^{*} p<0.05\right)$ using the Wilcoxon matched-pairs signed-rank test.

\subsubsection{IgG Concentration}

IgG concentration decreased significantly $(48 \%, p=0.026$, Figure 2D) from human milk to the term infant stomach but did not change in the preterm infant stomach ( $p=0.11$, Figure $2 \mathrm{C}$ ). IgG concentration in human milk from preterm-delivering mothers was $97 \%$ lower than in milks from term-delivering mothers ( $p<0.001$, Figure S1B) but did not differ in the gastric contents between preterm and term infants $(p=0.58)$. IgG concentration in preterm and term milk did not change across postnatal age, GA, PMA, BW $\mathrm{b}_{\mathrm{b}}$, or feed volume ( $p>0.05$, Table S3). IgG concentration in preterm gastric samples decreased with increasing PMA or $\mathrm{BW}_{\mathrm{s}}$ and increased with increasing $\mathrm{BW}_{\mathrm{b}}$ in term stomach but did not change across GA or feed volume. No IgG was detected in the fortifier. 


\subsection{Peptidomic Results}

No peptides (counts or abundance) for Ig alpha-chain, Ig gamma-chain, Ig mu-chain, Ig J-chain, Ig lambda-chain, and Ig kappa-chain were detected in human milk from either the mothers who delivered prematurely or at term, but peptides from each of these proteins appeared in the gastric samples from both preterm and term infants (Figures S2-S4). Peptides from SC (f19-603 of PIgR) was detected in both milk and gastric samples from both preterm and term infants.

Ig alpha-chain peptide counts in gastric samples were 1.2-fold lower in preterm infants than in term infants (not significant, but a tendency: $p=0.081$, Figure $3 \mathrm{~A}$ ). Ig alpha-chain peptide abundance in gastric contents was 16-fold lower in preterm infants than in term infants ( $p=0.038$, Figure 3D). Ig mu-chain peptide counts and abundance in gastric contents were $2.3-(p=0.032$, Figure 3B) and 13 -fold ( $p=0.010$, Figure 3E), respectively, lower in preterm infants than in term infants. Ig gamma-chain peptide counts and abundance in gastric contents were 1.9- ( $p=0.002$, Figure 3C) and 34-fold $(p<0.001$, Figure $3 \mathrm{~F}$ ), respectively, lower in preterm infants than in term infants. Peptide counts and abundance of Ig J-chain, lambda-chain and Ig kappa-chain did not differ between preterm and term infant gastric samples ( $p>0.05$, Table S2). Peptide counts of SC (f19-603 of PIgR) decreased 37\% and 56\% from human milk to the stomach for preterm and term infants, respectively $(p<0.05$, Figure $4 \mathrm{~A}, \mathrm{~B})$. Peptide abundance of SC decreased $89 \%$ and $68 \%$ from human milk to the stomach for preterm and term infants, respectively $(p<0.05$, Figure 4 C,D).
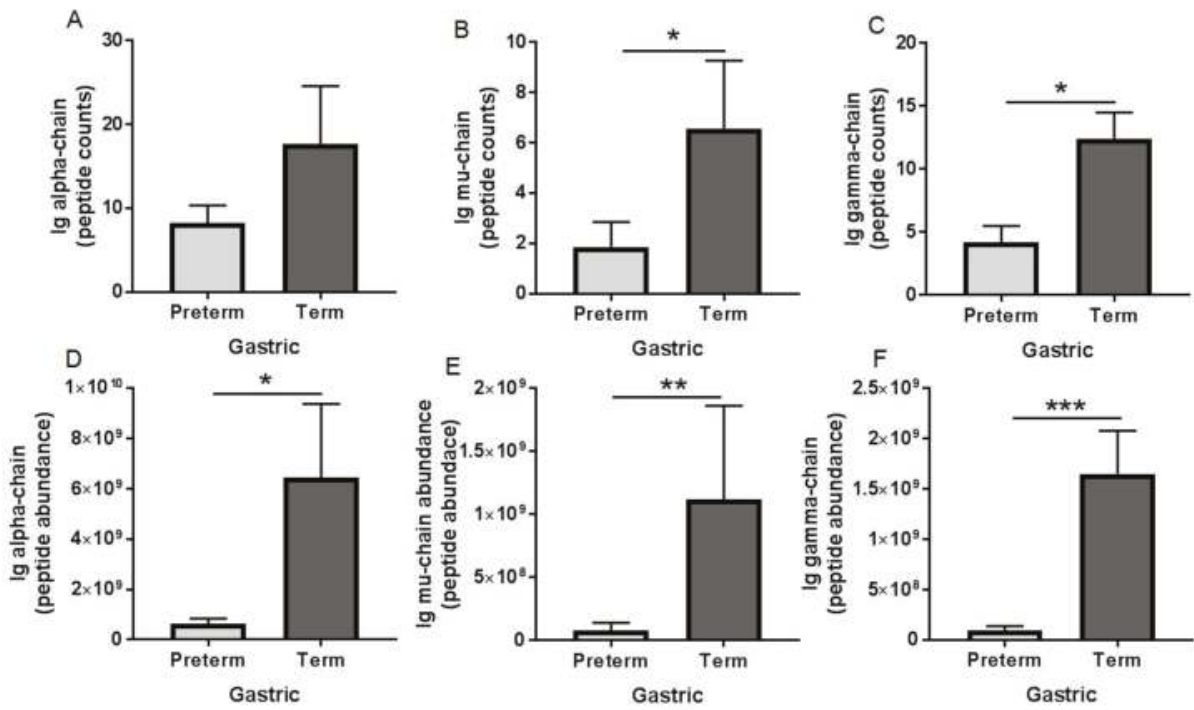

Figure 3. Peptide counts and abundance of human immunoglobulin fragments in human milk and gastric samples at $2 \mathrm{~h}$ postprandial time from paired mother-infant delivered prematurely (23-32 week of gestational age (GA), 7-98 days of postnatal age) and at term (38-40 week of GA, 16-42 days of postnatal age). (A,D) Ig alpha-chain (from SIgA/IgA) and (B,E) Ig mu-chain (from SIgM/IgM); $(\mathbf{C}, \mathbf{F})$ Ig gamma-chain (from IgG). Values are mean \pm SEM, $n=15$ for preterm infants and $n=8$ for term infants. Asterisks show statistical significant differences between variables ${ }^{* * *} p<0.001 ;{ }^{* *} p<0.01$; $\left.{ }^{*} p<0.05\right)$ using the Mann-Whitney test (unpaired samples). 

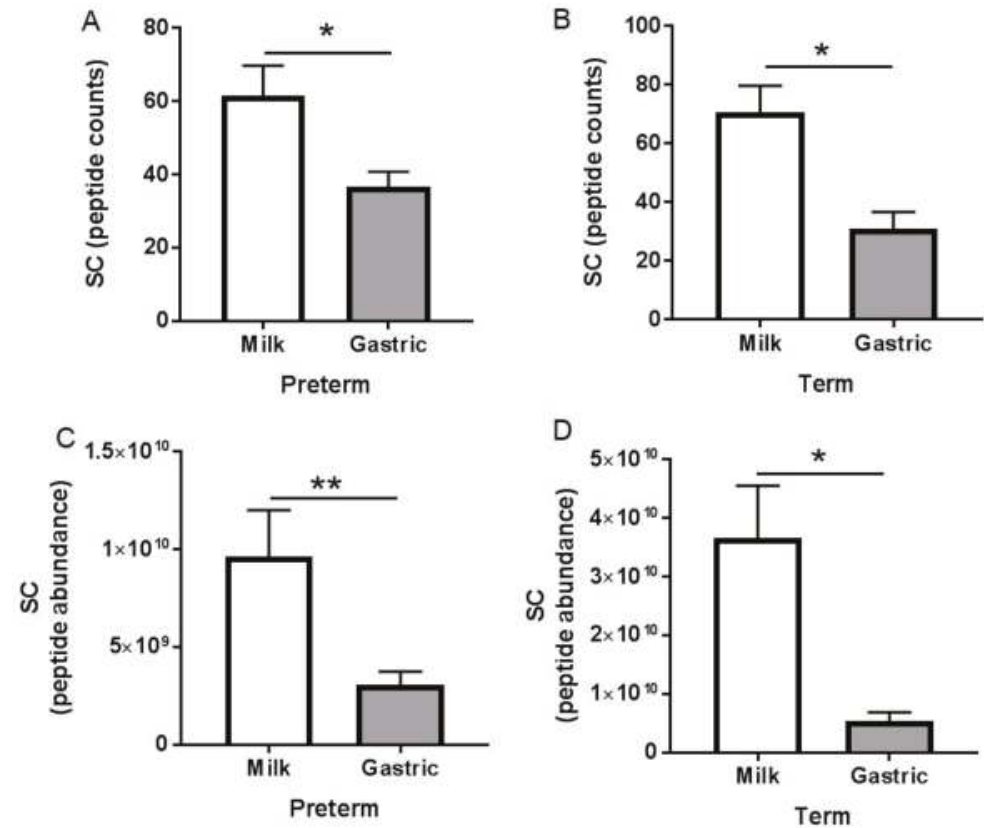

Figure 4. Peptides counts and abundance of SC (f19-603 of PIgR) in human milk and gastric samples from mother-infant pairs with preterm delivery (23-32 week of gestational age (GA), 7-98 days of postnatal age) and term delivery (38-40 week of GA, 16-42 days of postnatal age. Peptides counts of SC (A) in preterm infant samples; (B) in term infant samples. Peptide abundance of SC (C) in preterm infant samples and (D) in term infant samples. Values are mean \pm SEM, $n=15$ for preterm infants and $n=8$ for term infants. Asterisks show statistical significant differences between variables $(* * p<0.01$; $\left.{ }^{*} p<0.05\right)$ using the Wilcoxon matched-pairs signed-rank test.

\section{4. $p H$}

For preterm and term mother-infant samples, $\mathrm{pH}$ values in gastric samples were lower than those in human milk ( $p<0.001$, Figure S5). Neither milk nor gastric sample $\mathrm{pH}$ differed between preterm and term mother-infant pairs ( $p>0.05$, Table S2).

\section{Discussion}

The Ig concentrations in milk from preterm- and term-delivering mothers have been previously studied [34-37]. No study has compared the survival of Igs in the stomach or intestine between preterm and term infants. Our recent studies demonstrated that preterm infants partially degrade IgA but not IgG and IgM in the stomach [21,22]. A few studies have measured the survival of milk Igs to infant stool $[17,38]$. However, measuring Igs in infant stool samples does not accurately represent the biologically relevant survival of Igs within the upper GI tract, as Igs are exposed to proteases from the infant gastrointestinal tract as well as protein-fermenting colonic bacteria, which can degrade them before they are identified in stool [39]. To begin to address this lack of knowledge, the present study examined the stability of human milk Igs—-total IgA (SIgA/IgA), total IgM (SIgM/IgM), IgG, and total SC (SC/SIgA/SIgM)—during gastric digestion in preterm and term infants.

Concentrations of Igs in human milk from mothers delivering prematurely and at term were congruent with those found in previous studies [36,37,40,41]. Concentrations of total IgA or total SC did not differ between preterm and term milk, which matches with the observation in a previous study [34] that milk total IgA did not differ between preterm and term milk ( $3.2 \mathrm{mg} / \mathrm{mL})$ from 6 to 
28 days postnatal age. Ballabio et al. [34] observed a higher concentration of total IgA than measured in this present study and did not detect IgM or IgG in preterm and term milk (we detected IgM and $\operatorname{IgG}$ ). This difference could be due to their use of an immunoelectrophoretic technique (SDS-PAGE and immunoblotting) to determine the concentration of Igs in their milk samples. ELISA is more quantitative (lower detection limit) than SDS-PAGE and immunoblotting. We found that total IgA concentration decreased with increasing postnatal age, $\mathrm{PMA}$, and $\mathrm{BW}_{\mathrm{s}}$ in preterm milk, which matches with this same previous study [34] (total IgA concentration decreased from colostrum to mature milk from preterm-delivering mothers). A few studies found higher amounts of total IgA in colostrum from preterm-delivering mothers compared with colostrum from term-delivering mothers [3,34,35,42]; however, as no colostrum was collected in the present study, we cannot compare our results to that data. Another study found that preterm milk IgA concentration was 1.1- to 1.4-fold higher than in term milk from 3 to 15 days but found no differences in concentration from 28 to 56 days of postnatal age [3]. Thus, the observed lack of difference for total IgA concentration between preterm and term milk in the present study is likely due to the older postnatal age (average: 39 days for preterm and 23 days for term infants) for milk collection.

The concentration of total SC (mostly SIgA but can include SC and SIgM) represented $73.5 \%$ of total IgA in preterm milk and $63.6 \%$ of total IgA in term milk. These percentages are somewhat lower than the observations reported by Goldman et al. [4] that total SC (called "SIgA" by the authors but actually representing total SC, as an anti-SC primary antibody was used) concentration represented $90 \%$ of the total $\operatorname{IgA}$ in term milk.

The concentration of IgG and total IgM were lower in human milk from preterm-delivering mothers than term-delivering mothers. Previous studies observed IgG and IgM concentrations in human milk that were similar between mothers delivering prematurely and at term [36,41]. On the other hand, Chandra et al. [3] found that IgM and IgG concentrations were 1.5-fold higher in preterm milk than in term milk from 14 to 28 days of postnatal age.

The total IgA concentration decreased from human milk to the preterm and term infant stomach. The stability of SC/SIgA/SIgM during gastric digestion in preterm suggests that $\mathrm{SC}$ remains intact and detectable by the anti-SC even if it is released from the SIgA complex. Unlike in preterm infant, total SC concentration decreased in the term infant stomach. The reduction of total $\operatorname{IgA}(\mathrm{SIgA} / \operatorname{IgA})$ was likely due to degradation by proteases (pepsin and/or milk proteases [21]) and not acid-induced structural deterioration in the stomach, as incubation of standard IgA in acid conditions did not decrease its concentration (Figure S6). If we consider that $74 \%$ of total IgA in preterm milk is SIgA-we could not distinguish between SC/SIgA/SIgM, the decrease of total IgA likely derived from preterm gastric digestion of partly IgA and partly SIgA. We found higher peptide abundance of Ig alpha-chain (which could derive from either IgA or SIgA) in term infant gastric contents than in preterm infants, which suggests that $\operatorname{Ig} \mathrm{A} / \mathrm{SIgA}$ is more digested by term infants than preterm infants.

We also observed that human milk total IgM decreased and IgG tended to decrease in the term stomach ( $48 \%$ and $49 \%$ reduction, respectively) but were stable in the preterm stomach. We found higher peptide counts and abundance of Ig $\mathrm{mu}$ (which could derive from either IgM or SIgM) and Ig gamma (which derive from $\mathrm{IgG}$ ) in term infant gastric infants than preterm infants, which suggests that $\operatorname{SIgM} / \operatorname{IgM}$ and $\operatorname{IgG}$ is more digested by term infants than preterm infants. This decrease of Igs is unlikely to relate to gastric $\mathrm{pH}$ as preterm and term infants' gastric $\mathrm{pH}$ did not differ. We also observed that $\mathrm{pH} 4.5$ had no effect on the stability of Igs (Figure S2). Gastric proteases are likely responsible for observed differences, as we previously demonstrated that gastric pepsin activity and proteolysis were higher in term infants than in preterm infants [20].

We observed that the stability of $\operatorname{IgG}$ and total $\operatorname{IgM}$ during gastric preterm digestion was higher than total IgA. No previous study evaluated the digestibility of Igs in the preterm or term infant stomach or small intestine, but a previous study [17] found a greater reduction of IgA compared with IgG or IgM in preterm infant stools. When preterm infants (1-28 days of postnatal age (GA unknown) 0.8-2 kg BW) were fed only infant formula or infant formula plus pasteurized pooled human milk 
supplemented with $600 \mathrm{mg}$ daily of serum-derived human $\operatorname{IgA}(73 \%)$ and $\operatorname{IgG}(26 \%)$, the stool samples collected contained 1-10 mg IgG per $\mathrm{g}$ of dried feces (percentage reduction not calculated) and no IgA [17]. We showed that IgG was decreased in the term infant stomach but stable in the preterm infant stomach.

Using peptidomics, we demonstrated partial digestion (demonstrated by an increase in peptide counts and abundances from milk to the stomach) of Ig alpha-chain (from IgA and SIgA), Ig gamma-chain (from IgG), Ig mu-chain (from IgM or SIgM), and Ig J-chain (from IgA, SIgA, IgM or SIgM), Ig kappa-chain and Ig lambda-chain (from all Igs) in both preterm and term infants. Dallas et al. [43] also observed that alpha-1-chain (named IGHA1) was not detected in term milk but was detected in the term stomach. SC peptide counts decreased in both preterm and term gastric samples, suggesting that SIgA or SIgM were partially digested in the stomach from both infants. We found a decrease in peptide counts and abundance of SC (which could derive from either SIgA or SIgM) in both preterm and term infant gastric samples, suggesting a digestion of these Igs in the infant stomach.

A limitation of this study is the small number of samples for preterm and term infants. Another limitation is that we did not measure Ig concentrations in the infant intestine contents. Therefore, we plan to examine how human milk Ig concentrations change in the stomach and intestine in a future study with a larger number of preterm and term infants. The infants in our study had conditions that required a naso-gastric tube, which could affect Ig digestion. However, we included only infants with conditions that are not expected to interfere with protein digestion. Ideally, the study would include healthier infants; however, placing a feeding tube in infants that do not require them is very challenging from an ethical and patient enrollment perspective. Development of non-invasive techniques to collect digestive samples from fragile infants would be highly beneficial to this field. The placement of the nasogastric tube in these infants could also modify the gastric microbiome, which could influence Ig digestion. However, as we currently have no technique to assess gastric digestion in infants without a feeding tube, we cannot assess this potential impact.

\section{Conclusions}

The present study revealed that total $\operatorname{IgA}(\mathrm{SIgA} / \operatorname{Ig} \mathrm{A})$ was digested in the preterm and term infant stomach. Human milk total SC (SC/SIgA/SIgM), IgM, and IgG were stable in the preterm stomach but were digested in the term stomach. Ig-derived peptides from all different Ig isotypes were in higher amounts in the gastric contents of term infants than in preterm infants, demonstrating a higher overall gastric Ig digestion in term infants. Therefore, human milk Igs are less digested in the preterm infant stomach than the term infant stomach. As the stomach represents only the beginning of the digestive system, the concentrations of human milk Igs in intestinal samples from preterm and term infants need to be determined to clarify their potential survival during infant digestion. The longer that milk Igs survive through the digestive system, the longer they can act as passive immune system components, which is particularly important in the context of the immune system immaturity of the early postnatal period in preterm and term infants.

Supplementary Materials: The following are available online at http:/ /www.mdpi.com/2072-6643/10/5/631/s1, Table S1: Details for the assays performed in human milk and gastric samples; Table S2: Statistical results ( $p$-values) for Wilcoxon matched-pairs signed rank test to compare immunoglobulin concentrations (ELISA) and peptides (peptidomics) in human milk and gastric samples in preterm and term infants (PM vs. PG or TM vs. TG); Table S3: Statistical results ( $p$-value) from the linear regression of the $\mathrm{pH}$ and the concentration of total $\operatorname{IgA}(\mathrm{SIgA} / \operatorname{IgA})$, total SC (SC/SIgA/SIgM), IgG and total IgM (SIgM/IgM) across postnatal age (P), gestational age at birth (GA), postmenstrual age (PMA), $\mathrm{BW}_{\mathrm{b}}, \mathrm{BW}_{\mathrm{s}}$ and feed volume (FV) in human milk and in gastric contents at 1,2 and $3 \mathrm{~h}$ postprandial from preterm infants (23-32 week of gestational age, 7-98 days of postnatal age) and term infants (38-40 week of GA, 16-42 days of postnatal age); Figure S1: Immunoglobulin concentrations of (A) total IgM (SIgM/IgM) and (B) IgG in mother's milks delivering prematurely (23-32 week of gestational age (GA), 7-98 days of postnatal age) and at term (38-40 week of GA, 16-42 days of postnatal age). Figures S2-S4: Peptide counts and abundance of human immunoglobulin fragments in human milk and gastric samples at $2 \mathrm{~h}$ postprandial time from paired mother-infant delivered prematurely (23-32 week of gestational age (GA), 7-98 days of postnatal age) and at term (38-40 week of GA, 16-42 days of postnatal age). Figure S5: The pH in human milk and gastric 
samples in human milk and gastric samples at $2 \mathrm{~h}$ postprandial time from paired (A) mother-infant delivered prematurely (23-32 week of gestational age (GA), 7-98 days of postnatal age) and (B) at term (38-40 week of GA, 16-42 days of postnatal age). Figure S6: Stability of standard immunoglobulins (Igs) before and after the incubation at $\mathrm{pH} 4.5$.

Author Contributions: V.D.-M. conducted the ELISA analyses, analyzed data and conducted the statistical analysis. M.A.U. provided milk and gastric samples. R.L.B. and S.D.N. conducted the peptidomic analyses. V.D.-M. and D.C.D. designed the study and drafted the manuscript. V.D.-M. and D.C.D. have primary responsibility for the final content.

Acknowledgments: The authors acknowledge the Mass Spectrometry Center at Oregon State University, which is supported in part by the National Institute of Health grant S10OD020111. This study was supported by the K99/R00 Pathway to Independence Career Award, Eunice Kennedy Shriver Institute of Child Health \& Development of the National Institutes of Health (R00HD079561) (D.C.D.) and The Gerber Foundation.

Conflicts of Interest: The authors declare no conflict of interest.

\section{References}

1. Tha-In, T.; Bayry, J.; Metselaar, H.J.; Kaveri, S.V.; Kwekkeboom, J. Modulation of the cellular immune system by intravenous immunoglobulin. Trends Immunol. 2008, 29, 608-615. [CrossRef] [PubMed]

2. Palmeira, P.; Quinello, C.; Silveira-Lessa, A.L.; Zago, C.A.; Carneiro-Sampaio, M. IgG placental transfer in healthy and pathological pregnancies. Clin. Dev. Immunol. 2011, 2012, 985646. [CrossRef] [PubMed]

3. Chandra, R. Immunoglobulin and protein levels in breast milk produced by mothers of preterm infants. Nutr. Res. 1982, 2, 27-30. [CrossRef]

4. Goldman, A.S.; Garza, C.; Nichols, B.L.; Goldblum, R.M. Immunologic factors in human milk during the first year of lactation. J. Pediatr. 1982, 100, 563-567. [CrossRef]

5. Duijts, L.; Jaddoe, V.W.; Hofman, A.; Moll, H.A. Prolonged and exclusive breastfeeding reduces the risk of infectious diseases in infancy. Pediatrics 2010, 126, e18. [CrossRef] [PubMed]

6. Janeway, C.A., Jr.; Travers, P.; Walport, M.; Shlomchik, M.J. The distribution and functions of immunoglobulin isotypes. In Immunobiology, the Immune System in Health and Disease, 5th ed.; Garland Science: New York, NY, USA, 2001.

7. Melis, J.P.; Strumane, K.; Ruuls, S.R.; Beurskens, F.J.; Schuurman, J.; Parren, P.W. Complement in therapy and Disease: Regulating the complement system with antibody-based therapeutics. Mol. Immunol. 2015, 67, 117-130. [CrossRef] [PubMed]

8. Bakker-Zierikzee, A.; Tol, E.; Kroes, H.; Alles, M.; Kok, F.; Bindels, J. Faecal SIgA secretion in infants fed on pre-or probiotic infant formula. Pediatr. Allergy Immunol. 2006, 17, 134-140. [CrossRef] [PubMed]

9. Mantis, N.J.; Rol, N.; Corthésy, B. Secretory IgA's complex roles in immunity and mucosal homeostasis in the gut. Mucosal Immunol. 2011, 4, 603. [CrossRef] [PubMed]

10. Macpherson, A.J.; Hunziker, L.; McCoy, K.; Lamarre, A. IgA responses in the intestinal mucosa against pathogenic and non-pathogenic microorganisms. Microbes Infect. 2001, 3, 1021-1035. [CrossRef]

11. Yoshida, M.; Claypool, S.M.; Wagner, J.S.; Mizoguchi, E.; Mizoguchi, A.; Roopenian, D.C.; Lencer, W.I.; Blumberg, R.S. Human neonatal Fc receptor mediates transport of IgG into luminal secretions for delivery of antigens to mucosal dendritic cells. Immunity 2004, 20, 769-783. [CrossRef] [PubMed]

12. Perkkiö, M.; Savilahti, E. Time of appearance of immunoglobulin-containing cells in the mucosa of the neonatal intestine. Pediatr. Res. 1980, 14, 953. [CrossRef] [PubMed]

13. Robert-Guroff, M. IgG surfaces as an important component in mucosal protection. Nat. Med. 2000, 6, 129. [CrossRef] [PubMed]

14. Hansen, G.H.; Niels-Christiansen, L.-L.; Immerdal, L.; Danielsen, E.M. Antibodies in the small Intestine: Mucosal synthesis and deposition of anti-glycosyl IgA, IgM, and IgG in the enterocyte brush border. Am. J. Physiol. Gastrointest. Liver Physiol. 2006, 291, G82-G90. [CrossRef] [PubMed]

15. Hansen, G.H.; Pedersen, E.D.; Immerdal, L.; Niels-Christiansen, L.-L.; Danielsen, E.M. Anti-glycosyl antibodies in lipid rafts of the enterocyte brush Border: A possible host defense against pathogens. Am. J. Physiol. Gastrointest. Liver Physiol. 2005, 289, G1100-G1107. [CrossRef] [PubMed]

16. Roos, N.; Mahe, S.; Benamouzig, R.; Sick, H.; Rautureau, J.; Tome, D. 15N-labeled immunoglobulins from bovine colostrum are partially resistant to digestion in human intestine. J. Nutr. 1995, 125, 1238-1244. [PubMed] 
17. Eibl, M.M.; Wolf, H.M.; Fürnkranz, H.; Rosenkranz, A. Prevention of necrotizing enterocolitis in low-birth-weight infants by IgA-IgG feeding. N. Engl. J. Med. 1988, 319, 1-7. [CrossRef] [PubMed]

18. Schanler, R.J.; Goldblum, R.M.; Garza, C.; Goldman, A.S. Enhanced fecal excretion of selected immune factors in very low birth weight infants fed fortified human milk. Pediatr. Res. 1986, 20, 711. [CrossRef] [PubMed]

19. Goldblum, R.M.; Schanler, R.J.; Garza, C.; Goldman, A.S. Human milk feeding enhances the urinary excretion of immunologic factors in low birth weight infants. Pediatr. Res. 1989, 25, 184. [CrossRef] [PubMed]

20. Demers-Mathieu, V.; Qu, Y.; Underwood, M.; Borghese, R.; Dallas, D. Premature infants have lower gastric digestion capacity for human milk proteins than term infants. J. Pediatr. Gastroenterol. Nutr. 2018, 66, 816-821. [CrossRef] [PubMed]

21. Demers-Mathieu, V.; Nielsen, S.D.; Underwood, M.A.; Borghese, R.; Dallas, D.C. Changes in proteases, antiproteases and bioactive proteins from mother's breast milk to the premature infant stomach. J. Pediatr. Gastroenterol. Nutr. 2017, 66, 318-324. [CrossRef] [PubMed]

22. Demers-Mathieu, V.; Underwood, M.A.; Beverly, R.L.; David, D.C. Survival of immunoglobulins from human milk to preterm infant gastric samples at 1,2, and 3 hours postprandial. Neonatology 2018, in press.

23. Malek, A.; Sager, R.; Kuhn, P.; Nicolaides, K.H.; Schneider, H. Evolution of maternofetal transport of immunoglobulins during human pregnancy. Am. J. Reprod. Immunol. 1996, 36, 248-255. [CrossRef] [PubMed]

24. Zemlin, M.; Hoersch, G.; Zemlin, C.; Pohl-Schickinger, A.; Hummel, M.; Berek, C.; Maier, R.F.; Bauer, K. The postnatal maturation of the immunoglobulin heavy chain IgG repertoire in human preterm neonates is slower than in term neonates. J. Immunol. 2007, 178, 1180-1188. [CrossRef] [PubMed]

25. Langkamp, D.L.; Davis, J.P. Increased risk of reported pertussis and hospitalization associated with pertussis in low birth weight children. J. Pediatr. 1996, 128, 654-659. [CrossRef]

26. Groothuis, J.R.; Simoes, E.A.; Hemming, V.G. Respiratory syncytial virus (RSV) infection in preterm infants and the protective effects of RSV immune globulin (RSVIG). J. Pediatr. 1995, 95, 463-467.

27. Victor, Y. Effect of body position on gastric emptying in the neonate. Arch. Dis. Child. 1975, 50, 500-504. [CrossRef] [PubMed]

28. Arena, F.; Romeo, C.; Baldari, S.; Arena, S.; Antonuccio, P.; Campennì, A.; Zuccarello, B.; Romeo, G. Gastrointestinal sequelae in survivors of congenital diaphragmatic hernia. Pediatr. Int. 2008, 50, 76-80. [CrossRef] [PubMed]

29. Faingold, R.; Cassia, G.; Prempunpong, C.; Morneault, L.; Sant'Anna, G.M. Intestinal ultrasonography in infants with moderate or severe hypoxic-ischemic encephalopathy receiving hypothermia. Pediatr. Radiol. 2016, 46, 87-95. [CrossRef] [PubMed]

30. Demers-Mathieu, V.; Nielsen, S.D.; Underwood, M.A.; Borghese, R.; Dallas, D.C. Analysis of milk from mothers who delivered prematurely reveals few changes in proteases and protease inhibitors across gestational age at birth and infant postnatal age. J. Nutr. 2017, 147, 1152-1159. [CrossRef] [PubMed]

31. Siegel, M.; Lebenthal, E.; Topper, W.; Krantz, B.; Li, P.K. Gastric emptying in prematures of isocaloric feedings with differing osmolalities. Pediatr. Res. 1982, 16, 141-147. [CrossRef] [PubMed]

32. Nielsen, S.D.; Beverly, R.L.; Dallas, D.C. Peptides released from foremilk and hindmilk proteins by breastmilk proteases are highly similar. Front. Nutr. 2017, 4, 54. [CrossRef] [PubMed]

33. Nielsen, S.D.; Beverly, R.L.; Dallas, D.C. Milk proteins are human mammary gland. J. Mammary Gland. Biol. Neoplasia 2017, 22, 251-261. [CrossRef] [PubMed]

34. Ballabio, C.; Bertino, E.; Coscia, A.; Fabris, C.; Fuggetta, D.; Molfino, S.; Testa, T.; Sgarrella, M.; Sabatino, G.; Restani, P. Immunoglobulin-A profile in breast milk from mothers delivering full term and preterm infants. Int. J. Immunopathol. Pharmacol. 2007, 20, 119-128. [CrossRef] [PubMed]

35. Montagne, P.; Cuillière, M.L.; Molé, C.; Béné, M.C.; Faure, G. Immunological and nutritional composition of human milk in relation to prematurity and mothers' parity during the first 2 weeks of lactation. J. Pediatr. Gastroenterol. Nutr. 1999, 29, 75-80. [CrossRef] [PubMed]

36. Gross, S.J.; Buckley, R.H.; Wakil, S.S.; McAllister, D.C.; David, R.J.; Faix, R.G. Elevated IgA concentration in milk produced by mothers delivered of preterm infants. J. Pediatr. 1981, 99, 389-393. [CrossRef]

37. Castellote, C.; Casillas, R.; Ramírez-Santana, C.; Pérez-Cano, F.J.; Castell, M.; Moretones, M.G.; López-Sabater, M.C.; Franch, À. Premature delivery influences the immunological composition of colostrum and transitional and mature human milk. J. Nutr. 2011, 141, 1181-1187. [CrossRef] [PubMed] 
38. Blum, P.M.; Phelps, D.L.; Ank, B.J.; Krantman, H.J.; Stiehm, E.R. Survival of oral human immune serum globulin in the gastrointestinal tract of low birth weight infants. Pediatr. Res. 1981, 15, 1256-1260. [CrossRef] [PubMed]

39. Dallas, D.C.; Sanctuary, M.R.; Qu, Y.; Khajavi, S.H.; Van Zandt, A.E.; Dyandra, M.; Frese, S.A.; Barile, D.; German, J.B. Personalizing protein nourishment. Crit. Rev. Food Sci. Nutr. 2017, 57, 3313-3331. [CrossRef] [PubMed]

40. Evans, T.; Ryley, H.; Neale, L.; Dodge, J.; Lewarne, V. Effect of storage and heat on antimicrobial proteins in human milk. Arch. Dis. Child. 1978, 53, 239-241. [CrossRef] [PubMed]

41. Koenig, Á.; Diniz, E.M.d.A.; Barbosa, S.F.C.; Vaz, F.A.C. Immunologic factors in human Milk: The effects of gestational age and pasteurization. J. Hum. Lact. 2005, 21, 439-443. [CrossRef] [PubMed]

42. Araújo, E.D.; Gonçalves, A.K.; Cornetta, M.d.C.; Cunha, H.; Cardoso, M.L.; Morais, S.S.; Giraldo, P.C. Evaluation of the secretory immunoglobulin A levels in the colostrum and milk of mothers of term and pre-trerm newborns. Braz. J. Infect. Dis. 2005, 9, 357-362. [CrossRef] [PubMed]

43. Dallas, D.C.; Guerrero, A.; Khaldi, N.; Borghese, R.; Bhandari, A.; Underwood, M.A.; Lebrilla, C.B.; German, J.B.; Barile, D. A peptidomic analysis of human milk digestion in the infant stomach reveals protein-specific degradation patterns. J. Nutr. 2014, 144, 815-820. [CrossRef] [PubMed] 
Article

\title{
Fat Loss in Continuous Enteral Feeding of the Preterm Infant: How Much, What and When Is It Lost?
}

\author{
Carlos Zozaya ${ }^{1, *}$, Alba García-Serrano ${ }^{2}$, Javier Fontecha ${ }^{2}$, Lidia Redondo-Bravo ${ }^{3}$, \\ Victoria Sánchez-González ${ }^{1}$, María Teresa Montes ${ }^{1}$ and Miguel Saenz de Pipaón ${ }^{1,4}$ \\ 1 Neonatology Department, La Paz University Hospital, Autonomous University of Madrid, 28046 Madrid, \\ Spain; vickysg85@hotmail.com (V.S.-G.); maitemontesb@gmail.com (M.T.M.); \\ miguel.saenz@salud.madrid.org (M.S.d.P.) \\ 2 Bioactivity and Food Analysis Department, Institute of Food Science Research (CIAL, CSIC-UAM), \\ Autonomous University of Madrid, 28049 Madrid, Spain; albamaria.garcia.serrano@csic.es (A.G.-S.) \\ j.fontecha@csic.es (J.F.) \\ 3 Preventive Medicine and Public Health Department, La Paz University Hospital, \\ Autonomous University of Madrid, 28046 Madrid, Spain; lidiaredondobravo@gmail.com \\ 4 Carlos III Health Institute, Maternal and Child Health and Development Research Network, \\ 48903 Barakaldo, Bizkaia, Spain \\ * Correspondence: czozayan@gmail.com; Tel.: +34-91-727-74-16
}

Received: 6 June 2018; Accepted: 22 June 2018; Published: 23 June 2018

\begin{abstract}
Human milk fat is a concentrated source of energy and provides essential and long chain polyunsaturated fatty acids. According to previous experiments, human milk fat is partially lost during continuous enteral nutrition. However, these experiments were done over relatively short infusion times, and a complete profile of the lost fatty acids was never measured. Whether this loss happens considering longer infusion times or if some fatty acids are lost more than others remain unknown. Pooled breast milk was infused through a feeding tube by a peristaltic pump over a period of $30 \mathrm{~min}$ and 4, 12 and $24 \mathrm{~h}$ at $2 \mathrm{~mL} / \mathrm{h}$. Adsorbed fat was extracted from the tubes, and the fatty acid composition was analyzed by gas chromatography-mass spectrometry. Total fat loss (average fatty acid loss) after $24 \mathrm{~h}$ was $0.6 \pm 0.1 \%$. Total fat loss after $24 \mathrm{~h}$ infusion was $0.6 \pm 0.1 \%$ of the total fat infused, although the highest losses occur in the first $30 \mathrm{~min}$ of infusion $(13.0 \pm 1.6 \%)$. Short-medium chain $(0.7 \%, p=0.15)$, long chain $(0.6 \%, p=0.56)$, saturated $(0.7 \%, p=0.4)$, monounsaturated $(0.5 \%, p=0.15)$, polyunsaturated fatty $(0.7 \%, p=0.15)$, linoleic $(0.7 \%, p=0.25)$, and docosahexaenoic acids $(0.6 \%, p=0.56)$ were not selectively adsorbed to the tube. However, very long chain fatty $(0.9 \%, p=0.04)$, alpha-linolenic $(1.6 \%, p=0.02)$ and arachidonic acids $(1 \%, p=0.02)$ were selectively adsorbed and, therefore, lost in a greater proportion than other fatty acids. In all cases, the magnitude of the loss was clinically low.
\end{abstract}

Keywords: preterm infant; enteral nutrition; lipids; omega-3 fatty acids; omega-6 fatty acids; Docosahexaenoic acid; Arachidonic acid; long-chain polyunsaturated fatty acids

\section{Introduction}

Fat is an important nutrient for preterm infants [1]. Lipids provide infants most of their energy needs. Lipids also offer specific supplies critical for growth and development like long and very long chain polyunsaturated fatty acids (LCPUFA) including essential fatty acids (Alpha-linolenic and Linoleic acids) and their main derivatives: Docosahexaenoic acid (DHA) and Arachidonic acid (ARA). DHA and ARA seem to be semi-essential for the preterm infant [2]. Both are major components of the brain, and retinal cell membranes and might be related to neurodevelopment and visual function. In case of an early deficit of these fatty acids, there is an increased risk of prevalent preterm 
morbidities, like sepsis and bronchopulmonary dysplasia [3]. Unfortunately, this early deficit might be common, as current nutritional practices (early parenteral nutrition, with lipid emulsions not designed specifically for the preterm infant) do not deliver the same amount of LCPUFA than a fetus of the same gestational ages would receive in-utero [4].

Human milk (HM) is the recommended diet for all infants, including very low birth weight (VLBW) infants. For the latter, HM is usually delivered via an enteral feeding tube until the preterm infant can be fed orally. Continuous enteral feeding is used in the neonatal intensive care unit as an alternative to bolus/gavage feeding in some clinical scenarios (e.g., enteral intolerance or persistent hypoglycemia) [5]. In 1978, Brooke and Barley reported for the first time that human milk fat delivery was reduced when milk was continuously infused [6]. During the 80 's and 90 's different authors had similar results, reporting total fat losses up to $90 \%$ after $8 \mathrm{~h}$ of infusion of freshly collected human milk [7]. To limit fat loss, several strategies have been tested (higher infusion flow rates, syringe angulation, milk refrigeration, use of eccentric nozzle syringes, use of peristaltic syringe pumps, previous and frequent milk homogenization, etc.). These interventions proved to be useful to a certain degree [7-13]. However, concern about fat loss during continuous enteral nutrition is a recurrent issue that still appears to be a problem. Recent research studies have reported total fat losses between 4 and $25 \%$ [13-16]. Whether fat loss is important enough to be clinically relevant in real conditions remains to be clarified. It is important to note that the infusion time of all these studies has been shorter than $8 \mathrm{~h}$, whereas feeding tubes are usually used for longer times in real conditions. Previous reports suggested that lipid losses were not constant over time, but timing of the greater losses (at the beginning of the infusion or later on) is still a controversial issue $[6,15,17,18]$. Moreover, there are other aspects related to fat loss during continuous enteral nutrition that have not yet been investigated. To date, most reports have focused on total fat losses or have only described what happens to lipid fractions (i.e., triglycerides), but we have no data on possible different losses of individual fatty acids depending on fatty acid characteristics (i.e., chain length or degree of unsaturation). Not all fatty acids have the same biological functions, and some of them are essential in humans or semi-essential for the preterm infant.

We conducted an in vitro experiment, which mirrors in vivo current clinical practice, over a 24-h period. Our objectives were: (1) to determine whether fat losses are constant over the infusion time and, if not, when they are more pronounced over the 24-h period; and (2) to test whether there is a selective loss of individual or groups of fatty acids depending on chain length and degree of unsaturation.

\section{Materials and Methods}

Pooled donor, non-pasteurized HM (1100 mL) was used in this experiment. The HM used in this experiment was progressively collected and was kept frozen for a mean period of 2.5 months (range 1.9-4.7) at $-20^{\circ} \mathrm{C}$. It was defrosted before the experiment keeping the sample in refrigeration conditions $\left(5^{\circ} \mathrm{C}\right)$ over $24 \mathrm{~h}$.

The experiment reproduced our standard clinical practices. HM was infused through a 4-French diameter and 40-cm polyvinyl chloride (PVC) di-(2-ethylhexyl) phthalate (DEHP)-free feeding tube (Nutrisafe 2, Vygon, Écouen, France) attached to a PVC system $150 \mathrm{~cm}$ in length and $1.5 \times 2.5 \mathrm{~mm}$ in diameter (Nutrisafe 2, Vygon) by a peristaltic pump (Alaris Enteral, CareFusion, San Diego, CA, USA). The feeding tube and part of the attached system were inside an incubator (Incubator 8000 SC Dräger, Lübeck, Germany). The incubator was set at $33^{\circ} \mathrm{C}$ and $60 \%$ humidity. The syringe, pump and the rest of the connecting systems were outside the incubator. Average room temperature was $23.8^{\circ} \mathrm{C}$ (range $22.7-24.7^{\circ} \mathrm{C}$ ), and the humidity was $36.9 \%$ (range $35-39 \%$ ). The entire experiment was done within the same $24 \mathrm{~h}$. HM was loaded into 20-mL syringes for enteral nutrition. Then the syringe was hand shaken to homogenize the milk and placed in the pump, with the syringe maintained in a vertical position (tip upwards). Infusions then were programmed at $2 \mathrm{~mL} / \mathrm{h}$. When infusion finished, feeding tubes and systems were washed with a distilled water bolus $(5 \mathrm{~mL})$ to remove the remaining milk. Then, the feeding tubes and connecting systems were collected and immediately stored at $-20^{\circ} \mathrm{C}$. 
This whole procedure was repeated infusing milk over $30 \mathrm{~min}, 4,12$ and $24 \mathrm{~h}$, in quadruplicate for each infusion time. To reproduce a real 12 and 24-h infusion, both the HM and the syringes were changed every $4 \mathrm{~h}$. The pooled $\mathrm{HM}$ was kept refrigerated $\left(5^{\circ} \mathrm{C}\right)$ during the experiment day. Aliquots $(20 \mathrm{~mL})$ were extracted and then left at room temperature for $30 \mathrm{~min}$ before filling the syringes, to warm the HM, according to our standard practice. Pre-infusion HM samples were collected at time zero, and at 12 and $24 \mathrm{~h}$ from the beginning of the experiment. Following collection, the milk samples were stored at $-20{ }^{\circ} \mathrm{C}$.

\subsection{Total Fat Extraction and GC-MS Analysis}

The milk fat adsorbed inside the tubes was extracted using high-performance liquid chromatography-grade hexane as a solvent. More commonly used extraction solutions, such as chloroform 2/methanol 1 (Folch) and hexane/isopropanol (Hara and Radin), were initially used, but they extracted silicones from the tube's inner surfaces, contaminating the sample and raising concerns about chromatogram reliability. We subsequently verified that hexane allows extraction of the total fat, given no compounds of dairy fatty acids were detected at the retention times when the tubes were ultimately washed according to the Folch [19] or the Hara and Radin method [20]. Blank control tubes $(n=3)$ were washed with the same solvent but without having passed any milk.

The lipid extracts obtained were concentrated by removing the organic solvent under a gentle stream of nitrogen. Then, the lipid extracts were weighed and analyzed as fatty acid methyl esters (FAMEs) obtained by direct derivatization of samples, as described by Castro-Gómez et al. [21]. Briefly, lipid extracts were transferred to borosilicate glass tubes with an acid/heat resistant cap containing $100 \mu \mathrm{L}$ of tritridecanoin in hexane as internal standard $(1.3 \mathrm{mg} / \mathrm{mL})$. Then, $1 \mathrm{~mL}$ of $3 \mathrm{M} \mathrm{H}_{2} \mathrm{SO}_{4}$ in methanol was added to each tube and heated for $30 \mathrm{~min}$ at $98^{\circ} \mathrm{C}$. After incubation, the samples were cooled in ice for $5 \mathrm{~min}$, and $1 \mathrm{~mL}$ of hexane was added. The samples were vortexed for $30 \mathrm{~s}$, and the reaction was then stopped with $7.5 \mathrm{~mL}$ of $6 \%$ solution of sodium hydrogen carbonate and centrifuged at $1000 \times g$, at $4{ }^{\circ} \mathrm{C}$, for $5 \mathrm{~min}$. The upper organic layer containing the FAME was collected and transferred to amber vials for GC-MS injection and $1 \mu \mathrm{L}$ (at 1:10 split ratio) was injected into a 6890 Agilent gas chromatograph (Palo Alto, CA, USA) fitted with a mass spectrometry (MS) (Agilent $5973 \mathrm{~N}$ ) detector in a 100-m CPSil-88 capillary column $(100 \mathrm{~m} \times 0.25 \mathrm{~mm}$ inner diameter $\times 0.2 \mu \mathrm{m}$ film thickness (Chrompack, Middelburg, The Netherlands). The GC-MS temperature program and conditions were those previously reported by Rodriguez-Alcala and Fontecha [22]. Briefly, the column was maintained at $100{ }^{\circ} \mathrm{C}$ for $1 \mathrm{~min}$ after injection and temperature-programmed at $7{ }^{\circ} \mathrm{C} / \mathrm{min}$ to $170{ }^{\circ} \mathrm{C}$, maintained there for $55 \mathrm{~min}$, and then raised $10^{\circ} \mathrm{C} / \mathrm{min}$ to $230^{\circ} \mathrm{C}$ and maintained there for $33 \mathrm{~min}$. The injector temperature was set at $250{ }^{\circ} \mathrm{C}$. Helium was used as carrier gas with a column inlet pressure of $30 \mathrm{psi}$. MS detector conditions were a transfer line temperature of $250{ }^{\circ} \mathrm{C}$, a source temperature of $230^{\circ} \mathrm{C}$, a quad temperature of $150^{\circ} \mathrm{C}$ and electron impact ionization at $70 \mathrm{eV}$. For peak identification, mass spectra obtained in our analysis were compared with those in the National Institute of Standards and Technology Library (Gaithersburg, MD, USA). For the qualitative and quantitative analyses, response factors were calculated using anhydrous milk fat (reference material BCR-164) and Supelco 37 FAME mix (Sigma, St. Louis, MO, USA). Tritridecanoin as internal standard (200 $\mu \mathrm{L} ; 1.3 \mathrm{mg} / \mathrm{mL}$ ) was also used. Assays were performed in triplicate.

Losses of total fat and fatty acids at each time point were expressed as percentages of the total infused amount recovered from the tube. Percentages were calculated according to the formula:

$$
\% \text { Loss }=\mathrm{R} \times 100 /(\mathrm{C} \times \mathrm{V}),
$$

where " $\mathrm{R}$ " stands for the raw amount of fatty acid / total fat $(\mathrm{mg})$ recovered from the tube, " $\mathrm{C}$ " means milk's fatty acid concentration $(\mathrm{mg} / \mathrm{mL})$ and " $\mathrm{V}$ " is the volume infused through the tube over the set time (1 $\mathrm{mL}$ over $30 \mathrm{~min}, 8,24$ and $48 \mathrm{~mL}$ over 4,12 and $24 \mathrm{~h}$, respectively). 
A fatty acid was classified as very-long-chain fatty acid (VLCFA) if it had $>18$ carbon atoms, long-chain fatty acid (LCFA) when it had 16 or 18 carbon atoms and short-medium chain fatty acid (SMCFA) if it had between 6 and 14 carbon atoms.

\subsection{Statistical Analysis}

The statistical analysis was performed using SPSS 20 statistical software (IBM Corporation, Armonk, NY, USA). Descriptive data are presented as mean ( \pm standard deviation) and frequency (\%) as appropriate. The Mann-Whitney $\mathrm{U}$ test was used to calculate median differences between total fat (which is equivalent to mean fatty acid loss) and individual/families of fatty acid rate losses at $24 \mathrm{~h}$. The Kruskal-Wallis rank test was performed to compare median losses among more than two groups according to the fatty acid composition in various time periods ( $30 \mathrm{~min}, 4,12$ and $24 \mathrm{~h}$ ). A selected one-to-one post hoc analysis was performed correcting significance according to the Dunn-Bonferroni method. Losses of total fat (average fatty acid loss) and individual selected fatty acids over time were analyzed with a curvilinear regression model. For these models, we selected the most abundant fatty acids in the HM or those which seem to be more clinically relevant (essential fatty acids and LCPUFA).

\subsection{Ethical Issues}

The La Paz University Hospital research ethics committee approved the study, and our donors provided informed consent to use their milk for research purposes.

\section{Results}

The fatty acid composition of the pooled HM used in this study is described in Table 1. Samples were analyzed at time zero, 12 and $24 \mathrm{~h}$ on the experiment day to rule out oxidative changes affecting the relative fatty acid composition of the HM before it was infused. There were no statistically significant differences regarding saturated fatty acids (SFA), monounsaturated fatty acids (MUFA) and polyunsaturated fatty acids (PUFA) nor regarding chain length (short-medium, long and very long fatty acids) relative composition. Furthermore, individual LCPUFA: alpha-linolenic acid (ALA), linoleic acid (LNA), DHA and ARA concentrations (mg/100 mg of fat) remained stable throughout the study period of $24 \mathrm{~h}$ (differences were not statistically significant).

Table 1. The fatty acid composition of the pooled human milk used in this experiment.

\begin{tabular}{cc}
\hline Fatty Acids & $\mathbf{m g} / \mathbf{1 0 0} \mathbf{~} \mathbf{g}$ of Fat \\
\hline $6: 0$ & $0.2 \pm 0.10$ \\
$8: 0$ & $0.3 \pm 0.10$ \\
$10: 0$ & $1.4 \pm 0.20$ \\
$12: 0$ & $5 \pm 0.30$ \\
$14: 0$ & $5.2 \pm 0.10$ \\
$15: 0$ & $0.2 \pm 0.02$ \\
$16: 0$ & $21.5 \pm 0.20$ \\
$17 \mathrm{ai}$ & $0.1 \pm 0.01$ \\
$16: 1 \mathrm{t}$ & $0.3 \pm 0.03$ \\
$16: 1 \mathrm{n} 7$ & $1.3 \pm 0.10$ \\
$17: 0$ & $0.2 \pm 0.02$ \\
$18: 0$ & $8.3 \pm 0.20$ \\
$18: 1 \mathrm{n} 9$ & $40.6 \pm 0.30$ \\
$18: 1$ n11 & $1.7 \pm 0.10$ \\
18:2 n6 (LNA) & $11.6 \pm 0.20$ \\
20:0 & $0.1 \pm 0.02$ \\
18:3 n3 (ALA) & $0.3 \pm 0.05$ \\
\hline
\end{tabular}


Table 1. Cont.

\begin{tabular}{|c|c|}
\hline Fatty Acids & $\mathrm{mg} / 100 \mathrm{mg}$ of Fat \\
\hline 20:1 n9 & $0.3 \pm 0.10$ \\
\hline $20: 3 \mathrm{n} 6$ & $0.15 \pm 0.10$ \\
\hline 20:4 n6 (ARA) & $0.2 \pm 0.03$ \\
\hline $22: 6$ n3 (DHA) & $0.25 \pm 0.02$ \\
\hline Traces $(<0.2 \%)$ & $0.85 \pm 0.20$ \\
\hline$\sum$ SFA & $42.6 \pm 0.60$ \\
\hline$\sum \overline{M U F A}$ & $44.8 \pm 0.30$ \\
\hline$\sum$ PUFA & $12.5 \pm 0.30$ \\
\hline$\sum$ SMCFA & $12.4 \pm 0.70$ \\
\hline$\sum$ LCFA & $86.4 \pm 0.50$ \\
\hline$\sum$ VLCFA & $1.2 \pm 0.20$ \\
\hline
\end{tabular}

ALA: $\alpha$-linolenic acid; LNA: linoleic acid; AA: arachidonic acid; DHA: docosahexaenoic acid; SFA: saturated fatty acid; MUFA: monounsaturated fatty acid; PUFA: polyunsaturated fatty acid; SMCFA: short-medium chain fatty acids; LCFA: long-chain fatty acid, and VLCFA: Very long chain fatty acid.

Over $24 \mathrm{~h}$, the mean total fat loss was $0.6 \pm 0.1 \%$ of the total fat infused. However, fat loss was not constant. The highest fat losses occur in the first $30 \mathrm{~min}$ of infusion $(13.0 \pm 1.6 \%)$ and then fat loss progressively decreased at 4 and $12 \mathrm{~h}$ to $2 \% \pm 0.4 \%$ and $0.87 \% \pm 0.04 \%$, respectively $\left(R^{2}=0.98\right.$; $p<0.0001$ ) (Figure 1).

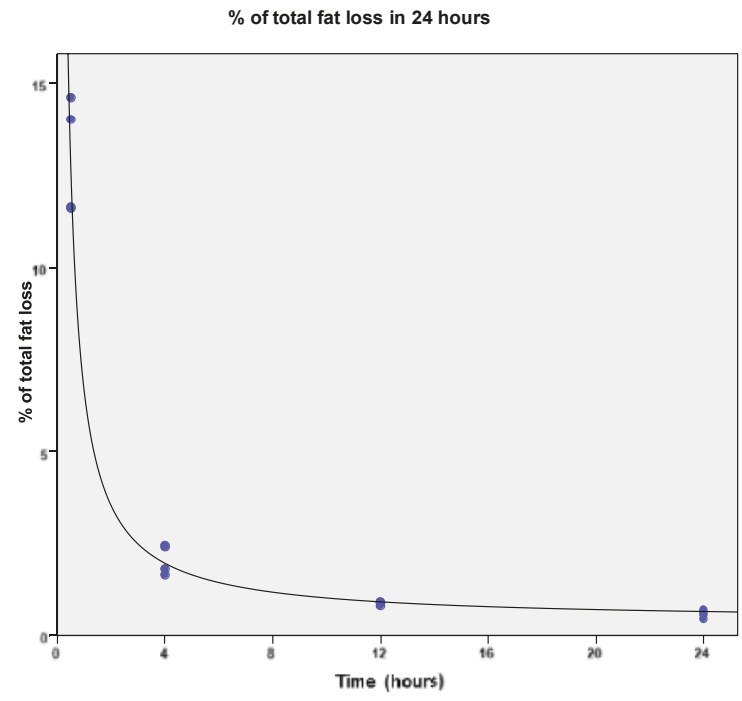

$Y=0.381+6.294 / X$

Figure 1. Amount of the total fat loss as \% of the fat adsorbed in the tube in relation with the total fat infused during $24 \mathrm{~h}$.

Fatty acids were lost in different percentages, at different time points, according to the degree of unsaturation of the fatty acid chain (Table 2). Saturated fatty acids were lost in a higher proportion than monounsaturated fatty acids at $30 \mathrm{~min}(30.6 \%$ vs. $5.8 \%, p=0.01)$ and $12 \mathrm{~h}$ of infusion $(1.1 \%$ vs. $0.6 \%, p=0.02$ ), whereas we found no differences when comparing saturated or monounsaturated with polyunsaturated fatty acids. At $24 \mathrm{~h}$, there were no significant differences among the three groups. 
Table 2. Percentage of fatty acid loss (\% of the total amount of FA initially infused that was recovered from the tube after the infusion time).

\begin{tabular}{ccccc}
\hline Fatty Acid & $\mathbf{3 0} \mathbf{~ m i n}$ & $\mathbf{4 h}$ & $\mathbf{1 2} \mathbf{h}$ & $\mathbf{2 4} \mathbf{h}$ \\
\hline $6: 0$ & $80.4 \pm 13.2$ & $8.3 \pm 2.90$ & $2.0 \pm 0.30$ & $1.2 \pm 0.20$ \\
$8: 0$ & $26.4 \pm 0.60$ & $4.5 \pm 0.80$ & $1.7 \pm 0.10$ & $1.1 \pm 0.30$ \\
$10: 0$ & $23.8 \pm 8.20$ & $5.5 \pm 1.10$ & $2.4 \pm 0.40$ & $1.5 \pm 0.40$ \\
$12: 0$ & $9.5 \pm 1.80$ & $2.4 \pm 0.60$ & $1.1 \pm 0.10$ & $0.8 \pm 0.10$ \\
$14: 0$ & $9.0 \pm 0.01$ & $1.3 \pm 0.40$ & $0.5 \pm 0.10$ & $0.4 \pm 0.10$ \\
$15: 0$ & $13.6 \pm 3.80$ & $2.0 \pm 0.50$ & $0.6 \pm 0.20$ & $0.4 \pm 0.10$ \\
$16: 0$ & $24.0 \pm 1.60$ & $2.9 \pm 0.40$ & $1.0 \pm 0.10$ & $0.6 \pm 0.10$ \\
$16: 1 \mathrm{n} 7$ & $8.0 \pm 2.00$ & $2.1 \pm 0.80$ & $1.1 \pm 0.10$ & $0.8 \pm 0.20$ \\
$17: 0$ & $23.4 \pm 2.00$ & $2.8 \pm 0.50$ & $1 \pm 0.10$ & $0.6 \pm 0.10$ \\
$18: 0$ & $31.4 \pm 2.10$ & $3.5 \pm 0.60$ & $1.2 \pm 0.10$ & $0.7 \pm 0.10$ \\
$18: 1 \mathrm{n} 9$ & $5.6 \pm 1.80$ & $1.3 \pm 0.50$ & $0.6 \pm 0.10$ & $0.5 \pm 0.10$ \\
$18: 1 \mathrm{n} 11$ & $4.7 \pm 2.00$ & $1.1 \pm 0.50$ & $0.5 \pm 0.10$ & $0.4 \pm 0.10$ \\
$18: 2$ n6 (LNA) & $7 \pm 2.10$ & $1.8 \pm 0.70$ & $0.9 \pm 0.10$ & $0.7 \pm 0.10$ \\
$18: 3$ n3 (ALA) & $10.5 \pm 2.60$ & $3.8 \pm 1.80$ & $2.2 \pm 0.30$ & $1.6 \pm 0.30$ \\
$20: 0$ & $64.4 \pm 5.20$ & $10.2 \pm 2.10$ & $3.4 \pm 0.50$ & $2.1 \pm 0.60$ \\
$20: 3$ n6 & $9.5 \pm 4.20$ & $2.5 \pm 1.00$ & $1.3 \pm 0.20$ & $0.9 \pm 0.10$ \\
$20: 4$ n6 (ARA) & $11.6 \pm 3.60$ & $3.0 \pm 1.00$ & $1.5 \pm 0.20$ & $1.0 \pm 0.20$ \\
$22: 6$ n3 (DHA) & $8.4 \pm 2.10$ & $1.6 \pm 0.80$ & $0.8 \pm 0.20$ & $0.6 \pm 0.10$ \\
$\sum$ SFA & $30.6 \pm 2.60 \mathrm{a}$ & $2.9 \pm 0.40$ & $1.1 \pm 0.10 \mathrm{~b}$ & $0.7 \pm 0.10$ \\
$\sum$ MUFA & $5.8 \pm 1.30 \mathrm{a}$ & $1.3 \pm 0.50$ & $0.6 \pm 0.10 \mathrm{~b}$ & $0.5 \pm 0.10$ \\
$\sum$ PUFA & $7.5 \pm 2.00$ & $1.9 \pm 0.70$ & $1.0 \pm 0.10$ & $0.7 \pm 0.10$ \\
$p$-value * & 0.015 & 0.06 & 0.02 & 0.08 \\
$\sum$ SMCFA & $13.8 \pm 6.10$ & $2.4 \pm 0.50$ & $1.0 \pm 0.10$ & $0.7 \pm 0.10$ \\
$\sum$ LCFA & $14 \pm 4.50$ & $2.0 \pm 0.40 \mathrm{c}$ & $0.8 \pm 0.01 \mathrm{~d}$ & $0.6 \pm 0.10 \mathrm{e}$ \\
$\sum$ VLCFA & $23.5 \pm 2.50$ & $3.7 \pm 0.50 \mathrm{c}$ & $1.4 \pm 0.20 \mathrm{~d}$ & $0.9 \pm 0.20 \mathrm{e}$ \\
$p$-value * & 0.09 & 0.02 & 0.007 & 0.04 \\
\hline
\end{tabular}

Results are expressed as mean $\% \pm$ standard deviation. Distributions were compared for degree of unsaturation and length chain at every time point by Kruskal-Wallis test ( ${ }^{*} p$-value is presented). A, b, c, d and e, indicate significant differences in the one to one post-hoc analysis (Dunn-Bonferroni test). ALA: $\alpha$-linolenic acid; LNA: linoleic acid; AA: arachidonic acid; DHA: docosahexaenoic acid; SFA: saturated fatty acid; MUFA: monounsaturated fatty acid; PUFA: polyunsaturated fatty acid; SMCFA: short-medium chain fatty acids; LCFA: long-chain fatty acid; VLCFA: Very long chain fatty acid.

Regarding the length of the chain, fatty acids were lost differently from $4 \mathrm{~h}$ of infusion onwards (Table 2). VLCFA loss was higher than that of LCFA at $4 \mathrm{~h}(3.7 \%$ vs. $2 \%, p=0.02)$, at $12 \mathrm{~h}(1.4 \%$ vs. $0.8 \%, p=0.005)$ and, at $24 \mathrm{~h}(0.9 \%$ vs. $0.6 \%, p=0.03)$. We did not find differences between VLCFA and SMCFA or between SMCFA and LCFA at any time.

We also studied the loss of some individual fatty acids. The most abundant fatty acids in the HM (16:0 and 18:1n-9) or those which are especially relevant for the preterm infant (essential fatty acids - LNA and ALA — and their derivatives DHA and ARA) were selected. Total fat loss after $24 \mathrm{~h}$ was considered as a reference. Some fatty acids were not lost in a higher proportion than total fat, as in the cases of $18: 1 \mathrm{n}-9(0.5 \% \pm 0.1 \%, p=0.15)$, LNA $(0.7 \% \pm 0.1 \%, p=0.25)$ and DHA $(0.6 \% \pm 0.1 \%$, $p=0.56)$. However, in other cases, losses of some fatty acids were significantly greater than total fat loss, as with ALA $(1.6 \% \pm 0.3 \%, p=0.02)$ and ARA $(1 \% \pm 0.2 \%, p=0.02)$. In all cases, the magnitude of the loss was small after $24 \mathrm{~h}$ (Table 2). In Table 3 we presented curvilinear regression models predicting losses after $24 \mathrm{~h}$ of these selected fatty acids. 
Table 3. Curvilinear regression models to predict loss of some selected fatty acids after $24 \mathrm{~h}$ of HM infusion. ALA: $\alpha$-linolenic acid; LNA: linoleic acid; AA: arachidonic acid; DHA: docosahexaenoic acid.

\begin{tabular}{cccc}
\hline Fatty Acids & Coefficient of Determination $\left.\mathbf{( R}^{2}\right)$ & Curvilinear Model Equation & $p$-Value \\
\hline $16: 0$ & 0.99 & $\mathrm{y}=-0.007+11.98 / \mathrm{x}$ & $<0.0001$ \\
$18: 1$ n9 & 0.86 & $\mathrm{y}=0.477+2.562 / \mathrm{x}$ & $<0.0001$ \\
$18: 2$ n6 (LNA) & 0.87 & $\mathrm{y}=0.732+3.143 / \mathrm{x}$ & $<0.0001$ \\
$18: 3 \mathrm{n} 3$ (ALA) & 0.85 & $\mathrm{y}=1.969+4.285 / \mathrm{x}$ & $<0.0001$ \\
20:4 n6 (ARA) & 0.87 & $\mathrm{y}=1.177+5.257 / \mathrm{x}$ & $<0.0001$ \\
22:6 n3 (DHA) & 0.92 & $\mathrm{y}=0.494+3.985 / \mathrm{x}$ & $<0.0001$ \\
\hline
\end{tabular}

\section{Discussion}

How much HM fat is lost during continuous enteral feeding, and when this loss occurs during the infusion, is controversial. In addition, there are no data in the literature about what is exactly lost, in terms of individual fatty acid losses. To answer these questions, we have introduced three different elements in our experimental design, compared to previous studies:

\subsection{Our Total Experiment Time Was Longer (up to 24 h of Infusion), Although We Still Included Intermediate Times}

Our first objective was to determine whether the previously reported fat losses percentages could be related, at least to a certain degree, with the duration of the experiments. Some authors have suggested losses were higher only after $4 \mathrm{~h}$ [17] or even after $8 \mathrm{~h}$ of infusion [18]. However, another recent article suggested that losses appear to be higher at the beginning of the infusion [15], although the experiments in that particular study did not last more than $60 \mathrm{~min}$. Our results are consistent with this last report and show that the duration of the feeding affects overall fat delivery efficiency. Significant fat loss in the first $30 \mathrm{~min}$ of infusion suggests that binding sites in the tubes become saturated at the beginning of the infusion. Later, percentage of fat loss is smaller maybe because there are not as many available binding sites. Thus, after some time of infusion, fat delivery efficiency increases, even though infusion flow velocity remains the same. Experiment duration has varied in previous studies but, to our knowledge, it has never been longer than $8 \mathrm{~h}$. However, feeding tubes are changed every 48-72 h in real practice [23]. Thus, we designed a study over a 24-h period to mimic longer infusion times, which is closer to actual practices. We did not prolong to $48-72 \mathrm{~h}$ to avoid potential fatty acid oxidation of the breast milk during the experiment. As we used the same pooled milk during the whole experiment, fatty acid oxidation could have limited our conclusions, because the pre-infusion milk would have become somewhat "different" during the experiment. We can assure that in our case this change in the milk fatty acid composition did not occur because the milk fatty acid profile remained unchanged at time zero, mid and end of the study day. The velocity of milk flow is another important factor associated with fat loss. The lower the velocity, the higher the losses [24]. We chose a very slow flow $(2 \mathrm{~mL} / \mathrm{h}$ ) to reproduce a worse-case-possible real scenario (less than $2 \mathrm{~mL} / \mathrm{h}$ would represent trophic feeds for many preterm infants). However, we adhered to the best standard of care, and we also implemented some proven preventive measures: an upward tip position and gentle homogenization of the milk before infusion, to minimize the loss as we would do in our patients $[8,13]$.

\subsection{Direct Measurement of Adhered Fat to Nasogastric Tubes Instead of a Pre-and Post-Infused Milk Analysis}

We recovered the fat remaining in the tube after the HM infusion and then, we determined the fatty acid composition on these samples. On the other hand, in previous studies, the milk coming out of the tubes was collected and then, the percentage of fat lost was calculated knowing the composition of the milk going in. We believe direct determination is more accurate than calculation. Moreover, we have proved this method is feasible. So far, no clinical studies involving real patients have been done. Therefore, the ultimate clinical relevance of fat loss during continuous enteral nutrition has never been reported. Our method could be used in future clinical studies, to measure fat loss affecting 
real patients, collecting feeding tubes after having been used in real patients, instead of recreating clinical practices in experimental conditions. Doing so, we could eventually relate fat losses with clinical outcomes.

\subsection{Outcome Variables (not only Total Fat but also Individual Fatty Acids)}

To our knowledge, this is the first report of the fatty acid composition of HM fat loss during continuous enteral nutrition. We speculate that the more fluid a fatty acid, the more easily it flows through the tube. Saturated and longer chain fatty acids were the more retained fatty acids over short infusion times (which would be the beginning of the continuous infusion in a real patient). This finding could be related to the fatty acid structure, which determines its fluidity [25]. Unsaturated fatty acids have lower melting points than saturated fatty acids of the same length. In other words, saturated fatty acids are less fluid. Also, the longer a fatty acid is, the less its fluidity.

This study has some limitations. We only measured fat adhered to the feeding and connecting tubes but not to the syringes. Nevertheless, the capacity of the syringe is greater, and contact between the milk and binding sites of its surface is less likely, so we think its effect would be neglectable. Only one flow rate $(2 \mathrm{~mL} / \mathrm{h})$ was tested. We cannot extrapolate our results directly to other flow speeds. However, we assumed that at higher velocities losses would be less based on previous studies. Finally, we did not run milk for more than $24 \mathrm{~h}$, although feeding tubes are used for longer times before being replaced in real patients. We do not know what the actual fat delivery would be after $48-72 \mathrm{~h}$ using the same feeding tube. However, our regression model suggests that as of $12 \mathrm{~h}$ of infusion the losses remain somewhat constant and low.

According to our results, the clinical significance of $\mathrm{HM}$ fat loss after $24 \mathrm{~h}$ of continuous infusion seems to be trivial, both quantitatively and qualitatively. When fat is oxidized, every gram of it produces $9 \mathrm{kcal}$. Considering our results, if an infant is fed $120-150 \mathrm{~mL} / \mathrm{kg} /$ day in continuous enteral infusion, fat loss would mean $0.2-0.3 \mathrm{kcal} / \mathrm{kg} /$ day. This represents around $0.2-0.25 \%$ of the total caloric intake recommended for a preterm infant on enteral nutrition. Regarding quality does not seem to be clinically significant either. As stated previously, LCPUFA delivery to the preterm infant is insufficient following current nutritional recommendations [26]; but this problem does not appear to be worsened significantly by additional fat losses during continuous enteral nutrition. Mean HM fat content is $3.2 \mathrm{~g}-3.6 \mathrm{~g} / 100 \mathrm{~mL}$ [27]. Worldwide, DHA and ARA fatty acids represent $0.32 \% \pm 0.22 \%$ and $0.47 \% \pm 0.13 \%$ of HM fat, respectively [28]. Fetal accretion rates are $95 \mathrm{mg}$ of ARA and $42 \mathrm{mg}$ of DHA per day during the last five weeks of gestation [29]. Thus, losses in continuous enteral feeding over 24-h infusion in a $1.5 \mathrm{~kg}$ infant would lead to a daily loss of $0.1-0.15 \mathrm{mg}$ of DHA and 0.3-0.4 mg of ARA.

\section{Conclusions}

We conclude that continuous enteral feeding over $24 \mathrm{~h}$ resulted in no substantial loss of human milk fat. Therefore, feeding over a 24-h period does not appear to be a barrier to the delivery of fatty acids, including DHA and ARA fatty acids.

Author Contributions: C.Z., J.F. and M.S.d.P. conceptualized the study; C.Z., V.S.-G. and M.T.M. performed the experiment and collected the samples; A.G.-S. and J.F. did the laboratory determinations; L.R.-B. work on the statistical analysis; C.Z. drafted the manuscript, which was critically reviewed by the rest of the authors.

Funding: This research received no external funding.

Acknowledgments: We thank the technician Javier Megino and all Hospital La Paz NICU nurses and auxiliary nurses for their valuable assistance. We also thank the mothers who donate the milk to conduct this research.

Conflicts of Interest: The authors declare no conflict of interest. 


\section{References}

1. Agostoni, C.; Buonocore, G.; Carnielli, V.P.; De Curtis, M.; Darmaun, D.; Decsi, T.; Domellöf, M.; Embleton, N.D.; Fusch, C.; Genzel-Boroviczeny, O.; et al. Enteral nutrient supply for preterm infants: Commentary from the European Society of Paediatric Gastroenterology, Hepatology and Nutrition Committee on Nutrition. J. Pediatr. Gastroenterol. Nutr. 2010, 50, 85-91. [CrossRef] [PubMed]

2. Martin, C.R. Fatty acid requirements in preterm infants and their role in health and disease. Clin. Perinatol. 2014, 41, 363-382. [CrossRef] [PubMed]

3. Martin, C.R.; Dasilva, D.A.; Cluette-Brown, J.E.; Dimonda, C.; Hamill, A.; Bhutta, A.Q.; Coronel, E.; Wilschanski, M.; Stephens, A.J.; Driscoll, D.F.; et al. Decreased postnatal docosahexaenoic and arachidonic acid blood levels in premature infants are associated with neonatal morbidities. J. Pediatr. 2011, 159, 743-749. [CrossRef] [PubMed]

4. Lapillonne, A.; Eleni Dit Trolli, S.; Kermorvant-Duchemin, E. Postnatal docosahexaenoic acid deficiency is an inevitable consequence of current recommendations and practice in preterm infants. Neonatology 2010, 98, 397-403. [CrossRef] [PubMed]

5. Klingenberg, C.; Embleton, N.D.; Jacobs, S.E.; O'Connell, L.A.F.; Kuschel, C.A. Enteral feeding practices in very preterm infants: An international survey. Arch. Dis. Child. 2012, 97, F56-F61. [CrossRef] [PubMed]

6. Brooke, O.; Barley, J. Loss of energy during continuous infusions of breast milk. Arch. Dis. Child. 1978, 53, 344-345. [CrossRef] [PubMed]

7. Lavine, M.; Clark, R.M. The effect of short-term refrigeration of milk and addition of breast milk fortifier on the delivery of lipids during tube feeding. J. Pediatr. Gastroenterol. Nutr. 1989, 8, 496-499. [CrossRef] [PubMed]

8. Spencer, S.A.; Hull, D. Fat content of expressed breast milk: A case for quality control. Br. Med. J. 1981, 282, 99-100. [CrossRef]

9. Narayanan, I.; Singh, B.; Harvey, D. Fat loss during feeding of human milk. Arch. Dis. Child. 1984, 59, 475-477. [CrossRef] [PubMed]

10. Greer, F.R.; McCormick, A.; Loker, J. Changes in fat concentration of human milk during delivery by intermittent bolus and continuous mechanical pump infusion. J. Pediatr. 1984, 105, 745-749. [CrossRef]

11. Martinez, F.E.; Desai, I.D.; Davidson, A.G.; Nakai, S.R.A. Ultrasonic homogenization of expressed human milk to prevent fat loss during tube feeding. J. Pediatr. Gastroenterol. Nutr. 1987, 6, 593-597. [CrossRef] [PubMed]

12. Rayol, M.R.S.; Martinez, F.E.; Jorge, S.M.; Goncalves, A.L.; Desai, I.D. Feeding premature infants banked human milk homogenized by ultrasonic treatment. J. Pediatr. 1993, 123, 985-988. [CrossRef]

13. García-Lara, N.R.; Escuder-Vieco, D.; Alonso Díaz, C.; Vázquez Román, S.; De la Cruz-Bértolo, J.; Pallás-Alonso, C.R. Type of Homogenization and Fat Loss during Continuous Infusion of Human Milk. J. Hum. Lact. 2014, 30, 436-441. [CrossRef] [PubMed]

14. Igawa, M.; Murase, M.; Mizuno, K.; Itabashi, K. Is fat content of human milk decreased by infusion? Pediatr. Int. 2014, 56, 230-233. [CrossRef] [PubMed]

15. Tabata, M.; Abdelrahman, K.; Hair, A.; Hawthorne, K.; Chen, Z.; Abrams, S. Fortifier and Cream Improve Fat Delivery in Continuous Enteral Infant Feeding of Breast Milk. Nutrients 2015, 7, 1174-1183. [CrossRef] [PubMed]

16. Jarjour, J.; Juarez, A.; Kocak, D.; Liu, N.J.; Tabata, M.M.; Hawthorne, K.M.; Ramos, R.F.; Abrams, S.A. A Novel Approach to Improving Fat Delivery in Neonatal Enteral Feeding. Nutrients 2015, 7, 5051-5064. [CrossRef] [PubMed]

17. Chan, M.M.; Nohara, M.; Chan, B.R.; Curtis, J.; Chan, G.M. Lecithin decreases human milk fat loss during enteral pumping. J. Pediatr. Gastroenterol. Nutr. 2003, 36, 613-615. [CrossRef] [PubMed]

18. Lemons, P.M.; Miller, K.; Eitzen, H.; Strodtbeck, F.; Lemons, J.A. Bacterial growth in human milk during continuous feeding. Am. J. Perinatol. 1983, 1, 76-80. [CrossRef] [PubMed]

19. Folch, J.M.; Lees, S.; Loane, S. A simple method for the isolation and purification of total lipides from animal tissues. J. Biol. Chem. 1957, 226, 497-509. [PubMed]

20. Hara, A.; Radin, N.S. Lipid extraction of tissues with a low-toxicity solvent. Anal. Biochem. 1978, 90, 420-426. [CrossRef] 
21. Castro-Gómez, P.; Fontecha, J.; Rodríguez-Alcalá, L.M. A high-performance direct transmethylation method for total fatty acids assessment in biological and foodstuff samples. Talanta 2014, 128, 518-523. [CrossRef] [PubMed]

22. Rodríguez-Alcalá, L.M.; Fontecha, J. Hot topic: Fatty acid and conjugated linoleic acid (CLA) isomer composition of commercial CLA-fortified dairy products: Evaluation after processing and storage. J. Dairy Sci. 2007, 90, 2083-2090. [CrossRef] [PubMed]

23. Hurrell, E.; Kucerova, E.; Loughlin, M.; Caubilla-Barron, J.; Hilton, A.; Armstrong, R.; Smith, C.; Grant, J.; Shoo, S.; Forsythe, S. Neonatal enteral feeding tubes as loci for colonisation by members of the Enterobacteriaceae. BMC Infect. Dis. 2009, 9, 146. [CrossRef] [PubMed]

24. Mehta, N.R.; Hamosh, M.; Bitman, J.; Wood, D.L. Adherence of medium-chain fatty acids to feeding tubes during gavage feeding of human milk fortified with medium-chain triglycerides. J. Pediatr. 1988, 112, 474-476. [CrossRef]

25. Mostofsky, D.I.; Yehuda, S.; Salem, N. Fatty Acids: Physiological and Behavioral Functions; Humana Press: New York, NY, USA, 2001.

26. Lapillonne, A.; Groh-Wargo, S.; Lozano Gonzalez, C.H.; Uauy, R. Lipid needs of preterm infants: Updated recommendations. J. Pediatr. 2013, 162, S37-S47. [CrossRef] [PubMed]

27. Ballard, O.; Morrow, A.L. Human Milk Composition. Pediatr. Clin. N. Am. 2013, 60, 49-74. [CrossRef] [PubMed]

28. Brenna, J.T.; Varamini, B.; Jensen, R.G.; Diersen-Schade, D.A.; Boettcher, J.A.; Arterburn, L.M. Docosahexaenoic and arachidonic acid concentrations in human breast milk worldwide. Am. J. Clin. Nutr. 2007, 85, 1457-1464. [CrossRef] [PubMed]

29. Kuipers, R.S.; Luxwolda, M.F.; Offringa, P.J.; Rudi Boersma, E.; Dijck-Brouwer, D.A.; Muskiet, F.A. Fetal intrauterine whole body linoleic, arachidonic and docosahexaenoic acid contents and accretion rates. Prostaglandins Leukot. Essent. Fat. Acids 2012, 86, 13-20. [CrossRef] [PubMed]

(C) 2018 by the authors. Licensee MDPI, Basel, Switzerland. This article is an open access article distributed under the terms and conditions of the Creative Commons Attribution (CC BY) license (http:/ / creativecommons.org/licenses/by/4.0/). 
Article

\title{
The Impact of Maternal Diet during Pregnancy and Lactation on the Fatty Acid Composition of Erythrocytes and Breast Milk of Chilean Women
}

\author{
Cynthia Barrera ${ }^{1}$, Rodrigo Valenzuela ${ }^{1,2, *}$, Rodrigo Chamorro ${ }^{1}$, Karla Bascuñán ${ }^{1}$, \\ Jorge Sandoval ${ }^{2,3}$, Natalia Sabag ${ }^{1}$, Francesca Valenzuela ${ }^{1}$, María-Paz Valencia ${ }^{1}$, \\ Claudia Puigrredon ${ }^{2,3}$ and Alfonso Valenzuela ${ }^{2}$ \\ 1 Department of Nutrition, Faculty of Medicine, University of Chile, Av. Independencia 1027, Independencia, \\ Casilla 70000, Santiago 8380453, Chile; cynthia.barrera@gmail.com (C.B.); \\ rodrigochamorro@med.uchile.cl (R.C.); kbascunan@med.uchile.cl (K.B.); n.sabag.c@gmail.com (N.S.); \\ francesca.valenzuelar@gmail.com (F.V.); mpvalencia@ug.uchile.cl (M.-P.V.) \\ 2 Lipid Center, Institute of Nutrition and Food Technology (INTA), University of Chile, Av. El Líbano 5524, \\ Macul, Santiago 8380453, Chile; jsandoval@hcuch.cl (J.S.); cpuigrredon@hcuch.cl (C.P.); \\ avalenzu@inta.uchile.cl (A.V.) \\ 3 Obstetrics and Gynecology Department, Clinical Hospital of the University of Chile, Av. Santos Dumont 999, \\ Independencia, Santiago 8380453, Chile \\ * Correspondence: rvalenzuelab@med.uchile.cl; Tel.: +56-2-29786014; Fax: +56-2-9786182
}

Received: 11 April 2018; Accepted: 26 June 2018; Published: 28 June 2018

\begin{abstract}
Maternal diet during pregnancy is relevant for fatty acid supply during fetal life and lactation. Arachidonic (AA) and docosahexaenoic (DHA) acids are also relevant for the normal growth and development of brain and visual system. AA and DHA provided by the mother to the fetus and infant are directly associated with maternal dietary intake and body stores. Our aim was to evaluate the impact of maternal diet, specially referring to the quality of fatty acid intake, in a sample of Chilean women during last stage of pregnancy and across the lactation period. Fifty healthy pregnant women (age range 20-33 years) were studied from the 6th month of pregnancy and followed until 6th month of lactation period. Diet characteristics were evaluated through food frequency questionnaires. Fatty acids composition of erythrocyte phospholipids and breast milk samples was assessed by gas-liquid chromatography. Overall, women had high saturated fatty acids intake with sufficient intake of mono- and polyunsaturated fatty acids (PUFA). Diet was high in $n-6$ PUFA and low in $n$-3 PUFA (mainly DHA), with imbalanced $n-6 / n-3$ PUFA ratio. Erythrocytes and breast milk DHA concentration was significantly reduced during lactation compared to pregnancy, a pattern not observed for AA. We concluded that is necessary to increase the intake of $n-3$ PUFA during pregnancy and lactation by improving the quality of consumed foods with particular emphasis on its DHA content.
\end{abstract}

Keywords: pregnancy; breast milk; lactation; maternal diet; $n-6$ and $n-3$ polyunsaturated fatty acid; docosahexaenoic acid

\section{Introduction}

Arachidonic acid (C20:4n-6, AA) and docosahexaenoic acid (C22:6n-3, DHA) are long chain polyunsaturated fatty acids (LCPUFA) that have a relevant role in different metabolic and physiological process during embryonic and fetal development, and the first years of life [1]. Highest concentrations of AA and DHA are found in nervous system, particularly in brain and retina, specifically in the phospholipids of cell membranes [2,3]. AA and DHA have an active role in brain development 
during neurogenesis, synaptogenesis, neuronal migration, neuronal differentiation, and also in gene expression and in the general metabolic energy status [4]. AA is formed from the precursor linoleic acid (C18:2n-6 LA) and DHA from the precursor alpha-linolenic acid (C18:3n-3, ALA) [5]. Humans can synthesize AA and DHA, mainly in the liver, through a complex metabolic process that includes different enzymatically catalyzed desaturations and elongations of their respective metabolic precursors (LA and ALA); $\Delta-5$ and $\Delta-6$ desaturases are the most relevant enzymes participating in these processes [6].

Hepatic synthesis of AA and DHA is a fundamental metabolic process necessary to ensure the constant supply of these LCPUFA to other tissues [7]. Synthesis of AA and DHA in women is more efficient than in men due the active positive control of estrogens over desaturase activities [8]. Women, in addition, can store LCPUFA during pregnancy and lactation to ensure an adequate flow of AA and DHA to the fetus and newborn [9]. During pregnancy, the transport of AA and DHA from the mother to embryo and fetus is facilitated by specific transporter proteins that enhance the transfer of these fatty acids through the placenta [10]. During this period, women can also incorporate AA and DHA in their diet by eating eggs and meat (as sources of preformed AA) and fatty fish (i.e., tuna, mackerel, and salmon, among others) as a sources of preformed DHA [11]). However, in western countries DHA intake from fish consumption is very low compared to the intake of LA (from vegetable oils) and AA (from eggs and meat) [11]. In addition to the high dietary intake of $n-6$ fatty acids, the synthesis of AA from LA when this fatty acid is highly consumed is more efficient than the synthesis of DHA from ALA [12].

Several studies have established the relevance of the fatty acid composition of breast milk and its direct association with the diet of the mother during pregnancy and lactation [13-15]. The aim of our research was to evaluate the impact of maternal diet, with specific reference to the quality of fatty acids intake, in a sample of Chilean women during the last stage of pregnancy and across the lactation period. The fatty acid composition of erythrocyte phospholipids (during pregnancy and lactation) and of breast milk (during the first sixth months of lactation) were assessed as analytical criteria for $n-6$ and $n-3$ LCPUFA availability.

\section{Materials and Methods}

\subsection{Study Design and Subjects}

Pregnant women $(n=50)$ who attended the Obstetrical and Gynecology Health Service of the Clinical Hospital, University of Chile, Santiago, Chile were included in the study. Inclusion criteria were women between 20 and 33 years; gestational age at least 22 and up to 25 weeks according to the date of the last menstrual period and confirmed by ultrasound; and history of successful lactation. Exclusion criteria were women with history of drug or alcohol consumption; with current consumption of $n-6$ and / or $n-3$ LCPUFA supplements; who were underweight (as defined by the Chilean chart for pregnant women [16]); who had a history of twins; who had been diagnosed with chronic diseases such as diabetes, arterial hypertension, or other illnesses that could affect fetal growth. Recruited women mainly belonged to the low and middle socioeconomic status according to the European Society for Opinion and Marketing Research (ESOMAR) [17]. All women were of Hispanic origin.

At the time of recruitment, all women who fulfilled the inclusion criteria were given general information about the study, and a dietitian explained the objectives and main characteristics of the research. The protocol was reviewed and approved by the Institutional Review Board of the Faculty of Medicine, University of Chile (Protocol \#073-2011), and by the Ethics Committee of the Clinical Hospital, University of Chile (Protocol \#507/11). All information regarding the study was given to each participant who voluntarily agreed to participate and signed the informed consent. 


\subsection{Clinical and Nutritional Assessment}

Participants were subject to a clinical evaluation when incorporated into the study. A physician and a nurse assessed each woman regarding her health by following the standard clinical approach for pregnant women. Anthropometric data of weight $(\mathrm{kg})$ and height $(\mathrm{m})$ were assessed to determine body-mass index (BMI, $\mathrm{kg} / \mathrm{m}^{2}$ ), which was then used to establish the maternal nutritional status according to gestational week following the Chilean reference [17]. Energy and nutrient requirements were established according to WHO criteria [18] and recommended dietary intakes according to the American Institute of Medicine, 2001 [19].

\subsection{Dietary Intake}

All women were interviewed by trained dietitians at the enrollment of the study and asked to report all groups of foods consumed at the first week after delivery and during the six-month of lactation, using a food frequency questionnaire. In addition to this questionnaire and in order to improve the estimation of eaten foods, dietitians used a photographic atlas of commonly consumed foods in Chile [20], which is a validated graphic instrument that helps to estimate the amount of each food/beverage consumed. Food intake data were checked by contrasting the energy/nutrient intake data composition with dietary questionnaires, identifying potential under- or over-reports. In that case, a careful review of each food frequency questionnaire was done. Dietary data were grouped into nine food groups (cereals, fruits and vegetables, dairy, meats and eggs, legumes, fish and shellfish, high-fat foods, oils and fats, sugars and processed foods), according to dietary analysis previously reported by Bascuñán et al. [21]. Dietary data was analyzed using the software Food Processor SQL ${ }^{\circledR}$ (ESHA Research, Salem, OR, USA), to calculate the daily intake of energy and nutrients. Nutritional composition of foods was obtained using a database from the USDA National Nutrient Database for Standard Reference, which also incorporated information from locally generated nutrient composition data.

\subsection{Collection and Fatty Acid Analysis from Erythrocytes and Breast Milk Samples}

Blood samples were obtained at the enrollment, immediately after the delivery and at the 1st and the 6 th month after lactation. The samples were immediately centrifuged to obtain the erythrocyte fraction $\left(3000 \times g\right.$ for $10 \mathrm{~min}$ at $\left.20^{\circ} \mathrm{C}\right)$ and then frozen at $-80^{\circ} \mathrm{C}$ until further analysis. Breast milk was extracted by the mothers themselves after the infant had been fed for at least 2 min and was collected in plastic vials. Breast milk samples $(5 \mathrm{~mL})$ were collected monthly from the 1 st until the 6th month of lactation. Once collected, the samples were immediately frozen at $-80{ }^{\circ} \mathrm{C}$ until further analysis. Details of the analysis of fatty acids of erythrocyte phospholipids and breast milk samples were previously described by Valenzuela et al. [15].

\subsection{Statistical Analyses}

Dietary data were checked by contrasting the energy/nutrient intake data composition with dietary questionnaires, identifying potential outliers. In that case, a careful review of each food frequency questionnaire was done. After a descriptive analysis, the distribution of variables was evaluated using the Shapiro-Wilk test. Results are expressed as the mean \pm SD. Assessment of significant differences between mean values was performed by one-way ANOVA and with Bonferroni post-hoc test. Statistical significance was set at an alpha level of $5 \%$. For all analyses, the statistical software used was SPSS v.24.0 (Chicago, IL, USA).

\section{Results}

\subsection{Background and Anthropometric Characteristics of the Sample}

Table 1 shows the main background characteristics of the sample (age, socio-economic status (SES), weight, BMI, nutritional status, and gestational age). $68.2 \%$ of women belonged to the SES 
medium. Regarding the nutritional status, $37.8 \%$ were overweight and $11.1 \%$ obese; therefore, $48.9 \%$ of women exhibited overnutrition at the study enrollment.

Table 2 shows the anthropometric characteristics of women during the pregnancy and lactation period. Significant modification was observed in the weight and BMI during the time course of the study. The weigh and BMI were higher $(p<0.05)$ than delivery compared with other times evaluated. At the beginning of the study, $4.4 \%$ were underweight. At the end of the study, $46.7 \%$ of women were overweight and $16.1 \%$ obese; therefore, $62.8 \%$ exhibited overnutrition, and no underweight was observed. The $45 \%$ of deliveries were after cesarean, condition that may modify the milk production in amount and nutritional quality (not evaluated in the present study). At 6th month of lactation, infant presented a normal increment of weight and height. An important aspect is the increase in weight of the women from the 6th month of pregnancy until the delivery (12.2 kg on average), along with the weight of infant at birth, which on average was $4.25 \mathrm{~kg}$.

Table 1. Background characteristics of the women at the study enrollment.

\begin{tabular}{|c|c|c|}
\hline Variable & & $(n=50)$ \\
\hline Age (Years) & & $29.4 \pm 6.2$ \\
\hline \multirow{3}{*}{ SES } & High (\%) & 20.4 \\
\hline & Medium (\%) & 68.2 \\
\hline & Low $(\%)$ & 11.4 \\
\hline Preconception Weight (kg) & & $64.9 \pm 9.3$ \\
\hline Preconception BMI $\left(\mathrm{kg} / \mathrm{m}^{2}\right)$ & & $24.9 \pm 3.3$ \\
\hline \multirow{4}{*}{ Nutritional Status } & Underweight (\%) & 4.4 \\
\hline & Normal Weight (\%) & 46.7 \\
\hline & Overweight (\%) & 37.8 \\
\hline & Obese (\%) & 11.1 \\
\hline Gestational Age (Weeks) * & & $24.2 \pm 3.8$ \\
\hline
\end{tabular}

Table 2. Anthropometric characteristics of the women during the pregnancy and lactation period.

\begin{tabular}{|c|c|c|c|c|}
\hline Variable & $\begin{array}{l}\text { 6th Month of } \\
\text { Pregnancy }\end{array}$ & Delivery & $\begin{array}{l}\text { 1th Month of } \\
\text { Lactation }\end{array}$ & $\begin{array}{l}\text { 6th Month of } \\
\text { Lactation }\end{array}$ \\
\hline Weight (kg) & $70.3 \pm 9.0^{b}$ & $82.5 \pm 10.8^{a, c, d}$ & $69.1 \pm 9.7^{b}$ & $66.2 \pm 9.4^{b}$ \\
\hline Height (m) & $1.61 \pm 0.1$ & $1.61 \pm 0.1$ & $1.61 \pm 0.1$ & $1.61 \pm 0.1$ \\
\hline BMI $\left(\mathrm{kg} / \mathrm{m}^{2}\right)$ & $27.1 \pm 3.2^{b}$ & $31.9 \pm 3.9^{\mathrm{a}, \mathrm{c}, \mathrm{d}}$ & $26.5 \pm 3.5^{b}$ & $25.5 \pm 3.4^{b}$ \\
\hline \multicolumn{5}{|l|}{ Nutritional Status } \\
\hline Underweight (\%) & 4.4 & 0 & 0 & 0 \\
\hline Normal Weight (\%) & 46.7 & 42.2 & 40.8 & 37.2 \\
\hline Overweight $(\%)$ & 37.8 & 38.3 & 44.4 & 46.7 \\
\hline Obese $(\%)$ & 11.1 & 19.5 & 14.8 & 16.1 \\
\hline Gestational age at birth (weeks) & & $39 \pm 1$ & & \\
\hline Vaginal delivery $(\%)$ & - & 55 & - & 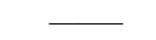 \\
\hline Cesarean delivery $(\%)$ & & 45 & - & 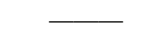 \\
\hline Gender: male (\%) & - & 53 & - & - \\
\hline Gender: female (\%) & 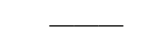 & 47 & - & - \\
\hline Infant Weight (g) & - & $4251 \pm 489^{d}$ & $4619 \pm 619$ & $7916 \pm 852^{b}$ \\
\hline Infant Height $(\mathrm{cm})$ & $\longrightarrow$ & $47.9 \pm 4.2^{\mathrm{d}}$ & $53.9 \pm 5.8$ & $66.9 \pm 6.4^{b}$ \\
\hline
\end{tabular}

Values are shown as mean \pm S.D., or as a percentage (\%); BMI, body mass index $=\mathrm{kg} / \mathrm{m}^{2}$. Statistical significance $(p<0.05){ }^{a}$ : Significantly different from the 6 th month of pregnancy; ${ }^{b}:$ significantly different at birth; ${ }^{c}$ : significantly different from 1st month of lactation; and ${ }^{d}$ : significantly different from the 6 th month of lactation. One-way ANOVA and Bonferroni test. 


\subsection{Daily Intake According to Food Groups during Pregnancy and Lactation}

Table 3 shows the dietary intake during the period studied. As expected at 6 th month of lactation, a general reduction of food intake compared to the evaluation at 6th month of pregnancy was observed not produced. However, fruits and vegetables, fish and seafood, oil and fats, and sugar and processed foods consumption were reduced $(p<0.05)$ at the 1st and 6th month of lactation when compared to the 6 th month of pregnancy. In addition, consumption of dairy, meat, and eggs was reduced $(p<0.05)$ at 6 th month of lactation. Consumption of high-fat foods was not significantly modified during the study. A reduction $(p<0.05)$ of the ingestion of fish and seafood $(39.3 \%$ at the 1 st month of lactation and $54 \%$ at the 6 th month of lactation) compared to the 6 th month of pregnancy was observed.

Table 3. Daily intake according to the food groups consumed by the women during the pregnancy and lactation period.

\begin{tabular}{|c|c|c|c|}
\hline \multirow[b]{2}{*}{ Food Groups } & \multicolumn{3}{|c|}{ Food Groups Intake (g/Day) } \\
\hline & 6th Month of Pregnancy & 1st Month of Lactation & 6th Month of Lactation \\
\hline Cereals & $347.0 \pm 59.8$ & $302.7 \pm 40.3$ & $299.7 \pm 31.7$ \\
\hline Fruits and Vegetables & $638.9 \pm 51.2^{b, c}$ & $371.5 \pm 54.6^{\mathrm{a}}$ & $303.6 \pm 38.9^{a}$ \\
\hline Dairy & $461.4 \pm 40.2^{c}$ & $383.6 \pm 38.6$ & $332.2 \pm 27.6^{\mathrm{a}}$ \\
\hline Meats and Eggs & $113.5 \pm 12.5^{c}$ & $112.0 \pm 10.6^{c}$ & $85.2 \pm 8.4^{a, b}$ \\
\hline Fish and Seafood & $28.5 \pm 6.9^{b, c}$ & $17.3 \pm 3.3^{\mathrm{a}}$ & $13.1 \pm 2.4^{\mathrm{a}}$ \\
\hline Legumes & $20.0 \pm 4.4^{b}$ & $10.5 \pm 2.6^{\mathrm{a}}$ & $15.9 \pm 4.4$ \\
\hline High-Lipid Foods & $47.1 \pm 10.9$ & $38.6 \pm 9.7$ & $37.1 \pm 8.9$ \\
\hline Oils and Fats & $39.8 \pm 7.3^{b, c}$ & $23.5 \pm 5.5^{\mathrm{a}}$ & $22.6 \pm 4.4^{\mathrm{a}}$ \\
\hline $\begin{array}{c}\text { Sugar and Processed } \\
\text { Foods }\end{array}$ & $537.7 \pm 53.5^{b, c}$ & $368.6 \pm 48.6^{\mathrm{a}}$ & $390.3 \pm 41.7^{\mathrm{a}}$ \\
\hline
\end{tabular}

Value are shown as mean \pm S.D. Statistical significance $(p<0.05){ }^{\text {a }}$ : Significantly different from the 6 th month of pregnancy; ${ }^{b}$ : significantly different from the 1 st month of lactation; and ${ }^{c}$ : significantly different the 6th month of lactation. The food was organized in nine groups according to the methodology described in the text. One-way ANOVA and Bonferroni test.

\subsection{Energy, Nutrients, and Most Relevant Fatty Acid Intake during Pregnancy and Lactation}

Table 4 shows the energy, nutrients, and the most relevant fatty acids intake of the sample. At the 1st and 6th month of lactation, a significant reduction in the energy and carbohydrate intake compared to the 6th month of pregnancy was observed. No significant modification in the intake of protein, fiber, and fat was observed. Table 4 also shows the daily intake of the most relevant fatty acids during pregnancy and lactation. During the study, women had a high intake of saturated fatty acid (SFA), total $n-6$ fatty acids, LA, and AA, together with an adequate intake of total monounsaturated fatty acid (MUFA) and total polyunsaturated fatty acid (PUFA) and a low intake of total n-3 fatty acids ALA, EPA, and DHA. At the 1st month of lactation a significant reduction of the intake of EPA and DHA (50\% and $33 \%$, respectively) was produced, and at the 6 th month this reduction was $50 \%$ for both LCPUFA, compared to the values at the 6th month of pregnancy. The rest of the most relevant fatty acids and the n-6/n-3 PUFA ratio were not significantly modified.

\subsection{Fatty Acid Composition of Erythrocyte Phospholipids}

Table 5 shows the fatty acid composition of erythrocyte phospholipids at the 6th month of pregnancy, at the delivery and at the 1st and 6th month of lactation. No significant changes were produced for SFA, MUFA, PUFA, LCPUFA, and n-6 LCPUFA when compared to the values for the 6th month of pregnancy and for the 6th month of lactation. However, docosapentaenoic acid (C22:5, n-6 DPA) was significantly increased (34.2\%) and DHA and total $n-3$ LCPUFA were significantly reduced for the same period $(27.6 \%$ reduction for DHA and $21.6 \%$ reduction for total $n-3$ LCPUFA, compared to the 6 th month of pregnancy). 


\subsection{Composition of the Most Relevant Fatty Acids of Breast Milk during the First Six Months of Lactation}

Data from Table 6 shows that the composition of the majority of the fatty acids from milk is maintained during the period studied, with the exception of DHA, LCPUFA, and $n-3$ LCPUFA, which were significantly reduced from the 4 th month of lactation. It is interesting to mention that at the 6 th month of lactation the levels of DHA, LCPUFA, and $n$-3 LCPUFA were even lower $(p<0.05)$ than the values observed at the 4 th month of lactation. In addition, the DHA was reduced by $38.5 \%$ at the 4 th month of lactation and by $64.1 \%$ at the 6 th month of lactation, compared to the values obtained at the 1st month of lactation. The reduction of $n-3$ LCPUFA is reflected in the significant increase of the $n-6 / n-3$ LCPUFA ratio.

\section{Discussion}

A woman's diet during pregnancy and lactation has a fundamental role in the adequate contribution of macro and micronutrients for her infant during the fetal life and during lactation [22,23]. The tissue levels of fatty acids in a woman during pregnancy and lactation are directly related to her diet, her reserve capacity, and her metabolic utilization of fatty acids (synthesis, oxidation, transport, etc.) $[24,25]$. Therefore, the diet and the metabolism of fatty acids of women during pregnancy and lactation have a relevant role in determining the levels of LCPUFA present in erythrocytes and breast milk [26,27]. The availability of LCPUFA for the infant is directly related to the transfer of these fatty acids from the mother to her offspring, first through the placenta (intra-uterine life) [28] and then through lactation [29]. Regarding AA and DHA, their availability will depend on the intake of foods that provided these fatty acids [30] and/or from the capacity of the mother to form these fatty acids from their metabolic precursors [31]. According to our results we concluded that the Chilean women evaluated have a high intake of $n-6$ PUFA, LA, and AA (Table 4), and a low intake of $n-3$ PUFA, ALA, EPA, and DHA (Table 4). In this context, is also remarkable that among LCPUFA, DHA is one the fatty acid with the most important metabolic characteristics for the physiological period studied [28,32]. The low intake of foods that are natural sources of DHA, such as fish or seafood, as was observed in our study (Table 3), added to an excessive intake of foods that are high in $n$ - 6 fatty acids, especially LA (e.g., consumption of soy or sunflower oil), which can produce a reduction of the capacity of the mother for transferring DHA to her offspring during pregnancy and breast feeding [13,26,27]. In our sample, DHA levels in breast milk were reduced by $38.5 \%$ at the 4 th month, by $48.7 \%$ at the 5 th month, and by $64.1 \%$ at the 6 th month of lactation compared with the 1st month of lactation (Table 6). This situation was not produced for AA and LA in erythrocytes and breast milk because of the adequate consumption of foods considered good sources of these fatty acids (Table 3).

The reserve capacity of women for LCPUFA, particularly DHA, is sensitive to the number of pregnancies, because it is produced by a significant decrease in the tissue levels and availability of DHA after frequent pregnancies [28,30,33]. In addition, another interesting aspect to consider is the activity of the $\Delta-5$ and $\Delta-6$ desaturases, key enzymes for the synthesis of LCPUFA from their specific precursors $[8,31]$. The presence of polymorphisms in the genes encoding these enzymes may produce a lower synthesis of both $n-6$ and $n-3$ LCPUFA, but being the diet sufficient in AA, the effect of polymorphism should be more deleterious for the availability of DHA than AA [34]. Is important to emphasize that the dietary imbalance of $n-6$ to $n-3$ PUFA can lower the synthesis of $n-3$ LCPUFA, particularly DHA, because of the competition generated between the respective precursors for the active sites of desaturase enzymes $[6,8,35]$. An excess of $n-6$ fatty acid (such as LA) may decrease the synthesis of DHA from ALA [36]. The increment in the values for DPAn-6 (Table 5) may be a metabolic compensatory mechanism to form LCPUFA because of the reduction of the $n-3$ LCPUFA (DHA), an aspect that remains to be studied. The synthesis of LCPUFA, as well as the availability of precursors and the activity of $\Delta-5$ and $\Delta-6$ desaturase enzymes, is also dependent on the availability of specific nutrients, such as zinc, magnesium, calcium, vitamin B6, and vitamin C [37]. It is also interesting that while the synthesis of DHA from ALA may be sufficient for the adult human in normal physiological conditions [38], some diseases, such as non-alcoholic fatty liver, a pathology 
very prevalent in populations that are overweight or obese [39] (in our study, women who were overweight or obese reached $48.9 \%$ at 6 th month of pregnancy), which reduces the activity of $\Delta-5$ and $\Delta-6$ desaturase enzymes [40], could adversely affect the synthesis of DHA, decreasing the levels of $n-3$ LCPUFA in erythrocytes and breast milk, as was observed in this work (Tables 5 and 6). Another aspect observed in this study was the increase in weight of the women from the 6th month of pregnancy until the delivery (average $12.2 \mathrm{~kg}$ ), in addition to the high prevalence of overweight and obese women at the end of pregnancy $(62.8 \%)$, along with the birth weight of children, which, on average, was $4.25 \mathrm{~kg}$. In this context, previous studies suggest that Chilean women during the fertile age show a fast and growing tendency towards obesity [41], and a high prevalence of being overweight (50\%) and obese $(20 \%)$ is observed in pregnant Chilean women [42]. This nutritional situation has been related to (i) the increasing tendency of the Chilean newborns to have a weight at birth higher than $4.0 \mathrm{~kg}$ [43] and (ii) a high number of births by caesarian intervention, which are associated with overweight and obesity of pregnant women (up to $35 \%$ ) [44].

In relation to the Chilean population, Bascunán et al. [21] reported that women in the 3rd trimester of pregnancy with a low intake of DHA showed low levels of this fatty acid in erythrocyte phospholipids. In the population evaluated in this study, it was observed that DHA levels in erythrocytes were significantly decreased at the 6th month of lactation (Table 5) and the content of DHA in breast milk also decreased $(p<0.05)$, starting from the 4th month of lactation (Table 6). In this same context, Valenzuela et al. [15] reported that in Chilean pregnant women, who included in their diet chia oil ("Salvia hispanica L.", 60\% ALA) instead of the traditionally consumed oils (soy and sunflower oil) from the 6th month of pregnancy and until the 6th month of lactation, it a significant increase of DHA levels in breast milk was produced, but only until the 3rd month of lactation, without modification of the AA levels during the 6th month of lactation. The same authors reported that AA and DHA in erythrocyte phospholipids were not modified during the dietary intervention [15], suggesting that the ingestion of oils with high content of ALA (such as chia oil) would not be entirely efficient to increase the levels of DHA in breast milk during a lactation period up to three months [15].

Concerning the actual background of $n-3$ LCPUFA, dietary strategies have been developed to improve the quality of the diet of women during pregnancy and lactation through educational programs focused on promoting consumption of foods that provide DHA (especially from marine origin) [45-47]. However, it is not easy to modify the dietary habits of women during these periods [45,47], which adds to the concern that currently exists about the contamination of foods from marine origin (heavy metals, dioxins, PGB, etc.) and the questioning in the population about "possible adverse effects" of seafood consumption [48-50]. It is remarkable that, in the present study, it was observed that women significantly decreased fish intake after the delivery (Table 3), even though they were advised by professional nutritionist that they should increase the consumption of fish and other foods from marine origin. In this regard, the decrease of DHA levels $(p<0.05)$ observed in erythrocytes and breast milk in women's sample (Tables 5 and 6) may be a concern, as it has been previously reported that higher levels of DHA in breast milk are associated with better academic performance, particularly in mathematics, in long-term studies on children [51]. Addressing this dietary and nutritional problem, various studies have used supplements containing DHA or DHA-added foods, evidencing that an increase in the intake of DHA increases the content of the fatty acid in erythrocytes and subsequently in breast milk in pregnant and lactating women [52-55]. In consistent with this same direction, the Chilean Ministry of Health has developed a food program that benefits (free of cost) all women during pregnancy and lactation, providing a dairy drink containing DHA $(60 \mathrm{mg} / 200 \mathrm{~mL}$ of product), and recommending the intake of two daily portions (120 mg DHA/day) [56]. Although the program began in 2009, there are no current results regarding the impact of this dairy product, either on DHA levels in erythrocytes and breast milk or on scholarly performance. The results of this program are currently being assessed. 


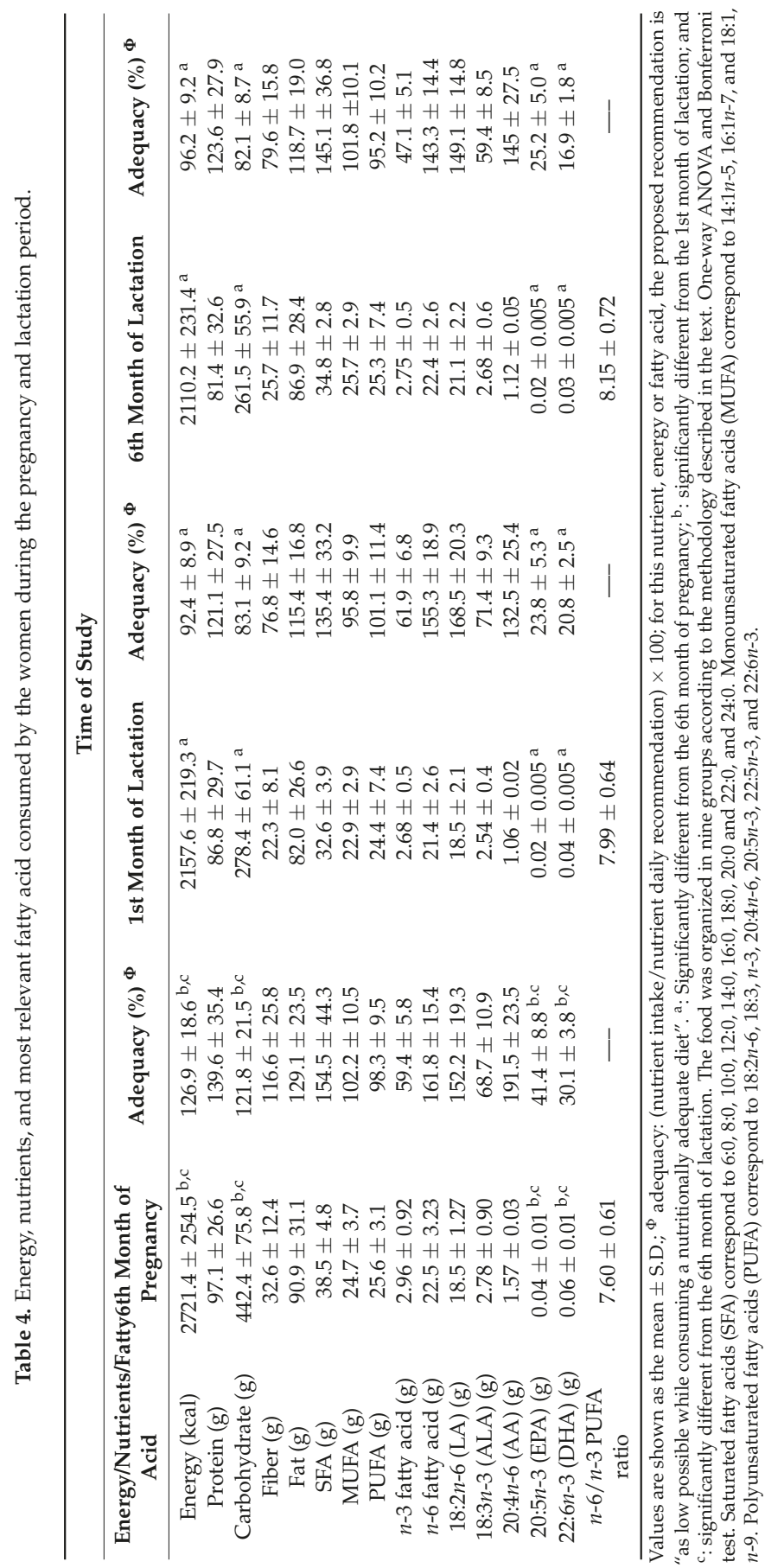




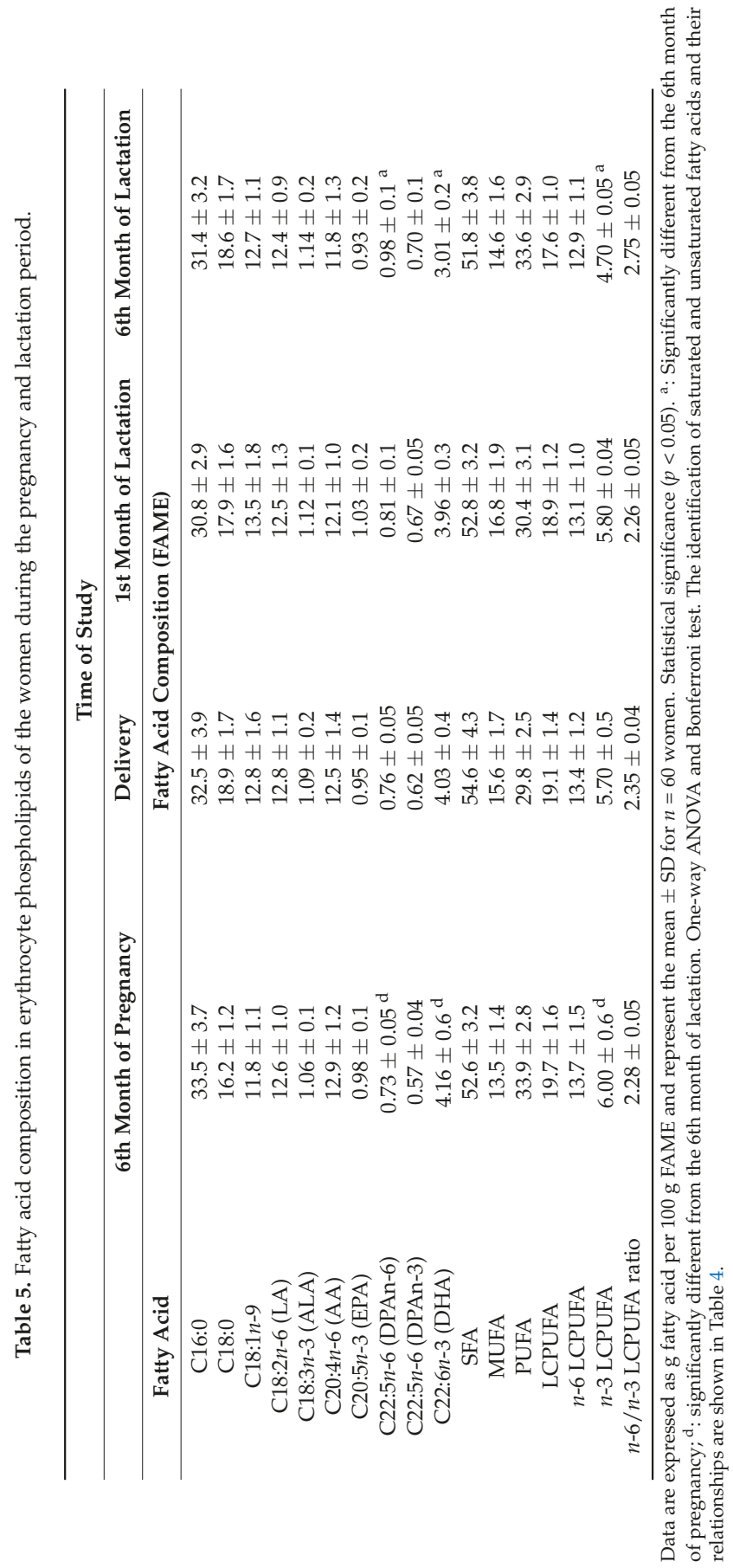




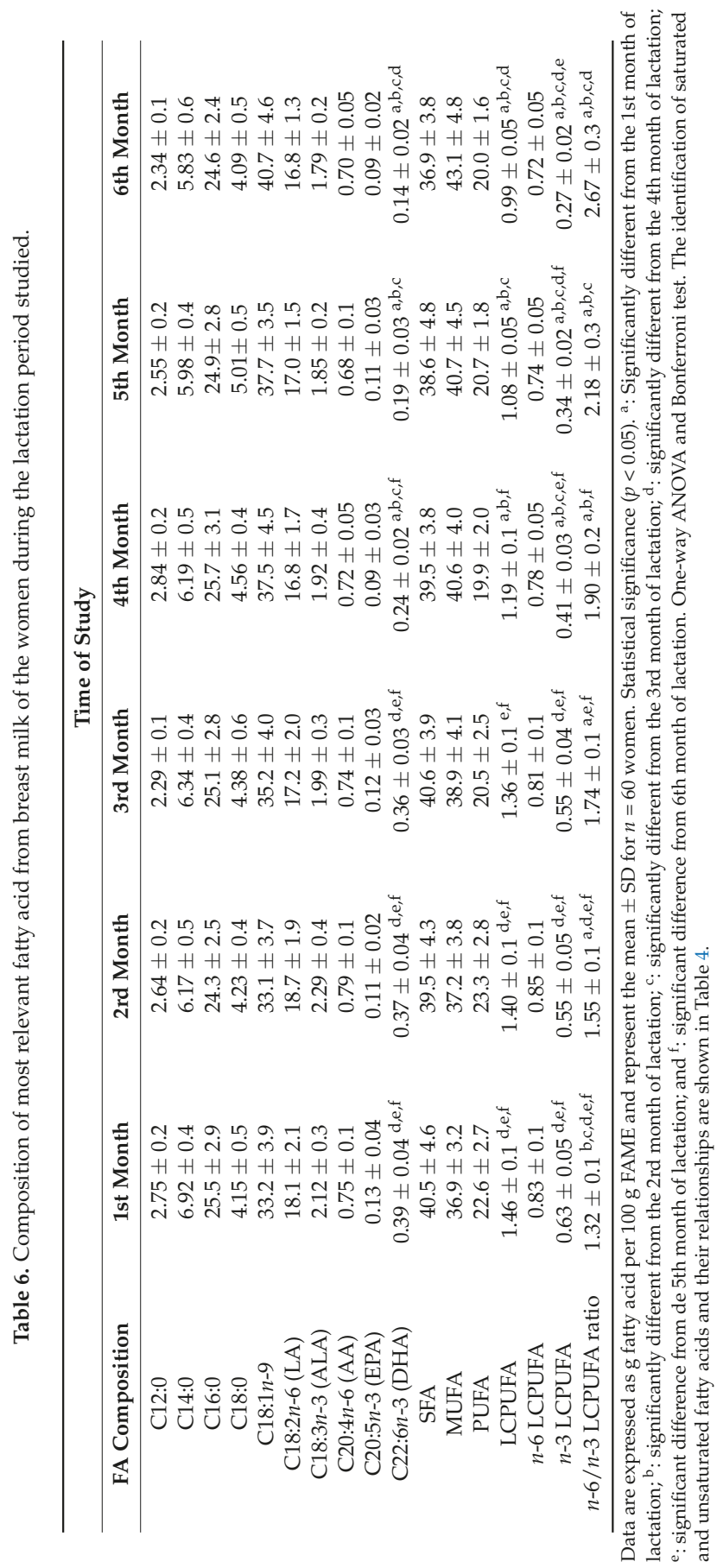




\section{Conclusions}

This study shows that Chilean women during pregnancy and lactation have a low intake of foods that are natural sources of $n-3$ fatty acids (vegetable oils, fish, and seafood) and a high intake of $n-6$ fatty acid (LA and AA). This dietary situation produced a significant reduction of DHA levels in erythrocytes and breast milk. Therefore, it is necessary to promote the consumption of foods that naturally contain DHA or are fortified with $n$-3 LCPUFA. Another alternative is direct supplementation with DHA during pregnancy and lactation through products now widely available (fish oil or krill oil capsules or microcapsules).

Author Contributions: R.V., K.B., and R.C. designed the study and analyzed and interpreted the data. K.B., R.V., C.B., J.S., and C.P. performed clinical and nutritional evaluations. C.B., K.B., R.C., N.S., F.V., and M.-P.V. conducted the dietary analysis. R.V., K.B., R.C., and A.V. wrote the manuscript. All authors reviewed and approved the final version of the manuscript.

Funding: This study was supported by the FONDECYT (National Fund for Scientific and Technological Development, Grant 11140174), granted to R.V., for supporting this study.

Acknowledgments: Authors are grateful to Department of Nutrition of the Faculty of Medicine of the University of Chile and the Obstetrics and Gynecology Department, Clinical Hospital of the University of Chile, for supporting this study.

Conflicts of Interest: Authors declare no conflict of interest.

\section{References}

1. Carlson, S.E.; Colombo, J. Docosahexaenoic Acid and Arachidonic Acid Nutrition in Early Development. Adv. Pediatr. 2016, 63, 453-471. [CrossRef] [PubMed]

2. Svennerholm, L.; Vanier, M.T. The distribution of lipids in the human nervous system. 3. Fatty acid composition of phosphoglycerides of human foetal and infant brain. Brain Res. 1973, 50, 341-351. [CrossRef]

3. Birch, E.E.; Hoffman, D.R.; Uauy, R.; Birch, D.G.; Prestidge, C. Visual acuity and the essentiality of docosahexaenoic acid and arachidonic acid in the diet of term infants. Pediatr. Res. 1998, 44, 201-209. [CrossRef] [PubMed]

4. Uauy, R.; Hoffman, D.R.; Peirano, P.; Birch, D.G.; Birch, E.E. Essential fatty acids in visual and brain development. Lipids 2001, 36, 885-895. [CrossRef] [PubMed]

5. González-Mañán, D.; Tapia, G.; Gormaz, J.G.; D’Espessailles, A.; Espinosa, A.; Masson, L.; Varela, P.; Valenzuela, A.; Valenzuela, R. Bioconversion of $\alpha$-linolenic acid to $n$-3 LCPUFA and expression of PPAR-alpha, acyl Coenzyme A oxidase 1 and carnitine acyl transferase I are incremented after feeding rats with $\alpha$-linolenic acid-rich oils. Food Funct. 2012, 3, 765-772. [CrossRef] [PubMed]

6. Nakamura, M.T.; Nara, T.Y. Structure, function, and dietary regulation of delta6, delta5, and delta9 desaturases. Annu. Rev. Nutr. 2004, 24, 345-376. [CrossRef] [PubMed]

7. Rincón-Cervera, M.A.; Valenzuela, R.; Hernandez-Rodas, M.C.; Marambio, M.; Espinosa, A.; Mayer, S.; Romero, N.; Barrera, C.; Valenzuela, A.; Videla, L.A. Supplementation with antioxidant-rich extra virgin olive oil prevents hepatic oxidative stress and reduction of desaturation capacity in mice fed a high-fat diet: Effects on fatty acid composition in liver and extrahepatic tissues. Nutrition 2016, 32, 1254-1267. [CrossRef] [PubMed]

8. Brenna, J.T.; Salem, N., Jr.; Sinclair, A.J.; Cunnane, S.C.; International Society for the Study of Fatty Acids and Lipids, ISSFAL. $\alpha$-Linolenic acid supplementation and conversion to $n-3$ long-chain polyunsaturated fatty acids in humans. Prostaglandins Leukot. Essent. Fat. Acids 2009, 80, 85-91. [CrossRef] [PubMed]

9. Lauritzen, L.; Carlson, S.E. Maternal fatty acid status during pregnancy and lactation and relation to newborn and infant status. Matern. Child Nutr. 2011, 7, 41-58. [CrossRef] [PubMed]

10. Lewis, R.M.; Desoye, G. Placental Lipid and Fatty Acid Transfer in Maternal Overnutrition. Ann. Nutr. Metab. 2017, 70, 228-231. [CrossRef] [PubMed]

11. Simopoulos, A.P. An Increase in the Omega-6/Omega-3 Fatty Acid Ratio Increases the Risk for Obesity. Nutrients 2016, 8, 128. [CrossRef] [PubMed] 
12. Metherel, A.H.; Lacombe, R.J.S.; Chouinard-Watkins, R.; Hopperton, K.E.; Bazinet, R.P. Complete assessment of whole-body $n-3$ and $n-6$ PUFA synthesis-secretion kinetics and DHA turnover in a rodent model. J. Lipid Res. 2018, 59, 357-367. [CrossRef] [PubMed]

13. Urwin, H.J.; Zhang, J.; Gao, Y.; Wang, C.; Li, L.; Song, P.; Man, Q.; Meng, L.; Frøyland, L.; Miles, E.A.; et al. Immune factors and fatty acid composition in human milk from river/lake, coastal and inland regions of China. Br. J. Nutr. 2013, 109, 1949-1961. [CrossRef] [PubMed]

14. Bravi, F.; Wiens, F.; Decarli, A.; Dal Pont, A.; Agostoni, C.; Ferraroni, M. Impact of maternal nutrition on breast-milk composition: A systematic review. Am. J. Clin. Nutr. 2016, 104, 646-662. [CrossRef] [PubMed]

15. Valenzuela, R.; Bascuñán, K.; Chamorro, R.; Barrera, C.; Sandoval, J.; Puigrredon, C.; Parraguez, G.; Orellana, P.; Gonzalez, V.; Valenzuela, A. Modification of Docosahexaenoic Acid Composition of Milk from Nursing Women Who Received Alpha Linolenic Acid from Chia Oil during Gestation and Nursing. Nutrients 2015, 7, 6405-6424. [CrossRef] [PubMed]

16. Atalah, E.; Castillo, C.; Castro, R.; Aldea, A. Proposalof a new standard for the nutritional assessment of pregnant women. Rev. Med. Chile 1997, 125, 1429-1436. [PubMed]

17. European Society for Opinion and Marketing Research. The ESOMAR Standard Demographic Classification; ESOMAR: Amsterdam, The Netherlands, 1997.

18. WHO/FAO/UNU. Human Energy Requirements, Report of a Joint FAO/WHO/UNU Expert Consultation; Food and Agriculture Organization: Rome, Italy, 2014.

19. Food and Nutrition Board, Institute of Medicine. Dietary Reference Intakes: Guiding Principles for Nutrition Labeling and Fortification; Institute of Medicine of the National Academies: Washington, DC, USA, 2001; pp. $1-224$.

20. Cerda, R.; Barrera, C.; Arena, M.; Bascuñán, K.A.; Jimenez, G. Photographic Atlas of Chilean Food and Typical Preparations. National Survey of Food Consumption, 1st ed.; Government of Chile, Ministry of Health: Santiago, Chile, 2010.

21. Bascuñán, K.A.; Valenzuela, R.; Chamorro, R.; Valencia, A.; Barrera, C.; Puigrredon, C.; Sandoval, J.; Valenzuela, A. Polyunsaturated fatty acid composition of maternal diet and erythrocyte phospholipid status in Chilean pregnant women. Nutrients 2014, 6, 4918-4934. [CrossRef] [PubMed]

22. Kashtanova, D.A.; Popenko, A.S.; Tkacheva, O.N.; Tyakht, A.B.; Alexeev, D.G.; Boytsov, S.A. Association between the gut microbiota and diet: Fetal life, early childhood, and further life. Nutrition 2016, 32, 620-627. [CrossRef] [PubMed]

23. Innis, S.M. Metabolic programming of long-term outcomes due to fatty acid nutrition in early life. Matern. Child Nutr. 2011, 2, S112-S123. [CrossRef] [PubMed]

24. Berti, C.; Cetin, I.; Agostoni, C.; Desoye, G.; Devlieger, R.; Emmett, P.M.; Ensenauer, R.; Hauner, H.; Herrera, E.; Hoesli, I.; et al. Pregnancy and Infants' Outcome: Nutritional and Metabolic Implications. Crit. Rev. Food Sci. Nutr. 2016, 56, 82-91. [CrossRef] [PubMed]

25. Larqué, E.; Demmelmair, H.; Gil-Sánchez, A.; Prieto-Sánchez, M.T.; Blanco, J.E.; Pagán, A.; Faber, F.L.; Zamora, S.; Parrilla, J.J.; Koletzko, B. Placental transfer of fatty acids and fetal implications. Am. J. Clin. Nutr. 2011, 94, S1908-S1913. [CrossRef] [PubMed]

26. Peng, Y.; Zhou, T.; Wang, Q.; Liu, P.; Zhang, T.; Zetterström, R.; Strandvik, B. Fatty acid composition of diet, cord blood and breast milk in Chinese mothers with different dietary habits. Prostaglandins Leukot. Essent. Fat. Acids 2009, 81, 325-330. [CrossRef] [PubMed]

27. Zhang, J.; Wang, C.; Gao, Y.; Li, L.; Man, Q.; Song, P.; Meng, L.; Du, Z.Y.; Miles, E.A.; Lie, Ø.; et al. Different intakes of $n-3$ fatty acids among pregnant women in 3 regions of China with contrasting dietary patterns are reflected in maternal but not in umbilical erythrocyte phosphatidylcholine fatty acid composition. Nutr. Res. 2013, 33, 613-621. [CrossRef] [PubMed]

28. Gil-Sánchez, A.; Koletzko, B.; Larqué, E. Current understanding of placental fatty acid transport. Curr. Opin. Clin. Nutr. Metab. Care 2012, 15, 265-272. [CrossRef] [PubMed]

29. Grote, V.; Verduci, E.; Scaglioni, S.; Vecchi, F.; Contarini, G.; Giovannini, M.; Koletzko, B.; Agostoni, C.; European Childhood Obesity Project. Breast milk composition and infant nutrient intakes during the first 12 months of life. Eur. J. Clin. Nutr. 2016, 70, 250-256. [CrossRef] [PubMed]

30. Larqué, E.; Gil-Sánchez, A.; Prieto-Sánchez, M.T.; Koletzko, B. Omega 3 fatty acids, gestation and pregnancy outcomes. Br. J. Nutr. 2012, 107, S77-S84. [CrossRef] [PubMed] 
31. De la Garza, A.; Montes, R.; Chisaguano, A.M.; Torres-Espínola, F.J.; Arias, M.; de Almeida, L.; Bonilla, M.; Guerendiain, M.; Castellote, A.I.; Segura, M.; et al. Association of maternal weight with FADS and ELOVL genetic variants and fatty acid levels- The PREOBE follow-up. PLoS ONE 2017, 12, e0179135. [CrossRef] [PubMed]

32. Herrera, E. Implications of dietary fatty acids during pregnancy on placental, fetal and postnatal development-A review. Placenta 2002, 23, S9-S19. [CrossRef] [PubMed]

33. Cetin, I.; Koletzko, B. Long-chain omega-3 fatty acid supply in pregnancy and lactation. Curr. Opin. Clin. Nutr. Metab. Care 2008, 11, 297-302. [CrossRef] [PubMed]

34. Glaser, C.; Lattka, E.; Rzehak, P.; Steer, C.; Koletzko, B. Genetic variation in polyunsaturated fatty acid metabolism and its potential relevance for human development and health. Matern. Child Nutr. 2011, 7, 27-40. [CrossRef] [PubMed]

35. Scholtz, S.A.; Kerling, E.H.; Shaddy, D.J.; Li, S.; Thodosoff, J.M.; Colombo, J.; Carlson, S.E. Docosahexaenoic acid (DHA) supplementation in pregnancy differentially modulates arachidonic acid and DHA status across FADS genotypes in pregnancy. Prostaglandins Leukot. Essent. Fat. Acids 2015, 94, 29-33. [CrossRef] [PubMed]

36. Lassek, W.D.; Gaulin, S.J. Linoleic and docosahexaenoic acids in human milk have opposite relationships with cognitive test performance in a sample of 28 countries. Prostaglandins Leukot. Essent. Fat. Acids 2014, 91, 195-201. [CrossRef] [PubMed]

37. Das, U.N. Essential fatty acids: Biochemistry, physiology and pathology. Biotechnol. J. 2006, 1, 420-439. [CrossRef] [PubMed]

38. Domenichiello, A.F.; Kitson, A.P.; Bazinet, R.P. Is docosahexaenoic acid synthesis from $\alpha$-linolenic acid sufficient to supply the adult brain? Prog. Lipid Res. 2015, 59, 54-66. [CrossRef] [PubMed]

39. Valenzuela, R.; Videla, L.A. The importance of the long-chain polyunsaturated fatty acid $n-6 / n-3$ ratio in development of non-alcoholic fatty liver associated with obesity. Food Funct. 2011, 2, 644-648. [CrossRef] [PubMed]

40. Araya, J.; Rodrigo, R.; Pettinelli, P.; Araya, A.V.; Poniachik, J.; Videla, L.A. Decreased liver fatty acid delta-6 and delta-5 desaturase activity in obese patients. Obesity 2010, 18, 1460-1463. [CrossRef] [PubMed]

41. Garmendia, M.L.; Alonso, F.T.; Kain, J.; Uauy, R.; Corvalan, C. Alarming weight gain in women of a post-transitional country. Public Health Nutr. 2014, 17, 667-673. [CrossRef] [PubMed]

42. Araya, B.M.; Padilla, O.; Garmendia, M.L.; Atalah, E.; Uauy, R. Prevalence of obesity among Chilean women in childbearing ages. Rev. Med. Chile 2014, 142, 1440-1448.

43. Lopez, P.O.; Bréart, G. Trends in gestational age and birth weight in Chile, 1991-2008. A descriptive epidemiological study. BMC Pregnancy Childbirth. 2012, 12, 121. [CrossRef] [PubMed]

44. Olmos, P.R.; Borzone, G.R.; Olmos, R.I.; Valencia, C.N.; Bravo, F.A.; Hodgson, M.I.; Belmar, C.G.; Poblete, J.A.; Escalona, M.O.; Gómez, B. Gestational diabetes and pre-pregnancy overweight: Possible factors involved in newborn macrosomia. J. Obstet. Gynaecol. Res. 2012, 38, 208-214. [CrossRef] [PubMed]

45. Miles, E.A.; Noakes, P.S.; Kremmyda, L.S.; Vlachava, M.; Diaper, N.D.; Rosenlund, G.; Urwin, H.; Yaqoob, P.; Rossary, A.; Farges, M.C.; et al. The Salmon in Pregnancy Study: Study design, subject characteristics, maternal fish and marine $n-3$ fatty acid intake, and marine $n-3$ fatty acid status in maternal and umbilical cord blood. Am. J. Clin. Nutr. 2011, 94, S1986-S1992. [CrossRef] [PubMed]

46. Fayet-Moore, F.; Baghurst, K.; Meyer, B.J. Four Models Including Fish, Seafood, Red Meat and Enriched Foods to Achieve Australian Dietary Recommendations for n-3 LCPUFA for All Life-Stages. Nutrients 2015, 7, 8602-8614. [CrossRef] [PubMed]

47. Rahmawaty, S.; Lyons-Wall, P.; Charlton, K.; Batterham, M.; Meyer, B.J. Effect of replacing bread, egg, milk, and yogurt with equivalent $\omega-3$ enriched foods on $\omega-3$ LCPUFA intake of Australian children. Nutrition 2014, 30, 1337-1343. [CrossRef] [PubMed]

48. Yeates, A.J.; Thurston, S.W.; Li, H.; Mulhern, M.S.; McSorley, E.M.; Watson, G.E.; Shamlaye, C.F.; Strain, J.J.; Myers, G.J.; Davidson, P.W.; et al. PUFA Status and Methylmercury Exposure Are Not Associated with Leukocyte Telomere Length in Mothers or Their Children in the Seychelles Child Development Study. J. Nutr. 2017, 147, 2018-2024. [CrossRef] [PubMed]

49. Golding, J.; Gregory, S.; Emond, A.; Iles-Caven, Y.; Hibbeln, J.; Taylor, C.M. Prenatal mercury exposure and offspring behaviour in childhood and adolescence. Neurotoxicology 2016, 57, 87-94. [CrossRef] [PubMed]

50. Gil, A.; Gil, F. Fish, a Mediterranean source of n-3 PUFA: Benefits do not justify limiting consumption. Br. J. Nutr. 2015, 113, S58-S67. [CrossRef] [PubMed] 
51. Lassek, W.D.; Gaulin, S.J. Maternal milk DHA content predicts cognitive performance in a sample of 28 nations. Matern. Child Nutr. 2015, 11,773-779. [CrossRef] [PubMed]

52. Smit, E.N.; Koopmann, M.; Boersma, E.R.; Muskiet, F.A. Effect of supplementation of arachidonic acid (AA) or a combination of AA plus docosahexaenoic acid on breastmilk fatty acid composition. Prostaglandins Leukot. Essent. Fat. Acids 2000, 62, 335-340. [CrossRef] [PubMed]

53. Sherry, C.L.; Oliver, J.S.; Marriage, B.J. Docosahexaenoic acid supplementation in lactating women increases breast milk and plasma docosahexaenoic acid concentrations and alters infant omega 6:3 fatty acid ratio. Prostaglandins Leukot. Essent. Fat. Acids 2015, 95, 63-69. [CrossRef] [PubMed]

54. Echeverría, F.; Valenzuela, R.; Hernandez-Rodas, M.C.; Valenzuela, A. Docosahexaenoic acid (DHA), a fundamental fatty acid for the brain: New dietary sources. Prostaglandins Leukot. Essent. Fat. Acids 2017, 124, 1-10. [CrossRef] [PubMed]

55. Atalah, S.E.; Araya, B.M.; Rosselot, P.G.; Araya, L.H.; Vera, A.G.; Andreu, R.R.; Barba, G.C.; Rodriguez, L. Consumption of a DHA-enriched milk drink by pregnant and lactating women, on the fatty acid composition of red blood cells, breast milk, and in the newborn. Arch. Latinoam. Nutr. 2009, 59, 271-277.

56. Contreras, A.; Herrera, Y.; Rodríguez, L.; Pizarro, T.; Atalah, E. Acceptability and consumption of a dairy drink with omega-3 in pregnant and lactating women of the national supplementary program. Rev. Chil. Nutr. 2011, 38, 313-320.

(C) 2018 by the authors. Licensee MDPI, Basel, Switzerland. This article is an open access article distributed under the terms and conditions of the Creative Commons Attribution (CC BY) license (http:/ / creativecommons.org/licenses/by/4.0/). 


\title{
Zinc Deficiency among Lactating Mothers from a Peri-Urban Community of the Ecuadorian Andean Region: An Initial Approach to the Need of Zinc Supplementation
}

\author{
Camila Narváez-Caicedo ${ }^{1}$, Gabriela Moreano ${ }^{1}$, Bernardo A. Sandoval ${ }^{1,2}$ and \\ Miguel Á. Jara-Palacios ${ }^{1, *}$ \\ 1 Escuela de Medicina, Facultad de Ciencias Médicas de la Salud y de la Vida, Universidad Internacional del \\ Ecuador, 170113 Quito, Ecuador; camilanarvaezca@gmail.com (C.N.-C.); \\ gaby.moreano94@gmail.com (G.M.); bsandoval@uide.edu.ec (B.A.S.) \\ 2 Servicio de Cirugía, Hospital Metropolitano, 170521 Quito, Ecuador \\ * Correspondence: migueljarapalacios@gmail.com; Tel.: +1-203-999-7089
}

Received: 26 May 2018; Accepted: 3 July 2018; Published: 5 July 2018

check for updates

\begin{abstract}
Zinc is an important mineral for biological and physiological processes. Zinc deficiency (ZD) is one of the most common micronutrient deficiencies worldwide and a crucial determinant of pregnancy outcomes and childhood development. Zinc levels and the zinc supplementation rate among lactating women have not been assessed neither in Ecuador nor in the Andean region. We conducted a pilot study including 64 mothers of infants between eight days to seven months old from a primary care center located in Conocoto, a peri-urban community of Quito, Ecuador. The mothers were interviewed and a fasting blood sample was taken to determine plasma zinc levels. The prevalence of ZD was calculated and compared with the prevalence of ZD among Ecuadorian non-pregnant non-lactating women, and the sample was analysed considering zinc supplementation during pregnancy. The prevalence of ZD among the participants was $81.3 \%$ (95\% CI: 71.7-90.9), higher than the reported among non-pregnant non-lactating women $\left(\mathrm{G}^{2}=18.2 ; p<0.05\right)$. Zinc supplementation rate was $31.2 \%$. No significant differences were found comparing the groups considering zinc supplementation. The insights obtained from this study encourage extending studies to document zinc levels and its interactions among breastfeeding women in areas with a high prevalence of ZD in order to determine the need of zinc supplementation.
\end{abstract}

Keywords: zinc deficiency; plasma zinc; lactating women; zinc supplementation; Quito; Ecuador; Andean region

\section{Introduction}

Zinc is an ubiquitous mineral within the body that has catalytic, structural, and regulatory biological functions [1]. This mineral is known to be an essential micronutrient for development and normal cell activity [2]. Zinc deficiency (ZD) is one of the most common micronutrient deficiencies worldwide, affecting around 2 billion people, especially among developing countries. ZD has important consequences especially during pregnancy, lactation, and childhood development [3,4].

In spite of the physiological adjustment, which increases the zinc absorption during pregnancy and lactation, women and children are still affected by zinc deficiency due to increased nutrient requirements [5,6]. During pregnancy, ZD can affect multiple systems and could increase the risk of infections, preeclampsia, miscarriages, and adverse fetal outcomes, such as fetal growth restriction, low weight at birth, neurological malformations, and/or neurological impairment [2,4-11]. If ZD in 
the mothers persists after birth, particularly if their infants are exclusively breastfed, adverse infant development outcomes may occur [2,8].

Approximately $82 \%$ of mothers around the world do not have an adequate zinc intake in their diet, but the prevalence of ZD during pregnancy worldwide has not been determined [12]. It has been reported a higher prevalence of zinc deficiency among pregnant, compared to non-pregnant, women in India $(65 ; 41 \%)$, Ethiopia $(56 ; 34 \%)$, and Pakistan $(48 ; 42 \%)$ [13-16]. In lactating women, the prevalence of ZD varied widely among small cohorts from different countries, such as Indonesia ( $25 \%)$ and Vietnam (55\%) [17,18].

In Ecuador, according to the Ecuadorian National Health and Nutrition Survey (ENSANUT-ECU, 2014), 56.1\% (95\% CI: 54.9-57.1) of women of reproductive age have ZD. Pregnant and lactating women were not sampled in this survey [19]. On the other hand, a study conducted previously by our research group, found a prevalence of ZD of 31.4\% (95\% CI: 17.1-48.6) in a small cohort of mothers of children with non-syndromic cleft lip with or without cleft palate. It is important to clarify that these women did not exclusively breastfed their babies due to the oral malformation [20].

Micronutrient deficiencies have been observed in Ecuador for at least two decades, particularly in vulnerable segments of the population [21]; in part, it could be affected by globalization through multiple pathways, such as urbanization, economic growth, trade, and investments [21,22]. Processes of urbanization are associated with higher inequality and changes in the food environment, subsequently affecting the nutritional status, especially in poor people who cannot afford food of high nutritional quality [22]. Nowadays, peri-urban areas of Quito are suffering accelerated urbanization, growing in population at a higher index than the urban area [23,24].

The suggested association between maternal zinc depletion and poor outcomes of pregnancy and lactation have raised the possibility of prevention by using supplementary zinc, but results of previous studies have not been conclusive and still need to be assessed, especially focusing on populations with low dietary zinc intake [25].

The objective of the present study was to assess plasma zinc (PZn) levels and zinc supplementation rate during pregnancy, among lactating women attending to a public care center from a peri-urban Andean community of Quito, Ecuador in order to make an initial approach to the zinc status of lactating women in the region.

\section{Materials and Methods}

\subsection{Study Setting and Participants}

Between October 2015 and July 2016, a case study series was conducted in order to determine the rate of ZD among Ecuadorian mothers who have a breastfeeding infant, lived in Conocoto, and attended to the Primary Health Centre of Conocoto for regular paediatric and/or gynaecological check-ups. This health center belongs to the Ministry of Public Health which offers its services to the general population especially to people who have no private or social insurance [26].

Conocoto is one of the 33 rural parishes of Quito, the capital of Ecuador, located in the Andean region. Today, it is a heterogeneous territory with extensively urbanized areas and a few rural neighbourhoods pressed by growing urbanization. According to the last national census conducted in 2010, in Conocoto there were 82,072 inhabitants of whom 42,381 were women, with a population density of 1594 people $/ \mathrm{km}^{2}$ and a birth rate of 16.98 [27]. Of the 33 rural parishes of Quito, eight have been characterised as peri-urbans [23].

We collected pertinent clinical information and a single blood sample to quantify zinc levels from the mothers; after they read and signed an informed consent.

Inclusion criteria were women who lived in Conocoto and had a child who was being fed mainly with breast milk. By mainly, we mean that at the time of the interview at least $90 \%$ of the children's diet was breast milk, but three or fewer times a week some of them received other types of foods, such as "coladas" (a beverage made out of water and any kind of cereal flour), water, or formula. 
As exclusion criteria, we considered the following: diagnosis of any chronic disease, any malformation in the index child, use of oral contraceptives, consumption of alcohol, cigarettes or any recreational drug at any time, any respiratory or gastrointestinal infection during the fifteen days prior to sampling and use of pharmacological therapy within this period. Additionally, we excluded pregnant women, non-fasting women, women with any disability that interferes with sample collection, and women who consumed alcohol or performed extreme exercise the night before sampling, factors that were contemplated by ENSANUT-ECU. We followed these guidelines following the methodology of a previous report of our research group [20].

This study was conducted following the Declaration of Helsinki, approved by the Bioethics Committee of the Universidad Internacional del Ecuador and registered it under the number 04-2013.

\subsection{Measurements}

Maternal variables recorded were age, educational level, number of pregnancy check-ups, and intake of vitamin and mineral supplements. In regards to vitamin and mineral supplements, we asked at what time during pregnancy they started consuming it, doses, frequency, and the form of the supplement ingested. Infant variables registered were age at the time of the interview, sex, weight at birth, length at birth, and gestational age at birth. The weight, length, and gestational age at birth were obtained from hospital records. All other information was based on self-report by the mothers.

\subsection{Collection of Blood Samples and Determination of Zinc Levels}

A single blood sample was obtained between 8 and 10 a.m. from fasting mothers using tubes coated with lithium heparin and a gel separator (BD Vacutainer ${ }^{\circledR}$ PST $^{\mathrm{TM}}$, Becton-Dickinson Inc, Franklin Lakes, NJ, USA). The tubes were centrifuged for $10 \mathrm{~min}$ and refrigerated at $2-8{ }^{\circ} \mathrm{C}$ during 3-6 h. In the laboratory, the plasma was separated into additional tubes and stored at $-80^{\circ} \mathrm{C}$. Plasma samples contaminated with lysed red blood cells were discarded. Then, prior to zinc quantification, the refrigerated samples were thawed at room temperature $\left(20-24^{\circ} \mathrm{C}\right)$, and mixed with a vortex. PZn were measured by flame-atomic-absorption spectrophotometry (AAnalyst 400, PerkinElmert, Billerica, MA, USA), the coefficient of variation for the essay was $6.2 \%$. A standard solution of zinc in $1000 \pm 4 \mathrm{mg} / \mathrm{L}$ of nitric acid (Fluka ${ }^{\mathrm{TM}}$ Analytical, Steinheim, Germany) (2\% p/p) was used for the calibration curve with dilutions from 25 to $300 \mathrm{ppb}$. All chemicals were of analytical grade purity (Type I water, nitric acid $69 \%$ ). Zinc quantification of all the samples was performed at the same time in dust-free fume hoods with minimal contact with air. We followed the same criteria that in our previous report [20].

Previous reports suggest $10.70 \mu \mathrm{mol} / \mathrm{L}$ as the cut-off point for zinc deficiency in fasting females over 10 years-old [6,28], and this value was utilized as the cut-off point by ENSANUT-ECU [19]. Therefore, we adopted this cut-off point in order to determine ZD in the present study and to compare it with the general population of Ecuadorian women of reproductive age.

\subsection{Data Processing}

The sample was evaluated as one group and divided in two subgroups based on the mothers' declaration of zinc consumption during pregnancy. The data were analyzed using the Statistical Package for Social Science (SPSS) Statistics for Windows, version 20.0 (IBM Corp., Armonk, NY, USA). Correlations were assessed by Spearman's rho, and $p<0.05$ was considered as statistical significant. The $t$-test was used to compare the plasma zinc level means of the supplemented and non-supplemented groups.

In order to compare the prevalence of ZD in our sample with the reported among women of reproductive age by ENSANUT-ECU, we used the likelihood ratio and its corresponding statistic $\left(\mathrm{G}^{2}\right)$; $p<0.05$ was considered as statistical significant. 


\section{Results}

We collected data and blood samples from 64 mothers, 3.2 (1.8) months postpartum. The mothers were between 15 and 39 years old with a mean age of 26.8 (6.4) years old. The majority of their infants were full-term (93.8\%), and had adequate weight (76.6\%) and length (62.5\%) at birth (Table 1$)$.

Table 1. Plasma zinc levels, maternal and infant characteristics of zinc supplemented and non-supplemented women during their pregnancies $(n=64)$.

\begin{tabular}{|c|c|c|c|c|c|}
\hline Recorded Variables & $\begin{array}{c}\text { All the } \\
\text { Sample } \\
n=64\end{array}$ & $\begin{array}{c}\text { Women Who Did } \\
\text { Not Received Zinc } \\
\text { Supplements } \\
n=44\end{array}$ & $\begin{array}{c}\text { Women Who } \\
\text { Received Zinc } \\
\text { Supplements } \\
n=20\end{array}$ & $\begin{array}{l}\rho \text { (Rho) de } \\
\text { Spearman }\end{array}$ & $p$-Value \\
\hline \multicolumn{6}{|l|}{ Maternal variables } \\
\hline Age (years, mean $\pm S D$ ) * & $26.8 \pm 6.5$ & $26.1 \pm 6.8$ & $28.4 \pm 5.7$ & 0.17 & 0.17 \\
\hline \multicolumn{6}{|l|}{ Level of education (\%) } \\
\hline Elementary school only & 21.9 & 22.7 & 20.0 & 0.03 & 0.81 \\
\hline $\begin{array}{l}\text { High school or any } \\
\text { superior level }\end{array}$ & 78.1 & 77.3 & 80.0 & & \\
\hline \multicolumn{6}{|l|}{$\begin{array}{l}\text { Number of prenatal } \\
\text { check-ups }(\%)\end{array}$} \\
\hline$<5$ & 6.2 & 6.8 & 5.0 & 0.04 & 0.79 \\
\hline$\geq 5$ & 93.8 & 93.2 & 95.0 & & \\
\hline \multicolumn{6}{|l|}{$\begin{array}{l}\text { Consumption of iron during } \\
\text { pregnancy }(\%)\end{array}$} \\
\hline Yes & 93.8 & 90.9 & 100 & 0.17 & 0.17 \\
\hline No & 6.2 & 9.1 & 0 & & \\
\hline \multicolumn{6}{|l|}{$\begin{array}{l}\text { Consumption of folic acid } \\
\text { during pregnancy }(\%)\end{array}$} \\
\hline Yes & 96.9 & 95.5 & 100 & 0.12 & 0.34 \\
\hline No & 3.1 & 4.5 & 0 & & \\
\hline $\mathrm{PZn}(\mu \mathrm{mol} / \mathrm{L}$, mean $\pm \mathrm{SD}) *$ & $9.3 \pm 1.8$ & $9.07 \pm 1.7$ & $9.72 \pm 2.0$ & & \\
\hline Zinc deficiency $n(\%)$ & $52(81.2)$ & $37(84.1)$ & $15(75)$ & -0.11 & 0.40 \\
\hline \multicolumn{6}{|l|}{ Infant variables } \\
\hline $\begin{array}{l}\text { Infants' age (months, mean } \\
\pm \mathrm{SD})^{*}\end{array}$ & $3.2 \pm 1.8$ & $3.3 \pm 1.8$ & $3.0 \pm 1.7$ & & \\
\hline Sex (Male/Female) & $32 / 32$ & $22 / 22$ & $10 / 10$ & & \\
\hline \multicolumn{6}{|l|}{ Gestational age at birth (\%) } \\
\hline 37 weeks or more & 92.2 & 90.9 & 95.0 & 0.07 & 0.58 \\
\hline Less than 37 weeks & 7.8 & 9.1 & 5.0 & & \\
\hline \multicolumn{6}{|l|}{ Weight at birth } \\
\hline $2500 \mathrm{~g}$ or more & 78.1 & 79.5 & 75.0 & -0.05 & 0.69 \\
\hline Less than $2500 \mathrm{~g}$ & 21.9 & 20.5 & 25.0 & & \\
\hline \multicolumn{6}{|l|}{ Length at birth (\%) } \\
\hline $48 \mathrm{~cm}$ or more & 62.5 & 61.4 & 65.0 & 0.04 & 0.79 \\
\hline Less than $48 \mathrm{~cm}$ & 37.5 & 38.6 & 35.0 & & \\
\hline
\end{tabular}


The percentages of women who consumed folic acid, iron, and zinc during pregnancy were $96.9 \%, 93.8 \%$, and $31.2 \%$, respectively (Table 1 ). The participants who reported ingesting zinc during pregnancy affirmed to have consumed zinc as part of a multivitamin supplement, but the majority of them were not able to accurately provide the doses and frequency of the supplements consumed and it was not possible to analyze this data.

The PZn levels were between 3.9 to $13.0 \mu \mathrm{mol} / \mathrm{L}$, the mean PZn concentration was 9.3 (1.8) $\mu \mathrm{mol} / \mathrm{L}$. The first, second, third, and fourth quartiles were 8.1, 9.2, 10.3, and $13.0 \mu \mathrm{mol} / \mathrm{L}$, respectively. We found ZD in 52 women with a prevalence of ZD of 81.2\% (95\% CI: 71.7-90.9), which is statically different to the reported among women of reproductive age by ENSANUT-ECU of 56.1\% (95\% CI: 54.9-57.1) $\left(\mathrm{G}^{2}=18.23 ; p<0.05\right)$ [19]. There was no difference between the means of plasma zinc levels of the supplemented and non-supplemented groups $(t=1.34 ; p>0.05)$.

\section{Discussion}

The prevalence of ZD worldwide is unknown. Nevertheless, a global inadequate zinc intake rate of $17.3 \%$ has been estimated with a high geographical variation. The Andean region, which Ecuador is part of, has an estimated inadequate zinc intake of 17.0\% [29]. ZD is widespread especially among developing countries affecting mainly children, and pregnant and lactating women [2,3,5,9], and it is expected to especially affect people living in areas of high urbanization that have been associated with higher levels of inequality and subsequent micronutrient deficiencies [21,22].

Many studies have found that ZD is higher among pregnant than non-pregnant women [13-16] and that zinc levels between pregnancy and postpartum periods do not differ significantly [30], which raises the need to evaluate zinc status among pregnant and lactating women in Ecuador, where high rates of ZD have been reported [19].

In Ecuador, the prevalence of ZD reported by ENSANUT-ECU among women of reproductive age from the general population is $56.1 \%$ and varies across the country, being higher in the urban coast $(62.9 \%)$ and lower in the rural highlands (46.6\%). In Quito, the prevalence of ZD is 55.6\% [19].

Our results showed a higher prevalence of ZD among lactating women than in non-pregnant non-lactating women of reproductive age reported by ENSANUT-ECU [19], which agrees with previous reports from India, Ethiopia, and Pakistan. However, it is worth clarifying that the studies performed in Ethiopia and Pakistan evaluated zinc deficiency in both groups which were part of the same cohort [14-16]. Additionally, it is important to note that we are comparing two groups with different physiological status because lactating women are in a period of high metabolic demand in order to supply milk for their offspring $[5,6]$.

Another factor that could be related to the high prevalence of ZD in our sample is the fact that nearly all the participants received iron supplements during pregnancy. The competition between iron and zinc absorption is well known, which means that PZn levels will be lower when iron is concurrently supplemented $[6,31,32]$.

Although a mother with ZD most likely would have breast milk with a normal zinc concentration due to a physiological adjustment that tries to supply enough zinc for their offspring, it has been found that children of mothers with profound ZD are at risk of ZD and its consequences [17,31,33]. Therefore, an adequate zinc nutrition is necessary for normal pregnancy outcomes, child growth, adequate immune function and neurocognitive development [2,4,5,7-11]. We could not find any association between maternal zinc levels, zinc supplementation, and the anthropometry of their infants at birth, probably because our sample was small with the majority having ZD; nevertheless, the rates of ZD, premature delivery, and low weight and length at birth are not negligible [11]. Despite this, comparing the groups based on the declaration of zinc ingestion during pregnancy, we observed a slightly lower prevalence of zinc deficiency among the supplemented group than the non-supplemented group which was not statistically significant. The aforementioned statements raise the necessity to extend studies in order to assess the need of zinc supplementation among lactating women in disadvantaged groups with similar conditions. 
Zinc supplementation has proven to reduce the rate of premature delivery, and improve linear growth and weight gain, especially in children of short stature, and decrease morbidity, duration, and severity of diarrhea and acute lower respiratory infection [1,8,34]. Supplementing zinc during pregnancy and lactation increases the mothers' PZn concentration by $3 \%$ and $1 \%$, respectively, which could protect the mothers' nutritional status and, therefore, their children during these periods of life [35].

In spite of the aforementioned health zinc benefits, the World Health Organization (WHO) recommends zinc supplementation for pregnant women only in the context of rigorous research due to the moderate to low certainty evidence related to maternal, fetal, and neonatal outcomes, and no guidelines for zinc supplementation during lactation exist $[7,36]$. Nevertheless, we found that $31.2 \%$ of the participants were supplemented with zinc during pregnancy and suspended after delivery. Maternal zinc supplementation during lactation has yielded inconsistent results regarding the improvement of breast milk zinc concentrations or to demonstrate benefits for mothers and their infants [37,38]; however, considering the high rates of ZD among lactating women, it is worth extending studies to address the need of zinc supplementation identifying a profile of mothers that could benefit from this.

It is important to mention that in a previous report following the same methodology, we evaluated PZn levels in a cohort of 35 women who had a child between 1 and 12-months-old with an oral malformation, reporting a prevalence of $\mathrm{ZD}$ of 31.4 ; these results were probably due to the use of formula to feed the children because the oral malformation causes breastfeeding difficulties [20]. Lactation is a risk factor for zinc deficiency and the recommended dietary allowance is higher among pregnant and lactating women than in non-pregnant non-lactating women [6]. Therefore, the use of formula could have protected the mothers of children with the oral malformation from developing ZD in the postpartum period and could explain its lower ZD prevalence compared with the women of the present study.

Our study had several limitations. First, the sample corresponds to a small group of women from a single peri-urban community of Quito which does not allow extending these results, as the prevalence of zinc deficiency could differ by taking into account differences in socio-economic status or geographical location, however, this could be an example of what is happening among women from communities under pressure of accelerated urbanization where inequality levels are high [21,22].

Second, even though women who were going through any type of clinical infection or disease were excluded from the study, we did not assess any inflammatory marker, such as alpha 1 glycoprotein, C-reactive protein and interleukin-6; that would allow exclusion of women with an inflammatory process that could be related to decreased PZn $[39,40]$. It is also important to note that postpartum women, in general, have a more active immune system and higher serum levels of proinflammatory cytokines, and it is somewhat more active among breastfeeding than formula-feeding women [41]. Third, we were unable to register the doses, frequency, and forms of zinc ingested as, most of time, it was part of a multivitamin and mineral supplement; this did not allow for a deep analysis regarding zinc supplementation. Finally, we included only healthy women, therefore, this prevalence would likely increase if women with any comorbidity were included.

The results of this study raise the necessity to evaluate zinc supplementation during pregnancy and the extension to the lactation period in breastfeeding women who attend public care centers for their controls after delivery living in communities experiencing accelerated urbanization, such as Conocoto. Furthermore, we recommend extending studies in the Andean region and in areas with a suspected or confirmed high prevalence of ZD to document levels of zinc among breastfeeding women.

Author Contributions: M.Á.J.-P. conceived the study, analyzed and interpreted the results, helped draft the manuscript, and provided overall coordination of the study. C.N.-C. collected data, analyzed the results, and drafted the manuscript. G.M. collected data and helped draft the manuscript. B.A.S. participated in the study design and analyzed the results. All authors read and approved the final manuscript.

Funding: This research was funded by Universidad Internacional del Ecuador. 
Acknowledgments: The authors wish to thank Angélica C. Cornejo for the critical review and Andrés A. Galvis for his help with the statistical analysis. We also thank David Acosta and the staff of the Public Medical Center of Conocoto who helped us planning the sampling, and to the mothers who participated in the study; contributing considerably.

Conflicts of Interest: The authors declare no conflict of interest.

\section{References}

1. Brown, K.H.; Wuehler, S.E.; Peerson, J.M. The importance of zinc in human nutrition and estimation of the global prevalence of zinc deficiency. Food Nutr. Bull. 2001, 22, 113-125. [CrossRef]

2. Colombo, J.; Zavaleta, N.; Kannass, K.; Lazarte, F.; Albornoz, C.; Kapa, L.; Caulfield, L. Zinc supplementation sustained normative neurodevelopment in a randomized, controlled trial of peruvian infants aged 6-18 months. J. Nutr. 2014, 144, 1298-1305. [CrossRef] [PubMed]

3. Bailey, R.L.; West, K.P.; Black, R.E. The epidemiology of global micronutrient deficiencies. Ann. Nutr. Metab. 2015, 66, 22-33. [CrossRef] [PubMed]

4. Institute of Medicine (US) Committee on Micronutrient Deficiencies; Howson, C.P.; Kennedy, E.T.; Horwitz, A. Prevention of Micronutrient Deficiencies: Tools for Policymakers and Public Health Workers; National Academies Press (US): Washington, DC, USA, 1998. Available online: https://www.ncbi.nlm.nih.gov/ books/NBK230107/ (accessed on 3 July 2018).

5. Ejezie, F.E.; Nwagha, U.I. Zinc Concentration During pregnancy and Lactation in Enugu, South-East Nigeria. Ann. Med. Health Sci. Res. 2011, 1, 69-76. [PubMed]

6. King, J.C.; Brown, K.H.; Gibson, R.S.; Krebs, N.F.; Lowe, N.M.; Siekmann, J.H.; Raiten, D.J. Biomarkers of Nutrition for Development (BOND)—Zinc Review. J. Nutr. 2016, 146, 858S. [CrossRef] [PubMed]

7. Ota, E.; Mori, R.; Middleton, P.; Tobe-Gai, R.; Mahomed, K.; Bhutta, Z. Zinc supplementation for improving pregnancy and infant outcome. Cochrane Database Syst. Rev. 2012, 1-97. [CrossRef] [PubMed]

8. Black, M. Zinc deficiency and child development. Am. J. Clin. Nutr. 1998, 68, 464S-469S. [CrossRef] [PubMed]

9. Liu, J.; Hanlon, A.; Ma, C.; Zhao, S.R.; Cao, S.; Compher, C. Low blood zinc, iron, and other sociodemographic factors associated with behavior problems in preschoolers. Nutrients 2014, 6, 530-545. [CrossRef] [PubMed]

10. Tamura, T.; Goldenberg, R.L.; Ramey, S.L.; Nelson, K.G.; Chapman, V.R. Effect of zinc supplementation of pregnant women on the mental and psychomotor development of their children at 5 years of age. Am. J. Clin. Nutr. 2003, 77, 1512-1516. [CrossRef] [PubMed]

11. Wang, H.; Hu, Y.-F.; Hao, J.-H.; Chen, Y.-H.; Su, P.-Y.; Wang, Y.; Yu, Z.; Fu, L.; Xu, Y.-Y.; Zhang, C.; et al. Maternal zinc deficiency during pregnancy elevates the risks of fetal growth restriction: A population-based birth cohort study. Sci. Rep. 2015, 5, 11262. [CrossRef] [PubMed]

12. Caulfield, L.E.; Zavaleta, N.; Shankar, A.H.; Merialdi, M. Potential contribution of maternal zinc supplementation during pregnancy to maternal and child survival 1-3. Am. J. Clin. Nutr. 1998, 68, 499S-508S. [CrossRef] [PubMed]

13. Pathak, P.; Kapil, U.; Dwivedi, S.N.; Singh, R. Serum zinc levels amongst pregnant women in a rural block of Haryana state, India. Asia Pac. J. Clin. Nutr. 2008, 17, 276-279. [PubMed]

14. Harika, R.; Faber, M.; Samuel, F.; Kimiywe, J.; Mulugeta, A.; Eilander, A. Micronutrient status and dietary intake of iron, Vitamin A, iodine, folate and zinc in women of reproductive age and pregnant women in Ethiopia, Kenya, Nigeria and South Africa: A systematic review of data from 2005 to 2015. Nutrients 2017, 9, 1096. [CrossRef] [PubMed]

15. Akhtar, S. Zinc status in South Asian populations-An update. J. Health Popul. Nutr. 2013, 31, 139-149. [CrossRef] [PubMed]

16. Bhutta, Z. Pakistan National Nutrition Survey 2011. Natl. Nutr. Surv. 2012, 1-84. Available online: http:/ / www.mhinnovation.net/sites/default/files/downloads/innovation/research/Pakistan\% 20National\%20Nutrition\%20Survey\%202011.pdf (accessed on 3 July 2018).

17. Dijkhuizen, M.A.; Wieringa, F.T.; West, C.E.; Muherdiyantiningsih; Muhilal. Concurrent micronutrient deficiencies in lactating mothers and their infants in Indonesia. Am. J. Clin. Nutr. 2001, 73, 786-791. [PubMed] 
18. Nakamori, M.; Ninh, N.X.; Isomura, H.; Yoshiike, N.; Hien, V.T.T.; Nhug, B.T.; Nhien, N.V.; Nakano, T.; Khan, N.C.; Yamamoto, S. Nutritional status of lactating mothers and their breast milk concentration of iron, zinc and copper in rural Vietnam. J. Nutr. Sci. Vitaminol. 2009, 55, 338-345. [CrossRef] [PubMed]

19. Freire, W.B.; Ramírez-Luzuriaga, M.J.; Belmont, P.; Mendieta, M.J.; Silva-Jaramillo, K.; Romero, N.; Sáenz, K.; Piñeiros, P.; Gómez, L.F.; Monge, R. Tomo I: Encuesta Nacional de Salud y Nutrición de la población ecuatoriana de cero a 59 años; ENSANUT-ECU 2012; Ministerio de Salud Pública/Instituto Nacional de Estadísticas y Censos: Quito, Ecuador, 2014. Available online: http:/ / www.ecuadorencifras.gob.ec/documentos/webinec/Estadisticas_Sociales/ENSANUT/MSP_ENSANUT-ECU_06-10-2014.pdf (accessed on 3 July 2018).

20. Jara-Palacios, M.A.; Cornejo, A.C.; Narváez-Caicedo, C.; Moreano, G.; Vásquez, K.P.; Moreno-Izquierdo, C.; Romero-Sandoval, N. Plasma zinc levels in Ecuadorian mothers of infants with nonsyndromic cleft lip with or without cleft palate: A case series. Birth Defects Res. 2018, 495-501. [CrossRef] [PubMed]

21. Freire, W.; Silva-jaramillo, K.; Ramírez-Luzuriaga, M.; Belmont, P.; Waters, W. The double burden of undernutrition and excess body weight in Ecuador. Am. J. Clin. Nutr. 2014, 100, 1636-1643. [CrossRef] [PubMed]

22. Schram, A.; Labonté, R.; Sanders, D. Urbanization and international trade and investment policies as determinants of noncommunicable diseases in sub-saharan Africa. Prog. Cardiovasc. Dis. 2013, 56, 281-301. [CrossRef] [PubMed]

23. Pinto-Valencia, P. Las parroquias rurales de Quito y sus interrelaciones con el espacio urbano. Revista del Instituto de la Ciudad 2013, 2, 71-119. Available online: http://repositorio.flacsoandes.edu.ec/bitstream/ 10469/6454/1/REXTN-QUR5-03-Pinto.pdf (accessed on 3 July 2018).

24. Diagnóstico del Territorio del Distrito Metropolitano de Quito. Unidad del Plan de Desarrollo y Ordenamiento Territorial del DMQ. Municipio del Distrito Metropolitano de Quito: Diagnóstico Estratégico-Eje Territorial. 2014. Available online: http://gobiernoabierto.quito.gob.ec/wp-content/uploads/documentos/pdf/ diagnosticoterritorio.pdf (accessed on 3 July 2018).

25. Mahomed, K.; James, D.K.; Golding, J.; McCabe, R. Zinc supplementation during pregnancy: A double blind randomised controlled trial. Br. Med. J. 1989, 299, 826-830. [CrossRef]

26. Lucio, R.; Villacrés, N.; Henríquez, R. Sistema de salud de Ecuador. Salud Pub. Mex. 2011, 53, $177-187$. [CrossRef]

27. Gobierno Autónomo Descentralizado Parroquial de Conocoto. Plan De Desarrollo y Ordenamiento Territorial de Conocoto 2012-2025; Dirección de Gestión de Desarrollo Comunitario e Inclusión Social/Dirección de Gestión de Planificación: Quito, Ecuador, 2012. Available online: http://181.112.151.230:8081/attachments/ download/558/PDOT\%20CONOCOTO\%202012.pdf (accessed on 3 July 2018).

28. Hotz, C.; Peerson, J.M.; Brown, K.H. Suggested lower cutoffs of serum zinc concentrations for assessing zinc status: Reanalysis of the second National Health and Nutrition Examination Survey data (1976-1980). Am. J. Clin. Nutr. 2003, 78, 756-764. [CrossRef] [PubMed]

29. Wessells, K.R.; Brown, K.H. Estimating the Global Prevalence of Zinc Deficiency: Results Based on Zinc Availability in National Food Supplies and the Prevalence of Stunting. PLoS ONE 2012, 7, e50568. [CrossRef] [PubMed]

30. Severi, C.; Hambidge, M.; Krebs, N.; Alonso, R.; Atalah, E. Zinc in plasma and breast milk in adolescents and adults in pregnancy and pospartum: A cohort study in Uruguay. Nutr. Hosp. 2013, 28, 223-228. [CrossRef] [PubMed]

31. Donangelo, C.M.; King, J.C. Maternal zinc intakes and homeostatic adjustments during pregnancy and lactation. Nutrients 2012, 4, 782-798. [CrossRef] [PubMed]

32. Swanson, C.A.; King, J.C. Zinc utilization in pregnant and nonpregnant women fed controlled diets providing the zinc RDA. J. Nutr. 1982, 112, 697-707. [CrossRef] [PubMed]

33. Donangelo, C.M.; Vargas-Zapata, C.L.; Woodhouse, L.R.; Shames, D.M.; Mukherjea, R.; King, J.C. Zinc absorption and kinetics during pregnancy and lactation in Brazilian women. Am. J. Clin. Nutr. 2005, 82, 118-124. [CrossRef] [PubMed]

34. Bhutta, Z.A.; Black, R.E.; Brown, K.H.; Meeks-Gardner, J.; Gore, S.; Hidayat, A.; Khatun, F.; Martorell, R.; Ninh, N.X.; Penny, M.E.; et al. Prevention of diarrhea and pneumonia by zinc supplementation in children in developing countries: Pooled analysis of randomized controlled trials. J. Pediatr. 1999, 135, 689-697. [CrossRef] 
35. Moran, V.H.; Skinner, A.L.; Medina, M.W.; Patel, S.; Dykes, F.; Souverein, O.W.; Dullemeijer, C.; Lowe, N.M. The relationship between zinc intake and serum/plasma zinc concentration in pregnant and lactating women: A systematic review with dose-response meta-analyses. J. Trace Elem. Med. Biol. 2012, 26, 74-79. [CrossRef] [PubMed]

36. World Health Organization (WHO). WHO Recommendation on Antenatal Care for a Positive Pregnancy Experience; WHO: Geneva, Switzerland, 2016.

37. Krebs, N. Zinc supplementation during lactation. Am. J. Clin. Nutr. 1998, 510S-512S. [CrossRef] [PubMed]

38. Sazawal, S.; Black, R.E.; Dhingra, P.; Jalla, S.; Krebs, N.; Malik, P.; Dhingra, U.; Bhan, M.K. Zinc Supplementation does not Affect the Breast Milk Zinc Concentration of Lactating Women Belonging to Low Socioeconomic Population. J. Hum. Nutr. Food Sci. 2013, 1, 1-6.

39. Karakochuk, C.D.; Barr, S.I.; Boy, E.; Bahizire, E.; Tugirimana, P.L.; Akilimali, P.Z.; Houghton, L.A.; Green, T.J. The effect of inflammation on serum zinc concentrations and the prevalence estimates of population-level zinc status among Congolese children aged 6-59 months. Eur. J. Clin. Nutr. 2017, 71, 1467-1470. [CrossRef] [PubMed]

40. MacDonell, S.O.; Miller, J.C.; Harper, M.J.; Reid, M.R.; Haszard, J.J.; Gibson, R.S.; Houghton, L.A. A comparison of methods for adjusting biomarkers of iron, zinc, and selenium status for the effect of inflammation in an older population: A case for interleukin 6. Am. J. Clin. Nutr. 2018, 932-940. [CrossRef] [PubMed]

41. Groer, M.W.; Davis, M.W.; Smith, K.; Casey, K.; Kramer, V.; Bulovsky, E. Immunity, inflammation and infection in post-partum breast and formula feeders. Am. J. Reprod. Immunol. 2005, 54, 222-231. [CrossRef] [PubMed]

(C) 2018 by the authors. Licensee MDPI, Basel, Switzerland. This article is an open access article distributed under the terms and conditions of the Creative Commons Attribution (CC BY) license (http:/ / creativecommons.org/licenses/by/4.0/). 


\title{
Lactation Duration and Long-Term Thyroid Function: A Study among Women with Gestational Diabetes
}

\author{
Pranati L. Panuganti ${ }^{1,2}$, Stefanie N. Hinkle ${ }^{1}$, Shristi Rawal ${ }^{1,3}$, Louise G. Grunnet ${ }^{4,5,6}$, \\ Yuan Lin ${ }^{1,7}$, Aiyi Liu ${ }^{1}$, Anne C. B. Thuesen ${ }^{5}$, Sylvia H. Ley ${ }^{8,9}$, Sjurdur F. Olesen ${ }^{4}$ and \\ Cuilin Zhang ${ }^{1, *}$
}

1 Epidemiology Branch, Division of Intramural Population Health Research, Eunice Kennedy Shriver National Institute of Child Health and Human Development, National Institutes of Health, Bethesda, MD 20817, USA; pranati_panuganti@brown.edu (P.L.P.); hinklesn@mail.nih.gov (S.N.H.); shristi.rawal@rutgers.edu (S.R.); linyy@iu.edu (Y.L.); liua@mail.nih.gov (A.L.)

2 The Warren Alpert Medical School of Brown University, Providence, RI 02903, USA

3 Department of Nutritional Sciences, School of Health Professions, Rutgers University, Newark, NJ 07107, USA

4 Centre for Fetal Programming, Department of Epidemiology Research, Statens Serum Institut, DK-2300 Copenhagen, Denmark; Louise.Groth.Grunnet@regionh.dk (L.G.G.); SFO@ssi.dk (S.F.O.)

5 Department of Endocrinology, Rigshospitalet University Hospital, DK-2200 Copenhagen, Denmark; anne.cathrine.baun.thuesen@regionh.dk

6 The Danish Diabetes Academy, DK-5000 Odense, Denmark

7 Department of Epidemiology, Richard M. Fairbanks School of Public Health, Indiana University, Indianapolis, IN 46202, USA

8 Harvard T.H. Chan School of Public Health, Harvard University, Boston, MA 02115, USA; sylvia.ley@channing.harvard.edu

9 Channing Division of Network Medicine, Harvard Medical School and Brigham and Women's Hospital, Boston, MA 02115, USA

* Correspondence: zhangcu@mail.nih.gov; Tel.: +1-301-435-6917

Received: 19 June 2018; Accepted: 17 July 2018; Published: 21 July 2018

\begin{abstract}
Lactation is associated with reduced postpartum weight retention and a lower risk of several cardiometabolic disorders in population-based studies. We examined the association between lactation and long-term thyroid function among women with history of gestational diabetes mellitus (GDM), a high-risk population for subsequent metabolic complications. The study included 550 women who developed GDM in the Danish National Birth Cohort (1996-2002) and followed-up in the Diabetes \& Women's Health Study (2012-2014). We assessed adjusted associations between cumulative lactation duration and concentrations of thyroid stimulating hormone (TSH), free triiodothyronine (fT3), and free thyroxine (fT4) measured at follow-up. Women with longer cumulative lactation duration tended to have higher fT3 levels (adjusted $\beta$ and $95 \%$ confidence interval (CI) for $\geq 12$ months vs. none: $0.19(0.03-0.36)$; $p$-trend $=0.05)$. When restricted to women with a single lifetime pregnancy to control for parity $(n=70)$, women who lactated for $>6$ months (vs. none) had higher fT3 levels $(0.46 \mathrm{pmol} / \mathrm{L}(0.12-0.80) ; p$-trend $=0.02)$ and a higher fT3:fT4 ratio (0.61 (0.17-1.05); $p$-trend $=0.007)$. Our findings suggested that a longer duration of lactation may be related to greater serum fT3 levels and fT3:fT4 ratio 9-16 years postpartum among Danish women with a history of GDM. The association was particularly pronounced among women who only had one lifetime pregnancy.
\end{abstract}

Keywords: GDM; lactation; thyroid; triiodothyronine; thyroxine; thyroid antibodies 


\section{Introduction}

The thyroid gland is involved in several physiological processes, including glucose metabolism, muscle repair, cardiovascular function, and thermogenesis [1]. Thyroid dysfunction may be manifested as clinical, subclinical, or autoimmune, depending on the levels of thyroid hormones (thyroid stimulating hormone (TSH), free triiodothyronine (fT3), and free thyroxine (fT4)) and anti-thyroid antibodies (e.g., thyroperoxidase antibody (anti-TPO), and thyroglobulin antibody (anti-TG)). Women who develop diabetes in pregnancy, or gestational diabetes mellitus (GDM), are particularly subject to a substantially increased risk for cardiometabolic disorders [2]. Given the importance of thyroid hormones in maintaining the function of multiple systems and regulatory pathways related to cardiometabolic functions, the identification of potentially modifiable factors related to thyroid function among GDM women is of important clinical and public health significance.

Lactation has been associated with several health benefits in women [3]. Foremost, postpartum weight retention is reduced in women who breastfed their children [3]. In addition, women with a longer lifetime duration of lactation are at lower risk for cardiometabolic disorders, including cardiovascular disease, type 2 diabetes mellitus (T2DM), and hypertension [3]. Interestingly, animal data also demonstrate that increasing lactation duration is associated with heightened thyroid activity, resulting in increased levels of biologically active thyroid hormone, triiodothyronine (T3) [4]. Specifically, in bovine models, mRNA transcripts for the enzyme responsible for generating $\mathrm{T} 3$ were 6-fold greater with 90 days of lactation compared to levels preceding birth [4]. A link between lactation and thyroid function may lie in the reduction of postpartum weight retention, as weight gain was previously highly associated with TSH and fT4 humans [5]. However, human data are lacking for the influence of lactation on long-term thyroid function among both the general population and women with a history of GDM in particular.

The objective of this study was to examine the association between lactation duration and long-term thyroid function among a high-risk cohort of women with a history of GDM [6-13]. We hypothesize that a longer cumulative lactation duration is associated with improved thyroid function, approximately 9-16 years after the index GDM pregnancy.

\section{Materials and Methods}

\subsection{Study Design}

In the Diabetes \& Women's Health (DWH) Study [14], we followed up women identified as having GDM during the index pregnancy of the Danish National Birth Cohort (DNBC) [15]. The DNBC collected data on maternal demographics, perinatal exposures, and health conditions through four telephone interviews conducted at gestational weeks 12 and 30, and 6 and 18 months postpartum (1996-2002). The DWH Study (2012-2014) enrolled 790 women who had GDM during their index pregnancy, 9-16 years after their enrollment in the DNBC. This analysis was limited to women who completed the DWH Study clinical exam in which biospecimens were collected $(n=619)$ and for whom thyroid markers were available $(n=611 ; 99.0 \%)$. Women reporting thyroid disease before the index DNBC pregnancy $(n=18 ; 3.0 \%)$ were excluded. We further excluded participants with missing data on breastfeeding duration $(n=43 ; 7.3 \%)$ to arrive at the final analytic sample $(n=550)$. All participants provided informed consent. The study was approved by the Regional Scientific Ethical Committee (VEK) of the Capital Region of Denmark (record No. H-4-2013-129).

\subsection{Ascertainment of Cumulative Lactation History after Index GDM Pregnancy}

At the DWH Study follow-up, women retrospectively reported the duration of lactation for each of their pregnancies. The cumulative duration of lactation was calculated by summing the number of lactating months following each birth and was categorized as: none, $<6,6$ to $<12$, or $\geq 12$ months. Because we lacked covariate information for pregnancies before the index DNBC pregnancy, we calculated lifetime lactation duration starting at the index pregnancy and thus, adjusted for parity at 
the index pregnancy and performed further analyses limited to women who were nulliparous at the index pregnancy.

To assess the validity of recalled lactation duration, we examined the correlation between lactation duration proximally reported for the index DNBC pregnancy at 6 and 18 months postpartum interviews, and the duration recalled for the corresponding pregnancy on the DWH Study follow-up questionnaire 9-16 years postpartum. The correlation was high $(r=0.81)$, and $69.9 \%$ of women accurately reported the duration within 1 month. In our analyses, we use the retrospectively reported lactation data as it captured the lactation history over all pregnancies as opposed to only the index pregnancy.

\subsection{Thyroid Function}

At the DWH Study follow-up clinical exam, fasting venous blood samples were collected, processed within $1 \mathrm{~h}$, and stored at $-80^{\circ} \mathrm{C}$ until being analyzed by a central laboratory following a standardized protocol. All samples were assayed in a single batch by a certified clinical laboratory at the University of Minnesota. Concentrations of thyroid stimulating hormone (TSH) (mIU/L) were measured using a sandwich immunoassay (Roche Diagnostics, Indianapolis, IN, USA). Concentrations of free triiodothyronine (fT3) (pmol/L), free thyroxine (fT4) (ng/dL), anti-TPO (IU/mL), and anti-TG (thyroglobulin antibody; IU $/ \mathrm{mL}$ ) were measured using a competitive immunoassay (Roche Diagnostics, Indianapolis, IN, USA). The fT3:fT4 ratio was calculated by dividing serum concentrations of fT3 (pmol/L) by fT4 (ng/dL). The inter-assay coefficients of variance (CV) were all $<6.2 \%$ for fT3, fT4, and TSH and $<15.1 \%$ for the anti-TPO and anti-TG antibodies.

Thyroid function was assessed based on continuous levels of thyroid hormones (TSH, fT3, fT4, fT3:fT4). Additionally, following the American Thyroid Association guidelines, subclinical hypothyroidism (SCH) was defined as having an elevated TSH (normal range 0.45-4.12 mIU/L) with normal fT4 levels (0.93-1.7 ng/dL) [16]. Subclinical hyperthyroidism was defined as having low to undetectable TSH with normal levels of fT3 (normal range 3.53-6.45 pmol/L) and fT4 (normal range $0.93-1.7 \mathrm{ng} / \mathrm{dL}$ ) $[16,17]$. Women with both TSH and fT4 levels within the normal range were classified as euthyroid. Participants who did not meet the criteria for euthyroid, SCH, or subclinical hyperthyroidism were classified as "other". Lastly, participants were categorized as positive for anti-TPO and anti-TG if their antibody levels were above lab reference ranges (anti-TPO: $\geq 35 \mathrm{IU} / \mathrm{mL}$; anti-TG: $\geq 115 \mathrm{IU} / \mathrm{mL}$ ).

\subsection{Covariates}

Data on potential confounders were available from interview responses at the baseline index DNBC pregnancy. Covariates selected a priori included age (years), socioeconomic status (high or medium level professional, skilled worker, other (student, unskilled, unemployed)), nulliparity (yes, no), smoking during pregnancy (any, none), alcohol during pregnancy (any, none) and pre-pregnancy body mass index (BMI) $\left(<25,25-29.9, \geq 30.0 \mathrm{~kg} / \mathrm{m}^{2}\right)$ calculated from self-reported height and pre-pregnancy weight. Pre-pregnancy weight was not reported at the time of the index pregnancy for a small sub-set of women $(n=41)$, however, pre-pregnancy weight was reported on the DWH Study follow-up questionnaire. For women who reported a pre-pregnancy weight at both instances the weight was highly correlated $(r=0.89)$ and thus, was used to supplement the missing data when available $(n=40)$.

We considered effect modification by several key variables at the DWH Study follow-up, including T2DM status, age, menopausal status, and long-term weight change since the index pregnancy. T2DM status was classified based on $\mathrm{HbA} 1 \mathrm{c}$ levels $\geq 6.5 \%$, fasting glucose $\geq 7 \mathrm{mmol} / \mathrm{L}, 2 \mathrm{~h}$ glucose after $75 \mathrm{~g}$ oral glucose tolerance test $\geq 11.1 \mathrm{mmol} / \mathrm{L}$, or self-report of physician diagnosis at follow-up [18]. Type 1 diabetes at follow-up was based on self-report of physician diagnosis. Women self-reported their menopausal status (yes, no). Age was categorized according to the median age in the cohort at follow-up ( $<45$ years vs. $\geq 45$ years). Long-term weight change was calculated as the difference in 
measured weight at the DWH follow-up and self-reported pre-pregnancy weight at the DNBC index pregnancy and was categorized according to the median level ( $<4.1 \mathrm{~kg} \mathrm{vs.} \geq 4.1 \mathrm{~kg})$.

\subsection{Statistical Analysis}

Characteristics of study participants were presented overall and by cumulative lactation duration after the index pregnancy. Differences across lactation duration were described as mean (standard deviation, SD) for continuous variables and frequency (\%) for categorical variables. Bivariate associations were evaluated using the one-way Analysis of Variance (ANOVA) for continuous variables and the $\chi^{2}$ test for categorical variables.

For each lactation category, we used generalized linear models to estimate the unadjusted and adjusted differences in concentrations of TSH, fT3, fT4 and fT3:fT4, by categories of cumulative lactation duration, with no lactation as the reference group. We assessed linear trends ( $p$-trend) by using the median for each category of lactation duration as a continuous exposure. We used logistic regression to estimate unadjusted and adjusted odds ratios (ORs) for $\mathrm{SCH}$, anti-TG positivity, and anti-TPO positivity. All multivariable models were adjusted for potential confounders measured at the index pregnancy and included age, socioeconomic status, pre-pregnancy BMI, parity, smoking during pregnancy, and alcohol consumption during pregnancy. An unknown category was used to account for the small proportion of missing covariate data. Due to a small number of women with $\mathrm{SCH}(n=17)$, the models were unstable and results for $\mathrm{SCH}$ are not reported.

To ensure that our findings were not biased by pregnancy or lactation characteristics prior to the index DNBC pregnancy and to remove the effect of parity, we repeated our analyses with only women who had one pregnancy overall and thus, were nulliparous at the index pregnancy. By limiting our sample, we also reduced recall bias from women with multiple pregnancies. In this subset of women, lactation was based on only a single pregnancy and was categorized as none, $>0$ to 6 months, and $>6$ months.

To understand whether the observed findings differed across several clinically distinct subgroups of individuals, we tested for a multiplicative interaction with the following characteristics measured at the DWH Study follow-up: age, menopausal status, T2DM status, and long-term weight change.

Two-tailed $p$-values $<0.05$ were considered significant. All statistical analyses were performed using SAS version 9.4 (SAS Institute, Cary, NC, USA).

\section{Results}

Overall, the median cumulative duration of lactation after a median of one (interquartile range $1-2)$ pregnancy was 9 (interquartile range 4-15) months, including a lactation duration of 0 months for women who reported never lactating $(n=62,11.3 \%)$. With an increasing duration of lactation, women tended to be lower in pre-pregnancy BMI, of older age, multiparous, and report no alcohol consumption during the index pregnancy. Thyroid biomarkers for most participants were within the euthyroid range $(n=476,86.6 \%)$, while the remainder were classified as SCH ( $n=19,3.5 \%)$, hyperthyroid $(n=7,1.3 \%)$, or other $(n=48,8.7 \%)$. Participants in the "other" category did not meet the criteria for $\mathrm{SCH}$, hyperthyroidism, or euthyroid. Several participants were positive for anti-TPO $(n=78,14.2 \%)$ and anti-TG $(n=69,12.6 \%)$ (Table 1$)$.

There was no significant association between lactation and odds of anti-TG positivity or anti-TPO positivity (Table 2). Table 3 shows the unadjusted and adjusted differences in women's thyroid marker levels for each category of cumulative lactation, compared to women who never lactated. Women with a longer lactation duration had higher fT3 levels at follow-up, (adjusted $\beta$ and 95\% confidence interval (CI) for $\geq 12$ months vs. none: $0.19(0.03,0.36)$; $p$-trend $=0.05)$. No significant associations were observed between lactation and concentrations of TSH, fT4, or the fT3:fT4 ratio at follow-up. 
Table 1. Participant characteristics overall and by lactation history from the Diabetes \& Women's Health Study.

\begin{tabular}{|c|c|c|c|c|c|c|}
\hline \multirow{2}{*}{$\begin{array}{c}\text { Characteristics } \\
\text { Ascertained at Index } \\
\text { Pregnancy (1996-2002) }\end{array}$} & \multirow[b]{2}{*}{$\begin{array}{l}\text { Overall } \\
(n=550)\end{array}$} & \multicolumn{4}{|c|}{ Cumulative Lactation Duration, Months } & \multirow[b]{2}{*}{$p^{*}$} \\
\hline & & $\begin{array}{c}\text { None } \\
(n=62)\end{array}$ & $\begin{array}{l}>0 \text { to }<6 \\
(n=106)\end{array}$ & $\begin{array}{l}6 \text { to }<12 \\
(n=171)\end{array}$ & $\begin{array}{c}\geq 12 \\
(n=211)\end{array}$ & \\
\hline Age, years & $31.5(4.5)$ & $31.1(4.5)$ & $31.8(4.0)$ & $32.2(4.6)$ & $30.8(4.4)$ & 0.01 \\
\hline Pre-pregnancy BMI, $\mathrm{kg} / \mathrm{m}^{2}$ & & & & & & $<0.001$ \\
\hline Unknown & $30(5.5)$ & $6(9.7)$ & $8(7.6)$ & $6(3.5)$ & $10(4.7)$ & \\
\hline$<25.0$ & $221(40.2)$ & $13(21.0)$ & $30(28.3)$ & $76(44.4)$ & $102(48.3)$ & \\
\hline $25.0-29.9$ & $143(26.0)$ & $16(25.8)$ & $26(24.5)$ & $45(26.3)$ & $56(26.5)$ & \\
\hline$\geq 30.0$ & $156(28.4)$ & $27(43.6)$ & $42(39.6)$ & $44(25.7)$ & $43(20.4)$ & \\
\hline Occupation & & & & & & $<0.001$ \\
\hline Unknown & $49(8.9)$ & $13(21.0)$ & $12(11.3)$ & $9(5.3)$ & $15(7.1)$ & \\
\hline Professional & $254(46.2)$ & $20(32.3)$ & $31(29.3)$ & $82(48.0)$ & $121(57.4)$ & \\
\hline Skilled worker & $152(27.6)$ & $16(25.8)$ & $41(38.7)$ & 47 (27.5) & $48(22.8)$ & \\
\hline $\begin{array}{l}\text { Other (unskilled worker, } \\
\text { unemployed, student) }\end{array}$ & 95 (17.3) & $13(21.0)$ & $22(20.8)$ & $33(19.3)$ & $27(12.8)$ & \\
\hline Parity & & & & & & $<0.001$ \\
\hline Unknown & $46(8.4)$ & $13(21.0)$ & $11(10.4)$ & $8(4.7)$ & $14(6.6)$ & \\
\hline 0 & $200(36.4)$ & $26(41.9)$ & $32(30.2)$ & $41(24.0)$ & $101(47.9)$ & \\
\hline$\geq 1$ & $304(55.3)$ & $23(37.1)$ & $63(59.4)$ & $122(71.4)$ & $96(45.5)$ & \\
\hline $\begin{array}{c}\text { Drank any alcohol while } \\
\text { pregnant }\end{array}$ & & & & & & $<0.001$ \\
\hline Unknown & $21(3.8)$ & $8(12.9)$ & $4(3.8)$ & $3(1.8)$ & $6(2.8)$ & \\
\hline No & $244(44.4)$ & $33(53.2)$ & $42(39.6)$ & $68(39.8)$ & $101(47.9)$ & \\
\hline Yes & $285(51.8)$ & $21(33.9)$ & $60(56.6)$ & $100(58.5)$ & $104(49.3)$ & \\
\hline Smoked while pregnant & & & & & & 0.002 \\
\hline Unknown & $21(3.8)$ & $8(12.9)$ & $4(3.8)$ & $3(1.8)$ & $6(2.8)$ & \\
\hline No & $383(69.6)$ & $34(54.8)$ & $69(65.1)$ & $124(72.5)$ & $156(73.9)$ & \\
\hline Yes & $146(26.6)$ & $20(32.3)$ & $33(31.1)$ & $44(25.7)$ & $49(23.2)$ & \\
\hline \multicolumn{7}{|l|}{$\begin{array}{l}\text { Characteristics ascertained } \\
\text { at follow-up (2012-2014) }\end{array}$} \\
\hline Age, year & $43.6(4.6)$ & $43(4.6)$ & $43.9(4.3)$ & $44.3(4.7)$ & $43.1(4.6)$ & \\
\hline Weight change, $\mathrm{kg}$ & & & & & & 0.36 \\
\hline Unknown & $8(1.5)$ & $2(3.2)$ & $2(1.9)$ & $2(1.2)$ & $2(1.0)$ & \\
\hline$<4.1$ & $269(48.9)$ & $35(56.5)$ & $52(49.1)$ & $89(52.1)$ & $93(44.1)$ & \\
\hline$\geq 4.1$ & $273(49.6)$ & $25(40.3)$ & $52(49.1)$ & $80(46.8)$ & $116(55.0)$ & \\
\hline Diabetes & & & & & & 0.02 \\
\hline Unknown & $6(1.1)$ & $2(3.2)$ & $2(1.9)$ & $1(0.6)$ & $1(0.5)$ & \\
\hline No & $388(70.6)$ & 37 (59.7) & $65(61.3)$ & $120(70.2)$ & $166(78.7)$ & \\
\hline Type 1 & $14(2.6)$ & $1(1.6)$ & $5(4.7)$ & $4(2.3)$ & $4(1.9)$ & \\
\hline Type 2 & $142(25.8)$ & $22(35.5)$ & $34(32.1)$ & $46(26.9)$ & $40(19)$ & \\
\hline Post-Menopausal & & & & & & 0.047 \\
\hline Unknown & $6(1.1)$ & $2(3.2)$ & $2(1.9)$ & $1(0.6)$ & $1(0.5)$ & \\
\hline No & $463(84.2)$ & $49(79)$ & $87(82.1)$ & $137(80.1)$ & $190(90.1)$ & \\
\hline Yes & $81(14.7)$ & $11(17.7)$ & $17(16)$ & 33 (19.3) & $20(9.5)$ & \\
\hline TSH (mIU/L) & $2.1(1.5)$ & $2.2(1.5)$ & $2(1.3)$ & $2.1(1.3)$ & $2.2(1.8)$ & 0.90 \\
\hline fT3 (pmol/L) & $4.6(0.6)$ & $4.5(0.6)$ & $4.6(0.7)$ & $4.6(0.5)$ & $4.7(0.6)$ & 0.16 \\
\hline fT4 (ng/dL) & $1.1(0.2)$ & $1.1(0.2)$ & $1.2(0.2)$ & $1.1(0.2)$ & $1.2(0.2)$ & 0.67 \\
\hline fT3:fT4 ratio & $4.1(0.6)$ & $4.1(0.7)$ & $4(0.6)$ & $4.1(0.7)$ & $4.1(0.6)$ & 0.59 \\
\hline Anti-TPO positive & $78(14.2)$ & $8(12.9)$ & $13(12.3)$ & $30(17.5)$ & $27(12.8)$ & 0.51 \\
\hline Anti-TG Positive & $69(12.6)$ & $5(8.1)$ & $9(8.5)$ & $26(15.2)$ & $29(13.7)$ & 0.25 \\
\hline
\end{tabular}

Data are presented as mean (standard deviation; SD) for continuous variables and $n(\%)$ for categorical variables.

* Bolded $p$ values represent significant global differences in participant characteristics across lactation duration categories. Abbreviations: BMI, body mass index; TSH, thyroid stimulating hormone; fT3, free triiodothyronine; fT4, free thyroxine; anti-TOP, thyroperoxidase antibody; anti-TG, thyroglobulin antibody. 
Table 2. Odds of thyroid autoimmunity 9 to 16 years postpartum according to lactation history among women with a history of gestational diabetes, Diabetes \& Women's Health Study.

\begin{tabular}{|c|c|c|c|c|c|}
\hline Outcome & $n$ & $\begin{array}{l}\text { Unadjusted } \\
\text { OR }(95 \% \text { CI) }\end{array}$ & $p^{*}$ & $\begin{array}{c}\text { Adjusted OR }{ }^{1} \\
(95 \% \mathrm{CI})\end{array}$ & $p^{*}$ \\
\hline \multicolumn{6}{|l|}{ TG-positive ${ }^{2}$} \\
\hline$\geq 12$ months & 211 & $1.82(0.67,4.91)$ & 0.23 & $2.63(0.74,9.37)$ & 0.13 \\
\hline 6 to $<12$ months & 171 & $2.04(0.75,5.58)$ & 0.16 & $2.88(0.80,10.27)$ & 0.10 \\
\hline$<6$ months & 106 & $1.06(0.34,3.31)$ & 0.92 & $1.72(0.44,6.82)$ & 0.43 \\
\hline None & 62 & 1.00 (reference) & & 1.00 (reference) & \\
\hline \multicolumn{6}{|l|}{ TPO-positive $^{2}$} \\
\hline$\geq 12$ months & 211 & $0.99(0.43,2.31)$ & 0.98 & $0.93(0.36,2.39)$ & 0.88 \\
\hline 6 to $<12$ months & 171 & $1.44(0.62,3.33)$ & 0.40 & $1.17(0.46,2.99)$ & 0.74 \\
\hline$<6$ months & 106 & $0.94(0.37,2.42)$ & 0.90 & $0.70(0.24,2.03)$ & 0.52 \\
\hline None & 62 & 1.00 (reference) & & 1.00 (reference) & \\
\hline
\end{tabular}

1 Model was adjusted for the following covariates, measured at the index pregnancy: age, pre-pregnancy body mass index (unknown, $<25,25-29.9, \geq 30.0 \mathrm{~kg} / \mathrm{m}^{2}$ ), occupation (unknown, professional, skilled worker, unskilled/unemployed/student), nulliparity (unknown, yes, no), smoking during pregnancy (unknown, any, none), and alcohol consumption during pregnancy (unknown, any, none). ${ }^{2} \mathrm{TG}$, thyroglobulin antibody; TPO, thyroid peroxidase antibody. ${ }^{*} p$-values represent significance in the difference in odds of TG- or TPO-positivity for each category of lactation duration compared to no lactation.

Table 3. Thyroid biomarker levels 9 to 16 years postpartum according to lactation history among women with a history of gestational diabetes, Diabetes \& Women's Health Study.

\begin{tabular}{|c|c|c|c|c|c|}
\hline $\begin{array}{c}\text { Thyroid } \\
\text { Biomarker }\end{array}$ & $n$ & $\begin{array}{c}\text { Unadjusted } \beta \\
\text { Estimate }(95 \% \mathrm{CI})\end{array}$ & $p$-Trend * & $\begin{array}{c}\text { Adjusted }{ }^{1} \beta \\
\text { Estimate }(95 \% \mathrm{CI})\end{array}$ & $p$-Trend * \\
\hline TSH (mIU/L) & & & 0.79 & & 0.87 \\
\hline$\geq 12$ months & 211 & $-0.05(-0.48,0.38)$ & & $-0.03(-0.47,0.41)$ & \\
\hline 6 to $<12$ months & 171 & $-0.07(-0.51,0.37)$ & & $-0.03(-0.49,0.43)$ & \\
\hline$<6$ months & 106 & $-0.17(-0.64,0.31)$ & & $-0.13(-0.61,0.35)$ & \\
\hline None & 62 & Reference & & Reference & \\
\hline fT3 (pmol/L) & & & 0.03 & & 0.05 \\
\hline$\geq 12$ months & 211 & $0.18(0.01,0.34)$ & & $0.19(0.03,0.36)$ & \\
\hline 6 to $<12$ months & 171 & $0.10(-0.07,0.27)$ & & $0.15(-0.02,0.32)$ & \\
\hline$<6$ months & 106 & $0.10(-0.08,0.28)$ & & $0.13(-0.05,0.31)$ & \\
\hline None & 62 & Reference & & Reference & \\
\hline fT4 (ng/dL) & & & 0.65 & & 0.55 \\
\hline$\geq 12$ months & 211 & $0.03(-0.02,0.07)$ & & $0.03(-0.02,0.07)$ & \\
\hline 6 to $<12$ months & 171 & $0.02(-0.03,0.07)$ & & $0.03(-0.02,0.07)$ & \\
\hline$<6$ months & 106 & $0.03(-0.02,0.08)$ & & $0.04(-0.01,0.09)$ & \\
\hline None & 62 & Reference & & Reference & \\
\hline fT3:fT4 ratio & & & 0.24 & & 0.30 \\
\hline$\geq 12$ months & 211 & $0.04-0.14,0.23)$ & & $0.06(-0.13,0.25)$ & \\
\hline 6 to $<12$ months & 171 & $-0.01(-0.20,0.18)$ & & $0.03(-0.16,0.23)$ & \\
\hline$<6$ months & 106 & $-0.06(-0.26,0.14)$ & & $-0.05(-0.25,0.15)$ & \\
\hline None & 62 & Reference & & Reference & \\
\hline
\end{tabular}

${ }^{1}$ Model was adjusted for the following covariates, measured at the index pregnancy: age, pre-pregnancy body mass index (unknown, $<25,25-29.9, \geq 30.0 \mathrm{~kg} / \mathrm{m}^{2}$ ), occupation (unknown, professional, skilled worker, unskilled/unemployed/student), nulliparity (unknown, yes, no), smoking during pregnancy (unknown, any, none), and alcohol during pregnancy (unknown, any, none). * Bolded $p$-trend values represent significant linear trends in thyroid biomarker levels with increasing lactation duration. We assessed $p$-trends by using the median for each category of lactation duration as a continuous exposure.

Although there were significant differences in mean levels of several thyroid biomarkers according to long-term weight change and diabetes status at follow-up (Table A1), there was no variation in the lactation duration-thyroid function association according to maternal weight change, diabetes status, or age at follow-up ( $p$ for interaction $>0.05$ ). There was a significant interaction between lactation duration and menopause status for the outcomes of fT3 $(p=0.02)$ and the fT3:fT4 ratio $(p=0.03)$. 
The results were null among pre-menopausal women, but stronger among post-menopausal women such that a lactation duration of $\geq 12$ months was associated with higher fT3 levels by $0.47 \mathrm{pmol} / \mathrm{L}$ compared to women who never lactated (Table A2).

To tease apart the effect of lactation from the number of pregnancies, we restricted the analyses to women with a single lifetime pregnancy $(n=70)$. Women with a cumulative lactation duration $>6$ months had significantly higher levels of fT3 $(0.46 \mathrm{pmol} / \mathrm{L}(0.12,0.80) ; p$-trend $=0.02)$ and a higher fT3:fT4 ratio $(0.61(0.17,1.05) ; p$-trend $=0.007)$ than women who had never lactated (Table 4$)$. In addition, there was a significant trend $(p=0.04)$ of increasing TSH with increasing lactation duration.

Table 4. Thyroid biomarker levels 9 to 16 years postpartum according to lactation history among women with a history of gestational diabetes who had a single lifetime pregnancy, Diabetes \& Women's Health Study.

\begin{tabular}{|c|c|c|c|c|c|}
\hline $\begin{array}{c}\text { Thyroid } \\
\text { Biomarker }\end{array}$ & $n$ & $\begin{array}{c}\text { Unadjusted } \beta \\
\text { Estimate }(95 \% \mathrm{CI})\end{array}$ & $p$-Trend * & $\begin{array}{c}\text { Adjusted }{ }^{1} \beta \\
\text { Estimate }(95 \% \mathrm{CI})\end{array}$ & $p$-Trend * \\
\hline TSH (mIU/L) & & & 0.045 & & 0.04 \\
\hline$>6$ months & 24 & $0.67(-0.09,1.44)$ & & $0.78(-0.03,1.58)$ & \\
\hline$>0$ to 6 months & 26 & $-0.04(-0.79,0.71)$ & & $0.14(-0.65,0.94)$ & \\
\hline None & 20 & Reference & & Reference & \\
\hline fT3 (pmol/L) & & & 0.17 & & 0.02 \\
\hline$>6$ months & 24 & $0.22(-0.12,0.56)$ & & $0.46(0.12,0.80)$ & \\
\hline$>0$ to 6 months & 26 & $0.04(-0.29,0.38)$ & & $0.27(-0.06,0.60)$ & \\
\hline None & 20 & Reference & & Reference & \\
\hline $\mathrm{fT} 4$ (ng/dL) & & & 0.07 & & 0.36 \\
\hline$>6$ months & 24 & $-0.09(-0.20,0.01)$ & & $-0.05(-0.15,0.06)$ & \\
\hline$>0$ to 6 months & 26 & $-0.03(-0.14,0.07)$ & & $-0.02(-0.12,0.09)$ & \\
\hline None & 20 & Reference & & Reference & \\
\hline fT3:fT4 ratio & & & 0.009 & & 0.007 \\
\hline$>6$ months & 24 & $0.55(0.12,0.99)$ & & $0.61(0.17,1.05)$ & \\
\hline$>0$ to 6 months & 26 & $0.12(-0.31,0.55)$ & & $0.25(-0.18,0.69)$ & \\
\hline None & 20 & Reference & & Reference & \\
\hline \multicolumn{6}{|c|}{$\begin{array}{l}1 \text { Model was adjusted for the following covariates, measured at the index pregnancy: age, pre-pregnancy } \\
\left.\text { body mass index (unknown, }<25,25-29.9, \geq 30.0 \mathrm{~kg} / \mathrm{m}^{2}\right), \text { occupation (unknown, professional, skilled worker } \\
\text { unskilled/unemployed/student), nulliparity (unknown, yes, no), smoking during pregnancy (unknown, any, none) } \\
\text { and alcohol during pregnancy (unknown, any, none). * Bolded } p \text {-trend values represent significant linear trends in } \\
\text { thyroid biomarker levels with increasing lactation duration. We assessed } p \text {-trends by using the median for each } \\
\text { category of lactation duration as a continuous exposure. }\end{array}$} \\
\hline
\end{tabular}

\section{Discussion}

In the present study following women 9-16 years after GDM pregnancy, a high-risk population for cardiometabolic complications, we examined whether cumulative duration of lactation is associated with long-term thyroid function. Findings from this study suggested that a longer cumulative lactation duration is associated with higher serum fT3 levels and fT3:fT4 ratio approximately 9-16 years after the index pregnancy. The positive association between lactation duration and fT3 levels was more pronounced among nulliparous women at the index pregnancy. While the strengths of these associations are modest, they highlight novel findings, extending the existing literature which demonstrates long-term associations between women's lifetime duration of lactation and cardiometabolic health to potentially include thyroid function as a novel endpoint [19-21].

An important feature of our study was distinguishing the effect of cumulative duration of lactation from parity, as these variables are highly correlated. We accomplished this by restricting the study specifically to women who were nulliparous at the index pregnancy and had only a single lifetime pregnancy. The results of our analyses were more robust among this subset, as we removed any residual confounding due to parity. It was meaningful to analyze this subset, as we observed an association between lactation and thyroid biomarker levels even within the shorter lactation periods following only a single birth. 
Prior studies have observed that a longer lactation duration is related to lower levels of risk for breast and ovarian cancer, cardiovascular disease, diabetes, hypertension, and hyperlipidemia [3]. There has been limited clinical or epidemiologic research on lactation and long-term maternal thyroid function. Our study adds an intriguing piece to the prior work by linking lactation duration with long-term thyroid function, as we provide suggestive evidence that women who lactated for longer durations of time had higher levels of serum fT3 later in life. Interestingly, we observed that women with even 6 months of lactation, compared to none, had higher levels of fT3 and the fT3:fT4 ratio.

Several hypotheses may explain the biological mechanisms associating lactation with long-term maternal metabolic health. To support the developing fetus and prepare the mother for lactation, there is an increase in visceral adiposity, circulating lipids, and insulin levels during pregnancy [22]. It is hypothesized that a prolonged duration of lactation helps to rapidly and more completely reverse these metabolic changes by mobilizing the fat stores accumulated during pregnancy [22]. This "reset" to the maternal metabolism may reduce women's risk for future metabolic diseases, possibly including thyroid dysfunction. Animal models also offer unique insight into the biological pathways linking lactation and thyroid function. In bovine models, the quantity of mRNA transcripts in the mammary gland for iodothyronine deiodinase type II (DIO2), the enzyme responsible for generating T3, the biologically active thyroid hormone, from inactive T4, increased with lactation duration [4]. This observation is consistent with our findings that a longer duration of lactation is associated with greater fT3 levels and fT3:fT4, as fT3 is the form of T3 that is in circulation, and the fT3:fT4 ratio is a proxy for the conversion from T4 to T3. Furthermore, we hypothesized that a potential mechanism for the association between lactation and thyroid function may have been through weight change, yet we did not observe any evidence for this. Lastly, the association between GDM and T2DM has been previously studied and served as the impetus for this research to look beyond cardiometabolic disorders and extend to thyroid metabolism. However, it should be noted that the results were consistent regardless of T2DM status at follow-up. Further research into the mechanisms is warranted.

Although the effect sizes observed in our study do not clinically alter euthyroid status, prior research has demonstrated an association between small changes in thyroid hormone levels and clinical outcomes, even within the euthyroid range. Specifically, the importance of T3 in overall health and metabolic function has been demonstrated among patients with end-stage renal disease, hemodialysis, chronic heart failure, and cerebral infarction who also had clinically low T3 (i.e., low T3 syndrome) and were receiving T3 replacement therapy [23-26]. Conversely, higher fT3 levels have been associated with several parameters of metabolic syndrome, non-alcoholic fatty liver disease (NAFLD), and insulin resistance among cohorts of healthy euthyroid individuals [27-30]. The conflicting results from these studies warrant further investigation of lifetime lactation and thyroid disease, beyond clinical biomarkers. Lactation was not associated with thyroid autoimmunity status (i.e., TGO-positivity and TG-positivity); however, the sample size in the current study may have been too small to detect an association. Further research, with a larger sample and longer follow-up time, may be necessary to understand the clinical significance of higher fT3 levels associated with a longer duration of cumulative lactation.

There are several strengths to our study, including the hybrid study design, combining DNBC pregnancy data with follow-up data from the DWH Study. This study design provided the unique opportunity to follow a large high-risk cohort of women with a history of GDM for over a decade, and to understand their long-term health consequences and modifiable risk factors for specific health outcomes. Additionally, lactation duration, which was reported by women immediately following the index DNBC pregnancy, was highly correlated with women's reported duration at the DWH Study follow-up 9-16 years later. This also helped to validate women's recall of lactation duration for the additional births. Our study was further strengthened by consideration of several risk factors for thyroid function reported during the index DNBC pregnancy, including pre-pregnancy BMI, age, socioeconomic status, smoking during pregnancy, and alcohol consumption during pregnancy. Finally, our study is generalizable to Danish women with a history of GDM. While our findings 
may not be generalizable to all women, women with GDM represent a unique high-risk model for metabolic dysfunction.

Several limitations warrant discussion. First, the cumulative duration of lactation was calculated following the index pregnancy because we did not have detailed lactation data and related covariates before the index pregnancy. However, to account for potential confounding from previous lactation history, we adjusted for the number of pregnancies before the index pregnancy (i.e., parity at the index pregnancy). In addition, we conducted an analysis restricted to women for whom the index pregnancy was their first pregnancy and thus had no prior lactation history. In these analyses, we observed a similar, but stronger positive association between lactation duration and thyroid marker levels. Second, we did not have clear data on how many additional pregnancies were complicated by GDM. Third, we assessed thyroid biomarkers only at a single follow-up and we were unaware of baseline thyroid marker levels before pregnancy or during the interim between the index GDM pregnancy and follow up. While we excluded women with known thyroid disorders prior to pregnancy, we did not have information on the development of, and treatment for, thyroid disease during the period between the index GDM pregnancy and follow up. Fourth, we did not have the power to test for associations between lactation and clinical end points related to thyroid function, such as $\mathrm{SCH}$ and other thyroid diseases. Lastly, we did not have data from women on reasons for discontinuing breastfeeding or choosing not to breastfeed, and thus we cannot tease out confounding from other factors (i.e., social factors, milk supply, etc.) that may have contributed to lactation duration.

\section{Conclusions}

In Danish women with a history of GDM, our findings suggest a positive association between a longer duration of lactation and higher levels of thyroid hormone 9-16 years postpartum, even among women with a single lifetime pregnancy. Here we identified lactation as a novel potential modifiable factor for thyroid function, and with replication, these findings may add thyroid function to the wide-array of long-term cardiometabolic outcomes associated with increased lactation duration. As with the findings related to metabolic health, more research is needed to understand the mechanisms behind our findings. A longer follow-up period may help further understanding of the clinical impact of elevated fT3 levels associated with longer lactation duration.

Author Contributions: The study was conceived and designed by C.Z. The data were analyzed by P.L.P. and S.N.H. Funding was obtained by C.Z. The first draft of the manuscript was prepared by P.L.P. and S.N.H., which was edited with the input from the writing team (S.N.H., S.R., L.G.G., S.H.L., Y.L., A.L., A.C.B.T., S.F.O., C.Z.). All authors revised the manuscript critically for important intellectual content. All authors gave final approval of the version to be published and have contributed to the manuscript. C.Z. and S.N.H. are the guarantors.

Funding: This work was supported by the Intramural Research Program of the Eunice Kennedy Shriver National Institute of Child Health and Human Development at the National Institutes of Health (contract numbers HHSN275201000020C, HHSN275201500003C, HHSN275201300026I, HSN275201100002I). Financial support for DNBC was received from: March of Dimes Birth Defects Foundation (6-FY-96-0240, 6-FY97-0553, 6-FY97-0521, 6-FY00-407), Innovation Fund Denmark (grant number 09-067124 and 11-115923), Danish Council for Independent Research (grant number 9601842 and 22-03-0536), the Health Foundation (11/263-96), and the Heart Foundation (96-2-4-83-22450). The funders had no role in the analysis and interpretation of data, in the writing of the report, or in the decision to submit the article for publication.

Conflicts of Interest: The authors report no conflict of interest. 


\section{Appendix A}

Table A1. Thyroid biomarkers according to participant characteristics at follow-up (2012-2014).

\begin{tabular}{cccccc}
\hline \multirow{2}{*}{$\begin{array}{c}\text { Characteristics Ascertained } \\
\text { at Follow-Up (2012-2014) }\end{array}$} & $n$ & \multicolumn{4}{c}{ Thyroid Biomarkers } \\
\cline { 3 - 5 } Age & & TSH (mIU/L) & fT3 (pmol/L) & fT4 (ng/dL) & fT3:fT4 Ratio \\
\hline Unknown & 6 & $1.73(1.10)$ & $4.0(1.38)$ & $1.09(0.41)$ & $3.74(0.46)$ \\
$<45$ & 314 & $2.09(1.66)$ & $4.65(0.60)$ & $1.14(0.15)$ & $4.12(0.66)$ \\
$\geq 45$ & 230 & $2.20(1.32)$ & $4.62(0.52)$ & $1.15(0.17)$ & $4.07(0.62)$ \\
$p^{*}$ & & 0.41 & 0.51 & 0.47 & 0.32 \\
Weight change, kg & & & & & \\
Unknown & 8 & $2.31(1.73)$ & $4.19(1.22)$ & $1.10(0.35)$ & $3.84(0.45)$ \\
$<4.1$ & 265 & $2.09(1.31)$ & $4.53(0.56)$ & $1.17(0.16)$ & $3.94(0.62)$ \\
$\geq 4.1$ & 277 & $2.17(1.70)$ & $4.74(0.56)$ & $1.13(0.15)$ & $4.25(0.64)$ \\
$p^{*}$ & & 0.57 & $<0.01$ & 0.01 & $<0.01$ \\
Diabetes & & & & & \\
Unknown & 6 & $1.73(1.10)$ & $4.0(1.38)$ & $1.09(0.41)$ & $3.74(0.46)$ \\
No & 388 & $2.15(1.63)$ & $4.61(0.57)$ & $1.14(0.15)$ & $4.09(0.60)$ \\
Type 1 & 14 & $2.69(1.44)$ & $4.55(0.48)$ & $1.04(0.09)$ & $4.37(0.43)$ \\
Type 2 & 142 & $2.04(1.19)$ & $4.72(0.58)$ & $1.18(0.18)$ & $4.09(0.76)$ \\
$p^{*}$ & & 0.29 & 0.13 & $<0.01$ & 0.28 \\
Post-menopausal & & & & & \\
Unknown & 6 & $1.73(1.10)$ & $4.0(1.38)$ & $1.09(0.41)$ & $3.74(0.46)$ \\
No & 463 & $2.14(1.55)$ & $4.63(0.56)$ & $1.14(0.15)$ & $4.11(0.65)$ \\
Yes & 81 & $2.13(1.36)$ & $4.70(0.59)$ & $1.18(0.19)$ & $4.05(0.62)$ \\
$p^{*}$ & & 0.99 & 0.29 & 0.05 & 0.43 \\
\hline
\end{tabular}

Data are presented as the mean (standard deviation; SD) of each thyroid biomarker (i.e., TSH, fT3, fT4, fT3:fT4), according to categories of participant characteristics (i.e., age, weight change, diabetes status, menopause status). * Bolded $p$-values represent significant global differences in the levels of each thyroid biomarker across non-missing categories of participant characteristics.

Table A2. Associations between lactation duration and fT3 and the fT3:fT4 ratio by menopausal status among women with a history of gestational diabetes, Diabetes \& Women's Health Study.

\begin{tabular}{|c|c|c|c|c|c|c|}
\hline \multirow{2}{*}{$\begin{array}{l}\text { Thyroid } \\
\text { Biomarker }\end{array}$} & \multicolumn{2}{|c|}{$\begin{array}{l}\text { Pre-Menopausal Women } \\
\qquad(n=463)\end{array}$} & \multirow[b]{2}{*}{$p$-Trend * } & \multicolumn{2}{|c|}{$\begin{array}{l}\text { Post-Menopausal Women } \\
\qquad(n=81)\end{array}$} & \multirow[b]{2}{*}{$p$-Trend * } \\
\hline & $n$ & $\begin{array}{c}\text { Adjusted }{ }^{1} \beta \\
\text { Estimate }(95 \% \mathrm{CI})\end{array}$ & & $n$ & $\begin{array}{c}\text { Adjusted }{ }^{1} \beta \\
\text { Estimate }(95 \% \mathrm{CI})\end{array}$ & \\
\hline fT3 (pmol/L) & & & 0.29 & & & 0.22 \\
\hline$\geq 12$ months & 190 & $0.10(-0.08,0.28)$ & & 20 & $0.47(0.04,0.90)$ & \\
\hline 6 to $<12$ months & 137 & $0.12(-0.07,0.03)$ & & 33 & $0.10(-0.30,0.50)$ & \\
\hline$<6$ months & 87 & $0.02(-0.17,0.22)$ & & 17 & $0.46(0.03,0.90)$ & \\
\hline None & 49 & Reference & & 11 & Reference & \\
\hline fT3:fT4 ratio & & & 0.43 & & & 0.33 \\
\hline$\geq 12$ months & 190 & $0.00(-0.20,0.21)$ & & 20 & $0.38(-0.06,0.82)$ & \\
\hline 6 to $<12$ months & 137 & $0.05(-0.17,0.27)$ & & 33 & $-0.12(-0.53,0.29)$ & \\
\hline$<6$ months & 87 & $-0.11(-0.34,0.12)$ & & 17 & $0.14(-0.30,0.59)$ & \\
\hline None & 49 & Reference & & 11 & Reference & \\
\hline
\end{tabular}

${ }^{1}$ Model was adjusted for the following covariates, measured at the index pregnancy: age, pre-pregnancy body mass index (unknown, $<25,25-29.9, \geq 30.0 \mathrm{~kg} / \mathrm{m}^{2}$ ), occupation (unknown, professional, skilled worker, unskilled/unemployed/student), nulliparity (unknown, yes, no), smoking during pregnancy (unknown, any, none), and alcohol during pregnancy (unknown, any, none). ${ }^{*} p$-trend values represent linear trends in thyroid biomarker levels with increasing lactation duration. We assessed $p$-trends by using the median for each category of lactation duration as a continuous exposure. 


\section{References}

1. Yen, P.M. Physiological and molecular basis of thyroid hormone action. Physiol. Rev. 2001, 81, 1097-1142. [CrossRef] [PubMed]

2. Reece, E.A. The fetal and maternal consequences of gestational diabetes mellitus. J. Matern. Fetal Neonatal Med. 2010, 23, 199-203. [CrossRef] [PubMed]

3. Stuebe, A.M.; Schwarz, E.B. The risks and benefits of infant feeding practices for women and their children. J. Perinatol. 2010, 30, 155-162. [CrossRef] [PubMed]

4. Capuco, A.V.; Connor, E.E.; Wood, D.L. Regulation of mammary gland sensitivity to thyroid hormones during the transition from pregnancy to lactation. Exp. Biol. Med. 2008, 233, 1309-1314. [CrossRef] [PubMed]

5. Knudsen, N.; Laurberg, P.; Rasmussen, L.B.; Bulow, I.; Perrild, H.; Ovesen, L.; Jorgensen, T. Small differences in thyroid function may be important for body mass index and the occurrence of obesity in the population. J. Clin. Endocrinol. Metab. 2005, 90, 4019-4024. [CrossRef] [PubMed]

6. Baker, J.L.; Gamborg, M.; Heitmann, B.L.; Lissner, L.; Sorensen, T.I.; Rasmussen, K.M. Breastfeeding reduces postpartum weight retention. Am. J. Clin. Nutr. 2008, 88, 1543-1551. [CrossRef] [PubMed]

7. Bano, A.; Dhana, K.; Chaker, L.; Kavousi, M.; Ikram, M.A.; Mattace-Raso, F.U.S.; Peeters, R.P.; Franco, O.H. Association of thyroid function with life expectancy with and without cardiovascular disease: The rotterdam study. JAMA Intern. Med. 2017, 177, 1650-1657. [CrossRef] [PubMed]

8. Fatima, S.S.; Rehman, R.; Butt, Z.; Tauni, M.A.; Munim, T.F.; Chaudhry, B.; Khan, T.A. Screening of subclinical hypothyroidism during gestational diabetes in pakistani population. J. Matern. Fetal Neonatal Med. 2016, 29, 2166-2170. [CrossRef] [PubMed]

9. Shahbazian, H.; Shahbazian, N.; Baniani, M.R.; Yazdanpanah, L.; Latifi, S.M. Evaluation of thyroid dysfunction in pregnant women with gestational and pre-gestational diabetes. Pak. J. Med. Sci. 2013, 29, 638-641. [CrossRef] [PubMed]

10. Speller, E.; Brodribb, W. Breastfeeding and thyroid disease: A literature review. Breastfeed. Rev. 2012, 20, 41-47. [PubMed]

11. Vafaeimanesh, J.; Asgarani, F.; Bagherzadeh, M.; Ebrahimi, G.; Parham, M. Thyroid function in pregnant women with gestational diabetes: Is screening necessary? Thyroid Res. Pract. 2015, 12, 3-7. [CrossRef]

12. Vitacolonna, E.; Lapolla, A.; Di Nenno, B.; Passante, A.; Bucci, I.; Giuliani, C.; Cerrone, D.; Capani, F.; Monaco, F.; Napolitano, G. Gestational diabetes and thyroid autoimmunity. Int. J. Endocrinol. 2012, 2012, 867415. [CrossRef] [PubMed]

13. Yang, S.; Shi, F.T.; Leung, P.C.; Huang, H.F.; Fan, J. Low thyroid hormone in early pregnancy is associated with an increased risk of gestational diabetes mellitus. J. Clin. Endocrinol. Metab. 2016, 101, 4237-4243. [CrossRef] [PubMed]

14. Zhang, C.; Hu, F.B.; Olsen, S.F.; Vaag, A.; Gore-Langton, R.; Chavarro, J.E.; Bao, W.; Yeung, E.; Bowers, K.; Grunnet, L.G.; et al. Rationale, design, and method of the diabetes \& women's health study-A study of long-term health implications of glucose intolerance in pregnancy and their determinants. Acta Obstet. Gynecol. Scand. 2014, 93, 1123-1130. [CrossRef] [PubMed]

15. Olsen, J.; Melbye, M.; Olsen, S.F.; Sorensen, T.I.; Aaby, P.; Andersen, A.M.; Taxbol, D.; Hansen, K.D.; Juhl, M.; Schow, T.B.; et al. The danish national birth cohort-Its background, structure and aim. Scand. J. Public Health 2001, 29, 300-307. [CrossRef] [PubMed]

16. Garber, J.R.; Cobin, R.H.; Gharib, H.; Hennessey, J.V.; Klein, I.; Mechanick, J.I.; Pessah-Pollack, R.; Singer, P.A.; Woeber, K.A. Clinical practice guidelines for hypothyroidism in adults: Cosponsored by the american association of clinical endocrinologists and the american thyroid association. Endocr. Pract. 2012, 18, 988-1028. [CrossRef] [PubMed]

17. Hollowell, J.G.; Staehling, N.W.; Flanders, W.D.; Hannon, W.H.; Gunter, E.W.; Spencer, C.A.; Braverman, L.E. Serum TSH, $\mathrm{T}_{4}$, and thyroid antibodies in the united states population (1988 to 1994): National health and nutrition examination survey (NHANES III). J. Clin. Endocrinol. Metab. 2002, 87, 489-499. [CrossRef] [PubMed]

18. Association, A.D. Diagnosis and classification of diabetes mellitus. Diabetes Care 2011, 34, S62-S69. [CrossRef] [PubMed]

19. Bianco, A.C.; Kim, B.W. Deiodinases: Implications of the local control of thyroid hormone action. J. Clin. Investig. 2006, 116, 2571-2579. [CrossRef] [PubMed] 
20. Hyatt, H.W.; Zhang, Y.; Hood, W.R.; Kavazis, A.N. Lactation has persistent effects on a mother's metabolism and mitochondrial function. Sci. Rep. 2017, 7, 17118. [CrossRef] [PubMed]

21. Stuebe, A.M.; Michels, K.B.; Willett, W.C.; Manson, J.E.; Rexrode, K.; Rich-Edwards, J.W. Duration of lactation and incidence of myocardial infarction in middle to late adulthood. Am. J. Obstet. Gynecol. 2009, 200, 138.e1-138.e8. [CrossRef] [PubMed]

22. Stuebe, A.M.; Rich-Edwards, J.W. The reset hypothesis: Lactation and maternal metabolism. Am. J. Perinatol. 2009, 26, 81-88. [CrossRef] [PubMed]

23. Chang, T.I.; Nam, J.Y.; Shin, S.K.; Kang, E.W. Low triiodothyronine syndrome and long-term cardiovascular outcome in incident peritoneal dialysis patients. Clin. J. Am. Soc. Nephrol. 2015, 10, 975-982. [CrossRef] [PubMed]

24. Fragidis, S.; Sombolos, K.; Thodis, E.; Panagoutsos, S.; Mourvati, E.; Pikilidou, M.; Papagianni, A.; Pasadakis, P.; Vargemezis, V. Low T3 syndrome and long-term mortality in chronic hemodialysis patients. World J. Nephrol. 2015, 4, 415-422. [CrossRef] [PubMed]

25. Wu, G.H.; Kong, F.Z.; Cheng, Q.Z.; Luo, W.F.; Du, X.D. Low T3 syndrome predicts severe neurological deficits of cerebral infarction inpatients with large artery artherosclerosis in internal carotid artery system. Neuro Endocrinol. Lett. 2014, 35, 149-153. [PubMed]

26. Zoccali, C.; Mallamaci, F.; Tripepi, G.; Cutrupi, S.; Pizzini, P. Low triiodothyronine and survival in end-stage renal disease. Kidney Int. 2006, 70, 523-528. [CrossRef] [PubMed]

27. Ferrannini, E.; Iervasi, G.; Cobb, J.; Ndreu, R.; Nannipieri, M. Insulin resistance and normal thyroid hormone levels: Prospective study and metabolomic analysis. Am. J. Physiol. Endocrinol. Metab. 2017, 312, E429-E436. [CrossRef] [PubMed]

28. Roef, G.L.; Rietzschel, E.R.; Van Daele, C.M.; Taes, Y.E.; De Buyzere, M.L.; Gillebert, T.C.; Kaufman, J.M. Triiodothyronine and free thyroxine levels are differentially associated with metabolic profile and adiposity-related cardiovascular risk markers in euthyroid middle-aged subjects. Thyroid 2014, 24, 223-231. [CrossRef] [PubMed]

29. Tarcin, O.; Abanonu, G.B.; Yazici, D.; Tarcin, O. Association of metabolic syndrome parameters with TT3 and FT3/FT4 ratio in obese turkish population. Metab. Syndr. Relat. Disord. 2012, 10, 137-142. [CrossRef] [PubMed]

30. Van den Berg, E.H.; van Tienhoven-Wind, L.J.; Amini, M.; Schreuder, T.C.; Faber, K.N.; Blokzijl, H.; Dullaart, R.P. Higher free triiodothyronine is associated with non-alcoholic fatty liver disease in euthyroid subjects: The lifelines cohort study. Metabolism 2017, 67, 62-71. [CrossRef] [PubMed]

(c) 2018 by the authors. Licensee MDPI, Basel, Switzerland. This article is an open access article distributed under the terms and conditions of the Creative Commons Attribution (CC BY) license (http:/ / creativecommons.org/licenses/by/4.0/). 


\title{
Article \\ Predictors of Continued Breastfeeding at One Year among Women Attending Primary Healthcare Centers in Qatar: A Cross-Sectional Study
}

\author{
Amal Nasser, Fadumo Omer, Fatima Al-Lenqawi, Rehab Al-awwa, Tamam Khan, \\ Asmaa El-Heneidy, Rana kurdi and Ghadir Al-Jayyousi * \\ Department of Public Health, College of Health Sciences, Qatar University, 2713 Doha, Qatar; \\ an1205803@student.qu.edu.qa (A.N.); fo1203580@student.qu.edu.qa (F.O.); \\ fa1203503@student.qu.edu.qa (F.A.-L.); ra1208955@student.qu.edu.qa (R.A.-a.); \\ tk1202457@student.qu.edu.qa (T.K.); mph.asmaa@gmail.com (A.E.-H.); rana.kurdi@qu.edu.qa (R.k.) \\ * Correspondence: g.aljayyousi@qu.edu.qa; Tel.: +974-4403-6556
}

Received: 31 May 2018; Accepted: 24 July 2018; Published: 27 July 2018

Abstract: The number of babies in Qatar being exclusively breastfed is significantly lower than the global target set by the World Health Organization. The purpose of this study was to assess knowledge, attitude, and practice (KAP), selected barriers, and professional support as well as their association with continued breastfeeding at one year of age. A sample of Qatari and non-Qatari mothers $(N=195)$ who attended a well-baby clinic held at primary health care centers in Qatar completed a self-administered questionnaire. Descriptive analysis, the Pearson Chi-squared test, and logistic regression were performed. Around $42 \%$ of the mothers stopped breastfeeding when their child was aged between 0 and 11 months old. Mothers who had only one or female child stopped breastfeeding between the ages of 0 and 6 months $(p=0.025,0.059)$. The more optimal the breastfeeding practices followed by the mothers, the older the age of the infant when they stopped breastfeeding $(p=0.001)$. The following factors were inversely associated with breastfeeding duration: the mother's perceptions that she "did not know how to breastfeed," or "wasn't making enough milk," and the need "to return to work/school", with $p=0.022,0.004$, and 0.022 , respectively. These findings present factors that should be considered when planning for health education and promotion programs to prolong breastfeeding duration in Qatar.

Keywords: breastfeeding; knowledge; practice; barriers; social support; professional support

\section{Introduction}

Exclusive breastfeeding contributes greatly to providing better health outcomes by preventing disease and promoting health in both the short and long term for mothers and their children [1]. It also reduces infant mortality from common childhood illnesses. Approximately 800,000 children's lives could be saved globally each year if every child was exclusively breastfed for the first six months of life [2].

Despite the established importance of breastfeeding, in the Middle East and North Africa (MENA), regional fact sheet-recommended levels are still not attained. The rate of exclusive breastfeeding continuation in Qatar is $12 \%$, which is low when compared with the global rate of $37 \%$. Qatar's rate is also significantly lower than the global target set by the World Health Organization, which calls for at least $50 \%$ of babies under six months of age to be exclusively breastfed by 2025 [3].

Mothers who do not breastfeed their infants and depend on formula milk may suffer from increased incidence of premenopausal breast cancer, ovarian cancer, retained gestational weight gain, type 2 diabetes, myocardial infarction, and metabolic syndrome. Additionally, infants who are not 
breastfed are more likely to suffer from infectious morbidity, childhood obesity, diabetes (types 1 and 2), leukemia, and sudden infant death syndrome [4].

Qatar ranks 10th in the world and third in the MENA region for diabetes. The nation has a comparative prevalence of diabetes of $22.9 \%$ and an estimated 283,000 diabetes patients between 20 and 75 years of age [5]. Therefore, understanding the factors affecting breastfeeding and how to enhance and prolong the duration of breastfeeding is vital for diabetes prevention in the region.

\subsection{Continued Breastfeeding at One Year: A Core Indicator to Assess Breastfeeding Practices}

Globally in 2015, the percentage of children with continued breastfeeding at one year of age was $74 \%$ [6]. Regionally, the proportion of children with continued breastfeeding up to one year in Oman was reported to be $95 \%$ in 2000. This is much higher than the rate of Qatar, where it was $4 \%$ among Qataris and 69.6\% among non-Qataris (non-Qataris refer to any immigrant in Qatar-not only limited to Middle Eastern immigrants, but also Arabs from the Gulf Cooperation Council (GCC) region, other Arab regions, and non-Arab nationalities [7]). The World Health Organization uses core indicators to assess breastfeeding practices among women [8]. Since the majority of mothers in Qatar stop breastfeeding within one year, it is crucial to study the "continued breastfeeding at one year" indicator, which is defined as the proportion of children aged 12-15 months who are fed breast milk.

\subsection{Factors Influencing Breastfeeding Continuation at One Year}

Qatar is a rapidly developing country with massive reserves of oil and natural gas. Globally, it is considered to have the second-largest natural gas reserves. It is part of the GCC region and has a population of 2,700,000, which includes only 320,000 Qatari citizens. The remaining are residents from a large range of nationalities and comprise the main work force in the country.

Previous research has found that nationality, education, and financial status are among the factors that influence breastfeeding duration. Al-Darweesh and colleagues found that non-Kuwaiti women with postgraduate degrees practiced breastfeeding longer than other Kuwaiti women $[9,10]$ and that rich families left traditional practices and used formula feeds [11].

Knowledge, attitude, and practice (KAP) of breastfeeding also contribute to continued breastfeeding at one year of age. In GCC countries, research found that breastfeeding knowledge among studied participants was highly adequate [12]. However, continued breastfeeding practices at one year were not sufficient, and was negatively influenced by mother's attitudes toward breastfeeding [13]. In addition, some breastfeeding practices have contributed to continued breastfeeding at one year of age, including mother-infant skin-to-skin contact (SSC) after birth, which was shown as an efficient method in supporting the continuation of breastfeeding [14]. However, pacifier usage and using only breast milk to feed infants six months and older without complementary foods were factors that were found to promote weaning $[15,16]$.

Factors that are considered as barriers to continued breastfeeding include mothers' perception of pain, body image, body changes, embarrassment from breastfeeding in public, and breastfeeding at work. Barriers arising from social norms are also documented in the literature: some mothers subscribe to the norm "bigger is better," which means fat babies are healthier. This leads them to use bottled milk and introduce solid food earlier than recommended [9,11,17].

Social support provided by family members also shapes continued breastfeeding at one year of age. Women rely on support and advice from their mothers and grandmothers, who may provide practical support to continue breastfeeding for up to two years. Research has reported that support from a spouse was also positively associated with longer duration of breastfeeding $[17,18]$. With regards to professional support, healthcare professionals can also provide a vital role for breastfeeding mothers. Face-to-face pre-natal and postnatal classes proved to be effective in reducing early cessation of breastfeeding and promoted breastfeeding prevalence [19].

The unique context in Qatar causes more challenges to continued breastfeeding than in other GCC countries. A previous study reported that mothers in Saudi Arabia than Qatar are more open to 
breastfeeding in front of the family and in public [11]. Relatively little is known about breastfeeding continuation practices up to one year of age among mothers in Qatar. The purpose of this research is to study demographics, assess KAP regarding breastfeeding, explore barriers to breastfeeding, and study selected aspects of professional support and their association with continued breastfeeding at one year of age among mothers visiting primary healthcare centers in Qatar.

\section{Materials and Methods}

A cross-sectional study was conducted between February and June 2017. Participants were randomly selected mothers attending well-baby clinics at five primary healthcare centers in Doha, Qatar. The study locations were selected based on geographical distribution; they were also training sites for the first five authors.

A selection of Qatari and non-Qatari mothers who attended well-baby clinics was chosen. The target sample was 200 with 40 mothers per center. To be eligible for the study, mothers had to have given birth to their youngest baby in Qatar (participant baby), lived in Qatar for at least two-thirds of the pregnancy, and attended a well-baby clinic at one of the chosen centers. A total of 195 questionnaires were completed by participants.

The study was approved by the Qatar University Institutional Review Board (Research Ethics Approval No is QU-IRB 387-E/17) and was conducted in full agreement with the rules and regulations of the Primary Healthcare Care Cooperation (PHCC) Research Section. A consent form was read and signed by participants who agreed to participate prior to filling out the questionnaire.

\subsection{Data Collection}

Data were collected anonymously using a self-administered questionnaire, which was prepared in English and Arabic. Participants were randomly selected from the different five centers. We approached mothers in the well-baby clinic and briefly explained the purpose of the study. Mothers who agreed to participate were handed a consent form to sign prior to completing the questionnaire. A cover letter was attached to each questionnaire, addressing the purpose of the study and emphasizing confidentiality.

The estimated time for completion of the survey was 15-20 min. The opportunity to ask any question was given to the participants. Mothers completed the questionnaire and returned it to the data collectors. Participation was voluntary, and mothers were able to refuse any question they did not wish to respond to.

\subsection{Overview of Survey}

An extensive literature review of studies on breastfeeding was conducted to identify potential items of the study instrument. Based on the literature review, the questionnaire was adapted from validated surveys that have been used locally, regionally, and globally. It was translated into Arabic and subjected to a process of forward and backward translation by a research group at Qatar University. It was pretested on 20 mothers who attended well-baby clinics at primary health care institutions in Doha, and modifications were made as necessary so that it was clear and would provide accurate information.

The first section of the questionnaire related to socio-demographic characteristics of the participants, such as age, nationality, religion, education level for mothers and husbands, occupation, living with a husband, total monthly income, number of children, mode of delivery, last age of child, sex of child, and order. These questions were adapted from previous studies conducted in Saudi Arabia and Iran $[9,10,17,19,20]$. The second section covered breastfeeding knowledge, attitude, and practice. The breastfeeding knowledge part was used to assess the participants' knowledge of four aspects: benefits to the baby (it provides more protection from allergies compared to formula milk and reduces the risk of childhood obesity and chronic diseases such as diabetes), benefits to mothers (exclusive breastfeeding is beneficial in spacing births, achieving pre-pregnancy weight faster, and leads to a lower risk of developing breast and ovarian cancer), duration of breastfeeding, and effective feeding 
(breastfeeding should initiate immediately after delivery, infants should be exclusively breastfed for the first 6 months, breastfeeding is recommended up to one year of age, and the optimal age to stop breastfeeding is two years of age). These questions were adapted from previous research in Kuwait and Saudi Arabia [21,22]. For benefits to baby statements, a five-point Likert scale ( $1=$ strongly agree and somewhat agree, $0=$ somewhat disagree, strongly disagree, and neutral) was used to evaluate the participants' responses. For the benefits to mothers, duration of breastfeeding, and effectiveness of breastfeeding, items had several categories of responses (yes, no, or do not know).

The part on breastfeeding attitudes focused on reasons behind the adoption of breastfeeding, and items had response categories of yes, no, and do not know [21,22]. The breastfeeding practice questions asked about the number of children who were breastfed, the starting time of breastfeeding, the age of the child when mothers introduced formula or other milk, the practice of skin-to-skin contact, amount of water given to the baby after breastfeeding, and pacifier usage after delivery $[7,9,14,21,23]$.

In the third section, participants were asked whether they had stopped breastfeeding, the age of the child when they completely stopped breastfeeding, and reasons that made women decide to stop breastfeeding. These questions were adapted from different studies [21,22]. Furthermore, there was a question reflecting social support ("Who had the most impact on you to continue breastfeeding?") and participants were able to select more than one answer. The choices included mother, partner, mother-in-law, and friends [21].

Section four was about professional support provided by healthcare providers for breastfeeding mothers. This section included questions about whether mothers received training about proper positioning during breastfeeding and support for feeding problems after delivery, if the importance of breast milk was explained by a physician, if they received breastfeeding health education, and if the hospital provided them with breastfeeding tools $[16,19,22]$. This section included five questions and items had two categories of responses (yes and no). At the end of the survey, an open-ended question was used to elicit the participants' opinion on how professionals can support mothers for prolonged breastfeeding.

\subsection{Statistical Analysis}

Data generated by the questionnaire were appropriately coded and analyzed using Software Package for Social Sciences (SPSS) version 23. Regarding the breastfeeding knowledge part, a correct response was scored as 1, while a wrong answer/"do not know" response was scored as 0 . The knowledge score was calculated by adding the correct responses to 14 statements and categorized into three levels, which were poor (0-4), moderate (5-9), and good (10-14).

The first and second statements in the breastfeeding attitude part ("The community prefers breastfeeding over artificial feeding" and "Breastfeeding reduces family expenses") were scored as "yes" = 1, "no", "do not know" = 0 . The third statement "It is difficult for a breast feeder to take care of her family") was scored as "yes" = 1 , "no", "do not know" $=0$. To calculate the total attitude score, we classified those that answered two or three statements correctly as having a positive attitude, while others who answered zero or one statement correctly as having a negative attitude.

The breastfeeding practice part included nine questions. Out of these questions, five featured "yes" and "no", options- the correct answer was scored as 1 while a wrong answer was scored as 0 . For the question "How many of your children did you breastfeed?", the response "all or some of them" was scored as 1 , and the response "only the first child only or last child only" was scored as 0. For the question "When did you start breastfeeding?", the answer was scored as 1 if they answered that it had occurred within 1 or $6 \mathrm{~h}$ of delivery and was scored as 0 if they responded that it had occurred after $6 \mathrm{~h}$ of delivery, after $24 \mathrm{~h}$ of delivery, or that they did not breastfeed. The overall practice score was categorized into two levels, indicated as non-optimal (0-4) and optimal (5-9). Optimal practice means that mothers breastfed all or some of their children, practiced skin-to-skin contact, practiced breastfeeding within 1 or $6 \mathrm{~h}$ of delivery, did not introduce formula or other milk 
before 6 months of age, did not give water to the infant after every breastfeed, and did not use a pacifier after delivery.

In the third section, the answers to the question "Did you stop breastfeeding?" were scored as "yes" $=1$ and "no" $=0$. For the question "How old was your infant when you totally stopped breastfeeding?", the answers were scored as "Have not stopped" $=0$, "0-6 months" $=1$, "7-11 months" $=2$, and "12 months or more" $=3$. Questions about the reasons that made women decide to stop breastfeeding included nine statements. We scored the participants' responses as "not at all" $=0$, and "a little", "somewhat", and "a lot" = 1 . The last section, regarding professional support, included five questions for which the possible responses were "yes" and "no". A correct response was scored as 1 while a wrong response was scored as 0 . Lastly, a thematic analysis was conducted for participants' responses to the open-ended question to understand the mothers' recommendations on how to prolong the duration of breastfeeding.

Descriptive analysis was performed for mothers' demographics, breastfeeding knowledge, attitude and practice, barriers, and professional support. The bivariate Chi-squared analysis was used to describe the categorical variables and study the association with outcome 1 "Did the mother stop breastfeeding?" and outcome 2 "Infant age when breastfeeding was stopped". A bar chart was used for breastfeeding adoption question, barriers, and professional support to show the comparison of each category in a frequency distribution. The significant variables from the Chi-squared test were used to fit a model about the possible predictors of outcomes one and two by performing multivariate logistic regression, $p$-values less than 0.05 were considered to be significant.

\section{Results}

The majority of the participants in this study were aged between 31 and 35 years $(31.8 \%)$, were non-Qatari (around $60 \%$ ), Muslim (87.7\%), and had university education or higher $(74.4 \%)$. In terms of the mother's occupation, $53.3 \%$ of the mothers were housewives and most of the participants (64.1\%) had a monthly income between $0-20,000$ QR (\$5500) (Table 1). The majority of the participant babies were aged 12 months or older (57.7\%), around $29.2 \%$ were aged $0-6$ months, and $12.8 \%$ of the babies were aged 7-11 months.

Table 1. Socio-demographic characteristics of participants.

\begin{tabular}{|c|c|}
\hline Characteristics & $N(\%)$ \\
\hline \multicolumn{2}{|l|}{ Age } \\
\hline $21-25$ & $36(18.5 \%)$ \\
\hline $26-30$ & $53(27.2 \%)$ \\
\hline $31-35$ & $62(31.8 \%)$ \\
\hline $36-40$ & $44(22.6 \%)$ \\
\hline \multicolumn{2}{|l|}{ Nationality } \\
\hline Qatari & $79(40.50 \%)$ \\
\hline Non-Qatari & $115(59.0 \%)$ \\
\hline \multicolumn{2}{|l|}{ Religion } \\
\hline Muslim & $171(87.7 \%)$ \\
\hline Non-Muslim & $24(12.3 \%)$ \\
\hline \multicolumn{2}{|l|}{ Mother's education } \\
\hline High school or less & $50(26.6 \%)$ \\
\hline University education or higher & $145(74.4 \%)$ \\
\hline \multicolumn{2}{|l|}{ Mother's occupation } \\
\hline Housewife & $98(53.3 \%)$ \\
\hline Employee & $86(46.7 \%)$ \\
\hline
\end{tabular}


Table 1. Cont.

\begin{tabular}{cc}
\hline Characteristics & $N$ (\%) \\
\hline Income & \\
\hline $0-20,000(\$ 5500)$ & $123(64.1 \%)$ \\
More than 20,000 & $69(35.9 \%)$ \\
\hline Smoking & $3(1.5 \%)$ \\
\hline Yes & \\
\hline Number of children & $58(29.7 \%)$ \\
\hline One & $52(26.7 \%)$ \\
Two & $85(43.6 \%)$ \\
\hline Three or more & $122(62.6 \%)$ \\
\hline Delivery mode & $73(37.4)$ \\
\hline Normal delivery & \\
Caesarean section & $91(46.7 \%)$ \\
\hline Sex of last child & $104(53.3 \%)$ \\
\hline Male &
\end{tabular}

\subsection{Descriptive Analysis}

Our study showed that overall breastfeeding knowledge based on four aspects (the benefits to the baby, the benefits to mothers, the duration of breastfeeding, and effective feeding) was possessed by $80 \%$ of the mothers.

The majority of the mothers $(89 \%)$ reported a positive attitude towards breastfeeding. Most of the respondents agreed that "The community prefers breastfeeding over artificial feeding" (78.9\%), and "Breastfeeding reduces family expenses" (82.5\%). Around 76\% of the mothers believed that breastfeeding did not affect taking care of the family when presented with the statement: "It is difficult for the breastfeeding mother to take care of her family." They also reported that the major reasons behind the adoption of breastfeeding were child health $(44.3 \%)$, followed by cleanliness $(20.9 \%)$, and religious reasons (19.3\%). The latter is related to Islamic beliefs stated in the Holy Quran that "... mothers shall breastfeed their children for two whole years for those who desire to complete the appropriate duration of breastfeeding" (2:223) (Figure 1).

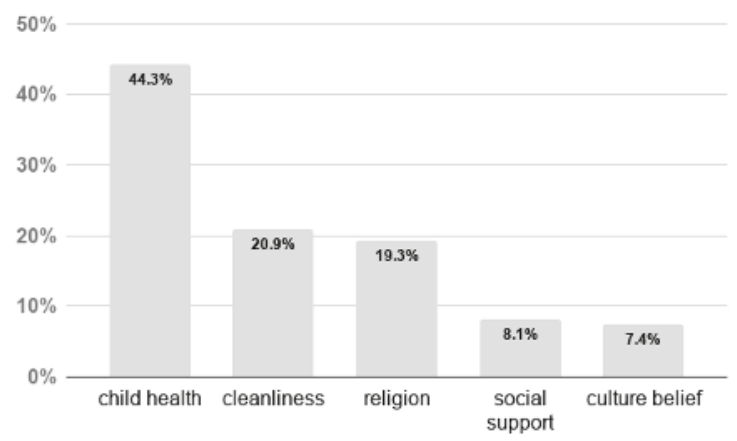

Figure 1. Reasons behind the adoption of breastfeeding among mothers attending the Primary Healthcare Care Cooperation (PHCC). 
Regarding breastfeeding practices, around $94 \%$ of participants breastfed their children, and the majority $(78.8 \%$ ) initiated breastfeeding within the first $6 \mathrm{~h}$ of delivery (Table 2 ). Yet, around $78 \%$ of participants gave their children formula or other milk and most of them started this between the first and third month of the infant's life (40.6\%). This may have influenced the exclusive breastfeeding rates among the participants at 1-3 months, 4-6 months, and after 6 months, which were $40 \%, 20 \%$, and $10 \%$, respectively.

Table 2. Breastfeeding practices by mothers attending primary healthcare centers

\begin{tabular}{cc}
\hline Statements (Variables) & $N(\%)$ \\
\hline Had breastfed a child & $182(93.8 \%)$ \\
\hline $\begin{array}{c}\text { Number of children breastfed } \\
\text { First child only or last child only } \\
\text { All of them or some of them }\end{array}$ & $53(28.2 \%)$ \\
\hline Practiced skin-to-skin contact & $135(71.8 \%)$ \\
\hline Timepoint when breastfeeding started & $162(85.7 \%)$ \\
Within 1 or 6 h of delivery & $152(78.8 \%)$ \\
Did not breastfeed, or breastfed after 6 h & $41(21.2 \%)$ \\
but within 24 h of delivery & $48(77.5 \%)$ \\
Used formula or other milk & \\
\hline Baby's age when the formula or other milk & was introduced \\
Less than 1 month & $1-3$ months \\
$4-6$ months & $65(40.6 \%)$ \\
More than 6 months & $32(20.0 \%)$ \\
\hline Giving water to the baby is encouraged & $16(10.0 \%)$ \\
\hline after every breastfeeding & $72(38.3 \%)$ \\
\hline Use a pacifier after delivery & $87(46.3 \%)$ \\
\hline
\end{tabular}

Table 3 shows that, among mothers who had already stopped breastfeeding (76.8\%), 42\% had stopped before the age of 12 months. Participants explained that major factors prohibited them from continuing to breastfeed their infants. These reasons include being uncomfortable with breastfeeding in public (59.4\%), not producing enough milk for their baby's needs (57\%) and having to go back to work or school (43.6\%) (Figure 2).

Table 3. Prevalence of outcomes 1 and 2.

\begin{tabular}{|c|c|c|c|c|}
\hline \multirow{2}{*}{ Did you stop breastfeeding? } & \multicolumn{4}{|c|}{ Yes } \\
\hline & \multicolumn{4}{|c|}{$139(76.8 \%)$} \\
\hline \multirow{2}{*}{$\begin{array}{l}\text { How old was your infant when you } \\
\text { totally stopped breastfeeding? }\end{array}$} & Have not stopped & $0-6$ months & 7-11 months & 12 months or more \\
\hline & $\begin{array}{c}42 \\
(23.2 \%)\end{array}$ & $\begin{array}{c}64 \\
(35.4 \%)\end{array}$ & $\begin{array}{c}12 \\
(6.6 \%)\end{array}$ & $\begin{array}{c}63 \\
(34.8 \%)\end{array}$ \\
\hline
\end{tabular}




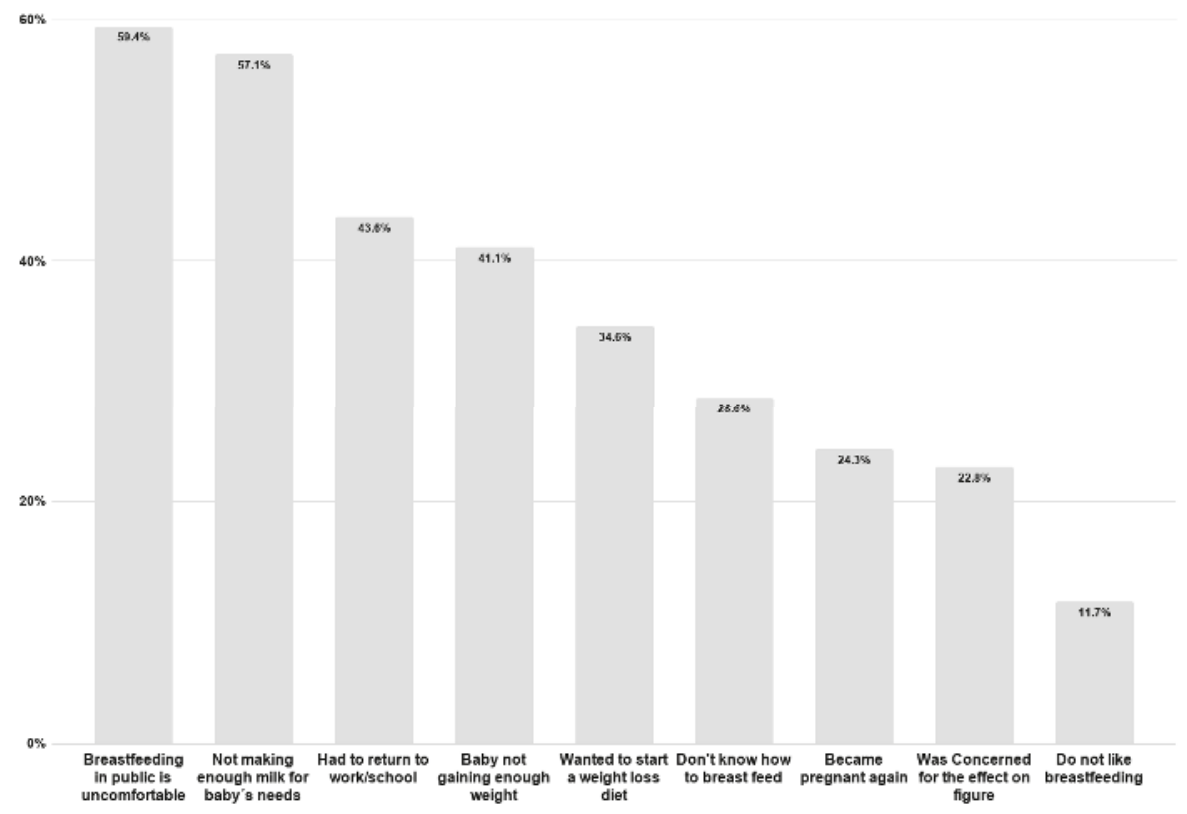

Figure 2. Barriers to continued breastfeeding as identified by mothers attending the PHCC.

However, participants reported that mothers had the most influence on their continuation of breastfeeding $(52.90 \%)$, followed by partners and mothers-in-law $(17.60 \%)$. Regarding professional support provided by healthcare providers at PHCCs, participants were mostly satisfied with the physicians' explanation regarding the importance of breastmilk, followed by breastfeeding health education messages provided during or after pregnancy. Only half of mothers received training about the proper position during breastfeeding and thought that there was enough support for feeding problems after delivery. Even though mothers received knowledge and information regarding the importance of breastfeeding, they were not provided the crucial skills needed to follow an effective feeding process and manage any feeding issue faced after delivery.

\subsection{Bivariate Analysis}

Table 4 presents the association of selected demographics of the participants, KAP, and barriers of continued breastfeeding with outcome 1 (if the mother stopped breastfeeding) and outcome 2 (infant age when stopped breastfeeding). The Chi-squared test showed that $81.3 \%$ of women who stopped breastfeeding were aged between 21 to 25 years old and, according to outcome 2 (infant age when stopped breastfeeding), $50 \%$ of these women stopped breastfeeding early (0-6 months of age). Qatari mothers who stopped breastfeeding when their infant was between 0 to 6 months of age represented $41.9 \%$ of the studied population. However, $36.8 \%$ of non-Qataris mothers continued breastfeeding to 12 months, which was longer compared to the Qataris mothers (31.1\%). 


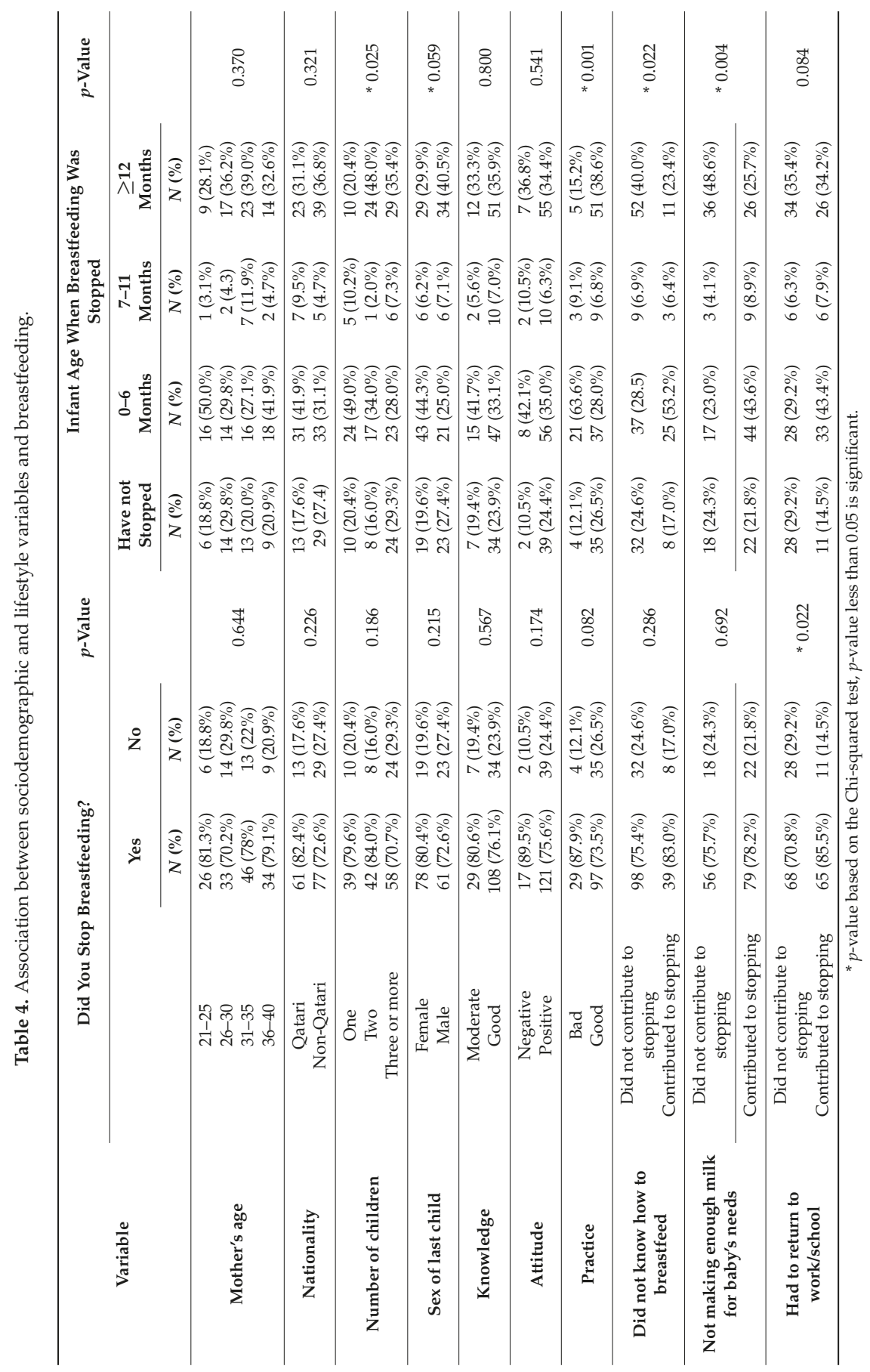


The work environment was a major barrier to continued breastfeeding, as the majority of employed mothers (80.2\%) reported that they had stopped breastfeeding. Additionally, $40.7 \%$ of employed mothers stopped breastfeeding when their infants were between 0 and 6 months of age, compared to $30 \%$ of housewives. With regard to family income, $43.3 \%$ of mothers who had a high income (more than 20,000 QR, approximately \$5500) reported that they stopped breastfeeding when the infant was between 0 and 6 months of age. A higher proportion of mothers (38.1\%) with a lower income stopped breastfeeding their infant before 12 months as compared to mothers with a higher income $(29.9 \%)$.

The results showed that $84 \%$ of participants with two children had stopped breastfeeding. There was a significant association $(p=0.025)$ between the number of children and outcome 2 (infant age when BF was stopped); $49 \%$ of mothers with only one child stopped breastfeeding within the first 6 months and they were the least likely to continue breastfeeding their infants to 12 months or more $(20.4 \%)$. The sex of the child could also play an important role in continued breastfeeding up to one year of age. Our results showed that $80.4 \%$ of mothers stopped breastfeeding their female child earlier than their male child. There was a slight significant association between the child's sex and outcome 2 ( $p$-value $=0.059)$. In addition, $44.3 \%$ of mothers stopped breastfeeding their female infants between the ages of 0 and 6 months, while $40.5 \%$ of mothers reported that their infant males received breast milk for up to 12 months.

The results showed that mothers tended to stop breastfeeding despite good breastfeeding knowledge because of the influence of other individual, sociocultural, and environmental factors. The majority of women who had a negative attitude toward breastfeeding $(42.1 \%)$ and disclosed non-optimal breastfeeding practices $(87.9 \%)$ stated that they stopped breastfeeding between 0 and 6 months. Among mothers who had optimal practice, $26.5 \%$ of them were still breastfeeding their babies $(p=0.001)$ and $(38.6 \%)$ of these mothers breastfed their children for 12 months or more.

The analysis showed that around $83 \%$ of the mothers stated that they stopped breastfeeding because they did not know how to breastfeed, and there was a statistically significant relationship between this barrier and outcome $2(p=0.022)$. Around $53 \%$ of mothers stopped breastfeeding their infant between the ages of 0 and 6 months because they did not know how to do it. Not making enough milk for the baby's needs was a barrier that many mothers faced. Around $78 \%$ of the respondents reported that they stopped breastfeeding due to this barrier and $43.6 \%$ of those stopped breastfeeding their infant between 0 and 6 months of age. There was a significant relationship between this barrier and outcome $2(p=0.001)$.

Considering the work environment, which acts as a huge barrier to breastfeeding for employed mothers, our results showed that there is a significant relationship between going back to work and outcome 1 (whether the mother had stopped breastfeeding) ( $p=0.022$ ). Around $86 \%$ of the respondents reported that they had stopped breastfeeding because they had to go back to work and $43.4 \%$ of those stopped breastfeeding between 0 and 6 months of age. Other barriers to continued breastfeeding were "disliking breastfeeding", preferring other methods (such as bottled milk), and mother's concerns about her figure. However, none of these factors showed a statistically significant relationship with the outcomes.

\subsection{Multivariate Logistic Regression of Barriers by Outcome 1}

The significant variables from the Chi-squared test were used to fit a model about the possible predictors of outcome 1 (whether the mother had stopped breastfeeding). The findings show that "Had to return to work/school" was the only significant predictor ( $p=0.021$ ), (odds ratio (OR) 2.698, $95 \%$ confidence interval (CI) $1.162,6.267)$. Therefore, mothers who had to go back to work were more likely to stop breastfeeding because of the work environment was prohibitive to practicing breastfeeding (see Table 5). 
Table 5. Association between significant predictors and breastfeeding.

\begin{tabular}{ccc}
\hline Variable & OR (95\% CI) & $p$-Value \\
\hline Number of children & $0.806(0.493-1.320)$ & 0.392 \\
Sex of last child & $1.602(0.741-3.464)$ & 0.231 \\
Practice & $0.468(0.141-1.546)$ & 0.213 \\
I did not know how to breastfeed & $1.356(0.535-3.435)$ & 0.521 \\
I was not making enough milk for & $0.782(0.345-1.773)$ & 0.556 \\
my baby's needs & $2.698(1.162-6.267)$ & $* 0.021$ \\
\hline I had to return to work/school &
\end{tabular}

$\mathrm{OR}=$ odd ratio, $\mathrm{CI}=$ confidence interval, ${ }^{*} p$-value based on Chi-squared test, $p$-value less than 0.05 is significant.

\subsection{Qualitative Analysis}

A thematic analysis was conducted for participants' responses to an open-ended question about their recommendations to prolong breastfeeding duration. Most of the participants thought that raising awareness about the importance and benefits of breastfeeding for the mother and her child was necessary. This applied especially to new mothers and one of the participants suggested the provision of "... awareness lectures for mothers before and after giving birth about the importance of breastfeeding."

Many mothers suggested having professional support aimed at providing training courses/practical sessions for new mothers about the appropriate positions to breastfeed and providing health advice about suitable nutritional intake for mothers during breastfeeding. One of the participants stated that health professionals should: "explain to mothers the importance of skin-to-skin contact to increase the quality and quantity of the breast milk and to not use formula milk for the baby during the first months."

Finally, one of the recommendations was to have longer maternity leave, since working mothers face difficulties in balancing work and breastfeeding. Some of the participants mentioned that "Maternity leave is only 60 days, which is not enough to give baby breast milk" while another suggested extending "... maternal leave to 4-6 months so the mother will not stop breastfeeding and will not leave her child for long hours."

\section{Discussion}

The present study assessed selected factors and their association with continued breastfeeding at one year of age among mothers attending well-baby clinics at five primary health care in Qatar. Around $77 \%$ of the participants in our study reported that they had stopped breastfeeding and $42 \%$ of those had stopped within the infant's first year of life. Mothers in our study were more likely to stop breastfeeding early (0-6 months of infant's age) if they were between 21 to 25 years of age, had only one infant (first-time mothers), were employed, and had high incomes (more than 20,000 QR). Similar findings were reported in Saudi Arabia, where it was found that increased maternal age, lower levels of education, and low income contributed to longer duration of breastfeeding [20]. Therefore, it is crucial that these young women are targeted for breastfeeding educational campaigns to enhance their awareness of the importance of breastfeeding for them and their infants [23].

Culture and social norms play a role in influencing the duration of breastfeeding depending on the sex of the child. Our results showed that male infants were more likely to be breastfed for up to 12 months than female infants. This aligns with findings from Turkey, which showed that male infants are breastfed for one or two months longer than females [24]. In an Arab culture, mothers are always pleased if they have a baby boy and they do their best to raise a healthy man. As a result, these cultural values shape their breastfeeding practices and lead them to spend more time breastfeeding their male infants than females. Addressing religion when communicating to mothers, indicating that 
Islam encourages mothers to breastfeed their infants for two years without preference for either sex, could help promote the duration of breastfeeding for both sexes.

At the individual level, we found that most of the participating mothers have adequate breastfeeding knowledge and a positive attitude. However, almost half of them stopped breastfeeding their infants before one year of age. These results are consistent with previous research in which having a high breastfeeding knowledge level did not enhance the rate of continued breastfeeding [25]. Other factors should thus be addressed to help explain why mothers stop breastfeeding, despite being aware of the benefits to them and their infants. Assessing practices followed by participants and other social and environmental factors could help explain what prohibits these mothers from continuing breastfeeding until the child is one year of age.

Participants reported following a variety of breastfeeding practices. Those who disclosed that they had good practices were more likely to breastfeed their children for 12 months or more, while others who had bad practices stopped breastfeeding after 0-6 months. Most of those who disclosed bad practices gave their infants formula or other milk between the first and the third month. Some mothers gave water to the child after every breastfeed and around half of the mothers used a pacifier after delivery, which may have accelerated the weaning process. Similar findings have been reported by previous research [26]. As a consequence, in our study only $10 \%$ of the participants exclusively breastfed their infants for the first six months, which is considered a low rate according to the WHO recommendations, but higher than exclusive breastfeeding rates from other regions. Kamudoni and colleagues (2007) reported that exclusive breastfeeding rates in Malawi at 2, 4, and 6 months of age were $39.1 \%, 27.5 \%$, and $7.5 \%$, respectively [27]. An explanation for these findings could be the lack of antenatal breastfeeding education and postnatal support provided by healthcare providers, which was strongly recommended by participants to enhance the duration of breastfeeding. Breastfeeding educators must contribute effectively to the promotion of breastfeeding and alleviate the current gap between knowledge and breastfeeding practice, so that it can be re-incorporated into the lives of Qatari women [11].

Other significant barriers to continued breastfeeding reported by participants were "not making enough milk for baby's needs" and "did not know how to breastfeed." These mothers were more likely to stop breastfeeding early, in the first 6 months. Our findings are consistent with previous research which showed that if women felt that their babies were not satisfied with breastfeeding, it might negatively influence breastfeeding duration [28]. Many women utilize infant satisfaction cues as their main indication of milk supply, since actual milk supply is difficult to measure $[29,30]$. A recent study conducted by Galipeau et al. has concluded that mother's perception of not having sufficient milk was not associated with an actual insufficiency of milk supply, as measured by the baby's weight loss or 24-h milk production. The authors found that factors associated with maternal perception versus actual insufficiency were different and therefore they recommended that interventions should be directed toward promoting early, optimal, and frequent feedings [31]. Training sessions about appropriate breastfeeding positions and practices to increase breastmilk should be incorporated into breastfeeding promotion campaigns. Another barrier to continued breastfeeding reported by the participants was feeling that "breastfeeding in public is uncomfortable." A study conducted in Saudi Arabia found that the chance of stopping breastfeeding increased among mothers who perceived lactation in public as a barrier [32].

In Qatar, one study found that women who practiced breastfeeding because they were aware of the benefits for them and their infants still struggle to commit to practice due to limited social support. Family has been recognized as a crucial context to promote breastfeeding duration by providing social support to mothers. It has been reported that mothers and mothers-in-law provide social support and encourage breastfeeding mothers to continue to one year of age [28]. These findings align with those of our study, which showed that mothers and mothers-in-law of the participants were the biggest providers of social support to continue breastfeeding for one year. In addition, partners provided 
less support. This indicates that various family members should be included in health education and promotion programs that aim to enhance the duration of breastfeeding among mothers in Qatar.

Multiple logistic regressions in our study showed that the most significant predictor of continued breastfeeding to one year was returning back to work or school. Mothers reported that if they had to go back to work, then they were more likely to stop breastfeeding their infants, which suggests that these women had difficulty combining work with breastfeeding. A Kuwaiti study disclosed work as a barrier to continued breastfeeding, with women reporting that they could not breastfeed and work. The Department of Health and Human Services in the US has claimed that returning to work is a barrier to continued breastfeeding, since mothers usually have less time and do not have an appropriate place to breastfeed at work. The creation of breastfeeding-conductive spaces in public and at work to increase social acceptability of breastfeeding and, as a consequence, enhance the duration of breastfeeding has been recognized by new initiatives in Gulf Cooperation Council (GCC) countries [30]. Additionally, participants in our study recommended increasing maternity leave to six months, which will enable them to spend more time with their infants and, therefore, enhance the probability of continued breastfeeding to one year.

Limitations in this study include the cross-sectional design, which limits the ability to infer the causation between the various factors and continued breastfeeding at one year. Using a self-administrated survey may lead to recall bias. The small sample size (this was a pilot study) limits the generalization to all mothers attending well-baby clinics at PHCCs in Qatar.

The current study provides baseline information and addresses a gap in the literature regarding factors influencing continued breastfeeding among mothers attending primary health care in Qatar. Understanding these factors will provide evidence for policy makers and breastfeeding educators to plan for effective health promotion programs that promote breastfeeding practices and prolong the duration, which align with the major goals of the national public health strategy in Qatar [33]. Improving the health and well-being of mothers and their infants will decrease the prevalence of major health issues the country is facing, including obesity and diabetes. Future research should focus on studying the factors and determinants associated with continued breastfeeding using a large, representative sample of mothers attending PHCCs in Qatar. More research is also needed to study actual breastfeeding practices in the country in relation to international recommendations.

\section{Conclusions}

Our study is the first of its kind in Qatar, assessing different factors on different levels and their association with continued breastfeeding at one year of age. Around $42 \%$ of the participants in our study had stopped breastfeeding within one year and only $10 \%$ of them did not use formula or other milk before six months of age. This is reflected in the low rate of exclusive breastfeeding in our sample. The findings suggested that continuation of breastfeeding is a process influenced by a variety of individual, sociocultural, and environmental factors which should be considered when planning for effective interventions to enhance continued breastfeeding until one year of age. First-time mothers, Qataris, and high-income mothers were more likely to stop breastfeeding when their child was between 0 and 6 months old. Working mothers and mothers who did not know how to effectively breastfeed their infants were also more likely to stop breastfeeding within one year.

Implementing the WHO and UNICEF (United Nations International Children's Emergency Fund) recommendations for breastfeeding-friendly hospitals in Qatar will improve continued breastfeeding to one year and beyond. Community-based interventions can facilitate the implementation of these recommendations and enhance exclusive and continued breastfeeding until one year of age. Breastfeeding educators and other healthcare providers can work with mothers to provide the skills needed for optimal breastfeeding practices, including appropriate breastfeeding positioning and practices that increase breastmilk. Educators can help mothers to seek family members for social support to enhance continued breastfeeding for at least one year and can help policy makers advocate for friendly breastfeeding work environments and longer maternity leave. 
Author Contributions: This is a capstone project for undergraduate public health students focused on health education who conducted the research, performed the analysis, and wrote the paper. The first five authors are the students. The following two authors are two teaching assistants who helped in data entry, management, and analysis. The last author is the supervising professor for this project and the corresponding author for this manuscript.

Funding: This research received no external funding.

Acknowledgments: We acknowledged the support provided by Primary Health Care Corporation in approving the data collection process from their centers.

Conflicts of Interest: The authors declare no conflict of interest.

\section{References}

1. World Health Organization: Exclusive Breastfeeding. Available online: http://www.who.int/nutrition/ topics/exclusive_breastfeeding/en/ (accessed on 4 March 2017).

2. Qin, H.; Zhang, L.; Zhang, L.; Zhang, W.; Li, L.; Deng, X.; Tian, D.; Deng, J.; Hu, G. Prevalence of Breastfeeding: Findings from the First Health Service Household Interview in Hunan Province, China. Int. J. Environ. Res. Public Health 2017, 14, 150. [CrossRef] [PubMed]

3. UNICEF: Breastfeeding in Qatar. Available online: http://www.sidra.org/breastfeeding-in-qatar/ (accessed on 4 March 2017).

4. Stuebe, A. The risks of not breastfeeding for mothers and infants. Rev. Obstet. Gynecol. 2009, 2, $222-231$. [PubMed]

5. International Diabetes Federation (IDF). IDF Diabetes Atlas, 6th ed.; IDF: Brussels, Belgium, 2013; ISBN 2-930229-85-3.

6. UNICEF. Adopting Optimal Feeding Practices is Fundamental to a Child's Survival, Growth and Development, but Too Few Children Benefit; UNICEF: New York, NY, USA, 2017. Available online: https:/ / data.unicef.org/ topic/nutrition/infant-and-young-child-feeding/\# (accessed on 4 March 2017).

7. Multiple Indicator Cluster Survey (MICS), UNICEF. Available online: http://mics.unicef.org/surveys (accessed on 27 February 2017).

8. World Health Organization: Indicators for Accessing Breastfeeding Practices. Available online: http: / /apps.who.int/iris/bitstream/10665/62134/1/WHO_CDD_SER_91.14.pdf (accessed on 4 March 2017).

9. Al-Darweesh, F.; RaAl-Hendyani, R.; Al-Shatti, K.; Abdullah, A.; Taqi, M.; Abbas, A.; Mitra, A. Knowledge, Intention, Practice, and Perceived Barriers of Breastfeeding among Married Working Women in Kuwait. Int. J. Community Fam. Med. 2016, 1, IJCFM-108. [CrossRef] [PubMed]

10. Karimi, B.; Sani, M.Z.; Ghorbani, R.; Danai, N. The Pregnant Mothers' Knowledge about Breastfeeding in Semnan, Iran. Middle East J. Rehabil. Health Stud. 2014, 1, e20833. [CrossRef]

11. Nikaiin, B.B.; Nazir, N.; Mohammad, A.; Donnelly, T.; Dorri, R.A.; Petal, N. Contextual Factors Influencing Breastfeeding Practices Among Arab Women in the State of Qatar. Qual. Sociol. Rev. 2013, 9, 74-95.

12. Kornides, M.; Kitsantas, P. Evaluation of breastfeeding promotion, support, and knowledge of benefits on breastfeeding outcomes. J. Child Health Care 2013, 17, 264-273. [CrossRef] [PubMed]

13. Bertino, E.; Varalda, A.; Magnetti, F.; Di Nicola, P.; Cester, E.; Occhi, L.; Preathoner, C.; Soldi, A.; Prandi, G. Is breastfeeding duration influenced by maternal attitude and knowledge? A longitudinal study during the first year of life. J. Matern. Fetal Neonatal Med. 2012, 25, 32-36. [CrossRef] [PubMed]

14. Aghdas, K.; Talat, K.; Sepideh, B. Effect of immediate and continuous mother-infant skin-to-skin contact on breastfeeding self-efficacy of primiparous women: A randomized control trial. Women Birth 2014, 27, 37-40. [CrossRef] [PubMed]

15. Riva, E.; Banderali, G.; Agostoni, C.; Silano, M.; Radaelli, G.; Giovannini, M. Factors associated with initiation and duration of breastfeeding in Italy. Acta Paediatr. 1999, 88, 411-415. [CrossRef] [PubMed]

16. Al-Kohji, S.; Said, H.A.; Selim, N.A. Breastfeeding practice and determinants among Arab mothers in Qatar. Saudi Med. J. 2012, 33, 436-443. [PubMed]

17. Nassar, M.F.; Abdel-Kader, A.M.; Al-Refaee, F.A.; Mohammad, Y.A.; Al Dhafiri, S.; Gabr, S.; Al-Qattan, S. Breastfeeding practice in Kuwait: Determinants of success and reasons for failure/Pratique de l'allaitement maternel au Koweï: Déterminants de réussite et motifs d'échec. East Mediterr. Health J. 2014, 20, 409-415. [CrossRef] [PubMed] 
18. Dashti, M.; Scott, J.A.; Edwards, C.A.; Al-Sughayer, M. Predictors of breastfeeding duration among women in Kuwait: Results of a prospective cohort study. Nutrients 2014, 6, 711-728. [CrossRef] [PubMed]

19. Al-Binali, A.M. Breastfeeding knowledge, attitude and practice among school teachers in Abha female educational district, southwestern Saudi Arabia. Int. Breastfeed. J. 2012, 7, 10. [CrossRef] [PubMed]

20. Al Juaid, D.A.; Binns, C.W.; Giglia, R.C. Breastfeeding in Saudi Arabia: A review. Int. Breastfeed. J. 2014, 9, 1. [CrossRef] [PubMed]

21. Begley, C. The National Infant Feeding Survey 2008/Prepared for the Health Service Executive by Cecily Begley (Rep.). Available online: http:/ / hdl.handle.net/10147/118910 (accessed on 13 March 2017).

22. Schwager, H. Why Do Women Stop after Successfully Breastfeeding for 3 Months? (Rep.). Available online: https:/ /hdl.handle.net/11244/9278 (accessed on 15 March 2017).

23. Radwan, H. Patterns and determinanats of breasfeeding and complementary feeding practices of Emirati mothers in the United Arab Emirates. BMC Public Health 2013, 13, 171. [CrossRef] [PubMed]

24. Hizel, S.; Ceyhun, G.; Tanzer, F.; Sanli, C. Traditional beliefs as forgotten influencing factors on breast-feeding performance in Turkey. Saudi Med. J. 2006, 27, 511-518. [PubMed]

25. Veghari, G.; Mansourian, A.; Abdollahi, A. Breastfeeding status and some related factors in Northern Iran. Oman Med. J. 2011, 26, 342-348. [CrossRef] [PubMed]

26. El Mouzan, M.I.; Al Omar, A.A.; Al Salloum, A.A.; Al Herbish, A.S.; Qurachi, M.M. Trends in infant nutrition in Saudi Arabia: Compliance with WHO recommendations. Ann. Saudi Med. 2009, 29, 20-23. [CrossRef] [PubMed]

27. Kamudoni, P.; Maleta, K.; Shi, Z.; Holmboe-Ottesen, G. Infant feeding practices in the first 6 months and associated factors in a rural and semiurban community in Manghotchi district, Malawi. J. Hum. Lact. 2007, 23, 325-332. [CrossRef] [PubMed]

28. Agunbiade, O.M.; Ogunleye, O.V. Constraints to exclusive breastfeeding practice among breastfeeding mothers in Southwest Nigeria: Implications for scaling up. Int. Breastfeed. J. 2012, 7, 5. [CrossRef] [PubMed]

29. Gatti, L. Maternal Perceptions of Insufficient Milk Supply in Breastfeeding. J. Nurs. Scholarsh. 2008, 40, 355-363. [CrossRef] [PubMed]

30. World Breastfeeding Trends Initiative. Saudi Arabia. 2012. Available online: www.wordbreastfeedingtrends. org/GenerateReports/report/WBTi-KSA-2012.pdf (accessed on 15 March 2017).

31. Galipeau, R.; Dumas, L.; Lepage, M. Perception of Not Having Enough Milk and Actual Milk Production of First-Time Breastfeeding Mothers: Is There a Difference? Breastfeed. Med. 2017, 12, 210-217. [CrossRef] [PubMed]

32. Saied, H.; Mohamed, A.; Suliman, A.; Al Anazi, W. Breastfeeding Knowledge, Attitude, and Barriers among Saudi Women in Riyadh. J. Natural Sci. Res. 2013, 3, 12.

33. National Health Strategy (NHS). Available online: http://www.nhsq.info/app/media/1541 (accessed on 4 May 2017).

(C) 2018 by the authors. Licensee MDPI, Basel, Switzerland. This article is an open access article distributed under the terms and conditions of the Creative Commons Attribution (CC BY) license (http:/ / creativecommons.org/licenses/by/4.0/). 
Article

\title{
Nutrition of Preterm Infants and Raw Breast Milk-Acquired Cytomegalovirus Infection: French National Audit of Clinical Practices and Diagnostic Approach
}

\author{
Anne-Aurelie Lopes ${ }^{1, *}$, Valerie Champion ${ }^{2}$ and Delphine Mitanchez ${ }^{2}$ \\ 1 Pediatric Emergency Department, AP-HP, Robert Debre Hospital, 48 Boulevard Serurier, 75019 Paris, France \\ 2 Neonatology Department, AP-HP, Armand Trousseau Hospital, 26 Avenue du Dr Arnold Netter, \\ 75012 Paris, France; valerie.champion@aphp.fr (V.C.); delphine.mitanchez@aphp.fr (D.M.) \\ * Correspondence: anne-aurelie.lopes@aphp.fr; Tel.: +33-140-032-272; Fax: +33-140-032-344
}

Received: 30 July 2018; Accepted: 14 August 2018; Published: 18 August 2018

\begin{abstract}
Raw breast milk is the optimal nutrition for infants, but it is also the primary cause of acquired cytomegalovirus (CMV) infection. Thus, many countries have chosen to contraindicate to feed raw breast milk preterm infants from CMV-positive mothers before a corrected age of 32 weeks or under a weight of $1500 \mathrm{~g}$. French national recommendations have not been updated since 2005. An audit of the French practices regarding the nutrition with raw breast milk in preterm infants was carried out using a questionnaire sent to all neonatal care units. Diagnosed postnatal milk-acquired CMV infections have been analysed using hospitalisation reports. Seventy-five percent of the neonatal units responded: $24 \%$ complied with the French recommendations, $20 \%$ contraindicated raw breast milk to all infants before 32 weeks regardless of the mothers' CMV-status, whereas $25 \%$ fed all preterm infants unconditionally with raw breast milk. Thirty-five cases of infants with milk-acquired CMV infections have been reported. The diagnosis was undeniable for five patients. In France, a high heterogeneity marks medical practices concerning the use of raw breast milk and the diagnostic approach for breast milk-acquired CMV infection is often incomplete. In this context, updated national recommendations and monitored CMV infections are urgently needed.
\end{abstract}

Keywords: raw breast milk; cytomegalovirus; milk-acquired infections; preterm infant

\section{Introduction}

In the last fifty years, the development of neonatology reversed the prognosis of preterm infants with a weight over $1500 \mathrm{~g}$ from a mortality rate of $85 \%$ to a survival rate without sequelae of $85 \%[1,2]$. This drop in mortality was accompanied by a steady decrease in severe morbidities [2]. It was mainly related to significant advances in lung maturation, respiratory support, and optimal nutrition [3]. Breast milk is a crucial part in the management of preterm infants with widely documented immunological and nutritional benefits [4]. Its composition adapts to the gestational age at birth to better protect preterm infants and to regulate their immune response [5-8]. It reduces the risk of infection and inflammatory phenomena, leading to a significant decrease in the incidence of bronchopulmonary dysplasia [9], retinopathy of prematurity [10] and necrotising enterocolitis (NEC) [11]. Nutritional values of breast milk also have a beneficial role in both short and long-term neurological development [12-14], and exposure to breast milk antigens promotes the development of tolerance and significantly reduces the risk of allergy and atopic diseases $[15,16]$. However, the long-term benefits of breast milk on the prevention of leukaemia [17], obesity [18], type 2 diabetes [19], and others are not yet fully assessed. 
The risk of transmitting infections remains a barrier to the use of raw breast milk. To limit this risk, methods have been developed. Freezing reduces the risk of infection (mainly viral risk) without eliminating it, and pasteurisation affects nutritional and antimicrobial properties of breast milk [20]. Whilst there are no differences in neurodevelopmental outcomes in preterm infant fed preterm formula compared with those fed breast milk there is a significantly higher risk of developing NEC with formula [21]. Thus, in light of the improvement of knowledge on the benefits of breast milk, the risk of transmission of the commonly feared infections has been carefully reassessed, and contraindications have been increasingly restricted. With the temporary contraindication of breastfeeding caused by Herpes simplex or Herpes zoster lesions on the breast [22], the only definite contraindication of raw breast milk in developed western countries is maternal HIV-positivity [23,24], and the most discussed one is the maternal cytomegalovirus (CMV)-positivity regarding preterm infants.

CMV (cytomegalovirus) is reactivated by lactation in the mammary gland with a prevalence greater than $95 \%$ and is then transmitted via macrophages, monocytes, and virions present in raw breast milk $[25,26]$. In the mother milk of full-term infants, CMV is excreted as early as colostrum and during the first three months of lactation. In the mother milk of preterm infants, CMV excretion begins with a lower viral load and the onset of excretion is more variable. It usually begins in the first ten days of life, but may be present from the colostrum $[27,28]$. The standard diagnostic method of CMV infection was viral isolation on fibroblasts culture from a urine sample, but current polymerase chain reaction (PCR) techniques have better sensitivity and specificity ( $98.8 \%$ and $99.9 \%$, respectively) and can be performed on urine or blood samples [29]. To conclude to a milk-acquired infection, congenital CMV infections should be eliminated by a negative CMV research on a blood or urine sample taken within the 21st days of life or on a salivary sample taken within the 21st days of life and before nutrition by unpasteurised breast milk $[29,30]$. In the case of CMV infection diagnosed after the 21st day of life, the positivity of the PCR could no longer differentiate congenital and postnatal infections. Then, only a negative CMV PCR on a specimen collected before the 21st day of life (cord blood or dried blood spots collected on blotting paper for newborn screening program) can eliminate the diagnosis of congenital infection. The reactivation of the virus in breast milk can be confirmed the same way, by viral isolation or PCR done on milk sample [31]. In the 1990s to 2000s, studies demonstrated that, in children born before a corrected age of 32 weeks or below a weight of $1500 \mathrm{~g}$, the CMV transmission rate was over $50 \%$ in the first three months of life [27]. Moreover, $50 \%$ of preterm infants had symptomatic infections, and $15 \%$ of these infections were severe [26]. The main symptoms were apnoea, bradycardia, pneumonia, hepatitis, gastrointestinal tract symptoms, and haematological signs (thrombocytopenia, neutropenia, and lymphocytosis). These infections appeared between four and eight weeks of life and were responsible for significant clinical degradations that could be life-threatening, whereas the level of C-reactive protein remained low (10 to $20 \mathrm{mg} / \mathrm{L})$. This clinical situation was called "sepsis-like". Thus, the international guidelines agreed not to feed preterm infants from CMV-positive mothers with raw breast milk before a corrected age of 32 weeks or below a weight of $1500 \mathrm{~g}$ [32-34].

In the 2010s, retrospective studies and reviews of the literature reassessed the risk of milk-acquired severe CMV infections and the prognosis of affected infants. The risk associated with symptomatic infections and "sepsis-like" were estimated to be low [35]. In particular, the risk of neurological sequelae (cognitive and motor) was similar to that of preterm infants without a history of postnatal CMV infections [25]. Therefore, since 2012, the American Academic of Pediatrics recommends nutrition with raw breast milk for all preterm infants [36]. However, publications have rapidly reported cases with severe "sepsis-like" and severe enteropathies suggestive of atypical NEC [37-40]. Several fatal cases have been reported [41,42]. Moreover, since 2015, large cohorts have shown that the incidence of bronchopulmonary dysplasia was significantly higher in infants with postnatal CMV infection [43]. The absence of long-term consequences has also been questioned [28].

Since 2005, the French recommendations maintained to not feed preterm infants from CMV-positive mothers with raw breast milk before a corrected age of 32 weeks or below a weight of $1500 \mathrm{~g}$. The breast milk must then be pasteurised before its administration [32]. This recommendation 
is problematic for several reasons. Routine CMV screening of pregnant women is not recommended in France [44], and not all neonatal units have access to pasteurisation. Furthermore, several cases of postnatal breast milk-acquired CMV infections in infants fed raw breast milk before a corrected age of 32 weeks or below a weight of $1500 \mathrm{~g}$ have been published by French neonatal units $[42,45,46]$. Thus, this study aimed to evaluate the French national current clinical practices about breast milk nutrition of preterm infants, to carry out a first national census of raw breast milk-acquired CMV infections and to check the validity of this diagnosis.

\section{Methods}

\subsection{Study Design}

An observational, transverse, prospective, multicentre, descriptive study was conducted via a questionnaire sent by e-mail to all NICU (neonatal intensive care unit) and neonatal non-ICU, in mainland France and French overseas territories.

\subsection{Outcome Measures}

The questionnaire was sent by email to at least one doctor or breastfeeding counsellor from each unit from June 2015 to June 2016. Reminders were sent out every four months for one year as long as there was no answer. The answers were collected by e-mail and by post. The questionnaire was written in French and translated into English for this publication (Figure S1).

The questionnaire consisted of four parts. In the first part, general data on the neonatal unit were collected. In the second part, the current clinical practices of each service were requested. The information was related to the use of breast milk (frozen, pasteurised, raw mother milk, or donation of breast milk), the promotion of breastfeeding, and the access to a human milk bank. The third part concerned the conditions of use of raw breast milk (maternal CMV status, infants' term or weight) and the barriers to its use (mainly infectious risks). Finally, the last part of the questionnaire identified diagnosed cases of postnatal CMV infections imputed to raw breast milk (clinical signs and course).

Subsequently, the neonatal units reporting cases of breast milk-acquired CMV infections were contacted again between June 2016 and June 2017 to obtain the hospitalisation reports of the concerned infants.

\subsection{Data Management}

CMV infections have been classified as "proven", "highly probable", "probable", or "unlikely" breast milk-acquired infections.

CMV infections have been classified as "proven" if the infection occurred in infants from CMV-positive mothers fed raw breast milk with documented CMV reactivation in breast milk and without any other mode of transmission possible. A congenital infection must have been rejected. Both viral isolation culture and PCR methods were accepted to reject the congenital infection and confirm the infection in the blood or the urine of the preterm infant and to confirm the CMV reactivation in breast milk. Other possible modes of transmission had to be eliminated (PCR on residual blood from transfusions), and no other cause of infection must be found.

CMV infections have been classified as "highly probable" if the infection occurred in infants from CMV-positive mothers fed raw breast milk, but one of the following items was not documented: reactivation of CMV in breast milk, elimination of all other possible modes of transmission, and evidence of absence of congenital infection except mothers' CMV-positivity prior to pregnancy.

CMV infections were classified as "probable" if the infection occurred in infants from CMV-positive mothers fed raw breast milk, but two of the following items were undocumented: reactivation of CMV in breast milk, elimination of all other possible modes of transmission, and evidence of absence of congenital infection except mothers' CMV-positivity prior to pregnancy.

CMV infections have been classified as "unlikely" for other cases. 


\subsection{Statistical Analysis}

The results for the quantitative variables were expressed in median (minimum-maximum). The results for the qualitative variables were expressed in numbers and percentages.

\section{Results}

The questionnaire was completed by 105 neonatal units including 58 NICU (88\%) and 47 non-ICU $(64 \%)$, representing an overall response rate of $75 \%$ (Figure 1). The participation rate was evenly distributed across regions, ranging from the smallest to the largest unit (Table S1).

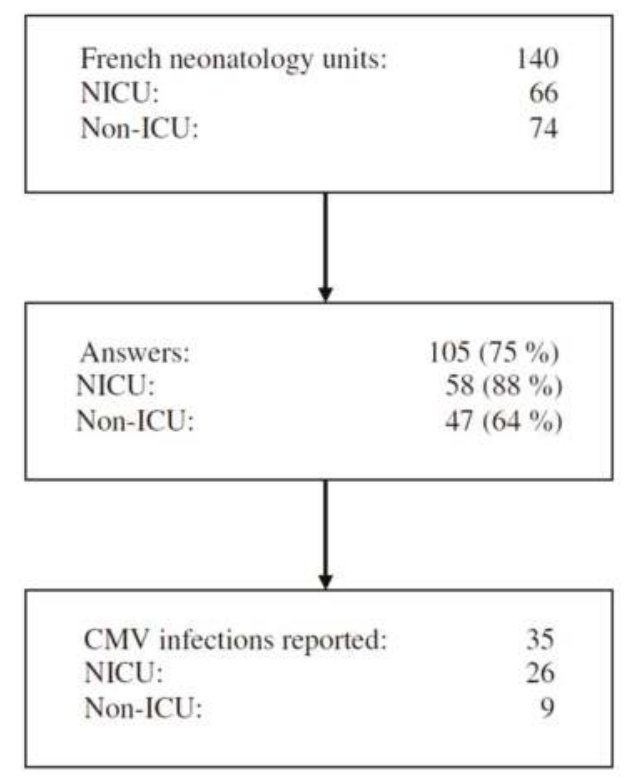

Figure 1. Flowchart. NICU: neonatal intensive care unit; ICU: intensive care unit; CMV: cytomegalovirus.

\subsection{Current Clinical Practices}

Ninety percent of NICU and $67 \%$ of non-ICU reported promoting breast milk nutrition with $70 \%$ of NICU and $63 \%$ of non-ICU having a breastfeeding counsellor, but only half of them with protocols to help initial breastfeeding. The storage methods of breast milk were freezing in 17 units, pasteurisation in 32 units, and both methods in 47 units.

The 36 neonatal units located in hospitals with a human milk bank responded to the questionnaire. Fifty-six other neonatal units (53\%), including $35 \mathrm{NICU}$, had access to a human milk bank outside their hospital. Thirteen services (12\%) reported not having access to a human milk bank. Among them, six neonatal units ( 5 NICU) were in overseas territories. The two NICU of the Reunion (overseas territory) were the only units to report the use of freeze-dried women's milk from the French human milk bank of Marmande.

Among the 92 neonatal units that had access to a human milk bank, if the infant's mother milk was unavailable, $87(95 \%)$ routinely used pasteurised women's milk instead of formulas for preterm infants before a corrected age of 32 weeks. This corresponded to 51 NICU and 36 non-ICU. All NICU systematically used pasteurised women's milk before a corrected age of 30 weeks, except for a unit that used it systematically only for infants before 28 weeks. For infant over a corrected age of 32 weeks, 37 units (35\%), including 19 NICU, used pasteurised women's milk for initial trophic nutrition, nutrition for infants with significant in utero growth retardation (birth weight $<10$ th percentile and 
less than $1500 \mathrm{~g}$ ) or for children with congenital digestive disorders, current digestive disorders, or renal failure.

\subsection{Raw Breast Milk Use}

Only two among 105 units declared never to use raw breast milk. Among the 103 units that used raw breast milk, $25 \%$ neonatal units fed all infants with raw milk, regardless of term, weight and the mother CMV status (Figure 2). Among them, seven units did not have access to a Human milk bank (including three overseas territories), and eight NICU decided to feed all preterm infants with raw breast milk, despite access to a Human milk bank.

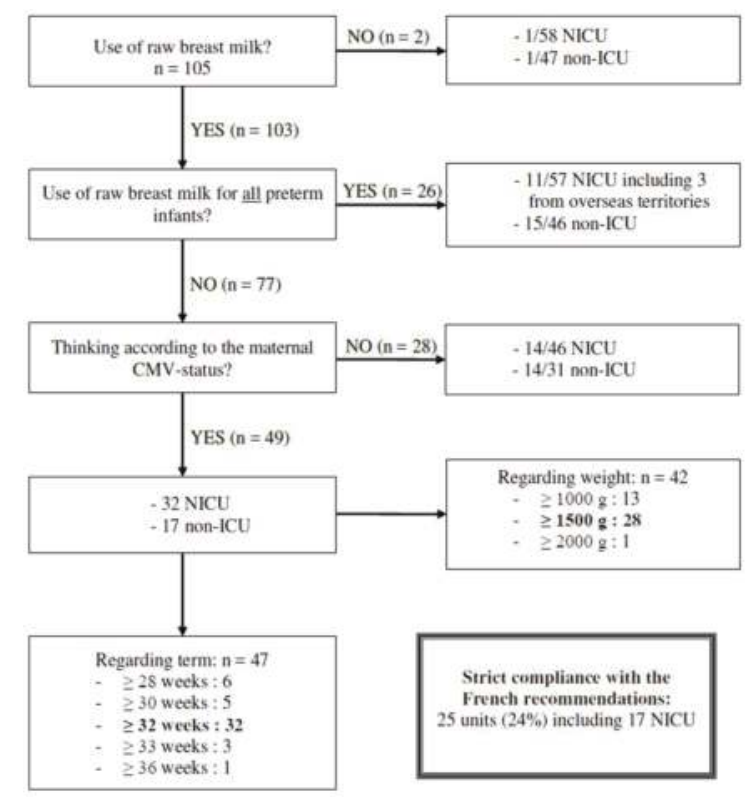

Figure 2. Summary of the current use of raw breast milk in France.

Seventy-three percent of the units used raw breast milk based on the infants' term or weight. Among them, 49 units gave raw milk according to the CMV maternal status. Thus, all infants born from CMV-negative mothers received raw breast milk from birth. On the other hand, in the case of maternal CMV-positivity, 32 units gave breast milk from a corrected age of 32 weeks, but some gave it either later or sooner (Figure 2). Although $1500 \mathrm{~g}$ was the most common weight limit used, $1000 \mathrm{~g}$ was also widely used, associated with a term limit of 28 weeks. Twenty-eight units gave raw breast milk based on infants' term, but not according to the maternal CMV status. Consequently, even infants born from CMV-negative mothers did not receive raw breast milk before a defined term or weight. The clear majority (21 including 11 NICU) gave raw breast milk from 32 weeks to all preterm infants. Other units gave it from terms ranging from 28 weeks to 35 weeks. Overall, 25 units (24\%) complied with the French recommendations.

Among the 77 units that did not give unconditionally raw breast milk, 95\% reported that the risk of transmission of infectious diseases was the main barrier. The most feared infections were caused by CMV (56 units including all NICU), HIV (43 units including 26 NICU), bacterial infections led by those caused by Staphylococcus aureus (33 units including 20 NICU) and HTLV-1 (10 NICU). Five units also reported that the current French recommendations were the principal barrier to the use of raw breast milk. Units that did not give raw breast milk before a corrected age of 32 weeks 
and below $1500 \mathrm{~g}$, regardless of the maternal CMV-status, highlighted the contradiction of the French recommendations as the maternal CMV serology is not recommended during pregnancy. On the other hand, those who gave raw breast milk to all infants, regardless of their term, weight, and their mother's CMV-status, justified this approach by the numerous studies on the benefits of breast milk and the latest American recommendations. Other units pointed out that the absence of a human milk bank was of great importance in their decision and that it could have been otherwise. Moreover, some units changed their practices because of cases of severe postnatal infections.

\subsection{Reported Postnatal CMV Infection Attributed to Raw Breast Milk Nutrition}

Twenty-one units (20\%) (17 NICU and 4 non-ICU) reported a total of 35 cases of postnatal CMV infections thought to be transmitted via raw breast milk between 2013 and 2016. Eight infants (23\%) had asymptomatic infections, 11 (31\%) had moderate signs (hepatic cytolysis, thrombocytopenia), and $16(46 \%)$ had significant signs including 10 infants (29\%) with "sepsis-like" infections. Two infants died in NICU during the infection, and another infant died a few months later from complications of this infection. Seventeen hospitalisation reports were obtained, including the reports of two of the three deceased infants and were classified as "proven", "highly probable", "probable", and "unlikely" (Table 1).

Table 1. Infection cases.

\begin{tabular}{|c|c|c|c|c|c|c|}
\hline $\begin{array}{c}\text { "Proven" } \\
\text { Infections }\end{array}$ & $\begin{array}{l}\text { Term at } \\
\text { Birth }\end{array}$ & $\begin{array}{l}\text { Weight at } \\
\text { Birth }\end{array}$ & $\begin{array}{c}\text { Age at } \\
\text { Diagnosis } \\
\text { (Day) }\end{array}$ & Symptoms & Missing Information & Source of Reference \\
\hline 1 & $\begin{array}{c}27 \text { weeks } \\
4 \text { days }\end{array}$ & $550 \mathrm{~g}$ & 50 & $\begin{array}{c}\text { "Sepsis-like", NEC, } \\
\text { death }\end{array}$ & / & Lopes et al., 2016 \\
\hline 2 & $\begin{array}{c}27 \text { weeks } \\
4 \text { days }\end{array}$ & $1000 \mathrm{~g}$ & 50 & Asymptomatic & / & Lopes et al., 2016 \\
\hline 3 & 26 weeks & $810 \mathrm{~g}$ & 70 & "Sepsis-like", NEC, & / & This study \\
\hline 5 & 29 weeks & $1200 \mathrm{~g}$ & 53 & Asymptomatic & / & $\begin{array}{l}\text { Croly-Labourlette et al., } \\
2006\end{array}$ \\
\hline \multicolumn{7}{|l|}{$\begin{array}{l}\text { "Highly } \\
\text { probable" } \\
\text { infections }\end{array}$} \\
\hline 8 & $\begin{array}{l}27 \text { weeks } \\
5 \text { days }\end{array}$ & $950 \mathrm{~g}$ & 41 & $\begin{array}{l}\text { "Sepsis-lik", } \\
\text { thrombocytopenia } \\
\text { NEC, death }\end{array}$ & $\begin{array}{c}\text { Elimination of } \\
\text { congenital origin }\end{array}$ & This study \\
\hline 9 & 28 weeks & $1125 \mathrm{~g}$ & 60 & Thrombocytopenia & $\begin{array}{c}\text { CMV reactivation in } \\
\text { breast milk } \\
\text { (stopped before) }\end{array}$ & Boumahni et al., 2014 \\
\hline 10 & 30 weeks & $1500 \mathrm{~g}$ & 15 and 40 & $\begin{array}{l}\text { Cholestasis } \\
\text { "Sepsis-like" }\end{array}$ & $\begin{array}{l}\text { CMV reactivation in } \\
\text { breast milk }\end{array}$ & Radi et al., 2007 \\
\hline 11 & 33 weeks & $>2000 \mathrm{~g}$ & 20 & "Sepsis-like", NEC & $\begin{array}{l}\text { Elimination of } \\
\text { congenital origin }\end{array}$ & This study \\
\hline 12 & 33 weeks & $>2000 \mathrm{~g}$ & 20 & Adenopathies & $\begin{array}{c}\text { Elimination of } \\
\text { congenital origin }\end{array}$ & This study \\
\hline
\end{tabular}

CMV: cytomegalovirus; PCR: polymerase chain reaction; NEC: necrotising enterocolitis. 
The five cases classified as "proven" infections were fed raw breast milk before their second week of life. The congenital origin of the infection was eliminated by a CMV PCR negative on the dried blood spots of the newborn screening program for all of them, excepted one infant with an intrauterine growth restriction who had negative research of CMV done on urine sample during his first week of life. The mean gestational age of birth of these children was 27 weeks (26-29 weeks). Two children had an asymptomatic infection diagnosed for one because of the symptomatic infection of his twin, and for the other during a pilot study. One infant received blood transfusions because of a win-to-twin transfusion syndrome with negative CMV PCR done on the residual blood (patient 1). His autopsy found typical CMV lesions in all organs including the entire digestive tract. Another infant who suffered from "sepsis-like" and NEC showed a CMV PCR positive on peritoneal liquid.

The seven cases classified as "highly probable" were all fed raw breast milk before their second week of life. When it was done, the congenital origin of the infection was eliminated by a CMV PCR negative on the dried blood spots of the newborn screening program. The children had a gestational age of birth between 25 and 33 weeks. Half of the infections were discovered on biological abnormalities. The mother of the twins born at 33 weeks with a weight over $2000 \mathrm{~g}$ was suffering from a CMV mastitis. Only two patients received treatment by ganciclovir: one died (patient 8 ), and the other showed numerous CMV reactivation (patient 10). The histological examination of the ileocaecal resection of patient 8 showed intense necrotic and pan-parietal inflammatory lesions with typical CMV lesions. This infant presented a persistent hepatocellular insufficiency associated with significant thrombocytopenia requiring numerous platelet transfusions. Four months later, during surgery for restoring the continuity of the gastrointestinal tract, his clinical condition deteriorated rapidly, and he died in the following hours.

The three cases classified as "probable" were all fed through raw breast milk from their first week of life. In these cases, even if the mothers were CMV-positive before pregnancy, the congenital origin was not eliminated, and the reactivation in the mother milk was not proven. The diagnostic was done after two months of life in the two cases.

Two reported cases considered as milk-acquired CMV infections were classified as "unlikely". Based on their history, they were probably congenital infections. One did not receive his mother milk but women pasteurised milk. For the second one, the CMV PCR done on the mother milk was negative.

\section{Discussion}

As a result of a high rate of participation, this work offers a global vision of clinical practices in France. All neonatal units recognised the fundamental issue of promoting breastfeeding and emphasised the importance of individual and adapted care, as shown by the higher importance given to the breastfeeding counsellor compared with the establishment of breastfeeding protocols. Regarding raw breast milk, $24 \%$ of units strictly complied with the French recommendations and $20 \%$ applied the same limits to all preterm infants, regardless of maternal CMV-status, whereas $25 \%$ of neonatal units fed all preterm infants unconditionally with raw breast milk. Most neonatal units believed that the French recommendations are outdated. Their current protocols were the result of reflections including French recommendations [32], recommendations from authorities of other countries [36] or by French experts [47], recent literature, possible access to a human milk bank, and their clinical experience. It resulted in a variety of protocols ranging from raw breast milk nutrition for all preterm infants to non-use of it before a corrected age of 36 weeks or below a weight of $2000 \mathrm{~g}$.

These protocols were mainly based on the fear of severe breast milk-acquired CMV infections in preterm infants. However, this audit shows that the diagnostic approach to conclude such an infection was often incomplete. Out of the 17 hospitalisation reports obtained, the diagnosis was confirmed in only five cases. In the group of infections classified as "highly probable", the missing step was most often the elimination of congenital infection. In the case of infections classified as "probable", the two missing elements were both the elimination of congenital infection and the confirmation of the CMV reactivation in breast milk. The diagnosis was always made in the first months of life when 
the information can be retreived. Indeed, the retrospective way to eliminate a congenital infection is to perform a CMV PCR on a sample taken before the 21st day of life. In France, blotting papers for the newborn screening program are kept for 18 months. Viral DNA testing on the dried blood spots collected on this blotting paper could be done up to five years after birth [48] and the technique to perform a CMV PCR on it has evolved to improve the sensitivity and specificity to $99.9 \%$ [49,50]. Thus, the congenital origin of a CMV infection can be confirmed or denied. The second missing element was the proof of CMV reactivation in the mother milk. CMV excretion occurs from the first to the eighth week with a peak of viral load between the third and the fifth week. Freezing decreases the viral load while preserving the viral DNA. Thus, this research can be conducted afterwards, including that on frozen milk.

In the absence of an exhaustive diagnostic approach, it is impossible to know the exact number of postnatal breast milk-acquired CMV infections, as well as their risk factors and prognosis. A possible exhaustive diagnostic approach is presented in Figure 3. The only mode of transmission that is not eliminated by this approach is perinatal transmission when passing the birth canal. Few studies have investigated this mode of transmission, but they showed a near-zero risk in term infant as in preterm infants [26]. However, this approach investigates and eliminates all other sources of transmission. In the 2000s, prospective studies focused on eliminating congenital origin and confirming postnatal infection, without systematically eliminating the risk of transmission through blood products or through confirming the CMV reactivation in breast milk. This fact has been emphasised in the review of the literature by Kurath et al. in 2010 [25] and a meta-analysis conducted in 2017 by Lanzieri et al. [35]. The latter analysed all the studies carried out since 1980 in English, French, Spanish, and Portuguese. Its inclusion factors were known old maternal immunity, birth before a gestational age of 32 weeks or below a weight of $1500 \mathrm{~g}$, elimination of congenital infection, confirmation of postnatal infection, and accuracy of the preservation mode of the breast milk received (pasteurised, frozen, or raw). Only 17 studies conducted between 2001 and 2011 could be included, and elimination of transmission via blood products was analysed, but was not an inclusion factor as few studies explicitly excluded it. These studies underlined the difficulty of analysing the results, considering the heterogeneity of the international recommendations and practices.

In our study, infants affected by CMV infections were born between 25 and 33 weeks and fed raw breast milk before their second week of life. Infections occurred between the 15th and 70th day of life. All reported cases of severe infections ("sepsis-like", ECUN, deaths) involved infants born before 30 weeks of age, except for one twin born at 33 weeks with a mother suffering from CMV mastitis. Eight cases involved infants born before 28 weeks with infections occurring until a corrected age of 36 weeks. Studies and reviews of the literature seem to agree that children born before a corrected age of 28 weeks or a weight below $1000 \mathrm{~g}$ are at higher risk of developing severe infections, and that $80 \%$ of "sepsis-like" concern infants born before 26 weeks $[27,41]$. One of the reasons is probably the reduced transplacental passage of protective maternal antibodies before the end of the 28th week [51]. The risk of severe infection appears to be increased if preterm infants receive raw breast milk during the first month of life and have comorbidities [52-54]. A group of French experts worked on the use of raw breast milk to harmonise practices and wrote the "First Recommendations for the Use of Raw Milk" [47]. The experts provided advice on all crucial steps in breastfeeding, from breastfeeding promotion to protein fortification and viral and bacteriological infectious contraindications. Regarding CMV, the contraindication only concerns infants born from CMV-positive mothers before a corrected age of 28 weeks or below a weight of $1000 \mathrm{~g}$. For these infants, although raw colostrum can be administered within the first 2-3 days of life, milk must be pasteurised up to a corrected age of 31 weeks and 6 days to protect children for at least the first month of life. Six NICU followed these recommendations. However, this expert opinion, like the current French recommendations, raises the problem of CMV screening in pregnant women. The main argument for not recommending this non-targeted screening is the lack of effective treatment for congenital infections. However, this screening would promote hygiene measures to CMV-negative women [55] 
and facilitate compliance with the French recommendations concerning the nutrition of preterm infants with raw breast milk. Moreover, although no curative treatment exists, early treatment of infants born with congenital infection can reduce the rate of neurosensory sequelae [56].

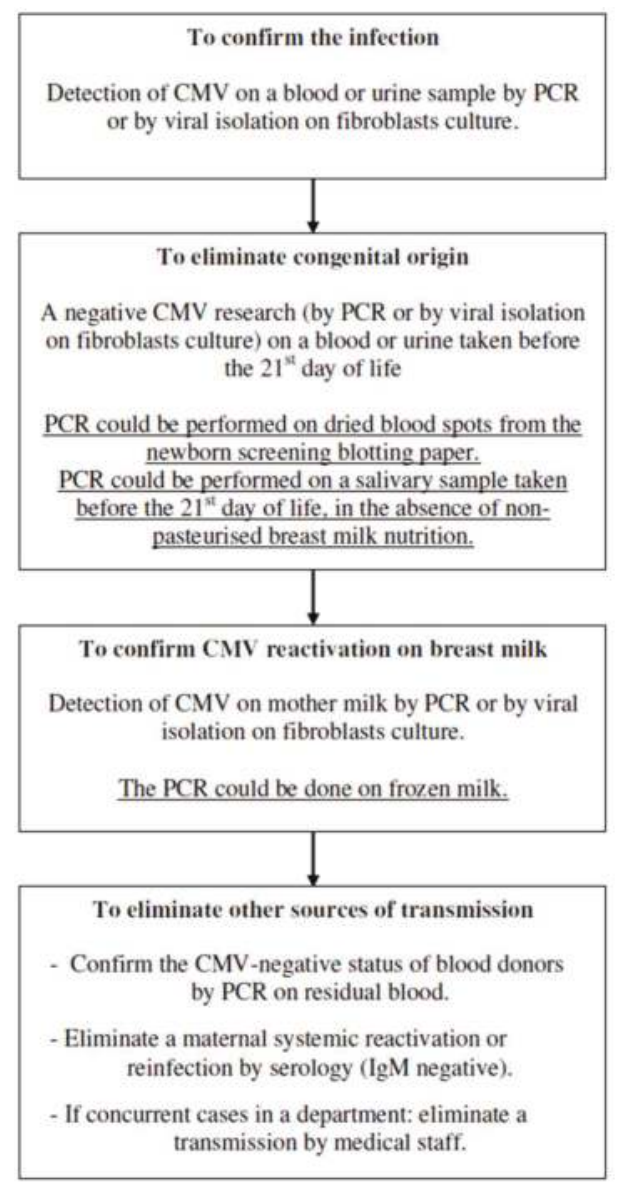

Figure 3. Possible exhaustive diagnostic approach of milk-acquired CMV infections. PCR: polymerase chain reaction.

Another issue raised by this study is the link between milk-acquired CMV infection and NEC. In our study, more than half of severe postnatal CMV infections were associated with NEC. The autopsy finding of one infant showed specific CMV lesions in the digestive tract [42], as the histological examination of the ileocaecal resection of a second infant, and a CMV PCR was positive on the peritoneal liquid of another infant. NEC is an acute inflammatory reaction with necrosis of the digestive tract and is the leading gastrointestinal cause of morbidity and mortality in preterm infants with, in very low birth weight, an incidence estimated at $11 \%$ [57]. Surgery is required for $50 \%$ of them and 35\% die [57]. In CMV infection, the involvement of the digestive tract is mainly described in immunocompromised patients where the entire digestive tract can be affected and can lead to digestive perforation with a poor prognosis [58]. Studies have shown that raw breast milk nutrition significantly reduces the risk of NEC [58]. However, the link between NEC and postnatal CMV infection transmitted via breast milk (and thus via the digestive tract) is controversial. Although many 
case reports highlight this association [37,42], some prospective studies have shown no link between NEC and viral infections [59], while others have shown a significant incidence of CMV infections in acute digestive tract pathologies in preterm infants [57]. The digestive manifestations associated with a postnatal or congenital CMV infection appear to be diverse and include, in term or premature infants, NEC (including atypical) or digestive perforations as volvulus in low birth weight infants [60].

Our study has a major limitation. The questionnaire was essentially empirical and could be completed by a physician, a nurse, or a breastfeeding counsellor. The high level of participation and its even distribution across regions should provide an overview of French practices and enable an optimal census of diagnosed milk-acquired CMV infections. However, we faced a reporting bias and probably an underestimation of the cases. While many units have reported milk-acquired CMV infections, half have finally agreed to send us the hospitalisation reports. Moreover, some known cases have not been reported. The human milk bank of Ile-de-France is often consulted to determine the probability that CMV infections are milk-acquired infections. In 2015-2016, it confirmed four cases. These cases concern four neonatal units who responded to the audit, but none of them has reported the infections in our questionnaire. These cases, like most of the cases reported without an obtained hospitalisation report, occurred in neonatal units using raw breast milk before a corrected age of 32 weeks or below a weight of $1500 \mathrm{~g}$. On the other hand, units that have turned back their practices because of the occurrence of milk-acquired CMV infections, or that continue voluntarily to not follow the French recommendations, have sent us the hospitalisation reports.

\section{Conclusions}

In the absence of an efficient technique to eliminate infectious risk while preserving the nutritional and immunological values of breast milk, consensus on the use of raw breast milk in preterm infants is needed. The French recommendations are indeed too restrictive but, given the heterogeneity of clinical practices and the likely underestimation of infectious risk, new recommendations seem challenging to formulate. The creation of a national registry of milk-acquired CMV infections with a structured diagnostic approach could be an effective way to assess the real infectious risk; identify a population at risk; and, in few years, write national recommendations. These recommendations will have, among others, to rule on the knowledge of maternal CMV status during pregnancy or in preterm births, as well as on the best screening methods for infants.

Supplementary Materials: The following are available online at http://www.mdpi.com/2072-6643/10/8/1119/ s1, Figure S1: Translation of the questionnaire in English, Table S1: Demographic data.

Author Contributions: Conceptualization, methodology, investigation, formal analysis and writing (original draft preparation) were done by A.-A.L. Writing (review and editing) and supervision were done by V.C. and D.M. All the authors critically reviewed the manuscript, approved the final manuscript as submitted and agree to be accountable for all aspects of the work.

Funding: This research received no external funding.

Conflicts of Interest: The authors declare no conflicts of interest.

\section{References}

1. Torchin, H.; Ancel, P.-Y.; Jarreau, P.-H.; Goffinet, F. Epidemiology of preterm birth: Prevalence, recent trends, short- and long-term outcomes. J. Gynecol. Obstet. Biol. Reprod. 2015, 44, 723-731. [CrossRef] [PubMed]

2. Ancel, P.-Y.; Goffinet, F.; EPIPAGE-2 Writing Group; Kuhn, P.; Langer, B.; Matis, J.; Hernandorena, X.; Chabanier, P.; Joly-Pedespan, L.; Lecomte, B.; et al. Survival and morbidity of preterm children born at 22 through 34 weeks' gestation in France in 2011: Results of the EPIPAGE-2 cohort study. JAMA Pediatr. 2015, 169, 230-238. [CrossRef] [PubMed]

3. Vidyasagar, D. Half a Century of Evolution of Neonatology: A Witness's Story. Indian J. Pediatr. 2015, 82, 1117-1125. [CrossRef] [PubMed]

4. Cleminson, J.S.; Zalewski, S.P.; Embleton, N.D. Nutrition in the preterm infant: What's new? Curr. Opin. Clin. Nutr. Metab. Care 2016, 19, 220-225. [CrossRef] [PubMed] 
5. Lewis, E.D.; Richard, C.; Larsen, B.M.; Field, C.J. The Importance of Human Milk for Immunity in Preterm Infants. Clin. Perinatol. 2017, 44, 23-47. [CrossRef] [PubMed]

6. Araújo, E.D.; Gonçalves, A.K.; da Cornetta, M.C.; Cunha, H.; Cardoso, M.L.; Morais, S.S.; Giraldo, P.C. Evaluation of the secretory immunoglobulin A levels in the colostrum and milk of mothers of term and pre-term newborns. Braz. J. Infect. Dis. 2005, 9, 357-362. [CrossRef] [PubMed]

7. Mehta, R.; Petrova, A. Biologically active breast milk proteins in association with very preterm delivery and stage of lactation. J. Perinatol. 2011, 31, 58-62. [CrossRef] [PubMed]

8. Koenig, A.; de Albuquerque Diniz, E.M.; Barbosa, S.F.C.; Vaz, F.A.C. Immunologic factors in human milk: The effects of gestational age and pasteurization. J. Hum. Lact. 2005, 21, 439-443. [CrossRef] [PubMed]

9. Spiegler, J.; Preuß, M.; Gebauer, C.; Bendiks, M.; Herting, E.; Göpel, W.; German Neonatal Network (GNN). Does Breastmilk Influence the Development of Bronchopulmonary Dysplasia? J. Pediatr. 2016, 169, 76-80. [CrossRef] [PubMed]

10. Bharwani, S.K.; Green, B.F.; Pezzullo, J.C.; Bharwani, S.S.; Dhanireddy, R. Systematic review and meta-analysis of human milk intake and retinopathy of prematurity: A significant update. J. Perinatol. 2016, 36, 913-920. [CrossRef] [PubMed]

11. Donovan, S.M. The Role of Lactoferrin in Gastrointestinal and Immune Development and Function: A Preclinical Perspective. J. Pediatr. 2016, 173, S16-S28. [CrossRef] [PubMed]

12. Horta, B.L.; Loret de Mola, C.; Victora, C.G. Breastfeeding and intelligence: A systematic review and meta-analysis. Acta Paediatr. 2015, 104, 14-19. [CrossRef] [PubMed]

13. Belfort, M.B.; Rifas-Shiman, S.L.; Kleinman, K.P.; Guthrie, L.B.; Bellinger, D.C.; Taveras, E.M.; Gillman, M.W.; Oken, E. Infant feeding and childhood cognition at ages 3 and 7 years: Effects of breastfeeding duration and exclusivity. JAMA Pediatr. 2013, 167, 836-844. [CrossRef] [PubMed]

14. Victora, C.G.; Horta, B.L.; de Mola, C.L.; Quevedo, L.; Pinheiro, R.T.; Gigante, D.P.; Gonçalves, H.; Barros, F.C. Association between breastfeeding and intelligence, educational attainment, and income at 30 years of age: A prospective birth cohort study from Brazil. Lancet Glob. Health 2015, 3, e199-e205. [CrossRef]

15. Van Odijk, J.; Kull, I.; Borres, M.P.; Brandtzaeg, P.; Edberg, U.; Hanson, L.A.; Høst, A.; Kuitunen, M.; Olsen, S.F.; Skerfving, S.; et al. Breastfeeding and allergic disease: A multidisciplinary review of the literature (1966-2001) on the mode of early feeding in infancy and its impact on later atopic manifestations. Allergy 2003, 58, 833-843. [CrossRef] [PubMed]

16. Dogaru, C.M.; Nyffenegger, D.; Pescatore, A.M.; Spycher, B.D.; Kuehni, C.E. Breastfeeding and childhood asthma: Systematic review and meta-analysis. Am. J. Epidemiol. 2014, 179, 1153-1167. [CrossRef] [PubMed]

17. Amitay, E.L.; Keinan-Boker, L. Breastfeeding and Childhood Leukemia Incidence: A Meta-analysis and Systematic Review. JAMA Pediatr. 2015, 169, e151025. [CrossRef] [PubMed]

18. Pudla, K.J.; Gonzaléz-Chica, D.A.; Vasconcelos, F.D.A.G.D. Effect of breastfeeding on obesity of schoolchildren: Influence of maternal education. Rev. Paul. Pediatr. 2015, 33, 295-302. [CrossRef] [PubMed]

19. Horta, B.L.; Loret de Mola, C.; Victora, C.G. Long-term consequences of breastfeeding on cholesterol, obesity, systolic blood pressure and type 2 diabetes: A systematic review and meta-analysis. Acta Paediatr. 2015, 104, 30-37. [CrossRef] [PubMed]

20. Van Gysel, M.; Cossey, V.; Fieuws, S.; Schuermans, A. Impact of pasteurization on the antibacterial properties of human milk. Eur. J. Pediatr. 2012, 171, 1231-1237. [CrossRef] [PubMed]

21. Quigley, M.; Embleton, N.D.; McGuire, W. Formula versus donor breast milk for feeding preterm or low birth weight infants. Cochrane Database Syst. Rev. 2018, 6. [CrossRef] [PubMed]

22. Berens, P.; Eglash, A.; Malloy, M.; Steube, A.M. ABM Clinical Protocol \#26: Persistent Pain with Breastfeeding. Breastfeed. Med. 2016, 11, 46-53. [CrossRef] [PubMed]

23. Little, K.M.; Hu, D.J.; Dominguez, K.L. HIV-1 and breastfeeding in the United States. Adv. Exp. Med. Biol. 2012, 743, 261-270. [CrossRef] [PubMed]

24. World Health Organization. UNICEF Update on HIV and Infant Feeding; World Health Organization: Geneva, Switzerland, 2016.

25. Kurath, S.; Halwachs-Baumann, G.; Müller, W.; Resch, B. Transmission of cytomegalovirus via breast milk to the prematurely born infant: A systematic review. Clin. Microbiol. Infect. 2010, 16, 1172-1178. [CrossRef] [PubMed]

26. Hamprecht, K.; Maschmann, J.; Vochem, M.; Dietz, K.; Speer, C.P.; Jahn, G. Epidemiology of transmission of cytomegalovirus from mother to preterm infant by breastfeeding. Lancet 2001, 357, 513-518. [CrossRef] 
27. Vochem, M.; Hamprecht, K.; Jahn, G.; Speer, C.P. Transmission of cytomegalovirus to preterm infants through breast milk. Pediatr. Infect. Dis. J. 1998, 17, 53-58. [CrossRef] [PubMed]

28. Hamprecht, K.; Goelz, R. Postnatal Cytomegalovirus Infection through Human Milk in Preterm Infants: Transmission, Clinical Presentation, and Prevention. Clin. Perinatol. 2017, 44, 121-130. [CrossRef] [PubMed]

29. Ross, S.A.; Ahmed, A.; Palmer, A.L.; Michaels, M.G.; Sánchez, P.J.; Bernstein, D.I.; Tolan, R.W.; Novak, Z.; Chowdhury, N.; Fowler, K.B.; et al. Detection of congenital cytomegalovirus infection by real-time polymerase chain reaction analysis of saliva or urine specimens. J. Infect. Dis. 2014, 210, 1415-1418. [CrossRef] [PubMed]

30. Boppana, S.B.; Ross, S.A.; Shimamura, M.; Palmer, A.L.; Ahmed, A.; Michaels, M.G.; Sánchez, P.J.; Bernstein, D.I.; Tolan, R.W.; Novak, Z.; et al. Saliva polymerase-chain-reaction assay for cytomegalovirus screening in newborns. N. Engl. J. Med. 2011, 364, 2111-2118. [CrossRef] [PubMed]

31. Romero-Gómez, M.P.; Cabrera, M.; Montes-Bueno, M.T.; Cendejas-Bueno, E.; Segovia, C.; Pastrana, N.; Mingorance, J.; Omeñaca, F. Evaluation of cytomegalovirus infection in low-birth weight children by breast milk using a real-time polymerase chain reaction assay. J. Med. Virol. 2015, 87, 845-850. [CrossRef] [PubMed]

32. Agence française de sécurité sanitaire des aliments. Recommandations d'hygiène pour la préparation et la conservation des biberons. Available online: https://www.anses.fr/fr/system/files/MIC-Ra-BIB.pdf (accessed on 4 July 2015).

33. Gartner, L.M.; Morton, J.; Lawrence, R.A.; Naylor, A.J.; O’Hare, D.; Schanler, R.J.; Eidelman, A.I. Breastfeeding and the Use of Human Milk. Pediatrics 2005, 115, 496-506. [CrossRef] [PubMed]

34. Omarsdottir, S.; Casper, C.; Akerman, A.; Polberger, S.; Vanpée, M. Breastmilk handling routines for preterm infants in Sweden: A national cross-sectional study. Breastfeed. Med. 2008, 3, 165-170. [CrossRef] [PubMed]

35. Lanzieri, T.M.; Dollard, S.C.; Josephson, C.D.; Schmid, D.S.; Bialek, S.R. Breast milk-acquired cytomegalovirus infection and disease in VLBW and premature infants. Pediatrics 2013, 131, e1937-e1945. [CrossRef] [PubMed]

36. Eidelman, A.I.; Schanler, R.J.; Johnston, M.; Landers, S.; Noble, L.; Szucs, K.; Viehmann, L. Se Breastfeeding and the use of human milk. Pediatrics 2012, 129, e827-e841. [CrossRef]

37. Cheong, J.L.Y.; Cowan, F.M.; Modi, N. Gastrointestinal manifestations of postnatal cytomegalovirus infection in infants admitted to a neonatal intensive care unit over a five year period. Arch. Dis. Child. Fetal Neonatal Ed. 2004, 89, F367-F369. [CrossRef] [PubMed]

38. Okulu, E.; Akin, I.M.; Atasay, B.; Ciftçi, E.; Arsan, S.; Türmen, T. Severe postnatal cytomegalovirus infection with multisystem involvement in an extremely low birth weight infant. J. Perinatol. 2012, 32, 72-74. [CrossRef] [PubMed]

39. Takahashi, R.; Tagawa, M.; Sanjo, M.; Chiba, H.; Ito, T.; Yamada, M.; Nakae, S.; Suzuki, A.; Nishimura, H.; Naganuma, M.; et al. Severe postnatal cytomegalovirus infection in a very premature infant. Neonatology 2007, 92, 236-239. [CrossRef] [PubMed]

40. Fischer, C.; Meylan, P.; Bickle Graz, M.; Gudinchet, F.; Vaudaux, B.; Berger, C.; Roth-Kleiner, M. Severe postnatally acquired cytomegalovirus infection presenting with colitis, pneumonitis and sepsis-like syndrome in an extremely low birthweight infant. Neonatology 2010, 97, 339-345. [CrossRef] [PubMed]

41. Hamele, M.; Flanagan, R.; Loomis, C.A.; Stevens, T.; Fairchok, M.P. Severe morbidity and mortality with breast milk associated cytomegalovirus infection. Pediatr. Infect. Dis. J. 2010, 29, 84-86. [CrossRef] [PubMed]

42. Lopes, A.-A.; Belhabri, S.; Karaoui, L. Erratum: Clinical Findings and Autopsy of a Preterm Infant with Breast Milk-Acquired Cytomegalovirus Infection. AJP Rep. 2016, 6, e367. [CrossRef] [PubMed]

43. Kelly, M.S.; Benjamin, D.K.; Puopolo, K.M.; Laughon, M.M.; Clark, R.H.; Mukhopadhyay, S.; Benjamin, D.K.; Smith, P.B.; Permar, S.R. Postnatal Cytomegalovirus Infection and the Risk for Bronchopulmonary Dysplasia. JAMA Pediatr. 2015, 169, e153785. [CrossRef] [PubMed]

44. Collège National des Gynécologues et Obstétriciens Français. Cytomégalovirus et grossesse. Available online: http:/ / www.cngof.fr/actualites/403-cytomegalovirus-et-grossesse (accessed on 2 March 2016).

45. Boumahni, B.; Robillard, P.-Y. Infection post-natale à cytomégalovirus chez l'enfant prématuré: Rôle du lait de mère. Arch. Pédiatr. 2014, 21, 1060-1061. [CrossRef] [PubMed]

46. Croly-Labourdette, S.; Vallet, S.; Gagneur, A.; Gremmo-Feger, G.; Legrand-Quillien, M.-C.; Ansquer, H.; Jacquemot, L.; Narbonne, V.; Lintanf, J.; Collet, N.; et al. Transmission du cytomégalovirus par le lait maternel cru aux enfants prématurés: Étude épidémiologique pilote. Arch. Pédiatr. 2006, 13, 1015-1021. [CrossRef] [PubMed] 
47. Picaud, J.C.; Buffin, R.; Gremmo-Feger, G.; Rigo, J.; Putet, G.; Casper, C.; Working group of the French Neonatal Society on fresh human milk use in preterm infants. Review concludes that specific recommendations are needed to harmonise the provision of fresh mother's milk to their preterm infants. Acta Paediatr. 2018, 107, 1145-1155. [CrossRef] [PubMed]

48. Barbi, M.; Binda, S.; Primache, V.; Caroppo, S.; Didò, P.; Guidotti, P.; Corbetta, C.; Melotti, D. Cytomegalovirus DNA detection in Guthrie cards: A powerful tool for diagnosing congenital infection. J. Clin. Virol. 2000, 17, 159-165. [CrossRef]

49. Binda, S.; Caroppo, S.; Didò, P.; Primache, V.; Veronesi, L.; Calvario, A.; Piana, A.; Barbi, M. Modification of CMV DNA detection from dried blood spots for diagnosing congenital CMV infection. J. Clin. Virol. 2004, 30, 276-279. [CrossRef] [PubMed]

50. Göhring, K.; Dietz, K.; Hartleif, S.; Jahn, G.; Hamprecht, K. Influence of different extraction methods and PCR techniques on the sensitivity of HCMV-DNA detection in dried blood spot (DBS) filter cards. J. Clin. Virol. 2010, 48, 278-281. [CrossRef] [PubMed]

51. Mussi-Pinhata, M.M.; Pinto, P.C.G.; Yamamoto, A.Y.; Berencsi, K.; de Souza, C.B.S.; Andrea, M.; Duarte, G.; Jorge, S.M. Placental transfer of naturally acquired, maternal cytomegalovirus antibodies in term and preterm neonates. J. Med. Virol. 2003, 69, 232-239. [CrossRef] [PubMed]

52. Mussi-Pinhata, M.M.; Yamamoto, A.Y.; do Carmo Rego, M.A.; Pinto, P.C.G.; da Motta, M.S.F.; Calixto, C. Perinatal or early-postnatal cytomegalovirus infection in preterm infants under 34 weeks gestation born to CMV-seropositive mothers within a high-seroprevalence population. J. Pediatr. 2004, 145, 685-688. [CrossRef] [PubMed]

53. Mehler, K.; Oberthuer, A.; Lang-Roth, R.; Kribs, A. High rate of symptomatic cytomegalovirus infection in extremely low gestational age preterm infants of 22-24 weeks' gestation after transmission via breast milk. Neonatology 2014, 105, 27-32. [CrossRef] [PubMed]

54. Remington, J.; Klein, J.; Wilson, C.; Nizet, V.; Maldonado, Y. Infectious Diseases of the Fetus and Newborn, 7th ed.; Elsevier Saunders: Philadelphia, PA, USA, 2011.

55. Vauloup-Fellous, C.; Picone, O.; Cordier, A.-G.; Parent-du-Châtelet, I.; Senat, M.-V.; Frydman, R.; Grangeot-Keros, L. Does hygiene counseling have an impact on the rate of CMV primary infection during pregnancy? Results of a 3-year prospective study in a French hospital. J. Clin. Virol. 2009, 46, S49-S53. [CrossRef] [PubMed]

56. Kimberlin, D.W.; Jester, P.M.; Sánchez, P.J.; Ahmed, A.; Arav-Boger, R.; Michaels, M.G.; Ashouri, N.; Englund, J.A.; Estrada, B.; Jacobs, R.F.; et al. National Institute of Allergy and Infectious Diseases Collaborative Antiviral Study Group Valganciclovir for symptomatic congenital cytomegalovirus disease. N. Engl. J. Med. 2015, 372, 933-943. [CrossRef] [PubMed]

57. Omarsdottir, S.; Agnarsdottir, M.; Casper, C.; Orrego, A.; Vanpée, M.; Rahbar, A.; Söderberg-Nauclér, C. High prevalence of cytomegalovirus infection in surgical intestinal specimens from infants with necrotizing enterocolitis and spontaneous intestinal perforation: A retrospective observational study. J. Clin. Virol. 2017, 93, 57-64. [CrossRef] [PubMed]

58. You, D.M.; Johnson, M.D. Cytomegalovirus infection and the gastrointestinal tract. Curr. Gastroenterol. Rep. 2012, 14, 334-342. [CrossRef] [PubMed]

59. Skeath, T.; Stewart, C.; Waugh, S.; Embleton, N.; Cummings, S.; Berrington, J. Cytomegalovirus and other common enteric viruses are not commonly associated with NEC. Acta Paediatr. 2016, 105, 50-52. [CrossRef] [PubMed]

60. Goelz, R.; Hamprecht, K.; Klingel, K.; Poets, C.F. Intestinal manifestations of postnatal and congenital cytomegalovirus infection in term and preterm infants. J. Clin. Virol. Off. Publ. Pan Am. Soc. Clin. Virol. 2016, 83, 29-36. [CrossRef] [PubMed]

(C) 2018 by the authors. Licensee MDPI, Basel, Switzerland. This article is an open access article distributed under the terms and conditions of the Creative Commons Attribution (CC BY) license (http:/ / creativecommons.org/licenses/by/4.0/). 
Article

\title{
Human Milk Adiponectin and Leptin and Infant Body Composition over the First 12 Months of Lactation
}

\author{
Zoya Gridneva ${ }^{1, *}$, Sambavi Kugananthan ${ }^{1,2}$, Alethea Rea ${ }^{3}$, Ching Tat Lai ${ }^{1}$, Leigh C. Ward ${ }^{4}$, \\ Kevin Murray ${ }^{5}$, Peter E. Hartmann ${ }^{1}$ and Donna T. Geddes ${ }^{1}$ \\ 1 School of Molecular Sciences, M310, The University of Western Australia, Crawley, Perth, WA 6009, \\ Australia; 21141062@student.uwa.edu.au (S.K.); ching-tat.lai@uwa.edu.au (C.T.L.); \\ peter.hartmann@uwa.edu.au (P.E.H.); donna.geddes@uwa.edu.au (D.T.G.) \\ 2 School of Human Sciences, The University of Western Australia, Crawley, Perth, WA 6009, Australia \\ 3 Centre for Applied Statistics, The University of Western Australia, Crawley, Perth, WA 6009, Australia; \\ alethea.rea@uwa.edu.au \\ 4 School of Chemistry and Molecular Biosciences, The University of Queensland, St. Lucia, \\ Brisbane, QLD 4072, Australia; l.ward@uq.edu.au \\ 5 School of Population and Global Health, The University of Western Australia, Crawley, \\ Perth, WA 6009, Australia; kevin.murray@uwa.edu.au \\ * Correspondence: zoya.gridneva@uwa.edu.au; Tel.: +61-8-6488-4467
}

Received: 31 July 2018; Accepted: 17 August 2018; Published: 20 August 2018

check for updates

\begin{abstract}
Human milk (HM) adipokines may influence infant feeding patterns, appetite regulation, and body composition (BC). The associations between concentrations/calculated daily intakes (CDI) of $\mathrm{HM}$ adipokines in the first 12 months postpartum and maternal/term infant $\mathrm{BC}$, and infant breastfeeding parameters were investigated. BC of breastfeeding dyads $(n=20)$ was measured at 2, 5, 9, and/or 12 months postpartum with ultrasound skinfolds (infants) and bioimpedance spectroscopy (infants/mothers). 24-h milk intake and feeding frequency were measured along with whole milk adiponectin and skim and whole milk leptin (SML and WML) and CDI were calculated. Statistical analysis used linear regression/mixed effects models; results were adjusted for multiple comparisons. Adipokine concentrations did not associate with infant BC. Higher CDI of adiponectin were associated with lower infant fat-free mass (FFM; $p=0.005)$ and FFM index (FFMI; $p=0.009$ ) and higher fat mass (FM; $p<0.001$ ), FM index (FMI; $p<0.001)$, and \%FM $(p<0.001)$. Higher CDI of SML were associated with higher infant FM $(p<0.001)$, FMI $(p<0.001)$, and \%FM $(p=0.002)$. At 12 months, higher CDI of WML were associated with larger increases in infant adiposity (2-12 month: FM, $p=0.0006$; \%FM, $p=0.0004$ ); higher CDI of SML were associated with a larger decrease in FFMI (5-12 months: $p=0.0004)$. Intakes of HM adipokines differentially influence development of infant $\mathrm{BC}$ in the first year of life, which is a critical window of infant programming and may potentially influence risk of later disease via modulation of BC.
\end{abstract}

Keywords: adipokines; adiponectin; leptin; breastfeeding; infant; body composition; bioelectrical impedance spectroscopy; ultrasound skinfolds; human milk; lactation

\section{Introduction}

A major research focus seeks to elucidate the developmental origins of adiposity and obesity and their health outcomes later in life, since convincing evidence exists of early programming effects on obesity and adiposity [1]. Postnatal feeding choices offer a window of opportunity to prevent obesity. Breastfeeding is implicated in the establishment of infant appetite regulation, feeding patterns, 
and body composition (BC) and is also associated with reduced risk of developing obesity and a range of other chronic noncommunicable diseases (NCD) [2]. The development of infant BC in early life is known to play an important role in the programming of these health outcomes [3], since deviation from the optimal growth trajectory in early infancy may have a significant effect on adult health later in life. Further, the different growth pattern of breastfed infants compared to those formula-fed has been linked to lower rates of obesity [4-7]. The reduction in risk may be an outcome of multiple synergetic mechanisms associated with human milk (HM) composition [8-10], infant breastfeeding patterns, and behavior [11-15], all of which are highly variable between breastfeeding dyads and may influence infant growth and development of BC.

HM is multifunctional fluid shaped by many thousand years of evolution. As well as essential nutrients, it contains immunological and bioactive components which provide nutrition, protection against infections, developmental factors and, most recently discovered, a host of appetite control factors such as HM adipokines leptin and adiponectin [16]. Higher concentrations of HM adiponectin and leptin have an age-related association with infant weight suggesting an active role in energy homeostasis [17-20].

Although leptin is the most widely studied of HM appetite hormones, the research in this area is limited and has yet to establish clear relationships between HM leptin and infant BC. However, concentrations of this satiety hormone have been examined predominantly in skim milk where the concentration is significantly lower than in whole milk [21,22] and anthropometric measures or body mass index (BMI) were used, rather than BC measurements [20,23]. BMI is a limited index of adiposity that fails to reflect body shape, fat distribution, and density and may lead to misleading conclusions $[23,24]$. Therefore, a combination of accurate noninvasive methods to measure infant BC in conjunction with comprehensive HM composition is needed [25]. While leptin has been shown to associate positively with maternal adiposity $[22,26]$, the data on the relationship with infant growth and $\mathrm{BC}$ are not conclusive [27], due to heterogeneity in studies designs and few longitudinal studies.

Adiponectin is the appetite hormone present in the highest concentrations in HM and is more than 40-times higher than that of leptin [28]. Amongst its various functions adiponectin regulates lipid and glucose metabolism, stimulates food intake, participates in energy balance, and has anti-inflammatory effects $[29,30]$. HM adiponectin concentrations are positively associated with maternal serum levels [18,31], and generally maternal serum concentrations of adiponectin are inversely related to maternal body weight and BMI [32,33]. Further, some studies show a positive relationship between HM adiponectin and maternal adiposity [17,28,34], while others show no association [35-40]. Adiponectin initially was reported to associate negatively with infant growth and lean body mass accretion in earlier months postpartum [17,31,35,41,42], but recent findings of a few longitudinal studies also report positive associations emerging past $4-6$ months of life $[18,36,43]$. These studies support the notion of differential age-related effects of adiponectin, which modulate growth in early development and promote a growth pattern thought to be responsible for the reduced or increased incidence of adult obesity. This reversal of the initial trend in early life is speculated to be related to the cessation of breastfeeding [18]; high HM adiponectin levels may initially downregulate infant growth, and later promote adipogenesis and adipocyte hypertrophy [44], highlighting the necessity to measure the intake of these adipokines.

It is essential to understand the mechanisms by which breastfeeding and HM may impact infant $\mathrm{BC}$, as this will allow for more targeted interventions that may improve infant outcome and reduce infant and adult overweight and obesity. Thus, the aim of this longitudinal study was to investigate relationships of concentrations and daily intakes of HM adiponectin and leptin with anthropometrics and BC of healthy term breastfed infants and their mothers during first 12 months postpartum. Further, exploration of relationships of infant 24-h milk intake and feeding frequency with HM adipokines was carried out. 


\section{Materials and Methods}

\subsection{Study Participants}

Breastfed infants ( $n=20 ; 10$ males, 10 females) of English-speaking, predominantly Caucasian (18 Caucasian, 2 Asian), mothers of higher social-economic status from a developed country were recruited from the community, primarily from the West Australian branch of the Australian Breastfeeding Association. Inclusion criteria were: healthy singletons, gestational age $\geq 37$ weeks, exclusively breastfed [45] at 2 and 5 months, and maternal intention to breastfeed until 12 months. Exclusion criteria were: infant factors that could potentially influence growth and development of BC, maternal smoking, and low milk supply. All mothers provided written informed consent to participate in the study, which was approved by The University of Western Australia Human Research Ethics Committee (RA/1/4253, RA/4/1/2639) and registered with the Australian New Zealand Clinical Trials Registry (ACTRN12616000368437).

\subsection{Study Session}

Measurements were made when the infants were 2 and/or 5, 9, and 12 months of age. Participants visited our laboratory at King Edward Memorial Hospital for Women (Subiaco, Perth, WA, Australia) for up to four monitored breastfeeding sessions between March 2013 and September 2015.

At each study session, the infant was weighed prefeed, and then the mother breastfed her infant. Infant bioelectrical impedance spectroscopy (BIS) measurements were made prefeed, unless impractical, then they were taken postfeed [46]. Ultrasound skinfold (US) and anthropometric measurements were made postfeed. Clothing was removed for the measurements except for a dry diaper and a singlet.

Maternal weight, height, and BIS measurements were recorded. Small (1-2 mL) pre-/postfeed milk samples were collected into 5-mL polypropylene vials (Disposable Products, Adelaide, SA, Australia) from the breast/s that the infant was fed from and samples were frozen at $-20{ }^{\circ} \mathrm{C}$ for biochemical analysis. Current feeding frequency (FFQ) of the infants was self-reported by mothers.

\subsection{Anthropometric Measurements}

Infants weight was determined before breastfeeding using Medela Electronic Baby Weigh Scales ( \pm 2.0 g; Medela Inc., McHenry, IL, USA). Infant crown-heel length was measured once to the nearest $0.1 \mathrm{~cm}$ using nonstretch tape and a headpiece and a footpiece, both applied perpendicularly to the hard surface. Infant head circumference was measured with a nonstretch tape to the nearest $0.1 \mathrm{~cm}$.

Maternal weight was measured using Seca electronic scales $( \pm 0.1 \mathrm{~kg}$; Seca, Chino, CA, USA). Height was self-reported by participants or measured against a calibrated marked wall (accuracy $\pm 0.1 \mathrm{~cm}$ ).

Infant and maternal BMI were calculated as $\mathrm{kg} / \mathrm{m}^{2}$.

\subsection{Body Composition with Bioelectrical Impedance Spectroscopy}

The methods for measuring maternal and infant BC with the Impedimed SFB7 bioelectrical impedance analyzer (ImpediMed, Brisbane, QLD, Australia) as well as equations for calculations of infant BC parameters have been published previously [14]. The within participant coefficient of variation $(\mathrm{CV})$ for maternal \%FM was $0.21 \%$ [22]. Within participant CV for infant resistance measurements at $50 \mathrm{kHz}\left(\mathrm{R}_{50}\right)$ was $1.5 \%$ [46].

\subsection{Ultrasound Skinfold Measurements}

The method for measuring infant skinfolds using the Aplio XG (Toshiba, Tokyo, Japan) ultrasound machine with a 14-8 MHz transducer (PLT-1204BX) and sterile water-based ultrasonic 
gel (Parker Laboratories Inc., Fairfield, NJ, USA) as well as equations for calculations of infant BC parameters during this study have been published previously $[14,25]$.

\subsection{Body Composition Indices}

The indices of height-normalized BC were calculated for mothers and infants: FM index (FMI) was calculated as FM/length ${ }^{2}$, and FFM index (FFMI) was calculated as FFM/length ${ }^{2}$; both expressed as $\mathrm{kg} / \mathrm{m}^{2}[47]$.

\subsection{4-h Milk Intake and Feeding Frequency}

Infant 24-h milk intake (MI) was measured by mothers using the 24-h milk production (MP) protocol, weighing infants at home with the Medela Electronic Baby Weigh Scales pre- and post each breastfeed during a 24-h period plus one breastfeeding, and recording amounts of HM (g) consumed by the infant (including expressed HM if any) [48]. 24-h MI was determined as previously described with potential underestimation of 3-10\% [48] and FFQ (meals per 24-h) was recorded [49]. 24-h MI was measured at 3 time points: between 2 and 5 (4.0 \pm 1.3$)$ months, when MI is shown to be stable [49], and within two weeks of $9(9.4 \pm 0.3)$ and $12(12.2 \pm 0.4)$ months. Given that measuring $24-\mathrm{h}$ MI is not always practical, particularly at the later stages of lactation, mothers were also asked how frequently the infant fed, and self-reported (SR) the typical time between the meals (e.g., each $2 \mathrm{~h}$ ) during the week prior to the study session as a proxy measure of FFQ.

\subsection{Calculated Daily Intakes of Adipokines}

24-h MI values from the 24-h MP, and HM adipokine concentrations (averaged pre-/postfeed) from samples taken at the study sessions were used for the calculation of daily intakes (CDI). These CDI were considered representative of a typical daily intake due to absence of significant short-term changes in HM adiponectin and leptin concentrations [22].

\subsection{Sample Preparation}

Prior to further analysis, $\mathrm{HM}$ samples were thawed for $2 \mathrm{~h}$ at room temperature, mixed on Intelli-Mixer RM-2M (ELMI, Riga, Latvia) at 50 revolutions per min in "UU" mode for $15 \mathrm{~s}$, then, after gentle inversion (3 times), aliquoted into $1.5 \mathrm{~mL}$ tubes (Sarstedt, Numbrecht, Germany). Pre- and postfeed samples of whole HM were used for measuring whole milk leptin (WML) and whole milk adiponectin (WMA) concentrations. Milk samples were defatted by centrifugation at room temperature in a Beckman Microfuge 11 (Aberdon Enterprise Inc, Elk Grove Village, IL, USA) at 10,000 $\times g$ for $10 \mathrm{~min}$ and removing the fat layer by clipping it off together with the top of the tube [50]. Skim HM was used for measuring skim milk leptin (SML). Standard assays were adapted for and carried out using a JANUS workstation (PerkinElmer, Inc., Waltham, MA, USA) and measured on EnSpire (PerkinElmer, Inc., Waltham, MA, USA).

\subsection{Leptin}

Leptin concentration in whole and skim HM was measured using the R\&D Systems Human Leptin ELISA DuoSet kit (R \& D system, Minneapolis, MN, USA) with a protocol to measure leptin in skim HM optimized by Cannon et al. [51], and further modified for measurement of leptin in skim and whole HM by Kugananthan et al. [21]. Recovery of leptin was $97.1 \pm 9.1 \%(n=10)$ with a detection limit of $0.05 \mathrm{ng} / \mathrm{mL}$ and an inter-assay $\mathrm{CV}$ of $<7.2 \%$.

\subsection{Adiponectin}

Adiponectin concentration was measured in whole HM using the Biovendor Human Adiponectin Sandwich ELISA kit (Life Technologies, Asheville, North Carolina, NC, USA). WMA recovery was $96.2 \pm 3.2 \%(n=10)$ with a detection limit of $1 \mathrm{ng} / \mathrm{mL}$ and an inter-assay $\mathrm{CV}$ of $<2.5 \%$. 


\subsection{Statistical Analyses}

Data for this analysis came from the longitudinal study, the details of which, including power calculation, have been described previously [14]. During this longitudinal study participants were measured at 4 time points ( 2 and/or 5, 9, and 12 months). Descriptive statistics are reported as mean \pm standard deviation (SD) and range; model parameters as estimates \pm SE (standard errors).

The analyses for systematic differences in concentrations and CDI of adipokines at different months after birth used linear mixed model with age as effect factor and mother as a random factor. Differences between each month were analyzed using general linear hypothesis tests (Tukey's all pair comparisons).

Relationships between: (a) maternal BC and adipokines' concentrations/CDI, (b) adipokines' concentrations/CDI and infant BC, (c) adipokines' concentrations and breastfeeding parameters (24-h MI/FFQ), and (d) FFQ and CDI of adipokines were analyzed using linear mixed effects models. Each adipokine concentration/CDI or infant BC measure/index was considered separately as the response variable, and each model contained fixed effects of infant age (months), a single predictor (either maternal BC measure/index, adipokine concentration/CDI, or breastfeeding parameters), and an interaction between infant age and predictor, as well as a random intercept per participant. If the interaction is not significant results were reported for the same model fitted without the interaction to assist in understanding the nature of the relationship between the predictor and outcome. As interactions between infant sex and BC measurement methods were nonsignificant $(p>0.52)$ [14], reported associations are for combined male and female data.

Relationships between CDI of adipokines measured between 2 and 5, and at 9 and 12 months after birth and changes $(\Delta)$ in infant $\mathrm{BC}$ and anthropometric parameters between the time points were analyzed using linear regression models.

Owing to the large number of comparisons, a false discovery rate adjustment [52] was applied to the subgroupings of results to the interaction $p$-value if it was less than 0.05 or to the main effect $p$-value; the adjusted significance levels are reported in Results and Tables and set at the $5 \%$ level otherwise. Missing data was dealt with using available case analysis. Statistical analysis was performed in R 3.1.2 [53]. Additional packages were used for linear mixed effects models (nlme, lme4 and car) [54-56], intra-class correlations (irr) [57], Tukey's all pair comparisons (multcomp) [58], and graphics (ggplot2) [59].

\section{Results}

\subsection{Subjects}

Twenty-two two infants were recruited; two infants (one male, one female) were excluded from the study after the 2-month visit (commenced weaning; personal circumstances) and one female infant weaned at 6 months and was therefore excluded from further analysis. The 19 remaining infants were breastfed at 2, 5 and 9 months and 17 infants continued to breastfeed at 12 months. Out of 18 infants measured at 12 months, 16 infants $(89 \%)$ still continued to breastfeed; one male infant ceased breastfeeding 2 weeks before the 12-month appointment and one female infant stopped at 10 months after birth.

Therefore, overall, 6 infants missed one study session and one infant missed two study sessions. Five of these infants were not recruited until 5 months, one infant did not attend the study session at 9 months, and two did not attend the study session at 12 months. Recruitment of participants at the 5 months point was introduced, as many mothers would not commit to a study that required breastfeeding to 12 months, when approached at 2 months.

Overall 80 measures were expected, however some were missing, specifically: infant weight $(n=9)$; infant BC parameters measured with US 2SF, and maternal age, weight, height, BMI, and BC parameters measured with BIS ( $n=10)$; infant head circumference $(n=11)$; infant length, BMI, and BC parameters measured with US 4SF, concentrations of WMA, SML, and WML $(n=12)$; infant BC 
parameters measured with BIS $(n=13)$; self-reported FFQ $(n=20)$. Missing data also occurred due to difficulties with conducting 24-h MI measurements at later stages of lactation. The following measurements from the 60 expected were missing: FFQ from 24-h MP $(n=26), 24-\mathrm{h} \mathrm{MI}$, and CDI of WMA, SML, and WML ( $n=27)$. Missing data were spread across the time points (Table 1 ).

Table 1. Participant anthropometric and breastfeeding characteristics.

\begin{tabular}{|c|c|c|c|c|}
\hline Characteristic & $\begin{array}{l}2 \text { Months }{ }^{a} \\
\text { Mean } \pm \text { SD } \\
\text { (Range) }\end{array}$ & $\begin{array}{l}5 \text { Months }{ }^{b} \\
\text { Mean } \pm \text { SD } \\
\text { (Range) }\end{array}$ & $\begin{array}{l}9 \text { Months } \\
\text { Mean } \pm \text { SD } \\
\text { (Range) }\end{array}$ & $\begin{array}{l}12 \text { Months }{ }^{\mathrm{d}} \\
\text { Mean } \pm \mathrm{SD} \\
\text { (Range) }\end{array}$ \\
\hline \multicolumn{5}{|c|}{ Mothers } \\
\hline Weight (kg) & $\begin{array}{l}78.8 \pm 19.3 \\
(57.5-116.2)\end{array}$ & $\begin{array}{l}70.1 \pm 17.8 \\
(53.7-115.3)\end{array}$ & $\begin{array}{c}63.0 \pm 10.0 \\
(50.4-121.9)\end{array}$ & $\begin{array}{c}64.2 \pm 17.3 \\
(51.4-121.9)\end{array}$ \\
\hline $\operatorname{BMI}\left(\mathrm{kg} / \mathrm{m}^{2}\right)$ & $\begin{array}{l}27.2 \pm 5.5 \\
(20.4-35.5)\end{array}$ & $\begin{array}{l}24.8 \pm 5.0 \\
(19.0-35.2)\end{array}$ & $\begin{array}{l}22.7 \pm 3.9 \\
(17.9-37.2)\end{array}$ & $\begin{array}{l}23.9 \pm 5.9 \\
(18.2-37.2)\end{array}$ \\
\hline \multicolumn{5}{|c|}{ Infants } \\
\hline $\operatorname{Sex}(M / F)$ & $9 \mathrm{M} / 6 \mathrm{~F}$ & $10 \mathrm{M} / 10 \mathrm{~F}$ & $10 \mathrm{M} / 9 \mathrm{~F}$ & $9 \mathrm{M} / 9 \mathrm{~F}$ \\
\hline Age (months) & $\begin{array}{l}2.04 \pm 0.14 \\
(1.87-2.33)\end{array}$ & $\begin{array}{l}5.16 \pm 0.22 \\
(4.77-5.47)\end{array}$ & $\begin{array}{l}9.22 \pm 0.27 \\
(8.83-9.77)\end{array}$ & $\begin{array}{c}12.26 \pm 0.28 \\
(11.63-12.67)\end{array}$ \\
\hline Length $(\mathrm{cm})$ & $\begin{array}{l}58.1 \pm 1.9 \\
(54.2-60.0)\end{array}$ & $\begin{array}{c}64.8 \pm 2.3 \\
(60.5-69.5)\end{array}$ & $\begin{array}{l}71.7 \pm 1.9 \\
(66.0-74.0)\end{array}$ & $\begin{array}{l}73.6 \pm 3.2 \\
(69.0-78.5)\end{array}$ \\
\hline Weight $(\mathrm{kg})$ & $\begin{array}{l}5.630 \pm 0.660 \\
(4.420-7.400)\end{array}$ & $\begin{array}{l}7.431 \pm 1.134 \\
(5.808-9.510)\end{array}$ & $\begin{array}{l}8.836 \pm 0.975 \\
(6.675-10.095)\end{array}$ & $\begin{array}{c}9.650 \pm 0.618 \\
(7.165-11.085)\end{array}$ \\
\hline $\operatorname{BMI}\left(\mathrm{kg} / \mathrm{m}^{2}\right)$ & $\begin{array}{l}16.6 \pm 1.2 \\
(14.5-18.1)\end{array}$ & $\begin{array}{c}17.6 \pm 1.9 \\
(14.9-20.4)\end{array}$ & $\begin{array}{c}17.7 \pm 1.7 \\
(14.2-20.2)\end{array}$ & $\begin{array}{c}17.8 \pm 0.9 \\
(13.7-19.2)\end{array}$ \\
\hline Head circumference $(\mathrm{cm})$ & $\begin{array}{l}39.7 \pm 1.6 \\
(37.0-42.0)\end{array}$ & $\begin{array}{l}42.1 \pm 1.5 \\
(40.0-45.9)\end{array}$ & $\begin{array}{l}45.6 \pm 1.7 \\
(43.0-48.5)\end{array}$ & $\begin{array}{c}46.6 \pm 1.7 \\
(44.2-49.5)\end{array}$ \\
\hline \multicolumn{5}{|c|}{ Breastfeeding characteristics } \\
\hline 24-h milk intake (g) & $\mathrm{n} / \mathrm{a}^{\mathrm{e}}$ & $\begin{array}{c}818.8 \pm 204.9 \\
(498-1185)\end{array}$ & $\begin{array}{c}478.3 \pm 154.0 \\
(300-775)\end{array}$ & $\begin{array}{c}451.1 \pm 215.7 \\
(255-795)\end{array}$ \\
\hline 24-h feeding frequency (MP) & $\mathrm{n} / \mathrm{a}^{\mathrm{e}}$ & $\begin{array}{c}8.1 \pm 1.4 \\
(6-11)\end{array}$ & $\begin{array}{c}5.4 \pm 1.3 \\
(4-7)\end{array}$ & $\begin{array}{c}4.4 \pm 2.1 \\
\quad(2-8)\end{array}$ \\
\hline Feeding frequency (SR) & $\begin{array}{c}2.3 \pm 0.4^{\mathrm{f}} \\
(1.5-3.0)\end{array}$ & $\begin{array}{c}2.8 \pm 0.8 \\
(1.5-4.0)\end{array}$ & $\begin{array}{l}3.7 \pm 1.2 \\
(2.0-6.0)\end{array}$ & $\begin{array}{l}5.4 \pm 2.9 \\
(2.2-12.0)\end{array}$ \\
\hline
\end{tabular}

Data are mean \pm standard deviation (SD) and ranges. ${ }^{\mathrm{a}} n=15 ;{ }^{\mathrm{b}} n=20 ;{ }^{\mathrm{c}} n=19 ;{ }^{\mathrm{d}} n=18 .{ }^{\mathrm{e}}$ Milk intake and feeding frequency as meals per 24-h was determined from 24-h milk production (MP) measured between 2 and 5 months (presented at 5 months here, $n=17)$ and within 2 weeks of $9(n=6)$ and 12 months $(n=8)$; n/a-not applicable.

${ }^{\mathrm{f}}$ Maternal self-report (SR) of feeding frequency at the time of the visit as a typical time between meals (e.g., each $2 \mathrm{~h}$ )

( $n=11, n=19, n=17, n=13$ at 2,5,9, and 12 months respectively). BMI-body mass index.

Mean maternal age at the start of the study was $33.3 \pm 4.7$ (24-44) years, mean height was $167.4 \pm 7.4(150-181) \mathrm{cm}$ and mean parity was $2.3 \pm 0.9(1-4)$. Infant male/female ratio was $1: 1$, mean birth weight was $3.486 \pm 0.498(2.660-4.455) \mathrm{kg}$, and mean gestational age was 39.4 (37.6-43) weeks. Demographic, anthropometric, and breastfeeding characteristics measured at the four study sessions are presented in Table 1. The more detailed determinants of maternal and infant BC as well as description of longitudinal changes in infant and maternal BC and breastfeeding parameters, and the associations between them have been reported previously [14].

\subsection{Breastfeeding Parameters and Milk Components}

HM adipokines concentrations and CDI at 4 time points are detailed in Table 2. Concentration of SML, CDI of WMA, SML and WML, 24-h MI, and both SR and MP FFQ decreased across the lactation (see Table 3 for estimates and significances). 
Table 2. Human milk adipokines presented as concentration and 24-h intakes at the months after birth ${ }^{\mathrm{a}}$.

\begin{tabular}{|c|c|c|c|c|}
\hline Components & $\begin{array}{c}2 \text { Months } \\
\text { Mean } \pm \text { SD } \\
\text { (Range) }\end{array}$ & $\begin{array}{c}5 \text { Months } \\
\text { Mean } \pm \text { SD } \\
\text { (Range) }\end{array}$ & $\begin{array}{l}9 \text { Months } \\
\text { Mean } \pm \text { SD } \\
\text { (Range) }\end{array}$ & $\begin{array}{c}12 \text { Months } \\
\text { Mean } \pm \text { SD } \\
\text { (Range) }\end{array}$ \\
\hline \multicolumn{5}{|c|}{ Concentrations $^{b}$} \\
\hline Whole milk adiponectin $(\mathrm{ng} / \mathrm{mL})$ & $\begin{array}{c}11.14 \pm 5.79^{c} \\
(6.61-21.56)\end{array}$ & $\begin{array}{l}8.42 \pm 1.69 \\
(6.18-22.58)\end{array}$ & $\begin{array}{c}8.44 \pm 1.33 \\
(6.41-12.86)\end{array}$ & $\begin{array}{l}11.22 \pm 4.22 \\
(5.66-19.38)\end{array}$ \\
\hline Whole milk leptin (ng/mL) & $\begin{array}{c}0.50 \pm 0.18 \\
(0.24-0.77)\end{array}$ & $\begin{array}{l}0.49 \pm 0.17 \\
(0.23-0.71)\end{array}$ & $\begin{array}{l}0.56 \pm 0.11 \\
(0.42-0.67)\end{array}$ & $\begin{array}{l}0.50 \pm 0.11 \\
(0.34-0.74)\end{array}$ \\
\hline Skim milk leptin (ng/mL) & $\begin{array}{c}0.34 \pm 0.20 \\
(0.20-0.84)\end{array}$ & $\begin{array}{l}0.26 \pm 0.08 \\
(0.20-0.40)\end{array}$ & $\begin{array}{c}0.21 \pm 0.02 \\
(0.19-0.27)\end{array}$ & $\begin{array}{c}0.21 \pm 0.03 \\
(0.19-0.40)\end{array}$ \\
\hline \multicolumn{5}{|c|}{$\mathrm{CDI}^{\mathrm{d}}$} \\
\hline Whole milk adiponectin (ng) & $\mathrm{n} / \mathrm{a}^{\mathrm{e}}$ & $\begin{array}{c}7976 \pm 4480^{d} \\
(3771-22,439)\end{array}$ & $\begin{array}{c}4446 \pm 1645 \\
(2142-6673)\end{array}$ & $\begin{array}{l}3922 \pm 1431 \\
(2511-6352)\end{array}$ \\
\hline Whole milk leptin (ng) & $\mathrm{n} / \mathrm{a}$ & $\begin{array}{c}362 \pm 173 \\
(162-841)\end{array}$ & $\begin{array}{c}280 \pm 73 \\
(132-349)\end{array}$ & $\begin{array}{l}219 \pm 90 \\
(122-350)\end{array}$ \\
\hline Skim milk leptin (ng) & $\mathrm{n} / \mathrm{a}$ & $\begin{array}{c}200 \pm 81 \\
(106-402)\end{array}$ & $\begin{array}{l}114 \pm 38 \\
(62-172)\end{array}$ & $\begin{array}{l}93 \pm 36 \\
(51-159)\end{array}$ \\
\hline
\end{tabular}

a Milk components' concentrations and 24-h components' intakes are presented grouped by the month after birth. ${ }^{\mathrm{b}}$ Concentrations as measured at various months postpartum $(n=15, n=20, n=18$, and $n=15$ at 2, 5, 9, and 12 months respectively). ${ }^{\mathrm{c}}$ Data are mean $\pm \mathrm{SD}$ and ranges. ${ }^{\mathrm{d}} \mathrm{CDI}$ of adipokines were calculated between 2 and 5 months (presented at 5 months here, $n=17)$ and within 2 weeks of $9(n=8)$ and 12 months $(n=8)$.

${ }^{\mathrm{e}} \mathrm{n} / \mathrm{a}-$ not applicable.

Table 3. Differences by infant age/lactation duration within measured human milk adipokines and breastfeeding parameters ${ }^{a}$.

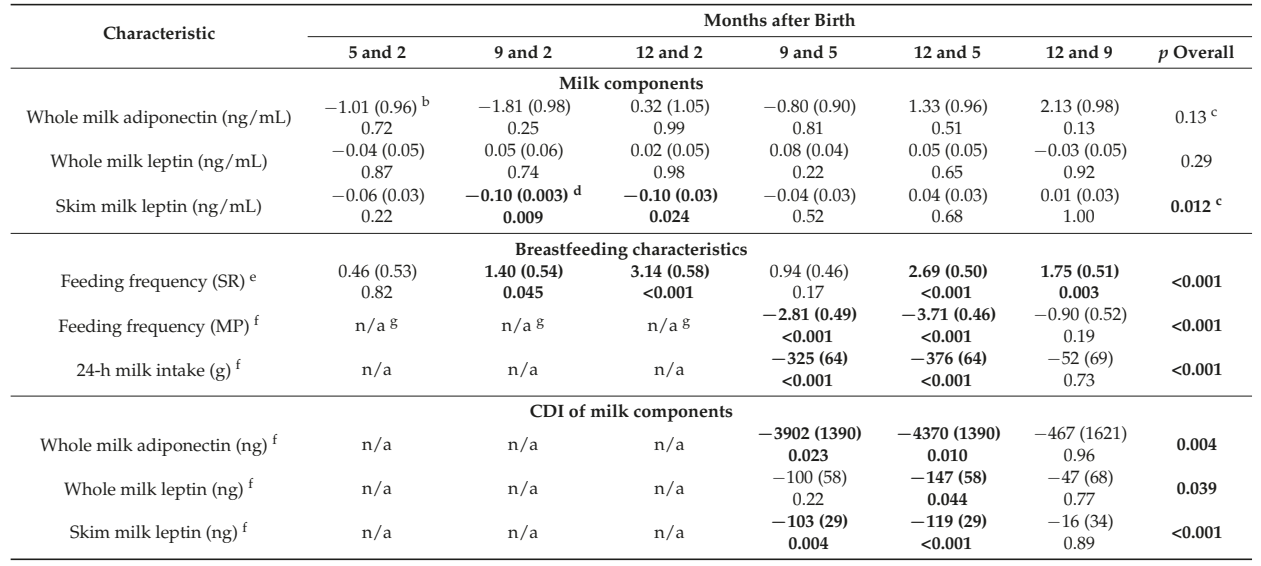

a Systematic differences in the measured variables between different months after birth were calculated using general linear hypothesis test (Tukey's all pair comparisons). ${ }^{\mathrm{b}}$ Data are parameter estimate \pm standard error of estimate and $p$-value. ${ }^{c}$ Overall $p$-value is associated with age as reported in linear mixed model. ${ }^{\mathrm{d}}$ Bold text indicates significant difference $(p<0.05)$ between two time points or overall. ${ }^{\mathrm{e}}$ Feeding frequency was self-reported (SR) by mothers at the time of the visit as an average time between meals (e.g., each $2 \mathrm{~h}$ ) $(n=11, n=19, n=17$, and $n=13$ at 2, 5, 9, and 12 months respectively). ${ }^{\mathrm{f}}$ 24-h milk intake and feeding frequency as meals per 24-h was measured at 24-h milk production (MP) and CDI calculated between 2 and 5 months (presented at 5 months here, $n=17)$ and within 2 weeks of $9(n=8)$ and 12 months $(n=8)$. ${ }^{g}$ Results are not presented for impractical combinations; $\mathrm{n} / \mathrm{a}-$ not applicable.

\subsection{Maternal Body Composition and Adipokines}

Significant interactions between maternal characteristics and the month after birth were seen only for SML concentration (Table A1). The changes in slope for maternal characteristics from 
positive (2 months) to negative $(5,9$, and 12$)$ and the decrease in slope indicate that associations between maternal characteristics and SML concentration weaken over the first 12 months of lactation (Figure 1). No significant associations between concentrations of WML and maternal characteristics were seen after adjusting for the false discovery rate. No associations were seen between maternal characteristics and concentrations of WMA as well as CDI of the adipokines (see Table A1 for estimates and significances).
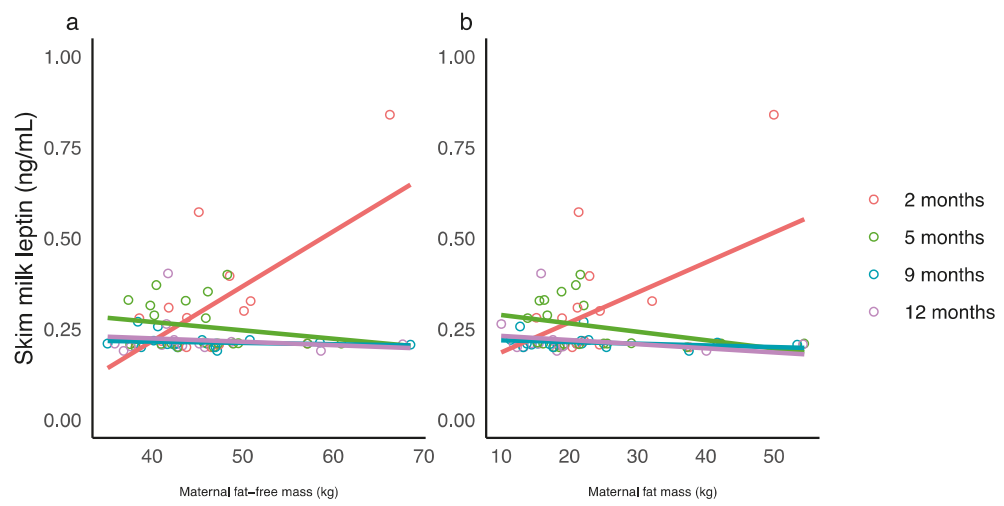

Figure 1. Significant interaction between maternal body composition and the month after birth for skim milk leptin concentration. (a) Maternal fat-free mass and (b) maternal fat mass measured with bioelectrical impedance spectroscopy. Lines represent linear regression and grouped by the month of lactation.

\subsection{Infant Body Composition and Concentrations of Adipokines}

No significant associations between concentrations of adipokines and infant characteristics were seen after adjusting for the false discovery rate (see Table A2 for estimates and significances).

\subsection{Infant Body Composition and Calculated Daily Intakes of Adipokines}

Higher CDI of WMA were associated with lower infant FFM and FFMI and with an increased infant FM, FMI, and \%FM (measured with US 4SF) (Table A3, Figure 2).

Higher CDI of SML were associated with an increase in infant BMI at 5 months, a small decrease in at 9 months, and a larger decrease at 12 months. Higher CDI of SML were associated with small decreases in FFMI (US 4SF) at 5 months and 9 months and a larger decrease at 12 months. Higher CDI of SML were associated with an increase in FM (US 2SF) at 5 months, decrease at 9 months, and an increase at 12 months. Higher CDI of SML were associated with an increase in infant FM (US 4SF), FMI (US 2SF, 4SF), and \%FM (US 2SF, 4SF) (Figure 3).

No associations were seen between infant characteristics and CDI of WML (see Table A3 for estimates and significances). 

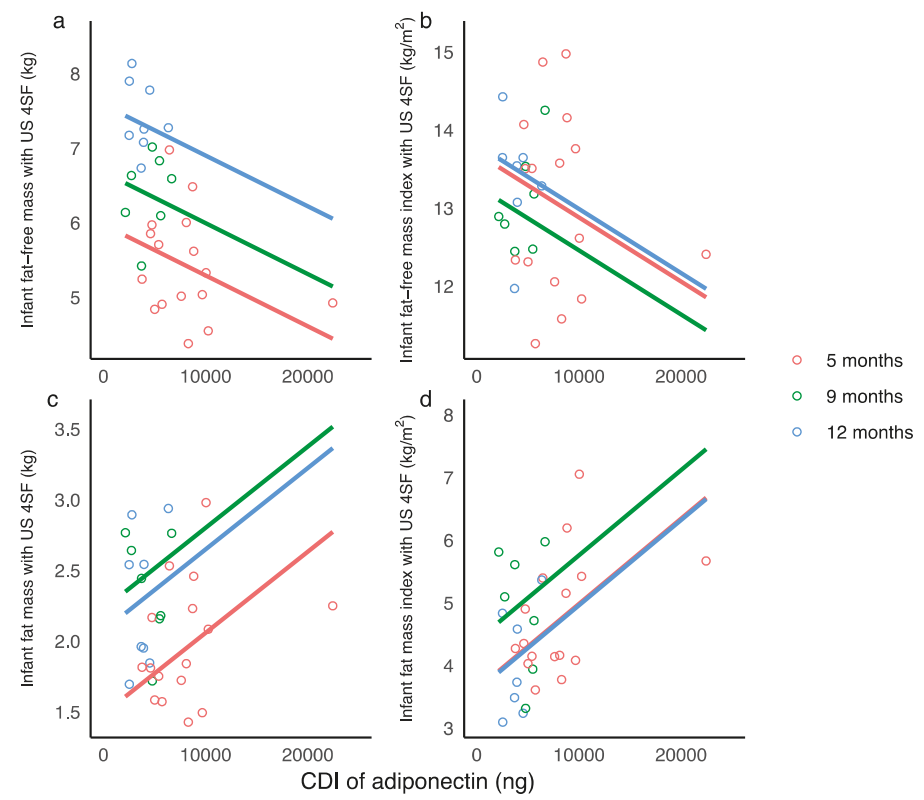

Figure 2. Significant associations between calculated daily intakes (CDI) of whole milk adiponectin. (a) Infant fat-free-mass measured with ultrasound 4-skinfolds (US 4SF); (b) infant fat-free mass index with US 4SF; (c) infant fat mass with US 4SF; (d) infant fat mass index with US 4SF. Lines represent linear regression and grouped by the month of lactation.
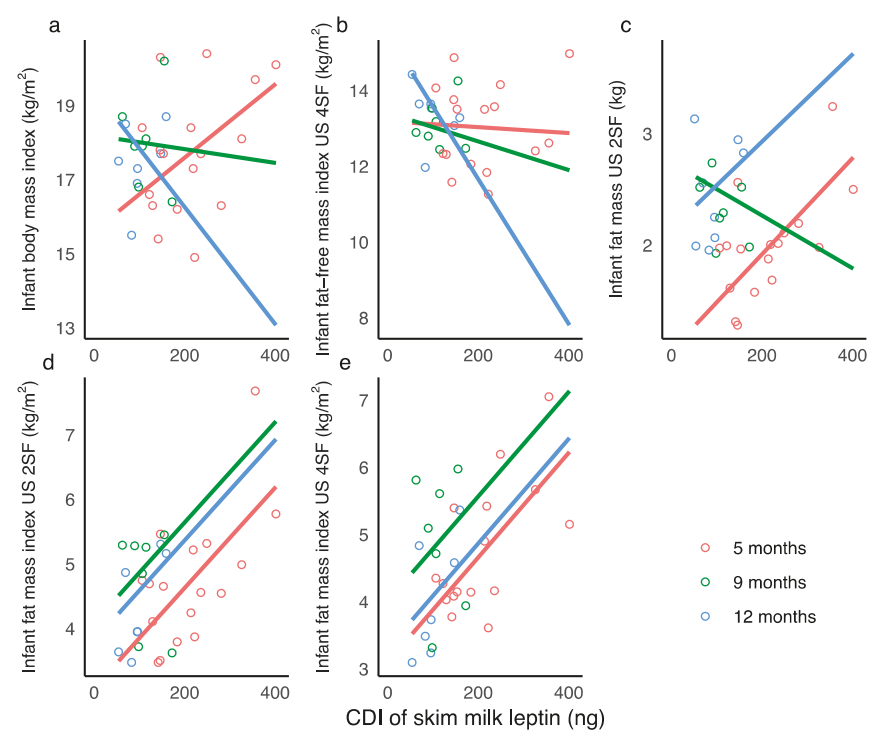

Figure 3. Significant associations between calculated daily intakes (CDI) of skim milk leptin. (a) Infant body mass index; (b) infant fat-free mass index measured with ultrasound 4-skinfolds (US 4SF); (c) infant fat mass with US 2-skinfolds (US 2SF); (d) infant fat mass index with US 2SF; (e) infant fat mass index with US 4SF. Lines represent linear regression and grouped by the month of lactation. 


\subsection{Breastfeeding Parameters and Adipokines}

A higher concentration of WMA was associated with no change in infant 24-h MP FFQ between 2 and 5 months and a decrease at 9 and 12 months. A higher concentration of WMA was associated with an increase in infant 24-h MI at 5 and 9 months and a decrease at 12 months (Figure S1; Table A4). No significant associations were seen between concentration of WMA and SR FFQ or between concentrations of leptin and breastfeeding parameters. Higher 24-h MP FFQ was associated with an increase in CDI of WML (Figure S2; see Table A4 for estimates and significances). Breastfeeding parameters were not associated with CDI of WMA or SML.

\subsection{Changes in Infant Characteristics and Calculated Daily Intakes of Adipokines}

After accounting for the false discovery rate, significant associations were seen between changes in infant $\mathrm{BC}(\Delta)$ between the time points and CDI of leptin at the later stages of lactation. Higher CDI of WML at 12 months were associated with larger increase in infant FM (US 2 SF) and \%FM (US 2SF) between 2 and 12 months (Table A5), while higher CDI of SML at 12 months were associated with larger decrease in FFMI (US 4SF) between 5 and 12 months (Table A6). No significant associations were seen between infant $\mathrm{BC}$ and $\mathrm{CDI}$ of WMA after accounting for the false discovery rate (Table A7).

\section{Discussion}

This study sheds new light on the complex mechanisms by which breastfeeding may influence infant BC and confer some degree of protection from obesity. For the first time, daily intakes of HM adiponectin and leptin have been associated with development of infant BC and are differentially related to infant FM and FFM (Figure 4) at different stages of lactation. Furthermore, infant FFQ was associated with both the concentration of WMA and CDI of WML emphasizing the critical role of breastfeeding in programming of infant appetite control and growth in the first year of life.

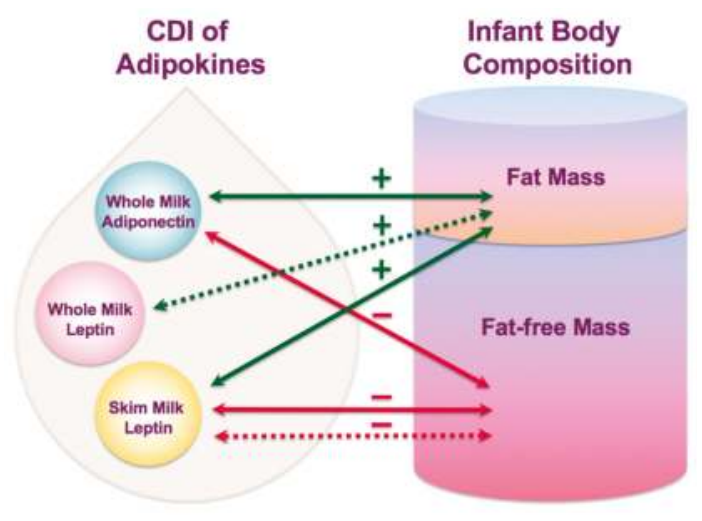

Figure 4. Interconnecting pathways of lactocrine programming of the infant body composition as researched. Solid arrows indicate associations of calculated daily intakes of adipokines with measured body composition parameters and dotted arrows indicate associations with changes in body composition between time points (green-positive associations; red-negative associations). CDI-calculated daily intakes.

Small bioactive HM peptides believed to be involved in the development of appetite regulation and infant BC include adiponectin and leptin. Whilst several studies have investigated longitudinal changes in both HM leptin and adiponectin, only two studies have measured these hormones at several time points up to 12 months of lactation, one reporting lower concentration of skim milk adiponectin (SMA) [17], and another higher concentrations of SML and WMA [37] at 12 months. We found that 
although CDI of these adipokines decreased over the 12 months of lactation, the concentrations of both HM leptin and adiponectin measured in whole milk did not change, despite reduction of SML concentration (Section 3.2; Table 3). In this study, higher WML concentrations were associated with higher maternal weight, fat, and lean body mass, but were rendered nonsignificant with adjustment for multiple comparisons (Section 3.3; Table A1). Nevertheless, they are consistent with our earlier whole HM leptin results from a larger cross-sectional cohort [22]. This represents a potential pathway by which HM composition may be improved by maintaining maternal adiposity within the normal range during pregnancy and lactation.

For the first time, we found a higher CDI of adiponectin to be associated with both lower infant lean body mass and higher adiposity (Section 3.5; Table A3). One study has investigated the effect of CDI of adiponectin on infant growth velocity reporting higher CDI of SMA by 3 months in infants with higher weight gain compared with low and normal weight gain groups [10], although no differences in concentrations were detected. We found no association with weight gain in our study, however our study of whole milk is representative of the amount of adiponectin consumed by the infant.

We found a weak relationship between WMA concentration and infant FFM (correction for multiple comparisons eliminated statistical significance) over the first 12 months of lactation (Section 3.4; Table A2). Our results are in contrast to a past study [37], that reported a positive association of WMA with infant weight gain at 6, but not at 12 months. Statistical methods may account for differences where Spearman correlations were employed, with not accounting for stage of lactation or adjusting for multiple comparisons in the previous study. Studies of SMA give conflicting results when compared to WMA studies, with higher skim HM adiponectin concentrations in the first 6 months postpartum being associated with lower weight and lean tissue accretion [36] and lower weight-for-age and weight-for-length z-scores at 6 months of age [17]. Others have shown positive $[35,37,39]$ or no association $[38,60,61]$ with infant growth characteristics. These skim milk studies however showed either positive associations of SMA with weight and adipose tissue accretion at 1 year of life [36] or no associations with weight-for-age and weight-for-length z-scores at 1 year and a positive association during the 2nd year of life [18]. We did not find a positive trend of WMA after 6 months of age calling into question the validity of the skim milk studies.

HM leptin is the most studied adipokine and is implicated in short and long term satiety and regulation of energy intake and body weight [19], still the role of HM leptin in the development of infant $B C$ is yet to be fully understood [27]. For the first time CDI of WML has been linked to infant $\mathrm{BC}$, with higher CDI of both WML and SML associating with greater deposition of adipose tissue (FM, FMI, and \%FM), although the results were not comparable (WML, accretion between time points: Section 3.7, Table A5; SML, overall association: Section 3.5, Table A3). These results extend the findings of Kon at al. [10], who reported higher total daily consumption of SML in a group of 3-month-old infants with higher weight gain compared with groups with low and normal weight gain. Other studies report inverse correlations of HM leptin with infant weight or BMI [19,36,42,60,62-64], no association $[41,65,66]$, or positive associations $[10,35,67]$.

We found that higher CDI of SML were associated with a lower accrual of infant FFM over 12 months postpartum (Section 3.5; Table A3) and greater reduction in FFMI between 5 and 12 months of age (Section 3.7, Table A6). Leptin from rat adipocytes and osteoblasts is known to both supress and stimulate bone growth [68], and therefore may affect infant lean mass. However, SML concentration/CDI results should be interpreted with caution as associations of SML with infant FFM were different to WML, and SML concentration differed by the month of lactation yet WML concentration did not (Table 3). In addition, skim milk, which excludes the fat and cellular components of $\mathrm{HM}$, has a lower concentration of leptin $[21,22,69,70]$ and is therefore not representative of the milk consumed by the infant. Further study of larger number of infants should be carried out to confirm these new-found relationships.

Breastfeeding frequency and volumes are highly variable between infants and is a reflection of the storage capacity of the breast [49] and likely, of infant appetite regulation [71]. In this study we 
found a positive relationship between FFQ and CDI of WML (Section 3.7; Table A4), which were also associated with higher FM accretion. Furthermore, the concentration of WMA in our study showed a differential effect on FFQ and 24-h MI (Section 3.7; Table A4), with higher WMA concentrations during earlier months associating with an increase in MI. During later months higher concentrations were associated with reduced FFQ and MI, and may be due to the intake of solids at weaning, although this was not investigated. Recently we have shown increased FFQ was associated with increased 24-h MI, and both of these breastfeeding parameters were related to higher infant adiposity and lower lean body mass [14], suggesting that HM components may differentially influence lean and fat mass compartments, supported by a recent study [72]. Thus, adiponectin and leptin may play active roles in BC development via appetite regulation. These relationships add to the possible pathways of the mechanisms of infant $\mathrm{BC}$ regulation.

The strength of this proof-of-concept study is the wide variation of maternal adiposity, that measurements were performed on breastfeeding dyads feeding on demand over 12 months of lactation, and that adipokines were measured in whole HM. The limitations are the small number of 24-h MP at the later stages of lactation, the modest number of participants associated with multiple measurement time points, and the absence of infant dietary data between 6 and 12 months of age. Our population was predominantly Caucasian term healthy fully-breastfed singletons from mothers of higher social-economic status therefore, the results may not be applicable to dyads from other backgrounds.

\section{Conclusions}

These results confirm that the first year of life is a critical window of infant developmental programming and show a differential effect of concentrations and doses of HM leptin and adiponectin on development of infant lean and fat mass during this time. CDI may be a more relevant factor than concentrations when examining the nutritional physiology of the breastfed infant. Given the appetite and $\mathrm{BC}$ regulating effects of these adipokines, there is a potential to improve the outcome for the infant through interventions, such as the continuation of breastfeeding during the first year of life and beyond, which may facilitate favorable developmental programming and reduce risk of obesity later in life.

Supplementary Materials: The following are available online at http://www.mdpi.com/2072-6643/10/8/1125/ s1, Figure S1: Significant associations between concentration of whole milk adiponectin and (a) infant feeding frequency (meals /24-h); (b) infant 24-h milk intake (g), Figure S2: Significant associations between calculated daily intakes (CDI) of whole milk leptin and infant feeding frequency (meals/24-h) measured during 24-h milk productions.

Author Contributions: All authors critically reviewed and approved the final manuscript. Conceptualization, Z.G., A.R., L.W., P.H. and D.G.; Data curation, Z.G., A.R., K.M. and D.G.; Formal analysis, Z.G., A.R., L.W. and K.M.; Funding acquisition, D.G.; Investigation, Z.G., A.R., C.T.L. and D.G.; Methodology, Z.G., C.T.L. and D.G.; Resources, D.G.; Supervision, K.M., P.H. and D.G.; Visualization, Z.G. and A.R.; Writing-original draft, Z.G., A.R., K.M. and D.G.; Writing-review \& editing, S.K., C.T.L., L.W. and P.H.

Funding: This research was funded by an unrestricted research grant from Medela AG (Switzerland). Zoya Gridneva was supported by an Australian Postgraduate Award from The University of Western Australia (Australia). Sambavi Kugananthan was supported by a Margaret Lomann-Hall Scholarship from the School of Human Sciences, The University of Western Australia (Australia).

Acknowledgments: Authors wish to thank Anna R. Hepworth for consultation on research design and assistance with data analyses. Many thanks are extended to all mothers and infants who participated in this study.

Conflicts of Interest: The authors declare that Medela AG provided an unrestricted research grant to Donna T. Geddes, from which salaries to Donna T. Geddes, Zoya Gridneva, and Ching Tat Lai were paid. Medela AG provided a Top-up Scholarship for Zoya Gridneva and has provided speaker's fees to Donna T. Geddes for educational lectures. The funding sponsors had no role in the design of the study; in the collection, analyses, or interpretation of data; in the writing of the manuscript, or in the decision to publish the results. Author Leigh C. Ward provides consultancy services to ImpediMed Ltd. ImpediMed Ltd. was not involved in the inception and conducting of this research or in the writing of the manuscript. The other authors declare no conflicts of interest. 


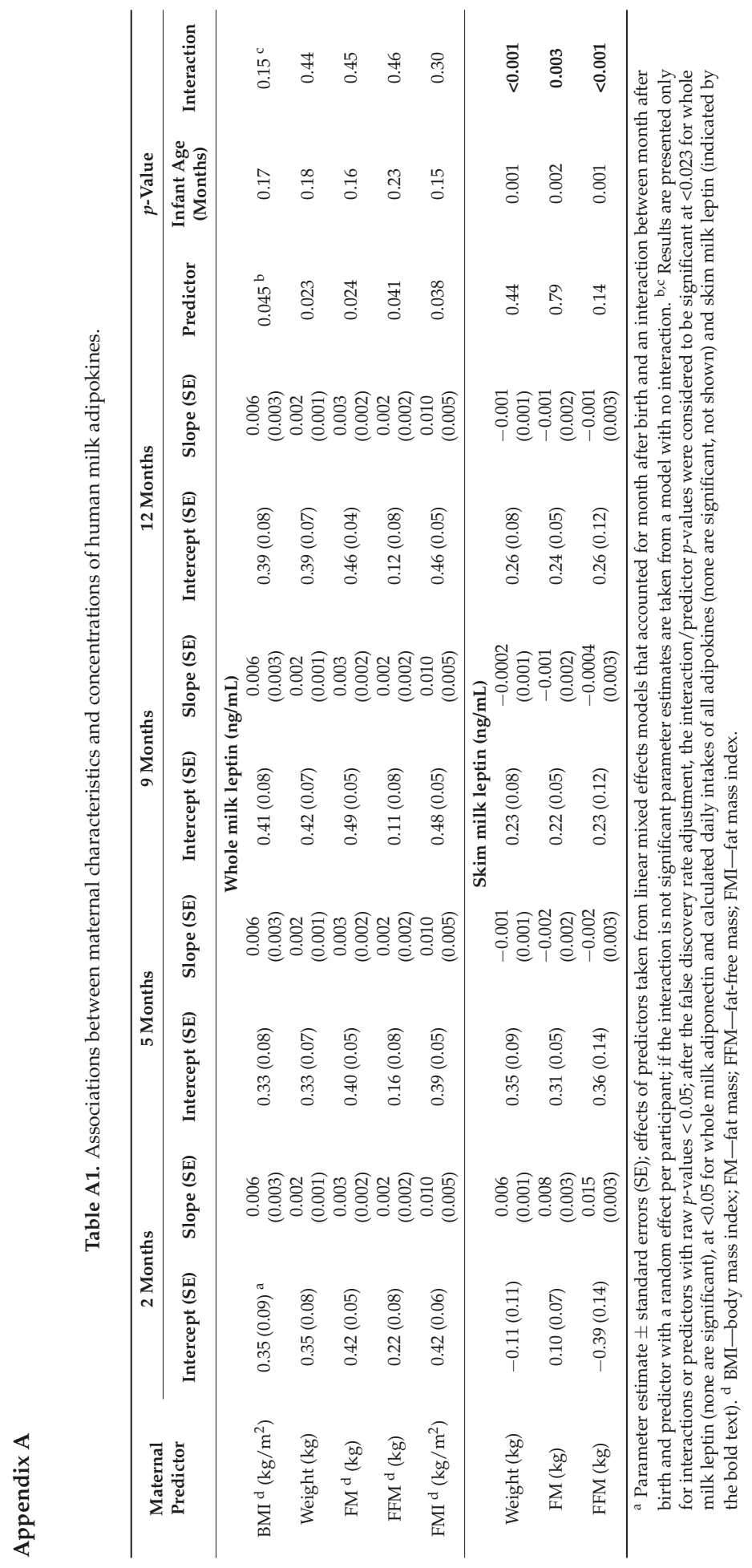




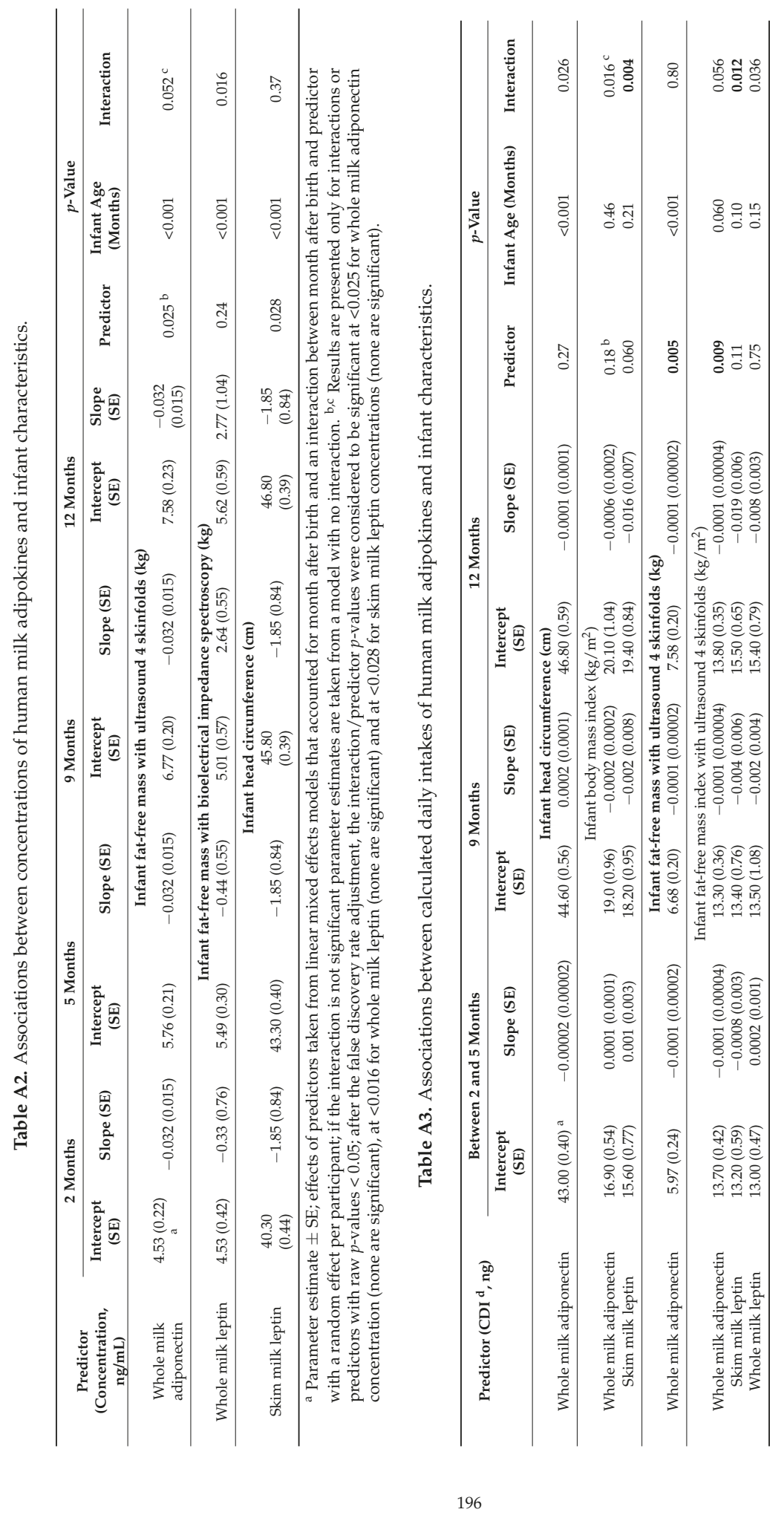




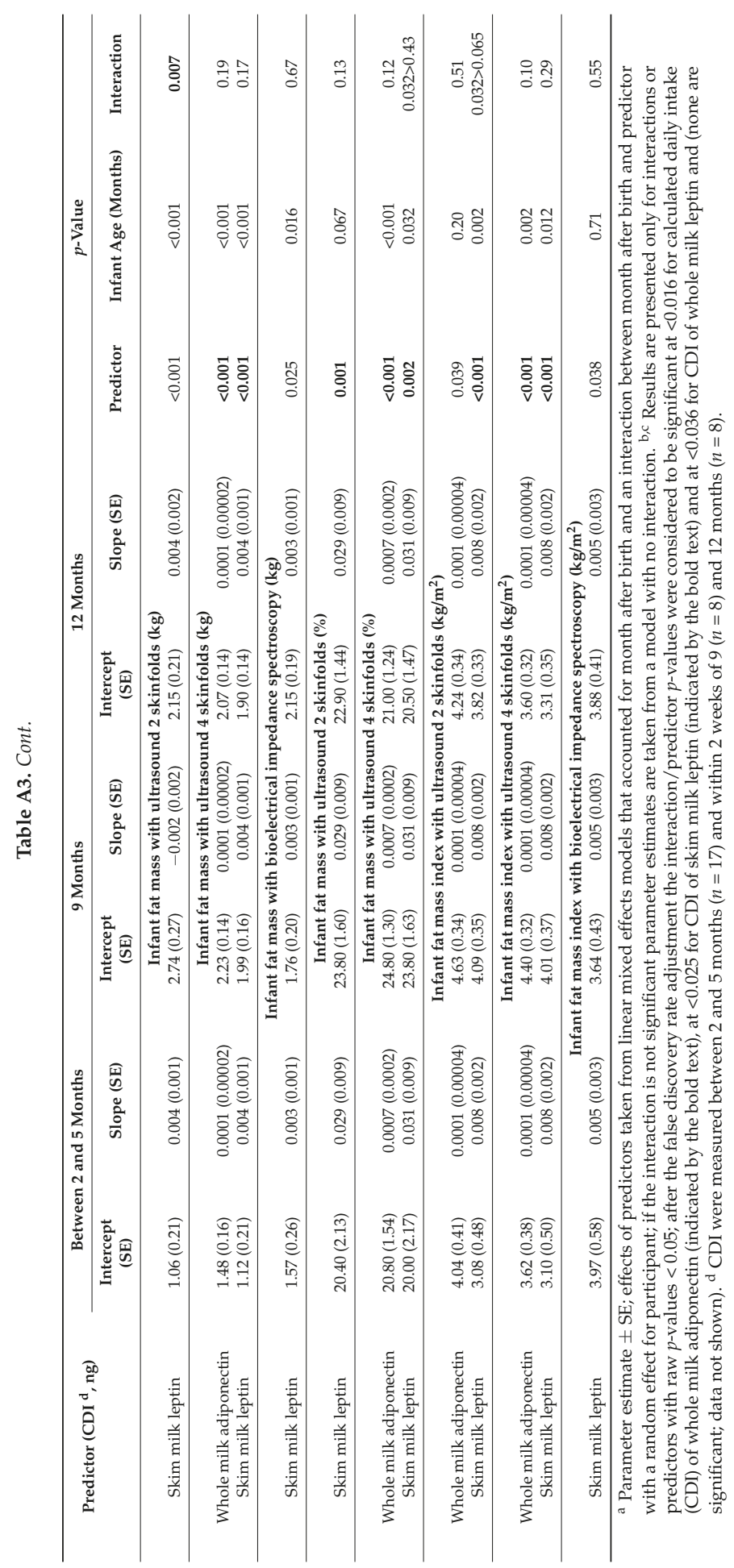


Nutrients 2018, 10, 1125

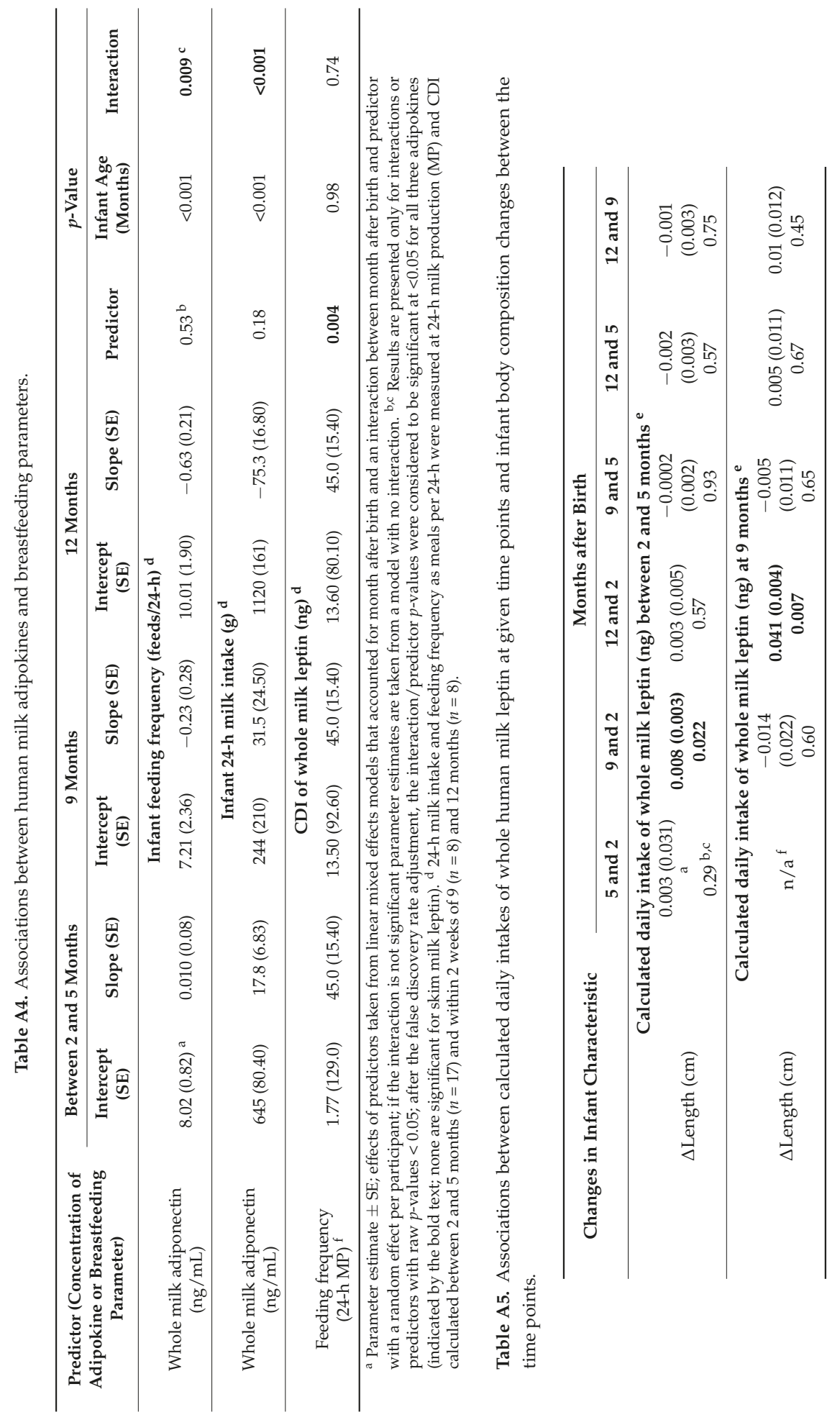




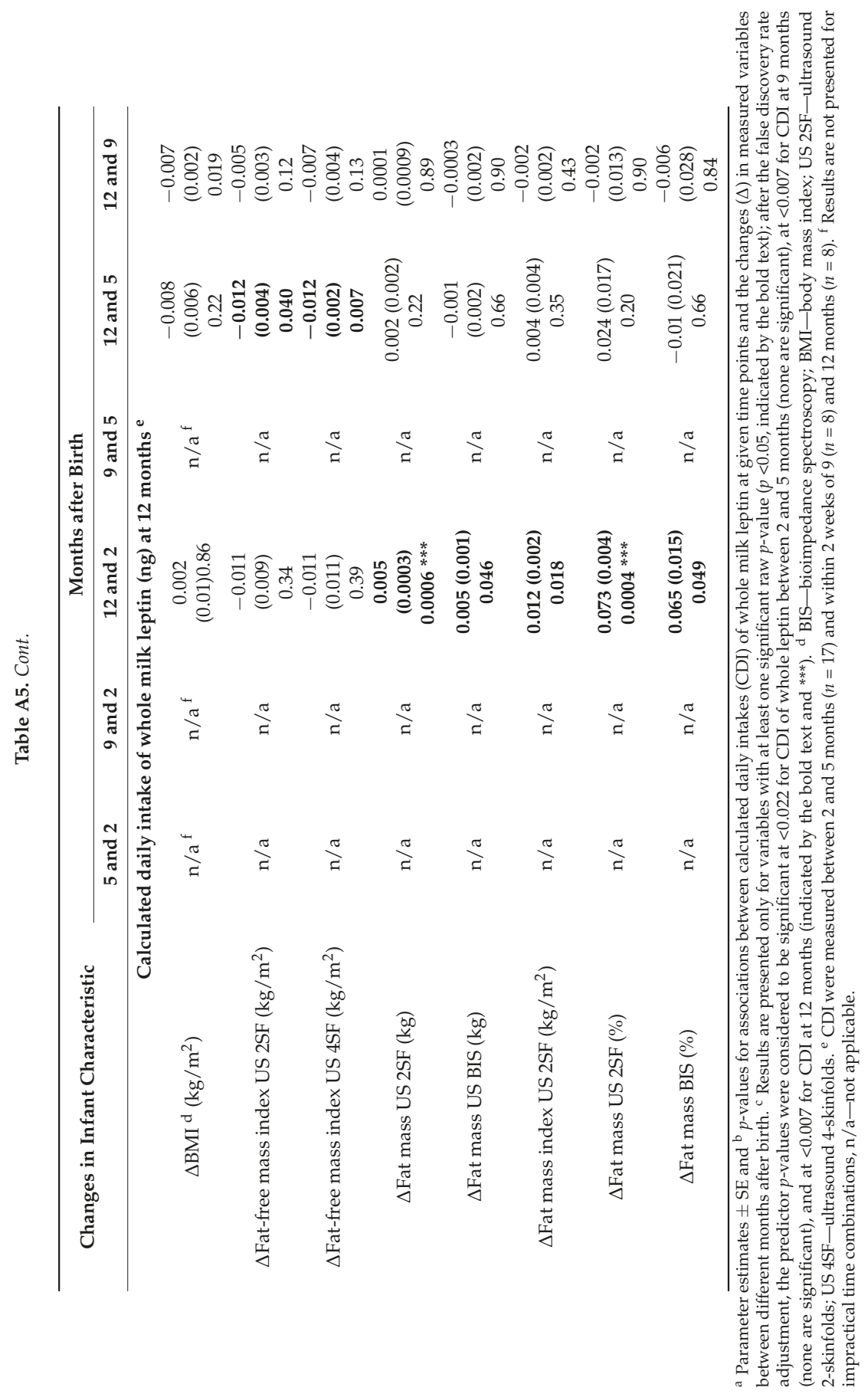




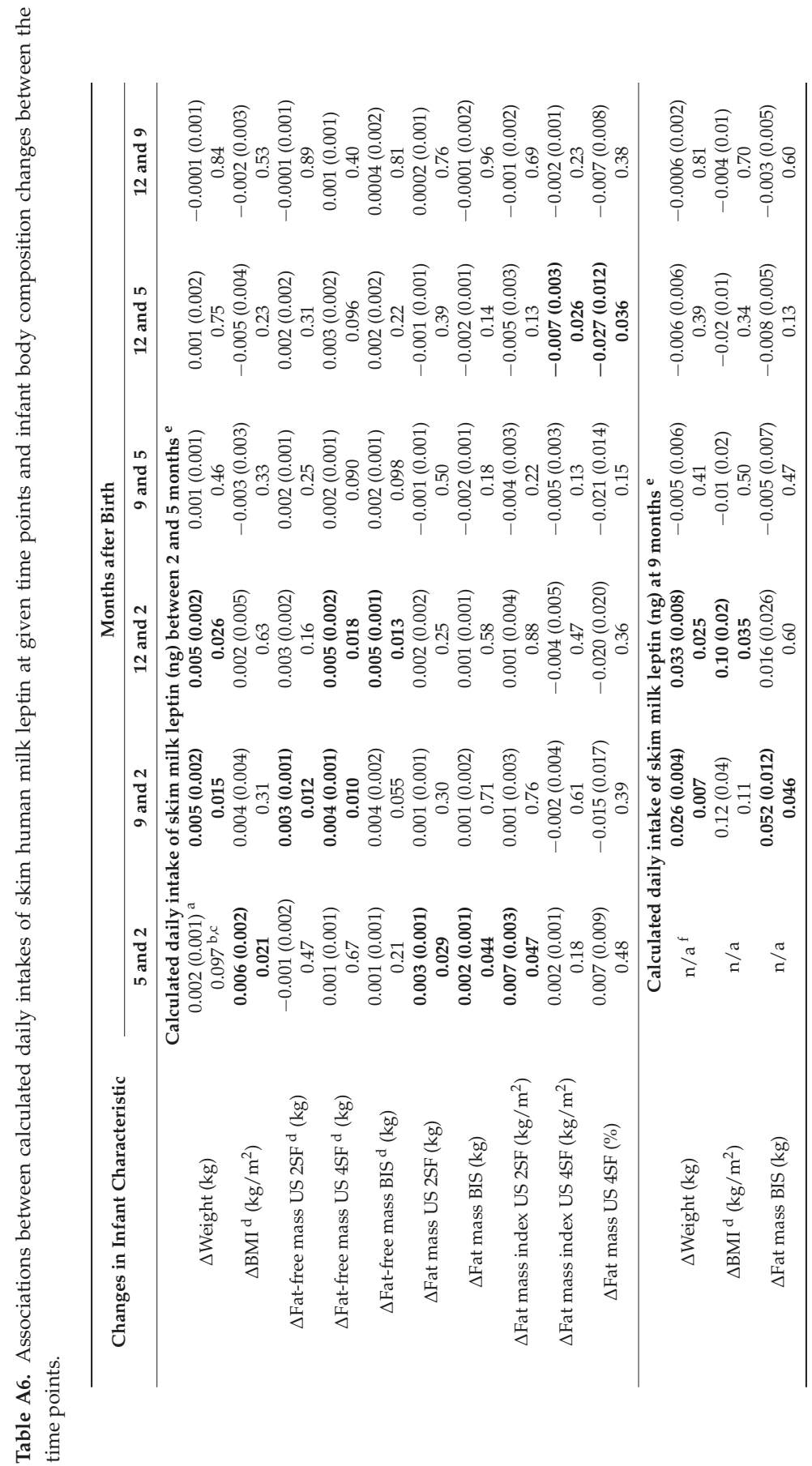




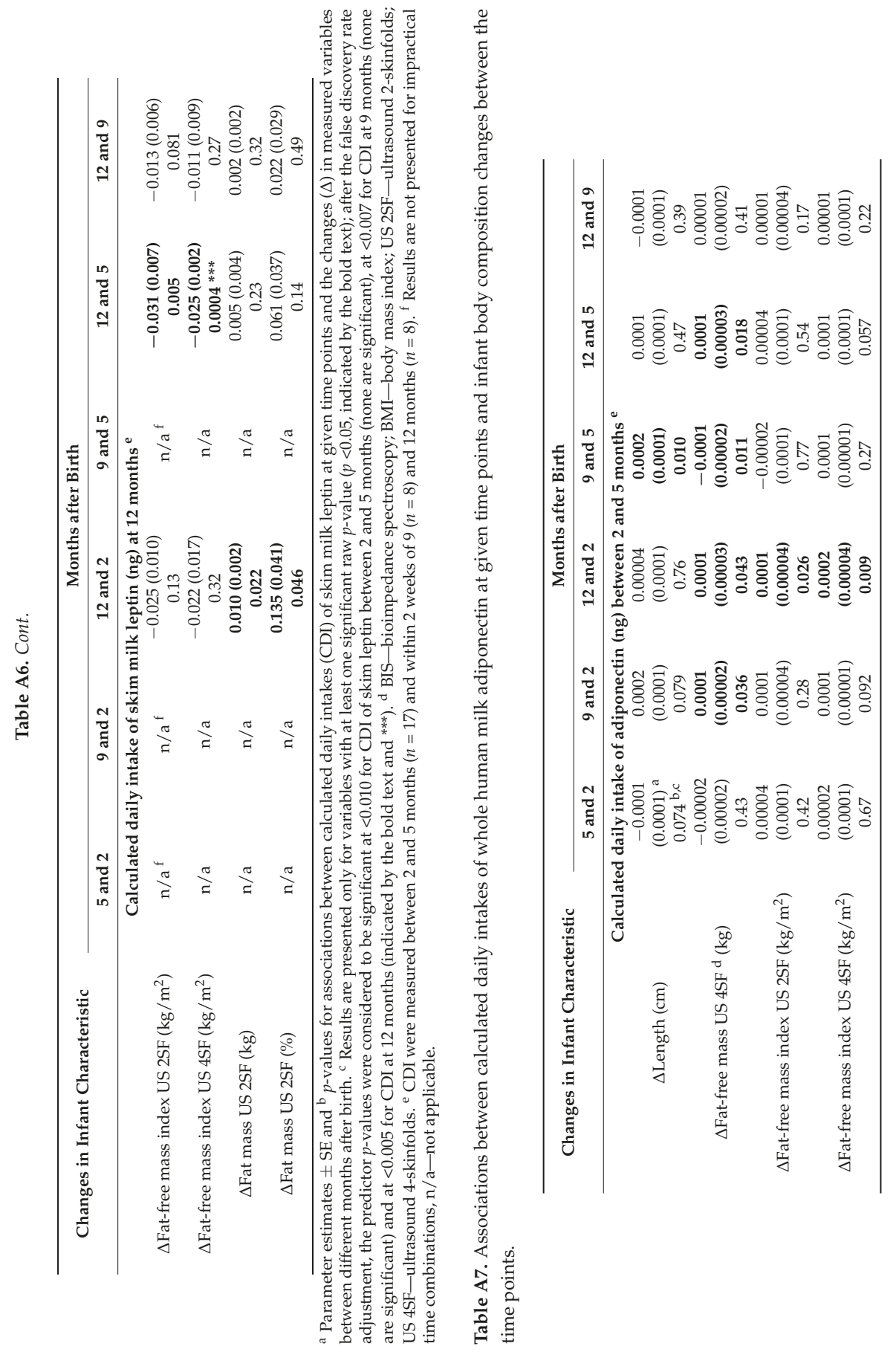




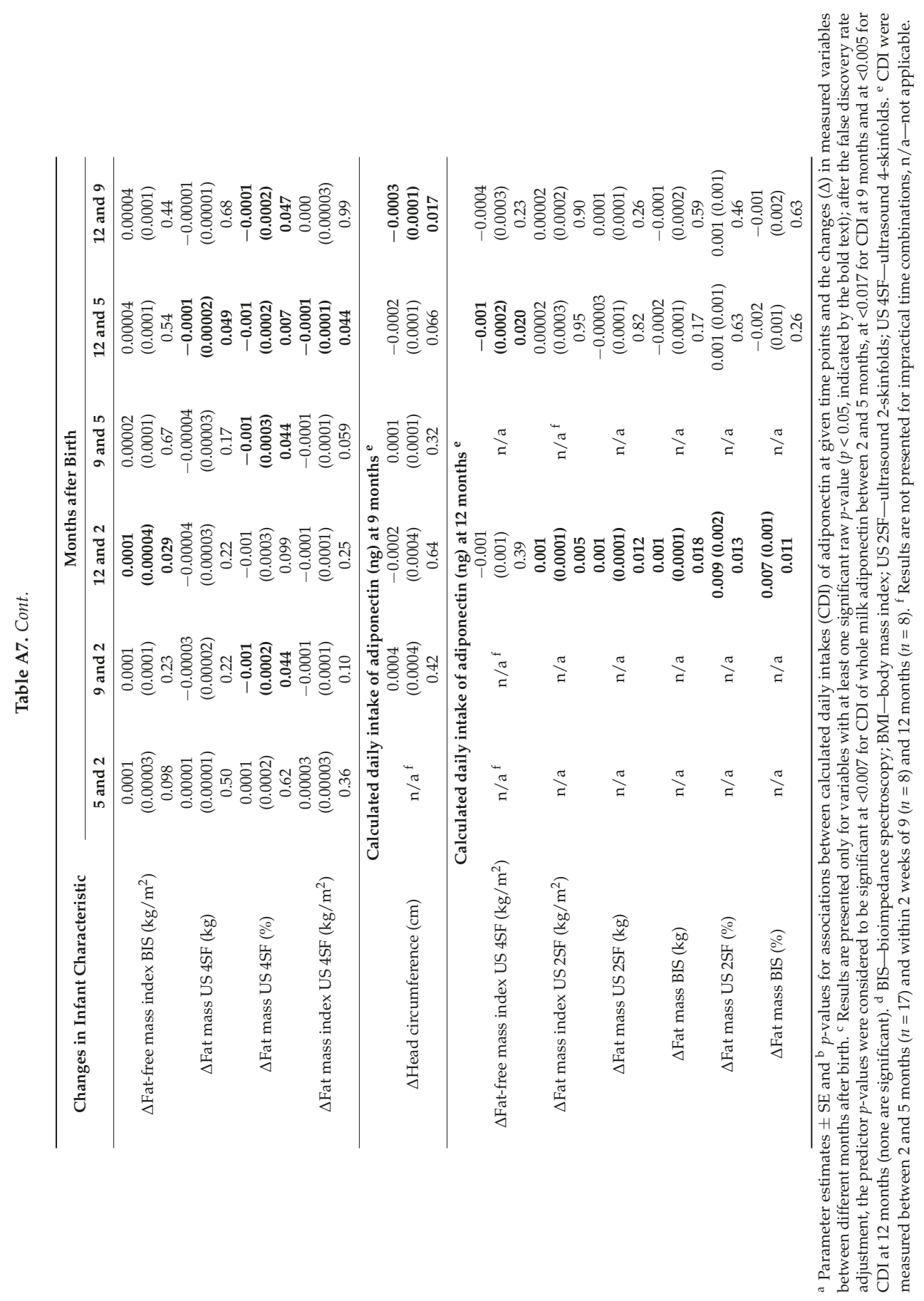




\section{References}

1. Koletzko, B.; Brands, B.; Chourdakis, M.; Cramer, S.; Grote, V.; Hellmuth, C.; Kirchberg, F.; Prell, C.; Rzehak, P.; Uhl, O.; et al. The power of programming and the early nutrition project: Opportunities for health promotion by nutrition during the first thousand days of life and beyond. Ann. Nutr. Metab. 2014, 64, 187-196. [CrossRef] [PubMed]

2. Geddes, D.; Prescott, S. Developmental origins of health and disease: The role of human milk in preventing disease in the 21(st) century. J. Hum. Lact. 2013, 29, 123-127. [CrossRef] [PubMed]

3. Wells, J.C.; Chomoto, S.; Fewtrell, M.S. Programming of body composition by early growth and nutrition. Proc. Nutr. Soc. 2007, 66, 423-434. [CrossRef] [PubMed]

4. Hypponen, E.; Kenward, M.; Virtanen, S.; Piitulainen, A.; Virta-Autio, P.; Tuomilehto, J.; Knip, M.; Akerblom, H. Infant feeding, early weight gain, and risk of type 1 diabetes. Childhood diabetes in Finland (DiMc) study group. Diab. Care 1999, 22, 1961-1965. [CrossRef]

5. Andersson, S.; Bengtsson, C.; Hallberg, L.; Lapidus, L.; Niklasson, A.; Wallgren, A.; Hulthen, L. Cancer risk in Swedish women: The relation to size at birth. Br. J. Cancer 2001, 84, 1193-1198. [CrossRef] [PubMed]

6. Stettler, N.; Kumanyika, S.; Katz, S.; Zemel, B.; Stallings, V. Rapid weight gain during infancy and obesity in young adulthood in a cohort of African Americans. Am. J. Clin. Nutr. 2003, 77, 1374-1378. [CrossRef] [PubMed]

7. Stettler, N.; Stallings, V.; Troxel, A.; Zhao, J.; Schinnar, R.; Nelson, S.; Ziegler, E.; Strom, B. Weight gain in the first week of life and overweight in adulthood: A cohort study of European American subjects fed infant formula. Circulation 2005, 111, 1897-1903. [CrossRef] [PubMed]

8. Butte, N.; Wong, W.; Hopkinson, J.; Smith, E.; Ellis, K. Infant feeding mode affects early growth and body composition. Pediatrics 2000, 16, 1355-1366. [CrossRef]

9. Luque, V.; Closa-Monasterolo, R.; Escribano, J.; Ferre, N. Early programming by protein intake: The effect of protein on adiposity development and the growth and functionality of vital organs. Nutr. Metab. Insights 2015, 8, 49-56. [CrossRef] [PubMed]

10. Kon, I.Y.; Shilina, N.M.; Gmoshinskaya, M.V.; Ivanushkina, T.A. The study of breast milk IGF-1, leptin, ghrelin and adiponectin levels as possible reasons of high weight gain in breast-fed infants. Ann. Nutr. Metab. 2014, 65, 317-323. [CrossRef] [PubMed]

11. Sievers, E.; Oldigs, H.D.; Santer, R.; Schaub, J. Feeding patterns in breast-fed and formula-fed infants. Ann. Nutr. Metab. 2002, 46, 243-248. [CrossRef] [PubMed]

12. Savino, F.; Liguori, S.A.; Fissore, M.F.; Oggero, R. Breast milk hormones and their protective effect on obesity. Int. J. Pediatr. Endocrinol. 2009, 2009, 327505. [CrossRef] [PubMed]

13. Bartok, C. Babies fed breastmilk by breast versus by bottle: A pilot study evaluating early growth patterns. Breastfeed. Med. 2011, 6, 117-124. [CrossRef] [PubMed]

14. Gridneva, Z.; Rea, A.; Hepworth, A.R.; Ward, L.C.; Lai, C.T.; Hartmann, P.E.; Geddes, D.T. Relationships between breastfeeding patterns and maternal and infant body composition over the first 12 months of lactation. Nutrients 2018, 10, 45. [CrossRef] [PubMed]

15. Khan, S.; Hepworth, A.R.; Prime, D.K.; Lai, C.T.; Trengove, N.J.; Hartmann, P.E. Variation in fat, lactose, and protein composition in breast milk over 24 hours: Associations with infant feeding patterns. J. Hum. Lact. 2013, 29, 81-89. [CrossRef] [PubMed]

16. Hennet, T.; Borsig, L. Breastfed at Tiffany's. Trends Biochem. Sci. 2016, 41, 508-518. [CrossRef] [PubMed]

17. Woo, J.; Guerrero, M.; Altaye, M.; Ruiz-Palacios, G.; Martin, L.; Dubert-Ferrandon, A.; Newburg, D.; Morrow, A. Human milk adiponectin is associated with growth in two independent cohorts. Breastfeed. Med. 2009, 4, 101-109. [CrossRef] [PubMed]

18. Woo, J.; Guerrero, M.; Guo, F.; Martin, L.; Davidson, B.; Ortega, H.; Ruiz-Palacios, G.; Morrow, A. Human milk adiponectin affects infant weight trajectory during the second year of life. J. Pediatr. Gastroenterol. Nutr. 2012, 54, 532-539. [CrossRef] [PubMed]

19. Miralles, O.; Sanchez, J.; Palou, A.; Pico, C. A physiological role of breast milk leptin in body weight control in developing infants. Obesity 2006, 14, 1371-1377. [CrossRef] [PubMed]

20. Fields, D.; Demerath, E. Relationship of insulin, glucose, leptin, IL-6 and TNF-a in human breast milk with infant growth and body composition. Pediatr. Obes. 2012, 7, 304-312. [CrossRef] [PubMed] 
21. Kugananthan, S.; Lai, C.T.; Gridneva, Z.; Mark, P.J.; Geddes, D.T.; Kakulas, F. Leptin levels are higher in whole compared to skim human milk, supporting a cellular contribution. Nutrients 2016, 8, 711. [CrossRef] [PubMed]

22. Kugananthan, S.; Gridneva, Z.; Lai, C.T.; Hepworth, A.R.; Mark, P.J.; Kakulas, F.; Geddes, D.T. Associations between maternal body composition and appetite hormones and macronutrients in human milk. Nutrients 2017, 9, 252. [CrossRef] [PubMed]

23. Uysal, F.; Onal, E.; Aral, Y.; Adam, B.; Dilmen, U.; Ardicolu, Y. Breast milk leptin: Its relationship to maternal and infant adiposity. Clin. Nutr. 2002, 21, 157-160. [CrossRef] [PubMed]

24. Prentice, A.M.; Jebb, S.A. Beyond body mass index. Obes. Rev. 2001, 2, 141-147. [CrossRef] [PubMed]

25. Gridneva, Z.; Hepworth, A.R.; Ward, L.C.; Lai, C.T.; Hartmann, P.E.; Geddes, D.T. Determinants of body composition in breastfed infants using bioimpedance spectroscopy and ultrasound skinfolds—methods comparison. Pediatr. Res. 2016, 81, 423-433. [CrossRef] [PubMed]

26. Andreas, N.J.; Hyde, M.J.; Gale, C.; Parkinson, J.R.C.; Jeffries, S.; Holmes, E.; Modi, N. Effect of maternal body mass index on hormones in breast milk: A systematic review. PLoS ONE 2014, 9, e115043. [CrossRef] [PubMed]

27. Fields, D.A.; Schneider, C.R.; Pavela, G. A narrative review of the associations between six bioactive components in breast milk and infant adiposity. Obesity 2016, 24, 1213-1221. [CrossRef] [PubMed]

28. Martin, L.J.; Woo, J.G.; Geraghty, S.R.; Altaye, M.; Davidson, B.S.; Banach, W.; Dolan, L.M.; Ruiz-Palacios, G.M.; Morrow, A.L. Adiponectin is present in human milk and is associated with maternal factors. Am. J. Clin. Nutr. 2006, 83, 1106-1111. [CrossRef] [PubMed]

29. Nigro, E.; Scudiero, O.; Monaco, M.L.; Palmieri, A.; Mazzarella, G.; Costagliola, C.; Bianco, A.; Daniele, A. New insight into adiponectin role in obesity and obesity-related diseases. BioMed Res. Int. 2014, 2014, 658913. [CrossRef] [PubMed]

30. Catli, G.; Dundar, N.O.; Dundar, B.N. Adipokines in breast milk: An update. J. Clin. Res. Pediatr. Endocrinol. 2014, 6, 192-201. [CrossRef] [PubMed]

31. Weyermann, M.; Beermann, C.; Brenner, H.; Dietrich, R. Adiponectin and leptin in maternal serum, cord blood, and breast milk. Clin. Chem. 2006, 52, 2095-2102. [CrossRef] [PubMed]

32. Arita, Y.; Kihara, S.; Ouchi, N.; Takahashi, M.; Maeda, K.; Miyagawa, S.; Hotta, K.; Shimomura, I.; Nakamura, T.; Miyaoka, K.; et al. Paradoxical decrease of an adipose-specific protein, adiponectin, in obesity. Biochem. Biophys. Res. Commun. 1999, 257, 79-83. [CrossRef] [PubMed]

33. Savino, F.; Lupica, M.M.; Benetti, S.; Petrucci, E.; Liguori, S.A.; Cordero Di Montezemolo, L. Adiponectin in breast milk: Relation to serum adiponectin concentration in lactating mothers and their infants. Acta Pediatr. 2012, 101, 1058-1062. [CrossRef] [PubMed]

34. Hamdy, A.M.; Algendy, Y.G.; Shaaban, M.A.; El Shalabi, A.M. Relation of breast milk adiponectin level to infant growth. Egypt. J. Hosp. Med. 2017, 69, 3036-3041. [CrossRef]

35. Weyermann, M.; Brenner, H.; Rothenbacher, D. Adipokines in human milk and risk of overweight in early childhood: A prospective cohort study. Epidemiology 2007, 18, 722-729. [CrossRef] [PubMed]

36. Brunner, S.; Schmid, D.; Zang, K.; Much, D.; Knoeferl, B.; Kratzsch, J.; Amann-Gassner, U.; Bader, B.L.; Hauner, H. Breast milk leptin and adiponectin in relation to infant body composition up to 2 years. Pediatr. Obes. 2014, 10, 67-73. [CrossRef] [PubMed]

37. Bronsky, J.; Mitrova, K.; Karpisek, M.; Mazoch, J.; Durilova, M.; Fisarkova, B.; Stechova, K.; Prusa, R.; Nevoral, J. Adiponectin, AFABP, and leptin in human breast milk during 12 months of lactation. J. Pediatr. Gastroenterol. Nutr. 2011, 52, 474-477. [CrossRef] [PubMed]

38. Dundar, N.O.; Dundar, B.; Cesur, G.; Yilmaz, N.; Sutcu, R.; Ozguner, F. Ghrelin and adiponectin levels in colostrum, cord blood and maternal serum. Pediatr. Int. 2010, 52, 622-625. [CrossRef] [PubMed]

39. Anderson, J.; McKinley, K.; Onugha, J.; Duazo, P.; Chernoff, M.; Quinn, E.A. Lower levels of human milk adiponectin predict offspring weight for age: A study in a lean population of Filipinos. Matern. Child. Nutr. 2016, 12, 790-800. [CrossRef] [PubMed]

40. Luoto, R.; Kalliomaki, M.; Laitinen, K.; Delzenne, N.M.; Cani, P.D.; Salmimen, S.; Isolauri, E. Initial dietary and microbiological environments deviate in normal-weight compared to overweight children at 10 years of age. J. Pediatr. Gastroenterol. Nutr. 2011, 52, 90-95. [CrossRef] [PubMed] 
41. Khodabakhshi, A.; Ghayour-Mobarhan, M.; Rooki, H.; Vakili, R.; Hashemy, S.-I.; Mirhafez, S.R.; Shakeri, M.-T.; Kashanifar, R.; Pourbafarani, R.; Mirzaei, H.; et al. Comparative measurement of ghrelin, leptin, adiponectin, EGF and IGF-1 in breast milk of mothers with overweight/obese and normal-weight infants. Eur. J. Clin. Nutr. 2015, 69, 614-618. [CrossRef] [PubMed]

42. Quinn, E.A.; Childs, G. Ecological pressures and milk metabolic hormones of ethnic Tibetans living at different altitudes. Ann. Hum. Biol. 2016, 44, 34-45. [CrossRef] [PubMed]

43. Meyer, D.M.; Brei, C.; Stecher, L.; Much, D.; Brunner, S.; Hauner, H. The relationship between breast milk leptin and adiponectin with child body composition from 3 to 5 years: A follow-up study. Pediatr. Obes. 2017, 12, 125-129. [CrossRef] [PubMed]

44. Bieswal, F.; Ahn, M.T.; Reusens, B.; Holvoet, P.; Raes, M.; Rees, W.D.; Remacle, C. The importance of catch-up growth after early malnutrition for the programming of obesity in male rat. Obesity 2006, 14, 1330-1343. [CrossRef] [PubMed]

45. Binns, C.W.; Fraser, M.L.; Lee, A.H.; Scott, J. Defining exclusive breastfeeding in Australia. J. Paediatr. Child Health 2009, 45, 174-180. [CrossRef] [PubMed]

46. Gridneva, Z.; Hepworth, A.; Ward, L.; Lai, C.T.; Hartmann, P.; Geddes, D.T. Bioimpedance spectroscopy in the infant: Effect of milk intake and extracellular fluid reservoirs on resistance measurements in term breastfed infants. Eur. J. Clin. Nutr. 2016, 70, 843-851. [CrossRef] [PubMed]

47. Van Itallie, T.B.; Yang, M.U.; Heymsfield, S.B.; Funk, R.C.; Boileau, R.A. Height-normalized indices of the body's fat-free mass and fat mass: Potentially useful indicators of nutritional status. Am. J. Clin. Nutr. 1990, 52, 953-959. [CrossRef] [PubMed]

48. Arthur, P.; Hartmann, P.; Smith, M. Measurement of the milk intake of breast-fed infants. J. Pediatr. Gastroenterol. Nutr. 1987, 6, 758-763. [CrossRef] [PubMed]

49. Kent, J.C.; Mitoulas, L.R.; Cregan, M.D.; Ramsay, D.T.; Doherty, D.A.; Hartmann, P.E. Volume and frequency of breastfeedings and fat content of breast milk throughout the day. Pediatrics 2006, 117, e387-e395. [CrossRef] [PubMed]

50. Keller, R.; Neville, M. Determination of total protein in human milk: Comparison of methods. Clin. Chem. 1986, 32, 120-123. [PubMed]

51. Cannon, A.; Kakulas, F.; Hepworth, A.; Lai, C.; Hartmann, P.; Geddes, D. The effects of leptin on breastfeeding behaviour. Int. J. Environ. Res. Public. Health 2015, 12, 12340-12355. [CrossRef] [PubMed]

52. Curran-Everett, D. Multiple comparisons: Philosophies and illustrations. Am. J. Physiol. Reg Integr. Compar. Physiol. 2000, 279, R1-R8. [CrossRef] [PubMed]

53. R Core Team. R: A language and Environment for Statistical Computing; R Foundation for Statistical Computing: Vienna, Austria, 2017.

54. Pinheiro, J.B.; Bates, D.; DebRoy, S.; Sarkar, D.; R Development Core Team. nlme: Linear and Nonlinear Mixed Effects Models, R package version 3.1-102; R Foundation for Statistical Computing: Vienna, Austria, 2011.

55. Bates, D.; Maechler, M.; Bolker, B.; Walker, S. Ime4: Linear Mixed-Efects Models Using Eigen and s4, R package version 1.1-7; R Foundation for Statistical Computing: Vienna, Austria, 2014.

56. Fox, J.; Weisberg, S. An R Companion to Applied Regression; Sage: Thousand Oaks, CA, USA, 2011.

57. Gamer, M.; Lemon, J.; Fellows, I.; Singh, P. Various Coefficients of Interrater Reliability and Agreement; 0.84; R Foundation for Statistical Computing: Vienna, Austria, 2012.

58. Hothorn, T.; Bretz, F.; Westfall, P. Simultaneous inference in general parametric models. Biom. J. 2008, 50, 346-363. [CrossRef] [PubMed]

59. Wickham, H. Ggplot2: Elegant Graphics for Data Analysis; Springer: New York, NY, USA, 2009.

60. Chan, D.; Goruk, S.; Becker, A.B.; Subbarao, P.; Mandhane, P.J.; Turvey, S.E.; Lefebvre, D.; Sears, M.R.; the CHILD Study investigators; Field, C.J.; et al. Adiponectin, leptin and insulin in breast milk: Associations with maternal characteristics and infant body composition in the first year of life. Int. J. Obes. 2018, 42, 36-43. [CrossRef] [PubMed]

61. Cesur, G.; Ozguner, F.; Yilmaz, N.; Dundar, B. The relationship between ghrelin and adiponectin levels in breast milk and infant serum and growth of infants during early postnatal life. J. Physiol. Sci. 2012, 62, 185-190. [CrossRef] [PubMed]

62. Schuster, S.; Hechler, C.; Gebauer, C.; Kiess, W.; Kratzsch, J. Leptin in maternal serum and breast milk: Association with infants' body weight gain in a longitudinal study over 6 months of lactation. Pediatr. Res. 2011, 70, 633-637. [CrossRef] [PubMed] 
63. Quinn, E.A.; Largado, F.; Borja, J.B.; Kuzawa, C.W. Maternal characteristics associated with milk leptin content in a sample of Filipino women and associations with infant weight for age. J. Hum. Lact. 2015, 31, 273-281. [CrossRef] [PubMed]

64. Fields, D.A.; George, B.; Williams, M.; Whitaker, K.; Allison, D.B.; Teague, A.; Demerath, E.W. Associations between human breast milk hormones and adipocytokines and infant growth and body composition in the first 6 months of life. Pediatr. Obes. 2017, 12, 78-85. [CrossRef] [PubMed]

65. De Luca, A.; Frasquet-Darrieux, M.; Gaud, M.A.; Christin, P.; Boquien, C.-Y.; Millet, C.; Herviou, M.; Darmaun, D.; Robins, R.J.; Ingrand, P.; et al. Higher leptin but not human milk macronutrient concentration distinguishes normal-weight from obese mothers at 1-month postpartum. PLoS ONE 2016, 11, e0168568. [CrossRef] [PubMed]

66. Ucar, B.; Kirel, B.; Bor, O.; Kilic, F.S.; Dogruel, N.; Aydogdu, S.D.; Tekin, N. Breast milk leptin concentrations in initial and terminal milk samples: Relationships to maternal and infant plasma leptin concentrations, adiposity, serum glucose, insulin, lipid and lipoprotein levels. J. Pediatr. Endocrinol. Metab. 2000, 13, 149-156. [CrossRef] [PubMed]

67. Bielicki, J.; Huch, R.; von Mandach, U. Time-course of leptin levels in term and prteterm human milk. Eur. J. Endocrinol. 2004, 151, 271-276. [CrossRef] [PubMed]

68. Whitfield, J.F.; Morley, P.; Willick, G.E. Bone growth stimulators: New tools for treating bone loss and mending fractures. Vitam. Horm. 2002, 65, 1-80. [PubMed]

69. Smith-Kirwin, S.M.; O'Connor, D.M.; Johnston, J.; De Lancey, E.; Hassink, S.G.; Funanage, V.L. Leptin expression in human mammary epithelial cells and breast milk. J. Clin. Endocrinol. Metab. 1998, 83, 1810-1813. [CrossRef] [PubMed]

70. Houseknecht, K.L.; McGuire, M.K.; Portocarrero, C.P.; McGuire, M.A.; Beerman, K. Leptin is present in human milk and is related to maternal plasma leptin concentration and adiposity. Biochem. Biophys. Res. Commun. 1997, 240, 742-747. [CrossRef] [PubMed]

71. Hassiotou, F.; Geddes, D.T. Programming of appetite control during breastfeeding as a preventative strategy against the obesity epidemic. J. Hum. Lact. 2014, 30, 136-142. [CrossRef] [PubMed]

72. Young, B.E.; Levek, C.; Reynolds, R.M.; Rudolph, M.C.; MacLean, P.; Hernandez, T.L.; Friedman, J.E.; Krebs, N.F. Bioactive components in human milk are differentially associated with rates of lean and fat mass deposition in infants of mothers with normal vs. elevated BMI. Pediatr. Obes. 2018. [CrossRef] [PubMed]

(C) 2018 by the authors. Licensee MDPI, Basel, Switzerland. This article is an open access article distributed under the terms and conditions of the Creative Commons Attribution (CC BY) license (http:/ / creativecommons.org/licenses/by/4.0/). 


\title{
Hourly Breast Expression to Estimate the Rate of Synthesis of Milk and Fat
}

\author{
Jacqueline C. Kent ${ }^{1, *}$, Hazel Gardner ${ }^{1}$, Ching-Tat Lai ${ }^{1}$, Peter E. Hartmann ${ }^{1}$, Kevin Murray ${ }^{2}$, \\ Alethea Rea ${ }^{3}$ and Donna T. Geddes ${ }^{1}$ \\ 1 School of Molecular Sciences, The University of Western Australia, 35 Stirling Hwy, \\ Crawley, WA 6009, Australia; Hazel.Gardner@uwa.edu.au (H.G.); Ching-Tat.Lai@uwa.edu.au (C.-T.L.); \\ Peter.Hartmann@uwa.edu.au (P.E.H.); Donna.Geddes@uwa.edu.au (D.T.G.) \\ 2 School of Population and Global Health, The University of Western Australia, 35 Stirling Hwy, \\ Crawley, WA 6009, Australia; Kevin.Murray@uwa.edu.au \\ 3 Centre of Applied Statistics, The University of Western Australia, 35 Stirling Hwy, \\ Crawley, WA 6009, Australia; Alethea.Rea@uwa.edu.au \\ * Correspondence: Jacqueline.Kent@uwa.edu.au; Tel.: +61-8-6488-1208
}

Received: 25 July 2018; Accepted: 15 August 2018; Published: 22 August 2018

check for updates

\begin{abstract}
Objective measurement of the rate of synthesis of breast milk and fat in breastfeeding mothers requires test-weighing of each breastfeed and the measurement of each expression from each breast over $24 \mathrm{~h}$, with the collection of milk samples before and after each breastfeed and expression. We sought an abbreviated technique for measuring these rates of synthesis. Participants completed a 24-h breastfeeding milk profile, and expressed their breasts on arrival at the research room and each hour thereafter for $3 \mathrm{~h}$ (4 expressions). The hourly rate of milk synthesis, as measured by the yield of milk from the fourth expression, was closely related to the hourly rate of milk synthesis calculated from the 24-h milk profile. The hourly rate of fat synthesis, calculated from the fat content of small samples of the first and last milk expressed during the fourth expression, was different from the rate of fat synthesis calculated from the fat content and volumes of all the breastfeeds and expressions during the 24-h milk profile. The study confirms the use of an abbreviated technique to measure the rate of breast milk synthesis, but is not reliable as a measure of the rate of fat synthesis for an individual.
\end{abstract}

Keywords: human lactation; expressing; milk synthesis; fat synthesis

\section{Introduction}

Many mothers are not achieving the recommendations of the World Health Organization, the National Health and Medical Research Council, and the American Academy of Pediatrics of exclusive breastfeeding for 6 months [1-5]. Over $90 \%$ of mothers in Perth, Western Australia commence breastfeeding, but fewer than $1 \%$ are exclusively breastfeeding at 6 months [3]. In the United States $81 \%$ of mothers ever breastfed, and $22 \%$ are exclusively breastfeeding at 6 months [5]. A common reason given for early weaning is a perception of insufficient milk [6] that in turn is associated with a lack of confidence in breastfeeding [7]. Clinical indications of infants receiving enough breast milk include sufficient bowel movements and urine output [8] and satisfactory weight gains [9]. These are believed to be sufficient to provide reassurance for both parents and health professionals. However, the high frequency mothers who have a perception of insufficient milk indicates that further objective measures of milk production are required.

Objective measurement of milk supply can be made by carrying out a 24-h milk profile that involves weighing the fully-clothed infant before and after every breastfeed at home and recording 
volumes of milk expressed (if applicable) for 24-26 $\mathrm{h}$ [10]. This allows calculation of both the total daily milk intake of the infant over $24 \mathrm{~h}$, and the total daily milk production of each breast [10]. The average hourly rate of milk synthesis can then be calculated from the total milk production in $24 \mathrm{~h}$ divided by 24. If a mother is reluctant to measure her milk profile over a full $24 \mathrm{~h}$, a small study has demonstrated that when the breasts are expressed using an electric breast pump each hour, the hourly rate of milk production from the 2 nd to the 7 th hour (the 3rd to the 8th expression) represents the longer-term physiological rate of milk synthesis [11]. The authors suggested that further studies are required to confirm this finding.

In addition to the volume of milk ingested by the infant, total caloric intake is important for energy expenditure and growth. Fat provides $49 \%$ of the energy in breast milk [12], and therefore the contribution of fat to the energy intake of the infant is significant. The daily fat intake of breastfed infants has been underestimated when unsuitable sampling regimes have been used. For example, calculations based on daily milk intake by test-weighing and fat content of milk samples collected before and after the first breastfeed of the morning [13] will underestimate the daily fat intake of the breastfed infant, because the fat content of milk in the morning is less than during the day and afternoon [14]. Another approach was to feed the infant from one breast only at each breastfeeding session and pump the other breast, alternating the breasts, for a 24-h period and measure the fat content of the pooled pumped milk [12]. The yield of milk from the pumping sessions averaged $49 \%$ of the 24-h milk production determined by test-weighing, but some were $<40 \%$ and others $>60 \%$ [12], indicating that this technique may be sufficiently accurate for a population study, but not useful to predict the daily fat intake for an individual infant.

When mothers collect small samples $(<1 \mathrm{~mL})$ of breast milk before and after each breastfeed during the 24-h milk profile, the total fat intake can be calculated from the fat content of the samples and the volume of each breastfeed. If the mother is also expressing breast milk, the measurement of the fat content of small samples $(<1 \mathrm{~mL})$ of breast milk collected before and after each expression and the volume of each expression, in addition to the data from the 24-h breastfeeds, allows calculation of the mother's total daily fat production. The hourly rate of fat synthesis can then be calculated by dividing the total fat production in $24 \mathrm{~h}$ by 24 .

We aimed to provide further data to investigate if the technique of hourly pumping can be used to estimate the rate of milk synthesis, and ascertain if the same technique could be used to estimate the rate of milk fat synthesis if mothers prefer this technique to 24 -h test-weighing with milk samples.

\section{Materials and Methods}

We recruited lactating mothers between 5 and 11 months from birth of term singleton infants who agreed to measure their 24-h milk profile with milk samples, and come to the research room at The University of Western Australia for a three-and-a-half-hour study session. Participants were only included in the study if their infants had previously accepted breast milk from a bottle. The study was approved by the Human Research Ethics Committee of The University of Western Australia (RA/4/1/4492) and all participants provided written informed consent.

Demographics were recorded and the participants were loaned accurate digital scales (BabyWeigh $^{\mathrm{TM}}$, Medela Inc., McHenry, IL, USA, resolution $2 \mathrm{~g}$, accuracy $\pm 0.034 \%$ ) to measure their 24-h milk profile. All measurements of breastfeed volumes and milk production were made in grams but expressed in $\mathrm{mL}$ because the density of milk is $1.03 \mathrm{~g} / \mathrm{mL}$ [15]. Data were recorded either on paper or entered on a secure password-protected website accessed by invitation only. The corrected 24-h milk production, for participants who were either exclusively breastfeeding or breastfeeding and expressing, was calculated by the method of Arthur et al. [10]. However, no correction for infant insensible water loss was made, and therefore milk production may be underestimated by an average of $10 \%$ (range $3-55 \%$ ) [10]. The mean rate of milk production for each breast was calculated by dividing the corrected 24-h milk production for each breast by 24 . 
During this 24-h period, the mothers hand-expressed small milk samples $(<1 \mathrm{~mL})$ into 5-mL polypropylene plastic vials (Disposable Products, Adelaide, SA, Australia), immediately before and after each breastfeed or expression from each breast. Samples were frozen as soon as possible and kept at $-15^{\circ} \mathrm{C}$ until analyzed. The cream content was measured using the creamatocrit method [16]. The cream content of the milk of each feed or expression during the measurement of milk profile was calculated as ([0.53 $\times$ pre-feed creamatocrit $+0.47 \times$ post-feed creamatocrit $] / 2)$ [17] . The creamatocrit was converted to fat using the following formula: fat $(\mathrm{g} / \mathrm{L})=3.968+(5.917 \times$ creamatocrit $)$ [16]. The amount of fat in each breastfeed or expression was calculated from the fat content of the milk and the volume of the feed or expression. The total amount of fat synthesized by each breast in the 24-h period was calculated by summing amount of fat in all the breastfeeds and expressions from that breast, and the mean rate of fat synthesis was calculated by dividing the total amount of fat synthesized for each breast by 24 .

For the hourly-pumping study the participants pumped either both breasts simultaneously or one breast only according to the preference of the participant. On arrival at the research room at The University of Western Australia, the mothers expressed their breast(s) using a Medela Symphony breast pump at their own maximum comfortable vacuum for $10 \mathrm{~min}$ after milk ejection was detected by an increase in milk flow. For each breast, milk was conveyed via a connecting tube from the breast shield to one of three bottles placed on the weigh platform of a continuous weigh balance (ShowMilk, Carag AG, Baar, Switzerland). The first $1 \mathrm{~mL}$ (first milk) was collected into the first bottle, the bulk of the expressed milk was collected into the second bottle (pooled milk), and the last milk expressed $(\sim 1 \mathrm{~mL})$ was collected into the third bottle (last milk). The ShowMilk device measures the cumulative weight of milk at $50 \mathrm{~Hz}$ with a resolution of $0.1 \mathrm{~g}$ and accuracy $0.02 \%$ to a maximum of $2 \mathrm{~kg}$ [18]. The expression was repeated 1,2 and $3 \mathrm{~h}$ after the commencement of the first expression. The total volume of each expression $(\mathrm{mL})$ from each breast was recorded.

The creamatocrit of the first, pooled, and last milk of each expression was measured as above and converted to fat content (g/L) [16]. This, with the total volume of milk expressed, was used to calculate the total amount of fat in the expressed milk for each hour.

From the 24-h milk profile, the fat content of all the milk samples and the volumes of all the breastfeeds were used to estimate the breastfeeding storage capacity of the breasts [19]. If the participants expressed their breast milk on one or more occasions during the $24 \mathrm{~h}$, the data from the expressions were included with the breastfeeding data to calculate the potential storage capacity of the breasts [20]. The fat content of the first milk samples was used to calculate the degree of fullness of the breast before the expression [21].

\section{Statistics}

Data are presented as mean $\pm \mathrm{SD}$ or median (IQR). A paired $t$-test was used to compare fat content of first milk and last milk for each expression, and unpaired $t$-test was used to compare total milk production of participants who were exclusively breastfeeding with those who were breastfeeding and expressing. Linear mixed models were used to analyse the responses volume of fourth expression, rate of synthesis, amount of fat fourth expression, and rate of fat synthesis using a fixed effect of method (hourly pumping vs. 24-h profile) and random effects of participant and breast within participant. The volume of each expression, and the fat content of the first and last milk at time points $0,1,2$, and $3 \mathrm{~h}$ of the hourly pumping study were included in the fixed effect of method. Key comparisons were considered using general linear hypothesis tests (with a Tukeys adjustment). Significance was set at the $5 \%$ level, and data were analysed using the R environment for statistical computing [22].

\section{Results}

Data were collected from 15 participants, of whom 10 were feeding male infants and 5 female infants. A 24-h milk profile was measured by 11 participants on a day during which they were exclusively breastfeeding and 4 participants on a day during which they were breastfeeding and 
expressing (1 participant on two occasions). The demographics of the participants are presented in Table 1. A single study session occurred within 11 weeks of the measurement of 24-h milk profile for 14 of the participants. One participant measured her 24-h milk profile when her infant was 5 weeks old and participated in a study session 6 weeks later. She measured her 24-h milk profile again when her infant was 17 weeks old and participated in a second study session 4 weeks later. During the study sessions 3 participants expressed from one breast, 11 expressed from both breasts, and 1 expressed from both breasts for 2 study sessions, providing data for 29 breasts. Milk samples before and after each breastfeeding or expression during the 24-h milk profile were collected by 11 of the participants, and 4 participants did not collect milk samples. The breastfeeding storage capacity [20] (calculated from the fat content of samples collected before and after breastfeeds only) was $165 \pm 42 \mathrm{~mL}$. When the fat content of all samples from all breastfeeds and expressions, including those from the study sessions, was included the potential storage capacity [20] was $182 \pm 58 \mathrm{~mL}$. On average, the breastfeeding infants took $67 \pm 11 \%$ of the milk that was available in the breast when the 24 -h milk profile was measured.

Table 1. Demographics of participants $(n=15)$.

\begin{tabular}{|c|c|}
\hline & Median (IQR) \\
\hline \multicolumn{2}{|l|}{ Infant } \\
\hline Age (weeks) & $22(12)$ \\
\hline Birth weight $(\mathrm{g})$ & $3650(726)$ \\
\hline Fat intake $(\mathrm{g} / 24 \mathrm{~h})(n=11)$ & $34(13)$ \\
\hline \multicolumn{2}{|l|}{ Mother } \\
\hline Age (years) & $35(37)$ \\
\hline \multicolumn{2}{|c|}{ Exclusive breastfeeding during $24-\mathrm{h}$ milk profile measurement $(n=11)$} \\
\hline 24-h milk production $(\mathrm{mL})$ & $860(237)$ \\
\hline \multicolumn{2}{|c|}{ Breastfeeding and expressing during 24-h milk profile measurement $\left(n=5^{*}\right)$} \\
\hline Milk transfer breastfeeding (breast to infant) (mL) & $565(311)$ \\
\hline Milk transfer (breast to bottle) (mL) & $180(124)$ \\
\hline Total 24-h milk production (mL) & $810(224)$ \\
\hline
\end{tabular}

There was no significant difference in $24-\mathrm{h}$ milk production between participants who were exclusively breastfeeding and those who were breastfeeding and expressing (difference $=68.6 \mathrm{~mL}$, 95\% CI: 101.9-239.1, $p=0.400$ )

The yield of milk from each expression during the study session and the hourly rate of milk synthesis calculated from the 24-h milk profile data are presented in Figure 1.

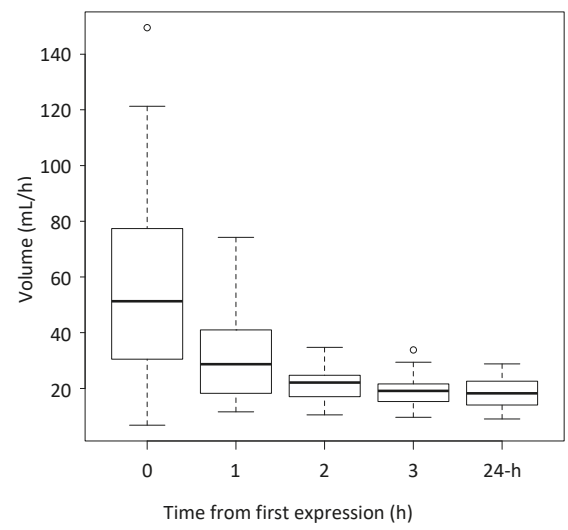

Figure 1. The yield of milk from hourly expressions, and the hourly rate of milk synthesis calculated from the 24-h milk profile data (24-h). $n=29$ breasts. 
On average the yield of milk from each of the second, third and fourth expressions $(1,2$ and $3 \mathrm{~h}$ after the first expression) was lower than for the expression the hour before (mean expression $59.0 \mathrm{~mL}$ at time $0,32.0 \mathrm{~mL}$ at time $1,22.0 \mathrm{~mL}$ at time 2 and $18.9 \mathrm{~mL}$ at time 3 ).

The yield of milk from the fourth expression ( $3 \mathrm{~h}$ after the first expression) $(18.9 \pm 5.8 \mathrm{~mL}$ ) was not significantly different from the hourly rate of milk synthesis $(18.2 \pm 5.4 \mathrm{~mL} / \mathrm{h})$ calculated from the 24 -h milk profile data (mean difference $=0.966, \mathrm{SE}=0.995, p=0.997$ ). There was also a statistically significant relationship between the volume of the fourth expression and rate of synthesis $(p=0.002)$ (Figure 2).

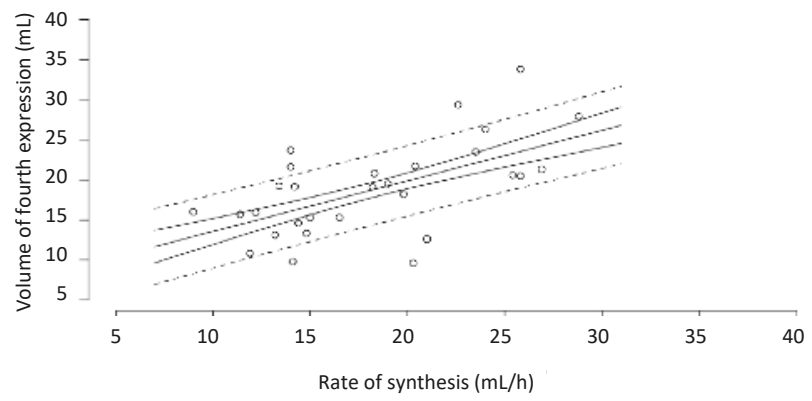

Figure 2. Relationship between the volume of milk from the fourth expression ( $3 \mathrm{~h}$ after the first expression) and the hourly rate of milk synthesis calculated from the 24 -h milk profile data. Fitted model (solid line), with the confidence (solid line) and prediction intervals (dashed lines). $n=29$ breasts.

The fat content of the first and last milk collected at each expression and the average fat content of milk samples collected during the 24-h milk profile are presented in Figure 3. The fat content of the last milk was higher than the first milk for each expression but not statistically different at time 3 (time 0: mean difference $=34.9 \mathrm{~g} / \mathrm{L}, \mathrm{SE}=8.1, p=0.001$; time 1: mean difference $=22.0 \mathrm{~g} / \mathrm{L}, \mathrm{SE}=8.1$, $p<0.001$; time 2: mean difference $=17.8 \mathrm{~g} / \mathrm{L}, \mathrm{SE}=8.1, p=0.021$; time 3: mean difference $=15.7 \mathrm{~g} / \mathrm{L}$, $\mathrm{SE}=8.1, p=0.092)$. There was no significant difference in the fat content of milk collected at the end of each of the first, second, third or fourth expressions $(63.7 \pm 27.6 \mathrm{~g} / \mathrm{L}, 69.1 \pm 28.2 \mathrm{~g} / \mathrm{L}, 69.1 \pm 29.4 \mathrm{~g} / \mathrm{L}$, $58.4 \pm 27.0 \mathrm{~g} / \mathrm{L}$ respectively). There was no significant difference between the fat content of pooled milk from the fourth expression $(52.5 \pm 23.5 \mathrm{~g} / \mathrm{L})$ and the average fat content of milk collected over the 24 -h period $(46.6 \pm 8.1 \mathrm{~g} / \mathrm{L})$ (mean difference $=10.5 \mathrm{~g} / \mathrm{L}, 95 \%$ CI: 7.2-20.1, $p=0.18$ ).

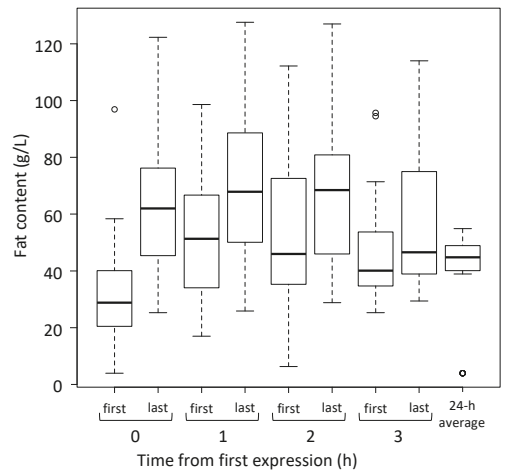

Figure 3. The fat content of first and last milk collected at each expression and the average fat content of milk samples collected during the 24 -h milk profile data (24-h average). $n=29$ breasts. 
The amount of fat in the milk from the fourth expression was $1.02 \pm 0.50 \mathrm{~g}$. This was different from the hourly rate of fat synthesis $(0.79 \pm 0.23 \mathrm{~g} / \mathrm{h})$ calculated from the 24 -h milk profile (mean difference $=0.23 \mathrm{~g} / \mathrm{h}, \mathrm{SE}=0.10, p=0.034)$. There was no significant relationship between the two measurements $(p=0.054)$ (Figure 4$)$.

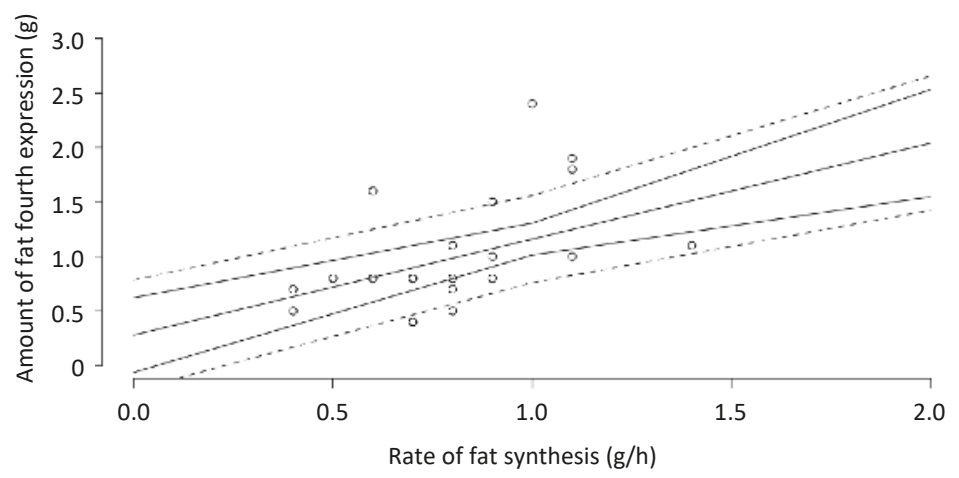

Figure 4. Relationship between the amount of fat from the fourth expression and the hourly rate of milk fat synthesis calculated from the 24 -h milk profile data $(p=0.054)$. Fitted model (solid line), with the confidence (solid line) and prediction interval (dashed line). $n=29$ breasts.

The degree of fullness of the breast before the first expression (time 0), calculated from the fat content of the first milk and the potential storage capacity of the breast, was $0.6 \pm 0.2$. The first expression removed $53 \pm 26 \%$ of the available milk, with 6 sessions classified as low outcome $(<40 \%$ of the available milk removed), and 5 sessions classified as high outcome ( $>70 \%$ of the available milk removed).

\section{Discussion}

The data suggest that all the milk from the fourth expression had been synthesized since the end of the third expression, and the yield of milk from that hour (50 minutes after the last expression plus 10 minutes expressing) was closely related to the hourly rate of milk synthesis calculated from the 24-h milk profile data. Therefore, this study has confirmed the findings of Lai et al. [11] that for mothers who are fully breastfeeding the technique of hourly pumping for 4 expressions can be used as a measure of the hourly rate of milk synthesis. It was established by Lai et al. that hourly pumping for a further $3 \mathrm{~h}$ yielded the same volume as the fourth expression, indicating that the steady state of milk synthesis and removal had been reached, and more than three hourly expressions after the first expression are not required. It has been established that an accurate measure of 24-h breast milk production cannot be calculated by test-weighing an infant for a 12-h period ( 6 a.m. to 6 p.m.) and doubling the total $[14,23]$, nor by taking the mean milk intake for two consecutive feedings and multiplying it by the number of feedings in the 24-h period [23]. If mothers are reluctant to weigh their infants before and after every breastfeed for a full $24 \mathrm{~h}$, or if they urgently need to know their rate of breast milk synthesis and are prepared to express their breasts every hour for $3 \mathrm{~h}$ after the first expression, this technique can provide reliable information. If milk production is less than optimal, early intervention is beneficial [24]. An objective measurement of the rate of milk synthesis can guide advice from a clinician on increasing milk production if required. It must be borne in mind that the infant will probably need to be given milk by another means unless there is usually an interval between breastfeeds of more than $3 \mathrm{~h}$.

The proportion of mothers in Australia who combine breastfeeding and expressing is increasing [25], either to leave expressed breast milk with a carer, or to increase milk supply [26]. 
Mothers with concerns about their infants' transfer of milk from the breast need to undertake a full 24-h period of test-weighing to determine the efficacy of their breastfeeding. Using the hourly pumping technique will provide information about their current total rate of milk production, and can be used to monitor the effects of alterations in their expression regime to increase milk production.

The finding that the milk removed during the fourth expression represents the hourly rate of milk secretion suggests that the yield of fat should represent the hourly rate of fat secretion. Although there was a trend towards a relationship between the amount of fat in the last expression and the hourly rate of fat synthesis calculated from the 24 -h profile data, the mean difference was 0.23 (a reduction of $23 \%$ ), indicating that this technique is not reliable for predicting the rate of fat synthesis for an individual mother.

The changes in fat content before and after each expression are interesting, and an explanation is worthwhile. The breast reaches its maximum degree of fullness following the longest interval after the previous removal of milk. This long interval allows time for the fat globules in the alveoli to partition, as they tend to adhere to the surface of the lactocytes, resulting in the first milk expressed being low in fat content [27]. The wide range in fat content before the first expression is a result of both the variable time since the previous removal of milk from the breast and inter-individual differences in fat content of milk [28]. The fat content increases as milk is removed from the breast, reaching a maximum when the breast is fully drained [27,29]. The wide range in fat content after the first expression is a result of the variability between mothers and the effectiveness of milk removal by a breast pump [18]. In the $50 \mathrm{~min}$ between the end of the first expression and the start of the second expression, the fat content of the milk within the alveoli $(69.1 \mathrm{~g} / \mathrm{L})$ was diluted by the newly-secreted milk with an average fat content of (41.1 g/L) [14] to reach 51.3 g/L (Figure 3). Compared with the previous interval, there was less time for the fat globules to partition between the lumen of the alveoli and the luminal wall of the lactocytes, resulting in a smaller difference between the first and last milk of the second expression (Figure 3). The higher fat content of the last milk of the second expression occurred because the first expression did not drain the breast completely ( $53 \%$ of the available milk removed), leaving milk with a high fat content in the alveoli. The wide variability is the result of the 6 sessions that were low outcome ( $<40 \%$ of the available milk removed). The yield of milk from the third expression was significantly higher than from the fourth expression, indicating that the third expression did not quite drain the breast, and only the fourth expression comprised only milk secreted in the previous hour. This is consistent with the fat content of the last milk from the third expression remaining elevated (Figure 3). There was a small difference in the fat content between the first and last milk from the fourth expression, which was consistent with some partitioning of the fat globules during the $50 \mathrm{~min}$ after the end of the third expression.

It is puzzling that, although the volume of the last expression was closely related to the hourly rate of milk synthesis calculated from the 24-h milk profile data, the amount of fat in the last expression was not an accurate reflection of the rate of fat synthesis for an individual. We suggest that it is the result of the steep rise in fat content as the breast is drained $[29,30]$ such that the final small volume of milk expressed makes a disproportionately large contribution to the fat content of the final milk sample.

The higher fat content of the second expression could make it suitable for feeding low-birth-weight infants when the mother has an abundant supply, with the milk from the first expression being stored for later use. This may be an alternative to fractionating an expression [31].

\section{Conclusions}

These data confirm that the fourth hourly expression can be used to estimate the average rate of breast milk synthesis for individuals and may be useful when low milk production is suspected. However, while there is a tendency to an overall relationship between hourly pumping and 24-h milk profile for estimating the rate of synthesis of milk fat, this technique is less accurate for individuals.

Author Contributions: Conceptualization, P.E.H., C.-T.L. and J.C.K.; Methodology, P.E.H., C.-T.L. and J.C.K.; Formal Analysis, A.R. and K.M.; Investigation, J.C.K., C.-T.L., H.G.; Resources, P.E.H. and D.T.G. Data Curation, 
J.C.K.; Writing-Original Draft Preparation, J.C.K.; Writing-Review \& Editing, D.T.G., C.-T.L., H.G., P.E.H. and A.R.; Supervision, P.E.H. and D.G.; Project Administration, P.E.H. and D.G.; Funding Acquisition, P.E.H. and D.T.G.

Funding: The study was funded by an unrestricted research grant from Medela AG.

Acknowledgments: We thank the participants, and the Australian Breastfeeding Association (WA Branch) for their support (Approval 2016-4).

Conflicts of Interest: J.C.K., H.G., C.-T.L. and D.T.G. are supported by an unrestricted research grant from Medela AG., P.E.H., A.R. and K.M. have no conflict of interest to declare.

\section{References}

1. Gartner, L.M.; Morton, J.; Lawrence, R.A.; Naylor, A.J.; O’Hare, D.; Schanler, R.J.; Eidelman, A.I. American Academy of Pediatrics. Breastfeeding and the use of human milk. Pediatrics 2005, 115, 496-506. [PubMed]

2. National Health and Medical Research Council. Dietary Guidelines for Children and Adolescents in Australia; Australian Government Publishing Service: Canberra, Australia, 2003.

3. Scott, J.A.; Binns, C.W.; Oddy, W.H.; Graham, K.I. Predictors of breastfeeding duration: Evidence from a cohort study. Pediatrics 2006, 117, e646-e655. [CrossRef] [PubMed]

4. World Health Organization. Global Strategy for Infant and Young Child Feeding; World Health Organization: Geneva, Switzerland, 2003; p. 30.

5. Centers for Disease Control and Prevention CDC Breastfeeding Report Card 2016. Available online: www. cdc.gov/breastfeeding/pdf/2016breastfeedingreportcard.pdf (accessed on 10 July 2018).

6. Gatti, L. Maternal perceptions of insufficient milk supply in breastfeeding. J. Nurs. Scholarsh. 2008, 40, 355-363. [CrossRef] [PubMed]

7. Dennis, C.L. The breastfeeding self-efficacy scale: Psychometric assessment of the short form. J. Obstet. Gynecol. Neonatal Nurs. 2003, 32, 734-744. [CrossRef] [PubMed]

8. Neifert, M.R. Prevention of breastfeeding tragedies. Pediatr. Clin. N. Am. 2001, 48, 273-297. [CrossRef]

9. WHO Multicentre Growth Reference Study Group. WHO Child Growth Standards: Growth Velocity Based on Weight, Length and Head Circumference. Methods and Development; World Health Oragnization: Geneva, Switzerland, 2009.

10. Arthur, P.G.; Hartmann, P.E.; Smith, M. Measurement of the milk intake of breast-fed infants. J. Pediatr. Gastroenterol. Nutr. 1987, 6, 758-763. [CrossRef] [PubMed]

11. Lai, C.T.; Hale, T.W.; Simmer, K.; Hartmann, P.E. Measuring milk synthesis in breastfeeding mothers. Breastfeed. Med. 2010, 5, 103-107. [CrossRef] [PubMed]

12. Butte, N.F.; Garza, C.; Smith, E.O.; Nichols, B.L. Human milk intake and growth in exclusively breast-fed infants. J. Pediatr. 1984, 104, 187-195. [CrossRef]

13. Grote, V.; Verduci, E.; Scaglioni, S.; Vecchi, F.; Contarini, G.; Giovannini, M.; Koletzko, B.; Agostoni, C. Breast milk composition and infant nutrient intakes during the first 12 months of life. Eur. J. Clin. Nutr. 2016, 70, 250-256. [CrossRef] [PubMed]

14. Kent, J.C.; Mitoulas, L.R.; Cregan, M.D.; Ramsay, D.T.; Doherty, D.A.; Hartmann, P.E. Volume and frequency of breastfeedings and fat content of breast milk throughout the day. Pediatrics 2006, 117, e387-e395. [CrossRef] [PubMed]

15. Moya, J.; Phillips, L.; Schuda, L.; Wood, P.; Diaz, A.; Lee, R.; Clickner, R.; Birch, R.J.; Adjei, N.; Blood, P. Human Milk Intake. In Exposure Factors Handbook; US Environmental Protection Agency: Washington, WA, USA, 2011.

16. Meier, P.P.; Engstrom, J.L.; Zuleger, J.L.; Motykowski, J.E.; Vasan, U.; Meier, W.A.; Hartmann, P.E.; Williams, T.M. Accuracy of a user-friendly centrifuge for measuring creamatocrits on mothers' milk in the clinical setting. Breastfeed. Med. 2006, 1, 79-87. [CrossRef] [PubMed]

17. Mitoulas, L.R. Short- and Long-term Variation in the Production, Content and Composition of Human Milk Fat. Ph.D. Thesis, The University of Western Australia, Perth, WA, Austalia, 2000.

18. Prime, D.K.; Garbin, C.P.; Hartmann, P.E.; Kent, J.C. Simultaneous breast expression in breastfeeding women is more efficacious than sequential breast expression. Breastfeed. Med. 2012, 7, 442-447. [CrossRef] [PubMed]

19. Cox, D.B.; Kent, J.C.; Casey, T.M.; Owens, R.A.; Hartmann, P.E. Breast growth and the urinary excretion of lactose during human pregnancy and early lactation: Endocrine relationships. Exp. Physiol. 1999, 84, 421-434. [CrossRef] [PubMed] 
20. Kent, J.C.; Mitoulas, L.R.; Cregan, M.D.; Geddes, D.T.; Larsson, M.; Doherty, D.A.; Hartmann, P.E. Importance of vacuum for breastmilk expression. Breastfeed. Med. 2008, 3, 11-19. [CrossRef] [PubMed]

21. Cox, D.B.; Owens, R.A.; Hartmann, P.E. Blood and milk prolactin and the rate of milk synthesis in women. Exp. Physiol. 1996, 81, 1007-1020. [CrossRef] [PubMed]

22. R: A Language and Environment for Statistical Computing. Available online: https://www.gbif.org/tool/ 81287/r-a-language-and-environment-for-statistical-computing (accessed on 10 July 2018).

23. Matheny, R.J.; Picciano, M.F. Assessment of abbreviated techniques for determination of milk volume intake in the human milk-fed infant. J. Pediatr. Gastroenterol. Nutr. 1985, 4, 808-812. [CrossRef] [PubMed]

24. Hill, P.D.; Aldag, J.C.; Chatterton, R.T.; Zinaman, M. Comparison of milk output between mothers of preterm and term infants: The first 6 weeks after birth. J. Hum. Lact. 2005, 21, 22-30. [CrossRef] [PubMed]

25. Johns, H.M.; Forster, D.A.; Amir, L.H.; McLachlan, H.L. Prevalence and outcomes of breast milk expressing in women with healthy term infants: A systematic review. BMC Pregnancy Childbirth 2013, 13, 212. [CrossRef] [PubMed]

26. Johns, H.M.; Amir, L.H.; McLachlan, H.L.; Forster, D.A. Breast pump use amongst mothers of healthy term infants in Melbourne, Australia: A prospective cohort study. Midwifery 2016, 33, 82-89. [CrossRef] [PubMed]

27. Atwood, C.S.; Hartmann, P.E. Collection of fore and hind milk from the sow and the changes in milk composition during suckling. J. Dairy Res. 1992, 59, 287-298. [CrossRef] [PubMed]

28. Neville, M.C.; Keller, R.P.; Seacat, J.; Casey, C.E.; Allen, J.C.; Archer, P. Studies on human lactation. I. Within-feed and between-breast variation in selected components of human milk. Am. J. Clin. Nutr. 1984, 40, 635-646. [CrossRef] [PubMed]

29. Hytten, F.E. Clinical and chemical studies in human lactation. I. Collection of milk samples. Br. Med. J. 1954, 23, 175-182. [CrossRef]

30. Daly, S.E.; Di Rosso, A.; Owens, R.A.; Hartmann, P.E. Degree of breast emptying explains changes in the fat content, but not fatty acid composition, of human milk. Exp. Physiol. 1993, 78, 741-755. [CrossRef] [PubMed]

31. Valentine, C.J.; Hurst, N.M.; Schanler, R.J. Hindmilk improves weight gain in low-birth-weight infants fed human milk. J. Pediatr. Gastroenterol. Nutr. 1994, 18, 474-477. [CrossRef] [PubMed]

(C) 2018 by the authors. Licensee MDPI, Basel, Switzerland. This article is an open access article distributed under the terms and conditions of the Creative Commons Attribution (CC BY) license (http:/ / creativecommons.org/licenses/by/4.0/). 
Article

\title{
Worldwide Variation in Human Milk Metabolome: Indicators of Breast Physiology and Maternal Lifestyle?
}

\author{
Melvin C. L. Gay ${ }^{1,+}{ }^{\dagger}$ Petya T. Koleva ${ }^{2,+}$, Carolyn M. Slupsky ${ }^{3, \dagger}$, Elloise du Toit ${ }^{4,+}$, \\ Merete Eggesbo ${ }^{5,+}$, Christine C. Johnson ${ }^{6,+}$, Ganesa Wegienka ${ }^{6,+}$, Naoki Shimojo ${ }^{7,+}$, \\ Dianne E. Campbell ${ }^{8, \dagger}$, Susan L. Prescott ${ }^{9,10, \dagger}$, Daniel Munblit ${ }^{11,12,+}$, Donna T. Geddes ${ }^{1, \dagger}$, \\ Anita L. Kozyrskyj ${ }^{13, *,+}$ and InVIVO LactoActive Study Investigators ${ }^{\dagger}$ \\ 1 School of Molecular Sciences, University of Western Australia, Perth, WA 6009, Australia; \\ melvin.gay@uwa.edu.au (M.C.L.G.); donna.geddes@uwa.edu.au (D.T.G.) \\ 2 Department of Pediatrics, University of Alberta, Edmonton, Alberta, AB T6G 1C9, Canada; \\ koleva@ualberta.ca \\ 3 Departments of Nutrition/Food Science \& Technology, University of California Davis, \\ California, CA 95616-5270, USA; cslupsky@ucdavis.edu \\ 4 Division of Medical Microbiology, University of Cape Town, Cape Town, Rondebosch 7701, South Africa; \\ elloisedutoit@gmail.com \\ 5 Department of Environmental Exposure and Epidemiology, Norwegian Institute of Public Health, \\ Oslo N-0213, Norway; merete.eggesbo@fhi.no \\ 6 Department of Public Health Sciences, Henry Ford Hospital, Detroit, MI 48208, USA; \\ cjohnso1@hfhs.org (C.C.J.); gwegien1@hfhs.org (G.W.) \\ 7 Department of Pediatrics, Chiba University, Chiba 260-8677, Japan; shimojo@faculty.chiba-u.jp \\ 8 Department of Allergy and Immunology, Children's Hospital at Westmead, University of Sydney, \\ Sydney, NSW 2145, Australia; dianne.campbell1@health.nsw.gov.au \\ 9 School of Medicine, University of Western Australia, Nedlands, WA 6009, Australia; \\ Susan.Prescott@telethonkids.org.au \\ 10 ORIGINS Project, Telethon Kids Institute, Perth Children's Hospital, Perth, WA 6009, Australia \\ 11 Department of Paediatrics, Imperial College London, London W2 1NY, UK; \\ daniel.munblit08@imperial.ac.uk \\ 12 Faculty of Pediatrics, Sechenov University, Moscow 119991, Russia \\ 13 Departments of Pediatrics/Obstetrics \& Gynecology, School of Public Health, University of Alberta, \\ Edmonton, Alberta AB T6G 1C9, Canada \\ * Correspondence: kozyrsky@ualberta.ca; Tel.: +1-780-248-5508 \\ † InVIVO Planetary Health of the Worldwide Universities Network (WUN), West New York, NJ 10704, USA.
}

Received: 1 August 2018; Accepted: 20 August 2018; Published: 23 August 2018

\begin{abstract}
Human milk provides essential substrates for the optimal growth and development of a breastfed infant. Besides providing nutrients to the infant, human milk also contains metabolites which form an intricate system between maternal lifestyle, such as the mother's diet and the gut microbiome, and infant outcomes. This study investigates the variation of these human milk metabolites from five different countries. Human milk samples $(n=109)$ were collected one month postpartum from Australia, Japan, the USA, Norway, and South Africa and were analyzed by nuclear magnetic resonance. The partial least squares discriminant analysis (PLS-DA) showed separation between either maternal countries of origin or ethnicities. Variation between countries in concentration of metabolites, such as 2-oxoglutarate, creatine, and glutamine, in human milk, between countries, could provide insights into problems, such as mastitis and/or impaired functions of the mammary glands. Several important markers of milk production, such as lactose, betaine, creatine, glutamate, and glutamine, showed good correlation between each metabolite. This work highlights the importance of milk metabolites with respect to maternal lifestyle and the environment, and also provides the framework for future breastfeeding and microbiome studies in a global context.
\end{abstract}


Keywords: human milk; milk metabolites; lactation; milk metabolomics

\section{Introduction}

Human milk provides all of the essential nutrients and bioactive substrates required for optimal growth and development of the nursing infant [1]. This includes not only large immunoglobulins and proteins, but also numerous low molecular weight substances, such as simple sugars and complex human milk oligosaccharides (HMOs), amino acids, short-chain fatty acids, and other energy metabolic pathway intermediates [2]. These nutrients work as part of a complex functional unit, operating in concert with intestinal enzymes to influence infant physiology. Many metabolites, such as HMOs, lactose, and other milk sugars, are also fermented by gut microbiota to generate additional metabolites. Variation in the human milk metabolome, namely with HMOs, is seen by maternal phenotype and diet [3]. Despite this, there are still only a few studies reporting on the determinants of small molecule concentrations in human milk or their role, and only limited studies on global regional differences in other milk components [4,5].

Patterns of infant growth are strong predictors of future cognitive performance and cardiometabolic health [6-8]. Breastfeeding may protect from rapid growth during infancy and the risk for overweight/obesity, diabetes, and high blood pressure [9]; however, this may vary with compositional factors in human milk [10]. Because human milk metabolites influence infant gut microbial composition, and gut microbial dysbiosis has been linked to future overweight [11,12], geographic variations in small milk molecules can conceivably predict regional differences in infant gut microbial development, infant growth, and future health. The activity of some milk metabolites directly reflects maternal diet, and hence would be a function of regional differences in dietary intake [13]. Taking as an example immune system activation of spillover lactose in the colon secondary to lactase deficiency in the small intestine [14], the activity of still other milk metabolites may be subject to regional differences in host genetics. With an aim to learn more about these less well-studied small molecular weight metabolites, we undertook a descriptive study of human milk metabolomics, comparing women from diverse geographical locations. Healthy women without atopic conditions were the focus of the comparison. We found regional differences in milk metabolites related to lactation performance that differentiated South African women from women from other countries.

\section{Materials and Methods}

With representation across ethnicity, maternal atopy status, and infant sex, 109 milk samples collected one month after birth were selected from six existing international cohorts: Perth, Australia $(n=29$ from 2 cohorts which include 21 atopic mothers, i.e., Perth \#1, [15] and 8 non-atopic mothers, i.e., Perth \#2) [16]; Chiba, Japan ( $n=12)$; Detroit, USA $(n=18)$ [17]; Oslo, Norway $(n=40)$ [18]; and Cape Town, South Africa $(n=10)$ [4]. The one-month postpartum time period was selected because human milk composition has stabilized by then [2]. Maternal atopy status was defined according to maternal report of asthma, eczema, allergies, or other atopic diseases, or at least one blood allergen-specific IgE level $\geq 35 \mathrm{kU} / \mathrm{L}$ for dust mite, dog, cat, Timothy grass, ragweed, Alternaria alternate, egg, or German cockroach. Research ethics approval was obtained from the Human Research Ethics Committee of The University of Western Australia, Human Research Ethics Committee of the Princess Margaret Hospital, Committee on Human Research of Chiba University, Institutional Review Board at Henry Ford Health System, Norwegian Regional Committees for Medical and Health Research Ethics, and University of Cape Town Human Research Ethical Committee.

Before sample collection, the mothers were given oral and written instructions for standardized collection of samples. Human milk samples were collected manually or with an electric breast pump into a sterile tube. Prior to collection, nipples and mammary areola were cleaned with soap and sterile water, and for the samples from South Africa, the area was additionally soaked with chlorhexidine to 
reduce contamination by skin microbes. All of the samples were kept frozen at $-20^{\circ} \mathrm{C}$ until delivery to the laboratory and then stored at $-20^{\circ} \mathrm{C}$ or $-80^{\circ} \mathrm{C}$ until further analysis. All the samples were shipped to Edmonton, Canada for storage, processing and Nuclear Magnetic Resonance (NMR) analysis at The Metabolomics Innovation Centre.

Milk metabolite levels were determined by NMR because of its high reproducibility and coverage of a large range of metabolites. Prior to NMR spectroscopy, milk samples were thawed on ice and mixed thoroughly. Approximately $500 \mu \mathrm{L}$ of each sample was filtered to remove residual lipids and protein using Amicon Ultra 0.5 mL 3-kDa cutoff spin filter (Millipore Sigma, Burlington, MA, USA). The filtration was performed at $10,000 \times g$ for $15 \mathrm{~min}$ at $4{ }^{\circ} \mathrm{C}$. Then, a $350 \mu \mathrm{L}$ clear filtrate was placed in a 1.5-mL Eppendorf tube, followed by the addition of $70 \mu \mathrm{L}$ of $\mathrm{D}_{2} \mathrm{O}$ and $60 \mu \mathrm{L}$ of standard buffer solution (585 mM NaHPO 4 ( $\mathrm{pH} 7.0$ ), $11.667 \mathrm{mM}$ disodium-2,2-dimethyl-2-silapentane-5-sulfonate (DSS), and $0.47 \% \mathrm{NaN}_{3}$ in $\left.\mathrm{H}_{2} \mathrm{O}\right)$. The samples $(460 \mu \mathrm{L})$ were then transferred to a regular NMR tube for subsequent NMR spectral analysis. All ${ }^{1} \mathrm{H}-\mathrm{NMR}$ spectra were collected on a Varian $500 \mathrm{MHz}$ Inova spectrometer equipped with a 5-mm HCN Z-gradient pulsed-field gradient cryogenic probe. ${ }^{1} \mathrm{H}-\mathrm{NMR}$ spectra were acquired at $25^{\circ} \mathrm{C}$ using the first transient of the Varian tnnoesy pulse sequence, which was chosen for its high degree of selective water suppression and quantitative accuracy of resonances around the solvent. Water suppression pulses were calibrated to achieve a bandwidth of 80 G. Spectra were collected with 128 transient and 8 steady-state scans using a 4-s acquisition time (48,000 complex points) and a 1-s recycle delay. Quality control (QC) mixtures which consisted of 4 metabolites at $1 \mathrm{mM}$ were analyzed for every 20 to 25 samples, and a relative standard deviation of $<2 \%$ was observed.

Before spectral analysis, all free induction decays were zero-filled to 64,000 data points and line broadened to $0.5 \mathrm{~Hz}$. The methyl singlet produced by a known quantity of DSS was used as an internal standard for chemical shift referencing (set to $0 \mathrm{ppm}$ ) and for quantification. All ${ }^{1} \mathrm{H}-\mathrm{NMR}$ spectra were processed and analyzed using the Chenomx NMR Suite Professional software package version 8.1 (Chenomx Inc., Edmonton, AB, Canada). The Chenomx NMR Suite software allows for qualitative and quantitative analysis of an NMR spectrum by manually fitting spectral signatures from an internal database to the spectrum. Typically, $90 \%$ of visible peaks were assigned to a compound, and more than $90 \%$ of the spectral area could be routinely fit using the Chenomx spectral analysis software. Most of the visible peaks were annotated with a compound name and expressed as $\mu \mathrm{mol} / \mathrm{L}$.

\section{Statistical Analysis}

Partial Least Square Discriminant Analysis (PLS-DA) was created using the Excel add-in Multibase 2015 package (Numerical Dynamics, Tokyo, Japan). PLS-DA was performed in order to maximize the separation between the different countries as well as the ethnicities of the mothers. Data preparation was made using the scaling method of standard deviations. Sample scatterplot and loading plots were compared where significant variables which contribute to sample distribution can be easily identified. Statistical analyses were carried out using R studio 1.1.414 (Rstudio Inc., Boston, MA, USA) with package nlme for linear mixed models to test for significant differences between the milk metabolites among different countries, and among ethnicities of the mothers. Milk metabolite levels were compared with each other using the Pearsons correlation across all the non-atopic mothers using Package corrplot [19]. Differences are considered to be significant if $p<0.05$.

\section{Results}

Of the 109 participating women, 69\% were Caucasian, 51\% were nursing male infants, and $43 \%$ had a history of atopy; this comparison oversampled atopic women and their additional results are the subject of another paper. The majority of South African women were of mixed race and all were non-atopic; most of the comparator cohorts were of Caucasian ancestry. Of the women in the US cohort, 39\% were African American (labelled as Black) and only one African-American woman was non-atopic. All women had delivered vaginally and did not receive intrapartum antibiotics. 


\subsection{Milk Metabolite Clusters by Country}

A total of 28 metabolites were identified in the human milk of our descriptive study, including sugars (fucose, glucose, lactose), amino acids (alanine, glutamine, glutamate, glycine, isoleucine, leucine, valine), choline and its metabolites, and energy metabolites (acetone, citrate, creatine, creatine phosphate, creatinine, lactate, 2-oxoglutarate, pyruvate, succinate), as shown in Figure 1. Most of the measured metabolites were within the range of those reported in other studies at a comparable time postpartum $[20,21]$.

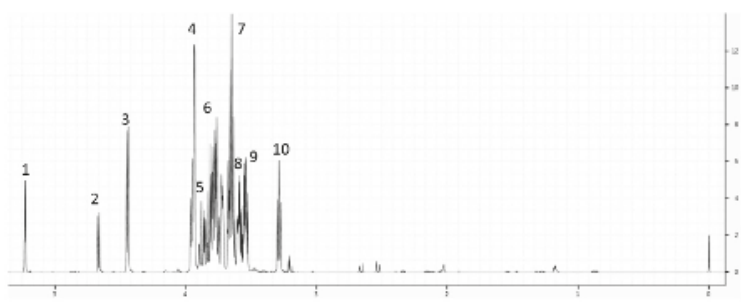

Figure 1. A representative ${ }^{1} \mathrm{H}$ nuclear magnetic resonance (NMR) spectrum of human milk. The ${ }^{1} \mathrm{H}$ chemical shifts for lactose are annotated 1 to 10 .

The PLS plot in Figure 2 shows three main clusters of milk metabolites in women by country as follows: (1) South African, (2) Australian atopic and US, and (3) Australian non-atopic and Norwegian cohorts. Milk metabolites of Japanese women overlapped between those of Norwegian and South African women. Creatine and 2-oxoglutarate were the main drivers of milk metabolite differences between South Africa and other countries. Glutamine and phosphocholine differentiated the milk of Norwegian and US women.
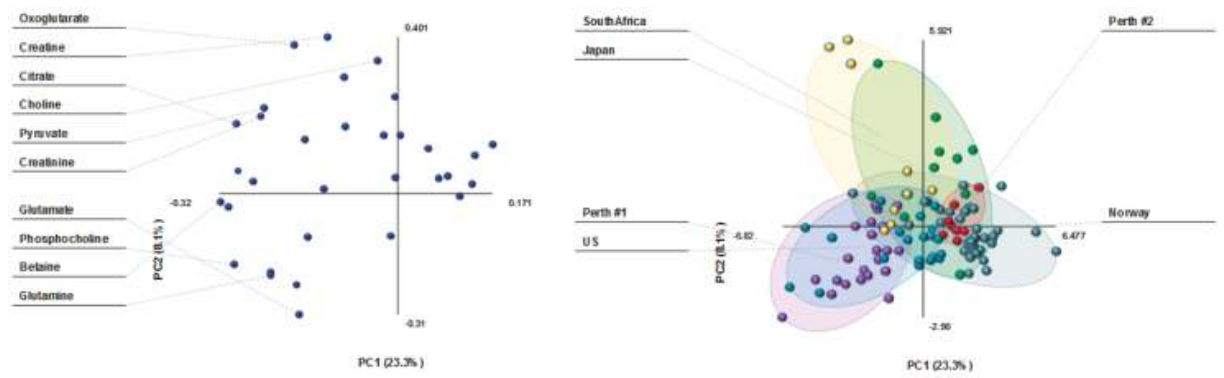

Figure 2. Partial Least Square Discriminant Analysis (PLS-DA) loading plot (left) and scatterplot (right) of human milk metabolites from 109 women in various countries. The score plot shows separation based on maternal country of origin (purple: Perth \#1, atopic mothers; red: Perth \#2, non-atopic mothers; green: Japan; aqua: US; dark green: Norway; and yellow: South Africa). The loading plot shows the milk metabolites that influence the separation based on maternal country of origin.

\subsection{Milk Metabolite Differences in Healthy, Non-Atopic Women}

Milk metabolite composition varied to the greatest extent between South African, and Norwegian or Australian women (Table 1). When compared to those of Norwegian women, the following milk metabolites were higher in concentration in South African women: lactose $(p=0.02), 2$-oxoglutarate $(p<0.001)$, citrate $(<0.001)$ and creatine $(p \leq 0.001) /$ creatine-phosphate $(p=0.02) /$ creatinine $(p<0.001)$, as well as betaine $(p<0.001)$ and glycerol-phosphocholine $(p=0.02)$. Except for 2-oxoglurate and glycerol-phosphocholine, similar differences in these milk metabolites were observed between South 
African and Australian women. No country differences were observed in other milk sugars (glucose or fucose) or other energy metabolite (i.e., succinate) concentrations.

In addition, a statistical significance was also noted for lower milk levels of glutamine $(p<0.001)$ in Norwegian versus US women. Japanese women had significantly higher milk levels of pyruvate $(p<0.01)$ and lactate $(p<0.01)$ than Norwegian women. Milk levels of methanol were significantly lower $(p<0.02)$ in Norway versus all other countries.

Table 1. Comparison of milk metabolites concentrations ( $\mu \mathrm{mol} / \mathrm{L})$ by country of origin.

\begin{tabular}{|c|c|c|c|c|c|}
\hline $\begin{array}{l}\text { MEAN (SD) } \\
\text { Metabolites/Countries }\end{array}$ & South Africa & Japan & Norway & US & Perth \#2 \\
\hline 2-Oxoglutarate & $79.6(25.6)$ & $79.1(49.2){ }^{\# \#}$ & $30.5(31.2) * * *$ & $39.0(12.7)$ * & $47.5(19.9)$ \\
\hline Acetone & $13.2(6.4)$ & $19.8(15.7)$ & $10.8(7.6)$ & $11.4(3.3)$ & $13.3(7.5)$ \\
\hline Alanine & $161.0(93.5)$ & $233.6(96.8)$ & $208.1(84.5)$ & $240.1(91.9)$ & $203.2(48.4)$ \\
\hline Betaine & $668.1(201.8)$ & $355.3(367.1)$ & $180.4(318.8)^{* * *}$ & $408.4(567.8)$ & $58.8(6.6)^{* * *}$ \\
\hline Caprate & $108.6(124.7)$ & $193.1(216.3)$ & $121.5(120.3)$ & $60.0(90.6)$ & $114.0(42.9)$ \\
\hline Caprylate & $157.1(137.2)$ & $357.6(497.0)$ & $131.6(207.1)$ & $92.7(132.5)$ & $82.7(47.1)$ \\
\hline Choline & 322.1 (194.9) & $192.0(128.3)$ & $184.1(163.7)$ & $133.0(77.0)$ & $138.5(71.1)$ \\
\hline Citrate & $5614(3920)$ & $2830(1313)$ * & $2718(1361)^{* * *}$ & $3642(1272)$ & $2856(836.1) *$ \\
\hline Creatine & $274.7(342.9)$ & $60.3(28.4) *$ & $63.9(26.9)^{* * *}$ & $56.8(23.4)$ * & $66.5(19.0)^{*}$ \\
\hline Creatine phosphate & $49.6(54.3)$ & $45.2(31.1)$ & $20.6(13.1) *$ & $42.0(12.7)$ & $23.9(7.6)$ \\
\hline Creatinine & $108.8(82.7)$ & $42.9(15.4) * *$ & $43.7(15.6)^{* * *}$ & $57.3(22.9)$ * & $45.0(5.7)^{* *}$ \\
\hline Formate & $83.4(79.4)$ & $112.2(127.8)$ & $902.0(1695.2)$ & $91.9(55.7)$ & $127.1(24.9)$ \\
\hline Fucose & $278.5(373.5)$ & $570.9(482.9)$ & $330.5(252.5)$ & $344.4(216.4)$ & $382.3(277.5)$ \\
\hline Glucose & 1347 (852.9) & 1563 (1327) & $926.4(747.8)$ & $1697(413.5)$ & 1699 (935.9) \\
\hline Glutamate & $858.9(491.3)$ & $1296(565.8)$ & $1467(835.2)$ & $1573(830.2)$ & $1554(369.4)$ \\
\hline Glutamine & $282.0(150.9)$ & $103.0(88.1)$ & $101.9(119.0)$ & $514.2(618.2)$ & $207.6(178.2)$ \\
\hline Glycine & 2796 (759.9) & 3135 (1373) & 2512 (1191) & $1641(1777)$ & 1919 (949.5) \\
\hline Guanidoacetate & 4771 (1048) & 3777 (1747) & $3373(2944)$ & 3435 (1770) & $1622(286.5)$ * \\
\hline Isoleucine & $20.8(12.2)$ & $24.1(25.9)$ & $24.2(21.1)$ & $10.9(2.6)$ & $12.0(4.0)$ \\
\hline Lactate & $213.7(63.1)$ & $3215(5561) * * \#$ & $443.5(693.6)$ & $137.8(102.0)$ & $125.7(41.5)$ \\
\hline Lactose & $189,874(34,234)$ & $139,161(83,333)$ & $101,351(84,404)$ * & $161,160(123,638)$ & $83,524(23,509)$ * \\
\hline Leucine & $33.7(21.1)$ & $49.9(61.2)$ & $66.2(76.2)$ & $30.9(8.9)$ & $34.0(7.8)$ \\
\hline Methanol & $78.0(17.6)$ & 97.7 (49.2) \#\#\# & $46.1(19.3)^{* * *}$ & $89.2(6.7)$ & $72.0(6.8)^{\#}$ \\
\hline Phosphocholine & $636.9(328.6)$ & $784.7(470.6)$ & $463.7(326.7)$ & $698.3(231.2)$ & $488.4(145.6)$ \\
\hline Pyruvate & $53.3(29.5)$ & $68.6(69.8)$ & $20.0(26.3) *$ & 31.7 (39.7) & $6.3(2.5)$ * \\
\hline Succinate & $48.3(67.0)$ & $47.9(25.9)$ & $210.8(515.7)$ & $21.2(9.6)$ & $49.3(15.7)$ \\
\hline Valine & $35.3(11.9)$ & $63.9(33.5)$ & $65.6(32.7) *$ & $50.2(21.1)$ & $57.4(17.6)$ \\
\hline Glycerophosphocholine & $797.6(439.3)$ & $645.0(393.0)$ & $414.9(346.0) *$ & $589.2(239.7)$ & $517.4(118.5)$ \\
\hline $\begin{array}{l}\text { \# metabolite differences } \\
\text { with South Africa }\end{array}$ & na & 4 & 11 & 3 & 7 \\
\hline $\begin{array}{l}\text { \# metabolite differences } \\
\text { with Norway }\end{array}$ & - & 4 & na & 2 & 1 \\
\hline
\end{tabular}

${ }^{*} p<0.05,{ }^{* *} p<0.01,{ }^{* * *} p<0.001$ when reference $=$ South Africa; ${ }^{\#} p<0.05,{ }^{\# \#} p<0.01,{ }^{\# \# \#} p<0.001$ when reference = Norway; na: not applicable.

\subsection{Milk Metabolite Correlations}

Correlations between milk metabolites are reported in Figure 3. Both lactose and betaine were positively correlated with citrate, creatine, and the phosphocholines; milk lactose levels significantly rose with those of 2-oxoglutarate. 


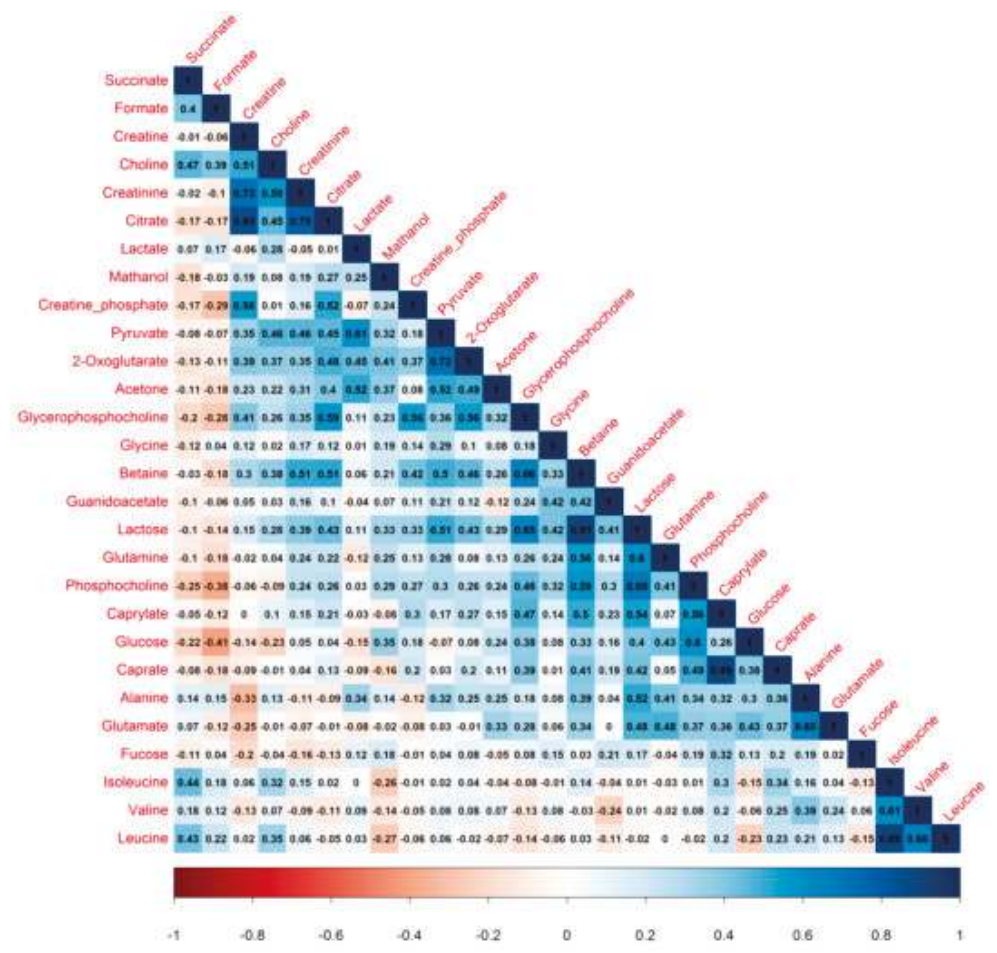

Figure 3. Correlation plot of 28 human milk metabolites from non-atopic mothers. Values shows Pearson correlation coefficients between pairs of metabolites. Positive correlation, zero correlation and negative correlation are represented by colors ranging from blue to white to red, respectively.

\subsection{Milk Metabolite Clusters by Race/Ethnicity}

Metabolite variation by ethnicity in the PLS plots was similar to country differences, showing three clusters of milk composition: Black, Caucasian and Asian (Figure 4). Milk lactate differentiated the Asian population from the rest. 2-Oxoglutarate and creatine were the main drivers of the milk cluster in the Black population, whereas the main milk metabolites in the Caucasian cluster were glutamate, glutamine, glucose, and phosphocholine.
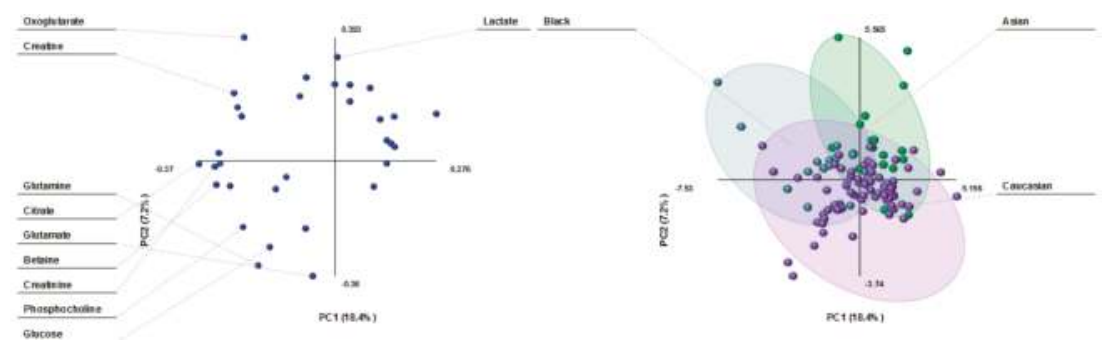

Figure 4. PLS-DA loading plot (left) and scatterplot (right) of human milk metabolites from women of different ethnic groups. The score plot shows separation based on maternal ethnicity (purple: Caucasian; green: Asian; and dark green: Black). The loading plot shows the milk metabolites that influence the separation based on maternal ethnicity. 


\subsection{Milk Metabolite Variation by Ethnicity in Healthy, Non-Atopic Women}

2-Oxoglutarate $(p=0.008)$ and creatine $(p=0.02) /$ creatine phosphate $(p=0.008) /$ creatinine $(p=0.03)$, as well as betaine $(p=0.01)$ and glycerophosphocholine $(p=0.009)$ concentrations were higher in the milk of Black compared to Caucasian women (Table 2). Although lactose levels were also higher, this difference did not reach statistical significance $(p=0.07)$. In contrast, milk valine levels were lower in Black than in Caucasian women. In essence, these results compare mixed-race South African to Caucasian women, because only one US Black-race woman was a member of the Black race group. Compared to that of Asian women, the milk of Caucasians had significantly lower levels of lactate $(p=0.03)$ and fucose $(p=0.03)$.

Table 2. Comparison of milk metabolite concentrations ( $\mu \mathrm{mol} / \mathrm{L})$ by maternal ethnicity.

\begin{tabular}{|c|c|c|c|}
\hline $\begin{array}{l}\text { MEAN (SD) } \\
\text { Metabolites/Countries }\end{array}$ & Black & Caucasian & Asian \\
\hline 2-Oxoglutarate & $75.4(31.1)$ & $37.6(29.2) * *$ & $55.3(47.1)$ \\
\hline Acetone & $12.9(5.3)$ & $11.9(7.4)$ & $14.2(13.0)$ \\
\hline Alanine & $164.4(87.9)$ & $210.6(82.1)$ & $222.3(90.9)$ \\
\hline Betaine & $581.1(283.6)$ & $204.9(352.7) *$ & $354.3(390.1)$ \\
\hline Caprate & $77.2(87.8)$ & $109.8(112.3)$ & $185.1(168.3)$ \\
\hline Caprylate & $134.7(136.2)$ & $118.1(184.1)$ & $266.9(375.3)$ \\
\hline Choline & $285.1(167.0)$ & $170.2(154.4)$ & 218.7 (145.5) \\
\hline Citrate & $4892(3687)$ & $3123(1833)$ & $2776(1061)$ \\
\hline Creatine & $229.4(326.5)$ & $77.6(101.1)$ * & $63.2(28.5) *$ \\
\hline Creatine phosphate & $54.0(56.2)$ & $24.1(13.7)^{* *}$ & $35.9(26.8)$ \\
\hline Creatinine & $89.7(68.2)$ & $51.6(37.0) *$ & $44.2(15.0)$ * \\
\hline Formate & $86.5(79.3)$ & $582.6(1363)$ & $534.5(1350)$ \\
\hline Fucose & $328.0(376.9)$ & $306.0(236.6)^{\#}$ & $556.4(391.5)$ \\
\hline Glucose & $1264(672.2)$ & $1263(866.0)$ & 1148 (1083) \\
\hline Glutamate & $1021(614.5)$ & 1478 (791.6) & $1272(536.3)$ \\
\hline Glutamine & $252.4(122.0)$ & $204.7(310.6)$ & $89.5(79.2)$ \\
\hline Glycine & $2646(1051)$ & $2360(1307)$ & 2596 (1169) \\
\hline Guanidoacetate & 4405 (1378) & $2912(2538)$ & 4544 (1916) \\
\hline Isoleucine & $17.3(11.5)$ & $20.5(18.4)$ & $23.4(21.8)$ \\
\hline Lactate & $198.2(82.2)$ & $339.5(600.5)^{\#}$ & $1918(4213)$ \\
\hline Lactose & $178,365(50,934)$ & $110,060(86,787)$ & $131,284(85,509)$ \\
\hline Leucine & $31.5(14.8)$ & $52.1(56.4)$ & $64.0(87.0)$ \\
\hline Methanol & $80.4(15.7)$ & $59.3(23.6)$ & $71.0(46.6)$ \\
\hline Phosphocholine & $584.6(305.1)$ & $521.9(304.1)$ & $642.5(435.6)$ \\
\hline Pyruvate & $46.5(25.3)$ & $21.4(29.6)$ & $47.2(58.8)$ \\
\hline Succinate & $36.1(53.0)$ & $152.7(446.7)$ & $100.8(124.4)$ \\
\hline Valine & $34.6(12.0)$ & $61.1(26.2) *$ & $63.9(40.5)$ \\
\hline Glycerophosphocholine & $846.4(424.6)$ & $467.7(319.9) * *$ & $518.9(327.7)$ \\
\hline \# metabolite differences with Black race & na & 7 & 2 \\
\hline \# metabolite differences with Asian race & 3 & na & 2 \\
\hline
\end{tabular}

${ }^{*} p<0.05,{ }^{* *} p<0.01$, when reference $=$ Black; ${ }^{\#} p<0.05$, when reference $=$ Asian; na: not applicable.

\section{Discussion}

In this comparison of 109 human milk samples from five countries, clustering by country of origin was observed, such that milk metabolites in South African women differed substantially from those in Norwegian and US women, and women in either of the two Australian cohorts. Milk metabolites in Japanese women formed an overlapping cluster between Norway and South Africa. Creatine and 2-oxoglutarate were the milk metabolites mainly responsible for these regional differences; they were also the highest in South African women among the cohort. Differences in these milk metabolites were evident in a comparison of healthy, non-atopic women in all countries studied; lactose milk levels were also the highest in South African women. Since several low molecular weight metabolites levels 
are tightly controlled in human milk [3], identifying differences between maternal countries of origin are noteworthy. In the pursuant paragraphs, we summarize what is known about these metabolites, highlight other findings, and offer candidate explanations for the higher levels seen in South African women when compared to women living in Australia or the northern hemisphere.

After protein, lactose is the most plentiful component of human milk and often measured to reflect the carbohydrate energy content. Human milk lactose is found to be associated with infant growth in observational and simulation studies [22,23]. Since levels rise during the postpartum period in breastfeeding women [24], lactose has also been labelled as a marker for milk production. In our comparison, all human milk samples were obtained within one month of birth, removing timing of collection as an explanation for differences in milk lactose or other metabolite concentrations. In two to five months old infants, Gridneva et al. documented higher lactose milk levels in Australian women who breastfed more frequently [16]. Their results are congruent with a metabolomics study of sow milk, whereby Tan et al. reported higher milk lactose levels with higher rather than lower lactation performance [25]. Glucose and fucose, other sugars typically found in human milk, did not differ in their levels across country cohorts in our study. Lactose has also been found to have antimicrobial and innate immunity-inducing properties $[2,26,27]$. Hence, the much greater levels of milk lactose in South African women than in women living in more industrialized societies may reflect a greater breastfeeding frequency, or maternal programming to prevent infection.

Although milk sugars are the main substrates for energy generation and the production of oligosaccharides [28], little is known about these intermediate metabolites of the tricarboxylic acid energy cycle (TCA cycle), such as 2-oxoglutarate, citrate, or succinate. In our study, lactose levels were positively correlated with milk citrate and 2-oxoglutarate. Milk 2-oxoglutarate and citrate levels were also highest in South African women in our comparison; however, no differences were observed in milk succinate concentrations. As shown in a study of US women, the levels of milk 2-oxoglutarate in women feeding term infants typically decline within one month of birth [20]. Of note, lower 2-oxoglutarate levels have been detected in the milk of dairy cows with mastitis (breast duct infection), which is interpreted to be a function of greater consumption of this TCA cycle intermediate by resident microbiota or infecting microbes [3,29]. Mastitis and subclinical mastitis are common infections postpartum in industrialized countries [30], which offers another explanation for 2-oxoglutarate consumption and lower milk concentrations in US, Australian, and Norwegian women. Japanese women had higher milk levels of other energy-related intermediates such as lactate and pyruvate than Norwegian women; not much is known about the milk levels of these metabolites in humans. Compared to non-lactating cows, the TCA and related cycles, including pyruvate metabolism, were found to be most activated in the mammary glands of lactating cows [31]; pyruvate and lactate were among the 118 metabolites shared between the mammary gland and milk.

Lactose is produced by mammary cells, but other low-weight molecules are speculated to diffuse into human milk and serve as indicators of maternal plasma levels [2]. Methanol is found in human milk and can originate from maternal circulation following the consumption of fruits, vegetables, alcohol, and artificial sweeteners, and from exposure to environmental tobacco smoke [32]. Other examples are betaine, a choline metabolite whose levels increase with dietary intake of choline [33]. Consistently, milk betaine and the phosphocholines were positively correlated in our study. We also found milk methanol to be lowest in Norwegian women, whereas betaine levels were lowered in Australian milk. Milk creatinine (derived from creatine, an energy metabolite) levels are also related to circulating blood levels [34]; urinary creatinine also reportedly rises postpartum during breastfeeding [24]. In lactating cows, creatine and lactose are the two metabolites found in the stomach, serum, milk, and urine, denoting their importance in the lactation process [31]. Of note, betaine is an osmolytic and its supplementation can increase milk yield in cows [35]. Together with the higher levels of milk lactose and betaine, elevated levels of milk creatine or its metabolite, creatinine, may indicate higher milk production in South African women. 
In Tan et al.'s study of sow milk [25], glutamate and glutamine were additional markers of high milk production. Glutamate is produced when 2-oxoglutarate combines with glutamine. Glutamine and glutamate are the most abundant amino acids in human milk, increasing with each stage of lactation, and are largely derived during lactation from muscle protein breakdown of glutamine $[21,36]$. Higher levels of the amino acid valine have also been found to be associated with higher bovine milk yield [37]. It is then noteworthy that glutamate was the metabolite which distinguished US human milk composition from that of Norwegian and Japanese women. Milk glutamine levels were significantly lower in Norwegian and Japanese than in US women, which is consistent with the general observation that glutamine levels are higher in North America than in Asia [21]. Both glutamine and glutamate are important energy sources for intestinal cells and are needed for infant growth. When Dangat et al. compared healthy women in India to women with maternal conditions such as pre-eclampsia, which are associated with growth retardation in offspring, they found healthy women to have higher glutamine and glutamate levels in their milk, as well as higher lactose concentrations [38].

Ethnic differences between the same milk metabolome of mixed Black and Caucasian women were consistent with the country variation we observed, and further indicated a similarity among Caucasian women in Norway, Australia, and the US, potentially in terms of breastfeeding practices and/or maternal diet, health status, or genetics. Maternal diet has a reported influence on some human milk constituents, such as fatty acids, vitamins, and minerals; maternal intake of carbohydrates or fat is unrelated to milk lactose levels, but little is known about other low molecular weight substrates [13]. Since several low molecular metabolites detected in milk can be consumed or produced by resident microbiota, ethnic differences in these milk metabolites may also be a function of variation in human milk microbiota. As shown by Kumar et al., human milk microbial composition can vary according to the degree of societal industrialization, whereby the abundance of Proteobacteria is observed to be higher in milk samples of South African women [4]. In this regard, human milk lactose levels have been reported by others to vary inversely with milk Enterobacteria [26]. Further, maternal intake of lactose has been inversely correlated with milk concentrations within the Firmicutes phylum [39]. Unfortunately, data on maternal diet was not collected at the time that human milk samples were obtained in our study. Additionally, no women of Asian ethnicity were present in the Australian or US cohorts, and too few non-atopic women of Black race were recruited in the US cohort to speculate on genetic versus environmental origins of the milk metabolite variation.

The strengths of this international comparative study are that all human milk samples were selected at the same stage of lactation with similar maternal characteristics, and processed with NMR spectroscopy in one center. As has been reported for other milk constituents [40], despite not following an identical protocol for milk collection, the individual country samples were similar across several milk metabolites whose levels have been reported to be tightly controlled [20].

\section{Conclusions}

Human milk composition is optimal for infant growth and development. As we and others seek to determine how societal factors impact on the infant gut microbiome, immune development and subsequent health, understanding population differences in early nutrition is essential. This present work should be considered a first step in helping to frame breastfeeding/microbiome studies in a global context. It reminds us that regional maternal diet and breastfeeding practices have the capacity to influence milk composition. In addition to trying to understand this variation, we must also leverage study findings to inform policy, especially in times of change. In our study, the milk composition of South African women had higher levels of lactose, creatine and energy metabolites. How will South African infants fare against the rising trends of not being fed colostrum, receiving short exclusive breastfeeding and being introduced to solids at an early stage [41] in a country historically known for lower infant growth failure rates than it neighbors [42]? Understanding people and their place and purpose on this planet is essential to understanding the complexity we face in harnessing the health of the microbiome. 
Author Contributions: Formal analysis, M.C.L.G.; Funding acquisition, E.d.T., M.E., C.C.J., G.W., N.S., D.E.C., S.L.P., D.M., D.T.G., and A.L.K.; Investigation, P.T.K.; Supervision, E.d.T., M.E., C.C.J., G.W., N.S., D.E.C., S.L.P., D.M., D.T.G., and A.L.K.; Visualization, M.C.L.G.; Writing—review \& editing, M.C.L.G., P.T.K., C.M.S., E.d.T., M.E., C.C.J., G.W., N.S., D.E.C., S.L.P., D.M., D.T.G., and A.L.K.

Funding: This research was funded by the World Universities Network (WUN) and Canadian Institutes of Health Research (CIHR). D.T.G. and M.C.L.G. received an unrestricted research grant from Medela A.G.

Acknowledgments: We are grateful to the mothers who provided their valuable data in the individual study cohorts and the research assistants who collected it. We would also like to acknowledge the contributions of Sarah Bridgman and Jennifer Petrie in formatting the manuscript. inVIVO LactoActive study investigators: Anita L. Kozyrskyj (lead), University of Alberta; Dianne E. Campbell, Children's Hospital at Westmead \& University of Sydney; Cecilie Dahl, Norwegian Institute of Public Health; Elloise du Toit, University of Cape Town; Merete Eggesbo, Norwegian Institute of Public Health; Melvin C. L. Gay, University of Western Australia; Donna T. Geddes, University of Western Australia; Aveni Haynes, University of Western Australia; Peter Hsu, Children's Hospital at Westmead \& University of Sydney; Petya T. Koleva, University of Alberta; Christine C. Johnson, Henry Ford Hospital; Charles Mackay, Monash University; Daniel Munblit, Imperial College London \& Sechenov University; John Penders, Maastricht University; Harald Renz, University of Marburg; Susan L. Prescott, Perth Children's Hospital \& University of Western Australia; Naoki Shimojo, Chiba University; Carolyn M. Slupsky, University of California Davis; Carel Thijs, Maastricht University; Ganesa Wegienka, Henry Ford Hospital; Christina West, Umea University.

Conflicts of Interest: The authors declare no conflict of interest.

\section{References}

1. Hennet, T.; Borsig, L. Breastfed at tiffany's. Trends Biochem. Sci. 2016, 41, 508-518. [CrossRef] [PubMed]

2. Demmelmair, H.; Koletzko, B. Variation of metabolite and hormone contents in human milk. Clin. Perinatol. 2017, 44, 151-164. [CrossRef] [PubMed]

3. Smilowitz, J.T.; O'Sullivan, A.; Barile, D.; German, J.B.; Lonnerdal, B.; Slupsky, C.M. The human milk metabolome reveals diverse oligosaccharide profiles. J. Nutr. 2013, 143, 1709-1718. [CrossRef] [PubMed]

4. Kumar, H.; du Toit, E.; Kulkarni, A.; Aakko, J.; Linderborg, K.M.; Zhang, Y.; Nicol, M.P.; Isolauri, E.; Yang, B.; Collado, M.C.; et al. Distinct patterns in human milk microbiota and fatty acid profiles across specific geographic locations. Front. Microbiol. 2016, 7, 1619. [CrossRef] [PubMed]

5. Fu, Y.; Liu, X.; Zhou, B.; Jiang, A.C.; Chai, L. An updated review of worldwide levels of docosahexaenoic and arachidonic acid in human breast milk by region. Public Health Nutr. 2016, 19, 2675-2687. [CrossRef] [PubMed]

6. Victora, C.G.; Adair, L.; Fall, C.; Hallal, P.C.; Martorell, R.; Richter, L.; Sachdev, H.S. Maternal and child undernutrition: Consequences for adult health and human capital. Lancet 2008, 371, 340-357. [CrossRef]

7. Adair, L.S.; Fall, C.H.; Osmond, C.; Stein, A.D.; Martorell, R.; Ramirez-Zea, M.; Sachdev, H.S.; Dahly, D.L.; Bas, I.; Norris, S.A.; et al. Associations of linear growth and relative weight gain during early life with adult health and human capital in countries of low and middle income: Findings from five birth cohort studies. Lancet 2013, 382, 525-534. [CrossRef]

8. Garza, C.; Borghi, E.; Onyango, A.W.; de Onis, M. Parental height and child growth from birth to 2 years in the WHO multicentre growth reference study. Matern. Child Nutr. 2013, 9, 58-68. [CrossRef] [PubMed]

9. Victora, C.G.; Bahl, R.; Barros, A.J.; Franca, G.V.; Horton, S.; Krasevec, J.; Murch, S.; Sankar, M.J.; Walker, N.; Rollins, N.C. Breastfeeding in the 21st century: Epidemiology, mechanisms, and lifelong effect. Lancet 2016, 387, 475-490. [CrossRef]

10. Munblit, D.; Peroni, D.G.; Boix-Amoros, A.; Hsu, P.S.; Van't Land, B.; Gay, M.C.L.; Kolotilina, A.; Skevaki, C.; Boyle, R.J.; Collado, M.C.; et al. Human milk and allergic diseases: An unsolved puzzle. Nutrients 2017, 9, 894. [CrossRef] [PubMed]

11. Davis, J.C.; Totten, S.M.; Huang, J.O.; Nagshbandi, S.; Kirmiz, N.; Garrido, D.A.; Lewis, Z.T.; Wu, L.D.; Smilowitz, J.T.; German, J.B.; et al. Identification of oligosaccharides in feces of breast-fed infants and their correlation with the gut microbial community. Mol. Cell Proteomics 2016, 15, 2987-3002. [CrossRef] [PubMed]

12. Koleva, P.T.; Bridgman, S.L.; Kozyrskyj, A.L. The infant gut microbiome: Evidence for obesity risk and dietary intervention. Nutrients 2015, 7, 2237-2260. [CrossRef] [PubMed]

13. Bravi, F.; Wiens, F.; Decarli, A.; Dal Pont, A.; Agostoni, C.; Ferraroni, M. Impact of maternal nutrition on breast-milk composition: A systematic review. Am. J. Clin. Nutr. 2016, 104, 646-662. [CrossRef] [PubMed]

14. Wahlqvist, M.L. Lactose nutrition in lactase nonpersisters. Asia Pac. J. Clin. Nutr. 2015, 24, S21-S25. [PubMed] 
15. Taylor, A.L.; Dunstan, J.A.; Prescott, S.L. Probiotic supplementation for the first 6 months of life fails to reduce the risk of atopic dermatitis and increases the risk of allergen sensitization in high-risk children: A randomized controlled trial. J. Allergy Clin. Immunol. 2007, 119, 184-191. [CrossRef] [PubMed]

16. Gridneva, Z.; Kugananthan, S.; Hepworth, A.R.; Tie, W.J.; Lai, C.T.; Ward, L.C.; Hartmann, P.E.; Geddes, D.T. Effect of human milk appetite hormones, macronutrients, and infant characteristics on gastric emptying and breastfeeding patterns of term fully breastfed infants. Nutrients 2016, 9, 15. [CrossRef] [PubMed]

17. Wegienka, G.; Havstad, S.; Joseph, C.L.; Zoratti, E.; Ownby, D.; Woodcroft, K.; Johnson, C.C. Racial disparities in allergic outcomes in african americans emerge as early as age 2 years. Clin. Exp. Allergy 2012, 42, 909-917. [CrossRef] [PubMed]

18. Eggesbo, M.; Thomsen, C.; Jorgensen, J.V.; Becher, G.; Odland, J.O.; Longnecker, M.P. Associations between brominated flame retardants in human milk and thyroid-stimulating hormone (TSH) in neonates. Environ. Res. 2011, 111, 737-743. [CrossRef] [PubMed]

19. Package "Corrplot". Available online: https://cran.r-project.org/web/packages/corrplot/corrplot.pdf. (accessed on 23 August 2018).

20. Spevacek, A.R.; Smilowitz, J.T.; Chin, E.L.; Underwood, M.A.; German, J.B.; Slupsky, C.M. Infant maturity at birth reveals minor differences in the maternal milk metabolome in the first month of lactation. J. Nutr. 2015, 145, 1698-1708. [CrossRef] [PubMed]

21. Zhang, Z.; Adelman, A.S.; Rai, D.; Boettcher, J.; Lonnerdal, B. Amino acid profiles in term and preterm human milk through lactation: A systematic review. Nutrients 2013, 5, 4800-4821. [CrossRef] [PubMed]

22. Prentice, P.; Ong, K.K.; Schoemaker, M.H.; van Tol, E.A.; Vervoort, J.; Hughes, I.A.; Acerini, C.L.; Dunger, D.B. Breast milk nutrient content and infancy growth. Acta Paediatr. 2016, 105, 641-647. [CrossRef] [PubMed]

23. Nilsson, A.; Mardinoglu, A.; Nielsen, J. Predicting growth of the healthy infant using a genome scale metabolic model. NPJ Syst. Biol. Appl. 2017, 3, 3. [CrossRef] [PubMed]

24. Sachse, D.; Baerug, A.; Sletner, L.; Birkeland, K.I.; Nakstad, B.; Jenum, A.K.; Berg, J.P. Urine nmr metabolomics analysis of breastfeeding biomarkers during and after pregnancy in a large prospective cohort study. Scand. J. Clin. Lab. Investig. 2014, 74, 264-272. [CrossRef] [PubMed]

25. Tan, C.; Zhai, Z.; Ni, X.; Wang, H.; Ji, Y.; Tang, T.; Ren, W.; Long, H.; Deng, B.; Deng, J.; et al. Metabolomic profiles reveal potential factors that correlate with lactation performance in sow milk. Sci. Rep. 2018, 8, 10712. [CrossRef] [PubMed]

26. Boix-Amoros, A.; Collado, M.C.; Mira, A. Relationship between milk microbiota, bacterial load, macronutrients, and human cells during lactation. Front. Microbiol. 2016, 7, 492. [CrossRef] [PubMed]

27. Cederlund, A.; Kai-Larsen, Y.; Printz, G.; Yoshio, H.; Alvelius, G.; Lagercrantz, H.; Stromberg, R.; Jornvall, H.; Gudmundsson, G.H.; Agerberth, B. Lactose in human breast milk an inducer of innate immunity with implications for a role in intestinal homeostasis. PLoS ONE 2013, 8, e53876. [CrossRef] [PubMed]

28. Cocinero, E.J.; Carcabal, P. Carbohydrates. Top. Curr. Chem. 2015, 364, 299-333. [PubMed]

29. Xi, X.; Kwok, L.Y.; Wang, Y.; Ma, C.; Mi, Z.; Zhang, H. Ultra-performance liquid chromatography-quadrupoletime of flight mass spectrometry ms(e)-based untargeted milk metabolomics in dairy cows with subclinical or clinical mastitis. J. Dairy Sci. 2017, 100, 4884-4896. [CrossRef] [PubMed]

30. Axelsson, D.; Blomberg, M. Prevalence of postpartum infections: A population-based observational study. Acta. Obstet. Gynecol. Scand. 2014, 93, 1065-1068. [CrossRef] [PubMed]

31. Sun, H.Z.; Shi, K.; Wu, X.H.; Xue, M.Y.; Wei, Z.H.; Liu, J.X.; Liu, H.Y. Lactation-related metabolic mechanism investigated based on mammary gland metabolomics and 4 biofluids' metabolomics relationships in dairy cows. BMC Genom. 2017, 18, 936. [CrossRef] [PubMed]

32. Dorokhov, Y.L.; Shindyapina, A.V.; Sheshukova, E.V.; Komarova, T.V. Metabolic methanol: Molecular pathways and physiological roles. Physiol. Rev. 2015, 95, 603-644. [CrossRef] [PubMed]

33. Fischer, L.M.; da Costa, K.A.; Galanko, J.; Sha, W.; Stephenson, B.; Vick, J.; Zeisel, S.H. Choline intake and genetic polymorphisms influence choline metabolite concentrations in human breast milk and plasma. Am. J. Clin. Nutr. 2010, 92, 336-346. [CrossRef] [PubMed]

34. Balzer, M.S.; Gross, M.M.; Lichtinghagen, R.; Haller, H.; Schmitt, R. Got milk? Breastfeeding and milk analysis of a mother on chronic hemodialysis. PLOS ONE 2015, 10, e143340. [CrossRef] [PubMed]

35. Peterson, S.E.; Rezamand, P.; Williams, J.E.; Price, W.; Chahine, M.; McGuire, M.A. Effects of dietary betaine on milk yield and milk composition of mid-lactation holstein dairy cows. J. Dairy Sci. 2012, 95, 6557-6562. [CrossRef] [PubMed] 
36. Manso, H.E.; Filho, H.C.; de Carvalho, L.E.; Kutschenko, M.; Nogueira, E.T.; Watford, M. Glutamine and glutamate supplementation raise milk glutamine concentrations in lactating gilts. J. Anim. Sci. Biotechnol. 2012, 3, 2. [CrossRef] [PubMed]

37. Wu, X.; Sun, H.; Xue, M.; Wang, D.; Guan, L.L.; Liu, J. Serum metabolome profiling revealed potential biomarkers for milk protein yield in dairy cows. J. Proteomics 2018, 184, 54-61. [CrossRef] [PubMed]

38. Dangat, K.; Upadhyay, D.; Kilari, A.; Sharma, U.; Kemse, N.; Mehendale, S.; Lalwani, S.; Wagh, G.; Joshi, S.; Jagannathan, N.R. Altered breast milk components in preeclampsia; an in-vitro proton nmr spectroscopy study. Clin. Chim. Acta 2016, 463, 75-83. [CrossRef] [PubMed]

39. Williams, J.E.; Carrothers, J.M.; Lackey, K.A.; Beatty, N.F.; York, M.A.; Brooker, S.L.; Shafii, B.; Price, W.J.; Settles, M.L.; McGuire, M.A.; et al. Human milk microbial community structure is relatively stable and related to variations in macronutrient and micronutrient intakes in healthy lactating women. J. Nutr. 2017, 147, 1739-1748. [PubMed]

40. Munblit, D.; Treneva, M.; Peroni, D.G.; Colicino, S.; Chow, L.; Dissanayeke, S.; Abrol, P.; Sheth, S.; Pampura, A.; Boner, A.L.; et al. Colostrum and mature human milk of women from London, Moscow, and Verona: Determinants of immune composition. Nutrients 2016, 8, e695. [CrossRef] [PubMed]

41. Budree, S.; Goddard, E.; Brittain, K.; Cader, S.; Myer, L.; Zar, H.J. Infant feeding practices in a south African birth cohort-a longitudinal study. Matern. Child Nutr. 2017, 13, 13. [CrossRef] [PubMed]

42. Osgood-Zimmerman, A.; Millear, A.I.; Stubbs, R.W.; Shields, C.; Pickering, B.V.; Earl, L.; Graetz, N.; Kinyoki, D.K.; Ray, S.E.; Bhatt, S.; et al. Mapping child growth failure in Africa between 2000 and 2015. Nature 2018, 555, 41-47. [CrossRef] [PubMed]

(C) 2018 by the authors. Licensee MDPI, Basel, Switzerland. This article is an open access article distributed under the terms and conditions of the Creative Commons Attribution (CC BY) license (http:/ / creativecommons.org/licenses/by/4.0/). 


\title{
Review \\ Human Milk Lipidomics: Current Techniques and Methodologies
}

\author{
Alexandra D. George ${ }^{1}$, Melvin C. L. Gay ${ }^{1}$, Robert D. Trengove ${ }^{2}$ and Donna T. Geddes ${ }^{1, *}$ \\ 1 School of Molecular Sciences, The University of Western Australia, Crawley, Perth, WA 6009, Australia; \\ alexandra.george@research.uwa.edu.au (A.D.G.); melvin.gay@uwa.edu.au (M.C.L.G.) \\ 2 Separation Science and Metabolomics Laboratory, Murdoch University, Murdoch, Perth, WA 6150, Australia; \\ R.Trengove@murdoch.edu.au \\ * Correspondence: donna.geddes@uwa.edu.au; Tel.: +61-8-6488-7006
}

Received: 30 July 2018; Accepted: 23 August 2018; Published: 26 August 2018

check for updates

\begin{abstract}
Human milk contains a complex combination of lipids, proteins, carbohydrates, and minerals, which are essential for infant growth and development. While the lipid portion constitutes only $5 \%$ of the total human milk composition, it accounts for over $50 \%$ of the infant's daily energy intake. Human milk lipids vary throughout a feed, day, and through different stages of lactation, resulting in difficulties in sampling standardization and, like blood, human milk is bioactive containing endogenous lipases, therefore appropriate storage is critical in order to prevent lipolysis. Suitable sample preparation, often not described in studies, must also be chosen to achieve the aims of the study. Gas chromatography methods have classically been carried out to investigate the fatty acid composition of human milk lipids, but with the advancement of other chromatographic techniques, such as liquid and supercritical fluid chromatography, as well as mass spectrometry, intact lipids can also be characterized. Despite the known importance, concise and comprehensive analysis of the human milk lipidome is limited, with gaps existing in all areas of human milk lipidomics, discussed in this review. With appropriate methodology and instrumentation, further understanding of the human milk lipidome and the influence it has on infant outcomes can be achieved.
\end{abstract}

Keywords: human milk; breastfeeding; lactation; lipids; lipidomics; mass spectrometry; chromatography; NMR spectroscopy

\section{Introduction}

Human milk (HM) is vital to the infant, providing both immune protection and energy required for optimal infant growth. Breastfeeding is associated with multiple benefits for both the infant and the mother, such as decreased risk of asthma, pneumonia, type 1 diabetes, and obesity and decreased incidence of breast and ovarian cancer, respectively [1-3]. Further, these breastfeeding benefits increase with the duration of breastfeeding $[1,4]$.

The macronutrient composition of HM consists of approximately $7 \%$ carbohydrates, $5 \%$ lipids, $0.9 \%$ protein, and $0.2 \%$ minerals emulsified in an aqueous milk matrix [5]. While the lipid portion of HM makes up only $5 \%$ of mature milk, it contributes to over $50 \%$ of the infant's daily energy requirement [6]. These lipids are known to be involved in both neural and retinal tissue development as well as immune system development and defense in the infant [7-9]. Furthermore, the HM lipid profile impacts early growth in preterm infants [10].

Despite the importance of these lipids, the total lipid content in HM is highly variable, with large changes occurring throughout the day, between breasts, between women, and throughout the whole lactation period [11]. Interestingly, the total HM lipid content is not believed to be changed by maternal diet; however, diet influences the specific fatty acid (FA) composition. One example of 
this is docosahexaenoic acid (DHA)-containing triacylglycerides (TAGs) which have been found to be in higher concentrations in HM of women with high seafood intake [12,13]. The concentrations of DHA, docosapentaenoic acid (DPA), and arachidonic acid (AA) are also observed to decrease over the lactation period and these are the three FAs implicated in infant neural and retinal development [14].

Along with the variability of HM lipids, the complexity of the milk matrix and lipid hydrophobicity adds to the difficulty of a comprehensive lipidomic analysis. Further, over 40,000 biological lipid structures have been identified in various biological matrices such as human blood and plant material, leaving the possibility for thousands of lipids to be identified and deconvoluted in HM [15].

A number of basic analytical techniques have been employed over the years to investigate the lipid composition of HM; however, with the recent advancement of analytical techniques such as chromatography coupled with mass spectrometry and nuclear magnetic resonance spectroscopy, current analysis promises to be more comprehensive. Lipidomics is the research field in which complex lipidome analyses are carried out to produce a comprehensive and quantitative description of the lipid species present in a given matrix. While lipidomics is expanding exponentially in biological research, it is only recently being applied to HM. Lipids can be defined as FAs and their derivatives, or by their solubility in organic solvents and insolubility in inorganic solvents. Fat-soluble vitamins such as vitamin $\mathrm{D}$ are often included within this definition of lipids, but for the purpose of this review only standard lipid classes such as FAs, glycerolipids, glycerophospholipids, sphingolipids, sterols, prenols, which have been identified in HM will be discussed [16-20].

Additionally, this review will investigate the current status of HM lipidomic analysis and the new emerging techniques, methods, and instruments being used. It will focus on analysis of HM lipidome composition, rather than simply total lipids, which has commonly been estimated using creamatocrit or gravimetric methods [21]. With the present-day state of 'omics' techniques, the ability to comprehensively and quantitatively analyse the HM lipidome will allow a greater understanding of HM lipids. However, in order to make significant advances in HM analysis, quality control and standardised sampling must be routinely employed. Lipidomics platforms hold great promise to further elucidate HM lipid composition and the role of lipids with respect to infant health and disease.

\section{Sampling}

HM lipid content and composition, as mentioned above, is highly variable and constantly changing to meet the demands of the infant. The total lipid content of HM varies widely between women, throughout a feed, a day, and lactation, with reported values ranging widely from $11.4 \mathrm{~g} / \mathrm{L}$ to $61.8 \mathrm{~g} / \mathrm{L}[11,22,23]$. While Jensen suggests that maternal age may influence HM lipid content, this has not been validated [11]. Similarly, diet has previously been suggested to influence lipid content, yet no studies exist to confirm this. In contrast, the FA composition of the lipids is influenced by maternal diet, where areas of China with high fish intake have significantly higher HM DHA than other provinces with lower fish intake, and DHA supplementation of breastfeeding women in Australia also led to an increase in HM DHA content [24,25]. While different ethnicity is thought to be another contributor to lipid composition variability, this too is most probably related to maternal diet. Other maternal health conditions, such as infections or metabolic diseases, have also been noted to reduce the total lipids in HM [6]. An obvious limitation to sampling protocols is that these studies are dealing with human participants, a mother feeding her infant, therefore sampling protocols should not negatively impact or interrupt infant feeding and sleeping patterns. Sampling protocols are non-invasive, involving expression of milk from the nipple either manually or using a breast pump. Differences between sampling methods and timing of collection of the sample may also contribute to complexity and variations within these results, therefore strict collection protocols should be implemented in order to obtain representative samples for HM studies. Details of the methods used in HM lipidomics studies, as well as the other methodology and identified lipids of existing studies, are summarised in Table 1. 


\subsection{Sampling with Respect to the Feed}

Fat content increases as the breast is drained of milk, during a feed, therefore sampling pre-feed HM will give lower total fat content than mid- or post-feed samples [26]. Studies often do not take this into account and do not specify when samples are taken, often accepting random samples from nonspecified time points. Some studies will sample at a single time point with no further details, prescribed time points or will attempt to investigate feeds more thoroughly by collecting pre-, mid- and/or post-feed samples [27-31]. One frequently used sampling method to interrogate the entire feed is to drain the whole breast using a breast pump and then sample from the pumped milk [20,32,33]. However, as infants rarely drain the whole breast [34,35], this method will remove more milk from the end of the feed which is higher in fat content leading to an overestimation of the infant consumption [36].

\subsection{Sampling over $24 \mathrm{~h}$}

As fat content increases with removal of milk from the breast subsequently the HM lipid content varies over a 24-h period, increasing from the first to the last feed of the day, higher in the evening than in the morning [37]. By sampling and test-weighing the infant before and after each feed in a 24-h period, milk production can be measured in addition to the actual amount of milk lipid ingested by the infant [38].

\subsection{Sampling through Stages of Lactation}

In general, the total HM lipid content increases throughout lactation, with Mitoulas et al. showing that lipids decrease from the first to second month but increase up to month 9 of lactation [26]. However, the mean amount of fat delivered to the infant remains constant as maternal milk production and infant intake changes across the months [26]. In order to account for the fat variations at different lactation stages, prescribed time points for sampling within a study, such as sampling on certain days (e.g., day 1, 14, and 42 post-partum) or sampling over a period of lactation (e.g., first 22-25 days of lactation) should be chosen, depending on the research question [13,29]. However, many studies either collect at different stages of lactation and pool their samples (such as [39]), or fail to mention when the samples are collected which makes comparison with other studies and understanding the lipidome difficult.

\subsection{Ideal Sampling Routine}

Due to these variations of both the total lipid content and lipid composition, lipidomic analysis at any given time has the potential to be very different. It is important that the aforementioned factors are all taken into account when sampling HM and that the study is defined in order to control these influences. This is rarely the case in HM studies, clearly outlined by the missing data in Table 2. We suggest defining the research question and then determining the appropriate samples in order to define and standardize sampling to minimize variables and confounding factors. Given that we know about the lipid variations at any given time, it is important that studies use sampling with 24-h test-weighing of the infant during breastfeeding (and expression) to provide more accurate interpretation of infant intake to determine the influence of these lipids on infant development [38]. This technique is not yet widely used but would greatly improve interpretation of research studies. Taking into account the published sampling methods, these are likely to contribute greatly to the large variation in reported lipids values [40]. 


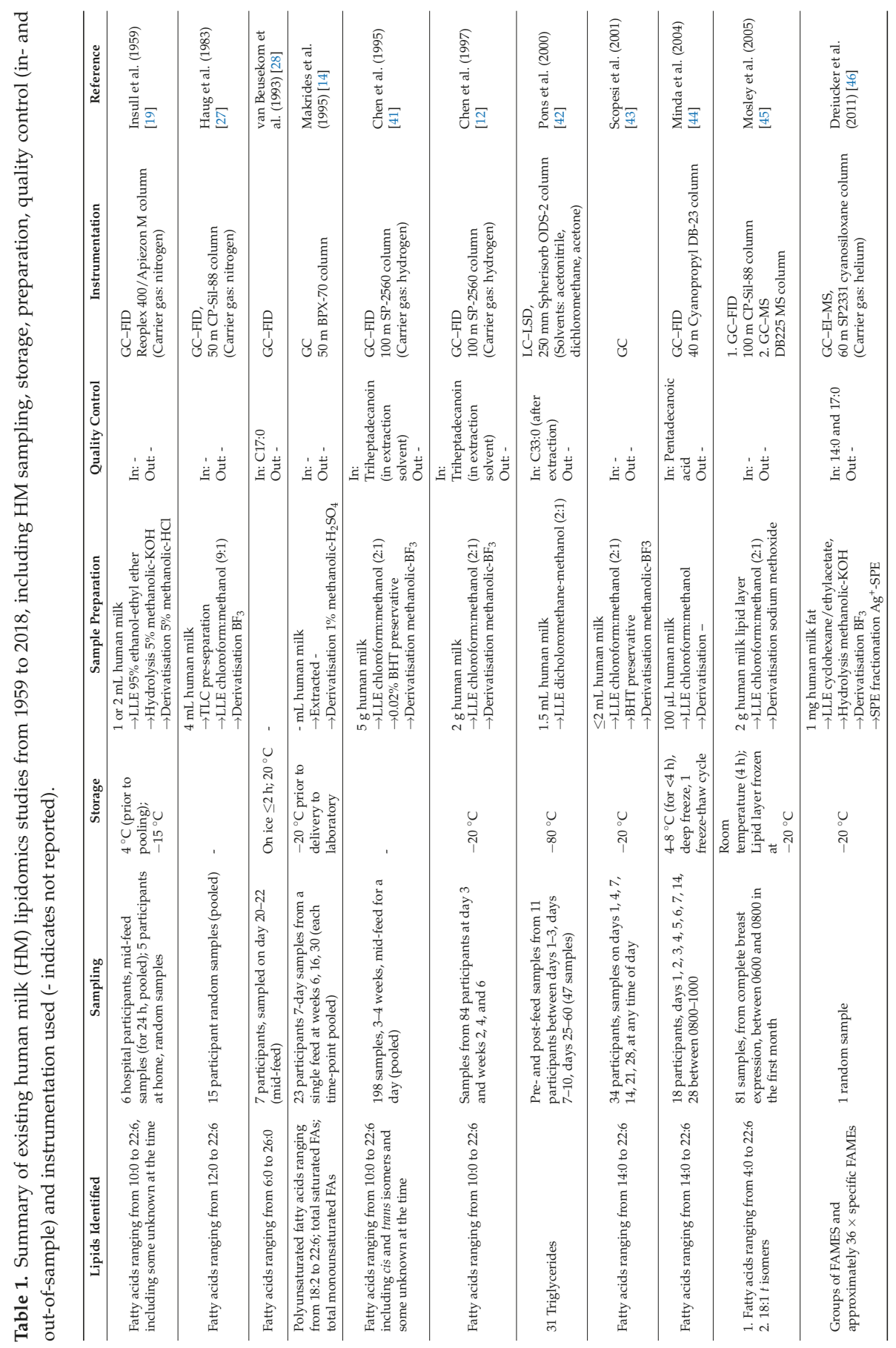




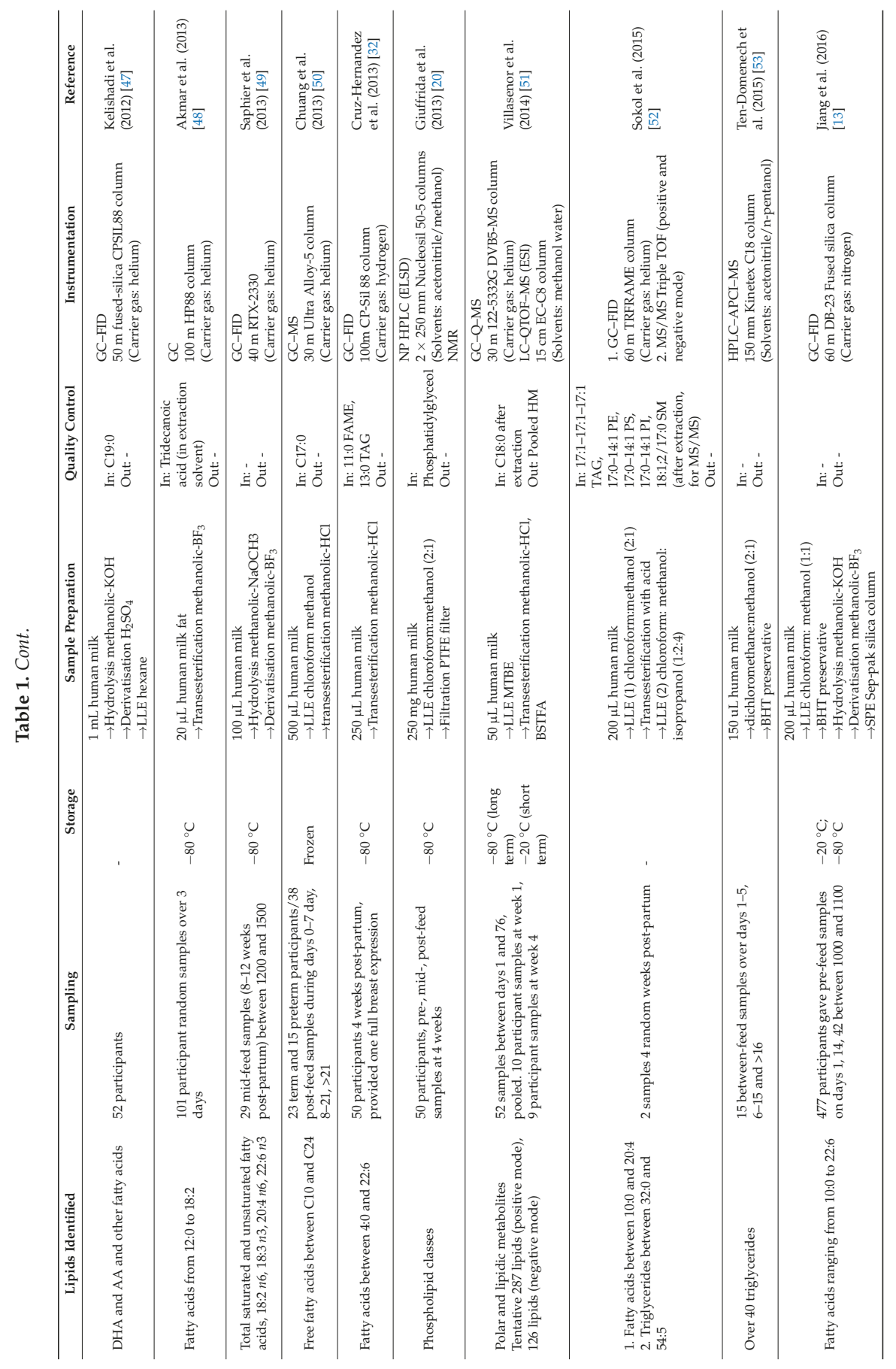




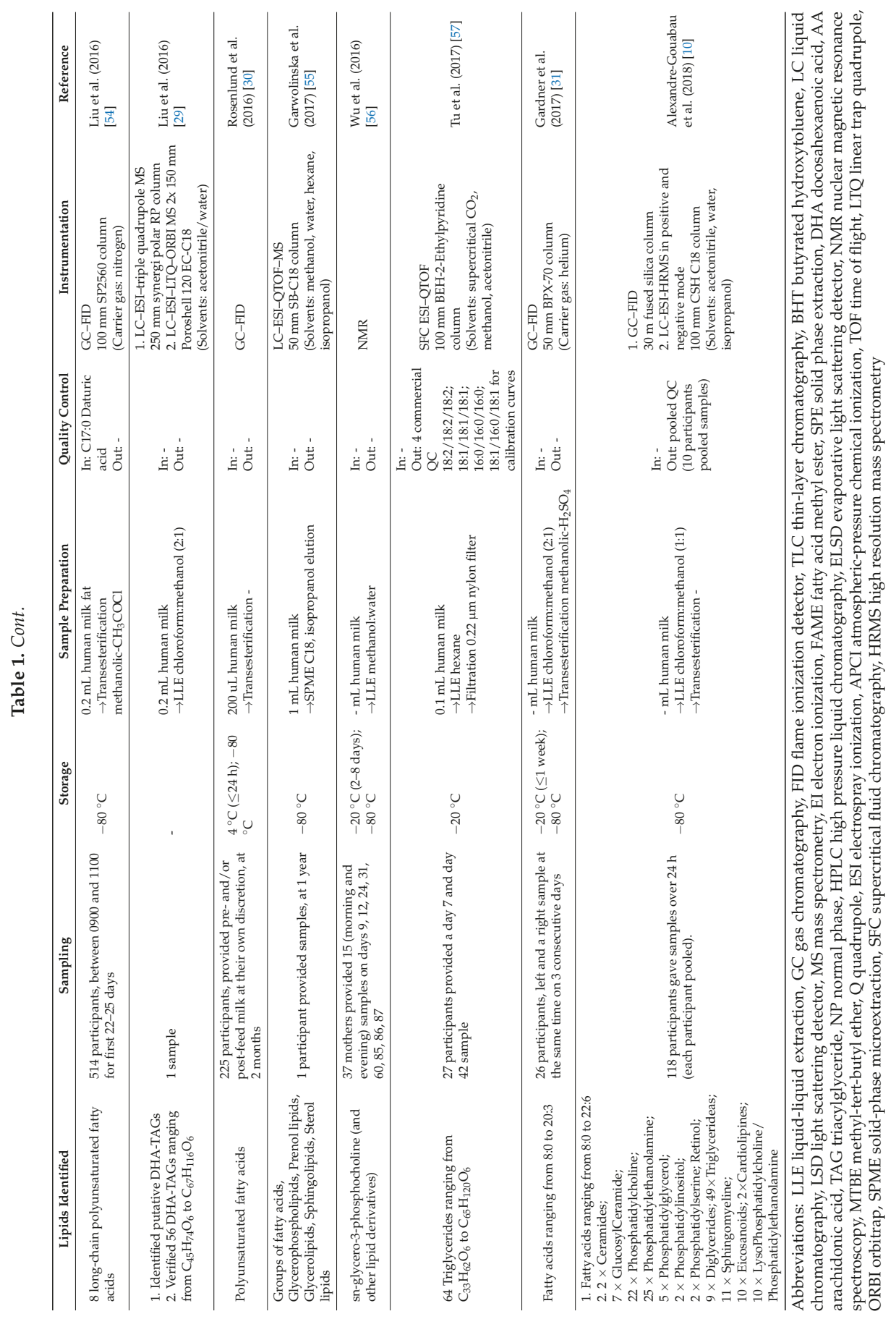




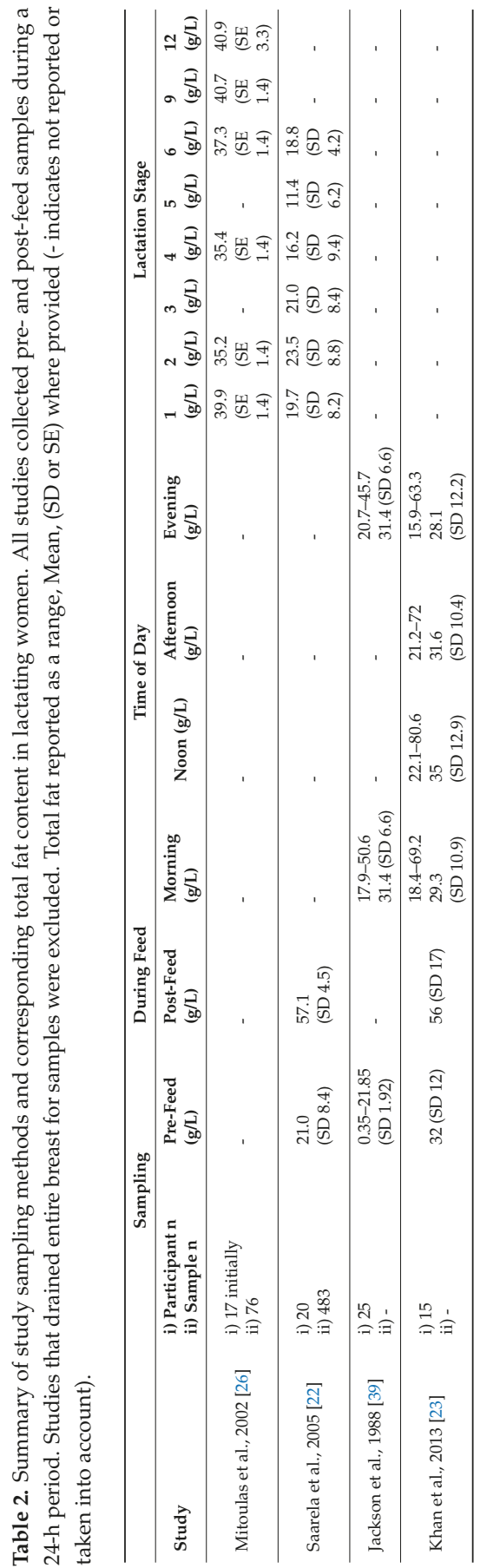




\section{Storage}

As with lipidomic analysis of all biological samples, care must be taken to minimise lipolysis and lipogenesis during storage due to enzymes, such as lipase (bile salt-stimulated lipase and lipoprotein lipase), which are present endogenously in HM $[58,59]$. While immediate analysis of the lipidome is ideal to minimize any compositional changes by lipase activity, in reality this is not practical, therefore correct storage and sample preservation is imperative. Poor consideration of adequate storage affects the reproducibility and interpretation of HM study results and, as shown in Table 1, is something rarely considered in HM lipidomics.

\subsection{Freezing}

Maintaining the integrity of a HM sample is carried out by freezing samples at temperatures such as $-20^{\circ} \mathrm{C},-70{ }^{\circ} \mathrm{C}$ or $-80^{\circ} \mathrm{C}$. If the sample is not frozen adequately, endogenous lipases have the opportunity to cause lipid hydrolysis resulting in inaccurate and misrepresentative HM lipid content for measurement. Studies have shown that while freezing $\mathrm{HM}$ at $-20^{\circ} \mathrm{C}$ for 3 months resulted in a significant loss of lipids (up to $20 \%$ ), storage at $-70{ }^{\circ} \mathrm{C}$ or $-80^{\circ} \mathrm{C}$ stops enzyme activity within the samples and HM lipid integrity is best preserved [60-62]. Although one study showed major lipid loss in $\mathrm{HM}$ samples stored at $-80^{\circ} \mathrm{C}$, Fusch et al. reported that this is likely an effect of poor experimental controls $[63,64]$. The duration of storage is not routinely reported in published studies but is obviously another factor affecting results. Another key factor is the number of freeze-thaw cycles that the sample underwent prior to analysis. In a study by Bitman et al., up to $20 \%$ fat loss was observed when HM underwent two freeze-thaw cycles, due to the resulting increase in lipolytic activity in HM during each of these cycles [65]. Therefore, steps during sample handling should be carefully planned such that all samples undergo the same number of freeze-thaw cycles.

\subsection{Preservatives}

HM has inherent antioxidant capacity to reduce and prevent oxidative degradation [66]. This degradation most commonly occurs in unsaturated fats, where the double bonds undergo cleavage by free radicals. In addition to freezing HM samples, antioxidant preservation of HM samples has also been used to maintain sample integrity. Phenol derivatives such as butyrated hydroxytoluene (BHT) have been used in previous studies to prevent lipid peroxidation [13,67]. BHT works by preferentially reacting with any oxygen present so that there is no opportunity for the lipids to be oxidatively degraded. There are currently no HM studies examining BHT efficacy for lipid preservation; however, studies of other biological samples such as red blood cells have used BHT with success, resulting in increased red blood cell FA preservation from 4 weeks to at least 17 weeks [68].

\section{Lipid Extraction}

Following appropriate HM sampling and storage for lipid analysis, sample preparation is essential to ensure accuracy and reproducibility of the results. For lipidomics analysis, mass spectrometry techniques, which will be discussed in Section 7.2, are commonly used. Therefore, clean-up steps such as liquid-liquid extraction and/or solid-phase extraction are essential to remove interferences such as proteins and sugars, as well as concentrate the lipids of interest. Sample preparation methods used in HM lipidomics studies are described in Table 1. Prior to lipid extraction, the sample must be homogenised, to ensure a uniform distribution of milk fat globules throughout the sample.

\subsection{Liquid-Liquid Extraction}

Liquid-liquid extraction (LLE) techniques are used to separate analyses by their relative solubility in different immiscible liquids. LLE is the classical choice of lipid extraction method used in HM analysis, with variations of the 1950s methods such as Folch [14,69] and Bligh-Dyer [70], using chloroform, methanol, and water in ratios 8:4:3 and 1:2:0.8 respectively, being most commonly used. 
Other than the solvent ratio, the difference in these methods is that Bligh-Dyer uses smaller volumes of solvent and is a less time-consuming protocol [70]. While the Bligh-Dyer extraction was first developed on fish muscle, Folch extraction was developed on brain tissue, however both quoted as being easily adapted to other tissue types. When these solvents are added to HM, the lipids are dissolved into the organic phase (chloroform) and are separated from the aqueous phase (methanol and water, containing carbohydrates and salts) by a layer of cell debris and protein (Figure 1i).

While the use of these methods is well established, the drawbacks include the use of hazardous solvent, such as chloroform, and also the risk of contaminating or losing the lipid-containing lower phase when sampling through the aqueous phase or separating layers. These methods have been directly translated into HM studies or modified to either replace the use of hazardous solvent, such as chloroform with dichloromethane; or increase extraction efficiency with the introduction of centrifugation to enhance phase separation and the omission of water [27,29]. Recently a methyl-tert-butyl ether (MTBE) extraction method, initially developed for plasma lipid extraction, has been employed for HM lipid extraction for the analysis of both lipids and other HM metabolites [51]. This extraction, similar to the Folch and Bligh and Dyer method, separates lipids using phase separation. However, using the MTBE method, the organic phase containing lipids instead forms the upper layer, and the aqueous phase (containing the matrix pallet) forms the lower layer (Figure 1ii). This method has made extraction of lipids simpler and minimizes the potential of cross contamination.

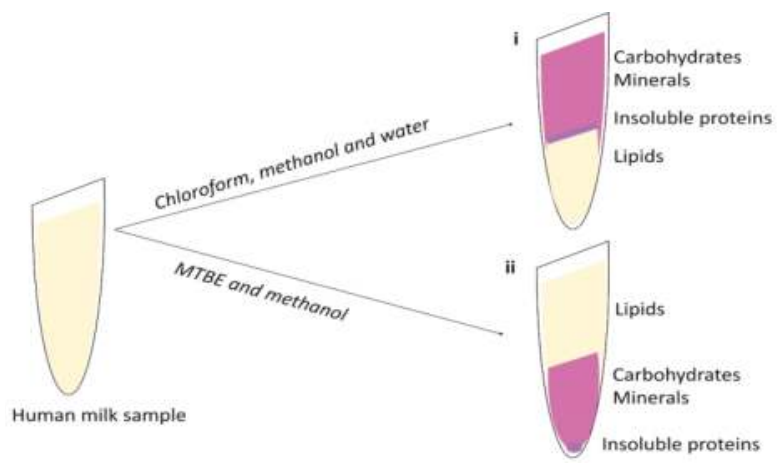

Figure 1. Liquid-liquid extraction of human milk lipids using (i) Folch extraction or (ii) Methyl-tert-butyl ether (MTBE) extraction.

\subsection{Solid-Phase Extraction}

The use of solid-phase extraction (SPE), a type of column chromatography, is gaining popularity for its rapid and efficient lipid extraction from biological fluids. In this process, HM is loaded into the cartridge with lipid analyses retained on the solid-phase sorbent, such as C18, packed in a cartridge, meanwhile the interfering milk matrix components are washed out. Lipids can then be eluted from the bonded phase using organic solvents (Figure 2) [71]. Only two published milk lipidome studies have successfully used SPE for lipid extraction from HM, extracting fatty acyls, glycolipids, sphingolipids, prenol lipids and sterol lipids for analysis [46,55]. The first study by Dreiucker and Vetter uses a silver-ion SPE to extract FAs separating them by their degree of saturation and isomeric configuration [46]. The FAs were then eluted with acetone-based solvents, which then allowed better measurement of preseparated FA isomers by GC-MS than in standard LLE extraction. While this silver-ion SPE method is more quantitative, it has limitations with reproducibility and standardization to ensure complete lipid extraction. In another study, a solid-phase micro extraction (SPME) technique was used. This SPME involves the immersion of a solid-phase sorbent-coated fiber into HM and then use of organic solvent (such as isopropanol) to desorb the lipids [55]. This technique has poor reproducibility for the amount and type of lipids absorbed by the fiber, even when other 
parameters such as time and elution solvent are standardized, thus rendering this method suitable for qualitative analyses only. These factors limit the current use of SPE in HM lipidomics; however, further optimisation could offer the possibility of SPE automation in a plate format, which would make this technique ideal for routine, high-throughput extraction of HM for lipidomics.

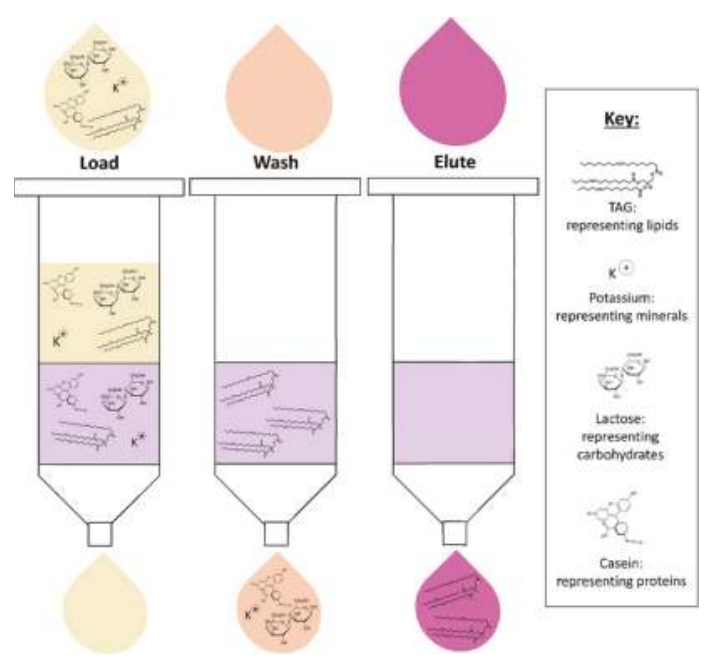

Figure 2. Solid-phase extraction of human milk lipids.

\section{Lipid Transesterification}

Following lipid extraction from HM samples, lipid transformation may be required for the analysis of non-volatile free or lipid-bound FAs. It is generally accepted that free FA in HM are artefacts of lipolysis, although only one study has investigated the FA from lipase hydrolysis of TAGs and other lipids (such as phospholipids and sphingolipids) [72]. This section will discuss only the analysis of FA that make up lipids, more specifically the FA composition of TAGs, which make up $98 \%$ of the lipids in HM, despite the methodology being poorly described (Table 1) [73]. Prior to the analysis of these FA, a two-part chemical transesterification is carried out, first hydrolysing the TAG, releasing three FA (Figure 3i), followed by derivatisation of the resulting FA to methyl esters (FAMEs) for GC analysis (Figure 3ii). This reaction can be either acid or base catalyzed. Derivatisation of FA is necessary for GC analysis as the high polarity of nonderivatised FA can result in hydrogen bond formation and therefore adsorption issues on a GC column, leading to band broadening and retention time shifting [74]. The resulting FAMEs have reduced polarity, able to be separated by a polar GC column.

The transesterification method is well-established and has been widely applied in FA analysis, where acidic transesterification using boron trifluoride $\left(\mathrm{BF}_{3}\right)$ is most commonly used, as first described in 1964 [75]. The early HM FA transesterification methods frequently use this $\mathrm{BF}_{3}$ and methanol approach $[10,12,13,43,48]$. In other HM studies, transesterifications have used acid catalysis (methanolic-hydrogen chloride) or base catalysis (using methanolic-potassium hydroxide or sodium-methoxide) $[19,32,45,72]$. Although $\mathrm{BF}_{3}$ is a hazardous chemical and could also interact with BHT preservatives in a sample, it is still widely used in HM preparation [32,76]. The primary drawbacks of transesterification for FAME analysis are the laborious and time-consuming steps involved, supporting the movement towards methods not involving such preparations (such as liquid chromatography-mass spectrometry). 

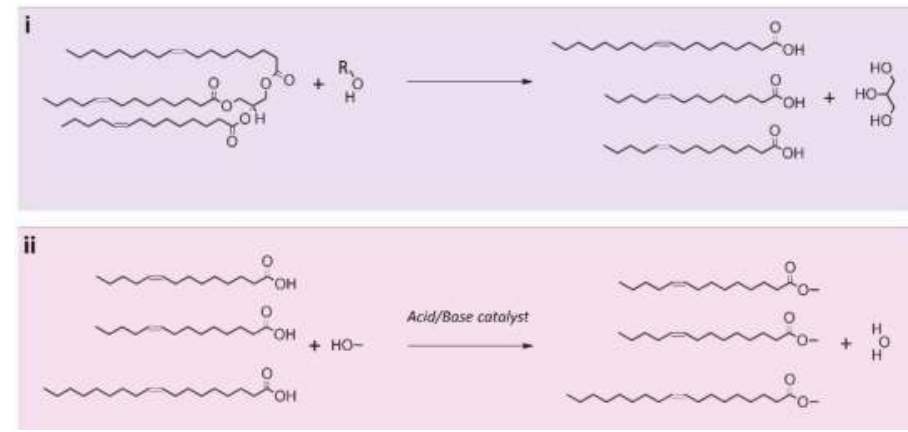

Figure 3. Transesterification reactions of triglyceride 14:1/14:1/18:1, one triglyceride commonly found in human milk. (i) Triglyceride hydrolysis, carried out with a base (such as $\mathrm{KOH}$ ), resulting in glycerol and three free fatty acids; (ii) Resulting free fatty acid reaction with methanol and an acid/base catalyst producing three fatty acid methyl esters and water.

\section{Quality Control}

The use of quality control (QC) is essential to minimize influences, such as sample matrix effects and instrument variations that could cause issues with method accuracy and reproducibility. Despite the importance of QC in lipidomics, it is often overlooked in almost all, not just in HM, studies (as can be seen in Table 1). The QC measures are generally determined by several factors including the target lipid class of the study, the availability and cost of the standards and researcher preference. Several types of QC, which we have categorized as 'in-sample' and 'out-of-sample' QCs, should also be in place when lipidomic analyses are carried out and these are described below.

\subsection{In Sample}

This QC is added in known concentrations to HM during sample preparation and is also referred to as the internal standard (IS). For optimal lipidomics, more than one compound should be used as an IS. If these IS are added to HM prior to extraction, they can be used to assess variability that may occur in sample storage and extraction recovery. If the IS is added after sample extraction, it is used to monitor instrument performance and variability. The compound selected as an IS should be a labelled compound that is, or behaves as, the compound/s of interest. Due to limited availability of expensive commercial labelled lipid standards, to date no HM lipidomics studies have used labelled lipid standards. HM studies have, however, used a variety of unlabelled commercial lipids which are presumed not to be present in HM as an IS, for example heptadecanoic acid (C17:0) [28].

\subsection{Out of Sample}

QC samples should also be analyzed periodically within an experiment to monitor for any instrument abnormalities, such as sample degradation or loss of response. QCs are typically a pooled QC or commercial QC. A pooled QC is prepared by pooling aliquots of $\mathrm{HM}$ samples from the laboratory and analyzing these alongside a batch of samples. These QCs need to be rigorously prepared and stored in order to achieve reproducibility and for accurate monitoring of intra- and inter-batch variations. The pooled QC is the simplest and cheapest to prepare. Commercial QCs are known lipid analytes purchased to be run within a batch, like other out-of-sample QCs, confirming and identifying the retention time, $m / z$ values and identity of these analyses. Additionally, these are often used to test an instrument for suitability. Out-of-sample QC should always be matrix matched to account for biological matrix effects, a condition that no HM lipidomic studies have yet met [77].

While there is currently no general consensus on the type of QC that should be used and the limits of variability within a lipidomics experiment, many studies will predefine the limits based on 
experience and the instruments used. Because of the vast number of lipids, untargeted HM lipidomics can only ever be semiquantitative [77].

\section{Analytical Instrumentation for Lipidomic Analysis}

Due to the complexity of lipids, complete lipidomic analysis requires more than one instrument platform. The choice of instrumentation for HM lipidomics therefore depends upon the study aims and the lipids of interest. Simple separation techniques have previously been used for qualitative analysis of lipids, such as thin-layer chromatography and gas chromatography (GC). Although GC is thought of as the gold standard for HM FA lipidomics, the availability and increasing prevalence of other separation techniques such as liquid chromatography (LC) and high-resolution mass analyzers, such as time-of-flight and Fourier Transform, means that the HM lipidome can be more comprehensively characterized [78]. The advantages and disadvantages of the instrumentation used in HM lipidomic analysis are summarized in Table 3. Consistent GC use in HM lipidomics can be seen in Table 1, with the slow emergence of mass spectrometry in recent years.

Table 3. Advantages and disadvantages of analytical instrumentation used in human milk lipidomics.

\begin{tabular}{|c|c|c|}
\hline Separation/Detection Method & Advantages & Disadvantages \\
\hline Gas chromatography & $\begin{array}{l}\text { 1. Fatty acid methyl ester analysis is well } \\
\text { characterized } \\
\text { 2. Flame ionisation detector is robust and easy to } \\
\text { maintain }\end{array}$ & $\begin{array}{l}\text { 1. Sample derivatisation is required } \\
\text { 2. Destructive } \\
\text { 3. Isomers separation requires longer column } \\
\text { and run time } \\
\text { 4. Flame ionisation detector lacks mass } \\
\text { selectivity }\end{array}$ \\
\hline Liquid chromatography & $\begin{array}{l}\text { 1. No sample derivatisation required } \\
\text { 2. Large selection of column chemistry available }\end{array}$ & $\begin{array}{l}\text { 1. Solvent system must be compatible with } \\
\text { detector type }\end{array}$ \\
\hline $\begin{array}{l}\text { Supercritical fluid } \\
\text { chromatography }\end{array}$ & $\begin{array}{l}\text { 1. No derivatisation required } \\
\text { 2. Compatible with almost any detector type } \\
\text { 3. Relatively inexpensive } \\
\text { 4. Low waste output } \\
\text { 5. Faster separation than in GC/LC } \\
\text { 6. Higher resolution than in GC/LC }\end{array}$ & $\begin{array}{l}\text { 1. Polar lipid separation requires organic } \\
\text { modifier }\end{array}$ \\
\hline Thin-layer chromatography & 1. Inexpensive & $\begin{array}{l}\text { 1. Qualitative lipid class separation only } \\
\text { 2. Low separating resolution compared to GC } \\
\text { and LC. }\end{array}$ \\
\hline Mass spectrometry & $\begin{array}{l}\text { 1. High sensitivity and specificity } \\
\text { 2. Qualitative and quantitative (with standards) }\end{array}$ & $\begin{array}{l}\text { 1. Expensive } \\
\text { 2. Destructive }\end{array}$ \\
\hline NMR spectroscopy & $\begin{array}{l}\text { 1. Non-destructive } \\
\text { 2. Highly reproducible }\end{array}$ & $\begin{array}{l}\text { 1. Expensive } \\
\text { 2. Signal overlapping in complex samples } \\
\text { 3. Lower sensitivity than MS } \\
\text { 4. Requires larger samples volume }\end{array}$ \\
\hline
\end{tabular}

\subsection{Separation Methods}

\subsubsection{Gas Chromatography}

GC coupled with a flame ionization detector (GC-FID) is the most routinely used separation method for FA analysis since the 1950s and is widely accepted for quantification of FA in many sample types, including HM [19]. Cyanopropyl-based columns ranging from 30 to $60 \mathrm{~m}$ in length are typically employed for FAME analysis. However, longer columns (up to $100 \mathrm{~m}$ ) are used if separation of dietary FAME isomers such as cis C18:1 and trans C18:1 is desired. Therefore, the requirement for a longer GC column can extend both the method preparation and run time. The FID is generally used in FAME analysis as it is considerably cheaper to purchase and maintain compared to mass spectrometry (MS) detectors. Furthermore, the robustness of the FID allows the analysis of large numbers of samples before the need for any maintenance and does not have the same requirements and issues as MS (such as ionization source cleaning and ionization issues, as seen in mass spectrometry), discussed in Section 7.2. Mass Spectrometry [79]. Additionally, HM FAME analysis using GC is well characterized based on elution order and retention time, either requiring a limited number of standards 
or using Kovats retention index, as described in a HM study by Villasenor et al., for identification by comparing experimental and established retention indices [51]. Further, retention time locking can add to method reproducibility. However, GC-FID lacks mass selectivity, unlike MS, so it has been known to misidentify FAMEs in the presence of co-eluting compounds or contaminants that may be present in the sample, although this has not been investigated in HM studies [80,81].

\subsubsection{Liquid Chromatography}

While GC is widely used for FA analysis, LC, with an evaporative light-scattering detector (ELSD), charged aerosol detector (CAD), electrochemical detector, or coupled to mass spectrometry (MS), has been used in the analysis of intact lipids, such as TAGs and phospholipids [79]. Currently only one study has used LC-ELSD in HM lipidomics, to quantify phospholipids, while other LC methodology is most commonly carried out using mass spectrometry [20,53]. Due to the wide variety of lipids in HM, various stationary phases and solvent combinations are employed depending on the type of lipids and separation required. Lipid separation in biofluids, including HM, is most often carried out using a C18 stationary phase column but other silica-based stationary phases, such as $\mathrm{C} 8$, have also been used in HM analysis for separation of all lipid classes and phospholipids, respectively [20,51]. Reversed-phase LC separates intact lipids and free FA based on their specific FA polarity, degree of saturation and chain length, while normal-phase LC will separate lipids, such as glycerophospholipids, by their class [82]. In LC analyses, the solvent and stationary phase must be compatible with the detection method, for example MS, where ratios of organic and inorganic solvents such as acetonitrile, alcohol, and water are most commonly used. When MS is the chosen detector ammonium salts (formate or acetate) and formic acid will be added (discussed in Section 7.2. Mass Spectrometry). The main advantage of LC over GC is that transformation is not required and intact lipids such as triglycerides can be analyzed [83].

\subsubsection{Supercritical Fluid Chromatography}

Supercritical fluid chromatography (SFC) is another separation technique similar to LC, which, instead of using a liquid mobile phase, uses a supercritical fluid, such as carbon dioxide $\left(\mathrm{CO}_{2}\right)$, as the mobile phase. Supercritical fluids are formed when dense compressed gas is subjected to a specific pressure and temperature. $\mathrm{CO}_{2}$ is the most commonly used supercritical solvent and its non-polar properties make it ideal for separating non-polar lipids like TAGs, shown by Laakso and Manninen in cow's milk, to separate TAGs by their molecular size [84]. Although SFC has been widely used in dairy milk fat research and oil separation, its use in HM is limited to one study where SFC was coupled to mass spectrometry [57]. Advantages of SFC include no requirement for derivatisation, and the ability for SFC to be coupled with all detector types, such as FID or MS, as well as its low cost and waste output relative to LC, using less organic solvents than LC, and allowing faster separation and higher resolution than LC and GC in metabolomics analyses [85]. These features all make SFC well suited to the analysis of multiple lipid classes in one sample that have a range of polarities [79].

\subsubsection{Thin-Layer Chromatography}

Like LC, thin-layer chromatography (TLC) may be qualitatively analytical but is more commonly used as a preparative step in human studies. HM studies often use TLC for separation of lipids into their individual classes, for example separation of short- and long-chain FAs prior to analysis [72]. This inexpensive technique is classically carried out using a silica plate and non-polar solvent for lipid class separation, and the classes can then be collected and analyzed using platforms such as GC or LC. As TLC does not have the separating resolution of GC or LC, its ability to perform identification is limited and thus may be the reason why TLC is not frequently used in HM lipidomics. 


\subsection{Mass Spectrometry}

Mass spectrometry (MS) is the detection technique that identifies ionized compounds based on their mass-to-charge ratio $(\mathrm{m} / \mathrm{z})$. This is a destructive technique in which the sample is destroyed and cannot be used for future analysis. In HM lipidomics analysis, various types of mass analyzers, such as quadrupole, triple-quadrupole and time-of-flight, have been employed to identify and quantitate different lipids [79]. Given the increased sensitivity and specificity of MS in contrast to other detector types, such as FID and ELSD, it is possible to confirm the identity of known lipids, identify unknown lipids and to elucidate structural information of lipids using MS.

In order for lipids to be detected by MS, the compound needs to be ionized first using one of a variety of ionization techniques such as EI (electron ionization), ESI (electrospray ionization), CI (chemical ionization) or MALDI (matrix assisted laser desorption/ionization), which have been extensively reviewed $[78,82]$. These ionization methods can be carried out in either positive (EI, CI, or ESI) or negative (CI or ESI) mode, producing cations or anions respectively. In HM lipidomics, EI and ESI methods are commonly used. The EI technique is commonly used in conjunction with GC separation for FA analysis, where lipids are bombarded with a high-energy electron beam causing them to be ionized and fragmented in characteristic patterns. This is a hard ionization technique and generally only the fragment ions are observed [82]. Three HM studies have employed GC-MS since 2011, identifying and quantifying a large number of FAs as derivatised FAMEs, with MS having the added advantage of identifying many glycerolipids, glycerophospholipids, sphingolipids, prenol lipids, and sterol lipids not previously identified using GC-FID [46,50,51]. In contrast to EI-MS, ESI-MS is widely used in LC for HM lipidomics analysis $[10,29,51,55]$. This soft ionization technique involves pushing samples through a capillary with a voltage applied to it, creating a fine aerosol where ions are formed by desolvation. As ESI is a soft ionization technique, it is able to provide information on both the molecular ion (intact lipid, such as a triglyceride) as well as additional structural information by fragmenting the molecular ion, such as the FA composition of a specific triglyceride [82].

Additionally, LC-MS often uses additives such as ammonium formate and formic acid in the mobile phase as modifiers to promote ammonium adduct formation, these adducts being more stable than hydrogen adducts and easier to fragment than metal ions, and prevent retention time shifting [83]. The use of both positive and negative ionization mode in ESI-MS covers even more lipids, for example, identifying FAs using negative mode and phospholipids using positive mode [10].

Shotgun MS, which involves introducing a sample directly into the ion source and carrying out both positive and negative ionization mode MS, is a common technique for untargeted identification and structural characterization of lipids having been recently used for HM [52]. While this method is fast, sensitive and only requires a small amount of sample to be injected, the lack of chromatographic separation and ion suppression makes interpretation difficult. Ion suppression is a common effect where the response of a species of interest is suppressed due to endogenous matrix species such as proteins, or exogenous species such as plasticizers from plastic tubes/tube caps, in the sample compete for ionization [77]. This can be minimized with efficient lipid extraction during sample preparation, resulting in a cleaner and purer lipid extract. As lipid mixtures are challenging to interpret, chromatographic preseparation (GC or LC) is usually employed to further assist in separating lipids/isomers, providing additional orthogonal data for easier identification and more accurate quantification compared to the shotgun approach [79]. Additionally, untargeted analysis results in a large number of compounds to interrogate and often requires very specialized and expensive software.

\subsection{Nuclear Magnetic Resonance Spectroscopy}

Since the introduction of nuclear magnetic resonance spectroscopy (NMR) to the world of metabolomics, it has been used frequently in analyses of various biofluids and tissues, including muscle tissue and milk (such as in cows and camels) [86]. NMR is widely used in HM metabolomics to measure sugars, amino acids, and nucleotides; however, only one lipid (sn-glycero-3-phosphocholine), 12 phospholipid classes and a small number of lipid derivatives 
have been identified in HM by NMR [86,87]. NMR uses atomic magnetic properties, detecting every hydrogen/carbon/phosphorus-containing molecule and has the ability to provide valuable structural information for the intact lipid, such as structural differences between intact phospholipids [62,79]. In contrast to MS, NMR is a non-destructive technique, samples can be re-analyzed with NMR or other techniques [88]. However the drawbacks of NMR include signal overlapping, which can make discrimination of resonances from complex samples difficult, as well as larger sample volume requirements. While the use of NMR may be limited by its lower sensitivity than MS, NMR is highly reproducible and simple for a trained user to run [56]. Sample preparation may involve lipid extraction, such as with Folch extraction method, or simply whole milk may be analyzed. While preparation is simple, it can be difficult to run large numbers of samples with the same high-throughput capability of MS methods, unless an autosampler is available. The detected analyses can then be quantitated using the direct relationship between intensity of resonance and concentration [89].

\section{Limitations and Future Perspectives}

In addition to lipids being the most variable portion of HM, lipidomic analyses are limited by the number of samples analyzed, limiting the conclusions and relationships that can be identified in studies. Further, HM lipidomics would greatly benefit from standardized workflows for sample collection and preparation, analytical methodology on a wide number of platforms, data acquisition and data processing. The future of HM lipidomics needs higher lipid coverage on multiple platforms, allowing development of a HM metabolome/lipidome database similar to that of the Human Metabolome Database [90].

\section{Conclusions}

HM lipids are an essential macronutrient for the growth, development, and health of the infant; therefore, HM lipidomics are essential to provide a deeper understanding of short- and long-term infant health. The recent advances in instrumentation and methods in lipidomics will result in more comprehensive HM lipidomic investigations. Chromatography, MS, and NMR methods also offer potential for further lipid identification, structural elucidation, and investigation in HM. To develop better knowledge of the lipid changes in HM throughout lactation, more rigorous studies need to be carried out, employing stringent sampling and storage routines and advanced methodology with strict quality control. Rigorous protocols in HM investigations will allow more accurate assessment and investigation of the HM lipidome and the impact these lipids have on the infant.

Author Contributions: A.D.G. wrote the manuscript. M.C.L.G., R.D.T. and D.T.G. all critically reviewed the manuscript. All authors have read and approved the final manuscript.

Funding: Alexandra receives a Postgraduate Scholarship from Medela AG, Switzerland. Donna Geddes and Melvin Gay receive an unrestricted research grant from Medela AG. Medela AG had no input into the design or publication of this review.

Conflicts of Interest: The authors declare no conflict of interest.

\section{References}

1. Eidelman, A.I. Breastfeeding and the use of human milk: An analysis of the American academy of pediatrics 2012 breastfeeding policy statement. Breastfeed. Med. 2012, 7, 323-324. [CrossRef] [PubMed]

2. Layde, P.M.; Webster, L.A.; Baughman, A.L.; Wingo, P.A.; Rubin, G.L.; Ory, H.W. The independent associations of parity, age at first full term pregnancy, and duration of breastfeeding with the risk of breast cancer. Cancer and steroid hormone study group. J. Clin. Epidemiol. 1989, 42, 963-973. [CrossRef]

3. Collaborative Group on Hormonal Factors in Breast Cancer. Breast cancer and breastfeeding: Collaborative reanalysis of individual data from 47 epidemiological studies in 30 countries, including 50302 women with breast cancer and 96973 women without the disease. Lancet 2002, 360, 187-195. [CrossRef] 
4. Victora, C.G.; Bahl, R.; Barros, A.J.; Franca, A.V.; Horton, S.; Krasevec, J.; Murch, S.; Sankar, M.J.; Walker, N.; Rollins, N.C. Breastfeeding in the 21st century: Epidemiology, mechanisms, and lifelong effect. Lancet 2016, 387, 475-490. [CrossRef]

5. Jenness, R. The composition of human milk. Semin. Perinatol. 1979, 3, 225-339. [PubMed]

6. Jensen, R.G. Lipids in human milk. Lipids 1999, 34, 1243-1271. [CrossRef] [PubMed]

7. Koletzko, B.; Agostoni, C.; Bergmann, R.; Ritzenthaler, K.; Shamir, R. Physiological aspects of human milk lipids and implications for infant feeding: A workshop report. Acta Paediatr. 2011, 100, 1405-1415. [CrossRef] [PubMed]

8. Innis, S.M. Dietary triacylglycerol structure and its role in infant nutrition. Adv. Nutr. 2011, 2, $275-283$. [CrossRef] [PubMed]

9. Lauritzen, L.; Fewtrell, M.; Agostoni, C. Dietary arachidonic acid in perinatal nutrition: A commentary. Pediatr. Res. 2015, 77, 263-269. [CrossRef] [PubMed]

10. Alexandre-Gouabau, M.-C.; Moyon, T.; Cariou, V.; Antignac, J.-P.; Qannari, E.M.; Croyal, M.; Soumah, M.; Guitton, Y.; David-Sochard, A.; Billard, H.; et al. Breast milk lipidome is associated with early growth trajectory in preterm infants. Nutrients 2018, 10, 164. [CrossRef] [PubMed]

11. Jensen, R.G. The lipids in human milk. Prog. Lipid Res. 1996, 35, 53-92. [CrossRef]

12. Chen, Z.Y.; Kwan, K.Y.; Tong, K.K.; Ratnayake, W.M.N.; Li, H.Q.; Leung, S.S.F. Breast milk fatty acid composition: A comparative study between Hong Kong and Chongqing Chinese. Lipids 1997, 32, 1061-1067. [CrossRef] [PubMed]

13. Jiang, J.; Wu, K.; Yu, Z.; Ren, Y.; Zhao, Y.; Jiang, Y.; Xu, X.; Li, W.; Jin, Y.; Yuan, J.; et al. Changes in fatty acid composition of human milk over lactation stages and relationship with dietary intake in Chinese women. Food Funct. 2016, 7, 3154-3162. [CrossRef] [PubMed]

14. Makrides, M.; Simmer, K.; Neumann, M.; Gibson, R. Changes in the polyunsaturated fatty acids of breast milk from mothers of full-term infants over $30 \mathrm{wk}$ of lactation. Am. J. Clin. Nutr. 1995, 61, 1231-1233. [CrossRef] [PubMed]

15. Sud, M.; Fahy, E.; Cotter, D.; Brown, A.; Dennis, E.A.; Glass, C.K.; Merrill, A.H., Jr.; Murphy, R.C.; Raetz, C.R.H.; Russell, D.W.; et al. LMSD: Lipid Maps structure database. Nucleic Acids Res. 2007, 35, D527-D532. [CrossRef] [PubMed]

16. Dotson, K.D.; Jerrell, J.P.; Picciano, M.F.; Perkins, E.G. High-performance liquid chromatography of human milk triacylglycerols and gas chromatography of component fatty acids. Lipids 1992, 27, 933-939. [CrossRef] [PubMed]

17. Kallio, M.J.; Siimes, M.A.; Perheentupa, J.; Salmenperä, L.; Miettinen, T.A. Cholesterol and its precursors in human milk during prolonged exclusive breast-feeding. Am. J. Clin. Nutr. 1989, 50, 782-785. [CrossRef] [PubMed]

18. Andreas, N.J.; Hyde, M.J.; Gomez-Romero, M.; Lopez-Gonzalvez, M.A.; Villaseñor, A.; Wijeyesekera, A.; Barbas, C.; Modi, N.; Holmes, E.; Garcia-Perez, I. Multiplatform characterization of dynamic changes in breast milk during lactation. Electrophoresis 2015, 36, 2269-2285. [CrossRef] [PubMed]

19. Insull, W. The fatty acids of human milk from mothers on diets taken ad libitum. Biochem. J. 1959, 72, 27-33. [CrossRef] [PubMed]

20. Giuffrida, F.; Cruz-Hernandez, C.; Flück, B.; Tavazzi, I.; Thakkar, S.K.; Destaillats, F.; Braun, M. Quantification of phospholipids classes in human milk. Lipids 2013, 48, 1051-1058. [CrossRef] [PubMed]

21. Du, J.; Gay, M.C.L.; Lai, C.T.; Trengove, R.D.; Hartmann, P.E.; Geddes, D.T. Comparison of gravimetric, creamatocrit and esterified fatty acid methods for determination of total fat content in human milk. Food Chem. 2017, 217, 505-510. [CrossRef] [PubMed]

22. Saarela, T.; Kokkonen, J.; Koivisto, M. Macronutrient and energy contents of human milk fractions during the first six months of lactation. Acta Paediatr. 2007, 94, 1176-1181. [CrossRef]

23. Khan, S.; Hepworth, A.R.; Prime, D.K.; Lai, C.T.; Trengove, N.J.; Hartmann, P.E. Variation in fat, lactose, and protein composition in breast milk over 24 hours: Associations with infant feeding patterns. J. Hum. Lact. 2013, 29, 81-89. [CrossRef] [PubMed]

24. Makrides, M.; Neumann, M.A.; Gibson, R.A. Effect of maternal docosahexaenoic acid (DHA) supplementation on breast milk composition. Eur. J. Clin. Nutr. 1996, 50, 352-357. [PubMed] 
25. Ruan, C.; Liu, X.; Man, H.; Ma, X.; Lu, G.; Duan, G.; DeFrancesco, C.A.; Connor, W.E. Milk composition in women from five different regions of China: The great diversity of milk fatty acids. J. Nutr. 1995, 125, 2993-2998. [PubMed]

26. Mitoulas, L.R.; Kent, J.C.; Cox, D.B.; Owens, R.A.; Sherriff, J.L.; Hartmann, P.E. Variation in fat, lactose and protein in human milk over $24 \mathrm{~h}$ and throughout the first year of lactation. Br. J. Nutr. 2002, 88, $29-37$. [CrossRef] [PubMed]

27. Haug, M.; Dieterich, I.; Laubach, C.; Reinhardt, D.; Harzer, G. Capillary Gas Chromatography of Fatty Acid Methyl Esters from Human Milk Lipid Subclasses. J. Chromatogr. A 1983, 279, 549-553. [CrossRef]

28. van Beusekom, C.M.; Nijeboer, H.J.; van der Veere, C.N.; Luteyn, A.J.; Offringa, P.J.; Muskiet, F.A.J.; Boersma, E.R. Indicators of long chain polyunsaturated fatty acid status of exclusively breastfed infants at delivery and after 20-22 days. Early Hum. Dev. 1993, 32, 207-218. [CrossRef]

29. Liu, Z.; Cocks, B.G.; Rochfort, S. Comparison of molecular species distribution of DHA-containing triacylglycerols in milk and different infant formulas by liquid Chromatography-Mass spectrometry. J. Agric. Food Chem. 2016, 64, 2134-2144. [CrossRef] [PubMed]

30. Rosenlund, H.; Fagerstedt, S.; Alm, J.; Mie, A. Breastmilk fatty acids in relation to sensitization-the ALADDIN birth cohort. Allergy 2016, 71, 1444-1452. [CrossRef] [PubMed]

31. Gardner, A.S.; Rahman, I.A.; Lai, C.T.; Hepworth, A.; Trengove, N.; Hartmann, P.E.; Geddes, D.T. Changes in fatty acid composition of human milk in response to cold-like symptoms in the lactating mother and infant. Nutrients 2017, 9, 1034. [CrossRef] [PubMed]

32. Cruz-Hernandez, C.; Goeuriot, S.; Giuffrida, F.; Thakkar, S.K.; Destaillats, F. Direct quantification of fatty acids in human milk by Gas Chromatography. J. Chromatogr. A 2013, 1284, 174-179. [CrossRef] [PubMed]

33. Daly, S.E.; Di Rosso, A.; Owens, R.A.; Hartmann, P.E. Degree of breast emptying explains changes in the fat content, but not fatty acid composition, of human milk. Exp. Physiol. 1993, 78, 741-755. [CrossRef]

34. Prime, D.K.; Kent, J.C.; Hepworth, A.R.; Trengove, N.J.; Hartmann, P.E. Dynamics of milk removal during simultaneous breast expression in women. Breastfeed. Med. 2012, 7, 100-106. [CrossRef] [PubMed]

35. Kent, J.C.; Ramsay, D.T.; Doherty, D.; Larsson, M.; Hartmann, P.E. Response of breasts to different stimulation patterns of an electric breast pump. J. Hum. Lact. 2003, 19, 179-186. [CrossRef] [PubMed]

36. Jensen, R.G.; Lammi-Keefe, C.J.; Koletzko, B. Representative sampling of human milk and the extraction of fat for analysis of environmental lipophilic contaminants. Toxicol. Environ. Chem. 1997, 62, 229-247. [CrossRef]

37. Lubetzky, R.; Littner, Y.; Mimouni, F.B.; Dollberg, S.; Mandel, D. Circadian variations in fat content of expressed breast milk from mothers of preterm infants. J. Am. Coll. Nutr. 2006, 25, 151-154. [CrossRef] [PubMed]

38. Kent, J.C.; Mitoulas, L.R.; Cregan, M.D.; Ramsay, D.T.; Doherty, D.A.; Hartmann, P.E. Volume and frequency of breastfeedings and fat content of breast milk throughout the day. Pediatrics 2006, 117, E387-e395. [CrossRef] [PubMed]

39. Jackson, D.A.; Imong, S.M.; Silprasert, A.; Ruckphaopunt, S.; Woolridge, M.W.; Baum, J.D.; Amatayakul, K. Circadian variation in fat concentration of breast-milk in a rural northern Thai population. Br. J. Nutr. 1988, 59, 349-363. [CrossRef] [PubMed]

40. Hassiotou, F.; Hepworth, A.R.; Williams, T.M.; Twigger, A.J.; Perrella, S.; Lai, C.T.; Filgueira, L.; Geddes, D.T.; Hartmann, P.E. Breastmilk cell and fat contents respond similarly to removal of breastmilk by the infant. PLoS ONE 2013, 8, E78232. [CrossRef] [PubMed]

41. Chen, Z.-Y.; Pelletier, G.; Hollywood, R.; Ratnayake, W.M. Trans fatty acid isomers in Canadian human milk. Lipids 1995, 30, 15-21. [CrossRef] [PubMed]

42. Pons, S.M.; Bargallo, A.C.; Folgoso, C.C.; Lopez Sabater, M.C. Triacylglycerol composition in colostrum, transitional and mature human milk. Eur. J. Clin. Nutr. 2000, 54, 878-882. [CrossRef] [PubMed]

43. Scopesi, F.; Ciangherotti, S.; Lantieri, P.B.; Risso, D.; Bertini, I.; Campone, F.; Pedrotti, A.; Bonacci, W.; Serra, G. Maternal dietary pufas intake and human milk content relationships during the first month of lactation. Clin. Nutr. 2001, 20, 393-397. [CrossRef] [PubMed]

44. Minda, H.; Kovacs, A.; Funke, S.; Szasz, M.; Burus, I.; Molnar, S.; Marosvolgyi, T.; Decsi, T. Changes of fatty acid composition of human milk during the first month of lactation: A day-to-day approach in the first week. Ann. Nutr. Metab. 2004, 48, 202-209. [CrossRef] [PubMed] 
45. Mosley, E.E.; Wright, A.L.; McGuire, M.K.; McGuire, M.A. Trans fatty acids in milk produced by women in the United States. Am. J. Clin. Nutr. 2005, 82, 1292-1297. [CrossRef] [PubMed]

46. Dreiucker, J.; Vetter, W. Fatty acids patterns in camel, moose, cow and human milk as determined with GC/MS after silver ion solid phase extraction. Food Chem. 2011, 126, 762-771. [CrossRef]

47. Kelishadi, R.; Hadi, B.; Iranpour, R.; Khosravi-Darani, K.; Mirmoghtadaee, P.; Farajian, S.; Poursafa, P. A study on lipid content and fatty acid of breast milk and its association with mother's diet composition. J. Res. Med. Sci. 2012, 17, 824-827. [PubMed]

48. Daud, A.Z.; Mohd-Esa, N.; Azlan, A.; Chan, Y.M. The 'trans' fatty acid content in human milk and its association with maternal diet among lactating mothers in Malaysia. Asia Pac. J. Clin. Nutr. 2013, 22, 431-442. [PubMed]

49. Saphier, O.; Blumenfeld, J.; Silberstein, T.; Tzor, T.; Burg, A. Fatty acid composition of breastmilk of Israeli mothers. Indian Pediatr. 2013, 50, 1044-1046. [CrossRef] [PubMed]

50. Chuang, C.-K.; Yeung, C.-Y.; Jim, W.-T.; Lin, S.-P.; Wang, T.J.; Huang, S.-F.; Liu, H.-L. Comparison of free fatty acid content of human milk from Taiwanese mothers and infant formula. Taiwan. J. Obstet. Gynecol. 2013, 52, 527-533. [CrossRef] [PubMed]

51. Villasenor, A.; Garcia-Perez, I.; Garcia, A.; Posma, J.M.; Fernandez-Lopez, M.; Nicholas, A.J.; Modi, N.; Holmes, E.; Barbas, C. Breast Milk metabolome characterization in a single-phase extraction, multiplatform analytical approach. Anal. Chem. 2014, 86, 8245-8252. [CrossRef] [PubMed]

52. Sokol, E.; Ulven, T.; Færgeman, N.J.; Ejsing, C.S. Comprehensive and quantitative profiling of lipid species in human milk, cow milk and a phospholipid-enriched milk formula by GC and MS/MS(All). Eur. J. Lipid Sci. Technol. 2015, 117, 751-759. [CrossRef] [PubMed]

53. Ten-Doménech, I.; Beltrán-Iturat, E.; Herrero-Martínez, J.M.; Sancho-Llopis, J.V.; Simó-Alfonso, E.F. Triacylglycerol analysis in human milk and other mammalian species: Small-scale sample preparation, characterization, and statistical classification using HPLC-ELSD profiles. J. Agric. Food Chem. 2015, 63, 5761-5770.

54. Liu, G.; Ding, Z.; Li, X.; Chen, X.; Wu, Y.; Xie, L. Relationship between polyunsaturated fatty acid levels in maternal diets and human milk in the first month post-partum. J. Hum. Nutr. Diet. 2016, 29, 405-410. [CrossRef] [PubMed]

55. Garwolińska, D.; Hewelt-Belka, W.; Namieśnik, J.; Kot-Wasik, A. Rapid characterization of the human breast milk lipidome using a Solid-Phase Microextraction and liquid Chromatography-Mass Spectrometry-based approach. J. Proteom. Res. 2017, 16, 3200-3208. [CrossRef] [PubMed]

56. Wu, J.; Domellöf, M.; Zivkovic, A.M.; Larsson, G.; Öhman, A.; Nording, M.L. NMR-based metabolite profiling of human milk: A pilot study of methods for investigating compositional changes during lactation. Biochem. Biophys. Res. Commun. 2016, 469, 626-632. [CrossRef] [PubMed]

57. Tu, A.; Ma, Q.; Bai, H.; Du, Z. A comparative study of triacylglycerol composition in Chinese human milk within different lactation stages and imported infant formula by SFC Coupled with Q-TOF-MS. Food Chem. 2017, 221, 555-567. [CrossRef] [PubMed]

58. Zechner, R. Rapid and simple isolation procedure for lipoprotein lipase from human milk. Biochim. Biophys. Acta (BBA) Lipids Lipid MeTable 1990, 1044, 20-25. [CrossRef]

59. Freudenberg, E. Lipase of human milk; Studies on its enzymological and nutritional significance. Bibl. Paediatr. 1953, 54, 1-68.

60. Berkow, S.E.; Freed, L.M.; Hamosh, M.; Bitman, J.; Wood, D.L.; Happ, B.; Hamosh, P. Lipases and lipids in human milk: Effect of freeze-thawing and storage. Pediatr. Res. 1984, 18, 1257-1262. [CrossRef] [PubMed]

61. Chang, Y.-C.; Chen, C.-H.; Lin, M.-C. The macronutrients in human milk change after storage in various containers. Pediatr. Neonatol. 2012, 53, 205-209. [CrossRef] [PubMed]

62. Garcia-Lara, N.R.; Escuder-Vieco, D.; Garcia-Algar, O.; De la Cruz, J.; Lora, D.; Pallas-Alonso, C. Effect of freezing time on macronutrients and energy content of breastmilk. Breastfeed. Med. 2012, 7, 295-301. [CrossRef] [PubMed]

63. Lev, H.M.; Ovental, A.; Mandel, D.; Mimouni, F.B.; Marom, R.; Lubetzky, R. Major losses of fat, carbohydrates and energy content of preterm human milk frozen at $-80^{\circ} \mathrm{C}$. J. Perinatol. 2014, 34, 396-398. [CrossRef] [PubMed] 
64. Fusch, G.; Rochow, N.; Choi, A.; Fusch, S.; Poeschl, S.; Ubah, A.O.; Lee, S.-Y.; Raja, P.; Fusch, C. Rapid measurement of macronutrients in breast milk: How reliable are infrared milk analyzers? Clin. Nutr. 2015, 34, 465-476. [CrossRef] [PubMed]

65. Bitman, J.; Wood, D.L.; Mehta, N.R.; Hamosh, P.; Hamosh, M. Lipolysis of triglycerides of human milk during storage at low temperatures: A note of caution. J. Pediatr. Gastroenterol. Nutr. 1983, 2, 521-524. [CrossRef] [PubMed]

66. Tijerina-Saenz, A.; Innis, S.M.; Kitts, D.D. Antioxidant capacity of human milk and its association with vitamins A and E and fatty acid composition. Acta Paediatr. 2009, 98, 1793-1798. [CrossRef] [PubMed]

67. Boris, J.; Jensen, B.; Dalby Salvig, J.; Secher, N.J.; Olsen, S.F. A randomized controlled trial of the effect of fish oil supplementation in late pregnancy and early lactation on the n-3 fatty acid content in human breast milk. Lipids 2004, 39, 1191-1196. [CrossRef] [PubMed]

68. Magnusardottir, A.R.; Skuladottir, G.V. Effects of storage time and added antioxidant on fatty acid composition of red blood cells at $-20^{\circ} \mathrm{C}$. Lipids 2006, 41, 401-404. [CrossRef] [PubMed]

69. Folch, J.; Lees, M.; Sloane Stanley, G.H. A simple method for the isolation and purification of total lipides from animal tissues. J. Bio.l Chem. 1957, 226, 497-509.

70. Bligh, E.G.; Dyer, W.J. A rapid method of total lipid extraction and purification. Can. J. Biochem. Physiol. 1959, 37, 911-917. [CrossRef] [PubMed]

71. Ruiz-Gutierrez, V.; Perez-Camino, M.C. Update on solid-phase extraction for the analysis of lipid classes and related compounds. J. Chromatogr. A 2000, 885, 321-341. [CrossRef]

72. Chappell, J.E.; Clandinin, M.T.; McVey, M.A.; Chance, G.W. Free fatty acid content of human milk: Physiologic significance and artifactual determinants. Lipids 1985, 20, 216-221. [CrossRef] [PubMed]

73. Meurant, G. Handbook of Milk Composition, 1st ed.; Jensen, R.G., Ed.; Academic Press: San Diego, CA, USA, 1995.

74. Orata, F. Derivatization reactions and reagents for Gas Chromatography analysis. Adv. Gas Chromatogr. 2012, 5, 83-108.

75. Morrison, W.R.; Smith, L.M. Preparation of fatty acid methyl esters and dimethylacetals from lipids with boron fluoride-Methanol. J. Lipid Res. 1964, 5, 600-608. [PubMed]

76. Christie, W.W. Preparation of ester derivatives of fatty acids for chromatographic analysis. Adv. Lipid Method. 1993, 2, 69-111.

77. Broadhurst, D.; Goodacre, R.; Reinke, S.N.; Kuligowski, J.; Wilson, I.D.; Lewis, M.R.; Dunn, W.B. Guidelines and considerations for the use of system suitability and quality control samples in mass spectrometry assays applied in untargeted clinical metabolomic studies. Metabolomics 2018, 14, 72. [CrossRef] [PubMed]

78. Yang, K.; Han, X. Lipidomics: Techniques, applications, and outcomes related to biomedical sciences. Trends Biochem. Sci. 2016, 41, 954-969. [CrossRef] [PubMed]

79. Jurowski, K.; Kochan, K.; Walczak, J.; Baranska, M.; Piekoszewski, W.; Buszewski, B. Analytical techniques in lipidomics: State of the art. Crit. Rev. Anal. Chem. 2017, 47, 418-437. [CrossRef] [PubMed]

80. Ackman, R.G. Misidentification of fatty acid methyl ester peaks in liquid canola shortening. J. Am. Oil Chem. Soc. 1990, 67, 1028. [CrossRef]

81. Hawrysh, Z.J.; Shand, P.J.; Lin, C.; Tokarska, B.; Hardin, R.T. Efficacy of tertiary butylhydroquinone on the storage and heat stability of liquid canola shortening. J. Am. Oil Chem. Soc. 1990, 67, 585-590. [CrossRef]

82. Waston, A.D. Thematic review series: Systems biology approaches to metabolic and cardiovascular disorders. Lipidomics: A global approach to lipid analysis in biological systems. J. Lipid Res. 2006, 47, 2101-2111.

83. Sommer, U.; Herscovitz, H.; Welty, F.K.; Costello, C.E. LC-MS-based method for the qualitative and quantitative analysis of complex lipid mixtures. J. Lipid Res. 2006, 47, 804-814. [CrossRef] [PubMed]

84. Laakso, P.; Manninen, P. Identification of milk fat triacylglycerols by capillary supercritical fluid chromatography-atmospheric pressure chemical ionization mass spectrometry. Lipids 1997, 32, 1285-1295. [CrossRef] [PubMed]

85. Taylor, L.T. Supercritical fluid chromatography. Anal. Chem. 2010, 82, 4925-4935. [CrossRef] [PubMed]

86. Garcia, C.; Lutz, N.W.; Confort-Gouny, S.; Cozzone, P.J.; Armand, M.; Bernard, M. Phospholipid fingerprints of milk from different mammalians determined by ${ }^{31} \mathrm{p}$ NMR: Towards specific interest in human health. Food Chem. 2012, 135, 1777-1783. [CrossRef] [PubMed]

87. Sundekilde, U.K.; Larsen, L.B.; Bertram, H.C. NMR-based milk metabolomics. Metabolites 2013, 3, $204-222$. [CrossRef] [PubMed] 
88. Guillén, M.D.; Ruiz, A. ${ }^{1} \mathrm{H}$ nuclear magnetic resonance as a fast tool for determining the composition of acyl chains in acylglycerol mixtures. Eur. J. Lipid Sci. Technol. 2003, 105, 502-507.

89. Wishart, D.S. Metabolomics: Applications to food science and nutrition research. Trends Food Sci. Technol. 2008, 19, 482-493. [CrossRef]

90. Wishart, D.S.; Feunang, Y.D.; Marcu, A.; Guo, A.C.; Liang, K.; Vazquez-Fresno, R.; Sajed, T.; Johnson, D.; Li, C.; Karu, N.; et al. HMDB 4.0: The human metabolome database for 2018. Nucleic Acids Res. 2018, 46, D608-D617. [CrossRef] [PubMed]

(C) 2018 by the authors. Licensee MDPI, Basel, Switzerland. This article is an open access article distributed under the terms and conditions of the Creative Commons Attribution (CC BY) license (http://creativecommons.org/licenses/by/4.0/). 
Review

\title{
Sex-Specific Human Milk Composition: The Role of Infant Sex in Determining Early Life Nutrition
}

\author{
Laura Galante ${ }^{1}$, Amber M. Milan ${ }^{1}$, Clare M. Reynolds ${ }^{1}$, David Cameron-Smith ${ }^{1,2,3}$, \\ Mark H. Vickers ${ }^{1}$ and Shikha Pundir ${ }^{1, *}$ \\ 1 Liggins Institute, the University of Auckland, Auckland 1142, New Zealand; 1.galante@auckland.ac.nz (L.G.); \\ a.milan@auckland.ac.nz (A.M.M.); c.reynolds@auckland.ac.nz (C.M.R.); \\ d.cameron-smith@auckland.ac.nz (D.C.-S.); m.vickers@auckland.ac.nz (M.H.V.) \\ 2 Riddet Institute, Massey University, Palmerston North 4442, New Zealand \\ 3 Food \& Bio-Based Products Group, AgResearch, Palmerston North 4442, New Zealand \\ * Correspondence: s.pundir@auckland.ac.nz; Tel.: +64-9-923-4087
}

Received: 3 August 2018; Accepted: 29 August 2018; Published: 1 September 2018

\begin{abstract}
Male and female infants respond differentially to environmental stimuli, with different growth and neurodevelopmental trajectories. Male infants are more likely to be disadvantaged when subjected to adversity and show a higher risk of perinatal complications. However, the underlying causes of this sex-bias are not well defined and optimising the early life nutritional care may be necessary to minimise the "male disadvantage" that may be experienced early in life. Experimental models have demonstrated that animal milk composition differs according to offspring sex, suggesting that the tailoring of early life nutrition may be one mechanism to maximise health protection and development to infants of both sexes. However, evidence for a sex-specificity in human milk composition is limited and conflicting, with studies documenting higher milk energy content for either male or female infants. These data show sex differences, however, there has been limited compositional analysis of the current data nor strategies proposed for how sex-specific compositional differences in early life nutrition may be used to improve infant health. The present narrative review highlights that an improved understanding of sex-specific human milk composition is essential for promoting optimal infant growth and development.
\end{abstract}

Keywords: human milk; sex-specificity; infant growth; early life nutrition; postnatal outcomes; breastfeeding

\section{Introduction}

Human milk (HM) is the gold standard for infant nutrition, providing the necessary building blocks required for postnatal growth and development [1,2]. The biochemical composition of HM is highly dynamic and varies between mothers and within the same mother throughout the different stages of lactation [3-6] and within a feed, such that foremilk and hindmilk have differing biochemical compositions [7]. HM composition changes most notably during the transition from colostrum to mature milk, with the intermediate of transitional milk, as lactation proceeds [8]. However, the dynamic regulation of HM composition is also impacted by maternal, environmental, and infant factors. Changes in maternal diet significantly influence HM macronutrient composition, particularly within the lipid fraction. This is evident in mothers consuming a western-style diet, where high dietary consumption of omega- 6 is directly reflected in the HM lipid profile [9]. Further, maternal BMI has also documented impacts on HM lipid profile with different fatty acid profile between obese and lean mothers $[10,11]$. Along with macronutrient composition, non-nutritive milk bioactives are also impacted by maternal factors such as stress [12] and BMI [13]. HM bioactives, such as 
growth [14] and satiety factors [15,16], and different classes of hormones, such as steroids [17], play important roles in human physiology, including the regulation of energy intake $[18,19]$ and somatic growth $[20,21]$. Interestingly, most of these bioactive compounds have been reported to follow sex-specific secretion pathways in adults, children [22,23], and in cord blood [24,25], yet, little is known about their sex-specific distribution in HM.

Despite international recognition of the importance of breastfeeding, not all infants can be exclusively breastfed. Some mothers indeed encounter challenges in establishing and / or maintaining lactation over the first six months of life [26-29], which results in early weaning or formula-feeding for their infants. Nutritional and hormonal exposures during the first 1000 days of life are known to be important for both short- and long-term health outcomes [30]. In this context, the observation that male and female infants have a sexually-dimorphic response to their early nutritional environment [31] suggests that there are sex-specific requirements during this early phase of life for optimal growth and development. Yet, there are currently no practice guidelines establishing different nutritional strategies or requirements for male and female infants [32]. For this reason, a better understanding of whether male and female infants receive different nutritional and hormonal intakes through maternal HM would provide important information around the potential need to tailor nutritional strategies for clinical and community settings in a sex-specific manner.

The present work constitutes a narrative review of the existing evidence regarding sex-specificity in HM composition in relation to sex-specific health outcomes such as the higher perinatal risk of mortality and morbidity observed in male neonates.

\section{The "Male Disadvantage": A Consequence of Sex-Specific Requirements?}

Differences in perinatal outcomes between males and females have been recognised since the 1970's as the "male disadvantage" [33]. The concept arose from the observation that male infants in the United States had a higher risk of neonatal mortality compared to females, and that this was not related to specific disease processes. Newborn males are known to be more vulnerable than females to postnatal complications including respiratory distress syndrome [34], neonatal anaemia and mineral deficiencies, particularly in high-risk populations such as low birthweight (LBW) infants [35]. Stevenson et al., later examined the "male disadvantage" to understand whether technological advances in neonatal care, such as improved ventilation, enhanced surfactant therapies and administration of antenatal steroids, would improve outcomes in male infants. However, despite an overall decrease in mortality following the implementation of these strategies, a sex-bias was still observed in male infants [36].

At present, sex-specific differences in perinatal health outcomes, including neurological, metabolic and respiratory complications, with males having higher risk for poorer health [37,38], remain of concern as a cause of higher mortality and morbidity in male newborns. While the main cause for this bias is unknown, the observation that female and male offspring react differently to early life nutritional stimuli $[39,40]$ may be a crucial factor in our understanding of the mechanisms underlying these differences in postnatal outcomes. Research carried out on ovine model has shown that offspring of different sexes respond differently to a standard supplemental nutrition [41]. When newborn lambs were randomised to receive maternal milk and either milk fortifiers or water for two weeks they showed differential outcomes compared to same-sex controls, with supplemented males displaying increased insulin in response to the intravenous glucose tolerance test (IVGTT) received at four months. Conversely, insulin was lower after the IVGTT in supplemented females compared to female controls. Divergent responses to infant feeding have also been documented in humans. Lucas et al., demonstrated that in humans, male preterm neonates were more responsive than females to preterm formula with higher protein, energy content and micronutrients to meet higher preterm requirements. These infants displayed higher Bayley's test scores, indicative of improved neurodevelopment, at 18 months of age, in comparison to male infants receiving a standard term formula [31]. Given the evidence of sex-specific infant outcomes based on differences in early life nutritional support, 
an improved understanding of the innate compositional differences in HM produced for these infants is essential to establishing requirements and guidelines for supplemental support.

\section{Sex-Specific Composition of Maternal Milk}

Evidence from animal models (Table 1) suggests that infant sex is a predictive determinant of maternal milk composition. Primate [41-43] and bovine [44] models have demonstrated that mothers produce different milk for male and female singleton offspring. For example, macaque (Macaca mulatta) mothers of male offspring produced a lower volume of milk but with a higher energy content, whereas mothers of female offspring had greater volume of milk which was less energy-dense, resulting in similar total energy content of the milk produced for both sexes [42]. Yet, milk produced for female macaque offspring had higher calcium content [41]. In a bovine model, mothers have been shown to produce considerably more milk and of higher energy content for their female offspring [44]. Evidence of sex-specific milk production has also been reported for other ruminants, as well as marsupials. Wild eastern kangaroos (Macropus giganteus) [45] and Tammar wallabies (Macropus eugenii) [46] were found to produce milk with higher protein for male offspring but same energy content and volume for offspring of different sex. Similarly, Iberian red deer (Cervus elaphus hispanicus) mothers were observed to produce greater yields of milk with higher energy content for male calves, reflected by higher protein, fat and lactose content [47]. While these studies provide evidence that the sex of offspring has an impact on the nutritional composition of maternal milk across very different taxa, the range of milk-borne compounds analysed in each study is limited and no information is available around hormonal concentration in milk produced for male and female offspring. Furthermore, the mechanisms driving sex-specific milk synthesis are currently unclear and need to be investigated in order to enable a better understanding of the phenomenon. 


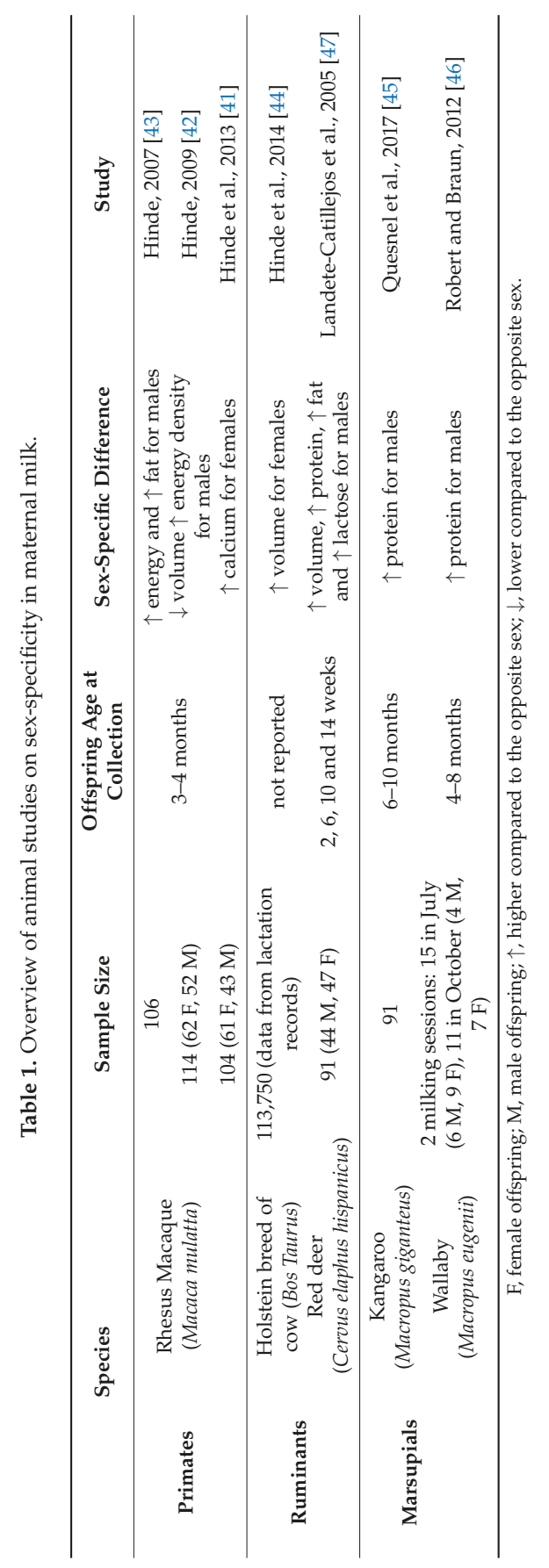


Despite data derived from experimental models, there remains a paucity of data around the potential for sex-specificity in HM composition in regards to nutrients and bioactive compounds. The potential for a sex-specificity has however been suggested by clinical research on twins [48]. In this setting, opposite-sex twins, that receive HM which cannot simultaneously be tailored for both sexes, have been hypothesised to have different outcomes in comparison with same-sex twins in regards to postnatal growth [48]. Kanazawa and Segal observed indeed that breastfed opposite-sex twins were on average 1 inch shorter and 12 pounds lighter than same-sex twins during adolescence and early adulthood. This may suggest that the sex-specificity of HM might have a role in the early growth of the infant and exerts effects that persist throughout the life course of the individual.

Nonetheless to date only six human studies have reported on the relationship between HM composition and infant sex [49-54]. These studies primarily focused on macronutrient profile and energy content of HM and reported conflicting relationships in regard to infant sex. In particular, one study on Filipino mothers found no association between HM composition and infant sex [52]. Another study reported that American mothers of male infants produce HM with a greater energy content [49]; whereas, other research groups found that HM was higher in energy for female infants in Korean and Kenyan mothers [50,51]. Interestingly, the study on Kenyan mothers reported a conditional association between female sex and higher fat content in HM, that was displayed only by mothers with low socioeconomic status [50], supporting the Trivers-Willard hypothesis of unequal parental investment between female and male offspring depending on maternal condition [55]. Mirroring experimental models, HM for female infants in Iraqi mothers was found to have a higher calcium content; yet phosphorus content and total volume were reduced relative to the HM produced for male infants [53]. However, these studies reported several limitations and were conducted in different contexts and with very different sampling and analytical methods, as summarized in Table 2, which likely explain the disparity in findings. Nevertheless, despite being limited by collection methods, Fujita's study [50] highlights the fact that HM composition is affected not only by multiple factors but also by the interactions between factors. A similar conditional association of the infant sex with HM composition has been reported by Fields et al., while looking at the influence of maternal BMI on HM composition [54]. Here, similarly to what was observed in Kenyan mothers, where fat content in $\mathrm{HM}$ was conditionally altered for female infants by the socioeconomic status of the family, Fields et al., found that insulin and leptin concentrations in maternal HM were the highest for mothers of females only when maternal BMI was very high [54].

Importantly, there is emerging research demonstrating a sex-specific differential effect of animal milk bioactives on offspring outcomes. As an example, elevated cortisol in the milk of lactating macaques has been found to correlate with a more nervousness and less confident temperament, impacting male offspring to a greater extent than female [56]. However, existing evidence supporting the idea of a sex-specific response to the nutrition received during early life (Table 3) is limited. The available findings often relate to subjects at risk, such as those born preterm or small for gestational age [31,57] and only assess the effect of the nutrition received immediately after birth [40]. Furthermore, in most of these studies the compositional analysis of maternal milk is not included, preventing the possibility of any association between specific milk-borne compounds and sex-specific response. This suggests the need for further research to fully understand the impact of nutritional and hormonal intakes during this sensitive period on sex-specific infant growth and development. 


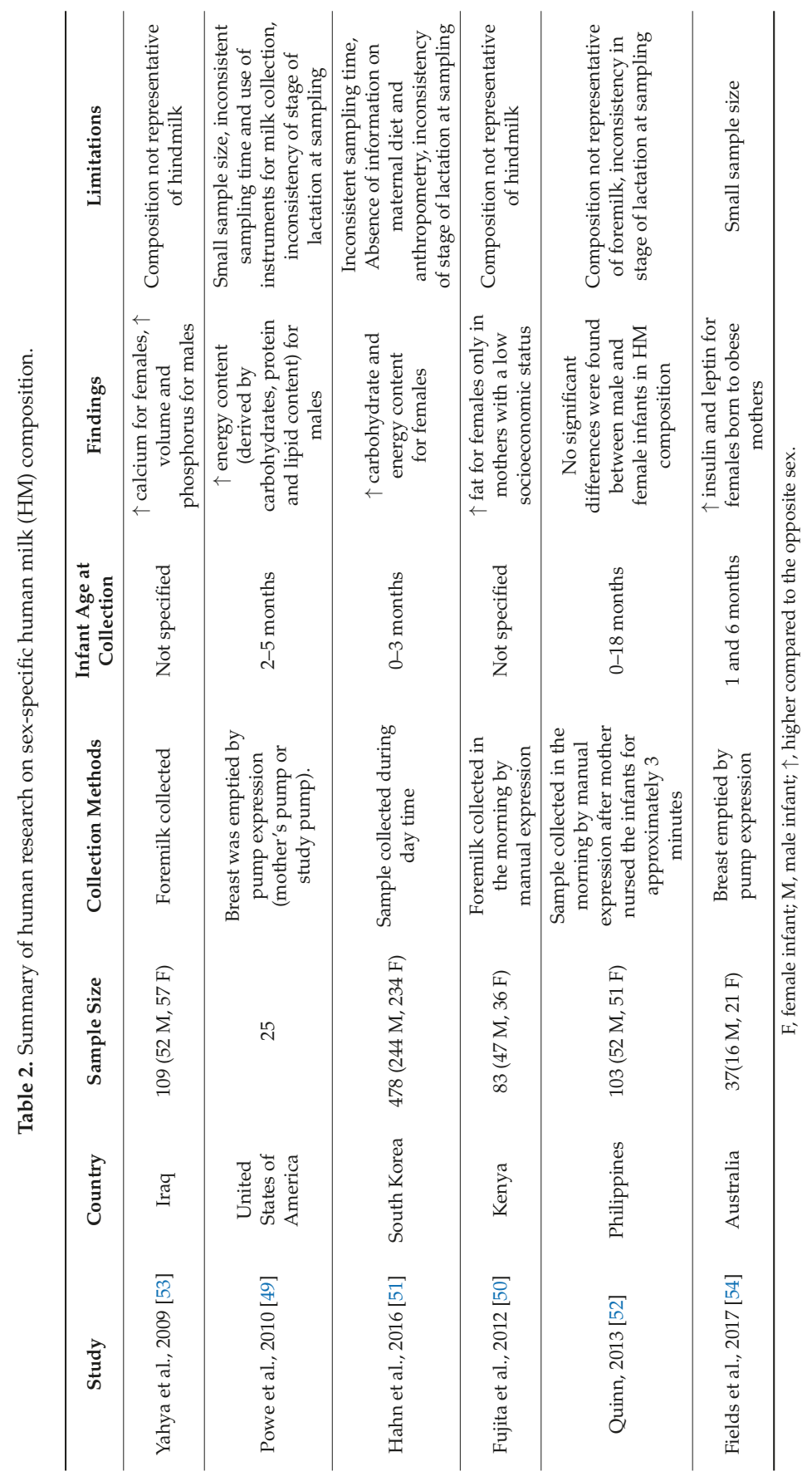




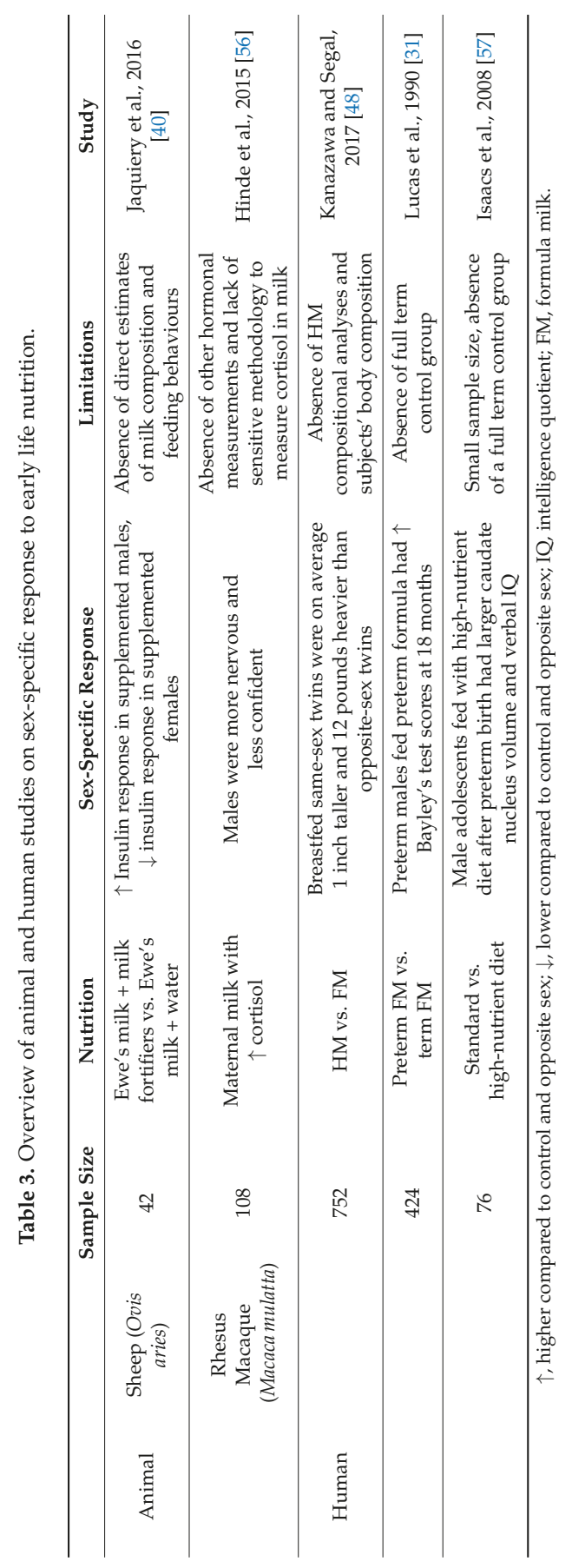




\section{Conclusions}

Evidence to date suggests that infants of different sex may have different nutritional and hormonal requirements. This underpins a need for sex-specific nutritional strategies for male and female infants, in order to optimise their growth and development. Experimental and limited clinical observations that maternal HM composition changes dependent on male or female sex, support this hypothesis. However, these reported sex-specific differences in HM composition require validation and clarification in additional human cohorts. These studies should aim to describe sex-specific differences in HM composition across the course of lactation and correlate differences with sex-specificity of infant outcomes, including sex-specific growth trajectories and sex-specific morbidity risk.

Author Contributions: L.G. wrote the manuscript. A.M.M., C.M.R., D.C.-S., M.H.V., and S.P. critically evaluated and edited the manuscript.

Funding: Funding for L.G., PhD student at Liggins Institute, University of Auckland, was obtained from Liggins Institute PhD Funding. Funding for publishing the manuscript was received from "Liggins Institute Journal Publication Funds" and was supported by the Liggins Institute FRDF (Faculty Research Development Fund) 3716954 grant.

Conflicts of Interest: The authors declare no conflict of interest.

\section{References}

1. Dieterich, C.M.; Felice, J.P.; O'Sullivan, E.; Rasmussen, K.M. Breastfeeding and health outcomes for the mother-infant dyad. Pediatr. Clin. N. Am. 2013, 60, 31-48. [CrossRef] [PubMed]

2. Martin, C.R.; Ling, P.R.; Blackburn, G.L. Review of infant feeding: Key features of breast milk and infant formula. Nutrients 2016, 8, 279. [CrossRef] [PubMed]

3. Zou, X.Q.; Guo, Z.; Huang, J.H.; Jin, Q.Z.; Cheong, L.Z.; Wang, X.G.; Xu, X.B. Human milk fat globules from different stages of lactation: A lipid composition analysis and microstructure characterization. J. Agric. Food Chem. 2012, 60, 7158-7167. [CrossRef] [PubMed]

4. Dewey, K.G.; Finley, D.A.; Lönnerdal, B. Breast milk volume and composition during late lactation (7-20 months). J. Pediatr. Gastroenterol. Nutr. 1984, 3, 713-720. [CrossRef] [PubMed]

5. Jiang, J.; Wu, K.; Yu, Z.; Ren, Y.; Zhao, Y.; Jiang, Y.; Xu, X.; Li, W.; Jin, Y.; Yuan, J.; et al. Changes in fatty acid composition of human milk over lactation stages and relationship with dietary intake in Chinese women. Food Funct. 2016, 7, 3154-3162. [CrossRef] [PubMed]

6. Saarela, T.; Kokkonen, J.; Koivisto, M. Macronutrient and energy contents of human milk fractions during the first six months of lactation. Acta Paediatr. 2005, 94, 1176-1181. [CrossRef] [PubMed]

7. Mizuno, K.; Nishida, Y.; Taki, M.; Murase, M.; Mukai, Y.; Itabashi, K.; Debari, K.; Liyama, A. Is increased fat content of hindmilk due to the size or the number of milk fat globules? Int. Breastfeed J. 2009, 4, 7. [CrossRef] [PubMed]

8. Ballard, O.; Morrow, A.L. Human milk composition: Nutrients and bioactive factors. Pediatr. Clin. N. Am. 2013, 60, 49-74. [CrossRef] [PubMed]

9. Martin, M.A.; Lassek, W.D.; Gaulin, S.J.C.; Evans, R.W.; Woo, J.G.; Geraghty, S.R.; Davidson, B.S.; Morrow, A.L.; Kaplan, H.S.; Gurven, M.D. Fatty acid composition in the mature milk of Bolivian forager-horticulturalists: Controlled comparisons with a US sample. Matern. Child Nutr. 2012, 8, 404-418. [CrossRef] [PubMed]

10. Mäkelä, J.; Linderborg, K.; Niinikoski, H.; Yang, B.; Lagström, H. Breast milk fatty acid composition differs between overweight and normal weight women: the STEPS Study. Eur. J. Nutr. 2013, 52, 727-735. [CrossRef] [PubMed]

11. Panagos, P.G.; Vishwanathan, R.; Penfield-Cyr, A.; Matthan, N.R.; Shivappa, N.; Wirth, M.D.; Hebert, J.R.; Sen, S. Breastmilk from obese mothers has pro-inflammatory properties and decreased neuroprotective factors. J. Perinatol. J. Calif. Perinat. Assoc. 2016, 36, 284-290. [CrossRef] [PubMed]

12. Thibeau, S.; D'Apolito, K.; Minnick, A.F.; Dietrich, M.S.; Kane, B.; Cooley, S.; Groer, M. Relationships of maternal stress with milk immune components in African American mothers of healthy term infants. Breastfeed Med. 2016, 11, 6-14. [CrossRef] [PubMed] 
13. Houseknecht, K.L.; McGuire, M.K.; Portocarrero, C.P.; McGuire, M.A.; Beerman, K. Leptin is present in human milk and is related to maternal plasma leptin concentration and adiposity. Biochem. Biophys. Res. Commun. 1997, 240, 742-747. [CrossRef] [PubMed]

14. Blum, J.W.; Baumrucker, C.R. Insulin-like growth factors (IGFs), IGF binding proteins, and other endocrine factors in milk: Role in the newborn. Adv. Exp. Med. Biol. 2008, 606, 397-422. [CrossRef] [PubMed]

15. Çatlı, G.; Olgaç Dündar, N.; Dündar, B.N. Adipokines in breast milk: An update. J. Clin. Res. Pediatr. Endocrinol. 2014, 6, 192-201. [CrossRef] [PubMed]

16. Savino, F.; Sorrenti, M.; Benetti, S.; Lupica, M.M.; Liguori, S.A.; Oggero, R. Resistin and leptin in breast milk and infants in early life. Early Hum. Dev. 2012, 88, 779-782. [CrossRef] [PubMed]

17. Pundir, S.; Wall, C.R.; Mitchell, C.J.; Thorstensen, E.B.; Lai, C.T.; Geddes, D.T.; Cameron-Smith, D. Variation of Human Milk Glucocorticoids over 24 hour Period. J. Mammary Gland Biol. Neoplasia 2017, 22, 85-92. [CrossRef] [PubMed]

18. Klok, M.D.; Jakobsdottir, S.; Drent, M.L. The role of leptin and ghrelin in the regulation of food intake and body weight in humans: A review. Obes. Rev. J. Int. Assoc. Study Obes. 2007, 8, 21-34. [CrossRef] [PubMed]

19. Dallman, M.F.; Warne, J.P.; Foster, M.T.; Pecoraro, N.C. Glucocorticoids and insulin both modulate caloric intake through actions on the brain. J. Physiol. 2007, 583, 431-436. [CrossRef] [PubMed]

20. Laron, Z. Insulin-like growth factor 1 (IGF-1): A growth hormone. Mol. Pathol. 2001, 54, 311-316. [CrossRef] [PubMed]

21. Murray, P.G.; Clayton, P.E. Endocrine control of growth. Am. J. Med. Genet. C Semin. Med. Genet. 2013, 163C, 76-85. [CrossRef] [PubMed]

22. Geary, M.P.P.; Pringle, P.J.; Rodeck, C.H.; Kingdom, J.C.P.; Hindmarsh, P.C. Sexual dimorphism in the growth hormone and insulin-like growth factor axis at birth. J. Clin. Endocrinol. Metab. 2003, 88, 3708-3714. [CrossRef] [PubMed]

23. Rosenbaum, M.; Leibel, R.L. Role of gonadal steroids in the sexual dimorphisms in body composition and circulating concentrations of leptin. J. Clin. Endocrinol. Metab. 1999, 84, 1784-1789. [CrossRef] [PubMed]

24. Vatten, L.J.; Nilsen, S.T.; Ødegård, R.A.; Romundstad, P.R.; Austgulen, R. Insulin-like growth factor I and leptin in umbilical cord plasma and infant birth size at term. Pediatrics 2002, 109, 1131-1135. [CrossRef] [PubMed]

25. Pardo, I.M.; Geloneze, B.; Tambascia, M.A.; Pereira, J.L.; Barros Filho, A.A. Leptin as a marker of sexual dimorphism in newborn infants. J. Pediatr. 2004, 80, 305-308. [CrossRef]

26. Chapman, D.J.; Pérez-escamilla, R. Identification of risk factors for delayed onset of lactation. J. Am. Diet Assoc. 1999, 99, 450-454. [CrossRef]

27. Hruschka, D.J.; Sellen, D.W.; Stein, A.D.; Martorell, R. Delayed onset of lactation and risk of ending full breast-feeding early in rural guatemala. J. Nutr. 2003, 133, 2592-2599. [CrossRef] [PubMed]

28. Grajeda, R.; Pérez-Escamilla, R. Stress during labor and delivery is associated with delayed onset of lactation among urban Guatemalan women. J. Nutr. 2002, 132, 3055-3060. [CrossRef] [PubMed]

29. Hauck, Y.L.; Fenwick, J.; Dhaliwal, S.S.; Butt, J.A. Western Australian survey of breastfeeding initiation, prevalence and early cessation patterns. Matern. Child Health J. 2011, 15, 260-268. [CrossRef] [PubMed]

30. Ford, N.D.; Behrman, J.R.; Hoddinott, J.F.; Maluccio, J.A.; Martorell, R.; Ramirez-Zea, M.; Stein, A.D. Exposure to improved nutrition from conception to age 2 years and adult cardiometabolic disease risk: A modelling study. Lancet Glob. Health 2018, 6, 875-884. [CrossRef]

31. Lucas, A.; Morley, R.; Cole, T.J.; Lucas, P.J.; Gore, S.M.; Crowle, P.; Pearse, R.; Boon, A.J.; Powell, R. Early diet in preterm babies and developmental status at 18 months. Lancet 1990, 335, 1477-1481. [CrossRef]

32. Harding, J.E.; Cormack, B.E.; Alexander, T.; Alsweiler, J.M.; Bloomfield, F.H. Advances in nutrition of the newborn infant. Lancet Lond. Engl. 2017, 389, 1660-1668. [CrossRef]

33. Naeye, R.L.; Burt, L.S.; Wright, D.L.; Blanc, W.A.; Tatter, D. Neonatal mortality, the male disadvantage. Pediatrics 1971, 48, 902-906. [PubMed]

34. Brothwood, M.; Wolke, D.; Gamsu, H.; Benson, J.; Cooper, D. Prognosis of the very low birthweight baby in relation to gender. Arch. Dis. Child 1986, 61, 559-564. [CrossRef] [PubMed]

35. Libster, R.; Hortoneda, J.B.; Laham, F.R.; Casellas, J.M.; Israele, V.; Polack, N.R.; Delgado, M.F.; Klein, M.I.; Polack, F.P. Breastfeeding prevents severe disease in full term female infants with acute respiratory infection. Pediatr. Infect. Dis. J. 2009, 28, 131. [CrossRef] [PubMed] 
36. Stevenson, D.K.; Verter, J.; Fanaroff, A.A.; Oh, W.; Ehrenkranz, R.A.; Shankaran, S.; Donovan, E.F.; Wright, L.L.; Lemons, J.A.; Tyson, J.E.; et al. Sex differences in outcomes of very low birthweight infants: The newborn male disadvantage. Arch. Dis. Child Fetal. Neonatal. Ed. 2000, 83, 182-185. [CrossRef]

37. Peacock, J.L.; Marston, L.; Marlow, N.; Calvert, S.A.; Greenough, A. Neonatal and infant outcome in boys and girls born very prematurely. Pediatr. Res. 2012, 71, 305-310. [CrossRef] [PubMed]

38. García-Patterson, A.; Aulinas, A.; Sojo, L.; Ginovart, G.; Adelantado, J.M.; de Leiva, A.; Corcoy, R. Poorer perinatal outcome in male newborns of women with pregestational diabetes mellitus. Diabet. Med. J. Br. Diabet. Assoc. 2011, 28, 436-439. [CrossRef] [PubMed]

39. Cheng, T.S.; Loy, S.L.; Cheung, Y.B.; Chan, J.K.Y.; Pang, W.W.; Godfrey, K.M.; Gluckman, P.D.; Kwek, K.; Saw, S.M.; Chong, Y.-S.; et al. Sexually dimorphic response to feeding mode in the growth of infants. Am. J. Clin. Nutr. 2016, 103, 398-405. [CrossRef] [PubMed]

40. Jaquiery, A.L.; Park, S.S.; Phua, H.H.; Berry, M.J.; Meijler, D.; Harding, J.E.; Oliver, M.H.; Bloomfield, F.H. Brief neonatal nutritional supplementation has sex-specific effects on glucose tolerance and insulin regulating genes in juvenile lambs. Pediatr. Res. 2016, 80, 861-869. [CrossRef] [PubMed]

41. Hinde, K.; Foster, A.B.; Landis, L.M.; Rendina, D.; Oftedal, O.T.; Power, M.L. Daughter dearest: Sex-biased calcium in mother's milk among rhesus macaques. Am. J. Phys. Anthropol. 2013, 151, 144-150. [CrossRef] [PubMed]

42. Hinde, K. Richer milk for sons but more milk for daughters: Sex-biased investment during lactation varies with maternal life history in rhesus macaques. Am. J. Hum. Biol. 2009, 512-519. [CrossRef] [PubMed]

43. Hinde, K. First-time macaque mothers bias milk composition in favor of sons. Curr. Biol. 2007, 17, 958-959. [CrossRef] [PubMed]

44. Hinde, K.; Carpenter, A.J.; Clay, J.S.; Bradford, B.J. Holsteins favor Heifers, not bulls: Biased milk production programmed during pregnancy as a function of fetal sex. PLoS ONE 2014, 9, e86169. [CrossRef] [PubMed]

45. Quesnel, L.; MacKay, A.; Forsyth, D.M.; Nicholas, K.R.; Festa-Bianchet, M. Size, season and offspring sex affect milk composition and juvenile survival in wild kangaroos. J. Zool. 2017, 302, 252-262. [CrossRef]

46. Robert, K.A.; Braun, S. Milk Composition during lactation suggests a mechanism for male biased allocation of maternal resources in the tammar wallaby (Macropus eugenii). PLoS ONE 2012, 7, e51099. [CrossRef] [PubMed]

47. Landete-Castillejos, T.; García, A.; López-Serrano, F.R.; Gallego, L. Maternal quality and differences in milk production and composition for male and female Iberian red deer calves (Cervus elaphus hispanicus). Behav. Ecol. Sociobiol. 2005, 57, 267-274. [CrossRef]

48. Kanazawa, S.; Segal, N.L. Same-sex twins are taller and heavier than opposite-sex twins (but only if breastfed): Possible evidence for sex bias in human breast milk. J. Exp. Child Psychol. 2017, 156, 186-191. [CrossRef] [PubMed]

49. Powe, C.E.; Knott, C.D.; Conklin-Brittain, N. Infant sex predicts breast milk energy content. Am. J. Hum. Biol. Off. J. Hum. Biol. Assoc. 2010, 22, 50-54. [CrossRef] [PubMed]

50. Fujita, M.; Roth, E.; Lo, Y.-J.; Hurst, C.; Vollner, J.; Kendell, A. In poor families, mothers' milk is richer for daughters than sons: A test of Trivers-Willard hypothesis in agropastoral settlements in Northern Kenya. Am. J. Phys. Anthropol. 2012, 149, 52-59. [CrossRef] [PubMed]

51. Hahn, W.-H.; Song, J.-H.; Song, S.; Kang, N. mi. Do gender and birth height of infant affect calorie of human milk? An association study between human milk macronutrient and various birth factors. J. Matern. Fetal Neonatal. Med. 2017, 30, 1608-1612. [CrossRef] [PubMed]

52. Quinn, E.A. No evidence for sex biases in milk macronutrients, energy, or breastfeeding frequency in a sample of filipino mothers. Am. J. Phys. Anthropol. 2013, 152, 209-216. [CrossRef] [PubMed]

53. Yahya, A.; Altufaily, C.A.B.P. The effect of infant gender on the quality of breast milk. Kufa Med. J. 2009, 1, 12.

54. Fields, D.A.; George, B.; Williams, M.; Whitaker, K.; Allison, D.B.; Teague, A.; Demerath, E.W. Associations between human breast milk hormones and adipocytokines and infant growth and body composition in the first 6 months of life. Pediatr. Obes. 2017, 1. [CrossRef] [PubMed]

55. Trivers, R.L.; Willard, D.E. Natural selection of parental ability to vary the sex ratio of offspring. Science 1973, 179, 90-92. [CrossRef] [PubMed] 
56. Hinde, K.; Skibiel, A.L.; Foster, A.B.; Del Rosso, L.; Mendoza, S.P.; Capitanio, J.P. Cortisol in mother's milk across lactation reflects maternal life history and predicts infant temperament. Behav. Ecol. 2015, 26, 269-281. [CrossRef] [PubMed]

57. Isaacs, E.B.; Gadian, D.G.; Sabatini, S.; Chong, W.K.; Quinn, B.T.; Fischl, B.R.; Lucas, A. The effect of early human diet on caudate volumes and IQ. Pediatr. Res. 2008, 63, 308-314. [CrossRef] [PubMed]

(C) 2018 by the authors. Licensee MDPI, Basel, Switzerland. This article is an open access article distributed under the terms and conditions of the Creative Commons Attribution (CC BY) license (http:/ / creativecommons.org/licenses/by/4.0/). 
Article

\title{
Levels of Growth Factors and IgA in the Colostrum of Women from Burundi and Italy
}

\author{
Daniel Munblit ${ }^{1,2,3, *,+}$, Priya Abrol ${ }^{1,+}{ }^{\dagger}$, Shreya Sheth ${ }^{1}$, Li Yan Chow ${ }^{1}$, Ekaterina Khaleva ${ }^{3,4}$, \\ Alan Asmanov ${ }^{5}$, Silvana Lauriola ${ }^{6}$, Ezio M. Padovani ${ }^{6}$, Pasquale Comberiati ${ }^{6}$, Attilio L. Boner ${ }^{6}$, \\ John O. Warner ${ }^{1,3}$, Robert J. Boyle ${ }^{1,3, \dagger}$ and Diego G. Peroni ${ }^{3,7, *, \dagger}$ \\ 1 Department of Paediatrics, Imperial College London, London W2 1NY, UK; p.abrol@doctors.org.uk (P.A.); \\ shreyasheth@me.com (S.S.); lychow8@gmail.com (L.Y.C.); j.o.warner@imperial.ac.uk (J.O.W.); \\ r.boyle@nhs.net (R.J.B.) \\ 2 Faculty of Pediatrics, Sechenov University, 119991 Moscow, Russia \\ 3 inVIVO Planetary Health, Group of the Worldwide Universities Network (WUN), 6010 Park Ave, \\ West New York, NJ 07093, USA \\ 4 Department of Paediatrics, Saint-Petersburg State Paediatric Medical University, \\ 194353 Saint-Petersburg, Russia; doctor.khaleva@gmail.com \\ 5 The Research and Clinical Institute for Pediatrics named after Academician Yuri Veltischev of the Pirogov \\ Russian National Research Medical University, 125412 Moscow, Russia; alan-asmanov@yandex.ru \\ 6 Department of Life and Reproduction Sciences, Section of Paediatrics, University of Verona, \\ 37124 Verona, Italy; silvana.lauriola@ospedaleuniverona.it (S.L.); ezio.padovani@univr.it (E.M.P.); \\ pasquale.comberiati@gmail.com (P.C.); attilio.boner@univr.it (A.L.B.) \\ 7 Department of Clinical and Experimental Medicine, Section of Paediatrics, University of Pisa, 56126 Pisa, Italy \\ * Correspondence: daniel.munblit08@imperial.ac.uk (D.M.); diego.peroni@unipi.it (D.G.P.) \\ + These authors contributed equally to this work.
}

Received: 31 July 2018; Accepted: 27 August 2018; Published: 3 September 2018

check for updates

\begin{abstract}
Colostrum is produced in the first days postpartum. It is a known source of immune mediators for a newborn within the first week of life. Although it is still unclear if colostrum composition varies between populations, recent data suggest differences. Hepatocyte growth factor (HGF); transforming growth factor- $\beta$ (TGF- $\beta$ ) 1, 2, and 3; and immunoglobulin A (IgA) are key immunological components of colostrum that stimulate neonatal gastrointestinal and immune system development. We aimed to investigate the differences in the concentration between immune markers in the colostrum of mothers living in Burundi and Italy, and to identify the factors associated with differences. In this cross-sectional birth cohort study, a total of 99 colostrum samples from Burundian $(n=23)$ and Italian $(n=76)$ women were collected at 0 to 6 days postpartum. A clinical chemistry analyser was used for IgA quantification and electro-chemiluminescence, for HGF and TGF $\beta 1-3$ assessment. A univariate analysis and multivariate linear regression model were used for statistical testing. The concentrations of TGF- $\beta 2(p=0.01)$ and $\operatorname{IgA}(p<0.01)$ were significantly higher in the colostrum from the women residing in Burundi than in Italy, both in a univariate analysis and upon the adjustment for confounding factors. A similar trend is seen for HGF, reaching statistical significance upon a multivariate analysis. We found a moderate to strong positive correlation between the TGF- $\beta$ isoforms and IgA concentration in both countries $(p<0.01)$, with stronger concentration in the colostrum from Burundi. The results of this study are in support of previous data, suggesting that concentration of the immune active molecules is higher in the human milk of women residing in developing countries. However, with a small sample size, caution must be applied, as the findings require further confirmation. Future work should also be focused on other factors (e.g., lipid and microbial composition), as well as the investigation into colostrum and between populations comparison, adjusting for potential confounders.
\end{abstract}

Keywords: breast milk; human milk; colostrum; IgA; HGF; TGF- $\beta$; growth factors; geographical location 


\section{Introduction}

Human milk is a first source of nutrition for a newborn child and is globally accepted to be beneficial for the developing infant [1]. The advantages of breastfeeding include the transference of multiple immune factors, maturation of gut immunity, and anti-inflammatory effects [2]. The rising burden of non-communicable diseases increased an interest in their association with human milk (HM) cytokine composition [3,4]. It has been hypothesised that immune mediators in HM may play an important role in both the maturation of the newborn intestine and in stimulating immune system activation [5]. Previous prospective and longitudinal studies provided conflicting evidence on a breastfeeding protective effect on non-communicable [6] and communicable [7] outcomes, and have led to a suggestion that it may be due to the diversity of human milk composition between individuals [8].

Colostrum is the first human milk produced within the first days postpartum, and it allows for the transport of a high concentration of growth factors per unit volume. Hepatocyte growth factor (HGF) is secreted into human colostrum by multipotent mesenchymal stem cells [9]. HGF is expressed in BM as well as in the epithelial cells of the female reproductive tract and the gastrointestinal tract [10]. Patki et al. found greater concentrations of HGF in colostrum compared with paired umbilical cord serum samples [11]. It has been postulated that an elevated concentration of HGF in human milk is needed to maintain proliferation, angiogenesis, and intestinal tissue maturation through paracrine and endocrine signaling [9]. Additionally, HGF may regulate the proinflammatory vascular endothelial growth factor (VEGF) production from endothelial cells [12], and provide a complementary effect with VEGF on the neonatal gut [9].

Transforming growth factor- $\beta$ (TGF- $\beta$ ) is an anti-inflammatory cytokine found in human colostrum. Throughout lactation, all three isoforms of the TGF- $\beta$ superfamily (TGF- $\beta 1$, TGF- $\beta 2$, and TGF- $\beta 3$ ) are produced, of which $95 \%$ is TGF- $\beta 2$. The known effects of maternal TGF- $\beta$ include oral and gut tolerance through the immunosuppressive action on neonatal T-lymphocytes $[13,14]$. Enteral exposures trigger neonatal antigen presenting cells in mucosal surfaces, to suppress inflammatory immune responses to common antigens via TGF- $\beta 2$ mediated tolerance [15], which may lead to the inhibition of antigen specific T-lymphocytes' proliferation [16]. It has been shown that intact cow's milk protein (bovine alpha-S1 casein) is found in human colostrum, and a failure to tolerate this antigen could lead to a sensitisation for cow's milk protein in the infant [17]. Some authors suggest that the TGF- $\beta$ presence in colostrum is of a particular importance, as it may be partially responsible for the control of inflammatory processes that could lead to atopic sensitisation [18-20].

TGF- $\beta$ is also responsible for the antibodies produced by B-lymphocytes' class-switching, in particular, secretory immunoglobulin A (IgA). Japanese researchers found a correlation between the TGF- $\beta 1$ concentration in human milk and IgA levels in the infant serum [21]. IgA secretion in human milk is vital to provide passive immune protection, as the newborn infant is unable to synthesise antibodies until 30 days postpartum [22]. Therefore, TGF- $\beta$ mediated tolerance and IgA antibody synthesis are both key components for infant immune development.

Previous studies have highlighted the importance of maternal and environmental factors' influence on BM composition. These include both genetic and environmental factors, such as diet, psychological background, and maternal atopic status [23-26]. Very few studies have assessed the levels of immune mediators in the human milk of women from Africa and Europe; Holmlund and colleagues identified that Swedish mothers born in Mali retained higher breast milk TGF- $\beta$ concentrations in comparison to women of Swedish origin [27]. The difference in association between the level of TGF- $\beta$ in colostrum and ethnicity was supported further by Aihara Y. et al., explaining it by variations in the consumption of animal protein and the mode of delivery of lactating mothers in Japan and Nepal [28]. Recently, Ruiz and Espinosa-Martos et al. assessed the human milk immunological 
composition of women residing in different geographical locations, including Europe and Africa, showing a substantial variation within and, particularly among, human subpopulations [29].

Based on the The United Nations Children's Fund (UNICEF) reported childhood mortality rate under the age of five, in Italy, it is 4 per 1000, whereas in Burundi, the rate is 104 per 1000, with $52 \%$ of these deaths being attributable to fatal childhood infections [30,31]. We hypothesised that the levels of immune active components, which may be able to reduce neonatal infection risk, in colostrum will be higher in the colostrum of Burundi women. Evidence suggests that not only bacterial exposures, but also a large range of other maternal and environmental factors, may be attributed to changes in the growth factors and IgA concentration [32], subsequently impacting the risk of non-communicable diseases [33] or infection [34] development.

The aim of the study was to investigate the levels of all of the TGF- $\beta$ isoforms (TGF- $\beta 1,2$, and 3 ), HGF, and IgA in the colostrum samples from Italy and Burundi, and to assess the potential factors responsible for the differences.

\section{Materials and Methods}

\subsection{Design}

This cross-sectional birth cohort study involved mother-newborn pairs recruited from postnatal wards. The inclusion criteria for the study were healthy term infants and their mothers willing to comply with the study procedures. The exclusion criteria were maternal immunosuppressive treatment during lactation, or severe illness; infants with a major birth defect, admitted to neonatal intensive care; other severe illnesses; and inability to express colostrum.

Informed consent was obtained from all of the participants who volunteered to take part, along with a colostrum sample and a questionnaire.

The investigations and sample collection were conducted following ethical approval by the ethics committees in the two countries participating in this study, the Ethical Committee of the Azienda Ospedaliera di Verona (Italy) (approval $\mathrm{N}^{\circ} 1288$ ) and the Ngozi Burundi Hospital approval, which was in accordance with Italian standards.

\subsection{Setting}

The women were approached at postnatal wards in two very different environments, namely: in a developed country, G.B. Rossi Hospital, Verona, Italy, and in a developing country, the Hospital of Ngozi, Republic of Burundi. All of the samples were collected in the hospital by a highly skilled member of a research team. The clinical data on the participating infants and mothers were extracted from the hospital notes.

\subsection{Colostrum Samples Collection}

The participants were given sterile tubes to collect their own colostrum (once, in the first six days of life). Instructions were given for the collection of samples by manual expression or by collecting the drip from the contralateral breast during feeding. The colostrum samples were frozen at $-20^{\circ} \mathrm{C}$ in Burundi and $-50{ }^{\circ} \mathrm{C}$ in Verona, within $12 \mathrm{~h}$ of collection, until their transfer to London. All of the samples were transported to Imperial College London, at $-70{ }^{\circ} \mathrm{C}$, where the samples were stored at $-80^{\circ} \mathrm{C}$ until analysis. All of the samples were analysed at Imperial College London facilities to ensure standardisation. After thawing, the samples were centrifuged at $1500 \times \mathrm{g}$ for $15 \mathrm{~min}$ at $4{ }^{\circ} \mathrm{C}$. The lipid layer was removed with a pipette tip, and the aqueous fraction was analysed for immune modulators. 


\subsection{Immune Mediators Measurement}

We used electro-chemiluminescence to measure the immune mediators in the colostrum samples, for HGF and TGF $\beta 1-3$ (MesoScale Discovery, Rockville, MD, USA). LThe laboratory experiments were described in detail elsewhere [26]. In brief, the samples were run in duplicate, according to the manufacturer's protocol, using an 8-point standard curve. No dilution was used for the HGF, and a 1:2 dilution was used for the TGF $\beta$ assays, following pilot experiments, which showed that the TGF $\beta 2$ level in the undiluted milk samples was often greater than the upper limit of detection.

Using an Abbott Architect clinical chemistry analyser ${ }^{\circledR}$ (Abbott, Abbott Park, IL, USA.), the IgA concentration analysis was conducted as described earlier [35]. The colostrum samples were centrifuged at $3000 \times g$ for $15 \mathrm{~min}$ at $4{ }^{\circ} \mathrm{C}$ to remove the lipaemic interference on analysis. Then, $300 \mu \mathrm{L}$ of the supernatant was transferred into the cuvettes using pipettes. The samples were diluted to 1:5 for the IgA quantification. The IgA analysis determined the immunoturbidity of the immune insoluble complexes formed in the presence of the reagent. The IgA analysis with the corresponding reagents was performed according to the manufacturer's protocol.

\subsection{Data Analysis}

Based on previously published research statistical power calculations [36], the sample size of $n=48$ in the Italy group and $n=22$ in Burundi group allows for $80 \%$ power at a 0.05 significance level, which would allow for detecting a $34 \%$ difference between the groups for IgA and $36 \%$ for TGF- $\beta$ in the colostrum. For the purpose of this study, only those samples with all data points available were included in the statistical analysis. The differences between the concentrations of HGF; TGF- $\beta 1,2$, and 3; and IgA in the Burundian and Italian colostrum samples were calculated with GraphPad Prism, Version 6.0 (GraphPad Software, La Jolla, CA, USA), for parametric data, using an unpaired $t$-test, Pearson's chi-squared test, and Fisher's exact test. The Mann-Whitney U test and Kruskal-Wallis one-way analysis of variance (ANOVA) were used for the non-parametric data. The correlations between TGF- $\beta 1,2$, and 3 with IgA were determined by Spearman's rank correlation coefficient. The multivariate linear regression using a backward stepwise entry, was conducted using IBM SPSS Statistics software for Windows, Version 20 (IBM Corp, Armonk, NY, USA). The factors in the regression model included the maternal age, country of origin, animal contact, and parity. The results were considered significant when the $p$-values were reported at a level less than 0.05 .

\section{Results}

\subsection{Study Participants Demographics}

Based on the questionnaire completed by all of the participants in the study, the demographic characteristics of the cohort have been assessed. Out of the total 117 women providing colostrum (Italy, $n=76$; Burundi, $n=41$ ), the full demographic details were available for 99 mothers only, and were included in the analysis (Italy, $n=76$; Burundi, $n=23$ ). Their demographic data is shown in Table 1. The maternal and parturition characteristics were different between the samples from Burundi and Italy. The women in Burundi were younger than the women in Italy $(p<0.01)$ and had more pregnancies $(p<0.01)$. The infants from Burundi were characterised by a lower weight and gestational age ( $p<0.01$ for both). 
Table 1. Characteristics of study participants between sites of collection.

\begin{tabular}{|c|c|c|c|}
\hline Characteristics & Burundi & Italy & $p$-Value \\
\hline Maternal age (years), mean (SD) ${ }^{1}$ & $24.30(5.57)$ & $37.39(5.38)$ & $<0.01$ \\
\hline Birth weight (grams), mean (SD) ${ }^{1}$ & $2831(746.8)$ & $3328(476.9)$ & $<0.01$ \\
\hline Gestational age (weeks), mean (SD) ${ }^{1}$ & $36.48(1.12)$ & $39.36(1 / 34)$ & $<0.01$ \\
\hline Gender (male), $n(\%)^{2}$ & $15 / 25(60)$ & $41 / 76(54)$ & 0.60 \\
\hline Mode of delivery (c-section), $n(\%)^{3}$ & $7 / 21(33)$ & $14 / 76(18)$ & 0.14 \\
\hline Parity, mean (SD) ${ }^{4}$ & $2.04(1.15)$ & $0.81(0.80)$ & $<0.01$ \\
\hline Maternal smoking, $n(\%)^{5}$ & $2 / 23(9)$ & $3 / 76(4)$ & 0.33 \\
\hline Antenatal infections 6 & $6 / 23(26)$ & $29 / 76(38)$ & 0.29 \\
\hline Regular animal contact ${ }^{6}$ & $10 / 23(43)$ & $27 / 76(36)$ & 0.49 \\
\hline Time of colostrum collection (hours), mean (SD) ${ }^{1}$ & $58.29(26.4)$ & $51.13(32.61)$ & 0.28 \\
\hline
\end{tabular}

Statistical methods used: ${ }^{1}$ Unpaired $t$ tests. ${ }^{2}$ Pearson chi-squared and data dichotomised into groups of female and male. ${ }^{3}$ Pearson chi-squared test and data dichotomised into groups of vaginal delivery and caesarean section (c-section) delivery. ${ }^{4}$ Mann-Whitney U test. ${ }^{5}$ Fisher's exact test. ${ }^{6}$ Pearson chi-squared test. Statistically significant results presented in bold. SD—standard deviation.

\subsection{Quantification of Factors in Colostrum Samples}

\subsubsection{Univariate Analysis Results}

The analysis of the TGF- $\beta 1$ and TGF- $\beta 3$ raw concentrations found no significant difference between the Burundian and Italian women. The HGF levels did not differ significantly, but there was a trend for higher levels in the colostrum of the Burundian women $(2785 \mathrm{pg} / \mathrm{mL}$ and $697.4-10,107$ vs. $1316,785-2628$, for the median and interquartile range (IQR), respectively, $p=0.08$ ) (Figure 1a). The TGF- $\beta 2$ concentrations in the Burundi women's colostrum were significantly higher than the colostrum samples from the Italian women $(59,708$ and $36,865-113,221 \mathrm{pg} / \mathrm{mL}$ vs. 33,176 and $18,046-66,520 \mathrm{pg} / \mathrm{mL}$, for the median and interquartile range (IQR), respectively, $p=0.01$ ) (Figure 1c). The principle concentration of the growth factor found in the human colostrum was dominated by TGF- $\beta 2$, followed by HGF, TGF- $\beta 3$, and TGF- $\beta 1$, respectively.

The concentration of IgA in the colostrum from Burundian mothers was significantly higher than in the Italian colostrum samples $(2.78$ and $1.45-22.2 \mathrm{~g} / \mathrm{L}$ vs. 1.48 and $0.89-2.67 \mathrm{~g} / \mathrm{L}$, for the median and interquartile range (IQR), respectively, $p<0.01$ ) (Figure 1e). 
(a)

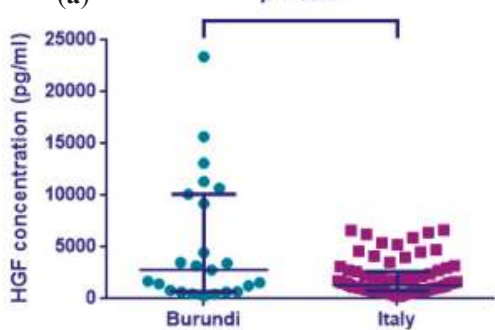

(c)

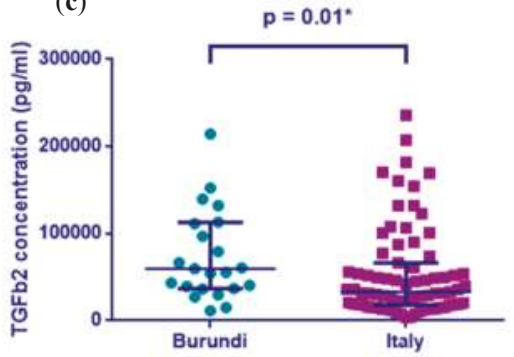

(b)

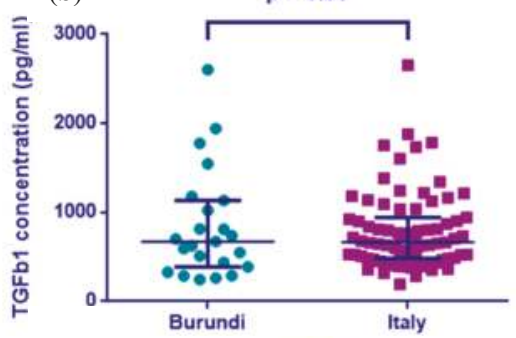

(d)

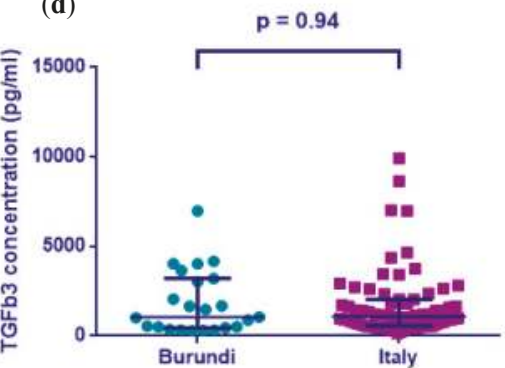

(e)

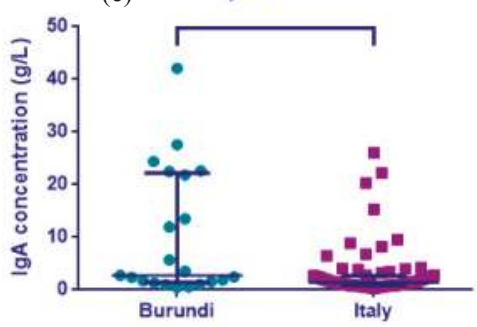

Figure 1. Comparison of growth factors: (a) hepatocyte growth factor (HGF), (b) transforming growth factor- $\beta 1$ (TGF- $\beta 1$ ), (c) TGF- $\beta 2$, (d) TGF- $\beta 3$, and (e) immunoglobulin A (IgA) raw concentrations in the colostrum samples from Burundi and Italy, using the Mann-Whitney $U$ test. The results were considered significant when the $p$-values were reported at a level less than 0.05 *.

\subsubsection{Multivariate Analysis Results}

The multivariate linear regression analysis was used to adjust for the factors that may affect the immune mediator concentration in human milk. The backwards regression models calculated the most significant factors contributing to the differences in concentration, shown in Table 2. The difference in the TGF- $\beta 2$ and IgA concentrations between the countries remained significant upon adjustment for potential confounders. The levels of HGF, which were at a borderline significance at the univariate analysis $(p=0.08)$, showed a significant difference upon adjustment. 
Table 2. Univariate and adjusted analysis for comparison of growth factors and IgA levels in the colostrum of women from Burundi and Italy.

\begin{tabular}{|c|c|c|c|c|c|}
\hline \multirow{2}{*}{ Immune Factor } & \multirow{2}{*}{ Burundi Median (IQR) } & \multirow{2}{*}{ Italy Median (IQR) } & \multirow{2}{*}{$\begin{array}{c}\text { Unadjusted } \\
p \text {-Values }{ }^{1}\end{array}$} & \multicolumn{2}{|c|}{ Adjusted Analysis ${ }^{2}$} \\
\hline & & & & Most Important Factor ${ }^{3}$ & $p$-Value \\
\hline HGF (pg/mL) & $2785(697.4-10,107)$ & $1316(785-2628)$ & 0.08 & Country of residence & $<0.01$ \\
\hline TGF $\beta 1(\mathrm{pg} / \mathrm{mL})$ & $673.1(387.5-1133)$ & $663(483.1-940.4)$ & 0.80 & Parity & 0.13 \\
\hline TGF $\beta 2(\mathrm{pg} / \mathrm{mL})$ & $59,708(36,865-113,221)$ & $33,176(18,046-66,520)$ & 0.01 & Country of residence & 0.03 \\
\hline TGF $\beta 3(\mathrm{pg} / \mathrm{mL})$ & $1056(456.5-3212)$ & $1066(566.3-2038)$ & 0.94 & Parity & 0.11 \\
\hline $\operatorname{Ig} \mathrm{A}(\mathrm{g} / \mathrm{L})$ & $2.78(1.45-22.2)$ & $1.48(0.89-2.67)$ & $<0.01$ & Country of residence & $<0.01$ \\
\hline
\end{tabular}

${ }^{1}$ Statistical analysis for unadjusted comparisons between women from Italy and Burundi, using Mann-Whitney $\mathrm{U}$ test; ${ }^{2}$ adjusted analysis (multivariate linear regression). Confounding factors included in the model: country of residence, parity (binary), maternal age, gestational age, and animal contact during pregnancy; ${ }^{3}$ According to multivariate regression model; Statistically significant results (at $p$ value $<0.05$ ) presented in bold. interquartile range-IQR; TGF- $\beta$ - transforming growth factor- $\beta$; HGF-hepatocyte growth factor; IgA-immunoglobulin A.

\subsubsection{Correlation of TGF- $\beta 1,2$, and 3 with IgA Levels}

To assess the correlation between the levels of TGF- $\beta$, we used Spearman's rank correlation coefficients. We found moderate positive correlations between the growth factor and IgA concentrations in the colostrum of Italian mothers $(p<0.01)$ and a moderate to strong correlation in the samples from the Burundian mothers. The growth factors correlated with IgA were apparently stronger in the colostrum samples from Burundi, as seen in Table 3.

Table 3. Correlation between levels of growth factors (TGFß1, 2, and 3) with IgA.

\begin{tabular}{ccc}
\hline Correlating Factors & Burundi $\left(r^{\mathbf{1}}\right)$ & Italy $(r)$ \\
\hline HGF/IgA & $0.71^{* *}$ & $0.38^{* *}$ \\
TGF- $\beta 1 /$ IgA & $0.52^{*}$ & $0.28^{*}$ \\
TGF- $\beta 2 /$ IgA & $0.68^{* *}$ & $0.32^{* *}$ \\
TGF- $\beta 3 /$ IgA & $0.72^{* *}$ & $0.51^{* *}$ \\
\hline
\end{tabular}

Statistical analysis was performed using Spearman's rank correlation coefficient. ${ }^{1}$ Spearman $r$ values are presented; *-results are statistically significant at a level less than 0.05 ; ${ }^{* *}$-results are statistically significant at a level less than 0.01 .

\section{Discussion}

This study aimed to evaluate the differences in the growth factors (TGF- $\beta$ isoforms and HGF) and IgA levels in the colostrum of women from two distinct cohorts, West African and a Southern European. A great variability in the microbial composition, risk of severe infections, and non-communicable disease rates is seen in these two populations. We found a few apparent differences between the sites following the unadjusted and adjusted analysis. The raw concentrations assessment identified significantly higher concentrations of TGF- $\beta 2$ and $\operatorname{IgA}$ in the Burundian samples and a cursory trend for the HGF concentration in the colostrum samples from Burundi, which reached statistical significance upon adjustment.

Our results showed no significant difference in the TGF- $\beta 1$ and 3 concentrations between the two groups of women. In contrast, a few studies previously found higher levels of TGF- $\beta 1$ in the human milk of mothers from developing countries [27,37], or from those residing in a farming environment [38]. The authors suggested that maternal contact with high microbial environments was attributed to an increase of TGF- $\beta 1$. As multiple factors may influence the immune marker concentration in human milk, it can be speculated that the differences between the studies are a result of ethnic and dietary differences. A major limitation of all of the abovementioned studies, as well as our own work, is a limited sample size. To fully understand the cause of the TGF- $\beta 1$ variations, the assessment of the dietary and genetic profiles of mothers from different geographical locations is required. 
We found higher concentrations of TGF- $\beta 2$, the predominant isoform of TGF- $\beta$ in human milk, and IgA in the Burundian colostrum samples. Existing differences in concentration between the sites were confirmed upon adjustment for potential confounders. This finding is in agreement with the results of the study comparing human milk samples from Estonia and Sweden, with higher secretory IgA levels found in Estonia, a country with a greater microbial burden [39]. The high bacterial and viral load in Western Africa may trigger the maternal immune responses and amplify the production of immune mediators; this induces in utero epigenetic alterations via placental Fc receptors [40]. Postnatally, the maternal immune mediators are passed to the infant through breast milk. Thus, the increased TGF- $\beta 2$ and IgA concentration in the colostrum reflects their purpose, to target microbial antigens and improve mucosal barrier function [41,42]. It may be hypothesised that the elevated IgA concentrations are a potential 'compensation' for the high microbial burden in Burundi.

The surroundings in sub-Saharan Africa offer a microbial load and may possibly stimulate the elevated HGF concentration that was found in colostrum from the Burundian mothers, as a result of the immune-protective effects of HGF on the newborn mucosal barriers [11].

The gestational age of the Burundian infants was lower than their Italian peers. This could potentially result in a greater immunological need, and the mothers' milk is more likely to resemble a preterm milk composition, with higher levels of IgA and TGF 32 [43], which could be another plausible explanation for the increased levels of these markers in the Burundian women's milk.

In Italy, a more industrialised country with fewer microbial exposures, there is decreased maternal immune stimulation and we detected lower levels of TGF- $\beta 2$ and IgA in the colostrum of the Italian women. This is supported by the 'hygiene hypothesis', which associated Western lifestyles with rising non-communicable disease prevalence as a consequence of decreased microbial exposures in childhood [44]. Previously, Hawkes and colleagues established that the maternal plasma concentrations of TGF- $\beta 2$ do not correlate to the levels found in paired breast milk samples [45]. It was also suggested that throughout pregnancy and lactation, the immune cells infiltrate the mammary tissue to upregulate the production of key soluble factors, including TGF- $\beta 2$ [46]. These studies propose that the local mammary gland production of TGF- $\beta 2$ may be responsible for the high concentrations seen in breast milk. Therefore, our results cannot unambiguously confirm that systemic immune responses due to a higher maternal microbial exposure in Burundi are a causal factor for the differences in the TGF- $\beta 2$ levels between the sites. Population studies and genome wide analysis can be used to assess the relationship of host-pathogen responses in human milk components between the countries. However, our results provide additional data, improving the understanding of the selective pressures that lead to immune component variability in human milk and the factors responsible for the differences observed.

The levels of each of the TGF- $\beta$ isoforms significantly correlated with the IgA concentration in the colostrum from both Burundi and Italy, with stronger correlations observed in the colostrum of the Burundi women. Correlations of immunological markers in human milk are a well-known phenomenon [47], however, it is worth noting that the strength of the correlation in different geographical locations is rarely assessed and should be considered as a potential topic of interest in the future. TGF- $\beta$ is known to mediate the class-switching of antibodies and therefore regulates the production of secretory IgA [48]. Some authors have suggested that additional signaling factors are required for complete IgA differentiation and class-switching, such as interleukin (IL)-2 and IL-5 [49,50]. We are far from completely understanding the IgA production trigger, but it is necessary to consider the contributing signals for antibody class-switching. This is a possible area that may be considered for further investigation.

Understanding the immune mediator composition of human milk and its' relationships with communicable and non-communicable disease progression is an area of great interest, as it could lead to the development of various therapeutic applications. This includes manipulation with the maternal diet or the environment so as to promote 'healthier' human milk composition, as well as the fortification of formula milk for those infants unable to receive breast milk. Some animal model studies show promising outcomes [51], and there are targets for application in humans [34]. It is 
widely believed that airborne allergens in human milk can be used to induce TGF- $\beta 1$ mediated oral tolerance [52]. This may be a possible intervention to prevent antigen-specific allergic airway diseases, but also may have applications in infectious diseases. By understanding the transfer of maternal plasma immune mediators to breast milk, there is vast potential for the development of vaccines that could immunise both mother and infant [53].

With the emergence of data showing that the differences in human milk immune composition most likely exist between the countries $[26,29,38,39,54]$, it becomes more apparent that it should be considered as an important confounding factor in breastfeeding research. At present, most of the studies evaluating the associations between breastfeeding and health outcomes do not take differences in the breast milk composition into account, which may lead to a serious bias. This becomes particularly important when the data from the developed and developing world is compared. The diversity of the human milk composition may account for the conflicting data coming from breastfeeding studies.

More intense research of the differences between the human milk immunological composition may result in an improvement of donor human milk routine use approaches. This is particularly important for a high-income, well developed countries, when necrotizing enterocolitis (NEC) is concerned [55]. A recently published Cochrane systematic review suggests that infant formula use in preterm infants results in an almost doubled risk of NEC development, when compared with donor human milk [56]. A better understanding of human milk immune composition may help to highlight the important markers that are able to further reduce the risk of NEC development and select the most appropriate milk for the infant at risk.

Our study had several limitations; the maternal dietary patterns have not been assessed using a food frequency questionnaire and the maternal body mass index (BMI) has not been measured; we did not evaluate the maternal genotype as a potentially important determinant or modulator of human milk composition; and the small cohort size from Burundi represented another limitation within our study. Although a larger number of samples were collected from the Burundi mothers, because of missing data from the mother-newborn pairs, some data were excluded from the statistical analysis, which led to a reduced statistical power. A small sample size could potentially skew the outcomes obtained on a multivariate linear regression analysis. We sampled single colostrum specimens from each subject, which can also be considered as a limitation. The time before freezing has not been recorded, but it is extremely unlikely to have influenced the results as the samples were frozen within a few hours of collection, and it has been previously shown that the immune active molecules are very stable and do not degrade rapidly $[57,58]$. The difference in the initial storage conditions $\left(-20{ }^{\circ} \mathrm{C}\right.$ and $-50{ }^{\circ} \mathrm{C}$ ), for a period of less than 12 months, is also very unlikely to have had a significant effect on the immunological composition [58]. The outcomes of the statistical analysis are somewhat promising, as the univariate analysis results were subsequently confirmed when the multivariate testing was applied. Another advantage of this study is the rigorous standardisation of the sample collection and analysis, as well as the measurement of important immunological markers, using a very sensitive technique.

\section{Conclusions}

This cross-sectional study has found differences in the colostrum immune composition between women residing in Burundi and Italy, with higher levels and stronger correlations between the markers found in the milk of the African women. Taken together, these results suggest that differences in the environment and a higher bacterial upload in the developing countries may lead to a higher excretion of factors in to the human milk, in order to provide better protection against infections, as a compensatory mechanism. A stronger correlation between the growth factors and the IgA in colostrum of the women residing in Africa needs further investigation.

The results of this research support the idea that maternal and environmental differences between the populations may result in human milk compositional changes. However, the generalisability of these results is subject to certain limitations. For instance, a small sample size does not allow us to make definitive conclusions. What is now needed is a cross-national study involving participants 
from very diverse geographical locations, in order to fully determine the cause of the differences in the colostrum composition, taking into account multiple factors that may influence the outcomes.

Author Contributions: J.O.W., R.J.B., A.L.B., D.G.P., and D.M. conceived and designed the experiments; D.M., L.Y.C., P.A., and S.S. performed the experiments; S.L., P.C., and E.M.P. collected data; D.M., P.A., E.K., A.A., R.J.B., and D.G.P. wrote the paper.

Funding: This research received no external funding.

Acknowledgments: We are indebted to Roberta Pigozzi for collecting the human milk samples in Verona. We are grateful to the mothers who kindly volunteered to donate their breast milk. R.J.B. is supported by the National Institute for Health Research Biomedical Research Centre (BRC). Both J.O.W. and R.J.B. have received research grant income from Danone, in relation to studies of the value of prebiotics in allergy prevention, and Airsonette, to evaluate the temperature-controlled laminar airflow for asthma. J.O.W. is on a Danone, UCB, and Airsonette scientific advisory board, and both J.O.W. and R.J.B. have given paid lectures for the companies. D.M. has received a consultancy payment from Dairy Goat Co-Operative (NZ) Ltd., and has given paid lectures for the Merck Sharp and Dohme (MSD). J.O.W. is supported by the NIHR CLAHRC for NW London and is its early years theme lead. The views expressed in this paper are those of the authors and not the NIHR or the Department of Health. The founding sponsors/pharmaceutical companies had no role in the design of the study; in the collection, analyses, or interpretation of data; in the writing of the manuscript; and in the decision to publish the results.

Conflicts of Interest: The authors declare no conflict of interest.

\section{References}

1. D'Alessandro, A.; Scaloni, A.; Zolla, L. Human milk proteins: An interactomics and updated functional overview. J. Proteome Res. 2010, 9, 3339-3373. [CrossRef] [PubMed]

2. Lawrence, R.M.; Pane, C.A. Human breast milk: Current concepts of immunology and infectious diseases. Curr. Probl. Pediatr. Adolesc. Health Care 2007, 37, 7-36. [CrossRef] [PubMed]

3. Verhasselt, V. Neonatal tolerance under breastfeeding influence. Curr. Opin. Immunol. 2010, 22, 623-630. [CrossRef] [PubMed]

4. Munblit, D.; Verhasselt, V. Allergy prevention by breastfeeding: Possible mechanisms and evidence from human cohorts. Curr. Opin. Allergy Clin. Immunol. 2016, 16, 427-433. [CrossRef] [PubMed]

5. Khodayar-Pardo, P.; Mira-Pascual, L.; Collado, M.C.; Martinez-Costa, C. Impact of lactation stage, gestational age and mode of delivery on breast milk microbiota. J. Perinatol. 2014, 34, 599-605. [CrossRef] [PubMed]

6. Lodge, C.J.; Tan, D.J.; Lau, M.X.; Dai, X.; Tham, R.; Lowe, A.J.; Bowatte, G.; Allen, K.J.; Dharmage, S.C. Breastfeeding and asthma and allergies: A systematic review and meta-analysis. Acta Paediatr. 2015, 104, 38-53. [CrossRef] [PubMed]

7. Doherty, A.M.; Lodge, C.J.; Dharmage, S.C.; Dai, X.; Bode, L.; Lowe, A.J. Human milk oligosaccharides and associations with immune-mediated disease and infection in childhood: A systematic review. Front. Pediatr. 2018, 6, 91. [CrossRef] [PubMed]

8. Munblit, D.; Peroni, D.G.; Boix-Amoros, A.; Hsu, P.S.; Land, B.V.; Gay, M.C.L.; Kolotilina, A.; Skevaki, C.; Boyle, R.J.; Collado, M.C.; et al. Human milk and allergic diseases: An unsolved puzzle. Nutrients 2017, 9, 894. [CrossRef] [PubMed]

9. Kobata, R.; Tsukahara, H.; Ohshima, Y.; Ohta, N.; Tokuriki, S.; Tamura, S.; Mayumi, M. High levels of growth factors in human breast milk. Early Hum. Dev. 2008, 84, 67-69. [CrossRef] [PubMed]

10. Galimi, F.; Brizzi, M.F.; Comoglio, P.M. The hepatocyte growth factor and its receptor. Stem Cells 1993, 11, 22-30. [CrossRef] [PubMed]

11. Patki, S.; Patki, U.; Patil, R.; Indumathi, S.; Kaingade, P.; Bulbule, A.; Nikam, A.; Pishte, A. Comparison of the levels of the growth factors in umbilical cord serum and human milk and its clinical significance. Cytokine 2012, 59, 305-308. [CrossRef] [PubMed]

12. Min, J.K.; Lee, Y.M.; Kim, J.H.; Kim, Y.M.; Kim, S.W.; Lee, S.Y.; Gho, Y.S.; Oh, G.T.; Kwon, Y.G. Hepatocyte growth factor suppresses vascular endothelial growth factor-induced expression of endothelial icam-1 and vcam-1 by inhibiting the nuclear factor-kappab pathway. Circ. Res. 2005, 96, 300-307. [CrossRef] [PubMed]

13. Cummins, A.G.; Thompson, F.M. Postnatal changes in mucosal immune response: A physiological perspective of breast feeding and weaning. Immunol. Cell Biol. 1997, 75, 419-429. [CrossRef] [PubMed] 
14. Donnet-Hughes, A.; Duc, N.; Serrant, P.; Vidal, K.; Schiffrin, E.J. Bioactive molecules in milk and their role in health and disease: The role of transforming growth factor-beta. Immunol. Cell Biol. 2000, 78, 74-79. [CrossRef] [PubMed]

15. Penttila, I.A. Milk-derived transforming growth factor-beta and the infant immune response. J. Pediatr. 2010, 156, S21-S25. [CrossRef] [PubMed]

16. Tiemessen, M.M.; Kunzmann, S.; Schmidt-Weber, C.B.; Garssen, J.; Bruijnzeel-Koomen, C.A.; Knol, E.F.; van Hoffen, E. Transforming growth factor-beta inhibits human antigen-specific CD4+ T cell proliferation without modulating the cytokine response. Int. Immunol. 2003, 15, 1495-1504. [CrossRef] [PubMed]

17. Jarvinen, K.M.; Suomalainen, H. Development of cow's milk allergy in breast-fed infants. Clin. Exp. Allergy 2001, 31, 978-987. [CrossRef] [PubMed]

18. Oddy, W.H.; Rosales, F. A systematic review of the importance of milk TGF-beta on immunological outcomes in the infant and young child. Pediatr. Allergy Immunol. 2010, 21, 47-59. [CrossRef] [PubMed]

19. Morita, Y.; Campos-Alberto, E.; Yamaide, F.; Nakano, T.; Ohnisi, H.; Kawamoto, M.; Kawamoto, N.; Matsui, E.; Kondo, N.; Kohno, Y.; et al. TGF-beta concentration in breast milk is associated with the development of eczema in infants. Front. Pediatr. 2018, 6, 162. [CrossRef] [PubMed]

20. Munblit, D.; Treneva, M.; Peroni, D.G.; Colicino, S.; Chow, L.Y.; Dissanayeke, S.; Pampura, A.; Boner, A.L.; Geddes, D.T.; Boyle, R.J.; et al. Immune components in human milk are associated with early infant immunological health outcomes: A prospective three-country analysis. Nutrients 2017, 9, 532. [CrossRef] [PubMed]

21. Ogawa, J.; Sasahara, A.; Yoshida, T.; Sira, M.M.; Futatani, T.; Kanegane, H.; Miyawaki, T. Role of transforming growth factor-beta in breast milk for initiation of IgA production in newborn infants. Early Hum. Dev. 2004, 77, 67-75. [CrossRef] [PubMed]

22. Iyengar, S.R.; Walker, W.A. Immune factors in breast milk and the development of atopic disease. J. Pediatr. Gastroenterol. Nutr. 2012, 55, 641-647. [CrossRef] [PubMed]

23. Arnould, V.M.; Soyeurt, H. Genetic variability of milk fatty acids. J. Appl. Genet. 2009, 50, 29-39. [CrossRef] [PubMed]

24. Kondo, N.; Suda, Y.; Nakao, A.; Oh-Oka, K.; Suzuki, K.; Ishimaru, K.; Sato, M.; Tanaka, T.; Nagai, A.; Yamagata, Z. Maternal psychosocial factors determining the concentrations of transforming growth factor-beta in breast milk. Pediatr. Allergy Immunol. 2011, 22, 853-861. [CrossRef] [PubMed]

25. Kuitunen, M.; Kukkonen, A.K.; Savilahti, E. Impact of maternal allergy and use of probiotics during pregnancy on breast milk cytokines and food antibodies and development of allergy in children until 5 years. Int. Arch. Allergy Immunol. 2012, 159, 162-170. [CrossRef] [PubMed]

26. Munblit, D.; Treneva, M.; Peroni, D.G.; Colicino, S.; Chow, L.; Dissanayeke, S.; Abrol, P.; Sheth, S.; Pampura, A.; Boner, A.L.; et al. Colostrum and mature human milk of women from London, Moscow, and Verona: Determinants of immune composition. Nutrients 2016, 8, 695. [CrossRef] [PubMed]

27. Holmlund, U.; Amoudruz, P.; Johansson, M.A.; Haileselassie, Y.; Ongoiba, A.; Kayentao, K.; Traore, B.; Doumbo, S.; Schollin, J.; Doumbo, O.; et al. Maternal country of origin, breast milk characteristics and potential influences on immunity in offspring. Clin. Exp. Immunol. 2010, 162, 500-509. [CrossRef] [PubMed]

28. Aihara, Y.; Oh-oka, K.; Kondo, N.; Sharma, J.; Ishimaru, K.; Hara, M.; Yamagata, Z.; Nakao, A. Comparison of colostrum TGF- $\beta 2$ levels between lactating women in Japan and Nepal. Asian Pac. J. Allergy Immunol. 2014, 32, 178-184. [CrossRef] [PubMed]

29. Ruiz, L.; Espinosa-Martos, I.; Garcia-Carral, C.; Manzano, S.; McGuire, M.K.; Meehan, C.L.; McGuire, M.A.; Williams, J.E.; Foster, J.; Sellen, D.W.; et al. What's normal? Immune profiling of human milk from healthy women living in different geographical and socioeconomic settings. Front. Immunol. 2017, 8, 696. [CrossRef] [PubMed]

30. UNICEF. Country Statistics-Burundi. 2013. Available online: https://www.unicef.org/infobycountry/ burundi_statistics.html (accessed on 17 April 2014).

31. UNICEF. Unicef-Italy Population Statistics. 2013. Available online: https://www.unicef.org/infobycountry/ italy_statistics.html (accessed on 5 April 2014).

32. Munblit, D.; Boyle, R.J.; Warner, J.O. Factors affecting breast milk composition and potential consequences for development of the allergic phenotype. Clin. Exp. Allergy 2015, 45, 583-601. [CrossRef] [PubMed] 
33. Munblit, D.; Boyle, R.J. Modulating breast milk composition-the key to allergy prevention? Int. Arch. Allergy Immunol. 2012, 159, 107-108. [CrossRef] [PubMed]

34. Le Doare, K.; Bellis, K.; Faal, A.; Birt, J.; Munblit, D.; Humphries, H.; Taylor, S.; Warburton, F.; Heath, P.T.; Kampmann, B.; et al. SIgA, TGF- $\beta 1$, IL-10, and TNF $\alpha$ in colostrum are associated with infant group B streptococcus colonization. Front. Immunol. 2017, 8, 1269. [CrossRef] [PubMed]

35. Munblit, D.; Sheth, S.; Abrol, P.; Treneva, M.; Peroni, D.G.; Chow, L.Y.; Boner, A.L.; Pampura, A.; Warner, J.O.; Boyle, R.J. Exposures influencing total IgA level in colostrum. J. Dev. Orig. Health Dis. 2016, 7, 61-67. [CrossRef] [PubMed]

36. Ismail, I.H.; Licciardi, P.V.; Oppedisano, F.; Boyle, R.J.; Tang, M.L. Relationship between breast milk scd14, TGF-beta1 and total IgA in the first month and development of eczema during infancy. Pediatr. Allergy Immunol. 2013, 24, 352-360. [CrossRef] [PubMed]

37. Amoudruz, P.; Holmlund, U.; Schollin, J.; Sverremark-Ekström, E.; Montgomery, S.M. Maternal country of birth and previous pregnancies are associated with breast milk characteristics. Pediatr. Allergy Immunol. 2009, 20, 19-29. [CrossRef] [PubMed]

38. Peroni, D.G.; Pescollderungg, L.; Piacentini, G.L.; Rigotti, E.; Maselli, M.; Watschinger, K.; Piazza, M.; Pigozzi, R.; Boner, A.L. Immune regulatory cytokines in the milk of lactating women from farming and urban environments. Pediatr. Allergy Immunol. 2010, 21, 977-982. [CrossRef] [PubMed]

39. Tomicic, S.; Johansson, G.; Voor, T.; Bjorksten, B.; Bottcher, M.F.; Jenmalm, M.C. Breast milk cytokine and iga composition differ in estonian and swedish mothers-relationship to microbial pressure and infant allergy. Pediatr. Res. 2010, 68, 330-334. [CrossRef] [PubMed]

40. Jenmalm, M.C. Childhood immune maturation and allergy development: Regulation by maternal immunity and microbial exposure. Am. J. Reprod. Immunol. 2011, 66, 75-80. [CrossRef] [PubMed]

41. Chatterton, D.E.; Nguyen, D.N.; Bering, S.B.; Sangild, P.T. Anti-inflammatory mechanisms of bioactive milk proteins in the intestine of newborns. Int. J. Biochem. Cell Biol. 2013, 45, 1730-1747. [CrossRef] [PubMed]

42. Rogier, E.W.; Frantz, A.L.; Bruno, M.E.; Wedlund, L.; Cohen, D.A.; Stromberg, A.J.; Kaetzel, C.S. Secretory antibodies in breast milk promote long-term intestinal homeostasis by regulating the gut microbiota and host gene expression. Proc. Natl. Acad. Sci. USA 2014, 111, 3074-3079. [CrossRef] [PubMed]

43. Trend, S.; Strunk, T.; Lloyd, M.L.; Kok, C.H.; Metcalfe, J.; Geddes, D.T.; Lai, C.T.; Richmond, P.; Doherty, D.A.; Simmer, K.; et al. Levels of innate immune factors in preterm and term mothers' breast milk during the 1st month postpartum. Br. J. Nutr. 2016, 115, 1178-1193. [CrossRef] [PubMed]

44. Umetsu, D.T. Early exposure to germs and the hygiene hypothesis. Cell Res. 2012, 22, 1210-1211. [CrossRef] [PubMed]

45. Hawkes, J.S.; Bryan, D.L.; Gibson, R.A. Variations in transforming growth factor beta in human milk are not related to levels in plasma. Cytokine 2002, 17, 182-186. [CrossRef] [PubMed]

46. Reed, J.R.; Schwertfeger, K.L. Immune cell location and function during post-natal mammary gland development. J. Mammary Gland Biol. Neoplasia 2010, 15, 329-339. [CrossRef] [PubMed]

47. Bottcher, M.F.; Jenmalm, M.C.; Garofalo, R.P.; Bjorksten, B. Cytokines in breast milk from allergic and nonallergic mothers. Pediatr. Res. 2000, 47, 157-162. [CrossRef] [PubMed]

48. Stavnezer, J. Regulation of antibody production and class switching by TGF-beta. J. Immunol. 1995, 155, 1647-1651. [PubMed]

49. Ehrhardt, R.O.; Strober, W.; Harriman, G.R. Effect of transforming growth factor (TGF)-beta 1 on IgA isotype expression. TGF-beta 1 induces a small increase in sIgA+ B cells regardless of the method of B cell activation. J. Immunol. 1992, 148, 3830-3836. [PubMed]

50. Sonoda, E.; Hitoshi, Y.; Yamaguchi, N.; Ishii, T.; Tominaga, A.; Araki, S.; Takatsu, K. Differential regulation of IgA production by TGF- $\beta$ and IL-5: TGF- $\beta$ induces surface IgA -positive cells bearing IL -5 receptor, whereas IL-5 promotes their survival and maturation into IgA-secreting cells. Cell. Immunol. 1992, 140, 158-172. [CrossRef]

51. Jain, S.K.; Baggerman, E.W.; Mohankumar, K.; Namachivayam, K.; Jagadeeswaran, R.; Reyes, V.E.; Maheshwari, A. Amniotic fluid-borne hepatocyte growth factor protects rat pups against experimental necrotizing enterocolitis. Am. J. Physiol. Gastrointest. Liver Physiol. 2014, 306, G361-G369. [CrossRef] [PubMed] 
52. Verhasselt, V.; Milcent, V.; Cazareth, J.; Kanda, A.; Fleury, S.; Dombrowicz, D.; Glaichenhaus, N.; Julia, V. Breast milk-mediated transfer of an antigen induces tolerance and protection from allergic asthma. Nat. Med. 2008, 14, 170-175. [CrossRef] [PubMed]

53. Shahid, N.S.; Steinhoff, M.C.; Roy, E.; Begum, T.; Thompson, C.M.; Siber, G.R. Placental and breast transfer of antibodies after maternal immunization with polysaccharide meningococcal vaccine: A randomized, controlled evaluation. Vaccine 2002, 20, 2404-2409. [CrossRef]

54. Orivuori, L.; Loss, G.; Roduit, C.; Dalphin, J.C.; Depner, M.; Genuneit, J.; Lauener, R.; Pekkanen, J.; Pfefferle, P.; Riedler, J.; et al. Soluble immunoglobulin a in breast milk is inversely associated with atopic dermatitis at early age: The pasture cohort study. Clin. Exp. Allergy 2014, 44, 102-112. [CrossRef] [PubMed]

55. Battersby, C.; Santhalingam, T.; Costeloe, K.; Modi, N. Incidence of neonatal necrotising enterocolitis in high-income countries: A systematic review. Arch. Dis. Child. Fetal Neonatal Ed. 2018, 103, F182-F189. [PubMed]

56. Quigley, M.; Embleton, N.D.; McGuire, W. Formula versus donor breast milk for feeding preterm or low birth weight infants. Cochrane Database Syst. Rev. 2018, 6, CD002971. [CrossRef] [PubMed]

57. Lawrence, R.A. Storage of human milk and the influence of procedures on immunological components of human milk. Acta. Paediatr. Suppl. 1999, 88, 14-18. [CrossRef] [PubMed]

58. Lawrence, R.A. Milk banking: The influence of storage procedures and subsequent processing on immunologic components of human milk. Adv. Nutr. Res. 2001, 10, 389-404. [PubMed]

(C) 2018 by the authors. Licensee MDPI, Basel, Switzerland. This article is an open access article distributed under the terms and conditions of the Creative Commons Attribution (CC BY) license (http:/ / creativecommons.org/licenses/by/4.0/). 
Article

\title{
Comparison of Inductively Coupled Plasma Optical Emission Spectrometry with an Ion Selective Electrode to Determine Sodium and Potassium Levels in Human Milk
}

\author{
Ching Tat Lai *, Hazel Gardner and Donna Geddes \\ School of Molecular Sciences, University of Western Australia, 35 Stirling Highway, Crawley, 6009 WA, \\ Australia; hazel.gardner@uwa.edu.au (H.G.); donna.geddes@uwa.edu.au (D.G.) \\ * Correspondence: ching-tat.lai@uwa.edu.au; Tel.: +61-08-6488-3200
}

Received: 1 August 2018; Accepted: 27 August 2018; Published: 3 September 2018

\begin{abstract}
Sodium (Na), potassium (K), and the ratio Na:K in human milk (HM) may be useful biomarkers to indicate secretory activation or inflammation in the breast. Previously, these elements have been measured in a laboratory setting requiring expensive equipment and relatively large amounts of HM. The aim of this study was to compare measurements of $\mathrm{Na}$ and $\mathrm{K}$ in $\mathrm{HM}$ using inductively coupled plasma optical emission spectrometry (ICP-OES) with small portable ion selective electrode probes for $\mathrm{Na}$ and $\mathrm{K}$. Sixty-five lactating women donated $5 \mathrm{~mL}$ samples of HM. Samples were analyzed with two ion selective probes (Na and K) and also ICP-OES. The data were analyzed using paired $t$-test and Bland-Altman plots. Na concentrations were not significantly different when measured with ion selective electrode $(6.18 \pm 2.47 \mathrm{mM}$; range: $3.59-19.8)$ and ICP-OES (5.91 $\pm 3.37 \mathrm{mM}$; range: $2.59-21.5)(p=0.20)$. K concentrations measured using the ion selective electrode (11.7 $\pm 2.21 \mathrm{mM}$ : range: 7.69-18.1) and ICP-OES (11.1 $\pm 1.55 \mathrm{mM}$ : range: $7.91-15.2)$ were significantly different $(p=0.01)$. However, the mean differences of $0.65 \mathrm{mM}$ would not be clinically relevant when testing at point of care. Compared to ICP-OES, ion selective electrode is sufficiently accurate to detect changes in concentrations of $\mathrm{Na}$ and $\mathrm{K}$ in $\mathrm{HM}$ associated with secretory activation and inflammation in the mammary gland.
\end{abstract}

Keywords: human milk; potassium; sodium; ICP-OES; ion selective electrode

\section{Introduction}

Human milk (HM) sodium (Na) and potassium (K) concentrations change dramatically during the first week postpartum at the onset of secretory activation. Sodium, in particular, follows a rapid downward trajectory after birth as a result of tight junction closure, which is essential for secretory activation of the onset of copious milk production [1]. It has been reported that human milk sodium drops from $60 \mathrm{mM}$ to $10 \mathrm{mM}$ between days 1 and 5 postpartum, this precipitous drop reflects tight junction closure [2]. Delayed onset of secretory activation is established as a risk factor for poor lactation outcomes [3] with high sodium concentrations at day 7 considered a risk factor for the cessation of breastfeeding [4].

The Na:K ratio is another marker that has been historically used to define secretory activation. The Na:K ratio is greater than 2.0 after birth and then declines as sodium concentrations decline with closure of the tight junctions. The Na:K ratio has been used to biochemically define lactation stages as follows: $\geq 2$ colostral milk, $<2$ transitional milk, and $<0.6$ mature milk [1,5]. This is mirrored by changes in the human milk transcriptome during this period and is therefore considered more accurate than using time postpartum to define lactation stage [5]. Changes in the Na:K ratio form a continuum 
over the first week postpartum, with a high Na:K ratio on day 7 (>0.8) [4] indicative of suboptimal milk supply or feeding problems and consequently a higher risk of breastfeeding cessation. An elevated ratio on day 7 is associated with 3.3 times greater odds of stopping breastfeeding in mothers reporting concerns about milk supply [4].

A high $\mathrm{Na}: \mathrm{K}$ ratio is also indicative of the increased mammary epithelial permeability associated with breast inflammation, breast engorgement or mastitis [4,6-8]. The Na:K ratio is viewed as superior to sodium alone as it negates the variation found in proportions of aqueous and fat layers of the milk which occur when different sampling methods are used. Interestingly, the $\mathrm{Na}: \mathrm{K}$ ratio has been found to be positively associated with the inflammatory chemokine interleukin- 8 concentrations suggesting that this ratio is also a marker for inflammation of the mammary epithelium [7].

Immediate measurement of HM sodium and potassium concentrations would be advantageous in situations of suspected delayed secretory activation and mammary infection allowing rapid intervention. Currently these components are measured with instruments such as flame photometry [4], atomic absorption spectroscopy [7], ion chromatography, inductively coupled plasma optical emission spectroscopy (ICP-OES), and inductively coupled plasma mass spectroscopy (ICP-MS). All of these techniques, which require hazardous chemicals, laboratory facilities, and highly skilled operators, are not immediate and are also costly. Alternatively, the ion selective electrode (ISE) is easy to use, portable, and provides immediate results and therefore has potential as a point of care device for screening of high-risk lactating women. Ion specific electrodes have been used previously, but have not been compared directly to other methods to determine their accuracy [9].

The objective of this study was to compare the accuracy of the sodium and potassium ion selective electrodes against inductively coupled plasma optical emission spectrometry (ICP-OES).

\section{Materials and Methods}

\subsection{Biochemical Analysis}

Sixty-five preterm/term mothers provided written informed consent to participate in the study, which was approved by the Human Ethics Committee at the University of Western Australia (RA/4/1/2369). Five mothers who participated in this study had a premature delivery $(<37$ weeks gestation). Each participant completed a $24 \mathrm{~h}$ milk profile using the test weighing method [10]. Mothers were issued with a set of baby weigh scales (Medela AG, Baar, Switzerland). Samples were collected in $5 \mathrm{~mL}$ polypropylene tubes (P5016SL, Techno Plas Pty Ltd, St Marys, SA, Australia) before and after each feed or breast expression. Milk samples collected from the $24 \mathrm{~h}$ milk production were then pooled and approximately $5 \mathrm{~mL}$ of the pooled samples were used in this study.

\subsubsection{Ion Selective Electrode Measurement of $\mathrm{HM} \mathrm{Na}$ and $\mathrm{K}$}

Concentrations of sodium and potassium in the milk samples were determined by ion selective electrodes (Sodium: B-722; potassium: B-731; Horiba, Japan). Calibration of the electrodes was conducted according to the manufacturer's recommendations. The calibration range for both $\mathrm{Na}$ and K electrodes were set between 150 to 2000 ppm according to the manufacturer's instructions. For each assay, the whole milk samples were thawed at $37^{\circ} \mathrm{C}$ for $1 \mathrm{~h}$. Prior to the measurement, the milk samples were shaken with an Intelli-mixer (RM-2, ELMI Ltd, Riga, Latvia) using tube stand mode for $15 \mathrm{~s}$ at $50 \mathrm{rpm}$ followed by 3 inversions. Three hundred microliters of the mixed milk was pipetted onto the electrode sensor. The sample was allowed to stabilize for $15 \mathrm{~s}$ before the reading was taken. After each measurement, the milk sample was removed from the sensor and returned to the storage tube. The sensor was then rinsed with double deionized water and wiped with KimWipes (Kimberly-Clark Worldwide, Irving, TX, USA) prior to the next measurement. All samples were analyzed together, in duplicate. The same procedures were applied to both electrodes. The results of $\mathrm{Na}$ and $\mathrm{K}$ obtained from the electrodes were averaged and were converted from ppm to $\mathrm{mM}$ using the molecular weight of $\mathrm{Na}$ and $\mathrm{K}$ ( 23 and $39 \mathrm{~g} / \mathrm{mol}$, respectively). 
2.1.2. Inductively Coupled Plasma Optical Emission Spectrometry (ICP-OES) Measurement of HM Na and $\mathrm{K}$ Reagents

Standard solutions of Na and K (High Purity Standard, USA, $1000 \mu \mathrm{g} / \mathrm{mL}$ ) were in the range of 50-500 $\mu \mathrm{g} / \mathrm{mL}$. Yittrium and scandium were used as internal standards $(1000 \mu \mathrm{g} / \mathrm{mL})$ from Sigma Aldrich (Castle Hill, NSW, Australia). All standards were diluted with $18.2 \mathrm{M} \Omega$ water.

Sample Preparation and Measurement

Whole milk or standard solution $(200 \mu \mathrm{L})$ was mixed with $300 \mu \mathrm{L}$ of nitric acid $\left(65 \%\right.$, Suprapur ${ }^{\circledR}$, Merck, Kenilworth, NJ, USA) into disposable borosilicate glass tubes $(10 \times 75 \mathrm{mM}$, Kimble Chase, Rockwood, TN, USA). The tubes were placed into a dry heating block (DBH40D, Ratek, Boronia, Victoria, Australia) and a dry acid washed marble was placed on top of the tube. The tubes were incubated at $110{ }^{\circ} \mathrm{C}$ for $1 \mathrm{~h}$ to allow for the completion of acid digestion. The hot tubes were allowed to cool down in an ice bath before adding the internal standard $(2.1 \mu \mathrm{g} / \mathrm{mL})$ and topped up to $2 \mathrm{~mL}$ with 18.2 M $\Omega$ water. The concentrations of $\mathrm{Na}$ and $\mathrm{K}$ in the digested milk samples were determined in triplicate by ICP-OES (5100, Agilent technologies, Santa Clara, CA, USA). Na and K were detected and measured at $568.263 \mathrm{~nm}$ and $568.821 \mathrm{~nm}$ for $\mathrm{Na}$; and $766.491 \mathrm{mM}$ and $769.897 \mathrm{mM}$ for $\mathrm{K}$. The results of $\mathrm{Na}$ and K obtained from those specific wavelengths were averaged and were converted from $\mu \mathrm{g} / \mathrm{mL}$ to $\mathrm{mM}$ using the molecular weight of $\mathrm{Na}$ and $\mathrm{K}(23$ and $39 \mathrm{~g} / \mathrm{mol}$, respectively).

\subsubsection{Validation of Analytical Methods}

Both methods were validated using a spike/recovery assay. In each set of recovery assay, there was 3 tubes: (a) known standard solution + milk; (b) water + milk; (c) known standard solution + water. The mixture in each tube was 1:1 (v:v). The concentrations of $\mathrm{Na}$ or $\mathrm{K}$ in the tubes were measured and the following formula was applied: $((a)-(b)) /(c) \times 100 \%$ to obtain the recovery $(\%)$ for the set. Five sets were measured for each method to ensure the recovery was between 95-105\% with a coefficient of variation (CV) $<10 \%$. The recovery (\%) for the electrode method was $95 \pm 4.1$ for $\mathrm{Na}$ and $99 \pm 3.9 \%$ for K with a CV of $4.1 \%$ and $3.9 \%$, respectively $(n=5)$, and for ICP-OES was $99.0 \pm 9.9$ for $\mathrm{Na}$ and $99 \pm 9.3 \%$ for K with a CV of $9.9 \%$ and $9.4 \%$, respectively $(n=5)$.

\subsection{Statistical Analysis}

Statistical analyses were carried out using R 3.4.4 [11] and R Studio 1.1.419 [12] with package Lattice [13] for Bland-Altman plots. A paired sample t-test was used the compare the Na or K concentrations between the two methods. The limits of agreement and the precision of the estimated limits of agreement between the two measuring methods were calculated [14]. Bland-Altman plots were created to illustrate the limits of agreement. Pearson correlation was used to determine the correlation between concentrations of $\mathrm{Na}$ or $\mathrm{K}$ measured by the two methods. Boxplots were used to illustrate the medians, quartiles, and the 5th and 95th percentiles. The results were expressed as mean \pm standard deviation (SD) unless stated otherwise. Differences were considered significant if $p<0.05$.

\section{Results}

Sixty-five participants were recruited to include a range of lactation stages and milk productions as shown in Table 1.

The correlation coefficient $\left(r^{2}\right)$ of the Na measurement between the ISE and ICP-OES was 0.76, $p<0.001$ (Figure 1A). The mean difference in the Na measurement between ISE and ICP-OES was within \pm 2 standard derivations (SD) (Figure 1B). There was no significant difference between the measurement of Na between the ISE and ICP-OES ( $p=0.20$, Figure 1C). 
Table 1. Participant Characteristics.

\begin{tabular}{cccc}
\hline $\boldsymbol{n}=\mathbf{6 5}$ & Mean & Standard Deviation & Range \\
\hline Maternal age (years) & 34 & 4 & $24-43$ \\
Length of gestation (weeks) & 39 & 2 & $30-41$ \\
Stage of lactation (weeks) & 10 & 5 & $0.5-27$ \\
24 h milk production (millilitres) & 780 & 318 & $36-1932$ \\
\hline
\end{tabular}

A

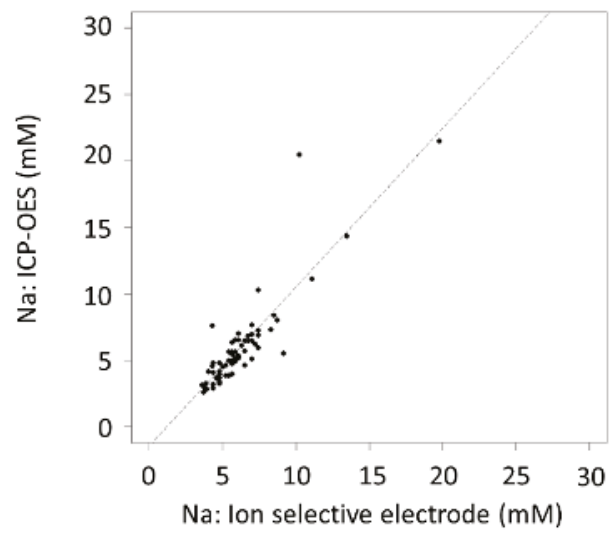

B

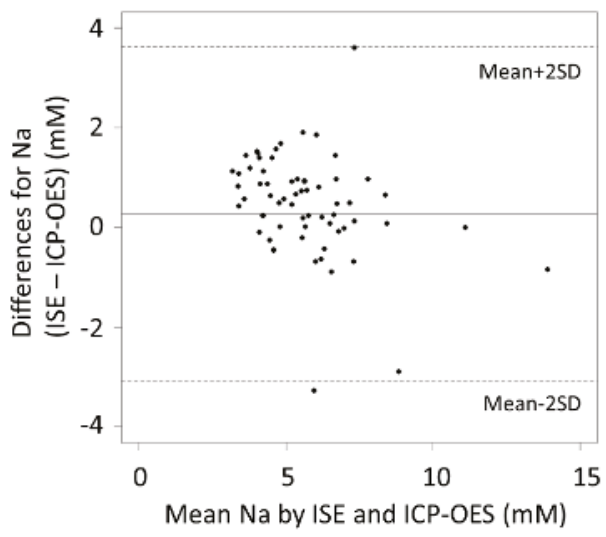

C

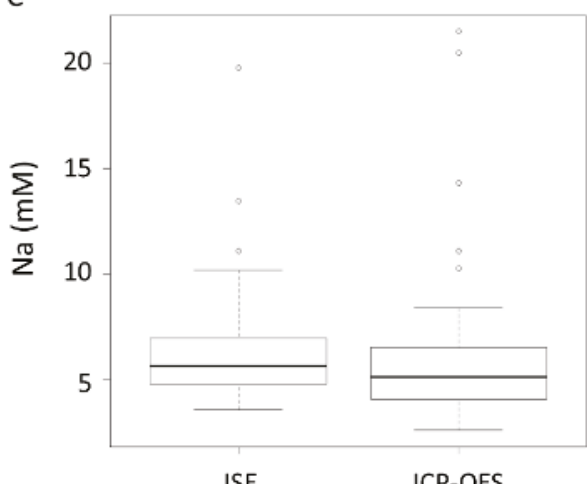

Figure 1. (A) Correlation of $\mathrm{Na}$ concentrations as measured by the ion selective electrode and inductively coupled plasma optical emission spectrometry (ICP-OES), $r^{2}=0.76, p<0.001$. (B) Bland-Altman plot showing the mean differences and limits of agreement. (C) Boxplot of $\mathrm{Na}$ concentrations, ion selective electrode (ISE).

The correlation coefficient $\left(r^{2}\right)$ of the K measurement between ISE and ICP-OES was $0.26, p<0.001$ (Figure 2A). The mean difference in the K measurement between ISE and ICP-OES was within \pm 2 standard deviations (SD) (Figure 2B). Measurement of K by the K ISE was significantly higher than ICP-OES ( $p=0.01$, Figure 2C). 
A

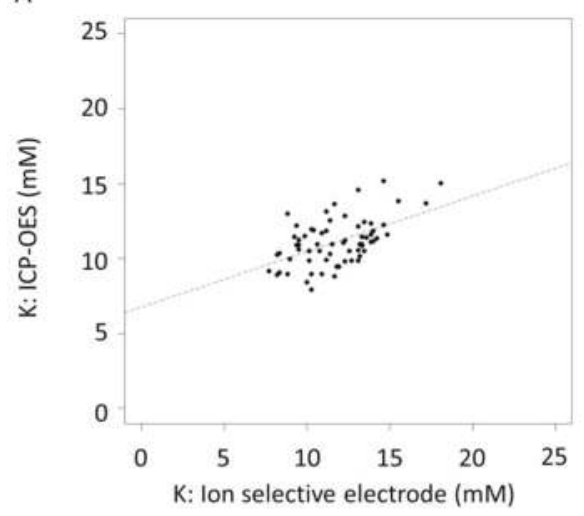

B

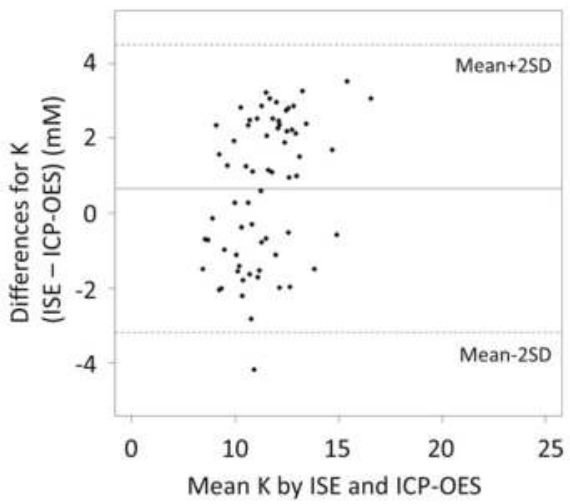

C

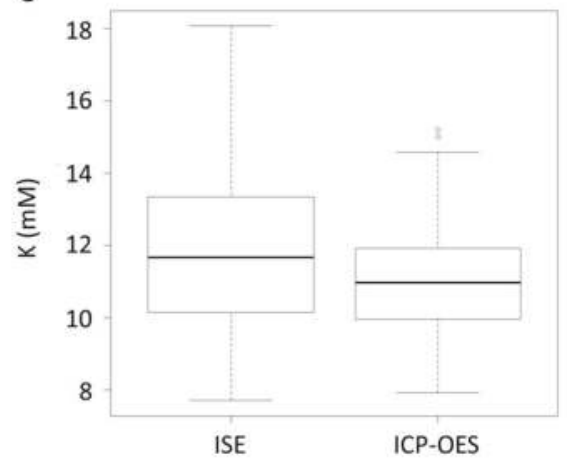

Figure 2. (A) Correlation of $\mathrm{K}$ concentrations as measured by the ion selective electrode and ICP-OES, $r^{2}=0.26, p<0.001$. (B) Bland-Altman plot showing the mean differences and limits of agreement. (C) Boxplot of K concentrations.

Twelve scenarios were considered (high to low measures for both $\mathrm{Na}$ and $\mathrm{K}$ ) to evaluate the impact of the change in the measurement of $\mathrm{K}$ on the Na:K ratio as shown in Table 2. In all 12 scenarios a movement equivalent to the mean difference in $\mathrm{K}$ is not enough to move the Na:K ratio over either the 0.8 or 2 thresholds.

Table 2. Calculated Na:K ratio with or without the mean difference of $\mathrm{K}$ measurement between ISE and ICP-OES.

\begin{tabular}{cccccc}
\hline & Na $(\mathbf{m M})$ & $\mathbf{K}(\mathbf{m M})$ & $\mathbf{K}+\mathbf{0 . 6 5} \mathbf{~ m M}$ & Na:K Ratio & Na:K + 0.65 mM Ratio \\
\hline Low K & 60 & 4 & 4.65 & 15.00 & 12.90 \\
& 40 & 4 & 4.65 & 10.00 & 8.60 \\
& 20 & 4 & 4.65 & 5.00 & 4.30 \\
Medium K & 12 & 4 & 4.65 & 3.00 & 2.58 \\
& 60 & 12 & 12.65 & 5.00 & 4.74 \\
& 40 & 12 & 12.65 & 3.33 & 3.16 \\
High K & 20 & 12 & 12.65 & 1.67 & 1.58 \\
& 12 & 12 & 12.65 & 1.00 & 3.95 \\
& 60 & 18 & 18.65 & 3.33 & 2.14 \\
& 40 & 18 & 18.65 & 2.22 & 1.07 \\
& 20 & 18 & 18.65 & 1.11 & 0.64 \\
\hline
\end{tabular}




\section{Discussion}

Inductively coupled plasma optical emission spectrometry is regarded as one of the "gold standard" techniques for trace element analysis. It utilizes specific wavelengths to detect and measure $\mathrm{Na}(568.263 \mathrm{~nm}$ and $568.821 \mathrm{~nm})$ and $\mathrm{K}(766.491 \mathrm{~nm}$ and $769.897 \mathrm{~nm})$ in the acid digested fluid, in this case HM, during the process. We found no statistically significant differences between $\mathrm{Na}$ concentrations as measured by the Na specific electrode and the ICP-OES in 65 whole HM samples (Figure 1C, Table 3).

Table 3. Human milk (HM) sodium and potassium concentrations measured using ion selective electrode (ISE) and inductively coupled plasma optical emission spectrometry (ICP-OES).

\begin{tabular}{ccccccc}
\hline$n=65$ & \multicolumn{3}{c}{ Sodium $(\mathbf{m M})$} & \multicolumn{3}{c}{ Potassium $(\mathbf{m M})$} \\
\hline & ISE & ICP-OES & $p$ value & ISE & ICP-OES & $p$ value \\
Mean SD & 6.182 .47 & 5.913 .37 & 0.20 & 11.702 .21 & 11.101 .55 & 0.01 \\
Range & $3.59-19.80$ & $2.59-21.50$ & & $7.69-18.10$ & $7.91-15.20$ & \\
\hline
\end{tabular}

For $\mathrm{K}$, measurements were significantly higher on average with the ion specific electrode (ISE) compared to the ICP-OES measurements (Figure 2C, Table 3). Whilst the ISE and ICP-OES were significantly correlated (Figure 2A) and the values fell within $2 \mathrm{SD}$ of the mean (Figure 2B) the confidence intervals of the mean difference also suggest a significant difference between the two methods. The design of the ISE for $\mathrm{Na}$ and $\mathrm{K}$ is based on a polymeric membrane such that the size of the molecular cavity on the membrane matches the size of the targeted ion for the measurement [15]. The only difference between $\mathrm{Na}$ and $\mathrm{K}$ ion selective electrodes is the type of polymeric membrane used. The Na ion selective electrode membrane is sodium ionophore II, while valinomycin is the polymeric membrane for the $\mathrm{K}$ ion selective electrode. The mean difference of $0.65 \mathrm{mM}$ in the measurement of $\mathrm{K}$ between the two methods may be related the effect of the milk matrix [16,17]. Components in milk, such as proteins and other ions, could interfere with the interaction of $\mathrm{K}$ and the membrane of the ISE by partially blocking some of the molecular pores in the membrane. The matrix effect of milk may be more prominent on the ISE of K as the molecular cavity of the membrane of the K ISE is greater than that of the Na ISE.

Nevertheless, the measurement of $\mathrm{Na}$ and $\mathrm{K}$ with both methods was comparable and the mean differences of $\mathrm{Na}$ and $\mathrm{K}$ with both methods were within the limits of agreement (Table 4). Furthermore, within the first year of lactation, the mean concentrations of $\mathrm{Na}$ and $\mathrm{K}$ in milk ranged between 11 to $60 \mathrm{mM}$ and 4 to $18.2 \mathrm{mM}$, respectively [18]. We calculated Na:K ratios using all permeations of high, medium, and low levels of $\mathrm{Na}$ and $\mathrm{K}$ to determine if the ratio would shift dramatically to alter diagnosis particularly in the case of identifying secretory activation. Indeed, we found the ratio not to be altered enough to change a clinical diagnosis. (Table 2).

Table 4. Limits of agreement for the measurement of $\mathrm{Na}$ and $\mathrm{K}$ concentrations using ICP-OES and the ion selective electrode.

\begin{tabular}{ccccccc}
\hline \multirow{2}{*}{ Element } & \multirow{2}{*}{$\begin{array}{c}\text { Mean } \\
\text { Difference }\end{array}$} & \multirow{2}{*}{$\begin{array}{c}\text { CI mean } \\
\text { Difference }\end{array}$} & \multicolumn{2}{c}{ Limits of Agreement } & \multicolumn{2}{c}{ CI of Limits of Agreement } \\
\cline { 4 - 7 } & 0.26 & $-0.15,0.68$ & -3.08 & 3.61 & $-3.81,-2.37$ & $2.90,4.34$ \\
& $\mathrm{Na}(\mathrm{mM})$ & $0.17,1.13$ & -3.19 & 4.49 & $-4.01,-2.37$ & $3.67,5.31$ \\
$\mathrm{~K}(\mathrm{mM})$ & 0.65 & \multicolumn{2}{c}{ CI-95\% confidence interval. }
\end{tabular}

The accuracy of the ISE was comparable to ICP-OES with a significant correlation (Figures 1A and $2 \mathrm{~A}$ ) and good limits of agreement (Figures $1 \mathrm{~B}$ and 2B). The ISE could therefore be used to measure the high sodium concentrations found in antepartum secretions, colostrum, and the milk from mastitic breast or milk during the involution phase [6,19]. Under all these conditions the integrity of the tight 
junctions is compromised resulting in widening of the paracellular pathway between the mammary epithelial cells allowing transfer of components between the circulation and milk [19].

Human milk sodium concentrations decrease rapidly in the first three days post-partum as secretory activation occurs in response to the withdrawal of progesterone. However, milk sodium levels have a nonlinear relationship with milk volume, suggesting changes are not due to dilution as milk volume increases [1]. Delayed secretory activation is a risk factor for reduced breastfeeding success [1]. HM sodium drops from $60 \mathrm{mM}$ to $10 \mathrm{mM}$ between days 1 and 5 postpartum [2]. As such, the ion selective probe is accurate enough to monitor HM sodium levels postpartum to confirm secretory activation particularly in high risk mothers such as primiparous mothers, those with maternal obesity, preterm birth, caesarean deliveries, and those who have had long and complicated deliveries [20-24].

Low milk supply is also a frequent concern for mothers, particularly primiparous mothers $[3,25]$. This concern may be either perceived or actual milk supply insufficiency. It is known that milk production at week 2 is predictive of milk production at week 6; therefore, the first 14 days are critical to the establishment of a good milk supply. Na:K ratios during the first week postpartum have been shown to be useful biochemical indicators of suboptimal milk production, and as a result, are predictors of shorter breastfeeding duration $[4,9,26]$. High Na:K ratios are indicative of incomplete tight junction closure. This may impact the volume of milk produced and thus the transition to full lactation [9]. Higher breastfeeding frequency is associated with lower sodium levels, and increased production. Dewey et al. [20] recommend that all mother and infant dyads be followed up at 72-96 h postpartum to ensure that secretory activation has occurred and a biochemical indicator such as ion selective probes that measure $\mathrm{Na}$ and $\mathrm{K}$ may provide rapid results to ensure early intervention to improve milk production.

The ISE may also be useful in immediate detection of mastitis particularly subclinical mastitis [27] via increases in Na:K (>1.0). Mastitis is a debilitating inflammatory breast disease, reported to result in cessation of breastfeeding in as many as $20 \%$ of cases; therefore, rapid detection would allow early treatment and resolution resulting in better breastfeeding outcomes. HM sodium levels decrease over time with mature milk to $4-5 \mathrm{mM}$, whereas $\mathrm{HM}$ Na levels of $12 \mathrm{mM}$ and upwards are indicative of subclinical mastitis and mastitis. The $\mathrm{Na}: \mathrm{K}$ ratio has been categorized as $<0.6$ normal, $\geq 0.6$ and $\leq 1$ slightly elevated, $>1$ very high [7]. Na:K above 1.0 is commonly used in the diagnosis of subclinical or clinical mastitis $[7,27,28]$.

In conclusion, ion selective probes are sufficiently accurate to determine secretory activation by measurement of $\mathrm{Na}$ and $\mathrm{K}$ in the milk of lactating women. The use of ion selective probes may provide a useful point of care instrument to diagnose low milk supply and/or mammary infection. Early detection of these issues would allow timely intervention to ensure a successful lactation.

Author Contributions: Conceptualization, C.T.L. and D.G.; Methodology, C.T.L. and D.G.; Formal Analysis, C.T.L. and H.G.; Investigation, C.T.L.; Resources, D.G.; Data Curation, C.T.L. \& H.G.; Writing-Review \& Editing: C.T.L., H.G., D.G.; Visualization, C.T.L.; Supervision, D.G.; Project Administration, D.G.; Funding Acquisition, D.G.

Funding: The study was funded by an unrestricted research grant from Medela AG.

Acknowledgments: We thank the participated mothers who donated their milk for this study. We thank Oscar Del Borrello for his technical support on the ICP-OES instrument.

Conflicts of Interest: C.T.L., H.G. and D.G. receive salaries an unrestricted research grant from Medela AG.

\section{References}

1. Neville, M.C.; Allen, J.C.; Archer, P.C.; Casey, C.E.; Seacat, J.; Keller, R.P.; Lutes, V.; Rasbach, J.; Neifert, M. Studies in human lactation: milk volume and nutrient composition during weaning and lactogenesis. Am. J. Clin. Nutr. 1991, 54, 81-92. [CrossRef] [PubMed]

2. Cregan, M.D.; De Mello, T.R.; Kershaw, D.; McDougall, K.; Hartmann, P.E. Initiation of lactation in women after preterm delivery. Acta. Obstet. Gynecol. Scand. 2002, 81, 870-877. [CrossRef] [PubMed]

3. Wagner, E.A.; Chantry, C.J.; Dewey, K.G.; Nommsen-Rivers, L.A. Breastfeeding concerns at 3 and 7 days postpartum and feeding status at 2 months. Pediatrics 2013, 132, e865-e875. [CrossRef] [PubMed] 
4. Murase, M.; Wagner, E.A.; Chantry, C.J.; Dewey, K.G.; Nommsen-Rivers, L.A. The relation between breast milk sodium to potassium ratio and maternal report of a milk supply concern. J. Pediatr. 2017, 181, 294-297.e3. [CrossRef] [PubMed]

5. Lemay, D.G.; Ballard, O.A.; Hughes, M.A.; Morrow, A.L.; Horseman, N.D.; Nommsen-Rivers, L.A. RNA sequencing of the human milk fat layer transcriptome reveals distinct gene expression profiles at three stages of lactation. PLoS ONE 2013, 8, e67531. [CrossRef] [PubMed]

6. Fetherston, C.M.; Lai, C.; Hartmann, P.E. Relationships between symptoms and changes in breast physiology during lactation mastitis. Breastfeed. Med. 2006, 1, 136-145. [CrossRef] [PubMed]

7. Filteau, S.M.; Rice, A.L.; Ball, J.J.; Chakraborty, J.; Stoltzfus, R.; de Francisco, A.; Willumsen, J.F. Breast milk immune factors in Bangladeshi women supplemented postpartum with retinol or beta-carotene. Am. J. Clin. Nutr. 1999, 69, 953-958. [CrossRef] [PubMed]

8. Prentice, A.; Prentice, A. Unilateral breast dysfunction in lactating Gambian women. Ann. Trop. Paediatr. 1984, 4, 19-23. [CrossRef] [PubMed]

9. Humenick, S.; Hill, P.; Thompson, J.; Hart, A. Breast-milk sodium as a predictor of breastfeeding patterns. Can. J. Nurs. Res. 1997, 30, 67-81.

10. Arthur, P.; Hartmann, P.; Smith, M. Measurement of the milk intake of breast-fed infants. J. Pediatr. Gastroenterol. Nutr. 1987, 6, 758-763. [CrossRef] [PubMed]

11. R Core Team. R: A Language and Environment for Statistical Computing. Available online: ftp:/ /ftp.uvigo. es/CRAN/web/packages/dplR/vignettes/intro-dplR.pdf (accessed on 1 August 2018).

12. R Studio Team. R Studio: Integrated Development for R. Available online: https://www.rstudio.com/ (accessed on 1 August 2018).

13. Sarkar, D. Lattice: Trellis Graphics for R. Available online: https://cran.r-project.org/web/packages/lattice/ index.html (accessed on 1 August 2018).

14. Bland, J.M.; Altman, D.G. Statistical methods for assessing agreement between two methods of clinical measurement. Lancet 1986, 1, 307-310. [CrossRef]

15. Hauser, P.C. Determination of alkali ions in biological and environmental samples. In The Alkali Metal Ions: Their Role for Life; Sigel, A., Sigel, H., Sigel, R.K.O., Eds.; Springer: Cham, Germany, 2016; pp. 11-25.

16. Payne, R.; Buckley, B.; Rawson, K. Protein interference with ion-selective electrode measurement depends on reference electrode composition and design. Ann. Clin. Biochem. 1991, 28, 68-72. [CrossRef] [PubMed]

17. Dimeski, G.; Badrick, T.; St John, A. Ion selective electrodes (ISEs) and interferences-A review. Clin. Chim. Acta 2010, 411, 309-317. [CrossRef] [PubMed]

18. Atkinson, S.; Alston-Mills, B.; Lönnerdal, B.; Neville, M.C. Major minerals and ionic constituents of human and bovine milks. In Handbook of Milk Composition; Elsevier: Newyork, NY, USA, 1995; pp. 593-622.

19. Neville, M.C.; Keller, R.P.; Seacat, J.; Casey, C.E.; Allen, J.C.; Archer, P. Studies on human lactation. I. Within-feed and between-breast variation in selected components of human milk. Am. J. Clin. Nutr. 1984, 40, 635-646. [CrossRef] [PubMed]

20. Dewey, K.G.; Nommsen-Rivers, L.A.; Heinig, M.J.; Cohen, R.J. Risk factors for suboptimal infant breastfeeding behavior, delayed onset of lactation, and excess neonatal weight loss. Pediatrics 2003, 112, 607-619. [CrossRef] [PubMed]

21. Chapman, D.J.; Perez-Escamilla, R. Identification of risk factors for delayed onset of lactation. J. Am. Diet. Assoc. 1999, 99, 450-454. [CrossRef]

22. Hildebrandt, H. Maternal perception of lactogenesis time: A clinical report. J. Hum. Lact. 1999, 15, 317-323. [CrossRef] [PubMed]

23. Hall, R.T.; Mercer, A.M.; Teasley, S.L.; McPherson, D.M.; Simon, S.D.; Santos, S.R.; Meyers, B.M.; Hipsh, N.E. A breast-feeding assessment score to evaluate the risk for cessation of breast-feeding by 7 to 10 days of age. J. Pediatr. 2002, 141, 659-664. [CrossRef] [PubMed]

24. Meier, P.P.; Engstrom, J.L.; Mingolelli, S.S.; Miracle, D.J.; Kiesling, S. The rush mothers' milk club: Breastfeeding interventions for mothers with very-low-birth-weight Infants. J. Obstet. Gynecol. Neonatal Nurs. 2004, 33, 164-174. [CrossRef] [PubMed]

25. Li, R.; Fein, S.B.; Chen, J.; Grummer-Strawn, L.M. Why mothers stop breastfeeding: Mothers' self-reported reasons for stopping during the first year. Pediatrics 2008, 122 (Suppl. 2), S69-S76. [CrossRef] 
26. Morton, J.A. The clinical usefulness of breast milk sodium in the assessment of lactogenesis. Pediatrics 1994, 93, 802-806. [PubMed]

27. Aryeetey, R.N.; Marquis, G.S.; Timms, L.; Lartey, A.; Brakohiapa, L. Subclinical mastitis is common among Ghanaian women lactating 3 to 4 months postpartum. J. Hum. Lact. 2008, 24, 263-267. [CrossRef] [PubMed]

28. Buescher, E.S.; Hair, P.S. Human milk anti-inflammatory component contents during acute mastitis. Cell. Immunol. 2001, 210, 87-95. [CrossRef] [PubMed]

(C) 2018 by the authors. Licensee MDPI, Basel, Switzerland. This article is an open access article distributed under the terms and conditions of the Creative Commons Attribution (CC BY) license (http:/ / creativecommons.org/licenses/by/4.0/). 


\title{
Lactoferrin: A Critical Player in Neonatal Host Defense
}

\author{
Sucheta Telang ${ }^{1,2, *}$ \\ 1 Division of Neonatology, Department of Pediatrics, University of Louisville, Louisville, KY 40202, USA \\ 2 Division of Hematology/Oncology, Department of Medicine, James Graham Brown Cancer Center, \\ University of Louisville, Louisville, KY 40202, USA
}

Received: 31 July 2018; Accepted: 30 August 2018; Published: 4 September 2018

\begin{abstract}
Newborn infants are at a high risk for infection due to an under-developed immune system, and human milk has been shown to exhibit substantial anti-infective properties that serve to bolster neonatal defenses against multiple infections. Lactoferrin is the dominant whey protein in human milk and has been demonstrated to perform a wide array of antimicrobial and immunomodulatory functions and play a critical role in protecting the newborn infant from infection. This review summarizes data describing the structure and important functions performed by lactoferrin in protecting the neonate from infection and contributing to the maturation of the newborn innate and adaptive immune systems. We also briefly discuss clinical trials examining the utility of lactoferrin supplementation in the prevention of sepsis and necrotizing enterocolitis in newborn infants. The data reviewed provide rationale for the continuation of studies to examine the effects of lactoferrin administration on the prevention of sepsis in the neonate.
\end{abstract}

Keywords: lactoferrin; human milk; infection; immunity

\section{Introduction}

The neonatal period is an exceptionally vulnerable period of life, during which term and preterm infants are at high risk for morbidity and mortality. According to recent data from the World Health Organization, 2.6 million neonates died globally in 2016 alone-accounting for $46 \%$ of the deaths under the age of five years [1]. Infections are responsible for approximately $36 \%$ of the deaths that occur in the newborn period [1], and there thus exists an urgent need for better strategies and approaches to improve neonatal outcomes worldwide.

The increased susceptibility of the newborn infant to infection is largely due to the immaturity of the neonatal immune system. Limited antigenic exposure in the predominantly sterile in utero environment is a dominant factor contributing to the underdevelopment of the adaptive immune response. Additional contributory factors are deficiencies in the cells responsible for adaptive immunity themselves- they are present in smaller numbers and show great variability in their adaptive responses [2]. As a result, to combat early infectious threats, newborn rely on their innate immune response, which is also not yet fully developed $[3,4]$.

Neonatal deficiencies in immunity and host defense are compensated by several mechanisms. An early mechanism is the acquisition of antibodies passively transferred through the placenta from the mother [5]. Since this transfer occurs largely in the third trimester, the term infant is able to benefit from these antibodies but the preterm infant is unfortunately deprived of their protection.

A critical component of the armamentarium of the term and preterm neonate against infection is contributed by human milk. Human milk contains a wide array of bioactive proteins, growth factors, cells, and other constituents that modulate the development of a competent immune system to defend the term and preterm newborn against infections [6]. Of the bioactive factors present in human milk, 
lactoferrin has emerged as a key player that performs wide-ranging functions to directly and indirectly protect the neonate against infection.

\section{Lactoferrin Distribution and Properties}

Lactoferrin (or lactotransferrin, Lf) is a glycoprotein from the transferrin family of proteins. Lf was first identified in bovine milk by Sørensen and Sørensen in 1939 [7], then isolated from human and bovine milk by several investigators in 1960 [8-10]. Human Lf is a $78 \mathrm{kDa}$ glycoprotein which contains 691 amino acids and is expressed and secreted by epithelial cells in many exocrine secretions, including saliva, tears, and milk [11,12].

In human milk, Lf is the most abundant protein in the whey fraction, with a concentration varying from $1 \mathrm{gm} / \mathrm{L}$ to $7 \mathrm{gm} / \mathrm{L}$ (in colostrum) [12]. Multiple studies have evaluated Lf concentrations in colostrum and mature milk and in term and preterm milk. An early study that compared Lf levels between colostrum and mature milk in 30-32 week and >39 week neonates found trends towards higher initial Lf levels in the term infant group and higher sustained Lf levels in the preterm mature milk, but the differences did not reach significance [13]. A recent and comprehensive study has examined maternal milk samples from 24 week to term infants, and from birth to $>10$ days after birth, and found that Lf levels were highest in milk samples from mothers with infants $<1400 \mathrm{~g}$ and that the levels varied significantly over time and with gestation [14]. Interestingly, the variation between samples within groups appeared fairly uniform, indicating that Lf concentrations in maternal milk at similar gestations may be relatively similar [14].

Lf levels are also sensitive to low and high temperatures. Studies (from our group) found that refrigeration of human milk samples (at $4{ }^{\circ} \mathrm{C}$ ) for up to 5 days did not significantly lower Lf levels, but freezing (to -18 to $-20{ }^{\circ} \mathrm{C}$ ) decreased Lf dramatically to $\sim 35 \%$ of the levels in fresh milk by 6 months, with a similarly significant decrease in its activity (by $\sim 43 \%$, measured by nitric oxide production) $[15,16]$. Heating also appears to decrease Lf levels, indicated by data showing that pasteurization $\left(62.5^{\circ} \mathrm{C}\right.$ for $30 \mathrm{~min}$, Holder method) significantly decreased the total protein (and thus presumably Lf) in human milk samples [17,18]. Further studies, in donor milk samples, showed an even more dramatic decrease (up to 88\%) in Lf levels due to pasteurization [19]. This, when coupled with the freezing that these samples are exposed to, may indicate why donor milk has not shown the advantages of fresh maternal milk in terms of reduction in sepsis and necrotizing enterocolitis [18-20]. The detrimental effects of Holder pasteurization on immunological proteins in human milk have led to the active exploration of alternative methods to process donor human milk. Of these methods, exposure to $72{ }^{\circ} \mathrm{C}$ for $15 \mathrm{~s}$ (high temperature/short time or HTST pasteurization) has been demonstrated to preserve the integrity of Lf to a greater extent than the Holder method, although a significant decline in Lf relative to untreated milk is still noted [21-24]. Interestingly, studies have found that human Lf exposure to HTST conditions had only mild effects on its anti-bacterial activity [25], which may indicate that isolated and recombinant Lf may be less susceptible to temperature variations. Non-thermal alternatives to process donor milk are also under evaluation, such as high pressure processing, which has been shown to efficiently destroy microorganisms and allow greater retention of the immune components of human milk, including Lf [21,24]. A highly promising method that is currently under study is ultraviolet-C (UV-C) radiation. Recent data have indicated that UV-C radiation causes significant retention of Lf relative to Holder pasteurization and additionally induces greater resistance to bacterial infections in vivo [26-28]. In addition, these studies have described a technique to deliver UV-C radiation that has successfully overcome the limitations imposed by the high absorption coefficient of human milk [27]. These alternative processing methods will require extensive further investigation before reaching clinical application but certainly carry great promise.

The crystal structure of human Lf (hLf) was first solved in 1987 [29] and the protein has since been well described [30]. HLf contains two homologous lobes, each of which binds one ferric iron $\left(\mathrm{Fe}^{3+}\right)$ with high affinity, making hLf a strong scavenger of iron. Lf is also able to retain bound iron down to a $\mathrm{pH}$ of $~ 3.5$ [31] due to interactions between the 2 lobes, allowing it to be an effective anti-oxidant 
and bacteriostatic agent. Depending on its metal ion status, Lf can adopt either an iron-bound closed (holo-Lf) or a metal-free open conformation (apo-Lf) — both states have been demonstrated to perform functions in host defense. Lf additionally carries a high positive charge, with an isoelectric point of 9-10 that provides a high propensity for binding to negatively charged molecules on cell surfaces or in solution. Of particular importance are the basic residues at the N-terminus of Lf, at which proteolytic cleavage releases a potent antimicrobial peptide termed lactoferricin ( $\mathrm{Lfc}$ ) [32] that is highly exposed in both apo- and holo-Lf and may enable binding to bacterial cell membranes. A second peptide sequence, lactoferrampin, also has been identified as a major binding site with potential antibacterial properties. Additional data indicate that the glycan chains of Lf may mediate certain anti-bacterial and anti-viral activities as well [30].

Human Lf shares $70 \%$ sequence homology with bovine Lf (bLf) [33], which has a molecular weight of $\sim 76 \mathrm{kDa}$ [34] and consists of 689 amino acids, and is both folded into $\mathrm{N}$ and $\mathrm{C}$ lobes and has antigenic determinants highly similar to its human counterpart [35]. Bovine Lf has a lower iron affinity than hLf, potentially due to altered interdomain interactions in its structure driven by the orientation and domains of its lobes and by its oligosaccharide units (particularly a glycan chain at Asn 545) [35,36]. Despite this difference, near-identical functions of human and bovine Lf [11] against multiple pathogenic organisms have been well documented [11]. Similar to human Lf, bovine Lf generates Lfc by cleavage at the cationic $\mathrm{N}$-terminal region, which has been shown to cause a rapid loss of colony-forming capability [37]. Interestingly, the bovine Lf-generated Lfc was observed to have greater efficacy than human Lf against Gram-negative and Gram-positive bacteria [37]. Since bovine Lf is generally recognized as safe by the United States Food and Drug Administration (GRAS), it is easily available commercially and has therefore been widely used in vitro and in vivo for the examination of the various functions of this protein. In a recent study, commercial bLf added to infant formula was compared with hLf in an intestinal enterocyte model [38]. Commercial bLf was found to bind to the cells, be taken up by the human lactoferrin receptor, internalize, and promote proliferation and differentiation, indicating that it will likely exert bioactivities similar to hLf if supplemented in infant formula [38]. Several clinical trials examining the effects of bLf on infection have been conducted in preterm and term neonates, where bLf has been tolerated well (see Section 7). Bovine Lf-containing formula is also currently under active study in a clinical trial (NCT\#02103205) evaluating the effects of the addition of bLf on the immune system, the microbiota composition, metabolomics, growth, body composition, and cognitive development.

The variable Lf levels in maternal milk likely indicate the evolving requirement for this protein with gestational and post-natal age, and these data form an important basis for the development of optimal strategies for infants who require supplementation. Although the susceptibility of Lf in milk to heat and cold may hamper the use of stored human milk for such strategies, the stability of isolated hLf and the similarities between hLf and bLf structure and function indicate the potential utility of these proteins in formula supplementation.

\section{Direct Anti-Microbial Effects}

The anti-microbial effects attributed to Lf (Figure 1) were initially believed to be entirely due to the ability of unsaturated Lf to avidly bind iron and thereby cause bacteriostatic effects in iron-requiring pathogens. Early studies indicated that human milk and Lf purified from human milk had bacteriostatic effects on the growth of E. coli that were lost on saturation with iron [39]. These investigators went on to examine the effects of Lf against E. coli in vivo by gavage-feeding guinea pig pups with E. coli, and then either allowing the pups to suckle or feeding them with a milk substitute diet. They found that the suckled pups had substantially lower intestinal $E$. coli counts, interestingly with a corresponding increase in Lactobacillus numbers, and that the decrease in counts was reversed by feeding the pups hematin [39]. Iron-dependent anti-microbial effects of human and bovine Lf have been observed against a number of pathogens, including $S$. mutans, V. cholerae, and also P. aeruginosa, where iron 
chelation by Lf was found to stimulate a form of cell motility that inhibited biofilm formation by these bacteria [40-44].

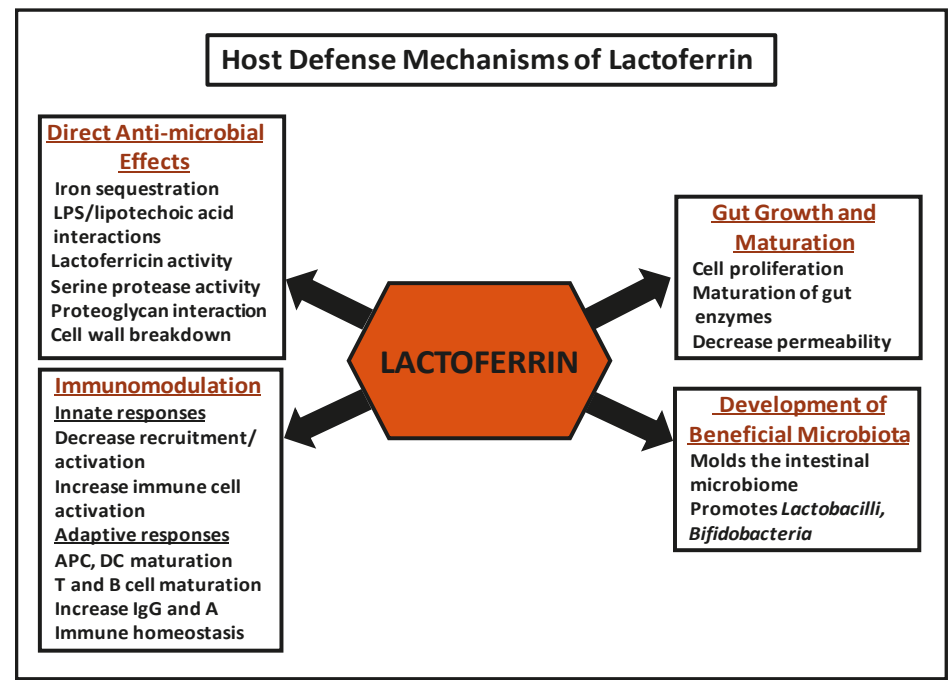

Figure 1. Functions of Lactoferrin in Neonatal Host Defense.

Several studies demonstrate that, independent of its iron-binding capabilities, Lf is bactericidal to several pathogens $[45,46]$ through interactions with the lipopolysaccharide (LPS) of Gram negative and the lipotechoic acid of Gram-positive bacteria [11]. In E. coli, Lf inhibits adherence and biofilm formation potentially by binding to lipid portions of the LPS layer, with a resultant increase in membrane permeability and disruption of virulence proteins anchored to the outer membrane [47]. These activities may be due to the action of Lfc-the peptide formed by the cleavage of Lf [32]. Further studies have determined another distinct anti-microbial function of the N-lobe of Lf due to the formation of a catalytic dyad by Ser259 and Lys73 that has a serine protease activity shown to successfully cleave and remove adherence elements of $H$. influenzae, thus attenuating its pathogenic potential [48,49]. An additional potential anti-bacterial mechanism has been proposed for Lf wherein it may enhance anoikis of infected enterocytes [50], but this activity requires significant further investigation. Taken together, these data indicate that the ability of Lf to affect bacterial attachment and invasion proteins may play a role in protecting suckling animals from infection by preventing the attachment and colonization of bacteria in the intestinal epithelium. In support of this hypothesis, studies have demonstrated that neonatal rodents pretreated with Lf had less bacteremia and less severe disease due to intestinal E. coli infection [51]. In addition, Lf has shown potent synergistic activity in killing Gram-negative bacteria in vitro with lysozyme-a second important component of the human milk whey fraction that is able to degrade bacterial membrane peptidoglycans. By binding LPS and removing it from the outer cell membrane, Lf allows lysozyme to access and degrade the inner membrane proteoglycans and kill the bacteria [52]. The bactericidal activity against Gram-positive bacteria appears to be caused by the same residues as with Gram-negative bacteria [53]. Of interest, a recent study examined the effects of $S$. aureus bacteremia in piglets pre-treated with dietary bovine Lf [54] and found that bLf pretreatment effectively reduced S. aureus systemic infection. BLf additionally decreased IL-10 and increased interferon- $\gamma$ mRNA in these animals, indicating a type $1 \mathrm{~T}$ helper (Th1) immune response and, thus, effects on the innate and adaptive immunity of these animals. These results may explain some of the beneficial effects of bLf observed in preterm infants. 
Lf also has direct inhibitory effects on viruses and other microbes. Against viruses, these effects may involve the attachment of Lf to surface proteoglycans, such as heparan sulfate, to which Lf has a high affinity through its $\mathrm{N}$-terminus glycosaminoglycan-binding domains [55], thus blocking the entry of certain viruses, e.g., HSV. Other mechanisms may involve direct interactions of Lf with viral envelope proteins [56]. In fungi such as Candida, Lf has been shown to have effects as well, and was observed to cause cell wall perturbations, with the formation of surface blebs, swelling, and the collapse of the cell [57].

Variable responses to Lf-driven inhibition have been observed in different micro-organisms that are likely driven by differences in their iron requirement and their strategies to increase iron uptake and, additionally, by structural variations that may serve to limit direct access by Lf. As examples, several bacterial species have developed mechanisms to evade the iron-limiting effects of Lf. Neisseria and Moraxella species express specific Lf receptors that bind Lf to induce a conformational change in its structure and release iron into the bacteria [58]. Other micro-organisms have developed strategies to resist direct Lf-driven killing, such as $S$. pneumoniae, which binds Lf by pneumococcal surface protein A (PspA) and thereby evades the bactericidal effects of Lf [59], and V. vulnificus, which expresses a metalloprotease (Vvpe) that destroys Lf and facilitates the ability of the bacteria to invade the mucosa [60].

The studies indicate that further investigation into the anti-microbial functions of Lf is required. The examples described above notwithstanding, the available scientific evidence demonstrates the widespread inhibitory effects of Lf on the proliferation and survival of pathogenic micro-organisms - either by the sequestration of iron or direct activity on virulence factors-and strongly supports a protective role for Lf against infection in the newborn.

\section{Immunomodulatory Functions of Lactoferrin}

Lf plays a key role in neonatal host defense by modulating the innate and adaptive immune response of the neonate to infections (Figure 1). In addition, a growing body of evidence suggests that Lf facilitates mechanisms whereby adaptive immune changes may influence the innate immune system.

\subsection{Mechanisms of Interaction of Lactoferrin with Immune Cells}

The effects of Lf on immune cells are modulated by binding to a variety of targets. Among the most abundant are the glycosaminoglycans on membrane peptidoglycans [61], which are critical for the binding of many cytokines and factors, and it has been postulated that Lf may alter immune cell function by displacing these factors [62]. Other receptors described include lectins (e.g., TLR-4) which recognize the glycan chains of Lf, receptors recognizing the Lfc or N1 domain, intelectin-1 (found on enterocytes and immune cells), and nucleolin which may serve the additional function of transporting Lf to the nucleus [61,63]. All these receptors may potentially internalize Lf with downstream activation of signaling pathways e.g., the phosphoinositide 3-kinase (PI3K)/Akt and the mitogen activated protein kinase (MAPK)/extracellular signal-regulated kinase (ERK) signaling pathways, whereby Lf has been shown to activate cell cycle progression, proliferation, and downstream cellular responses [64-66] or, following nuclear localization, the NFkB pathway $[61,67]$. In addition, Lf has been found to bind to a receptor on (transformed) hematopoietic cells and translocate to the nucleus, leading to transcriptional activation with downstream effects $[68,69]$.

\subsection{Innate Immune Effects of Lactoferrin}

The effects of Lf on the innate immune response are related in part to its ability to bind to conserved structures, termed pathogen-associated molecular patterns (PAMPs), present on pathogens (e.g., LPS on Gram-negative bacteria and peptidoglycans on Gram-positive bacteria). PAMPs are recognized by pattern recognition receptors or PRRs such as Toll-like receptors (TLRs) [70], that are critical for the activation of innate immunity. TLR4 has been demonstrated to bind and transfer LPS, with the assistance of the transfer molecule LPS-binding protein (LBP), to CD14. CD14 is a 
glycosylphosphatidylinositol-anchored membrane protein present on myeloid cells which leads to their activation and the release of pro-inflammatory cytokines e.g., TNF- $\alpha$, IL-6, and IL-1 $\beta$ [71,72]. Lf is demonstrated to bind to several PAMPs, including LPS, and thereby compete with LBP to inhibit the release of pro-inflammatory cytokines [62,73]. Lf may also modulate recruitment of immune cells by interfering with the expression of endothelial cell adhesion molecules required for the recruitment of these cells to sites of inflammation as shown by data indicating that the interference of Lf in the LPS-CD14 interaction may inhibit the expression of E-selectin, ICAM-1, and IL-8 by human umbilical vein endothelial cells (HUVECs) [62,74]. Lf may cause further suppressive effects on immune cells by binding to other molecular cell surface targets, as evident by its function in competing with the chemokine IL-8 for binding to endothelial cell proteoglycans to inhibit the activation and recruitment of leukocytes to sites of inflammation [74].

Lf is also capable of enhancing the activation of immune cells. Following bacterial invasion, LPS binds to TLR4 on sentinel cells to cause the release of potent cytokines including TNF- $\alpha$, IL- $1 \beta$, and IL-6 [62,75]. These molecules will activate and modify the permeability of endothelial cells to allow the passage of complement and antibodies and recruit neutrophils to the site of inflammation. Activated neutrophils will release Lf from their secondary granules to exert its direct microbicidal effects [62]. Lf may also enhance the cytotoxic functions of NK and lymphokine-activated killer cells, potentially through binding to RNA and DNA [76].

Promotion of lytic cell activity is a key role played by Lf. Lf receptors are found on macrophages [77] and Lf is shown to activate macrophages to release pro-inflammatory molecules e.g., TNF- $\alpha$, IL-8, and nitric oxide $[15,78]$ and to increase their phagocytic activity when infected [79]. Lf is also expressed on the membranes of resting PMNs and may enable interaction between Lf-bound microbes and PMNs [80]. Bovine Lf was noted to increase phagocytic killing of S. aureus-potentially by activation of the alternate complement pathway by its Lfc domain $[81,82]$.

\subsection{Effects on Adaptive Immune Responses}

Lf plays an important immunomodulatory role in activation and antigen presentation by antigen-presenting cells (APCs) and in their functions in the adaptive immune response by affecting $\mathrm{T}$ cell development. Macrophages function as APCs to stimulate the development of antigen-specific CD4+ T cells, and Lf enhances their ability to function as APCs by stimulating the production of cytokines, such as IL-12, responsible for modulating development of Th1 cells [83,84].

Lf also assists in the maturation of dendritic cells (DCs) - by enhancing their release of IL-8 and CXCL10, decreasing antigen internalization, increasing their capacity to trigger proliferation and release IFN- $\gamma$ in the presence of allogeneic human $\mathrm{T}$ cells, and to prime naive $\mathrm{T}$ cells in response to several antigenic stimuli [85]. Recent studies indicate that Lf may function similarly to an alarmin to promote the activation of APCs and antigen-specific immune responses [86,87]. These studies demonstrate that, similar to the previous study, Lf is able to chemoattract and cause the maturation of monocyte-derived DCs, and also stimulate the production of pro-inflammatory cytokines. Lf additionally may prompt Th1 polarized antigen-specific immune responses in immunized mice and the recruitment of macrophages and neutrophils when injected into the mouse peritoneal cavity [86].

More recent data indicate a role for $\mathrm{Lf}$ in immune homeostasis as well. Studies indicate that DCs differentiated in the presence of Lf showed decreased responsiveness towards TLR ligands $[88,89]$ and reduced cytokine production demonstrating a potential role for Lf in immune homeostasis. These results indicate a potent anti-inflammatory function for Lf by skewing monocyte differentiation into DCs with impaired capacity for activation and for promotion of Th1 responses and may represent a strategy to block excessive DC activation upon TLR-induced inflammation, adding further evidence for a critical role of Lf in directing host immune function.

Lf has been shown to modulate the production of pro-inflammatory cytokines, such as TNF- $\alpha$, IL-1 $\beta$, and IL-6, from leukocyte populations, which may be increased or decreased depending on the 
condition recognized by the immune system. In addition, Lf may increase the production of IL-12 by APCs when presented with pathogens. IL-12 enhances IFN- $\gamma$ production and proliferation, augments cytotoxic activity of lymphocytes responsible for innate (NK cells) and adaptive (CD4+and CD8+ T-cells) immunity, and is a major driver of Th1 cell development $[83,84]$.

Lf also influences $\mathrm{T}$ and $\mathrm{B}$ lymphocyte maturation. Lf is able to bind to surface receptors and be internalized by human Jurkat lymphoblastic T cells [90], where it accelerates $\mathrm{T}$ cell maturation by induction of CD4 via activation of the MAPK pathway [91]. Human milk-derived Lf is observed to cause maturation of $\mathrm{CD} 4^{-} \mathrm{CD} 8^{-}$murine T-cells, with a preference towards expression of CD4 [92]. When administered orally, Lf has the ability to restore the host $\mathrm{T}$ cell compartment, evident by an increase in splenic cellularity and enrichment of CD3+ CD4+ T cells, and suggesting a possible role for Lf in the reconstitution of the cellular immune response [93]. As noted with other cell types, Lf also appears to exert anti-inflammatory effects. The addition of Lf to mitogen-activated T-cells decreases overall cytokine production demonstrated by the decreased production of IFN- $\gamma$ and IL-2 by ConA-stimulated murine splenocytes cultured with Lf [94]. Similarly, Lf is able to promote the maturation of immature B lymphocytes, shown by an increase in surface Ig D and complement receptor expression. In addition, Lf was shown to enable B cells from normal newborn and adult immunodeficient mice to present antigen to an antigen-specific T-helper type 2 (Th2) cell line [95]. Orally administered Lf has been demonstrated to increase the pool of CD4+ T cells, immunoglobulin levels ( $G$ and A), as well as proliferation in the Peyer's patches of the intestine, suggesting that Lf may act as an immunostimulatory factor on the mucosal immune system [96-98]. In addition, in a chemotherapy-induced immune suppression murine model, Lf administered intraperitoneally was able to decrease the suppression of antibody forming cells and facilitate the restoration of the immune response [99].

Taken together, these studies illustrate the multiple activities performed by Lf to modulate the nascent neonatal immune system and highlight the importance of this protein in the development of a mature immune response. The growing body of scientific evidence suggests that the effects of Lf vary depending on the threat faced by the immune system and thereby emphasizes the importance of this glycoprotein in the protection of the newborn from infection.

\section{Effects of Lactoferrin on the Development of Beneficial Microbiota}

The bacterial flora colonizing human milk fed infants have been demonstrated to be different from those of formula-fed infants. Higher concentrations of Lactobacillus and Bifidobacteria species are observed with comparatively fewer bacteria with high pathogenic potential e.g., E. coli, Campylobacter, and Bacteroides [100]. In vitro studies have demonstrated that Lf from human and bovine milk promotes the growth of intestinal bifidobacteria without the requirement for binding of the Lf molecule to the bacterial cell surface or a dependence on the acquisition and utilization of iron [101]. Bifidogenic peptides have been isolated from human milk (derived from hLf) that demonstrate strong bifidogenic effects on several bifidobacterial species (B. bifidum, B. breve, and B. longum) and are resistant to digestive enzymes $[102,103]$. The importance of Lf in the development of beneficial bacteria is underscored by data from breastfed term and preterm infants, showing high fecal Lf levels and a significant association of bifidobacteria and lactobacilli with fecal Lf levels on day three of life, suggesting that Lf may be a key factor in the initiation, development, and composition of the neonatal gut microbiota [104]. These data indicate that Lf is a tremendously important influence on the development of the intestinal microbiome (Figure 1). The importance of this function of Lf is magnified in critically ill and hospitalized term and preterm infants who are at risk for colonization and infection with highly pathogenic bacteria [105], and where Lf administration may be able to play a critical role in decreasing invasive infection and necrotizing enterocolitis. Interestingly, recent studies have examined the effect of Lf on probiotic bacterial growth in vitro and found that both hLf and bLf may retard the growth of certain bifidobacteria $[106,107]$. In view of the importance of the development of beneficial gut bacteria 
and ongoing clinical examination of Lf supplementation with probiotics, the further delineation of the precise effects of Lf in probacterial growth is a critical avenue of investigation.

\section{Effects on Gut Growth and Maturation}

Lf has been found to directly stimulate intestinal growth and proliferation [108,109] (Figure 1). Studies conducted on Caco-2 (transformed) enterocytes in vitro have found that exposure to high Lf concentrations led to a dose-dependent increase in cell proliferation, while low Lf levels stimulated intestinal cell differentiation [108]. These data suggest that Lf may actively modulate enterocyte growth and development in vivo due to variations in its concentration from colostrum to mature milk, in addition to its stimulatory effects on intestinal enzyme maturation [108]. Of interest, these studies found that bLf was a more potent effector of growth than hLf, which provides rationale for its supplementation in infant formula [108]. Beneficial effects of bLf administration were also noted in vivo. Neonatal piglets fed formula that contained physiological levels of bLf relative to controls fed low bLf showed an increase in intestinal cellular proliferation and, additionally, increased $\beta$-catenin levels, indicating a potential role for Wnt signaling in gut proliferation [110]. Other studies have observed that Lf is taken up by enterocytes via the Lf receptor and stimulates enterocyte proliferation through the Ras-MAPK pathway [68], the strong mitogenic effect of which also may drive the rapid development of the intestinal mucosa in newborns fed maternal milk. An additional possible function for Lf in intestinal maturation is in regulation of gut permeability. In its support, studies have shown that preterm infants fed maternal milk had decreased gastrointestinal permeability relative to formula-fed controls, which indicates a potential role for components of human milk in intestinal maturation [111]. This mechanism of action will require further examination in the newborn population.

The functions performed by Lf in the growth and maturation of the gut are critical for the development and maintenance of the intestinal barrier to infection. The breakdown of this barrier may expose the newborn to potentially highly pathogenic bacteria. These findings therefore support the importance of early and continued exposure of the newborn gut to Lf in human milk or as a supplement in formula.

\section{Examination for Clinical Efficacy of Lf in Neonates}

An overwhelming body of experimental evidence supports the beneficial anti-infective properties of Lf, providing strong rationale for its use against infection in newborn infants.

Based on the significant anti-microbial and immunomodulatory effects caused by Lf, this protein may be particularly useful in host defense in critically ill and very low birth weight (VLBW) neonates. VLBW infants carry an enhanced risk for bacterial sepsis and potentially devastating sequelae [112] and are frequently unable to tolerate feeds, thus depriving them of the protective benefits of maternal milk. Based on this rationale, several studies have examined the efficacy of Lf supplementation against sepsis [113] in the neonatal period. An early study where healthy, formula-fed infants ( $\geq 34$ weeks gestation and $\leq 4$ weeks old) were fed formula supplemented with bovine Lf vs. cow milk-based formula and followed for 12 months found significantly fewer lower respiratory tract illnesses in the Lf-fed group [114] (Table 1). In 2009, Manzoni's group performed a multicenter, double-blinded, placebo-controlled, randomized trial in VLBW infants $(<1500 \mathrm{~g})$ comparing administration of bLf alone or in combination with Lactobacillus rhamnosus GG (LGG) to placebo [115]. They found significantly lower invasive infections in the treatment groups, with an effect on infection-related mortality $(0 \%$ for bLf and $0.7 \%$ for bLf plus LGG, vs. $4.8 \%$ for placebo). A follow-up study from the same group in 2014 found that bLf supplementation alone or in combination with LGG significantly reduced the incidence of $\geq$ stage 2 necrotizing enterocolitis (NEC), and of death-and/or $\geq$ stage 2 NEC in VLBW neonates [116]. Apart from these, several other studies (Table 1) have also examined bovine Lf and found that treatment with bLf led to a reduction in infection in both VLBW and 500-2500 g neonates $[117,118]$. Importantly, none of these investigations noted any adverse effects or intolerance 
with bovine Lf. BLf has also been evaluated in a recent study that confirmed that it was well tolerated [119]. Several other studies examining the efficacy of bovine Lf are currently underway [113]. Of note, a multicenter trial of enteral bovine Lf in $2200<32$ week infants (the ELFIN trial UK) that has recently completed recruitment, will primarily evaluate effects on late onset invasive infection but also mortality, NEC, and several later sequelae [120]. The results of this large trial may serve to further validate the utility of bovine Lf supplementation in this vulnerable population.

Table 1. Clinical Studies of Lactoferrin in Neonates. The $n$ values denote the number of patients in the treatment groups. Significant study outcomes are in bold type. LOS, Late-onset sepsis.

\begin{tabular}{|c|c|c|c|c|c|}
\hline Year & Study Population & Study Design & Lf Type & Outcomes & Investigator, Site \\
\hline 2007 & $\begin{array}{l}\text { Neonates } \geq 34 \text { weeks, } \\
\leq 4 \text { weeks of life }(n=26)\end{array}$ & $\begin{array}{l}\text { Formula }+ \text { Lf }(850 \mathrm{mg} / \mathrm{L}) \mathrm{vs} . \\
\text { cow-milk formula }+ \text { Lf }(102 \\
\mathrm{mg} / \mathrm{L})(\leq 4 \text { weeks }-12 \text { months })\end{array}$ & Bovine & $\begin{array}{l}\text { Lower incidence of lower } \\
\text { respiratory tract infections }\end{array}$ & King, USA \\
\hline 2009,2012 & $\begin{array}{l}\text { VLBW Neonates }<1500 \mathrm{~g} \\
(\mathrm{Lf}, n=153, \mathrm{Lf}+\mathrm{LGG}, \\
n=151)\end{array}$ & $\begin{array}{l}\text { Lf }(100 \mathrm{mg} / \text { day }) \pm \text { LGG vs. } \\
\text { placebo, } 0-30 \text { days }(0-45 \text { days } \\
\text { for }<1000 \mathrm{~g} \text { at birth })\end{array}$ & Bovine & $\begin{array}{l}\text { Lower incidence of first } \\
\text { LOS episode (in Lf } \pm \text { LGG) } \\
\text { Lower incidence of } \\
\text { Candida LOS }\end{array}$ & Manzoni, Italy \\
\hline 2014 & $\begin{array}{l}\text { VLBW neonates, }<1500 \mathrm{~g} \\
\text { or }<32 \text { weeks }(n=25)\end{array}$ & $\begin{array}{l}\text { Lf ( } 200 \mathrm{mg} / \text { day) vs. placebo, } \\
\text { through hospitalization period }\end{array}$ & Bovine & $\begin{array}{l}\text { Decreased nosocomial sepsis } \\
\text { episodes }\end{array}$ & Akin, Turkey \\
\hline 2015 & $\begin{array}{l}\text { Neonates, } 500-2500 \mathrm{~g} \\
(n=95)\end{array}$ & $\begin{array}{l}200 \mathrm{mg} / \mathrm{kg} / \text { day vs. placebo } \\
\text { from } 2-28 \text { days }\end{array}$ & Bovine & $\begin{array}{l}\text { Sepsis less frequent in Lf } \\
\text { group (Primary outcome: } \\
\text { incidence of LOS, no } \\
\text { statistical significance but CI } \\
\text { suggestive of effect) }\end{array}$ & Ochoa, Peru \\
\hline 2015 & $\begin{array}{l}\text { Neonates }<2000 \mathrm{~g} \\
(n=65)\end{array}$ & $\begin{array}{l}\mathrm{Lf}(80-140 \mathrm{mg} / \mathrm{kg} / \text { day }) \mathrm{vs} . \\
\text { placebo from } 1-28 \text { days }\end{array}$ & Bovine & $\begin{array}{l}\text { Lower incidence of first } \\
\text { LOS episode, reduction in } \\
\text { sepsis-attributable } \\
\text { mortality }\end{array}$ & Kaur, India \\
\hline 2016 & $\begin{array}{l}\text { Neonates } 750-1500 \mathrm{~g} \\
(n=60)\end{array}$ & $\begin{array}{l}\mathrm{Lf}(150 \mathrm{mg} / \mathrm{kg} \mathrm{q} 12 \mathrm{~h}) \mathrm{vs} . \\
\text { placebo from 1-28 days }\end{array}$ & Human & $\begin{array}{l}\text { Trend towards decreased } \\
\text { infectious morbidities } \\
\text { (primary outcomes: } \\
\text { bacteremia, NEC } \\
\text { pneumonia, UTI, } \\
\text { meningitis) }\end{array}$ & Sherman, USA \\
\hline 2016 & $\begin{array}{l}\text { Neonates }<32 \text { weeks } \\
(n=40)\end{array}$ & $\begin{array}{l}\text { Lf ( } 100 \mathrm{mg} / \text { day) vs. placebo, } \\
\text { until } 36 \text { weeks PMA or } \\
\text { discharge }\end{array}$ & Bovine & $\begin{array}{l}\text { No difference in feeding } \\
\text { tolerance }\end{array}$ & Barrington, Canada \\
\hline
\end{tabular}

Multiple invitro and animal studies have demonstrated potent anti-microbial and immunomodulatory effects with Lf isolated from human milk. A recombinant human lactoferrin, generated in Aspergillus oryzae, was demonstrated to have an amino acid structure and functions highly similar to the human milk molecule [121]. Based on this expression system, commercial amounts of this protein were generated, leading to the development of a clinical candidate (talactoferrin) that differs from the native human protein in its glycosylation due to the fungal expression system but is otherwise unchanged $[122,123]$. Studies have demonstrated that talactoferrin is well tolerated in adult patients [124]. A single multicenter trial was conducted using talactoferrin in 750-1500 g neonates, which examined 120 infants and showed a trend towards decreased infectious morbidity but did not achieve statistical significance [125] (Table 1). Further trials with this protein, however, currently appear to be on hold following recent data showing no benefit in a trial in adult ICU patients [126].

Based on the pre-clinical data, the potential benefits of Lf supplementation are clear-with strong evidence supporting its direct anti-microbial and immune-boosting properties and effects on gut proliferation, maturation, and the development of beneficial bacteria. The clinical studies done thus far have shown uniformly positive results that have reached statistical significance in certain studies (Table 1). Based on the clinical data, early commencement of Lf may be associated with greater clinical benefits, demonstrated by examining study results from Ochoa et al. (Lf started with enteral feeds at $4 \pm 1.4$ days [118]), Akin et al. (with feeds at $20 \mathrm{~mL} / \mathrm{kg} /$ day [117]), and Manzoni et al. (at < $72 \mathrm{~h} \mathrm{[115]).}$ 
Early supplementation may mimic the higher Lf in human colostrum and, as shown in vitro, may allow for early gut proliferation. The addition of a probiotic $[115,116]$ appeared to substantially improve outcomes and should be further explored. However, as indicated by recent in vitro data described above $[106,107]$, the administration of Lf in conjunction with a probiotic requires further careful study. Additionally, the use of a standard dose of Lf for all patients may not be optimal for delivering adequate concentrations of Lf to each patient and weight-based dosing regimens should be evaluated for clinical efficacy. Last, although the results with recombinant human Lf were not significant, the use of a human Lf might be revisited in the future.

\section{Conclusions}

Taken together, the experimental and pre-clinical studies examining the functions of Lf present overwhelming evidence, supporting a pivotal role for this multifaceted glycoprotein in preventing infection, in immunomodulation, and bolstering host defense. Many questions remain to be answered regarding the function of this glycoprotein at the molecular level and the extent of direct and immune modulatory effects caused by supplementation of Lf in the diet. Several of these questions are best addressed by in vivo studies in patients. These are challenging studies, particularly as they are targeted towards the critical VLBW infant. However, the clinical data obtained thus far have been promising and certainly support the utility of continuation of studies to examine the effects of Lf supplementation on modulating the immune response and decreasing life-threatening infections in the highly vulnerable neonatal population. Several studies are currently underway, and their results will serve to clarify the benefits of Lf supplementation in the diet of the term and preterm infant, and potentially pave the way to using Lf in the clinical setting.

Author Contributions: S.T. researched and wrote the manuscript.

Funding: We gratefully acknowledge the support of the University of Louisville Department of Pediatrics and the James Graham Brown Cancer Center.

Acknowledgments: We thank John Eaton and Paula Radmacher for helpful discussions.

Conflicts of Interest: The authors declare no conflict of interest.

\section{References}

1. UNIGME. Inter-Agency Group for Child Mortality Estimation: Levels and Trends in Child Mortality, Report 2017; UNICEF: New York, NY, USA, 2017.

2. Adkins, B.; Leclerc, C.; Marshall-Clarke, S. Neonatal adaptive immunity comes of age. Nat. Rev. Immunol. 2004, 4, 553-564. [CrossRef] [PubMed]

3. Strunk, T.; Currie, A.; Richmond, P.; Simmer, K.; Burgner, D. Innate immunity in human newborn infants: Prematurity means more than immaturity. J. Matern. Fetal. Neonatal Med. 2011, 24, 25-31. [CrossRef] [PubMed]

4. De Jong, E.; Strunk, T.; Burgner, D.; Lavoie, P.M.; Currie, A. The phenotype and function of preterm infant monocytes: Implications for susceptibility to infection. J. Leukocyte Biol. 2017, 102, 645-656. [CrossRef] [PubMed]

5. Fouda, G.G.; Martinez, D.R.; Swamy, G.K.; Permar, S.R. The Impact of IgG Transplacental Transfer on Early Life Immunity. ImmunoHorizons 2018, 2, 14-25. [CrossRef] [PubMed]

6. Ballard, O.; Morrow, A.L. Human milk composition: Nutrients and bioactive factors. Pediatr. Clin. N. Am. 2013, 60, 49-74. [CrossRef] [PubMed]

7. Sorensen, M.; Sorensen, S.P.L. The proteins in whey. C. R. Trav. Lab. Carlsberg 1939, 23, 55-99.

8. Groves, M.L. The isolation of a red protein from milk. J. Am. Chem. Soc. 1960, 83, 3345-3350. [CrossRef]

9. Johansson, B. Isolation of an iron-containing red protein from human milk. Acta Chem. Scand. 1960, 14, 510-512. [CrossRef]

10. Montreuil, J.; Tonnelat, J.; Mullet, S. Preparation and properties of lactosiderophilin (lactotransferrin) of human milk. Biochim. Biophys. Acta 1960, 45, 413-421. [CrossRef] 
11. Rosa, L.; Cutone, A.; Lepanto, M.S.; Paesano, R.; Valenti, P. Lactoferrin: A Natural Glycoprotein Involved in Iron and Inflammatory Homeostasis. Int. J. Mol. Sci. 2017, 18, 1985. [CrossRef] [PubMed]

12. Liao, Y.; Alvarado, R.; Phinney, B.; Lonnerdal, B. Proteomic characterization of human milk whey proteins during a twelve-month lactation period. J. Proteome Res. 2011, 10, 1746-1754. [CrossRef] [PubMed]

13. Ronayne de Ferrer, P.A.; Baroni, A.; Sambucetti, M.E.; Lopez, N.E.; Ceriani Cernadas, J.M. Lactoferrin levels in term and preterm milk. J. Am. Coll. Nutr. 2000, 19, 370-373. [CrossRef] [PubMed]

14. Albenzio, M.; Santillo, A.; Stolfi, I.; Manzoni, P.; Iliceto, A.; Rinaldi, M.; Magaldi, R. Lactoferrin Levels in Human Milk after Preterm and Term Delivery. Am. J. Perinatol. 2016, 33, 1085-1089. [PubMed]

15. Raoof, N.A.; Adamkin, D.H.; Radmacher, P.G.; Telang, S. Comparison of lactoferrin activity in fresh and stored human milk. J. Perinatol. Off. J. Calif. Perinat. Assoc. 2016, 36, 207-209. [CrossRef] [PubMed]

16. Rollo, D.E.; Radmacher, P.G.; Turcu, R.M.; Myers, S.R.; Adamkin, D.H. Stability of lactoferrin in stored human milk. J. Perinatol. Off. J. Calif. Perinat. Assoc. 2014, 34, 284-286. [CrossRef] [PubMed]

17. Koenig, Á.; Diniz, E.M.D.A.; Barbosa, S.F.C.; Vaz, F.A.C. Immunologic Factors in Human Milk: The Effects of Gestational Age and Pasteurization. J. Hum. Lact. 2005, 21, 439-443. [CrossRef] [PubMed]

18. Manzoni, P. Clinical Benefits of Lactoferrin for Infants and Children. J. Pediatr. 2016, 173. [CrossRef] [PubMed]

19. Meier, P.P.; Patel, A.L.; Esquerra-Zwiers, A. Donor Human Milk Update: Evidence, Mechanisms and Priorities for Research and Practice. J. Pediatr. 2017, 180, 15-21. [CrossRef] [PubMed]

20. Underwood, M.A.; Scoble, J.A. Human Milk and the Premature Infant: Focus on Use of Pasteurized Donor Human Milk in the NICU; Springer: New York, NY, USA, 2015; pp. 795-806.

21. Picaud, J.C.; Buffin, R. Human Milk-Treatment and Quality of Banked Human Milk. Clin. Perinatol. 2017, 44, 95-119. [CrossRef] [PubMed]

22. Mayayo, C.; Montserrat, M.; Ramos, S.J.; Martínez-Lorenzo, M.J.; Calvo, M.; Sánchez, L.; Pérez, M.D. Kinetic parameters for high-pressure-induced denaturation of lactoferrin in human milk. Int. Dairy J. 2014, 39, 246-252. [CrossRef]

23. Baro, C.; Giribaldi, M.; Arslanoglu, S.; Giuffrida, M.G.; Dellavalle, G.; Conti, A.; Tonetto, P.; Biasini, A.; Coscia, A.; Fabris, C.; et al. Effect of two pasteurization methods on the protein content of human milk. Front. Biosci. (Elite Ed.) 2011, 3, 818-829. [CrossRef] [PubMed]

24. Peila, C.; Emmerik, N.E.; Giribaldi, M.; Stahl, B.; Ruitenberg, J.E.; van Elburg, R.M.; Moro, G.E.; Bertino, E.; Coscia, A.; Cavallarin, L. Human Milk Processing: A Systematic Review of Innovative Techniques to Ensure the Safety and Quality of Donor Milk. J. Pediatr. Gastroenterol. Nutr. 2017, 64, 353-361. [CrossRef] [PubMed]

25. Conesa, C.; Rota, C.; Castillo, E.; PÉRez, M.-D.; Calvo, M.; SÁNchez, L. Antibacterial Activity of Recombinant Human Lactoferrin from Rice: Effect of Heat Treatment. Biosci. Biotechnol. Biochem. 2009, 73, 1301-1307. [CrossRef] [PubMed]

26. Li, Y.; Nguyen, D.N.; de Waard, M.; Christensen, L.; Zhou, P.; Jiang, P.; Sun, J.; Bojesen, A.M.; Lauridsen, C.; Lykkesfeldt, J.; et al. Pasteurization Procedures for Donor Human Milk Affect Body Growth, Intestinal Structure, and Resistance against Bacterial Infections in Preterm Pigs. J. Nutr. 2017, 147, 1121-1130. [CrossRef] [PubMed]

27. Christen, L.; Lai, C.T.; Hartmann, B.; Hartmann, P.E.; Geddes, D.T. Ultraviolet-C Irradiation: A Novel Pasteurization Method for Donor Human Milk. PLoS ONE 2013, 8, e68120. [CrossRef] [PubMed]

28. Christen, L.; Lai, C.T.; Hartmann, B.; Hartmann, P.E.; Geddes, D.T. The Effect of UV-C Pasteurization on Bacteriostatic Properties and Immunological Proteins of Donor Human Milk. PLoS ONE 2013, 8, e85867. [CrossRef] [PubMed]

29. Anderson, B.F.; Baker, H.M.; Dodson, E.J.; Norris, G.E.; Rumball, S.V.; Waters, J.M.; Baker, E.N. Structure of human lactoferrin at 3.2-A resolution. Proc. Natl. Acad. Sci. USA 1987, 84, 1769-1773. [CrossRef] [PubMed]

30. Baker, H.M.; Baker, E.N. A structural perspective on lactoferrin function. Biochem. Cell Biol. 2012, 90, 320-328. [CrossRef] [PubMed]

31. Mazurier, J.; Spik, G. Comparative study of the iron-binding properties of human transferrins. I. Complete and sequential iron saturation and desaturation of the lactotransferrin. Biochim. Biophys. Acta 1980, 629, 399-408. [CrossRef]

32. Tomita, M.; Bellamy, W.; Takase, M.; Yamauchi, K.; Wakabayashi, H.; Kawase, K. Potent antibacterial peptides generated by pepsin digestion of bovine lactoferrin. J. Dairy Sci. 1991, 74, 4137-4142. [CrossRef] 
33. Pierce, A.; Colavizza, D.; Benaissa, M.; Maes, P.; Tartar, A.; Montreuil, J.; Spik, G. Molecular Cloning and Sequence Analysis of Bovine Lactoferrin. Eur. J. Biochem. 2005, 196, 177-184. [CrossRef]

34. Castellino, F.J.; Fish, W.W.; Mann, K.G. Structural Studies on Bovine Lactoferrin. J. Biol. Chem. 1970, 245, 4269-4275. [PubMed]

35. Moore, S.A.; Anderson, B.F.; Groom, C.R.; Haridas, M.; Baker, E.N. Three-dimensional structure of diferric bovine lactoferrin at $2.8 \AA$ resolution11Edited by D. Rees. J. Mol. Biol. 1997, 274, 222-236. [CrossRef] [PubMed]

36. Magnuson, J.S.; Henry, J.F.; Yip, T.T.; Hutchens, T.W. Structural homology of human, bovine, and porcine milk lactoferrins: Evidence for shared antigenic determinants. Pediatr. Res. 1990, 28, 176-181. [CrossRef] [PubMed]

37. Bellamy, W.; Takase, M.; Yamauchi, K.; Wakabayashi, H.; Kawase, K.; Tomita, M. Identification of the bactericidal domain of lactoferrin. Biochim. Biophys. Acta Protein Struct. Mol. Enzymol. 1992, 1121, 130-136. [CrossRef]

38. Lonnerdal, B.; Jiang, R.; Du, X. Bovine lactoferrin can be taken up by the human intestinal lactoferrin receptor and exert bioactivities. J. Pediatr. Gastroenterol. Nutr. 2011, 53, 606-614. [CrossRef] [PubMed]

39. Bullen, J.J.; Rogers, H.J.; Leigh, L. Iron-binding proteins in milk and resistance to Escherichia coli infection in infants. Br. Med. J. 1972, 1, 69-75. [CrossRef] [PubMed]

40. Acosta-Smith, E.; Viveros-Jimenez, K.; Canizalez-Roman, A.; Reyes-Lopez, M.; Bolscher, J.G.M.; Nazmi, K.; Flores-Villasenor, H.; Alapizco-Castro, G.; de la Garza, M.; Martinez-Garcia, J.J.; et al. Bovine Lactoferrin and Lactoferrin-Derived Peptides Inhibit the Growth of Vibrio cholerae and Other Vibrio species. Front. Microbiol. 2017, 8, 2633. [CrossRef] [PubMed]

41. Allison, L.M.; Walker, L.A.; Sanders, B.J.; Yang, Z.; Eckert, G.; Gregory, R.L. Effect of Human Milk and its Components on Streptococcus Mutans Biofilm Formation. J. Clin. Pediatr. Dent. 2015, 39, 255-261. [CrossRef] [PubMed]

42. Berlutti, F.; Ajello, M.; Bosso, P.; Morea, C.; Petrucca, A.; Antonini, G.; Valenti, P. Both lactoferrin and iron influence aggregation and biofilm formation in Streptococcus mutans. Biometals 2004, 17, 271-278. [PubMed]

43. Singh, P.K.; Parsek, M.R.; Greenberg, E.P.; Welsh, M.J. A component of innate immunity prevents bacterial biofilm development. Nature 2002, 417, 552-555. [CrossRef] [PubMed]

44. Arnold, R.R.; Cole, M.F.; McGhee, J.R. A bactericidal effect for human lactoferrin. Science 1977, 197, $263-265$. [CrossRef] [PubMed]

45. Arnold, R.R.; Russell, J.E.; Champion, W.J.; Brewer, M.; Gauthier, J.J. Bactericidal activity of human lactoferrin: Differentiation from the stasis of iron deprivation. Infect. Immun. 1982, 35, 792-799. [PubMed]

46. Arnold, R.R.; Russell, J.E.; Champion, W.J.; Gauthier, J.J. Bactericidal activity of human lactoferrin: Influence of physical conditions and metabolic state of the target microorganism. Infect. Immun. 1981, 32, 655-660. [PubMed]

47. Ochoa, T.J.; Brown, E.L.; Guion, C.E.; Chen, J.Z.; McMahon, R.J.; Cleary, T.G. Effect of lactoferrin on enteroaggregative E. coli (EAEC). Biochem. Cell Biol. 2006, 84, 369-376. [CrossRef] [PubMed]

48. Qiu, J.; Hendrixson, D.R.; Baker, E.N.; Murphy, T.F.; St Geme, J.W., 3rd; Plaut, A.G. Human milk lactoferrin inactivates two putative colonization factors expressed by Haemophilus influenzae. Proc. Natl. Acad. Sci. USA 1998, 95, 12641-12646. [CrossRef] [PubMed]

49. Hendrixson, D.R.; Qiu, J.; Shewry, S.C.; Fink, D.L.; Petty, S.; Baker, E.N.; Plaut, A.G.; St Geme, J.W., 3rd. Human milk lactoferrin is a serine protease that cleaves Haemophilus surface proteins at arginine-rich sites. Mol. Microbiol. 2003, 47, 607-617. [CrossRef] [PubMed]

50. Sherman, M.P.; Petrak, K. Lactoferrin-enhanced anoikis: A defense against neonatal necrotizing enterocolitis. Med. Hypotheses 2005, 65, 478-482. [CrossRef] [PubMed]

51. Edde, L.; Hipolito, R.B.; Hwang, F.F.; Headon, D.R.; Shalwitz, R.A.; Sherman, M.P. Lactoferrin protects neonatal rats from gut-related systemic infection. Am. J. Physiol. Gastrointest. Liver Physiol. 2001, 281, G1140-G1150. [CrossRef] [PubMed]

52. Ellison, R.T., 3rd; Giehl, T.J. Killing of gram-negative bacteria by lactoferrin and lysozyme. J. Clin. Investig. 1991, 88, 1080-1091. [CrossRef] [PubMed]

53. Valenti, P.; Antonini, G. Lactoferrin: An important host defence against microbial and viral attack. Cell. Mol. life Sci. 2005, 62, 2576-2587. [CrossRef] [PubMed] 
54. Reznikov, E.A.; Comstock, S.S.; Hoeflinger, J.L.; Wang, M.; Miller, M.J.; Donovan, S.M. Dietary Bovine Lactoferrin Reduces Staphylococcus aureus in the Tissues and Modulates the Immune Response in Piglets Systemically Infected with S. aureus. Curr. Dev. Nutr. 2018, 2, nzy001. [CrossRef] [PubMed]

55. Wu, H.F.; Monroe, D.M.; Church, F.C. Characterization of the glycosaminoglycan-binding region of lactoferrin. Arch. Biochem. Biophys. 1995, 317, 85-92. [CrossRef] [PubMed]

56. Jenssen, H.; Hancock, R.E.W. Antimicrobial properties of lactoferrin. Biochimie 2009, 91, 19-29. [CrossRef] [PubMed]

57. Xu, Y.Y.; Samaranayake, Y.H.; Samaranayake, L.P.; Nikawa, H. In vitro susceptibility of Candida species to lactoferrin. Med. Mycol. 1999, 37, 35-41. [CrossRef] [PubMed]

58. Beddek, A.J.; Schryvers, A.B. The lactoferrin receptor complex in Gram negative bacteria. Biometals 2010, 23, 377-386. [CrossRef] [PubMed]

59. Shaper, M.; Hollingshead, S.K.; Benjamin, W.H., Jr.; Briles, D.E. PspA protects Streptococcus pneumoniae from killing by apolactoferrin, and antibody to PspA enhances killing of pneumococci by apolactoferrin [corrected]. Infect. Immun. 2004, 72, 5031-5040. [CrossRef] [PubMed]

60. Kim, C.M.; Park, R.Y.; Chun, H.J.; Kim, S.Y.; Rhee, J.H.; Shin, S.H. Vibrio vulnificus metalloprotease VvpE is essentially required for swarming. FEMS Microbiol. Lett. 2007, 269, 170-179. [CrossRef] [PubMed]

61. Legrand, D. Overview of Lactoferrin as a Natural Immune Modulator. J. Pediatr. 2016, 173, S10-S15. [CrossRef] [PubMed]

62. Legrand, D.; Mazurier, J. A critical review of the roles of host lactoferrin in immunity. BioMetals 2010, 23, 365-376. [CrossRef] [PubMed]

63. Losfeld, M.E.; Khoury, D.E.; Mariot, P.; Carpentier, M.; Krust, B.; Briand, J.P.; Mazurier, J.; Hovanessian, A.G.; Legrand, D. The cell surface expressed nucleolin is a glycoprotein that triggers calcium entry into mammalian cells. Exp. Cell Res. 2009, 315, 357-369. [CrossRef] [PubMed]

64. Lee, S.H.; Pyo, C.W.; Hahm, D.H.; Kim, J.; Choi, S.Y. Iron-saturated lactoferrin stimulates cell cycle progression through PI3K/Akt pathway. Mol. Cells 2009, 28, 37-42. [CrossRef] [PubMed]

65. Liu, M.; Fan, F.; Shi, P.; Tu, M.; Yu, C.; Yu, C.; Du, M. Lactoferrin promotes MC3T3-E1 osteoblast cells proliferation via MAPK signaling pathways. Int. J. Biol. Macromol. 2018, 107, 137-143. [CrossRef] [PubMed]

66. Oh, S.-M.; Hahm, D.H.; Kim, I.-H.; Choi, S.-Y. Human Neutrophil Lactoferrin trans-Activates the Matrix Metalloproteinase 1 Gene through Stress-activated MAPK Signaling Modules. J. Biol. Chem. 2001, 276, 42575-42579. [CrossRef] [PubMed]

67. Oh, S.M.; Pyo, C.W.; Kim, Y.; Choi, S.Y. Neutrophil lactoferrin upregulates the human p53 gene through induction of NF-kappaB activation cascade. Oncogene 2004, 23, 8282-8291. [CrossRef] [PubMed]

68. Jiang, R.; Lopez, V.; Kelleher, S.L.; Lonnerdal, B. Apo- and holo-lactoferrin are both internalized by lactoferrin receptor via clathrin-mediated endocytosis but differentially affect ERK-signaling and cell proliferation in Caco-2 cells. J. Cell. Physiol. 2011, 226, 3022-3031. [CrossRef] [PubMed]

69. He, J.; Furmanski, P. Sequence specificity and transcriptional activation in the binding of lactoferrin to DNA. Nature 1995, 373, 721-724. [CrossRef] [PubMed]

70. Akira, S.; Hemmi, H. Recognition of pathogen-associated molecular patterns by TLR family. Immunol. Lett. 2003, 85, 85-95. [CrossRef]

71. Elass-Rochard, E.; Legrand, D.; Salmon, V.; Roseanu, A.; Trif, M.; Tobias, P.S.; Mazurier, J.; Spik, G. Lactoferrin Inhibits the Endotoxin Interaction with CD14 by Competition with the Lipopolysaccharide-Binding Protein. Infect. Immun. 1998, 66, 486-491. [PubMed]

72. Lee, W.J.; Farmer, J.L.; Hilty, M.; Kim, Y.B. The Protective Effects of Lactoferrin Feeding against Endotoxin Lethal Shock in Germfree Piglets. Infect. Immun. 1998, 66, 1421-1426. [PubMed]

73. Appelmelk, B.J.; An, Y.Q.; Geerts, M.; Thijs, B.G.; de Boer, H.A.; MacLaren, D.M.; de Graaff, J.; Nuijens, J.H. Lactoferrin is a lipid A-binding protein. Infect. Immun. 1994, 62, 2628-2632. [PubMed]

74. Elass, E.; Masson, M.; Mazurier, J.; Legrand, D. Lactoferrin inhibits the lipopolysaccharide-induced expression and proteoglycan-binding ability of interleukin-8 in human endothelial cells. Infect. Immun. 2002, 70, 1860-1866. [CrossRef] [PubMed]

75. Andonegui, G.; Zhou, H.; Bullard, D.; Kelly, M.M.; Mullaly, S.C.; McDonald, B.; Long, E.M.; Robbins, S.M.; Kubes, P. Mice that exclusively express TLR4 on endothelial cells can efficiently clear a lethal systemic Gram-negative bacterial infection. J. Clin. Investig. 2009, 119, 1921-1930. [CrossRef] [PubMed] 
76. Shau, H.; Kim, A.; Golub, S.H. Modulation of natural killer and lymphokine-activated killer cell cytotoxicity by lactoferrin. J. Leukocyte Biol. 1992, 51, 343-349. [CrossRef] [PubMed]

77. Roseanu, A.; Chelu, F.; Trif, M.; Motas, C.; Brock, J.H. Inhibition of binding of lactoferrin to the human promonocyte cell line THP-1 by heparin: The role of cell surface sulphated molecules. Biochim. Biophys. Acta 2000, 1475, 35-38. [CrossRef]

78. Sorimachi, K.; Akimoto, K.; Hattori, Y.; Ieiri, T.; Niwa, A. Activation of macrophages by lactoferrin: Secretion of TNF-alpha, IL-8 and NO. Biochem. Mol. Biol. Int. 1997, 43, 79-87. [CrossRef] [PubMed]

79. Tanida, T.; Rao, F.; Hamada, T.; Ueta, E.; Osaki, T. Lactoferrin peptide increases the survival of Candida albicans-inoculated mice by upregulating neutrophil and macrophage functions, especially in combination with amphotericin B and granulocyte-macrophage colony-stimulating factor. Infect. Immun. 2001, 69, 3883-3890. [CrossRef] [PubMed]

80. Deriy, L.V.; Chor, J.; Thomas, L.L. Surface Expression of Lactoferrin by Resting Neutrophils. Biochem. Biophys. Res. Commun. 2000, 275, 241-246. [CrossRef] [PubMed]

81. Miyauchi, H.; Hashimoto, S.; Nakajima, M.; Shinoda, I.; Fukuwatari, Y.; Hayasawa, H. Bovine lactoferrin stimulates the phagocytic activity of human neutrophils: Identification of its active domain. Cell. Immunol. 1998, 187, 34-37. [CrossRef] [PubMed]

82. Kai, K.; Komine, K.I.; Komine, Y.; Kuroishi, T.; Kozutsumi, T.; Kobayashi, J.; Ohta, M.; Kitamura, H.; Kumagai, K. Lactoferrin stimulates A Staphylococcus aureus killing activity of bovine phagocytes in the mammary gland. Microbiol. Immunol. 2002, 46, 187-194. [CrossRef] [PubMed]

83. Actor, J.K.; Hwang, S.A.; Kruzel, M.L. Lactoferrin as a natural immune modulator. Curr. Pharm. Des. 2009, 15, 1956-1973. [CrossRef] [PubMed]

84. Trinchieri, G. Interleukin-12 and the regulation of innate resistance and adaptive immunity. Nat. Rev. Immunol. 2003, 3, 133-146. [CrossRef] [PubMed]

85. Spadaro, M.; Caorsi, C.; Ceruti, P.; Varadhachary, A.; Forni, G.; Pericle, F.; Giovarelli, M. Lactoferrin, a major defense protein of innate immunity, is a novel maturation factor for human dendritic cells. FASEB J. 2008, 22, 2747-2757. [CrossRef] [PubMed]

86. De la Rosa, G.; De, Y.; Tewary, P.; Varadhachary, A.; Oppenheim, J.J. Lactoferrin acts as an alarmin to promote the recruitment and activation of antigen-presenting cells and antigen-specific immune responses. J. Immunol. 2008, 180, 6868-6876. [CrossRef] [PubMed]

87. Kruzel, M.L.; Zimecki, M.; Actor, J.K. Lactoferrin in a Context of Inflammation-Induced Pathology. Front. Immunol. 2017, 8, 1438. [CrossRef] [PubMed]

88. Perdijk, O.; van Neerven, R.J.J.; van den Brink, E.; Savelkoul, H.F.J;; Brugman, S. Bovine Lactoferrin Modulates Dendritic Cell Differentiation and Function. Nutrients 2018, 10, 848. [CrossRef] [PubMed]

89. Puddu, P.; Carollo, M.G.; Belardelli, F.; Valenti, P.; Gessani, S. Role of endogenous interferon and LPS in the immunomodulatory effects of bovine lactoferrin in murine peritoneal macrophages. J. Leukocyte Biol. 2007, 82, 347-353. [CrossRef] [PubMed]

90. Bi, B.Y.; Liu, J.L.; Legrand, D.; Roche, A.C.; Capron, M.; Spik, G.; Mazurier, J. Internalization of human lactoferrin by the Jurkat human lymphoblastic T-cell line. Eur. J. Cell Biol. 1996, 69, 288-296. [PubMed]

91. Dhennin-Duthille, I.; Masson, M.; Damiens, E.; Fillebeen, C.; Spik, G.; Mazurier, J. Lactoferrin upregulates the expression of CD4 antigen through the stimulation of the mitogen-activated protein kinase in the human lymphoblastic T Jurkat cell line. J. Cell. Biochem. 2000, 79, 583-593. [CrossRef]

92. Zimecki, M.; Mazurier, J.; Machnicki, M.; Wieczorek, Z.; Montreuil, J.; Spik, G. Immunostimulatory activity of lactotransferrin and maturation of CD4- CD8- murine thymocytes. Immunol. Lett. 1991, 30, 119-123. [CrossRef]

93. Artym, J.; Zimecki, M.; Kruzel, M.L. Reconstitution of the cellular immune response by lactoferrin in cyclophosphamide-treated mice is correlated with renewal of T cell compartment. Immunobiology 2003, 207, 197-205. [CrossRef] [PubMed]

94. Kobayashi, S.; Sato, R.; Inanami, O.; Yamamori, T.; Yamato, O.; Maede, Y.; Sato, J.; Kuwabara, M.; Naito, Y. Reduction of concanavalin A-induced expression of interferon-gamma by bovine lactoferrin in feline peripheral blood mononuclear cells. Vet. Immunol. Immunopathol. 2005, 105, 75-84. [CrossRef] [PubMed]

95. Zimecki, M.; Mazurier, J.; Spik, G.; Kapp, J.A. Human lactoferrin induces phenotypic and functional changes in murine splenic B cells. Immunology 1995, 86, 122-127. [PubMed] 
96. Siqueiros-Cendón, T.; Arévalo-Gallegos, S.; Iglesias-Figueroa, B.F.; García-Montoya, I.A.; Salazar-Martínez, J.; Rascón-Cruz, Q. Immunomodulatory effects of lactoferrin. Acta Pharmacol. Sin. 2014, 35, 557-566. [CrossRef] [PubMed]

97. Debbabi, H.; Dubarry, M.; Rautureau, M.; Tome, D. Bovine lactoferrin induces both mucosal and systemic immune response in mice. J. Dairy Res. 1998, 65, 283-293. [CrossRef] [PubMed]

98. Sfeir, R.M.; Dubarry, M.; Boyaka, P.N.; Rautureau, M.; Tome, D. The mode of oral bovine lactoferrin administration influences mucosal and systemic immune responses in mice. J. Nutr. 2004, 134, 403-409. [CrossRef] [PubMed]

99. Artym, J.; Zimecki, M.; Kuryszko, J.; Kruzel, M.L. Lactoferrin accelerates reconstitution of the humoral and cellular immune response during chemotherapy-induced immunosuppression and bone marrow transplant in mice. Stem Cells Dev. 2005, 14, 548-555. [CrossRef] [PubMed]

100. Kleessen, B.; Bunke, H.; Tovar, K.; Noack, J.; Sawatzki, G. Influence of two infant formulas and human milk on the development of the faecal flora in newborn infants. Acta Paediatr. 1995, 84, 1347-1356. [CrossRef] [PubMed]

101. Petschow, B.W.; Talbott, R.D. Response of bifidobacterium species to growth promoters in human and cow milk. Pediatr. Res. 1991, 29, 208-213. [CrossRef] [PubMed]

102. Liepke, C.; Adermann, K.; Raida, M.; Magert, H.J.; Forssmann, W.G.; Zucht, H.D. Human milk provides peptides highly stimulating the growth of bifidobacteria. Eur. J. Biochem. 2002, 269, 712-718. [CrossRef] [PubMed]

103. Oda, H.; Wakabayashi, H.; Yamauchi, K.; Abe, F. Lactoferrin and bifidobacteria. Biometals 2014, 27, $915-922$. [CrossRef] [PubMed]

104. Mastromarino, P.; Capobianco, D.; Campagna, G.; Laforgia, N.; Drimaco, P.; Dileone, A.; Baldassarre, M.E. Correlation between lactoferrin and beneficial microbiota in breast milk and infant's feces. Biometals 2014, 27, 1077-1086. [CrossRef] [PubMed]

105. Claud, E.C.; Walker, W.A. Hypothesis: Inappropriate colonization of the premature intestine can cause neonatal necrotizing enterocolitis. FASEB J. 2001, 15, 1398-1403. [CrossRef] [PubMed]

106. Woodman, T.; Strunk, T.; Patole, S.; Hartmann, B.; Simmer, K.; Currie, A. Effects of lactoferrin on neonatal pathogens and Bifidobacterium breve in human breast milk. PLoS ONE 2018, 13, e0201819. [CrossRef] [PubMed]

107. Chen, P.W.; Ku, Y.W.; Chu, F.Y. Influence of bovine lactoferrin on the growth of selected probiotic bacteria under aerobic conditions. Biometals 2014, 27, 905-914. [CrossRef] [PubMed]

108. Buccigrossi, V.; de Marco, G.; Bruzzese, E.; Ombrato, L.; Bracale, I.; Polito, G.; Guarino, A. Lactoferrin induces concentration-dependent functional modulation of intestinal proliferation and differentiation. Pediatr. Res. 2007, 61, 410-414. [CrossRef] [PubMed]

109. Nichols, B.L.; McKee, K.S.; Henry, J.F.; Putman, M. Human lactoferrin stimulates thymidine incorporation into DNA of rat crypt cells. Pediatr. Res. 1987, 21, 563-567. [CrossRef] [PubMed]

110. Reznikov, E.A.; Comstock, S.S.; Yi, C.; Contractor, N.; Donovan, S.M. Dietary bovine lactoferrin increases intestinal cell proliferation in neonatal piglets. J. Nutr. 2014, 144, 1401-1408. [CrossRef] [PubMed]

111. Shulman, R.J.; Schanler, R.J.; Lau, C.; Heitkemper, M.; Ou, C.-N.; Smith, E.O.B. Early Feeding, Antenatal Glucocorticoids, and Human Milk Decrease Intestinal Permeability in Preterm Infants. Pediatr. Res. 1998, 44, 519. [CrossRef] [PubMed]

112. Stoll, B.J.; Hansen, N.I.; Adams-Chapman, I.; Fanaroff, A.A.; Hintz, S.R.; Vohr, B.; Higgins, R.D. Neurodevelopmental and growth impairment among extremely low-birth-weight infants with neonatal infection. JAMA 2004, 292, 2357-2365. [CrossRef] [PubMed]

113. Pammi, M.; Suresh, G. Enteral Lactoferrin Supplementation for Prevention of Sepsis and Necrotizing Enterocolitis in Preterm Infants. Cochrane Database Syst. Rev. 2017, 6, CD007137.

114. King, J.C.J.; Cummings, G.E.; Guo, N.; Trivedi, L.; Readmond, B.X.; Keane, V.; Feigelman, S.; De Waard, R. A Double-Blind, Placebo-Controlled, Pilot Study of Bovine Lactoferrin Supplementation in Bottle-fed Infants. J. Pediatr. Gastroenterol. Nutr. 2007, 44, 245-251. [CrossRef] [PubMed]

115. Manzoni, P.; Rinaldi, M.; Cattani, S.; Pugni, L.; Romeo, M.G.; Messner, H.; Stolfi, I.; Decembrino, L.; Laforgia, N.; Vagnarelli, F.; et al. Bovine lactoferrin supplementation for prevention of late-onset sepsis in very low-birth-weight neonates: A randomized trial. JAMA 2009, 302, 1421-1428. [CrossRef] [PubMed] 
116. Manzoni, P.; Meyer, M.; Stolfi, I.; Rinaldi, M.; Cattani, S.; Pugni, L.; Romeo, M.G.; Messner, H.; Decembrino, L.; Laforgia, N.; et al. Bovine lactoferrin supplementation for prevention of necrotizing enterocolitis in very-low-birth-weight neonates: A randomized clinical trial. Early Hum. Dev. 2014, 90 (Suppl. 1), S60-S65. [CrossRef]

117. Akin, I.M.; Atasay, B.; Dogu, F.; Okulu, E.; Arsan, S.; Karatas, H.D.; Ikinciogullari, A.; Turmen, T. Oral lactoferrin to prevent nosocomial sepsis and necrotizing enterocolitis of premature neonates and effect on T-regulatory cells. Am. J. Perinatol. 2014, 31, 1111-1120. [PubMed]

118. Ochoa, T.J.; Zegarra, J.; Cam, L.; Llanos, R.; Pezo, A.; Cruz, K.; Zea-Vera, A.; Carcamo, C.; Campos, M.; Bellomo, S. Randomized controlled trial of lactoferrin for prevention of sepsis in peruvian neonates less than 2500 g. Pediat. Infect. Dis. J. 2015, 34, 571-576. [CrossRef] [PubMed]

119. Barrington, K.J.; Assaad, M.A.; Janvier, A. The Lacuna Trial: A double-blind randomized controlled pilot trial of lactoferrin supplementation in the very preterm infant. J. Perinatol. 2016, 36, 666-669. [CrossRef] [PubMed]

120. Summary Protocol for a Multi-Centre Randomised Controlled Trial of Enteral Lactoferrin Supplementation in Newborn Very Preterm Infants (ELFIN). Neonatology 2018, 114, 142-148. [CrossRef] [PubMed]

121. Ward, P.P.; Lo, J.Y.; Duke, M.; May, G.S.; Headon, D.R.; Conneely, O.M. Production of biologically active recombinant human lactoferrin in Aspergillus oryzae. Biotechnology (N. Y.) 1992, 10, 784-789. [CrossRef]

122. Ward, P.P.; Cunningham, G.A.; Conneely, O.M. Commercial production of lactoferrin, a multifunctional iron-binding glycoprotein. Biotechnol. Genet. Eng. Rev. 1997, 14, 303-319. [CrossRef] [PubMed]

123. Sun, X.L.; Baker, H.M.; Shewry, S.C.; Jameson, G.B.; Baker, E.N. Structure of recombinant human lactoferrin expressed in Aspergillus awamori. Acta Crystallogr. Sect. D Biol. Crystallogr. 1999, 55, 403-407. [CrossRef]

124. Lyons, T.E.; Miller, M.S.; Serena, T.; Sheehan, P.; Lavery, L.; Kirsner, R.S.; Armstrong, D.G.; Reese, A.; Yankee, E.W.; Veves, A. Talactoferrin alfa, a recombinant human lactoferrin promotes healing of diabetic neuropathic ulcers: A phase 1/2 clinical study. Am. J. Surg. 2007, 193, 49-54. [CrossRef] [PubMed]

125. Sherman, M.P.; Adamkin, D.H.; Niklas, V.; Radmacher, P.; Sherman, J.; Wertheimer, F.; Petrak, K. Randomized Controlled Trial of Talactoferrin Oral Solution in Preterm Infants. J. Pediatr. 2016, 175, 68-73.e63. [CrossRef] [PubMed]

126. Vincent, J.L.; Marshall, J.C.; Dellinger, R.P.; Simonson, S.G.; Guntupalli, K.; Levy, M.M.; Singer, M.; Malik, R. Talactoferrin in Severe Sepsis: Results From the Phase II/III Oral tAlactoferrin in Severe sepsIS Trial. Crit. Care Med. 2015, 43, 1832-1838. [CrossRef] [PubMed]

(C) 2018 by the author. Licensee MDPI, Basel, Switzerland. This article is an open access article distributed under the terms and conditions of the Creative Commons Attribution (CC BY) license (http:/ / creativecommons.org/licenses/by/4.0/). 


\title{
The Antisecretory Factor in Plasma and Breast Milk in Breastfeeding Mothers-A Prospective Cohort Study in Sweden
}

\author{
Anna Gustafsson ${ }^{1,2, *}$, Elisabeth Granström ${ }^{3}$, Christina Stecksén-Blicks ${ }^{3}$, Christina E. West ${ }^{4}$ and \\ Sven-Arne Silfverdal ${ }^{4}$ \\ 1 Department of Clinical Science, Intervention and Technology, Karolinska Institutet, \\ SE 14186 Stockholm, Sweden \\ 2 Department of Neonatology, Karolinska University Hospital, SE 17176 Stockholm, Sweden \\ 3 Department of Odontology, Pediatric dentistry, Umeå university, SE 90187 Umeå, Sweden; \\ elisabeth.granstrom@umu.se (E.G.); christina.stecksen-blicks@umu.se (C.S.-B.) \\ 4 Department of Clinical Sciences, Pediatrics, Umeå University, SE 90187 Umeå, Sweden; \\ christina.west@umu.se (C.E.W.); sven.arne.silfverdal@umu.se (S.-A.S.) \\ * Correspondence: anna.gustafsson.1@ki.se; Tel.: +46-70856641
}

Received: 31 July 2018; Accepted: 3 September 2018; Published: 4 September 2018

check for updates

\begin{abstract}
Inflammation and infection postpartum threaten the mother and her infant. Human milk provides a defense for the infant, but inflammatory complications like mastitis may lead to the cessation of breastfeeding. Antisecretory factor (AF) has a role in the regulation of secretory processes and inflammation. The objective of the study was to describe AF-levels in plasma and breast milk, and in relation to breast complications. Breastfeeding mothers $(n=95)$ were consecutively recruited at a Well Baby Clinic in Umeå, Sweden. At inclusion four weeks postpartum, samples of venous blood $(10 \mathrm{~mL})$ and breast milk $(10 \mathrm{~mL})$ were collected. Active AF was analyzed with ELISA using a monoclonal antibody mAb43, and was detected in all samples of plasma and breast milk with a positive correlation (Spearman coefficient $=0.40, p<0.001$; Pearson correlation $=0.34$, $p<0.01$ ). High AF-levels in plasma correlated with high AF-levels in breast milk. The results suggest a co-regulation between active AF in plasma and breastmilk, and/or a local regulation of AF in the breast. Further studies are needed to determine the pathways for the activation of AF-levels in breast milk and plasma.
\end{abstract}

Keywords: antisecretory factor; human milk; breast milk; breastfeeding; inflammation; lactoferrin; candida

\section{Introduction}

Infection and inflammation constitute a threat to the newborn infant and its mother. The gut of the human newborn infant is susceptible to inflammation and inflammatory conditions that may affect the growth of the infant and cause complications, especially in infants born preterm [1,2]. Human milk provides a broad anti-inflammatory defense [3], but sometimes lactation is threatened by inflammatory complications like mastitis that can lead to the cessation of breastfeeding [4]. Mastitis in lactating women is defined by the World Health Organization (WHO) as an inflammatory condition of the breast, which may or may not be accompanied by infection [5]. It varies in severity, ranging from mild symptoms with local inflammation in the affected breast to more serious symptoms including fever, abscess, and septicemia, requiring interventions [6] that may lead to the cessation of breastfeeding [4]. Recent studies have suggested that inflammation in subclinical mastitis may cause a low milk supply and hence increase the risk of impaired growth of the infant, as well as the cessation of breastfeeding [7]. 
Human milk contains a variety of biologically active components and is involved in the development of the infant immune system and intestinal microbiota [8,9]. The bioactivity of breast milk has been shown to influence gene expression in the neonatal gastrointestinal tract, with a different expression in formula-fed infants when compared with breast-fed infants [10]. Secretory IgA antibodies bind the microbes on the infant's mucosal membranes, preventing activation of the pro-inflammatory defense. Lactoferrin, a major milk protein, is an important protein for protection against inflammation and disease and reduces inflammatory responses. Additionally, the non-absorbed human milk oligosaccharides block the attachment of microbes to the infant's mucosa, preventing infectious diseases [11]. Transfer of numerous cytokines and growth factors via milk may also activate the infant's immune system. Recent studies have detected a human milk microbiome [12], which is suggested to be influenced by factors like mode of delivery, duration of breastfeeding, and place of habitation.

Antisecretory factor (AF) is a protein which regulates secretory processes and inflammation and that might be of importance not only in animals, but also in infants in the postnatal period [13]. AF is present in most human tissues and body fluids, as well as in the placenta and in breast milk [14,15], with a suggested role in the immune system due to expression on macrophages, B-cells, and dendritic cells, and in all secondary lymphoid organs [16]. A high expression of AF is restricted to specific cell populations, such as certain types of epithelia, neuron, endocrine cells, and subgroups of leukocytes [13]. Cells that store AF also have the capacity to synthesize AF. It probably exerts its effects via nerves, but other mechanisms via receptors, binding proteins, and transport channels in the cellular membrane might be involved. AF can be detected in plasma, and is mostly present in an inactive form in healthy persons [13]. Active endogenous AF in plasma increases by exposure to enterotoxins and certain food constituents. An enhanced activation of endogenous AF synthesis improves the clinical outcome in diseases characterized by inflammation and secretory dysfunction in both humans and animals $[13,14,17]$. In animals, the levels of active AF in breast milk are positively correlated to the levels in plasma, with a higher concentration in milk than in plasma, probably due to the active transport of active AF across the epithelial lining of the mammary gland [13]. Studies of animals have demonstrated that levels of active AF in plasma and milk can be enhanced through an AF inducing diet, with a protective effect in the offspring related to growth and health [18].

In humans, an intervention study in breastfeeding mothers showed that the induction of endogenous AF in breast milk with specially processed cereals (SPC-flakes ${ }^{\circledR}$ ) prevented mastitis [15]. An increased AF plasma level has so far not been reported to induce any form of medical side effects [19].

There is, to our knowledge, no previous descriptive study on the basic levels of active AF in plasma and breast milk in breastfeeding women. An increased knowledge of the basic levels of AF in plasma and breastmilk, and their relation, may be a base for intervention studies, aiming to prevent inflammatory complications in both the mother and infant and promote breastfeeding, as well as infant growth and health. The objective of the study was to describe the basic levels of AF in plasma and breast milk in a cohort of breastfeeding mothers in Sweden.

\section{Materials and Methods}

\subsection{Study Population and Ethical Approvals}

This study was prospective, explorative, and descriptive, and a sub-study of an investigation of oral candida infection in infants [20] with 100 mother-infant pairs included. The levels of the outcome parameters, candida colonization in newborns, and antisecretory factor in the mothers were not known. We estimated that in this exploratory study, 100 children needed to be included to obtain an acceptable accuracy. A total of 120 mothers and their four-week old infants were invited to participate in a consecutive order at a single Well Baby Clinic in Umeå, Northern Sweden, between April 2011 and September 2012, of whom 20 did not consent to participate. Samples from plasma and breast milk 
were collected from 95 of the mothers. The study was approved by the Regional Ethical Review Board in Umeå, Sweden (Dnr 2010-218-31M and 2014-341-32M). Before entry, both parents signed written consent.

\subsection{Questionnaire}

At inclusion, mothers were asked to fill in a questionnaire about dietary habits on the consumption of an AF-inducing diet consisting of SPC-flakes ${ }^{\circledR}$ and other cereals on the market (i.e., breakfast cereals, grain, porridge) that also might induce/activate AF. Questions were also asked about living conditions, delivery mode, infant weight and length at birth, infant feeding, smoking in the family, and use of antibiotics in the mother and infant. Data on the mother's BMI $\left(\mathrm{kg} / \mathrm{m}^{2}\right)$ were calculated using data on weight and length collected from charts at the last visit during pregnancy at the maternity clinic, and data were available for 86 mothers. At 12 months postpartum, the mother was asked about a history of maternal infections and inflammation, including mastitis and sore nipples.

\subsection{Samplings and Analysis of Antisecretory Factor (AF), Lactoferrin and Calcium}

From the mothers, samples of venous blood $(10 \mathrm{~mL})$ and breast milk $(10 \mathrm{~mL})$ were collected at entry (when the infant was four-weeks old). AF appears in both active and inactive forms [21], which was why it was purified by affinity chromatography for optimal binding sensitivity in the subsequent enzyme-linked immunosorbent assay (ELISA) [22]. In brief, $1 \mathrm{~mL}$ of breast milk or plasma was purified by running it through a column of Sepharose 6B CL (GE Healthcare BioSciences AB, Uppsala, Sweden), during which AF binds to the matrix by affinity binding. After washing with physiological phosphate buffered saline (PBS), AF was eluted with $1 \mathrm{~mol} \cdot \mathrm{L}^{-1} 1$ methyl-alfa-D-glucopyronoside in PBS. The eluate was kept at $-20^{\circ} \mathrm{C}$ until ELISA was performed. Ass. Prof Ewa Johansson and Prof Stefan Lange (Clinical microbiology, Sahlgrenska University Hospital, Gothenburg, Sweden) provided a monoclonal antibody of the IgM isotype (mAb43) to detect the active form of the protein [22]. All samples were run in duplicate and the mean was calculated. The concentrations of AF were expressed as equivalents against the reference peptide AF1-105, a peptide at the N-terminal part of AF including the active part of the full length protein.

Lactoferrin in breastmilk was analyzed using a Human Lactoferrin Elisa kit, HK329 (Hyocult, Biotec Inc., Uden, The Netherlands). The milk samples were diluted to $1.2 \times 10^{5}$, run in duplicate, and the mean of each sample was calculated.

The analyses of AF (purification and ELISA) and lactoferrin were performed by EG, who is an experienced biomedical analyst at the Biochemical laboratory at the Department of Odontology, Faculty of Medicine at Umeå University.

Calcium in plasma was analyzed at the Department of Clinical Chemistry at Umeå University Hospital according to accredited methods.

\subsection{Samplings and Analysis of Oral Candida Colonization in Infants}

The inside of the cheeks and tongue of the infants were sampled for Candida using a cotton swab. The samples were then cultivated on a selective media Oricult-N semi-quantitative dipslide (Orion Diagnostica, Espoo, Finland) and scored; Score $0=$ no growth, Score $1=10^{3} \mathrm{CFU} \mathrm{mL}^{-1}$, Score $2=10^{4} \mathrm{CFU} \cdot \mathrm{mL}^{-1}$, Score $3=10^{5} \mathrm{CFU} \cdot \mathrm{mL}^{-1}$.

\subsection{Data Analyzes}

AF-levels in plasma and breast milk were analyzed descriptively in relation to each other and to the background factors in the mothers (i.e., age, parity BMI, mode of delivery, vaginal candida infection, and tobacco use) and infants (i.e., gestational age, birth weight, and sex). The distribution of levels of active AF in plasma and breast milk cannot be assumed to be normally distributed, since $\mathrm{AF}$ is predominantly present in the inactive form in healthy persons in a healthy environment [13] and the study cohort mainly consisted of healthy mothers. Therefore, we used non-parametric tests 
(Spearman's correlation, Wilcoxon signed rank test), as well as both non-parametric and parametric tests after log transformation (Pearson's correlation, Independent $t$-test, paired samples $t$-test). The log transformed values were used for the analytical analysis. A $p$-value of $<0.05$ was regarded as statistically significant. The Kolmogorov-Smirnov test was used to test for the normality distribution. Fisher's exact test was used in the analysis when the numbers of observations were small. The software SPSS (version 24) was used in the analyses.

\section{Results}

\subsection{Descriptive Results}

The characteristics of mothers and infants at entry of the study are shown in Table 1. Fifty-four women were multipara, eleven infants were born by caesarean section, and four were born before gestational week 37. None of the women reported the consumption of an AF-inducing diet consisting of SPC-flakes ${ }^{\circledR}$.

Table 1. Maternal and infant characteristics.

\begin{tabular}{cc}
\hline Factor & Total \\
\hline & $N=95$ \\
\hline Maternal characteristics & \\
Age (years) Mean (SD) (range) & $32(4.5) 21-44$ \\
Primiparous, $n$ & 44 \\
BMI* $\left(\mathrm{kg} / \mathrm{m}^{2}\right)$ Median (range) & $23(19.6-30.4)$ \\
Cesarean Section, $n$ & 9 \\
Vaginal Candida Infection, $n$ & 16 \\
Treatment for Candida Infection, $n$ & 15 \\
Tobacco Use & \\
Maternal Smoking, $n$ & 2 \\
Paternal Smoking, $n$ & 1 \\
Maternal Use of Snuff, $n$ & 2 \\
Infant characteristics & 3 \\
Gestational Age $<37$ Weeks & \\
Birth Weight, Gram Mean (SD) & $3552(481.5)$ \\
Female $n$ & 50 \\
Oral Candida, $n$ & 11 \\
Breast complications & \\
Pain in the Breast, $n$ & 19 \\
Sore Nipples, $n$ & 25 \\
Mastitis, $n$ & 17 \\
Infection, $n$ & 3 \\
\hline
\end{tabular}

* BMI data were collected from 84 of the mothers.

Active AF was present in all samples of plasma and breast milk. The AF-levels were not normally distributed, neither in the plasma (Figure 1a) nor in breast milk (Figure 1b). Furthermore, there was a wider distribution of the level of active AF in the plasma than in the breast milk (Table 2).

Figure 1a,b show the distribution of AF-levels (arbitrary units) in plasma and breastmilk.

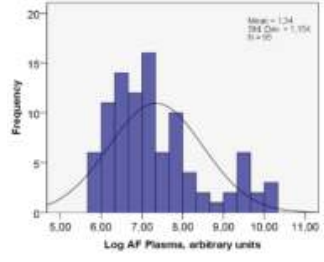

(a)

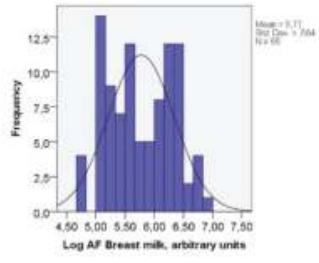

(b)

Figure 1. (a) Distribution of log AF-levels (arbitrary units) in the plasma Kolmogorov-Smirnov test of normality, $p<0.05$. (b) Distribution of log AF-levels (arbitrary units) in breastmilk. Kolmogorov-Smirnov test of normality, $p<0.05$. 
Table 2. Distribution of AF (arbitrary units) in plasma and breast milk, before and after log-transformation.

\begin{tabular}{cc}
\hline AF Plasma Median (range) & $1240(27,835)$ \\
log AF Plasma, Mean (SD) & $7.34(1.15)$ \\
AF Breast Milk, Median (range) & $293(904)$ \\
log AF Breast Milk, Mean (SD) & $5.77(0.56)$ \\
\hline
\end{tabular}

\subsection{Analytical Results}

There was a positive correlation between the levels of active AF in plasma and breast milk (Spearman's rho $=0.403, p<0.001$ ) (Figure 2).

Figure 2 shows the correlation between AF-levels in the plasma and breast milk.

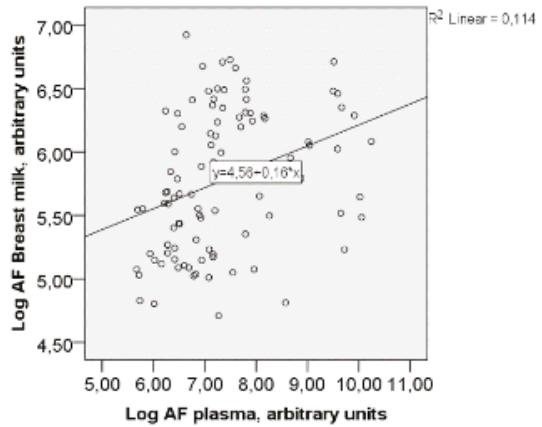

Figure 2. Correlation between the AF-levels (arbitrary units) in plasma and breast milk, Spearman's rho $=0.403, p<0.001$.

We found that there was a positive correlation between AF in plasma and the BMI of the mother (Spearman's rho 0.252, $p<0.05$, Figure 3a), while the correlation to AF in breast milk was not statistically significant (Figure 3b). There were no differences in AF-levels depending on parity, gestational age, or mode of delivery.

None of the mothers reported the intake of specially processed cereals (SPC-Flakes ${ }^{\circledR}$ ), and we found no relation between $\mathrm{AF}$ and the intake of other cereals on the market (i.e., breakfast cereals, grain, porridge). Seventeen mothers reported a history of vaginal fungal infection during pregnancy and 14 of 17 received anti-fungal treatment. No correlation was found between the level of active AF in plasma or breast milk with neither vaginal fungal infection during pregnancy nor treatment. Furthermore, there was no correlation between infant Candida colonization and AF-levels in plasma or breastmilk $(p>0.05)$.

Twenty-five women reported having had sore nipples. There was a positive correlation between having had sore nipples and pain in the breast (Spearman's rho 0.387, $p<0.01$ ), inflammatory mastitis (Spearman's rho 0.443, $p<0.01$ ), and breast infection (Spearman's rho 0.302, $p<0.01$ ), but no correlation was demonstrated between having had sore nipples or pain in the breast and AF-level in plasma or breast milk.

Figure 3a,b show the AF-levels in plasma and breastmilk in correlation to maternal BMI. 


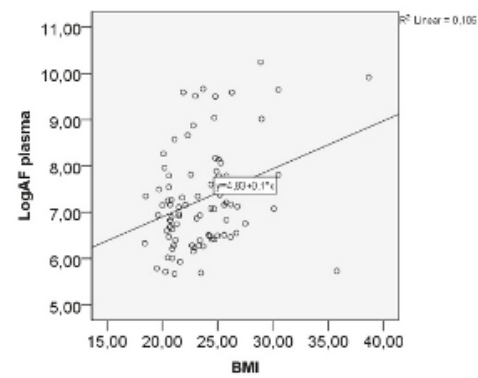

(a)

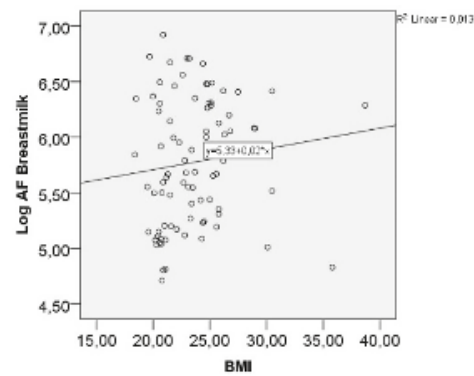

(b)

Figure 3. (a) AF-levels in plasma correlating to BMI, Spearman's rho 0.252, $p<0.05$; (b) AF-levels in breast milk in correlation to BMI, Spearman's rho 0.193, $p=0.08$.

Three women reported having had a history of breast infection, and there was a correlation between breast-infection and low levels of active AF in plasma (Pearson's correlation coefficient 0.220 , $p<0.05$ Pearson correlation), but not in breast milk (Pearson correlation coefficient $0.065, p>0.05$ ). In women who experienced a breast infection, the AF-levels in plasma were lower when compared to those without a history of infection $(p<0.05$, independent samples $t$-test) (Figure $4 a)$, while there was no difference in breast milk $(p>0.05)$ (Figure $4 b)$. All three women with breast infections had AF-levels in plasma below the 25th percentile ( $p<0.05$, independent samples $t$-test).

Figure 4a,b show the AF-levels and reported breast infection.

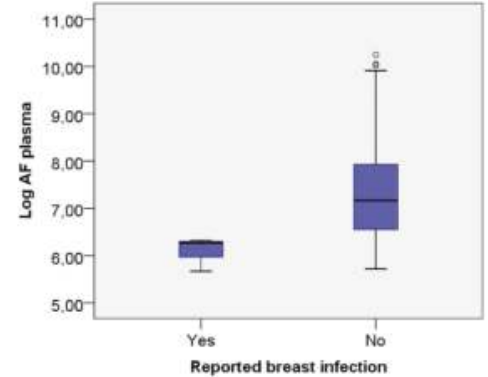

(a)

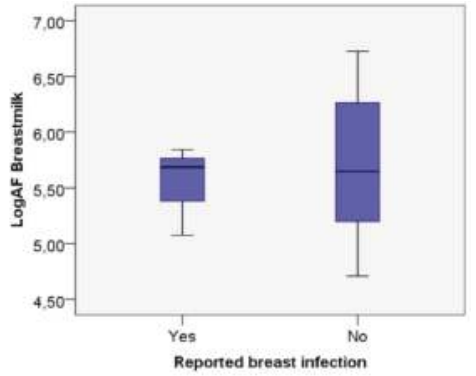

(b)

Figure 4. (a) Lower AF-levels in plasma related to having reported a breast infection $(n=3)$ compared with not reporting a breast infection $(p<0.05)$, unfilled circles indicate values $1.5 \times I Q R$ or more above the third quartile; (b) AF-levels in breast milk related to having reported breast infection $(n=3)$ compared with not reporting a breast infection $(p>0.05)$.

There was a positive correlation between the calcium levels in plasma and having had a breast infection (Spearman's rho-0.255, $p=0.05$ ). Figure 5 displays the elevated calcium levels in mothers with a history of breast infection.

Furthermore, we found no association between reported breast complications and oral Candida colonization of the infant (Chi-Square test and Fischer exact test, $p>0.05$ ) (Table 3).

Figure 5 shows the reported breast infection and calcium-levels. 


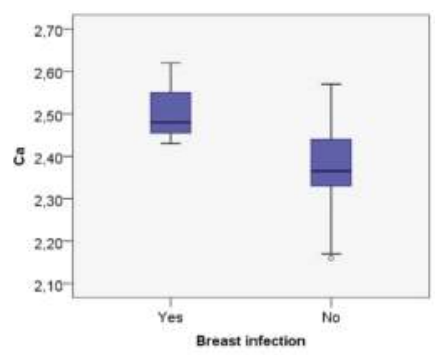

Figure 5. Calcium-levels related to reported breast infection, $t$-test, independent samples $p<0.01$, unfilled circle indicates a value $1.5 \times I Q R$ or more below the third quartile.

Table 3. Maternal breast complication and infant oral Candida colonization.

\begin{tabular}{|c|c|c|c|c|}
\hline \multirow{2}{*}{\multicolumn{2}{|c|}{ Maternal Breast Complication }} & \multicolumn{3}{|c|}{ Infant Oral Candida Colonization } \\
\hline & & $\frac{\text { No Candida }}{N}$ & Candida & $p$-Value \\
\hline \multirow{2}{*}{ Mastitis } & yes & 17 & 0 & \multirow{2}{*}{$>0.05$} \\
\hline & no & 56 & 9 & \\
\hline \multirow{2}{*}{ Breast Infection } & yes & 3 & 0 & \multirow[b]{2}{*}{$>0.05$} \\
\hline & no & 69 & 9 & \\
\hline \multirow{2}{*}{ Sore Nipples } & yes & 23 & 2 & \multirow{2}{*}{$>0.05$} \\
\hline & no & 50 & 6 & \\
\hline \multirow{2}{*}{ Pain in Breast/Nipples } & yes & 17 & 2 & \multirow{2}{*}{$>0.05$} \\
\hline & no & 56 & 7 & \\
\hline
\end{tabular}

The mean lactoferrin level in breastmilk was $2.19 \mathrm{~g} / \mathrm{L}, \mathrm{SD} 0.68$. A positive correlation was demonstrated between the AF-levels in breast milk and lactoferrin levels (Spearman's rho 0.341, $p<0.01$, Figure 6a). There was also a positive correlation between the AF-levels in plasma and lactoferrin; however, it did not reach statistical significance (Spearman's rho 0.193, $p=0.06$, Figure 6b).

Figure 6 shows the correlations between lactoferrin and AF-levels in breast milk and plasma.

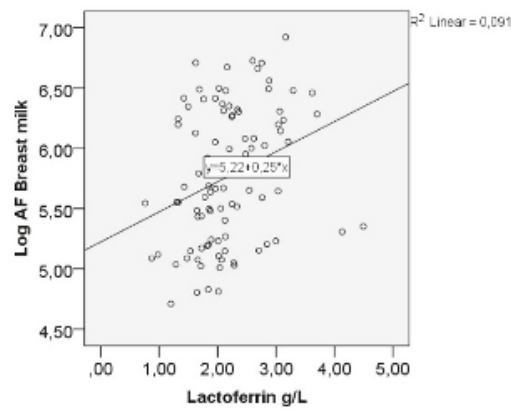

(a)

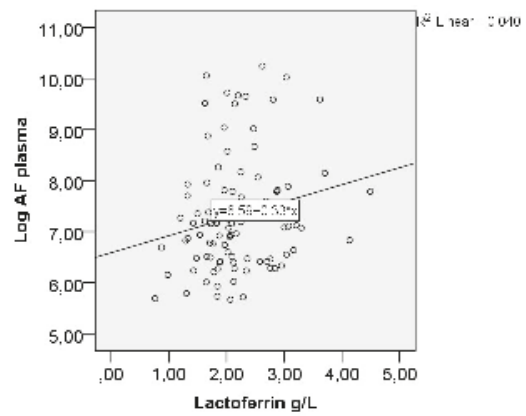

(b)

Figure 6. (a) Correlation between the AF-levels in breast milk and lactoferrin, Spearman's rho 0.341, $p<0.01$; (b) Correlation between the AF-levels in plasma and lactoferrin, Spearman's rho 0.193, $p=0.06$. 


\section{Discussion}

Active AF was present in all samples of plasma and breast milk from the mothers at one month post-partum. A positive relation was found between the levels of active AF in plasma and in breast milk, generally with lower levels in breast milk when compared to plasma.

In the majority of the mothers, the levels of active AF in plasma were low, possibly due to a generally healthy population with a high standard of living in an industrialized setting. We found a positive correlation between the BMI and level of AF in plasma. A higher BMI had previously been associated with an increased level of inflammation [23], and a higher AF-level might be a feed-back response to a higher degree of inflammation in general. However, we found no correlation between the AF-levels in breast milk and maternal BMI.

Furthermore, the levels of active AF in breast milk were also low in the majority of mothers. These results were consistent with the results from the intervention study by Svensson et al. [15]. The difference between the factors that correlated with the AF-levels in plasma and breast milk may suggest a local regulation of the AF-levels in the breast due to the health status of the breast and/or the infant. A relationship between the health status of both the mother and the infant and leucocyte count in breast milk has previously been described [24,25], where there were low baseline levels of leukocytes with increasing leukocyte levels, a proxy for the immune response, if the mother or infant had an infection. When the mother and infant are healthy, the origin of maternal cells in human milk is mainly from the mammary epithelium. However, during the first days postpartum and during periods of infection in either the mother or the infant, the human milk cells are dominated by immune cells from the maternal circulation $[24,26,27]$.

The level of active AF was higher in the breast milk from mothers of infants colonized with Candida when compared to mothers of non-colonized infants, but the difference did not reach statistical significance [20]. Active AF in breast milk and lactoferrin were significantly correlated, as demonstrated by a weakly positive correlation between the AF in plasma and lactoferrin, but did not reach statistical significance. Lactoferrin has a role in the innate immunity. A study has shown enhanced levels of lactoferrin, expressed by endothelial cells and activated neutrophils, in breast milk from mothers breastfeeding sick infants [27]. The increased levels of AF in the present study did not protect the infant from Candida colonization [20], but the higher AF-level in breast milk may be due to the colonialization itself and might protect the infant and the mother from active disease.

Three women reported having experienced breast infection, and also had low AF-levels in plasma. The correlation between breast infection and low AF-levels in plasma may indicate an increased risk of breast infection in mothers with low AF-levels. However, there were only three mothers with a breast infection in our material, which is why larger and more detailed studies are needed before firm conclusions can be drawn, although our finding was consistent with the result from the intervention study by Svensson et al. [15]. Furthermore, in this study, we found no correlation between the oral Candida colonization of the infants and breast complications in the mother. The role of infant oral Candida colonization for maternal breast complications during lactation has previously been described with conflicting results [28,29], and there are ongoing studies [30].

Hence, AF is present in plasma and breast milk. Our hypothesis is that AF is involved in the immune defense and regulation of inflammation in the breastfeeding mother and her infant. The correlation between AF-levels in plasma and breast milk was positive and statistically significant. The pathways of the regulation of AF-levels between plasma and breast milk are yet to be fully understood. Is the level of active AF regulated by both the level in maternal plasma and by a local regulation in the breast due to the presence of colonization and/or infection in the breast or infant? Further studies are needed to determine the relation of AF in plasma and AF in breast milk, and the pathways of influence between them.

This study adds to the knowledge about AF in lactating mothers and how it associates with background factors, which has previously been very sparsely studied. The study was performed from 2011 to 2012, and the AF-levels demonstrated in it may be different in a different cohort or time period. 
However, in this cohort, the results demonstrated the basic levels of generally healthy woman in an industrialized setting. No consumption of specially processed cereals (SPC-flakes ${ }^{\circledR}$ ) was reported and we found no relationship between the AF-levels and intake of other cereals on the market.

The ELISA method we used was a non-commercial, in house version, which has now been further developed as a sandwich ELISA. Basic levels of AF may differ between individuals, and longitudinal studies with repeated measurements may show individual variability. Furthermore, the variability of AF in lactating mothers is unknown. Blood samples were only collected once in the study, at four weeks postpartum. Unfortunately, information on breast complications was not collected at the time of blood sampling, only at 12 months postpartum, and changes in AF-levels may have happened several times during this period of time. However, samples of plasma and samples of breast milk were collected at the same time, and breast complications most commonly appeared in the first weeks to months postpartum. The exact time for reported breast complications and relation in time to the collection of breast milk and plasma samples is unknown, which makes it difficult to establish firm conclusions about the causality between mastitis or sore nipples and AF-levels. Only three mothers in the cohort reported having had a breast infection, which is a low prevalence. However, all three mothers who experienced a breast infection during the first year after pregnancy had low AF-levels in plasma at one month post-partum. Furthermore, we found a positive correlation between calcium in plasma and having experienced a breast infection, which was consistent with the results from the study by Li et al. [31], who showed higher levels of calcium related to subclinical mastitis. They also demonstrated a positive correlation between calcium and the pro-inflammatory cytokine IL-6. Calcium is known to be involved in the recruitment of neutrophils to sites of inflammation. Changes in intracellular calcium levels play an important role in neutrophil activation and function [32].

\section{Conclusions}

Active AF was present in plasma and breast milk in all mothers and their levels were positively correlated. Active AF was predominantly low in both plasma and breast milk, but there was a broader distribution of AF-levels in plasma when compared with the AF-levels in breast milk. The results suggest a possible local regulation of AF-levels in the breast, as well as a co-regulation with AF-levels in plasma. This study contributes to the knowledge regarding the basic patterns of active AF in plasma and breastmilk. The study was explorative and further longitudinal studies on active AF in breast milk and plasma are needed.

Author Contributions: C.S.-B., CW., and S.-A.S. planned and designed the study and performed the data collection. E.G. performed the laboratory analysis. AG performed the statistical analysis, prepared tables and figures, and wrote the first draft of the manuscript. C.S.-B., C.E.W., A.G., and S.-A.S. reviewed and edited the manuscript. S.-A.S. supervised the process.

Funding: Data collection and laboratory analysis were funded by a regional agreement between Umeå University and Västerbotten County Council in the field of Medicine Odontology and Health (TUA), while data analysis and draft preparation were funded by the Mjölkdroppen Foundation.

Acknowledgments: We express our gratitude to the parents and their children for their participation, and the nurses at Teg Child Health Centre/Well Baby Clinic, Umeå who recruited and sampled the children: Birgitta Isaksson, Ann-Sofie Gustafsson, Britt Stenmark, and Åsa Granberg. We also thank Prof. Lange and Ass. Prof Johansson of Gothenburg University for providing the monoclonal antibody against AF.

Conflicts of Interest: The authors declare no conflict of interest.

\section{References}

1. Goldenberg, R.L.; Culhane, J.F.; Iams, J.D.; Romero, R. Epidemiology and causes of preterm birth. Lancet 2008, 371, 75-84. [CrossRef]

2. Blencowe, H.; Cousens, S.; Chou, D.; Oestergaard, M.; Say, L.; Moller, A.B.; Kinney, M.; Lawn, J.; Born Too Soon Preterm Birth, A. Born too soon: The global epidemiology of 15 million preterm births. Reprod. Health 2013, 10, S2. [CrossRef] [PubMed] 
3. Ballard, O.; Morrow, A.L. Human milk composition: Nutrients and bioactive factors. Pediatr. Clin. N. Am. 2013, 60, 49-74. [CrossRef] [PubMed]

4. Berens, P.D. Breast pain: Engorgement, nipple pain, and mastitis. Clin. Obstet. Gynecol. 2015, 58, 902-914. [CrossRef] [PubMed]

5. Inch, S.; Fisher, C. Breastfeeding: Early problems. Pract. Midwife 2000, 3, 12-15. [PubMed]

6. Jahanfar, S.; Ng, C.J.; Teng, C.L. Antibiotics for mastitis in breastfeeding women. Cochrane Database Syst. Rev. 2009. [CrossRef]

7. Ingman, W.V.; Glynn, D.J.; Hutchinson, M.R. Inflammatory mediators in mastitis and lactation insufficiency. J. Mammary Gland Biol. Neoplasia 2014, 19, 161-167. [CrossRef] [PubMed]

8. Andreas, N.J.; Kampmann, B.; Mehring Le-Doare, K. Human breast milk: A review on its composition and bioactivity. Early Hum. Dev. 2015, 91, 629-635. [CrossRef] [PubMed]

9. Lonnerdal, B. Bioactive proteins in breast milk. J. Paediatr. Child Health 2013, 49 (Suppl. 1), 1-7. [CrossRef] [PubMed]

10. Donovan, S.M.; Wang, M.; Li, M.; Friedberg, I.; Schwartz, S.L.; Chapkin, R.S. Host-microbe interactions in the neonatal intestine: Role of human milk oligosaccharides. Adv. Nutr. 2012, 3, 450s-455s. [CrossRef] [PubMed]

11. Hanson, L.A. Session 1: Feeding and infant development breast-feeding and immune function. Proc. Nutr. Soc. 2007, 66, 384-396. [CrossRef] [PubMed]

12. Cabrera-Rubio, R.; Collado, M.C.; Laitinen, K.; Salminen, S.; Isolauri, E.; Mira, A. The human milk microbiome changes over lactation and is shaped by maternal weight and mode of delivery. Am. J. Clin. Nutr. 2012, 96, 544-551. [CrossRef] [PubMed]

13. Lange, S.; Lönnroth, I. The antisecretory factor: Synthesis, anatomical and cellular distribution, and biological action in experimental and clinical studies. Int. Rev. Cytol. 2001, 210, 39-75. [PubMed]

14. Zaman, S.; Aamir, K.; Lange, S.; Jennische, E.; Silfverdal, S.A.; Hanson, L.A. Antisecretory factor effectively and safely stops childhood diarrhoea: A placebo-controlled, randomised study. Acta Paediatr. 2014, 103, 659-664. [CrossRef] [PubMed]

15. Svensson, K.; Lange, S.; Lönnroth, I.; Widström, A.M.; Hanson, L. Induction of anti-secretory factor in human milk may prevent mastitis. Acta Paediatr. 2004, 93, 1228-1231. [CrossRef] [PubMed]

16. Davidson, T.S.; Hickey, W.F. Distribution and immunoregulatory properties of antisecretory factor. Lab. Investig. 2004, 84, 307-319. [CrossRef] [PubMed]

17. Ulgheri, C.; Paganini, B.; Rossi, F. Antisecretory factor as a potential health-promoting molecule in man and animals. Nutr. Res. Rev. 2010, 23, 300-313. [CrossRef] [PubMed]

18. Lange, S.; Martinsson, K.; Lonnroth, I.; Goransson, L. Plasma level of antisecretory factor (ASF) and its relation to post-weaning diarrhoea in piglets. J. Vet. Med. Ser. B 1993, 40, 113-118. [CrossRef]

19. Bjorck, S.; Bosaeus, I.; Ek, E.; Jennische, E.; Lonnroth, I.; Johansson, E.; Lange, S. Food induced stimulation of the antisecretory factor can improve symptoms in human inflammatory bowel disease: A study of a concept. Gut 2000, 46, 824-829. [CrossRef] [PubMed]

20. Stecksen-Blicks, C.; Granstrom, E.; Silfverdal, S.A.; West, C.E. Prevalence of oral candida in the first year of life. Mycoses 2015, 58, 550-556. [CrossRef] [PubMed]

21. Johansson, E.; Lange, S.; Lonnroth, I. Identification of an active site in the antisecretory factor protein. Biochim. Biophys. Acta 1997, 1362, 177-182. [CrossRef]

22. Johansson, E.; Lonnroth, I.; Jonson, I.; Lange, S.; Jennische, E. Development of monoclonal antibodies for detection of antisecretory factor activity in human plasma. J. Immunol. Methods 2009, 342, 64-70. [CrossRef] [PubMed]

23. Cox, A.J.; West, N.P.; Cripps, A.W. Obesity, inflammation, and the gut microbiota. Lancet Diabetes Endocrinol. 2015, 3, 207-215. [CrossRef]

24. Hassiotou, F.; Hartmann, P.E. At the dawn of a new discovery: The potential of breast milk stem cells. Adv. Nutr. 2014, 5, 770-778. [CrossRef] [PubMed]

25. Bode, L.; McGuire, M.; Rodriguez, J.M.; Geddes, D.T.; Hassiotou, F.; Hartmann, P.E.; McGuire, M.K. It's alive: Microbes and cells in human milk and their potential benefits to mother and infant. Adv. Nutr. 2014, 5, 571-573. [CrossRef] [PubMed]

26. Hassiotou, F.; Hepworth, A.R.; Metzger, P.; Tat Lai, C.; Trengove, N.; Hartmann, P.E.; Filgueira, L. Maternal and infant infections stimulate a rapid leukocyte response in breastmilk. Clin. Transl. Immunol. 2013, 2, e3. [CrossRef] [PubMed] 
27. Riskin, A.; Almog, M.; Peri, R.; Halasz, K.; Srugo, I.; Kessel, A. Changes in immunomodulatory constituents of human milk in response to active infection in the nursing infant. Pediatr. Res. 2012, 71, 220-225. [CrossRef] [PubMed]

28. Andrews, J.I.; Fleener, D.K.; Messer, S.A.; Hansen, W.F.; Pfaller, M.A.; Diekema, D.J. The yeast connection: Is candida linked to breastfeeding associated pain? Am. J. Obstet. Gynecol. 2007, 197, 424. [CrossRef] [PubMed]

29. Jimenez, E.; Arroyo, R.; Cardenas, N.; Marin, M.; Serrano, P.; Fernandez, L.; Rodriguez, J.M. Mammary candidiasis: A medical condition without scientific evidence? PLoS ONE 2017, 12, e0181071. [CrossRef] [PubMed]

30. Amir, L.H.; Cullinane, M.; Garland, S.M.; Tabrizi, S.N.; Donath, S.M.; Bennett, C.M.; Cooklin, A.R.; Fisher, J.R.; Payne, M.S. The role of micro-organisms (staphylococcus aureus and candida albicans) in the pathogenesis of breast pain and infection in lactating women: Study protocol. BMC Pregnancy Childbirth 2011, 11, 54. [CrossRef] [PubMed]

31. Li, C.; Solomons, N.W.; Scott, M.E.; Koski, K.G. Subclinical mastitis (SCM) and proinflammatory cytokines are associated with mineral and trace element concentrations in human breast milk. J. Trace Elem. Med. Biol. 2018, 46, 55-61. [CrossRef] [PubMed]

32. Immler, R.; Simon, S.I.; Sperandio, M. Calcium signaling and related ion channels in neutrophil recruitment and function. Eur. J. Clin. Investig. 2018, e12964. [CrossRef] [PubMed]

(C) 2018 by the authors. Licensee MDPI, Basel, Switzerland. This article is an open access article distributed under the terms and conditions of the Creative Commons Attribution (CC BY) license (http:/ / creativecommons.org/licenses/by/4.0/). 
Article

\title{
Expression of Granulisyn, Perforin and Granzymes in Human Milk over Lactation and in the Case of Maternal Infection
}

\author{
Alecia-Jane Twigger ${ }^{1, *, \dagger}$, Gwendoline K. Küffer ${ }^{2,+}{ }^{+}$Donna T. Geddes ${ }^{3}$ and Luis Filgueria ${ }^{2}$ \\ 1 Institute for Stem Cell Research, Helmholtz Center Munich, 85764 Munich, Germany \\ 2 Faculty of Science and Medicine, University of Fribourg, 1700 Fribourg, Switzerland; \\ gwendoline.kueffer@gmail.com (G.K.K.); luis.filgueira@unifr.ch (L.F.) \\ 3 School of Molecular Sciences, Faculty of Science, The University of Western Australia, Perth 6009, Australia; \\ donna.geddes@uwa.edu.au \\ * Correspondence: alecia-jane.twigger@helmholtz-muenchen.de; Tel.: +49-(0)89-3187-3756 \\ + These authors contributed equally to this work.
}

Received: 31 July 2018; Accepted: 31 August 2018; Published: 4 September 2018

check for updates

\begin{abstract}
Human milk has been previously found to contain various types of leukocytes however specific characteristics of these cells, such as whether they contain cytolytic antimicrobial proteins that may induce pathogen directed cell death, are unknown. This project aims to examine the presence and localization of immune proteins such as perforin, granulysin and granzymes in human milk cells at the protein and mRNA level. Genes encoding these proteins were confirmed in human milk cell samples, which were particularly enriched in early milk and in the case of maternal infection. Fluorescence activated cell sorting (FACS) was used to investigate the co-expression of these proteins with pan-immune cell marker CD45 and epithelial marker EPCAM. Co-expression of antimicrobial proteins was found predominantly in CD45 positive cells, also increasing in the case of maternal infection. Our study suggests that human milk contains cells that carry hallmarks of activated or memory T-cells which are enriched early in lactation and in the case of maternal infection. Presence and prevalence of these cells in human milk may indicate a role in the protection of the maternal breast or for delivery to the vulnerable infant.
\end{abstract}

Keywords: human milk; milk cells; immune cells; antimicrobial proteins

\section{Introduction}

Human milk contains all the necessary components required to satisfy the nutritional requirements of the infant, as well as immunological factors that support survival and thriving of the child. At birth, full term newborns are exposed to both vaginal bacteria and gut microbiota, some of which may be pathogenic [1]. Whilst infants have more or less a complete immune system, it is still highly immature [1-3] and requires contact with foreign antigens and stimulation such as from human milk to develop effective and specific defence mechanisms [2,3]. Human milk proteins, such as lactoferrin, certain caseins $[4,5]$ and lysozyme, along with other bioactive molecules such as oligosaccharides, defensins, cytokines, chemokines, growth factors and anti-oxidants [1,3] support the development of the immune system at the same time as providing antimicrobial and antiviral effects [2]. Of note, secretory IgA (sIgA) antibodies found in human milk and produced by maternal plasma cells is an important antibody directed against microbes and food antigens that have challenged the maternal immune response [2]. The presence of immune cells, such as lymphocytes, has been shown in human milk [1-3,6,7], which increase in number when the infant has an infection even in an asymptomatic mother [8]. Moreover, the immune cell content of milk increases to a much greater degree during 
maternal infection, particularly during mastitis. It is therefore hypothesized that leukocytes entering human milk might play a role for the infant but may also result from protection of the breast tissue [3].

Generally, if a pathogenic or non-host cell (such as bacteria) is detected, the immune system is activated, leading primed leukocytes such as cytotoxic t-cells and natural killer cells to release cytolytic granules. These granules contain the bioactive molecules perforin [9] and granulysin (synthetic drugs include novobiocin [10]), which work together to form pores in the cell to allow the entry of a third component, granzymes into pathogenic cells, and trigger bacterial cell programmed death and possibly also apoptosis of the host cell [11-14]. Granzymes are serine proteases and of the twelve granzymes already described, five have been found in the human (A, B, H, K and M) and ten have been identified in rodents (A-G, K, M and N) [13]. Whilst immune cells are known to exist in human milk with their numbers and distribution changing in relation to the health status of either the mother or the child $[3,7,8,15]$, the presence of associated cytotoxic immune proteins perforin, granzymes and granulysin have not yet been identified in human milk cells. Presence of these proteins in human milk leukocytes would indicate the existence of activated or memory t-cells which have recently or may be actively fighting pathogenic cells which may be of importance either for the protection of the maternal breast or infant. The aim of this study is to determine the presence of these antimicrobial proteins in human milk cells and determine whether they are normally expressed across different stages of lactation in healthy participants or in the case of breast inflammatory conditions such as mastitis.

\section{Materials and Methods}

\subsection{Human Milk Collection}

The study was approved by the Swissethics Committee (2016-00309, Switzerland), the Human Research Ethics Committee of The University of Western Australia (UWA, RA/4/1/4397) and the Australian Breastfeeding Association (ABA; 2014-5). All participants provided informed written consent to engage in the study and all methods were carried out according to the approved guidelines. Thirty-six mothers were recruited through the Hospital of Fribourg, ABA meetings and the website humanlactationresearchgroup.com. Mothers were on average 34.4 years of age (range from 27 to 45 years of age) (Table 1). All infants, with $53 \%$ being male, were born term with a median of 278 days of gestation (range from 249 to 301 days) with 60\% delivered vaginally (Table 1). Milk samples were collected 4-142 days post-partum and a single pre-partum secretion was collected 5 days before birth (Table 1). After collection, milk samples were brought directly to the laboratory to be processed.

Table 1. Demographics of study participants engaged in the study.

\begin{tabular}{llll}
\hline & \multicolumn{2}{c}{ Median (Range) } \\
\cline { 2 - 4 } & \multicolumn{1}{c}{ All Participants } & \multicolumn{1}{c}{ PCR Participants } & \multicolumn{1}{c}{ Flow Cytometry Participants } \\
\hline Maternal characteristics & $n=36$ & $n=24$ & $n=13$ \\
Age (years) & $34(27-45)$ & $33(27-45)$ & $35.5(34-38)$ \\
Body Mass Index (BMI) & $24(19.8-31.9)$ & $22.5(19.8-27.9)$ & $25.6(22-31.9)$ \\
Parity & $2(1-4)$ & $2(1-4)$ & $2(1-3)$ \\
\hline Infant characteristics & $n=36$ & $n=24$ & $n=13$ \\
Gestational age (days) & $278(249-301)$ & $278(249-301)$ & $275(252-281)$ \\
Infant age at collection (days) & $47(4-142)$ & $45(4-142)$ & $57(37-84)$ \\
\hline Milk characteristics & $n=85$ & $n=70$ & $n=15$ \\
Volume of milk (mL) & $50(0.61-490)$ & $50(0.61-490)$ & $62(35-195)$ \\
Total cell count (cells/mL) & $16.4(1.9-214.5)$ & $16.55(1.9-214.5)$ & $13.8(4.9-63.8)$ \\
Viability $(\%)$ & $97.4(53.2-100)$ & $98.1(53.2-100)$ & $93.6(84.7-96.5)$ \\
\hline
\end{tabular}

\subsection{Milk Cell Isolation}

Each milk sample was diluted in equal volume of Phosphate Buffer Saline (PBS) (Gibco, Thermo Fisher Scientific, Wilmington, DE, USA) and centrifuged at $800 \mathrm{~g}$ for $20 \mathrm{~min}$ at $20^{\circ} \mathrm{C}$. The fat and skim layer of the milk was removed before washing the cell pellet twice in sterile PBS and the cells were 
resuspended in 5-10 mL of PBS. Cells were used fresh for flow cytometry or frozen and stored at $-80^{\circ} \mathrm{C}$ for RNA extraction and corresponding analysis.

\subsection{RNA Extraction}

Total RNA was extracted from frozen cell pellets, previously collected as part of a larger study. Mini RNeasy extraction kit (Qiagen, Valencia, CA, USA) was used according to the manufacturer's protocol. The concentration and purity of RNA was measured using NanoDrop 2000 (Thermo Fisher Scientific, Wilmington, DE, USA). All extracted RNA was of a high quality with a 260/280 ratio between 1.8 and 2.2. Pooled resting mammary tissue RNA taken from five donors aged 40-55 was purchased from Aligent Technologies (Catalogue number: 540045, lot number: 0006135096, Aligent Technologies, Santa Clara, CA, USA).

\section{4. cDNA Generation}

RNA was reverse transcribed into cDNA using the cDNA archive kit (Life Technologies, Carlsbad, CA, USA) following the manufacturer's instructions. $50 \mu \mathrm{L}$ reactions were incubated in a Bio-Rad C1000 96-well gradient block thermal cycler and held at $25^{\circ} \mathrm{C}$ for $10 \mathrm{~min}$, followed by $37^{\circ} \mathrm{C}$ for $120 \mathrm{~min}, 85^{\circ} \mathrm{C}$ for $5 \mathrm{~min}$ and finally at $4{ }^{\circ} \mathrm{C}$ until collection.

\subsection{Quantitative Real Time Polymerase Chain Reaction ( $q R T-P C R$ )}

Gene expression was investigated through quantitative real time PCR using Taqman probes (Table S1, Life Technologies, Thermo fisher, CA, USA) with the 7500 Fast qRT-PCR system (Life Technologies). Each sample was measured in triplicate or where necessary, in duplicate. Cycle time (CT) values were obtained for each sample and subsequently, relative quantitation (RQ) was calculated using $2^{\mathrm{Ct}(\text { control)-Ct(sample) }} \pm \mathrm{SD}$, where genes were normalized to resting breast tissue and GAPDH was used as a housekeeping control gene.

\subsection{Sequencing Library Research}

Genes coding for cytolytic immune proteins perforin (PRF1), granulysin (GNLY) and granzymes A, B, H and M (GZMA, GZMB, GZMH, GZMM) were searched in an RNA-sequencing dataset [16], which explored the transcriptome of prepartum secretions (PS) and human milk (HM) cells as well as resting mammary tissue (RMT). Previously, $1.1 \times 10^{5}-19.3 \times 10^{5}$ cells $/ \mathrm{mL}$ were isolated from PS samples collected from four women at 38-40 weeks of pregnancy. All participants provided follow-up samples of $0.4 \times 10^{6}-43.5 \times 10^{6}$ cells $/ \mathrm{mL}$ HM at 1, 3, 6 and 12 months of lactation [16]. mRNA was extracted from the isolated cells, the quantity was then standardized $[17,18]$ and the samples were processed for library preparation. Moreover, RMT taken from five women aged 40-55 years (Catalogue number: 540045, Lot number: 0006135096, Agilent Technologies, Santa Clara, CA, USA) was pooled and mRNA was likewise processed for library preparation. Illumina HiSeq2500 version 3 was used to sequence all samples with a production of a minimum of 20 million 50 base paired single end reads. SOAP aligner 2 was used to align $865,913,217$ clean reads to the human genome where only 2 mismatches were allowed, resulting in 414,203,980 clean transcripts. Gene expression levels were expressed as RPKM (Reads Per Kilobase per Million mapped reads) [19] and annotated with the algorithm Basic Local Alignment Search Tools (BLAST) (2.2.23). Plots of the genes of interest expression patterns were made, as described below.

\subsection{Flow Cytometry}

Flow cytometry was performed in cells isolated from fresh milk samples by either staining immediately $(n=11)$ or fixed in $1 \%$ paraformaldehyde $2 / 3 \%$ sucrose in PBS for subsequent staining the following day $(n=4)$. When immediately stained, 2 million cells were separated into Eppendorf tubes. Conjugated extracellular antibodies were added to cells (Table S2) in $100 \mu \mathrm{L}$ of $2 \%$ foetal bovine 
serum (Fisher Biotec, Wembley, WA, Australia) PBS and incubated for 30 min at $4{ }^{\circ} \mathrm{C}$ shielded from light. When immediately fixed, the cells were stained the next day with antibodies against membrane proteins (Table S2), diluted in PBS for 30 min on ice and in the dark. All stained cells were then washed twice in PBS $(10,000 \mathrm{~g}$ for $30 \mathrm{~s})$ and fixed in $3 \%$ paraformaldehyde $2 / 3 \%$ sucrose in PBS for $20 \mathrm{~min}$. Subsequently, cells were washed again twice in PBS. While optimizing the technique, it was observed that the antibody against granzyme A worked better when the permeabilization was conducted with $0.05 \%$ Tween, therefore antibodies for intracellular staining were diluted in $0.3 \%$ saponin or $0.05 \%$ Tween in PBS, were added for $30 \mathrm{~min}$, at room temperature, in the dark. Cells were subsequently washed once with $0.1 \%$ saponin or $0.05 \%$ Tween in PBS (10000 $\mathrm{g}$ for $30 \mathrm{~s}$ ) and then in PBS before resuspending the cells in PBS for subsequent data acquisition. Cells were either analyzed with a BD Accuri C6 plus flow cytometer or in case of triple stainings, cells were measured with a BD FACS Canto. FCS files were then analyzed with the version 10.2 of the software FlowJo ${ }^{\mathrm{TM}}$. To avoid doublets, single cells were gated using forward scatter area (FSC-A) versus forward scatter height (FSC-H) (Figure S1). Single cells were further separated in three populations (Figure S1) to examine the presence of granzyme A, granzyme B, granulysin and perforin in immune and epithelial cells.

\subsection{Statistical Analysis}

Statistical analyses were carried out using R 3.3.2. for Mac OSX, with additional packages ggplot2 [20], lattice [21], nlme [22], FactoMineR [23] and factoextra for longitudinal plots, box and whisker plots, mixed linear effects models, principal component analysis (PCA) and plots respectively. Longitudinal spaghetti plots of PS cells, HM cells and RMT gene expression obtained from the sequencing dataset of PRF1, GNLY, GZMA, GZMB, GZMH and GZMM were plotted. Linear mixed effects modeling was used to investigate correlation between qRT-PCR analyzed gene expression products and lactation stage, with participant being a fixed effect. Box and whisker plots were created for gene expression products of all genes resulting from qRT-PCR analysis of HM cells taken from healthy participants. Correlations between expression of the different genes in HM cells taken from healthy participants assessed with qRT-PCR was examined using pairs plots and PCA analysis. Differences in gene expression products assessed with qRT-PCR between healthy and mastitis participants were investigated using PCA and dot plots.

\section{Results}

\subsection{Analysis of mRNA Encoding Antimicrobial Proteins in Human Milk Cells}

3.1.1. Higher Expression of Immune Cell Genes in the Mammary Gland and Milk Cells Taken during Pregnancy and Early Lactation

Analysis of a prior human mammary transcriptome dataset (GEO Series accession number GSE85494) for immune related genes perforin (PRF1), granulysin (GNLY), granzyme A, B, H and M (GZMA, GZMB, GZMH, GZMM) found the highest expression in pre-partum secretions compared with purchased resting mammary tissue (RMT) mRNA (see Section 2, RNA extraction) and human milk (HM) cells (Figure 1). Overall, the average Reads Per Kilobase per Million mapped reads (RPKM) expression of HM cells for these genes were highest in the prepartum secretions, except for PRF1 which had a similar expression in RMT. Interestingly, whilst post-partum the levels of gene expression generally decreased over the course of 12 months, it appeared there was a slight increase in the expression of GZMA, GZMB, GZMH and PRF1 from 6 to 12 months in 1-2 samples. 


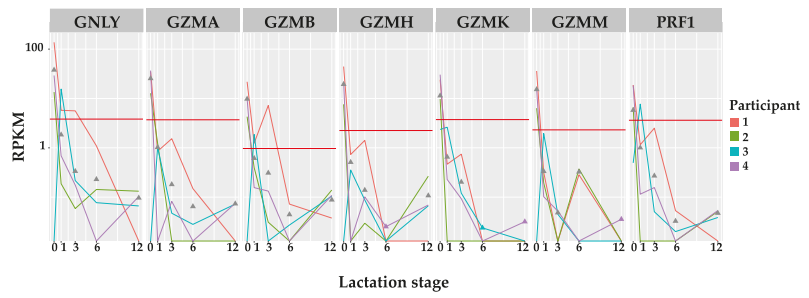

Figure 1. Expression of the genes encoding granzyme A (GZMA), granzyme B (GZMB), granzyme H (GZMH), granzyme M (GZMM), granulysin (GNLY) and perforin (PRF1) expressed in Reads Per Kilobase per Million mapped reads (RPKM) in longitudinal human milk cells (coloured lines) and pooled resting mammary tissue (RMT) (straight red line). Four lactating participants provided pre-partum secretion (PS) cells and four subsequent human milk cell samples at months 1, 3, 6 and 12 of lactation. Average expression at each time point is represented by grey triangles. In general, there is a decrease over lactation period with the highest expression in the pre-partum secretions. Moreover, PS cells show higher gene expression for all immune proteins in comparison with RMT.

\subsubsection{Expression Level of Immune Protein Encoding Genes Shows Little Association with Time Post-Partum}

Expression analysis of the immune cell gene products was expanded in a larger pool of participants ( $n=24)$ who provided multiple (1-3) HM cell samples within the first six months of lactation for qRT-PCR analysis (Figure 2). A total of $65 \mathrm{HM}$ cell samples from healthy participants were analysed for PRF1, GZMA, GZMB, GZMH, GZMM, GNLY, the gene encoding the immune cell marker CD45 (PTPRC) and epithelial cell adhesion marker (EPCAM). Many of the HM cell samples expressed all genes, where EPCAM was the most highly expressed gene and GZMM was the least expressed gene (Figure 2). The highest variation of gene expression between participants was found in GZMB (Figure 2, Table S3). Interestingly GZMB expression was found to be negatively associated with lactation stage ( $p$-value 0.047, Table S4, Figure S2). GZMA, GZMH, GZMM, GNLY and PTPRC showed no association between expression levels and time post-partum (Table S4, Figure S2) whereas PRF1 had a borderline significant decrease ( $p$-value 0.068) (Table S4). Analysis between expression of selected gene products with infant post-partum age revealed inter-individual differences, with participant as an influencing factor on the levels of gene expression.

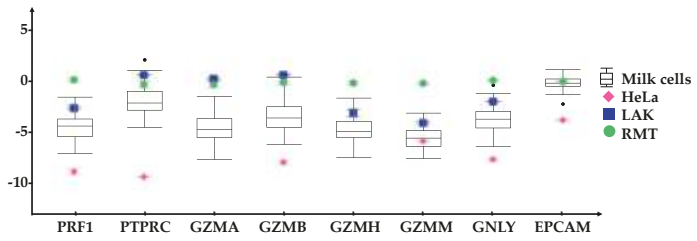

Figure 2. Box and whisker plots of the expression of the immune related genes PRF1, PTPRC, GZMA, GZMB, GZMH, GZMM, GNLY and the epithelial marker EPCAM in 65 HM cell samples (百). For each gene, the range of expression is shown by the whiskers of the plot and the interquartile ranges are displayed as the upper and lower sections of the boxes. The median expression in milk cells for each gene is represented by the horizontal line going through the box. In the case of milk cell outliers, these are represented by black circles. HeLa cells $(\checkmark)$ were used as a negative control whereas Lymphokine Activated Killer cells (LAK, $\mathbf{\square})$ were a positive control for immune markers. All genes were normalized to resting mammary tissue (RMT, $\bullet$ ). The measured standard error of the mean for these reference samples are represented by error bars. All immune related genes were expressed in the HM cells, although in some cases having a lower expression compared to RMT and LAK. 


\subsubsection{Negative Association between EPCAM and Immune Markers}

Principal component analysis (PCA) revealed a negative association between expression of EPCAM and the immune cell related gene products PTPRC, GZMA, GZMB, GZMH, GZMM, GNLY and PRF1 (Figure 3) with 51.3\% of total variation explained by the differences in these genes (Figure 3a). Linear analysis of the markers (Figure $3 b$ ) showed an association between mRNA levels of PRF1 with GZMA $\left(r^{2}=0.74\right)$, GZMH $\left(r^{2}=0.72\right)$, GZMM $\left(r^{2}=0.61\right)$, PTPRC $\left(r^{2}=0.54\right)$ or GNLY $\left(r^{2}=0.72\right)$. Furthermore, associations between expression of PTPRC and GZMA $\left(r^{2}=0.58\right)$ as well as GNLY with GZMH $\left(r^{2}=0.81\right)$ or GZMM $\left(r^{2}=0.78\right)$ were found. There also appears to be a correlation between expression of GZMH with GZMM $\left(r^{2}=0.71\right)$ or GZMB $\left(r^{2}=0.51\right)$ (Figure 3b). In contrast, there is a negative association between expression of EPCAM and PTPRC $\left(r^{2}=0.18\right)$, GNLY $\left(r^{2}=0.19\right)$ and GZMA $\left(r^{2}=0.22\right)$ (Figure 3b).
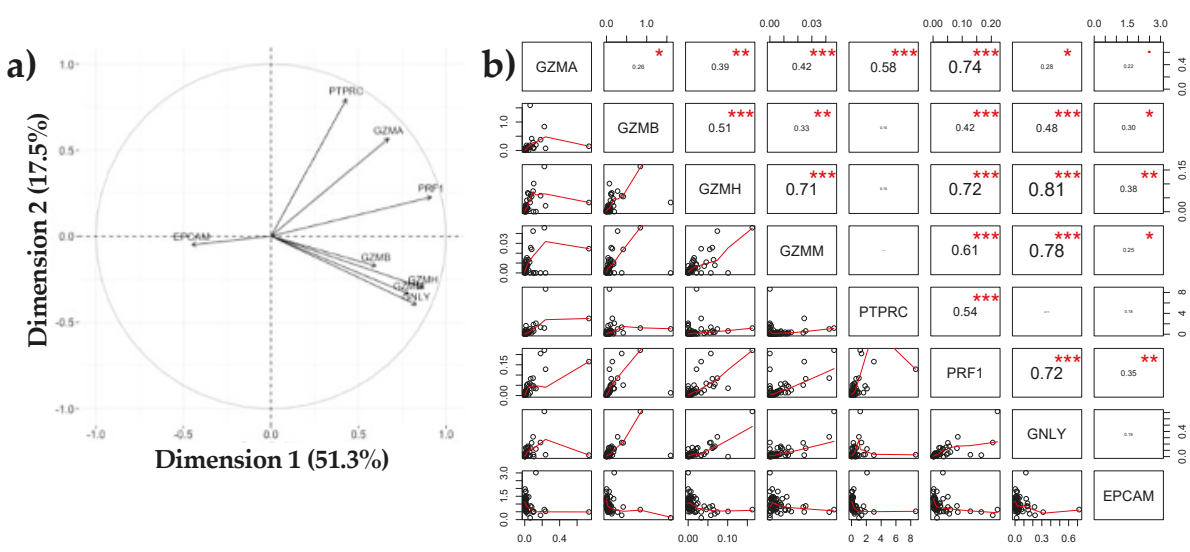

Figure 3. Associations between immune (GZMA, GZMB, GZMH, GZMM, GNLY, PRF1, PTPRC) and epithelial cell markers (EPCAM) analysed with (a) principal component analysis (PCA) and (b) Linear modelling. Half of the variation could be explained by difference between EPCAM and the immune markers. Moreover, $17.5 \%$ was due to the difference between expression of genes encoding granzyme B (GZMB), granzyme H (GZMH), granzyme M (GZMM) and granulysin (GNLY) compared to granzyme A (GZMA), perforin (PRF1) and immune cell marker PTPRC. Linear associations displayed a positive association of gene expression between PRF1 and GZMA, PRF1 and GZMB, PRF1 and PTPRC as well as between GZMA and GZMB. On the other hand a negative association exists between expression of EPCAM and GNLY. ${ }^{* * *}$ represents highly correlative genes $\left(r^{2}>0.5\right)$, ${ }^{* *}$ moderately correlative $\left(0.5>r^{2}>0.3\right)$ and ${ }^{*}$ low correlation between genes $\left(r^{2}<0.3\right)$.

3.1.4. Participants with Mastitis Show a Higher Expression of Immune Genes Compared to Healthy Participants

Three participants with mastitis (one with an abscess) provided HM cell samples from the affected breast. Moreover, two of the participants provided an additional sample from the adjacent healthy breast. Compared to the healthy breast, the two mastitis samples from the infected breast displayed a higher expression of immune cell related genes (Figure 4). There was much higher expression of PTPRC in the mastitis sample compared to the adjacent breast in both participants (Figure 4). In addition, the expression of GZMA, GZMB and PRF1 was increased in the mastitis sample of one participant when compared to the adjacent breast (Figure $4 \mathrm{a}(\mathrm{i})$ ). Principal component analysis (PCA) of gene expression of all healthy and mastitic HM cell samples revealed great variation between healthy participants and the mastitis or breast abscess samples (Figure $4 b$ ). 
a) $\mathrm{i}$

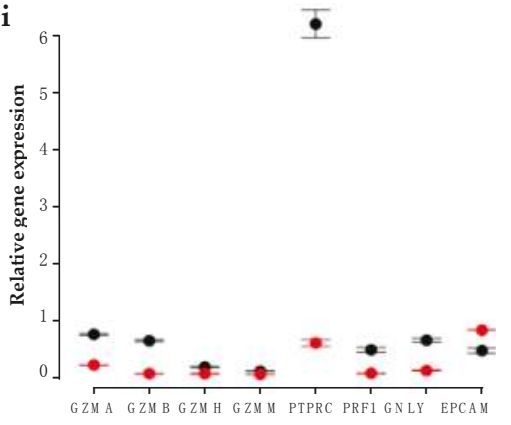

ii

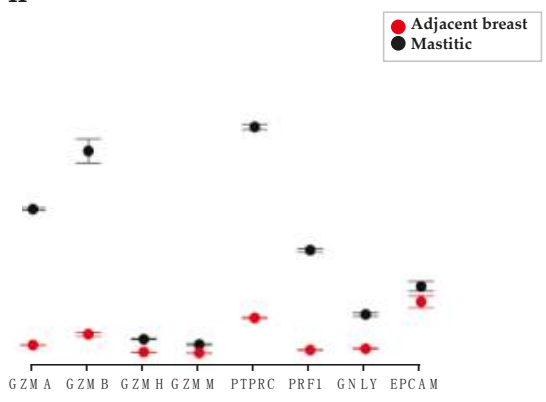

Marker

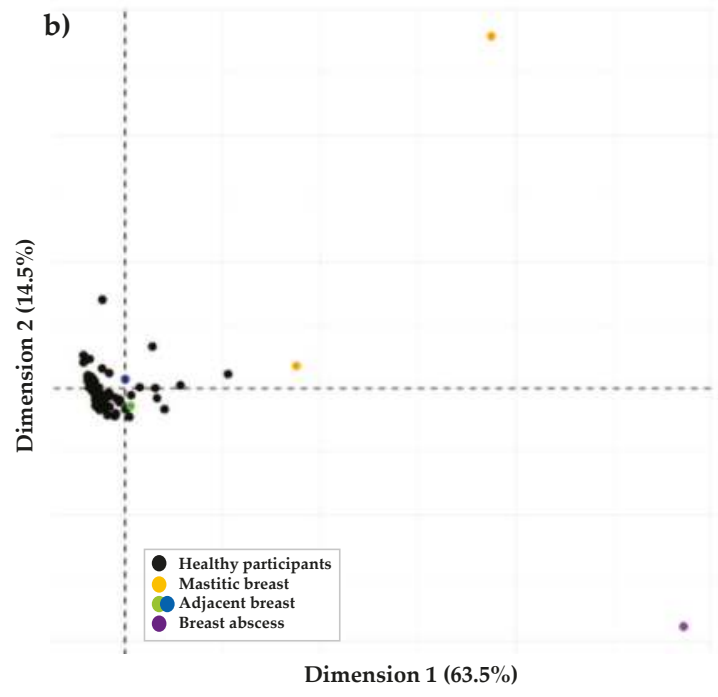

Figure 4. Comparisons of gene expression of immune genes in HM cells taken under different conditions. (a) Compares gene expression of HM cells taken from two participants (i and ii) with breasts affected by mastitis compared to the adjacent breast. PTPRC gene expression was upregulated in both participants (i and ii), whereas GZMA, GZMB and PRF1 was higher only in the second participant. Error bars represent the calculated standard error of the mean. (b) Principal component analysis (PCA) of the variance found between immune related gene expression in human milk cells taken from healthy participants compared to those from participants with mastitis or a breast abscess.

\subsection{Analysis of Antimicrobial Proteins in Human Milk Cells}

\subsubsection{Expression at the Protein Level of All Immune Proteins in Healthy Participants}

Flow cytometry was conducted to investigate the presence of the immune proteins in a healthy lactating population with infants under three months post-partum. Single cells were gated in each sample before separation into three cell populations (Figure S1). Both epithelial (EPCAM) and immune cell (CD45) markers were expressed in all samples with a median of $4.5 \%$ and $7.8 \%$ respectively, in the total cell population (Table 2). The presence of granulysin and perforin was also confirmed in all samples with a median of $1.6 \%$ and $1.4 \%$ of cells (Table 2), respectively. Granzyme A was not found in one out of eight samples, whereas granzyme B was not found in two out of twelve samples. 
The median percentage of cells of healthy participants expressing granzyme A was $0.3 \%$ (Table 2). When the sample not expressing granzyme A was taken out, the median was still similar with $0.4 \%$ of cells expressing the immune protein granzyme A. In contrast, the median for granzyme B expression in all samples was $2.7 \%$ (Table 2) whereas when the samples not expressing granzyme B were taken out, the median increased to 3.01\%. When looking at the different cell populations, a high presence of CD45 positive cells (51.5\%) exists in cell population 1 (Table 2). Moreover this population included a large cell population expressing granzyme B (11.9\%), granulysin (11.7\%) and perforin (8.4\%) positive cells (Table 3). Epithelial cell marker EPCAM was present in all three gated populations at a similar level (population 1: $18.2 \%$, population 2: $20.2 \%$ and population 3: $19.9 \%$, Table 2 ). All immune related proteins were more highly expressed in CD45 positive cells in comparison to EPCAM positive cells (Table 3), as shown for granzyme B (CD45: 5.2\%, EPCAM: 0.5\%) (Figure 5). Moreover, there seem to be a co-localization between granzyme $\mathrm{B}$, granulysin and perforin with the highest percentage in population 1 (Table S5).

Table 2. Flow cytometric analysis of immune and epithelial cell proteins in HM cells (\%) taken from healthy, mastitis and non-mammary post-surgery participants. Single marker expression.

\begin{tabular}{|c|c|c|c|c|}
\hline & \multirow{2}{*}{$\begin{array}{l}\text { Healthy }(n=12) \\
\text { Median (Range) }\end{array}$} & \multicolumn{2}{|c|}{ Mastitis Sample $(n=1)$} & \multirow{2}{*}{$\begin{array}{l}\text { Non-Mammary Surgery Sample } \\
\qquad(n=1)\end{array}$} \\
\hline & & Mastitis Breast & Non-Mastitic Adjacent Breast & \\
\hline \multicolumn{5}{|l|}{$\mathrm{CD} 45^{+}(\%)$} \\
\hline All single cells & $7.8(2.1-13.4)$ & 12.2 & 8.4 & 26.7 \\
\hline Population 1 & $51.5(1.5-66.7)$ & 78.2 & 64.8 & 66.8 \\
\hline Population 2 & $11.8(1.9-51.5)$ & 69.6 & 73.5 & 71.8 \\
\hline Population 3 & $2.7(1.2-8.7)$ & 3.2 & 1.5 & 17.3 \\
\hline \multicolumn{5}{|l|}{ Granzyme $\mathrm{A}^{+}(\%)$} \\
\hline All single cells & $0.3(0.0-10.6)$ & & 0.8 & 0 \\
\hline Population 1 & $1.9(0.4-82.0)$ & & 7.1 & 4.3 \\
\hline Population 2 & $0.5(0.0-19.2)$ & & 1.6 & 0 \\
\hline Population 3 & $0.9(0.00-1.3)$ & & 0.4 & 0 \\
\hline \multicolumn{5}{|l|}{ Granzyme B+ $(\%)$} \\
\hline All single cells & $2.7(0.0-4.1)$ & 0.4 & 1.2 & 2.6 \\
\hline Population 1 & $11.9(0.3-39.6)$ & 37.7 & 17.4 & 33.1 \\
\hline Population 2 & $0.8(0.0-9.5)$ & 1.1 & 1.7 & 7.8 \\
\hline Population 3 & $0.4(0.0-4.3)$ & 0.3 & 0.6 & 10.1 \\
\hline \multicolumn{5}{|l|}{ Granulysin $^{+}(\%)$} \\
\hline All single cells & $1.6(0.3-12.3)$ & & 1.6 & 2.2 \\
\hline Population 1 & $11.7(1.2-18.8)$ & & 4.3 & 13 \\
\hline Population 2 & $2.0(0.4-12.8)$ & & 4.5 & 12.4 \\
\hline Population 3 & $15.5(0.6-30.3)$ & & 1.1 & 15.7 \\
\hline \multicolumn{5}{|l|}{ Perforin $^{+}(\%)$} \\
\hline All single cells & $1.4(0.5-2.5)$ & 2.3 & 1.4 & 2.4 \\
\hline Population 1 & $8.4(0.0-31.7)$ & 37.4 & 8.6 & 27 \\
\hline Population 2 & $2.2(0.5-13.9)$ & 4.5 & 9.7 & 10.3 \\
\hline Population 3 & $1.3(0.8-9.5)$ & 0.3 & 0.6 & 1.1 \\
\hline \multicolumn{5}{|l|}{$\mathrm{EPCAM}^{+}(\%)$} \\
\hline All single cells & $4.5(3.8-12.8)$ & 6.6 & 5.1 & 3.4 \\
\hline Population 1 & $18.2(3.5-36.8)$ & 18.2 & 11.4 & 13.4 \\
\hline Population 2 & $20.2(5.8-26.0)$ & 23.4 & 12.9 & 9.5 \\
\hline Population 3 & $19.9(9.4-30.3)$ & 19.3 & 16.5 & 7.1 \\
\hline
\end{tabular}

3.2.2. Increased Number of Immune Cells and Higher Expression of Immune Proteins in Mastitic Milk Samples in Comparison to Healthy Milk Samples

HM cells taken from a single participant with mastitis from both the affected and tender adjacent breast were examined for protein expression of immune, antimicrobial and epithelial markers and compared to results from the healthy population (Tables 2 and 3, Figure 6). Cells taken from the mastitic breast were stained for the markers CD45, granzyme B, perforin and EPCAM due to the low cell number. CD45 positive cells were in a higher proportion in the mastitic sample $(12.2 \%$ of the whole cell population, Table 2) compared to $8.4 \%$ and $7.8 \%$ in the adjacent breast and healthy 
population respectively (Table 2). Similarly, population 1 in healthy participant's milk showed the highest expression of immune components (Table 2). In this particular population (population 1), the mastitis sample contained a significantly higher number of CD45 positive cells (78.2\%) and higher amount of granzyme B positive cells $(37.7 \%)$ in comparison with the sample from the adjacent breast $\left(64.8 \% \mathrm{CD} 45^{+}, 17.4 \%\right.$ granzyme $\left.\mathrm{B}^{+}\right)$or healthy population $\left(51.5 \% \mathrm{CD}^{+} 5^{+}, 11.9 \%\right.$ granzyme $\left.\mathrm{B}^{+}\right)($Table 2$)$. It also appeared that the sample from the adjacent breast contained slightly higher amounts of CD45 positive cells as well as cells expressing the immune proteins granzyme A and B in comparison to the cohort of healthy population $(n=12)$ (Table 2). Colocalization of granzyme B and perforin in CD45 and EPCAM positive cells from population 1 is shown in Figure 6. In the mastitis sample, clear co-expression of granzyme B in CD45 cells (27.0\%, Table 3) as well as perforin in CD45 cells (34.1\%, Table 3$)$ is evident. Interestingly, there was also expression of the immune proteins granzyme B $(2.2 \%$, Table 3 ) and perforin (2.1\%, Table 3 ) in cells expressing the epithelial marker EPCAM.

Table 3. Flow cytometric analysis of immune and epithelial cell proteins in HM cells (\%) taken from healthy, mastitis and non-mammary post-surgery participants. Double stainings between either CD45 or EPCAM positive cells and immune proteins.

\begin{tabular}{|c|c|c|c|c|}
\hline & \multirow{2}{*}{$\begin{array}{l}\text { Healthy }(n=12) \\
\text { Median (Range) }\end{array}$} & \multicolumn{2}{|c|}{ Mastitis Sample $(n=1)$} & \multirow{2}{*}{$\begin{array}{l}\text { Non-Mammary Surgery Sample } \\
\qquad(n=1)\end{array}$} \\
\hline & & Mastitis Breast & Adjacent Breast & \\
\hline \multicolumn{5}{|c|}{ CD45-Granzyme A (\%) } \\
\hline All single cells & $0.2(0.0-14.9)$ & & 0.2 & 0.4 \\
\hline Population 1 & $1.0(0.3-32.6)$ & & 10.9 & 5.4 \\
\hline Population 2 & $0.2(0.0-50.0)$ & & 3.5 & 1.3 \\
\hline Population 3 & $0.2(0.0-0.4)$ & & 0.2 & 0 \\
\hline \multicolumn{5}{|c|}{ CD45-Granzyme B (\%) } \\
\hline All single cells & $0.7(0.0-5.5)$ & 0.5 & 0.4 & 4.4 \\
\hline Population 1 & $5.2(0.0-26.8)$ & 27 & 17.2 & 33.4 \\
\hline Population 2 & $0.3(0.0-13.9)$ & 0.3 & 4.2 & 20.3 \\
\hline Population 3 & $0.0(0.0-0.3)$ & 0 & 0.3 & 1.4 \\
\hline \multicolumn{5}{|c|}{ CD45-Granulysin (\%) } \\
\hline All single cells & $0.7(0.0-10.4)$ & & 0.3 & 4.1 \\
\hline Population 1 & $3.2(0.8-21.1)$ & & 4.4 & 12.9 \\
\hline Population 2 & $3.6(0.0-13.4)$ & & 5.8 & 34 \\
\hline Population 3 & $13.6(0.2-27.1)$ & & 0.1 & 0.9 \\
\hline \multicolumn{5}{|l|}{ CD45-Perforin (\%) } \\
\hline All single cells & $0.9(0.6-8.3)$ & 0.8 & 0.9 & 23.7 \\
\hline Population 1 & $11.0(0.1-35.2)$ & 34.1 & 8.2 & 27.7 \\
\hline Population 2 & $2.1(0.1-26.8)$ & 3.9 & 10.6 & 48.2 \\
\hline Population 3 & $0.7(0.1-1.8)$ & 0.7 & 0.1 & 4.8 \\
\hline \multicolumn{5}{|c|}{ EPCAM-Granzyme A (\%) } \\
\hline All single cells & $0.0(0.0-0.2)$ & & 0.1 & 0 \\
\hline Population 1 & $0.1(0.0-35.2)$ & & 0.1 & 0.1 \\
\hline Population 2 & $0.4(0.0-1.5)$ & & 0.6 & 0 \\
\hline Population 3 & $1.0(0.2-1.8)$ & & 0.2 & 0 \\
\hline \multicolumn{5}{|c|}{ PCAM-Granzyme B (\%) } \\
\hline All single cells & $0.2(0.1-1.0)$ & 1.3 & 0 & 1.5 \\
\hline Population 1 & $0.5(0.3-3.5)$ & 2.2 & 0.6 & 0.9 \\
\hline Population 2 & $1.4(0.0-3.0)$ & 0.3 & 0.5 & 6.1 \\
\hline Population 3 & $1.3(0.2-2.3)$ & 0 & 0.2 & 1.1 \\
\hline \multicolumn{5}{|c|}{ EPCAM-Granulysin (\%) } \\
\hline All single cells & $0.2(0.0-2.1)$ & & 0.1 & 2.4 \\
\hline Population 1 & $1.5(0.2-3.1)$ & & 1.8 & 1 \\
\hline Population 2 & $0.6(0.1-1.4)$ & & 1.4 & 7.9 \\
\hline Population 3 & $2.6(0.2-5.0)$ & & 0.1 & 1.9 \\
\hline \multicolumn{5}{|c|}{ EPCAM-Perforin (\%) } \\
\hline All single cells & $0.2(0.0-5.5)$ & 0.2 & 0.1 & 5.2 \\
\hline Population 1 & $3.3(1.1-9.5)$ & 2.1 & $\begin{array}{l}0.1 \\
0.5\end{array}$ & 2.2 \\
\hline Population 2 & $1.7(0.3-8.4)$ & 0.7 & 1.1 & 7.4 \\
\hline Population 3 & $0.4(0.3-0.4)$ & 0.3 & 0 & 1.8 \\
\hline
\end{tabular}


a)

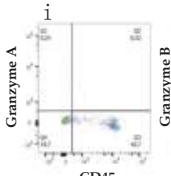

b)

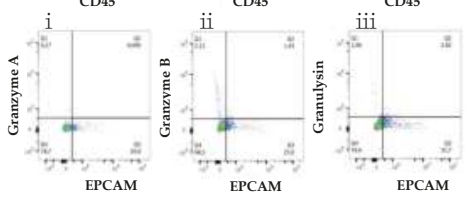

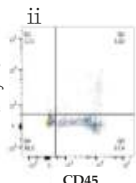

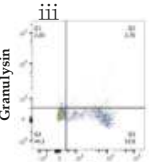

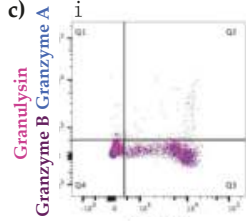

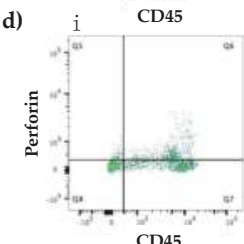

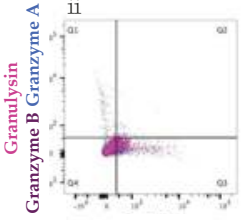

EPCAM

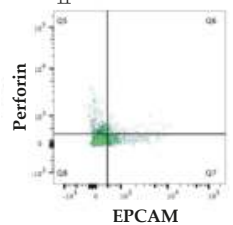

Figure 5. Co-expression of immune proteins granulysin, granzyme A, granzyme B and perforin with immune cell marker CD45 or epithelial marker EPCAM. Granzyme A, granzyme B and granulysin were all co-expressed with CD45 (0.3\%, 5.0\% and 2.8\% respectively) positive cells (a(i-iii)), whereas little co-expression was found between the immune proteins and the epithelial cell marker EPCAM $(0.1 \%$, $1.4 \%$ and $2.8 \%$ respectively) (b(i-iii)). Superposition of the co-expression of granulysin, granzyme B and granzyme A in $\mathrm{CD}_{4} 5^{+}(\mathbf{c}(\mathbf{i}))$ and $\operatorname{EPCAM}^{+}(\mathbf{c}(\mathbf{i i}))$. Perforin co-expression with $\mathrm{CD}^{+} 5^{+}(\mathbf{d}(\mathbf{i}))$ cells at a level of $23.7 \%$ whereas only $5.2 \%$ with $\operatorname{EPCAM}^{+}(\mathbf{d}(\mathbf{i i}))$ cells.

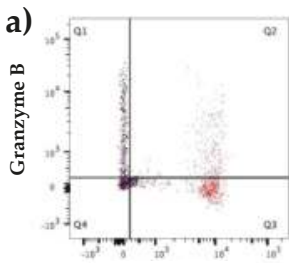

EPCAM CD45

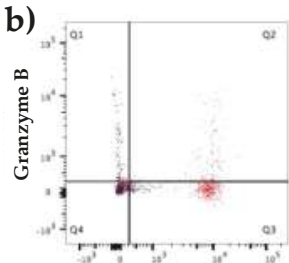

EPCAM CD45

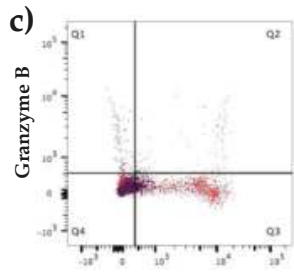

EPCAM CD45

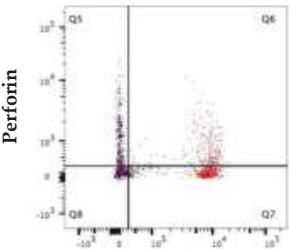

EPCAM CD45

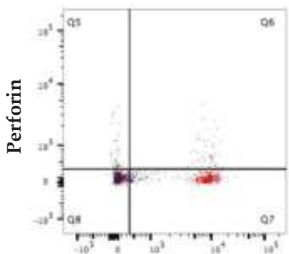

EPCAM CD45

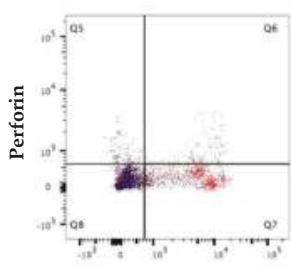

EPCAM CD45

Figure 6. Flow cytometry analysis of human milk cells taken from a participant suffering from (a) mastitis with comparison sample of the (b) adjacent breast of the same participant and (c) compared to a healthy participant. Increased numbers of CD45 positive cells expressing of granzyme B and perforin, whereas no co-expression observed in EPCAM positive cells. Higher expression of immune proteins in CD45 cells was evident in the mastitis affected participant when compared to healthy participants. 
3.2.3. Participant Recovering from Non-Mammary Surgery Shows Higher Expression of Immune Proteins Compared to Healthy Participants

HM cells (84 days post-partum) isolated from a participant recovering from a reparative surgery of the hand after a household accident, showed a higher expression of antimicrobial proteins (Figure 7) compared to the median expression of healthy HM cells (Table 2) indicative of heightened levels of activated or memory t-cells. Higher levels of granzyme B, granulysin and perforin were observed in HM cells from the participant that had undergone non-mammary surgery (Figure 7, Table 2). Curved gating was chosen for the double staining of CD45 and granzyme A, CD45 and granzyme B as well as CD45 and granulysin to better exclude the false positive measurements (Figure 7). CD45 positive cells expressed granzyme B (4.4\%) as well as granulysin (4.1\%) (Table 3). Similar to the mastitis sample, the presence of these immune proteins in EPCAM positive cells was minor. The same tendency is observed with perforin, where the protein was found in higher amounts in CD45 positive cells (23.7\%) compared to EPCAM positive cells (5.2\%) (Table 3$)$.
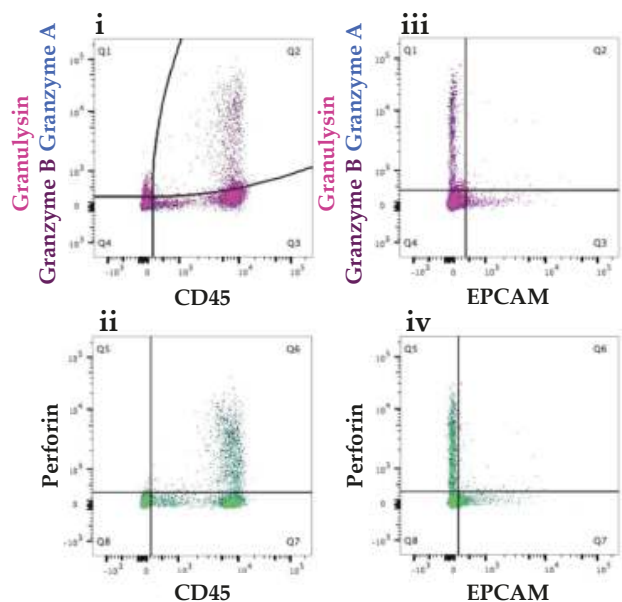

Figure 7. Expression of granzyme A, granyme B, granulysin and perforin in CD45 positive cells and EPCAM positive cells in population 1 in a participant recovering from a non-breast related surgery. Dot plots revealed increased number of $\mathrm{CD} 45^{+}$cells (i,ii) expressing the immune proteins compared with $\mathrm{EPCAM}^{+}$cells (iii,iv). Expression of the immune proteins in the EPCAM positive cells was low and similar in both participant populations.

\section{Discussion}

Human milk is known to contain immune cells [7] and display antimicrobial properties [24], little is known about the purpose of the cells in the milk or the mechanisms involved in the antibacterial activity. To better understand the actions of immune cells in milk, this study examined the presence of the known antimicrobial proteins perforin, granzymes and granulysin in HM. This study confirms for the first time the expression of the genes GZMA, GZMB, GZMH, GZMM, GNLY and PRF1 in resting mammary tissue, prepartum secretion cells and human milk cells taken from a larger population of women at different stages of lactation. All HM cell samples within this study contained cells positive for the protein CD45 that co-stained with granulysin and perforin and in most cases for granzyme A and B. Whilst EPCAM positive cells were also identified in all samples, co-staining with the investigated immune proteins was minimal. Further investigation revealed that HM cells from participants with local or systemic inflammation had a higher protein expression of CD45 positive cells compared with healthy participants, which is consistent with data from previous 
studies [8]. The presence and variation of immunological factors in human milk cells in both healthy and participants with inflammation suggests a selected prevalence of activated immune T-cells in HM that also expresses the cytotoxic immune proteins, likely involved both in maternal mammary gland and overall infant protection.

Extending previous studies examining immune cells in human milk, antimicrobial proteins not previously identified in milk cells were found and varied depending on the stage of mammary gland maturation. Investigation of the immune cell related genes identified in mammary transcriptomic data revealed a similar expression pattern between milk leukocyte content and lactation stage with previous data, showing a decreased immune cell content over lactation period $[3,7,8]$. Pre-partum cells, resting mammary tissue extracts and milk cells at month 1 and 3 had the highest levels of perforin, granulysin and granzymes whilst lower levels were observed in milk samples of the later months. Despite this, gene expression analysis using qRT-PCR did not find a linear relationship between immune gene expression and lactation stage over the first four months except for GZMB where a significant decrease over lactation stage was found. This may reflect the elevated levels of immune cells naturally present in milk from the first 4 months of lactation, which was specifically examined in the qRT-PCR experiments or may suggest that immune protein content is different between women despite infant age in early lactation. It was found that participant was an influencing factor on antimicrobial protein expression but further investigation should consider a larger cohort of women with samples examined at the same lactation stage.

According to previous studies $[3,25,26]$, different populations of $\mathrm{T}$ lymphocytes are present in the milk compared to the peripheral blood circulation. Associations between the expression of PRF1 and the genes encoding for granzymes and granulysin (Figure 3) in HM cells supports previous findings identifying increased expression of effector and memory T-cells in HM in comparison to peripheral blood [25,27], as these proteins are only present after activation of the lymphocytes [28]. Further investigations should include specific antibodies against activated $\mathrm{T}$ lymphocytes markers such as CD45RO+ or HLA-DR [29]. A linear association analysis of the immune related gene products showed a positive correlation between PRF1 and the genes encoding for granzymes and granulysin. This observation could mean that the expression of these proteins might be linked, possibly by the same expression control mechanisms. Co-expression of perforin with granulysin and granzymes in CD45 positive HM cells found with flow cytometry also confirms this theory as the efficiency of the proteins is higher when all three proteins are working together [11,12]. Surprisingly, some co-expression was found between EPCAM and the immune proteins (Figure 6) which may indicate low level expression of immune proteins in epithelial cells, epithelial cell uptake of exocytosed cytotoxic granules from activated leukocytes $[28,30]$ or low levels of cell aggregates in the FACS data. Future studies should further investigate the presence of immunological proteins in epithelial cells or whether granules containing immune proteins are released into the milk in cases of infection. Results from this study suggest that leukocytes in the milk are increased not only in the case of mammary inflammation but also in the case of systemic inflammation.

Investigated immune components (cells and immune proteins) were not only more prevalent in $\mathrm{HM}$ taken from the breast affected by mastitis but also in the adjacent breast. Interestingly immune components were also elevated in a participant who had undergone non-mammary surgery, compared to HM cells from healthy participants. Mastitis, being an infection of the breast tissue [8], creates a local inflammation and a systemic response leading to an increase of circulating immune cells in the blood [31]. Consequently, a higher quantity of lymphocytes infiltrating mammary tissue likely being the cause of heightened immune cells in the milk during mastitis [8]. As shown by flow cytometry, there were a heightened number of $\mathrm{CD} 45$ positive HM cells with co-expression of granzymes, granulysin and perforin from a participant with mastitis (Figure 6). As mastitis is usually a bacterial infection, the increased presence of immune cells and increased expression of antimicrobial proteins having an antibacterial effect was an expected outcome. In addition, elevated levels of CD45 cells with co-expression of the investigated immune proteins was also found in the milk obtained from the 
non-mastitic adjacent breast, although they were at much lower levels (Figure 6). The presence of immune related proteins in healthy participants, may suggest that they might not only play a role in the fight against an infection, but also in the prevention of one. This indicates that immune cells may not only have a role in the development of the immune system of the infant [32], but also in the protection of the lactating breast [3]. Follow-up studies should consider including complimentary blood samples alongside with milk to have an appropriate comparison point between milk and blood leukocytes.

\section{Conclusions}

This study showed for the first time the expression of the antimicrobial proteins perforin, granulysin and different granzymes at the protein and mRNA level in HM cells, RMT and PS cells. Furthermore, it provided confirmation that HM from healthy women is enriched in cells that carry hallmarks of activated or memory T-cells, which are elevated in case of maternal infection. Presence of these cells may indicate a purpose in the protection of the vulnerable infant or as a mechanism to defend the maternal breast against infection however further investigations should done to clarify this.

Supplementary Materials: The following are available online at http://www.mdpi.com/2072-6643/10/9/1230/ s1, Figure S1: Gating around single cells using forward scatter area (FSC-A) and forward scatter height (FSC-H). Three populations were then gated using only single cells. Figure S2: Relative quantitation (RQ) of the expression of the epithelial marker EPCAM, the immune cell marker PTPRC and the genes coding for granzyme A (GZMA), granzyme $B(G Z M B)$, granzyme H (GZMH), granzyme M (GZMM), granulysin (GNLY) and perforin (PRF1) distributed according to the time period post-partum. Table S1: Taqman probes from Life Technologies. Table S2: Antibodies used for FACS analysis. Table S3: Expression of selected genes in human milk cells (HMC) measured via RT-PCR, normalised to either lymphokine activated killer cells (LAK) or resting tissue (RT). Table S4: Univariate linear mixed modelling of days post-partum and antimicrobial protein genes, with participant as an influencing factor on gene expression. Table S5: Flow cytometric analysis of immune and epithelial cell proteins in HM cells (\%) taken from healthy, mastitis and non-related surgical patient participants.

Author Contributions: Conceptualization, G.K.K., A.-J.T., D.T.G. and L.F.; Data curation, A.-J.T.; Formal analysis, G.K.K.; Funding acquisition, D.T.G. and L.F.; Investigation, G.K.K., A.-J.T. and L.F.; Methodology, G.K.K. and A.-J.T.; Project administration, A.-J.T., D.T.G. and L.F.; Resources, D.T.G. and L.F.; Supervision, A.-J.T., D.T.G. and L.F.; Visualization, G.K.K.; Writing-original draft, G.K.K. and A.-J.T.; Writing-review \& editing, G.K. K., A.-J.T., D.T.G. and L.F.

Funding: Internal funding from the department of Anatomy at The University of Fribourg supported this work and the salaries of A.-J.T. and D.T.G. were provided from an unrestricted research grant from Medela AG (Switzerland).

Acknowledgments: Many thanks to the Australian Breastfeeding Association for their support and to all participating mothers of the study. The Centre for Microscopy, Characterisation \& Analysis (UWA) provided access to facilities and technical assistance. Many thanks to Solange Kharoubi-Hess, Alethea Rea and Michelle Trevenen for their technical and statistical support.

Conflicts of Interest: The authors declare no conflict of interest. The founding sponsors had no role in the design of the study; in the collection, analyses, or interpretation of data; in the writing of the manuscript, and in the decision to publish the results.

\section{References}

1. Hale, T.W.; Hartmann, P.E. Textbook of Human Lactation, 1st ed.; Hale Publishing L.P.: Amarillo, TX, USA, 2007; p. 662.

2. Hanson, L.; Silfverdal, S.A.; Stromback, L.; Erling, V.; Zaman, S.; Olcen, P.; Telemo, E. The immunological role of breast feeding. Pediatr. Allergy Immunol. 2001, 12 (Suppl. S14), 15-19. [CrossRef]

3. Hassiotou, F.; Geddes, D.T. Immune cell-mediated protection of the mammary gland and the infant during breastfeeding. Adv. Nutr. 2015, 6, 267-275. [CrossRef] [PubMed]

4. Liepke, C.; Zucht, H.D.; Forssmann, W.G.; Standker, L. Purification of novel peptide antibiotics from human milk. J. Chromatogr. B Biomed. Sci. Appl. 2001, 752, 369-377. [CrossRef]

5. Zhang, F.; Cui, X.; Fu, Y.; Zhang, J.; Zhou, Y.; Sun, Y.; Wang, X.; Li, Y.; Liu, Q.; Chen, T. Antimicrobial activity and mechanism of the human milk-sourced peptide casein201. Biochem. Biophys. Res. Commun. 2017, 485, 698-704. [CrossRef] [PubMed] 
6. Hanson, L.A.; Korotkova, M.; Lundin, S.; Haversen, L.; Silfverdal, S.A.; Mattsby-Baltzer, I.; Strandvik, B.; Telemo, E. The transfer of immunity from mother to child. Ann. N. Y. Acad. Sci. 2003, 987, 199-206. [CrossRef] [PubMed]

7. Hassiotou, F.; Geddes, D.T.; Hartmann, P.E. Cells in human milk: State of the science. J. Hum. Lact. 2013, 29, 171-182. [CrossRef] [PubMed]

8. Hassiotou, F.; Hepworth, A.R.; Metzger, P.; Tat Lai, C.; Trengove, N.; Hartmann, P.E.; Filgueira, L. Maternal and infant infections stimulate a rapid leukocyte response in breastmilk. Clin. Transl. Immunol. 2013, 2. [CrossRef] [PubMed]

9. Xu, Q.; Abdubek, P.; Astakhova, T.; Axelrod, H.L.; Bakolitsa, C.; Cai, X.; Carlton, D.; Chen, C.; Chiu, H.J.; Clayton, T.; et al. Structure of a membrane-attack complex/perforin (macpf) family protein from the human gut symbiont bacteroides thetaiotaomicron. Acta Crystallogr. Sect. F Struct. Biol. Cryst. Commun. 2010, 66, 1297-1305. [CrossRef] [PubMed]

10. Nobre, T.M.; Martynowycz, M.W.; Andreev, K.; Kuzmenko, I.; Nikaido, H.; Gidalevitz, D. Modification of salmonella lipopolysaccharides prevents the outer membrane penetration of novobiocin. Biophys. J. 2015, 109, 2537-2545. [CrossRef] [PubMed]

11. Walch, M.; Dotiwala, F.; Mulik, S.; Thiery, J.; Kirchhausen, T.; Clayberger, C.; Krensky, A.M.; Martinvalet, D.; Lieberman, J. Cytotoxic cells kill intracellular bacteria through granulysin-mediated delivery of granzymes. Cell 2014, 157, 1309-1323. [CrossRef] [PubMed]

12. Dotiwala, F.; Mulik, S.; Polidoro, R.B.; Ansara, J.A.; Burleigh, B.A.; Walch, M.; Gazzinelli, R.T.; Lieberman, J. Killer lymphocytes use granulysin, perforin and granzymes to kill intracellular parasites. Nat. Med. 2016, 22, 210-216. [CrossRef] [PubMed]

13. Lieberman, J. Granzyme a activates another way to die. Immunol. Rev. 2010, 235, 93-104. [CrossRef] [PubMed]

14. Stenger, S.; Hanson, D.A.; Teitelbaum, R.; Dewan, P.; Niazi, K.R.; Froelich, C.J.; Ganz, T.; Thoma-Uszynski, S.; Melian, A.; Bogdan, C.; et al. An antimicrobial activity of cytolytic t cells mediated by granulysin. Science 1998, 282, 121-125. [CrossRef] [PubMed]

15. Bryan, D.L.; Hart, P.H.; Forsyth, K.D.; Gibson, R.A. Immunomodulatory constituents of human milk change in response to infant bronchiolitis. Pediatr. Allergy Immunol. 2007, 18, 495-502. [CrossRef] [PubMed]

16. Twigger, A.J.; Kakulas, F. RNA-Sequencing of Milk Cells Extracted from Pre-Partum Secretions and Longitudinally from Mature Human Milk accros the First Year of Lactation. NCBI: Gene Expression Omnibus, 2016. Available online: https://www.ncbi.nlm.nih.gov/geo/ (accessed on 25 August 2016).

17. Twigger, A.J.; Hepworth, A.R.; Lai, C.T.; Chetwynd, E.; Stuebe, A.M.; Blancafort, P.; Hartmann, P.E.; Geddes, D.T.; Kakulas, F. Gene expression in breastmilk cells is associated with maternal and infant characteristics. Sci. Rep. 2015, 5. [CrossRef] [PubMed]

18. Hassiotou, F.; Beltran, A.; Chetwynd, E.; Stuebe, A.M.; Twigger, A.J.; Metzger, P.; Trengove, N.; Lai, C.T.; Filgueira, L.; Blancafort, P.; et al. Breastmilk is a novel source of stem cells with multilineage differentiation potential. Stem Cells 2012, 30, 2164-2174. [CrossRef] [PubMed]

19. Mortazavi, A.; Williams, B.A.; McCue, K.; Schaeffer, L.; Wold, B. Mapping and quantifying mammalian transcriptomes by rna-seq. Nat. Methods 2008, 5, 621-628. [CrossRef] [PubMed]

20. Wickham, H. Ggplot2: Elegant Graphics for Data Analysis; Springer: New York, NY, USA, 2009.

21. Sarkar, D. Lattice: Multivariate Data Visualization with R; Springer: New York, NY, USA, 2008.

22. Pinheiro, J.; Bates, D.; DebRoy, S.; Sarkar, D.; Team, R.C. Nlme: Linear and Nonlinear Mixed Effects Models. R Package Version 3.1-131 2017. Available online: https://mran.microsoft.com/snapshot/2017-02-20/web/ packages/nlme/index.html (accessed on 20 February 2017).

23. Lê, S.; Josse, J.; Husson, F. Factominer: An r package for multivariate analysis. J. Stat. Softw. 2008, 25, 1-18. [CrossRef]

24. Chirico, G.; Marzollo, R.; Cortinovis, S.; Fonte, C.; Gasparoni, A. Antiinfective properties of human milk. J. Nutr. 2008, 138, 1801s-1806s. [CrossRef] [PubMed]

25. Sabbaj, S.; Ghosh, M.K.; Edwards, B.H.; Leeth, R.; Decker, W.D.; Goepfert, P.A.; Aldrovandi, G.M. Breast milk-derived antigen-specific cd8+ t cells: An extralymphoid effector memory cell population in humans. J. Immunol. 2005, 174, 2951-2956. [CrossRef] [PubMed]

26. Wirt, D.P.; Adkins, L.T.; Palkowetz, K.H.; Schmalstieg, F.C.; Goldman, A.S. Activated and memory t lymphocytes in human milk. Cytometry 1992, 13, 282-290. [CrossRef] [PubMed] 
27. Peroni, D.G.; Chirumbolo, S.; Veneri, D.; Piacentini, G.L.; Tenero, L.; Vella, A.; Ortolani, R.; Raffaelli, R.; Boner, A.L. Colostrum-derived $b$ and $t$ cells as an extra-lymphoid compartment of effector cell populations in humans. J. Matern. Fetal Neonatal Med. 2013, 26, 137-142. [CrossRef] [PubMed]

28. Cullen, S.P.; Martin, S.J. Mechanisms of granule-dependent killing. Cell Death Differ. 2007, 15, $251-262$. [CrossRef] [PubMed]

29. Costello, P.; Bresnihan, B.; O'Farrelly, C.; FitzGerald, O. Predominance of cd8+t lymphocytes in psoriatic arthritis. J. Rheumatol. 1999, 26, 1117-1124. [PubMed]

30. Veugelers, K.; Motyka, B.; Goping, I.S.; Shostak, I.; Sawchuk, T.; Bleackley, R.C. Granule-mediated killing by granzyme $\mathrm{b}$ and perforin requires a mannose 6-phosphate receptor and is augmented by cell surface heparan sulfate. Mol. Biol. Cell 2006, 17, 623-633. [CrossRef] [PubMed]

31. Coutinho, A.E.; Chapman, K.E. The anti-inflammatory and immunosuppressive effects of glucocorticoids, recent developments and mechanistic insights. Mol. Cell. Endocrinol. 2011, 335, 2-13. [CrossRef] [PubMed]

32. Field, C.J. The immunological components of human milk and their effect on immune development in infants. J. Nutr. 2005, 135, 1-4. [CrossRef] [PubMed]

(C) 2018 by the authors. Licensee MDPI, Basel, Switzerland. This article is an open access article distributed under the terms and conditions of the Creative Commons Attribution (CC BY) license (http:/ / creativecommons.org/licenses/by/4.0/). 
Article

\title{
Human Milk Composition and Dietary Intakes of Breastfeeding Women of Different Ethnicity from the Manawatu-Wanganui Region of New Zealand
}

\author{
Christine A. Butts ${ }^{1, *}$, Duncan I. Hedderley ${ }^{1}$, Thanuja D. Herath ${ }^{1}$, Gunaranjan Paturi ${ }^{2}$, \\ Sarah Glyn-Jones ${ }^{3}$, Frank Wiens ${ }^{4}$, Bernd Stahl ${ }^{4}$ and Pramod Gopal ${ }^{1,5}$ \\ 1 The New Zealand Institute for Plant and Food Research Limited, Private Bag 11600, \\ Palmerston North 4442, New Zealand; duncan.hedderley@plantandfood.co.nz (D.I.H.); \\ hmth4@hotmail.com (T.D.H.); pramod.gopal@plantandfood.co.nz (P.G.) \\ 2 The New Zealand Institute for Plant and Food Research Limited, Private Bag 92169, \\ Auckland 1142, New Zealand; gunaranjan.paturi@plantandfood.co.nz \\ 3 Danone Nutricia NZ Limited, 56-58 Aintree Avenue, Mangere, Auckland 2022, New Zealand; \\ sarah.glyn-jones@danone.com \\ 4 Danone Nutricia Research, Upsalalaan 12, 3584 CT Utrecht, The Netherlands; \\ frank.wiens@danone.com (F.W.); bernd.stahl@danone.com (B.S.) \\ 5 Riddet Institute, Massey University, Palmerston North 4442, New Zealand \\ * Correspondence: chrissie.butts@plantandfood.co.nz; Tel.: +64-6-355-6147
}

Received: 15 August 2018; Accepted: 31 August 2018; Published: 4 September 2018

check for updates

\begin{abstract}
Human milk is nutrient rich, complex in its composition, and is key to a baby's health through its role in nutrition, gastrointestinal tract and immune development. Seventy-eight mothers (19-42 years of age) of Asian, Māori, Pacific Island, or of European ethnicity living in Manawatu-Wanganui, New Zealand (NZ) completed the study. The women provided three breast milk samples over a one-week period (6-8 weeks postpartum), completed a three-day food diary and provided information regarding their pregnancy and lactation experiences. The breast milk samples were analyzed for protein, fat, fatty acid profile, ash, selected minerals (calcium, magnesium, selenium, zinc), and carbohydrates. Breast milk nutrient profiles showed no significant differences between the mothers of different ethnicities in their macronutrient (protein, fat, carbohydrate, and moisture) content. The breast milk of Asian mothers contained significantly higher levels of polyunsaturated fatty acids (PUFAs), omega-3 (n-3) and omega-6 (n-6) fatty acids, docosahexaenoic acid (DHA), and linoleic acids. Arachidonic acid was significantly lower in the breast milk of Māori and Pacific Island women. Dietary intakes of protein, total energy, saturated and polyunsaturated fat, calcium, phosphorus, zinc, iodine, vitamin A equivalents, and folate differed between the ethnic groups, as well as the number of serves of dairy foods, chicken, and legumes. No strong correlations between dietary nutrients and breast milk components were found.
\end{abstract}

Keywords: human milk; breastfeeding; ethnicity; composition; diet

\section{Introduction}

Human milk usually provides all the nutrients a human infant requires for the first 6 months of life. As well as the essential macro and micro-nutrients, breast milk contains many distinctive bioactive molecules that protect the new-born against pathogens and inflammation, and contribute to immune system maturation, organ development, and healthy microbial colonization [1,2]. The benefits of breastfeeding on the health and wellbeing of the infant are well recognized and include the prevention of infections, optimal neurodevelopment, and may limit the development of allergy, obesity and 
diabetes later in life [3-5]. The World Health Organization (WHO) [6] and the national advisory bodies of many countries, including New Zealand (NZ) [7], actively support and promote breastfeeding by their strong recommendations that all infants should be exclusively breastfed for the first 6 months of life and that breastfeeding be continued with appropriate complementary foods for 2 years and beyond postpartum. For infants who are not breastfed, human milk composition is used as an important reference in decisions on the adequacy of surrogate infant nutrition products.

Human milk composition varies considerably within and between mothers and even within a single milk expression. This multidimensional variation in composition is believed to be an adaptation to the infants' changing needs [8-10], and geographical region and food supply [11,12]. The variations in human milk composition between individual women and populations have been reported to be in response to cultural differences such as diet and other lifestyle factors $[13,14]$, environmental factors, such as mineral content of the soil that is then reflected in the mineral density of the foods grown there [15], and human genetic differences [16]. However, human milk composition data has not been collected from all world regions and populations. Therefore, studies of human milk composition in other regions and populations are important, particularly with regard to micronutrient concentrations and the proportions of specific lipids where a large variability has been noted from existing studies [14,17-19].

There is limited information available on the nutrient composition of breast milk from NZ mothers. Early research on breast milk from NZ women, by Deem [20,21], investigated diurnal variation in fat content and the influence of dietary macronutrient content on breast milk composition. Recent published information on breast milk composition in NZ women has focused on the levels of environmental contaminants in breast milk [22], the micronutrient iodine [23-26] and the macronutrient and amino acid compositions [27]. In the present study, we investigated the composition of breast milk of an ethnically mixed population of NZ women as a first representation of the New Zealand national population. The main ethnic groups in NZ are the indigenous Māori (14.9\%) and three major immigrant populations from the Pacific Islands (7.4\%), Asia (11.8\%) and Europe (74.0\%) [28]. We note here that some NZ citizens identify with more than one ethnic group resulting in the total being greater than $100 \%$. The secondary aims of this study were to determine the dietary nutrient intakes of breastfeeding women, compare these to recommended intakes, to assess if the diets were different between different ethnic groups, and if this had any impact on breastmilk composition.

\section{Materials and Methods}

\subsection{Study Design}

This was an observational study with participating women providing samples of their breast milk as well as stool samples from themselves and their babies at 6-8 weeks postpartum. All participants gave their informed consent for inclusion before taking part in the study. The study was conducted in accordance with the Declaration of Helsinki and the protocol was approved by the New Zealand Human Disability and Ethics Committee (Application number 13/CEN/79/AM01).

One hundred and forty-six participants living in the Manawatū-Wanganui region of the North Island of New Zealand were screened for this study; 66 participants did not meet the recruitment criteria. A total of 80 women who fulfilled the inclusion and exclusion criteria were recruited into the study (Figure 1). The study was advertised through newspaper and radio, flyers displayed on community noticeboards, in midwifery and in childcare centers in and around the Palmerston North area. Interested participants were first contacted by phone and then visited in their homes to obtain informed consent and complete their enrolment into the study. Participant information regarding their ethnicity (self-identified), anthropometry, parity, recent pregnancy and childbirth experiences, general medical history, and recent breast-feeding practices, as well as previous pregnancies and birth history, were collected through questionnaires. Only breastfeeding women aged 18-55 years of Māori, Pacific Island, European, or Asian ethnicity permanently living in New Zealand were included. 
Recruitment focused initially on women who were breast-feeding exclusively, however, if recruitment was slow we accepted women who were primarily breast-feeding and included no more than two formula feeds a day or water or medication. The mothers were asked to record exactly what method of feeding they used. Women with a pre-term childbirth or with infants who had required neonatal care were excluded from the study. Other exclusion criteria were active dieting, clinically significant renal, hepatic, endocrine, cardiac, pulmonary, pancreatic, neurological, hematologic, biliary, and mental health disorders as identified through their medical history.

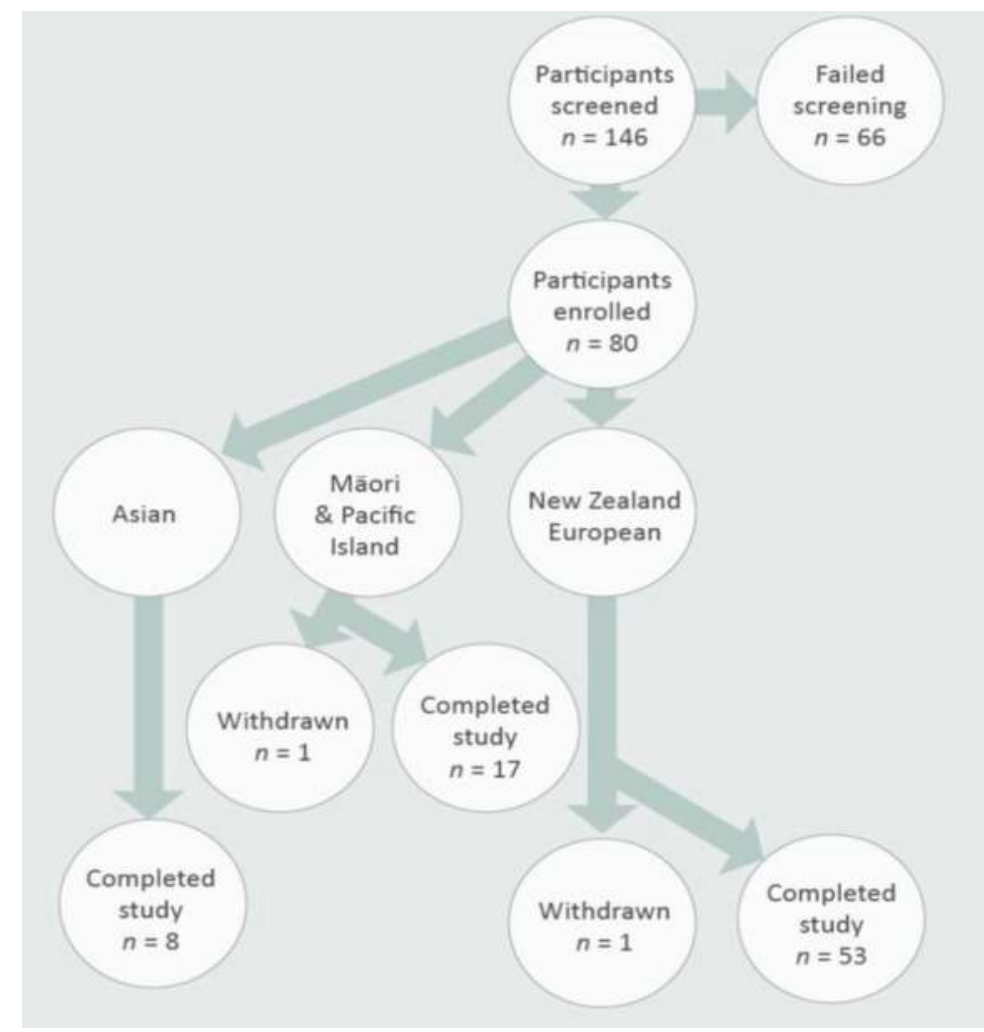

Figure 1. Study participant recruitment flow chart.

Following enrolment, each woman was asked to express three breast milk samples over a one-week period between six and eight weeks post-partum. Each sample of approximately $50 \mathrm{~mL}$ was collected from the first feed of the day (first feed after sunrise) into sterile containers and immediately frozen in household freezers at $-18{ }^{\circ} \mathrm{C}$. In order to guarantee enough milk supply to the infant, mothers fed their baby immediately before the collection of the expressed breast milk. Breast milk was collected by hand or breast pump, and the mode of expression was recorded. Subsequently, the three samples from each mother were thawed, pooled, aliquoted into smaller containers, and refrozen at -80 ${ }^{\circ} \mathrm{C}$ until analysis. In addition to breast milk, a fecal sample from both mother and infant was collected and frozen during the same week (rationale, methods and analyses will be reported elsewhere).

Each participating mother provided a diet record of every item she ate or drank and the quantities consumed over three consecutive days (two working days one non-working day) during the one week period of breast milk collection. Participants were reimbursed with grocery or fuel vouchers to compensate for their time commitment to this study. 


\subsection{Analysis of Breast Milk}

Breast milk samples were analyzed for selected macronutrients (protein, carbohydrates, fat, polyunsaturated fatty acids; PUFAs) and micronutrients (calcium, magnesium, selenium, zinc). These analyses were carried out by the Nutrition Laboratory, Massey University, Palmerston North, NZ. Total protein was determined by the combustion method using a LECO analyzer (AOAC 968.06) [29] and the factor 6.38 to convert nitrogen content to protein. Total fat was measured using the Mojonnier method (AOAC 954.02) [29], and fatty acids were measured as their methyl esters by gas chromatography (Sukhija and Palmquist 1988). Ash was measured following incineration in a furnace at $550{ }^{\circ} \mathrm{C}$ (AOAC 942.05) [29]. Inductively coupled plasma mass spectroscopy (ICP-MS) was used to measure the individual minerals in breast milk. Following acid digestion, the samples were analyzed on a PerkinElmer Sciex Elan 6000 ICP-MS (PerkinElmer, Waltham, MA, USA). The system comprised a variable speed peristaltic pump, nebulizer, argon gas plasma (1500 W), vacuum chambers, quadrapole, and a combined pulse counting/analog detector. Each element was monitored at an isotope(s) chosen for its abundance/sensitivity and freedom from known interferences. The total carbohydrate content was estimated by the difference using the determined values for protein, fat, water, and ash [30].

\subsection{Dietary Intake Analysis}

The dietary intakes of the macro and micronutrients were calculated from the three-day food diaries completed by all participants. The data were entered into FoodWorks (Professional version 7.0 Xyris Software package, Brisbane, Australia) using the New Zealand Food Composition Database (2014).

\subsection{Statistical Analysis}

All data were transferred into an Excel database and summary statistics (means and standard deviations or standard errors) were calculated. The participants were grouped by ethnicity into three groups: Asians, Māori and Pacific Island, and NZ European. In NZ, population and government statistics distinguishes between indigenous Māori and immigrants from the Pacific Islands, however, for this study, we combined these two groups as both share a Polynesian background and the numbers of participants of Pacific Island ethnicity were small $(n=2)$. Demographic data, breast milk nutrients, dietary nutrient intakes, food serves, and the dietary supplements taken were compared between ethnic groups using an analysis of variance (ANOVA); where there was a significant $(p<0.05)$ difference between groups and multiple comparisons were made using the least significant difference. Where data were skewed, Kruskal-Wallis non-parametric ANOVA was also carried out. Nutrient data from the breast milk samples were analyzed in the same way. Analyses were carried out using Genstat (version 17, 2014, VSNi Ltd., Hemel Hempstead, UK) and the $R$ package gplots (R Foundation for Statistical Computing, Vienna, Austria).

\section{Results}

\subsection{Study Population}

The demographic and baseline characteristics of all participants in this study are summarized in Tables 1 and 2. Of the 80 participants enrolled in the study, 78 completed the study; $68 \%$ of these were NZ Europeans, 22\% Māori and Pacific Island and 10\% Asian. One participant each of Māori and NZ European ethnicities withdrew from the study before completion as they were no longer able to provide the samples requested.

The mean age of the participants was 31 years, their mean body mass index (BMI) was 27, and the mean infant birth weight was $3.6 \mathrm{~kg}$ (Table 1). When analyzed based on ethnicity, there were no statistically significant differences in the age and heights of the women (Table 2), whereas there were significant differences in body weight $(p<0.001)$ and BMI $(p=0.003)$ of the mothers from the different ethnic groups. Asian women had the lowest mean body weight and BMI, and the Māori and Pacific Island mothers had the highest mean body weight and BMI (Table 2). It is important to note that 
the NZ Ministry of Health guidelines use different BMI [31] values to classify women of different ethnicities into normal, overweight and obese categories to those recommended by the WHO [32] (Table 3). Demographic distribution and baseline characteristics of the study population based on BMI classifications recommended by the NZ Ministry of Health are summarized in Table 4. Based on these criteria, the proportions of all participants in the normal, overweight and obese categories were 35\%, $40 \%$, and 25\%, respectively. The Asian mothers had the highest proportion of women with normal BMI while the Māori and Pacific Island mothers had the lowest proportion in the normal BMI range.

Table 1. Demographics and baseline characteristics of the study participants.

\begin{tabular}{ccc}
\hline Baseline Characteristics & Mean & Range \\
\hline Mothers $(n=78)$ & $31 \pm 5$ & $19-42$ \\
Age (years) & $74 \pm 14$ & $48-109$ \\
Weight $(\mathrm{kg})$ & $1.65 \pm 0.06$ & $1.52-1.87$ \\
Height $(\mathrm{m})$ & $27 \pm 5$ & $20-39$ \\
Body mass index $\left(\mathrm{kg} / \mathrm{m}^{2}\right)$ & $3.6 \pm 0.5$ & $2.4-4.6$ \\
Babies $(n=79)$ & $4.8 \pm 0.6$ & $3.3-6.2$ \\
Birth weight $(\mathrm{kg})$ &
\end{tabular}

Table 2. Demographics and baseline characteristics of the study participants according to ethnicity.

\begin{tabular}{ccccc}
\hline & Asian & Māori \& Pacific Island & New Zealand European & $p$ Value \\
\hline Participants in group $(n)$ & 8 & 17 & 53 & \\
Age (years) & $30.4 \pm 1.2$ & $31.2 \pm 1.5$ & $30.7 \pm 0.7$ & 0.917 \\
Weight $(\mathrm{kg})$ & $58.4 \pm 3.1^{\mathrm{a}}$ & $80.8 \pm 4.2^{\mathrm{b}}$ & $74.5 \pm 1.6^{\mathrm{b}}$ & $<0.001$ \\
Height $(\mathrm{m})$ & $1.61 \pm 0.02$ & $1.65 \pm 0.01$ & $1.66 \pm 0.01$ & 0.162 \\
Body mass index $\left(\mathrm{kg} / \mathrm{m}^{2}\right)$ & $22.5 \pm 1.1^{\mathrm{a}}$ & $29.6 \pm 1.5^{\mathrm{b}}$ & $27.2 \pm 0.6^{\mathrm{b}}$ & 0.003 \\
Birth weight $(\mathrm{kg})$ & $3.32 \pm 0.13$ & $3.63 \pm 0.13$ & $3.60 \pm 0.06$ & 0.255 \\
\hline
\end{tabular}

Data expressed as mean \pm standard error of the mean. Mean values with a different letter differ significantly, $p<0.05$.

Table 3. World Health Organisation and New Zealand Ministry of Health classifications of body mass index $\left(\mathrm{kg} / \mathrm{m}^{2}\right)$.

\begin{tabular}{ccccc}
\hline & World Health Organisation $^{\mathbf{1}}$ & \multicolumn{3}{c}{ New Zealand Ministry of Health ${ }^{\mathbf{2}}$} \\
\cline { 2 - 5 } & All Populations & Asian & Māori \& Pacific & New Zealand European \\
\hline Underweight & $<18.50$ & $<18.50$ & $<18.50$ & $<18.50$ \\
Normal & $18.50-24.99$ & $18.5-22.9$ & $18.5-26$ & $18.5-25$ \\
Overweight & $\geq 25.00$ & $23-27.4$ & $26-32$ & $25-30$ \\
Obese & $\geq 30.00$ & $>27.5$ & $>32$ & $>30$ \\
\hline
\end{tabular}

${ }^{1}$ Adapted from World Health Organisation 1995, 2000 and 2004 [32]. ${ }^{2}$ Ministry of Health, New Zealand [31].

Table 4. Demographics and baseline characteristics of the study participants according to body mass index (BMI) classifications outlined by Ministry of Health, New Zealand.

\begin{tabular}{ccccc}
\hline & Normal & Overweight & Obese & $p$ Value \\
\hline Age (years) & $30.3 \pm 1.0$ & $31.5 \pm 0.8$ & $30.2 \pm 1.4$ & 0.559 \\
Weight $(\mathrm{kg})$ & $60.4 \pm 1.2^{\mathrm{a}}$ & $74.7 \pm 1.7^{\mathrm{b}}$ & $92.0 \pm 2.0^{\mathrm{c}}$ & $<0.001$ \\
Height $(\mathrm{cm})$ & $165.0 \pm 1.4$ & $165.2 \pm 1.0$ & $164.6 \pm 1.1$ & 0.943 \\
Baby's weight $(\mathrm{kg})$ & $3.52 \pm 0.08$ & $3.65 \pm 0.09$ & $3.54 \pm 0.14$ & 0.592 \\
Participants in BMI category (\%) & 35 & 40 & 25 &
\end{tabular}

Data expressed as mean \pm standard error of the mean. Mean values with a different letter differ significantly, $p<0.05$. 


\subsection{Nutrient Composition of Breast Milk}

The nutrient profiles of the mothers' breast milk are presented in Table 5. The mean values for the three main macronutrients (protein, fat, carbohydrates) and water in the breast milk across all ethnicities were not significantly different between women of different ethnicities. There were no significant differences in the mean breast milk concentrations of the minerals calcium, selenium, and zinc, but there were significant differences in magnesium concentrations, where NZ European mothers had significantly higher concentrations than Māori and Pacific Island mothers $(p=0.049)$.

There were significant differences in the total PUFAs, $n-3$ and $n-6$ fatty acids present in the breast milk. Asian mothers had higher concentrations of these fatty acids than Māori and Pacific Island and NZ European mothers. The fatty acids contributing to these differences were docosahexaenoic acid (DHA) $(p<0.001)$, arachidonic acid $(p=0.023)$, and linoleic acid $(C 18: 2 n 6 c)(p=0.009)$. DHA was significantly higher in Asian mothers' breast milk compared to Māori and Pacific Island and NZ European mothers, but there was no significant difference between the Māori and Pacific Island and NZ European mothers. For arachidonic acid, however, breast milk from Māori and Pacific Island mothers had significantly lower concentrations than Asian and NZ European mothers, and there was no significant difference between the breast milk concentrations from Asian and NZ European mothers.

The nutrient intakes of the study participants in this study determined from their 3-day diet records are summarized in Table 6. Protein intakes of Māori and Pacific Island mothers were significantly lower $(p=0.023)$ than the NZ European mothers. There were no significant differences in the intakes of energy, total fat, saturated, polyunsaturated or monounsaturated fats, carbohydrate, sugars, starch, or dietary fiber between the mothers from different ethnic groups. There were, however, some significant differences in the total energy and different types of fats consumed. The energy from saturated fat $(p=0.019)$ and the proportion of fat from saturated fat $(p=0.010)$ was significantly lower in the diets of Asian women compared to Māori and Pacific Island and NZ European women. Asian mothers consumed a significantly higher proportion of their total fat intake as monounsaturated fats $(p=0.042)$ than the other ethnic groups, and significantly more PUFAs $(p=0.026)$ than NZ European mothers.

Dietary intakes of calcium $(p=0.007)$, phosphorus $(p=0.024)$, and zinc $(p=0.029)$ were significantly higher in NZ European mothers than Asian and Māori and Pacific Island mothers. Iodine intakes were highest for the Asian mothers ( $p=0.027)$. Dietary intakes of vitamins were similar except for folate (food; $p=0.025$ ) and vitamin A equivalents $(p=0.009)$, where Asian mothers consumed significantly higher amounts than Māori and Pacific Island and NZ European mothers.

The association between specific dietary intakes of nutrients and breast milk composition was analyzed by Spearman rank-correlation (Figure 2). There were positive associations with breast milk concentrations of omega $6(n-6)$ and PUFAs, and linoleic acid with polyunsaturated and monounsaturated fat consumption. Trans-fatty acid concentrations in breast milk were positively correlated with saturated fat intakes. Breast milk magnesium was positively associated with dietary magnesium intake as well as carbohydrate, energy, iodine, caffeine, iron, fiber, folate, and potassium dietary intake.

To further understand the dietary sources of nutrients eaten by the mothers, we examined the number of serves per day of the main food groups (Table 7) and found that these were similar across the ethnic groups-except for dairy products where NZ European mothers consumed significantly $(p=0.009)$ more serves. The numbers of serves of protein and fatty acid rich foods consumed by the mothers from the different ethnic groups were similar for lamb, beef, pork, fish, egg, and nuts (Table 8). Asian mothers, however, ate significantly $(p=0.036)$ more serves of chicken than Māori and Pacific Island mothers and more serves $(p=0.027)$ of legumes than NZ European mothers.

The percent recommended daily intake (RDI) of key nutrients are shown in Table 9. Recommended daily intake is the average amount of each nutrient that meets the daily needs of healthy people at a particular age, metabolic status (e.g., pregnant, lactating), and gender. For all the mothers in the study, the percent daily intakes for folate, selenium, iodine, and molybdenum were lower than the recommended levels for lactating mothers. Iodine intake for the Asian, Māori and Pacific Island, and NZ European mothers was particularly low at 53\%, 23\%, and 30\%, respectively, 
of the recommended intake. In addition, Māori and Pacific Island mothers consumed less energy, protein, vitamin $\mathrm{B}_{6}$, vitamin $\mathrm{A}$, calcium, and zinc; Asian mothers consumed less calcium, and NZ European mothers consumed less energy, vitamin $B_{6}$, and vitamin $A$ than recommended. There were significant differences in the percent RDI between mothers of different ethnicity for protein, vitamin C, vitamin A, calcium, phosphorus, and iodine. Asian mothers consumed a significantly higher percentage RDI's for vitamin C $(p=0.016)$, vitamin A $(p=0.002)$, and iodine $(p=0.010)$. Māori and Pacific Island mother's protein intake was the lowest $(p=0.003)$, and NZ European mothers consumed the highest RDI's for calcium $(p=0.012)$ and phosphorus $(p=0.017)$. Some mothers in the study consumed supplements (Table 10), which could have improved their \%RDI's from those calculated from their diet records. Multivitamin, iodine and iron supplements were the most frequently taken dietary supplements. There were no significant differences $(p>0.05)$ in supplement consumption by the mothers of different ethnicity.

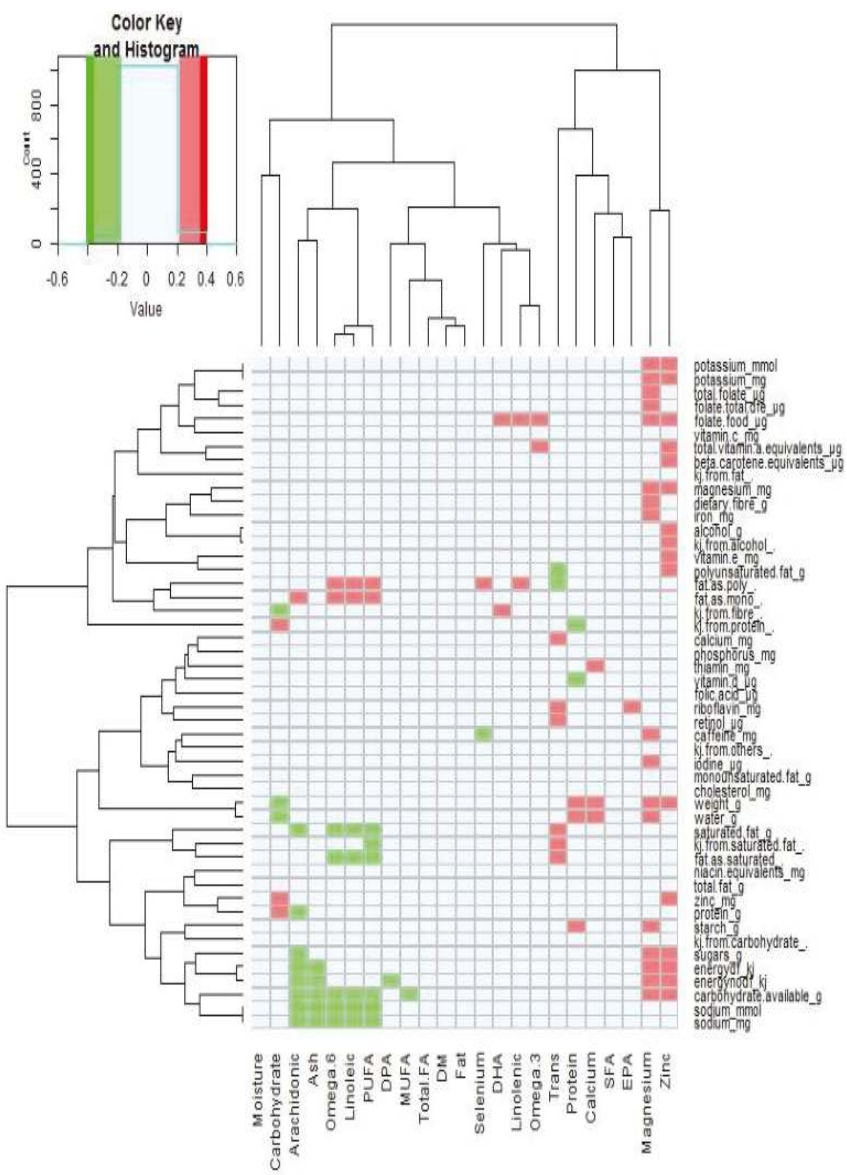

Figure 2. Spearman's rank-correlations between mother's dietary intake and breast milk nutrients. PUFAs, Polyunsaturated fatty acids; DPA, Docosapentaenoic acid; MUFA, Monounsaturated fatty acids; FA, fatty acid; DM, dry matter; DHA, Docosahexaenoic acid; SFA, Saturated fatty acids; and EPA, Eicosapentaenoic acid. 


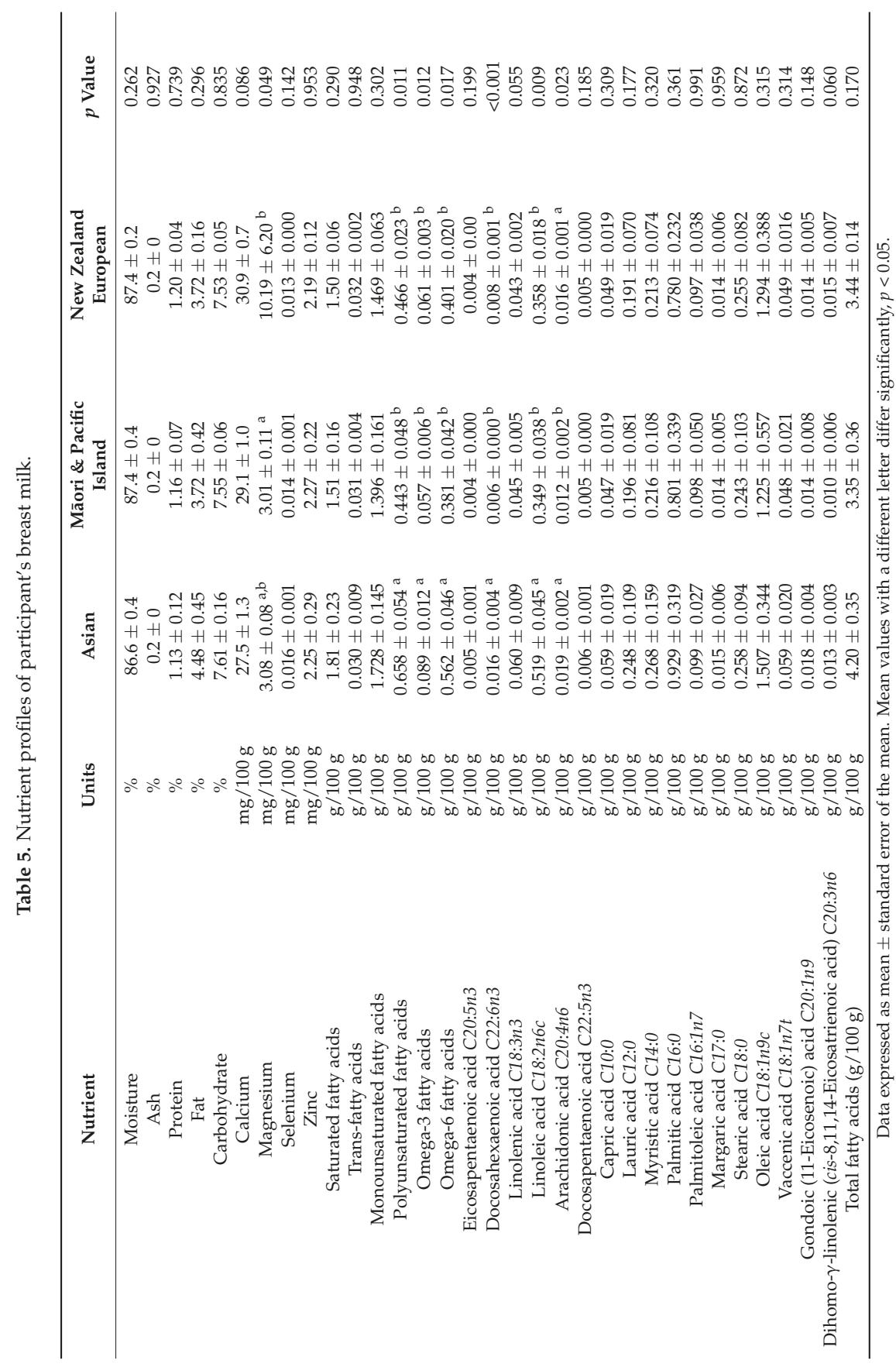


Table 6. Nutrient intakes of the participants.

\begin{tabular}{|c|c|c|c|c|}
\hline & Asian & Māori \& Pacific Island & New Zealand European & $p$ Value \\
\hline Food weight $(\mathrm{g})$ & $3615 \pm 369$ & $2771 \pm 254$ & $3656 \pm 263$ & 0.175 \\
\hline Energy (no dietary fibre) (kJ) & $9732 \pm 1159$ & $8762 \pm 574$ & $9940 \pm 282$ & 0.178 \\
\hline Energy dietary fibre (kJ) & $10,008 \pm 1208$ & $8979 \pm 586$ & $10,124 \pm 285$ & 0.207 \\
\hline Protein $(\mathrm{g})$ & $85.4 \pm 6.3^{\mathrm{a}, \mathrm{b}}$ & $82.5 \pm 5.0^{\mathrm{a}}$ & $97.8 \pm 3.0^{\mathrm{b}}$ & 0.023 \\
\hline Total fat (g) & $100.3 \pm 14.0$ & $88.9 \pm 6.0$ & $99.2 \pm 3.8$ & 0.407 \\
\hline Saturated fat (g) & $34.6 \pm 9.2$ & $37.3 \pm 2.3$ & $41.7 \pm 1.7$ & 0.231 \\
\hline Polyunsaturated fat (g) & $16.4 \pm 2.5$ & $12.8 \pm 1.3$ & $12.7 \pm 1.0$ & 0.338 \\
\hline Monounsaturated fat (g) & $38.9 \pm 6.0$ & $32.7 \pm 2.8$ & $35.7 \pm 1.7$ & 0.471 \\
\hline Cholesterol (mg) & $313 \pm 32$ & $286 \pm 31$ & $301 \pm 15$ & 0.831 \\
\hline Carbohydrate available (g) & $275 \pm 36$ & $244 \pm 19$ & $272 \pm 10$ & 0.412 \\
\hline Sugars $(\mathrm{g})$ & $111 \pm 24$ & $105 \pm 9$ & $128 \pm 6$ & 0.132 \\
\hline Starch $(g)$ & $164 \pm 14$ & $139 \pm 11$ & $144 \pm 6$ & 0.408 \\
\hline Water (g) & $3069 \pm 348$ & $2300 \pm 235$ & $3116 \pm 266$ & 0.221 \\
\hline Alcohol (g) & $0.13 \pm 0.08$ & $0.74 \pm 0.51$ & $2.19 \pm 1.05$ & 0.555 \\
\hline Dietary fibre (g) & $33.58 \pm 5.80$ & $25.96 \pm 2.01$ & $27.13 \pm 1.18$ & 0.155 \\
\hline Thiamine (mg) & $1.56 \pm 0.25$ & $1.92 \pm 0.19$ & $1.75 \pm 0.10$ & 0.507 \\
\hline Riboflavin (mg) & $2.14 \pm 0.32$ & $1.95 \pm 0.14$ & $2.31 \pm 0.11$ & 0.227 \\
\hline Niacin equivalents (mg) & $37.25 \pm 3.54$ & $35.44 \pm 2.61$ & $42.44 \pm 2.05$ & 0.150 \\
\hline Vitamin C (mg) & $156.80 \pm 25.01$ & $87.90 \pm 18.03$ & $118.70 \pm 9.10$ & 0.060 \\
\hline Vitamin D ( $\mu \mathrm{g})$ & $3.83 \pm 1.07$ & $3.50 \pm 0.52$ & $4.42 \pm 0.49$ & 0.576 \\
\hline Vitamin E (mg) & $13.37 \pm 2.31$ & $9.97 \pm 0.82$ & $11.91 \pm 1.06$ & 0.451 \\
\hline Total folate $(\mu \mathrm{g})$ & $421.30 \pm 68.69$ & $359.10 \pm 36.03$ & $348.40 \pm 16.17$ & 0.357 \\
\hline Folic acid $(\mu \mathrm{g})$ & $28.14 \pm 13.47$ & $87.27 \pm 23.03$ & $65.97 \pm 9.50$ & 0.173 \\
\hline Folate food $(\mu \mathrm{g})$ & $395 \pm 67^{\mathrm{a}}$ & $273 \pm 25^{b}$ & $285 \pm 13^{b}$ & 0.025 \\
\hline Folate, total dietary folate equivalents $(\mu \mathrm{g})$ & $440 \pm 72$ & $418 \pm 49$ & $393 \pm 20$ & 0.693 \\
\hline Total Vitamin A equivalents $(\mu \mathrm{g})$ & $1583 \pm 370^{a}$ & $937 \pm 80^{b}$ & $988 \pm 63^{b}$ & 0.009 \\
\hline Retinol $(\mu \mathrm{g})$ & $668 \pm 309$ & $339 \pm 32$ & $427 \pm 28$ & 0.066 \\
\hline Beta-carotene equivalents $(\mu \mathrm{g})$ & $5483 \pm 1880$ & $3584 \pm 462$ & $3389 \pm 312$ & 0.118 \\
\hline Sodium $(\mathrm{mg})$ & $3138 \pm 483$ & $2914 \pm 235$ & $2889 \pm 130$ & 0.804 \\
\hline Sodium (mmol) & $137 \pm 21$ & $127 \pm 10$ & $126 \pm 6$ & 0.804 \\
\hline Potassium (mg) & $3609 \pm 574$ & $2971 \pm 203$ & $3551 \pm 174$ & 0.218 \\
\hline Potassium (mmol) & $92 \pm 15$ & $76 \pm 5$ & $91 \pm 4$ & 0.218 \\
\hline Magnesium (mg) & $406 \pm 68$ & $318 \pm 24$ & $401 \pm 22$ & 0.128 \\
\hline Calcium (mg) & $736 \pm 162^{a}$ & $758 \pm 56^{\mathrm{a}}$ & $1041 \pm 53^{b}$ & 0.007 \\
\hline Phosphorus (mg) & $1489 \pm 170^{\mathrm{a}, \mathrm{b}}$ & $1356 \pm 84^{a}$ & $1648 \pm 53^{b}$ & 0.024 \\
\hline Iron $(\mathrm{mg})$ & $16.1 \pm 1.9$ & $13.3 \pm 1.0$ & $14.8 \pm 0.7$ & 0.328 \\
\hline Zinc (mg) & $11.0 \pm 0.9^{\mathrm{a}, \mathrm{b}}$ & $10.7 \pm 0.7^{\mathrm{a}}$ & $13.1 \pm 0.5^{b}$ & 0.029 \\
\hline Iodine $(\mu \mathrm{g})$ & $133.1 \pm 56.4^{\mathrm{a}}$ & $61.1 \pm 5.7^{b}$ & $80.0 \pm 5.8^{b}$ & 0.027 \\
\hline $\mathrm{KJ}$ from protein $(\%)$ & $15.3 \pm 1.1$ & $15.8 \pm 0.5$ & $16.6 \pm 0.4$ & 0.346 \\
\hline $\mathrm{KJ}$ from fat $(\%)$ & $36.4 \pm 2.2$ & $36.6 \pm 1.1$ & $36.1 \pm 0.8$ & 0.956 \\
\hline $\mathrm{KJ}$ from saturated fat (\%) & $11.9 \pm 1.6^{\mathrm{a}}$ & $15.0 \pm 0.4^{b}$ & $15.2 \pm 0.4^{b}$ & 0.019 \\
\hline KJ from carbohydrate $(\%)$ & $45.8 \pm 2.4$ & $44.9 \pm 1.3$ & $44.2 \pm 0.9$ & 0.753 \\
\hline KJ from alcohol (\%) & $0.03 \pm 0.02$ & $0.21 \pm 0.14$ & $0.62 \pm 0.30$ & 0.561 \\
\hline KJ from fibre $(\%)$ & $2.55 \pm 0.24$ & $2.32 \pm 0.12$ & $2.14 \pm 0.07$ & 0.104 \\
\hline KJ from others (\%) & $0.12 \pm 0.11$ & $0.19 \pm 0.05$ & $0.22 \pm 0.03$ & 0.531 \\
\hline Fat as monounsaturated (\%) & $44.2 \pm 3.4^{\mathrm{a}}$ & $39.0 \pm 1.2^{b}$ & $39.5 \pm 0.7^{b}$ & 0.042 \\
\hline Fat as polyunsaturated (\%) & $18.6 \pm 1.8^{a}$ & $15.7 \pm 1.6^{\mathrm{a}, \mathrm{b}}$ & $13.9 \pm 0.6^{b}$ & 0.026 \\
\hline Fat as saturated $(\%)$ & $37.2 \pm 4.7^{a}$ & $45.3 \pm 1.6^{b}$ & $46.7 \pm 1.1^{b}$ & 0.010 \\
\hline Caffeine (mg) & $13.9 \pm 5.8$ & $41.0 \pm 10.8$ & $118.5 \pm 28.8$ & 0.122 \\
\hline
\end{tabular}

Data expressed as mean \pm standard error of the mean. Mean values with a different letter differ significantly, $p<0.05$. kJ-kilojoules.

Table 7. Number of food serves per day consumed by the participants.

\begin{tabular}{ccccc}
\hline & Asian & Māori \& Pacific Island & New Zealand European & $p$ Value \\
\hline Fruit & $2.48 \pm 0.68$ & $1.56 \pm 0.55$ & $1.48 \pm 0.15$ & 0.226 \\
Vegetables & $1.72 \pm 0.46$ & $2.38 \pm 0.29$ & $2.28 \pm 0.17$ & 0.437 \\
Whole grains & $2.88 \pm 1.27$ & $1.98 \pm 0.34$ & $1.84 \pm 0.20$ & 0.300 \\
Meat and fish & $1.21 \pm 0.22$ & $1.54 \pm 0.13$ & $1.58 \pm 0.08$ & 0.258 \\
Egg & $0.38 \pm 0.12$ & $0.27 \pm 0.08$ & $0.33 \pm 0.06$ & 0.849 \\
Dairy & $0.85 \pm 0.26^{\mathrm{a}}$ & $1.09 \pm 0.17^{\mathrm{a}}$ & $1.66 \pm 0.13^{\mathrm{b}}$ & 0.009 \\
Nuts and legumes & $0.88 \pm 0.25$ & $0.28 \pm 0.11$ & $0.38 \pm 0.10$ & 0.107 \\
\hline
\end{tabular}

Data expressed as mean \pm standard error of the mean. Mean values with a different letter differ significantly, $p<0.05$. 
Table 8. Number of serves per day of foods rich in protein and fats consumed by the participants.

\begin{tabular}{ccccc}
\hline & Asian & Māori \& Pacific Island & New Zealand European & $p$ Value \\
\hline Lamb & $0.11 \pm 0.11$ & $0.49 \pm 0.19$ & $0.24 \pm 0.09$ & 0.231 \\
Beef & $0.67 \pm 0.36$ & $1.27 \pm 0.37$ & $1.77 \pm 0.20$ & 0.070 \\
Pork & $0.44 \pm 0.29$ & $1.58 \pm 0.39$ & $1.01 \pm 0.18$ & 0.082 \\
Chicken & $1.64 \pm 0.33^{\mathrm{a}}$ & $0.54 \pm 0.23^{\mathrm{b}}$ & $1.19 \pm 0.19^{\mathrm{a}, \mathrm{b}}$ & 0.036 \\
Fish & $0.90 \pm 0.30$ & $0.40 \pm 0.24$ & $0.58 \pm 0.12$ & 0.112 \\
Egg & $1.38 \pm 0.32$ & $1.27 \pm 0.38$ & $0.95 \pm 0.19$ & 0.255 \\
Legumes & $1.75 \pm 0.74^{\mathrm{a}}$ & $0.48 \pm 0.18^{\mathrm{a}, \mathrm{b}}$ & $0.29 \pm 0.09 \mathrm{~b}$ & 0.027 \\
Nuts & $0.55 \pm 0.23$ & $0.67 \pm 0.29$ & $0.72 \pm 0.25$ & 0.394 \\
\hline
\end{tabular}

Data expressed as mean \pm standard error of the mean. Mean values with a different letter differ significantly, $p<0.05$.

Table 9. Recommended daily intake (\%) of the participants.

\begin{tabular}{ccccc}
\hline & Asian & Māori \& Pacific Island & New Zealand European & $p$ Value \\
\hline Energy & $107 \pm 15$ & $85 \pm 6$ & $96 \pm 3$ & 0.093 \\
Protein & $145 \pm 18^{\mathrm{a}}$ & $96 \pm 8^{\mathrm{b}}$ & $122 \pm 4^{\mathrm{a}}$ & 0.003 \\
Thiamine & $116 \pm 18$ & $137 \pm 14$ & $124 \pm 7$ & 0.570 \\
Riboflavin & $148 \pm 32$ & $122 \pm 9$ & $143 \pm 7$ & 0.292 \\
Niacin & $225 \pm 21$ & $208 \pm 15$ & $247 \pm 12$ & 0.208 \\
Vitamin C & $209 \pm 41^{\mathrm{a}}$ & $104 \pm 21^{\mathrm{b}}$ & $92 \pm 5$ & 0.016 \\
Vitamin B6 & $111 \pm 17$ & $88 \pm 8$ & $140 \pm 38$ & 0.303 \\
Vitamin B12 & $118 \pm 30$ & $202 \pm 94$ & $78 \pm 4$ & 0.786 \\
Folate, total dietary folate equivalents & $92 \pm 17^{\mathrm{b}}$ & $83 \pm 10$ & $89 \pm 6^{\mathrm{b}}$ & 0.496 \\
Total Vitamin A equivalents & $154 \pm 35^{\mathrm{a}}$ & $84 \pm 7^{\mathrm{b}}$ & $126 \pm 7$ & 0.002 \\
Magnesium & $129 \pm 22$ & $99 \pm 7$ & $104 \pm 5^{\mathrm{b}}$ & 0.111 \\
Calcium & $74 \pm 16^{\mathrm{a}}$ & $78 \pm 6^{\mathrm{a}}$ & $164 \pm 5^{\mathrm{b}}$ & 0.012 \\
Phosphorus & $149 \pm 17^{\mathrm{a}, \mathrm{b}}$ & $132 \pm 9^{\mathrm{a}}$ & $163 \pm 7$ & 0.017 \\
Iron & $164 \pm 21$ & $147 \pm 11$ & $108 \pm 4$ & 0.523 \\
Zinc & $99 \pm 12^{2}$ & $94 \pm 6$ & $70 \pm 4$ & 0.228 \\
Selenium & $68 \pm 12^{\mathrm{a}}$ & $62 \pm 6$ & $30 \pm 2^{\mathrm{b}}$ & 0.662 \\
Iodine & $53 \pm 21^{\mathrm{a}}$ & $23 \pm 2^{\mathrm{b}}$ & $74 \pm 4$ & 0.010 \\
Molybdenum & $80 \pm 12^{\mathrm{b}}$ & $90 \pm 11$ & 0.219 \\
\hline
\end{tabular}

Data expressed as mean \pm standard error of the mean. Mean values with a different letter differ significantly, $p<0.05$.

Table 10. Number of participants taken dietary supplements.

\begin{tabular}{ccccc}
\hline & Asian & Māori \& Pacific Island & New Zealand European & $p$ Value \\
\hline Total supplements & 7 & 7 & 36 & 0.065 \\
Multivitamin & 3 & 3 & 8 & 0.361 \\
Iodine & 2 & 4 & 22 & 0.351 \\
Iron & 4 & 4 & 13 & 0.342 \\
Vitamin C & 1 & 1 & 11 & 0.405 \\
Fish oil & 2 & 1 & 5 & 0.342 \\
Probiotics & 2 & 0 & 4 & 0.099 \\
Other & 2 & 2 & 12 & 0.684 \\
\hline
\end{tabular}

\section{Discussion}

This study is the first to measure and compare breast milk composition and nutrient intakes from an ethnically representative proportion of NZ mothers. We found that the breast milk nutrient profiles of women from different ethnicities were similar in their macronutrient composition, but there were differences in the concentrations of some fatty acids and magnesium. Dietary intakes were different for protein, total energy, saturated and polyunsaturated fat, calcium, phosphorus, zinc, iodine, vitamin A equivalents, and folate. The serves of dairy products, chicken and legumes consumed by the mothers were different between the ethnic groups. There were weak positive associations with breast milk concentrations of some fatty acids and magnesium with dietary fatty acid and magnesium intakes. 
Our study population was representative of the main ethnic groups present in NZ. Recent census figures [28], reported that $74.0 \%$ of the NZ population identifies themselves as Europeans, $11.8 \%$ as Asian and $22.3 \%$ as Māori and Pacific Island. This is very similar to the proportions in our study population: 68\% NZ European, 10\% Asian, and 22\% Māori and Pacific Island. Other demographic characteristics of the participants were also similar across the different ethnicities. Categorization of the participants' BMI was also representative of the NZ population with $40 \%$ classified as overweight and $30 \%$ as obese - reflecting the results reported by the NZ Ministry of Health [33] of 35\% overweight and $30 \%$ obese. While the Māori and Pacific Island participants had significantly higher body weights and BMI in the present study, the actual values were lower or similar to those reported (BMI 28.7 vs. 32.8) in a recent national health survey [33]; as were the BMI's for Asian (BMI 22.5 vs. 24.4) and NZ European (BMI 27.2 vs. 27.9) participants. Weight gain during pregnancy is normal due to the growth of the fetus, placenta, and amniotic fluid [34], and postpartum weight loss may be influenced by infant nursing mode [35]. In normal weight mothers, the gestational weight gain has been found to be approximately $13 \mathrm{~kg}$ [36], and weight loss has been reported to be variable with between 8 and $9 \mathrm{~kg}$ at 1 month postpartum and 4 and $11 \mathrm{~kg}$ at 3 months postpartum [35,37]. Gestational weight gain is associated with ethnicity, socio-demographic, lifestyle, and pregnancy characteristics within populations but which of these factors is predominant is unknown [38]. We weighed the mothers in the present study at six weeks postpartum when postpartum weight loss may not be completed.

The macronutrient composition of human milk is known to vary within mothers and during lactation, and yet it is conserved across populations despite variations in maternal nutritional status $[39,40]$. We found no statistically significant differences in the macronutrient concentrations in the breast milk of NZ women of different ethnicity. Breast milk samples collected in this study had similar protein $(1.2 \%)$, carbohydrate $(7.5 \%)$, and fat $(3.8 \%)$ concentrations to those reported in the literature for mature hind milk [6,8,40-42]. Lipids can be the most variable macronutrient of human breast milk. For example, hind milk, defined as the last milk of a feed, may contain higher concentrations (4.79-6.07 g/100 mL) of milk fat than that found in foremilk (1.14-2.63 g/100 mL), defined as the initial milk of a feed [43]. Milk fat content has also been reported to be significantly lower in night (37.2 g/L; 10:01 pm to 4:00 am) and morning (37.1 g/L; 4:01 am to 10:00 am) feed samples than those from day (42.8 g/L; 10:01 am to 4:00 pm) and evening (43.2 g/L; 4:01 to 10:00 pm) feeds [21,44]. Total fat, dry matter, and energy contents of human milk are also known to increase markedly during the feed (water content decreases accordingly) as the breast is emptied [45]. The breast milk samples in the present study were collected from the first feed of the day (first feed after sunrise) and after the baby was fed and were, therefore, samples of hind milk. The mean fat content of 3.79\% found in the present study is within the ranges for hind milk and milk collected in the morning when milk fat content is lower [44].

Calcium, phosphorous and magnesium concentrations in maternal serum are tightly regulated and it has been reported that there is little effect of maternal dietary intake of these minerals on their concentrations in human milk [46,47]. The mean concentrations of calcium and magnesium in mature milk reported in the literature are approximately $280 \mathrm{mg} / \mathrm{L}$ and $35 \mathrm{mg} / \mathrm{L}$, respectively [46,48,49]. The observed concentrations of calcium and magnesium in breast milk reported here are in agreement with these values. However, we found that the mean magnesium concentration of breast milk from NZ European mothers was significantly higher than for the Asian and Māori and Pacific Island mothers, though there was not a statistically significant difference in dietary magnesium intake between ethnicities. We did observe a weak positive association with breast milk magnesium content and dietary intake which is in contrast to the literature $[46,47]$ and may warrant further investigation.

The mean concentrations of zinc and selenium in the breast milk collected in the present study were $2.21 \mathrm{mg} / \mathrm{kg}$ and $0.014 \mathrm{mg} / \mathrm{kg}$, respectively, and there were no significant differences between mothers of different ethnicity. Zinc concentrations in human milk decrease over lactation and steeply decline over the first month of lactation from that found in colostrum (>10 mg/L) and then gradually to $0.5 \mathrm{mg} / \mathrm{L}$ by the twelfth month of lactation (Casey 1989). The dietary intake of zinc has mostly been 
reported in the literature as having little impact on the concentrations found in breast milk $[14,48,50]$. Two studies, however, reported that zinc supplementation may influence zinc concentration in late lactation [51,52], which is in agreement for the positive association found here (Figure 2). The selenium concentrations in mature breast milk have been reported to be between 10-30 $\mu \mathrm{g} / \mathrm{L}$ [53], with higher concentrations found at the initiation of lactation $(41 \mu \mathrm{g} / \mathrm{L})$ and decreasing as lactation progresses [54]. Worldwide, there are major differences in the selenium content of soils and therefore in the food supply [15], and NZ has one of the lowest estimated adult selenium intakes and blood serum concentrations in the world [55]. Rural African women's selenium breast milk concentrations were low when their dietary selenium intakes were low [56]. In contrast, Debski et al. [57] reported that the selenium breast milk concentrations of lacto-ovo-vegetarian women $(22.2 \mathrm{ng} / \mathrm{mL})$ were greater than that of non-vegetarian women $(16.8 \mathrm{ng} / \mathrm{mL})$, but there was no significant differences in selenium intake between the two groups. We found no significant differences in breast milk selenium concentrations between the mothers of different ethnicity in the present study. No selenium dietary intake data are reported here as this data was not available for dietary analysis.

The composition of human milk has been observed to be consistent across ethnicities and countries in many parameters [58], but it is also known to be influenced by diet and particularly by intakes of fatty acids [14]. In the present study, we found that levels of PUFAs, $n-3$ and $n-6$ fatty acids, docosahexaenoic acid, and linoleic acid in the breast milk of Asian women were significantly higher compared to the other two ethnicities. While the intakes of the different types of dietary fat (monounsaturated, polyunsaturated, saturated) were similar between the different ethnic groups, the Asian women consumed fewer saturated fat and the proportion of dietary monounsaturated and polyunsaturated fats of total fat consumed was higher. This is supported by the lower number of dairy serves (higher in saturated fats) and higher number of serves of chicken (higher in polyunsaturated $n-6$ fatty acids) observed here in the Asian mothers. A similar result was observed for $n-6$ fatty acid contents in the breast milk of rural African women who consumed little animal fat [59].

Studies linking diet and breast milk fatty acid contents have not shown consistent results. Su et al. [60] found differences in breast milk fatty acid content between ethnicities, but the dietary intakes of $n-3$ and n-6 PUFAs for the different ethnicities were similar. Glew et al. [61] found no correlation between dietary intakes of $\alpha$-linoleic acid and DHA and the amounts of these fatty acids in the breast milk of women from New Mexico. In contrast, a study in South Korea found that the dietary intakes of eicosapentaenoic acid (EPA), docosahexaenoic acid (DHA), omega 3 (n-3) fatty acids, omega 6 (n-6) fatty acids, saturated fatty acids (SFAs), and polyunsaturated fatty acids (PUFAs) were highly positively correlated, with the corresponding fatty acids in the breast milk samples [62], while a study in China found that dietary intakes and breast milk content of long chain $n-3$ PUFAs and linolenic were positively correlated [63]. Furthermore, other studies have shown that women who consume fish and other foods containing high levels of PUFA have relatively higher breast milk $n-3$ fatty acids and DHA concentrations compared to milk from women who consume diets that are low in these components $[59,64,65]$. In our study, the consumption of monounsaturated and polyunsaturated fats and fish were similar between the three ethnic groups. There were, however, correlations between dietary kilojoules from saturated fat, and $n-6$, linoleic and PUFAs in the breast milk. Trans-fatty acids in the milk were positively correlated with dietary saturated fat intake, and negatively correlated with polyunsaturated fat intake. Fatty acids in human milk are sourced not only from dietary fat but are also mobilized from maternal body fat and synthesized in the milk glands and hepatic cells. Therefore, the fatty acids found in human milk are likely to be influenced by short term and long term fatty acid dietary intake. The lack of consistency on the effect of dietary fatty acid intake on breast milk fatty acid composition in the literature is likely due to the collection of only short term fatty acid intake data, and not long term intakes, and the complex metabolic interdependencies between dietary and milk fatty acids.

The main strength of our study is that the breast milk nutrient composition and dietary nutrient intakes has been measured in NZ mothers of different ethnicity for the first time. 
A strength and a limitation of this study is that our participant population was in only one region (Manawatū-Whanganui) of New Zealand. While the ethnic composition of our study population was similar to that found in the overall population of NZ, the study region included urban and rural areas but no major cities where diet and lifestyle could be different. The second limitation is the collection of breast milk after the infant had fed, as the time of milk collection is known to affect the measurement of the breast milk fat content where the concentration differs between the beginning and end of feeding and over the day night cycle. The breast milk samples in the present study comprised $1.2 \%$ protein, $3.8 \%$ fat, and $7.5 \%$ total carbohydrate, which is very similar to the data from mature breast milk $(\mathrm{g} / \mathrm{dL}$; protein 0.9-1.2, fat 3.2-3.6, lactose 7.2-7.8) collected from a number of studies reviewed by Ballard and Morrow [40]. The third limitation is the quantity of milk collected at each sampling (30 mL), which limited the quantity and therefore the range of nutrients that could be analyzed. This timing and quantity of breast milk collection were selected to ensure the infant had been fed and the infant's and mother's welfare were not compromised by the breast milk sampling.

\section{Conclusions}

We found that the nutrient composition of breast milk differed between ethnic groups for PUFAs, $n-3, n-6$, DHA, linoleic and arachidonic fatty acids and the mineral magnesium. Dietary intakes of protein, total energy, saturated and polyunsaturated fat, calcium, phosphorus, zinc, iodine, vitamin A equivalents, and folate differed between the ethnic groups, as well as the number of serves of dairy foods, chicken, and legumes. There were positive associations between breast milk concentrations of $n-6$, polyunsaturated and linoleic acid with dietary polyunsaturated and monounsaturated fats. The percent daily dietary intakes of folate, selenium, iodine, and molybdenum for the mothers in this study were less than that recommended for lactating women, which may negatively affect the health of these mothers and their infants. Additional dietary advice from health professionals such as midwives, registered nutritionists, and dietitians for pregnant and lactating mothers may improve their nutrient intakes ensuring the on-going health and well-being of NZ mothers and their babies.

Author Contributions: Data curation, T.D.H. and G.P.; Formal analysis, C.A.B., D.I.H. and G.P.; Investigation, C.A.B. and T.D.H.; Methodology, C.A.B., T.D.H. and G.P.; Project administration, C.A.B., S.G.-J. and P.G.; Resources, C.A.B.; Visualization, G.P.; Writing-original draft, C.A.B., D.I.H., T.D.H., P.G., and G.P.; Writing-review \& editing, C.A.B., D.I.H., G.P., S.G.-J., F.W., B.S. and P.G.

Funding: We thank Nutricia New Zealand for funding this study.

Acknowledgments: We thank Juliet Ansell, Greg Ward and Berneace Steffens for the initial study design and plan. Our thanks to Alison Wallace and Sarah Eady for their contributions to the project scope and study design. We are grateful to Sheridan Martell and Hannah Dinnan for recruiting the study participants and encouraging and supporting the mothers to complete the questionnaires and collect the samples. We are grateful to Shila Shafaeizadeh and Leilani L. Muhardi of Nutricia Research Singapore for their comments on early versions of this manuscript.

Conflicts of Interest: The authors, C.A.B., D.I.H., T.D.H., G.P. and P.G. declare no conflict of interest. S.G.-J., F.W., and B.S. provided guidance and support to the presentation and writing of the manuscript.

\section{References}

1. Lönnerdal, B. Breast milk: A truly functional food. Nutrition 2000, 16, 509-511. [CrossRef]

2. Field, C.J. The immunological components of human milk and their effect of immune development in infants. J. Nutr. 2005, 135, 1-4. [CrossRef] [PubMed]

3. Victora, C.G.; Bahl, R.; Barros, A.J.D.; França, G.V.A.; Horton, S.; Krasevec, J.; Murch, S.; Sankar, M.J.; Walker, N.; Rollins, N.C. Breastfeeding in the 21st century: Epidemiology, mechanisms, and lifelong effect. Lancet 2016, 387, 475-490. [CrossRef]

4. Marseglia, L.; Manti, S.; D’Angelo, G.; Cuppari, C.; Salpietro, V.; Filippelli, M.; Trovato, A.; Gitto, E.; Salpietro, C.; Arrigo, T. Obesity and breastfeeding: The strength of association. Women Birth 2015, 28, 81-86. [CrossRef] [PubMed] 
5. Manti, S.; Lougaris, V.; Cuppari, C.; Tardino, L.; Dipasquale, V.; Arrigo, T.; Salpietro, C.; Leonardi, S. Breastfeeding and IL-10 levels in children affected by cow's milk protein allergy: A restrospective study. Immunobiology 2017, 222, 358-362. [CrossRef] [PubMed]

6. World Health Organization. 10 Facts on Breastfeeding. Available online: http://www.who.int/features/ factfiles/breastfeeding/en/ (accessed on 22 February 2018).

7. National Breastfeeding Advisory Committee of New Zealand. National Strategic Plan of Action for Breastfeeding 2008-2012: National Breastfeeding Advisory Committee of New Zealand's Advice to the Director-General of Health; Ministry of Health: Wellington, The New Zealand, 2009. Available online: http://www.moh.govt.nz (accessed on 20 February 2018).

8. Michaelsen, K.F.; Skafte, L.; Badsberg, J.H.; Jorgensen, M. Variation in macronutrients in human bank milk: Influencing factors and implications for human-milk banking. J. Pediatr. Gastroenterol. Nutr. 1990, 11, 229-239. [CrossRef] [PubMed]

9. Sauer, C.W.; Boutin, M.A.; Kim, J.H. Wide Variability in Caloric Density of Expressed Human Milk Can Lead to Major Underestimation or Overestimation of Nutrient Content. J. Hum. Lact. 2017, 33, 341-350. [CrossRef] [PubMed]

10. Fujita, M.; Roth, E.; Lo, Y.-J.; Hurst, C.; Vollner, J.; Kendell, A. In poor families, mothers' milk is richer for daughters than sons: A test of Trivers-Willard hypothesis in agropastoral settlements in Northern Kenya. Am. J. Phys. Anthropol. 2012, 149, 52-59. [CrossRef] [PubMed]

11. Hinde, K.; German, J.B. Food in an evolutionary context: Insights from mother's milk. J. Sci. Food Agric. 2012, 92, 2219-2223. [CrossRef] [PubMed]

12. Morrow, A.L.; Ruiz-Palacios, G.M.; Altaye, M.; Jiang, X.; Lourdes Guerrero, M.; Meinzen-Derr, J.K.; Farkas, T.; Chaturvedi, P.; Pickering, L.K.; Newburg, D.S. Human milk oligosaccharides are associated with protection against diarrhea in breast-fed infants. J. Pediatr. 2004, 145, 297-303. [CrossRef] [PubMed]

13. Prentice, A.; Prentice, A.M.; Whitehead, R.G. Breast-milk fat concentrations of rural african women: 1. Short-term variations within individuals. Br. J. Nutr. 1981, 45, 483-494. [CrossRef] [PubMed]

14. Bravi, F.; Wiens, F.; Decarli, A.; Dal Pont, A.; Agostoni, C.; Ferraroni, M. Impact of maternal nutrition on breast-milk composition: A systematic review. Am. J. Clin. Nutr. 2016, 104, 646-662. [CrossRef] [PubMed]

15. Zachara, B.A.; Pilecki, A. Selenium concentration in the milk of breast-feeding mothers and its geographic distribution. Environ. Health Perspect. 2000, 108, 1043-1046. [CrossRef] [PubMed]

16. Ameur, A.; Enroth, S.; Johansson, Å.; Zaboli, G.; Igl, W.; Johansson, A.C.; Rivas, M.A.; Daly, M.J.; Schmitz, G.; Hicks, A.A.; et al. Genetic Adaptation of Fatty-Acid Metabolism: A Human-Specific Haplotype Increasing the Biosynthesis of Long-Chain Omega-3 and Omega-6 Fatty Acids. Am. J. Hum. Genet. 2012, 90, 809-820. [CrossRef] [PubMed]

17. Yang, T.; Zhang, L.S.; Bao, W.; Rong, S. Nutritional composition of breast milk in Chinese women: A systematic review. Asia Pac. J. Clin. Nutr. 2018, 27, 491-502. [PubMed]

18. Fu, Y.Q.; Liu, X.; Zhou, B.; Jiang, A.C.; Chai, L.Y. An updated review of worldwide levels of docosahexaenoic and arachidonic acid in human breast milk by region. Public Health Nutr. 2016, 19, 2675-2687. [CrossRef] [PubMed]

19. Su, M.Y.; Jia, H.X.; Chen, W.L.; Qi, X.Y.; Liu, C.P.; Liu, Z.M. Macronutrient and micronutrient composition of breast milk from women of different ages and dietary habits in Shanghai area. Int. Dairy J. 2018, 85, 27-34. [CrossRef]

20. Deem, H.E. Effect of diet on human milk secretion. Br. Med. J. 1935, 1935, 80-81. [CrossRef]

21. Deem, H.E. Observations on the milk of New Zealand Women. Arch. Dis. Child. 1931, 6, 53-70. [CrossRef] [PubMed]

22. Bates, M.N.; Hannah, D.J.; Buckland, S.J.; Taucher, J.A.; Vanmaanen, T. Chlorinated organic contaminants in breast-milk of new-zealand women. Environ. Health Perspect. 1994, 102, 211-217. [CrossRef] [PubMed]

23. Brough, L.; Jin, Y.; Shukri, N.H.; Wharemate, Z.R.; Weber, J.L.; Coad, J. Iodine intake and status during pregnancy and lactation before and after government initiatives to improve iodine status, in Palmerston North, New Zealand: A pilot study. Matern. Child Nutr. 2015, 11, 646-655. [CrossRef] [PubMed]

24. Skeaff, S.A.; Ferguson, E.L.; McKenzie, J.E.; Valeix, P.; Gibson, R.S.; Thomson, C.D. Are breast-fed infants and toddlers in New Zealand at risk of iodine deficiency? Nutrition 2005, 21, 325-331. [CrossRef] [PubMed]

25. Mulrine, H.M.; Skeaff, S.A.; Ferguson, E.L.; Gray, A.R.; Valeix, P. Breast-milk iodine concentration declines over the first 6 mo postpartum in iodine-deficient women. Am. J. Clin. Nutr. 2010, 92, 849-856. [CrossRef] [PubMed] 
26. Johnson, L.A.; Ford, H.C.; Doran, J.; Richardson, V.F. A survey of the iodide concentration of human-milk. N. Z. Med. J. 1990, 103, 393-394. [PubMed]

27. Darragh, A.J.; Moughan, P.J. The amino acid composition of human milk corrected for amino acid digestibility. Br. J. Nutr. 1998, 80, 25-34. [CrossRef] [PubMed]

28. Statistics New Zealand. 2013 Census QuickStats about Culture and Identity; Statistics New Zealand: Wellington, New Zealand, 2014.

29. AOAC International. Official Methods of Analysis of AOAC International; AOAC International: Gaithersburg, MD, USA, 2005.

30. Food and Agriculture Organization of the United Nations. Analytical Methods for Carbohydrates in Foods. In Food Energy - Methods of Analysis and Conversion Factors; Food and Agriculture Organization of the United Nations: Rome, Italy, 2003; Volume 77.

31. Ministry of Health. Food and Nutrition Guidelines for Healthy Pregnant and Breastfeeding Women: A Background Paper; Ministry of Health: Wellington, New Zealand, 2006.

32. WHO. Global Datebase on Body Mass Index. Available online: http://apps.who.int/bmi/index.jsp? introPage=intro_3.html (accessed on 8 October 2017).

33. Ministry of Health. Annual Update of Key Results 2014/15: New Zealand Health Survey; Ministry of Health: Wellington, New Zealand, 2015.

34. Ministry of Health. Guidance for Healthy Weight Gain in Pregnancy; Ministry of Health: Wellington, New Zealand, 2014.

35. Martin, J.E.; Hure, A.J.; Macdonald-Wicks, L.; Smith, R.; Collins, C.E. Predictors of post-partum weight retention in a prospective longitudinal study. Matern. Child Nutr. 2014, 10, 496-509. [CrossRef] [PubMed]

36. Nomura, K.; Kido, M.; Tanabe, A.; Nagashima, K.; Takenoshita, S.; Ando, K. Investigation of optimal weight gain during pregnancy for Japanese Women. Sci Rep. 2017, 7, 2569. [CrossRef] [PubMed]

37. Whitaker, K.M.; Marino, R.C.; Haapala, J.L.; Foster, L.; Smith, K.D.; Teague, A.M.; Jacobs, D.R.; Fontaine, P.L.; McGovern, P.M.; Schoenfuss, T.C.; et al. Associations of Maternal Weight Status Before, During, and After Pregnancy with Inflammatory Markers in Breast Milk. Obesity 2017, 25, 2092-2099. [CrossRef] [PubMed]

38. Bahadoer, S.; Gaillard, R.; Felix, J.F.; Raat, H.; Renders, C.M.; Hofman, A.; Steegers, E.A.P.; Jaddoe, V.W.V. Ethnic disparities in maternal obesity and weight gain during pregnancy. The Generation R Study. Eur. J. Obstet. Gynecol. Reprod. Biol. 2015, 193, 51-60. [CrossRef] [PubMed]

39. Prentice, A.N.N. D-Regional Variations in the Composition of Human Milk A2-Jensen, Robert G. In Handbook of Milk Composition; Academic Press: San Diego, CA, USA, 1995; pp. 115-221. Available online: https:/ / doi.org/10.1016/B978-012384430-9/50012-3pp (accessed on 23 February 2018).

40. Ballard, O.; Morrow, A.L. Human Milk Composition Nutrients and Bioactive Factors. Pediatr. Clin. N. Am. 2013, 60, 49-74. [CrossRef] [PubMed]

41. Hester, S.N.; Hustead, D.S.; Mackey, A.D.; Singhal, A.; Marriage, B.J. Is the Macronutrient Intake of Formula-Fed Infants Greater Than Breast-Fed Infants in Early Infancy? J. Nutr. Metab. 2012, 2012, 13. [CrossRef] [PubMed]

42. Wojcik, K.Y.; Rechtman, D.J.; Lee, M.L.; Montoya, A.; Medo, E.T. Macronutrient Analysis of a Nationwide Sample of Donor Breast Milk. J. Am. Diet. Assoc. 2009, 109, 137-140. [CrossRef] [PubMed]

43. Saarela, T.; Kokkonen, J.; Koivisto, M. Macronutrient and energy contents of human milk fractions during the first six months of lactation. Acta Paediatr. 2005, 94, 1176-1181. [CrossRef] [PubMed]

44. Kent, J.C.; Mitoulas, L.R.; Cregan, M.D.; Ramsay, D.T.; Doherty, D.A.; Hartmann, P.E. Volume and frequency of breastfeedings and fat content of breast milk throughout the day. Pediatrics 2006, 117, E387-E395. [CrossRef] [PubMed]

45. Michaelsen, K.F.; Larsen, P.S.; Thomsen, B.L.; Samuelson, G. The Copenhagen cohort study on infant nutrition and growth: Breast-milk intake, human-milk macronutrient content, and influencing factors. Am. J. Clin. Nutr. 1994, 59, 600-611. [CrossRef] [PubMed]

46. Vitolo, M.R.; Soares, L.M.V.; Carvalho, E.B.; Cardoso, C.B. Calcium and magnesium concentrations in mature human milk: Influence of calcium intake, age and socioeconomic level. Arch. Latinoam. Nutr. 2004, 54, 118-122. [PubMed]

47. Dorea, J.G. Calcium and phosphorus in human milk. Nutr. Res. 1999, 19, 709-739. [CrossRef]

48. Feeley, R.M.; Eitenmiller, R.R.; Jones, J.B.; Barnhart, H. Copper, iron, and zinc contents of human-milk at early stages of lactation. Am. J. Clin. Nutr. 1983, 37, 443-448. [CrossRef] [PubMed] 
49. Fransson, G.B.; Lonnerdal, B. Zinc, copper, calcium, and magnesium in human-milk. J. Pediatr. 1982, 101, 504-508. [CrossRef]

50. Moser, P.B.; Reynolds, R.D. Dietary zinc intake and zinc concentrations of plasma, erythrocytes, and breast-milk in antepartum and postpartum lactating and nonlactating women-A longitudinal-study. Am. J. Clin. Nutr. 1983, 38, 101-108. [CrossRef] [PubMed]

51. Krebs, N.F.; Hambidge, K.M.; Jacobs, M.A.; Rasbach, J.O. The effects of a dietary zinc supplement during lactation on longitudinal changes in maternal zinc status and milk zinc concentrations. Am. J. Clin. Nutr. 1985, 41, 560-570. [CrossRef] [PubMed]

52. Karra, M.V.; Kirksey, A.; Galal, O.; Bassily, N.S.; Harrison, G.G.; Jerome, N.W. Effect of short-term oral zinc supplementation on the concentration of zinc in milk from american and egyptian women. Nutr. Res. 1989, 9, 471-478. [CrossRef]

53. Kumpulainen, J. Selenium: Requirement and supplementation. Acta Paediatr. Scand. 1989, $114-117$. [CrossRef]

54. Smith, A.M.; Picciano, M.F.; Milner, J.A. Selenium intakes and status of human-milk and formula fed infants. Am. J. Clin. Nutr. 1982, 35, 521-526. [CrossRef] [PubMed]

55. Combs, G.F. Selenium in global food systems. Br. J. Nutr. 2001, 85, 517-547. [CrossRef] [PubMed]

56. Funk, M.A.; Hamlin, L.; Picciano, M.F.; Prentice, A.; Milner, J.A. Milk selenium of rural african women: Influence of maternal nutrition, parity, and length of lactation. Am. J. Clin. Nutr. 1990, 51, 220-224. [CrossRef] [PubMed]

57. Debski, B.; Finley, D.A.; Picciano, M.F.; Lonnerdal, B.; Milner, J. Selenium content and glutathione-peroxidase activity of milk from vegetarian and nonvegetarian women. J. Nutr. 1989, 119, 215-220. [CrossRef] [PubMed]

58. Jenness, R. Composition of human-milk. Semin. Perinatol. 1979, 3, 225-239. [PubMed]

59. Koletzko, B.; Thiel, I.; Abiodun, P.O. The fatty acid composition of human milk in Europe and Africa. J. Pediatr. 1992, 120, S62-S70. [CrossRef]

60. Su, L.L.; Sk, T.C.; Lim, S.L.; Chen, Y.; Tan, E.A.; Pai, N.N.; Gong, Y.H.; Foo, J.; Rauff, M.; Chong, Y.S. The influence of maternal ethnic group and diet on breast milk fatty acid composition. Ann. Acad. Med. Singap. 2010, 39, 675-679. [PubMed]

61. Glew, R.H.; Wold, R.S.; Herbein, J.H.; Wark, W.A.; Martinez, M.A.; VanderJagt, D.J. Low Docosahexaenoic Acid in the Diet and Milk of Women in New Mexico. J. Am. Diet. Assoc. 2008, 108, 1693-1699. [CrossRef] [PubMed]

62. Kim, H.; Kang, S.; Jung, B.-M.; Yi, H.; Jung, J.A.; Chang, N. Breast milk fatty acid composition and fatty acid intake of lactating mothers in South Korea. Br. J. Nutr. 2017, 117, 556-561. [CrossRef] [PubMed]

63. Urwin, H.J.; Zhang, J.; Gao, Y.; Wang, C.; Li, L.; Song, P.; Man, Q.; Meng, L.; Frøyland, L.; Miles, E.A.; et al. Immune factors and fatty acid composition in human milk from river/lake, coastal and inland regions of China. Br. J. Nutr. 2012, 109, 1949-1961. [CrossRef] [PubMed]

64. Wang, L.W.; Shimizu, Y.; Kaneko, S.; Hanaka, S.; Abe, T.; Shimasaki, H.; Hisaki, H.; Nakajima, H. Comparison of the fatty acid composition of total lipids and phospholipids in breast milk from Japanese women. Pediatr. Int. 2000, 42, 14-20. [CrossRef] [PubMed]

65. Innis, S.M.; Kuhnlein, H.V. Long-chain $n$-3 fatty acids in breast milk of Inuit women consuming traditional foods. Early Hum. Dev. 1988, 18, 185-189. [CrossRef]

(C) 2018 by the authors. Licensee MDPI, Basel, Switzerland. This article is an open access article distributed under the terms and conditions of the Creative Commons Attribution (CC BY) license (http:/ / creativecommons.org/licenses/by/4.0/). 


\title{
Mother-Infant Physical Contact Predicts Responsive Feeding among U.S. Breastfeeding Mothers
}

\author{
Emily E. Little $^{1, *}$, Cristine H. Legare ${ }^{2}$ and Leslie J. Carver ${ }^{1}$ \\ 1 Department of Psychology, University of California, San Diego, CA 92093, USA; ljcarver@ucsd.edu \\ 2 Department of Psychology, The University of Texas at Austin, TX 78712, USA; legare@austin.utexas.edu \\ * Correspondence: emelysialittle@gmail.com; Tel.: +1-707-391-7477
}

Received: 31 July 2018; Accepted: 4 September 2018; Published: 6 September 2018

\begin{abstract}
Responsive feeding-initiating feeding in response to early hunger cues—supports the physiology of lactation and the development of infant feeding abilities, yet there is a dearth of research examining what predicts responsive feeding. In non-Western proximal care cultures, there is an association between responsive feeding and mother-infant physical contact, but this has not been investigated within Western populations. In two studies, we tested whether mother-infant physical contact predicted feeding in response to early hunger cues versus feeding on a schedule or after signs of distress among U.S. breastfeeding mothers. With an online questionnaire in Study $1(n=626)$, physical contact with infants (via co-sleeping and babywearing) predicted increased likelihood of self-reported responsive feeding. Mothers who reported responsive feeding were more likely to exclusively breastfeed for the first six months, breastfeed more frequently throughout the day, and had a longer planned breastfeeding duration than mothers who reported feeding on a schedule or after signs of infant distress. In Study $2(n=96)$, a three-day feeding log showed that mother-infant physical contact predicted feeding in response to early hunger cues but mother-infant proximity (without physical contact) did not. In sum, our results demonstrate that physical contact with infants may shape breastfeeding behavior among U.S. mothers, highlighting a connection between social interaction and infant nutrition that warrants further investigation.
\end{abstract}

Keywords: responsive feeding; breastfeeding; breastmilk; babywearing; co-sleeping; mother-infant interaction; feeding cues; maternal responsiveness; mother-infant physical contact; proximal care

\section{Introduction}

Breastfeeding is internationally recognized as the optimal nutrition for infant health and development [1,2], yet most U.S. mothers do not meet the World Health Organization's recommendation of exclusive breastfeeding for six months and continued breastfeeding for 24 months and beyond [3-5]. Responsive feeding-initiating feeding in response to early hunger cues such as lip smacking and bringing hands to mouth-decreases breastfeeding challenges by supporting the physiology of lactation and the development of infant feeding abilities [6-8]. Most mothers in the U.S. report crying as the primary reason for initiating feeding, which is an indication of infant distress rather than an early cue for hunger [9]. Ethnographic accounts of infant care report high levels of responsive feeding among mothers in proximal care cultures in which infants are in near-constant physical contact with mothers $[10,11]$. No research to date has systematically documented the association between mother-infant physical contact and responsive feeding among U.S. mothers. Here, we use convergent methods - an online questionnaire and an at-home feeding log-to examine whether mother-infant physical contact facilitates increased responsive feeding among U.S. breastfeeding mothers.

Ethnographic accounts of infant care in non-Western cultures show that responsive feeding is associated with proximal caretaking practices, a style of parenting characterized by mother-infant 
physical contact through the day and night. For example, Konner and colleagues note that !Kung San caregivers of Northwestern Botswana are in near-constant physical contact with infants and respond to their needs quickly [12]. Hewlett and colleagues have noted similar practices among the Aka foragers of Central Africa, who also keep infants close throughout the majority of the day and respond promptly to signs of distress [13]. Responsiveness in this context of physical closeness often manifests in the form of offering the breast for nursing [14-16]. Breastfeeding promptly in response to early hunger cues may preclude the need for infants to display overt signs of distress $[17,18]$. When in sustained body contact, mothers can sense infants' needs via subtle physical movements and do not wait to see or hear overt signs of discomfort $[19,20]$. This leads to high frequency of breastfeeding in proximal care cultures, up to several times per hour [21,22]. Mothers also show acute awareness of subtle elimination signals, demonstrated by moving infants into an appropriate position immediately before infants empty their bowels [23].

A limitation of the ethnographic literature is that the connection between high levels of physical contact and increased maternal responsiveness is embedded within the broader parenting profile of proximal care, comprising a distinct set of parenting beliefs that may also be driving increased responsiveness. These beliefs are referred to as parental ethnotheories, or cultural parenting models used to define parental roles and goals for children [24]. It is an open question whether the mother-infant physical contact characteristic of proximal care facilitates increased responsiveness or whether the increased responsiveness is simply part of the psychological model of socialization goals and parenting beliefs.

The connection between mother-infant physical contact and maternal responsiveness has not been investigated outside of the proximal care context of small-scale, indigenous communities. Infant care among middle-class Euro-American parents in the U.S. is described as distal care, which is characterized by face-to-face interaction and object stimulation [25]. Yet a movement within many Western countries to adopt philosophies of "natural parenting" and "attachment parenting" has motivated some parents to adopt a parenting style that resembles proximal care, emphasizing high levels of physical contact and extended breastfeeding. There is a dearth of information about these practices in Western culture. One study reported that parents in London who identify with proximal care parenting philosophies had over 50\% more physical contact with their infant than parents practicing distal care, which resulted in the proximal care infants crying $50 \%$ less and breastfeeding for longer [26]. The extent to which the practices of proximal care-including babywearing (carrying infants for extended periods on the body using a sling or wrap) and co-sleeping (bed-sharing with infants to maintain physical contact throughout the night)—predict increased maternal responsiveness during feeding among U.S. mothers is currently unknown.

Our objective in the current investigation was to test whether mother-infant physical contact predicts variation in responsive feeding among U.S. breastfeeding mothers. Though very little is known about the predictors of responsive feeding among U.S. mothers, mothers who breastfeed directly from the breast - in comparison with bottle-feeding - are more likely to be responsive to early hunger cues [27] and are also more likely to breastfeed for a longer duration [28,29]. This disparity in responsive feeding between direct breast- versus bottle-feeding has been explained by several different factors, including the salient visual cue of the emptying bottle, prompting mothers to use quantity consumed to guide feeding rather than infant hunger and fullness cues [30]. Another potential explanation is the increased maternal sensitivity promoted by the oxytocin release during skin-to-skin contact [31]. We examined predictors of responsive feeding solely among mothers feeding directly from the breast.

In two studies, we used convergent evidence-a self-report questionnaire (Study 1) and an at-home feeding $\log$ (Study 2) - to test the hypothesis that mother-infant physical contact predicts responsiveness to infant hunger cues among U.S. breastfeeding mothers. Whereas questionnaires can assess overall reported responsiveness or philosophies about feeding, evidence can be strengthened if it is combined with live documentation of each feeding session, allowing us to capture not only variation 
between individuals in feeding strategies but also variation within individuals in their likelihood of responding to early hunger cues. Cross-cultural variation in mother-infant physical contact is associated with a set of socialization goals characteristic of proximal care culture, and even subtle differences in beliefs and intentions regarding breastfeeding can affect breastfeeding behavior [32]. We therefore used Keller's [33] parental ethnotheories questionnaire to assess maternal beliefs about breastfeeding and responsiveness in Studies 1 and 2. We hypothesized that mother-infant physical contact would be associated with increased maternal responsiveness to infant hunger cues in the context of breastfeeding.

\section{Study 1}

Responsive feeding supports the supply and demand physiology of lactation and works in accordance with the range of individual variation in infant feeding needs [34]. It may also help protect against perceived insufficient milk syndrome, one of the primary reasons mothers end breastfeeding earlier than planned [35]. Though many lactation education programs now recommend responsive feeding as best practice for successful breastfeeding [36], alternate recommendations also exist. For example, starting in the 18th century, European male pediatricians recommended that infants be fed on a strictly regulated schedule [37], a practice which is still promoted today, both informally by parenting blogs and in certain health care settings. Waiting for crying and feeding on a set schedule are both problematic, because they neglect the ability of infants to communicate their hunger, creating a mismatch between infant hunger and feeding time. This mismatch has been linked to problems with early self-regulation and childhood obesity [38-40], in addition to breastfeeding-specific problems of latching difficulties and perceived insufficient milk supply, all of which may contribute to ending breastfeeding earlier than recommended.

Despite the known consequences of not practicing responsive feeding, there is a dearth of information regarding what individual level factors predict responsive feeding, especially among middle-class Euro-American mothers. Study 1 addressed two research question. First, do behaviors consistent with proximal care (i.e., babywearing, co-sleeping) predict a responsive breastfeeding philosophy? We hypothesized that mothers who practiced high level of physical contact through the day (via babywearing) and through the night (via co-sleeping) would be more likely to report a responsive feeding philosophy. Second, does having a responsive feeding philosophy predict improved breastfeeding outcomes? We predicted that self-reported responsive feeding would be associated with an increased likelihood of exclusive breastfeeding for the first six months, increased feeding frequency, and longer planned breastfeeding duration.

\subsection{Materials and Methods}

This study was conducted in accordance with the Declaration of Helsinki, and the protocol was approved by the Institutional Review Board of University of California, San Diego (protocol number 130567 "Culture and Infant-Caregiver Interactions"). We recruited mothers $(n=626)$ of newborn to 24-month-old infants to fill out an online questionnaire. These dyads were recruited from social media postings within U.S.-based parenting groups. After mothers expressed interest in participating in the study, they were contacted electronically by a research assistant who explained the protocol and obtained consent. Participants filled out the anonymous online questionnaire from their home.

Demographic information for the sample is included in Table 1. 
Table 1. Demographic information for the participants in Study 1.

\begin{tabular}{lccc}
\hline Maternal and Infant Characteristics & Range & $\boldsymbol{M}$ & $\boldsymbol{S D}$ \\
\hline Infant Age & $0.23-24.91$ & 9.36 & 5.92 \\
Maternal Age & $20-44$ & 30.71 & $(4.29)$ \\
Daycare & $0-60$ & 9.70 & $(15.59)$ \\
\hline & $n$ & $\%$ \\
\hline Maternal Education & & \\
High School & 174 & $30.16 \%$ \\
College Degree & 204 & $35.36 \%$ \\
Graduate Degree & 199 & $34.49 \%$ \\
Maternal Employment & & \\
Home & 345 & $55.11 \%$ \\
Working & 281 & $44.89 \%$ \\
\hline
\end{tabular}

$M$ is the mean response of each category; $S D$ is the standard deviation of each category; $n$ is number of caregivers in the sample who fit into each category; percentages provided are based on the total sample. Infant age was measured in months, maternal age was measured in years, and daycare was measured as hours per week that the child spends in daycare.

The online questionnaire (administered through Google Forms) assessed demographic factors; parenting practices that facilitate mother-infant physical contact (babywearing, co-sleeping); maternal beliefs; and infant feeding philosophies, practices, and outcomes. We collected basic sociodemographic information from all mothers and controlled for these factors in each statistical model, including infant age (in months), maternal education level (high school, college, or graduate degree), current employment status (at home not working, working outside of the home), and hours per week that the child spends in daycare. These factors were chosen because past research has indicated these variables may be important for predicting breastfeeding behavior [41], and they play a role in how much time the mother spends with her child, potentially impacting ability to recognize or respond to feeding cues.

To assess mother-infant physical contact during the day, mothers were asked about infant carrying practices: "What is the primary method you use to transport your baby?" with the following response options: babywearing or other (i.e., "arms", "stroller/seat"). To assess nighttime physical contact, mothers were asked "Where does your baby currently sleep?" with the following response categories: co-sleeping ("In the same bed as me") or mixed/other ("in the same room, but a separate bed", "in a separate room", or "mixed").

Maternal beliefs about responsiveness were assessed with Keller's 10-question parental ethnotheory questionnaire that solicits degree of agreement with parenting statements regarding the care of a three-month-old infant. Responses to each item were on a scale from one (completely disagree) to five (completely agree). Responses from each participant were compiled to form a proximal care belief score, calculated by summing responses from all questions aimed at measuring alignment with goals of proximal care parenting culture then subtracting the sum of responses to all questions designed to test alignment with goals of distal care parenting culture. The range of possible scores was negative 20 to positive 20. Positive scores indicated that mothers were more aligned with the values of proximal care culture than distal care culture, and a higher score indicated a greater agreement with the parenting goals characteristic of proximal care culture.

To assess feeding philosophy, each mother was asked to choose the option that best described her feeding strategy with the options: responsive ("on demand") or schedule/mixed ("feeding schedule", "mix of both"). To assess breastfeeding outcomes, mothers of infants six months of age and younger were asked about exclusive breastfeeding (only breastmilk, as recommended by the World Health Organization and other international health organizations for the first six months of life) versus non-exclusive breastfeeding (supplementing breastmilk with formula, solids, or other liquids). Breastfeeding frequency throughout the day was assessed by asking mothers how many times per day 
they usually breastfeed their child (number). We assessed planned breastfeeding duration by asking mothers how many months they planned to breastfeed their child for (number in months).

In our analyses, we first sought to describe the beliefs and practices of U.S. breastfeeding mothers. We then examined the degree to which engagement in the beliefs and practices of proximal care predicted responsive feeding and whether responsive feeding predicted breastfeeding behavior. To examine whether proximal care beliefs (proximal care belief score) and practices (babywearing, co-sleeping) predicted self-reported responsive breastfeeding, we conducted multistep logistic regressions with feeding philosophy (responsive, scheduled/mixed) as the outcome measures and proximal care beliefs (proximal care beliefs score) and practices (babywearing, co-sleeping, or both) as the predictor measures, controlling for infant age, maternal education and employment, and hours per week that the child spends in daycare.

To examine whether reporting a responsive feeding philosophy predicted improved breastfeeding outcomes, we conducted separate logistic regressions with feeding strategy (responsive, scheduled/mixed) as the predictor measure - controlling for infant age, maternal education, maternal employment, and hours per week that the child spent in daycare-and exclusive breastfeeding (yes versus no) as the outcome measure (for mothers of infants six months of age and younger, $n=217$ ). Controlling for the same demographic variables, we conducted linear regressions with breastfeeding frequency (number of times per day) and planned duration of breastfeeding (in months) as continuous outcome measures.

\subsection{Results}

All descriptive statistics for Study 1 are included in Table 2.

Table 2. Descriptive statistics for the participants in Study 1.

\begin{tabular}{|c|c|c|}
\hline Maternal Characteristics & $n$ & $\%$ \\
\hline \multicolumn{3}{|l|}{ Feeding Philosophy } \\
\hline Responsive Feeding & 441 & $71.13 \%$ \\
\hline Other (Schedule/Mixed) & 179 & $28.87 \%$ \\
\hline \multicolumn{3}{|l|}{ Co-sleeping } \\
\hline Yes & 266 & $42.42 \%$ \\
\hline No & 361 & $57.58 \%$ \\
\hline \multicolumn{3}{|l|}{ Babywearing } \\
\hline Yes & 439 & $73.41 \%$ \\
\hline No & 159 & $26.59 \%$ \\
\hline \multicolumn{3}{|c|}{ Exclusive Breastfeeding (for infants 6 months and younger, $n=217$ ) } \\
\hline Yes & 177 & $81.94 \%$ \\
\hline \multirow[t]{2}{*}{ No } & 39 & $18.06 \%$ \\
\hline & $M$ & $S D$ \\
\hline \multicolumn{3}{|l|}{ Maternal Beliefs } \\
\hline Proximal Care Belief Score & 6.85 & 5.73 \\
\hline Breastfeeding Duration & 21.55 & 11.76 \\
\hline Breastfeeding Frequency & 6.92 & 4.33 \\
\hline
\end{tabular}

$M$ is the mean response of each category; $S D$ is the standard deviation of each category; $n$ is number of participants in the sample.

We first tested proximal care predictors of responsive breastfeeding. In Step 1 of the model, controlling for infant age, maternal education, maternal employment, and hours per week in daycare, proximal care belief score predicted a self-reported responsive feeding style, $\beta=0.10, S E=0.02$, $\chi^{2}=24.37, p<0.0001$ ( $\beta$ is the effect estimate, $S E$ is the standard error, $\chi^{2}$ is the chi-squared statistic, and $p$ is the calculated probability). In Step 2, physical contact throughout the day and night (via babywearing and co-sleeping) predicted reporting an on-demand feeding philosophy, $\beta=0.62$, $S E=0.21, \chi^{2}=9.13, p<0.001$; see Table 3 . 


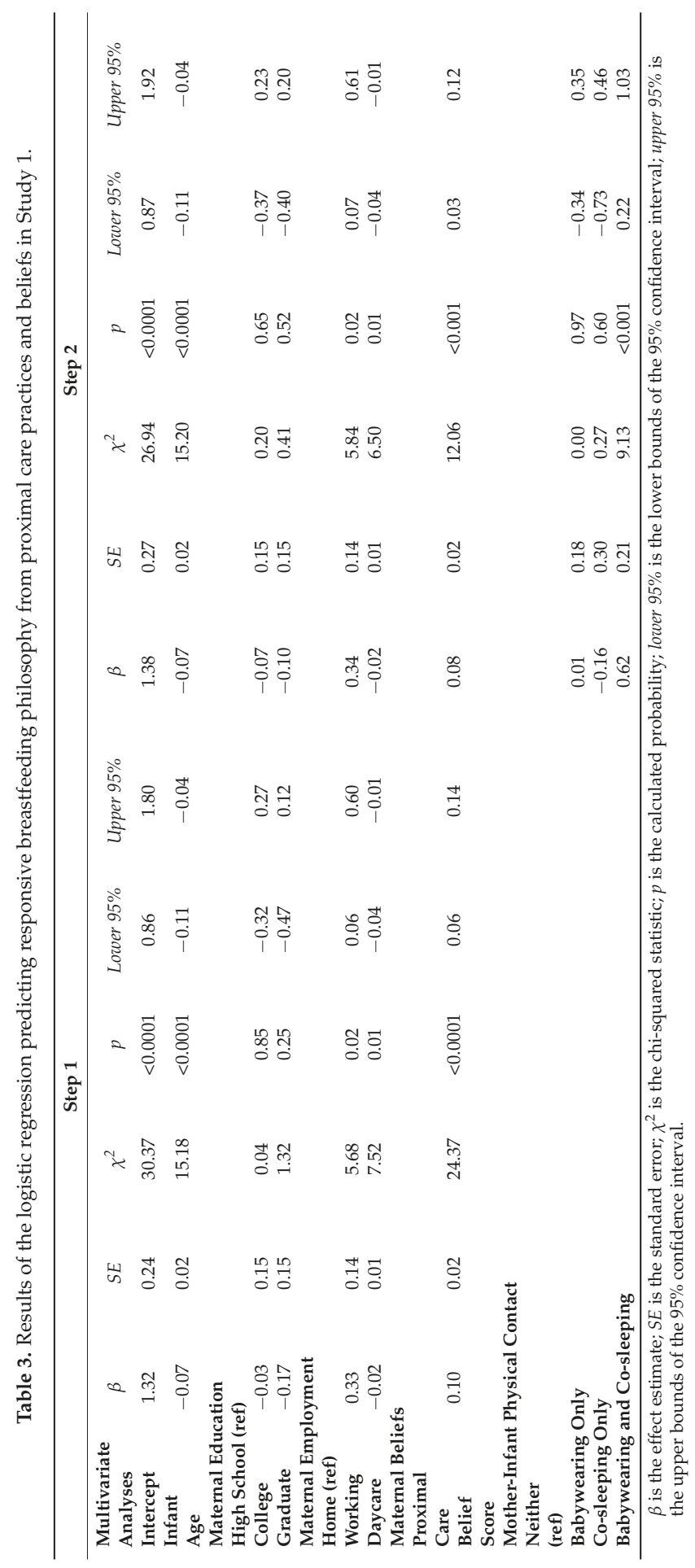


We next tested breastfeeding outcomes associated with responsive breastfeeding philosophy with three separate models. Controlling for infant age, maternal education, maternal employment, and hours per week in daycare, responsive feeding philosophy predicted increased likelihood of exclusive breastfeeding (for infants under six months), $\beta=0.50, S E=0.24, \chi^{2}=4.33, p=0.04$ (Model 1, see Table 4); increased frequency of breastfeeding times per day $\beta=0.84, S E=0.23, \chi^{2}=3.64, p<0.001$ (Model 2, see Table 4); and longer planned breastfeeding duration, $\beta=2.40, S E=0.70, \chi^{2}=3.44$, $p<0.001$ (Model 3, see Table 4).

Table 4. Results of the logistic regression predicting exclusive breastfeeding (feeding only breastmilk to infants under six months) from self-reported responsive feeding (Model 1), results of the linear regression predicting feeding frequency (average number of breastfeeding sessions per day) from self-reported responsive feeding (Model 2), and results of the linear regression predicting planned breastfeeding duration (in months) from self-reported responsive feeding (Model 3) in Study 1.

\begin{tabular}{|c|c|c|c|c|c|c|}
\hline Model 1: Exclusive Breastfeeding & $\beta$ & $S E$ & $\chi^{2}$ & $p$ & Lower $95 \%$ & Upper $95 \%$ \\
\hline Intercept & 2.19 & 0.54 & 16.41 & $<0.0001$ & 1.18 & 3.32 \\
\hline Infant Age & -0.27 & 0.12 & 4.78 & 0.03 & -0.53 & -0.03 \\
\hline \multicolumn{7}{|l|}{ Maternal Education } \\
\hline \multicolumn{7}{|l|}{ High School (ref) } \\
\hline College & 0.10 & 0.32 & 0.10 & 0.75 & -0.50 & 0.75 \\
\hline Graduate & -0.30 & 0.30 & 1.00 & 0.32 & -0.89 & 0.30 \\
\hline \multicolumn{7}{|l|}{ Maternal Employment } \\
\hline \multicolumn{7}{|l|}{ Home (ref) } \\
\hline Working & 0.11 & 0.25 & 0.20 & 0.65 & -0.37 & 0.63 \\
\hline Daycare & 0.01 & 0.02 & 0.37 & 0.54 & -0.02 & 0.05 \\
\hline \multicolumn{7}{|l|}{ Feeding Philosophy } \\
\hline \multicolumn{7}{|l|}{ Schedule/Other (ref) } \\
\hline Responsive Feeding & 0.50 & 0.24 & 4.33 & 0.04 & 0.02 & 0.97 \\
\hline Model 2: Breastfeeding Frequency & $\beta$ & $S E$ & $\chi^{2}$ & $p$ & Lower 95\% & Upper 95\% \\
\hline Intercept & 8.58 & 0.43 & 19.72 & $<0.0001$ & 7.73 & 9.43 \\
\hline Infant Age & -0.18 & 0.03 & -5.36 & $<0.0001$ & -0.25 & -0.12 \\
\hline \multicolumn{7}{|l|}{ Maternal Education } \\
\hline \multicolumn{7}{|l|}{ High School (ref) } \\
\hline College & 0.24 & 0.30 & 0.80 & 0.42 & -0.34 & 0.82 \\
\hline Graduate & -0.26 & 0.29 & -0.91 & 0.36 & -0.82 & 0.30 \\
\hline \multicolumn{7}{|l|}{ Maternal Employment } \\
\hline \multicolumn{7}{|l|}{ Home (ref) } \\
\hline Working & -0.29 & 0.25 & -1.15 & 0.25 & -0.77 & 0.20 \\
\hline Daycare & -0.03 & 0.02 & -1.85 & 0.07 & -0.06 & 0.00 \\
\hline \multicolumn{7}{|l|}{ Feeding Philosophy } \\
\hline \multicolumn{7}{|l|}{ Schedule/Other (ref) } \\
\hline Responsive Feeding & 0.84 & 0.23 & 3.64 & $<0.001$ & 0.39 & 1.29 \\
\hline Model 3: Breastfeeding Duration & $\beta$ & $S E$ & $\chi^{2}$ & $p$ & Lower 95\% & Upper $95 \%$ \\
\hline Intercept & 15.75 & 1.41 & 11.17 & $<0.0001$ & 12.98 & 18.52 \\
\hline Infant Age & 0.45 & 0.11 & 4.15 & $<0.0001$ & 0.24 & 0.67 \\
\hline \multicolumn{7}{|l|}{ Maternal Education } \\
\hline \multicolumn{7}{|l|}{ High School (ref) } \\
\hline College & -1.80 & 0.94 & -1.92 & 0.06 & -3.64 & 0.05 \\
\hline Graduate & 1.11 & 0.86 & 1.28 & 0.20 & -0.59 & 2.81 \\
\hline \multicolumn{7}{|l|}{ Maternal Employment } \\
\hline \multicolumn{7}{|l|}{ Home (ref) } \\
\hline Working & -2.60 & 0.82 & -3.19 & $<0.001$ & -4.21 & -0.99 \\
\hline Daycare & 0.00 & 0.05 & 0.04 & 0.97 & -0.10 & 0.10 \\
\hline \multicolumn{7}{|l|}{ Feeding Philosophy } \\
\hline \multicolumn{7}{|l|}{ Schedule/Other (ref) } \\
\hline Responsive Feeding & 2.40 & 0.70 & 3.44 & $<0.001$ & 1.03 & 3.77 \\
\hline
\end{tabular}

Exclusive breastfeeding was defined as feeding only breastmilk to infants and this model only included a sub-sample of infants under six months of age $(n=217)$; breastfeeding frequency was defined as the average number of breastfeeding sessions per day; breastfeeding duration was the planned number of months of breastfeeding.

\subsection{Discussion}

We documented beliefs and practices consistent with proximal care and their relation to self-reported breastfeeding behavior among U.S. mothers. Our first research aim was to assess whether behaviors consistent with proximal care (i.e., mother-infant physical contact throughout the day and 
night via babywearing and co-sleeping) predicted increased likelihood of reporting a responsive breastfeeding philosophy. Consistent with our predictions, mothers who reported both babywearing and co-sleeping (but not babywearing or co-sleeping only) had an increased likelihood of reporting a responsive feeding philosophy. This finding aligns with ethnographic work showing high levels of breastfeeding responsiveness among populations that practice physical contact throughout the day and night. It is possible that mothers who only practice babywearing or only practice co-sleeping may engage in these practices for convenience, rather than for the desire to have constant physical closeness to infants. This distinction between constant day and night physical contact versus just babywearing or just co-sleeping warrants further investigation.

Our second research aim was to examine whether having a responsive feeding philosophy predicted improved breastfeeding outcomes. Reporting a responsive feeding philosophy predicted increased likelihood of exclusive breastfeeding during the first six months of life, increased feeding frequency, and longer planned breastfeeding duration. The finding regarding planned breastfeeding duration was limited by the fact that this was only in relation to the planned-rather than actual-breastfeeding duration. Future work should employ a longitudinal design to see if responsive feeding does in fact predict actual breastfeeding duration.

One general limitation of this study is that it only reports whether the mother would describe herself as a responsive feeder, which may be closer to her ideal behavior rather than reflecting the mother's actual behavior at time of feeding. To address this in Study 2, we had mothers fill out a three-day at-home feeding log. At the time of each feeding, mothers documented the reason for feeding their child, as well as their distance from their child (i.e., in physical contact versus not in physical contact) preceding feeding onset, with the aim of capturing a more accurate depiction of the mother's feeding behavior and how it relates to mother-infant physical contact.

\section{Study 2}

The primary objective of Study 2 was to examine whether individual variation in mother-infant physical contact predicted increased likelihood of feeding in response to early hunger cues (e.g., rooting, lip smacking) rather than waiting for the onset of distress (i.e., crying) or feeding for other reasons (comfort, schedules). Mothers filled out an at-home feeding log for three days. For each feeding, mothers documented the location of the infant (i.e., mother-infant contact) preceding feed onset and the reason for initiating feeding. In line with past ethnographic work citing an association between mother-infant physical contact and increased breastfeeding frequency [42], we predicted increased responsiveness to infant hunger cues when a feeding was preceded by mother-infant physical contact in comparison with mother-infant proximity (without direct physical contact).

Hunger is not the only reason a mother might breastfeed her baby, as feeding may be motivated by mother-led contextual reasons (e.g., work constraints, doctor-recommended schedules, or concerns about breastfeeding in public). Mothers may also feed for infant-led contextual reasons (e.g., use nursing as a strategy for comforting infants, as well as decreasing crying and helping infants get to sleep) [43]. Our second objective was to examine whether increased physical contact predicted increased likelihood of feeding to comfort the infant (as reported by the mother) rather than for adult-led contextual reasons. Based on the proposal that physical contact facilitates mother-infant bonding [44], we predicted that when mothers report feeding for non-hunger reasons, the feeding session would be more likely to be preceded by physical contact when feeding in response to infant-led (i.e., comfort) reasons versus adult-led contextual reasons.

As in Study 1, we tested mothers' degree of alignment with the beliefs of proximal care culture with Keller's parental ethnotheory questionnaire. We controlled for these beliefs in all analyses to test whether feeding responsiveness could be attributed to increased mother-infant physical contact, beyond the variation attributed to maternal beliefs. We also tested-and controlled for-the same demographic factors that were included as controls in Study 1 (infant age, maternal education, maternal employment, and hours per week in daycare). These societal factors are some of the primary differences 
between proximal care and distal care cultures, have been identified as shaping breastfeeding outcomes in the breastfeeding literature, and also may be important because they affect the amount of time a mother spends with her infant.

\subsection{Materials and Methods}

This study was conducted in accordance with the Declaration of Helsinki, and the protocol was approved by the Institutional Review Board of University of California, San Diego (protocol number 130567 "Culture and Infant-Caregiver Interactions"). Study 2 comprised a subset of the participants from Study 1 (recruitment methods and eligibility were identical to Study 1). Only mothers who logged at least 12 breastfeeding sessions over a period of three consecutive days were included in the sample. Because we were only sampling from populations of breastfeeding mothers, the participants in this study are a unique sample and are not representative of U.S. mothers at large.

We used an online questionnaire (Google Forms) to solicit demographic information from each mother, including infant age, maternal age, maternal education, maternal employment (currently working outside of the home versus not), and average hours per week that the infant spends in daycare. Maternal beliefs about responsiveness were assessed with the same questions from Keller's parental ethnotheory questionnaire that was used in Study 1.

The feeding log consisted of three questions: (1) feed method (breastmilk from breast, breastmilk from bottle, formula in bottle, other liquids, other solids, and other), (2) location of the infant before feeding onset (in physical contact, in visual proximity, and no contact), and (3) reason for feeding (hunger: early cues, hunger: distress, non-hunger: infant-led, and non-hunger: mother-led). The date and time of the feeding session was automatically recorded by the online form. For each of these questions, a list of options was provided and only one response could be chosen for each question. For the question: "Where was your baby when you decided to feed him/her?", there were three pre-determined mutually exclusive categories of responses with regard to mother-infant contact: (1) physical contact (mother was in direct physical contact with the infant), (2) visual proximity (the mother was near enough to see the infant, but not in physical contact), and (3) no contact (the infant was out of sight or with another caregiver). For the question: "Why did you decide to start feeding your baby?", there were four pre-determined mutually exclusive categories of responses: (1) hunger: early cues, (2) hunger: distress, (3) non-hunger: infant-led, and (4) non-hunger: mother-led.

Feedings were coded as being in response to cues if the mother indicated that the feeding was initiated because the infant had shown either visual communication (e.g., facial expression), vocal communication (e.g., lip smacking), or physical communication (e.g., breast nuzzling, squirming) that indicated hunger (but not to the point of distress or crying). Feedings were coded as being in response to distress if the mother indicated she had decided to feed because the infant was crying or showing clear distress. Non-hunger feedings were coded as infant-led if the mother initiated feeding for a reason other than hunger that was centered around the well-being of the baby (e.g., wanted to comfort the baby, wanted to calm the baby before getting shots). Non-hunger feedings were coded as mother-led if the mother initiated feeding for a reason other than hunger that was centered around adult-dictated logistical reasons such as schedules (e.g., needing to leave for work) or other maternally-motivated reasons (e.g., breasts feeling engorged).

After indicating interest in the study, mothers were contacted electronically by a research assistant to give details about the feeding log procedure and obtain informed consent. Mothers were instructed to fill out the maternal questionnaire first, then fill out the feeding log during a consecutive three-day period of their choice. Both the questionnaire and the feeding log were administered online via a web browser or smartphone app.

We used generalized mixed-effects logistic regression models to test whether maternal beliefs (proximal care belief score) and immediate physical contact (versus visual contact or no contact) predicted reason for feeding. We analyzed hunger-related reasons for feeding (early cues versus distress) separately from non-hunger reasons for feeding (infant-led versus mother-led). In these 
models, we controlled for infant age, maternal education, maternal employment, and infant hours in daycare by including these as fixed effects. We included random intercepts for subject, as well as random slopes to account for the multiple responses for each participant [45]. These analyses were conducted using the lme4 package within R Studio software, Version 1.0.44 (RStudio, Inc., Boston, MA, USA) [46].

\subsection{Results}

Ninety-nine breastfeeding mothers completed the feeding log and were included in the final analyses. Infants were 0- to 12-month-olds (51 female, 5.66 months, standard deviation $(S D)=3.25$ ). Mothers were 21 to 42 years old $(M=30.97$ years, $S D=4.64)$ and had completed high school $(30.61 \%)$, college $(38.78 \%)$ or a graduate program (30.61\%). The average household income of the sample was $\$ 78,703(S D=\$ 50,064)$. Mothers were multiparous (had more than one child, $75.26 \%$ ) and were exclusively breastfeeding (65.66\%). Many of the mothers were not currently working $(60.20 \%)$, and infant hours in daycare ranged from zero to $55 \mathrm{~h}$ per week $(M=4.41 \mathrm{~h}, S D=11.52)$.

Mothers logged from 12-47 breastfeeding sessions over the course of three days $(M=25.86$, $S D=8.34$ ); see Table 5. An average of 14.15 of the feedings were initiated when the infant was in physical contact with the mother $(S D=7.37,3-39$ feeds). The most common reason for feeding was early hunger cues $(M=30.52 \%, S D=16.91 \%)$, followed by late cues $(M=34.59 \%, S D=21.53 \%)$, infant-led non-hunger reasons $(M=17.42 \%, S D=15.34 \%)$, and mother-led non-hunger reasons $(M=17.26 \%, S D=14.33 \%)$. Proximal care belief scores ranged from -8 to $+17(M=5.84, S D=5.88)$ out of a possible range of -20 to +20 .

Table 5. Descriptive statistics for Study 2.

\begin{tabular}{|c|c|c|c|}
\hline Feeding and Infant Care Characteristics & Range & $M$ & $S D$ \\
\hline Total Breastfeeding Sessions & $12-47$ & 23.63 & 7.77 \\
\hline Initiated in Physical Contact & $3-39$ & 13.07 & 6.74 \\
\hline Initiated in Visual Contact & $0-24$ & 7.85 & 4.54 \\
\hline Hunger-Early Cues & $0-23$ & 6.06 & 4.19 \\
\hline Hunger-Distress & $2-22$ & 10.85 & 5.45 \\
\hline Non-Hunger-Infant-Led & $0-9$ & 3.02 & 2.79 \\
\hline \multirow[t]{2}{*}{ Non-Hunger-Mother-Led } & $0-9$ & 3.05 & 2.62 \\
\hline & & $n$ & $\%$ \\
\hline \multicolumn{4}{|l|}{ Exclusive Breastfeeding for 6 Months } \\
\hline Yes & & 35 & $85.37 \%$ \\
\hline No & & 6 & $14.63 \%$ \\
\hline \multicolumn{4}{|l|}{ Babywearing } \\
\hline Yes & & 29 & $70.73 \%$ \\
\hline No & & 12 & $29.27 \%$ \\
\hline \multicolumn{4}{|l|}{ Co-sleeping } \\
\hline Yes & & 17 & $41.46 \%$ \\
\hline No & & 24 & $58.54 \%$ \\
\hline
\end{tabular}

$M$ is the mean response of each category; $S D$ is the standard deviation of each category; $n$ is number of participants in the sample.

Mother-infant physical contact predicted feeding in response to early hunger cues in comparison with distress, $\beta=0.991, S E=0.315, z=3.149, p=0.002$; see Table 6. Mothers who initiated more feedings while in physical contact (i.e., the median $53 \%$ or more) had a higher percentage of feeds initiated in response to early cues $(M=33.24 \%, S E=2.41)$ than mothers who initiated fewer feedings (less than $53 \%$ ) while in physical contact $(M=25.67 \%, S E=2.59)$. Visual contact did not predict feeding in response to early cues versus distress, $\beta=0.002, S E=0.200, z=0.007, p=0.994$. 
Table 6. Model Predicting Responsiveness to Cues. Fixed effects for the mixed-effects model predicting initiating hunger-related feedings in response to early cues (in comparison with crying) in Study 2.

\begin{tabular}{ccccc}
\hline Multivariate Analyses & $\beta$ & $S E$ & $z$ & $p$ \\
\hline $\begin{array}{c}\text { Infant Age } \\
\text { Maternal Education } \\
\text { High School (ref) } \\
\quad \text { College }\end{array}$ & -0.030 & 0.044 & -0.665 & 0.506 \\
$\quad$ Graduate & -0.081 & 0.343 & -0.235 & 0.814 \\
$\begin{array}{c}\text { Maternal Employment } \\
\quad \text { Home (ref) }\end{array}$ & 0.740 & 0.390 & 1.898 & 0.058 \\
$\quad$ Working & & & & \\
$\quad$ Daycare & -0.604 & 0.326 & -1.853 & 0.064 \\
$\quad \begin{array}{c}\text { Maternal Beliefs } \\
\text { Proximal Care Belief Score }\end{array}$ & 0.044 & 0.016 & 2.844 & 0.004 \\
$\begin{array}{c}\text { Mother-Infant Physical Contact } \\
\text { No Contact (ref) } \\
\quad \text { Visual Contact }\end{array}$ & 0.030 & 0.027 & 1.106 & 0.269 \\
Physical Contact & 0.002 & 0.300 & 0.007 & 0.994 \\
\hline
\end{tabular}

$\beta$ is the effect estimate; $S E$ is the standard error; $z$ is the $z$-score; $p$ is the calculated probability.

For non-hunger feedings, the bivariate regression analysis revealed that physical contact predicted feeding for infant-led versus mother-led reasons, $\beta=1.271, S E=0.261, z=4.868, p<0.0001$. Controlling for demographic factors and multiple responses (i.e., feeding log entries), we found that physical contact predicted feeding for infant-led versus mother-led reasons, $\beta=1.246, S E=0.304, z=4.095$, $p<0.0001$; see Table 7. Mothers with more feedings initiated in physical contact had a lower percentage of feeds initiated for mother-led reasons $(M=14.54 \%, S E=1.94)$ than mothers with fewer feedings initiated in physical contact $(M=22.22 \%, S E=2.08)$. In contrast, visual contact did not predict feeding for infant-led versus mother-led reasons, $\beta=0.397, S E=0.288, z=1.379, p=0.168$.

Table 7. Model Predicting Responsiveness to Cues. Fixed effects for the mixed-effects model predicting initiating non-hunger feedings in response to infant comfort (in comparison with adult-determined reasons) in Study 2.

\begin{tabular}{|c|c|c|c|c|}
\hline Multivariate Analyses & $\beta$ & $S E$ & $z$ & $p$ \\
\hline Infant Age & 0.067 & 0.044 & 1.514 & 0.130 \\
\hline \multicolumn{5}{|l|}{ Maternal Education } \\
\hline \multicolumn{5}{|l|}{ High School (ref) } \\
\hline College & 0.027 & 0.345 & 0.079 & 0.937 \\
\hline Graduate & 0.085 & 0.403 & 0.210 & 0.833 \\
\hline \multicolumn{5}{|l|}{ Maternal Employment } \\
\hline \multicolumn{5}{|l|}{ Home (ref) } \\
\hline Working & -0.446 & 0.319 & -1.397 & 0.162 \\
\hline Daycare & -0.013 & 0.015 & -0.897 & 0.369 \\
\hline \multicolumn{5}{|l|}{ Maternal Beliefs } \\
\hline Proximal Care Belief Score & 0.057 & 0.022 & 2.665 & 0.008 \\
\hline \multicolumn{5}{|l|}{ Mother-Infant Physical Contact } \\
\hline \multicolumn{5}{|l|}{ No Contact (ref) } \\
\hline Visual Contact & 0.397 & 0.288 & 1.379 & 0.168 \\
\hline Physical Contact & 1.246 & 0.304 & 4.095 & $<0.0001$ \\
\hline
\end{tabular}

\subsection{Discussion}

These data provide support for the proposal that mother-infant physical contact influences maternal responsiveness to early hunger cues during breastfeeding. Consistent with our predictions, mothers were more likely to respond to early hunger cues when in physical contact with their infant. Visual contact did not predict reason for feeding, suggesting that it is something unique about physical 
contact that facilitates increased maternal responsiveness. In addition, increased responsiveness to hunger cues was not simply attributed to increased feeding frequency overall.

We tested whether physical contact predicted infant-led non-hunger reasons for feeding. When feeding for non-hunger contextual reasons, mothers were more likely to feed for infant-led reasons (e.g., to comfort the infant) rather than adult-led reasons (e.g., schedules) if the feeding was preceded by mother-infant physical contact. Visual contact was not associated with feeding for non-hunger reasons, suggesting that there is something special about direct physical contact that facilitates infant-led motivations for feeding above and beyond just having the infant in proximity.

In addition to testing specific research questions, we also sought to document proximal care parenting practices among Euro-American middle class parents. Though caregiving in U.S. culture is typically characterized as distal care; the mothers in our sample participated in many parenting practices typical of proximal care culture, including babywearing and co-sleeping. This study provides insight into how proximal care practices might shape other components of infant-caregiver interaction during feeding. More research is needed to examine the implications of these practices for infant health and nutrition.

\section{General Discussion}

Convergent methods were used to test whether mother-infant physical contact predicts increased responsiveness to early hunger cues during breastfeeding. Both the self-report questionnaire and the at-home feeding log showed that maternal beliefs and practices characteristic of proximal care culture predicted increased maternal responsiveness to infant hunger cues during breastfeeding. We discuss potential mechanisms underlying the connection between physical contact and maternal responsiveness, present potential directions for future research, and discuss broader implications of this work for protecting and promoting breastfeeding.

\subsection{Mechanisms Underlying the Effect of Physical Contact}

Our data show that mother-infant physical contact predicts increased responsiveness to infant hunger cues during breastfeeding, beyond any variation explained by underlying beliefs about responsiveness. Thus, mother-infant physical contact may facilitate increased maternal awareness of her infant's emotional state and communicative intentions, allowing her to increase her responsiveness to subtle movements or physiological changes in the infant that cannot be observed but can be felt. The release of oxytocin-a neuropeptide involved in mammalian social bonds—during mother-infant physical contact may also underlie the effect of physical contact on maternal responsiveness. Oxytocin is associated with some aspects of responsiveness, including responding to infant crying [47] and infant laughing [48]. Because oxytocin is released during skin-to-skin contact [49], infant holding without direct skin-to-skin [50], and even in response to infant vocalizations [51], oxytocin release likely plays a role in the relationship between mother-infant physical contact and maternal responsiveness demonstrated in these studies. Though measuring oxytocin was outside of the scope of the current project, future research should measure the effect of mother-infant contact on maternal responsiveness while accounting for potential changes in oxytocin levels.

\subsection{Limitations and Broader Implications}

Because we did not directly manipulate mother-infant physical contact in these studies, we cannot determine the causal relationship between mother-infant physical contact and responsive feeding. We hope these studies motivate controlled experimental studies to continue investigating our hypothesis that mother-infant physical contact facilitates increased responsiveness to infant hunger cues during breastfeeding. Because we specifically sampled from the population of breastfeeding mothers, mothers in our study were more likely to be older, more educated, and have a higher income than the general population. We therefore cannot determine whether these findings generalize to the population of U.S. mothers at large. Many studies have reported that crying is the most commonly reported indication 
of hunger used by U.S. mothers to initiate feeding [52]. The fact that mothers in this sample initiated feeding more often due to early hunger cues rather than distress in Study 2 demonstrates higher levels of responsiveness than the average population. Past research shows that breastfeeding mothers show different patterns of interaction and responsiveness than bottle-feeding mothers. Breastfeeding mothers-in comparison with bottle-feeding mothers-are more likely to show an increase in oxytocin levels after holding their infant [53] and are more likely to show neural activation in response to their infant's cry [54], suggesting that the variation in responsiveness found in these studies may be specific to breastfeeding mothers.

Populations show substantial variation in the modality of infant-caregiver interaction, especially with regard to physical contact [55]. The amount of physical contact with infants in Western, educated, industrialized, rich, and democratic- “WEIRD" societies, which comprise the majority of research on infant nutrition and development [56,57]—is substantially lower than in many other human populations [58,59]. Infant care in WEIRD societies is increasingly dominated by products that limit physical contact between infants and caregivers (e.g., cribs, strollers, playpens, and bouncers). Because human infants are like all other primates in their need to maintain close contact with mothers, this lack of physical contact represents a caregiving method that is unique from a cultural and historical perspective [60]. Though past research has identified the importance of cultural ecologies on breastfeeding behavior-including both cultural beliefs and behaviors [61] —our data suggest that amount of physical contact with infants may shape breastfeeding behavior, presenting a new avenue for exploring the intersection between social interaction and early nutrition.

Skin-to-skin contact is beneficial for physiological stability, physical growth, and breastfeeding initiation for both preterm and full-term infants, yet investigating the implications of mother-infant physical contact for responsiveness to hunger cues during breastfeeding is surprisingly understudied. Randomized controlled trials with preterm infants show that skin-to-skin contact immediately after birth increases the likelihood of breastfeeding in the hospital and throughout the first postpartum months [62], while also leading to a more stable heartbeat, respiratory rate, body temperature, and other benefits [63]. Intervention studies with full-term infants show that increased physical contact through carrying facilitates more secure attachment [64] and increased frequency of breastfeeding [65]. There are still substantial gaps in our knowledge of the processes underlying the effects of skin-to-skin and physical contact, leaving many questions unanswered about how and why physical contact can be used to improve breastfeeding outcomes for infants. Because increasing mother-infant physical contact is both a viable and inexpensive potential intervention, this area warrants further research.

\subsection{Conclusions}

Breastmilk is internationally recognized as the optimal nutrition for infant health and development. Neglecting to recognize and respond to subtle feeding cues exacerbates both physiological and psychological breastfeeding challenges, yet many mothers feed on a schedule or report crying rather than early hunger cues as the primary motivation for initiating feeding. Our data suggest that culturally-mediated parenting practices like mother-infant physical contact may shape maternal responsiveness to early hunger cues, providing a new potential opportunity for intervention to support breastfeeding mothers in meeting their goals.

Author Contributions: Conceptualization, E.E.L.; Methodology, E.E.L.; Formal Analysis, E.E.L.; Investigation, E.E.L.; Writing-Original Draft Preparation, E.E.L.; Writing-Review \& Editing, C.H.L. and L.J.C.; Supervision, C.H.L. and L.J.C.

Funding: This research was supported by a fellowship from the Center for Academic Research \& Training in Anthropogeny (CARTA) to the first author.

Conflicts of Interest: The authors declare no conflict of interest. 


\section{References and Note}

1. Victora, C.G.; Bahl, R.; Barros, A.J.; França, G.V.; Horton, S.; Krasevec, J. Breastfeeding in the 21st century: Epidemiology, mechanisms, and lifelong effect. Lancet 2016, 387, 475-490. [PubMed]

2. Bode, L.; McGuire, M.; Rodriguez, J.M.; Geddes, D.T.; Hassiotou, F.; Hartmann, P.E.; McGuire, M.K. It's alive: Microbes and cells in human milk and their potential benefits to mother and infant. Adv. Nutr. 2014, 5, 571-573. [CrossRef] [PubMed]

3. World Health Organization. Health Topics-Breastfeeding. Available online: http://www.who.int/topics/ breastfeeding/en/ (accessed on 16 August 2018).

4. Bartick, M.C.; Schwarz, E.B.; Green, B.D.; Jegier, B.J.; Reinhold, A.G.; Colaizy, T.T.; Bogen, D.L.; Schaefer, A.J.; Stuebe, A.M. Suboptimal breastfeeding in the United States: Maternal and pediatric health outcomes and costs. Matern. Child Nutr. 2017, 13, e12366. [CrossRef]

5. Centers for Disease Control and Prevention. Breastfeeding Report Card-United States. 2018. Available online: https:/ / www.cdc.gov/breastfeeding/pdf/2018breastfeedingreportcard.pdf (accessed on 16 August 2018).

6. Shloim, N.; Vereijken, C.M.; Blundell, P.; Hetherington, M.M. Looking for cues-infant communication of hunger and satiation during milk feeding. Appetite 2017, 108, 74-82. [PubMed]

7. Woolridge, M.W. Baby-controlled breastfeeding: Biocultural implications. In Breastfeeding: Biocultural Perspectives; Stuart-Macadam, P., Dettwyler, K.A., Eds.; Walter de Gruyter: Hawthorne, NY, USA, 1995; pp. 217-242.

8. Kent, J.C. How breastfeeding works. J. Midwifery Women's Health 2007, 52, 564-570. [CrossRef]

9. Kirk, A.T.; Alder, S.C.; King, J.D. Cue-based oral feeding clinical pathway results in earlier attainment of full oral feeding in premature infants. J. Perinatol. 2007, 27, 572-578. [PubMed]

10. Hodges, E.A.; Hughes, S.O.; Hopkinson, J.; Fisher, J.O. Maternal decisions about the initiation and termination of infant feeding. Appetite 2008, 50, 333-339. [CrossRef] [PubMed]

11. Konner, M. Hunter-gatherer infancy and childhood. In Hunter-Gatherer Childhoods: Evolutionary, Developmental, \& Cultural Perspectives; Hewlett, B.S., Lamb, M.E., Eds.; Aldine Transaction: New Brunswick, NJ, USA, 2005; pp. 19-64.

12. Hewlett, B.S.; Lamb, M.E. Integrating evolution, culture and developmental psychology: Explaining caregiver-infant proximity and responsiveness in central Africa and the USA. In Between Culture and Biology: Perspectives on Ontogenetic Development; Cambridge University Press: Cambridge, UK, 2002; Volume 8, pp. 241-269.

13. Konner, M. Hunter-gatherer infancy and childhood: The! Kung and others. In Hunter-Gatherer Childhoods; Routledge: Oxford, UK, 2017; pp. 19-64.

14. Hewlett, B.S.; Lamb, M.E.; Shannon, D.; Leyendecker, B.; Schölmerich, A. Culture and early infancy among central African foragers and farmers. Dev. Psychol. 1998, 34, 653-661. [CrossRef] [PubMed]

15. Richman, A.L.; Miller, P.M.; LeVine, R.A. Cultural and educational variations in maternal responsiveness. Dev. Psychol. 1992, 28, 614-621.

16. Morelli, G.A.; Rogoff, B.; Oppenheim, D.; Goldsmith, D. Cultural variation in infants' sleeping arrangements: Questions of independence. Dev. Psychol. 1992, 28, 604-613. [CrossRef]

17. Kärtner, J.; Keller, H.; Yovsi, R.D. Mother-infant interaction during the first 3 months: The emergence of culture-specific contingency patterns. Child Dev. 2010, 81, 540-554. [CrossRef] [PubMed]

18. LeVine, R.A.; Miller, P.M.; Richman, A.L.; LeVine, S. Education and mother-infant interaction: A Mexican case study. In Parents' Cultural Belief Systems: Their Origins, Expressions, and Consequences; Harkness, S., Super, M.K., Eds.; The Guilford Press: San Antonio, TX, USA, 1996; pp. 254-269.

19. Caudill, W.A.; Schooler, C. Child behavior and child rearing in Japan and the United States: An interim report. J. Nerv. Ment. Dis. 1973, 157, 323-338. [CrossRef]

20. Kärtner, J.; Keller, H.; Lamm, B.; Abels, M.; Yovsi, R.D.; Chaudhary, N.; Su, Y. Similarities and differences in contingency experiences of 3-month-olds across sociocultural contexts. Infant Behav. Dev. 2008, 31, 488-500. [CrossRef] [PubMed]

21. Barr, R.G.; Konner, M.; Bakeman, R.; Adamson, L. Crying in! Kung San infants: A test of the cultural specificity hypothesis. Dev. Med. Child Neurol. 1991, 33, 601-610. [CrossRef] [PubMed]

22. Dettwyler, K.A. More than nutrition: Breastfeeding in urban Mali. Med. Anthropol. Q. 1988, 2, 172-183. [CrossRef] 
23. Chapin, B.L. Attachment in rural Sri Lanka: The shape of caregiver sensitivity, communication, and autonomy. In Attachment Reconsidered; Palgrave Macmillan: New York, NY, USA, 2013; pp. 143-163.

24. Harkness, S.; Super, C.M. Themes and variations: Parental ethnotheories in Western cultures. In Parenting Beliefs, Behaviors, and Parent-Child Relations: A Cross-Cultural Perspective; Rubin, K.H., Chung, O.B., Eds.; Psychology Press: Florence, KY, USA, 2006; pp. 61-79.

25. Keller, H.; Borke, J.; Staufenbiel, T.; Yovsi, R.D.; Abels, M.; Papaligoura, Z.; Jensen, H.; Lohaus, A.; Chaudhary, N.; Lo, W.; et al. Distal and proximal parenting as alternative parenting strategies during infants' early months of life: A cross-cultural study. Int. J. Behav. Dev. 2009, 33, 412-420. [CrossRef]

26. St James-Roberts, I.; Alvarez, M.; Csipke, E.; Abramsky, T.; Goodwin, J.; Sorgenfrei, E. Infant crying and sleeping in London, Copenhagen and when parents adopt a "proximal" form of care. Pediatrics 2006, 117, e1146-e1155. [CrossRef] [PubMed]

27. Hodges, E.A.; Johnson, S.L.; Hughes, S.O.; Hopkinson, J.M.; Butte, N.F.; Fisher, J.O. Development of the responsiveness to child feeding cues scale. Appetite 2013, 65, 210-219. [CrossRef] [PubMed]

28. Pinchevski-Kadir, S.; Shust-Barequet, S.; Zajicek, M.; Leibovich, M.; Strauss, T.; Leibovitch, L.; Morag, I. Direct feeding at the breast is associated with breast milk feeding duration among preterm infants. Nutrients 2017, 9, 1202. [CrossRef] [PubMed]

29. Pang, W.W.; Bernard, J.Y.; Thavamani, G.; Chan, Y.H.; Fok, D.; Soh, S.E.; Chua, M.C.; Lim, S.B.; Shek, L.P.; Yap, F.; et al. Direct vs. expressed breast milk feeding: Relation to duration of breastfeeding. Nutrients 2017, 9, 547. [CrossRef] [PubMed]

30. Dewey, K.G.; Lonnerdal, B. Infant self-regulation of breast milk intake. Acta Paediatr. 1986, 75, 893-898. [CrossRef]

31. Uvnäs-Moberg, K. Oxytocin may mediate the benefits of positive social interaction and emotions. Psychoneuroendocrinology 1998, 23, 819-835. [CrossRef]

32. Perrella, S.L.; Williams, J.; Nathan, E.A.; Fenwick, J.; Hartmann, P.E.; Geddes, D.T. Influences on breastfeeding outcomes for healthy term and preterm/sick infants. Breastfeed. Med. 2012, 7, 255-261. [CrossRef]

33. Keller, H.; Poortinga, Y.H.; Scholmerich, A.; Butterworth, G.; Schölmerich, A. Between Culture and Biology: Perspectives on Ontogenetic Development; Cambridge University Press: Cambridge, UK, 2002; Volume 8.

34. Kent, J.C.; Mitoulas, L.R.; Cregan, M.D.; Ramsay, D.T.; Doherty, D.A.; Hartmann, P.E. Volume and frequency of breastfeedings and fat content of breast milk throughout the day. Pediatrics 2006, 117, e387-e395. [CrossRef] [PubMed]

35. Colombo, L.; Crippa, B.L.; Consonni, D.; Bettinelli, M.E.; Agosti, V.; Mangino, G.; Bezze, E.N.; Mauri, P.A.; Zanotta, L.; Roggero, P.; et al. Breastfeeding determinants in healthy term newborns. Nutrients 2018, 10, 48. [CrossRef] [PubMed]

36. Ventura, A.K. Associations between breastfeeding and maternal responsiveness: A systematic review of the literature. Adv. Nutr. 2017, 8, 495-510. [CrossRef] [PubMed]

37. Manz, F.; Manz, I.; Lennert, T. Zur Geschichte der ärztlichen Stillempfehlungen in Deutschland. Monatsschrift Kinderheilkunde 1997, 145, 572-587. [CrossRef]

38. Iacovou, M.; Sevilla, A. Infant feeding: The effects of scheduled vs. on-demand feeding on mothers' wellbeing and children's cognitive development. Eur. J. Public Health 2012, 23, 13-19. [CrossRef] [PubMed]

39. Li, R.; Fein, S.B.; Grummer-Strawn, L.M. Do infants fed from bottles lack self-regulation of milk intake compared with directly breastfed infants? Pediatrics 2010. [CrossRef] [PubMed]

40. Ventura, A.K.; Mennella, J.A. An experimental approach to study individual differences in infants' intake and satiation behaviors during bottle-feeding. Child. Obes. 2017, 13, 44-52. [CrossRef] [PubMed]

41. Piper, S.; Parks, P.L. Predicting the duration of lactation: Evidence from a national survey. Birth 1996, 23, 7-12. [CrossRef] [PubMed]

42. Fouts, H.N.; Hewlett, B.S.; Lamb, M.E. A biocultural approach to breastfeeding interactions in Central Africa. Am. Anthropol. 2012, 114, 123-136. [CrossRef] [PubMed]

43. True, M.; Pisani, L.; Oumar, F. Infant-mother attachment among the Dogon of Mali. Child Dev. 2001, 72, 1451-1466. [CrossRef] [PubMed]

44. Tessier, R.; Cristo, M.; Velez, S.; Girón, M.; de Calume, Z.F.; Ruiz-Paláez, J.G.; Charpak, Y.; Charpak, N. Kangaroo mother care and the bonding hypothesis. Pediatrics 1998, 102, e17. [CrossRef] [PubMed]

45. Pinheiro, J.; Bates, D.; DebRoy, S.; Sarkar, D. Linear and nonlinear mixed effects models. R package version 3. 2014. pp. 1-137. 
46. Bates, D.; Maechler, M.; Bolker, B. Ime4: Linear Mixedeffects Models Using S4 Classes; R Package Version 0.999375-42; R Foundation for Statistical Computing: Vienna, Austria, 2011.

47. Bakermans-Kranenburg, M.J.; van IJzendoorn, M.H.; Riem, M.M.; Tops, M.; Alink, L.R. Oxytocin decreases handgrip force in reaction to infant crying in females without harsh parenting experiences. Soc. Cogn. Affect. Neurosci. 2011, 7, 951-957. [CrossRef] [PubMed]

48. Riem, M.M.; Bakermans-Kranenburg, M.J.; van IJzendoorn, M.H.; Out, D.; Rombouts, S.A. Attachment in the brain: Adult attachment representations predict amygdala and behavioral responses to infant crying. Attach. Hum. Dev. 2012, 14, 533-551. [CrossRef] [PubMed]

49. Neumann, I.D. Brain oxytocin: A key regulator of emotional and social behaviours in both females and males. J. Neuroendocrinol. 2008, 20, 858-865. [CrossRef] [PubMed]

50. Light, K.C.; Smith, T.E.; Johns, J.M.; Brownley, K.A.; Hofheimer, J.A.; Amico, J.A. Oxytocin responsivity in mothers of infants: A preliminary study of relationships with blood pressure during laboratory stress and normal ambulatory activity. Health Psychol. 2000, 19, 560-567. [CrossRef] [PubMed]

51. Seltzer, L.J.; Ziegler, T.E.; Pollak, S.D. Social vocalizations can release oxytocin in humans. Proc. R. Soc. Lond. B Biol. Sci. 2010, 277, 2661-2666. [CrossRef] [PubMed]

52. Gross, R.S.; Fierman, A.H.; Mendelsohn, A.L.; Chiasson, M.A.; Rosenberg, T.J.; Scheinmann, R.; Messito, M.J. Maternal perceptions of infant hunger, satiety, and pressuring feeding styles in an urban Latina WIC population. Acad. Pediatr. 2010, 10, 29-35. [CrossRef] [PubMed]

53. Heinrichs, M.; Baumgartner, T.; Kirschbaum, C.; Ehlert, U. Social support and oxytocin interact to suppress cortisol and subjective responses to psychosocial stress. Biol. Psychiatry 2003, 54, 1389-1398. [CrossRef]

54. Kim, P.; Feldman, R.; Mayes, L.C.; Eicher, V.; Thompson, N.; Leckman, J.F.; Swain, J.E. Breastfeeding, brain activation to own infant cry, and maternal sensitivity. J. Child Psychol. Psychiatry 2011, 52, 907-915. [CrossRef] [PubMed]

55. Little, E.E.; Carver, L.J.; Legare, C.H. Cultural variation in triadic infant caregiver object exploration. Child Dev. 2016, 87, 1130-1145. [CrossRef] [PubMed]

56. Henrich, J.; Heine, S.J.; Norenzayan, A. Most people are not WEIRD. Nature 2010, 466, 29. [CrossRef] [PubMed]

57. Nielsen, M.; Haun, D.; Kärtner, J.; Legare, C.H. The persistent sampling bias in developmental psychology: A call to action. J. Exp. Child Psychol. 2017, 162, 31-38. [CrossRef] [PubMed]

58. Bartick, M.; Tomori, C.; Ball, H.L. Babies in boxes and the missing links on safe sleep: Human evolution and cultural revolution. Matern. Child Nutr. 2018, 14, e12544. [CrossRef] [PubMed]

59. Trevathan, W.R.; McKenna, J.J. Evolutionary environments of human birth and infancy: Insights to apply to contemporary life. Children's Environ. 1994, 1, 88-104.

60. Lozoff, B.; Brittenham, G. Infant care: Cache or carry. J. Pediatr. 1979, 95, 478-483. [CrossRef]

61. Bazzano, A.N.; Kaji, A.; Felker-Kantor, E.; Bazzano, L.A.; Potts, K.S. Qualitative studies of infant and young child feeding in lower-income countries: A systematic review and synthesis of dietary patterns. Nutrients 2017, 9, 1140. [CrossRef] [PubMed]

62. Moore, E.R.; Anderson, G.C.; Bergman, N.; Dowswell, T. Early skin-to-skin contact for mothers and their healthy newborn infants. Cochrane Database Syst. Rev. 2012. [CrossRef]

63. Ludington-Hoe, S.M.; Morgan, K.; Abouelfettoh, A. A clinical guideline for implementation of kangaroo care with premature infants of 30 or more weeks' postmenstrual age. Adv. Neonatal Care 2008, 8, S3-S23. [CrossRef]

64. Anisfeld, E.; Casper, V.; Nozyce, M.; Cunningham, N. Does infant carrying promote attachment? An experimental study of the effects of increased physical contact on the development of attachment. Child. Dev. 1990, 61, 1617-1627. [CrossRef] [PubMed]

65. Pisacane, A.; Continisio, P.; Filosa, C.; Tagliamonte, V.; Continisio, G.I. Use of baby carriers to increase breastfeeding duration among term infants: The effects of an educational intervention in Italy. Acta Paediatr. 2012, 101, e434-e438. [CrossRef] [PubMed]

(c) 2018 by the authors. Licensee MDPI, Basel, Switzerland. This article is an open access article distributed under the terms and conditions of the Creative Commons Attribution (CC BY) license (http:/ / creativecommons.org/licenses/by/4.0/). 


\title{
Article \\ Endocannabinoid Metabolome Characterization of Transitional and Mature Human Milk
}

\author{
Adriana V. Gaitán ${ }^{1, *}$, JodiAnne T. Wood ${ }^{2}$, Fan Zhang ${ }^{1}$, Alexandros Makriyannis ${ }^{2}$ \\ and Carol J. Lammi-Keefe ${ }^{1,3}$ \\ 1 Louisiana State University and Louisiana State University Agricultural Center, Baton Rouge, LA 70803, USA; \\ fzhan14@lsu.edu (F.Z.); clammi-keefe@agcenter.lsu.edu (C.J.L.-K.) \\ 2 Center for Drug Discovery, Northeastern University, Boston, MA 02115, USA; \\ j.wood@northeastern.edu (J.T.W.), a.makriyannis@northeastern.edu (A.M.) \\ 3 Pennington Biomedical Research Center, Baton Rouge, LA 70803, USA \\ * Correspondence: gaitan.adri@gmail.com; Tel.: +1-225-993-1234
}

Received: 31 July 2018; Accepted: 11 September 2018; Published: 12 September 2018

\begin{abstract}
Recognized as the gold standard, human milk (HM) is an extremely complex yet fascinating biofluid tailored to meet an infant's nutritional requirements throughout development. Endocannabinoids and endocannabinoid-like compounds (endocannabinoid metabolome, ECM) are endogenous lipid mediators derived from long-chain polyunsaturated fatty acids that have been identified in HM. Previous research has shown that arachidonoylglycerol might play a role in establishing the infant's suckling response during lactation by activating the type 1 cannabinoid receptor in the infant's brain. The mechanisms of action and the role of the ECM in HM are not fully understood. Transitional and mature milk samples were collected from lactating women $(n=24)$ for ECM characterization, quantification, and to evaluate differences among the two stages. HM samples were analyzed by liquid chromatography-mass spectrometry. Identified members of the ECM were: arachidonoylethanolamine, palmitoylethanolamine, oleoylethanolamine, docosahexaenoylethanolamine, eicoapentaenoylethanolamine, eicosenoylethanolamine, arachidonoylglycerol, palmitoyglycerol, oleoylglycerol, docosahexaenoylglycerol, eicosapentaenoylglycerol, eiconenooylglycerol, arachidonic acid, docosahexaenoic acid, and eicosapentaenoic acid. Only docosahexaenoylglycerol was different across transitional and mature milk $(p \leq 0.05)$. Data from this cohort suggest that bioactive constituents in HM may also play a role in infant health and development. Future studies can be developed based on this study's data to help elucidate specific roles for each ECM member in addition to understanding how the ECM modulates infant health.
\end{abstract}

Keywords: fatty acids; long-chain polyunsaturated fatty acids; endocannabinoids; infant health; breast milk

\section{Introduction}

According to the Center for Disease Control and Prevention (2018) [1], 83.2\% of infants in the United States are breastfed, with almost $60 \%$ breastfeeding at six months, almost $36 \%$ breastfeeding at 12 months, and only $24.9 \%$ meeting the global recommendation to breastfeed exclusively for six months [2,3]. The recommendation for exclusive breastfeeding during the first months following delivery is based in part on the knowledge that breast milk provides the infant with nutrients that meet his requirements during development. These beneficial nutrients include the long-chain polyunsaturated fatty acids (LCPUFAs), docosahexaenoic acid (DHA, 22:6n3), and arachidonic acid (ARA, 20:4n6), that play a role in cognitive and retinal development and growth of the infant [4]. These nutrients are transferred to the infant across the placenta during pregnancy and through breast milk after birth. 
It has been shown that LCPUFAs are precursors to endocannabinoids (EC) which are endogenous lipid mediators that bind to the same receptors as Cannabis sativa (marijuana) [5]. Endocannabinoids have been shown to play a role in appetite and food intake [6] by activating cannabinoid receptor 1 (CB1) which is present in the central nervous system [7]. Cannabinoid receptor 1 is activated by two different EC, arachidonoylethanolamide (anandamide, AEA) and arachidonoyl glycerol (AG), both derived from $n-6$ ARA. In particular, for infant feeding behavior, AG has been demonstrated to play a role in establishing the suckling response of the neonate when nursing [8]. Evidence in mouse pups suggest that $\mathrm{CB} 1$ activation by $\mathrm{AG}$ is needed to establish the suckling response by activating the oral-motor musculature behavior needed for milk suckling [8-10]. Establishment of this role for AG was demonstrated after administration of a CB1 antagonist (SR141716A) to mouse pups which resulted in growth inhibition and even death by day eight after birth [8].

Recent work has indicated that EC and EC-like compounds (collectively referred to as the endocannabinoid metabolome, ECM) are present in human milk [11-13]. Endocannabinoid-like compounds, referred to as entourage metabolites [14], may support the activity and physiologic responses of the EC system by interacting with AEA and AG, their enzymes, or their receptors. These entourage metabolites exert cannabimimetic effects (similar pharmacological effects to those of cannabis) [15]. The ECM encompasses 15 metabolites identified to date: (i) ethanolamide derivatives: AEA, palmitoyl ethanolamide (PEA), oleoyl ethanolamide (OEA), docosahexaenoyl ethanolamide (DHEA), eicosapentaenoyl ethanolamide (EPEA), and eicosenoyl ethanolamide (EEA); (ii) glycerol derivatives: AG, palmitoyl glycerol (PG), oleoyl glycerol (OG), docosahexaenoyl glycerol (DHG), eicosapenaenoyl glycerol (EPG), eicosenoyl glycerol (EG); and (iii) precursor LCPUFAs: ARA, DHA, and eicosapentaenoic acid (EPA, 20:5n3). There is limited information regarding the ECM of human milk and its role in infant development. Thus, in the present study, we characterized and quantified the ECM in human milk in transitional and mature milk and evaluated if the concentrations of these metabolites changed over time.

\section{Materials and Methods}

\subsection{Study Design}

This research project was an exploratory-longitudinal study to evaluate if there was a difference in the ECM of transitional milk (two weeks postpartum) and the ECM of mature milk (four weeks postpartum).

\subsection{Subject Recruitment}

Pregnant women from the greater Baton Rouge, Louisiana area who were planning to breastfeed for a minimum of four weeks were invited to participate in this study. Recruitment was based on intent to breastfeed. Subjects were invited to participate before delivery through private physicians' offices and hospital prenatal clinics or by posting flyers describing the study around the community. Women who demonstrated interest in participation in the study were contacted to explain the study and for pre-screening based on the inclusion criteria: maternal age of 18-40 years at the time of delivery, full term delivery ( $\geq 37$ gestational weeks), singleton birth, plan to breastfeed for at least 4 weeks, willing to provide a breast milk sample (complete breast emptying from one breast) during the morning (6-10 am), have not been breastfeeding or pregnant in the previous year. Before delivery, women were contacted again to schedule the consent process (thorough explanation of the study and for signature of the consent form). The exclusion criteria were discussed at the time of consent: any tobacco use during lactation, alcohol consumption ( $>1$ drink per week), presumed or confirmed congenital birth defects.

Materials provided to the subjects for the study included two breast milk storage bags, instruction on how to collect the breast milk sample, and a schedule card for visits. These were provided the same 
day that the consent was obtained. The Louisiana State University Agricultural Center Institutional Review Board approved the study.

\subsection{Sample Collection}

Participants provided written consent and filled out a health history questionnaire that included questions about previous and current pregnancies, pregravid body mass index (BMI), and prior lactation experience. Details regarding infant birth weight and length were completed following the infant's birth. In addition, participants provided information about education and socioeconomic status. This information was confirmed by their health care providers.

Breast milk samples were collected at two and four weeks postpartum at the participants' homes. Participants were asked to provide a breast milk expression from a single breast (emptying a full mammary gland by collecting all the milk from that breast) [16] by using an electric breast pump. In preparation for milk collection, participants fasted for at least two hours and collections were made between $6 \mathrm{am}$ and $10 \mathrm{am}$. The sample was stored under refrigeration at the participant's house (for a maximum of $24 \mathrm{~h}$ ) in the breast milk storage bag provided by the researcher. Samples were transported on ice to the laboratory where the milk was warmed in a $37^{\circ} \mathrm{C}$ water bath, manually gently swirled to mix, and $\sim 15 \mathrm{~mL}$ aliquots were made in small glass vials with Teflon-lined caps and stored at $-80{ }^{\circ} \mathrm{C}$ until analyses. Information including the breast pump brand used, exclusive breastfeeding, and use of formula for supplementation were also recorded. Samples were shipped overnight on dry ice to the Center for Drug Discovery, Northeastern University, Boston, MA, USA and kept at $-80^{\circ} \mathrm{C}$ until analysis.

\subsection{Sample Analysis}

The breast milk samples were analyzed by liquid chromatography-mass spectrometry (LC-MS) with a state-of-the-art methodology established at the Center for Drug Discovery at Northeastern University, Boston, MA, USA. Milk samples were thawed in a $37^{\circ} \mathrm{C}$ water bath and vortexed at medium speed for $10 \mathrm{~s}$ at room temperature. Protein precipitation was carried out with chilled acetonitrile and PBS ( $\mathrm{pH} 7.4$ ) and the addition of an internal standard mixture containing the same 15 metabolites identified followed by centrifugation $\left(14,000 \times g, 5 \mathrm{~min}, 4^{\circ} \mathrm{C}\right)$. The resulting supernatant was diluted with four volumes of $5 \%$ phosphoric acid followed by solid phase extraction using OASIS HLB reverse-phase chromatography cartridges (Waters Corp., Mildford, MA, USA) which were previously rinsed with methanol and water prior to loading the diluted samples. Loaded cartridges were washed with $40 \%$ aqueous methanol prior to eluting the absorbed lipids with acetonitrile. The acetonitrile fraction was evaporated to dryness under nitrogen, reconstituted in ethanol, vortexed and sonicated, and centrifuged prior to LC-MS analysis. The autosampler was kept at $4{ }^{\circ} \mathrm{C}$ to prevent analyte degradation. A TSQ Quantum Ultra triple quadrupole mass spectrometer (Thermo Electron, San Jose, CA, USA) with an Agilent 1100 liquid chromatograph (Agilent Technologies, Wilmington, DE, USA) at the front end was used for identification and quantification. Separation of analytes was carried out using an Agilent $2.1 \times 50 \mathrm{~mm}, 5 \mu \mathrm{m}$ Zorbax SB-CN column $[17,18]$ with gradient elution using $10 \mathrm{mM}$ ammonium acetate ( $\mathrm{pH} 7.3$ ) and methanol (flow rate, $0.5 \mathrm{~mL} / \mathrm{min}$ ). Elution of fatty acids was achieved while the mass spectrometer was in negative ionization mode, followed by a change in the mass spectrometer to positive ionization mode for elution of ethanolamine and glycerol esters. Eluted peaks were ionized via atmospheric pressure chemical ionization in multiple reaction monitoring mode as previously described [18]. Deuterated internal standards were used to derive a standard curve for each analyte and concentrations $(\mathrm{ng} / \mathrm{mL}$ ) of breast milk were calculated. Each sample was analyzed in triplicate and concentrations were averaged.

\subsection{Statistical Analyses}

Statistical analyses were performed using SAS by SAS Institute, Inc., version 9.4 (Cary, NC, USA). The level of significance was set at $\leq 0.05$. Descriptive statistics (mean, standard deviation, and range) 
were used for numeric variables. Repeated measures analysis of variance using proc mixed was used to assed the effect of time across the two different time points on the concentrations of members of the ECM.

\section{Results}

One hundred thirty-one potential participants were invited to participate in the study from which 31 consented to participate. Seven women dropped out during the study; thus, data from 24 participants was included in the study. Table 1 provides the participants' characteristics. Lactating women in the study were between 18 years old and 39 years old.

Table 2 shows the constituents of the ECM at two weeks (transitional milk) and four weeks (mature milk) postpartum. Standard curves for each metabolite were linear and had regression values $\geq 0.99$, except for PG which was 0.98. Extraction efficiencies were greater than $80 \%$, except for OG which was greater than $78 \%$. The main metabolite present in the fatty acids group was ARA accounting for more than $60 \%$ of that fraction. In the ethanolamide group, OEA accounted for more than $50 \%$ of that portion, and PG in the glycerol group accounted for more than $90 \%$. Eicosenoyl ethanolamide and EPG were present in the lowest concentrations in the ethanolamide and glycerol groups, respectively.

Table 1. Maternal-Infant Characteristics $(n=24)$.

\begin{tabular}{lc}
\hline \multicolumn{1}{c}{ Characteristic } & $\begin{array}{c}\text { Mean } \pm \text { SD or } \\
\% \text { (Frequency) }\end{array}$ \\
\hline Maternal Characteristics & \\
\hline Age (year) & $30.5 \pm 5.0$ \\
Pre-pregnancy BMI $\left(\mathrm{kg} / \mathrm{m}^{2}\right)$ & $28.0 \pm 5.8$ \\
Race & $71(17)$ \\
White & $17(4)$ \\
Black & $8(2)$ \\
Hispanic & $4(1)$ \\
Asian & $39.2 \pm 1.3$ \\
Gestational age at delivery (weeks) & $71(17)$ \\
Previous breastfeeding experience & $4(1)$ \\
No & $4(1)$ \\
Education & $21(5)$ \\
Some high school & $25(6)$ \\
High school & $46(11)$ \\
Some college & \\
4-year post-high school & $79(19)$ \\
Post-graduate & \\
Marital Status & $88(21)$ \\
Married & \\
WIC participation & \\
No & $33(8)$ \\
\hline Infant characteristics & \\
\hline Sex $\quad$ Girls & \\
Birth weight (lbs) & \\
Feeding type & $(18)$ \\
Exclusively breastfed & \\
\hline
\end{tabular}

BMI, body mass index; WIC, Woman, Infant, and Children Special Supplemental Nutrition Program.

Twenty-one percent of the samples for EPEA and 27\% of the samples for EEA were below the standard curve and $21 \%$ of the samples for PG were above although values were close to the curve for the latter. Therefore, those results should be interpreted with caution. Only DHEA demonstrated a 
time effect ( $p \leq 0.05$ ) across the two different time points postpartum (transitional (two weeks) versus mature (four weeks) milk) with higher concentrations in transitional milk. Overall, breast milk glycerol group concentrations were higher than those of the ethanolamides.

Table 2. Endocannabinoid Metabolome of Human Milk.

\begin{tabular}{cccc}
\hline Metabolite & Transitional Milk ${ }^{\mathbf{1}}$ & Mature Milk $^{\mathbf{1}}$ & $p$ Value $^{2}$ \\
\hline Fatty Acids & \multicolumn{3}{c}{} \\
\hline ARA & $2818.96 \pm 580.77$ & $7030.33 \pm 3638.67$ & 0.2451 \\
DHA & $2031.17 \pm 486.39$ & $2384.71 \pm 1140.13$ & 0.7569 \\
EPA & $381.49 \pm 131.91$ & $1362.93 \pm 933.24$ & 0.2979 \\
\hline Ethanolamides & & \\
\hline AEA & $0.15 \pm 0.05$ & $0.08 \pm 0.01$ & 0.1772 \\
PEA & $0.90 \pm 0.10$ & $0.74 \pm 0.08$ & 0.1095 \\
OEA & $1.48 \pm 0.24$ & $1.12 \pm 0.10$ & 0.0841 \\
DHEA & $0.11 \pm 0.01$ & $0.07 \pm 0.01$ & 0.0022 \\
EPEA ${ }^{3}$ & $0.07 \pm 0.03$ & $0.11 \pm 0.04$ & 0.5184 \\
EEA ${ }^{3}$ & $0.03 \pm 0.01$ & $0.03 \pm 0.00$ & 0.2382 \\
\hline Glycerol esters & & & \\
\hline AG & $166.85 \pm 36.30$ & $312.11 \pm 119.97$ & 0.2550 \\
PG & $37477.67 \pm 7296.61$ & $110,091.70 \pm 54,443.90$ & 0.1905 \\
OG & $4059.33 \pm 716.85$ & $7719.96 \pm 2269.68$ & 0.1225 \\
DHG & $673.50 \pm 198.01$ & $866.30 \pm 383.60$ & 0.6352 \\
EPG & $24.70 \pm 7.70$ & $61.99 \pm 28.85$ & 0.2161 \\
EG & $242.48 \pm 66.75$ & $899.37 \pm 509.86$ & 0.2078 \\
\hline
\end{tabular}

All data are presented in $\mathrm{ng} / \mathrm{mL}$ and are mean \pm SE. Significant difference marked in bold. ${ }^{1}$ Two and four weeks postpartum. ${ }^{2} p$ value represents the effect of time across the two time points. ${ }^{3}$ Some values were below the standard curve. ${ }^{4}$ Some values were above the standard curve. ARA, arachidonic acid; DHA, docosahexaenoic acid; EPA, eicosapentaenoic acid; AEA, Anandamide; PEA, palmitoyl ethanolamide; OEA, oleoyl ethanolamide; DHEA, docosahexaenoyl ethanolamide; EPEA, eicosapentaenoyl ethanolamide; EEA, eicosenoyl ethanolamide; AG, arachidonoyl glycerol; PG, palmitoyl glycerol; OG, oleoyl glycerol; DHG, docosahexaenoyl glycerol; EPG, eicosapenaenoyl glycerol; EG, eicosenoyl glycerol.

Combining the two time points together to evaluate relationships, it was observed that there were significant correlations between the precursor LCPUFA and its derived EC. Results are showed in Table 3.

Table 3. Correlations between the Parent Fatty Acid and its Derived Metabolites.

\begin{tabular}{ccc}
\hline Fatty Acid & Metabolite & Pearson Correlation Coefficient ${ }^{\mathbf{1}}$ \\
\hline ARA & AG & 0.88 \\
DHA & DHEA & 0.69 \\
DHA & DHG & 0.95 \\
EPA & EPEA & 0.80 \\
EPA & EPG & 0.91 \\
\hline
\end{tabular}

${ }^{1} p \leq 0.01$. ARA, arachidonic acid; DHA, docosahexaenoic acid; EPA, eicosapentaenoic acid; AG, arachidonoyl glycerol; DHEA, docosahexaenoyl ethanolamide; DHG, docosahexaenoyl glycerol; EPEA, eicosapentaenoyl ethanolamide; EPG, eicosapenaenoyl glycerol.

\section{Discussion}

Our study has characterized the ECM in transitional and mature human milk to explore differences in the ECM concentrations at these two stages of breast milk production. The mechanisms of action and the roles of the ECM in both breast milk and for infant development are not fully described/ understood. Understanding the bioactive components (i.e., ECM) in breast milk contributes to the body of research that supports the importance of breast milk for infant nourishment and development. 
In this exploratory study, we have characterized the ECM of transitional and mature milk. Our results showed that only DHEA, a derivative of DHA conjugated with ethanolamine, was different across the two different time points $(p \leq 0.05)$ with higher concentrations in transitional milk. Research evaluating the role of the endocannabinoid system (ECS) in infant feeding behavior has been focused on the activation of $\mathrm{CB} 1$ when binding to AG, which in turn activates the oral-motor musculature needed for milk suckling [8]. However, DHEA has also been shown to be an agonist to CB1 [19]. Although the role of DHEA in food intake has not been studied, it may be hypothesized that by binding to the same receptor as AG, DHEA exerts some of the same activities. In addition, as DHA plays a key role in infant cognitive development $[20,21]$ and is a precursor to DHEA, it is plausible that DHEA also supports brain development. Moreover, the development of the hippocampus, a brain area related to learning and memory, has been shown to be supported by DHEA [22].

Scarce data are available for a comparison with our current results. However, the earliest study by Fride et al. (2001) [8] that established a role for the ECS in mouse pup suckling and growth, also analyzed milk from various sources including human milk. Even though the study by Fride et al. (2001) did not specify the number of milk samples analyzed, our results follow the same pattern in demonstrating that PG is present in human milk in higher concentrations than AG. Furthermore, a study by Di Marzo et al. (1998) [23] reported $330 \mathrm{ng} / \mathrm{mL}$ of AG in mature human milk, a concentration very similar to our result of $312.11 \mathrm{ng} / \mathrm{mL}$, and indicated that AG is found in human milk in higher concentrations than AEA which is also demonstrated in our present results. Similarly, a study by Schuel et al. (2002) [24] in which ethanolamides were analyzed in human fluids, including mature milk, demonstrated that OEA was present in higher concentrations than PEA, and PEA in higher concentrations than AEA, as also shown in our results. In addition, our results are in line with preliminary data from our laboratory [11,12] that included the same members of the ECM that were investigated in the current study. Our results follow the same pattern in terms of the proportion of each member within each group: fatty acids, ethanolamides, and glycerols. In summary, there are only a few studies available for a comparison to the findings of our current study that support the presence of EC and EC-like metabolites in human milk.

Correlations between the precursor LCPUFAs and their ethanolamide- and glycerol-derivatives showed a more robust correlation for the precursor LCPUFA and its derived glycerol metabolites. This strong correlation between the precursor LCPUFA with its glycerol- but not its ethanolamidederivatives, may support a more important role for the glycerols (AG, PG, OG, DHG, EPG, and EG) in establishing the suckling response of the newborn by modulating motor development and behavior. In addition, the presence of entourage metabolites (PEA, OEA, DHEA, EPEA, EEA, PG, OG, DHG, EPG, and EG), which exhibit cannabimimetic responses [14], may enhance the activity of the two most thoroughly studied EC, AG, and AEA. For example, PG has been shown to increase AG affinity to CB2 by acting as a lipid signaling mediator [18]; and PEA and OEA reduce enzymatic breakdown, cellular uptake, and degradation of AEA. These entourage metabolites may interfere with enzymatic activity as they can also act as substrates for catabolic and anabolic enzymes. In addition, the presence of these lipid mediators may prevent EC activation or deactivation. All of these interactions may also explain our finding that $n-3$ LCPUFA derivatives, both ethanolamides and glycerols, correlated with each other (DHEA-DHG $(r=0.61, p \leq 0.01)$ and EPEA-EPG $(r=0.84, p \leq 0.01))$. The associations among the $n-3$ LCPUFA derivatives, but not for the $n-6$ derivatives (AEA and AG), leads to the speculation that they support the role of DHA in infant cognitive development, although their roles have not yet been fully elucidated.

To date, the mechanisms of action regarding how the ECM as a whole interacts with the ECS and its role in infant feeding behavior, and therefore infant development and growth, are still poorly understood. Our results provide evidence that there are metabolites similar to the previously described EC [5], i.e., AEA and AG, present in human milk. With an understanding of the role of the ECM and its interactions with the ECS in human milk and the infant's brain, potential interventions could be developed for infants with difficulties latching on and for preterm infants who could be aided by the 
countless benefits of breast milk to ensure continued development outside the womb. While this is out of the scope of this study, it merits further exploration.

This study was limited by its small sample size $(n=24)$. Having a relatively small group of participants did not allow for further explorations between the concentrations for some of the ECM members and demographic data such as BMI and race, for example. However, this study provides an opportunity to develop hypotheses for future studies to evaluate how the ECM of breast milk may be modulated on the basis of maternal and/or infant factors.

\section{Conclusions}

Our study provides evidence that EC and EC-like metabolites are present in human milk. The findings in this study not only support the role of AG in establishing the suckling response of the newborn by activating oral-motor musculature needed for milk suckling, but also suggest that other bioactive constituents in breast milk may also play a role in infant health and development. In addition, knowing that EC-like metabolites are present in breast milk, future studies can be developed to elucidate specific roles for each member of the ECM.

Author Contributions: Study conceptualization and design, A.G. and C.J.L.-K.; Participant recruitment and sample collections, A.G.; Sample methodology of analysis, J.T.W. and A.M.; Statistical analyses, F.Z. and A.G.; Writing-Original Draft Preparation, A.G.; Writing-Review \& Editing, C.J.L.-K.; Supervision, C.J.L.-K.; Project Administration, A.G.; Funding Acquisition, C.J.L.-K. All authors read and approved the final manuscript.

Funding: This research was funded in part by LSU College of Agriculture and LSU AgCenter.

Acknowledgments: We would like to thank the participants in this study and physicians' offices in the community, especially at Woman's Hospital of Baton Rouge. Thank you to Georgianna Tuuri of Louisiana State University who edited an initial version of this manuscript.

Conflicts of Interest: The authors declare no conflict of interest.

\section{References}

1. Centers for Disease Control and Prevention. Breastfeeding Report Card. Available online: https://www.cdc. gov/breastfeeding/pdf/2018breastfeedingreportcard.pdf (accessed on 26 August 2018).

2. World Health Organization. Infant and Young Child Feeding. Available online: http://www.who.int/ mediacentre/factsheets/fs342/en/ (accessed on 13 February 2018).

3. American Academy of Pediatrics. Breastfeeding and the Use of Human Milk. Pediatrics 2012, 129, e827-e841. [CrossRef] [PubMed]

4. Koletzko, B. Human Milk Lipids. Ann. Nutr. Metab. 2016, 69 (Suppl. S2), 27-40. [CrossRef] [PubMed]

5. Di Marzo, V. Endocannabinoids: Synthesis and degradation. Rev. Physiol. Biochem. Pharmacol. 2008, 160, 1-24. [CrossRef] [PubMed]

6. Cascio, M.G. PUFA-derived endocannabinoids: An overview. Proc. Nutr. Soc. 2013, 72, 451-459. [CrossRef] [PubMed]

7. Grant, I.; Cahn, B.R. Cannabis and endocannabinoid modulators: Therapeutic promises and challenges. Clin. Neurosci. Res. 2005, 5, 185-199. [CrossRef] [PubMed]

8. Fride, E.; Ginzburg, Y.; Breuer, A.; Bisogno, T.; Di Marzo, V.; Mechoulam, R. Critical role of the endogenous cannabinoid system in mouse pup suckling and growth. Eur. J. Pharmacol. 2001, 419, 207-214. [CrossRef]

9. Fride, E. The endocannabinoid-CB1 receptor system in pre- and postnatal life. Eur. J. Pharmacol. 2004, 500, 289-297. [CrossRef] [PubMed]

10. Fride, E.; Foox, A.; Rosenberg, E.; Faigenboim, M.; Cohen, V.; Barda, L.; Blau, H.; Mechoulam, R. Milk intake and survival in newborn cannabinoid CB1 receptor knockout mice: Evidence for a "CB3" receptor. Eur. J. Pharmacol. 2003, 461, 27-34. [CrossRef]

11. Durham, H.A.; Wood, J.T.; Vadivel, S.K.; Makriyannis, A.; Lammi-Keefe, C.J. Detection of the endocannabinoid metabolome in human plasma and breast milk. FASEB J. 2013, 27 (Suppl. S1), 45.8.

12. Wood, J.T.; Durham, H.A.; Vadivel, S.K.; Makriyannis, A.; Lammi-Keefe, C.J. Postpartum changes in the endocannabinoid metabolome of human breast milk. FASEB J. 2013, 27 (Suppl. S1), 629.15. 
13. Wu, J.; Gouveia-Figueira, S.; Domellöf, M.; Zivkovic, A.M.; Nording, M.L. Oxylipins, endocannabinoids, and related compounds in human milk: Levels and effects of storage conditions. Prostaglandins Other Lipid Mediat. 2016, 122, 28-36. [CrossRef] [PubMed]

14. Ben-Shabat, S.; Fride, E.; Sheskin, T.; Tamiri, T.; Rhee, M.-H.; Vogel, Z.; Bisogno, T.; De Petrocellis, L.; Di Marzo, V.; Mechoulam, R. An entourage effect: Inactive endogenous fatty acid glycerol esters enhance 2-arachidonoyl-glycerol cannabinoid activity. Eur. J. Pharmacol. 1998, 353, 23-31. [CrossRef]

15. Mechoulam, R.; Fride, E.; Di Marzo, V. Endocannabinoids. Eur. J. Pharmacol. 1998, 359, 1-18. [CrossRef]

16. Miller, E.M.; Aiello, M.O.; Fujita, M.; Hinde, K.; Milligan, L.; Quinn, E.A. Field and laboratory methods in human milk research. Am. J. Hum. Biol. 2013, 25, 1-11. [CrossRef] [PubMed]

17. Williams, J.; Pandarinathan, L.; Wood, J.; Vouros, P.; Makriyannis, A. Endocannabinoid metabolomics: A novel liquid chromatography-mass spectrometry reagent for fatty acid analysis. AAPS J. 2006, 8, E655-E660. [CrossRef] [PubMed]

18. Williams, J.; Wood, J.; Pandarinathan, L.; Karanian, D.A.; Bahr, B.A.; Vouros, P.; Makriyannis, A. Quantitative Method for the Profiling of the Endocannabinoid Metabolome by LC-Atmospheric Pressure Chemical Ionization-MS. Anal. Chem. 2007, 79, 5582-5593. [CrossRef] [PubMed]

19. Brown, I.; Cascio, M.G.; Wahle, K.W.J.; Smoum, R.; Mechoulam, R.; Ross, R.A.; Pertwee, R.G.; Heys, S.D. Cannabinoid receptor-dependent and -independent anti-proliferative effects of omega- 3 ethanolamides in androgen receptor-positive and -negative prostate cancer cell lines. Carcinogenesis 2010, 31, 1584-1591. [CrossRef] [PubMed]

20. Carlson, S.E.; Colombo, J. Docosahexaenoic Acid and Arachidonic Acid Nutrition in Early Development. Adv. Pediatr. 2016, 63, 453-471. [CrossRef] [PubMed]

21. Jensen, C.L.; Voigt, R.G.; Llorente, A.M.; Peters, S.U.; Prager, T.C.; Zou, Y.L.; Rozelle, J.C.; Turcich, M.R.; Fraley, J.K.; Anderson, R.E.; et al. Effects of Early Maternal Docosahexaenoic Acid Intake on Neuropsychological Status and Visual Acuity at Five Years of Age of Breast-Fed Term Infants. J. Pediatr. 2010, 157, 900-905. [CrossRef] [PubMed]

22. Kim, H.-Y.; Moon, H.-S.; Cao, D.; Lee, J.; Kevala, K.; Jun, S.B.; Lovinger, D.M.; Akbar, M.; Huang, B.X. $\mathrm{N}$-Docosahexaenoylethanolamide promotes development of hippocampal neurons. Biochem. J. 2011, 435, 327-336. [CrossRef] [PubMed]

23. Di Marzo, V.; Sepe, N.; De Petrocellis, L.; Berger, A.; Crozier, G.; Fride, E.; Mechoulam, R. Trick or treat from food endocannabinoids? Nature 1998, 396, 636. [CrossRef] [PubMed]

24. Schuel, H.; Burkman, L.J.; Lippes, J.; Crickard, K.; Forester, E.; Piomelli, D.; Giuffrida, A. N-Acylethanolamines in human reproductive fluids. Chem. Phys. Lipids 2002, 121, 211-227. [CrossRef]

(C) 2018 by the authors. Licensee MDPI, Basel, Switzerland. This article is an open access article distributed under the terms and conditions of the Creative Commons Attribution (CC BY) license (http:/ / creativecommons.org/licenses/by/4.0/). 
Article

\title{
Human Milk Casein and Whey Protein and Infant Body Composition over the First 12 Months of Lactation
}

\author{
Zoya Gridneva ${ }^{1, *}$, Wan J. Tie ${ }^{1}$, Alethea Rea ${ }^{2}$, Ching Tat Lai ${ }^{1}$, Leigh C. Ward ${ }^{3}$, Kevin Murray ${ }^{4}$, \\ Peter E. Hartmann ${ }^{1}$ and Donna T. Geddes ${ }^{1}$ \\ 1 School of Molecular Sciences, M310, The University of Western Australia, Crawley, Perth, WA 6009, \\ Australia; ash.tie@uwa.edu.au (W.J.T.); ching-tat.lai@uwa.edu.au (C.T.L.); \\ peter.hartmann@uwa.edu.au (P.E.H.); donna.geddes@uwa.edu.au (D.T.G.) \\ 2 Centre for Applied Statistics, The University of Western Australia, Crawley, Perth, WA 6009, Australia; \\ alethea.rea@uwa.edu.au \\ 3 School of Chemistry and Molecular Biosciences, The University of Queensland, St. Lucia, Brisbane, \\ QLD 4072, Australia; l.ward@uq.edu.au \\ 4 School of Population and Global Health, The University of Western Australia, Crawley, Perth, WA 6009, \\ Australia; kevin.murray@uwa.edu.au \\ * Correspondence: zoya.gridneva@uwa.edu.au; Tel.: +61-8-6488-4467
}

Received: 1 August 2018; Accepted: 17 September 2018; Published: 19 September 2018

check for updates

\begin{abstract}
Human milk (HM) influences infant feeding patterns and body composition (BC). This small proof-of concept longitudinal study investigated relationships between infant/maternal BC and HM casein, whey and total protein during the first 12 months of lactation. BC of breastfeeding dyads $(n=20)$ was measured at $2(n=15), 5(n=20), 9(n=19)$, and/or $12(n=18)$ months postpartum with ultrasound skinfolds (infants) and bioimpedance spectroscopy (infants/mothers). Proteins concentrations and 24-h milk intake were measured and calculated daily intakes (CDI) determined. Higher maternal weight, body mass index, fat-free mass, fat-free mass index, and fat mass index were associated with higher concentration of whey protein $(p \leq 0.034, n=20)$. There were no associations between infant BC and concentrations of all proteins, and CDI of whey and total protein. Higher CDI of casein were associated with lower infant fat-free mass ( $p=0.003, n=18)$ and higher fat mass $(p<0.001)$, fat mass index $(p=0.001, n=18)$, and $\%$ fat mass $(p<0.001, n=18)$ measured with ultrasound skinfolds. These results show a differential effect of HM casein on development of infant $\mathrm{BC}$ during the first year of life, suggesting that there is a potential to improve outcome for the infant through interventions, such as continuation of breastfeeding during the first 12 months of life and beyond, which may facilitate favourable developmental programming that could reduce risk of non-communicable diseases later in life.
\end{abstract}

Keywords: casein; whey; protein; breastfeeding; infant; body composition; bioelectrical impedance spectroscopy; ultrasound skinfolds; human milk; calculated daily intakes; lactation

\section{Introduction}

An epidemic of childhood obesity and the associated non-communicable diseases (NCD), such as diabetes and cardiovascular disease are of increasing international concern [1]. Increasingly, data suggests the lifelong risk of NCD could be modified through early programming effects on obesity and adiposity [2,3]. It is evident that breastfed infants are at $15-20 \%$ reduced risk of obesity and obesity related disease later in life [4,5]. Whilst the protective mechanisms of breastfeeding are not fully understood [6], the development of body composition (BC) in early life [7], composition of 
human milk (HM) [8-10], and infant breastfeeding patterns and behavior [11-13] are all known to play an important role in the programming of these health outcomes.

An increased risk of obesity has been associated with both rapid weight gain [14] and the elevated protein content in infant formulas [9,15]. Compared with $9 \mathrm{~g} / \mathrm{L}$ in term HM $(6-12 \mathrm{~g} / \mathrm{L}$, week 10/12) [16], protein content ranged from 12 to $19 \mathrm{~g} / \mathrm{L}$ in infant formulas and from 16 to $27 \mathrm{~g} / \mathrm{L}$ in follow-up formulas [15]. Thus, limiting protein intake from infant foods could be an effective strategy in reduction of childhood obesity, leading to the development of lower protein formulas to mimic growth rates of HM fed infants [15]. HM is recognized as the best form of nutrition for optimal growth and development of the human infant and as such is species specific in composition. The stark compositional differences between HM and formula have been implicated in the differences in weight and $\mathrm{BC}$ between breastfed and formula-fed infants and decreased risk of later obesity for breastfed infants $[8,9]$.

The protein content of HM, which is low yet highly bioavailable, appears to play a key role in infant growth [9] and might provide a rationale for the reduced risk of being in rapid-growth trajectory [17] and for the lower fat-free mass (FFM) and higher fat mass (FM) and percentage FM (\%FM) of breastfed infants compared with formula-fed [18] during early infancy. In term infants, the protein intakes from HM (three months, boys: $6.4 \pm 1.2 \mathrm{~g}$ /day; girls: $5.8 \pm 1.0 \mathrm{~g} /$ day) were found to be almost half that of formula (boys: $11.4 \pm 2.0 \mathrm{~g} /$ day; girls: $10.5 \pm 1.8 \mathrm{~g} /$ day), and at three and six months differences in protein intake and weight gain were associated with disproportionate gain in FM and FFM, resulting in \%FM difference between the groups [8]. These variations suggest a self-regulatory mechanism of milk intake (MI), which may be in part driven by HM components [10] that are associated with feeding frequency (FFQ) [19].

Despite MI and protein intakes being associated with breastfed infants' weight and FFM gain [8] very few comprehensive longitudinal HM protein intake studies are available through the exclusive breastfeeding and weaning periods. Also, there has been no investigation of the effect of either concentrations or daily intakes of specific HM fractions-such as casein and whey-on infant BC, yet these are highly variable between breastfeeding dyads. Daily intakes of HM components are the true reflection on what infant receives, unlike the concentrations, which can be misleading, especially if the milk intake is inadequate or if mixed feeding is present.

The protective role of $\mathrm{HM}$ and breastfeeding may be attributable to the effect of specific protein fractions on development of infant $\mathrm{BC}$. Thus, the aim of this longitudinal study was to investigate relationships of concentrations and daily intakes of HM casein, whey and total protein with anthropometrics and BC of healthy term breastfed infants and their mothers during first 12 months postpartum. Further exploration of relationships of infant 24-h MI and FFQ with HM proteins was carried out.

\section{Materials and Methods}

\subsection{Study Participants}

Breastfed infants ( $n=20 ; 10$ males, 10 females) of English-speaking, predominantly Caucasian, mothers of higher social-economic status from a developed country were recruited from the community, primarily from the West Australian branch of the Australian Breastfeeding Association. Inclusion criteria were: healthy singletons, gestational age $\geq 37$ weeks, exclusively breastfed [20] at 2 and 5 months, and maternal intention to breastfeed until 12 months. Exclusion criteria were: infant factors that could potentially influence growth and development of BC, maternal smoking, and low milk supply. All mothers provided written informed consent to participate in the study, which was approved by The University of Western Australia Human Research Ethics Committee (RA/1/4253, RA/4/1/2639) and registered with the Australian New Zealand Clinical Trials Registry (ACTRN12616000368437). 


\subsection{Study Session}

Measurements were made when the infants were 2 and/or 5, 9, and 12 months of age. Participants visited our laboratory at King Edward Memorial Hospital for Women (Subiaco, Perth, WA, Australia) for up to four monitored breastfeeding sessions between March 2013 and September 2015. At each study session, the infant was weighed pre-feed, and then the mother breastfed her infant. Infant bioelectrical impedance spectroscopy (BIS) measurements were made pre-feed, unless impractical, then they were taken post-feed [21]. Ultrasound skinfold and anthropometric measurements were made post-feed. Clothing was removed for the measurements except for a dry diaper and a sleeveless shirt.

Maternal weight, height, and BIS measurements were recorded. Small (1-2 mL) pre- and post-feed milk samples were collected into $5 \mathrm{~mL}$ polypropylene vials (Disposable Products, Adelaide, SA, Australia) from the breast/s that infant was fed from and samples were frozen at $-20{ }^{\circ} \mathrm{C}$ for biochemical analysis. Current feeding frequency (FFQ) of the infants was self-reported by mothers.

\subsection{Anthropometric Measurements}

Infants weight was determined before breastfeeding using Medela Electronic Baby Weigh Scales ( \pm 2.0 g; Medela Inc., McHenry, IL, USA). Infant crown-heel length was measured once to the nearest $0.1 \mathrm{~cm}$ using non-stretch tape and a headpiece and a footpiece, both applied perpendicularly to the hard surface. Infant head circumference was measured with a non-stretch tape to the nearest $0.1 \mathrm{~cm}$.

Maternal weight was measured using Seca electronic scales $( \pm 0.1 \mathrm{~kg}$; Seca, Chino, CA, USA). Height was self-reported by participants or measured against a calibrated marked wall (accuracy $\pm 0.1 \mathrm{~cm}$ ).

Infant and maternal body mass index (BMI) were calculated as $\mathrm{kg} / \mathrm{m}^{2}$.

\subsection{Body Composition with Bioelectrical Impedance Spectroscopy}

The methods for measuring maternal and infant BC with BIS using Impedimed SFB7 bioelectrical impedance analyser (ImpediMed, Brisbane, QLD, Australia) as well as age-specific equations used for calculations of infant $\mathrm{BC}$ parameters during this study have been published previously [22]. The within participant coefficient of variation (CV) for maternal \%FM was $0.21 \%$ [23]. Within participant CV for infant resistance measurements at $50 \mathrm{kHz}\left(\mathrm{R}_{50}\right)$ was $1.5 \%$ [21].

\subsection{Ultrasound Skinfold Measurements}

The method for measuring infant skinfolds using the Aplio XG ultrasound machine (Toshiba, Tokyo, Japan) with a 14-8 MHz transducer (PLT-1204BX) and sterile water-based ultrasonic gel (Parker Laboratories Inc., Fairfield, NJ, USA) as well as the equations for calculations of infant BC parameters during this study have been published previously [22,24]. Briefly, single ultrasound scans of four anatomical sites (biceps, subscapular, suprailiac and triceps) were performed on the left side of the body with minimal compression. At all tome points, infant BC was calculated with both, 2-skinfolds (US 2SF: triceps, subscapular) and 4-skinfolds (US 4SF: biceps, subscapular, suprailiac and triceps).

\subsection{Body Composition Indices}

The indices of height-normalized BC were calculated for mothers and infants: FM index (FMI) was calculated as FM/length ${ }^{2}$, and FFM index (FFMI) was calculated as FFM/length ${ }^{2}$; both expressed as $\mathrm{kg} / \mathrm{m}^{2}$ [25].

\subsection{4-h Milk Intake and Feeding Frequency}

Infant 24-h MI was measured by mothers using the 24-h milk production (MP) protocol, weighing infants at home with the Medela Electronic Baby Weigh Scales pre- and post each breastfeed during a 24-h period plus one breastfeeding, and recording amounts of $\mathrm{HM}(\mathrm{g})$ consumed by the 
infant (including expressed HM if any) [26]. 24-h MI was determined as previously described with potential underestimation of 3-10\% [26] and FFQ (meals per 24-h) was recorded [27]. 24-h MI was measured at three time points: between 2 and $5(4.0 \pm 1.3)$ months, when MI is shown to be stable [27], and within 2 weeks of $9(9.4 \pm 0.3)$ and $12(12.2 \pm 0.4)$ months. Given that measuring $24-\mathrm{h}$ MI is not always practical, particularly at the later stages of lactation, mothers were also asked to estimate how frequently the infant fed, and self-reported (SR) the typical time between the meals (e.g., each $2 \mathrm{~h}$ ) during the week prior to the study session as a proxy measure of FFQ.

\subsection{Calculated Daily Intakes of Human Milk Proteins}

24-h MI values from the 24-h MP, and casein, whey and total protein concentrations (pooled pre-/post-feed) from samples taken at the study sessions were used for determination of calculated daily intakes (CDI). These CDI were considered representative of a typical daily intake due to the absence of significant short-term (weekly) [28] and circadian [29,30] variations in HM protein concentrations during the established lactation.

\subsection{Sample Preparation}

Prior to further analysis, HM samples were thawed for $2 \mathrm{~h}$ at room temperature, mixed on Intelli-Mixer RM-2M (ELMI, Riga, Latvia) at 50 revolutions per min in "UU" mode for $15 \mathrm{~s}$, then, after gentle inversion (three times), aliquoted into $1.5 \mathrm{~mL}$ tubes (Sarstedt, Numbrecht, Germany). Pre- and post-feed samples were pooled for measuring casein and whey and total protein. Milk samples were defatted by centrifugation at room temperature in a Beckman Microfuge 11 (Aberdon Enterprise Inc., Elk Grove Village, IL, USA) at 10,000 $\times \mathrm{g}$ for $10 \mathrm{~min}$ and removing the fat layer by clipping it off together with the top of the tube [31]. Skim HM was used for measuring protein concentrations. Standard assays were adapted for and carried out using a JANUS workstation (PerkinElmer, Inc., Waltham, MA, USA) and measured on EnSpire (PerkinElmer, Inc., Waltham, MA, USA).

\subsection{Human Milk Fractions}

Casein and whey proteins were separated by the method described by Kunz and Lonnerdal [32] and Khan et al. [30]. Protein concentrations (total protein, casein, and whey proteins) were measured using the Bradford Protein Assay adapted from Mitoulas et al. [29]. Recovery of protein was $100.6 \pm 5.2 \%(n=5)$ with a detection limit of $0.031 \mathrm{~g} / \mathrm{L}$ and an inter-assay CV of $7.8 \%(n=18)$. Casein-whey ratio was calculated as follows:

$$
\text { Casein }- \text { whey ratio }=\text { casein concentration } / \text { whey protein concentration. }
$$

\subsection{Statistical Analyses}

Data for this analysis came from the longitudinal study, the details of which have been described previously [22]. Descriptive statistics are reported as mean \pm standard deviation (SD) (range); model parameters as estimate \pm SE (standard error).

During this longitudinal study infants were measured at four time points ( 2 and/or 5, 9 and 12 months). An approximate sample size was calculated using the ' $\mathrm{F}$ tests-Linear multiple regression: Fixed model: $R^{2}$ increase' option in $G^{*}$ Power [33] as if this was a cross-sectional study with equal numbers at each time. Allowing four predictors (three for age comparisons), $\alpha=0.05$ and 14 participants ( 56 sample points $=14$ participants $\times 4$ time points) would give the study power of 0.80 to detect an effect size of 0.15 . This approach was selected, as there is no closed form expression suitable for the calculation of sample sizes for this research design [34], with the consideration that longitudinal study design is more powerful. Recruitment of participants at the 5-months point was introduced, as many mothers would not commit to a study that required breastfeeding to 12 months, when approached at 2 months. As a result, required number of participants was increased to 20 in 
order to maintain predicted power; this also addressed issues relating to missed visits. Missing data was dealt with using available case analysis.

The analyses for systematic differences in concentrations and CDI of adipokines at different months after birth used linear mixed model with age as effect factor and participant as a random factor. Differences between each month were analysed using general linear hypothesis tests (Tukey's all pair comparisons).

Relationships between: (a) maternal BC and protein concentrations/CDI, and (b) protein concentrations/CDI and infant BC, (c) protein concentrations and breastfeeding parameters (24-h $\mathrm{MI} / \mathrm{FFQ}$ ), and (d) FFQ and CDI of proteins were analysed using linear mixed effects models. Each protein concentration/CDI or infant BC measure/index was considered separately as the response variable, and each model contained fixed effects of infant age (months), a predictor (maternal BC measure/index, protein concentration/CDI and breastfeeding parameters (24-h MI/FFQ)) and an interaction between infant age and predictor, as well as a random intercept per participant. If the interaction was not significant results were reported for the same model fitted without the interaction to assist in understanding the nature of the relationship between the predictor and outcome.

To investigate if there were differences by sex, relationships between infant characteristics and protein concentrations were also analysed using linear mixed effects models accounting for age and sex and an interaction between infant age and predictor, as well as a random intercept per participant.

Relationships between CDI of proteins measured between 2 and 5, and at 9 and 12 months after birth and changes $(\Delta)$ in infant BC and anthropometric parameters between the time points were analysed using linear regression models.

Owing to the large number of comparisons, a false discovery rate adjustment [35] was performed on associated subgroupings of results to the interaction $p$-value if it was less than 0.05 or to the main effect $p$-value. The adjusted significance levels are reported in Results and Tables and set at the 5\% level otherwise. Statistical analysis was performed in R 3.1.2 [36]. Additional packages were used for linear mixed effects models (nlme, lme4, and car) [37-39], intra-class correlations (irr) [40], Tukey's all pair comparisons (multcomp) [41] and graphics (ggplot2) [42].

\section{Results}

\subsection{Subjects}

Twenty-two infants were recruited; two infants (one male, one female) were excluded from the study after the 2-months visit (commenced weaning; personal circumstances). One female infant weaned after the 5-months visit and was not followed up further; 19 remaining infants were breastfed at 9 months; 17 infants continued to breastfeed at 12 months. Out of 18 infants measured at 12 months, 16 infants $(89 \%)$ still continued to breastfeed; one male infant ceased breastfeeding 2 weeks before the 12-month appointment and one female infant stopped at 10 months after birth.

Therefore, overall six infants missed one study session and one infant missed two study sessions. Five of these infants were not recruited until 5 months, one infant did not attend the study session at 9 months and two did not attend the study session at 12 months.

Overall, 80 measures were expected however some were missing, specifically: infant weight $(n=9)$; infant BC parameters measured with US 2SF, and maternal age, weight, height, BMI and BC parameters measured with BIS $(n=10)$; infant head circumference $(n=11)$; infant length, BMI and BC parameters measured with US 4SF, concentrations of casein, whey and total protein $(n=12)$; infant BC parameters measured with BIS $(n=13)$; self-reported FFQ $(n=20)$. Missing data also occurred due to difficulties with conducting 24-h MI measurements at later stages of lactation. The following measurements from the 60 expected were missing: FFQ from 24-h MP $(n=26)$, 24-h MI and CDI of casein, whey and total protein $(n=27)$. Missing data were spread across the time points (Table 1$)$.

Participant demographic characteristics collected at the start of the study are presented in Table 2, anthropometric and breastfeeding characteristics measured at the four study sessions are presented 
in Table 1. The more detailed determinants of maternal and infant BC as well as description of longitudinal changes in infant and maternal BC and breastfeeding parameters, and the associations between them have been reported previously [22].

Table 1. Participant anthropometric and breastfeeding characteristics throughout 12 months of lactation.

\begin{tabular}{|c|c|c|c|c|}
\hline & 2 Months & 5 Months & 9 Months & 12 Months \\
\hline \multirow[t]{2}{*}{ Characteristics } & Mean \pm SD & Mean \pm SD & Mean \pm SD & Mean \pm SD \\
\hline & (Range) & (Range) & (Range) & (Range) \\
\hline \multicolumn{5}{|c|}{ Mothers } \\
\hline & $n=14$ & $n=20$ & $n=18$ & $n=18$ \\
\hline Weight (kg) & $\begin{array}{c}78.8 \pm 19.3^{\mathrm{a}} \\
(57.5-116.2)\end{array}$ & $\begin{array}{c}70.1 \pm 17.8 \\
(53.7-115.3)\end{array}$ & $\begin{array}{c}63.0 \pm 10.0 \\
(50.4-121.9)\end{array}$ & $\begin{array}{c}64.2 \pm 17.3 \\
(51.4-121.9)\end{array}$ \\
\hline BMI $\left(\mathrm{kg} / \mathrm{m}^{2}\right)$ & $\begin{array}{l}27.2 \pm 5.5 \\
(20.4-35.5)\end{array}$ & $\begin{array}{l}24.8 \pm 5.0 \\
(19.0-35.2)\end{array}$ & $\begin{array}{c}22.7 \pm 3.9 \\
(17.9-37.2)\end{array}$ & $\begin{array}{l}23.9 \pm 5.9 \\
(18.2-37.2)\end{array}$ \\
\hline \multicolumn{5}{|c|}{ Infants } \\
\hline & $n=14$ & $n=20$ & $n=19$ & $n=18$ \\
\hline $\operatorname{Sex}(M / F)$ & $9 \mathrm{M} / 6 \mathrm{~F}$ & $10 \mathrm{M} / 10 \mathrm{~F}$ & $10 \mathrm{M} / 9 \mathrm{~F}$ & $9 \mathrm{M} / 9 \mathrm{~F}$ \\
\hline Age (months) & $\begin{array}{l}2.04 \pm 0.14 \\
(1.87-2.33)\end{array}$ & $\begin{array}{l}5.16 \pm 0.22 \\
(4.77-5.47)\end{array}$ & $\begin{array}{l}9.22 \pm 0.27 \\
(8.83-9.77)\end{array}$ & $\begin{array}{c}12.26 \pm 0.28 \\
(11.63-12.67)\end{array}$ \\
\hline Length $(\mathrm{cm})$ & $\begin{array}{l}58.1 \pm 1.9 \\
(54.2-60.0)\end{array}$ & $\begin{array}{l}64.8 \pm 2.3 \\
(60.5-69.5)\end{array}$ & $\begin{array}{l}71.7 \pm 1.9 \\
(66.0-74.0)\end{array}$ & $\begin{array}{l}73.6 \pm 3.2 \\
(69.0-78.5)\end{array}$ \\
\hline Weight (kg) & $\begin{array}{l}5.630 \pm 0.660 \\
(4.420-7.400)\end{array}$ & $\begin{array}{l}7.431 \pm 1.134 \\
(5.808-9.510)\end{array}$ & $\begin{array}{l}8.836 \pm 0.975 \\
(6.675-10.095)\end{array}$ & $\begin{array}{c}9.650 \pm 0.618 \\
(7.165-11.085)\end{array}$ \\
\hline BMI $\left(\mathrm{kg} / \mathrm{m}^{2}\right)$ & $\begin{array}{l}16.6 \pm 1.2 \\
(14.5-18.1)\end{array}$ & $\begin{array}{l}17.6 \pm 1.9 \\
(14.9-20.4)\end{array}$ & $\begin{array}{l}17.7 \pm 1.7 \\
(14.2-20.2)\end{array}$ & $\begin{array}{l}17.8 \pm 0.9 \\
(13.7-19.2)\end{array}$ \\
\hline Head circumference (cm) & $\begin{array}{l}39.7 \pm 1.6 \\
(37.0-42.0)\end{array}$ & $\begin{array}{l}42.1 \pm 1.5 \\
(40.0-45.9)\end{array}$ & $\begin{array}{l}45.6 \pm 1.7 \\
(43.0-48.5)\end{array}$ & $\begin{array}{l}46.6 \pm 1.7 \\
(44.2-49.5)\end{array}$ \\
\hline \multicolumn{5}{|c|}{ Breastfeeding Characteristics } \\
\hline & & $n=17$ & $n=8$ & $n=8$ \\
\hline \multirow[t]{2}{*}{ 24-h milk intake (g) } & $\mathrm{n} / \mathrm{a}^{\mathrm{b}}$ & $\begin{array}{c}818.8 \pm 204.9 \\
(498-1185)\end{array}$ & $\begin{array}{c}478.3 \pm 154.0 \\
(300-775)\end{array}$ & $\begin{array}{c}451.1 \pm 215.7 \\
(255-795)\end{array}$ \\
\hline & & $n=17$ & $n=8$ & $n=9$ \\
\hline \multirow[t]{2}{*}{ 24-h feeding frequency (MP) } & $\mathrm{n} / \mathrm{a}^{\mathrm{b}}$ & $\begin{array}{c}8.1 \pm 1.4 \\
(6-11)\end{array}$ & $\begin{array}{l}5.4 \pm 1.3 \\
\quad(4-7)\end{array}$ & $\begin{array}{l}4.4 \pm 2.1 \\
\quad(2-8)\end{array}$ \\
\hline & $n=11$ & $n=19$ & $n=17$ & $n=13$ \\
\hline Feeding frequency (SR) & $\begin{array}{c}2.3 \pm 0.4^{\mathrm{c}} \\
(1.5-3.0)\end{array}$ & $\begin{array}{l}2.8 \pm 0.8 \\
(1.5-4.0)\end{array}$ & $\begin{array}{l}3.7 \pm 1.2 \\
(2.0-6.0)\end{array}$ & $\begin{array}{l}5.4 \pm 2.9 \\
(2.2-12.0)\end{array}$ \\
\hline
\end{tabular}

a Data are mean \pm SD and ranges. ${ }^{b}$ Milk intake and feeding frequency as meals per 24-h was determined from 24-h milk production (MP) measured between 2 and 5 months (presented at 5 months here) and within 2 weeks of 9 and 12 months; $\mathrm{n} / \mathrm{a}$, not applicable. ${ }^{\mathrm{c}}$ Maternal self-report (SR) of feeding frequency at the time of the visit as a typical time between meals (e.g., each $2 \mathrm{~h}$ ). BMI, body mass index. M/F, male/female.

Table 2. Participant demographic characteristics at the start of the study.

\begin{tabular}{ll}
\hline Characteristics $n=\mathbf{2 0}$ & Mean \pm SD (Range) \\
\hline Maternal age (years) & $33.3 \pm 4.7(24-44)^{\mathrm{a}}$ \\
Maternal height $(\mathrm{cm})$ & $167.4 \pm 7.4(150-181)$ \\
Parity & $2.3 \pm 0.9(1-4)$ \\
Infant sex (Male/Female) & $10 / 10$ \\
Infant birth weight (kg) & $3.486 \pm 0.498(2.660-4.455)$ \\
Infant gestational age (weeks) & $39.4 \pm 1.3(37.6-43.0)$ \\
\hline
\end{tabular}

${ }^{a}$ Data are mean \pm standard deviation (SD) and ranges. 


\subsection{Breastfeeding Parameters and Human Milk Proteins}

HM protein concentrations and CDI at 4 time points are detailed in Table 3. CDI of casein, whey and total protein, 24-h MI, and both SR and MP FFQ decreased across the lactation (see Table 4 for estimates and significances).

Table 3. Human milk proteins presented as concentrations and 24-h intakes at months after birth ${ }^{\text {a }}$

\begin{tabular}{|c|c|c|c|c|}
\hline & 2 Months & 5 Months & 9 Months & 12 Months \\
\hline \multirow[t]{2}{*}{ Components } & Mean \pm SD & Mean \pm SD & Mean \pm SD & Mean \pm SD \\
\hline & (Range) & (Range) & (Range) & (Range) \\
\hline \multicolumn{5}{|c|}{ Concentrations ${ }^{b}$} \\
\hline & $n=15$ & $n=20$ & $n=19$ & $n=15$ \\
\hline Total protein $(\mathrm{g} / \mathrm{L})$ & $\begin{array}{c}11.03 \pm 1.40^{\mathrm{c}} \\
(7.60-12.32)\end{array}$ & $\begin{array}{l}11.90 \pm 4.31 \\
(7.93-24.16)\end{array}$ & $\begin{array}{c}9.69 \pm 1.12 \\
(7.25-14.96)\end{array}$ & $\begin{array}{l}10.72 \pm 2.84 \\
(5.89-16.80)\end{array}$ \\
\hline Casein $(\mathrm{g} / \mathrm{L})$ & $\begin{array}{l}1.24 \pm 0.24 \\
(0.69-1.57)\end{array}$ & $\begin{array}{l}1.51 \pm 0.44 \\
(0.78-3.45)\end{array}$ & $\begin{array}{l}1.11 \pm 0.38 \\
(0.49-2.00)\end{array}$ & $\begin{array}{l}1.07 \pm 0.35 \\
(0.65-1.87)\end{array}$ \\
\hline Whey protein $(\mathrm{g} / \mathrm{L})$ & $\begin{array}{l}6.44 \pm 1.62 \\
(4.12-9.08)\end{array}$ & $\begin{array}{l}5.43 \pm 0.90 \\
(3.82-7.38)\end{array}$ & $\begin{array}{l}5.43 \pm 0.93 \\
(3.94-9.40)\end{array}$ & $\begin{array}{l}7.61 \pm 1.85 \\
(4.49-9.76)\end{array}$ \\
\hline Casein-whey ratio & $\begin{array}{l}0.21 \pm 0.07 \\
(0.10-0.31)\end{array}$ & $\begin{array}{l}0.28 \pm 0.05 \\
(0.13-0.73)\end{array}$ & $\begin{array}{l}0.21 \pm 0.08 \\
(0.07-0.38)\end{array}$ & $\begin{array}{l}0.16 \pm 0.09 \\
(0.09-0.36)\end{array}$ \\
\hline \multicolumn{5}{|c|}{$\mathrm{CDI}^{\mathrm{d}}$} \\
\hline & & $n=17$ & $n=8$ & $n=8$ \\
\hline Total protein (g) & $\mathrm{n} / \mathrm{a}^{\mathrm{e}}$ & $\begin{array}{c}9.19 \pm 3.82^{\mathrm{d}} \\
(4.51-20.34)\end{array}$ & $\begin{array}{l}5.24 \pm 1.84 \\
(2.18-7.48)\end{array}$ & $\begin{array}{l}4.18 \pm 2.11 \\
(1.93-7.23)\end{array}$ \\
\hline Casein (g) & $\mathrm{n} / \mathrm{a}$ & $\begin{array}{l}1.45 \pm 0.82 \\
(0.56-3.63)\end{array}$ & $\begin{array}{l}0.60 \pm 0.23 \\
(0.17-0.95)\end{array}$ & $\begin{array}{l}0.54 \pm 0.34 \\
(0.24-1.19)\end{array}$ \\
\hline Whey protein (g) & $\mathrm{n} / \mathrm{a}$ & $\begin{array}{l}4.23 \pm 1.14 \\
(2.65-6.76)\end{array}$ & $\begin{array}{l}3.02 \pm 1.11 \\
(1.70-4.64)\end{array}$ & $\begin{array}{l}2.78 \pm 1.34 \\
(1.15-4.40)\end{array}$ \\
\hline
\end{tabular}

a Milk components' concentrations and 24-h components' intakes are presented grouped by the month after birth. ${ }^{b}$ Concentrations as measured at 2, 5, 9, and 12 months. ${ }^{\mathrm{c}}$ Data are mean $\pm \mathrm{SD}$ and ranges. ${ }^{\mathrm{d}}$ Calculated daily intakes (CDI) of proteins were calculated between 2 and 5 months (presented at 5 months here) and within 2 weeks of 9 and 12 months. ${ }^{\mathrm{e}} \mathrm{n} / \mathrm{a}$, not applicable. 


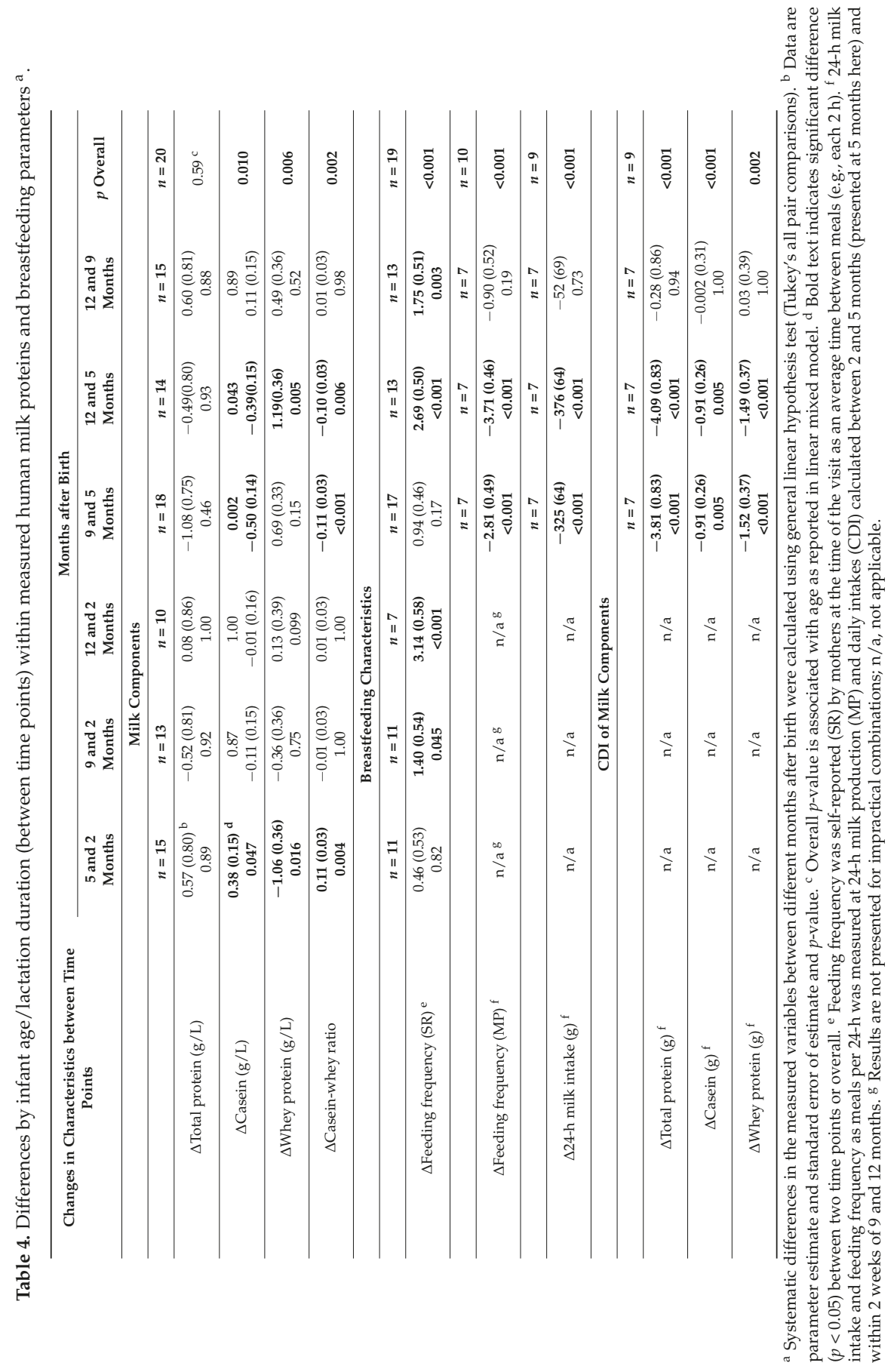




\subsection{Maternal Body Composition and Human Milk Proteins}

Higher maternal characteristics and BC measures (weight, BMI, FFM, FFMI, and FMI) were associated with higher whey protein concentrations (Figure 1).
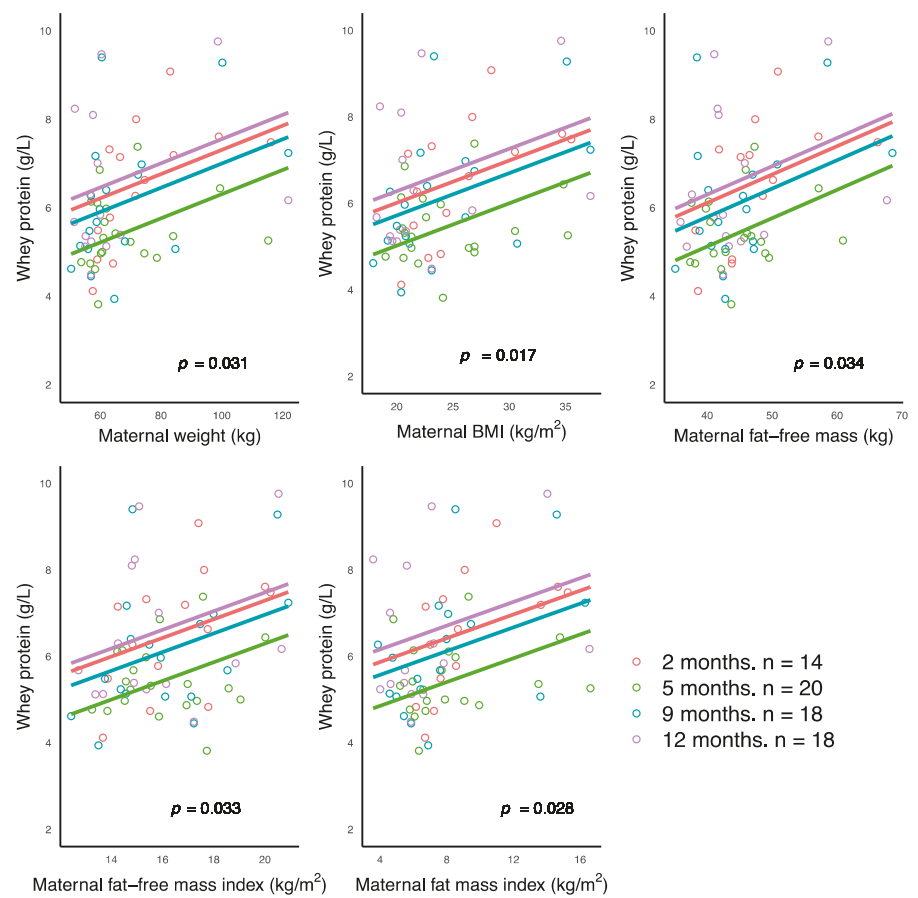

Figure 1. Significant positive associations between concentrations of human milk whey protein and maternal anthropometrics and body composition parameters measured with bioelectrical impedance spectroscopy. Lines represent linear regression and grouped by the month of lactation.

No associations were seen between maternal characteristics and casein-whey ratio, total protein and casein concentrations, and CDI of all proteins after adjusting for the false discovery rate (see Table A1 for estimates and significances).

\subsection{Infant Body Composition and Concentrations of Proteins}

No significant associations between concentrations of proteins and infant characteristics were seen after adjusting for the false discovery rate (see Table A2 for estimates and significances).

When infant sex was included in the models, significant differences were found for infant head circumference, weight, FFM (US 2SF, 4SF, and BIS) and FFMI (US 4SF and BIS), but none of the models reported an effect of either total protein, whey protein and casein concentrations or whey-to-casein ratio (details are not reported).

\subsection{Infant Body Composition and Calculated Daily Intakes of Proteins}

Higher CDI of casein were associated with lower infant FFM (US 4SF) and increased FM (US 4SF), $\%$ FM (US 4SF), and FMI (US 4SF) after adjusting for the false discovery rate (Figure 2).

No associations were seen between infant characteristics and both CDI of total protein and whey protein (see Table A3 for estimates and significances). 

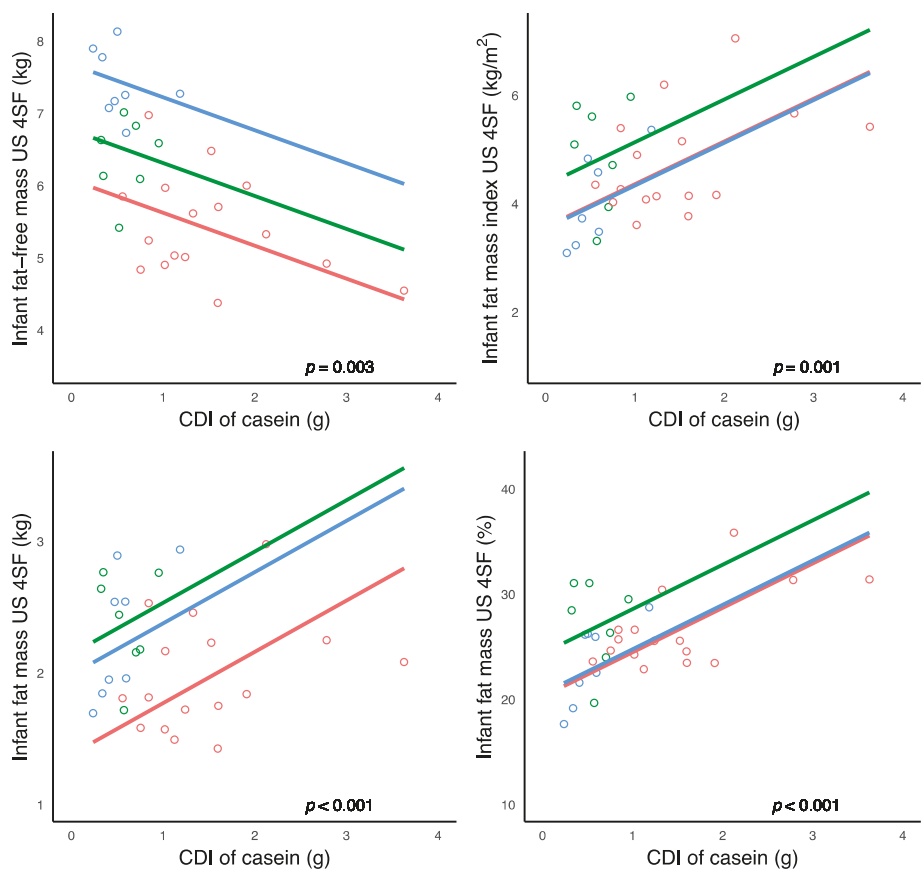

- 5 months. $n=16 \quad \circ \quad 9$ months. $n=7 \quad \circ \quad 12$ months. $n=8$

Figure 2. Significant associations between calculated daily intakes (CDI) of human milk casein and infant body composition parameters measured with ultrasound four-skinfolds. Lines represent linear regression and grouped by the month of lactation.

\subsection{Breastfeeding Parameters and Human Milk Proteins}

No significant associations were seen between concentrations of proteins and 24-h MI, 24-h MP FFQ, and SR FFQ after adjusting for the false discovery rate (see Table A4 for estimates and significances).

Higher FFQ (both, SR and 24-h MP) were associated with higher CDI of all, casein, whey and total protein (Figure 3) after adjusting for the false discovery rate (see Table A4 for estimates and significances).

\subsection{Changes in Infant Characteristics and Calculated Daily Intakes of Human Milk Proteins}

After adjusting for the false discovery rate, no significant associations were seen at any time points between changes in infant $\mathrm{BC}(\Delta)$ between the time points and CDI of either total protein, casein or whey protein (see Tables A5-A7 for estimates and significances). 

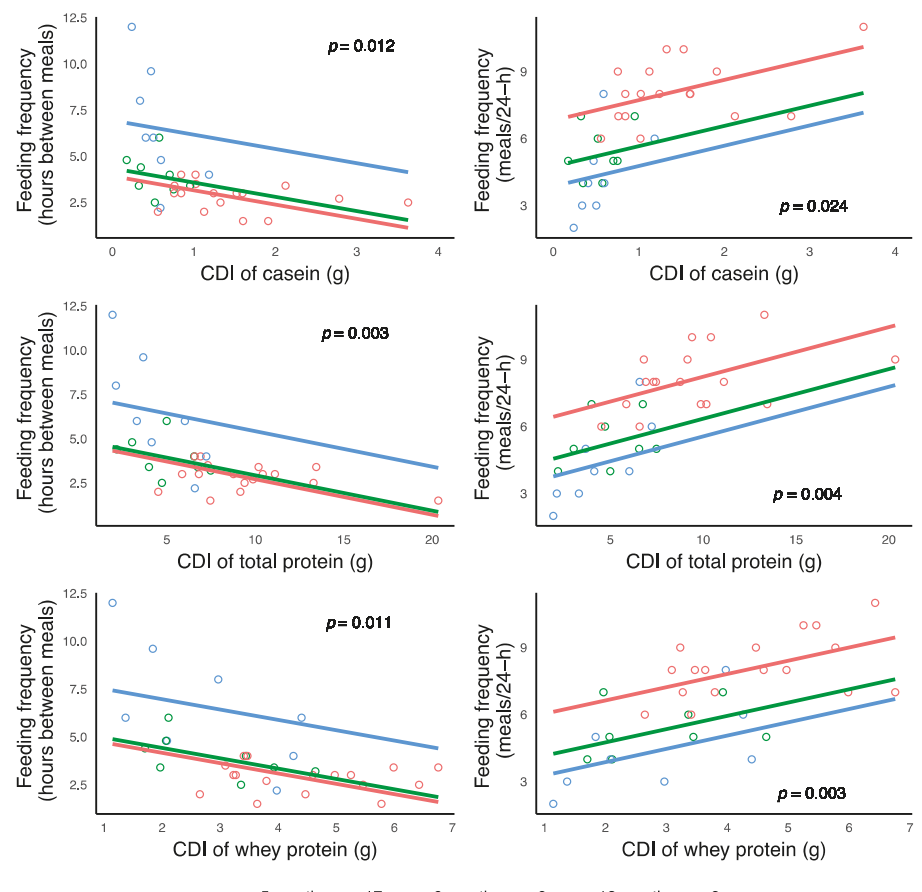

- 5 months. $n=17 \quad \circ \quad 9$ months. $n=8 \quad \circ \quad 12$ months. $n=8$

Figure 3. Significant associations between infant feeding frequency (self-reported feeding frequency (hours between meals) or 24-h milk production feeding frequency (meals per 24-h)) and calculated daily intakes (CDI) of casein and total and whey protein. Lines represent linear regression and grouped by the month of lactation.

\section{Discussion}

A dose-response relationship between breastfeeding duration and childhood obesity has been previously confirmed [43] and this longitudinal study provides more knowledge on the complex mechanisms by which breastfeeding and HM may influence infant BC and confer some degree of protection from obesity. In this small proof-of-concept study, calculated daily intakes of HM casein have been associated with the development of infant BC and are differentially related to infant FM and FFM (Figure 4). Furthermore, infant FFQ was associated with higher CDI of casein, whey and total protein, emphasizing the critical role of HM and breastfeeding in programming of infant appetite control and growth in the first year of life.

Within the normal developmental context of breastfeeding we found that concentrations of total protein (approximately $11 \mathrm{~g} / \mathrm{L}$ ) were not associated with infant anthropometrics or BC during first 12 months of lactation (Section 3.4; Table A2). However, in formula-fed infants high protein formulas (15-19 g/L) are related to accelerated growth trajectory with greater weight gain [15], while breastfed infants display reduced lean body mass and increased adiposity during the early infancy compared to formula-fed [18]. Also in contrast to our results, higher HM protein concentrations at four to eight weeks postpartum were related to higher infant BMI at 12 months but not weight gain or BC [44]. These contrasting results are likely due to the differences in protein composition of formulas and shorter breastfeeding durations. Our study focused on women who breastfed for 12 months and therefore is more reflective of normative development of $\mathrm{BC}$ of the breastfed infant. 


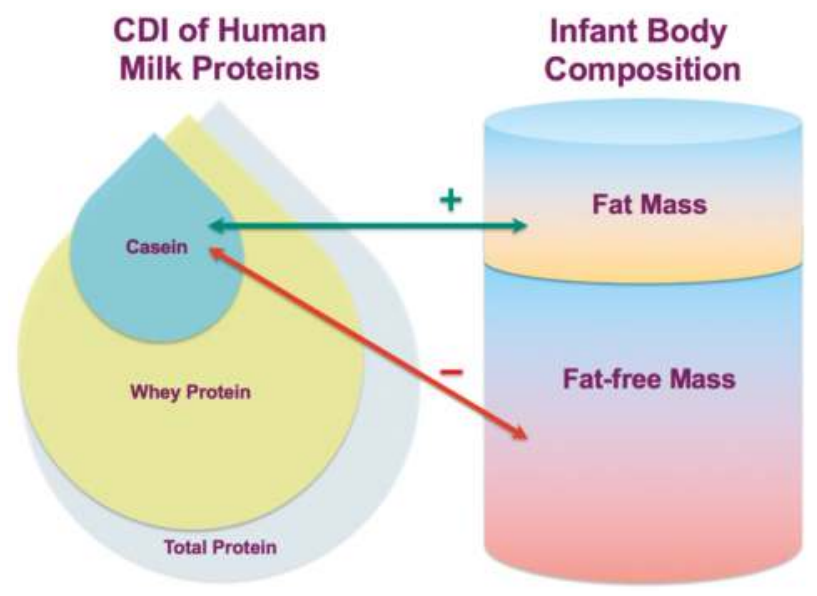

Figure 4. Possible lactocrine programming of the infant body composition during the first year of life as researched. Green arrow indicates positive associations of calculated daily intakes (CDI) of casein with measured body composition parameters and red arrow—-negative associations.

To account for variation in infant MI we calculated the CDI of total protein and found no association with infant anthropometrics or BC during first 12 months of lactation (Section 3.5; Table A3). Whilst volume of HM is known to be related to infant growth rate $[29,45,46]$, it has been shown that infants consuming higher daily intakes of protein at various times during first 12 months have higher weight and FFM $[8,47,48]$. These studies however included formula-fed infants and concentrated on differences between breastfeeding and formula feeding by calculating combined protein intakes from $\mathrm{HM}$ /formula and solids. Our study showed multiple positive associations between CDI of total protein between two and five months and changes in infant BC, but statistical significance did not remain after correction for multiple comparisons (Table A5). One recent study measured protein intakes from HM during the first three months in two groups of breastfed infants, with higher and lower weight gain [10] and found protein intakes were not different between the groups. It therefore remains to be elucidated if total protein intake in breastfed infants does impact BC development and larger longitudinal trials are required to do this.

Notwithstanding the absence of infant dietary data, we found that higher CDI of HM casein are associated with both lower infant lean body mass and higher adiposity (Section 3.5; Table A3), with associations strengthening at the later months of lactation. Casein is present in $\mathrm{HM}$ at a very low concentrations, compared with other species [49] and is not only a source of amino acids and trace elements (calcium and phosphorus) for the infant [50] but also breaks down to the bioactive peptides that have an array of functions including antimicrobial, gastrointestinal, immuno-modulating and opioid effects [51]. Indeed, increased CDI of casein was also related to increased FFQ. Previously we have shown that higher FFQ was related to lower FFM and higher 24-h MI and adiposity [22]. Furthermore, we have also found higher casein-whey ratios of HM are associated with shorter gastric emptying time in a cross-sectional cohort of exclusively breastfed infants [52]. Casein-whey ratios also interacted with feed volumes, with higher casein-whey ratios associating with faster gastric emptying of smaller feed volumes and slower gastric emptying of larger feed volumes [52]. Thus, HM casein may be modulating the development of infant $\mathrm{BC}$ via gastric emptying and breastfeeding frequency that in turn influences infant MI.

$\mathrm{CDI}$ of $\mathrm{HM}$ whey protein produced similar associations with infant $\mathrm{BC}$ to $\mathrm{CDI}$ of casein; negative with lean mass and positive with adiposity (Table A3) however, correction for multiple comparisons eliminated significance. We have found previously that higher HM whey protein concentrations are associated with larger post-feed stomach volumes in exclusively breastfed infants [52], and may 
therefore have an effect on infant BC, in combination with casein, via modulation of breastfeeding patterns. Studies in animal models indicate that whey from bovine milk may promote the growth of the soft (and adipose) tissue [53]. Furthermore, infants fed whey-dominant formula had higher weight-for-age and BMI-for-age z-scores at four months compared with breastfed infants despite the protein content being reduced to match HM [54], although it should be noted that these differences could be explained by the lower feed volumes in the breastfed group. In contrast infants fed a wheyand casein-dominant formula displayed no differences in BC or anthropometrics at four months of age [55], compared to breastfed infants. Another study has reported greater fat deposition in males and greater gains in lean mass in females fed whey-dominant formula during first three months compared to breastfed infants [56]. In this study, we did not see any effect of infant sex on relationships of either casein or whey protein concentrations with infant anthropometrics or BC. Larger longitudinal studies that focus on the array of HM whey proteins and sex-specific analysis may resolve these conflicting results.

We have examined the casein and total whey proteins of HM however, the HM whey fraction contains proteins that remain soluble in the liquid portion after precipitation of caseins-such as $\alpha$-lactalbumin, lactoferrin, lysozyme, and secretory IgA [57]—as well as various hormones, enzymes, and binding proteins [49]. Many of these proteins have not been studied in relation to infant growth and $\mathrm{BC}$ and may either act synergistically or in an antagonistic manner. Administered bovine and HM lysozyme [58,59] have been linked to increased weight gain in preterm infants. Furthermore, high levels of lactoferrin in bovine formula was associated with lower weight gain rate in healthy term female infants during first two months after birth compared with control [60]. Interestingly, $\alpha$-lactalbumin-enriched formula did not result in any differences in weight gain, weight-for-age and weight-for-length z-scores compared with breastfed infants or infants fed standard formula at four months of age [61]. This suggests that $\alpha$-lactalbumin, a rich source of essential amino acids, may play optimal nutritive but not necessarily programming role in infant growth. It is yet to be determined whether the proportions of the most abundant whey proteins impact growth and $\mathrm{BC}$ development, in particular lysozyme, which may act via promotion of the colonization of infant gut by the infant-type human-residential bifidobacteria [62] and via change in gut microbiome composition through both, detrimental microbe reduction and beneficial microbe enrichment as shown in porcine model [63].

The number of studies showing associations between maternal characteristics and HM components is increasing. We found that higher whey protein concentrations are related to higher maternal weight, BMI, and both fat and lean body mass throughout the first 12 months of lactation (Section 3.3, Table A1). This represents a potential pathway by which the protein composition of HM may be altered and potentially improved via optimal maternal BC which may impact infant development. The absence of an association with HM casein concentration suggests it is not modifiable, and reflects the intrinsic synthesis of casein in the breast [64] as opposed to movement of whey components from the maternal circulation into the milk [65]. Whilst we did not find any associations between maternal characteristics and total protein concentrations, total protein has previously been positively related to maternal \%FM [23] and BMI [66-69]. Other studies have shown no associations [70] or a negative association [71]. Larger longitudinal studies would help to clarify these associations.

Breastfeeding frequency is likely a reflection of appetite regulation of the infant [72] and is highly variable between breastfeeding dyads [27]. Recently we have shown increased FFQ was associated with increased 24-h MI, and both of these breastfeeding parameters were related to higher infant adiposity [22]. Both methods that we used to measure infant FFQ have some limitations, especially self-reported FFQ which has been shown to be biased towards reporting higher numbers of feeds in infants that feed more frequently in comparison with 24-h MP FFQ [22], which is again limited to one measure at the time point of data collection. Nevertheless, positive relationships between FFQ (which were also associated with higher FM accretion) and CDI of HM proteins casein in particular (Section 3.6; Table A4) help us to assemble the possible pathways of the mechanisms of infant BC regulation. These results emphasize the importance of including these measures in order to elucidate 
the mechanisms by which HM components affect infant growth and BC and the degree by which the mother influences HM composition and volumes.

Daily intakes may be a more relevant factor than concentrations of HM components for examining the nutritional physiology of the breastfed infant. In this study, daily intakes were calculated and are considered representative of a typical daily intake due to the absence of significant within-feed [23], short-term (weekly) [28], and circadian [29] variations in HM protein concentrations, including casein and whey [30], during the established lactation. Furthermore, measurement of 24-h milk intake by test-weighing is an accurate [73] and reproducible procedure from within two to four days to within one to two weeks between measurements [45,74-76]. Although no dietary data was collected in this study, the evidence is emerging that protein from dairy/milk sources may have different and/or more potent effects on infant growth and adiposity than the vegetable and meat proteins probably due to the bioactive factors present in milks $[77,78]$. The major source of milk protein in this study was HM, as no formula was used by mothers. Furthermore, differences in protein intake between breastfed and formula-fed infants during first 12 months are mainly attributed to a lower intake of protein from HM, not to a difference in protein intake from solid foods [48].

The strength of this proof-of-concept study is that infants were breastfed on demand up to 12 months and the broad range of adiposity levels among the mothers. The limitations are the small number of participants, the small number of 24-h MP at 9 and 12 months and the absence of infant dietary data between 5 and 12 months of age. Our population was term healthy fully-breastfed singletons from predominantly Caucasian mothers of higher social-economic status therefore, our results may not be applicable to dyads from other backgrounds.

\section{Conclusions}

The results of this small proof-of-concept study provide more evidence that HM impacts infant developmental programming and show differential associations between HM casein intakes and development of infant fat and fat-free mass during the first 12 months of life. Thus, the continuation of breastfeeding past the exclusive breastfeeding period may facilitate developmental programming that could potentially reduce risk of obesity and NCD later in life.

Author Contributions: All authors critically reviewed and approved the final manuscript. Conceptualization, Z.G., L.C.W., K.M., P.E.H., and D.T.G.; Data curation, Z.G.; Formal analysis, Z.G., A.R., and K.M.; Funding acquisition, D.T.G.; Investigation, Z.G., W.J.T., C.T.L., and D.T.G.; Methodology, C.T.L. and D.T.G.; Resources, D.T.G.; Supervision, C.T.L., L.C.W., K.M., P.E.H., and D.T.G.; Visualization, Z.G., A.R., and K.M.; Writing-Original draft, Z.G., A.R., and D.T.G.; Writing-Review \& editing, W.J.T., C.T.L., L.C.W., K.M., and P.E.H.

Funding: This study was supported by an unrestricted research grant from Medela AG (Switzerland). Z.G. was supported by an Australian Postgraduate Award from The University of Western Australia (Australia).

Acknowledgments: Authors wish to thank Anna R. Hepworth for consultation on research design and assistance with data analyses. Many thanks are extended to all mothers and infants who participated in this study.

Conflicts of Interest: The authors declare that Medela AG provided an unrestricted research grant to D.T.G., from which salaries to D.T.G., Z.G., W.J.T., and C.T.L. were paid. Medela AG provided a Top-up Scholarship for Z.G. and has provided speaker's fees to D.T.G. for educational lectures. The funding sponsors had no role in the design of the study; in the collection, analyses, or interpretation of data; In the writing of the manuscript, or in the decision to publish the results. Author L.C.W. provides consultancy services to ImpediMed Ltd. ImpediMed Ltd. was not involved in the inception and conducting of this research or in the writing of the manuscript. Other authors declare no conflict of interest. 


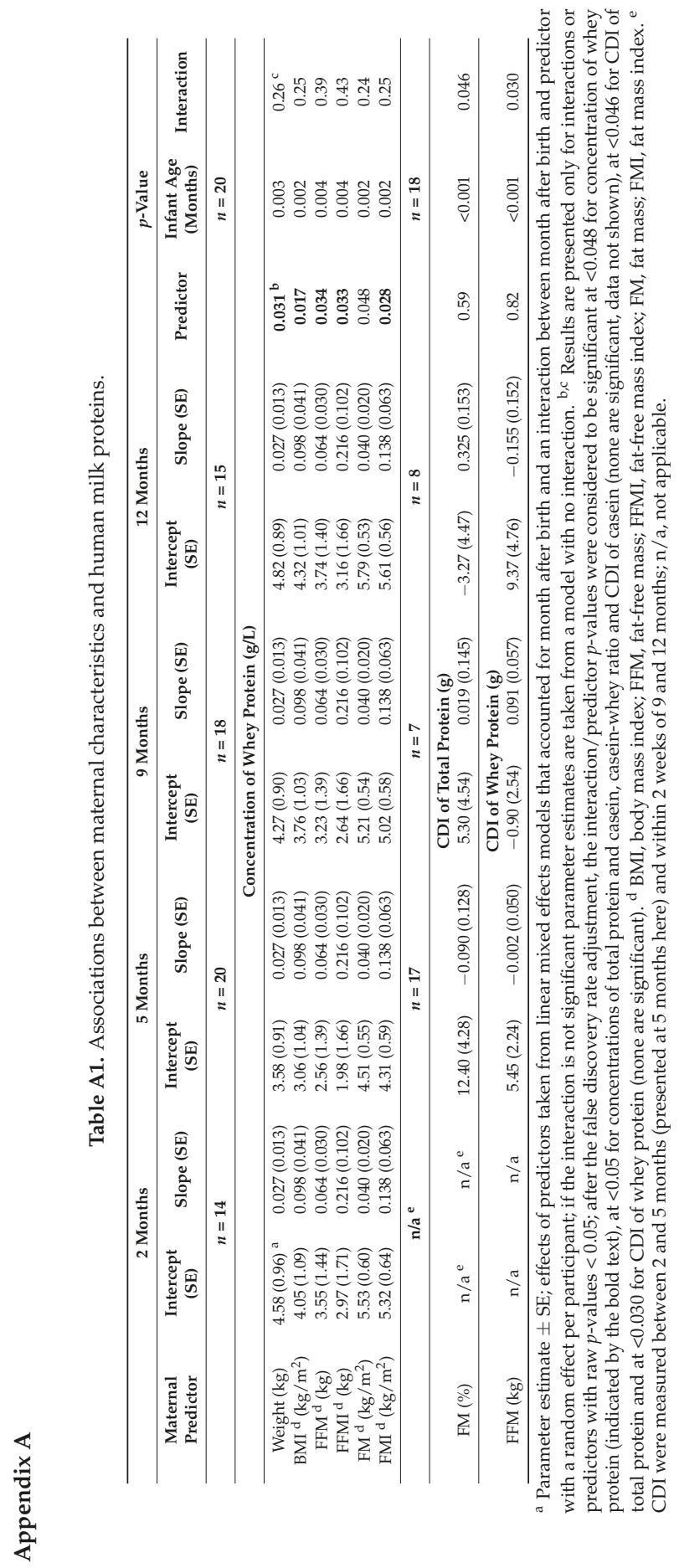


Nutrients 2018, 10, 1332

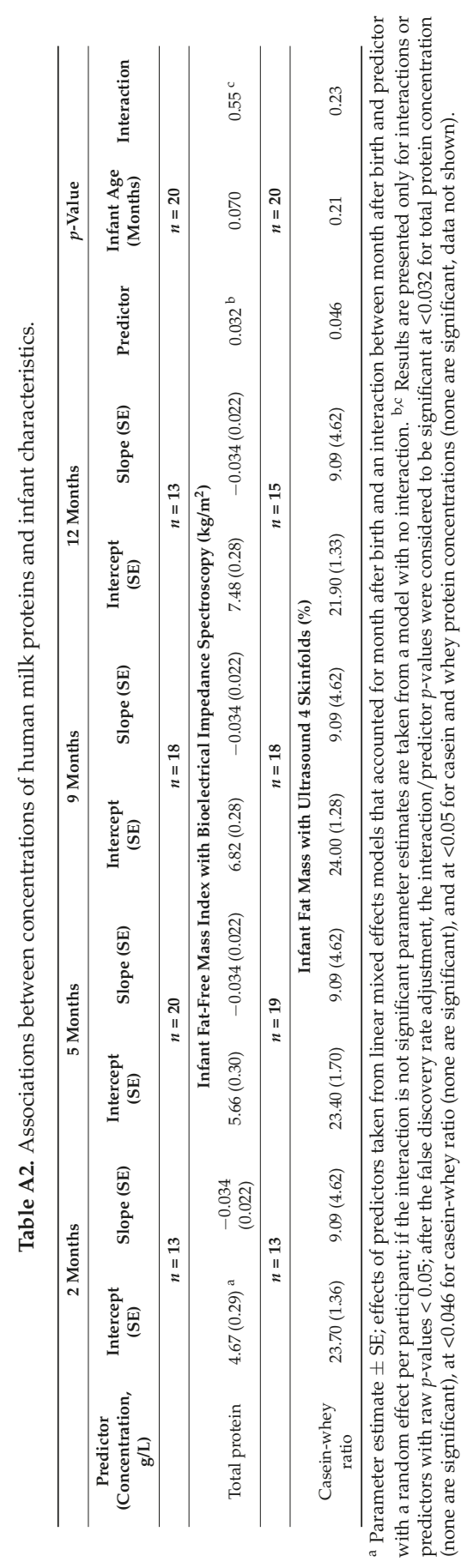


Nutrients 2018, 10, 1332

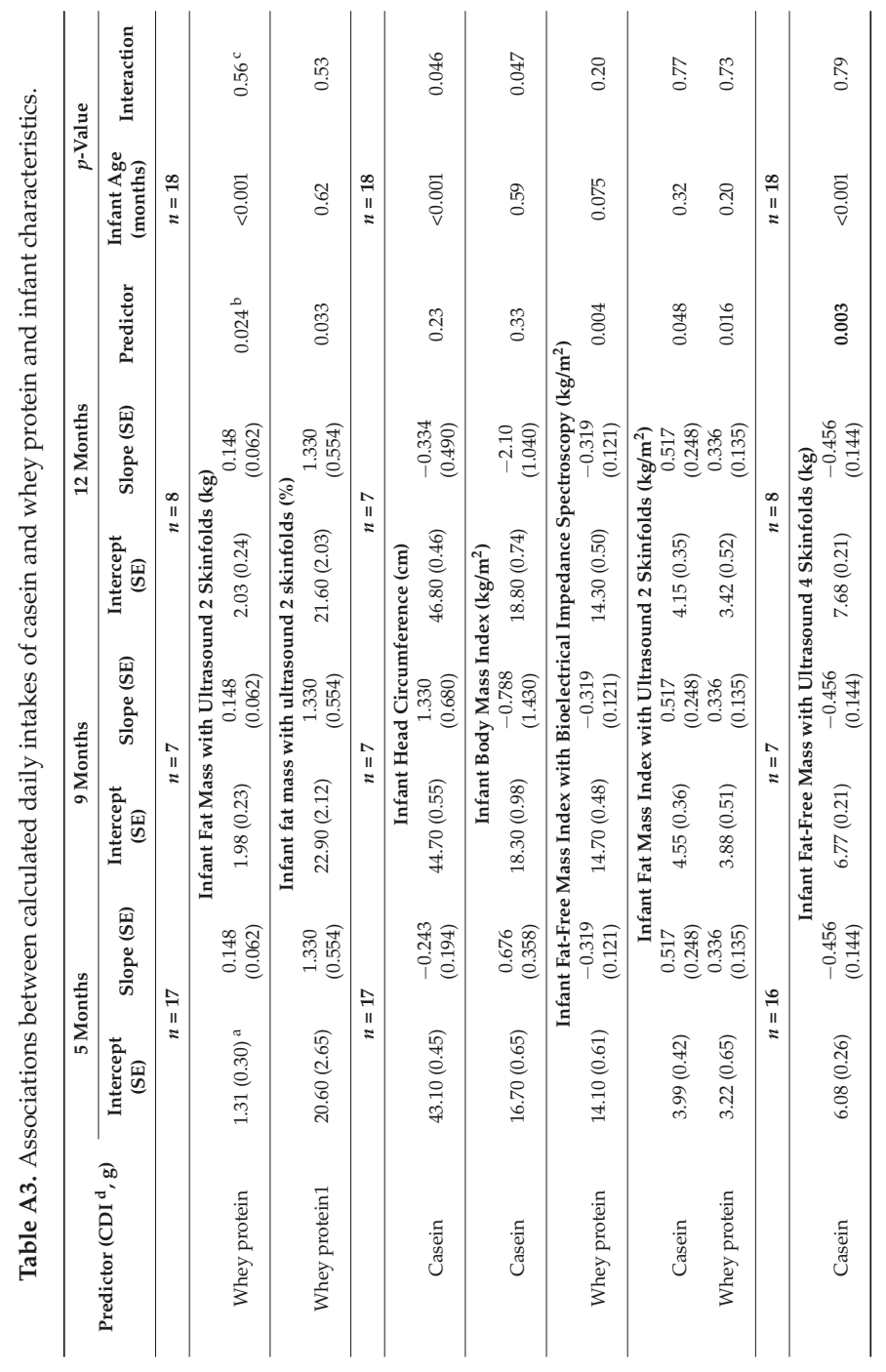




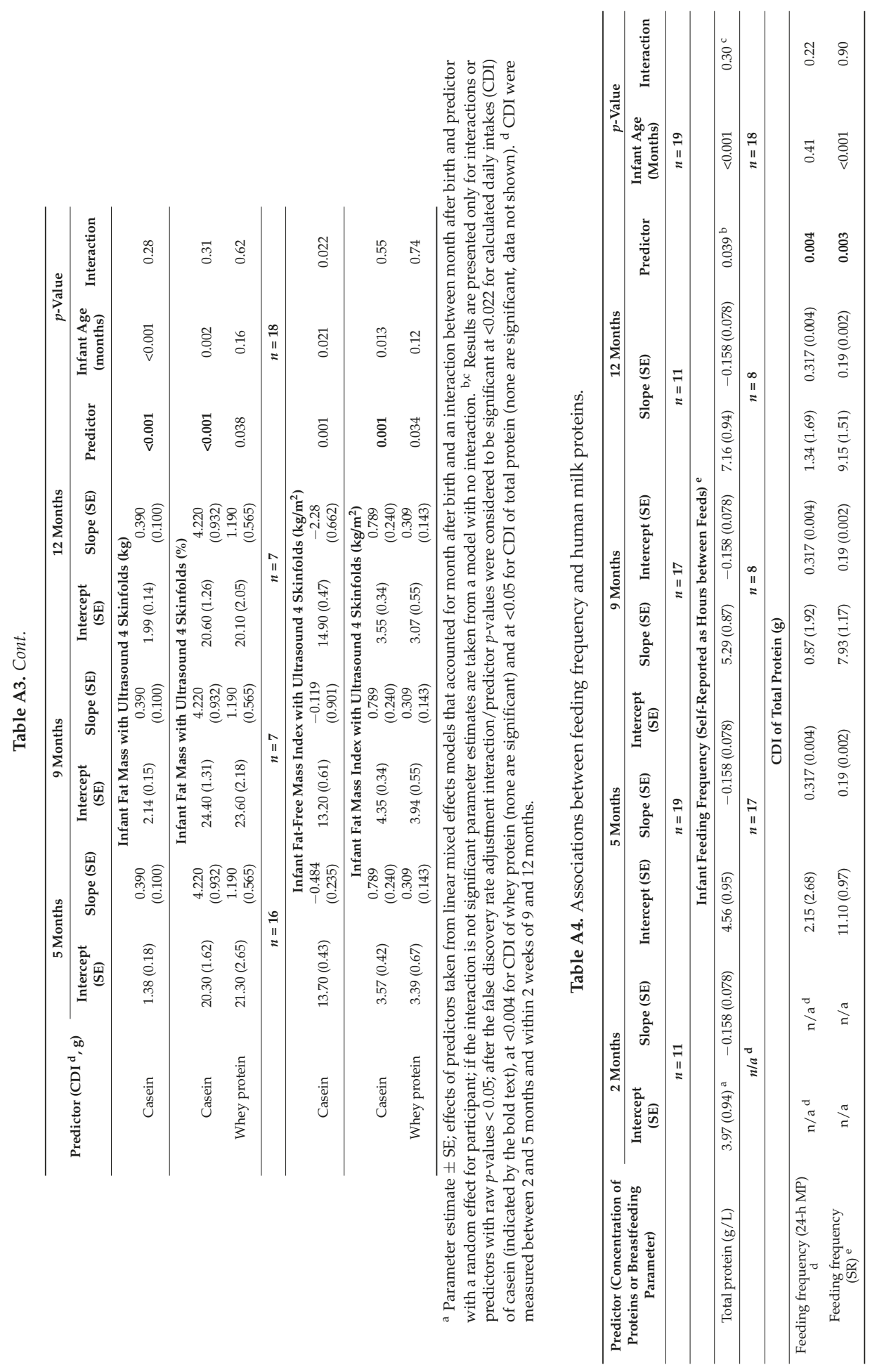




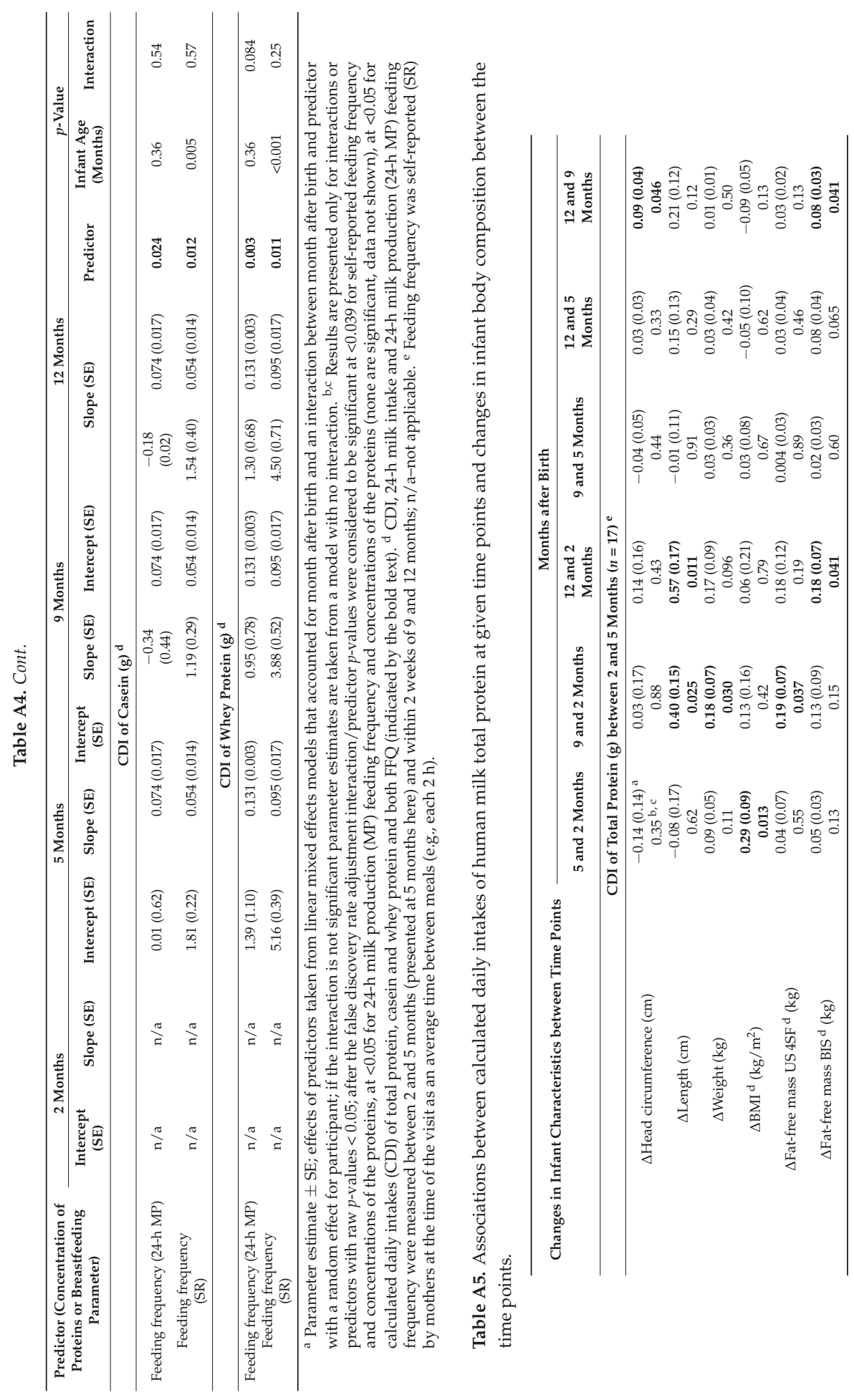




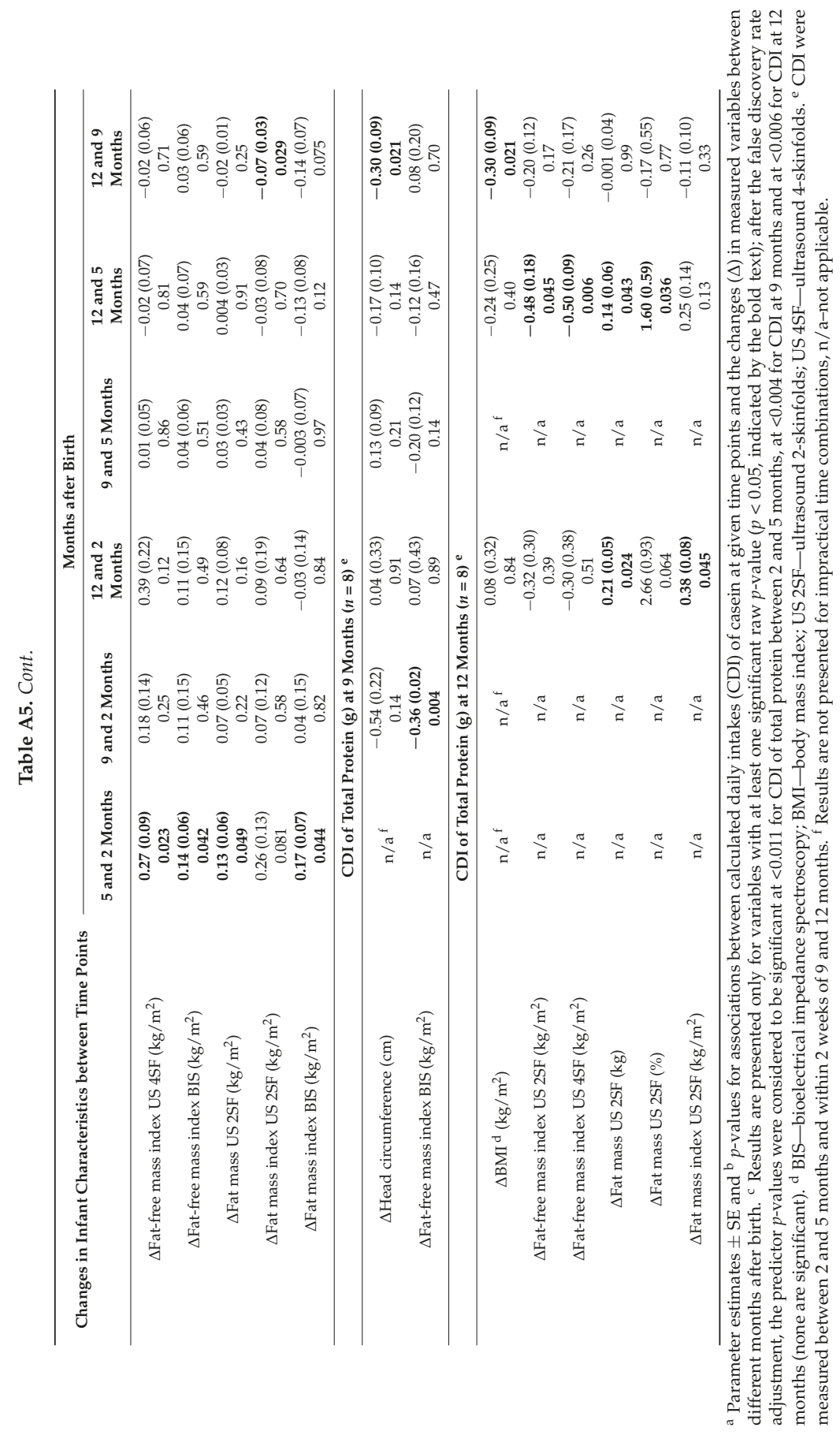




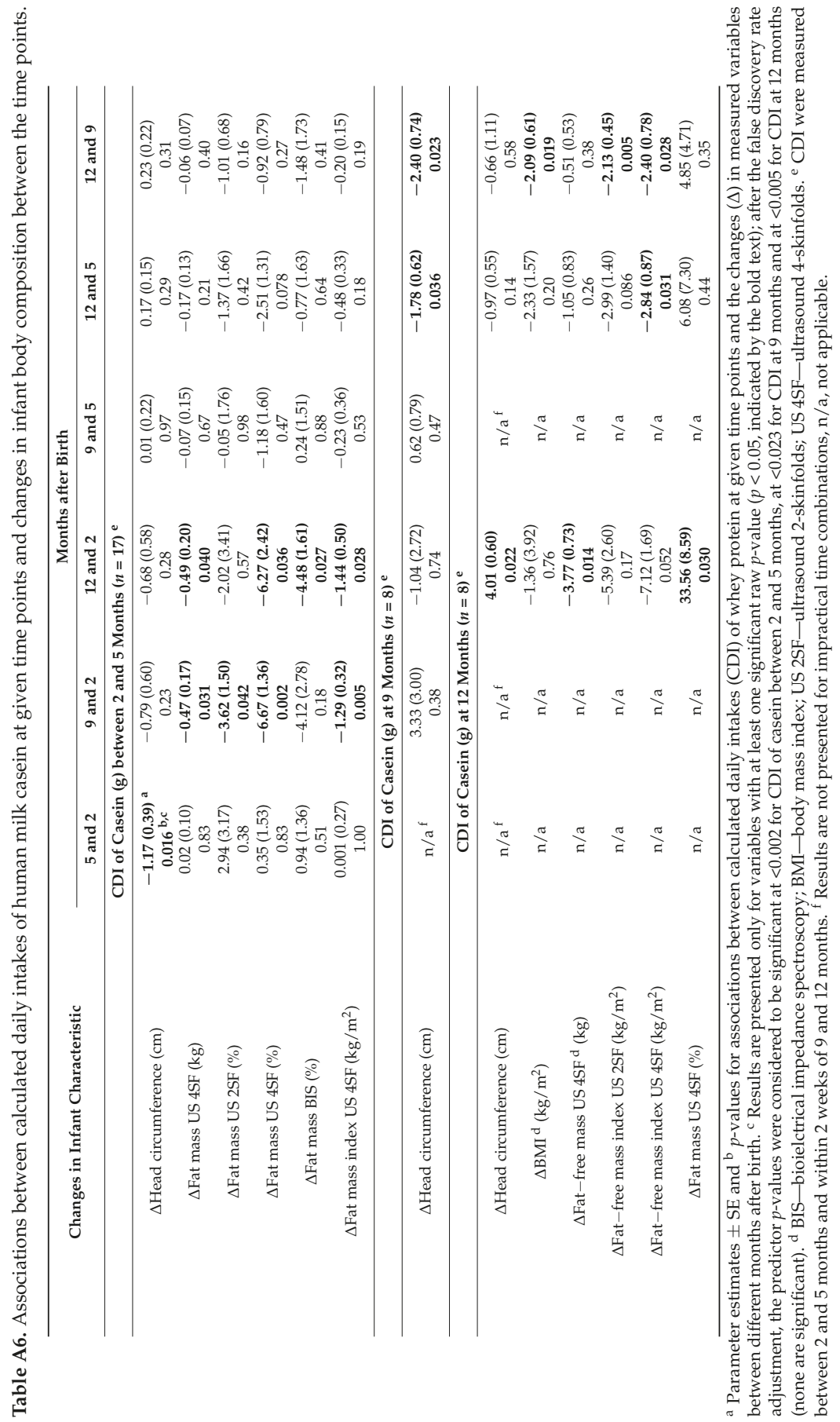


Nutrients 2018, 10, 1332

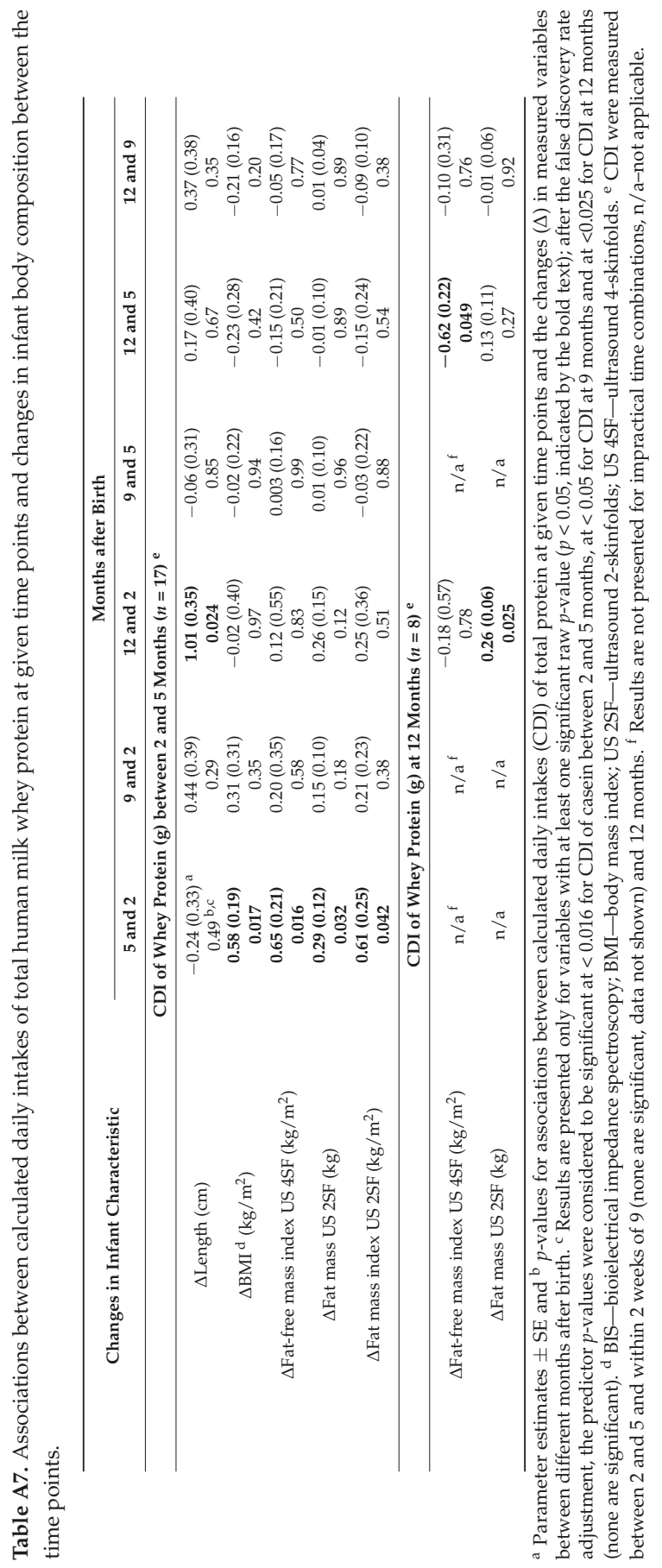




\section{References}

1. Wells, J.C.K.; Shirley, M.K. Body composition and the monitoring of non-communicable chronic disease risk. Glob. Health Epidemiol. Genom. 2016, 1, e18. [CrossRef] [PubMed]

2. Koletzko, B.; Brands, B.; Chourdakis, M.; Cramer, S.; Grote, V.; Hellmuth, C.; Kirchberg, F.; Prell, C.; Rzehak, P.; Uhl, O.; et al. The power of programming and the EarlyNutrition project: Opportunities for health promotion by nutrition during the first thousand days of life and beyond. Ann. Nutr. Metab. 2014, 64, 187-196. [CrossRef] [PubMed]

3. Ratnasingham, A.; Eiby, Y.; Dekker Nitert, M.; Donovan, T.; Lingwood, B.E. Review: Is rapid fat accumulation in early life associated with adverse later health outcomes? Placenta 2017, 54, 125-130. [CrossRef] [PubMed]

4. Weng, S.F.; Redsell, S.A.; Swift, J.A.; Yang, M.; Glazebrook, C.P. Systematic review and meta-analyses of risk factors for childhood overweight identifiable during infancy. Arch. Dis. Child. 2012, 97, 1019-1026. [CrossRef] [PubMed]

5. Koletzko, B.; von Kries, R.; Monasterolo, R.C.; Subias, J.E.; Scaglioni, S.; Giovannini, M.; Beyer, J.; Demmelmair, H.; Gruszfeld, D. Infant feeding and later obesity risk. Adv. Exp. Med. Biol. 2009, 646, 15-29. [PubMed]

6. Thompson, A.L. Developmental origins of obesity: Early feeding environments, infant growth, and the intestinal microbiome. Am. J. Hum. Biol. 2012, 24, 350-360. [CrossRef] [PubMed]

7. Wells, J.C.; Chomoto, S.; Fewtrell, M.S. Programming of body composition by early growth and nutrition. Proc. Nutr. Soc. 2007, 66, 423-434. [CrossRef] [PubMed]

8. Butte, N.; Wong, W.; Hopkinson, J.; Smith, E.; Ellis, K. Infant feeding mode affects early growth and body composition. Pediatrics 2000, 16, 1355-1366. [CrossRef]

9. Luque, V.; Closa-Monasterolo, R.; Escribano, J.; Ferre, N. Early programming by protein intake: The effect of protein on adiposity development and the growth and functionality of vital organs. Nutr. Metab. Insights 2015, 8, 49-56. [CrossRef] [PubMed]

10. Kon, I.Y.; Shilina, N.M.; Gmoshinskaya, M.V.; Ivanushkina, T.A. The study of breast milk IGF-1, leptin, ghrelin and adiponectin levels as possible reasons of high weight gain in breast-fed infants. Ann. Nutr. Metab. 2014, 65, 317-323. [CrossRef] [PubMed]

11. Sievers, E.; Oldigs, H.D.; Santer, R.; Schaub, J. Feeding patterns in breast-fed and formula-fed infants. Ann. Nutr. Metab. 2002, 46, 243-248. [CrossRef] [PubMed]

12. Savino, F.; Liguori, S.A.; Fissore, M.F.; Oggero, R. Breast milk hormones and their protective effect on obesity. Int. J. Pediatr. Endocrinol. 2009, 2009, 327505. [CrossRef] [PubMed]

13. Bartok, C. Babies fed breastmilk by breast versus by bottle: A pilot study evaluating early growth patterns. Breastfeed. Med. 2011, 6, 117-124. [CrossRef] [PubMed]

14. Druet, C.; Stettler, N.; Sharp, S.; Simmons, R.K.; Cooper, C.; Smith, G.D.; Ekelund, U.; Levy-Marchal, C.; Jarvelin, M.R.; Kuh, D.; et al. Prediction of childhood obesity by infancy weight gain: An individual-level meta-analysis. Paediatr. Perinat. Epidemiol. 2011, 26, 19-26. [CrossRef] [PubMed]

15. Koletzko, B.; von Kries, R.; Closa, R.; Escribano, J.; Scaglioni, S.; Giovannini, M.; Beyer, J.; Demmelmair, H.; Gruszfeld, D.; Dobrzanska, A.; et al. Lower protein in infant formula is associated with lower weight up to age 2 y: A randomized clinical trial. Am. J. Clin. Nutr. 2009, 89, 1836-1845. [PubMed]

16. Gidrewicz, D.A.; Fenton, T.R. A systematic review and meta-analysis of the nutrient content of preterm and term breast milk. BMC Pediatr. 2014, 14, 216. [CrossRef] [PubMed]

17. Rzehak, P.; Oddy, W.H.; Mearin, M.L.; Grote, V.; Mori, T.A.; Szajewska, H.; Shamir, R.; Koletzko, S.; Weber, M.; Beilin, L.J.; et al. Infant feeding and growth trajectory patterns in childhood and body composition in young adulthood. Am. J. Clin. Nutr. 2017, 102, 568-580. [CrossRef] [PubMed]

18. Gale, C.; Logan, K.; Santhakumaran, S.; Parkinson, J.; Hyde, M.; Modi, N. Effect of breastfeeding compared with formula feeding on infant body composition: A systematic review and meta-analysis. Am. J. Clin. Nutr. 2012, 95, 656-669. [CrossRef] [PubMed]

19. Khan, S.; Hepworth, A.R.; Prime, D.K.; Lai, C.T.; Trengove, N.J.; Hartmann, P.E. Variation in fat, lactose, and protein composition in breast milk over 24 hours: Associations with infant feeding patterns. J. Hum. Lact. 2013, 29, 81-89. [CrossRef] [PubMed]

20. Binns, C.W.; Fraser, M.L.; Lee, A.H.; Scott, J. Defining exclusive breastfeeding in Australia. J. Paediatr. Child Health 2009, 45, 174-180. [CrossRef] [PubMed] 
21. Gridneva, Z.; Hepworth, A.; Ward, L.; Lai, C.T.; Hartmann, P.; Geddes, D.T. Bioimpedance spectroscopy in the infant: Effect of milk intake and extracellular fluid reservoirs on resistance measurements in term breastfed infants. Eur. J. Clin. Nutr. 2016, 70, 843-851. [CrossRef] [PubMed]

22. Gridneva, Z.; Rea, A.; Hepworth, A.R.; Ward, L.C.; Lai, C.T.; Hartmann, P.E.; Geddes, D.T. Relationships between breastfeeding patterns and maternal and infant body composition over the first 12 months of lactation. Nutrients 2018, 10, 45. [CrossRef] [PubMed]

23. Kugananthan, S.; Gridneva, Z.; Lai, C.T.; Hepworth, A.R.; Mark, P.J.; Kakulas, F.; Geddes, D.T. Associations between maternal body composition and appetite hormones and macronutrients in human milk. Nutrients 2017, 9, 252. [CrossRef] [PubMed]

24. Gridneva, Z.; Hepworth, A.R.; Ward, L.C.; Lai, C.T.; Hartmann, P.E.; Geddes, D.T. Determinants of body composition in breastfed infants using bioimpedance spectroscopy and ultrasound skinfolds-Methods comparison. Pediatr. Res. 2016, 81, 423-433. [CrossRef] [PubMed]

25. Van Itallie, T.B.; Yang, M.U.; Heymsfield, S.B.; Funk, R.C.; Boileau, R.A. Height-normalized indices of the body's fat-free mass and fat mass: Potentially useful indicators of nutritional status. Am. J. Clin. Nutr. 1990, 52, 953-959. [CrossRef] [PubMed]

26. Arthur, P.; Hartmann, P.; Smith, M. Measurement of the milk intake of breast-fed infants. J. Pediatr. Gastroenterol. Nutr. 1987, 6, 758-763. [CrossRef] [PubMed]

27. Kent, J.C.; Mitoulas, L.R.; Cregan, M.D.; Ramsay, D.T.; Doherty, D.A.; Hartmann, P.E. Volume and frequency of breastfeedings and fat content of breast milk throughout the day. Pediatrics 2006, 117, e387-e395. [CrossRef] [PubMed]

28. Khan, S.; Prime, D.K.; Hepworth, A.R.; Lai, C.T.; Trengove, N.J.; Hartmann, P.E. Investigation of short-term variations in term breast milk composition during repeated breast expression sessions. J. Hum. Lact. 2013, 29, 196-204. [CrossRef] [PubMed]

29. Mitoulas, L.R.; Kent, J.C.; Cox, D.B.; Owens, R.A.; Sherriff, J.L.; Hartmann, P.E. Variation in fat, lactose and protein in human milk over $24 \mathrm{~h}$ and throughout the first year of lactation. Br. J. Nutr. 2002, 88, 29-37. [CrossRef] [PubMed]

30. Khan, S.; Casadio, Y.; Lai, C.; Prime, D.; Hepworth, A.; Trengove, N.; Hartmann, P. Investigation of short-term variations in casein and whey proteins in breast milk of term mothers. Hepatol. Nutr. 2012, 55, 136-141. [CrossRef] [PubMed]

31. Keller, R.; Neville, M. Determination of total protein in human milk: Comparison of methods. Clin. Chem. 1986, 32, 120-123. [PubMed]

32. Kunz, C.; Lonnerdal, B. Human milk proteins: Separation of whey proteins and their analysis by polyacrylamide gel electrophoresis, fast protein liquid chromatography (FPLC) gel filtration, and anion-exchange chromatography. Am. J. Clin. Nutr. 1989, 49, 464-470. [CrossRef] [PubMed]

33. Faul, F.; Erdfelder, E.; Buchner, A.; Lang, A.-G. Statistical power analyses using G*power 3.1: Tests for correlation and regression analyses. Behav. Res. Methods 2009, 41, 1149-1160. [CrossRef] [PubMed]

34. Diggle, P.J.; Heagerty, P.J.; Liang, K.-Y.; Zeger, S.L. Analysis of Longitudinal Data; Oxford University Press Inc.: New York, NY, USA, 2002.

35. Curran-Everett, D. Multiple comparisons: Philosophies and illustrations. Am. J. Physiol. Regul. Integr. Comp. Physiol. 2000, 279, R1-R8. [CrossRef] [PubMed]

36. R Core Team. R: A Language and Environment for Statistical Computing; R Foundation for Statistical Computing: Vienna, Austria, 2017.

37. Pinheiro, J.B.; Bates, D.; DebRoy, S.; Sarkar, D.; R Core Team. nlme: Linear and Nonlinear Mixed Effects Models; R Package Version 3.1-131; R Foundation for Statistical Computing: Vienna, Austria, 2017.

38. Bates, D.; Maechler, M.; Bolker, B.; Walker, S. Ime4: Linear Mixed-Efects Models Using Eigen and s4; R package Version 1.1-7; R Foundation for Statistical Computing: Vienna, Austria, 2014.

39. Fox, J.; Weisberg, S. An R Companion to Applied Regression; Sage: Thousand Oaks, CA, USA, 2011.

40. Gamer, M.; Lemon, J.; Fellows, I.; Singh, P. Various Coefficients of Interrater Reliability and Agreement; R package Version 0.84; R Foundation for Statistical Computing: Vienna, Austria, 2012.

41. Hothorn, T.; Bretz, F.; Westfall, P. Simultaneous inference in general parametric models. Biom. J. 2008, 50, 346-363. [CrossRef] [PubMed]

42. Wickham, H. Ggplot2: Elegant Graphics for Data Analysis; Springer: New York, NY, USA, 2009. 
43. Yan, J.; Liu, L.; Zhu, Y.; Huang, G.; Wang, P.P. The association between breastfeeding and childhood obesity: A meta-analysis. BMC Public Health 2014, 14, 1267. [CrossRef] [PubMed]

44. Prentice, P.; Ong, K.K.; Schoemaker, M.H.; van Tol, E.A.F.; Vervoort, J.; Hughes, I.A.; Acerini, C.L. Breast milk nutrient content and infancy growth. Acta Paediatr. 2016, 105, 641-647. [CrossRef] [PubMed]

45. Dewey, K.; Heinig, M.; Nommsen, L.; Lonnerdal, B. Maternal versus infant factors related to breast milk intake and residual milk volume: The Darling study. Pediatrics 1991, 87, 829-837. [PubMed]

46. Kent, J.; Mitoulas, L.; Cox, D.B.; Owens, R.; Hartmann, P. Breast volume and milk production during extended lactation in women. Exp. Physiol. 1999, 84, 435-447. [CrossRef] [PubMed]

47. De Bruin, N.C.; Degenhart, H.J.; Gal, S.; Westerterp, K.R.; Stijen, T.; Visser, H.K.A. Energy utilization and growth in breast-fed and formula-fed infants measured prospectively during the first year of life. Am. J. Clin. Nutr. 1998, 67, 885-896. [CrossRef] [PubMed]

48. Heinig, M.J.; Nommsen, L.A.; Peerson, J.M.; Lonnerdal, B.; Dewey, K.G. Energy and protein intakes of breast-fed and formula-fed infants during the first year of life and their association with growth velocity: The Darling study. Am. J. Clin. Nutr. 1993, 58, 152-161. [CrossRef] [PubMed]

49. Artym, J.; Zimecki, M. Milk-derived proteins and peptides in clinical trials. Postepy Hig. Med. Dosw. 2013, 67, 800-816. [CrossRef]

50. Czank, C.; Mitoulas, L.R.; Hartmann, P.E. Human milk composition: Nitrogen and energy content. In Hale and Hartmann's Text Book of Human Lactation; Hartmann, P.E., Hale, T.W., Eds.; Hale Publishing, L.P.: Dallas, TX, USA, 2007; pp. 89-102.

51. Clare, D.A.; Swaisgood, H.E. Bioactive milk peptides: A prospectus. J. Dairy Sci. 2000, 83, 1187-1195. [CrossRef]

52. Gridneva, Z.; Kugananthan, S.; Hepworth, A.R.; Tie, W.J.; Lai, C.T.; Ward, L.C.; Hartmann, P.E.; Geddes, D.T. Effect of human milk appetite hormones, macronutrients, and infant characteristics on gastric emptying and breastfeeding patterns of term fully breastfed infants. Nutrients 2017, 9, 15. [CrossRef] [PubMed]

53. Budek, A.Z.; Bjornvad, C.R.; Molgaard, C.; Bugel, S.; Vestergaard, M.; Pulkkinen, P.; Michaelsen, K.F.; Sangild, P.T. Effects of casein, whey and soy proteins on volumetric bone density and bone strength in immunocompromised piglets. Eur. J. Clin. Nutr. Metab. 2007, 2, 57-62. [CrossRef]

54. Alexander, D.D.; Yan, J.; Bylsma, L.C.; Northigton, R.S.; Grathwohl, D.; Steenhout, P.; Erdmann, P.; Spivey-Krobath, E.; Haschke, F. Growth of infants consuming whey-predominant term infant formulas with a protein content of $1.8 \mathrm{~g} / 100 \mathrm{kcal}$ : A multicenter pooled analysis of individual participant data. Am. J. Clin. Nutr. 2016, 104, 1083-1092. [CrossRef] [PubMed]

55. Harrison, G.G.; Graver, E.J.; Vargas, M.; Churella, H.R.; Paule, C.L. Growth and adiposity of term infants fed whey-predominant or casein-predominant formulas or human milk. J. Pediatr. Gastroenterol. Nutr. 1987, 6, 739-747. [CrossRef] [PubMed]

56. Shepherd, R.W.; Oxborough, D.B.; Holt, T.L.; Thomas, B.J.; Thong, Y.H. Longitudinal study of the body composition of weight gain in exclusively breast-fed and intake-measured whey-based formula-fed infants to age 3 months. J. Pediatr. Gastroenterol. Nutr. 1988, 7, 732-739. [CrossRef] [PubMed]

57. Ballard, O.; Morrow, A.L. Human milk composition: Nutrients and bioactive factors. Pediatr. Clin. N. Am. 2013, 60, 49-74. [CrossRef] [PubMed]

58. Bol'shakova, A.M.; Shcherbakova, E.G.; Ivanova, S.D.; Medvedeva, M.M.; Zhuravleva, T.P. Lysozyme in the feeding of premature infants with mixed pathology. Antibiotiki 1984, 29, 784-790. [PubMed]

59. Braun, O.H.; Sandkuhler, H. Relationships between lysozyme concentration of human milk, bacteriologic content, and weight gain of premature infants. J. Pediatr. Gastroenterol. Nutr. 1985, 4, 583-586. [CrossRef] [PubMed]

60. Johnston, W.H.; Ashley, C.; Yeiser, M.; Harris, C.L.; Stolz, S.I.; Wampler, J.L.; Wittke, A.; Cooper, T.R. Growth and tolerance of formula with lactoferrin in infants through one year of age: Double-blind, randomized, controlled trial. BMC Pediatr. 2015, 15, 173. [CrossRef] [PubMed]

61. Trabulsi, J.; Capeding, R.; Lebumfacil, J.; Ramanujam, K.; Feng, P.; McSweeney, S.; Harris, B.; DeRusso, P. Effect of an $\alpha$-lactalbumin-enriched infant formula with lower protein on growth. Eur. J. Clin. Nutr. 2011, 65, 167-174. [CrossRef] [PubMed]

62. Wong, C.B.; Xiao, J. Breast milk on the infant faecal microbiome: Components of breast milk orchestrating the establishment of bifidobacteria species. J. Hum. Nutr. Food Sci. 2018, 6, 1123. 
63. Maga, E.A.; Desai, P.T.; Weimer, B.C.; Dao, N.; Kultz, D.; Murray, J.D. Consumption of lysozyme-rich milk can alter microbial fecal populations. Appl. Environ. Microbiol. 2012, 78, 6153-6160. [CrossRef] [PubMed]

64. Neville, M.C.; Allen, J.C.; Watters, C. The mechanisms of milk secretion. In Lactation: Physiology, Nutrition, and Breast-Feedin; Neville, M.C., Neifert, M.R., Eds.; Plenum Press: New York, NY, USA, 1983; pp. 49-102.

65. Mather, I.H.; Keenan, T.W. Function of endomembranes and the cell surface in the secretion of organic milk constituents. In Biochemistry of Lactation; Mepham, T.B., Ed.; Elsevier: Amsterdam, The Netherlands, 1983; pp. 231-283.

66. Chang, N.; Jung, J.A.; Kim, H.; Jo, A.; Kang, S.; Lee, S.-W.; Yi, H.; Kim, J.; Yim, J.-G.; Jung, B.-M. Macronutrient composition of human milk from Korean mothers of full term infants born at 37-42 gestational weeks. Nutr. Res. Pract. 2015, 9, 433-438. [CrossRef] [PubMed]

67. Grote, V.; Verduci, E.; Scaglioni, S.; Vecchi, F.; Contarini, G.; Giovannini, M.; Koletzko, B.; Agostoni, C. Breast milk composition and infant nutrient intakes during the first 12 months of life. Eur. J. Clin. Nutr. 2016, 70, 250-256. [CrossRef] [PubMed]

68. Innis, S.M. Impact of maternal diet on human milk composition and neurological development of infants. Am. J. Clin. Nutr. 2014, 99, S734-S741. [CrossRef] [PubMed]

69. Nommsen, L.A.; Lovelady, C.A.; Heinig, M.; Lonnerdal, B.; Dewey, K.G. Determinants of energy, protein, lipid, and lactose concentrations in human milk during the first 12 mo of lactation: The Darling study. Am. J. Clin. Nutr. 1991, 53, 457-465. [CrossRef] [PubMed]

70. De Luca, A.; Frasquet-Darrieux, M.; Gaud, M.A.; Christin, P.; Boquien, C.-Y.; Millet, C.; Herviou, M.; Darmaun, D.; Robins, R.J.; Ingrand, P.; et al. Higher leptin but not human milk macronutrient concentration distinguishes normal-weight from obese mothers at 1-month postpartum. PLoS ONE 2016, 11, e0168568. [CrossRef] [PubMed]

71. Bachour, P.; Yafawi, R.; Jaber, F.; Choueiri, E.; Abdel-Razzak, Z. Effects of smoking, mother's age, body mass index, and parity number on lipid, protein, and secretory immunoglobulin a concentrations of human milk. Breastfeed. Med. 2012, 7, 179-188. [CrossRef] [PubMed]

72. Hassiotou, F.; Geddes, D.T. Programming of appetite control during breastfeeding as a preventative strategy against the obesity epidemic. J. Hum. Lact. 2014, 30, 136-142. [CrossRef] [PubMed]

73. Meier, P.P.; Engstrom, J.L. Test weighing for term and premature infants is an accurate procedure. Arch. Dis. Child. Fetal Neonatal Ed. 2007, 92, F155-F156. [CrossRef] [PubMed]

74. Butte, N.; Garza, C.; Smith, E.; Nichols, B. Human milk intake and growth in exclusively breast-fed infants. J. Pediatr. 1984, 104, 187-195. [CrossRef]

75. Rattigan, S.; Ghisalberti, A.V.; Hartmann, P.E. Breast-milk production in Australian women. Br. J. Nutr. 1981, 45, 243-249. [CrossRef] [PubMed]

76. Kent, J.; Hepworth, A.; Sherriff, J.; Cox, D.; Mitoulas, L.; Hartmann, P. Longitudinal changes in breastfeeding patterns from 1 to 6 months of lactation. Breastfeed. Med. 2013, 8, 401-407. [CrossRef] [PubMed]

77. Hoppe, C.; Udam, T.R.; Lauritzen, L.; Molgaard, C.; Juul, A.; Michaelsen, K.F. Animal protein intake, serum insulin-like growth factor I, and growth in healthy 2.5-yold danish children. Am. J. Clin. Nutr. 2004, 80, 447-452. [CrossRef] [PubMed]

78. Gunther, A.L.B.; Remer, T.; Kroke, A.; Buyken, A.E. Early protein intake and later obesity risk: Which protein sources at which time points throughout infancy and childhood are important for body mass index and body fat percentage at $7 \mathrm{y}$ of age? Am. J. Clin. Nutr. 2007, 86, 1765-1772. [CrossRef] [PubMed]

(C) 2018 by the authors. Licensee MDPI, Basel, Switzerland. This article is an open access article distributed under the terms and conditions of the Creative Commons Attribution (CC BY) license (http:/ / creativecommons.org/licenses/by/4.0/). 


\title{
Human Breast Milk NMR Metabolomic Profile across Specific Geographical Locations and Its Association with the Milk Microbiota
}

\author{
Carlos Gómez-Gallego ${ }^{1, *}$, Jose Manuel Morales ${ }^{2,3,4}$, Daniel Monleón ${ }^{2,4}$, Elloise du Toit ${ }^{5}$, \\ Himanshu Kumar ${ }^{1}$, Kaisa M. Linderborg ${ }^{6}$, Yumei Zhang ${ }^{7}$, Baoru Yang ${ }^{6}$, Erika Isolauri ${ }^{8}$, \\ Seppo Salminen ${ }^{1}$ and Maria Carmen Collado ${ }^{1,9, *}$
}

1 Functional Foods Forum, Faculty of Medicine, University of Turku, 20014 Turku, Finland; kumar.himanshu@utu.fi (H.K.); sepsal@utu.fi (S.S.)

2 Laboratory of Metabolomics, Institute of Health Research-INCLIVA, 46010 Valencia, Spain; J.Manuel.Morales@uv.es (J.M.M.); daniel.monleon@uv.es (D.M.)

3 Unidad Central de Investigación en Medicina, University of Valencia, 46010 Valencia, Spain

4 Pathology Department, School of Medicine, University of Valencia, 46100 Valencia, Spain

5 Division of Medical Microbiology, Department of Pathology, University of Cape Town, 7925 Cape Town, South Africa; elloisedutoit@gmail.com

6 Food Chemistry and Food Development, Department of Biochemistry, University of Turku, 20014 Turku, Finland; kamayl@utu.fi (K.M.L.); bayang@utu.fi (B.Y.)

7 Department of Nutrition and Food Hygiene, School of Public Health, Peking University, 100191 Beijing, China; zhangyumei@bjmu.edu.cn

8 Department of Paediatrics, University of Turku and Turku University Hospital, 20520 Turku, Finland; eriiso@utu.fi

9 Department of Biotechnology, Institute of Agrochemistry and Food Technology-National Research Council (IATA-CSIC), 46980 Valencia, Spain

* Correspondence: cargom@utu.fi (C.G.-G.); mcolam@iata.csic.es (M.C.C.)

Received: 24 July 2018; Accepted: 18 September 2018; Published: 21 September 2018

\begin{abstract}
The composition of human breast milk is highly variable, and it can be influenced by genetics, diet, lifestyle, and other environmental factors. This study aimed to investigate the impact of geographical location and mode of delivery on the nuclear magnetic resonance spectroscopy (NMR) metabolic profile of breast milk and its relationship with the milk microbiome. Human milk metabolic and microbiota profiles were determined using NMR and 16S rRNA gene sequencing, respectively, in 79 healthy women from Finland, Spain, South Africa, and China. Up to 68 metabolites, including amino acids, oligosaccharides, and fatty acid-associated metabolites, were identified in the milk NMR spectra. The metabolite profiles showed significant differences between geographical locations, with significant differences $(p<0.05)$ in the levels of galactose, lacto- $\mathrm{N}$-fucopentaose III, lacto- $\mathrm{N}$-fucopentaose I and 2-fucosyllactose, 3-fucosyllactose, lacto- $N$-difucohexaose II, lacto- $N$-fucopentaose III, 2-hydroxybutyrate, 3-hydroxybutyrate, proline, $\mathrm{N}$-acetyl lysine, methyl-histidine, dimethylamine, kynurenine, urea, creatine and creatine phosphate, formate, lactate, acetate, phosphocholine, acetylcholine, LDL, VLDL, ethanolamine, riboflavin, hippurate, spermidine, spermine and uridine. Additionally, the effect of caesarean section on milk metabolome was dependent on the geographical region. Specific interrelations between human milk metabolites and microbiota were also identified. Proteobacteria, Actinobacteria, and Bacilli were most significantly associated with the milk metabolites, being either positively or negatively correlated depending on the metabolite. Our results reveal specific milk metabolomic profiles across geographical locations and also highlight the potential interactions between human milk's metabolites and microbes.
\end{abstract}


Keywords: human milk; metabolites; microbiome; mode of delivery; caesarean section; proton nuclear magnetic resonance

\section{Introduction}

Arguably, the cornerstone of healthy growth and development in children is breast feeding, since breast milk offers myriad physiological advantages when compared to other sources of nutrition. Indeed, the optimal model of infant feeding is the healthy breastfed child [1]. When compared with formula-fed infants, breastfed children exhibit a reduced risk of gastrointestinal and respiratory infections [2], allergic disease [3,4], and being overweight or obese, with the benefits actually extending beyond infancy $[5,6]$.

Breast milk contains many biologically active compounds, such as growth factors, antimicrobial and immune-enhancing substances, oligosaccharides (HMOs), as well as a diverse and rich bacterial community [7-10].

The composition of human breast milk is determined by genetic factors, lifestyle, diet, and the age of the mother [11,12]. Furthermore, human milk's macronutrient composition varies across lactation, although it is relatively conserved between populations [13]. However, the determinants of the bioactive compounds are currently less well understood. While some components of human milk appear to be relatively stable across different locations, some, such as polyunsaturated fatty acids, vary according to the mother's diet [9,14], while others, such as polyamines [15], HMOs [16], and milk bacteria [9,17], seem to vary depending of multiple factors. The mode of birth has also been reported to be a modulating factor for human milk's composition, acting in a differential manner in different countries $[9,15]$. Yet, the complex interactions between the constituents of milk, the biological impact, and the consequences for the infant's health in the short- and long-term remain unclear.

In addition, the host-milk microbial interactions may be influenced by the presence and/or concentration of milk metabolites, which may in turn influence the intestinal bacterial communities as well as the immune cell populations in breastfed children due to favoring the growth of specific microbial genera [8].

The present study aimed to compare the metabolomic profile of human milk obtained from different regions and different delivery modes, as well as to ascertain the potential interaction with milk microbiota.

\section{Materials and Methods}

\subsection{Breast Milk Sample Collection}

This study's population comprised 79 healthy women volunteers representing different populations from around the world, including China (Beijing area), South Africa (Cape Town area), Finland (southwestern area), and Spain (Valencia area). The women were enrolled in the study according to previously described inclusion criteria [9]. Further, the subjects from each country $(n=20)$ were grouped into two sub-groups according to the mode of delivery, namely either vaginal delivery ( $\mathrm{n}=10$ for each country) or caesarean section ( $\mathrm{n}=10$ for China, Finland, and Spain; $\mathrm{n}=9$ for South Africa). Data regarding age of the mother and perinatal body max index (BMI) are presented in Table S1. The mothers from China had significantly lower BMI $(p<0.001)$, but there were no differences in other parameters between countries and mode of delivery. Parity was between 1 and 3, mostly 1 and 2, with no differences among countries or between mode of delivery. Exclusively breastfeeding was reported at time of the sampling.

All the participating women received written, complete, and detailed information about the study. Written informed consent was obtained from all the participants, and the ethics committees of the respective participating countries (Spain [Bioethics Committee of CSIC and the Regional 
Ethics Committee for Biomedical Research, Ref: ERC-639226], Finland [Turku University Hospital, Ref: 24/1801/2013], China [Medical Research Board of Peking University, Ref: IRB00001052-16038], and South Africa [University of Cape Town, Human Research Ethics Committee, Ref: HREC 649/2016]), approved the study protocol. The study was conducted in accordance with the Declaration of Helsinki. Inclusion criteria requested exclusive breastfeeding practices at sampling time and healthy status of the mother-child pairs. Exclusion criteria included antibiotics use after birth, perinatal probiotic consumption, and presence of disease.

Prior to the sample collection, the mothers were given oral and written instructions regarding the standardized collection of samples. The mature milk samples (one month postpartum) were collected manually in the morning into a sterile tube using the same protocol in all the countries. Before the collection, the mothers' nipples and mammary areola were cleaned with soap and sterile water and then soaked in chlorohexidine in order to reduce the presence of skin bacteria. Samples were collected in the morning, from one breast, before baby feeding. The first drops of milk (approx. $500 \mu \mathrm{L}$ ) were discarded. The average collected volume was $10 \mathrm{~mL}$. All the samples were kept frozen at $-20^{\circ} \mathrm{C}$ until delivery to the laboratory. They were then stored at $-80^{\circ} \mathrm{C}$ for further analysis.

The breast milk samples were thawed, carefully mixed by means of inversion, and then centrifuged at $14,000 \mathrm{rpm}$ for $20 \mathrm{~min}$ at $4{ }^{\circ} \mathrm{C}$. The fat was removed and the pellet was used for the total DNA extraction. Avoiding the outer layer of fat, the whey milk was transferred to a clean Falcon tube and then centrifuged again. This procedure was repeated. A clear supernatant was used for the metabolomic profile analysis.

\subsection{Breast Milk Metabolite Profiling}

A proton nuclear magnetic resonance (NMR) analysis of all the collected samples was performed. For each group, the milk samples $(455 \mu \mathrm{L})$ were mixed with $45 \mu \mathrm{L}$ of sodium-3'-trimethylsilylpropionate2,2,3,3-d4 (TSP) solved in deuterium oxide and then placed in a $5 \mathrm{~mm}$ NMR tube. The final TSP concentration in each sample was $2.5 \mathrm{mM}$. All the spectra were recorded on a Bruker Avance DRX 600 spectrometer (Bruker $\mathrm{GmbH}$, Rheinstetten, Germany) operating at a ${ }^{1} \mathrm{H}$ frequency of $600.13 \mathrm{MHz}$. The spectrometer was equipped with a triple resonance ${ }^{1} \mathrm{H} /{ }^{13} \mathrm{C} /{ }^{31} \mathrm{P}$ probe. The nominal temperature of the samples was kept at $310 \mathrm{~K}$. A single-pulse pre-saturation experiment was performed for all the samples. A total of 64 transients were collected into $65 \mathrm{k}$ data points for all the experiments, with a spectral width of $14 \mathrm{ppm}$. Water presaturation was performed for one second during the recycling delay for the solvent signal suppression. Prior to the Fourier transformation, the free induction decay was multiplied with a $0.3 \mathrm{~Hz}$ exponential line-broadening function. All the spectra were processed using MestReNova 8.1 software (Mestrelab Research S.L., Santiago de Compostela, Spain) and then transferred to MATLAB R2013a (The MathWorks Inc., Natick, MA 2013) using in-house scripts for data analysis. The metabolite spin systems and resonances were identified using data obtained from both the literature and the commercial resonances database Chenomx NMR Suite Profiler (Chenomx NMR Suite 8.1, Chenomx Inc., Edmonton, AB, Canada). The spectra were manually phase corrected and baseline adjusted, referenced to the TSP, and normalized to the total aliphatic spectral area ( 0.50 and $4.40 \mathrm{ppm}$ ) in order to eliminate any differences in the total metabolite concentration. The signals belonging to the identified metabolites were then integrated and quantified using the semi-automated ${ }^{1} \mathrm{H}$ NMR signal deconvolution routines in MestReNova 8.1 (Mestrelab Research SL, Santiago de Compostela, Spain). The final metabolite levels were calculated in arbitrary units as the area under the peak. In addition, two-dimensional NMR methods, including homonuclear correlation spectroscopy (TOCSY) and heteronuclear single quantum correlation spectroscopy (HSQC), were applied to a selected group of samples so as to confirm the assessment of the metabolites.

\subsection{Breast Milk DNA Extraction and Microbial $16 S$ rRNA Gene Sequencing}

The process of microbial DNA extraction and sequencing using an Illumina MiSeq sequencer was described in a previous study by Kumar et al. [9]. The sequencing data were submitted to the 
National Center for Biotechnology Information with the Sequence Read Archive accession: SRP082263 and submission ID: SUB1772296.

\subsection{Statistical Analysis}

A chemometrics statistical analysis was performed using in-house MATLAB scripts and the PLS_Toolbox 8.0.2 (Eigenvector Research, Inc., Wenatchee, WA, USA) statistical multivariate analysis library. The normalization of the NMR spectra was done using the total aliphatic spectral area $(0.50$ and $4.40 \mathrm{ppm}$ ) in order to eliminate any differences in the total metabolite concentration. Mean-centered and Pareto data scaling were used prior to multivariate analysis. A principal component analysis (PCA) was applied to the NMR spectra data sets. A PCA is able to identify low-dimensional embeddings of multivariate data in such a way that optimally preserves the structure of the data. The main advantage of PCA models is that the key sources of variability within the data are modeled by the so-called principal components (PCs) and, consequently, their associated scores and loadings allow for the visualization and understanding of different patterns and relations in the data. The principal components were chosen to explain at least $70 \%$ of the variance. The loading plots of the corresponding principal components were then used to detect the positions of most discriminative variables in the NMR spectra. In order to maximize the separation between the samples, a partial least squares discriminant analysis (PLS-DA) was conducted. A permutation test was performed to check the overfitting of the PLS-DA models. The multivariate chemometric models were cross-validated using ten-fold leave-one-out cross-validation. In each run, $10 \%$ of the data were left out of the training and used to test the model. The entire cross-validation process was run ten times. The spectral regions responsible for the classification of the models were identified using the variable importance in projections (VIP) coefficients obtained during the PLS-DA. The threshold used for VIP selection was $\geq 1$. Spectral regions with high VIP coefficients are more important in terms of providing class separation during the analysis, while those with very small VIP coefficients provide only a small contribution to the classification.

SPSS 25.0.0.1 software (IBM Corp., Armonk, NY, USA) was employed for the statistical analysis of the milk metabolites. Differences were considered significant at $p \leq 0.05$. Due to the non-normal distribution of the data and high presence of outliers, nonparametric tests were used. Comparisons among the data between the different countries were made by applying the Kruskal-Wallis test, while comparisons between the modes of birth were made by applying the Mann-Whitney U test. The significance values in the pairwise comparison were adjusted using the Bonferroni correction for multiple tests.

Calypso online software version 8.50 was used for data normalized via cumulative sum scaling in order to generate heat maps for the Spearman's correlations between the microbial groups and milk metabolites.

\section{Results}

A total of 68 metabolites were identified in the human milk one month after delivery, as detailed in Table 1 . The metabolites included 23 amino acids and derivatives, 18 sugars and derivatives, ten lipids and fatty acid-associated metabolites, and seven metabolites associated with energy metabolism, while the rest were linked to metabolic processes involving vitamins or nucleic acids, microbial metabolism, and food additives.

The most abundant metabolite was lactose, followed by lipids, with high amounts of the lipoproteins LDL (low-density lipoprotein) and VLDL (very low-density lipoproteins), and then, HMOs, and amino acids. The accurate relative quantitation of several metabolites by means of NMR was difficult due to the presence of multiple peaks or severe spectral overlapping, and it was thus not included in statistical analysis. Therefore, of the 68 metabolites identified, 37 were employed for statistical analysis in SPSS 25.0.0.1 software. Maternal factor and metabolites association were analyzed as shown in Table S2. 
The presence of LDL and VLDL as milk metabolites can be controversial. The particles detected in milk have similar nuclear magnetic resonances (NMR), physicochemical properties and mobility than those lipoproteins detected in plasma. However, they might be also different lipid and protein conjugates with similar composition and hydrodynamic properties; lipids similar to those present in LDL and VLDL enclosed in phospholipids, free cholesterol and proteins; or free lipids similar to those enclosed in LDL and VLDL attached to large proteins. For this reason, they should be considered LDLand VLDL-like particles.

Table 1. Human milk metabolites identified in breast milk samples and their probable origin. Chemical shifts in ppm are presented (in brackets).

\begin{tabular}{|c|c|}
\hline Metabolite & Origin \\
\hline \multicolumn{2}{|c|}{ Amino acids and derivatives } \\
\hline 2-Hydroxybutyrate (3.99) & endogenous \\
\hline 2-Hydroxyisovalerate $(0.95)$ & endogenous \\
\hline Alanine (1.47) & endogenous \\
\hline Anserine (8.91) & diet \\
\hline Creatinine (3.03) & endogenous \\
\hline Dimethylamine (2.72) & endogenous \\
\hline Glutamate (2.34) & endogenous \\
\hline Glutamine (2.47) & endogenous \\
\hline Carnitine (3.21) & endogenous \\
\hline Histidine (7.09) & endogenous \\
\hline Isoleucine (0.99) & diet \\
\hline Kynurenine (6.81) & endogenous \\
\hline Leucine $(0.94)$ & diet \\
\hline Methionine (2.62) & diet \\
\hline Methyl-histidine (7.88) & endogenous \\
\hline$N$-Acetyl lysine (1.79) & endogenous \\
\hline Phenylalanine (7.36) & endogenous \\
\hline Proline (3.34) & endogenous \\
\hline Taurine (3.25) & endogenous \\
\hline Tryptophan (7.70) & diet \\
\hline Tyrosine (3.06) & endogenous \\
\hline Urea (5.77) & endogenous \\
\hline Valine $(0.98)$ & diet \\
\hline \multicolumn{2}{|c|}{ Energy metabolites } \\
\hline 3-hydroxybutyrate (1.17) & endogenous \\
\hline Citrate (2.69) & endogenous \\
\hline Creatine (3.01) & endogenous \\
\hline Creatine-phosphate (3.02) & endogenous \\
\hline Formate $(8.44)$ & endogenous \\
\hline Lactate (1.32) & endogenous \\
\hline NADH (8.46) & endogenous \\
\hline \multicolumn{2}{|c|}{ Neurotransmitters, growth factors and second messengers } \\
\hline 4-Aminobutyrate (2.29) & endogenous \\
\hline Putrescine (1.75) & microbial \\
\hline Spermidine (2.61) & endogenous \\
\hline Spermine (2.70) & endogenous \\
\hline \multicolumn{2}{|c|}{ Fatty acids and associated metabolites } \\
\hline 4-Aminohippurate (2.29) & endogenous \\
\hline Acetate (1.91) & endogenous \\
\hline Acetylcholine (3.21) & endogenous \\
\hline Butyrate (2.16) & microbial \\
\hline Choline (4.06) & diet \\
\hline Ethanolamine (3.13) & endogenous \\
\hline
\end{tabular}


Table 1. Cont.

\begin{tabular}{|c|c|}
\hline Metabolite & Origin \\
\hline Glycero-3-phosphocholine (3.22) & endogenous \\
\hline LDL * (1.29) & endogenous \\
\hline Phosphocholine (3.2) & endogenous \\
\hline VLDL * (1.27) & endogenous \\
\hline \multicolumn{2}{|l|}{ Sugars and derivatives } \\
\hline 1,6 -anhydro-B-glucose (5.44) & diet \\
\hline 2-fucosyllactose (5.31) & endogenous \\
\hline 3-fucosyllactose (5.37) & endogenous \\
\hline Arabinose (4.51) & microbial \\
\hline Fucosyl- $\alpha-1,3-N$-acetylglucosamine (5.14) & endogenous \\
\hline Fucosyl- $\alpha-1,4-N$-acetylglucosamine (5.01) & endogenous \\
\hline Fucose $(4.55)$ & endogenous \\
\hline Galactose (4.57) & endogenous \\
\hline Glucose (3.23) & endogenous \\
\hline Glucose-1-phosphate (5.45) & endogenous \\
\hline Lactose (3.75) & endogenous \\
\hline Lactodifucotetraose (5.27) & endogenous \\
\hline Lacto- $N$-difucohexaose I (5.18) & endogenous \\
\hline Lacto- $N$-difucohexaose II (5.36) & endogenous \\
\hline Lacto- $N$-fucopentaose I (5.31) & endogenous \\
\hline Lacto- $N$-fucopentaose III (5.1) & endogenous \\
\hline Myo-inositol (4.05) & endogenous \\
\hline$N$-Acetylglucosamine (3.91) & endogenous \\
\hline \multicolumn{2}{|l|}{ Vitamins and nucleosides } \\
\hline Riboflavin (B2) (7.96) & diet \\
\hline Uridine (5.92) & endogeonous \\
\hline \multicolumn{2}{|l|}{ Others } \\
\hline Ethanol (1.16) & diet \\
\hline Hippurate (7.55) & microbial \\
\hline Propylene glycol (1.14) & diet \\
\hline
\end{tabular}

Classification and probable origin inferred from "The Human Metabolome Database" [18]. Metabolites employed for statistical comparison are highlighted with bold letters. * LDL- and VLDL-like particles with similar NMR resonances, structure and mobility than plasma LDL and VLDL.

\subsection{Differences in Milk Metabolites between Countries}

The global metabolic profile of breast milk was found to be different between countries, as seen in Figure 1. The PLS-DA analysis showed the Spanish samples to be widely dispersed, being more similar to the Finnish and South African samples and totally separate from the Chinese samples. After the exclusion of the Spanish milk samples from the analysis, total separation with only minimal overlapping between the samples was observed. PCA plots and loadings are available as supplementary material in Figures S1 and S2.

Significant differences in the sugars and HMOs between the countries can be seen in Figure 2. When compared to the breast milk samples from Finland, the Chinese samples exhibited significantly higher levels of 3-fucosyllactose ( $\left.3^{\prime} \mathrm{FL}\right)$ and lacto- $N$-fucopentaose III (LNFP III). A higher abundance of lacto- $N$-fucopentaose I (LNFP I) and 2-fucosyllactose ( $2^{\prime} \mathrm{FL}$ ) was observed in Finland and Spain, respectively, while $3^{\prime} \mathrm{FL}$ and LNFP III were more highly abundant in South Africa and China, respectively. 


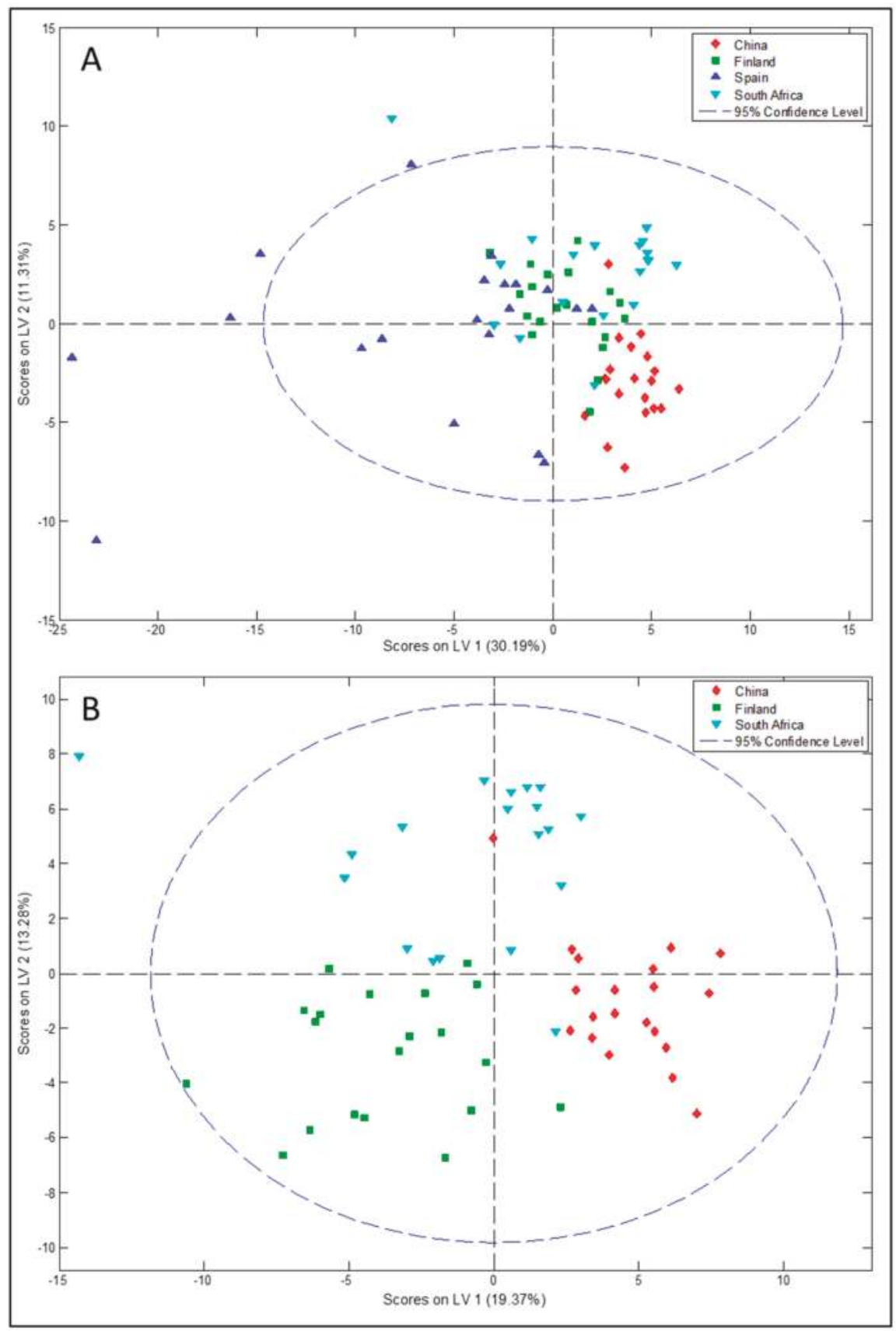

Figure 1. Partial least squares discriminant analysis (PLSDA) scores plot scaling nuclear magnetic resonance (NMR) data from all participant countries (A); participant countries without Spain (B). Countries are indicated as red diamonds (China), green squares (Finland), navy blue triangles (Spain), pale blue triangles (South Africa). 

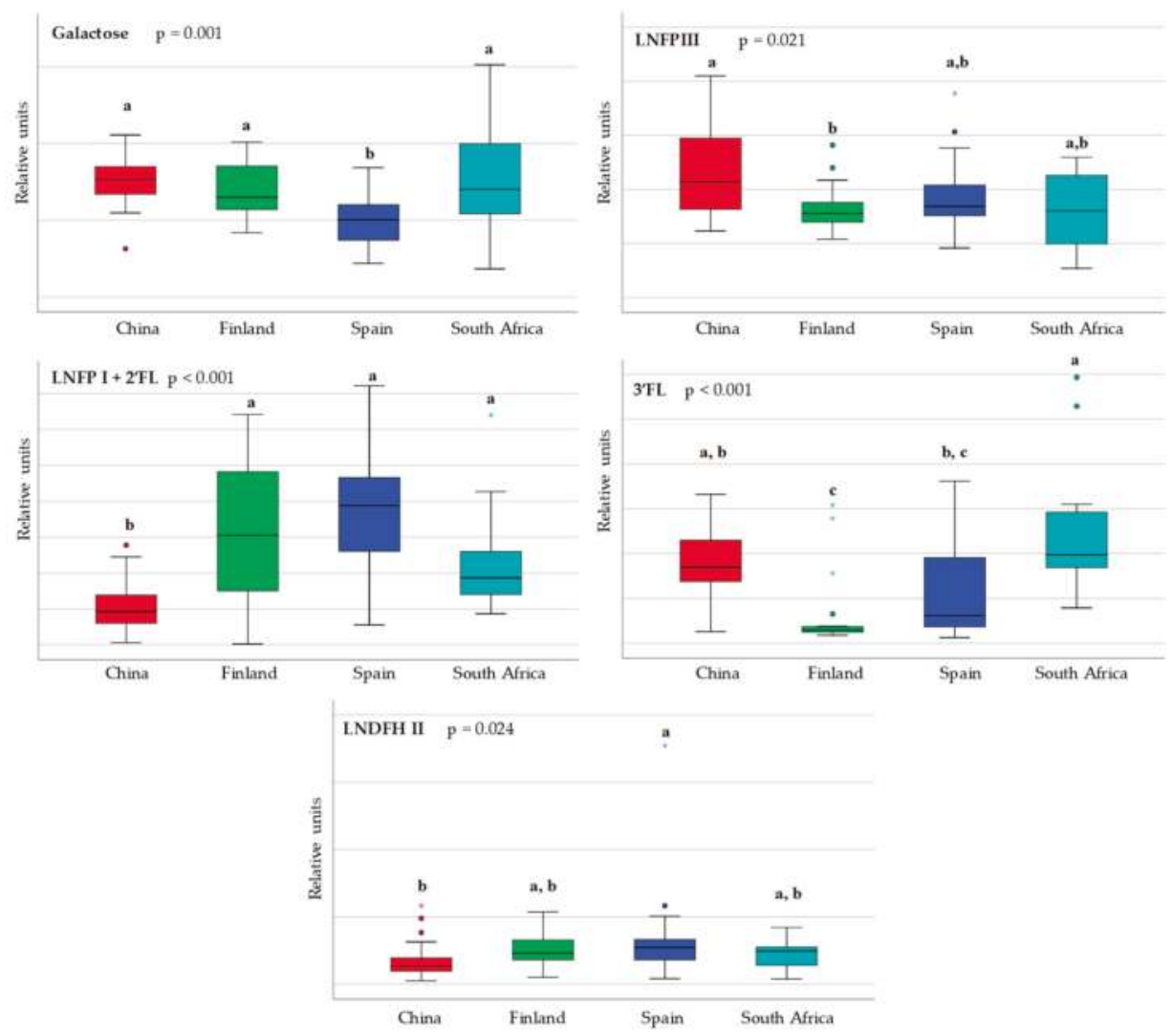

Figure 2. Box and whisker plot showing statistical significant differences in sugars and derivatives in breast milk obtained from China (red), Finland (green), Spain (blue) and South Africa (pale blue). Each bar represents the smallest observation, lower quartile (Q1), median, upper quartile (Q3) and largest observation. Differences among countries were calculated using the Kruskal-Wallis test. Unlike letters indicate statistically significant differences among countries. Circles and stars indicate outlier data. 3'FL: 3-fucosyllactose; LNFP I + 2'FL: lacto- $N$-fucopentaose I and 2-fucosyllactose; LNDFHII: lacto- $N$-difucohexaose II; LNFP III: lacto- $N$-fucopentaose III. Data are expressed in relative units.

Figure 3 shows statistically significant regional differences in the amino acids and derivatives found in the human milk samples. All of them have an endogenous origin and might therefore be the result of dietary and/or metabolic differences between geographical locations.

With regard to the content of the energy metabolites, fatty acids, and associated metabolites, the differences between countries are shown in Figures 4 and 5. The Finnish and Spanish samples were characterized by higher levels of lipoproteins (LDL and VLDL). Short-chain fatty acids (SCFA) were also detected with higher levels of acetate and formate in the Spanish samples. 


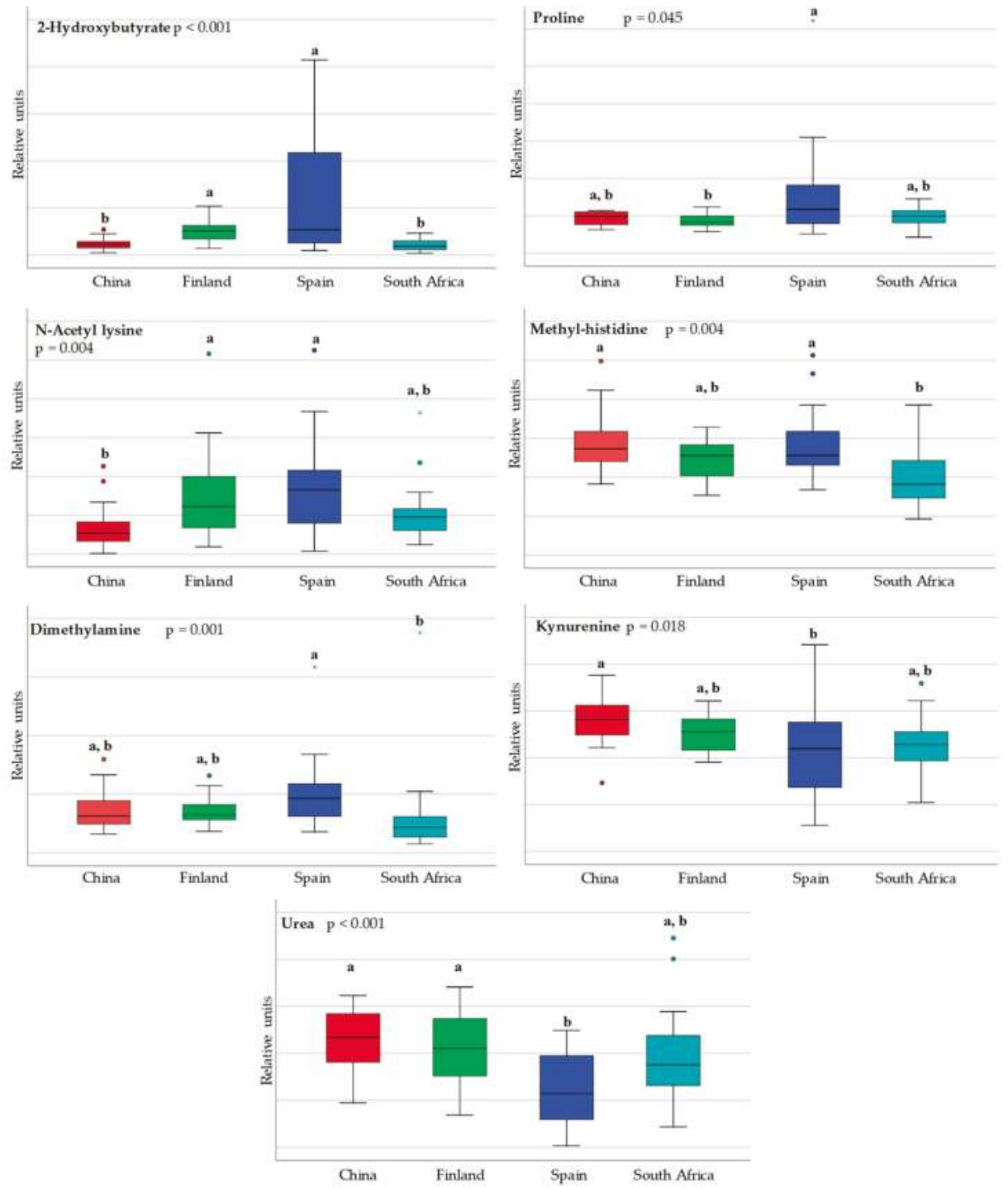

Figure 3. Box and whisker plot showing statistical significant differences in amino acids and derivatives in breast milk obtained from China (red), Finland (green), Spain (blue) and South Africa (pale blue). Data are expressed in relative units. Each bar represents the smallest observation, lower quartile (Q1), median, upper quartile (Q3) and largest observation. Differences among countries were calculated using the Kruskal-Wallis test. Unlike letters indicate statistically significant differences among countries. Circles and stars indicate outlier data.

The statistically significant differences identified in the other metabolites are presented in Figure 6. Some of them have a dietary origin, such as vitamin B2 (riboflavin), while others, such as the polyamines (spermidine and spermine), could have an endogenous, dietary, or microbial origin. 

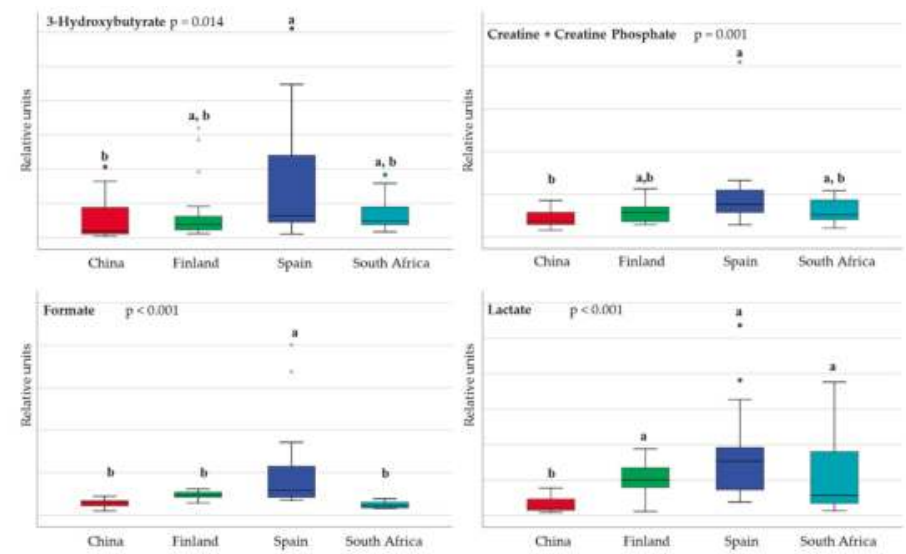

Figure 4. Box and whisker plot showing statistical significant differences in energy metabolites in breast milk in samples from China (red), Finland (green), Spain (blue) and South Africa (pale blue). Data are expressed in relative units. Each bar represents the smallest observation, lower quartile (Q1), median, upper quartile (Q3) and largest observation. Circles and stars indicate outlier data. Differences among countries were calculated using the Kruskal-Wallis test. Unlike letters indicate statistically significant differences among countries.
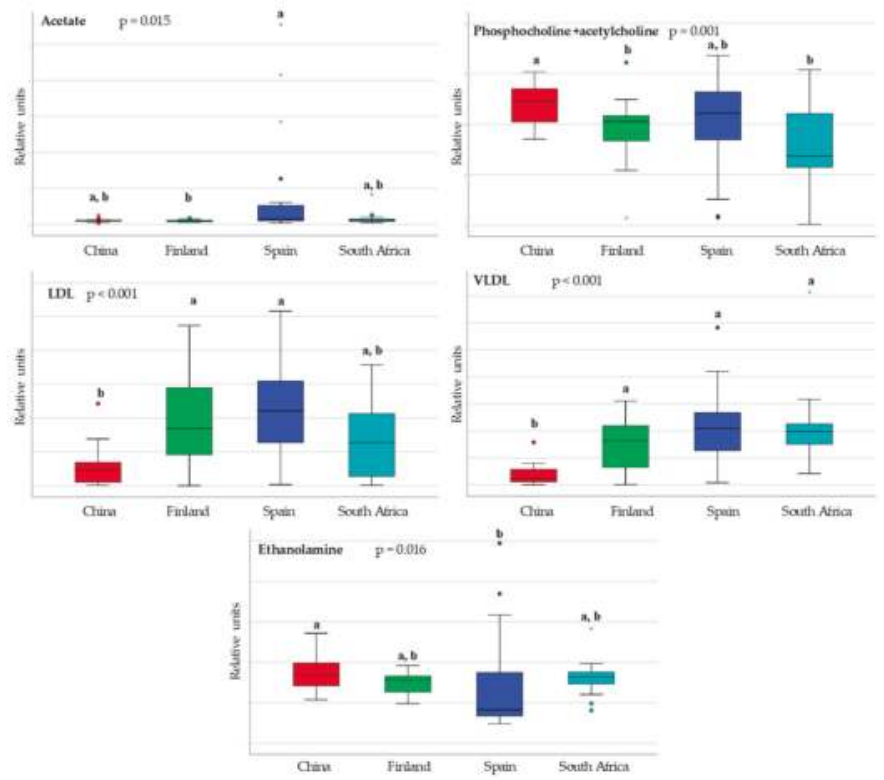

Figure 5. Box and whisker plot showing statistical significant differences in fatty acids and related metabolites in breast milk in samples from China (red), Finland (green), Spain (blue) and South Africa (pale blue). Data are expressed in relative units. Each bar represents the smallest observation, lower quartile (Q1), median, upper quartile (Q3) and largest observation. Circles and stars indicate outlier data. Differences among countries were calculated using the Kruskal-Wallis test. Unlike letters indicate statistically significant differences among countries. LDL and VLDL should be consider LDL- and VLDL-like particles with similar nuclear magnetic resonances (NMR), structure and mobility than plasma LDL and VLDL. 

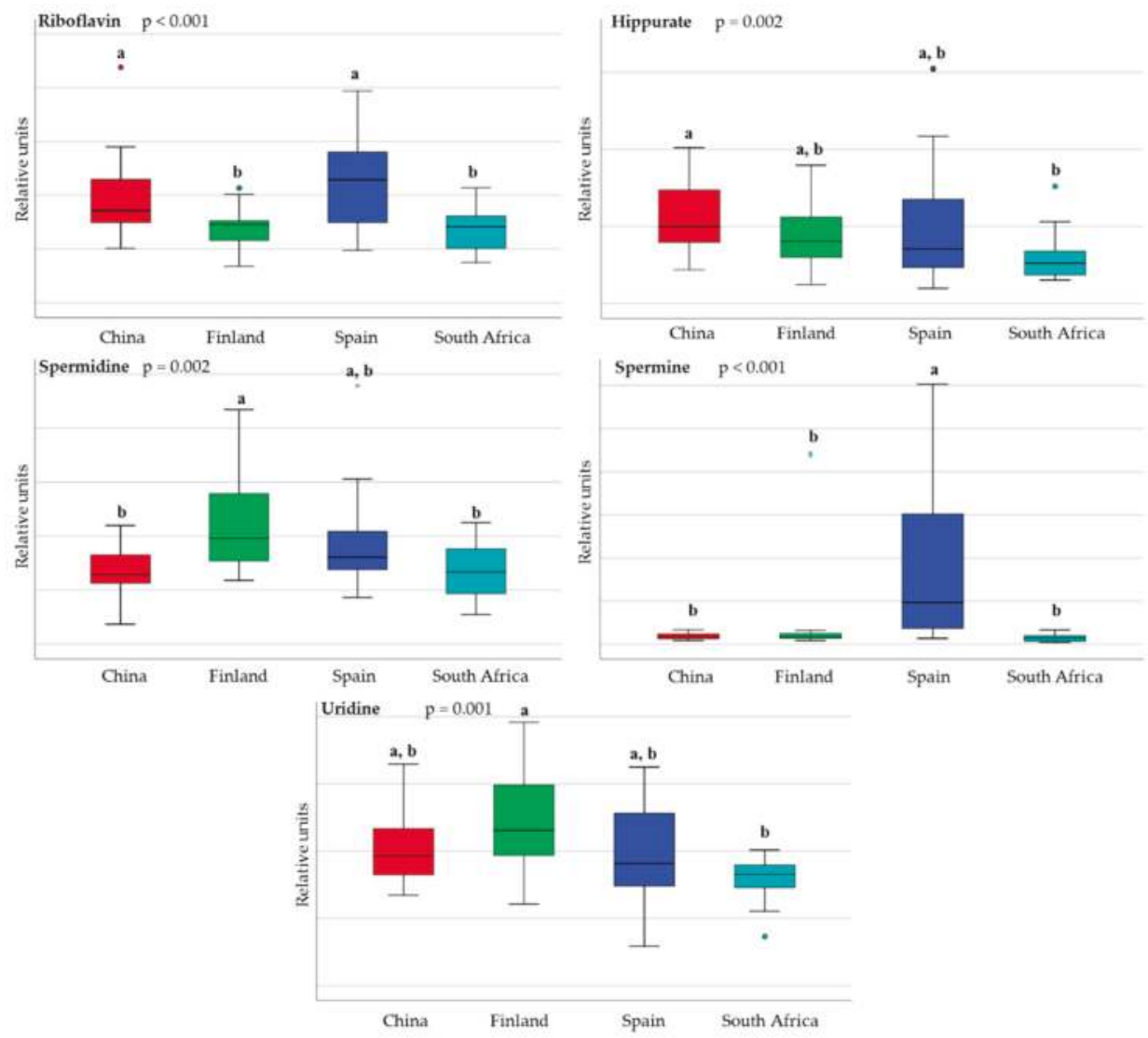

Figure 6. Box and whisker plot showing statistical significant differences in other metabolites in breast milk in samples from China (red), Finland (green), Spain (blue) and South Africa (pale bue). Data are expressed in relative units. Each bar represents the smallest observation, lower quartile (Q1), median, upper quartile $(\mathbf{Q} 3)$ and largest observation. Circles and stars indicate outlier data. Differences among countries were calculated using the Kruskal-Wallis test. Unlike letters indicate statistically significant differences among countries.

\subsection{Impact of Mode of Delivery on Human Milk Metabolites}

Independent of the country, the mode of delivery has a distinct impact on the human milk metabolome, as seen in Figure 7.

When considering all the mothers included in the study, six of the 37 semi-quantified metabolites were statistically different in the milk samples obtained from the mothers who underwent vaginal delivery when compared to those who underwent caesarean section, as seen in Figure S3. The milk from the mothers who underwent vaginal delivery had higher levels of 3-hydroxybutyrate $(p=0.048)$ and LNFP III $(p=0.045)$, while the milk from the mothers who underwent caesarean section had higher relative abundances of butyrate $(p=0.043)$, ethanolamine $(p=0.004)$, proline $(p=0.018)$, and urea $(p=0.020)$. However, these differences depend on the country. In the present study, based on the mode of delivery, we found significant differences in 16 metabolites in the South African samples, 11 metabolites in the Spanish samples, four in the Finnish samples, and one in the Chinese samples, shown in Table 2, reflecting regional differences in terms of the impact of caesarean section on the milk metabolome. 


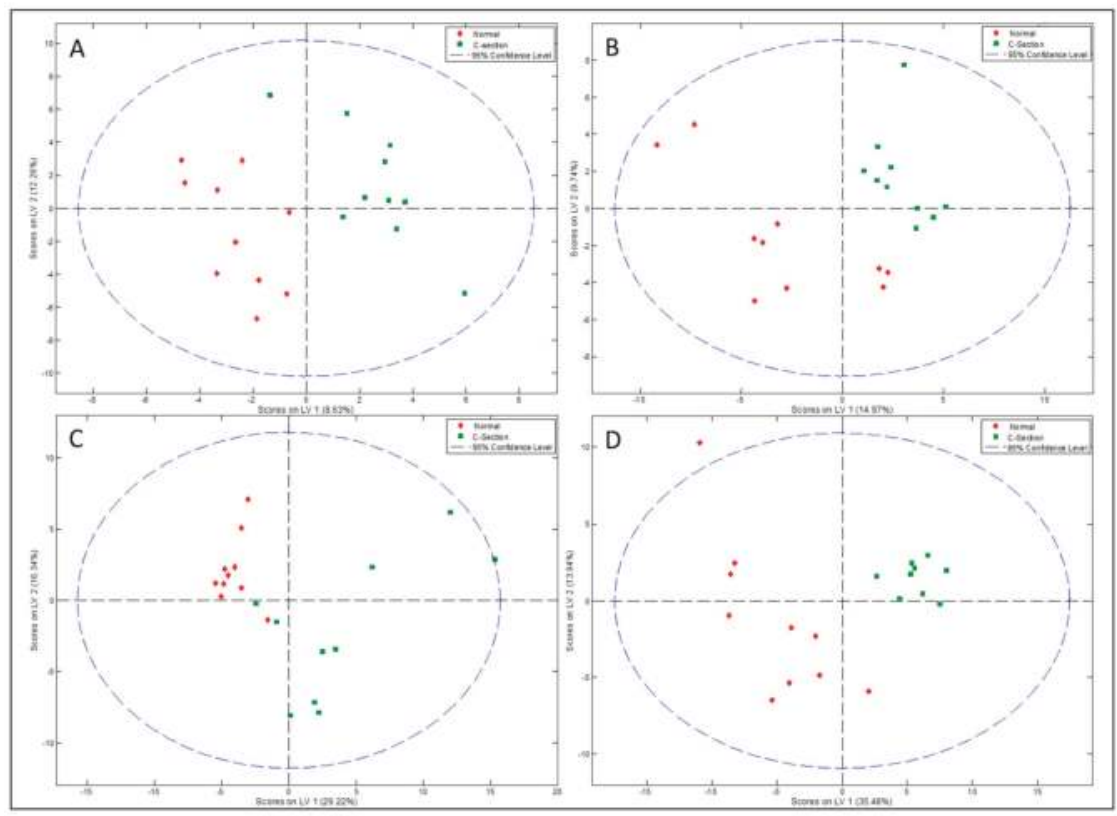

Figure 7. Partial least squares discriminant analysis scores plot scaling nuclear magnetic resonance (NMR) data from vaginal delivery samples (red) and caesarean section (green) from China (A), Finland (B), Spain (C), South Africa (D).

Table 2. Significant differences by country ( $p$-value) in specific metabolites in human milk after vaginal or caesarean delivery.

\begin{tabular}{|c|c|c|c|c|}
\hline Metabolite & China & Finland & Spain & South Africa \\
\hline 3-fucosyllactose & 0.739 & 0.971 & 0.190 & $\uparrow 0.035$ * \\
\hline Alanine & 0.853 & 0.739 & 0.280 & $\uparrow 0.022$ * \\
\hline Butyrate & 0.579 & 0.579 & 0.436 & $\uparrow 0.006$ * \\
\hline Dimethylamine & 0.971 & $0.035 *$ & $\uparrow 0.035 *$ & $0.002 *$ \\
\hline Ethanolamine & 0.631 & 0.579 & $\uparrow 0.000 *$ & 0.661 \\
\hline Formate & $\uparrow 0.035$ * & 0.684 & 0.796 & 0.006 * \\
\hline Fucosyl- $\alpha-1,4-N$-acetylglucosamine & 0.631 & $0.035 *$ & 0.247 & $0.002 *$ \\
\hline Galactose & 0.579 & 0.579 & 0.481 & $\uparrow 0.002 *$ \\
\hline Hippurate & 0.684 & 0.063 & $\uparrow 0.009 *$ & $0.022 *$ \\
\hline Kynurenine & 0.143 & 0.075 & $\uparrow 0.011 *$ & $0.028 *$ \\
\hline Lactodifucotetraose & 0.247 & $\uparrow 0.035$ * & 0.247 & 0.447 \\
\hline Leucine & 0.631 & $\uparrow 0.043 *$ & $\uparrow 0.009 *$ & 1.000 \\
\hline Lacto- $N$-fucopentaose I and 2-fucosyllactose & 0.353 & 1.000 & 0.971 & $0.035 *$ \\
\hline Lacto- $N$-fucopentaose III & 0.579 & 0.796 & 0.631 & $0.001 *$ \\
\hline Methyl-histidine & 0.353 & 0.684 & $\uparrow 0.023 *$ & $0.043 *$ \\
\hline Phosphocholine and acetylcholine & 0.393 & 0.796 & 1.000 & $0.002 *$ \\
\hline Proline & 0.393 & 0.739 & $\uparrow 0.002 *$ & 1.000 \\
\hline Propylene glycol & 0.912 & 0.089 & $\uparrow 0.023 *$ & $0.004 *$ \\
\hline Riboflavin & 0.529 & 0.353 & $\uparrow 0.002 *$ & 0.400 \\
\hline Spermidine & 0.579 & 0.353 & $\uparrow 0.000 *$ & 0.604 \\
\hline Spermine & 0.143 & 0.315 & 0.853 & 0.003 * \\
\hline Urea & 0.436 & 0.247 & 0.280 & 0.000 * \\
\hline Uridine & 0.529 & 0.912 & 0.019 * & 0.243 \\
\hline
\end{tabular}

* indicate $p$-values $<0.05$. $\uparrow$ indicate significant higher levels in milk from caesarean section donors. 


\subsection{Relationship between the NMR Metabolomic Profile and Milk Microbiota}

In order to explore the interrelations between the human milk metabolites and the milk microbiota profile, Spearman's rank correlations were determined and then represented in heat maps shown in Figures 8 and 9. Urea and galactose were positively correlated $(p<0.05)$ with Alpha- and Betaproteobacteria and Bacilli, although they were negatively correlated $(p<0.05)$ with Gammaproteobacteria. Yet, a group of metabolites were found to be positively correlated $(p<0.05)$ with Gammaproteobacteria, including lactate, creatine, proline, lacto- $N$-fucopentaose I, and 2-fucosyllactose VLDL, although they negatively correlated $(p<0.05)$ with Alpha- and Betaproteobacteria and Bacilli.

The Actinobacteria in the human breast milk were positively correlated $(p<0.05)$ with uridine, but negatively correlated with lacto- $N$-fucopentaose I, 2-fucosyllactose acetate, and spermidine $(p<0.05)$ as seen in Figure 8.

A few significant associations were found between the bacterial groups and HMOs, namely those related to fucosyl- $\alpha-1,4-N$-acetylglucosamine, lactodifucotetraose, lacto- $N$-fucopentaose III, lacto- $N$-fucopentaose I, and 2-fucosyllactose, shown in Figure 9.

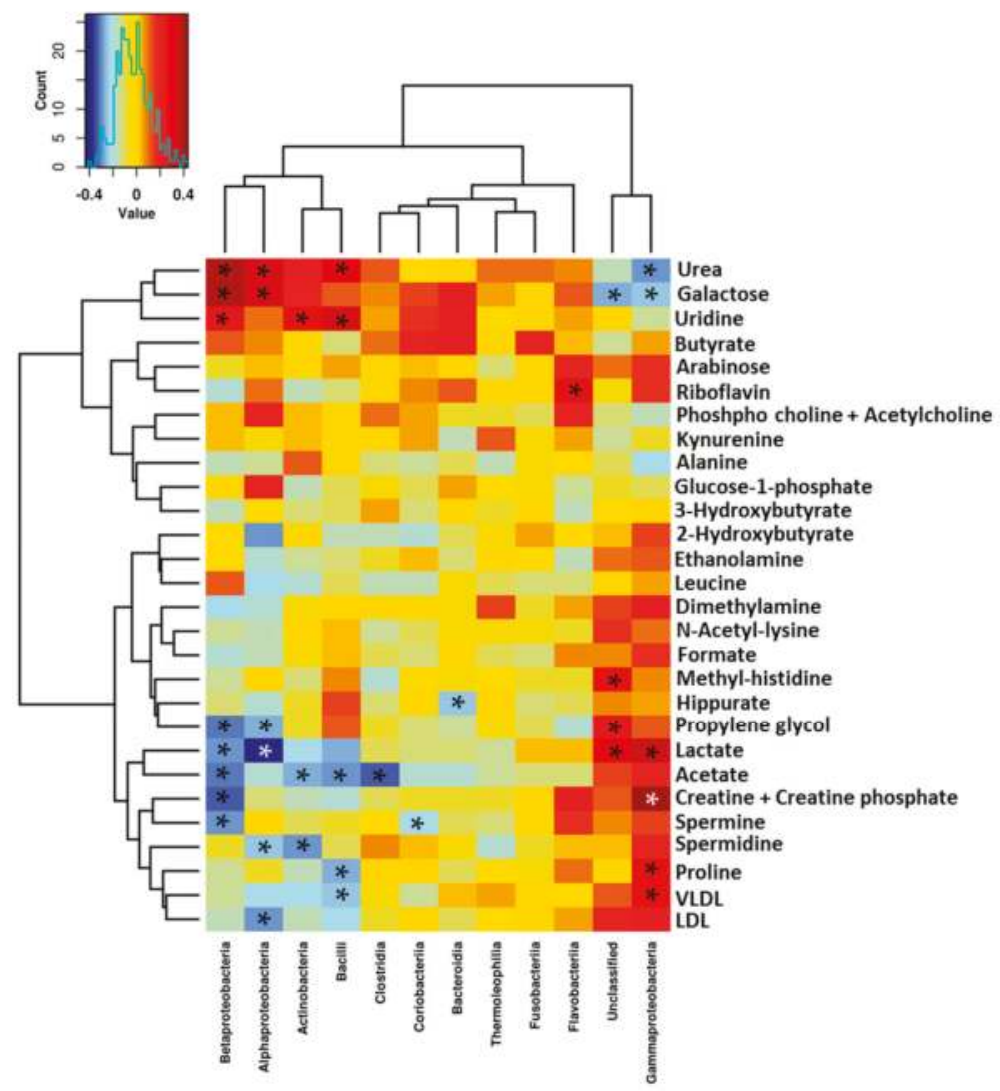

Figure 8. Heat map to show Spearman's correlation between metabolites and microbiota composition at class level. Asterisk indicates statistically significant correlation at the level of $(p<0.05)$. LDL and VLDL should be considered LDL- and VLDL-like particles with similar nuclear magnetic resonances (NMR), structure and mobility than plasma LDL and VLDL. 


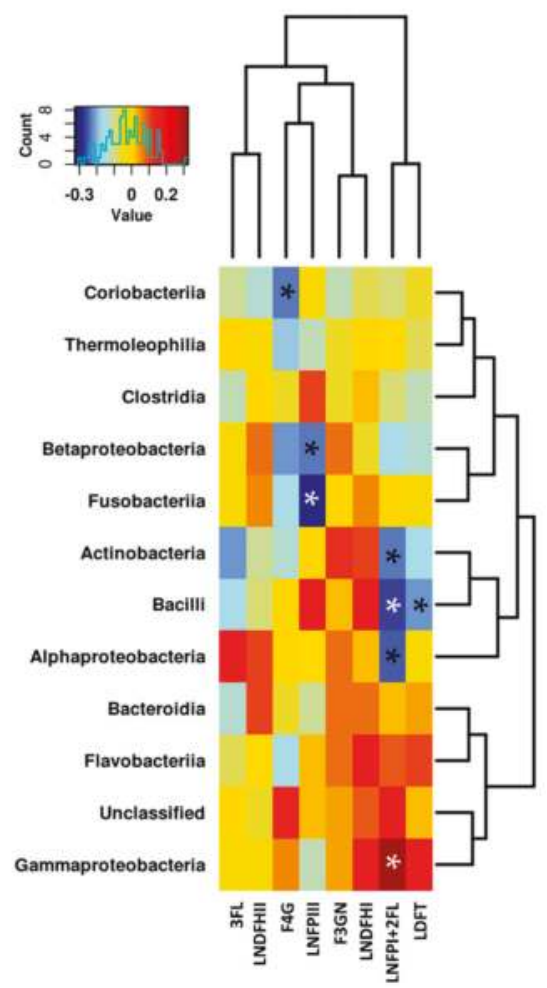

Figure 9. Heat map to show Spearman's correlation between oligosaccharides (HMOs) and microbiota composition at class level. 3FL: 3-fucosyllactose; LNDFHII: Lacto- $N$-difucohexaose; F4G: Fucosyl- $\alpha-1,4-N$-acetylglucosamine; LNFP III: lacto- $N$-fucopentaose; F3GN: Fucosyl- $\alpha-1,3-N-$ acetylglucosamine; LNDFH I: lacto- $N$-difucohexaose I; LNFP I: lacto- $N$-fucopentaose I; 2 FL: 2-fucosyllactose; LDFT: lactodifucotetraose. Asterisk indicates statistically significant correlation at the level of $(p<0.05)$.

\section{Discussion}

There is growing research interest in identifying and understanding the bioactive compounds found in human breast milk. The field of infant nutrition research is evolving and new methodologies are being developed to support exact knowledge concerning the composition of human milk. Moreover, a few prior studies have reported the composition of human milk using NMR-based metabolomics $[1,7,19,20]$. In this study, a total of 68 metabolites were identified in mature human milk. To the best of our knowledge, this is the first study to report the distinct milk metabolomics profile across different geographical locations, as well as to associate the complex interactions with human milk microbes. Spanish samples presented higher dispersion that was no associated with differences in maternal characteristics such as parity, age, or BMI between samples. Future studies to find the origin of this variability will be needed.

Different mode of delivery (vaginal vs caesarean section) influences the milk metabolite profile across locations. This study hence shows for the first time regional differences in the impact of caesarean section on human milk metabolites. The effect of caesarean section on the milk metabolome is dependent on the geographical region, with changes in different metabolites depending on the country. We suggest that the clinical intervention during caesarean and the alterations in the physiological and hormonal signals produced during normal vaginal delivery, would affected the milk metabolite profiles 
as already showed for milk microbiota [9]. In addition, we observed that the impact of the mode of delivery vary among geographical locations, maybe due to differences in the clinical procedures and antibiotic use [9].

Previous studies have shown inter-individual variations in human milk in terms of the macronutrients [21]. With regard to the metabolites, some of them, such as lactose, myo-inositol, and urea, have been reported to be conserved among mothers due to their important roles in infant growth and development, while those metabolites related to the genetic background or maternal diet are more variable, including HMOs, amino acids, choline, and vitamins [1,20]. No significant changes were found in the lactose and myo-inositol levels in relation to the geographical location and mode of delivery in this study, which suggests the strict regulation by the mammary gland, as has been proposed previously [1]. Yet, previous studies have found significant differences with regard to urea. For instance, Smilowitz et al. [1] analyzed 52 human milk samples collected at day 90 postpartum. They found that urea was one of the most abundant metabolites in human milk, and they further reported low variability between mothers, which suggested regulation at the level of the mammary gland, as well as an important role in developing infants as a source of nitrogen for intestinal microbiota. In the present study, the samples collected at day 30 postpartum showed differences in urea at different geographical locations in addition to relatively high variability. This may indicate differences in the regulation of the mammary gland depending on the number of days postpartum, as well as the influence of external factors, such as meat and dairy intake, or physical activity, during the early stages of lactation. The differences in the urea composition across the different geographical locations could also reflect variation in the gut microbiota composition of infants from the different locations $[9,22,23]$. A positive correlation between the colonic urea-nitrogen metabolism and the bifidobacteria concentration has previously been reported [24], although this association between the nitrogenated compounds and bifidobacteria might start early. We identified positive correlation between the nitrogenated compounds, such as urea, and uridine and Actinobacteria.

Similarly to urea, riboflavin has previously been reported to be a stable milk constituent in well-nourished mothers during the first month of lactation [19], although in the present study, its level varied with geographical locations. These differences might be explained by differences in maternal diet [20], and it might be extrapolated to other milk metabolites.

When compared with the other constituents, fat has previously been classified as the most variable component of breast milk due to being influenced by the time of day, inter-feeding interval, point of sampling during a feeding, stage of lactation, maternal weight, and differences between breasts [25]. In the present study, the differences in the LDL and VLDL in the milk might be partially explained by dietary impacts [26] or genetic factors, as previously reported [27]. However, their role in infant nutrition remains unclear, and the fatty acid content and their composition inside the lipoproteins might be more important for the infant than the content of lipoprotein particles. A recent study has shown the difference in the lipidomic profile across countries [9]. In this study, the lipid composition, particularly that of polyunsaturated fatty acids (PUFA), differed between the countries, with the highest level being observed in the case of omega-6 PUFA in Chinese women.

Human milk oligosaccharides, which are the third most abundant component in human milk [28,29], are a group of complex sugars that are non-digestible by infants. These HMOs contain a lactose core bound to one or more glucose, galactose, $\mathrm{N}$-acetylglucosamine, fucose, or sialic acid residues [30]. HMOs support the competitive growth of beneficial bacterial strains within the intestine [29], inhibit the adhesion of pathogens to the infant's epithelium, and interact directly with host immune cells [30]. Each HMO fulfils different roles and activities, and each is metabolized by different bacteria [31]. In addition, specific HMOs could render epithelial cells more resistant to bacterial colonization, and they can interact with immune cells in order to reduce the expression of pro-inflammatory cytokines [32]. This means that differences in the HMOs between geographical locations may result in different roles for human milk in relation to infant health and development. It has been suggested that HMO concentrations and profiles may vary geographically [16,32]. 
The present study confirmed significant differences in both sugars and HMOs between countries, which was also revealed by a study by McGuire et al. [16]. Their study revealed that milk from Sweden contained more than four times more 3-fucosyllactose (3FL) and lower disialyllacto- $N$-tetraose than milk collected in rural Gambia. Similarly, our study showed significant differences in the 3FL, lacto- $N$-fucopentaose III (LNFPIII), lacto- $N$-fucopentaose I (LNFPI), and 2-fucosyllactose (2FL) levels. The HMO profile and specific oligosaccharides, such as $2 \mathrm{FL}$, have also been linked to infant body composition and growth [33], the survival of children born to HIV-infected mothers [34], and allergic morbidity such as a cow milk protein allergy [33]. Furthermore, it was previously reported that specific HMOs, such as LNFPI and $3^{\prime}$-sialyllactose, were associated with infant morbidity and growth development, respectively, and at the same time, correlated with specific microbiota [31]. Another prior study reported an association between the total HMO concentrations and Actinobacteria (mainly Bifidobacterium spp.) counts [35]. More specifically, it was reported that B. breve was positively correlated with sialylated HMOs, while B. longum was positively correlated with non-fucosylated/non-sialylated HMOs. Furthermore, the same study reported positive correlations between fucosylated HMOs and the classes Verrucomicrobiae (Akkermansia muciniphila) and Bacilli, mainly Staphylococcus aureus [35]. Our results thus support the available, albeit limited, data concerning the complex interactions between microbiota and HMOs during lactation, with significant associations being found between the bacterial groups and fucosyl- $\alpha-1,4-N$-acetylglucosamine, lactodifucotetraose, LNFPIII, LNFPI, and 2FL. According to this study, geographical variations and changes in the metabolomics profile of human milk following caesarean section may involve differences in the protective activities of HMOs.

Previous studies have indicated that the neonatal primary gut colonizers include acetate- and lactate-producing bacteria from the classes Actinobacteria (Bifidobacterium), Bacteroidia (Bacteroides) and Bacilli (Lactobacillus, Streptococcus, Staphylococcus, and Enterococcus) [36]. These bacteria have also been found to be present in human breast milk in its viable form [37,38]. In this study, higher amounts of lactate, followed by acetate and propionate, were detected in the human milk samples across locations. The origin of this lactate may be both the mammary tissue and/or milk microbiota. In the mammary gland, lactate serves as an intermediate in carbohydrates metabolism [39], while lactate and acetate are intermediate fermentation products of the microbial metabolism $[40,41]$. The present study found significant differences in the acetate and lactate levels between locations, which were also correlated with several bacterial groups. The impact of milk lactate on infant health is not yet known, although recent studies have suggested that lactate must be efficiently metabolized during early life in order to avoid the potential negative consequences of lactate accumulation as acidosis, neurotoxicity, and cardiac arrhythmia [42]. In addition, it is known that acetate is a product of Bifidobacterium metabolism, and it serves to promote the defense functions of the host cells and exert a protective effect against infection [43]. It has also been established that acetate and lactate produced by Bifidobacterium and Lactobacillus contribute to SCFA-mediated health effects, although these two microorganisms do not directly produce butyrate and/or propionate [43,44].

Interestingly, ethanol was also identified in the breast milk samples analyzed in our study. Considering that mothers do not generally drink alcoholic beverages during lactation, the presence of this compound in small amounts in human milk might be a consequence of milk microbiota metabolism. Many microorganisms, including bacteria and yeasts, produce ethanol as the major fermentation product of carbohydrates. Furthermore, recent studies have reported the presence of yeasts, such as Saccharomyces and Candida, in breast milk samples obtained from healthy women [45], and the presence of ethanol in milk might be a product of their metabolism.

It has previously been reported that specific interactions take place between milk microbes and other milk components, including macro- and micro-nutrients as well as milk cells $[8,10]$. In this study, we found a group of metabolites to be positively correlated with Gammaproteobacteria, including lactate, creatine, proline, lacto- $N$-fucopentaose I, and 2-fucosyllactose VLDL, as well as negatively correlated with Alpha- and Betaproteobacteria, and Bacilli. 
The major limitation of this study is that we were unable to determine the effect of geographical location and caesarean section alone on the milk metabolome without the influence of other confounding variables (e.g., gestational age, diet, lifestyle, secretor status). To do so, future studies involving higher sample sizes and controlling for such variables should be performed. Despite this limitation, the present study contributes important information regarding regional variations in human milk metabolites, the impact of caesarean section, and the correlation of milk metabolites with milk microbiota. Understanding both the composition and function of the components of milk is vital to infant health. The data obtained from this study could form the basis for future studies concerning the milk metabolome and their contribution to infant health and development.

Taken together, ethnicity, diet, environment, and lifestyle could partially explain the different NMR metabolomic profiles seen in this study. It is well-known that diet influences the human metabolomics profile in the blood [46], urine [47], tissues [47], and fecal supernatants [48], and something similar may be expected in human milk. Moreover, only a few studies have investigated the relationships among the human milk microbiota and milk constituents [12,14]. Our findings confirm prior evidence showing that complex bacterial communities within milk are associated with variations in the nutritional composition and metabolites profiles $[9,15]$.

\section{Conclusions}

By means of NMR, we detected 68 metabolites in human milk sampled one month postpartum. The concentrations of carbohydrates, amino acids, short-chain fatty acids, and other metabolites reflected the external environment, that is, the geographical location. In addition, the internal environment, namely the mode of delivery, also impacted the metabolite profile. Our findings support the hypothesis that human milk metabolites from healthy women vary across locations. Shifts on metabolites are associated to milk microbiota profiles suggesting a complex interlink between milk compounds. It is known that breast-feeding provides a personalized infant nutrition driving the infant gut development, immune system maturation and metabolic activities. Climate, lifestyle, environmental exposure, circadian rhythms, ethnic origin, population-specific variations and genetics would have an impact on metabolite profile explaining some of the variation observed in this study and request further research. Furthermore, our study highlights the potential interactions between human milk metabolites and microbes.

Therefore, we need to understand the pivotal relationship between environment-host-nutrition during pregnancy, lactation and early infancy due its impact for human health. This knowledge would enable the design personalized interventions to modulate milk bioactive compounds to be transferred through breastfeeding to the infants. Our data highlight the need for controlled- large-scale human studies across locations and the need to associated changes on milk bioactive compounds to infant growth, development, and health outcomes.

On this basis, we suggest the hypothesis that breast milk provides optimal immunological and metabolic guidance to the extrauterine world, although the differences reported between the locations and modes of delivery in terms of the metabolite composition might involve variations in infant development.

Supplementary Materials: The following are available online at http:/ / www.mdpi.com/2072-6643/10/10/1355/ s1, Table S1: Age and pre-pregnancy body max index (BMI) of the participants in the study. Height was measured during the first visit at hospital and weight was self-reported, Table S2: Significantly $(p<0.05)$ spearman's rank-order correlation between metabolites and maternal characteristics, Figure S1: Principal component analysis (PCA) scores plot (A) and loadings of the first principal component (B) and second principal component (C) scaling NMR data from all participant countries. Countries are indicated as red diamonds (China), green squares (Finland), navy blue triangles (Spain), pale blue triangles (South Africa), Figure S2: Principal component analysis (PCA) scores plot (A) and loadings of the first principal component (B) and second principal component (C) scaling NMR data from all participant countries without Spain. Countries are indicated as red diamonds (China), green squares (Finland), pale blue triangles (South Africa), Figure S3: Box and whisker plot showing statistical significant differences in metabolityes in breast milk samples after vaginal or caesarean delivery. LNFP III: lacto-N-fucopentaose III. Mann-Whitney U test significant level was considered at 0.05 . Each bar represents the 
smallest observation, lower quartile (Q1), median, upper quartile (Q3) and largest observation. Circles and stars indicate outlier data.

Author Contributions: M.C.C. and S.S. conceived the idea and designed the study. J.M.M. carried out metabolomics data analysis and C.G.-G. analyzed metabolomics data, combined with microbiota, and wrote the manuscript. All authors contributed to the interpretation of data, critically reviewed the manuscript for intellectual content and approved the final version.

Funding: This work has been funded by the European Research Council (ERC) under the European Union's Horizon 2020 research and innovation program (ERC Starting Grant, project no. 639226). DM gratefully acknowledges financial support from the JPI HDHL (GUTMOM project) and the Ministerio de Ciencia, Innovación y Universidades from Spain (PCIN-2017-117 and SAF2014-52875R), and also from the 'Instituto de Salud Carlos III and co-funded by FEDER founds' (grant number PIE15/00013).

Acknowledgments: We are grateful for all the participant families providing biological samples for this study.

Conflicts of Interest: The authors declare no conflict of interest.

\section{References}

1. Smilowitz, J.T.; O'Sullivan, A.; Barile, D.; German, J.B.; Lonnerdal, B.; Slupsky, C.M. The human milk metabolome reveals diverse oligosaccharide profiles. J. Nutr. 2013, 143, 1709-1718. [CrossRef] [PubMed]

2. Stuebe, A. The risks of not breastfeeding for mothers and infants. Rev. Obstet. Gynecol. 2009, 2, $222-231$. [PubMed]

3. Lodge, C.J.; Tan, D.J.; Lau, M.X.; Dai, X.; Tham, R.; Lowe, A.J.; Bowatte, G.; Allen, K.J.; Dharmage, S.C. Breastfeeding and asthma and allergies: A systematic review and meta-analysis. Acta Paediatr. 2015, 104, 38-53. [CrossRef] [PubMed]

4. Luccioli, S.; Zhang, Y.; Verrill, L.; Ramos-Valle, M.; Kwegyir-Afful, E. Infant feeding practices and reported food allergies at 6 years of age. Pediatrics 2014, 134 (Suppl. 1), S21-S28. [CrossRef] [PubMed]

5. Jwa, S.C.; Fujiwara, T.; Kondo, N. Latent protective effects of breastfeeding on late childhood overweight and obesity: A nationwide prospective study. Obesity 2014, 22, 1527-1537. [CrossRef] [PubMed]

6. Marseglia, L.; Manti, S.; D'Angelo, G.; Cuppari, C.; Salpietro, V.; Filippelli, M.; Trovato, A.; Gitto, E.; Salpietro, C.; Arrigo, T. Obesity and breastfeeding: The strength of association. Women Birth 2015, 28, 81-86. [CrossRef] [PubMed]

7. Longini, M.; Tataranno, M.L.; Proietti, F.; Tortoriello, M.; Belvisi, E.; Vivi, A.; Tassini, M.; Perrone, S.; Buonocore, G. A metabolomic study of preterm and term human and formula milk by proton MRS analysis: Preliminary results. J. Matern. Fetal Neonatal Med. 2014, 27 (Suppl. 2), 27-33. [CrossRef] [PubMed]

8. Williams, J.E.; Price, W.J.; Shafii, B.; Yahvah, K.M.; Bode, L.; McGuire, M.A.; McGuire, M.K. Relationships among microbial communities, maternal cells, oligosaccharides, and macronutrients in human milk. J. Hum. Lact. 2017, 33, 540-551. [CrossRef] [PubMed]

9. Kumar, H.; du Toit, E.; Kulkarni, A.; Aakko, J.; Linderborg, K.M.; Yumei, Z.; Nicol, M.P.; Isolauri, E.; Yang, B.; Collado, M.C.; et al. Distinct patterns in human milk microbiota and fatty acid profiles across specific geographic locations. Front. Microbiol. 2016, 7, 1619. [CrossRef] [PubMed]

10. Boix-Amoros, A.; Collado, M.C.; Mira, A. Relationship between milk microbiota, bacterial load, macronutrients, and human cells during lactation. Front. Microbiol. 2016, 7, 492. [CrossRef] [PubMed]

11. Munblit, D.; Boyle, R.J.; Warner, J.O. Factors affecting breast milk composition and potential consequences for development of the allergic phenotype. Clin. Exp. Allergy 2015, 45, 583-601. [CrossRef] [PubMed]

12. Chung, M.Y. Factors affecting human milk composition. Pediatr. Neonatol. 2014, 55, 421-422. [CrossRef] [PubMed]

13. Ballard, O.; Morrow, A.L. Human milk composition nutrients and bioactive factors. Pediatr. Clin. N. Am. 2013, 60, 49-74. [CrossRef] [PubMed]

14. Yuhas, R.; Pramuk, K.; Lien, E.L. Human milk fatty acid composition from nine countries varies most in DHA. Lipids 2006, 41, 851-858. [CrossRef] [PubMed]

15. Gomez-Gallego, C.; Kumar, H.; Garcia-Mantrana, I.; du Toit, E.; Suomela, J.P.; Linderborg, K.M.; Zhang, Y.; Isolauri, E.; Yang, B.; Salminen, S.; et al. Breast milk polyamines and microbiota interactions: Impact of mode of delivery and geographical location. Ann. Nutr. Metab. 2017, 70, 184-190. [CrossRef] [PubMed] 
16. McGuire, M.K.; Meehan, C.L.; McGuire, M.A.; Williams, J.E.; Foster, J.; Sellen, D.W.; Kamau-Mbuthia, E.W.; Kamundia, E.W.; Mbugua, S.; Moore, S.E.; et al. What's normal? Oligosaccharide concentrations and profiles in milk produced by healthy women vary geographically. Am. J. Clin. Nutr. 2017, 105, 1086-1100. [CrossRef] [PubMed]

17. Li, S.W.; Watanabe, K.; Hsu, C.C.; Chao, S.H.; Yang, Z.H.; Lin, Y.J.; Chen, C.C.; Cao, Y.M.; Huang, H.C.; Chang, C.H.; et al. Bacterial composition and diversity in breast milk samples from mothers living in Taiwan and mainland China. Front. Microbiol. 2017, 8, 965. [CrossRef] [PubMed]

18. Wishart, D.S.; Feunang, Y.D.; Marcu, A.; Guo, A.C.; Liang, K.; Vazquez-Fresno, R.; Sajed, T.; Johnson, D.; Li, C.; Karu, N.; et al. HMDB 4.0: The human metabolome database for 2018. Nucleic Acids Res. 2018, 46, D608-D617. [CrossRef] [PubMed]

19. Sundekilde, U.K.; Downey, E.; O’Mahony, J.A.; O'Shea, C.A.; Ryan, C.A.; Kelly, A.L.; Bertram, H.C. The effect of gestational and lactational age on the human milk metabolome. Nutrients 2016, 8, 304. [CrossRef] [PubMed]

20. Spevacek, A.R.; Smilowitz, J.T.; Chin, E.L.; Underwood, M.A.; German, J.B.; Slupsky, C.M. Infant maturity at birth reveals minor differences in the maternal milk metabolome in the first month of lactation. J. Nutr. 2015, 145, 1698-1708. [CrossRef] [PubMed]

21. Mitoulas, L.R.; Kent, J.C.; Cox, D.B.; Owens, R.A.; Sherriff, J.L.; Hartmann, P.E. Variation in fat, lactose and protein in human milk over $24 \mathrm{~h}$ and throughout the first year of lactation. Br. J. Nutr. 2002, 88, 29-37. [CrossRef] [PubMed]

22. Kuang, Y.S.; Li, S.H.; Guo, Y.; Lu, J.H.; He, J.R.; Luo, B.J.; Jiang, F.J.; Shen, H.; Papasian, C.J.; Pang, H.; et al. Composition of gut microbiota in infants in china and global comparison. Sci. Rep. 2016, 6, 36666. [CrossRef] [PubMed]

23. Fallani, M.; Young, D.; Scott, J.; Norin, E.; Amarri, S.; Adam, R.; Aguilera, M.; Khanna, S.; Gil, A.; Edwards, C.A.; et al. Intestinal microbiota of 6-week-old infants across europe: Geographic influence beyond delivery mode, breast-feeding, and antibiotics. J. Pediatr. Gastroenterol. Nutr. 2010, 51, 77-84. [CrossRef] [PubMed]

24. De Preter, V.; Vanhoutte, T.; Huys, G.; Swings, J.; Rutgeerts, P.; Verbeke, K. Effect of lactulose and Saccharomyces boulardii administration on the colonic urea-nitrogen metabolism and the bifidobacteria concentration in healthy human subjects. Aliment. Pharmacol. Ther. 2006, 23, 963-974. [CrossRef] [PubMed]

25. Dror, D.K.; Allen, L.H. Overview of nutrients in human milk. Adv. Nutr. 2018, 9, 278s-294s. [CrossRef] [PubMed]

26. Jegou, M.; Gondret, F.; Lalande-Martin, J.; Tea, I.; Baeza, E.; Louveau, I. NMR-based metabolomics highlights differences in plasma metabolites in pigs exhibiting diet-induced differences in adiposity. Eur. J. Nutr. 2016, 55, 1189-1199. [CrossRef] [PubMed]

27. Garcia-Rios, A.; Perez-Martinez, P.; Mata, P.; Fuentes, F.; Lopez-Miranda, J.; Alonso, R.; Caballero, J.; Mata, N.; Perez-Jimenez, F.; Ordovas, J.M. Polymorphism at the TRIB1 gene modulates plasma lipid levels: Insight from the Spanish familial hypercholesterolemia cohort study. Nutr. Metab. Cardiovasc. Dis. 2011, 21, 957-963. [CrossRef] [PubMed]

28. Meredith-Dennis, L.; Xu, G.; Goonatilleke, E.; Lebrilla, C.B.; Underwood, M.A.; Smilowitz, J.T. Composition and variation of macronutrients, immune proteins, and human milk oligosaccharides in human milk from nonprofit and commercial milk banks. J. Hum. Lact. 2018, 34, 120-129. [CrossRef] [PubMed]

29. Chaturvedi, P.; Warren, C.D.; Altaye, M.; Morrow, A.L.; Ruiz-Palacios, G.; Pickering, L.K.; Newburg, D.S. Fucosylated human milk oligosaccharides vary between individuals and over the course of lactation. Glycobiology 2001, 11, 365-372. [CrossRef] [PubMed]

30. Kunz, C.; Rudloff, S.; Baier, W.; Klein, N.; Strobel, S. Oligosaccharides in human milk: Structural, functional, and metabolic aspects. Annu. Rev. Nutr. 2000, 20, 699-722. [CrossRef] [PubMed]

31. Davis, J.C.; Lewis, Z.T.; Krishnan, S.; Bernstein, R.M.; Moore, S.E.; Prentice, A.M.; Mills, D.A.; Lebrilla, C.B.; Zivkovic, A.M. Growth and morbidity of Gambian infants are influenced by maternal milk oligosaccharides and infant gut microbiota. Sci. Rep. 2017, 7, 40466. [CrossRef] [PubMed]

32. Thurl, S.; Munzert, M.; Boehm, G.; Matthews, C.; Stahl, B. Systematic review of the concentrations of oligosaccharides in human milk. Nutr. Rev. 2017, 75, 920-933. [CrossRef] [PubMed] 
33. Sprenger, N.; Lee, L.Y.; De Castro, C.A.; Steenhout, P.; Thakkar, S.K. Longitudinal change of selected human milk oligosaccharides and association to infants' growth, an observatory, single center, longitudinal cohort study. PLoS ONE 2017, 12, e0171814. [CrossRef] [PubMed]

34. Kuhn, L.; Kim, H.Y.; Hsiao, L.; Nissan, C.; Kankasa, C.; Mwiya, M.; Thea, D.M.; Aldrovandi, G.M.; Bode, L. Oligosaccharide composition of breast milk influences survival of uninfected children born to HIV-infected mothers in Lusaka, Zambia. J. Nutr. 2015, 145, 66-72. [CrossRef] [PubMed]

35. Aakko, J.; Kumar, H.; Rautava, S.; Wise, A.; Autran, C.; Bode, L.; Isolauri, E.; Salminen, S. Human milk oligosaccharide categories define the microbiota composition in human colostrum. Benef. Microbes 2017, 8 , 563-567. [CrossRef] [PubMed]

36. De Vadder, F.; Kovatcheva-Datchary, P.; Goncalves, D.; Vinera, J.; Zitoun, C.; Duchampt, A.; Backhed, F.; Mithieux, G. Microbiota-generated metabolites promote metabolic benefits via gut-brain neural circuits. Cell 2014, 156, 84-96. [CrossRef] [PubMed]

37. Martin, R.; Langa, S.; Reviriego, C.; Jiminez, E.; Marin, M.L.; Xaus, J.; Fernandez, L.; Rodriguez, J.M. Human milk is a source of lactic acid bacteria for the infant gut. J. Pediatr. 2003, 143, 754-758. [CrossRef] [PubMed]

38. Solis, G.; de Los Reyes-Gavilan, C.G.; Fernandez, N.; Margolles, A.; Gueimonde, M. Establishment and development of lactic acid bacteria and bifidobacteria microbiota in breast-milk and the infant gut. Anaerobe 2010, 16, 307-310. [CrossRef] [PubMed]

39. Mohammad, M.A.; Maningat, P.; Sunehag, A.L.; Haymond, M.W. Precursors of hexoneogenesis within the human mammary gland. Am. J. Physiol. Endocrinol. Metab. 2015, 308, E680-E687. [CrossRef] [PubMed]

40. Belenguer, A.; Duncan, S.H.; Calder, A.G.; Holtrop, G.; Louis, P.; Lobley, G.E.; Flint, H.J. Two routes of metabolic cross-feeding between bifidobacterium adolescentis and butyrate-producing anaerobes from the human gut. Appl. Environ. Microbiol. 2006, 72, 3593-3599. [CrossRef] [PubMed]

41. Schwab, C.; Ganzle, M. Lactic acid bacteria fermentation of human milk oligosaccharide components, human milk oligosaccharides and galactooligosaccharides. FEMS Microbiol. Lett. 2011, 315, 141-148. [CrossRef] [PubMed]

42. Ewaschuk, J.B.; Naylor, J.M.; Zello, G.A. D-lactate in human and ruminant metabolism. J. Nutr. 2005, 135, 1619-1625. [CrossRef] [PubMed]

43. Fukuda, S.; Toh, H.; Taylor, T.D.; Ohno, H.; Hattori, M. Acetate-producing bifidobacteria protect the host from enteropathogenic infection via carbohydrate transporters. Gut Microbes 2012, 3, 449-454. [CrossRef] [PubMed]

44. O'Callaghan, A.; van Sinderen, D. Bifidobacteria and their role as members of the human gut microbiota. Front. Microbiol. 2016, 7, 925. [CrossRef] [PubMed]

45. Boix-Amorós, A.; Martinez-Costa, C.; Querol, A.; Collado, M.C.; Mira, A. Multiple approaches detect the presence of fungi in human breastmilk samples from healthy mothers. Sci. Rep. 2017, 7, 13016. [CrossRef] [PubMed]

46. Guertin, K.A.; Moore, S.C.; Sampson, J.N.; Huang, W.Y.; Xiao, Q.; Stolzenberg-Solomon, R.Z.; Sinha, R.; Cross, A.J. Metabolomics in nutritional epidemiology: Identifying metabolites associated with diet and quantifying their potential to uncover diet-disease relations in populations. Am. J. Clin. Nutr. 2014, 100, 208-217. [CrossRef] [PubMed]

47. Playdon, M.C.; Sampson, J.N.; Cross, A.J.; Sinha, R.; Guertin, K.A.; Moy, K.A.; Rothman, N.; Irwin, M.L.; Mayne, S.T.; Stolzenberg-Solomon, R.; et al. Comparing metabolite profiles of habitual diet in serum and urine. Am. J. Clin. Nutr. 2016, 104, 776-789. [CrossRef] [PubMed]

48. Chow, J.; Panasevich, M.R.; Alexander, D.; Vester Boler, B.M.; Rossoni Serao, M.C.; Faber, T.A.; Bauer, L.L.; Fahey, G.C. Fecal metabolomics of healthy breast-fed versus formula-fed infants before and during in vitro batch culture fermentation. J. Proteome Res. 2014, 13, 2534-2542. [CrossRef] [PubMed] 


\title{
Maternal Nutrition and Body Composition During Breastfeeding: Association with Human Milk Composition
}

\author{
Agnieszka Bzikowska-Jura ${ }^{1}$, Aneta Czerwonogrodzka-Senczyna ${ }^{1}$, Gabriela Olędzka ${ }^{2, *}$, \\ Dorota Szostak-Węgierek ${ }^{1}$, Halina Weker ${ }^{1}$ and Aleksandra Wesołowska ${ }^{3}$ \\ 1 Department of Clinical Dietetics, Faculty of Health Science, Medical University of Warsaw, E Ciolka Str. 27, \\ 01-445 Warsaw, Poland; agnieszka.bzikowska@wum.edu.pl (A.B.-J.); aneta.senczyna@wum.edu.pl (A.C.-S.); \\ dorota.szostak-wegierek@wum.edu.pl (D.S.-W.); halina.weker@wum.edu.pl (H.W.) \\ 2 Department of Medical Biology, Faculty of Health Science, Medical University of Warsaw, Nowogrodzka Str. 73, \\ 02-018 Warsaw, Poland \\ 3 Laboratory of Human Milk and Lactation Research at Regional Human Milk Bank in Holy Family Hospital, \\ Faculty of Health Science, Department of Neonatology, Medical University of Warsaw, Zwirki i Wigury Str. 63A, \\ 02-091 Warsaw, Poland; aleksandra.wesolowska@wum.edu.pl \\ * Correspondence: gabriela.oledzka@wum.edu.pl; Tel.: +48-22-625-32-23
}

Received: 31 July 2018; Accepted: 25 September 2018; Published: 27 September 2018

\begin{abstract}
The composition of human milk is dynamic and can vary according to many maternal factors, such as diet and nutritional status. This study investigated the association of maternal nutrition and body composition with human milk composition. All measurements and analyses were done at three time points: during the first $(n=40)$, third $(n=22)$, and sixth $(n=15)$ month of lactation. Human milk was analyzed using the Miris human milk analyzer (HMA), body composition was measured with bioelectrical bioimpedance (BIA) using a Maltron BioScan 920-II, and the assessment of women's nutrition was based on a three-day dietary record. The correlation coefficient (Pearson's $r$ ) did not show a significant statistical relationship between human milk composition and nutrients in women's diet at three time points. For women in the third month postpartum, we observed moderate to strong significant correlations ( $r$ ranged from 0.47 to 0.64 ) between total protein content in milk and the majority of body composition measures as follows: positive correlations: \% fat mass $(r=0.60$; $p=0.003)$, fat-free mass expressed in $\mathrm{kg}(r=0.63 ; p=0.001)$, and muscle mass $(r=0.47 ; p=0.027)$; and negative correlation: \% total body water $(r=-0.60 ; p=0.003)$. The variance in milk fat content was related to the body mass index (BMI), with a significant positive correlation in the first month postpartum $(r=0.33 ; p=0.048)$. These findings suggest that it is not diet, but rather the maternal body composition that may be associated with the nutritional value of human milk.
\end{abstract}

Keywords: breastfeeding; human milk composition; body composition; maternal diet

\section{Introduction}

Human milk is the best source of nutrition for infants, as it contains essential nutrients in the right balance, and other bioactive factors (e.g., hormones, antibodies, bioactive molecules, stem cells) [1,2]. It is well documented that exclusive breastfeeding for the first six months of life is associated with a decreased incidence of infections and chronic diseases [3,4]. Exclusive breastfeeding has also been shown to trigger a protective effect against later obesity [5,6] and type 2 diabetes in the offspring [7,8]. Nevertheless, this protective effect is controversial, and it may differ in accordance with maternal phenotypes [9,10].

Compared to infant formulas, which have standardized compositions, human milk composition changes dynamically, as it is produced by women with significantly varying genotypes and phenotypes [11]. 
Table 1 summarizes the milk energy and macronutrient concentration from past studies on different human populations.

The composition of human milk is influenced by many maternal, infant, and physiological factors (Figure 1) [11]. Some of these factors are better investigated than others, involving $24 \mathrm{~h}$ variations (peak fat content occurs at midmorning) [12], lactational stage (colostrum is reported to be higher in protein but lower in lactose and fat in comparison to mature milk) [13], and time point in breastfeeding session (hindmilk is higher in fat than foremilk; lactose shows an inverse correlation with the stage of breastfeeding) [14,15]. The influence of other factors, including those contained in this study (maternal nutrition and body composition) do not have well-defined effects.

Previous studies assessing the relationship between maternal factors and human milk composition had some limitations, such as no specific time for the expression of milk samples (time of day, hind- or foremilk), and no information about exclusive breastfeeding or analysis of milk composition, excluding nonprotein nitrogen sources, which lead to inflated protein concentrations. Additionally, most of the existing studies were conducted in the 1980s and 1990s [16-18] and for milk composition analysis, they used separate analytical instruments for protein, fat, and lactose, which may have been flawed and was time-consuming. All of these limitations influence a true-value assessment of these associations.

We investigated the impact of maternal diet and body composition on human milk composition, analyzing women's diets and body composition and the nutritional value of human milk.

Table 1. Variations in mature human milk composition in different sample populations.

\begin{tabular}{|c|c|c|c|c|c|c|c|}
\hline Population & $\begin{array}{c}\text { Characteristics of } \\
\text { Participants }\end{array}$ & $\begin{array}{l}\text { Analytical } \\
\text { Technique }\end{array}$ & Energy $^{1}$ & Fat $^{2}$ & Lactose $^{2}$ & Protein $^{2}$ & Reference \\
\hline & & & \multicolumn{5}{|c|}{ Mean \pm SD } \\
\hline Korea & $\begin{array}{l}2632 \text { healthy lactating } \\
\text { mothers of full-term } \\
\text { infants }(32.0 \pm 3.3 \text { years) }\end{array}$ & $\begin{array}{c}\text { Infrared } \\
\text { spectrophotometry } \\
\text { (MilkoScan FT2) }\end{array}$ & $61.1 \pm 12.1$ & $3.0 \pm 1.4$ & $7.1 \pm 0.4$ & $1.4 \pm 0.3$ & Chang, 2015 [13] \\
\hline China & $\begin{array}{l}436 \text { urban Chinese } \\
\text { lactating mothers }\end{array}$ & $\begin{array}{c}\text { Infrared } \\
\text { spectrophotometry }\end{array}$ & 61.3 & 3.4 & 7.1 & 0.9 & Yang, 2014 [19] \\
\hline Tibet & $\begin{array}{l}82 \text { breastfeeding Tibetans } \\
\text { living at high and low } \\
\text { altitudes in rural villages }\end{array}$ & $\begin{array}{c}\text { Micro } \\
\text { Rose-Gottlieb }\end{array}$ & $81.4 \pm 17.4$ & $5.3 \pm 2.0$ & $7.4 \pm 0.5$ & $1.3 \pm 0.4$ & Quinn, 2016 [20] \\
\hline Philippines & $\begin{array}{l}102 \text { Filipino breastfeeding } \\
\text { mothers (24.6-25.4 years) }\end{array}$ & $\begin{array}{c}\text { Micro } \\
\text { Rose-Gottlieb }\end{array}$ & $68.6 \pm 15.0$ & $3.8 \pm 1.5$ & $7.3 \pm 0.6$ & $1.3 \pm 0.5$ & Quinn, 2012 [21] \\
\hline Brazil & $\begin{array}{c}34 \text { donors of the Human } \\
\text { Milk Bank }\end{array}$ & $\begin{array}{c}\text { Infrared } \\
\text { spectrophotometry } \\
\text { (MilkoScan Minor) }\end{array}$ & $56.7 \pm 11.7$ & $3.1 \pm 1.18$ & $6.1 \pm 0.6$ & $1.22 \pm 0.5$ & Abranches, 2014 [22] \\
\hline $\begin{array}{l}\text { United States } \\
\text { (DARLING study) }\end{array}$ & $\begin{array}{l}58 \text { healthy lactating } \\
\text { mothers planning to } \\
\text { continue nursing for } \\
\text { a minimum of } 12 \text { months }\end{array}$ & $\begin{array}{c}\text { Lowry assay } \\
\text { (protein), Folch } \\
\text { extraction (fat), } \\
\text { colorimetric } \\
\text { (lactose) }\end{array}$ & $69.7 \pm 6.7$ & $3.6 \pm 0.7$ & $7.4 \pm 0.15$ & $1.2 \pm 0.15$ & Nommsen, 1991 [18] \\
\hline Australia & $\begin{array}{l}23 \text { lactating mothers of } \\
\text { healthy term infants } \\
\text { (33.0 } 3 \text { years) }\end{array}$ & $\begin{array}{c}\text { Mid-infrared } \\
\text { spectrophotometry } \\
\text { (MIRIS) }\end{array}$ & 82.4 & 5.9 & 6.3 & 1.0 & Khan, 2013 [23] \\
\hline
\end{tabular}

${ }^{1}$ Energy is presented as kilocalories (kcal) per $100 \mathrm{~mL} .{ }^{2}$ Macronutrients are presented as grams per $100 \mathrm{~mL} . \mathrm{SD}$, standard deviation.

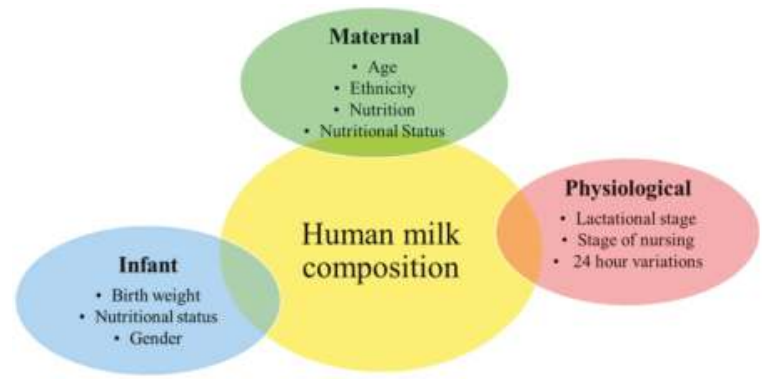

Figure 1. Maternal, infant, and physiological factors that may influence human milk composition. 


\section{Materials and Methods}

\subsection{Study Participants}

A convenience sample of breastfeeding women $(n=40)$ was recruited from the community, primarily from the Holy Family Hospital in Warsaw. Participants were enrolled during their first month of lactation. The inclusion criteria were as follows: age $\geq 18$ years, full-term delivery (gestational age $\geq 37$ weeks), exclusively breastfeeding, and no contraindications to body composition analysis (metal implants, pacemaker, defibrillator, stents, large implants, implanted devices that emit an electronic signal). Exclusion criteria included the following: preexisting chronic or gestational disease, smoking during pregnancy, multiple pregnancy, low birth weight of the newborn, and low milk supply. All mothers provided written informed consent to participate in the study. The study was approved by the Ethics Committee of the Medical University of Warsaw (KB/172/115).

\subsection{Study Session Design}

All measurements and analyses were made at three time points: during the first, third, and sixth months of lactation. Participants visited the Holy Family Hospital in Warsaw. At each study session, the mother was weighed, a body composition analysis was performed, and a $24 \mathrm{~h}$ milk collection was taken. Twenty-two women provided milk samples at two time points, and 15 women at three time points. A 3-day dietary record was self-reported by each mother and checked by a qualified dietitian.

\subsection{Anthropometric Measurements}

Body weight and height were measured using a Seca 799 measurement station and column scales $( \pm 0.1 \mathrm{~kg} / \mathrm{cm}$; Seca, Chino, CA, USA). The body mass index (BMI) was calculated as the ratio between the body weight and the height squared $\left(\mathrm{kg} / \mathrm{m}^{2}\right)$. Interpretation of these data followed the classification proposed by the World Health Organization (WHO): below $18.5 \mathrm{~kg} / \mathrm{m}^{2}$, underweight; $18.5-24.9 \mathrm{~kg} / \mathrm{m}^{2}$, normal weight; $25.0-29.9 \mathrm{~kg} / \mathrm{m}^{2}$, pre-obese; $\geq 30 \mathrm{~kg} / \mathrm{m}^{2}$, obese [24]

\subsection{Body Composition Analysis with Bioelectrical Impedance (BIA)}

Whole-body impedance (wrist to ankle) of the women was measured using the Maltron BioScan 920-II multifrequency bioelectrical impedance analyzer (Maltron Bioscan, Rayleigh, UK) according to the manufacturer's instructions [25]. Total body electrical impedance alternated with four frequencies: $5,50,100$, and $200 \mathrm{kHz}$. The subjects were measured in a supine position, on a nonconductive surface, after taking a rest for about $10 \mathrm{~min}$. Before the electrodes were placed, the sites were cleaned using isopropyl alcohol to limit possible errors and to ensure adherence. The whole-body impedance vector components resistance $(R)$ and reactance $\left(X_{c}\right)$ were measured at the same time. On this basis, body fat, lean mass, other components, and REE (resting energy expenditure) were calculated. Before taking the BIA measurement, the women were instructed with the following guidelines (according to Heyward and Stolarczyk [26]): no heavy exercise $12 \mathrm{~h}$ before the test, no large meals or caffeinated products $4 \mathrm{~h}$ before the test, consumption of liquids limited to $1 \%$ of body weight or two $8 \mathrm{oz}$. glasses of water $2 \mathrm{~h}$ before the test.

\subsection{Twenty-Four-Hour Human Milk Collection}

Human milk samples were collected by participants at home after they were given detailed instructions on taking, storing, and transporting samples to the Holy Family Hospital in Warsaw. Prefeed and postfeed samples were collected from all participants from all time periods (6:00-12:00, 12:00-18:00, 18:00-24:00, 24:00-6:00) to minimize possible circadian influences on the milk composition. A total of 5-10 mL of prefeed and postfeed milk samples were obtained from the breast(s) the infant fed from, by breast pump, or manually. Samples were stored at $-20{ }^{\circ} \mathrm{C}$ for later analysis. 


\subsection{Human Milk Composition}

Human milk was analyzed using the Miris human milk analyzer (HMA) (Miris, Uppsala, Sweden) with a validated protocol. The HMA is calibrated with human milk standards, and it can measure total and true proteins, fat, lactose, and total solids simultaneously. In addition, the macronutrient content of the milk was used to calculate digestible energy. The concentrations of all macronutrients were reported in grams per $100 \mathrm{~mL}$ and energy (kilocalories) was calculated at $4 \mathrm{kcal} / \mathrm{g}$ for protein, $9 \mathrm{kcal} / \mathrm{g}$ for fat, and $4 \mathrm{kcal} / \mathrm{g}$ for carbohydrates. Total protein refers to total nitrogen $\times 6.25$, and true protein is total protein minus $24 \%$ for nonprotein nitrogen. Total protein as reported by the Miris analyzer was converted to bioavailable protein (true protein) for the data analysis using the following equation: total protein $($ grams $) \times 0.825$ [26,27]. The HMA is based on semisolid mid-infrared (MIR) transmission spectroscopy, which is the certified method for milk analysis in the dairy industry according to ISO 9622:1999 by the Association of Official Analytical Chemists (AOAC) and the International Dairy Federation (IDF) [28]. Before analysis, each sample $(n=77)$ was warmed to $40{ }^{\circ} \mathrm{C}$ and homogenized for $1.5 \mathrm{~s} / 1 \mathrm{~mL}$ of probe using a sonicator (milk homogenizer, Miris, Uppsala, Sweden). From each pool, three samples $(\sim 12 \mathrm{~mL}$ in total) were taken to analyze the nutritional value, and for the result, we used the average of three measurements.

\subsection{Nutritional Value of Daily Food Consumption}

The assessment of women's nutrition was based on a 3-day dietary record. Sizes of declared food portions were verified using the "Album of Photographs of Food Products and Dishes" from the National Food and Nutrition Institute [29]. Collected data were used to estimate daily food consumption. Energy and nutritional value of daily food consumption (content of macronutrients, cholesterol, fatty acids, dietary fiber, minerals, and vitamins) were calculated using Dieta 5.0 nutritional software (National Food and Nutrition Institute, Warsaw, Poland).

\subsection{Statistical Analysis}

Statistical analyses were performed using Statistica 12PL, Tulusa, USA and IBM Statistics 21, New York, NY, USA. A p-value below 0.05 was adopted as statistically significant. Variable distributions were evaluated with Shapiro-Wilk test, and descriptive statistics (means and standard deviations as well as medians and interquartile ranges) were calculated. The mothers' anthropometric data and body composition, and the nutritional value of their diet in the first and sixth months of lactation were compared using a paired 2-sample Student's $t$-test (normal distribution of differences between all pairs), or a Wilcoxon signed-rank test for paired samples (nonnormally distributed differences between all pairs). A trend analysis of milk composition at three time points was performed with the Jonckheere-Terpstra test, and its effect size was estimated with Kendall's tau-b correlation coefficient. Correlations between milk composition and the mothers' body composition and diet were estimated with Pearson's r correlation coefficient.

\section{Results}

\subsection{Subjects and Human Milk Composition}

All participants had a university education and a high socioeconomic status. The subjects' anthropometric data and body composition measures are shown in Table 2. The mean maternal age was $31.1 \pm 4.4$ years. At the first month postpartum, none of the participants were classified as being underweight $\left(\mathrm{BMI}<18.5 \mathrm{~kg} / \mathrm{m}^{2}\right)$. Most of them $(n=32,80 \%)$ had normal body mass, and $20 \%(n=8)$ were overweight. We did not observe any statistically significant differences between maternal body composition at the first and third months postpartum. However, there were statistically significant differences between weight and BMI values in the first and sixth months of lactation. The Wilcoxon signed-rank test was 2.59, $p=0.009$ for weight and 2.67, $p=0.008$ for BMI. 
Table 2. Subjects' anthropometric data and body composition measures.

\begin{tabular}{|c|c|c|c|c|}
\hline \multirow{2}{*}{ Characteristic } & \multicolumn{3}{|c|}{ Month of Lactation } & \multirow{2}{*}{$\begin{array}{l}\text { Difference between } \\
\text { First and Sixth Month } \\
\text { of Lactation ( } p \text {-Value) }\end{array}$} \\
\hline & $1(n=40)$ & $3(n=22)$ & $6(n=15)$ & \\
\hline Weight (kg) & $\begin{array}{c}64.5 \pm 12.2 \\
62.3(54.8-70.9)\end{array}$ & $\begin{array}{c}65.1 \pm 13.2 \\
62.4(54.7-70.5)\end{array}$ & $\begin{array}{c}61.4 \pm 10.0 \\
59.5(54.6-67.0)\end{array}$ & $0.009^{2, *}$ \\
\hline Height (cm) & $\begin{array}{c}166.6 \pm 6.6 \\
166.5(162.0-172.5)\end{array}$ & $\begin{array}{c}166.6 \pm 6.6 \\
166.5(162.0-172.5)\end{array}$ & $\begin{array}{c}166.6 \pm 6.6 \\
166.5(162.0-172.5)\end{array}$ & - \\
\hline $\begin{array}{l}\text { Body mass index } \\
\qquad\left(\mathrm{kg} / \mathrm{m}^{2}\right)\end{array}$ & $\begin{array}{c}23.0 \pm 3.6 \\
22.7(20.4-24.8)\end{array}$ & $\begin{array}{c}23.3 \pm 4.0 \\
23.0(19.9-25.9)\end{array}$ & $\begin{array}{c}21.8 \pm 2.8 \\
21.5(19.8-23.7)\end{array}$ & $0.008^{2, *}$ \\
\hline Fat mass (kg) & $\begin{array}{c}19.8 \pm 10.3 \\
17.9(11.3-23.0)\end{array}$ & $\begin{array}{c}19.8 \pm 9.5 \\
19.3(12.6-23.3)\end{array}$ & $\begin{array}{c}16.9 \pm 6.6 \\
17.7(9.8-21.3)\end{array}$ & $0.064^{2}$ \\
\hline Fat mass (\%) & $\begin{array}{c}28.2 \pm 8.5 \\
28.5(20.6-33.0)\end{array}$ & $\begin{array}{c}28.9 \pm 8.6 \\
30.2(21.3-34.6)\end{array}$ & $\begin{array}{c}26.4 \pm 7.1 \\
26.7(19.2-32.4)\end{array}$ & $0.174^{1}$ \\
\hline Fat-free mass (kg) & $\begin{array}{c}45.4 \pm 3.9 \\
45.7(43.0-48.4)\end{array}$ & $\begin{array}{c}45.3 \pm 4.6 \\
45.1(40.9-49.2)\end{array}$ & $\begin{array}{c}45.0 \pm 4.4 \\
43.5(40.9-49.4)\end{array}$ & $0.701^{1}$ \\
\hline Total body water (L) & $\begin{array}{c}32.4 \pm 3.8 \\
31.2(29.4-35.2)\end{array}$ & $\begin{array}{c}32.2 \pm 4.1 \\
31.0(28.5-34.7)\end{array}$ & $\begin{array}{c}31.5 \pm 3.7 \\
30.6(28.5-34.1)\end{array}$ & $0.695^{2}$ \\
\hline Total body water (\%) & $\begin{array}{c}51.2 \pm 5.1 \\
50.3(46.9-55.3)\end{array}$ & $\begin{array}{c}50.3 \pm 4.9 \\
49.8(46.5-52.2)\end{array}$ & $\begin{array}{c}51.2 \pm 4.1 \\
50.9(46.9-54.8)\end{array}$ & $0.236^{1}$ \\
\hline Protein (kg) & $\begin{array}{c}9.0 \pm 1.4 \\
9.0(8.5-9.9)\end{array}$ & $\begin{array}{c}9.3 \pm 1.3 \\
9.2(8.4-10.6)\end{array}$ & $\begin{array}{c}9.6 \pm 1.0 \\
9.4(8.7-10.6)\end{array}$ & $0.638^{2}$ \\
\hline Muscles (kg) & $\begin{array}{c}19.9 \pm 1.9 \\
19.8(18.8-21.4)\end{array}$ & $\begin{array}{c}19.8 \pm 2.0 \\
19.7(18.3-21.7)\end{array}$ & $\begin{array}{c}19.3 \pm 1.7 \\
18.9(18.3-21.5)\end{array}$ & $0.084^{2}$ \\
\hline
\end{tabular}

Data are mean \pm standard deviation (SD), median, and ranges. ${ }^{1}$ Paired two-sample Student's $t$-test; ${ }^{2}$ Wilcoxon signed rank test for paired samples. ${ }^{*} p<0.05$.

Table 3 provides the average results of the nutritional value of human milk (energy, macronutrients, and dry matter) and changes in the concentrations of the components at three time points. We observed a statistically significant downward trend for total protein (tau-b $=-0.31 ; p=0.001)$ and true protein (tau-b $=-0.30 ; p=0.001)$ concentration in human milk. We also noted a decreasing energy value, but the trend was not statistically significant (tau-b $=-0.18 ; p=0.052)$.

Table 3. Composition of human milk.

\begin{tabular}{|c|c|c|c|c|c|}
\hline & \multicolumn{3}{|c|}{ Month of Lactation } & \multirow{2}{*}{$\begin{array}{l}\text { Linear Trend } \\
(p \text {-Value })\end{array}$} & \multirow{2}{*}{$\begin{array}{c}\text { Effect Size } \\
\text { (Kendall's Tau-b) }\end{array}$} \\
\hline & $1(n=40)$ & $3(n=22)$ & $6(n=15)$ & & \\
\hline $\begin{array}{c}\text { Energy } \\
(\mathrm{kcal} / 100 \mathrm{~mL})\end{array}$ & $\begin{array}{c}65.9 \pm 9.9 \\
65.5(62.0-72.5)\end{array}$ & $\begin{array}{c}61.8 \pm 14.4 \\
61.3(49.0-71.7)\end{array}$ & $\begin{array}{c}61.2 \pm 14.2 \\
60.0(47.0-73.7)\end{array}$ & 0.052 & -0.18 \\
\hline Fat $(\mathrm{g} / 100 \mathrm{~mL})$ & $\begin{array}{c}3.5 \pm 1.0 \\
3.5(3.1-4.3)\end{array}$ & $\begin{array}{c}3.2 \pm 1.5 \\
3.1(1.8-4.1)\end{array}$ & $\begin{array}{c}3.2 \pm 1.4 \\
3.1(1.8-4.5)\end{array}$ & 0.120 & -0.14 \\
\hline $\begin{array}{l}\text { Total protein } \\
(\mathrm{g} / 100 \mathrm{~mL})\end{array}$ & $\begin{array}{c}1.2 \pm 0.2 \\
1.1(1.1-1.2)\end{array}$ & $\begin{array}{c}1.1 \pm 0.1 \\
1.0(1.0-1.1)\end{array}$ & $\begin{array}{c}1.0 \pm 0.3 \\
1.0(0.9-1.1)\end{array}$ & 0.001 & -0.31 \\
\hline $\begin{array}{l}\text { True protein } \\
(\mathrm{g} / 100 \mathrm{~mL})\end{array}$ & $\begin{array}{c}0.9 \pm 0.2 \\
0.9(0.8-1.0)\end{array}$ & $\begin{array}{c}0.8 \pm 0.1 \\
0.8(0.7-0.9)\end{array}$ & $\begin{array}{c}0.8 \pm 0.2 \\
0.7(0.7-0.9)\end{array}$ & 0.001 & -0.30 \\
\hline $\begin{array}{c}\text { Carbohydrates } \\
\text { (g/100 mL) }\end{array}$ & $\begin{array}{c}7.0 \pm 0.3 \\
7.0(6.8-7.2)\end{array}$ & $\begin{array}{c}7.0 \pm 0.4 \\
7.1(6.9-7.2)\end{array}$ & $\begin{array}{c}7.1 \pm 0.4 \\
7.1(6.9-7.4)\end{array}$ & 0.236 & 0.11 \\
\hline $\begin{array}{l}\text { Dry matter } \\
(\mathrm{g} / 100 \mathrm{~mL})\end{array}$ & $\begin{array}{c}11.7 \pm 1.3 \\
11.8(11.2-12.7)\end{array}$ & $\begin{array}{c}11.4 \pm 1.7 \\
11.3(9.9-12.6)\end{array}$ & $\begin{array}{c}11.4 \pm 1.8 \\
11.0(9.7-12.9)\end{array}$ & 0.298 & -0.09 \\
\hline
\end{tabular}

\subsection{Nutritional Value of Daily Food Consumption}

Table 4 presents the results of energy and nutrient intake in relation to Polish nutritional standards [29]. The risk of deficient energy intake was observed in $100 \%$ of the women at three time points. There were no significant differences between the intake of macronutrients (protein, fat, and carbohydrates), minerals, 
and vitamins at each stage of the study. Among all of the women $(100 \%)$, at every time point we observed an insufficient intake of vitamin D. The majority of women $(60 \%, n=24)$ at the first month postpartum did not reach the estimated average requirement (EAR) value for calcium.

Table 4. Energy and nutrient intake in relation to Polish nutritional standards 2012 [29]

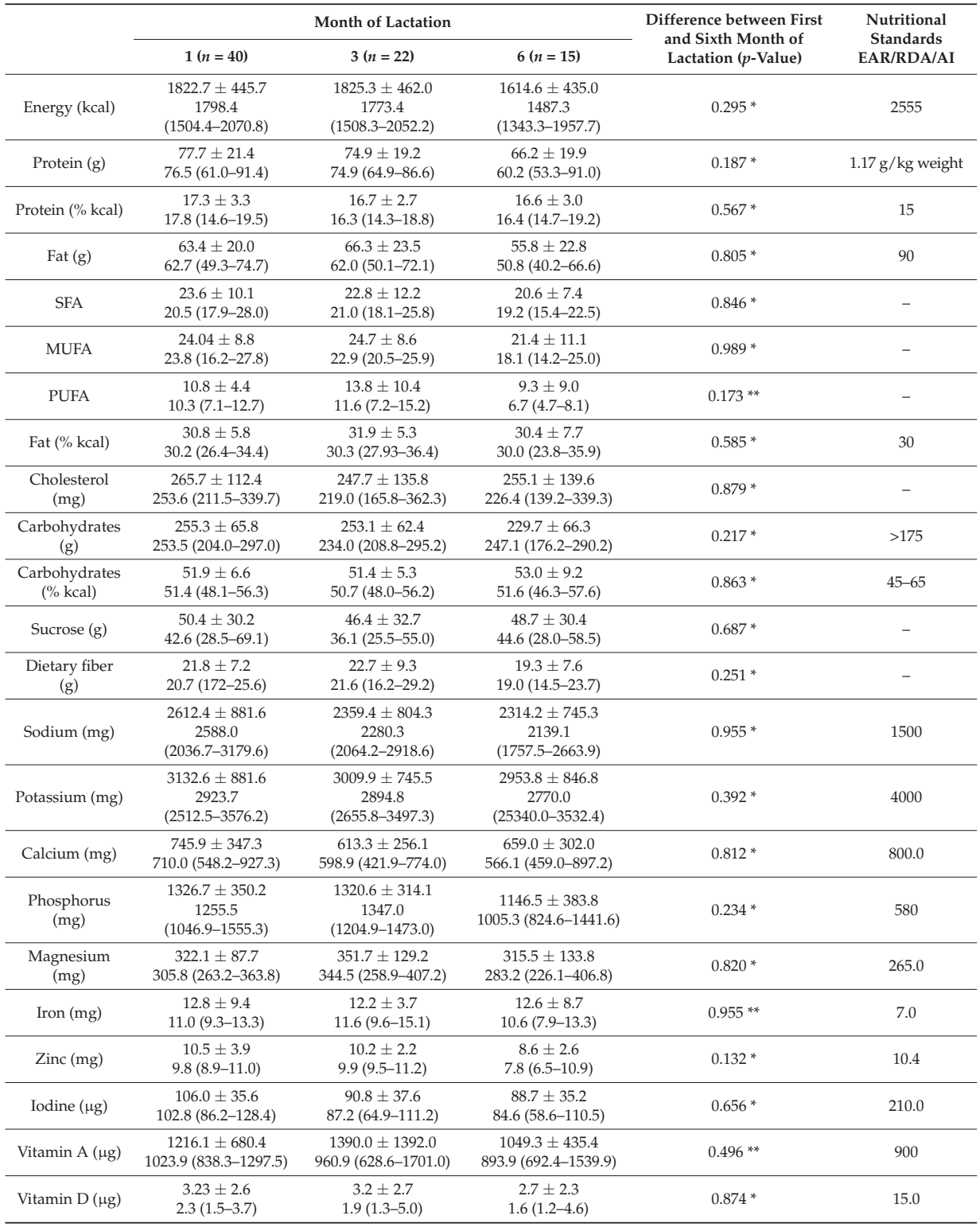


Table 4. Cont.

\begin{tabular}{|c|c|c|c|c|c|}
\hline & \multicolumn{3}{|c|}{ Month of Lactation } & \multirow{2}{*}{$\begin{array}{l}\text { Difference between First } \\
\text { and Sixth Month of } \\
\text { Lactation ( } p \text {-Value) }\end{array}$} & \multirow{2}{*}{$\begin{array}{l}\text { Nutritional } \\
\text { Standards } \\
\text { EAR/RDA/AI }\end{array}$} \\
\hline & $1(n=40)$ & $3(n=22)$ & $6(n=15)$ & & \\
\hline Vitamin E (mg) & $\begin{array}{c}10.4 \pm 4.6 \\
9.4(6.8-13.6)\end{array}$ & $\begin{array}{c}11.9 \pm 7.9 \\
9.3(7.2-16.0)\end{array}$ & $\begin{array}{c}10.4 \pm 6.8 \\
7.9(6.5-14.8)\end{array}$ & $0.873 *$ & 11.0 \\
\hline $\begin{array}{l}\text { Vitamin } B_{1} \\
(\mathrm{mg})\end{array}$ & $\begin{array}{c}1.2 \pm 0.4 \\
1.2(1.0-1.4)\end{array}$ & $\begin{array}{c}1.4 \pm 0.5 \\
1.2(1.0-1.7)\end{array}$ & $\begin{array}{c}1.2 \pm 0.5 \\
1.1(0.9-1.5)\end{array}$ & $0.825 *$ & 1.3 \\
\hline $\begin{array}{l}\text { Vitamin } B_{2} \\
(\mathrm{mg})\end{array}$ & $\begin{array}{c}1.8 \pm 0.6 \\
1.6(1.3-2.1)\end{array}$ & $\begin{array}{c}1.6 \pm 0.5 \\
1.6(1.2-1.9)\end{array}$ & $\begin{array}{c}1.7 \pm 0.6 \\
1.5(1.2-2.1)\end{array}$ & 0.920 * & 1.3 \\
\hline $\begin{array}{l}\text { Vitamin PP } \\
(\mathrm{mg})\end{array}$ & $\begin{array}{c}16.2 \pm 6.2 \\
14.9(11.0-18.5)\end{array}$ & $\begin{array}{c}16.8 \pm 6.0 \\
17.8(11.9-20.3)\end{array}$ & $\begin{array}{c}16.6 \pm 8.8 \\
13.0(11.0-20.3)\end{array}$ & $0.936 *$ & 13.0 \\
\hline$\underset{(\mathrm{mg})}{\text { Vitamin }} \mathrm{B}_{6}$ & $\begin{array}{c}1.9 \pm 0.7 \\
1.8(1.4-2.2)\end{array}$ & $\begin{array}{c}1.9 \pm 0.6 \\
1.9(1.4-2.2)\end{array}$ & $\begin{array}{c}4.8 \pm 8.3 \\
1.9(1.3-2.6)\end{array}$ & $1.00 * *$ & 1.7 \\
\hline Vitamin C (mg) & $\begin{array}{c}127.8 \pm 109.1 \\
92.3(57.6-139.9)\end{array}$ & $\begin{array}{c}135.6 \pm 83.9 \\
113.8(79.1-160.7)\end{array}$ & $\begin{array}{c}163.0 \pm 81.2 \\
139.8(120.7-253.7)\end{array}$ & 0.730 * & 100 \\
\hline $\begin{array}{c}\text { Vitamin } B_{12} \\
(\mu g)\end{array}$ & $\begin{array}{c}3.75 \pm 1.7 \\
3.4(2.3-4.8)\end{array}$ & $\begin{array}{c}4.04 \pm 3.1 \\
3.0(1.9-4.9)\end{array}$ & $\begin{array}{c}3.2 \pm 1.8 \\
2.9(1.9-4.1)\end{array}$ & 0.900 * & 2.4 \\
\hline Folic acid $(\mu \mathrm{g})$ & $\begin{array}{l}324.6 \pm 141.2 \\
(239.2-379.3)\end{array}$ & $\begin{array}{c}310.0 \pm 109.9288 .4 \\
(239.5-388.6)\end{array}$ & $\begin{array}{c}436.5 \pm 213.7364 .6 \\
\quad(284.6-672.8)\end{array}$ & 0.099 * & 450.0 \\
\hline
\end{tabular}

Data are mean \pm standard deviation (SD), median, and ranges. * Paired two-sample Student's $t$-test; ** Wilcoxon signed rank test for paired samples. SFA, saturated fatty acids; MUFA, monounsaturated fatty acids; PUFA, polyunsaturated fatty acids; EAR, estimated average requirement; RDA, recommended daily allowance; AI, adequate intake.

\subsection{Association between Maternal Diet and Milk Composition}

Table 5 presents correlation coefficient (Pearson's $r$ ) between human milk composition and nutrients in the mother's diet at three time points (first, third, and sixth month of lactation). We did not observe any statistically significant correlation between these factors $(p>0.05)$. The estimated energy value and the macronutrient content in lactating women's average daily food consumption recorded for three days did not allow for the prediction of the variance in their milk composition.

Table 5. Correlations between human milk composition and nutrients in mothers' diet.

\begin{tabular}{|c|c|c|c|c|c|c|c|}
\hline \multirow{2}{*}{$\begin{array}{l}\text { Energy and } \\
\text { Nutrients in } \\
\text { Mothers' Diet }\end{array}$} & \multirow{2}{*}{$\begin{array}{l}\text { Month of } \\
\text { Lactation }\end{array}$} & \multicolumn{6}{|c|}{ Composition of Human Milk } \\
\hline & & Energy ${ }^{1}$ & $\begin{array}{c}\text { Total } \\
\text { Protein } 2\end{array}$ & $\begin{array}{c}\text { True } \\
\text { Protein } 2\end{array}$ & Fat $^{2}$ & Carbohydrates $^{2}$ & $\begin{array}{c}\text { Dry } \\
\text { Matter }^{2}\end{array}$ \\
\hline \multirow{3}{*}{ Energy (kcal) } & 1 & 0.06 & 0.08 & 0.13 & 0.08 & -0.14 & 0.02 \\
\hline & 3 & 0.12 & -0.05 & 0.02 & 0.11 & 0.08 & 0.11 \\
\hline & 6 & -0.15 & -0.12 & -0.05 & -0.09 & -0.22 & -0.11 \\
\hline \multirow{3}{*}{ Protein $(\mathrm{g})$} & 1 & 0.08 & 0.02 & 0.02 & 0.08 & -0.05 & 0.06 \\
\hline & 3 & 0.10 & -0.19 & -0.12 & -0.13 & -0.20 & 0.05 \\
\hline & 6 & 0.01 & -0.7 & -0.05 & 0.05 & -0.24 & 0.03 \\
\hline \multirow{3}{*}{ Fat $(g)$} & 1 & 0.09 & 0.01 & 0.04 & 0.08 & -0.02 & 0.11 \\
\hline & 3 & 0.09 & 0.07 & 0.10 & 0.06 & 0.15 & 0.10 \\
\hline & 6 & -0.01 & -0.12 & -0.03 & 0.03 & -0.10 & 0.05 \\
\hline \multirow{3}{*}{ Carbohydrates (g) } & 1 & 0.01 & 0.13 & 0.20 & 0.05 & -0.22 & -0.09 \\
\hline & 3 & 0.15 & -0.06 & 0.04 & 0.14 & 0.06 & 0.13 \\
\hline & 6 & -0.27 & -0.08 & -0.03 & -0.21 & -0.26 & -0.25 \\
\hline \multirow{3}{*}{$\begin{array}{l}\text { Percent of energy } \\
\text { from protein }\end{array}$} & 1 & 0.02 & -0.06 & -0.13 & -0.01 & 0.12 & 0.06 \\
\hline & 3 & -0.01 & -0.18 & -0.16 & 0.05 & -0.42 & -0.06 \\
\hline & 6 & 0.25 & 0.13 & 0.05 & 0.24 & -0.01 & 0.25 \\
\hline \multirow{3}{*}{$\begin{array}{c}\text { Percent of energy } \\
\text { from fat }\end{array}$} & 1 & 0.13 & -0.07 & -0.09 & 0.09 & 0.21 & 0.24 \\
\hline & 3 & 0.01 & 0.16 & 0.14 & -0.02 & 0.22 & 0.05 \\
\hline & 6 & 0.23 & -0.08 & -0.04 & 0.22 & 0.13 & 0.27 \\
\hline \multirow{3}{*}{$\begin{array}{l}\text { Percent of energy } \\
\text { from carbohydrates }\end{array}$} & 1 & -0.13 & 0.09 & 0.15 & -0.08 & -0.24 & -0.24 \\
\hline & 3 & -0.01 & -0.06 & -0.06 & -0.01 & 0.01 & -0.02 \\
\hline & 6 & -0.27 & 0.03 & 0.02 & -0.26 & -0.10 & -0.30 \\
\hline
\end{tabular}


Table 5. Cont.

\begin{tabular}{|c|c|c|c|c|c|c|c|}
\hline \multirow{2}{*}{$\begin{array}{l}\text { Energy and } \\
\text { Nutrients in } \\
\text { Mothers' Diet }\end{array}$} & \multirow{2}{*}{$\begin{array}{l}\text { Month of } \\
\text { Lactation }\end{array}$} & \multicolumn{6}{|c|}{ Composition of Human Milk } \\
\hline & & Energy $^{1}$ & $\begin{array}{c}\text { Total } \\
\text { Protein }^{2}\end{array}$ & $\begin{array}{c}\text { True } \\
\text { Protein }^{2}\end{array}$ & Fat $^{2}$ & Carbohydrates $^{2}$ & $\begin{array}{c}\text { Dry } \\
\text { Matter }^{2}\end{array}$ \\
\hline \multirow{3}{*}{ Sodium (mg) } & 1 & 0.01 & 0.20 & 0.25 & -0.03 & -0.01 & 0.08 \\
\hline & 3 & -0.22 & 0.02 & 0.05 & -0.22 & -0.18 & -0.24 \\
\hline & 6 & -0.17 & 0.33 & 0.40 & -0.18 & 0.01 & -0.19 \\
\hline \multirow{3}{*}{ Potassium (mg) } & 1 & 0.09 & -0.02 & 0.04 & 0.11 & -0.04 & -0.05 \\
\hline & 3 & 0.21 & -0.01 & 0.20 & 0.22 & -0.07 & 0.18 \\
\hline & 6 & -0.37 & -0.03 & 0.04 & -0.34 & -0.48 & -0.35 \\
\hline \multirow{3}{*}{ Calcium (mg) } & 1 & 0.32 & 0.08 & 0.08 & 0.30 & 0.21 & 0.29 \\
\hline & 3 & 0.21 & -0.20 & -0.23 & 0.19 & 0.26 & 0.23 \\
\hline & 6 & 0.11 & -0.12 & -0.18 & 0.20 & -0.24 & 0.13 \\
\hline \multirow{3}{*}{ Phosphorus (mg) } & 1 & 0.12 & -0.03 & -0.03 & 0.13 & -0.01 & 0.09 \\
\hline & 3 & 0.19 & 0.02 & 0.08 & 0.19 & -0.06 & 0.17 \\
\hline & 6 & -0.04 & -0.05 & -0.02 & 0.02 & -0.40 & -0.02 \\
\hline \multirow{3}{*}{ Magnesium (mg) } & 1 & 0.04 & -0.04 & -0.02 & 0.03 & -0.02 & -0.11 \\
\hline & 3 & 0.27 & 0.26 & 0.37 & 0.27 & 0.00 & 0.27 \\
\hline & 6 & -0.34 & 0.01 & 0.06 & -0.37 & -0.32 & -0.33 \\
\hline \multirow{3}{*}{ Iron $(\mathrm{mg})$} & 1 & -0.04 & -0.06 & -0.01 & -0.02 & -0.11 & -0.13 \\
\hline & 3 & 0.26 & 0.24 & 0.43 & 0.26 & -0.01 & 0.24 \\
\hline & 6 & 0.01 & 0.10 & 0.17 & -0.04 & 0.08 & 0.03 \\
\hline \multirow{3}{*}{ Zinc (mg) } & 1 & -0.06 & -0.06 & -0.02 & -0.05 & -0.10 & -0.06 \\
\hline & 3 & 0.26 & 0.24 & 0.43 & 0.26 & -0.01 & 0.24 \\
\hline & 6 & -0.01 & 0.13 & 0.20 & 0.02 & -0.24 & 0.02 \\
\hline \multirow{3}{*}{ Iodine $(\mu g)$} & 1 & 0.10 & 0.20 & 0.18 & 0.08 & 0.01 & -0.03 \\
\hline & 3 & 0.10 & -0.28 & -0.24 & 0.14 & -0.21 & 0.04 \\
\hline & 6 & -0.12 & 0.10 & 0.14 & -0.12 & -0.18 & -0.20 \\
\hline \multirow{3}{*}{ Vitamin A $(\mu g)$} & 1 & -0.17 & -0.01 & 0.05 & -0.23 & -0.07 & -0.21 \\
\hline & 3 & -0.21 & -0.10 & 0.12 & -0.20 & -0.22 & -0.26 \\
\hline & 6 & -0.14 & -0.16 & -0.25 & -0.13 & -0.14 & -0.17 \\
\hline \multirow{3}{*}{ Vitamin D $(\mu g)$} & 1 & -0.09 & -0.09 & -0.08 & -0.15 & -0.09 & -0.14 \\
\hline & 3 & 0.23 & 0.16 & 0.18 & 0.28 & -0.41 & 0.16 \\
\hline & 6 & 0.33 & 0.06 & 0.08 & 0.35 & 0.03 & 0.33 \\
\hline \multirow{3}{*}{ Vitamin E (mg) } & 1 & 0.20 & 0.02 & 0.10 & 0.22 & 0.06 & 0.09 \\
\hline & 3 & 0.19 & 0.06 & 0.27 & 0.20 & -0.02 & 0.17 \\
\hline & 6 & -0.30 & -0.02 & 0.13 & -0.34 & -0.36 & -0.29 \\
\hline \multirow{3}{*}{ Vitamin $\mathrm{B}_{1}(\mathrm{mg})$} & 1 & -0.21 & -0.14 & -0.09 & -0.19 & -0.22 & -0.36 \\
\hline & 3 & 0.12 & 0.19 & 0.30 & 0.14 & -0.21 & 0.08 \\
\hline & 6 & -0.08 & -0.07 & -0.01 & -0.07 & 0.07 & -0.01 \\
\hline \multirow{3}{*}{ Vitamin $B_{2}(\mathrm{mg})$} & 1 & 0.15 & -0.02 & -0.02 & 0.18 & 0.08 & 0.09 \\
\hline & 3 & 0.08 & -0.18 & 0.02 & 0.10 & -0.10 & 0.05 \\
\hline & 6 & 0.17 & -0.01 & -0.02 & 0.21 & -0.04 & 0.20 \\
\hline \multirow{3}{*}{ Vitamin PP (mg) } & 1 & -0.20 & -0.18 & -0.15 & -0.18 & -0.30 & -0.24 \\
\hline & 3 & 0.14 & -0.02 & 0.10 & 0.18 & -0.25 & 0.09 \\
\hline & 6 & -0.27 & 0.02 & 0.05 & -0.30 & -0.08 & -0.19 \\
\hline & 1 & -0.08 & -0.10 & -0.05 & -0.07 & -0.14 & -0.24 \\
\hline Vitamin $\mathrm{B}_{6}(\mathrm{mg})$ & 3 & 0.10 & 0.02 & 0.21 & 0.13 & -0.26 & 0.04 \\
\hline & 6 & -0.06 & -0.00 & -0.07 & -0.09 & 0.16 & -0.07 \\
\hline & 1 & -0.01 & 0.03 & 0.09 & 0.02 & -0.02 & -0.39 \\
\hline Vitamin C (mg) & 3 & 0.08 & 0.02 & 0.27 & 0.11 & -0.22 & 0.03 \\
\hline & 6 & -0.19 & -0.36 & -0.23 & -0.11 & -0.18 & -0.25 \\
\hline & 1 & 0.21 & 0.08 & 0.03 & 0.15 & 0.06 & 0.02 \\
\hline Vitamin $B_{12}(\mu \mathrm{g})$ & 3 & 0.10 & -0.01 & 0.16 & 0.13 & -0.29 & 0.02 \\
\hline & 6 & 0.14 & -0.09 & -0.08 & 0.18 & -0.29 & 0.15 \\
\hline & 1 & 0.11 & -0.02 & 0.04 & 0.16 & -0.05 & -0.06 \\
\hline Folic acid $(\mu \mathrm{g})$ & 3 & 0.19 & 0.02 & 0.30 & 0.21 & -0.24 & 0.12 \\
\hline & 6 & -0.36 & -0.07 & -0.12 & -0.40 & 0.02 & -0.41 \\
\hline
\end{tabular}

1 Energy is presented as kilocalories (kcal) per $100 \mathrm{~mL} .{ }^{2}$ Macronutrients and dry matter are presented as grams per $100 \mathrm{~mL}$. Data are presented as Pearson's r coefficients. 


\subsection{Association between Maternal Body Composition and Milk Composition}

Table 6 presents the correlation coefficient (Pearson's $r$ ) between human milk composition and lactating mothers' body composition at three time points (first, third, and sixth month of lactation).

For women in the third month postpartum, we observed moderate to strong significant correlations ( $r$ ranged from 0.45 to 0.52 ) between the true protein content in their milk and the majority of body composition measures. In the third month of lactation, the total protein in milk correlated positively with the mothers' weight $(r=0.63 ; p=0.002)$, BMI $(r=0.59 ; p=0.004), \%$ fat mass $(r=0.60 ; p=0.003)$, fat-free mass expressed in $\mathrm{kg}(r=0.63 ; p=0.001)$, and muscle mass $(r=0.47 ; p=0.027)$ and negatively with percentage of total body water $(r=-0.60 ; p=0.003)$.

The variance in milk fat content was related to the lactating women's weight, with a significant positive correlation in the sixth month postpartum $(r=0.49 ; p=0.039)$, with BMI, and a significant positive correlation in the first month postpartum $(r=0.33 ; p=0.048)$.

Similar to the protein concentration, the energy value of human milk was highly correlated with the maternal body composition. In the third month postpartum, we found positive correlations with weight $(r=0.43 ; p=0.048)$, BMI $(r=0.39 ; p=0.049)$, and muscle mass $(r=0.44 ; p=0.041)$. There was a negative correlation with $\%$ total body water $(r=-0.60 ; p=0.032)$ in the third month of lactation.

We did not observe significant correlations between the carbohydrate content in human milk and measures of the body composition of lactating women, except for a positive correlation with the percentage of intracellular water and a negative correlation with the percentage of extracellular water in the third month postpartum.

Table 6. Correlations between human milk composition and mother's body composition.

\begin{tabular}{|c|c|c|c|c|c|c|c|}
\hline \multirow{2}{*}{$\begin{array}{l}\text { Mothers' Body } \\
\text { Composition }\end{array}$} & \multirow{2}{*}{$\begin{array}{l}\text { Month of } \\
\text { Lactation }\end{array}$} & \multicolumn{6}{|c|}{ Composition of Human Milk } \\
\hline & & Energy 1 & $\begin{array}{c}\text { Total } \\
\text { Protein }\end{array}$ & $\begin{array}{c}\text { True } \\
\text { Protein } 2\end{array}$ & Fat $^{2}$ & Carbohydrates $^{2}$ & $\begin{array}{c}\text { Dry } \\
\text { Matter }^{2}\end{array}$ \\
\hline \multirow{3}{*}{ Weight (kg) } & 1 & 0.32 * & 0.21 & 0.18 & 0.30 & 0.21 & $0.52 *$ \\
\hline & 3 & 0.43 * & 0.63 * & 0.51 * & 0.37 & 0.30 & $0.49 *$ \\
\hline & 6 & 0.41 & -0.14 & -0.21 & $0.49 *$ & 0.30 & 0.42 \\
\hline \multirow{3}{*}{$\begin{array}{l}\text { Body mass index } \\
\qquad\left(\mathrm{kg} / \mathrm{m}^{2}\right)\end{array}$} & 1 & $0.33 *$ & 0.27 & 0.24 & $0.33 *$ & 0.20 & $0.48 *$ \\
\hline & 3 & 0.39 * & 0.59 * & $0.45^{*}$ & 0.35 & 0.24 & 0.45 * \\
\hline & 6 & 0.44 & 0.01 & -0.05 & 0.52 & 0.34 & 0.45 \\
\hline \multirow{3}{*}{ Fat mass (kg) } & 1 & 0.17 & 0.19 & 0.14 & 0.15 & 0.14 & 0.33 * \\
\hline & 3 & 0.42 & 0.64 * & 0.51 * & 0.36 & 0.29 & 0.48 * \\
\hline & 6 & 0.43 & -0.10 & -0.12 & 0.51 & 0.24 & 0.43 \\
\hline \multirow{3}{*}{ Fat mass $(\%)$} & 1 & 0.32 * & 0.26 & 0.23 & 0.29 & 0.17 & $0.46^{*}$ \\
\hline & 3 & 0.39 & 0.60 * & $0.47^{*}$ & 0.33 & 0.32 & $0.46^{*}$ \\
\hline & 6 & 0.42 & 0.01 & -0.01 & 0.48 & 0.22 & 0.41 \\
\hline \multirow{3}{*}{ Fat free mass $(\mathrm{kg})$} & 1 & 0.24 & 0.10 & 0.06 & 0.22 & 0.25 & $0.52 *$ \\
\hline & 3 & 0.37 & 0.50 * & 0.42 & 0.32 & 0.29 & $0.43 *$ \\
\hline & 6 & 0.15 & -0.34 & -0.38 & 0.22 & 0.06 & 0.17 \\
\hline \multirow{3}{*}{$\begin{array}{c}\text { Total body water } \\
\text { (L) }\end{array}$} & 1 & 0.27 & 0.19 & 0.15 & 0.27 & 0.10 & 0.49 * \\
\hline & 3 & 0.35 & 0.54 * & 0.42 & 0.30 & 0.27 & 0.41 \\
\hline & 6 & 0.25 & -0.33 & -0.38 & 0.31 & 0.13 & 0.26 \\
\hline \multirow{3}{*}{$\begin{array}{c}\text { Total body water } \\
(\%)\end{array}$} & 1 & -0.27 & -0.20 & -0.20 & -0.23 & -0.22 & $-0.41 *$ \\
\hline & 3 & $-0.46^{*}$ & $-0.60 *$ & $-0.52 *$ & -0.40 & -0.35 & -0.52 * \\
\hline & 6 & -0.38 & -0.06 & -0.06 & -0.44 & -0.17 & -0.37 \\
\hline \multirow{3}{*}{ Protein (kg) } & 1 & -0.09 & -0.10 & -0.07 & -0.14 & 0.12 & -0.01 \\
\hline & 3 & 0.16 & 0.05 & 0.11 & 0.14 & 0.14 & 0.15 \\
\hline & 6 & -0.22 & -0.21 & -0.19 & -0.19 & -0.21 & -0.19 \\
\hline \multirow{3}{*}{ Muscles (kg) } & 1 & 0.26 & 0.10 & 0.04 & 0.22 & 0.28 & 0.53 * \\
\hline & 3 & 0.44 * & $0.47^{*}$ & 0.40 & 0.39 & 0.37 & 0.50 * \\
\hline & 6 & 0.03 & -0.10 & -0.12 & 0.08 & 0.10 & 0.13 \\
\hline
\end{tabular}

${ }^{1}$ Energy is presented as kilocalories (kcal) per $100 \mathrm{~mL} .{ }^{2}$ Macronutrients and dry matter are presented as grams per $100 \mathrm{~mL}$. Data are presented as Pearson's r coefficients. ${ }^{*} p<0.05$. 


\section{Discussion}

In our study, we found that protein (total and true) and carbohydrate concentrations in human milk were significantly different, depending on the period of lactation. From the first to the sixth month of lactation, total and true protein concentrations significantly decreased. We did not find any relationships between the nutritional value of maternal daily food consumption and milk composition. Maternal BMI and adiposity were positively associated with the protein content of milk.

The total milk protein content in our study was high compared to mature milk from Chinese (0.9 g/100 mL [19]), Brazilian (1.1 g/100 mL [22]), and Australian mothers (1.0 g/100 mL [23]). The analysis of carbohydrates by HMA MIRIS in human milk is affected by the presence of lactose and nonlactose carbohydrates, primarily human milk oligosaccharides (HMOs) [27]. Some of the divergence between the findings for lactose concentrations may be related to the inclusion of HMOs in the mid-infrared (mid-IR) transmission spectroscopy measurements [28]. Since the reference laboratory analysis for lactose concentration, high-pressure liquid chromatography (HPLC), does not measure HMOs, it is probable that lactose levels measured by mid-infrared transmission spectroscopy were a result of absorbing terminal or core lactose moieties of HMOs [30]. The measured concentration of carbohydrates in our study (7.0-7.1 g/100 mL) was consistent with the normal range in human milk [31]; however, it has been reported that lactose concentration can vary from 6.3 to $8.1 \mathrm{~g} / 100 \mathrm{~mL}$ [32]. The reasons for this variability may relate to the time point in the breastfeeding session (pre- or postfeeding), the time of feeding, feeding frequency, or the milk analysis method [33]. Fat is known to be the most variable macronutrient in human milk. In the first month postpartum, the median fat concentration in milk was $3.5 \mathrm{~g} / 100 \mathrm{~mL}$. Our finding was consistent with those in Japan (3.6 g/100 mL) [19], China (3.4 g/100 mL) [34], and the United States $(3.6 \mathrm{~g} / 100 \mathrm{~mL})$ [35].

It is reported that sampling protocols are of prime importance when investigating the association between human milk composition and maternal factors. The direct relationship between the dietary intake of single nutrients and their presence within human milk is hard to study for many reasons. These include difficulties in collecting nutrition data and the availability of reliable human milk samples. In the present study, the procedure of milk collection (using a 24-h period) was performed to minimize errors. We also confirmed a systemic change between fore- and hindmilk samples for concentrations of energy and macronutrients. Additionally, all of the milk samples were from mothers practicing exclusive breastfeeding.

We found no evidence for associations between the maternal intake of any dietary nutrients and the milk composition in this sample. This is consistent with past studies, which showed that milk nutrient composition appears to be mainly independent of the nutritional value of maternal daily food consumption [36]. This absence of an effect of maternal diet is evident in both observational and experimental studies, in which nutritional supplements were shown to result in minimal or trifling changes to milk macronutrient content $[21,35]$. Some compensatory physiological mechanisms might be responsible for the comparatively stable milk macronutrient composition related to the nutritional variations of maternal diet [33]. Tigas et al. [37] reported that increased glucose demands during lactation are met by increased glucose production as a result of increased glycogenolysis, but not gluconeogenesis, or by an increased use of free fatty acids. These results are consistent with the hypothesis that human milk composition might be buffered against variations in the maternal dietary intake of each component [15]. For instance, Rakicioğlu et al. [38] found that short-term fasting or dieting by lactating women has not been associated with milk composition, despite the fact that the nutritional status of lactating women was affected by Ramadan fasting, when all macronutrient intake decreased.

The concentration of lactose in human milk is known to be the least variable of the macronutrients [32]. No significant relationships were found between milk lactose and a maternal diet high in fat and low in carbohydrates, compared with a diet low in fat and high in carbohydrates [39]. Additionally, there were no significant differences between milk lactose and a high-protein diet [40], or between vegetarian and non-vegetarian diets [41]. Considering the total protein concentration, studies from Europe and the 
United States did not report any relationship between milk total protein and maternal intake of animal and vegetable protein [42]. The variation of total fat concentration in human milk also appears to be independent of maternal diet [30]. Nevertheless, the specific fatty acids that form the total lipid fraction are sensitive to maternal nutrition. These fatty acids are either taken up from the maternal plasma, or synthetized endogenously by the mammary glands. Both of these sources are influenced by maternal diet composition [37,43-45].

While there is a lack of a relationship between milk composition and maternal nutrition, we observed that concentrations of several nutrients in milk were correlated with maternal body composition, depending on the postpartum period.

Previous studies based on maternal BMI reported a positive relationship with fat concentration in human milk $[13,19,45]$, which is consistent with our results. We found that in the first month postpartum, maternal BMI was correlated with milk fat content $(0.33 ; p=0.048)$. Chang et al. [13] reported that the mother's current BMI was positively correlated with lipid levels at 1-2 weeks (0.151; $p<0.05), 2-3$ months $(0.151 ; p<0.05)$, and $7-8$ months $(0.153 ; p<0.05)$. Contrary to these findings, Bachour et al. [46] suggested that there were no associations between maternal body mass index and fat concentration in human milk. Interestingly, Quinn et al. [21] observed that women in Cebu with lower BMI tended to produce milk with higher fat contents than women with higher BMI. The significant inverse association between milk fat and BMI suggests that the sample BMI was indexing lean mass. In our study, we found no evidence for an association between maternal body composition and fat concentration in milk at any time point. Although past research has at times reported a relationship between maternal fat mass and milk fat content, these associations were often limited to overweight or obese women $[47,48]$. It has been also suggested that increasing maternal adiposity may be related to impaired milk sugar synthesis, and that the lipid increase reflects this decrease in lactose [49].

A few studies investigating the associations between human milk protein concentration and maternal nutritional status are contradictory, with some reporting a positive relationship between protein and maternal adiposity as assessed by BMI $[13,18,45,50]$ and one a negative association between total protein content and maternal BMI [21]. However, it must be stressed that BMI is not a direct measure of adiposity, so that the strength of the relationship between protein concentration and BMI may not reflect the true value of these associations. Kugananthan et al. [51] and Quinn et al. [52] observed that a higher maternal fat mass percentage, but not BMI, was associated with higher protein concentrations in milk. Using advanced techniques to evaluate maternal body composition, we found that maternal body composition was highly correlated with total protein concentration in milk. We reported a positive correlation with maternal weight $(p=0.002)$, BMI $(p=0.004), \%$ fat mass $(p=0.003)$, and muscles $(p=0.027)$, and a negative correlation with $\%$ total body water $(p=0.003)$ in the third month of lactation. A decreased total body water content is characteristic for women with more adipose tissue, explaining that negative correlation. It is also reported that several serum amino acid concentrations, in particular branched-chain amino acids (BCAAs), are increased in mothers with more adipose tissue [53], leading to more amino acids transferred to the breast and milk [54]. This may explain the positive relationship between maternal adiposity and milk protein concentration [18].

The concentration of carbohydrates in human milk is the least variable of the macronutrients. Considering that a stable concentration of lactose is important for maintaining a constant osmotic pressure in milk [55], maternal nutritional status is not expected to have a meaningful impact on total carbohydrate concentrations in milk. In our study, the measured concentrations of carbohydrates were not related to maternal BMI and body composition at any time point. This is in line with a previous study carried out by Kugananthan et al. [51], which showed that that the lactose concentration in human milk (measured by enzymatic spectrophotometric method) was not related to maternal adiposity profiles (BMI, $p=0.66$; \% fat mass, $p=0.48$ ). By contrast, Chang et al. [13] reported that maternal BMI was negatively correlated with lactose concentrations at $4-5$ months $(0.148 ; p<0.05)$ and 6-7 months $(0.242 ; p<0.01)$ postpartum. 
The strengths of this study are the use of advanced techniques to assess maternal body composition and the milk collection protocol, which allowed possible errors in human milk composition to be minimized. The limitations of this study are convenience sampling, the modest number of participants, mainly at six months postpartum, resulting from discontinuation of breastfeeding, and the constraints associated with multiple measurement time points. BIA analysis incorporates various assumptions, and it may also result in less precise estimates, mainly in situations in which the water-electrolyte balance is altered. If used to monitor individuals over time, it can indicate the direction, but not the magnitude of changes in lean mass. Further, our population was Caucasian, with university educations and a high socioeconomic status. All indicated limitations decreased the representativity of the study, and caution should be used when extrapolating the results.

Considering that human milk provides not only energy and nutrients, but also bioactive factors that are crucial for infant growth and development, human milk composition research should continue in order to identify factors that may be associated with changes in its composition. All of these efforts may contribute to accomplishing optimum growth, development, and health in infants.

Author Contributions: Conceptualization, A.B.-J., A.C.-S., G.O., D.S.-W., H.W., and A.W.; Data curation, A.B.-J.; Formal analysis, A.B.-J. and A.W.; Funding acquisition, A.C.-S. and A.W.; Investigation, A.B.-J.; Methodology, A.B.-J., D.S.-W., and A.W.; Project administration, A.B.-J., A.C.-S., and A.W.; Resources, D.S.-W. and A.W.; Supervision, A.C.-S., G.O., D.S.-W., and A.W.; Writing—original draft, A.B.-J.; Writing—review and editing, A.B.-J., A.C.-S., G.O., D.S.-W., H.W., and A.W.

Funding: This research was funded by a Polish Society of Clinical Child Nutrition research grant obtained in 2015 for the project titled "Association between human milk composition and the nutritional status and body composition of lactating women".

Acknowledgments: All authors read and approved the final manuscript. This study was supported by a Polish Society of Clinical Child Nutrition research grant obtained in 2015 for the project entitled "Association between human milk composition and the nutritional status and body composition of lactating women". Many thanks are extended to all of the mothers from the Holy Hospital in Warsaw who participated in this study.

Conflicts of Interest: The authors declare no conflict of interest.

\section{References}

1. Lonnerdal, B. Breast milk: A truly functional food. Nutrition 2000, 16, 509-511. [CrossRef]

2. Hassiotou, F.; Filgueira, L.; Trengove, N.; Lai, C.T.; Hartmann, P.E. Breastmilk contains primitive stem cells from lactating breast with multi-lineage differentiation potential. FASEB J. 2012, 26, 913.

3. Eidelman, A.I.; Schanler, R.J.; Johnston, M.; Landers, S.; Noble, L.; Szucs, K.; Viehmann, L. Breastfeeding and the use of human milk. Paediatrics 2012, 129, 827-841.

4. Kramer, M.S.; Kakuma, R. Optimal duration of exclusive breastfeeding. Cochrane Database Syst. Rev. 2012, 15, 8. [CrossRef]

5. Owen, C.G.; Martin, R.M.; Whincup, P.H.; Smith, G.D.; Cook, D.G. Effect of infant feeding on the risk of obesity across the life course: A quantitative review of published evidence. Paediatrics 2005, 115, 1367-1377. [CrossRef] [PubMed]

6. Victora, C.G.; Bahl, R.; Barros, A.J.; Franca, G.V.; Horton, S.; Krasevec, J.; Murch, S.; Sankar, M.J.; Walker, N.; Rollins, N.C. Breastfeeding in the 21st century: Epidemiology, mechanisms, and lifelong effect. Lancet 2016, 387, 475-490. [CrossRef]

7. Owen, C.G.; Martin, R.M.; Whincup, P.H.; Smith, G.D.; Cook, D.G. Does breastfeeding influence risk of type 2 diabetes in later life? A quantitative analysis of published evidence. Am. J. Clin. Nutr. 2006, 84, 1043-1054. [CrossRef] [PubMed]

8. Horta, B.L.; Loret de Mola, C.; Victora, C.G. Long-term consequences of breastfeeding on cholesterol, obesity, systolic blood pressure and type 2 diabetes: A systematic review and meta-analysis. Acta Paediatr. 2015, 104, 30-37. [CrossRef] [PubMed]

9. Buyken, A.E.; Karaolis-Danckert, N.; Remer, T.; Bolzenius, K.; Landsberg, B.; Kroke, A. Effects of breastfeeding on trajectories of body fat and BMI throughout childhood. Obesity 2008, 16, 389-395. [CrossRef] [PubMed] 
10. Beyerlein, A.; von Kries, R. Breastfeeding and body composition in children: Will there ever be conclusive empirical evidence for a protective effect against overweight? Am. J. Clin. Nutr. 2011, 94, 1772-1775. [CrossRef] [PubMed]

11. Andreas, N.J.; Kampmann, B.; Mehring Le Doare, K. Human breast milk: A review on its composition and bioactivity. Early Hum. Dev. 2015, 91, 629-635. [CrossRef] [PubMed]

12. Kent, J.C.; Mitoulas, L.R.; Cregan, M.D.; Ramsay, D.T.; Doherty, D.A.; Hartmann, P.E. Volume and frequency of breastfeedings and fat content of breast milk throughout the day. Paediatrics 2006, 117, 387-395. [CrossRef] [PubMed]

13. Chang, N.; Jung, J.A.; Kim, H.; Jo, A.; Kang, S.; Lee, S.W.; Yi, H.; Kim, J.; Yim, J.G.; Jung, B.M. Macronutrient composition of human milk from Korean mothers of full term infants born at 37-42 gestational weeks. Nutr. Res. Pract. 2015, 9, 433-438. [CrossRef] [PubMed]

14. Charpak, N.; Ruiz, J.G. Breast milk composition in a cohort of pre-term infants' mothers followed in an ambulatory programme in Colombia. Acta Paediatr. 2007, 96, 1755-1759. [CrossRef] [PubMed]

15. Mitoulas, L.R.; Kent, J.C.; Cox, D.B.; Owens, R.A.; Sherriff, J.L.; Hartmann, P.E. Variation in fat, lactose and protein in human milk over $24 \mathrm{~h}$ and throughout the first year of lactation. Br. J. Nutr. 2002, 88, 29-37. [CrossRef] [PubMed]

16. Lee, J.S. Changes in specific gravity, total solid and protein contents of human milk during the course of lactation in Korean women. Korean J. Nutr. 1988, 21, 129-133.

17. Lee, S.G.; Chung, T.H. Lipid content of breast milk in Korean women. J. Korean Pediatr. Soc. 1985, 28, 977-988.

18. Nommsen, L.A.; Lovelady, C.A.; Heinig, M.J.; Lönnerdal, B.; Dewey, K.G. Determinants of energy, protein, lipid, and lactose concentrations in human milk during the first 12 mo of lactation: The DARLING Study. Am. J. Clin. Nutr. 1991, 53, 457-465. [CrossRef] [PubMed]

19. Yang, T.; Zhang, Y.; Ning, Y.; You, L.; Ma, D.; Zheng, Y.; Yang, X.; Li, W.; Wang, J.; Wang, P. Breast milk macronutrient composition and the associated factors in urban Chinese mothers. Chin. Med. J. 2014, 127, 1721-1725. [PubMed]

20. Quinn, E.A.; Diki Bista, K.; Childs, G. Milk at Altitude: Human Milk Macronutrient Composition in a High-Altitude Adapted Population of Tibetans. Am. J. Phys. Anthropol. 2016, 159, 233-243. [CrossRef] [PubMed]

21. Quinn, E.A.; Largado, F.; Power, M.; Kuzawa, C.W. Predictors of breast milk macronutrient composition in Filipino mothers. Am. J. Hum. Biol. 2012, 24, 533-540. [CrossRef] [PubMed]

22. Abranches, A.D.; Soares, F.V.; Junior, S.C.; Moreira, M.E. Freezing and thawing effects on fat, protein, and lactose levels of human natural milk administered by gavage and continuous infusion. J. Pediatr. 2014, 90, 384-388. [CrossRef] [PubMed]

23. Khan, S.; Prime, D.K.; Hepworth, A.R.; Tat Lai, Ch.; Trengove, N.J.; Hartmann, P.E. Investigation of short-term variations in term breast milk composition during repeated breast expression sessions. J. Hum. Lact. 2013, 29, 196-204. [CrossRef] [PubMed]

24. Euro Who. Available online: http:/ / www.euro.who.int/en/health-topics/disease-prevention/nutrition/ahealthy-lifestyle/body-mass-index-bmi (accessed on 16 August 2018).

25. Maltron International. Maltron Bioscan 920-2 Operating and Service Manual. 1999, pp. 8-30. Available online: http:/ / www.bmgrp.at/fileadmin/user_upload/images/downloads/BioScan_920-II_new.pdf (accessed on 12 July 2018).

26. Heyward, V.H.; Stolarczyk, L.M. Applied Body Composition Assessment; Human Kinetics Publishers: Champaign, IL, USA, 1996; pp. 1-215.

27. Groh-Wargo, S.; Valentic, J.; Khaira, S.; Super, D.M.; Collin, M. Human Milk Analysis Using Mid-Infrared Spectroscopy. Nutr. Clin. Pract. 2016, 31, 266-272. [CrossRef] [PubMed]

28. Smilowitz, J.T.; Gho, D.S.; Mirmiran, M.; German, J.B.; Underwood, M.A. Rapid Measurement of Human Milk Macronutrients in the Neonatal Intensive Care Unit: Accuracy and Precision of Fourier Transform Mid-Infrared Spectroscopy. J. Hum. Lact. 2014, 30, 180-189. [CrossRef] [PubMed]

29. Szponar, L.; Wolnicka, K.; Rychlik, E. Album of Photographs of Food Products and Dishes; National Food and Nutrition Institute: Warsaw, Poland, 2011.

30. Casadio, Y.S.; Williams, T.M.; Lai, Ch.T.; Olsson, S.E.; Hepworth, A.R.; Ed, G.D.; Hartmann, P.E. Evaluation of a Mid-Infrared Analyzer for the Determination of the Macronutrient Composition of Human Milk. J. Hum. Lact. 2010, 26, 376-383. [CrossRef] [PubMed] 
31. Ballard, O.; Morrow, A.L. Human milk compostion: Nutrients and bioactive factors. Pediatr. Clin. N. Am. 2013, 60, 49-74. [CrossRef] [PubMed]

32. Miller, E.M.; Aiello, M.O.; Fujita, M.; Hinde, K.; Milligan, L.; Quinn, E.A. Field and laboratory methods in human milk research. Am. J. Hum. Biol. 2013, 25, 1-11. [CrossRef] [PubMed]

33. Keikha, M.; Bahreynian, M.; Saleki, M.; Kelishadi, R. Macro- and micronutrients of human milk composition: Are they related to maternal diet? A comprehensive systematic review. Breastfeed. Med. 2017, 12, 517-527. [CrossRef] [PubMed]

34. Fomon, S.J. Requirements and recommended dietary intakes of protein during infancy. Pediatr. Res. 1991, 30, 391-395. [CrossRef] [PubMed]

35. Prentice, A.M.; Prentice, A; Lamb, W.H.; Lunn, P.G.; Austin, S. Metabolic consequences of fasting during Ramadan in pregnant and lactating women. Hum. Nutr. Clin. Nutr. 1983, 37, 283-294. [PubMed]

36. Bravi, F.; Wiens, F.; Decarli, A.; Dal Pont, A.; Agostoni, C.; Ferraroni, M. Impact of maternal nutrition on breast-milk composition: A systematic review. Am. J. Clin. Nutr. 2016, 104, 646-662. [CrossRef] [PubMed]

37. Tigas, S.; Sunehag, A.; Haymond, M.W. Metabolic adaptation to feeding and fasting during lactation in humans. J. Clin. Endocrinol. Metab. 2002, 87, 302-307. [CrossRef] [PubMed]

38. Rakicioğlu, N.; Samur, G.; Topcu, A.; Topcu, A.A. The effect of Ramadan on maternal nutrition and composition of breast milk. Pediatr. Int. 2006, 48, 278-283. [CrossRef] [PubMed]

39. Mohammad, M.A.; Sunehag, A.L.; Haymond, M.W. Effect of dietary macronutrient composition under moderate hypocaloric intake on maternal adaptation during lactation. Am. J. Clin. Nutr. 2009, 89, 1821-1827. [CrossRef] [PubMed]

40. Forsum, E.; Lonnerdal, B. Effect of protein intake on protein and nitrogen composition of breast milk. Am. J. Clin. Nutr. 1980, 33, 1809-1813. [CrossRef] [PubMed]

41. Finley, D.A.; Lonnerdal, B.; Dewey, K.G.; Grivetti, L.E. Inorganic constituents of breast milk from vegetarian and nonvegetarian women: Relationships with each other and with organic constituents. J. Nutr. 1985, 115, 772-781. [CrossRef] [PubMed]

42. Boniglia, C.; Carratu, B.; Chiarotti, F.; Giammarioli, S.; Sanzini, E. Influence of maternal protein intake on nitrogen fractions of human milk. Int. J. Vitam. Nutr. Res. 2003, 73, 447-452. [CrossRef] [PubMed]

43. Innis, S.M. Human milk and formula fatty acids. J. Pediatr. 1992, 120, 56-61. [CrossRef]

44. Innis, S.M. Impact of maternal diet on human milk composition and neurological development of infants. Am. J. Clin. Nutr. 2014, 99, 734-741. [CrossRef] [PubMed]

45. Prentice, A.M.; Goldberg, G.R.; Prentice, A. Body mass index and lactation performance. Eur. J. Clin. Nutr. 1994, 48, 78-86.

46. Bachour, P.; Yafawi, R.; Jaber, F.; Choueiri, E.; Abdel-Razzak, Z. Effects of smoking, mother's age, body mass index and parity number on lipid, protein and secretory Immunoglobulin A concentrations of human milk. Breastfeed. Med. 2012, 7, 179-188. [CrossRef] [PubMed]

47. Barbosa, L.; Butte, N.F.; Villalpando, S.; Wong, W.W.; Smith, E.O. Maternal energy balance and lactation performance of Mesoamerindians as a function of body mass index. Am. J. Clin. Nutr. 1997, 66, 575-583. [CrossRef] [PubMed]

48. Villalpando, S.; del Prado, M. Interrelation among dietary energy and fat intakes, maternal body fatness, and milk total lipid in humans. J. Mammary Gland Biol. Neoplasia 1999, 4, 285-295. [CrossRef] [PubMed]

49. Rolls, B.A.; Gurr, M.I.; van Duijvenvoorde, P.M.; Rolls, B.J.; Rowe, E.A. Lactation in lean and obese rats: Effect of cafeteria feeding and of dietary obesity on milk composition. Physiol. Behav. 1986, 38, 185-190. [CrossRef]

50. Grote, V.; Verduci, E.; Scaglioni, S.; Vecchi, F.; Contarini, G.; Giovannini, M.; Koletzko, B.; Agostoni, C. Breast milk composition and infant nutrient intakes during the first 12 months of life. Eur. J. Clin. Nutr. 2016, 70, 250-256. [CrossRef] [PubMed]

51. Kugananthan, S.; Gridneva, Z.; Lai, Ch.T.; Hepworth, A.R.; Mark, P.J.; Kakulas, F.; Geddes, D.T. Associations between Maternal Body Composition and Appetite Hormones and Macronutrients in Human Milk. Nutrients 2017, 9, 252. [CrossRef] [PubMed]

52. Quinn, E.A.; Largado, F.; Borja, J.B.; Kuzawa, C.W. Maternal characteristics associated with milk leptin content in a sample of filipino women and associations with infant weight for age. J. Hum. Lact. 2015, 31, 273-281. [CrossRef] [PubMed] 
53. Xie, G.; Ma, X.; Zhao, A.; Wang, C.; Zhang, Y.; Nieman, D.; Nicholson, J.K.; Jia, W.; Bao, Y.; Jia, W. The metabolite profiles of the obese population are gender-dependent. J. Proteome Res. 2014, 13, 4062-4073. [CrossRef] [PubMed]

54. De Luca, A.; Hankard, R.; Alexandre-Gouabau, M.C.; Ferchaud-Roucher, V.; Darmaun, D.; Boquien, C.Y. Higher concentrations of branched-chain amino acids in breast milk of obese mothers. Nutrition 2016, 32, 1295-1298. [CrossRef] [PubMed]

55. Martin, C.R.; Ling, P.R.; Blackburn, G.L. Review of infant feeding: Key features of breast milk and infant formula. Nutrients 2016, 8, 279. [CrossRef] [PubMed]

(C) 2018 by the authors. Licensee MDPI, Basel, Switzerland. This article is an open access article distributed under the terms and conditions of the Creative Commons Attribution (CC BY) license (http:/ / creativecommons.org/licenses/by/4.0/). 
Article

\title{
Satiety Factors Oleoylethanolamide, Stearoylethanolamide, and Palmitoylethanolamide in Mother's Milk Are Strongly Associated with Infant Weight at Four Months of Age-Data from the Odense Child Cohort
}

\author{
Signe Bruun ${ }^{1,2,3, *}$, Sandra Gouveia-Figueira ${ }^{4}$, Magnus Domellöf ${ }^{5}$, Steffen Husby ${ }^{2,3}$, \\ Lotte Neergaard Jacobsen ${ }^{1}$, Kim F. Michaelsen ${ }^{6}$, Christopher J. Fowler ${ }^{7}$ and \\ Gitte Zachariassen 2,3 \\ 1 Strategic Business Unit Pediatric, Arla Foods Ingredients Group P/S, DK-8260 Viby J, Denmark; \\ lotte.neergaard.jacobsen@arlafoods.com \\ 2 Hans Christian Andersen Children's Hospital, Odense University Hospital, DK-5000 Odense C, Denmark; \\ steffen.husby@rsyd.dk (S.H.); gitte.zachariassen@rsyd.dk (G.Z.) \\ 3 Department of Clinical Research, Faculty of Health Sciences, University of Southern Denmark, \\ DK-5000 Odense C, Denmark \\ 4 Clinical Chemistry, County Council of Västerbotten, SE-901 85 Umeå, Sweden; sandra.gouveia@vll.se \\ 5 Department of Clinical Sciences, Pediatrics, Umeå University, SE-901 87 Umeå, Sweden; \\ magnus.domellof@umu.se \\ 6 Department of Nutrition, Exercise and Sports, Faculty of Science, University of Copenhagen, \\ DK-1958 Frederiksberg C, Denmark; kfm@nexs.ku.dk \\ 7 Department of Pharmacology and Clinical Neuroscience, Umeå University, SE-901 87 Umeå, Sweden; \\ christopher.fowler@umu.se \\ * Correspondence: signe.bruun@rsyd.dk; Tel.: +45-22-55-74-88
}

Received: 20 September 2018; Accepted: 6 November 2018; Published: 13 November 2018

\begin{abstract}
Regulation of appetite and food intake is partly regulated by $\mathrm{N}$-acylethanolamine lipids oleoylethanolamide (OEA), stearoylethanolamide (SEA), and palmitoylethanolamide (PEA), which induce satiety through endogenous formation in the small intestine upon feeding, but also when orally or systemic administered. OEA, SEA, and PEA are present in human milk, and we hypothesized that the content of OEA, SEA, and PEA in mother's milk differed for infants being heavy (high weight-for-age Z-score (WAZ)) or light (low WAZ) at time of milk sample collection. Ultra-high performance liquid chromatography-mass spectrometry was used to determine the concentration of OEA, SEA, and PEA in milk samples collected four months postpartum from mothers to high $(n=50)$ or low $(n=50)$ WAZ infants. Associations between OEA, SEA, and PEA concentration and infant anthropometry at four months of age as well as growth from birth were investigated using linear and logistic regression analyses, adjusted for birth weight, early infant formula supplementation, and maternal pre-pregnancy body mass index. Mean OEA, SEA, and PEA concentrations were lower in the high compared to the low WAZ group (all $p<0.02$ ), and a higher concentration of SEA was associated with lower anthropometric measures, e.g., triceps skinfold thickness $(\mathrm{mm})(\beta=-2.235,95 \% \mathrm{CI}=-4.04,-0.43, p=0.016)$, and weight gain per day since birth (g) $(\beta=-8.169,95 \% \mathrm{CI}=-15.26,-1.08, p=0.024)$. This raises the possibility, that the content of satiety factors OEA, SEA, and PEA in human milk may affect infant growth.
\end{abstract}

Keywords: infant growth; appetite regulation; $N$-acylethanolamines; OEA; SEA; PEA; breastfeeding; human milk composition; obesity 


\section{Introduction}

Globally, the prevalence of obesity has tripled since 1975 including a dramatic increase in the prevalence of overweight and obesity in children. In 2016, 41 million children under the age of 5 years, and more than 340 million children and adolescents aged 5-19 years were overweight or obese [1]. It is pronounced and acknowledged that rapid or excess weight gain, most often defined as a change in weight-for-age Z-score (WAZ) of $\geq 0.67$ [2,3], during the first two years of life is associated with a higher risk of being overweight or obese in later childhood [4], and that the association is even more pronounced for rapid weight gain during the first year of life [5]. Furthermore, childhood overweight and obesity track into adolescence and adulthood [6], resulting in an increased risk of noncommunicable diseases (e.g., cardiovascular diseases, diabetes, cancer) and premature death [1]. Increased intake of energy-dense foods (including a transition to a higher n- $6 / n-3$ ratio of the dietary polyunsaturated fatty acids intake [7]) and decreased levels of physical activity are major elements in the ongoing obesity epidemic.

In parallel, research and knowledge on appetite regulation has increased in the past decade. Appetite regulation is multifactorial, but—extremely simplified—appetite and energy homeostasis are regulated via stimulatory (orexigenic) or inhibitory (anorexigenic) signaling pathways, which are active in the central nervous system (CNS) in concert with the gastrointestinal system, adipose tissue (leptin, adiponectin), and the pancreas [8,9].

The regulation of appetite and food intake is partly regulated by the $\mathrm{N}$-acylethanolamine (NAE) lipid oleoylethanolamide (OEA) [10]. OEA acts peripherally and causes a state of satiety accompanied by prolonged inter-meal intervals, reduced size of feedings, and increased fatty acid uptake via interaction with the peroxisome proliferator-activated receptor $\alpha$ (PPAR- $\alpha$ ) and the transient receptor potential cation channel vanilloid-1 (TRPV1), which stimulate the vagal nerve [11], thereby indirectly signaling satiety to the hypothalamic nuclei [12]. However, the involvement of the vagal nerve is debated [13], and delayed gastric emptying and intestinal transit are other mechanisms described; independent of both PPAR- $\alpha$ and TRPV1 [14].

It is well described that feeding (especially dietary fat) promotes or activates the endogenous formation of OEA in the enterocytes of the small intestine, but (at least in rodents) orally or systemic administered OEA has shown effects similar to those of endogenous OEA, i.e., inducing satiety [14]. Related NAE lipids such as stearoylethanolamide (SEA) and the anti-inflammatory palmitoylethanolamide (PEA) have shown appetite-reducing effects in animal models, though these findings are less consistent and the effects may be weaker than those of OEA [10,15-17].

Human milk is considered ideal and appropriate as the only food for the first six months of life [18], since it contains a variety of components essential for infant growth, development and well-being, e.g., vitamins, minerals, carbohydrates, amino acids and proteins, hormones, growth factors, and antimicrobial factors. In the late 1990s, OEA, SEA, PEA, and other NAE lipids were detected in both human and animal milk [19-21].

The fact that orally administered OEA (and to some extent SEA and PEA) exert some of the same effects as endogenous OEA raises the possibility that the presence of these lipids in human milk play a role in the regulation of appetite and food intake in breastfed infants. In the present study, we aimed to determine the concentration of OEA, SEA, and PEA in human milk samples collected four months postpartum and investigate possible associations between the levels and concurrent infant anthropometry and growth from birth. Our hypothesis was that the concentration of the NAE lipids OEA, SEA, and PEA in mother's milk would differ between infants being relatively heavy at the time of the milk sample collection (i.e., having a high WAZ) and infants being relatively light (i.e., having a low WAZ). 


\section{Materials and Methods}

\subsection{Participants and Milk Sample Collection}

The Odense Child Cohort is an unselected, prospective birth cohort comprising infants born in the municipality of Odense, Denmark [22]. From January 2010 to December 2012, pregnant women of gestation $<16$ weeks were invited to participate; the only exclusion criterion was emigration from the municipality of Odense before birth. From March 2012, inclusion was extended to 2.5 months postpartum, but the majority of participants were included during pregnancy. The study consists of self-administered questionnaires and physical examinations including collection of biological material (blood samples, fecal samples, etc.) at 4 and 18 months, 3, 5, and 7 years of age. Further questionnaires and examinations are planned (yet not initiated) at 9, 12, 15, and 18 years of age.

As part of the physical examination, anthropometric data are collected in terms of e.g., length, weight, triceps and subscapular skinfold thickness. Abdominal circumference was measured in $\mathrm{cm}$ (one decimal) using a measuring tape (seca 212, Seca, Hamburg, Germany). Weight was measured in $\mathrm{g}$ (no decimals) using an electronic baby scale (seca 717, Germany). Length was measured in $\mathrm{cm}$ (one decimal) using a baby measuring rod (seca 231, Germany). Triceps and subscapular skinfold thickness was measured in mm (one decimal) using a skinfold caliper (Harpenden Skinfold Caliper, Baty International, West Sussex, England); the measurement was repeated three times, and the arithmetic mean calculated. In addition, 13 children in 2012 and 7 children in 2013 were measured by two examiners to determine inter-observer agreement. WAZ, height-for-age Z-score (HAZ), BMI-for-age Z-score (BMIZ), and weight-for-height Z-score (WHZ) are calculated based on the WHO's 2006 standards using the official STATA module, zscore06 [23].

From April 2012 and onwards, mothers were given the opportunity to deliver a milk sample, when their infant was seen for the first physical examination at four months of age. The mothers were to express the sample in the clinic, and a total of $30 \mathrm{~mL}$ was requested, but less was accepted. All physical examinations and thereby sample deliveries were scheduled between 8 a.m. and 5 p.m. There were no requirements regarding the sample being fore or hind milk; the use of a breast pump or manual expression; or recording of time since last feeding.

Milk samples from mothers to singleton infants with the lowest $(n=50)$ and highest $(n=50)$ WAZ were included, median (IQR) WAZ $-0.67(-0.98,-0.54)$ and $1.52(1.31,1.84)$, respectively. Since no a priori data concerning differences in the levels of NAE lipids in human milk exist, a power calculation was not performed, but the sample size chosen was considered sufficient to detect robust changes of biological (as opposed to purely statistical) significance. A flowchart of the inclusion is shown in Figure 1.

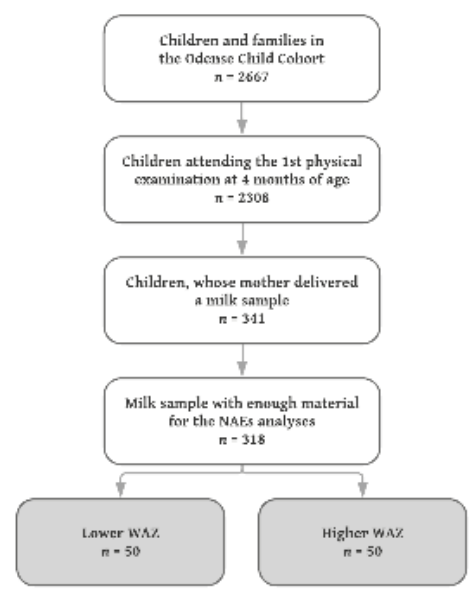

Figure 1. Flowchart of inclusion in the present study. WAZ: weight-for-age Z-score. 
Directly upon milk sample collection, the sample was split into $10 \mathrm{~mL}$ tubes $(100 \times 16 \mathrm{PP}$, Sarstedt, Nümbrecht, Germany) and stored at $5{ }^{\circ} \mathrm{C}$. Within a maximum of three days after delivery and if at least $10 \mathrm{~mL}$ had been delivered, macronutrient analysis regarding the content of protein, fat, lactose, and energy $(\mathrm{g} / 100 \mathrm{~mL}$ and $\mathrm{kJ} / 100 \mathrm{~mL}$, respectively) was performed (Miris HMA, Uppsala, Sweden); otherwise, macronutrient analysis was not prioritized. The remaining sample was centrifuged at $3600 \mathrm{rpm}$ and $21^{\circ} \mathrm{C}$ for $5 \mathrm{~min}$. (Eppendorf Centrifuge $5702 \mathrm{R}$, Eppendorf Corporate, Wesseling-Berzdorf, Germany). The resulting fat, skimmed, and solid fractions were manually aliquoted $\left(3.5 \mathrm{~mL}\right.$ transfer pipette, Sarstedt) into three different tubes $\left(3.6 \mathrm{~mL} \mathrm{Nunc}{ }^{\circledR}\right.$ CryoTubes ${ }^{\circledR}$, Thermo Fisher Scientific, Waltham, MA, USA) and stored at $-80{ }^{\circ} \mathrm{C}$. The skimmed fractions were shipped from Odense, Denmark to Umeå, Sweden on dry ice and remained frozen $\left(-80^{\circ} \mathrm{C}\right)$ upon arrival.

\subsection{Analysis and Quantification of NAE Lipids}

Analysis was performed according to a previously validated and published method [24] The samples had to be centrifuged prior to solid-phase extraction (SPE), since some particles were left in the suspension. In brief, the samples (spiked with $20 \mu \mathrm{L}$ internal standard (IS) solution at a concentration of $20 \mathrm{ng} / \mathrm{mL}$ for OEA-d4, SEA-d3, and PEA-d4) were applied to the SPE columns, and then washed by a solution of $5 \%$ methanol with $0.1 \%$ acetic acid. Afterwards, the metabolites were eluted using $2 \mathrm{~mL}$ of acetonitrile and $2 \mathrm{~mL}$ of methanol. Finally, the samples were dried using speed vacuum; reconstituted in $100 \mu \mathrm{L}$ of methanol, and spiked with $10 \mu \mathrm{L}$ of the recovery standard 12-[[(cyclohexylamino)carbonyl]amino]-dodecanoic acid (CUDA; $0.025 \mu \mathrm{g} / \mathrm{mL}$ ). An additional centrifugation was done with an Eppendorf tube filter.

The quantification was carried out by means of ultra-high performance liquid chromatographymass spectrometry (UPLC-MS). The system used was Agilent UPLC system (Infinity 1290) coupled with an electrospray ionization source (ESI) to an Agilent 6490 Triple Quadrupole system equipped with the iFunnel Technology (Agilent Technologies, Santa Clara, CA, USA), operating in positive mode. Metabolites separation was performed using a Waters BEH C18 column $(2.1 \times 150 \mathrm{~mm}, 130 \AA, 1.7 \mu \mathrm{m}$ particle size). A flow rate of $300 \mu \mathrm{L} / \mathrm{min}$. and $10 \mu \mathrm{L}$ injection volume were employed. The mobile phase consisted of (A) $0.1 \%$ acetic acid in MilliQ water and (B) acetonitrile:isopropanol (90:10), and the following gradient was used: $0.0-2.0 \mathrm{~min}, 30-45 \% \mathrm{~B} ; 2.0-2.5 \mathrm{~min}, 45-79 \% \mathrm{~B} ; 2.5-11.5 \mathrm{~min}, 79 \% \mathrm{~B}$; $11.5-12 \mathrm{~min}, 79-90 \%$ B; $12-14 \mathrm{~min}, 90 \%$ B; $14-14.5 \mathrm{~min}, 90-79 \%$ B; $14.5-15.5 \mathrm{~min}, 79 \% \mathrm{~B} ; 15.6-19 \mathrm{~min}$, $30 \%$ B. ESI applied conditions were optimized as described elsewhere [24]. The MassHunter Workstation software was used to control the instrument and to integrate all peaks manually.

The quantification was achieved by preparing a 10-point calibration curve using pure quantification standards. Furthermore, the recovery rates of each IS were calculated by adding the recovery standard (CUDA) to each sample. Using this technique, we were able robustly to quantify OEA, SEA, and PEA as well as other lower-abundance NAEs including the endocannabinoid anandamide (AEA). The related endocannabinoid 2-arachidonoylglycerol (2-AG), which belongs to the monoacylglycerol class of lipids, was also robustly measured. We decided not to present data on AEA and 2-AG due to their high degree of sensitivity to sample storage conditions including a rapid ex vivo synthesis and/or release from cells present in the samples prior to freezing $[20,25,26]$. The recovery rates (in \%) of each NAE were investigated using the IS, presented as mean \pm SD for low vs. high WAZ group respectively, $p$ values are for Welch's two-sample $t$-test: OEA, $44 \pm 20$ vs. $51 \pm 18$, $p=0.070$; SEA, $28 \pm 14$ vs. $33 \pm 13, p=0.073$; PEA, $33 \pm 16$ vs. $40 \pm 15, p=0.038$. As a consequence, the individual values reported in the following were corrected for recovery to negate this variability.

The following standards and internal standards were purchased from Cayman Chemicals (Ann Arbor, MI, USA); OEA, SEA, PEA, OEA-d4, SEA-d3, PEA-d4, and CUDA. Acetonitrile and methanol were purchased from Merck (Darmstadt, Germany), isopropanol from VWR PROLABO (Fontenay-sous-Bois, France), and acetic acid from Aldrich Chemical Company, Inc. (Milwaukee, WI, USA). All solvents and chemicals were of HPLC grade or higher. Water was purified by a Milli-Q 
Gradient system from Millipore (Milford, MA, USA), now Merck (Darmstadt, Germany). Oasis HLB cartridges (60 mg) were obtained from Waters (Milford, MA, USA).

\subsection{Ethics}

The study was approved by The Danish Data Protection Agency (ref. 12/26892), The Regional Committees on Health Research Ethics for Southern Denmark (ref. S-20090130, sub protocols 12, 18, and 37), and complied with the World Medical Association's Declaration of Helsinki II.

\subsection{Statistics}

Descriptive statistics were performed to describe the participating mother-infant dyads, categorized as either low or high WAZ. Continuous variables included were maternal pre-pregnancy BMI (termed $\mathrm{mBMI}, \mathrm{kg} / \mathrm{m}^{2}$ ), gestational age (days), birth weight $(\mathrm{g})$, infant weight at the time of milk sample collection (g), infant age at milk sample collection (weeks), and duration of exclusive breastfeeding (weeks), the latter based on weekly text message questions as recently described elsewhere [26]. Dichotomous or categorical variables were maternal educational level (three categories; low, intermediate, and high), maternal smoking (yes or no, the latter including those who stopped smoking during the first trimester), birth type (vaginal birth or Caesarean section), postdelivery parity (three categories; 1,2 , or $\geq 3$ ), sex (male or female), supplementation with infant formula within breastfeeding establishment; i.e., in the first few days after birth, but not necessarily later on (termed early infant formula, yes or no) [27], exclusive breastfeeding at the time of milk sample collection (yes or no) [27], and season at milk sample collection (either October-March or April-September). The latter was included, since we hypothesized that maternal dietary intake [28] and/or use of medications could differ between seasons, thereby influencing the NAE levels [29].

Maternal and infant baseline characteristics were compared using either two-sided $t$-test for normal distributed continuous variables; two-sample Kolmogorov-Smirnov test for not normal distributed continuous variables (comparing the cumulative distributions of data between the two WAZ groups); or Fisher's exact test for categorical variables. Normality of continuous variables were tested using Shapiro-Wilk W test.

To investigate the association between milk NAE concentration and WAZ group, a two-way ANOVA was run, examining the effect of WAZ group (low or high), NAE structure (OEA, SEA, or PEA, respectively), and the interaction WAZ group $\times$ NAE structure on the NAE levels. The ANOVA matched the NAE structures.

As an alternative approach, receiver operating characteristics (ROC) curves were constructed, and areas under the curve (AUC) were calculated to determine, whether or not the milk NAE concentration could discriminate the two WAZ groups. This approach is non-parametric in nature, therefore not sensitive to the (lack of) normality of the distribution. The AUC can range from 0.5 (no discriminatory power) to 1 (perfect discriminatory power) [30].

Finally, to investigate the correlations between the NAEs of interest and other milk components as well as maternal and infant characteristics, Spearman's correlation coefficients $\rho$ (rho) were calculated.

The inclusion of covariates in the adjusted analyses was based on the descriptive statistics, and covariates included were birth weight (data from $n=100)$, early infant formula $(n=81)$, and mBMI $(n=100)$.

As the primary inferential statistical analysis, associations between milk NAE concentration and WAZ groups were investigated using adjusted logistic regression analysis. As the secondary analysis, associations between milk NAE level and each of the following outcomes were investigated using adjusted linear regression analyses; abdominal circumference, weight, length, triceps skinfold thickness, subscapular skinfold thickness, WAZ, HAZ, WHZ, BMIZ, total weight gain since birth $(\Delta$ weight), weight gain per day ( $\Delta$ weight per day) since birth, and change from birth weight $Z$-score to WAZ at four months ( $\triangle \mathrm{WAZ}$ ). Lastly, stratification by sex was considered by comparing stratified results with overall results. 
Level of significance was set at $\alpha<0.05$. However, due to multiple testing and risk of false positives, a false discovery rate of $5 \%$ was pre-defined, and the Benjamini and Hochberg procedure was used to calculate the critical value of $p$ [31]. In consequence, and where appropriate, we have shown the unadjusted $p$ and indicated the critical value of $p$ in text, tables, and figures.

Descriptive and inferential statistics were conducted using STATA IC/15.1 (College Station, TX, USA), R Statistical Program vers. 3.4.1 (R Core Team, 2017), and GraphPad Prism 7.0b for Macintosh (GraphPad Software Inc., San Diego, CA, USA).

\section{Results}

\subsection{Participants}

An overview of maternal and infant characteristics across the two WAZ groups is shown in Table 1.

The two groups were well-matched regarding maternal educational level, smoking status, birth type, gestational age, postdelivery parity, infant sex, duration of exclusive breastfeeding, and - at the time of milk sample collection—infant age, season, and breastfeeding exclusivity. However, the two groups differed significantly regarding birth weight, mBMI, and number of infants supplemented with infant formula within breastfeeding establishment-i.e., early infant formula-but regarding the latter two, $p$-values were higher than the critical value of $p=0.008$, and should be considered in this light.

\subsection{NAES}

The individual values for OEA, SEA, and PEA are shown in the top row graphs in Figure 2. The upper panels show scatter plots (on a $\log _{10}$ scale) for the low and high WAZ groups (both $n=50$ ), with the mean of the logged values being indicated by the bars. $p$-values are for Welch's two-sample $t$-tests. The lower panels show the ROC analyses for each lipid with area under the curve (AUC), $95 \% \mathrm{CI}$, and $p$-values being given in each graph. There is a possible outlier for OEA, but the $P$-value remained significant $(0.035$, Welch's two-sample $t$-test) upon removal of this outlier. The critical value of $p$ at a false discovery rate of $5 \%$ was 0.05 .
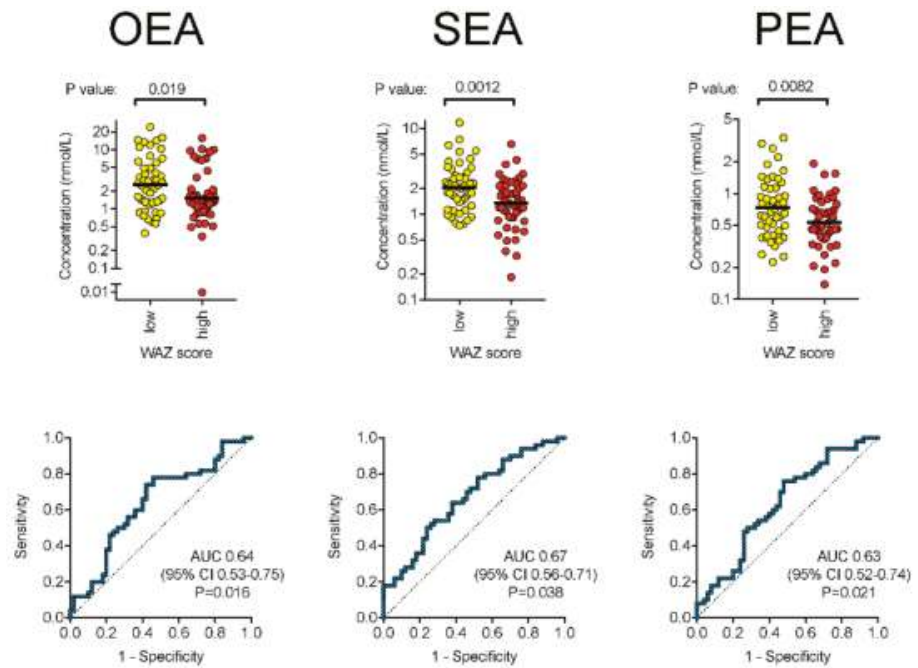

Figure 2. Oleoyl-(OEA), stearoyl-(SEA), and palmitoyl-(PEA) ethanolamine levels in milk samples. 
Table 1. Maternal and infant characteristics.

\begin{tabular}{|c|c|c|c|}
\hline & Lower WAZ & Higher WAZ & $p$ \\
\hline$N$ & 50 & 50 & \\
\hline \multicolumn{4}{|l|}{ Infant characteristics } \\
\hline \multicolumn{4}{|l|}{$\operatorname{Sex}^{1}, n(\%)$} \\
\hline female & $23(46)$ & $22(44)$ & \multirow{2}{*}{$>0.99$} \\
\hline male & $27(54)$ & $28(56)$ & \\
\hline Birth weight, $\mathrm{g}$ & $3253 \pm 544$ & $3894 \pm 487$ & $<0.001$ * \\
\hline Birth weight $Z$-score, SD & $-0.7 \pm 1.1$ & $0.6 \pm 1.1$ & $<0.001$ * \\
\hline Gestational age, days & $279(273-285)$ & $285(277-290)$ & $0.112^{2}$ \\
\hline \multicolumn{4}{|l|}{ Early infant formula ${ }^{3}, n(\%)$} \\
\hline yes & $7(14)$ & $15(30)$ & \multirow{3}{*}{0.048} \\
\hline no & $34(68)$ & $25(50)$ & \\
\hline unknown & $9(18)$ & $10(20)$ & \\
\hline Duration of exclusive breastfeeding, weeks & $17.0(11-19)$ & $19.0(4-22)$ & $0.105^{4}$ \\
\hline Infant age at time of milk sampling, weeks & $17.1 \pm 3.0$ & $17.3 \pm 3.3$ & 0.735 \\
\hline Infant weight at time of milk sampling, $\mathrm{g}$ & $6053(5770-6490)$ & $8140(7630-8565)$ & $<0.001 * 5$ \\
\hline \multicolumn{4}{|c|}{ Exclusive breastfeeding at time of milk sampling, $n(\%)$} \\
\hline yes & $17(34)$ & $15(30)$ & \multirow{3}{*}{0.076} \\
\hline no & $24(48)$ & $26(52)$ & \\
\hline unknown & $9(18)$ & $9(18)$ & \\
\hline \multicolumn{4}{|l|}{ Season at milk sampling, $n(\%)$} \\
\hline October-March & $19(38)$ & $21(42)$ & \multirow{2}{*}{0.838} \\
\hline April-September & $31(62)$ & $29(58)$ & \\
\hline \multicolumn{4}{|l|}{ Maternal characteristics } \\
\hline Maternal pre-pregnancy BMI (mBMI), kg/m² & $22.6(21.1-24.0)$ & $23.5(21.1-28.7)$ & $0.022^{6}$ \\
\hline \multicolumn{4}{|l|}{ Educational level ${ }^{7}, n(\%)$} \\
\hline low & $7(14)$ & $8(16)$ & \multirow{4}{*}{0.519} \\
\hline intermediate & $21(42)$ & $25(50)$ & \\
\hline high & $9(18)$ & $5(10)$ & \\
\hline unknown & $13(26)$ & $12(24)$ & \\
\hline \multicolumn{4}{|l|}{ Smoking status, $n(\%)$} \\
\hline no (or stopped during 1st trimester) & $50(100)$ & $49(98)$ & \multirow{2}{*}{$\mathrm{n} / \mathrm{a}$} \\
\hline unknown & $0(0)$ & $1(2)$ & \\
\hline \multicolumn{4}{|l|}{ Birth type, $n(\%)$} \\
\hline vaginal & $41(82)$ & $38(76)$ & \multirow{2}{*}{0.624} \\
\hline Caesarean section & $9(18)$ & $12(24)$ & \\
\hline \multicolumn{4}{|l|}{ Postdelivery parity, $n(\%)$} \\
\hline 1 & $25(50)$ & $22(44)$ & \multirow{3}{*}{0.457} \\
\hline 2 & $14(28)$ & $20(40)$ & \\
\hline$\geq 3$ & $11(22)$ & $8(16)$ & \\
\hline
\end{tabular}

Continuous variables are presented as mean \pm SD if normally distributed, otherwise as median (IQR); normality tested by Shapiro-Wilk W test. Statistical test used is two-sided $t$-test (unless otherwise stated) for continuous variables and Fisher's exact test for categorical variables. The critical value of $p$ at a false discovery rate of $5 \%$ was 0.008. $p$-values below this critical value are indicated with an asterisk. ${ }^{1}$ Two-way ANOVA stratifying for WAZ group and sex gave $p$ of $0.064,<0.001$, and 0.130 for the main effects of sex, WAZ group, and the interaction sex $\times$ WAZ group, respectively; ${ }^{2} p$ is for a two-sample Kolmogorov-Smirnov test for difference in distributions, since none of the distributions were normal; Shapiro-Wilk W test $p<0.001$ and $p=0.011$ for low and high WAZ, respectively; ${ }^{3}$ Supplementation with infant formula within breastfeeding establishment, i.e., first few days after birth; ${ }^{4} p$ is for two-sample Kolmogorov-Smirnov test; Shapiro-Wilk $W$ test $p=0.004$ and $p<0.001$ for low and high WAZ; ${ }^{5} p$ is for a two-sample Kolmogorov-Smirnov test; Shapiro-Wilk W test $p=0.039$ and $p=0.873$ for low and high WAZ; ${ }^{6} p$ is for a two-sample Kolmogorov-Smirnov test; Shapiro-Wilk W test $p=0.561$ and $p<0.001$ for low and high WAZ; ${ }^{7}$ Based on the highest, completed education; low = lower and upper secondary school or vocational education and training; intermediate = short-cycle higher education or medium-cycle higher education; high $=$ long-cycle higher education (i.e., university). 
The median (IQR) concentration was 1.54 (1.04-3.97) nmol/L for OEA, 1.7 (1.10-2.44) nmol/L for SEA, and $0.61(0.41-0.91) \mathrm{nmol} / \mathrm{L}$ for PEA. Due to non-normality of residuals, results were $\log _{10}$ transformed prior to statistical analysis and comparisons undertaken using the $\log _{10}$ means (i.e., corresponding to the geometric means of the untransformed data) as measures of central location. For all three NAE lipids, the mean concentration in the high WAZ group was significantly lower compared to the low WAZ group (all $p<0.02$, critical value of $p=0.05$ ). The mean values for the high group was on average $0.19 \log _{10}$ units lower, corresponding to a $35 \%$ lower geometric mean of the absolute values in the high versus low WAZ group.

In the two-way ANOVA matching for NAE structure, there was a significant main effect of WAZ group $(p=0.002)$ on the NAE concentration. The dataset failed Mauchly's test for sphericity; consequently Greenhouse-Geisser corrections were used, resulting in $p<0.001$ for the main effect of NAE structure, and $p=0.380$ for the interaction WAZ group $\times$ NAE structure. The lack of a significant interaction indicates that there is no evidence for different results for the different NAEs, i.e., the three NAE structures (OEA, SEA, and PEA) did not behave differently from each other.

The ROC curves were constructed for each NAE, and the area under the curve (AUC) was calculated. The data for the three lipids are shown in the lower row graphs in Figure 2. Unsurprisingly, the pattern of significance seen with the parametric $t$-tests was also seen in the ROC curves for all three NAEs.

Spearman's $\rho$ and the corresponding $p$-values for the 21 correlations obtained for each NAE are shown in Supplementary Figure S1. The three NAEs were highly correlated with one another, but did not correlate with macronutrients in the milk (fat, lactose, protein, total solid matter, or energy; data available for 62 of the 100 samples). In general, measures of infant growth were negatively correlated to the NAE levels, which are further detailed in the following section.

\subsection{NAEs and Infant Anthropometry and Growth}

The mean infant birth weight and the distribution of $\mathrm{mBMI}$ were significantly different between the two WAZ groups (Table 1). In order to investigate whether the difference in NAE concentrations between the low and high WAZ group was retained when these covariates were taken into account, multivariate logistic regressions were undertaken. For the whole dataset, the coefficients for $\log _{10}$ [SEA] and $\log _{10}[\mathrm{PEA}]$ were significant, as were the coefficients for $\mathrm{mBMI}$ and birth weight, whereas the coefficients for sex and the season of sampling (October-March vs. April-September) were not (Supplementary Table S1). In the case of OEA, the coefficient for $\log _{10}[\mathrm{OEA}]$ did not reach significance, and a similar $p$-value (0.084) was seen upon exclusion of the presumed outlier. However, the data for OEA is underpowered (explained in the next paragraph).

Supplementation with infant formula within breastfeeding establishment, i.e., early infant formula, also differed between the two groups. In this case, data were available for 81 of the 100 individuals. Given the smaller size of the dataset including this variable, we determined how robust the difference between the $\log _{10}[\mathrm{NAE}]$ concentrations (i.e., OEA, SEA, and PEA individually) in the two WAZ groups was at this sample size. This was undertaken by bootstrapping the data to generate 81 random samples from the 100 sample dataset, and then running a multivariate logistic regression analysis with $\log _{10}$ [NAE], mBMI, and birth weight as variables to see how often the coefficient for the $\log _{10}$ [NAE] was significant. Using 1000 iterations and the glm function available in $\mathrm{R}, p<0.05$ was seen in $32.6 \%$, $69.5 \%$, and $55.2 \%$ of the cases for OEA, SEA, and PEA, respectively. In consequence, we undertook the logistic regression analysis for the true dataset for 81 individuals to $\log _{10}$ [SEA] alone, since these data were the most robust with $p<0.05$ in $69.5 \%$ of the cases. The coefficient for $\log _{10}$ [SEA] remained significant, even when early infant formula was taken into account. The data are shown in Table 2. 
Table 2. Multivariate logistic regressions with higher WAZ as outcome.

\begin{tabular}{lccc}
\hline & Estimate & OR $(\mathbf{9 5} \% \mathrm{CI})$ & $p$ \\
\hline Complete dataset, $n=\mathbf{1 0 0}$ & & & \\
\hline $\log _{10}[\mathrm{SEA}]\left(\log _{10} \mathrm{pmol} / \mathrm{L}\right)$ & -2.82 & $0.06(0.01,0.50)$ & $0.009^{*}$ \\
birth weight $(\mathrm{g})$ & 0.00 & $1.00(1.00,1.00)$ & $<0.001^{*}$ \\
mBMI $\left(\mathrm{kg} / \mathrm{m}^{2}\right)$ & 0.21 & $1.24(1.05,1.45)$ & $0.009^{*}$ \\
\hline Reduced dataset; as above + information on early infant formula, $n=81$ \\
\hline $\log _{10}[\mathrm{SEA}]\left(\log _{10} \mathrm{pmol} / \mathrm{L}\right)$ & -3.49 & $0.03(0.00,0.51)$ & $0.015^{*}$ \\
birth weight $(\mathrm{g})$ & 0.00 & $1.00(1.00,1.00)$ & $0.002^{*}$ \\
mBMI $\left(\mathrm{kg} / \mathrm{m}^{2}\right)$ & 0.20 & $1.22(1.01,1.47)$ & $0.039^{*}$ \\
early infant formula ${ }^{1}$ & 0.88 & $2.42(0.52,11.30)$ & 0.261 \\
\hline
\end{tabular}

${ }^{1}$ Supplementation with infant formula within breastfeeding establishment, i.e., first few days after birth. The critical value of $p$ at a false discovery rate of $5 \%$ was 0.043 . $p$-values below this critical value are indicated with an asterisk.

Finally, concerning the exclusivity of breastfeeding at the time of sampling, i.e., if the infant was receiving any other food than mother's milk (e.g., infant formula or complementary foods), we speculated that exclusively breastfed infants were receiving more mother's milk than their partially breastfed counterparts. Information on breastfeeding exclusivity was available for 82 of the samples in the dataset, and even when including this in the logistic regression analysis, the coefficient for $\log _{10}[\mathrm{SEA}]$ was significant (OR $0.05,95 \% \mathrm{CI}=0.00,0.88, p=0.041$ ).

Due to insufficient amounts of sample material, macronutrient analysis was performed on 62 of the 100 milk samples. However, at this sample size, bootstrapping the data as described above, $p<0.05$ was found in only $29.1 \%, 57.3 \%$, and $43.2 \%$ of the 1000 iterations for OEA, SEA, and PEA, respectively, indicating that the sample size is underpowered for multivariate logistic regression analysis using macronutrients as covariates; and even with SEA, the analysis will be underpowered. In the actual 62 samples, the $\log _{10}$ [total NAE] concentrations in the two WAZ groups did not differ significantly $(p \geq 0.4)$.

As the secondary analysis, associations between $\log _{10}[\mathrm{SEA}]$ and other growth-related outcomes were investigated. Given the significant Spearman's correlation coefficients between the $\log _{10}$ [SEA] and several of these outcomes (Supplementary Figure S1), significant associations can be expected using a linear regression model. In the unadjusted analysis, $\log _{10}[\mathrm{SEA}]$ as the explanatory variable was inversely associated with several concurrent anthropometric measures; abdominal circumference in $\mathrm{cm}(\beta=-3.13, p=0.008)$, weight in $\mathrm{g}(\beta=-1.36, p=0.001)$, length in $\mathrm{cm}(\beta=-2.28, p=0.025)$, triceps skinfold thickness in mm $(\beta=-1.55, p=0.018), \operatorname{HAZ}(\beta=-1.10, p=0.006)$, WAZ $(\beta=-1.73$, $p<0.001)$, BMIZ ( $\beta=-1.51, p<0.001)$, WHZ ( $\beta=-1.40, p<0.001)$, total weight gain since birth in $\mathrm{g}$ $(\beta=-900.66, p=0.011)$, and weight gain per day in $\mathrm{g}(\beta=-6.66, p=0.010)$, but not to subscapular skinfold thickness in $\mathrm{mm}(\beta=-0.52, p=0.294)$; critical value of $p=0.045$. The pattern was retained for current weight, WAZ, WHZ, BMIZ, and total weight gain since birth (all $p<$ the critical value of $p=0.013)$ in the adjusted model including mBMI, birth weight, and early infant formula as covariates $(n=81)$. Triceps skinfold thickness $(p=0.016)$, subscapular skinfold thickness $(p=0.048)$, weight gain per day $(p=0.024)$, and change in WAZ ( $\triangle$ WAZ) since birth $(p=0.066)$ were not statistically significant according to the critical value of $p$, see Table 3 .

As for the latter, $\triangle \mathrm{WAZ}$, we did a post-hoc analysis, where children with a $\triangle \mathrm{WAZ}$ above the group mean were categorized as high weight gainers. We investigated the proportion of high weight gainers in the low and high WAZ group, respectively. Fourteen (28\%) of the low WAZ group were high weight gainers, i.e., had a $\triangle$ WAZ above the group mean, and $14(28 \%)$ of the high WAZ group had a $\triangle$ WAZ below the group mean. We believe this is partly responsible for the lack of significance regarding the observed associations between SEA and $\triangle \mathrm{WAZ}$. 
Table 3. Multivariate linear regressions for anthropometric and growth outcome measures.

\begin{tabular}{|c|c|c|c|c|c|}
\hline \multirow{2}{*}{$\begin{array}{c}\text { Explanatory Variables } \\
\text { Outcome Measure }\end{array}$} & \multicolumn{2}{|c|}{$\log _{10}[\mathrm{SEA}]\left(\log _{10} \mathrm{pmol} / \mathrm{L}\right)$} & \multirow{2}{*}{$\begin{array}{c}\mathrm{mBMI} \\
p\end{array}$} & \multirow{2}{*}{$\begin{array}{c}\text { Birth Weight } \\
p \\
\end{array}$} & \multirow{2}{*}{$\frac{{\text { Early } \mathrm{IF}^{1}}^{1}}{p}$} \\
\hline & $\beta(95 \% \mathrm{CI})$ & $p$ & & & \\
\hline Abdominal circumference $(\mathrm{cm})$ & $-2.28(-5.28,0.71)$ & 0.134 & 0.511 & $<0.001 *$ & 0.288 \\
\hline Weight at sampling $(\mathrm{g})$ & $-1.38(-2.35,-0.41)$ & $0.006^{*}$ & 0.098 & $<0.001 *$ & 0.201 \\
\hline Length at sampling $(\mathrm{cm})$ & $-1.28(-3.68,1.11)$ & 0.290 & 0.955 & $<0.001 *$ & 0.938 \\
\hline Triceps skinfold thickness (mm) & $-2.24(-4.04,-0.43)$ & 0.016 & 0.089 & 0.435 & 0.926 \\
\hline Subscapular skinfold thickness (mm) & $-1.27(-2.53,-0.01)$ & 0.048 & $0.009 *$ & 0.543 & 0.259 \\
\hline WAZ (SD) & $-1.56(-2.56,-0.56)$ & $0.003 *$ & 0.110 & $<0.001 *$ & 0.203 \\
\hline HAZ (SD) & $-0.37(-1.25,0.52)$ & 0.411 & 0.846 & $<0.001$ * & 0.784 \\
\hline WHZ (SD) & $-1.85(-2.85,-0.85)$ & $<0.001 *$ & 0.021 & 0.174 & 0.086 \\
\hline BMIZ (SD) & $-1.83(-2.85,-0.81)$ & $<0.001 *$ & 0.030 & 0.037 & 0.142 \\
\hline$\Delta$ weight since birth $(\mathrm{g})$ & $-1381(-2,350,-413)$ & $0.006^{*}$ & 0.098 & 0.698 & 0.201 \\
\hline$\Delta$ weight since birth per day $(\mathrm{g})$ & $-8.17(-15.26,-1.08)$ & 0.024 & 0.183 & 0.587 & 0.142 \\
\hline$\triangle$ WAZ since birth (SD) & $-1.04(-2.15,0.07)$ & 0.066 & 0.167 & $0.001 *$ & 0.320 \\
\hline
\end{tabular}

${ }^{1} \mathrm{IF}$ = infant formula; supplementation with infant formula within breastfeeding establishment, i.e., first few days after birth. Data shown are for the fully adjusted model including $\log 10[$ SEA], mBMI, birth weight, and early infant formula as explanatory variables $(n=81)$. Residual plots were acceptable in all cases. For the covariates included, only $p$ is shown (three last columns). The critical value of $p$ at a false discovery rate of $5 \%$ was 0.013 . $p$-values below this critical value are indicated with an asterisk.

\section{Discussion}

In the present study, we aimed to determine the concentration of NAE lipids-OEA, SEA, and PEA-in human milk samples collected at four months of age, and investigate associations to concurrent infant anthropometry as well as growth from birth. Mothers to infants with lower WAZ had a significant higher concentration of satiety factors OEA, PEA, and SEA in their milk compared to mothers to infants with higher WAZ. We observed significant inverse associations between NAE levels and anthropometric measures (in terms of weight, WAZ, WHZ, and BMIZ) at the time of milk sample collection, as well as growth from birth (in terms of total weight gain since birth), even after adjustment for possible confounders.

At the outset, it is important to consider the main strengths and weaknesses of the study. The strengths of the study are that the samples are from a well characterized cohort, the NAE analysis methodology is well validated, and the data are novel. The main weakness of the study is that the collection of the milk samples, collected as part of the cohort protocol, was not ideal for the present study.

With respect to the latter, most work on NAE and endocannabinoid stability and reproducibility of analysis has been undertaken in plasma samples, where levels of the related NAE (and endocannabinoid) AEA are very sensitive to ex vivo conditions due to release from intact cells in the samples [32]. In our hands, 45 min storage of plasma samples at $4{ }^{\circ} \mathrm{C}$ produced the expected increase in AEA levels, but also of OEA levels, whereas SEA levels were not affected [33]. The large variation in NAE concentrations between individuals is also seen in plasma [33,34]. In theory, such a large variation could be due to a measurement artefact. However, for SEA and OEA, measurement of plasma levels in separate batches by the current method gives a very high reproducibility [33], and in the case of AEA, the reproducibility between two different analysis methods is very high [34].

To our knowledge, only one study has investigated the stability of NAEs in human milk. The study group (which included two of us, S.G.-F. and M.D.) investigated NAE and oxylipin levels in three samples from the same mother stored for up to seven days prior to analysis [20]. Consistent with the blood plasma studies, levels of AEA and the related endocannabinoid 2-AG increased rapidly, i.e., a nearly five-fold increase was seen after one day of storage at $+4{ }^{\circ} \mathrm{C}$. OEA and PEA levels were not affected at this time point, but increased about five-fold after one week of storage, whilst SEA levels were about 2.5- and 3.5-fold higher after 1 and 7 days of storage, respectively. In contrast, the samples were stable for three months at $-80^{\circ} \mathrm{C}$. This was a small study on few milk samples from the same mother, but it raises the caveat that the observed changes in the present study could simply be due to differences in the storage times of the milk samples from the two groups. A simple linear regression of our $\log _{10}$ [NAE] data vs. days at $+4{ }^{\circ} \mathrm{C}$ from the mean values published by Wu et al. [19] gave slope 
values of $0.11,0.06$, and $0.11 \log _{10}[\mathrm{pmol} / \mathrm{L}]$ per day for OEA, SEA, and PEA, respectively. Although extremely approximate, this suggests that the observed $\log _{10}[\mathrm{nmol} / \mathrm{L}]$ differences between the low and high WAZ group $(0.19,0.23$, and 0.14 for OEA, SEA, and PEA, respectively) observed in the present study would require differences in storage times at $+4{ }^{\circ} \mathrm{C}$ of $\sim 2, \sim 3$, and $\sim 1$ days for OEA, SEA, and PEA, respectively, if explained solely by the sampling conditions. Such large differences in sampling times, particularly for SEA, are highly unlikely. Furthermore, if the abovementioned differences were due to differences in storage time, this would require a systematic error, e.g., where samples from mothers to lower WAZ infants were stored for a longer time than samples from mothers to higher WAZ infants. This is not likely, since the mother-infant dyads attended the physical examination (and thereby the milk sample collection) simply ordered by their cohort identification number.

A second criticism of the present study is that milk sample collection was at a single time-point. However, in a recent study published in this journal, OEA and PEA levels did not significantly differ from two to four weeks postpartum $(n=24)$. In that study, milk sample collection was standardized; two hours of fasting prior to expression, full expression of one breast using an electronic breast pump between 6 a.m. and 10 a.m. Levels of OEA and PEA were of the same order of magnitude as in our study, but levels of SEA were not reported [35]. To our knowledge, no studies have examined the circadian variation of the concentration of NAEs in human milk, which could be another issue [36].

The primary finding in the present study is the demonstration of an inverse association between the NAE levels and the WAZ groups, i.e., higher NAE level is associated to lower WAZ. NAEs are synthesized from $N$-acylphosphatidylethanolamines (NAPEs) through the $\mathrm{N}$-acyltransferase/ $\mathrm{N}$-acylphosphatidylethanolamine-hydrolyzing phospholipase D (NAPE-PLD) pathway $[17,37]$. The fact that the three NAE levels are highly correlated with each other and that there was no significant interaction between WAZ group and NAE structure in the analyses, suggests that the association may reflect a difference in the catalytic activity of one of the key enzymes responsible for the production of NAEs in milk, although this suggestion requires further investigation in future studies. Nonetheless, the consequence affects the levels and thereby the biological activities of the NAEs, as further examined below.

As stated in the Section 1, OEA—the more studied NAE-leads to prolonged inter-meal intervals, reduced size of feedings, delayed gastric emptying and intestinal transit, increased satiety through an increased fatty acid uptake and a higher oxidation rate. Some controversies exist regarding the intestinal response, since some reports indicate that feeding (especially dietary fat) activates the endogenous formation of OEA in the enterocytes of the small intestine [14], whereas others report a decrease in the small intestinal levels of OEA, PEA, and linoleoylethanolamide (LEA) in a timeand dose-dependent manner by dietary fat [13]. In addition, some indications exist, that CD36 (fatty acid translocase) gene polymorphisms correlate to plasma lipid level variations, i.e., CD36 gene polymorphisms lead to OEA synthesis inability, thereby being more prone to developing metabolic syndrome including obesity [38]. This is supported by the finding that OEA reduces plasma cholesterol and triglyceride levels in rodents [11]. OEA is considered a functional AEA antagonist, suppressing the appetite by stimulating satiety without altering the total motor activity [38], level of anxiety, alertness, or stress [12]. Other mechanisms described are the binding of OEA to glucagon-like peptide 1 (GLP-1), thereby increasing the anorectic properties of GLP-1 [12], but again some discrepancy exists, and others report that satiety signals like GLP-1, ghrelin, cholecystokinin (CKK), and peptide Y (PYY) are-in animal models-not affected by OEA [11].

SEA has been reported to inhibit food intake by downregulating the gene expression of a specific liver enzyme, stearoyl-coenzyme A desaturase-1 (SCD-1) [8]. SCD-1 is involved in the synthesis of monounsaturated fatty acids. SCD-1 deficient mice are lean and hypermetabolic, and leptin-deficient obese mice are significantly less obese when crossed with mice carrying a SCD-1 mutation. It has been suggested that downregulation of SCD-1 is an important component of the anorectic effect of leptin [16]. Orally administered SEA did not result in changes in serum glucose or serum leptin levels, 
and the degradation products of SEA (ethanolamine and stearic acid) are inactive in reducing food intake, as was also the case with OEA and its' degradation products [16].

PEA is primarily known for its anti-inflammatory effects [36]. Regarding an anorectic effect, findings are conflicting, but it is probable, that PEA has a less potent effect than OEA [17]. Interestingly, in clinical studies investigating the analgesic effects of PEA, weight loss (as a side effect) was not reported [13].

In our study, the geometric mean OEA and SEA concentrations found in the milk samples were $2.6 \mathrm{nmol} / \mathrm{L}$ and $2.1 \mathrm{nmol} / \mathrm{L}$, respectively, in the low; and $1.5 \mathrm{nmol} / \mathrm{L}$ and $1.3 \mathrm{nmol} / \mathrm{L}$, respectively, in the high WAZ group. Compared to the concentrations used in the animal experiments (see above), these concentrations are modest. Assuming an exclusively breastfed infant, the average daily milk intake at four months of age would be approx. 100-120 mL/kg, corresponding to a mean intake of $660 \mathrm{~mL}$ in the low and $890 \mathrm{~mL}$ in the high WAZ group. The total intake of OEA would be $\sim 1.7 \mathrm{nmol}$ in the low and $\sim 1.4 \mathrm{nmol}$ in high WAZ group, respectively, and the total intake of SEA would be $\sim 1.4 \mathrm{nmol}$ in the low and $\sim 1.2 \mathrm{nmol}$ in the high WAZ group, respectively. Since gastrointestinal epithelial cells express both the NAE hydrolytic enzymes NAAH and FAAH, they are well equipped to metabolize NAEs $[39,40]$. As a consequence, biological effects exerted by NAEs in human milk would presumably have to be mediated directly upon ingestion, rather than following absorption. If it is assumed, for the sake of argument, that the rather low levels in the present study are sufficient to evoke satiety responses in the infants, then these data are consistent with the speculation that infants adjust their intake of milk to the OEA and/or SEA satiety signals.

Finally, it is possible, that the NAEs are not active per se, but simply reflect the pattern of another (unknown) milk component, i.e., being a surrogate marker or variable. Clearly, more work is needed to establish the biological importance of NAEs in human milk.

\section{Conclusions}

In conclusion, the detection of appetite regulators (in this case NAE lipids) in human milk is not a novel finding, but-to our knowledge-this is the largest study investigating the concentration of satiety factors OEA, SEA, and PEA in human milk samples and associations to offspring anthropometry and growth. Based on human milk samples collected at four months of age, we observed statistically significant differences in the concentrations between mothers to infants with a low WAZ and mothers to infants with a high WAZ at time of the milk sample collection. The low WAZ group had a higher concentration of satiety factors OEA, PEA, and SEA compared to the high WAZ group, and even after adjustment for maternal pre-pregnancy BMI, birth weight, and supplementation with infant formula within breastfeeding establishment, a lower concentration of OEA, SEA, and PEA was associated with a higher weight gain since birth. These findings are of great interest in the research area of human milk, appetite regulation, and infant growth, and they could represent another piece of the puzzle of the functions of human milk. In future studies testing the reproducibility of our findings, we emphasize that milk samples should be processed immediately upon collection due to the pronounced ex vivo instability of these compounds.

Supplementary Materials: The following are available online at http://www.mdpi.com/2072-6643/10/11/1747/s1, Figure S1: Correlations between OEA, SEA, and PEA and maternal and infant characteristics; Table S1: Logistic regressions for $\log _{10}[\mathrm{NAE}]$ with higher WAZ as outcome.

Author Contributions: Conceptualization and methodology, S.B., S.G.-F., M.D., L.N.J., C.J.F., and G.Z.; Software, data curation, formal analysis, and validation, S.B., S.G.-F., and C.J.F.; Writing-original draft preparation, S.B., S.G.-F., and C.J.F.; Writing-review and editing, S.B., S.G.-F., M.D., S.H., L.N.J., K.F.M., C.J.F., and G.Z.; Visualization, S.B. and C.J.F.; Supervision, S.G.-F., M.D., S.H., L.N.J., K.F.M., C.J.F., and G.Z.; Project administration, S.B., S.H., L.N.J., K.F.M., and G.Z.; Funding acquisition, S.H., L.N.J., K.F.M., and G.Z.

Funding: The study was funded by Arla Foods Ingredients Group P/S, and is part of an industrial PhD funded by Arla Foods Ingredients Group P/S and Innovation Fund Denmark. 
Acknowledgments: Thanks to all participating children and their families as well as the employees at the Odense Child Cohort. Special thanks to scientific laboratory technician Mette Vogn Hviid for helping with the collection and processing of the milk samples. Finally, The Swedish Metabolomics Centre (www.swedishmetabolomicscentre. se) is acknowledged for valuable assistance with the UPLC-MS analyses.

Conflicts of Interest: As stated in the Affiliations, S.B. and L.N.J. are employees at Arla Foods Ingredients Group $\mathrm{P} / \mathrm{S}$, from where the present study is partially financed as an industrial PhD project. The other authors report no conflicts of interest.

\section{References}

1. World Health Organization: Fact Sheet on Obesity and Overweight. Available online: http://www.who.int/ mediacentre/factsheets/fs311/en/ (accessed on 20 March 2018).

2. Goodell, L.S.; Wakefield, D.B.; Ferris, A.M. Rapid weight gain during the first year of life predicts obesity in 2-3 year olds from a low-income, minority population. J. Community Health 2009, 34, 370-375. [CrossRef] [PubMed]

3. Ong, K.K.; Ahmed, M.L.; Emmett, P.M.; Preece, M.A.; Dunger, D.B. Association between postnatal catch-up growth and obesity in childhood: Prospective cohort study. BMJ 2000, 320, 967-971. [CrossRef] [PubMed]

4. Baird, J.; Fisher, D.; Lucas, P.; Kleijnen, J.; Roberts, H.; Law, C. Being big or growing fast: Systematic review of size and growth in infancy and later obesity. BMJ 2005, 331, 929. [CrossRef] [PubMed]

5. Zheng, M.; Lamb, K.E.; Grimes, C.; Laws, R.; Bolton, K.; Ong, K.K.; Campbell, K. Rapid weight gain during infancy and subsequent adiposity: A systematic review and meta-analysis of evidence. Obes. Rev. 2018, 19, 321-332. [CrossRef] [PubMed]

6. Péneau, S.; Giudici, K.V.; Gusto, G.; Goxe, D.; Lantieri, O.; Hercberg, S.; Rolland-Cachera, M.-F. Growth trajectories of body mass index during childhood: Associated factors and health outcome at adulthood. J. Pediatr. 2017, 186, 64-71. [CrossRef] [PubMed]

7. Freitas, H.R.; Isaac, A.R.; Malcher-Lopez, R.; Diaz, B.L.; Trevenzoli, I.H.; Reis, R.A.D.M. Polyunsaturated fatty acids and endocannabinoids in health and disease. Nutr. Neurosci. 2017, 7, 1-20. [CrossRef] [PubMed]

8. Huang, L.; Toyoshima, M.; Asakawa, A.; Inoue, K.; Harada, K.; Kinoshita, T.; Chen, S.; Koizumi, A. Levels of N-acylethanolamines in O,O,S-trimethylphosporothioate (OOS-TMP)-treated C57BL/6J mice and potential anti-obesity, anti-diabetic effects of OOS-TMP in hyperphagia and hyperglycemia mouse models. Pharmacol. Biochem. Behav. 2009, 92, 1-5. [CrossRef] [PubMed]

9. Lizarbe, B.; Benitez, A.; Paláez Brioso, G.A.; Sánchez-Montañes, M.; López-Larrubia, P.; Ballesteros, P.; Cerdán, S. Hypothalamic metabolic compartmentation during appetite regulation as revealed by magnetic resonance imaging and spectroscopy methods. Front. Neuroenerg. 2013, 5, 6. [CrossRef] [PubMed]

10. Witkamp, R.F. The role of fatty acids and their endocannabinoid-like derivatives in the molecular regulation of appetite. Mol. Asp. Med. 2018. [CrossRef] [PubMed]

11. Thabuis, C.; Tissot-Favre, D.; Bezelgues, J.-B.; Martin, J.-C.; Cruz-Hernandez, C.; Dionisi, F.; Destaillats, F. Biological functions and metabolism of oleoylethanolamide. Lipids 2008, 43, 887-894. [CrossRef] [PubMed]

12. Provensi, G.; Coccurello, R.; Umehara, H.; Munari, L.; Giacovazzo, G.; Galeotti, N.; Nosi, D.; Gaetani, S.; Romano, A.; Moles, A.; et al. Satiety factor oleoylethanolamide recruits the brain histaminergic system to inhibit food intake. Proc. Natl. Acad. Sci. USA 2014, 111, 11527-11532. [CrossRef] [PubMed]

13. Hansen, H.S.; Vana, V. Non-endocannabinoid N-acylethanolamines and 2-monoacylglycerols in the intestine. Br. J. Pharmacol. 2018. [CrossRef] [PubMed]

14. Piomelli, D. A fatty gut feeling. Trends Endocrinol. Metab. 2013, 24, 332-341. [CrossRef] [PubMed]

15. Bowen, K.J.; Kris-Etherton, P.M.; Shearer, G.C.; West, S.G.; Reddivari, L.; Jones, P.J.H. Oleic acid-derived oleoylethanolamide: A nutritional science perspective. Prog. Lipid Res. 2017, 67, 1-15. [CrossRef] [PubMed]

16. Terrazzino, S.; Berto, F.; Dalle Carbonare, M.; Fabris, M.; Guiotto, A.; Bernardini, D.; Leon, A. Stearoylethanolamide exerts anorexic effects in mice via down-regulation of liver stearoyl-coenzyme A desaturase-1 mRNA expression. FASEB J. 2004, 18, 1580-1582. [CrossRef] [PubMed]

17. Hansen, H.S. Role of anorectic N-acylethanolamines in intestinal physiology and satiety control with respect to dietary fat. Pharmacol. Res. 2014, 86, 18-25. [CrossRef] [PubMed]

18. World Health Organization: Breastfeeding. Available online: http://www.who.int/topics/breastfeeding/ en/ (accessed on 22 March 2018). 
19. Di Marzo, V.; Sepe, N.; De Petrocellis, L.; Berger, A.; Crozier, G.; Fride, E.; Mechoulam, R. Trick or treat from food endocannabinoids? Nature 1998, 396, 636-637. [CrossRef] [PubMed]

20. Wu, J.; Gouveia-Figueira, S.; Domellöf, M.; Zivkovic, A.M.; Nording, M.L. Oxylipins, endocannabinoids, and related compounds in human milk: Levels and effects of storage conditions. Prostaglandins Other Lipid Mediat. 2016, 122, 28-36. [CrossRef] [PubMed]

21. Gouveia-Figueira, S.; Nording, M.L. Development and validation of a sensitive UPLC-ESI-MS/MS method for the simultaneous quantification of 15 endocannabinoids and related componds in milk and other biofluids. Anal. Chem. 2014, 86, 1186-1195. [CrossRef] [PubMed]

22. Kyhl, H.B.; Jensen, T.K.; Barington, T.; Buhl, S.; Norberg, L.A.; Jørgensen, J.S.; Jensen, D.F.; Christesen, H.T.; Lamont, R.F.; Husby, S. The Odense Child Cohort: Aims, design, and cohort profile. Paediatr. Perinat. Epidemiol. 2015, 29, 250-258. [CrossRef] [PubMed]

23. Leroy, J.L. Zscore06: Stata Command for the Calculation of Anthropometric z-Scores Using the 2006 WHO Child Growth Standards. 2011. Available online: http://fmwww.bc.edu/RePEc/bocode/z/zscore06 (accessed on 1 February 2016).

24. Gouveia-Figueira, S.; Nording, M.L. Validation of a tandem mass spectrometry method using combined extraction of 37 oxylipins and 14 endocannabinoid-related compounds including prostamides from biological matrices. Prostaglandins Other Lipid Mediat. 2015, 121 Pt A, 110-121. [CrossRef]

25. Gabrielsson, L.; Mattsson, S.; Fowler, C.J. Palmitoylethanolamide for the treatment of pain: Pharmacokinetics, safety and efficacy. Br. J. Clin. Pharmacol. 2016, 82, 932-942. [CrossRef] [PubMed]

26. Ueda, N.; Tsuboi, K.; Uyama, T. Metabolism of endocannabinoids and related N-acylethanolamines: Canonical and alternative pathways. FEBS J. 2013, 280, 1874-1984. [CrossRef] [PubMed]

27. Bruun, S.; Buhl, S.; Husby, S.; Jacobsen, L.N.; Michaelsen, K.F.; Sørensen, J.; Zachariassen, G. Breastfeeding, infant formula, and introduction to complementary foods-Comparing data obtained by questionnaires and health visitors' reports to weekly short message service text messages. Breastfeed Med. 2017, 12, 554-560. [CrossRef] [PubMed]

28. Við Streym, S.; Højskov, C.S.; Møller, U.K.; Heickendorff, L.; Vestergaard, P.; Mosekilde, L.; Rejnmark, L. Vitamin D content in human breast milk: A 9-mo follow-up study. Am. J. Clin. Nutr. 2016, 103, 107-114. [CrossRef] [PubMed]

29. Fowler, C.J.; Tiger, G.; Stenström, A. Ibuprofen inhibits rat brain deamidation of anandamide at pharmacologically relevant concentrations. Mode of inhibition and structure-activity relationship. J. Pharmacol. Exp. Ther. 1997, 283, 729-734. [PubMed]

30. Grzybowski, M.; Younger, J.G. Statistical methodology: III. Receiver operating characteristics (ROC) curves. Acad. Emerg. Med. 1997, 4, 818-826. [CrossRef] [PubMed]

31. Benjamini, Y.; Hochberg, Y. Controlling the false discovery rate: A practical and powerful approach to multiple testing. J. R. Stat. Soc. Ser. B Stat. Methodol. 1995, 57, 289-300.

32. Vogeser, M.; Hauer, D.; Shahnaz, C.A.; Huber, E.; Storr, M.; Schelling, G. Release of anandamide from blood cells. Clin. Chem. Lab. Med. 2006, 44, 488-491. [CrossRef] [PubMed]

33. Gouveia-Figueira, S.; Goldin, K.; Hashemian, S.A.; Lindberg, A.; Persson, M.; Nording, M.L.; Laurell, K.; Fowler, C.J. Plasma levels of the endocannabinoid anandamide, related $\mathrm{N}$-acylethanolamines and linoleic acid-derived oxylipins in patients with migraine. Prostaglandins Leukot. Essent. Fat. Acids 2017, 120, 15-24. [CrossRef] [PubMed]

34. Marczylo, T.H.; Lam, P.M.; Nallendran, V.; Taylor, A.H.; Konje, J.C. A solid-phase method for the extraction and measurement of anandamide from multiple human biomatrices. Anal. Biochem. 2009, 384, 106-113. [CrossRef] [PubMed]

35. Gaitán, A.V.; Wood, J.T.; Zhang, F.; Makriyannis, A.; Lammi-Keefe, C.J. Endocannabinoid metabolome characterization of transitional and mature human milk. Nutrients 2018, 10. [CrossRef] [PubMed]

36. White, R.D. Circadian variation of breast milk components and implications for care. Breastfeed Med. 2017, 12, 398-400. [CrossRef] [PubMed]

37. Gillum, M.P.; Zhang, D.; Zhang, X.M.; Erion, D.M.; Jamison, R.A.; Choi, C.; Dong, J.; Shanabrough, M.; Duenas, H.R.; Frederik, D.W.; et al. N-acylphospatidylethanolamine, a gut-derived circulating factor induced by fat ingestion, inhibits food intake. Cell 2008, 135, 813-824. [CrossRef] [PubMed] 
38. Sihag, J.; Jones, P.J.H. Oleoylethanolamide: The role of a bioactive lipid amide in modulating eating behaviour. Obes. Rev. 2018, 19, 178-197. [CrossRef] [PubMed]

39. Alhouayek, M.; Bottemanne, P.; Subramanian, K.V.; Lambert, D.M.; Makriyannis, A.; Cani, P.D.; Muccioli, G.G. $\mathrm{N}$-Acylethanolamine-hydrolyzing acid amidase inhibition increases colon N-palmitoylethanolamine levels and counteracts murine colitis. FASEB J. 2015, 29, 650-661. [CrossRef] [PubMed]

40. Alhouayek, M.; Rankin, L.; Gouveia-Figueira, S.; Fowler, C.J. Interferon $\gamma$ treatment increases endocannabinoid and related $\mathrm{N}$-acylethanolamine levels in T84 human colon carcinoma cells. Br. J. Pharmacol. 2018. [CrossRef] [PubMed]

(C) 2018 by the authors. Licensee MDPI, Basel, Switzerland. This article is an open access article distributed under the terms and conditions of the Creative Commons Attribution (CC BY) license (http:/ / creativecommons.org/licenses/by/4.0/). 
MDPI

St. Alban-Anlage 66

4052 Basel

Switzerland

Tel. +41616837734

Fax +41 613028918

www.mdpi.com

Nutrients Editorial Office

E-mail: nutrients@mdpi.com

www.mdpi.com/journal/nutrients

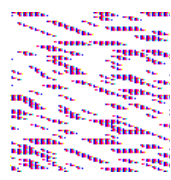



MDPI

St. Alban-Anlage 66

4052 Basel

Switzerland

Tel: +41 616837734

Fax: +41 613028918

www.mdpi.com 